(1)

8. 18 3.

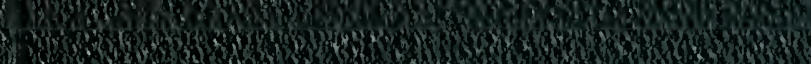

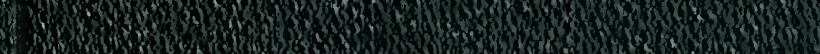
H.

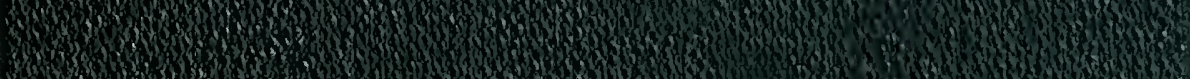

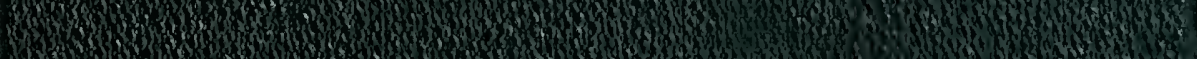

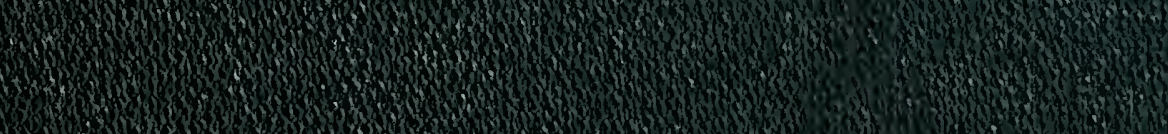

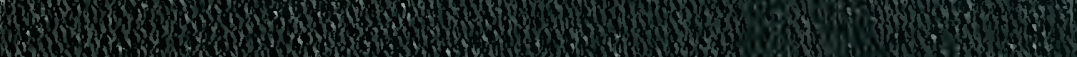

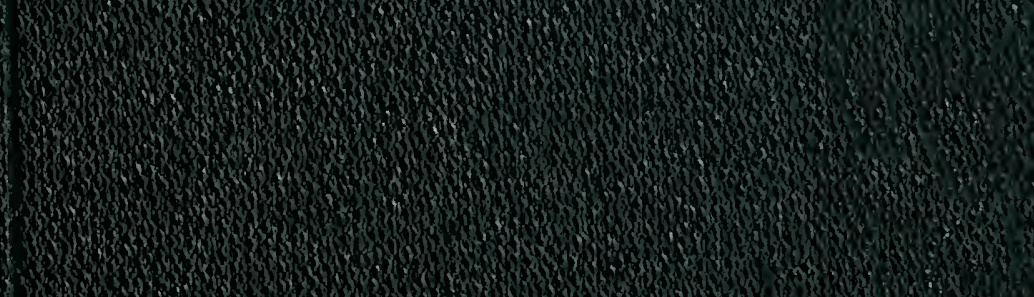






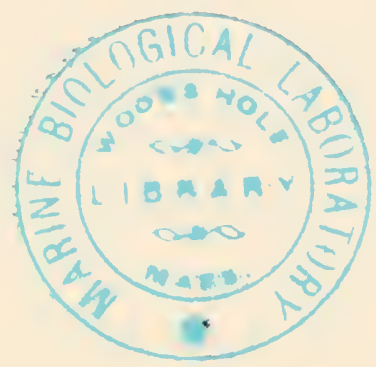

A TEXT-BOOK OF ZOOLOGY 


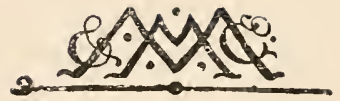

MAC'MII.L.IN AND CO., LIMUTED

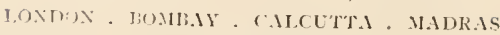
MILDERTENE

TII: MICMILL.IN COMPINY

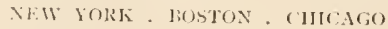

1).ILI.IS S SIN FRIXCISCO

THE MACMULAN CO. OF C.INADA LID.

TORONIO 


\section{A TEXT-BOOK OF ZOOLOGY}

BY THE LATE

T. JEFFERY PARKER, D.Sc,, F.R.S. PROFESSOR OF BIOLOGY IN THE UNIVERSITY OF OTAGO, DUNEDIN, N.z.

$$
\text { AND }
$$

WILLIAM A. HASWELL, M.A., D.Sc., F.R.S. EMERITUS PROFESSOR OF BIOLOGY IN THE UNIVERITY OF SYDNEY, N.S.W.

IN TWO VOLUMES VOL. II

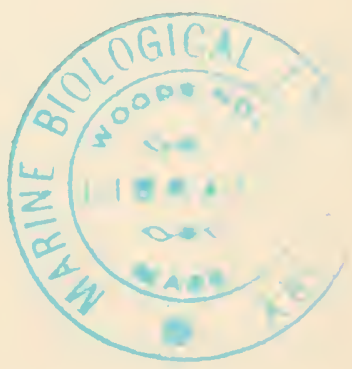

WITH ILLUSTRATIONS

MACMILLAN AND CO., LIMITED ST. MARTIN'S STREET, LONDON 


\section{COPYRIGHT}

First Edtition, 1898.

Second Edition, 1910

Third Edition, 1921 


\title{
CONTENTS
}

\author{
SECTION XIII
}

PAGE

Phylum Chordata . . . . . . . . . . . . . 1

Sub-phylum and Class I. Hemichorda (Adelochorda) . . . . . 2

Sub-phylum and Class II. Urochorda. $\quad . \quad$. $\quad . \quad$. $\quad . \quad 13$

1. Example of the Class-Ascidia . . . . . . . . 14

¿. Distinctive Characters and Classification. . . . . . 20

Systematic Position of the Example. . . . . . . 23

3. General Organisation . . . . . . . . . . 23

Sub-phylum III. Euchorda . . . . . . . . . . 42

Section I. ACRANTA . . . . . . . . . 43

Section II. Crantata . . . . . . . . . . . 63

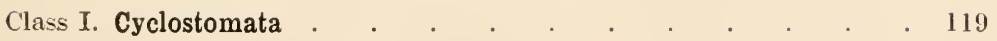

1. Example of the Class-Petromyzon . . . . . . . . 119

2. Distinctive Characters and Classification . . . . . . 133

3. Comparison of the Myxinoids with the Lamprey . . . . 134

4. Ceneral Remarks . . . . . . . . . . . 138

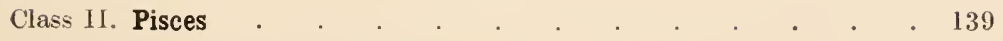

Sub-class I. Elasmobranchii . . . . . . . . . 140

1. Example of the'Sub-class-Scyllium canicula or Hemiscyllium modestum . . . . . . . . . . . . 140

2. Distinctive Characters and Classification . . . . . . 161

3. General Organisation . . . . . . . . 165 
Phylum Chordata-continued.

Class II. Pisces-continued.

Sub-class II. Holocephali 182

Sub-class III. Teleostomi

190

1. Example of the Sub-class - Salmo fario

2. Distinctive Characters and Classification Systematic Position of the Example

3. General Organisation

Sub-class IV. Dipnoi.

1. Example of the Class-Ceratodus (Neoceratodus or Epicera. todus) forsteri

2. Distinctive Characters and Classification . . . . . 249

3. General Remarks . . . . . . . . . . 250

Appendix to Pisces-Ostracodermi . . . . . . . 253

Class III. Amphibia . . . . . . . . . 256

1. Example of the Class-Rana temporaria or Rana esculenta . $\quad 257$

2. Distinctv e Characters and Classification . . . . . 283 Systematic Position of the Example. . . . . . . . 285

3. General Organisation . . . . . . . . . . 285

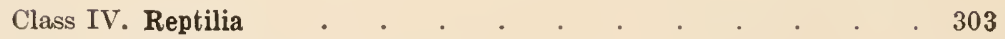

1. Example of the Class-Lacerta . . . . . . . . 304

2. Distinctive Characters and Classification . . . . . 323 Systematic Pasition of the Example. . . . . . . 327

3. General Organisation of Recent Reptilia . . . . . . 327

4. Extinct Groups of Reptiles. . . . . . . . 360

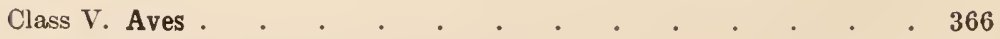

1. Example of the Class-Columba livia . . . . . . 367

2. Distinctive Characters and Classification . . . . 395 Systematic Position of the Example. . . . . . . 404

3. General Organisation . . . . . . . . . 405 Sub-class I. Archæornithes . . . . . . . . 405 II. Neornithes . . . . . . . . . 407 
Phylum Chordata-continued.

PAGE

Class VI. Mammalia . . . . . . . . . . 432

1. Example of the Class-Lepus cuniculus . . . . . . 432

2. Distinctive Characters and Classification of Recent Mammalia . 462

Sub-class I. Prototheria . . . . . . . . . 464

" II. Theria . . . . . . . . . . 464

Systematic Position of the Example. . . . . . . . 474

3. General Organisation . . . . . . . . . . 474

The Mutual Relationships of the Chordata . . . . . . 590

On the Mutual Relations of the Phyla of Animals . . . . . . 596

\section{SECTION XIV}

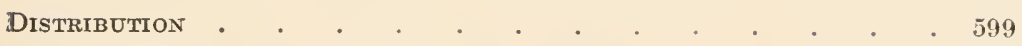

1. Goographical Distribution . . . . . . . . . 599

2. Bathymetrical Distribution . . . . . . . . 614

3. Geological Distribution . . . . . . . . . . 618

\section{SECTION XV}

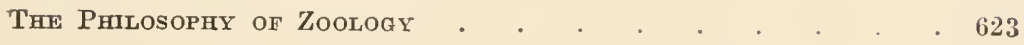

\section{SECTION XVI}

The History of Zoologx . . . . . . . . . . . . 647

APPENDIX-Zoological Literature . . . . . . . . 671

$\operatorname{INDEX} \quad . \quad . \quad . \quad . \quad . \quad . \quad . \quad .679$ 



\title{
LIST OF ILLUSTRATIONS
}

\author{
VOL. II
}

FIG.

PAGE

714. Balanoglossus . . . . . . . . . . . . . . . . 3

715.

anterior end . . . . . . . . . 4

716. Ptychodera bahamensis . . . . . . . . . . . . 5

717. Balanoglossus, development . . . . . . . . . . . . . 7

718. Tomaria . . . . . . . . . . . . . . . 7

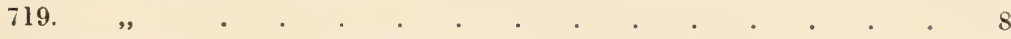

720. Cephalodiscus dodecalophus, gelatinous investment . . . $\quad 9$

721 .

722. zooid . . . . . . 10

sagittal section $\quad \cdot \ldots+1$

723. Rhabdopleura . . . . . . . . . . . . 12

724. Ascidia . . . . . . . . . . . . . 14

725., anatomy . . . . . . . . . . . . . . . . . 15

726., mesh of branchial sac . . . . . . . . 16

727. , diagrammatic longitudinal section . . . . . . 17

728. ,

729. , dorsal tubercle, ganglion, and associated parts . . $\quad 19$

730. ", sagittal and transverse sections . . . . . . . 20

731. Oikopleura. . . . . . . . . . . . . . . . . 23

732. Appendicularia, diagram . . . . . . . . . . . . . $\quad 24$

733. Botryllus violaceus . . . . . . . . . . . . . . . . . . . . . . .

734. Composite Ascidian, diagram of zooid . . . . . . . . . . 25

735. Doliolum . . . . . . . . . . . . . . . 26

736. Salpa democratica, ventral view . . . . . . . . . . 26

737. " lateral view . . . . . . . . . . . . . . . . 27

738. Pyrosoma . . . . . . . . . . . . . . . . . 27

$739 . \quad$, part of section . . . . . . . . . . . . 28

740. Salpa, lateral view of ganglion . . . . . . . . . . . 29

741. Ascidian, mature egg . . . . . . . . . . . . . 31

742. Development of Clavellina, early stages . . . . . . . . 32

T43., ,,$\quad$ later, . . . . . . . . . . 33

744. Larva of Ascidia mammillata . . . . . . . . . . . . . 35

745. Metamorphosis of Ascidian, diagrammatic . . . . . . 36

746. Doliolum, tailed larva . . . . . . . . . . . . . . 38

747., asexual stage, lateral view . . . . . . . . . 38

7.48 . $\quad$,, dorsal , . . . . . . . . 39 
749. Salpa, late stage in development . . . . . . . . . 40

750. Amphioxus lanceolatus . . . . . . . . . . 43

751. " ,,$\quad$ transverse sections of pharyngeal and intestinal regions . . . . . . 44

752. , , , , anatomy, diagrammatic . . . . 47

753. transverse section of pharyngeal region, diagrammatic . . . . . . 48

754. diagram of vascular system. . . . . $\quad .49$

755 . nephridium . . . . . . . 51

756 . brain and cerebral nerves . . . . $\quad .52$

757. anterior portion of neuron . . . . 53

758. segmentation of the oosperm . . . $\quad 54$

759 . formation of gastrula.

760. development of notochord, neuron, and mesoderm . . . . . . 56

761. advanced embryo . . . . . . $\quad$. 57

762. young larva . $5 \quad$. $\quad . \quad 5 \quad . \quad 59$

763.

764. more advanced larva . . . . . 60 development of atrium. . . . . 61

765.

766. Ideal Craniate .

transverse sections 62

767. Section of skin of Fish . . . . . . . . . . . 67

768. Muscular system of Dogfish . . . . . . . . . . . 68

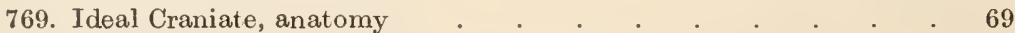

770. Vertebral column of embryo, transverse section. . . . . 70

771. Diagram illustrating segmentation of vertebral column . . . 71

772. Elements of embryonic cranium . . . . . . . . . 73

773. Diagrams of cartilaginous skull . . . . . . . . . . 74

774. Diagrams of bony skull . . . . . . . . . . . . 77

775. Development of pelvic fins, diagram . . . . . . . . . $\quad$. 79

776 and 777. Diagrams of limbs and limb-girdles . . . . . 80

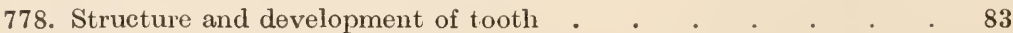

779. Structure of liver, diagrammatic . . . . . . . . . . . $\quad$. 85

780. Diagram of gills . . . . . . . . . . . . . 866

781. Diagram of vascular system of Fish . . . . . . . . . $\quad . \quad 48$

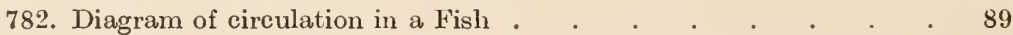

783. Diagram of vascular system of embryo of air-breathing Craniate . $\quad 92$

784. Diagram of heart of Amphibian and Crocodile . . . . . 93

785. Blood-corpuscles of Frog and Man . . . . . . . . . 94

786. Transverse section of spinal cord . . . . . . . . . . 95

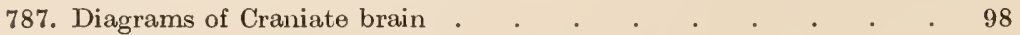

788. Diagram of cerebral and anterior spinal nerves . . . . . 101

789. Organs of touch . . . . . . . . . . . . . . . . 103

790. Sensory canals of head and organs of the lateral line . . . 104

791. Taste-buds. . . . . . . . . . . 105

792. Olf actory cells . . . . . . . . . . . . . . 106

793. Section of eye . . . . . . . . . . . . . . 106

794. Diagram of retina . . . . . . . . . . 107 
795. Development of eye

796. Muscles and nerves of eye

797. Pineal eye of Sphenodon.

798. Organ of hearing

799. Section of ampulla.

800. Urinary tubule .

801. Diagrams of urinogenital organs . . . . . . . . . 115

802. Development of mesoderm in Frog . . . . . . . 117

803. Petromyzon fluviatilis, external views of head . . . . . 120

804. " . marinus, skull, with branchial basket . . . . 121

$805 . \quad$ " $"$ " . . . . . . . 122

$806 . \quad, \quad$, dissection of female . . . . . 125

$807 . \quad, \quad$ brain . . . 127

808. , , , , with olfactory and pituitary sacs . 128

809. , , , development of olfactory and pituitary sacs. $\quad 129$

$810 . \quad, \quad, \quad$ auditory organ . . . . . . . 130

811. , , , transverse section of trunk . . . . 130

812. ., , . urinogenital sinus and related parts . . 131

813. , . . . . . . . . . . 131

814. , $\quad$ sections of embryos . . . . . . . . . 132

815.,$\quad$ fluviatilis, head of larva . . . . . . . . . . 133

816. Head of Myxine and of Bdellostoma . . . . . . . . 135

817. Myxine glutinosa, dissection . . . . . . . . . . . . 136

\$18. , auditory organ . . . . . . . . . . 137

819. Bdellostoma, kidney . . . . . . . . . . 137

820. Palæospondylus gumni . . . . . . . . . . 138

821. Hemiscyllium modestum. . . . . . . . . . 141

822. Scyllium canicula, vertebræ . . . . . . . . . . 142

823. Hemiscyllium, skull . . . . . . . . . . . . 143

824., visceral arches . . . . . . . . 146

825.,$\quad$ pectoral arch and fin . . . . . 147

826. ", . . . . . . . 147

827. " lateral dissection . . . . . 148

828. " branchial sac . . . . . . . . . 149

829. Scyllium, heart and branchial arteries . . . . . . . . 150

830. Hemiscyllium, blood-vessels . . . . . . . . . 152

831. Scyllium canicula, brain . . . . . . . . . . 154

832. Hemiscyllium, brain . . . . . . . . . . 155

833. Sycllium catulus, cranial nerves and branchial plexus . . . 156

834. " canicula, cerebral nerves. . . . . . . . . 157

835. " " urinogenital organs . . . . . 160

836. Hemiscyllium, right kidney and urinary sinus . . . . . 161

837. Dogfish, egg-case . . . . . . . . . . . 161

838. Cladoselache fyleri . . . . . . . . . . . 162

839. Pleuracanthus ducheni . . . . . . . . . . . . . 163

840. Acanthodes wardi . . . . . . . . . . . . 164

841. Chlamydoselachus anguineus . . . . . . . . . . 164

842. Lamna cornubica . . . . . . . . . . . 165 
FIG.

843. Urolophus cruciatus .

844. Centrophorus calceus, dermal denticles

845. Scymnus, spinal column .

846. Urolophus, skeleton

847. Heptanchus, skull . . . . . . . . . . 170

848. Torpedo-Ray, showing electric organ . . . . . . . . 172

849. Cestracion galeatus, egg-case . . . . . . . . . . . . . 176

850. Pristiurus, section of blastoderm . . . . . . . . . . 175

851. , formation of mesoderm . . . . . . . . 177

852. Elasmobranch embryo, sections . . . . . . . . . 178

853. Scyllium canicula, embryo . . . . . . . . . . . . 179

854. Ray, embryo . . . . . . . . . . . . 179

855. Elasmobranch embryo with yolk-sac . . . . . . . 180

856. Scyllium canicula, head of embryo . . . . . . . 181

857. " , , , , . . . . 181

858. Chimæra and Callorhynchus . . . . . . . . . . . 183

859. $"$ vertebral column . . . . . . . . . . . . . 185

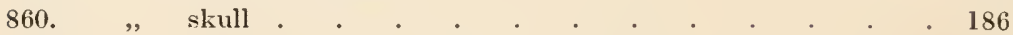

861. Callorhynchus antarcticus, skull . . . . . . . . . . 187

862. ", " brain . . . . . . 188

863. $\quad$ ", male urinogenital organs. . . . . 189

864. " , , embryo in egg-shell . . . . 191

865. Salmo fario . . . . . . . . . . 192

866. , , head . . . . . . . . . . . . 193

867. , , scale . . . . . . . . . . . . 194

868. , , vertebræ . . . . . . . . . . . . 195

869. " , , caudal end of vertebral column . . . . . . 196

870. ,, skull . . . . . . . . . . . . 197

871. „, fario, skull disarticulated . . . . . . . . . . . . 199

872 , salar, , of young individual . . . . . . 202

873. ", fario, fin-ray . . . . . . . . 202

874. , , , shoulder-girdle and pectoral fin . . . . . 203

875. , , " pelvic fin . . . . . . . . . . 204

876. ., ", side dissection . . . . . . . . . . . . . . . . 205

877. , " brain . . . . . . . . 207

878. " " $"$ eye . . . . . . . . . . . . . 208

879. " , " auditory organ . . . . . . . . . . 209

880. , , , urinary organs . . . . . . . . . . 209

881. " " . . . . . . . . . 210

882. , ,, section of blastoderm . . . . . . 210

883. Polypterus bichir . . . . . . . . . . . 212

884. Acipenser ruthenus . . . . . . . . . . . . . 212

885. Lepidosteus platystomus . . . . . . . . . 213

886. Amia calva . . . . . . . . . . . 213

887. Rita buchanani. . . . . . . . . . . . 214

888. Gadus morrhua. . . . . . . . . . . . 214

889. Sebastes percoides . . . . . . . . . . . . 215

890. Labrichthys psittacula . . . . . . . . . . 215 
891. Ostracion . 216

892. Hippocampus . . . . . . . . . . . . 217

893. Pleuronectes cynoglossus . . . . . . . . . . . 220

894. Stomias boa . . . . . . . . . 221

895. Ctenoid and ganoid scales . . . . . . . . . 221

896. Polypterus, part of vertebral column . . . . . . . . . 222

\$97. Sturgeon, skull . . . . . . . . . . . . . . . 223

898. Polypterus, skull . . . . . . . . . . . 224

s99., pectoral fin . . . . . . . . . . . 225

$900 . \quad$, pelvic fin . . . . . . . . . . 225

901. Gymnotus electricus . . . . . . . . . . . . . 226

902. Sargus, teeth . . . . . . . . . . . . 227

903. Anabas scandens . . . . . . . . . . . . . 228

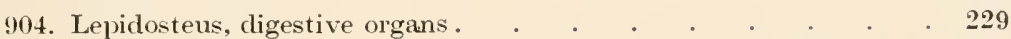

905. Pseudophycis bachus, relation of air-bladder to auditory organ . $\mathbf{2 3 0}$

906. Lepidosteus, brain . . . . . . . . . . . 231

$907 . \quad$, male organs . . . . . . . . . 232

$908 . \quad$,. $\quad$ and Amia, female organs _ . . . . . 233

909. Acipenser, Amia, and Lepidosteus, segmentation _ . . . 234

910. Polypterus bichir, head of larva . . . . . . . . . 235

911. Glyptolepis and Macropoma . . . . . . . . . . 236

912. Palæoniscus and Platysomus . . . . . . . . . 237

913. Lepidotus and Caturus . . . . . . . . . . . . . 238

914 . Ceratodus forsteri . . . . . . . . . . . 240

$915 . \quad$, , anterior portion of skeleton . . . . . 241

$916 . \quad, \quad$, skull, dorsal . . . . . . . . . 242

917., ",$\quad$, ventral . . . . . . . 242

918. ,, „, pelvic areh and fin . . . . . . . 243

$919 . \quad, \quad$, lung . . . . . . . 244

$920 . \quad, \quad, \quad$ heart and main blood-vessels . . . . 245

$921 . \quad$ ", brain . . . . . . . . 246

922. ., , $\quad$ reproductive organs, female . . . . . . 248

$923 . \quad$, , development . . . . . . . . 249

924. Protopterus annectens . . . . . . . . . . 251

925. , , , skull, shoulder-girdle, and fore-limb . . 252

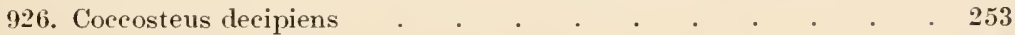

927. Pteraspis rostrata . . . . . . . . . . . . 254

928. Lanarkia spinosa . . . . . . . . . . . 254

929. Drepanaspis gemundenensis . . . . . . . . . 254

930. Cephalaspis . . . . . . . . . . . . . 255

931. Pterichthys testudinarius . . . . . . . . . 256

932. Rana temporaria . . . . . . . . . . . 257

933. ,,$\quad$ skeleton . . . . . . . . . . . . 259

934. „ ", skull . . . . . . . . . . . 261

935. , , ,, of tadpole . . . . . . . . 263

936. ", esculenta, shoulder-girdle . . . . . . . 263

937. " " , , , transverse section, diagrammatic 264

938. ", " . . . . . . . . . . 265 
FIG.

939. Rana esculenta, muscles

940. Rana temporaria, dissection from left side . esculenta, digestive organs . . .

942. ., temporaria, heart . . . . . . . . . . 270

943. .,$\quad$ arteries . . . . . . . . . 272

944. . ", veins . . . . . . . . . 273

945. , , $\quad$, course of blood and lymph, diagrammatic . . 275

946. .. esculenta, brain . . . . . . . . . . 276

947. . . accessory auditory apparatus . . . . . . . 277

948. ,, esculenta, urinogenital organs, male . . . . . . 278

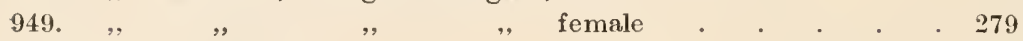

950. ," development . . . . . . . . . . . 281

951. ", temporaria, stages in life-history . . . . . . 282

952. Necturus maculatus . . . . . . . . . . . . 286

953. Siren lacertina . . . . . . . . . . . . . . 286

954. Amphiuma tridactyla . . . . . . . . . . . . 286

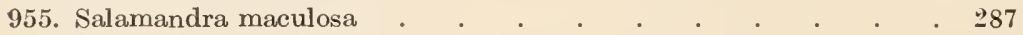

956. Cœcilia pachynema . . . . . . . . . . . . 288

957. Urodela, structure of vertebral column . . . . . . . 290

958. Proteus anguinus, chondrocranium . . . . . . . . . 291

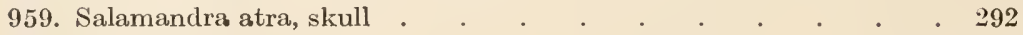

960. Ichthyophis glutinosa, skull . . . . . . . . . . . 292

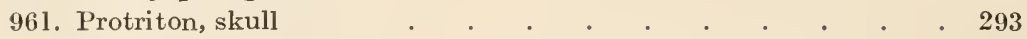

962. Salamandra and Amblystoma, shoulder-girdle and sternum . . 294

963.,$\quad$ pelvic girdle . . . . . . . . . 295

964. „, heart and chief arteries, larva and adult _ . . 296

965. " maculosa, venous system . . . . . . : 297

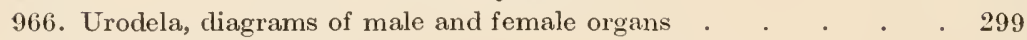

967. Nototrema marsupiatum . . . . . . . . . . 300

968. Pipa americana. . . . . . . . . . . . . 300

969. Ichthyophis glutinosa . . . . . . . . . . 301

970. Amblystoma tigrinum (axolotl) . . . . . . . . 301

971. Lacerta viridis . . . . . . . . . . . . . 304

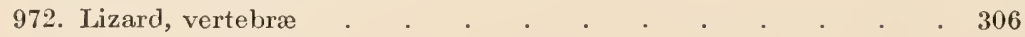

973. Lacerta agilis, skull . . . . . . . . . . . . . . . . 308

974. " , , pectoral arch and sternum . . . . . . 311

975. ", ", carpus . . . . . . . . . . 311

976. „, vivipara, pelvis . . . . . . . . . . . . 312

977. , agilis, tarsus . . . . . . . . . . 313

978. ", ", general view of viscera . . . . . . . 314

979. „, viridis, dissection from ventral aspect . . . . . 315

980. Lizard, lateral dissection. . . . . . . . . . . . . . . . $\quad . \quad 317$

981. Lacerta viridis, brain . . . . . . . . . . . 318

982. „, vivipara, brain of an embryo . . . . . . . . . 319

983. „, Jacobson's organs . . . . . . . . . . . . 320

984. ", sclerotic ossicles . . . . . . . . . . . 321

985. . " viridis, membranous labyrinth . . . . . . . 321

986. ", ", urinogenital organs, male . . . . . . . . 322 
FIG.

987. Lacerta viridis, urinogenital organs, female

988. Chamæleon vulgaris

989. Pygopus lepidopus

990. Sphenodon punctatum . . . . . . . . . . . . . . 330

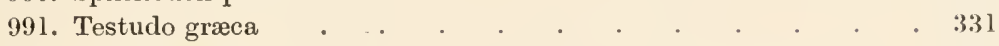

992. Sphenodon, vertebra . . . . . . . . . . . . . . 333

993. Python, vertebra . . . . . . . . . . . . . 333

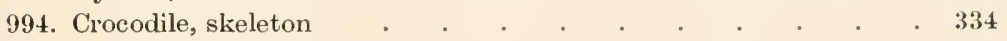

995. Sphenodon, skeleton . . . . . . . . . . . . 334

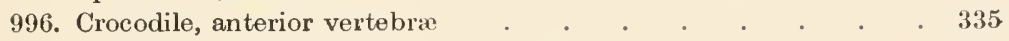

997. Cistudo lutaria, skeleton _ . . . . . . . . . 336

998. Chelone midas, transverse section of skeleton . . . . . . 336

999. Tropidonotus natrix, skull . . . . . . . . . . 337

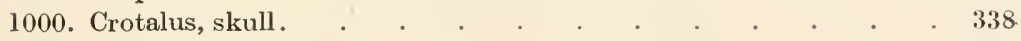

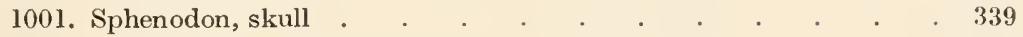

1002. Emys europæa, skull . . . . . . . . . . . 340

1003. Chelone mydas, skull . . . . . . . . . . . 341

1004. Crocodilus porosus, skull . . . . . . . . . 342

1005. Crocodile, skull . . . . . . . . . . . . . 34 ?

1006. Emys europæa, tarsus . . . . . . . . . . 343

1007. Alligator, carpus . . . . . . . . . . . 343

1008., pelvis . . . . . . . . . . . 344

1009. Crocodile, tarsus . . . . . . . . . . . . . . 344

1010. Monitor, Emys, and Alligator, tongues . . . . . . 346

1011. Chamæleon, lungs . . . . . . . . . . . . 347

1012. Lacerta muralis, heart . . . . . . . . . . 348

1013. Turtle, diagram of heart . . . . . . . . . . . . 348

1014. Crocodile, heart . . . . . . . . . . . 349

1015. Alligator, brain . . . . . . . . . . . . 350

1016. Sphenodon punctatum, pineal eye . . . . . . . . . 35l

1017. Alligator, early development . . . . . . . . . . . 352

1018. Lacerta . . . . . . . . . . . . 353

1019. Draco volans . . . . . . . . . . . . . . . . 355

1020. Rattlesnake, poison apparatus . . . . . . . . . . 356

1021. Belodon, skull . . . . . . . . . . . . . . . 359

1022. Galesaurus planiceps, skull . . . . . . . . . . 360

1023. Plesiosaurus macrocephalus . . . . . . . . . . 361

1024. ", pectoral arch . . . . . . . . . . 361

1025., pelvic arch . . . . . . . . . . . . . . . . . .

1026. Ichthyosaurus communis . . . . . . . . . . 362

1027. Iguanodon bernissartensis . . . . . . . . . . 363

1028. " mantelli, teeth . . . . . . . . 363

1029. Pterodactylus spectabilis . . . . . . . . . . 364

1030. Scaphognathus, skull . . . . . . . . . . . 365

1031. Ramphorhynchus . . . . . . . . . . . 365

1032. Edestosaurus . . . . . . . . . . . . 366

1033. Columba livia, external form . . . . . . . . . 368

1034. $"$, feathers . . . . . . . . . 369 
FIG.

1035. Structure of feather

1036. Development of feather

1037. Columba livia, pterylosis

1038.

" bones of trunk

1039.

, cervical vertebra.

374

1040.

, sacrum of nestling

375

1041. , , , skull of young specimen

1042. Diagram of Bird's skull.

1043. Columba livia, hyoid apparatus . . . . . . . . . 378

1044. columella auris

1045.,$\quad$ " bones of left wing . . . . . . . . . 379

1046. „ " . manus of nestling . . . . . . . 380

1047. . . . innominate of nestling . . . . . . . 380

1048. , , " bones of hind-limb . . . . . . . . 381

1049. , , , , foot of embryo . . . . . . . . . . 381

1050. " . muscles of wing . . . . . . . . 383

1051. , , dissection from right side . . . . . . 384

1052. " . " lungs and trachea . . . . . . . 386

1053. Diagram of air-sacs of a Bird . . . . . . . . 387

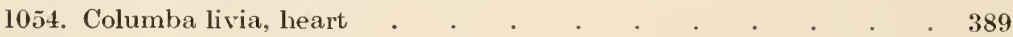

1055 .

, vascular system .

1057. ", ", dissections of brain . . . . . . . . 392

1058. ", , eye . . . . . . . . . . . 393

1059. " "

1060. " " " urinogenital organs, male . . . . . . . 395

1061. " " ",$\quad$, female . . . . . 395

1062. Apteryx australis . . . . . . . . . . . 398

$1063 . \quad, \quad, \quad$ skeleton . . . . . . . . . . 399

1064. Hesperornis regalis . . . . . . . . . . . 400

1065. Ichthyornis victor . . . . . . . . . . . 401

1066. Eudyptes antipodum . . . . . . . . . . 402

1067. Archæopteryx lithographica . . . . . . . . 406

1068. ,.,$\quad$ skull . . . . . . . 407

$1069 . \quad$..$\quad$ manus . . . . . . . 407

1070. Opisthocomus and Apteryx, wings . . . . . . . 408

1071. Gypaëtus and Ardea, pterylosis . . . . . . . . 410

1072. Casuarius, feather . . . . . . . . . . . 411

1073. Gallus, Turdus, Vultur, Procellaria, and Casuarius, sterna . . 412

1074. Eudyptes pachyrhynchus, skeleton . . . . . . . 413

1075. Apteryx mantelli, skull of young specimen, side view . . . 414

1076 .

dorsal view

1077. Anas boschas, skull 
1083. Galius bankiva, innominate of embryo

1084. Apteryx oweni, hind-limb of embryo

1085. Gallus bankiva, egg at time of laying

$1087 .$,

1090. Diagram illustrating the relationships of the chief groups of

\section{Birds}

1091. Iepus cuniculus, skcleton with outline of body

1099. ," ,,

1100. ,, .. digestive organs . . . . . . .

1101. ., , heart . . . . . . . . .

$1104 . \quad$.,$\quad$ transverse section of thorax . . . . . . 453

$1105 . \quad, \quad, \quad$ brain . . . . . . . 455

1106. , , , dissections of brain . . . . . . . 456

1107. ," , brain, vertical section . . . . . . 457

$1108 . \quad, \quad$, $\quad$ urinogenital organs $\quad . \quad$. . . . . . 459

1109. , , , female organs (part) . . . . . . . 460

1110. ", , diagrammatic scetion of advanced embryo . 461

1111. Section of human skin . . . . . . . . . . 474

111 . Longitudinal section of hair . . . . . . . . . 475

1113. Development of hair . . . . . . . . . . . . . 476

1114. Echidna aculeata, with pouch and mammary glands . . . 477

1115. Diagrams of development of nipple . . . . . . . . 478

1116. Ornithorhynchus anatinus . . . . . . . . . . . 479

1117. Echidna aculeata . . . . . . . . . . . . 479

1118. Didelphys virginiana . . . . . . . . . . 480

1119. Dasyurus viverrinus . . . . . . . . . . . . . . 480

11 0. Petrogale xanthopus . . . . . . . . . . . 481

1121. Notoryctes typhlops . . . . . . . . . . 481

11:2. Phascolomys mitchelli . . . . . . . . . . 482

1123. Phascolarctos cinereus . . . . . . . . . . . 482

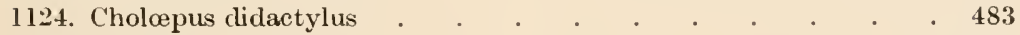

1125. Dasypus sexcinctus . . . . . . . . . . . . . 484

1126. Manis gigantea . . . . . . . . . . . . . 485

1127. Orycteropus capensis . . . . . . . . . . . . 485

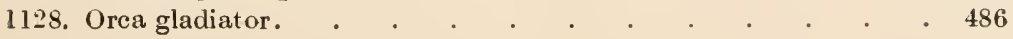


FIG.

1129. Hippopotamus amphibius

1130. Equus burchelli

1131. Tapirus terrestris

1132. Rhinoceros indicus

1134. Galeopithecus . . . . . . . . . . . . . 492

1135. Synotus barbastellus . . . . . . . . . . 493

1136. Gorilla . . . . . . . . . . . 495

1137. Diagram of Mammalian skull . . . . . . . . 497

1138. Sagittal sections of Mammalian skulls, diagrammatic . . . 499

1139. Ornithorhynchus, skeleton . . . . . . . . . 502

1140. Echidna aculeata, skull . . . . . . . . . 504

1141. Omithorhynchus, scapula . . . . . . . . . . 505

1142. Kangaroo, atlas . . . . . . . . . . . . . 506

1143. Halmaturus ualabatus, skeleton . . . . . . . . 507

1144. Dasyurus, skull . . . . . . . . . . . . 508

1145. Petrogale penicillata, skull . . . . . . . . . 508

1146. Phascolomys, skull. . . . . . . . . . . 509

1147. Phalanger, bones of leg and foot . . . . . . . 510

1148. Macropus, bones of foot . . . . . . . . . . 510

1149. Dasypus sexcinctus, skull . . . . . . . . . . 511

1150. Myrmecophaga, skull, lateral . . . . . . . . . 511

1151. ", ventral . . . . . . . . 512

1152. Bradypus tridactylus, skull . . . . . . . . . . 512

1153. Dasypus sexcinctus, shoulder-girdle . . . . . . . . 513

1154. Bradypus tridactylus, skeleton . . . . . . . . . 514

1155. ", , shoulder-girdle . . . . . . 515

1156.,$"$ manus . . . . . . . . 515

1157. $\quad, \quad$ pes . . . . . . . . . . . . . . . . .

1158. Dasypus sexcinctus, pelvis . . . . . . . . . 516

$1159 . \quad, \quad$, pes . . . . . . . . 516

1160. Phocæna communis, skeleton . . . . . . . . 517

1161. Balænoptera musculus, sternum . . . . . . . . 517

1162. Globiocephalus, skull . . . . . . . . . . 518

1163. Halicore australis, skeleton . . . . . . . . . 519

1164. Manatus senegalensis, skull . . . . . . . . . . 520

1165. Cervus elaphus, axis . . . . . . . . . . 520

1166. Equus caballus, posterior part of skull . . . . . . . 521

1167. Ovis aries, skull . . . . . . . . . . . . . . 523

1168. Hyrax, skull . . . . . . . . . . . . . 524

1169. Elephas africanus, skull . . . . . . . . . . . 524

1170. Cervus elaphus, scapula . . . . . . . . . . . . 525

1171. Tapirus indicus, manus . . . . . . . . . . . 526

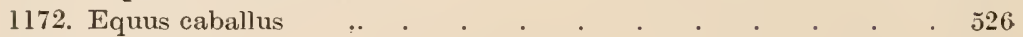

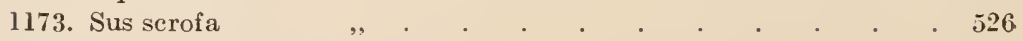

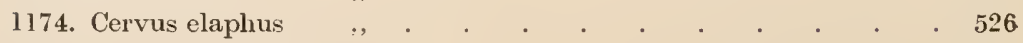

1175. Equus caballus, tarsus . . . . . . . . . . . . 527

1176. Cervus elaphus " . . . . . . . . . . . 527 
1177. Sus serofa, tarsus

1178 . Felis tigris, skull

1179. , . ., section of aurlitory bulla . . . . . . . . 529

1180. Canis familiaris, skull . . . . . . . . . . . 52!

1181. Ursus ferox, section of auditory bulla . . . . . . . . . . . . . .

1182. „, americanus, carpus . . . . . . . . . 530

1183. Felis leo, digit. . . . . . . . . . . . . . . 53)

1184. Phoca vitulina, skeleton . . . . . . . . . . . . . . . . . . . . . . .

1185. Centetes ecandatus, skull . . . . . . . . . . . . . 533

1186. Pteropus jubatus, skeleton . . . . . . . . . . . 534

1187. „, fuscus, skull . . . . . . . . 535

1188. Homo sapiens, skull . . . . . . . . . . . . . 536

1189. Anthropopithecus troglorlytes, skull . . . . . . . . 538

1190. Simia satyrus, skeleton _ . . . . . . . . . . . . . . 539

1191. Cynocephalus anubis, carpus . . . . . . . . . . 539

1192. Homo, Gorilla, and Simia, foot . . . . . . . . . 540

1193. Various forms of teeth, sections . . . . . . . . . 54l

1194. Development of Mammalian teeth . . . . . . . . . 5 . . . . . .

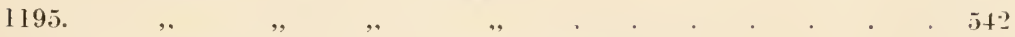

1196. Canis familiaris, milk and permanent dentitions . . . . . $5+3$

1197. Lagenorhynchus, teeth . . . . . . . . . . . jt4

1198. Perameles, teeth . . . . . . . . . . . . . 544

1199. Phaseolaretos einereus, front view of skull . . . . . . . $5+5$

1200. Macropus major, teeth . . . . . . . . . . . 546

1201. Sarcophilus ursinus, front view of skull . . . . . . . 5 5

1202. Didelphys marsupialis, teeth . . . . . . . . . . 546

1203. Orycteropus, section of lower jaw and tecth . . . . . . . . . . . .

1204. Sus serofa, teeth . . . . . . . . . . . . . . .

1205. Equus caballus, skull and teeth . . . . . . . . . 549

1206. Fleplıas af ricanus, molar teeth . . . . . . . . 550

1207. Balænoptera rostrata, lower jaw of fotus, with teeth . . . 550

1208. ., scetion of upper jaw, with baleen . . . . . 55l

1209. Lower carnassial teeth of Camivora . . . . . . . . . 552

1210. Different forms of stomach in Mammalia . . . . . . . . 5.5t

1211. Stomach of Ruminant . . . . . . . . . . . . . 555

1212. , , Porpoise . . . . . . . . . . 55.

1.213. Liver of Mammal, diagrammatic. . . . . . . . . 556

1214. Canis familiaris, brain . . . . . . . . . . 559

1215. Echidna aculeata, sagittal section of brain . . . . . . 560

1216. Petrogale penicillata ,, ,, , . . . . . . . 560

1217. Ornithorhynchus anatinus, brain. . . . . . . . . . . 561

1218. Echidna aculeata, brain . . . . . . . . . . 561

1219. Macropus major ., . . . . . . . . . . 562

1220. Cogia grayi * . . . . . . . . . . . . . . . . . . . . 562

1221. Homo sapiens, sagittal section of nasal and buceal cavities . . 563

1.22. . . . ear. . . . . . . . . . 564

1223. Female organs of Marsupials . . . . . . . . . . . . . . . . . . . . . . . . .

1224. Eteri of Eutheria . . . . . . . . . . . . . 567 
FIti.

1225. Homo, sagittal section of ovary . . . . . . . . 568

$1: 2$ (;. Development of Graafian follicle . . . . . . . . 568

1:27. Segmentation of Mammalian oosperm . . . . . . . . 570

1228. Lepus cuniculus, embryonic area. . . . . . . . . 571

1.29. , , , embryos . . . . . . . . . 572

1230. Formation of fotal membranes of Mammal . . . . . . 573

1:31. Lepus cuniculus, cmbryo with membranes . . . . . . 574

1.23.2. Erinaceus, formation of amnion and trophoblast . . . . 575

1233. Formation of amnion in Hammalia . . . . . . . . 575

1234. Macropus, mammary fotus . . . . . . . . . 577

1235. Hypsiprymus rufescens, embryo and fotal membrane . . . 578

1236. Phascolarctos cinereus , , , , , , . . . . 578

1237. Perameles obesula ., ., placenta . . . . . 578

12:38. Theria and Monotremata, blastula . . . . . . . 579

1239. Phascolotherium bucklandi, mandible . . . . . . 582

1240. Plagiaulax becklesi, mandible . . . . . . . . . 582

1241. Diprotodon australis, skeleton . . . . . . . . . . . . 583

1242. Nototherium mitchelli, skull . . . . . . . . . 584

1243. Thylacoleo carnifex, skull . . . . . . . . . . . . 584

1.24. Glyptodon clavipes, skeleton . . . . . . . . . . . 585

1:45. Mylodon robustus . . . . . . . . . . . . . 585

1246. Squalodon, teeth . . . . . . . . . . . . . . . 586

1247. Dinotherium giganteum, skull . . . . . . . . 587

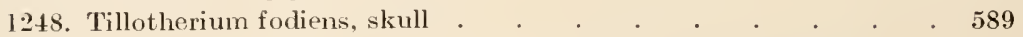

1249. Diagram illustrating the mutual relationships of the Chordata . 596

1250. , , , , , , , , , Phyla of

animals 598

1251. Map showing depths of sea between the British Isles and the Continent . . . . . . . . . . 603

1252. Map showing depths of sea between New Zealand and Australia . 604

1253. Diagram showing the relations of the Zoo-geographical Regions . 614 


\title{
ZOOLOGY
}

\author{
SECTION XIII
}

\section{PHYLUM CHORDATA}

IN the arrangement which it has been found convenient to follow in the present work, the Vertebrate animals (Fishes, Amphibians, Reptiles, Birds, and Nammals), together with the Cephalochorda or Lancelets, the Urochorda or Ascidians, and the Hemichorda or Balanoglossus and its allies, are all grouped together in a single phylum - the Chordata. The main groups comprised in this assemblage, however, differ so widely from one another in certain essential points, and the common features uniting them together are so few, that it has been thought advisable to depart from the plan of arrangement followed in connection with the rest of the phyla, and to make a primary division in this case not into classes, but into sub-phyla. In accordance with this scheme the phylum Chordata is regarded as made up of three sub-phyla-the Hemichorda, the Urochorda, and the Euchorda, the last-mentioned comprising the two sections Acrania and Craniata or Vertebrata, each of which receives separate treatment.

The name Chordata is derived from one of the few but striking common features by which the members of this extensive phylum are united together-the possession, either in the young condition or throughout life, of a structure termed the chorda dorsalis or notochord. This is a cord of specially modified vacuolated cells extending along the middle line on the dorsal side of the enteric cavity and on the ventral side of the central nervous system. In the lower Chordates (the Hemichorda, Urochorda, and Cephalochorda) the notochord is developed directly and ummistakably from the endoderm, and in the first-named group it remains permanently in continuity with that layer. But in the Craniata its origin is by no means so definite, and it may originate

vOL. II 
from cells which are not obviously of endodermal derivation. It may be enclosed in a firm sheath and thus be converted into a stiff, but elastic, supporting structure. In the Craniata (with a few exceptions among lower forms) it becomes in the adult replaced more or less completely by a segmented bony or cartilaginous axis-the spinal or vertebral column. Another nearly universal common feature of the Chordata is the perforation of the wall of the pharynx, either in the embryonic or larval condition only, or throughout life, by a system of clefts - the branchial clefts; and a third characteristic is the almost universal presence at all stages, or only in the larva, of a cavity or system of cavities, the neurocoele, in the interior of the central nervous system.

The Chordata are Cœlomata (Vol. I., p. 333), and the mode of development of the colome in the lower sub-phyla is essentially the same as in the Echinodermata (Vol. I., p. 421), the Chrtognatha (p. 313), and the Phoronida (p. 351): it is derived, that is to say, by direct outgrowth from the archenteron. In the Craniata this enterocœlic origin of the cavity is no longer definitely traceable, though what appear to be indications of it may be detected in some cases. The Urochorda are not segmented ${ }^{1}$ : in the Hemichorda there is a division of the cœlome into three parts, each occupying a definite region of the body, so that the view is sometimes maintained that these animals are tri-segmented: in the Cephalochorda and Craniata there are numerous segments, the nature of which will be referred to later.

\section{SUB-PHYLUM AND CLASS I.-HEMICHORDA (ADELOCHORDA).}

A number of worm-like, simply organised animals possessing a structure which is commonly regarded as of the nature of a rudimentary notochord, comprising Balanoglossus and certain allied genera, are so widely removed from the other members of the Chordata that, if we accept them as Chordates, it is advisable to consider them as constituting an independent sub-phylum, and to this the name of Hemichorda or Adelochorda has been applied. Resembling Balanoglossus in the condition of the supposed notochord, in the division of the body into three regions, sometimes looked upon as representing three segments, and in certain other features, are two genera of small marine animals-Cephalodiscus and Rhabdopleura. These are probably more nearly related to one another than they are to Balanoglossus, from which they are separated by well-marked differences, and the Hemichorda may, therefore, best be regarded as divisible into two classes-one, the Enteropneusta, comprising only Balanoglossus ${ }^{2}$ and its imme-

1 Though faint indications of serial repetition of parts are traceable in certain cases.

${ }_{2}$ The name Balanoglossus is here used as a general designation rather than as a strictly generic term. 
diate allies; the other, the Pterobranchia, including Cephalodiscus and Rhabdopleura.

External Characters and Cœlome of Enteropneusta.Balanoglossus (Fig. 714) is a softbodied, cylindrical, worm-like animal, the surface of which is uniformly ciliated. The size varies extremely in the different species, some being quite small-2 or 3 centimetres, while other species are of comparatively large size and may be as much as $2 \frac{1}{2}$ metres in length. It is divisible into three regions; in front there is a large club-shaped hollow organ-the proboscis $(p r$.$) ; immediately behind the$ proboscis and encircling its base is a prominent fold-the collar (co.); the third region or trunk is long and nearly cylindrical, but somewhat depressed.

Balanoglossus lives in the sea, usually in shallow water, burrowing in sand or mud by means of its proboscis: one species has been found swarming on the surface of the sea. Numerous glands in the integument secrete a viscid matter to which grains of sand adhere in such a way as to form a fragile temporary tube. The proboscis (Fig. 715, prob.) has muscular walls; its cavity (proboscis-cœelome) opens on the exterior usually by a single minute aperture -the proboscis-pore (prb. po.)-rarely by two. In some species the proboscis-pore does not communicate with the proboscis-coelome, but terminates blindly, and may send off a narrow tubular diverticulum which opens into the neurocole. The narrow posterior part or "neck" of the proboscis is strengthened by a layer of cartilage-like or chondroid tissue, which supports the blood-vessels. The collar is also muscular, and contains one cavity, or two (right and left) cavities

FIG. 714.-Balanoglossus. Entire animal. br. branchial region; co. collar; gen. genital ridges : hep. prominences formed by hepatic ceca ; pr. proboscis. (After Spengel.) 
separated from one another by dorsal and ventral mesenteries, and completely cut off from the proboscis-cavity. The collar-cavity and also that of the proboscis are crossed by numerous strands of connective-tissue of a spongy character. The collar-cavity communicates with the exterior by a pair of collar-pores - ciliated tubes leading into the first gill-slit or first gill-pouch.

On the dorsal surface of the anterior part of the trunk is a double row of small slits-the gill-slits (Fig. 714, br.) - each row situated

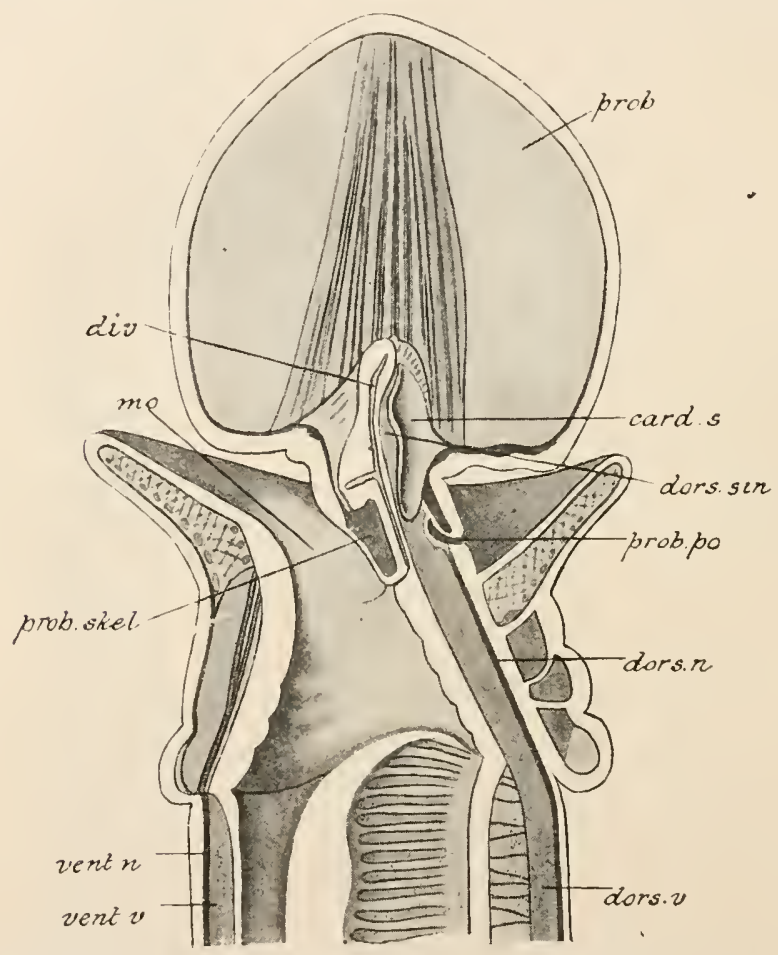

FIG. 715.-Balanoglossus. Diagrammatic sagittal section of anterior end. card. $s$. cardiac sac; div. diverticulum (supposed notochord); dors. $n$. dorsal nerve-strand ; dors. sin. dorsal sinus; dors. v. dorsal vessel; mo. mouth; prob. proboscis; prob. po. proboscis-pore ; prob. skel. proboscis-skeleton; vent. $n$. ventral nerve-strand; vent. $v$. ventral vessel. (After Spengel.)

in a longitudinal furrow; these slits increase in number throughout life. The most anterior are in some species overlapped by a posterior prolongation of the collar called the operculum. A pair of longitudinal genital ridges (gen.) - not recognisable in some species-which extend throughout a considerable part of the length of the body both behind and in the region of the gill-slits (branchial region), are formed by the internally situated gonads: these ridges are so prominent in some of the genera as to form a pair of wide wing-like lateral folds. Behind the branchial region 
are two rows of prominences (hep.) formed by the liepatic cæca. The trunk is irregularly ringed, this annulation, which is entirely superficial and does not correspond to an internal segmentation, being most strongly marked behind. The cœlome of the trunk is divided into two lateral closed cavities by a vertical partition (dorsal and ventral mesenteries).

Digestive Organs.-The mouth (Fig. 715, mo.) is situated ventrally at the base of the proboscis, within the collar. Into the dorsal half of the anterior portion of the alimentary canal open the internal gill-openings. Each of these is in the form of a long narrow $U$, the two limbs separated by a narrow process--the tongue -which contains a prolongation of the body-cavity. In most of the Enteropneusta the internal gill-openings $1 \mathrm{e}$ a d into gill-pouches which in turn communicate with the exterior by the gillslits. But in the genus Ptychodera (Fig. 716) there are no gill-pouches, the U-shaped internal gill-openings leading directly to the ex-

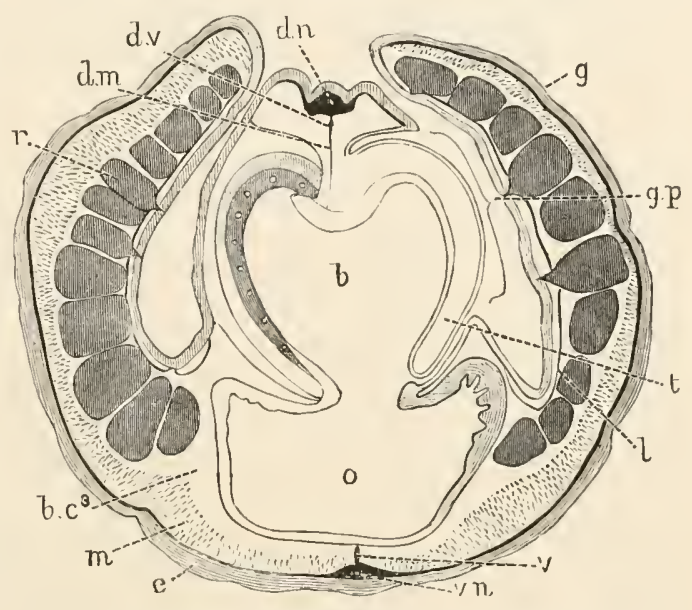
terior. The gillpouches are supported by a chitinoid skeleton consisting of a number of separate parts. Each of these consists of a dorsal

FIG. 716 - -Ptychodera bahamensis. Transverse section of the branchial region. $b$. branchial part of alimentary canal;
$b . c^{3}$, colone of trunk; $d . m$. dorsal mesentery; $d . n$. dorsal nerve; $d$. $v$. dorsal vessel; $e$. epidermis with nerve layer (black) at its base; g. genital wing; g. p. branchial aperture encroached upon by tongue $(t) ; l$. lateral septum; $m$. longitudinal muscles; $o$. digestive part of csophagus ; $r$. reproductive organ; $t$. tongue ; $v$. ventral mesentery' and ventral vessel; $v$. $n$. ventral nerve. (From Harmer, Cambridge Natural History, after Spengel.)

basal portion and three long narrow lamellæ, a median and two lateral; the median, which is bifurcated at the end, lies in the septum or interval between two adjoining gill-sacs; the two lateral lie in the neighbouring tongues. In most species a number of transverse rods - the synapticulce - connect together the tongues and the adjoining septa, and are supported by slender processes of the skeleton.

The posterior part of the alimentary canal is a nearly straight tube, giving off, in its middle part, paired hepatic caeca, which bulge outwards in the series of external prominences already mentioned. Posteriorly it terminates in an anal aperture situated at the posterior extremity of the body. In the posterior part of its 
extent in some Enteropneusta the intestine presents a ventral median ridge-like outgrowth of its epithelium-the pygochord. Throughout its length the intestine lies between the dorsal and ventral divisions of the vertical partition, which act as mesenteries.

As the animal forces its way through the sand, a quantity of the latter enters the digestive canal through the permanently open mouth, and is eventually passed out again by the anus in the shape of castings, which may be thrown out on the surface of the sand in a form resembling that taken by the castings of earthworms.

A series of pores (gastro-cutaneous pores), variously arranged in the different genera, connect the intestine with the surface.

Notochord or œsophageal diverticulum.--The dorsal wall of the part of the digestive canal immediately following upon the mouth gives off a diverticulum ( $d i v$.) that runs forwards some distance into the basal part of the proboscis after giving off a short ventral branch. The diverticulum contains a narrow lumen, and its wall is composed of a single layer of long and very narrow cells, each of which contains a vacuole. This layer of cells forming the wall of the diverticulum is continuous with the epithelium of the digestive canal itself, the cells being somewhat modified by the presence of the vacuoles. The diverticulum, owing partly to its structure, partly to its relations, is usually regarded as representing the notochord of the typical Chordata. In close relation with this on its ventral surface is the chitinoid proboscis-skeleton (prob. skel.), which consists of a median part of an hour-glass shape, and with a tooth-shaped process, bifurcating behind into two flattened bars which lie in the anterior region of the œsophagus and support the opening into the lumen of the diverticulum.

There is a blood-vascular system with dorsal and ventral longitudinal trunks. The dorsal vessel (dors. v.) lies above the notochord, and ends in front in a sinus, the dorsal sinus or heart (dors. $\sin$.), situated in the anterior part of the collar and the neck of the proboscis, in close contact with the notochord. From the posterior part of the sinus is given off a vessel which bifurcates to supply the proboscis. In communication with the sinus in front are a number of vessels of a bilateral plexus in the glomerulus, a glandular organ, probably excretory, situated at the anterior end of the œsophageal diverticulum. From the posterior end of each half of the glomerulus there passes backwards an efferent vessel which breaks up into a plexus; the two plexuses unite ventrally to form a median ventral plexus continuous behind with the ventral vessel. The dorsal sinus, having no definite walls, is not contractile ; but a closed sac, the cardiac sac ( $a r d$. $s$.), situated on the dorsal side of the sinus, has a muscular ventral wall, by the contractions of which the blood may be propelled.

The nervous system consists of dorsal and ventral strands 
(dors. n., vent. n.) which extend throughout the length of the body. These are merely thickenings of a layer of nerve-fibres which extends over the entire body in the deeper part of the epidermis. Here and there are giant nerve-cells. The part of the dorsal strand which lies in the collar (collar-cord) is detached from the epidermis ; it contains a larger number of the giant nerve-cells than the rest; in some species it encloses a canal, the neurocœle, opening in front and behind; in others a closed canal ; in most
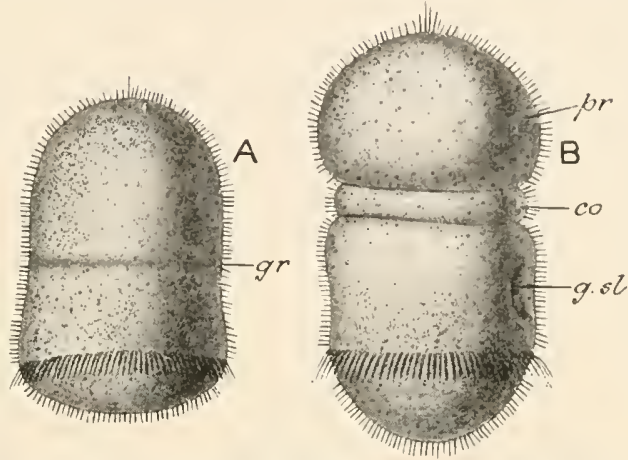

Fig. 717 - Development of Balanoglossus. $A$, stage of the formation of the first groove $(g r.) . \quad B$, stage in which the second groovelhas appeared, and the first gill-slit has become developed. co. collar; $g$. sl. gill-slit; pr. proboscis. (After Bateson.)

a number of separate cavities. At the posterior extremity of

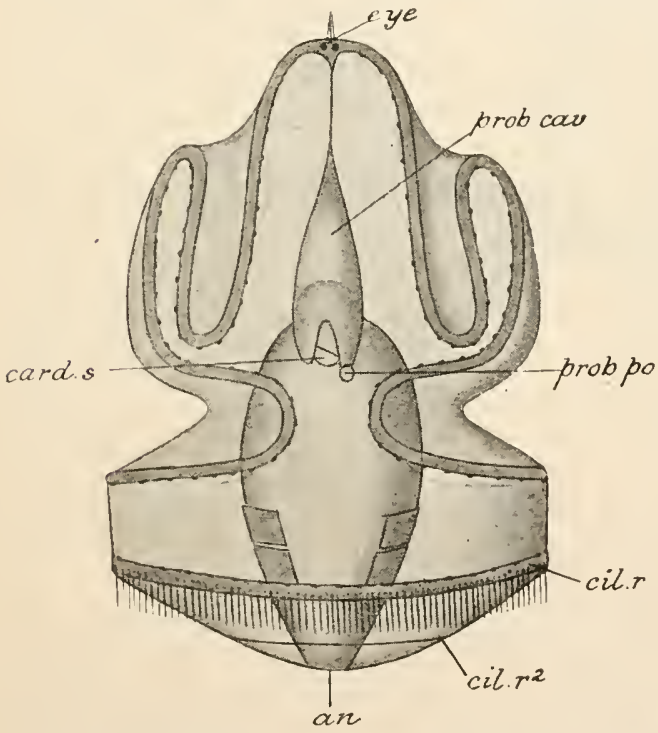

Fig. 718.-Tornaria. Dorsal view. an. anus ; card. s. cardiac sac ; cil. $r$. post-oral ciliated band (membranello); cil. $r^{2}$ posterior ciliated ring; cye, eye-spots on apical plate; prob. cav. proboscis-cavity; wrob. no. probosris-pore. (After s'pengel.) the collar the dorsal and ventral strands are connected by a ring-like thickening, and there is a thickening also round the neck of the proboscis. There are no organs of special sense; but some cells of the epidermis on certain parts of the proboscis and on the anterior edge of the collar seem to be of the character of sensory cells.

\section{Reproductive} Organs.-The sexes are separate, and often differ in shape and colour; the ovaries and testes are simple or branched saccular organs arranged in a double row along the branchial region of the trunk and further back; they open on the exterior by a series of pores. 
The course of the development (Figs. 717-719) differs in different species. In some it is comparatively direct; in others there is a metamorphosis. Impregnation is external. Segmentation is complete and fairly regular, resulting in the formation of a blastula, which is at first rounded, then flattened. On one side of the flattened blastula an invagination takes place. The embryo at this stage is covered with short cilia, with a ring of stronger cilia. The aperture of invagination closes and the ectoderm and endoderm become completely separate. The embryo elongates and a transverse groove $(g r$.$) appears (A)$ : the mouth is formed by an invagination in the position of the groove. The anus is

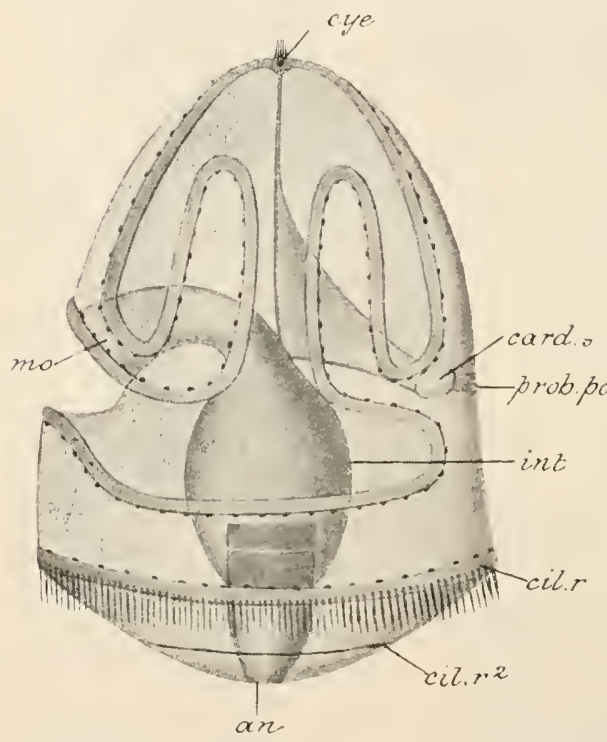

FIG. 719.-Tornaria. Lateral view, ' Letlering as in Fig. 718 : in addition, int. intestine, mo. mouth. (After Spengel.) developed in the position formerly occupied by the blastopore. Before the mouth appears there are formed two diverticula of the archenteron which become completely separated off, their cavities subsequently giving rise to the colomic cavities of the proboscis and of the collar, and the bodycavity of the trunk. By the appearance of a second transverse groove $(B)$ the body of the embryo becomes divided into three parts-an anterior, a middle, and a posterior - these being the beginnings respectively of the proboscis, the collar, and the trunk. The branchial region is marked off by the appearance of a pair of apertures-the first pair of branchial slits $(g . s l$.) - and other pairs subsequently develop behind these.

In the species that undergo a metamorphosis the embryo assumes a larval form termed Tornaria (Figs. 718 and 719). This is somewhat like an Echinoderm larva, with a looped ciliated band, sometimes lobed, sometimes produced into tentacles, running along its anterior part, and a ring of membranellæ (cil.r.), in some cases with a ring of smaller cilia (cil. $r^{2}$ ), round the posterior (anal) end. At the anterior end, in the middle of the pre-oral lobe, is an ectodermal thickening-the apical plate-containing nerve-cells and eye-spots, and, like the apical plate of a trochophore, constituting the nerve- 
centre of the larva : this disappears in the adult. There is a short alimentary canal with mouth and anus. The ciliated bands are lost; an outgrowth is formed to give rise to the proboscis, and a constriction separates it from the collar; the hinder part becomes elongated and narrow to form the body of the animal ; a series of perforations from the exterior give rise to the branchial pouches. A band of thickened epithelium has been described on the wall of the œesophagus and has been supposed to correspond to the structure termed endostyle to be subsequently met with in the Tunicata. The collar-cord is formed by the separating off of the deeperportion of the ectoderm along the middle line : or, in other species, by a sinking down of the whole thickness of the layer, which becomes cut off to form a medullary plate with its edges overlapped by the adjacent ectoderm.

Constituting the class Pterobranchia are only the two genera Cephalodiscus and Rhabdopleura. These both resemble Balanoglossus in having the body divided into three parts or regions-a proboscis with a proboscis-cavity, a collar with a collar-cavity communicating with the exterior by a pair of collar-pores (nephridia in Rhabdopleura), and a trunk with two distinct lateral cavities; and in the presence of a structure resembling a notochord with the same relations to the nervous system as in Balanoglossus. They both differ from Balanoglossus in having the alimentary canal bent on itself, so that the anal opening is

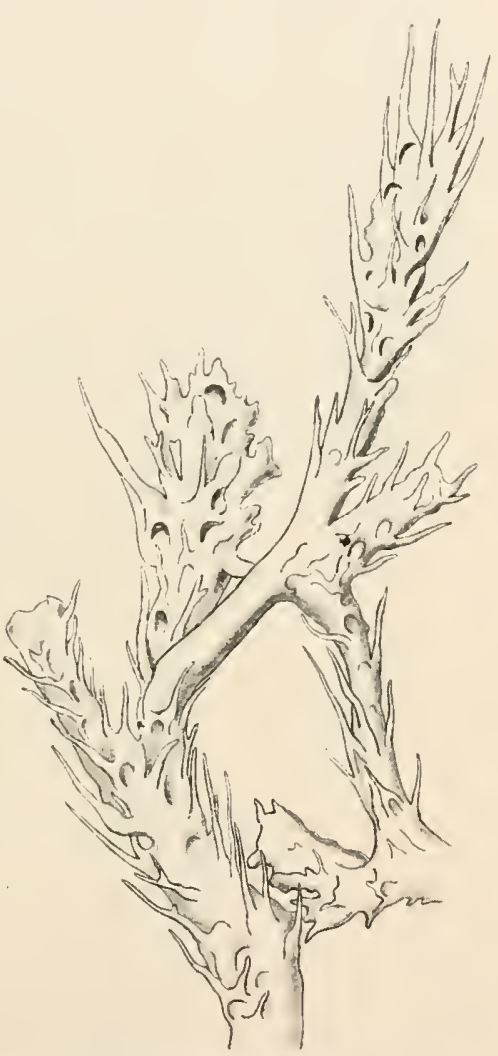

FIG. 720.-Cephalodiscus dodecalophus. Gelatinous investment. (After IIcIntosh.) situated not far from the mouth; in the presence of arms bearing tentacles arising from the collar; and in the comparatively small size of the proboscis. Cephalodiscus, moreover, has only a single pair of apertures which may be regarded as representing the gillslits; while in Rhabdopleura such openings are entirely absent, their places being taken, apparently, by a pair of ciliated grooves. Both forms occur in associations or colonies secreting 
a common case or investment. Both occur in the sea at various depths.

Cephalodiscus has an investment (Fig. 720) in the form of a branching gelatinous structure, which is in some species beset with

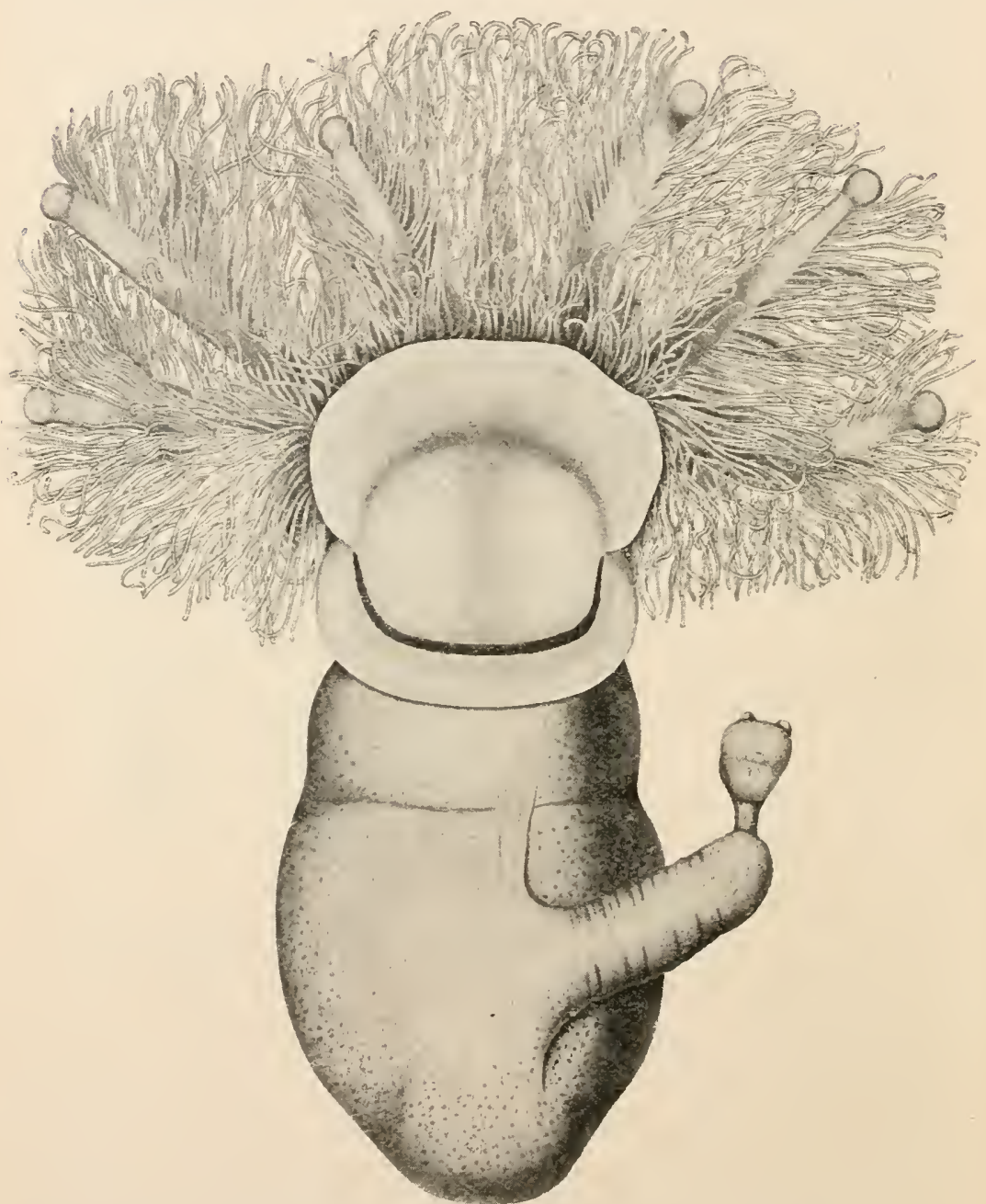

FIG. 721.-Cephalodiscus dodecalophus. Entire zooid. (After McIntosh.)

numerous short filiform processes, and contains a number of tubular cavities with external openings, occupied by zooids. The latter (Fig. 721) are not in organic continuity, so that, though enclosed in a common investment, they do not form a colony in the sense in which the word is used of the Polyzoa or the Hydroid Zoophytes. They 
have a feature in common with such a colony that they inultiply by the formation of buds; but these become detached before they are mature. With the collar-region are connected a series of usually eight to sixteen arms, each beset, except in the case of the male of one species, with numerous very fine pinnately-arranged tentacles, and containing a prolongation of the collar-cavity. The proboscis (Fig. 722, ps.) is a shield-shaped lobe overhanging the mouth; its cavity communicates with the exterior by two proboscis-pores $(p . p$.$) . The cavity of the collar communicates with the exterior by$ a pair of ciliated passages opening by the collar-pores. Behind the collar-region on each side is a small area in which the body-wall and that of the pharynx are coalescent; this area is perforated by an opening - the gill-slit. Cilia occur only on the arms, proboscis, and lateral lips. A nerve-strand, dorsal ganglion, or collar-cord, containing nerve-fibres and ganglion-cells, is situated on the dorsal side of the collar below the epidermis, and is prolonged on to the dorsal surface of the proboscis and the dorsal surface of the arms. On the ventral side of this nerve-strand is a very slender cylindrical cellular cord (nch.) continuous behind with the epithelium of the pharynx: this is supposed to represent the diverticulum of Balano-

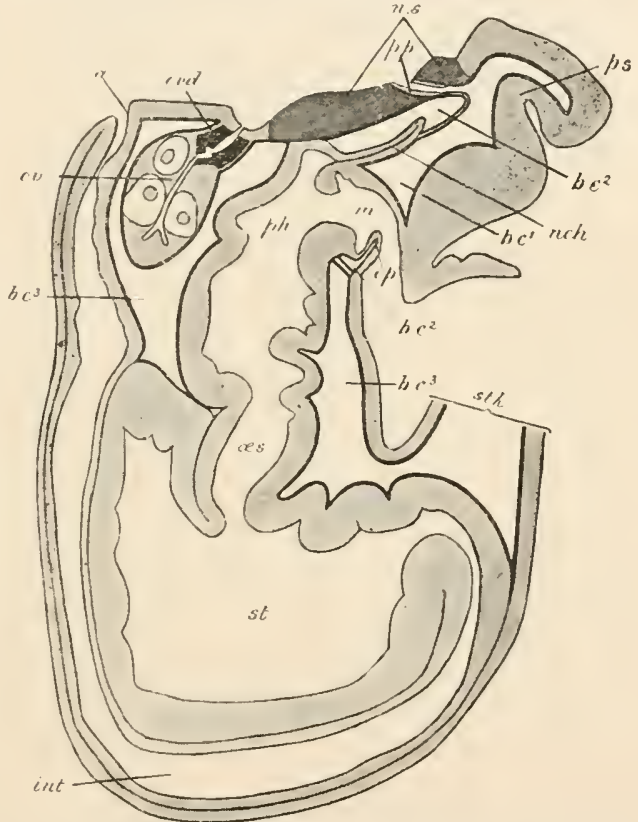

FIG. 72.2. Cephalodiscus. Jiagram, of longitudinal section. $a$. anus; $b c^{1}$. coelome of proboscis; $b c^{2}$. cœlome of collar; $b c^{3}$. ccelome of trunk; int. intestine ; m. mouth; nch. supposed notochord; $n$. $s$. nervestrand : op. operculum ; œs. œsophagus ; ov. ovary ; ovl.oviduct; $p h$. pharynx ; $p$. $p$. proboscis-pore; $p s$. proboscis ; st. stomach ; stk. stalk. (After Harmer.) glossus, and thus to be homologous with the notochord of the Chordata. A blood-vascular system with heart and cardiac sac like those of the Enteropneusta is present. In some species of Cephalodiscus the sexes are united, in most they are separate. The posterior end of the body is diawn out into a sort of stalk on which the buds are developed (Fig. 721). A pair of ovaries (ov.) lie in the trunkcavity, and there is a pair of oviducts (ovd.) lined by elongated, pigmented epithelium. The development, which is direct, without a 
free-swimming larval stage, takes place in passages in the investment. According to one account the seginentation is complete, but unequal, and a gastrula is formed by invagination: according to another, the segmentation is incomplete, and gastrula is formed by delamination. The larva bears a striking resemblance to that of Ectoproct Polyzoa.

Rhabdopleura (Fig. 723) occurs in colonies of zooids organically connected together, and enclosed in, though not in organic con-
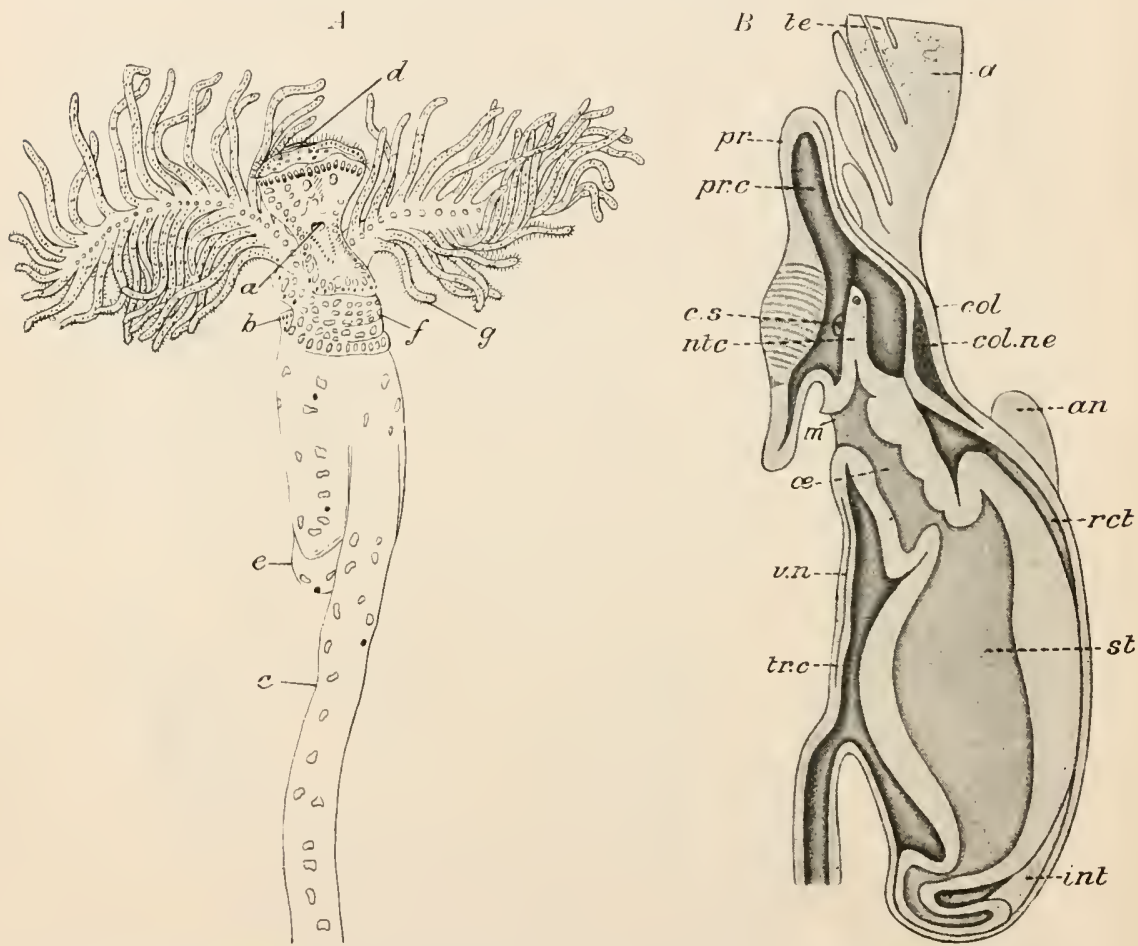

FIG. 723.-Rhabdopleura. A, Entire zooid. $a$, mouth; $b$, anus ; $c$, stalk of zooid ; $d$, proboscis: $e$ intestine ; $f$ anterior region of trunk: $g$, one of the tentacles. (After Ray Lankester.) $B$, Diagram of the organisation : median longitudinal section, seen from the left. a. arm; an. anal prominence; col. collar; col. ne. collar-nerve; $c$. s. cardiac sac; int. intestine ; $m$. mouth ; ntc. "notochord " : a. cesophagus ; pr. proboscis ; pr. c. probosciscœlome; rct. rectum; st. stomach; te. tcntacles; tr. $c$. trunk-colome; $n$. n. ventral nerve. (After Schepotieff.)

tinuity with, a system of branching membranous tubes connected with a creeping stolon. The collar-region bears a pair of hollow arms, each carrying a double row of slender tentacles-the whole supported by a system of firm internal (cartilaginous ?) rods. There are two collar-pores, each leading into a ciliated canal with an internal funnel, and a pair of proboscis-pores. The "notochord" and the nervous system resemble those of Cephalodiscus. The sexes are united. 
Cephalodiscus, of which there are three sub-genera with fifteen species, has been found at various widely separated localities in the Southern Hemisphere (Straits of Magellan, Borneo, Celebes, the Antarctic) : species occur also off the coast of Japan and Korea. Some live in shallow water: none have been found at a greater depth than about 300 fathoms. Rhabdopleura has been found at moderate depths in Norway, Shetland, the North Atlantic, France, the Azores, Tristan d'Acunha, Celebes, and South Australia. It seems doubtful if more than one species occurs.

Affinities.-The inclusion of the Hemichorda in the phylum Chordata is an arrangement the propriety of which is not universally admitted, and is carried out here partly to obviate the inconvenience of erecting the class into a separate phylum. On the whole, however, there seems to be sufficient evidence for the view that, if not the existing representatives of ancestral Chordates, they are at least a greatly modified branch, taking its origin from the base of the chordate tree. The presence of the presumed rudimentary representative of a notochord and of the gill-slits seems to point in this direction. It should, however, be stated that, by some of those zoologists by whom the members of this group have been most closely studied, their chordate affinities are altogether denied. If the Hemichorda are primitive Chordates, the fact is of special interest that they show remarkable resemblances in some points to a phylum-that of the Echinodermata-which it has been the custom to place very low down in the invertebrate series. The tornaria larva of Balanoglossus exhibits a striking likeness to an echinopædium (Vol. I., p. 422), and, though this likeness between the larvæ does not establish near connection, it suggests, at least, that an alliance exists. Between actinotrocha, the larva of Phoronis (Vol. I., p. 351) and tornaria there are some striking points of resemblance; and a pair of gastric diverticula in the former have sometimes been compared with the single notochord or œsophageal diverticulum of the Hemichorda.

\section{SUB-PHYLUM AND CLASS II.-UROCHORDA.}

The Class Urochorda or Tunicata comprises the Ascidians or Sea-Squirts, which are familiar objects on every rocky sea-margin. together with a number of allied forms, the Salpæ and others, all marine and for the most part pelagic. The Urochorda are specially interesting because of the remarkable series of changes which they undergo in the course of their life-history. Some present us with as marked an alternation of generations as exists among so many lower forms; and in most there is a retrogressive metamorphosis almost, if not quite, as striking as that which has been described among the parasitic Copepoda or the Cirripedia. In by far the greater number of cases it would be quite impossible by the study of the adult animal alone to guess at its relationship with the 
Chordata; its affinities with that phylum are only detected when the life-history is followed out, the notochord and other higher structures becoming lost in the later stages of the metamorphosis. Multiplication by budding, so common in the lower groups of Invertebrata, but exceptional or absent in the higher, is of very general occurrence in the Urochorda.

\section{Example of the Class-The Ascidian or Sea-Squirt. (Ascidia.)}

Sea-squirts are familiar objects on rocky sea-shores, where they occur, often in large associations, adhering firmly to the surface of the rock. When touched the Ascidian ejects with considerable

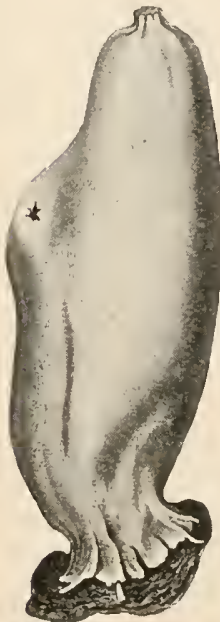

FIG. 72.-Ascidia, entire animal seen from the right side. (After Heriman.) force two fine jets of sea-water, which are found to proceed from two apertures on its upper end. The shape of the Ascidian, however, can only be profitably studied in the case of specimens that are completely immersed in the sea-water, specimens not so immersed always undergoing contraction. In an uncontracted specimen (Fig. 724), the general shape is that of a short cylinder with a broad base by which it is fixed to the rock. The free end presents a large rounded aperture, and some little distance from it on one side is a second of similar character; the former aperture is termed the oral, the latter the atrial. A strong current of water will be noticed, by watching the movements of floating particles, to be flowing steadily in at the former and out of the latter. When the animal is removed from the water both apertures become narrowed, so as to be almost completely closed, by the contraction of sphincters of muscular fibres which surround them. At the same time the walls of the body contract, streams of water are forced through the apertures, and the bulk is considerably reduced.

Body-wall and Atrial Cavity.-The outer layer of the body-wall is composed of a tough translucent substance forming a thick test or tunic (Fig. 725, test). This proves when analysed to consist largely of a substance called tunicine, which is apparently identical with the cellulose already referred to (Vol. I., p. 15) as a characteristic component of the tissues of plants, and of rare occurrence in the animal kingdom. The test of an Ascidian is frequently referred to as a cuticle, and it is a cuticle in the sense that it lies outside the ectoderm and is derived from that layer in the first instance. The cells, however, by the action of which its substance is added to in later stages, seem to be chiefly derived, not 
from the ectoderm, but from the underlying mesoderm, from which they migrate through the ectoderm to the outer surface. These formative cells of the test are to be found scattered through its substance. Running through it are also a number of branching tubes lined with cells, each terminal branch ending in a little bulb-like dilatation. The interior of each tube is divided into two channels by a longitudinal septum, which, however, does not completely divide the terminal bulb. Through these tubes (which are of the nature of looped blood-vessels) blood circulates, passing along one channel, through the terminal bulb, and back through the other channel.

When the test is divided (Fig. 725) the soft wall of the body or mantle (mant.), as it is termed, comes into view ; and the body is found to be freely suspended within the test, attached firmly to the latter only round the oral and atrial apertures. The mantle (body. wall) consists of the ectoderm with underlying layers of connective-tis s u e enclosing muscular fibres. It follows the general shape of the test, and

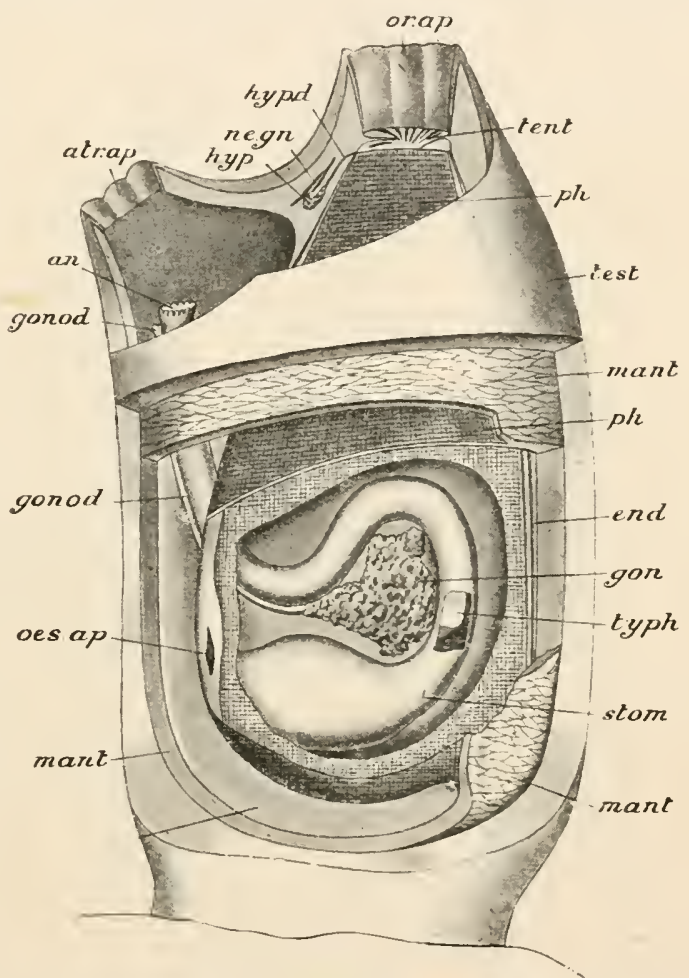

FIG. 725.-Dissection of Ascidia from the right side. The greater part of the test and mantle has been removed from that side so as to bring into view the relation of these layers and of the internal cavities and the course of the alimentary canal, etc. an. anus; atr. ap . atrial aperture ; end. endostyle; gon. gonad; gonod. gonoduct; hyp. neural gland; hyp. $d$. duct of neural gland; mant. mantle; ne. gn. nerve-ganglion ; $o s$. ap. aperture of œsophagus; or. $a p$. oral aperture; $p h$. pharynx; stom. stomach; tent. tentacles; test, test. (A fter Herdman.)

at the two apertures is produced into short and wide tubular prolongations, which are known respectively as the oral and atrial siphons (Fig. 727, or. siph., atr. siph.). These are continuous at their margins with the margins of the apertures of the test, and round the openings are the strong sphincter muscles by which closure is effected. In the rest of the mantle the muscular fibres 
are arranged in an irregular network, crossing one another in all directions, but for the most part either longitudinal or transverse. Within the body-wall is a cavity, the atrial or peribranchial cavity (atr. cav.), communicating with the exterior through the atrial aperture: this is not a cœlome, being formed to a great extent by involution from the outer surface.

Pharynx.-The oral aperture leads by a short and wide oral passage (stomodceum) into a chamber of large dimensions, the pharynx or branchial chamber (Fig. 725, ph.). This is a highly characteristic organ of the Urochorda. Its walls, which are thin and delicate, are pierced by a number of slit-like apertures, the stigmata (Fig. 727, stigm.), arranged in transverse rows. Through these the cavity of the pharynx communicates with the atrial or peribranchial cavity, ${ }^{1}$ which completely surrounds it except along one side. The edges of the stigmata are beset with numerous strong cilia, the action of which is to drive currents of water from the

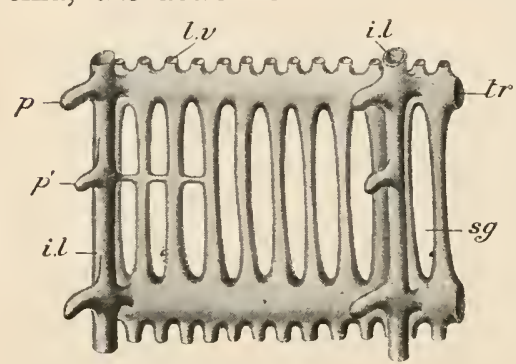

FIG. 726.-Ascidia, a single mesh of the branchial sac, seen from the inside. $i . l$. internal longitudinal bar; $l$. $v$. longitudinal vessel ; $p . p^{\prime}$. papillæ projecting inwards from the branchial bar; $s g$. stigma; tr. transverse vessel. (After Herdman.) pharynx into the atrial cavity. It is to the movements of these cilia lining the stigmata that are due the currents of water already mentioned as flowing into the oral and out of the atrial aperture, the ciliary action drawing a current in through the oral aperture, driving it through the stigmata into the atrial cavity, whence it reaches the exterior through the atrial aperture. The stigmata (Fig. 726) are all vertical in position; those of the same row are placed close together, separated only by narrow vertical bars; neighbouring rows are separated by somewhat thicker horizontal bars; in all of these bars run blood-vessels. Extending across the atrial cavity from the body-wall to the wall of the pharynx are a number of bands of vascular mesodermal tissue, the connectives.

It has been already mentioned that the atrial cavity does not completely surround the pharynx on one side. This is owing to the fact that on the side in question, which is ventral in position, the wall of the pharynx is united with the mantle along the middle line (Fig. 728). Along the line of adhesion the inner surface of the pharynx presents a thickening in the form of a pair of longitudinal folds separated by a groove: to this structure, consisting

1 A distinction is sometimes made between the lateral parts of this space (peribranchial cavities, right and left) and the median unpaired (dorsal) part (atrial cavity, or cloaca), in which the two peribranchial cavities coalesce, and which leads to the exterior through the atrial aperture. 
of the two ventral longitudinal folds with the groove between them, the term endostyle (end.) is applied. 'The cells covering the endostyle are large cells of two kinds-ciliuted cells and glandcells - the former beset at their free ends with cilia, the action of which is to drive floating particles that come within their influence outwards towards the oral aperture, the latter secreting and discharging a viscid mucous matter. Anteriorly the endostyle is continuous with a ciliated ridge which runs circularly round the anteriorend of the pharynx. In front of this circular ridge, and running parallel with it, separated from it only by a narrow groove, is another ridge of similar character: these are termed the peripharyngeal ridges; the groove between them is the peripharyngeal groove. Dorsally, i.e. opposite the endostyle, the posterior peripharyngea l ridge passes into a median, much more prominent, longit udinal ridge, the dorsal

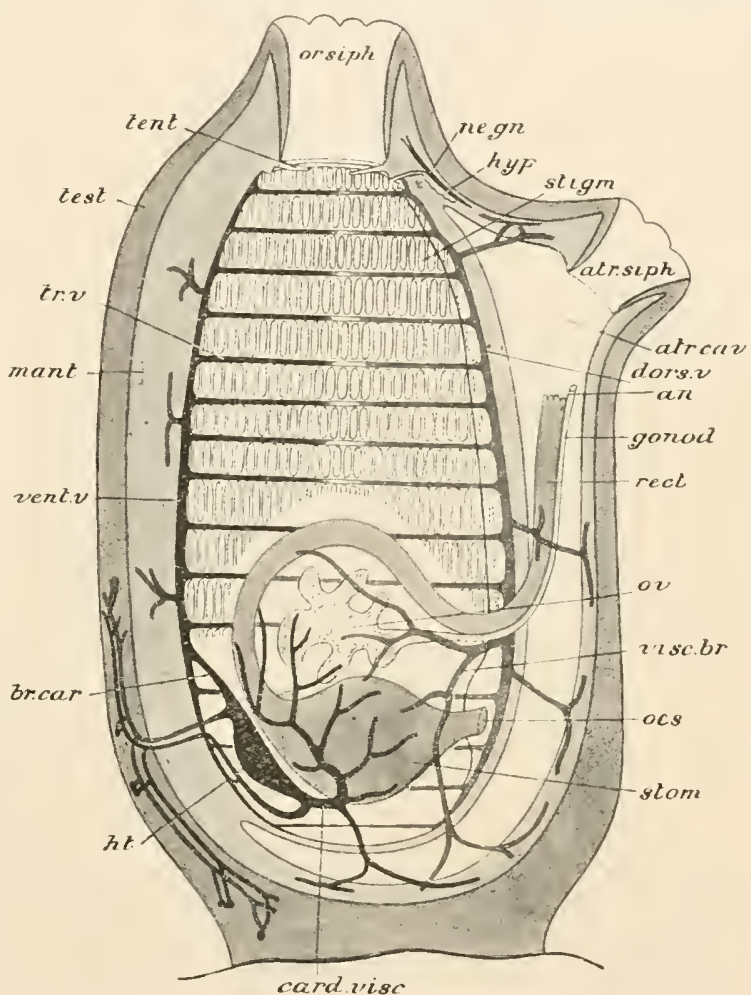

FIG. 727.-Ascidia, diagram of longitudinal section from the left side, the test and mantle removed. an. anus; atr. cav. atrial cavity ; atr. siph. atrial siphon; br. car. branchio-cardiac vessel ; card. visc. cardio-visceral vessel; dors. $v$, dorsal vessel ; gonod. gonoduct; ht. heart; hyp. neural gland; mant. mantle; ne. gn. nerve-ganglion ; ces. cesophagus ; or. siph. oral siphon ; ov. ovary; rect. rectum ; stigm. stigmata ; stom. stomach ; tent, tentacles ; test, test ; $t r . v$. transverse vessel ; vent. $v$. ventral vessel ; visc. br. viscero-branchial vessel. (From Herdman, after Perrier.)

lamina (dors. lam.), which runs along the middle of the dorsal surface of the pharynx to the opening of the œsophagus. In the living animal the lamina is capable of being bent to one side in such a way as to form a deep groove. The mucus secreted by the gland-cells of the endostyle forms viscid threads which entangle food-particles (microscopic organisms of various kinds); the cilia 
of its ciliated cells drive these forwards to the peribranchial groove, around which they pass to the dorsal lamina, and the cilia on the cells of the latter drive them backwards to the opening of the resophagus.

Some little distance in front of the anterior peripharyngeal ridge, at the inner or posterior end of the oral siphon, is a circlet of delicate tentacles (Fig. 725, tent.).

Enteric Canal. - The oesophagus (Figs. 725 and 7.27, oes.) leads from the pharynx (near the posterior end of the dorsal lamina) to the stomach (stom.), which, together with the intestine, lies embedded in the mantle on the left-hand side. The stomach is a

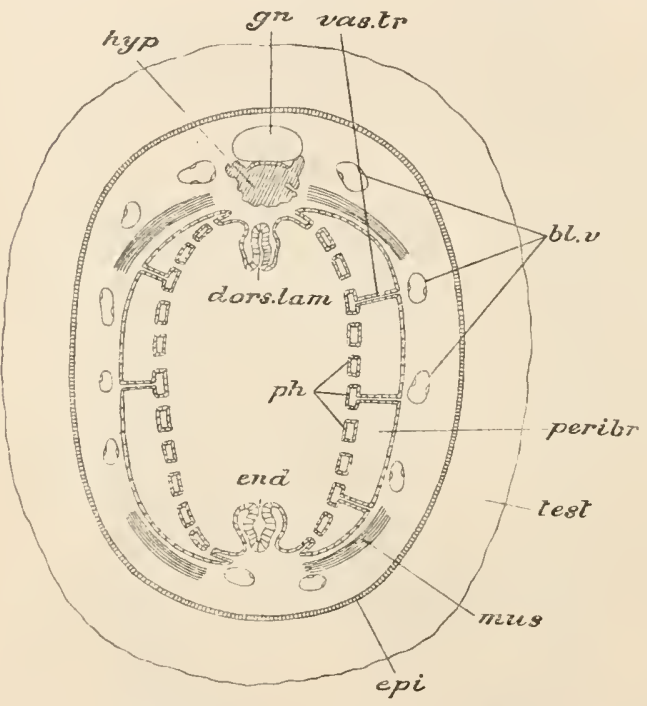

large fusiform sac with tolerably thick walls. The intestine is bent round into a double loop and runs forwards to terminate in an anal aperture (an.) situated in the atrial cavity. Along its inmer wall is a thickening - the typhlosole. There is no liver; but the walls of the stomach are glandular, and a system of delicate tubules, which ramify over the wall of the intestine and are connected with a duct

FIG. 728.-Ascidia, transrerse section. b7. r. blond-rensels; opening into the

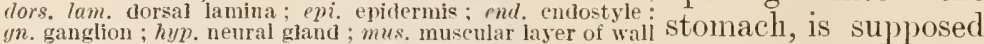
of body; peribr. peribranchial "avity; ph. pharynx; lest, to be of the nature
test; vas. tr. vascular trabeculie. (After.Julin.) of a digestive gland.

The Ascidian has a well-developed blood-system. The heart (Fig. $727, h t$.) is a simple muscular sac, situated near the stomach in the pericardium - a cavity entirely cut off from the surrounding spaces in which the blood is contained. Its mode of pulsation is very remarkable. The contractions are of a peristaltic character, and follow one another from one end of the heart to the other for a certain time; then follows a short pause, and when the contractions begin again they have the opposite direction. Thus the direction of the current of blood through the heart is reversed at regular intervals. There are no true vessels, the blood circulating through a system of channels or sinuses devoid of epithelial lining, and of spaces or lacunæ, forming a hæmocœle: in the 
description that follows, therefore, the word ressel is not used in its strict sense. At each end of the heart is given off a large "vessel." That given off ventrally, the branchio-cardiac vessel (br. car.), runs along the middle of the ventral side of the pharynx below (externally to) the endostyle, and gives off a number of branches which extend along the bars between the rows of stigmata, and give off smaller branches passing between the stigmata of each row. The vessel given off from the dorsal end of the heart - the cardio-visceral (card. visc.)-breaks up into branches which ramify over the surface of the alimentary canal and other organs. This system of visceral vessels or lacunæ opens into a large sinus, the viscero-branchial vessel, which runs along the middle of the dorsal wall of the pharynx externally to the dorsal lamina, and communicates with the dorsal ends of the series of transverse branchial vessels. In addition to these principal vessels there are numerous lacunæ extending everywhere throughout the body, and a number of branches, given off both from the branchiocardiac and cardio-visceral vessels, ramify, as already stated, in the substance of the test. The direction of the circulation through the main vessels differs according to the direction of the heart's contractions. When the heart contracts in a dorso-ventral direction, the blood flows through the branchiocardiac trunk to the ventral wall of the pharynx, and through the transverse vessels, after undergoing oxygenation in the finer branches between the stigmata, reaches the viscero-branchial vessel, by which it is carried to the system of visceral lacunæ, and from

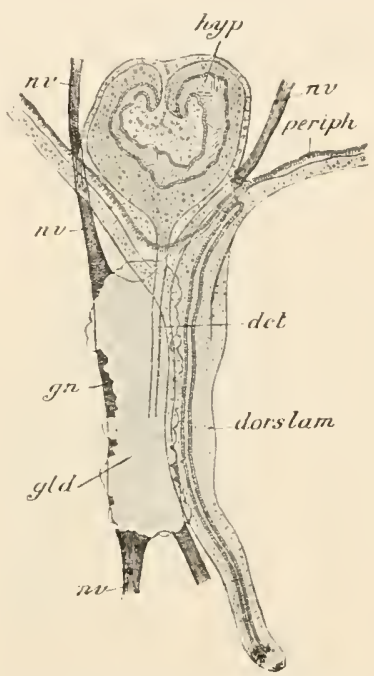

FIG. 729.-Ascidia. Dorsal tubercle, nerve-ganglion, and associated parts as seen from below. det. duet of neural gland; dors. lem. dorsal lamina; gke. neural gland; $g n$. ganglion; hyp. dorsal tubercle; ne., nv. nerves; periph. peri. pharyngeal band. (Ntter Julin.)

these back to the heart by the cardio-visceral vessel. When the contractions take the opposite direction, the course of this main current of the blood is reversed.

The nervous system is of an extremely simple character. There is a single nerve-ganglion (Figs. 725 and 727 , ne. gn., 729, gn., and $730, n . g$.$) which lies between the oral and atrial apertures, embedded$ in the mantle. This is elongated in the dorso-ventral direction, and gives off at each end nerves which pass to the various parts of the body.

Lying on the ventral side of the nerve-ganglion is a body-the neural gland (Figs. 725, 7:27, hyp. ; Fig. 729, gld., and Fig. 730, 
n.gl.) - which has sometimes been correlated with the hypophysis of the Craniata. A duct (Fig. 729, dct., and Fig. 730, gl.d) runs forward from it and opens into the cavity of the pharynx; the termination of the duct is dilated to form the ciliated funnel, and this is folded on itself to form a prominence, the dorsal tubercle, which projects into the cavity of the pharynx. The dorsal tubercle may be a sensory organ: the neural gland may have to do with excretion.

The excretory system seems to be mainly represented by a single mass of clear vesicles, without a duct, lying in the second loop of the intestine. In the interior of these are found concretions containing uric acid.

Reproductive System.-The sexes are united. The ovary and the testis are situated close together on the left-hand
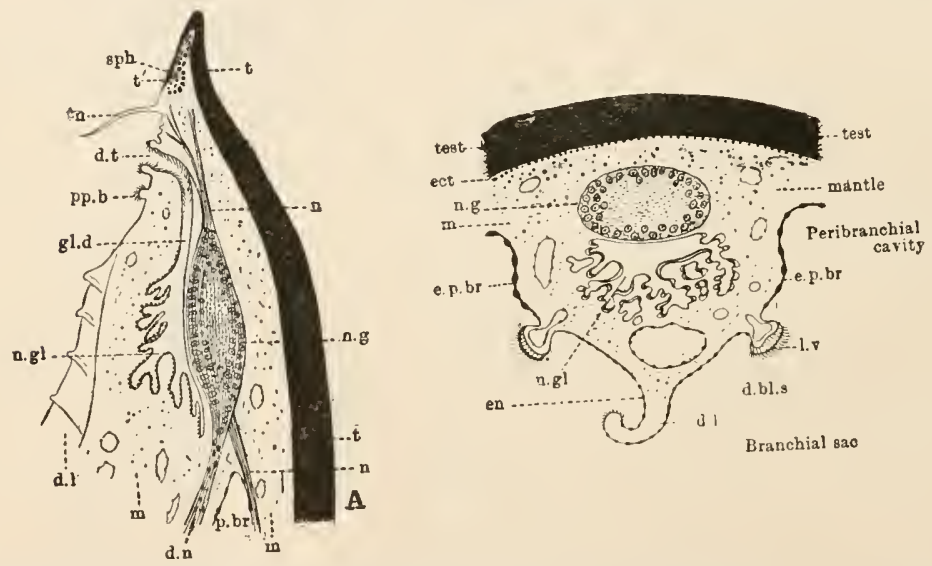

FIG. 730.-Antero-dorsal part of Ascidia, showing the relations of the layers of the body and of the nervous system, $A$, in sagittal section; $B$, in transverse section. $d . b l$. $s$. dorsal bloodsinus; $d . l$. dorsal lamina; $d$. $n$. dorsal nerve; $d$. $t$. dorsal tubercle; ect. ectoderm; $e n$. endoderm; $c . p$. br. epithelium of peribranchial cavity; $g l$. $d$. duct of neural gland ; $l . v$. points to the ciliated epithelium covering a longitudinal vessel of branchial sac (pharynx); $m$. mantle; $n$. nerve; $n$. $g$. ganglion; $n$. gl. neural gland ; $p$. br. peribranchial cavity ; $p p . b$. peripharyngeal bauds; $s p h$. oral sphincter; $t$., $t^{\prime}$. test ; $t n$. tentacle. (After Herdman.)

side of the body in the intestinal loop. Each of them contains a cavity which, like the pericardium and the cavities of the excretory vesicles, forms a part of the original coelome. Continuous with the cavity of each is a duct--oviduct or sperm-duct, as the case may be-which opens into the atrial cavity close to the anus.

The development of the Ascidian is described below (p. 30).

\section{Distinctive Characters and Classification.}

The Urochorda are Chordata in which the notochord is confined to the tail region, and, in all but the Larvacea, is found only in 
the larva. The adults, which for the most part are retrogressively metamorphosed in other respects besides the abortion of the notochord, are sometimes sessile, sometimes free and pelagic ; they frequently form colonies (fixed or free) by a process of budding, and in some instances exhibit a well-marked alternation of generations. The body is enclosed in a test consisting largely of cellulose. The proximal part of the enteric canal (pharynx) is enlarged to form a spacious sac with perforated walls, acting as an organ of respiration. There is a simple heart with a system of sinuses, all devoid of epithelial lining. The colome is represented, apparently, only by the pericardium and by spaces in the interior of the gonads and of the renal organ. The sexes are united. The larva is always free-swimming, and is nearly always provided with a caudal appendage.

Three orders of Urochorda are recognised :-

\section{Order 1.-Larvacea.}

Free-swimming pelagic Tunicata with a caudal appendage supported by a skeletal axis or notochord. The test is represented by a relatively large temporary envelope, the "house," formed with great rapidity as a secretion from the surface of the ectoderm and frequently thrown off and renewed. The pharynx has only two stigmata, and these lead directly to the exterior. There is no atrial or peribranchial cavity. The principal nerve-ganglion gives off a nerve-cord with ganglionic enlargements running to the tail, along the dorsal aspect of which it passes to the extremity. There is no reproduction by budding, and development takes place without metamorphosis.

This order contains only a single family, the Appendiculariida, with about nine genera, including Appendicularia and Oikopleura.

\section{Order 2.-Thaliacea.}

Free-swimming Tunicata, sometimes simple, sometimes colonial, never provided with a caudal appendage in the adult condition. The test is a permanent structure. The muscular fibres of the body-wall are arranged in complete or interrupted ring-like bands, or diffusely. The pharynx has either two large or many small stigmata leading into an atrial cavity which communicates with the exterior by the atrial aperture. There is usually an alternation of generations ; there may or may not be a tailed larval stage.

\section{Sub-Order a.-Cyclomyaria.}

Thaliacea with a cask-shaped body, having. the oral and atrial apertures at opposite ends, and surrounded by a series of complete rings of muscular fibres. There is a tailed larval stage. 
This sub-order contains only one family, the Doliolide, with the three genera, Doliolum, Anchinia, and Dolchinia.

\section{Sub-Order b.-Hemimyaria.}

Thaliacea with a more or less fusiform body, with sub-terminal oral and atrial apertures. The muscular fibres are arranged in bands which do not form complete rings. There is no tailed larval stage.

This sub-order is probably best looked upon as comprising only one family, the Salpidce.

\section{Order 3.-Ascidiacea.}

Mostly fixed Tunicata, either simple or forming colonies by a process of budding, and, in the adult condition, never provided with a tail. The test is a permanent structure, usually of considerable thickness. The muscular fibres of the mantle (bodywall) are not arranged in annular bands. The pharynx is large, and its walls are perforated by numerous stigmata leading into a surrounding atrium or peribranchial cavity, which communicates with the exterior by an atrial aperture. Most undergo a metamorphosis, the larva being provided with a caudal appendage, supported by a notochord similar to that of the Larvacea.

\section{Sub-Order a.--Ascidice simplices.}

Ascidians in which, when colonies are formed, the zooids are not embedded in a common gelatinous mass, but possess distinct tests of their own. They are nearly always permanently fixed and never free-swimming.

Including all the larger Ascidians or Sea-Squirts.

\section{Sub-Order b.-Ascidia compositce.}

Fixed Ascidians which form colonies of zooids, embedded in a common gelatinous material without separate tests.

This order includes Botryllus, Amarcecium, Diazona, and a number of other genera.

\section{Order 4.-LuCIDA.}

Pelagic Tunicata which reproduce by budding, so as to give rise to hollow cylindrical colonies, open at one or both ends, having the zooids embedded in the gelatinons wall in such a manner that the oral apertures open on the outer, the atrial on the inner surface of the cylinder. There is no tailed larval stage.

This order comprises only one family, the Pyrosomidce, with one genus, Pyrosoma. 


\section{Systematic Position of the Example.}

The genus Ascidia, of which there are very many species, is a member of the family Ascidiida of the Ascidice simplices. The Ascidiidæ differ from the other families of simple Ascidians by the union of the following characters :-The body is usually sessile, rarely elevated on a peduncle. The oral aperture is usually 8 -lobed and the atrial 6-lobed. The test is always of gelatinous or cartilaginous consistency. The wall of the pharynx is not folded; the tentacles are simple and filiform. The gonads are placed close to the intestine.

The genus Ascidia is characterised by having the oral and atrial apertures not close together, by the dorsal lamina being a continuous undivided fold, and by the ganglion and neural gland being situated at a little distance from the dorsal tubercle.

\section{General Organisation.}

General Features.-The Larvacea are minute transparent animals, in shape not unlike tadpoles, with a rounded body and a long tail-like appendage attached to the ventral side. At the extremity of the body most remote from the tail is the aperture of the mouth. This leads into a tolerably wide pharynx (Fig. 732, ph.), in the ventral wall of which (except in Kowalevskia) is

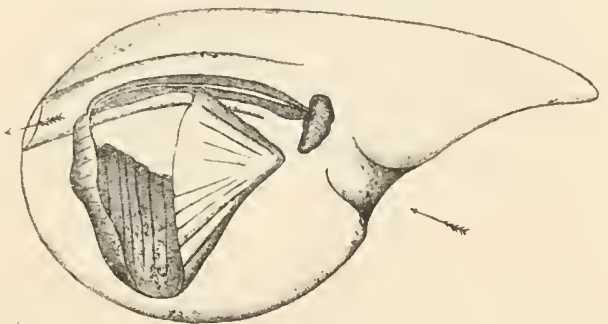

FIG. 731.-Oikopleura in "house." The arrows show the course of the current. (From Herdman, after Fol.)

an endostyle similar to that of the simple Ascidian, but comparatively short. Round the pharynx there run obliquely two bands covered with strong cilia-the peripharyngeal bands, which join a median dorsal ciliated band. On the ventral side of the pharynx there are two ciliated openings - the stigmata (stig.), which communicate with the exterior by short passages-the atrial canals, situated on either side behind the anus. The axis of the tail is occupied by a cylindrical rod-the notochord or urochord (noto.).

A remarkable peculiarity of the Larvacea is the power which they possess of secreting from the surface, by the agency of certain specially modified epidermal cells, a transparent envelope which is frequently discarded and quickly renewed. The chief object of this structure seems to be the capture of the very minute planktonorganisms on which the Larvacea feed. In Oitiopleura (Fig. 731) the "house" is a comparatively large structure within which the 
animal is enclosed: undulatory movements of the tail cause a current of water to flow in through a pair of incurrent apertures and out through a single excurrent aperture ; the former are closed by lattice-work of fine threads, preventing the passage of any but the smallest organisms. In the interior is an elaborate apparatus for filtering out the minute organisms from the water as it passes through. In Appendicularia and Kowalevskia the house also encloses the animal : in Fritillaria it does not do so.

Among the simple Ascidians there is a considerable degree of uniformity of structure, and little need be added here to the account given of the example. The shape varies a good deal : it is sometimes cylindrical, sometimes globular, sometimes compressed; usually sessile and attached by a broad base, often with root-like processes, but in other cases (e.g. Boltenia) elevated on

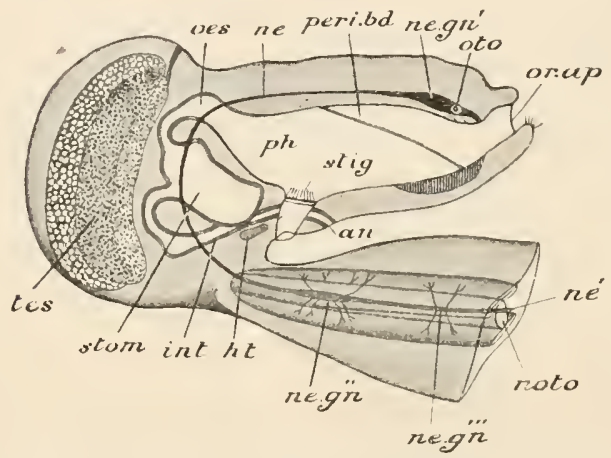

Fig. 732.-Diagram of Appendicularia from the right side. an. anus ; $h$. heart; int. intestine; ne. nerve ; $n e^{\prime}$. caudal portion of nerve; ne. gn.' principal nerveganglion; ne. gn.", ne. gn." first two ganglia of nerve of tail ; noto. notochord; as. cesophagus : or. ap. oral aperture ; oto otocyst (statocyst); peri. $b d$. neripharyngeal band; $p h$. pharynx ; tes. test is ; stig. one of the stigmata ; stom. stomach. (After Herdman.) a longer or shorter stalk. Most are solitary; but some(the so-called social Ascidians) multiply by budding, stolons being given off on which new zooids are developed, so that associations or colonies are formed; but the connection between the zooids is not close, and their tests remain distinct and separate. The testvaries considerably in consistency, being sometimes almost gelatinous, transparent or translucent, sometimes tough and leathery, occasionally hardened by encrusting sand-grains or fragments of shells, or by spicules of carbonate of lime. Calcareous spicules may be developed in the substance of the mantle. The apertures always have the same position and relations, varying only in their relative prominence. The pharynx varies in its size as compared with the rest of the internal organs, in the position which it occupies with regard to the various parts of the alimentary canal, and in the number and arrangement of the stigmata. The tentacles are sometimes simple, sometimes compound; and the dorsal lamina may or may not be divided up into a system of lobes or languets (Fig. 734, lang.).

In the composite Ascidians the zooids are embedded in a common gelatinous mass formed of their united tests. The gelatinous colony thus formed is sometimes flat and encrusting, sometimes branched 
or lobed, sometimes elevated on a longer or shorter stalk. In certain forms the gelatinous substance is hardened by the inclusion in it of numerous sand-grains. The arrangement of the zooids presents great differences. Sometimes they occur irregularly, dotted over the entire surface without exhibiting any definite arrangement; sometimes they are arranged in rows

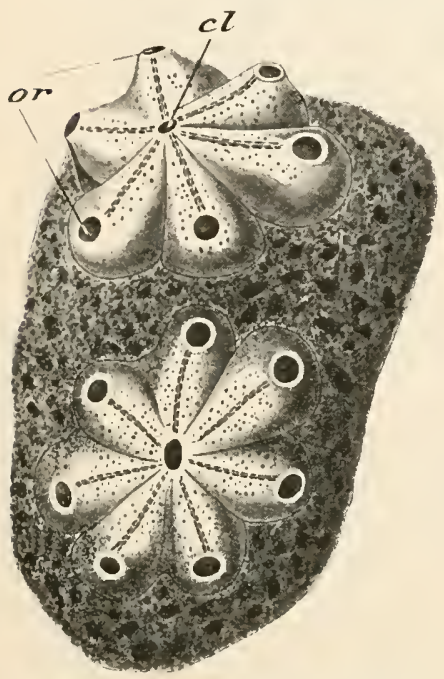

FIG. 733.-Botryllus violaceus. or. oral apertures; $\mathrm{cl}$. opening of common cloacal chamber. (After Milne-Edwards.)

or regular groups; in Botryllus (Fig. 733) they form star-shaped, radiating sets around a common cloacal chamber into which the atrial apertures of the zooids lead, while the oral apertures are towards their outer ends. In essential structure the zooids of such colonies (Fig. 734) resemble the simple Ascidians.

In the free-swimming pelagic Doliolum (Fig. 735) the shape is widely different from that of the ordinary fixed forms. The body
FIG. 734.-Diagram of a zooid of a colony of Composite Ascidians, in which the zooids are in pairs, as seen in a vertical section of the colony. an. anus; at. atrium; at'. atrium of adjoining zooid ; $c l$. cloaca common to the two zooids; end. endostyle; gld. digestive gland; $g n$. nerve-ganglion; ht. heart; hyp. neural gland; lang. languets; mant. mantle ; or. ap. oral aperture; ov. ovary; periph. peripharyngeal band ; ph. pharynx; rect. rectum ; stom. stomach ; te. testis; tent. tentacles; tst. test, or common gelatinous mass; $v$. $d$. vas deferens. (After Herdman.)

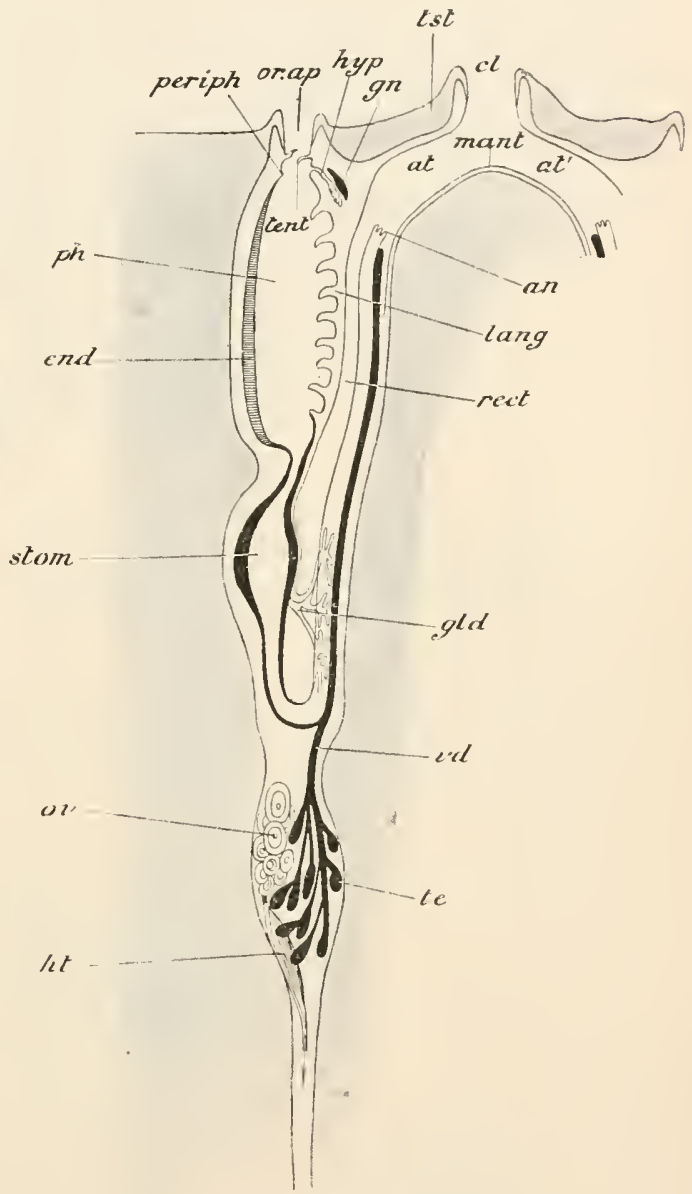


is cask-shaped, surrounded, as by hoops, by a series of annular bands of muscular fibres (mus. bds.). The oral and atrial apertures (or. ap., atr. ap.), instead of being situated near together at the same end of the body, are placed at opposite extremities, and the relations of the various organs have undergone a corresponding

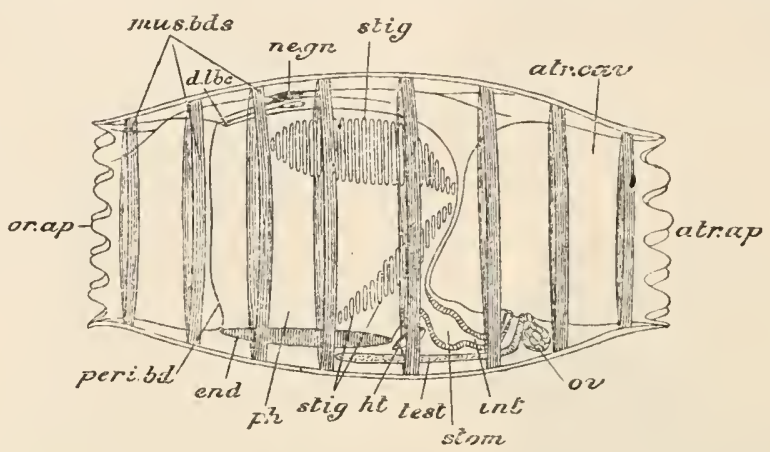

FIG. 735.-Doliolum. Diagram of the sexual form. atr. ap. atrial aperture surrounded by lobes; atr. cav. atrial cavity; $d$. thc. dorsal tubercle ; end. endontyle; $h$. heart ; int. intestine; mus. bds. muscular bands; ne.gn. nerve-ganglion; or, ap oral apert ure ; on. ovary ; peri. bd. peripharyngea! band; $p h$. pharynx; stig. stigma; stom. stomilch; test. testis. (After Herdman.)

modification. The test is thin and transparent. Surrounding each opening is a series of lobes-the oral and atrial lobes-in which there are sense-organs ; and the first and last of the muscular hoops serve as sphincters for the two orifices. The oral aperture

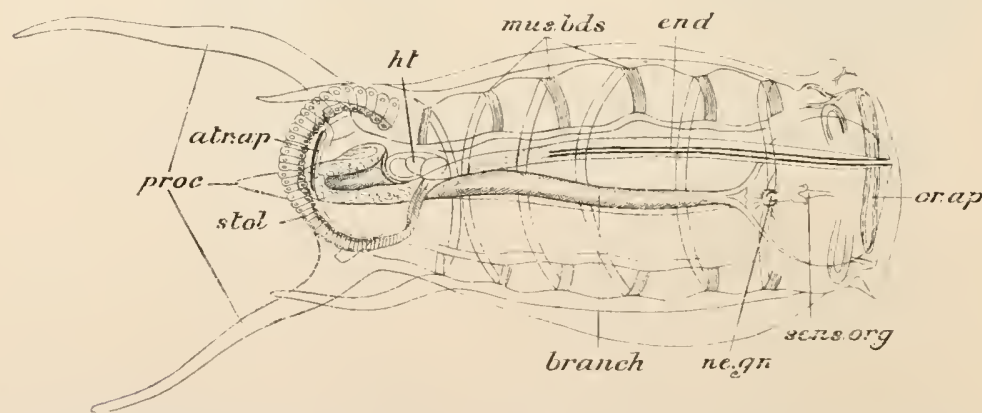

FIG. 736. - Salpa democratica, asexual form, ventral view, atr, ap. atrial aperture; branch. dorsal lamina ; end. endostyle; ht. heart ; mus. bds. muscular bands ; ne. gn. nerve-ganglion ; or. ap. oral a perture ; proc. processes at the posterior end; sens. org. sensory organ (ciliated tumnel and languet); stol. stolon. (After Vogt and Jung.)

leads into a wide pharyngeal sac $(p h$.) occupying at least the anterior half of the body; the posterior wall of the pharynx alone is usually perforated by stigmata (stig.). An endostyle (end.) is present, and a peripharyngeal band; but there is no dorsal lamina. Doliolum moves through the water by the contractions of the 
muscular bands, which have the effect of driving the water backwards out of the branchial sac.

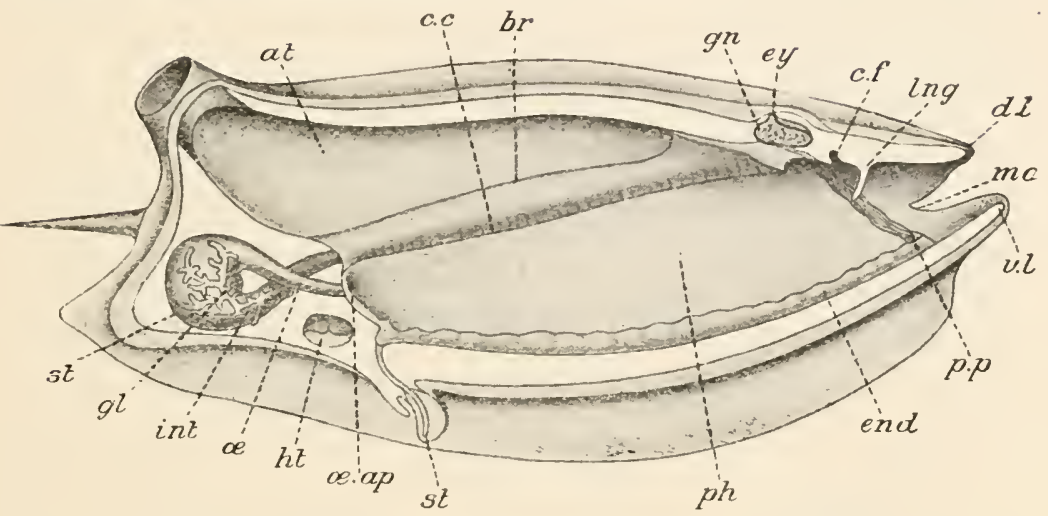

FIt. 737.-Salpa. Lateral view of a section-which is sagittal (longitudinal, vertical and median) in the oral two-thirds, and oblique in the atrial third. at. atrial cavity : $b r$. branchia ; $c$. $c$. ciliated crests on the edge of the branchia ; $c$. $f$. ciliated fumel ; $d$. $l$. dorsal lip ; end. endostyle ; $c y$. eye : $g l$. digestive gland; $g n$. ganglion; $h t$. heart; int. intestine ; Ing. languet : mo. mouth; $a$. cesophagus; $r$. ap. oesophageal aperture : $p h$. pharynx; $p p$. peripharyngeal band ; st. (right) stolon; st. (left) stomach; $v$. l. vent ral lip. (titer Delage and Hérollard.)

Salpa (Figs. 736-737) is nearly allied to Doliolum in its external features and internal structure. It has a fusiform body, usually somewhat compressed laterally, and with the oral and atrial apertures nearly terminal ; but the miuscular bands do not form complete hoops. The pharyngeal and atrial cavities take up the greater part of the space in the interior of the body, where they form an almost continuous cavity, being separated from one another only by an obliquely running vascular band, which represents the dorsal lamina of the fixed Ascidians and is frequently termed the branchia.

Octacnemus, sometimes regarded as allied to Salpa, appears to be fixed, is colonial in one

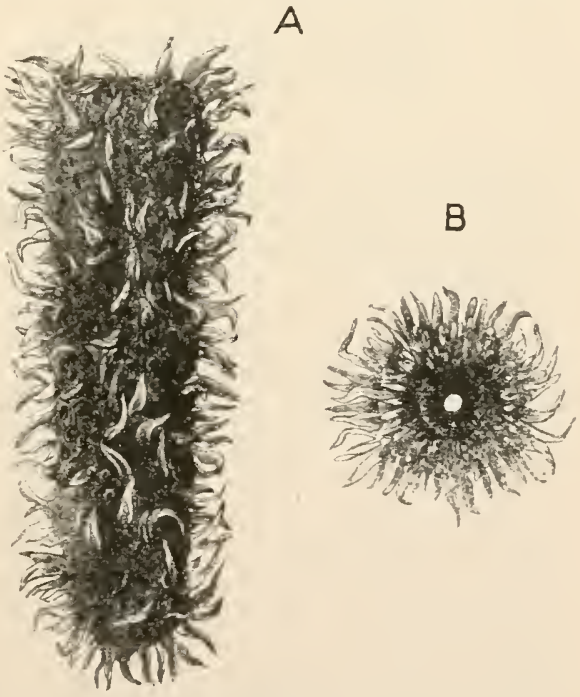

Fig. 73s.-Colony of Pyrosoma. A, sile view; $B$, end view. (After Herdman.) 
species, and has the oral and atrial apertures towards one end of the body, which is somewhat discoid, with its margin produced into eight tapering processes. It has no eye. In all probability Octacnemus is more nearly related to the social Ascidians (p. 24) than to Salpa.

Pyrosoma (Fig. 738) is a colonial Tunicate, the colony assuming the form of a cylinder, the internal cavity of which, closed at one end, open at the other, serves as the common cloaca for all the zooids. The oral apertures (Fig. 739, or. ap.) of the zooids are situated on the outer surface of the cylinder on a series of papillæ. The colonies of Pyrosoma, which may be from two or three inches to four feet in length, are pelagic, and are brilliantly phos-

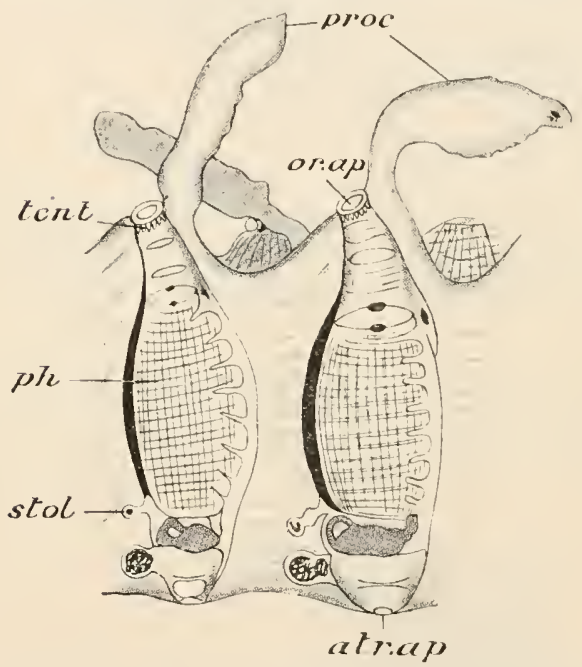

FIG. 739.-Part of a section through a Pyrosoma colony. atr. ap. atrial aperture; or. ap. oral aperture; proc. processes of test on outer surface of colony : ph. pharynx; stol. stolon on which are developed buds giving rise to new zooids; tent. tentacles. (After Herdman.) forms a relatively small dark mass-the so-called nucleus-towards the posterior end of the body; it consists of cesophagus, stomach, and intestine, the anal aperture being situated in the peribranchial or atrial part of the internal cavity.

The heart in all has the simple structure already described in the simple Ascidian. In one of the genera of Larvacea (Kowalevskia) it is absent.

The nervous system in Appendicularia consists of a cerebral ganglion (Fig. 732, ne. $g n^{\prime}$.) on the dorsal side of the mouth, of a dorsal nerve which passes from this to a caudal ganglion (ne. $g n^{\prime \prime}$.) at the root of the tail, and of a caudal nerve $\left(n e^{\prime}\right.$.) which extends 
from this to the extremity of the tail, presenting at intervals slight enlargements from which nerves are given off. An otocyst or statocyst (oto.) is placed in close relation to the cerebral ganglion, and close to it also is a ciliated funnel; but there is no neural gland opening into the pharynx. In one species of Oikopleura a simple light-perceiving organ, without pigment, is incorporated with the statocyst. In the simple Ascidians, as we have seen, there is a single flattened ganglion, representing the cerebral ganglion of Appendicularia, situated between the oral and atrial apertures; and the same holds good of the composite forms. Many of the simple Ascidians have pigment-spots, probably of a sensory character, around the oral and atrial apertures. The dorsal tubercle is always present, but varies in shape in accordance with variations in the form of the ciliated funnel, which opens on it usually in

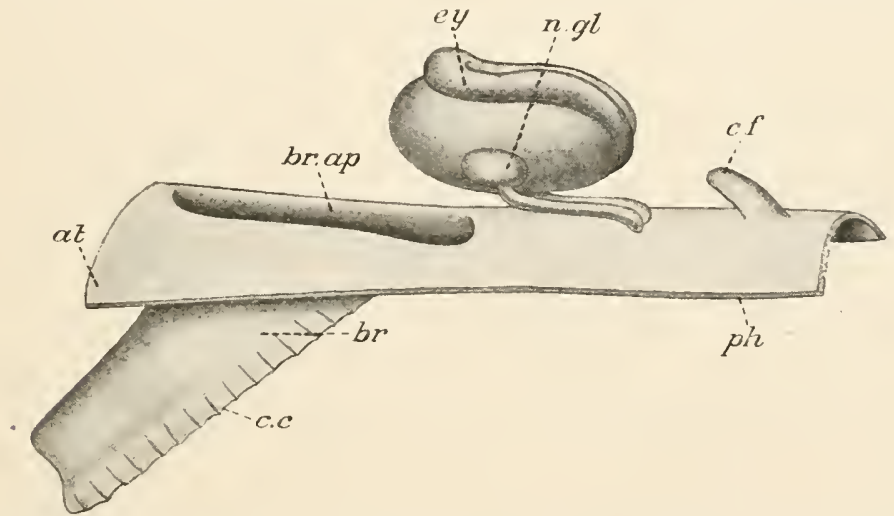

FIG. 740.-Salpa. Diagrammatic lateral view of the ganglion and neighbouring parts. at. wall of atrial cavity; $b r$. branchia: $b r$. ap. aperture of branchia; $c$. c ciliated crests of branchia; $c$. $f$. ciliated funnel; ey. eye; $n$. gl. gland (paired) that may represent neural gland; $p h$. wall of pharynx. (After Delage and Hérouard.)

conjunction with the duct of the neural gland, of which it forms the terminal part. The opening may be divided into several smaller apertures by fusion of its lips: rarely the duct gives off branches with independent openings. The tubercle with the ciliated funnel is supplied with nerve-fibres from the ganglion, and is probably a sensory organ of some kind. The neural gland is usually below the ganglion, but may be situated above it or on one side. Sometimes it coalesces with it.

In Salpa and Doliolum there is also a single ganglion (Figs. $735,736,737$, and 740) situated dorsally, giving off nerves to the various parts of the body. Salpa has a single tentacle, the so-called languet (Fig. 737, lng.), absent in Doliolum. In Salpa there is a median horse-shoe-shaped eye (Figs. 737, 740), and sometimes smaller accessory eyes. In Doliolum an eye is not developed, but there is a pair of otocysts or statocysts. A neural gland and duct 
with ciliated funnel are present in Doliolum ; but in Salpa, though there are a pair of glands which occupy a position similar to that occupied by the neural gland (Fig. $740, n . g l$.), their correspondence with the latter is not established, and their ducts have no connection with the ciliated fummel.

In the simple Ascidian we have seen that the renal organ consists of a number of large clear vesicles situated in the loop of the intestine and devoid of duct. In some forms the terminal portion of the sperm-duct has glandular walls in which concretions of nric acid have been found. The neural gland is by some zoologists looked upon as liaving an excretory function, but there is no positive evidence in favour of this view, and no definite conclusion has yet been reached as to the function which it performs.

Reproductive System.-The Urochorda are hermaphrodite. Ovary and testis are in all cases simple organs placed in close relation with one another. In Appendicularia (Fig. 732) they are situated in the aboral region of the body. In the simple Ascidians they may be either single or double, and their ducts, sometimes very short, sometimes more elongated. open close together into the atrial cavity. In Pyrosoma there are no gonoducts, the ovarywhich contains only a single ovum - and the testis being lodged in a diverticulum of the peribranchial cavity. In Salpa also the ovary contains usually only a single ovum : ovary and testis lie in close relation to the alimentary canal in the "nucleus," and their short ducts open into the peribranchial cavity. In Doliolum the elongated testis and oval ovary have a similar position to that which they occupy in Salpa, but the ovary consists of a number of ova.

Development and Metamorphosis.-In the Ascidiacea impregnation usually takes place after the ova have passed out from the atrial cavity. But in a few simple, and most, if not all, compound forms impregnation takes place in the atrium or in a special outgrowth of the latter serving as a brood-sac, and the ovum remains there until the tailed larval stage is attained. In certain composite forms there is a coalescence of the investing layers of the ovum with the wall of the atrium, forming a structure analogous to the placenta of Mammals and designated by that term. Selfimpregnation is usually rendered impossible by ova and sperms becoming mature at different times; but sometimes both ripen simultaneously, and self-impregnation is then possible.

A somewhat complicated series of membranes invests the ovum. The immature ovarian ovum is enclosed in a layer of flat cellsthe primitive follicle-cells-derived from indifferent cells of the ovary. On the surface of this is developed a structureless basal membrane. The follicle-cells increase by division and soon form a sphere of cubical cells. Certain of the cells migrate into the 
interior of the sphere so as to form a layer on the surface of the ovum. Others penetrate into the latter so as to lie in the superficial strata of the yolk. The laver of cells on the surface of the ovum are termed the test-cells (Fig. $741, e$ ) : they afterwards develop on the outer snrface a thin structureless laver, the chorion $(d)$, and internal to them is formed a gelatinous layer $(x)$ throngh which the test-cells in a degenerated condition become seattered. Meantime, external to the folliele-cells, between them and the basal membrane, has appeared a layer of flattened epithelial cells ; this. with the basal membrane, is lost before the egg is discharged. In all the simple Aseidians, with the exception of the few in which development takes place internally, the protoplasm of the follielecells (Fig. $741, c$ ) is greatly vaeuolated so as to appear frothy, and the eells become greatly enlarged, projeeting like papillx on the surface and buoying up the developing ovum.

Segmentation is eomplete and approximately equal, but in the eight-eell stage four of the cells are smaller and four larger: the smaller, situated on the future dorsal side, are the beginning of the endoderm; the four larger form the greater part, if not the whole, of the eetoderm. In the following stages the ectoderm eells multiply more rapidly than the endoderm, so that they soon beeome the smaller. In the sixteen-celled stage the embryo (Fig. $742, A$ ) lias the form of a flattened blastula

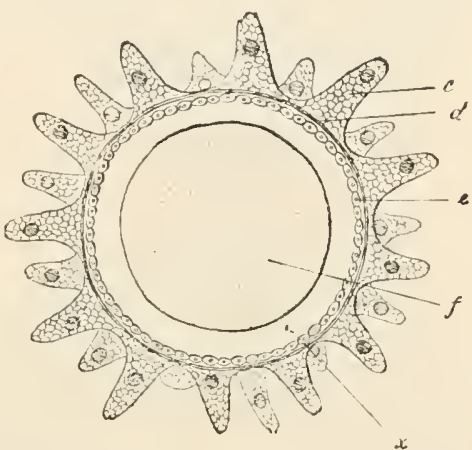

Fit. T 11.-Ascidian (Ciona). Mature egg irom the oviduct after the basal membrane and layer of flattened cells have been thrown off. $c$. follicle-cells ; $d$, chorion; $c$, test-cells : $f$, ovmu : $x$, gelatinous layer. (From Korschelt and Heider, after Kupfer.) (placula) with ectoderm on one side and endoderm on the other, and with a small segmentation-cavity. The transition to the gastrula stage is in most Aseidians effeeted by a process intermediate in charaeter between embolie and epibolic invagination; in some the invagination is of a distinetly epibolie character. In the former case the eetoderm cells eontinue to inerease more rapidly than the endoderm, the whole embryo beeomes eurved, with the coneavity on the endodermal side, and the eetoderm extends over the endoderm, the two layers coming to lie in close contaet and the segmentation-eavity thus becoming obliterated. The concavity deepens until the embryo assumes the form of a saucer-shaped gastrula with an archenteron and a blastopore which is at first a very wide aperture extending along the whole of the future dorsal side. The blastopore gradually beeomes eonstrieted (Fig. 742, B) -the closure taking plaee from 
before backwards, and the opening eventually being reduced to a small pore at the posterior end of the dorsal surface.

The embryo elongates in the direction of the future long axis. The dorsal surface becomes recognisable by being flatter, while the ventral remains convex. The ectoderm cells bordering the blastopore are distinguished from the rest by their more cubical shape ; these cells, which form the earliest rudiment of the nervous system, become arranged, as the blastopore undergoes contraction,
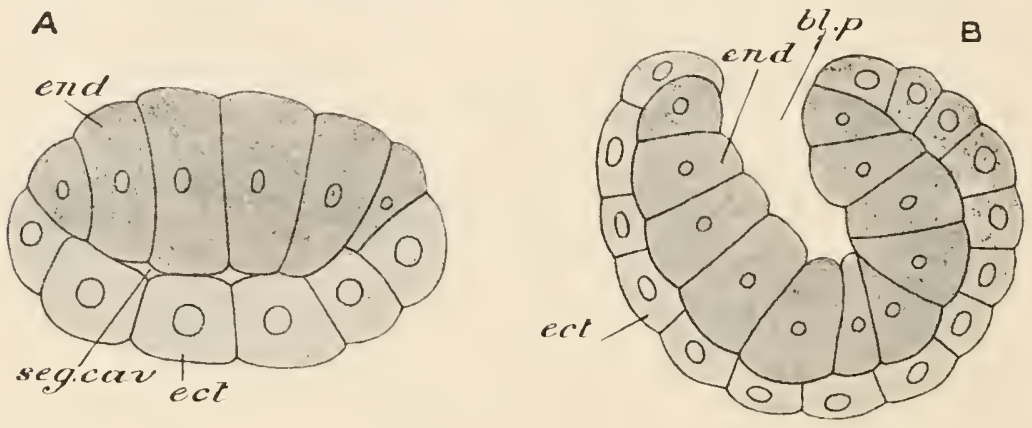

C
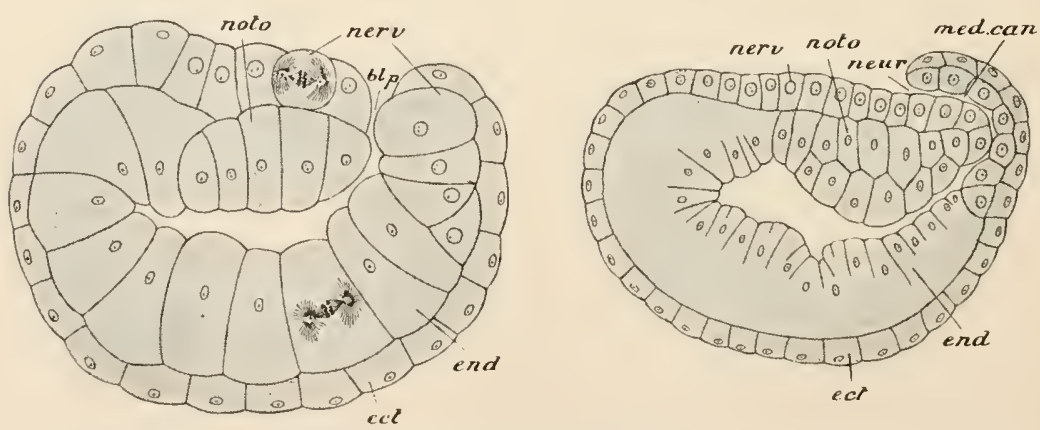

F10. 742.-Early stages in the development of Clavellina. $d$, flattened blastula ; $B$, early gastrula ; $C$, approximately median optical section of more advanced gastrula in which the blastopore has become greatly reduced and in which the first rudiment of the notochord is discernible $; D$, similar view of a later larva in which the medullary canal has begun to be closed in posteriorly. Ul. $p$. blastopore ; ect. ectoderm; end. endoderm; med, can. medullary canal ; nerv. cells destined to give rise to the nerve-cord; neur. neuropore; noto. notochord ; seg. cav. segmentation cavity. ( $A$ and $B$ from Korschelt and Heider, after Seeliger ; $C$ and $D$ ) after Van Beneden and Julin.)

in the form of a plate - the medullary plate - on the dorsal surface. On the surface of this plate appears a groove-the medullary groove -bounded by right and left medullary folds, which pass into one another behind the blastopore.

The medullary folds grow upwards and inwards over the medullary groove, and unite together $(D)$, the union beginning behind and progressing forwards in such a way as to form a canal, the neurocoele, in the hinder portion of which is the opening of the 
blastopore. In this process of closing-in of the medullary groove the fold which passes round behind the blastopore takes an important part, growing forwards. over the posterior portion of the canal. The blastopore, thus enclosed in the medullary canal, persists for a time as a small open ing-the neurenteric canal -by which the neurocoele and enteric cavity are placed in communication. At the anterior end of the medullary canal, owing to its incomplete closure in this region, there remains for a time an opening - the neuropore (Fig. 743 , neur.) leading to the exterior.

A notochord (Figs. 742, $C, D$, 743 and 744, noto.) is formed from certain of the cells of the wall of the archenteron along the middle line of the dorsal side. These are arranged to form an elongated cord of cells which
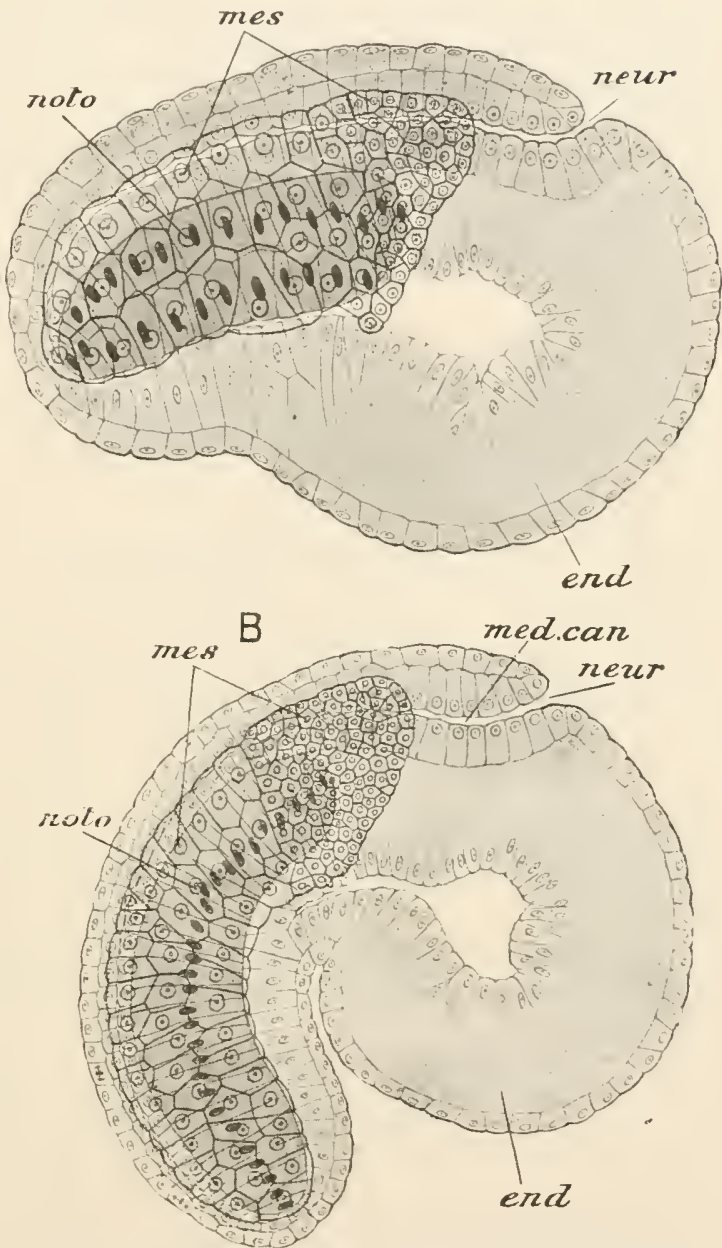

Fig. 743,-Later stages in the development of Clavellina. A, approximately median optical section of a larva in which the medullary canal (neurocele) has become enclosed throughout, communicating with the exterior only by the neuropore at the anterior end and with the archenteron by the neurenteric canal ; $B$, larva with a distinct rudiment of the tail and well-formed mesoderm-layer and notochord. Letters as in preceding figure : in addition, mes. mesoderm. (After Van Beneden and Julin.)

becomes completely constricted off from the endoderm of the wall of the archenteron, and comes to lie between the latter and the 
medullary groove. Laterally certain cells of the endoderm, in close relation to those forming the rudiment of the notochord, divide to give rise to a pair of longitudinal strands of cells - the rudiments of the mesoderm (Fig. 743, mes.). During this process of mesodernformation, there are no diverticula developed from the archenteron.

The embryo (Fig. 743, B) now becomes pear-shaped, the narrow part being the rudiment of the future tail. As this narrow portion elongates, the part of the enteric cavity which it contains soon disappears, coming to be represented only by a strand of endoderm cells, which gives rise in the middle to the extension backwards of the notochord, laterally to the mesoderm of the tail, and ventrally to a cord of endoderm cells continuous with the wall of the enteric cavity in front.

The caudal region increases in length rapidly, and the anterior or trunk region, at first round, becomes oval. At its anterior end there appear three processes of the ectoderm, the rudiments of the adhesive papillce (Fig. 744, adh.), organs by which the larva subsequently becomes fixed. The ectoderm cells at an early stage secrete the rudiments of the cellulose test; in the caudal region this forms longitudinal dorsal and ventral flaps having the function of unpaired fins.

The medullary canal becomes enlarged at its anterior end. A vesicular outgrowth from this enlarged anterior portion forms the sense-vesicle (sens. ves.) The posterior narrow part forms the caudal portion of the central nervous system (spinal cord). Masses of pigment in relation to the sense-vesicle early form the rudiment of the two larval sense-organs, otocyst (or statocyst) and eye. The part behind this presents a thickened wall with a narrow lumen. This is known as the ganglion of the trunk. The rudiment of the neural gland early appears on the ventral wall of a ciliated diverticulum (cil. gr.) of the anterior end of the archenteron (future pharynx), which subsequently unites with an outgrowth from the medullary canal.

The embryonic alimentary canal consists of two regions, a wide region situated altogether in front of the notochord, and a narrower portion situated behind in the region of the notochord. The wider anterior part gives rise to the pharynx, the posterior part to the osophagus, stomach, and intestine. The mouth-opening is formed shortly before the escape of the embryo from the egg; an ectodermal invagination is formed at the anterior end, and an endodermal diverticulum from the archenteron grows out to meet it ; the two coalesce, and the oral passage is thus formed.

The rudiments of the heart and pericardial cavity first appear as a hollow outgrowth from the archenteron: this subsequently becomes constricted off and involuted to form a double-walled sac, the inner layer of which forms the wall of the heart, while the outer gives rise to the wall of the pericardium. 
The first beginnings of the atrial cavity appear as a pair of invaginations of the ectoderm which grow inwards and form a pair of pouches, each opening on the exterior by an aperture. There is a difference of opinion as to some points in the history of these atrial pouches, and it remains uncertain to what extent the ectoderm and endoderm respectively share in the formation of the atrial cavity. Eventually spaces, into the formation of which the two ectodermal diverticula at least largely enter, grow round the pharynx and give rise to the atrial cavity; and perforations, the stigmata, primarily two in number, place the cavity of the pharynx in communication with the surrounding space. The two openings of the atrial pouches subsequently coalesce to form one-the permanent atrial aperture.

It will be useful now, at the cost of a little repetition, to summarise the various characteristics of the larval Ascidian at the stage when it escapes from the egg and becomes free-swinming (Fig. 744). In general shape it bears some resemblance to a

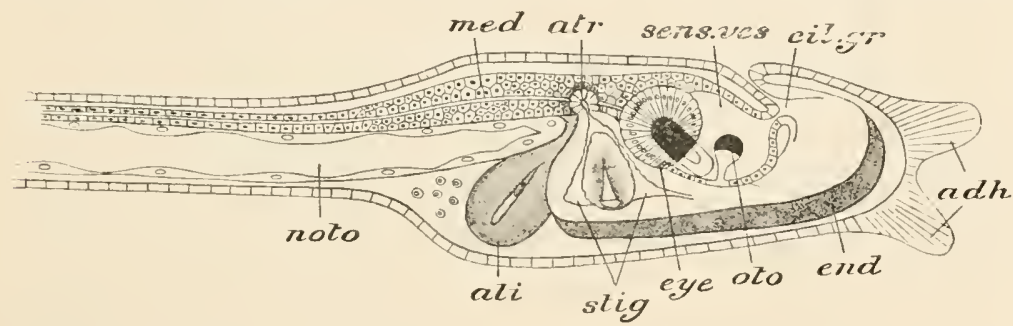

FIG, 7 th,- Hree-swimming larva of Ascidia mammilıta, lateral view, adh, adlesive papilla $a l i$, alimentary canal ; $a t r$. atrial aperture ; $c i$. $g r$. ciliated diverticulum, becoming ciliated funnel ; end. endostyle ; eye, eye; med. nerve-cord (ganglion of trunk); noto. notochord : oto. otocyst ; sens. ves. sense-vesicle ; stig. earliest stigmata. (From Korschelt and Heider, after Kowalevsky.)

minute tadpole, consisting of an oval trunk and a long laterallycompressed tail. The tail is fringed with a caudal fin, which is merely a delicate outgrowth of the thin test covering the whole of the surface; running through the delicate fringe are a series of striæ, presenting somewhat the appearance of the fin-rays of a Fish's fin. In the axis of the tail is the notochord (noto.), which at this stage consists of a cylindrical cord of gelatinous substance enclosed in a layer of cells. Parallel with this runs, on the dorsal side, the narrow caudal portion of the nerve-cord, and at the sides are bands of muscular-fibres. In the trunk the nerve-cord is dilated to form the ganglion of the trunk, and, further forwards, expands into the sense-vesicle (sens. ves.) with the otocyst or statocyst (oto.) and eye (eye). A prolongation of it unites, as already stated, with the ciliated diverticulum from the anterior part of the pharynx. From the walls of this at a later stage are developed, on the dorsal side, the ganglion, and, on the ventral, the neural gland; the pharyngeal opening (cil. gr.) becomes the 
ciliated funnel. The enteric canal is distinguishable into pharynx, oesophagus, stomach, and intestine. The pharynx opens on the exterior by the mouth: in its ventral floor the endostyle (end.) has become developed; its walls are pierced by stigmata, the
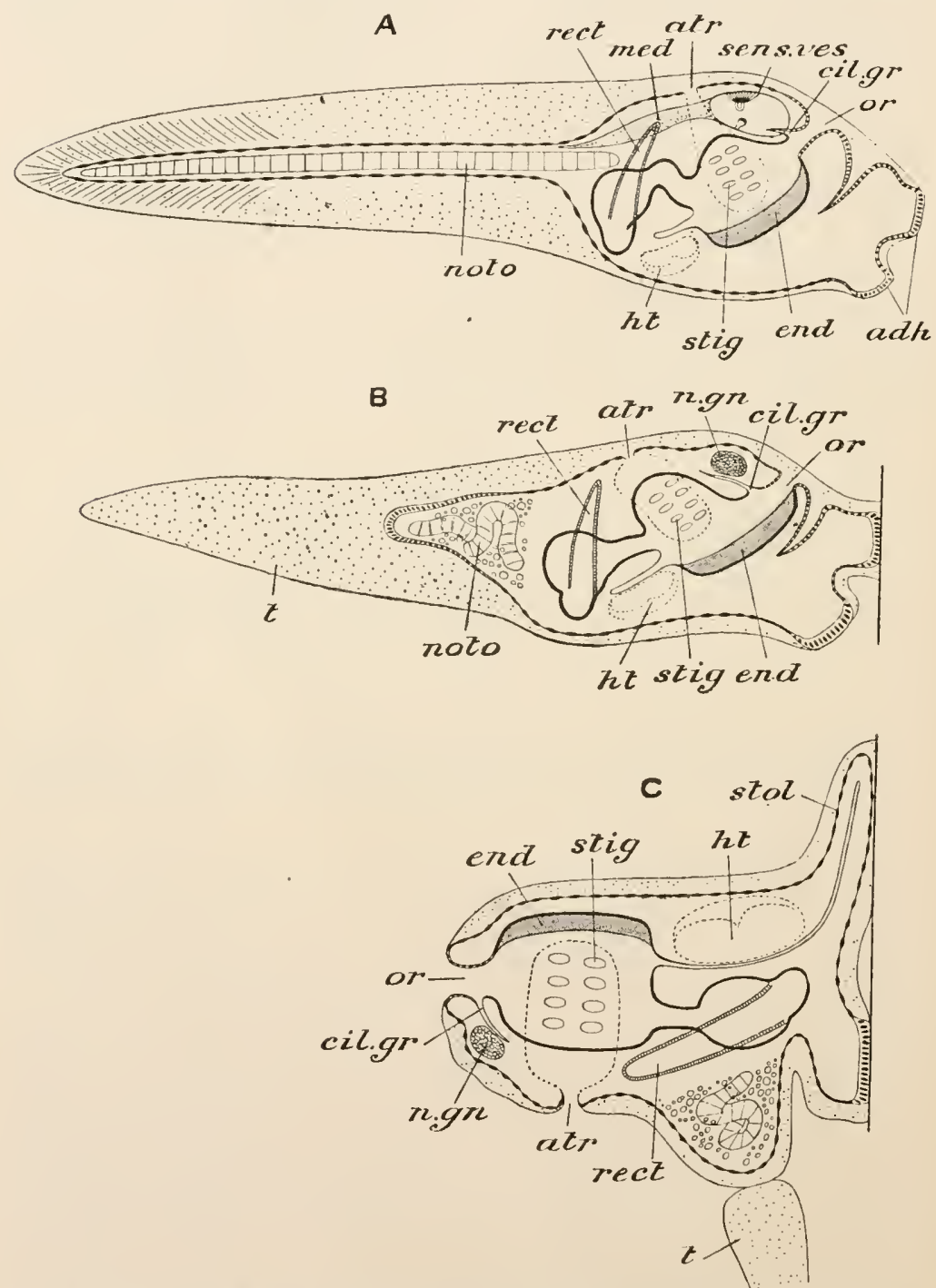

FIG. 745.-Diagram of the metamorphosis of the free-tailed larva into the fixed Ascidian. A, stage of free-swimming larva; $B$, larva recently fixed : $C$, older fixed stage, adh. adhesive papillx ; atr. atrial eavity ; $c i$. gr. ciliated diverticulum, beconuing ciliated funnel ; end. endostyle; $h t$. heart; med. ganglion of trunk; $n$. gn. nerve-ganglion; noto. notochord; or. oral apertire; rect. rectum; sens. ves. sense-vesicle; stig. stigmata; stol. stolon; $t$. tail. (From Korschelt and Heider, after Seeliger.) 
number of which varies. The atrial cavity has grown round the pliarynx, and opens on the exterior by a single aperture only (atr.). The heart and pericardial cavity have been formed. In this tailed free-swimming stage the larva remains only a few hours ; it soon becomes fixed by the adhesive papillæ and begius to undergo the retrogressive metamorphosis by which it attains the adult condition.

The chief changes involved in the retrogressive metamorphosis (Fig. 745) are the increase in the number of pharyngeal stigmata, the diminution, and eventually the complete disappearance, of the tail with the contained notochord and caudal part of the nervecord, the disappearance of the eye and the otocyst, the dwindling of the central part of the nervous system to a single ganglion, and the formation of the reproductive organs. Thus, from an active free-swimming larva, with complex organs of special sense, and provided with a notochord and well-developed nervous system, there is a retrogression to the fixed inert adult, in which all the parts indicative of affinities with the Vertebrata have become aborted. The significance of these facts will be considered when we come to discuss the general relationships of the Chordata.

In some simple Ascidians, and in the composite forms in which development takes place within the body of the parent, the metamorphosis may be considerably abbreviated, but there is always, so far as known, a tailed larva, except in the genus Anwiella of the Molgulide - a family of the simple forms - in which the tailed stage is wanting and there is only an obscure endodermal rudiment to represent the notochord.

In Pyrosoma development is direct, without a tailed larval stage, and takes place within the body of the parent. The ovum contains a relatively large quantity of food-yolk, and the segmentation is meroblastic. A process, developed at an early stage, elongates to form the so-called stolon, which divides, by the formation of constrictions, into four parts, each destined to give rise to a zooid (tetrazooid). The primary zooid (cyathozooid) undergoes atrophy, and at this stage the young colony, composed of four tetrazooids with the remains of the cyathozooid enclosing a mass of yolk-the whole invested in a common cellulose test-passes out from the brood-pouch in which it was developed and reaches the exterior through the cloaca of the parent colony. By a process of budding from the four primary tetrazooids, the entire adult colony is produced.

The development of Doliolum is, in all essential respects, very like that of the simple Ascidians. There is total segmentation, followed by the formation of an embolic gastrula; the larva (Fig. 746) has a tail with a notochord (noto.), and a body in which the characteristic muscular bands soon make their appearance. This tailed larva becomes the asexual stage or "nurse." By and by 
the tail aborts, and two processes, one postero-dorsal, the other ventral, known respectively as the cadophore (dors. st.) and the ventral stolon (vent. st.), grow out from the body of the larva. On the latter are formed a number of slight projections or buds. These becorne constricted off, and in the form of little groups of cells, each consisting of seven strings of cells with an ectodermal invest-

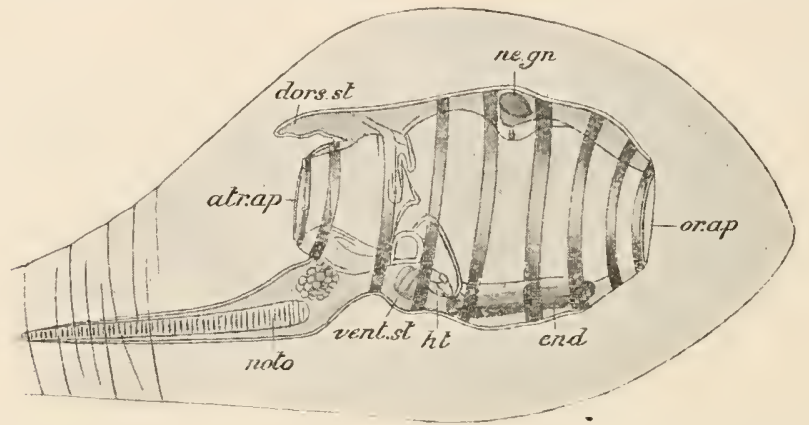

FId. 746.-Doliolu m, latestage in the development of theitailed larva. atr. ap. atrial aperture ; dors. st. caiophore; end. endostyle; ht. heart; ne. gn. nerve-ganglion; noto. notochord: or. ap. oral aperture; vent. st. ventral stolon. (Atter Uljanin.)

ment, creep over the surface of the parent (Fig. 747, e, and Fig. 748 ) till they reach the cadophore, to which they attach themselves after multiplying by division. The cadophore soon becomes elongated, and the bud-like bodies attached to it develop into zooids. As the long chain of zooids thus established is further developed,

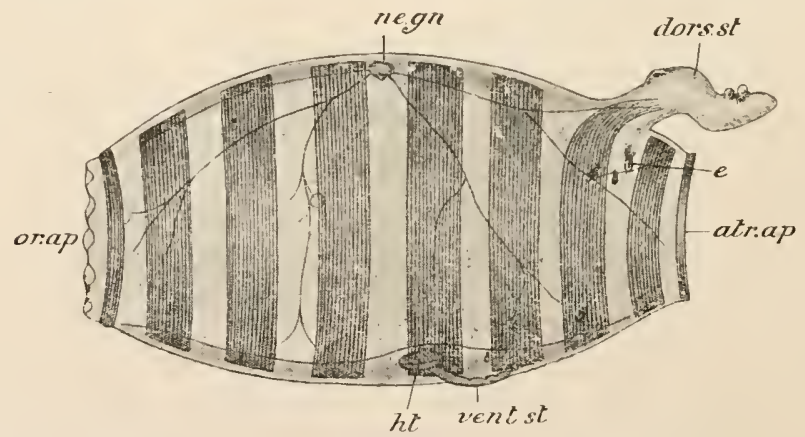

FIG. 747,-Doliolum, lateral view of asexual stage, showing the early development of the buds. atr. ap. atrial aperture; dors. st. cadophore; $e$. embryos passing over the surface from the ventral stolon to the cadophore; $h t$. heart; ne. $g n$. nerve-ganglion; or. ap. oral aperture : vent. st. ventral stolon. (After Uljanin.)

the parent Doliolum (Fig. 747) loses its branchiæ, its endostyle, and its alimentary canal; at the same time the muscle-bands increase in thickness and the nervous system attains a ligher development, until the whole parent comes to play a part like that of the nectocalyx of a Siphonophore (Vol. I., p. 161), its 
exclusive function being by its contractions to propel the colony through the water.

The zooids of the cadophore consist of two sets, differing from one another in position and in future history-the lateral zooids and the median zooids. The lateral zooids serve solely to carry on the nourishment and respiration of the colony, and do not undergo any further development. Some of the median buds, on the other hand, become detached and take on the special character of phorozooids. When free, each phorozooid carries with it the stalk by means of which it was attached to the stolon; on this stalk there have previously become attached a number of buds which are destined after a time to be developed into the sexual zooids.

The succession of stages in the life-history of Doliolum thus briefly sketched will be seen to succeed one another in the following order:-(1) sexual form; (2) tailed larva developed sexually from (1) ; (3) first asexual form or "nurse," the direct outcome of $(2)$; (4) second asexual form (phorozooid) developed on the cadophore of (3) from buds originating on the ventral stolon; (5) the young of the sexual form (1) which are developed on the stalk of (4).

Salpa, like Doliolum, presents a remarkable alternation of generations. In the sexual form, which has already been described, only one ovum is developed. The testis becomes mature later than the ovum, and the latter is impregnated by sperms from the testis of an individual of an older chain. The development is direct and takes place within the body of the parent, the embryo as it grows

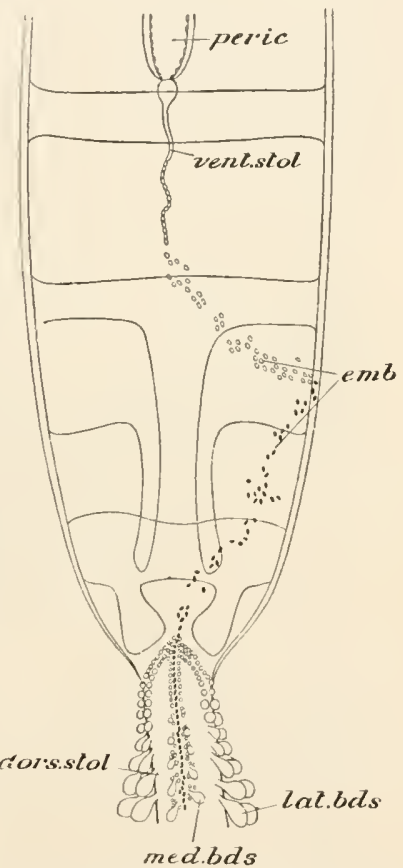

Fra. 748.-Doliolum, dorsal view of the posterior part of the body of an asexual zooid, showing the course taken by the buds (emb.) over the surface from the ventral stolon (ient. stol.) to the cadophore (dors. stol.) and their growth on the latter. lat. bds. lateral buds; med. bds. median buds; peric. pericardiun. (After Barrois.) projecting into the branchial cavity. The nourishment of the developing embryo (Fig. 749) is effected by the formation of a structure-the placenta-through which a close union is brought about between the vascular system of the parent and that of the embryo. The placenta of Salpa is partly formed from folliclccells and ectoderm-cells of the embryo, partly from the cells of the wall of the oviduct. Segmentation is complete. The study of the earlier stages is complicated by the very remarkable and unusual 
circumstance that during segmentation there is a migration inwards of some of the cells of the follicle and of the wall of the oviduct, which enter the segmenting ovum and pass among the blastomeres. There is uncertainty as to what part these inwardly migrating cells play in the development of the embryo; but probably they act merely as carriers of nourishment, and become broken up and eventually completely absorbed.

There is no tailed larval stage, and the embryo develops the muscle-bands and all the characteristic parts of the adult while still enclosed within the body of the parent and nourished by means of the placenta. 'This sexually-developed embryo, however, does not give rise to a form exactly like the parent, but to one which differs from the latter in certain less important features and

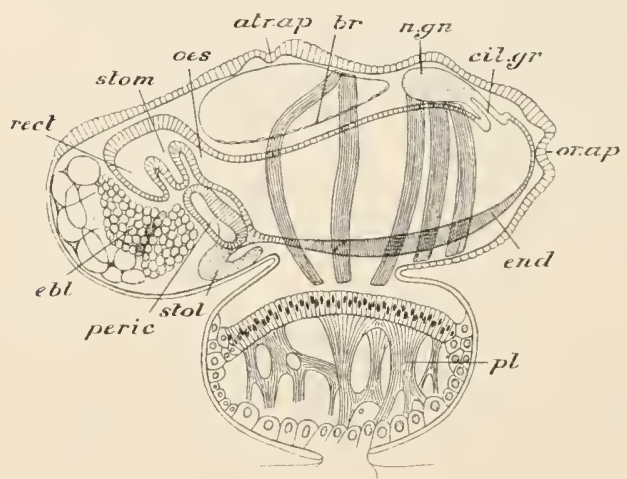

FIG. 749.-Late stage in the development of Salpa, showing the placental connection with the parent. atr. ap. atrial aperture ; $b r$. branchia; cil. gr. ciliated groove ; $e b l$. elæoblast (mass of tissue probably representing a vestige of the tail); end. endostyle ; n. gn. nerve-ganglion ; ces. cesophagus; or ap. oral aperture: peric pericardium; $p l$. placenta ; rect. rectum; stol. stolon; stom. stomach. (From Korscheit and Heider, after Salensky.) notably in the absence of reproductive organs. The sexually formed e m b ryo, in other words, gives rise to an asexual generation, which escapes to the exterior and becomes free - swimming (Fig. 736). After a time there is developed a process or stolon! (stol.), on the surface of which are formed a number of bud-like projections. These increase in size as the stolon elongates, and each eventually assumes the form of a sexual Salpa. The

stolon with the Salpæ attached becomes separated off and swims about as a chain of zooids in which the reproductive organs are developed.

Distribution, etc.-The pelagic forms are, as is the case with most pelagic organisms, of very wide distribution, and none of the genera are confined to particnlar oceanic areas. The fixed forms, both simple and composite, are also of world-wide distribution; they are much more abundant in the southern hemisphere than in the northern-the composite forms attaining their maximum in the South Pacific area. The depth to which the pelagic forms extend has not been determined. Fixed forms occur at all depths, but are much more numerous in shallow water than in deep, and at great depths are comparatively poorly represented, the simple forms extending to a greater depth than the composite. Sereral 
genera of pedunculated simple Ascidians seem to be confined to very great depths.

Though placed so high in the animal series, the Urochorda exhibit very low functional development. This is chiefly connected with the sessile condition of most of them. The movements performed by a fixed Ascidian are slow and very limited in character, being confined to contractions of the mantle; when the animal is detached, such contractions may sometimes be observed to result in a slow creeping locomotion. Even in the free forms the movements are limited to the contractions, of the tail muscles in Appendicularia, of the muscle-bands of the body-wall in Doliolum, by which swimming is effected. The mode of obtaining food resembles that which has already been described in the case of the Pelecypoda (Vol. I., p. 671), the currents which subserve respiration also bringing in microscopic organic particles to the mouth.

Affinities. - That the Urochorda are degenerate descendants of primitive Chordates admits of little doubt; the history of the development of the Ascidians, taken in connection with the occurrence of permanently chordate members of the group (Appendicularia and its allies), is quite sufficient to point to this conclusion. But the degree of degeneration which the class has undergonethe point in the line of development of the higher Chordata from which it diverged-is open to question. According to one view the Urochorda are all extremely degenerate, and have descended from ancestors which had all the leading features of the Craniata; according to another, the ancestors of the class were much lower than any existing Craniate- lower in the scale than even Amphioxus -and had not yet acquired the distinctive higher characteristics of the Craniates. The complete want of segmentation and the virtual absence of a colome seem to point in the latter direction : the presence in the larva of highly-developed central nervous system and sense-organs in the former. Appendicularia is hardly to be regarded as representing a primitive ancestral type; its close resemblance to the larva of the sessile Ascidians rather seems to indicate that it is a persistent larval form-a form in which sexual maturity has been reached at earlier and earlier stages in the life-history, and in which the final sessile stage has at last been lost, the animal having become completely adapted to a pelagic life. Probably the other pelagic forms-Salpa, Doliolum, Pyrosoma -were also descended from sedentary ancestors: none of them shows any character that can be interpreted as primitive.

The nearest existing ally of the Urochorda among lower forms is probably Balanoglossus. The similarity in the character of the pharynx, or anterior segment of the enteric canal, perforated by branchial apertures, is alone sufficient to point to such a connection; and further evidence is afforded by the occurrence of a "notochord" in both, and by the similarity in the development 
of the central part of the nervous system. But the notochord of the larval Ascidian, almost confined to a post-intestinal tail-region, differs very widely from the structure in Balanoglossus supposed to correspond to it, which is situated anteriorly and directed forwards ; moreover, the other differences are so great that the alliance cannot be a close one, and Balanoglossus and its allies can only be looked upon as very remotely connected with the stock from which the Urochorda are descended. Further consideration will be given to this subject in the general treatment of the relationships of the Chordata.

\section{SUB-PHYLUM III.--EUCHORDA.}

We have seen that the fundamental characters of the Chordata are the presence of a notochord, of a dorsal, hollow, nervous system, and of a pharynx perforated by apertures or gill-slits. In none of the lower Chordata, however, are these structures found in a typical condition, at least in the adult. In Balanoglossus, Cephalodiscus, and Rhabdopleura the "notochord" is rudimentary, and in nearly all Tunicata it is present only in the embryo. In Rhabdopleura the gill-slits are absent, and in that genus as well as in Cephalodiscus and the adult Tunicata the nervous system is represented by a single solid nerve-centre or ganglion, the neurocœle being absent. In Balanoglossus, moreover, there is a ventral as well as a dorsal nerve-cord, and it is only in the anterior portion of the latter that the neurocœle is represented.

In the Euchorda, on the other hand, what have been called the three fundamental chordate peculiarities are fully and clearly developed. There is always a distinct notochord extending as a longitudinal axis throughout the greater part of the elongated body, and either persisting throughout life, or giving place to an articulated vertebral column or backbone. The central nervous system remains throughout life in the form of a dorsal nerve-tube or neuron containing a longitudinal canal or neurocole, and the pharynx is always perforated, either throughout life or in the embryonic condition, by paired branchial apertures or gill-slits. In addition to these characters, the mouth is ventral and anterior, the anus ventral and posterior; the muscular layer of the bodywall is primarily segmented, and the renal organs arise as a series of paired tubules which may represent either nephridia or cœlomoducts. Moreover, there is always an important digestive gland, the liver, developed as a hollow outpushing of the gut, and distinguished by the fact that the blood from the alimentary canal circulates through it before passing into the general current, thus giving rise to what is called the hepatic portal system of blood-vessels.

The sub-phylum Euchorda comprises two sections of very unequal extent. 
Section I.-Acrania (Cephalochorda).

Including only the little fish-like Lancelets.

\section{Section II.-Craniata (Vertebrata).}

Including Cyclostomes, Fishes, Amphibians, Reptiles, Birds, and Mammals.

\section{SECTION I.-ACRANIA (CEPHALOCHORDA).}

The section Acrania includes only two families, the Branchiostomida - containing the genera Branchiostoma (usually known by the name of one of its sub-genera, Amphioxus), Epigonichthys, and perhaps some others; and the Amphioxidida-containing the pelagic genus Amphioxides, which, however, may not be an adult form. The differences between the genera and species of Branchiostomidæ are comparatively insignificant, and the following description will deal exclusively with the best known and most
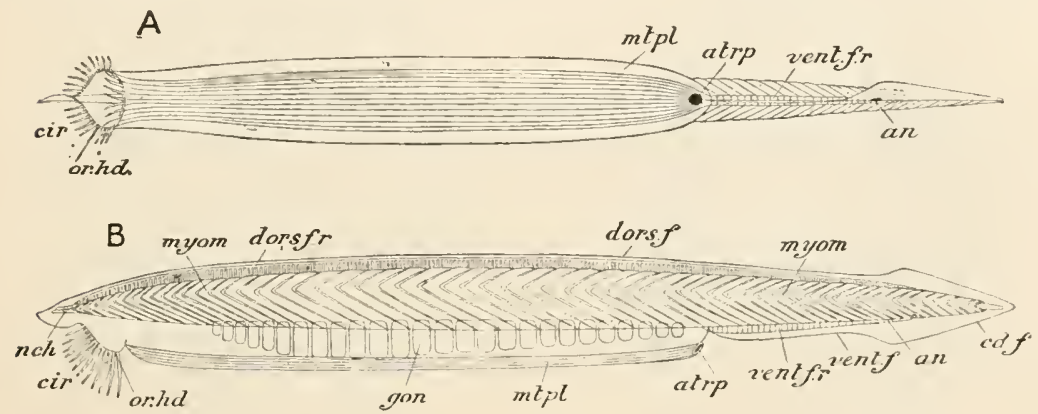

FIG. 750.-Amphioxus lanceolatus. $A$, ventral, $B$, "side view of the entire animal. an. anus; $a t r p$. atriopore; $c d . f$. caudal fin ; cir. cirri; dors. f. dorsal fin ; dors. $f$. $r$. dorsal finrays; non. gonads; mtpl. metapleure; myom. myomeres; nch. notochord; or. hd. oral hood ; vent. $f$. ventral fin; vent. $f$. $r$. ventral fin-rays. (After Kirkaldy.)

thoroughly investigated species, the Lancelet, Amphioxus lanceolatus, found in the English Channel, the North Sea, and the Mediterranean.

Amphioxus is a small transparent animal, occurring near the shore and burrowing in sand; its length does not exceed $5.8 \mathrm{~cm}$., or less than two inches. Its form will be obvious from Fig. 750 and from the transverse sections, Fig. 751, $A$ and $B$. The body is elongated, pointed at either end, and compressed. The anterior two-thirds is roughly triangular in transverse section, presenting right and left sides, inclined towards one another above, and a convex ventral surface. The posterior third is nearly oval in section, the right and left sides meeting above and below in a somewhat sharp edge.

Extending along the whole of the dorsal border is a median longitudinal fold, the dorsal fin (dors. $f$.) : this is continued round 
the posterior end of the body and extends forwards, as the ventral fin (vent. f.), as far as the region where the oval transverse section passes into the triangular. The portion of the continuous median fold which extends round the pointed posterior extremity of the body is somewhat wider than the rest, and may be distinguished as the caudal fin (cl. f.). In the anterior two-thirds of the body there is no median ventral fin, but at the junction of each lateral with the ventral surface is a paired longitudinal fold, the metapleure (mtpl.), which extends forwards to the oral hood mentioned in the next paragraph.

Below the pointed anterior extremity is a large median aperture surrounded by a frill-like membrane, the oral hood (or. $h d$.), the

A

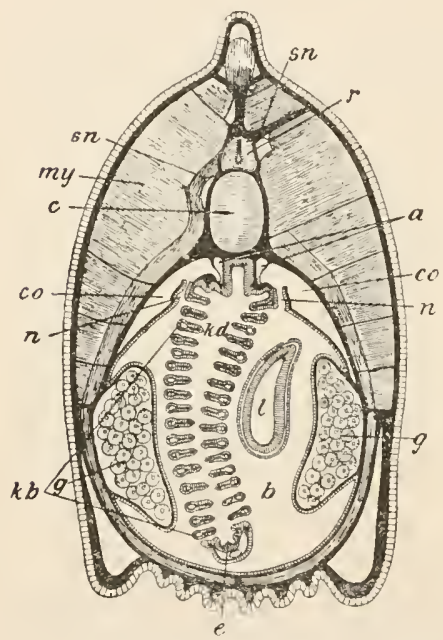

$\mathrm{B}$

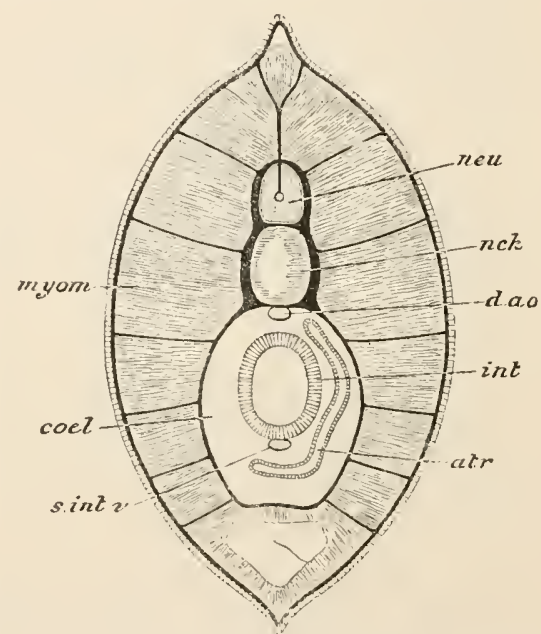

FIG. 75l.-Amphioxus lanceolatus. A, transverse section of the pharyngeal region. $a$. dorsal aorta ; $b$, atrium ; $c$ notochord ; $c o$ coetome; $e$ endostyle ; $g$ gonad ; $l \cdot b$ branchial

e lamelle; $k d$. pharynx; $l$. liver; my. myomere; $n$. nephridium; $r$. neuron; sn. spinal nerves. $B$, transverse section of the intestinal region. atr. atrium; col. coelome ; d. ao, dorsal aorta ; int. intestine : myom. myomere ; nch. notochord ; neu. neuron ; $s$, int. $v$. sub-intestinal vein. ( $A$, from Hertwig, aiter Lankester and Boveri; $B$, partly after Rolph.)

edge of which is beset with numerous tentacles or cirri (cir.). The oral hood encloses a cup-shaped cavity or vestibule, at the bottom of which is the mouth (Fig. 752, mth.). On the wall of the oral hood is a specially modified tract of the epithelium divided into finger-shaped lobes. The cells of this tract, which is known as the wheel-organ, are provided with long cilia, the movements of which drive currents of water with floating food-particles backwards into the pharynx. Along the roof of the vestibule runs a ciliated groove- the groove of Hatschel:. Immediately in front of the anterior termination of the ventral fin and partly enclosed by the metapleures is a rounded aperture of considerable size, the atriopore 
(atrp.), and a short distance from the posterior extremity of the body is the anus (an.), placed unsymmetrically on the left side of the ventral fin. The post-anal portion of the body is distinguished as the tail.

Amphioxus ordinarily lives with the greater part of the body buried in sand, only the anterior end with the expanded oral hood protruding. It also swims in the vertical position, and frequently lies on one side on the sand : it burrows, head foremost, with great rapidity. A current of water is constantly passing in at the mouth and out at the atriopore.

Body-wall.-The body is covered with an epidermis (Fig. 751) formed of a single layer of columnar epithelial cells, some of which are provided with sensory hairs, while some are unicellular glands. On the surface of the epidermis is a cuticle perforated by pores. The epithelium of the buccal cirri presents at intervals regular groups of sensory cells, some of them bearing stift sensory hairs, others cilia. Beneath the epidermis is the dermis, formed mainly of soft connective-tissue.

The muscular layer (my, myom.) is remarkable for exhibiting metameric segmentation. It consists of a large number-about sixty-of muscle-segments or myomeres, separated from one another by partitions of dense connective-tissue, the myocommas, and having the appearance, in a surface view, of a series of very open V's with their apices directed forwards (Figs. 750 and 752). Each myomere is composed of numerous flat, striated muscle-plates, arranged longitudinally, so that each is attached to two successive myocommas. In virtue of this arrangement the body can be bent from side to side with great rapidity. The myomeres of the right and left sides of the body are not opposite to one another, but have an alternate arrangement. A special set of transverse muscles (Fig. $751, A$ ) extends across the ventral surface of the anterior two-thirds of the body, lying in the floor of the atrial cavity presently to be described.

One striking and characteristic feature of the muscular layer of the body-wall is the immense thickness of its dorsal portion. In the higher Worms and many other Invertebrates the muscles form a layer of approximately equal thickness surrounding the bodycavity, which contains, amongst other organs, the central nervous system. In Vertebrates, on the other hand, the dorsal body-wall is greatly thickened, and in it are contained both the nervous system and the notochord.

Skeleton.-The chief of the skeletal or supporting structures of the Lancelet is the notochord (Figs. 751 and $752, c, n c h$.), a cylindrical rod, pointed at both ends, and extending from the anterior to the posterior end of the body in the median plane. It lies immediately above the enteric tract and between the right and $\mathrm{ft}$ myomeres. It is composed of a peculiar form of cellular 
tissue known as notochordal tissue, formed of large vacuolated cells extending from side to side of the notochord, and having the nuclei confined to its dorsal and ventral regions. Around these cells is a structureless layer, secreted by the cells, enclosed in a notochordal sheath of connective-tissue, which is produced dorsally into an investment for the canal enclosing the central nervous system. The notochord, like the parenchyma of plants, owes its resistant character to the vacuoles of its component cells being tensely filled with fluid, a condition of turgescence being thus produced.

The oral hood is supported by a ring (Fig. $752, s k$.) of cartilaginous consistency, made up of separate rod-like pieces arranged end to end, and corresponding in number with the cirri. Each piece sends an offshoot into the cirrus to which it is related, furnishing it with a skeletal axis.

The pharynx is supported by delicate oblique rods of a firm material, apparently composed of agglutinated elastic fibres, the gill-rods (br. r.). These will be most conveniently discussed in connection with the pharynx itself. The dorsal fin is supported by a single series and the ventral fin by a double series of fin-rays (dors. $f . r$., vent. $f . r$.), short rods of connective-tissue, continuous with the investment of the neural canal and separated from one another by small cavities (lymph-spaces).

Digestive and Respiratory Organs.-The mouth (mth.), as already mentioned, lies at the bottom of the vestibule or cavity of the oral hood (or. hd.). It is a small circular aperture surrounded by a membrane, the velum $(v l$.), which acts as a sphincter, and has its free edge produced into a number of velar tentacles (vl. $t$.).

The mouth leads into the largest section of the enteric canal, the pharynx ( $p h$.$) , a high, compressed chamber extending through$ the anterior half of the body. Its walls are perforated by more than a hundred pairs of narrow oblique clefts, the gill-slits or branchial apertures (br. cl.), which place the cavity of the pharynx in communication with the atrium (see below). From the posterior end of the pharynx goes off the tubular intestine (int.), which extends backwards almost in a straight line to the anus.

On the ventral wall of the pharynx is a longitudinal groove, the endostyle (Fig. 751, A, e.), lined by ciliated epithelium containing groups of gland-cells. Like the homologous organ in Ascidia (p. 17), the glands secrete a cord of mucus in which food-particles are entangled and carried by the action of the cilia to the intestine. A somewhat similar structure, the epipharyngeal groove, extends along the dorsal aspect of the pharynx: its sides are formed by ciliated cells, which, at the antcrior end of the groove, curve downwards, as the peripharyngeal bands, and join the anterior end of the endostyle.

From the ventral region of the anterior end of the intestine is 
given oft a blind pouch, the liver $\left(l r_{0}\right)$ or hepatic ccecum, which extends forwards to the right of the pharynx: it is lined with glandular epithelium and secretes a digestive fluid.

The gill-slits (br. cl.) are long narrow clefts, nearly vertical in the expanded condition, but very oblique in preserved and contracted specimens -hence the fact that a large number of clefts always appear in a single transverse section (Fig. $751, A, k d$.$) . The clefts$ are more numerous than the myomeres in the adult, but correspond in number with them in the larva: hence they are fundamentally meta meric, but undergo an increase in number as growth proceeds.

The branchial lamello (Fig. 752, br. sep., Fig. $751, A, k b$.$) , or portions$ of the pharyngeal wall separating the clefts from one another, are covered by an epithelium which is for the most part endodermal in origin, and is composed of greatly elongated and ciliated cells. On the outer face of each lamella, however, the cells are shorter and not ciliated, and are, as a matter of fact, portions of the epithelial lining of the atrium, and of ectodermal origin. Each

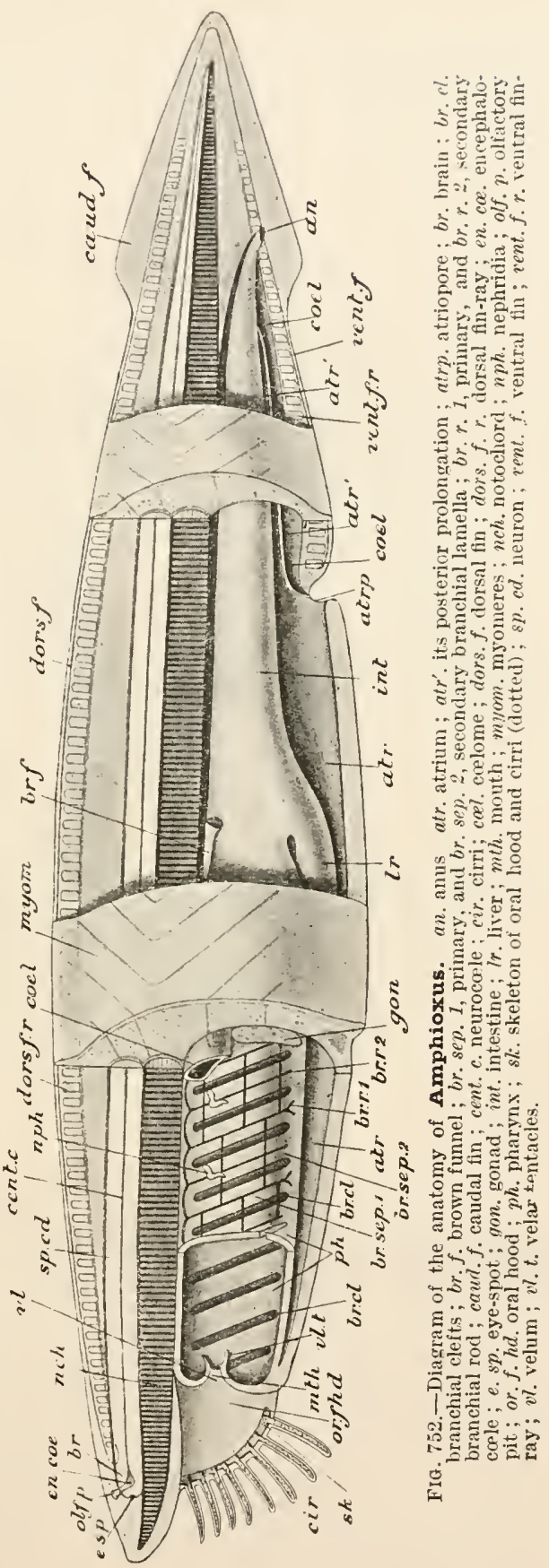


lamella is supported towards its outer edge by one of the branchial rods (Fig. 752, br. r.) already referred to. These are narrow bars united with one another dorsally by loops, but ending below in

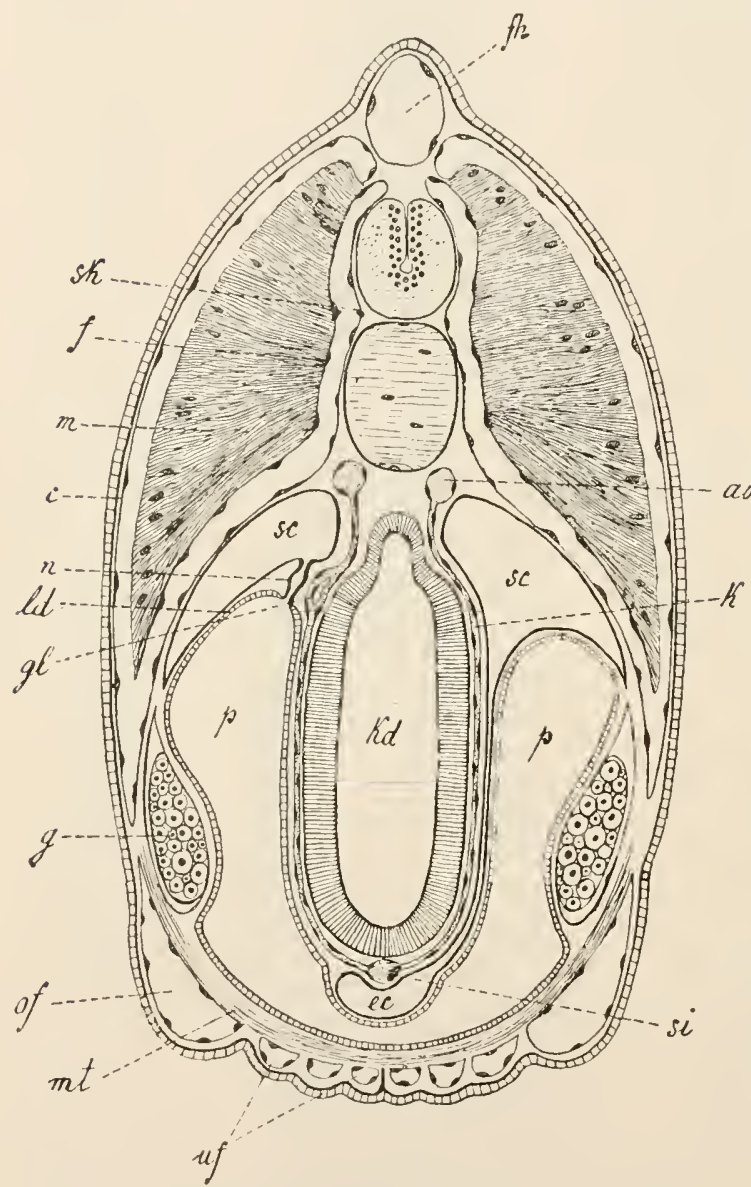

FIG.5753.-Amphioxus lanceolatus. Diagrammatic transverse section of the pharyngeal region, passing on the right through a primary, on the left, through a secondary branchial lamella. ao. dorsal aorta ; $c$. derm ; $e c$. endostylar portion of cœlome; $f$. fascia or investing layer of myomere; $f h$. compartment contalning fin-ray ; $g$. gonad : $g l$. glomerulus (modified part of branchial artery it relation to nephridium); $k$. branchial artery ; $k d$. pharynx; $l d$. combined atrial and coelomic wall (ligamentum denticulatum): $m$. myomere ; $m t$. transverse muscle; $n$. nephridium; of. metapleural lymph-space ; $p$. atrium ; sc. cœlome ; si. ventral aorta ; $s k$. sheath of notochord and neuron; $u f$. spaces in ventral wall. (From Korschelt and Heider, after Boveri and Hatschek.) free extr mities which are alternately simple and forked. The forked bars are the primary ( $b r$. $r .1$ ), those with simple ends the secondary (br. $r$. 2) branchial rods, and the lamellæ in which they are contained a $1 \mathrm{e}$ similarly to be distinguished as primary lamelle (br. sep. 1) and secondary or tongue-lamella (br. sep. 2). In the young condition the two clefts between any two primary lanellæ are represented by a single aperture : as development proceeds a downgrowth takes place from the dorsal edge of the a perture, forming, as in Balanoglossus (p. 5), a tongue which extends downwards, dividing the original cleft into two, and itself becoming a secondary lamella. A further complication is produced by the formation of transverse branchial junctions or synapticule, supported by rods connecting the primary septa with one another at tolerably regular intervals. 
The Atrium.-The gill-clefts lead into a wide chamber occupying most of the space between the body-wall and the pharynx, and called the atrium (Figs. 751, B, and 752, atr.). It is crescentic in section, surrounding the ventral and lateral regions of the pharynx, but not its dorsal portion. It ends blindly in front, opens externally, behind the level of the pharynx, by the atriopore ( $\left(t_{r p}^{\prime}.\right)$; and is continued backwards by a blind, pouch-like extension (atr.) lying to the right of the intestine (Fig. 751, B, atr.). The whole cavity is lined by an atrial epithelium of ectodermal origin. As in Ascidia, the cilia lining the gill-clefts produce a current setting in at the mouth, entering the pharynx, passing thence by the gill-slits into the atrium, and ont at the atriopore. The current, as in Tunicata and Balanoglossus, is both a respiratory and a food current, the animal feeding passively on the minute organisms in the surrounding water.

Cœlome.-Owing to the immense size of the atrium the bodycavity, which is a true colome, is much reduced. It is represented,

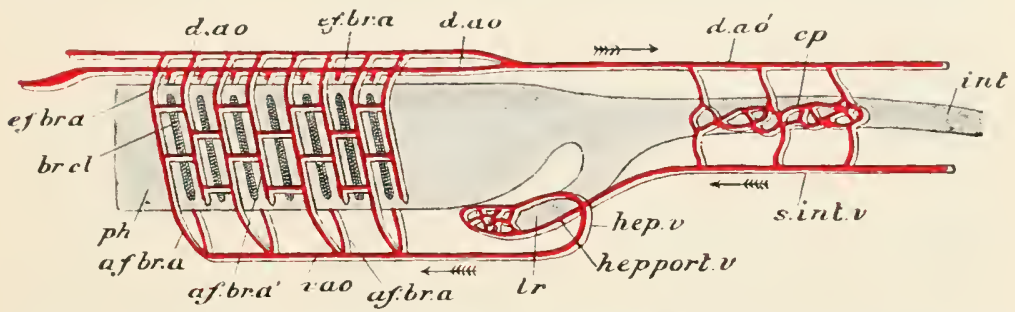

FIG. 754.-Diagram of the vascular system of Amphioxus. $a f$. br. $a$. afferent branchial arteries : br. cl. branchial cleft; $c p$. intestinal capiliaries; $d$. ao. paired ilorsal aortæ; $d . a o^{\prime}$. median dorial aorta; $e f$. br. a. efferent branchial arteries; hep. port. $t$. hepatic portal vein ; hep. $v$. hepatic vein; int. intestine; $l r$. liver; $p h$. pharynx; $s$. int. $i$. sub-intestinal vein; v. ao. ventral aorta.

in the pharyngeal region, by paired cavities (Fig. 752, coel., Fig. 751, $A$, co., Fig. 753, sc.) lying one on either side of the dorsal region of the pharynx above the atrium, and connected by narrow canals in the primary branchial lamellæ (Fig. 753, right side) with a median longitudinal space below the endostyle $(e c$.$) . In the intestinal$ region it entirely surrounds the intestine, but is much reduced on the right side, being displaced by the backward extension of the atrium (Fig. 751, B, atr., Fig. 752, atr' .) : on the left side a forward extension of it surrounds the liver (Fig. 751, A, l.). Separate cavities lie in the metapleures. The whole series of spaces is lined by cœelomic epithelium.

Blood-System.-The blood-vessels of Amphioxus are all of one kind, but, owing to certain undonbted homologies with the more complex vessels of the Craniata (see below), some of them receive the name of arteries, others of veins.

Lying in the ventral wall of the pharynx, below the endostyle, is a median longitudinal vessel, the veniral aorta (Fig. 754, v. ao, Fig. 753, si.); it is contractile, and drives the blood forwards. 
From it are given off, on each side, lateral branches, the afferent branchial arteries (Fig. 754, af. br. a. ; Fig. 753, k), with small contractile dilatations at their bases, which pass up the primary branchial lamellæ and communicate by cross-branches with similar vessels $\left(a f . b r . a^{\prime}\right.$.) in the secondary or tongue-lamellæ. The blood is exposed, while traversing these vessels, to the aërating influence of the respiratory current, and leaves the branchial lamellæ dorsally by efferent branchial arteries (ef. $b r$. a.) which open on each side into paired longitudinal vessels, the right and left dorsal aortoe (d. ao.), lying one on either side of the epipharyngeal groove. Anteriorly both dorsal aortæ are continued forwards to the region of the snout, the right being much dilated; posteriorly they unite with one another, behind the level of the pharynx, into an unpaired dorsal aorta $\left(d . a o^{\prime}.\right)$, which extends backwards in the middle line, immediately below the notochord and above the intestine.

The unpaired dorsal aorta sends off branches to the intestine, in the walls of which they break up to form a network of microscopic vessels or capillaries (cp.). From these the blood is collected and poured into a median longitudinal vessel, the sub-intestinal vein (Figs. 751, B, and 754, s. int. v.), lying beneath the intestine: in this trunk the blood flows forwards, and, at the origin of the liver, passes insensibly into a hepatic portal vein (hep. port. v.), which extends along the ventral side of the liver and breaks up into capillaries in that organ. From the liver the blood makes its way into a hepatic vein (hep. v.), which extends along the dorsal aspect of the digestive gland, and, turning downwards and forwards, joins the posterior end of the ventral aorta.

It will be seen that the vascular system of Amphioxus consists essentially of $(a)$ a dorsal vessel represented by the paired and unpaired dorsal aortæ, (b) a ventral vessel represented by the subintestinal vein and the ventral aorta, and $(c)$ commissural vessels represented by the afferent and efferent branchial arteries and the intestinal capillaries. So far the resemblance to the vascular system of Annulata is tolerably close ; but two important differences are to be noted. The blood in the ventral vessel travels forwards, that in the dorsal vessel backwards-the precise opposite of what occurs in Worms, and the ventral vessel is broken up, as it were, into two parts, by the interposition in its course of the capillaries of the liver, so that all the blood from the intestine has to pass through that organ before reaching the ventral aorta. This passage of the intestinal blood through the vessels of the liver constitutes what is called the hepatic portal system, and is eminently characteristic of Vertebrata.

The blood is almost colourless with a few red corpuscles, and appears to contain no leucocytes. It is not confined to the true blood-vessels just described, but occurs also in certain cavities or lymph-spaces, the most important of which are the cavities in the 
dorsal and ventral fins containing the fin-rays (Fig. 753, fh.), and paired canals in the metapleures (of.).

Excretory Organs.-The principal organs of excretion are about ninety pairs of peculiarly modified nephridia (Fig. 752 , neph.) situated above the pharynx and in relation with the main coelomic cavities. Each nephridium. (Fig. 755) is a bent tube consisting of an anterior vertical and a posterior horizontal limb. The vertical limb terminates in a large group of solenocytes (Vol. I., p. 468), and there are several smaller groups on the horizontal limb. The organ thus closely corresponds to the type ofnephridium with closed inner end bearing solenocytes already described as occurring in certain of the Polychæta. On the ventral surface of the horizontal limb, opposite a secondary branchial lamella, is a single aperture bearing long cilia and opening into the atrium: this corresponds with the nephridiopore or external aperture of the typical nephridium.

An excretory function has also been assigned to a single pair of

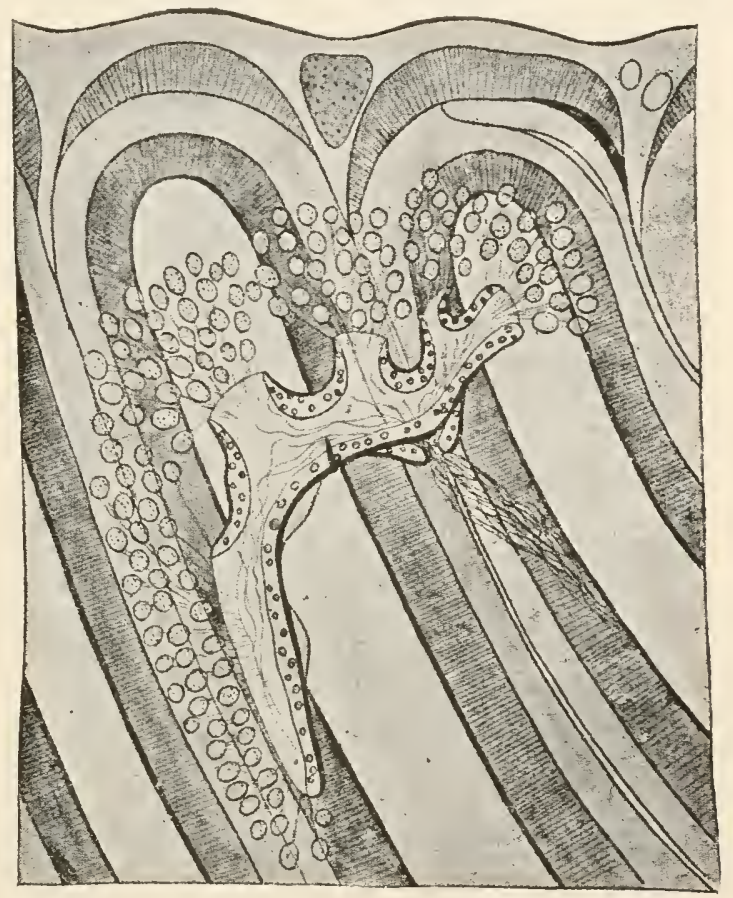

FIG. 755.-Amphioxus lanceolatus. Nephridium of the left side with part of the wall of the pharynx. (From Willey, aiter Boveri.)

organs called the brown funnels (Fig. 752, br.f.), also situated on the dorsal aspect of the pharynx at its posterior end. Their wide backwardly-directed ends open into the atrium; their narrow anterior ends probably communicate with the coelome. There are also groups of columnar excretory cells on the floor of the atrium.

Nervous System.-The central nervous system is a rod-like organ, the neuron or dorsal nerve-tube (Fig. $751, A, n . ; B$, neu., Figs. 752, 754), contained within and completely filling a median longitudinal neural canal which lies immediately above the noto- 
chord. It is roughly triangular in transverse section: anteriorly it ends abruptly, some distance behind the anterior end of the notochord, while posteriorly it tapers to a point over the hinder end of the latter. It is traversed by an axial cavity, the neurocoele (Fig. 752 , cent. c.), connected with the mid-dorsal region by a longitudinal cleft-the dorsal fissure. At the fore-end of the nervetube the neurocœle becomes dilated, forming a considerable cavity, the encephalocole or cerebral ventricle (Fig. 752, en. cœ., Fig. 756, c.v.), and a little behind this the dorsal fissure widens out above to form a trough-like dorsal dilatation (dil.) covered only by the delicate connective-tissue sheath which invests the whole nerve-tube. The anterior end of the neuron, containing these two cavities, is to be
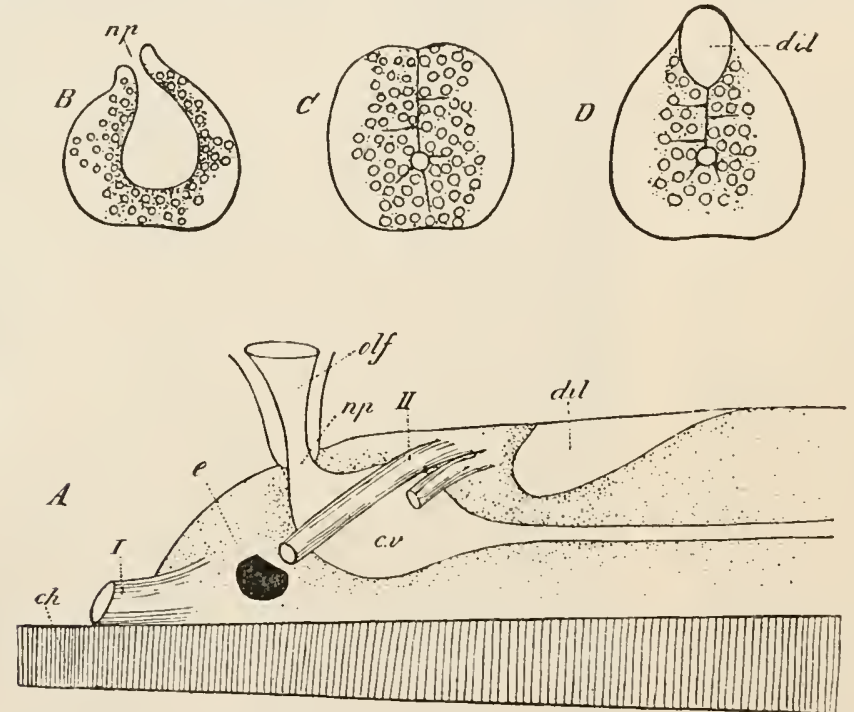

FIG. 756.-Amphioxus lanceolatus. $A$, brain and cerebral nerves of a young specimen; $B$, transverse section through neuropore ; $C$, behind cerebral ventricle ; $D$, through dorsal dilatation. $c h$. notochord; $c . v$. cerebral ventricle; dil. dorsal dilatation; $e$. eye-spot ; np. neuropore; olf. olfactory pit; $I, I I$, cerebral nerves. (From Willey, after Hatschek.)

looked upon as the brain, although not distinguishable externally from the remaining portion or spinal cord.

The anterior and dorsal region of the brain is produced into a small, hollow, pointed pouch which comes into relation with the olfactory organ and is called the median olfactory lobe. In its posterior and ventral region a depression has been described which appears to correspond with the infundibulum of the Craniata (vide, p. 99). In the young animal the cerebral ventricle opcns above by an aperture, the neuropore (Fig. $756, B, n p$.), which subsequently closes up.

The neuron.is mainly composed of longitudinal nerve-fibres with abundant nerve-cells mostly grouped around the neurocœle. At 
intervals giant nerve-cells occur-multipolar cells of immense proportional size, connected witl nerve-fibres of unusual thickness - the giant fibres. The latter appear to correspond with the giant fibres of Chætopods (Vol., I. p. 466), which have sometimes been supposed to have no nervous function and to be mere supporting structures.

The peripheral nervous system consists of the nerves given off from the neuron. They are divisible into two sets, the first consisting of two pairs of cerebral nerves (Fig. $756, I$. and $I I$.) arising from the brain, the second of a large number of spinal nerves arising from the spinal cord. The cerebral nerves take their origin in front of the first myomere, the first from the anterior extremity of the brain, the second from its dorsal region: they are both distributed to the snout, their branches being provided towards their extremities with numerous ganglia containing nervecells. The spinal nerves are segmentally arranged, and, in correspondence with the disposition of the myomeres, those of the right and left sides arise alternately, and not opposite one another (Fig. 757). In each segment there are two nerves on each side, a dorsal nerve, arising by a single root from the dorsal aspect of the spinal cord, and a ventral nerve, arising by numerous separate fibres: the dorsal nerves supply the skin and the transverse muscles and are therefore both sensory and motor, the ventral nerves are purely motor, supplying the myomeres.

Sensory Organs.-At the level of the anterior end of the brain is a narrow ciliated depression, the olfactory pit (Fig. 756, olf.), opening externally on the left side of the snout and connected at its lower end with the median olfactory lobe. This structure is supposed to be an organ of smell : in the larva

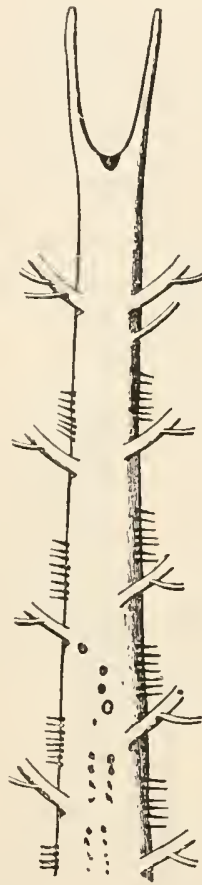

FIG. 757.-Amphioxus lanceolatus. - An. terior portion of neuron from above, showing nerves. (From Willey, after Schneirler.) its cavity is in direct communication with the neurocole through the neuropore (np.).

An unpaired pigment-spot (e) in the front wall of the brain is usually referred to as a median cerebral eye. There is no lens or other accessory apparatus, and experimental evidence seems to show that this so-called eye is not sensitive to light. Smaller eye-like organs with or without pigment occur in the spinal cord throughout the greater part of its length. There is no trace of auditory organs. The groove of Hatschek, on the roof of the buccal 
cavity, is supposed to have a sensory function, but this is very doubtful. Lastly, the sensory cells on the cirri of the oral hood give those organs an important tactile function.

Reproductive Organs.-The sexes are separate, but there is no distinction, apart from the organs of reproduction, between male and female. The gonads (Fig. 752, gon., Figs. 751, A, and 753,g.) are about twenty-six pairs of pouches arranged metamerically

B
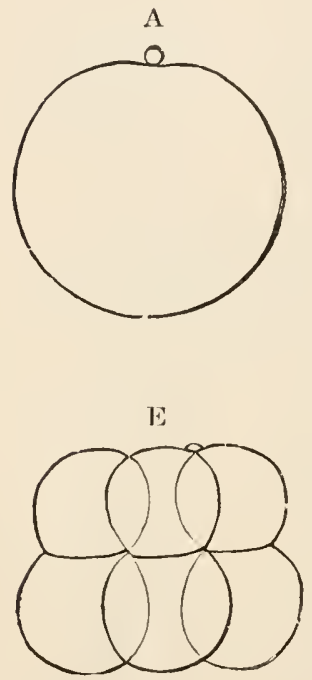

$\mathrm{H}$

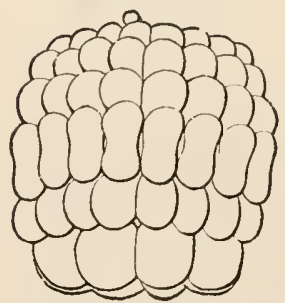

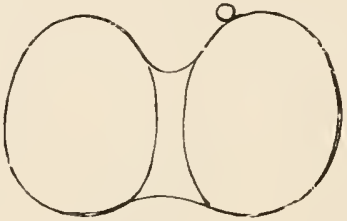

C

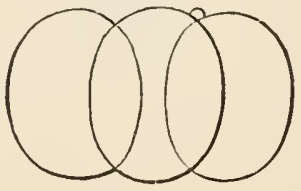

F

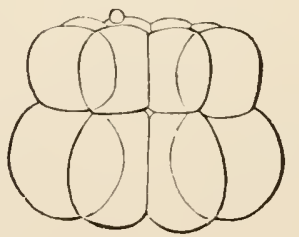

I

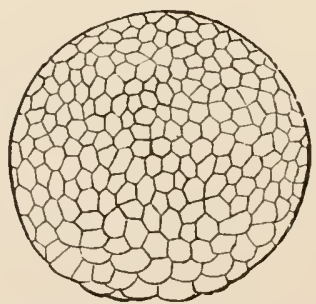

D

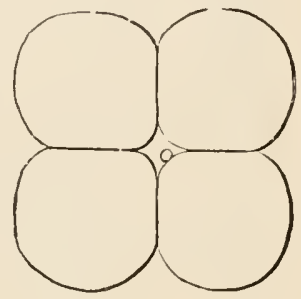

G

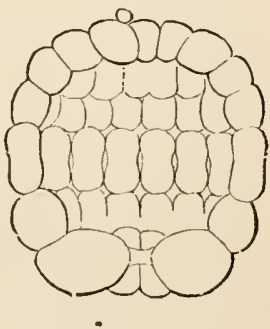

$\mathrm{K}$

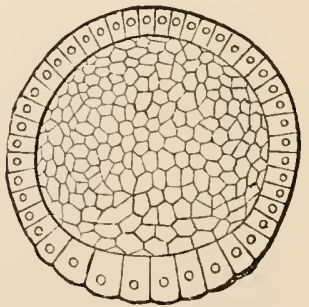

FIG. 758.-Amphioxus lanceolatus. Segmentation of the oosperm. $D$, the four-celled stage $(C)$ from above; $G$, vertical section of $H: K$, vertical section of the blastula stage $I$. (From Korschelt and'Heider, after Hatschek.)

along the body-wall, and projecting into the atrium so as largely to fill up its cavity. 'The inner or mesial face of each pouch is covered by atrial epithelium pushed inwards by the growth of the gonads; within this, and completely surrounding the reproductive organ, is a single layer of epithelium which is shown by 
development to be cœlomic. Hence each gonad is surrounded by a closed cœlomic sac.

When ripe the inner walls of the gonadic pouches burst, and the ova or sperms make their way into the atrium and thence by the atriopore to the external water. The laid eggs are covered by a thin vitelline membrane, to which a second, inner peri-vitelline membrane is added, the substance of which is derived from droplets in the protoplasm.

Development.-After maturation (Fig. 758, A) and impregnation, the membranes separate from the oosperm, leaving a wide space around the latter. Segmentation is complete, there being very little yolk; it begins by a meridional cleft dividing the oosperm into two $(B)$, and followed by a second cleft, also meridional, at right angles to the first $(C, D)$. Next, an (approximately) equatorial cleavage takes place, the embryo coming to be formed of eight cells $(E)$, of which the four belonging to the upper hemisphere, distinguished by the presence of the polar bodies, are smaller than the
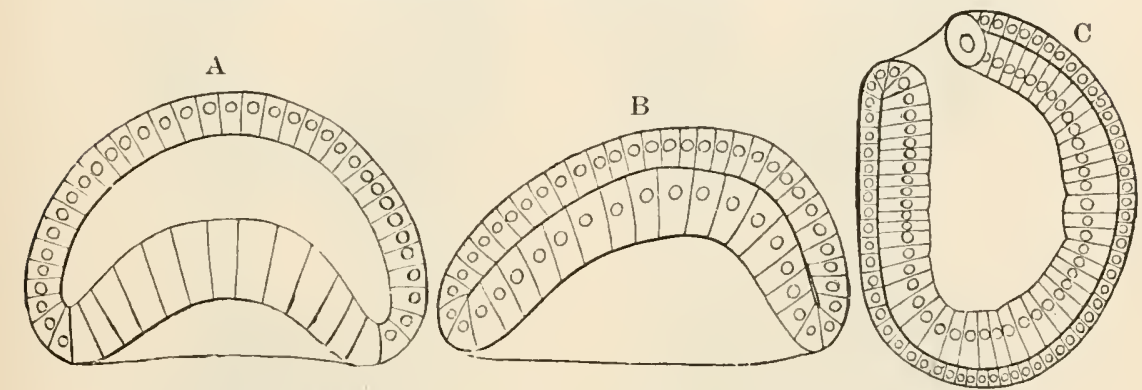

Frg, 759.-Amphioxus lanceolatus. Three stages in the formation of the gastrula. (From Korschelt and Heider, atter Hatschek.)

lower four. Apertures at the poles lead into a central cavity. Further meridional and latitudinal divisions take place, and the embryo becomes a blastula $(I, K)$, enclosing a spacious blastocoele, and having the cells on its lower pole (megameres) larger than the rest (micromeres). The polar apertures disappear owing to the closer approximation of the cells.

Invagination then takes place (Fig. 759, A), occasioned by the rapid multiplication of the micromeres; then the lower pole of the blastula becomes gradually pushed in until the whole lower hemisphere is in complete contact with the upper hemisphere and the blastocœle obliterated $(B)$. The gastrula thus formed is at first basin-shaped, having a very wide blastopore, but its cavity (the archenteron) gradually deepens, and the blastopore is reduced to a comparatively narrow aperture $(C)$, afterwards destined to form the anus. At the same time the aspects of the body are marked out; the dorsal surface becomes flattened, the ventral 
convex; the blastopore marks the posterior end and is distinctly dorsal in position. Cilia are developed from the ectoderm cells, and by their vibration cause the embryo to rotate within its membrane.

The ectoderm cells forming the median portion of the flattened dorsal surface now become differentiated and sink below the rest, giving rise to the medullary plate (Fig. 760, $A, m p$ ). The ordinary ectoderm cells on each side of this plate rise up as a pair of longitudinal medullary folds $(h b)$, extend towards the middle line and

A

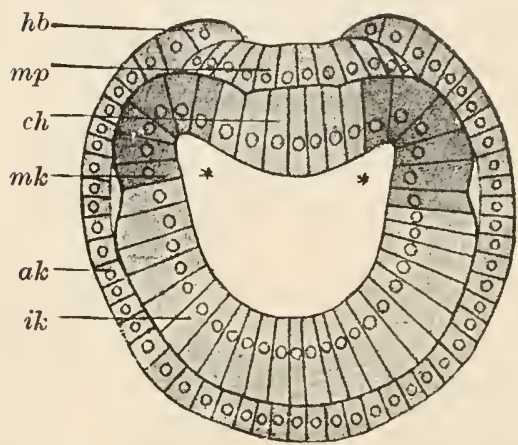

C

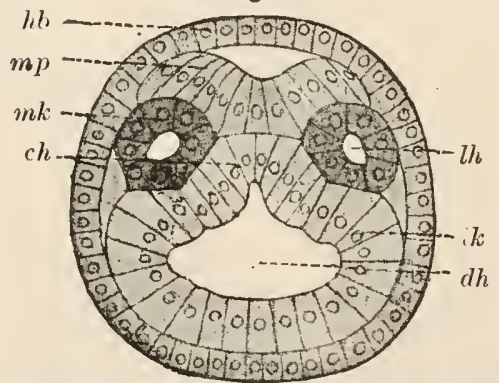

$\mathrm{B}$

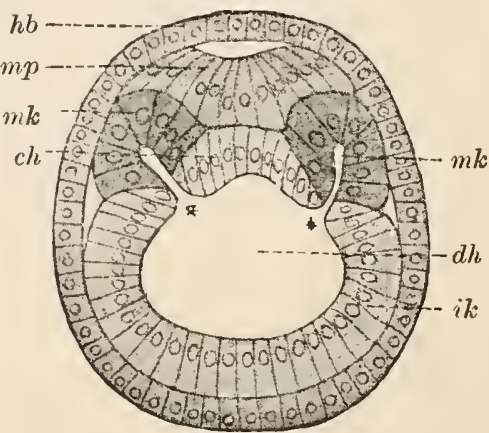

$\mathrm{D}$

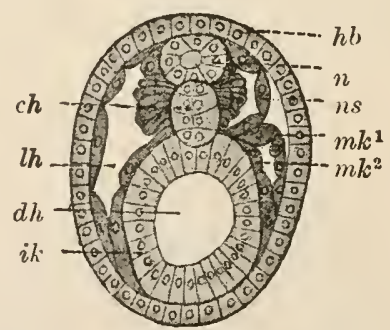

Fio. 760-Amphioxus lanceolatus. Four stages in the development of the notochord, nervous system, and mesoderm. $a k$, ectoderm; $c h$. notochord; $d h$. cavity of archenteron ; $h b$. ridge of ectoderm growing over nedullary plate; $i k$. endoderm; $l h$. colome; $m$ : cœlomic pouch ; $m k^{1}$. parietal layer of mesoderm ; $m k^{2}$. visceral layer ; $m p$. medullary plate ; $n$. neuron; ns. protovertebra. (From Korschelt and Heider, after Hatschek.)

unite $(B, h b)$, covering over the medullary plate. The latter bends upwards at the sides so as to become trough-like instead of flat $(C)$, and, its two sides coming in contact with one another above, the plate is converted into a tube, the neuron $(D, n)$, enclosing a central canal, the neurocole, continued dorsally into a narrow cleft. The medullary folds extend behind the blastopore so that, when they unite, the latter aperture opens into the neurocœle by a neurenteric canal (Fig. 761, A, cn). Anteriorly the folds remain apart up to a 
late period, so that the neurocœle opens externally in front by a wide aperture, the neuropore (Figs. 761, 762, and 763, np).

While the central nervous system is thus being formed, the endodermal wall of the archenteron develops dorso-laterally a pair of longitudinal folds (* in Fig. 760, $A$ and $B$ ), the cavities in which, continuous with the archenteron, are the beginnings of the enterocolic system. Transverse folds which appear divide the longitudinal folds into segments, with the result that the archenteron comes to have appended to it dorso-laterally a paired series of offshoots, the enterocoelic or colomic pouches (Fig. $760, \mathrm{mk}$ ), arranged metamerically. In this way segmentation is established, and it is at this period that the embryo ruptures its containing membrane and begins frea
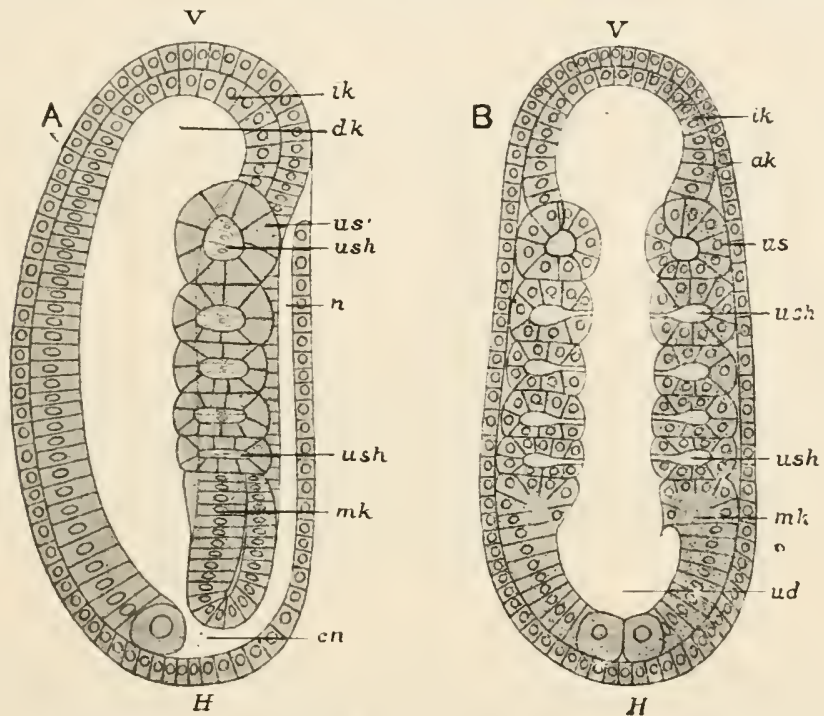

FIG. 761.- Amphioxus lanceolatus. Embryo. $A$, from the side; $B$, in horizontal section. $a k$. ectoderm ; $c n$. neurenteric canal ; $d k$. archenteron ; $i k$. endoderm ; $m k$. mesodermal folds; $n$. neural tube; ud. archenteron; us. first colomic pouch ; ush. cœelomic cavity; $I^{\prime}$. anterior; $H$. posterior end. (From Korschelt and Heider, after Hatschek.)

existence. Before long the colonic pouches separate from the archenteron and take on the form of a series of closed colomic sacs or somites (Fig. $760, C, D$ ), lying between ectoderm and endoderm. From the walls of these sacs the mesoderm is derived: their cavities uniting become the colome, which is therefore an enterocoele, like that of Sagitta and the Echinodermata.

While the coelomic sacs are in course of formation a median groove appears along the dorsal wall of the archenteron (Fig. $760, B, C, c h$ ): it deepens, loses its tubular character, and becones a solid rod, the notochord $(D, c h)$, lying immediately beneath the nerve-tube. The ordinary endoderm cells soon unite beneath it and so shut it off from the archenteron. It will be seen that the notochord, like the 
neuron, never exhibits any trace of segmentation. At its first formation it stops short of the anterior end of the archenteron : its final extension to the end of the snout is a subsequent process.

The significance of these early stages in the development of Amphioxus has been variously regarded by different embryologists, and it is impossible to give here more than a statement of the ascertained facts, leaving the question of their interpretation, which can only be profitably discussed on the broadest basis, as a matter for more advanced study. Since, however, the gastrulation and mesoderm-formation in Amphioxus are, almost universally, made the starting-point in the process of interpreting the plienomena of early development in all Vertebrates, it may be desirable at the present stage to state that though in the foregoing account the cells which are invaginated are referred to throughout simply as endoderm, this view of their nature is not the only one that may reasonably be held. If the inner layer of the gastrula be composed of endoderm and of endoderm only, certain consequences necessarily follow : the notochord must be purely endodermal in origin, and so must the whole of the mesoderm. This is the terminology which has been followed in the preceding pages. But it may be lield that the process of invagination is not so simple, and that the inner layer of the resulting gastrula is made up of two distinct parts, a dorsal part, which is ectodermal, and a ventral part, which consists of endoderm. On this view the notochord and the mesoderm, derived from the dorsal part of the invaginated layer, would both be of ectodermal origin, and only the enteric epithelium would be endodermal. These points will be referred to again at a later stage.

A further point about which there may be room for differences of opinion is the detailed development of the colome. According to one view of the facts the cœlome of Amphioxus may at one stage be compared to that of Balanoglossus (p. 3) :-with an unpaired anterior part, destined to form the headcolome and representing the proboscis-cavity of Balanoglossus ; a middle pair of pouches which form the first pair of somites, and a pair of canal-like backward extensions, which may be compared to the collar-cavities ; and a posterior pair corresponding with the trunk-colome of the Enteropneust-the last becoming divided up to form the cœlomic sacs.

New cœlomic pouches are formed in regular order from before backwards, the embryo at the same time elongating and becoming laterally compressed and pointed fore and aft. At the anterior end the mouth (Fig. 762, $m$ ) appears on the left side of the body as a small aperture, which soon increases greatly in size. On the ventral surface another small aperture, the first gill-slit (ks), makes its appearance, and soon shifts over to the right side: it forms a direct communication between the pharynx and the exterior, like the stigmata of Appendicularia (p. 23) : there is at present no trace of the atrium.

The anterior end of the archenteron has meanwhile grown out into a pair of pouches, which become shut off as closed sacs : of these the right gives rise to the cœlome of the head $(h)$, the left to a depression called the pre-oral pit $(w)$, which opens on the exterior, and from which the groove of Hatschek and the wheel-organ are afterwards formed. The pre-oral pit also gives rise to Hatschek's nephridium (Fig. 763, x), a narrow ciliated tube which opens into the anterior part of the pharynx, and runs forwards to terminate 
blindly in the roof of the oral hood: it disappears completely in the adult except in Amphioxides, in which it is said to contain solenocytes.

On the floor of the archenteron in the neighbourhood of the mouth a depression appears giving rise to a structure known as the $c l u b$ shaped gland $(k)$, which may be a modified gill-cleft. Posteriorly the neurenteric canal closes and the anus appears.

We left the mesoderm in the form of separate paired somites, arranged metamerically in the dorsal region of the embryo. These increase in size, and extend both upwards and downwards, each presenting a somatic layer (Fig. $760, D, m k^{1}$ ) in contact with the external ectoderm, and a splanchnic layer $\left(m k^{2}\right)$ in contact with the nervous

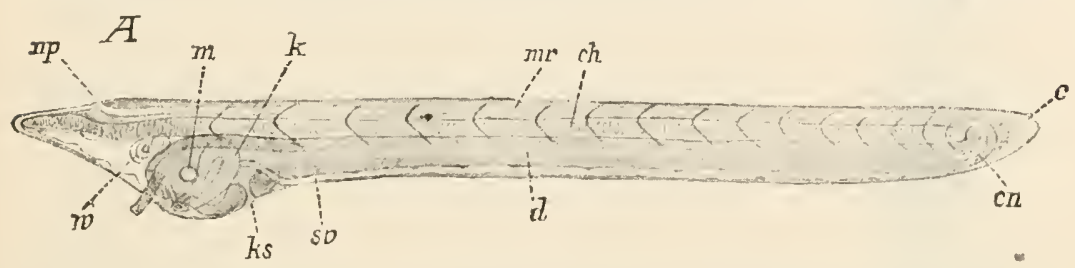

$B$

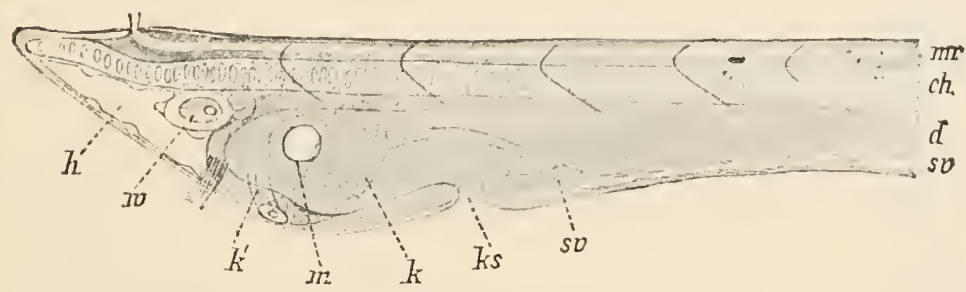

Fia. $76 \%$ - Amphioxus lanceolatus. A, young larva; $B$, anterior end more highly magnitied. $c$. provisional tail-fin ; $c h$. notochord; $c n$. neurenteric canal; $d$. enteric canal; $h$. colome of head; $k$. club-shaped gland ; $k^{\prime}$. its external aperture ; $k s$. first gill-slit ; $m$, mouth ; $m r$. neuron; $n p$. neuropore ; $s v$. sub-intestinal vein; $v$. pre-oral pit. (From Korschelt and Heider, after Hatschek.)

system and notochord dorsally, and with the enteric canal ventrally. At about the level of the ventral surface of the notochord a horizontal partition is formed in each cœlomic sac (Fig. 760, D), separating it into a dorsal and ventral portion. The dorsal section is distinguished as the protovertebra or myotome $(n s)$, and its cavity. as the myocole or muscle-cavity : the ventral section is called the lateral plate or splanchnotome, and its cavity forms a segment of the cœlome.

The lateral plates now unite with one another in pairs below the enteric canal, their cavities becoming continuous: at the same time the cavities of successive lateral plates are placed in communication with one another by the absorption of their adjacent (anterior and posterior) walls. In this way the cavities of the entire series 


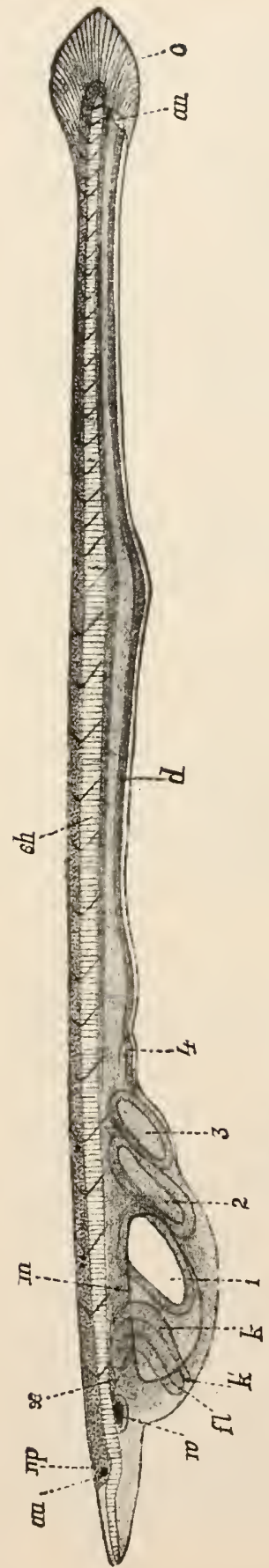

of ventral plates, right and left, unite to form the single unsegmented coelome of the adult, their walls giving rise to the cœlomic epithelium.

At the same time the cells of the splanchnic layer of the protovertebræ become converted into muscular fibres, which nearly fill the myocœle, and give rise to the myomeres: the myocommas arise from the adjacent anterior and posterior walls of the protovertebræ. An outpushing of the splanchnic layer, at about the level of the ventral surface of the notochord, grows upwards between the myomere externally and the notochord and nerve-tube internally: from the cells lining this pouch the connective-tissue sheath of the notochord and neural canal arises, and perhaps also the fin-rays. From the parietal layer of the protovertebræ is formed the derm or connective-tissue layer of the skin.

The larva increases in size, and becomes very long and narrow, with a pointed anterior end and a provisional caudal fin posteriorly (Fig. 763, c). As growth proceeds, new segments are added behind those already formed, the notochord grows forwards to the anterior end of the snout, and the eye-spot (au.) and olfactory pit appear, the latter as an ectodermal pit which communicates with the neurocœle by the still open neuropore $(n p$.$) . The mouth (m$.) attains a relatively immense size, still remaining on the left side.

Additional gill-slits arise behind the one already mentioned: they all make their appearance near the middle ventral line, and gradually shift over to the right side: at first they correspond with the myomeres, so that the segmentation of the pharynx is part of the general metamerism of the body. Altogether fourteen clefts are produced in a single longitudinal series. Above, i.e., 
dorsal to them, a second longitudinal series makes its appearance, containing eight clefts, so that at this stage there are two parallel rows of gill-slits on the rightside of the body, and none on the left. But as growth goes on, the first or ventral series gradually travels over to the left side, producing a symmetrical arrangement, and at the same time the first slit and the last five of the first or definitively left series close up and disappear, so that the numbers are equalised on the two sides. At first each gill-slit is simple, but before long a fold grows down from its dorsal edge, and, extending ventrally, divides the single aperture into two: this fold is the secondary or tongue-lamella, the original bars of tissue between the undivided slits becoming the primary lamellæ.

While the development of the gill-slits is proceeding, the atrium is in course of formation. Paired longitudinal ridges, the metapleural folds (Fig. 764, lf. rf., Fig. 765, sf.), appear on the ventral side of the body, behind the gill-slits, and gradually extend forwards,

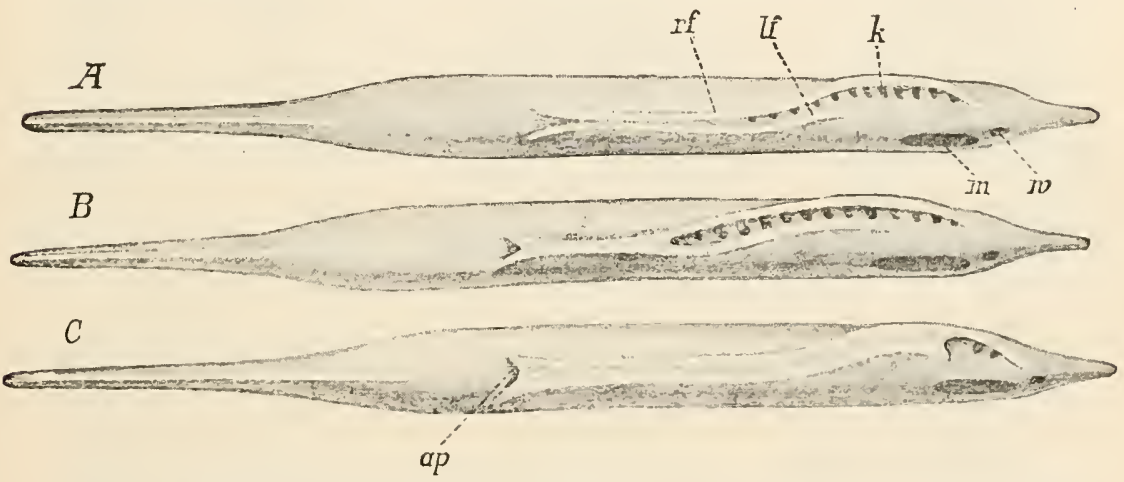

Fia. 764.-Amphioxus lanceolatus. Ventral aspect of three larve showing the development of the atrium. ap. atriopore; $k$. gill-slits ; lf. left metapleural fold ; $m$. inouth ; $r f$. right metapleural fold ; $w$. pre-oral pit. (From Korschelt and Heider, after Lankester and Willey.)

dorsal to the latter, their arrangement being very unsymmetrical in correspondence with that of the clefts themiselves. On the inner face of each fold, i.e. the face which looks towards its fellow of the opposite side, a longitudinal sub-atrial ridge (Fig. $765, A, s l$ ) appears, and the two sub-atrial ridges meeting and coalescing, a canal $(B, p)$ is formed immediately below the ventral body-wall. This canal is the commencement of the atrium : it is at first quite narrow, but gradually extends upwards on each side $(C, p)$ until it attains its full dimensions. It is open, at first, both in front and behind: the posterior opening remains as the atriopore: the anterior opening becomes gradually shifted forwards as the fusion of the sub-atrial ridges proceeds (Fig. 764, $B$ and $C$ ), and is finally completely closed. In this way the gill-slits come to open, not directly on the exterior, but into a cavity formed by the union of paired ridges of the body-wall, and therefore lined by ectoderm. 
The mouth gradually passes to the ventral surface, and undergoes a relative diminution in size : a fold of integument develops round it and forms the oral hood, which is probably to be looked upon as a stomodæum. The endostyle appears on the right of the pharynx (Fig. 763, $f$ ), and is at first rod-shaped, then $\mathrm{V}$-shaped : ultimately the limbs of the $V$ unite in the middle ventral line. The gill-slits increase in number and become more and more vertically elongated. The provisional caudal fin disappears. The gonads arise from the outer and ventral regions of the protovertebræ in the form of pouches, which gradually assume their permanent form.

Distribution.-The Branchiostomidæ are very widely distributed in tropical and warm-temperate seas. Amphioxides has only been obtained with the tow-net and is, seemingly, of
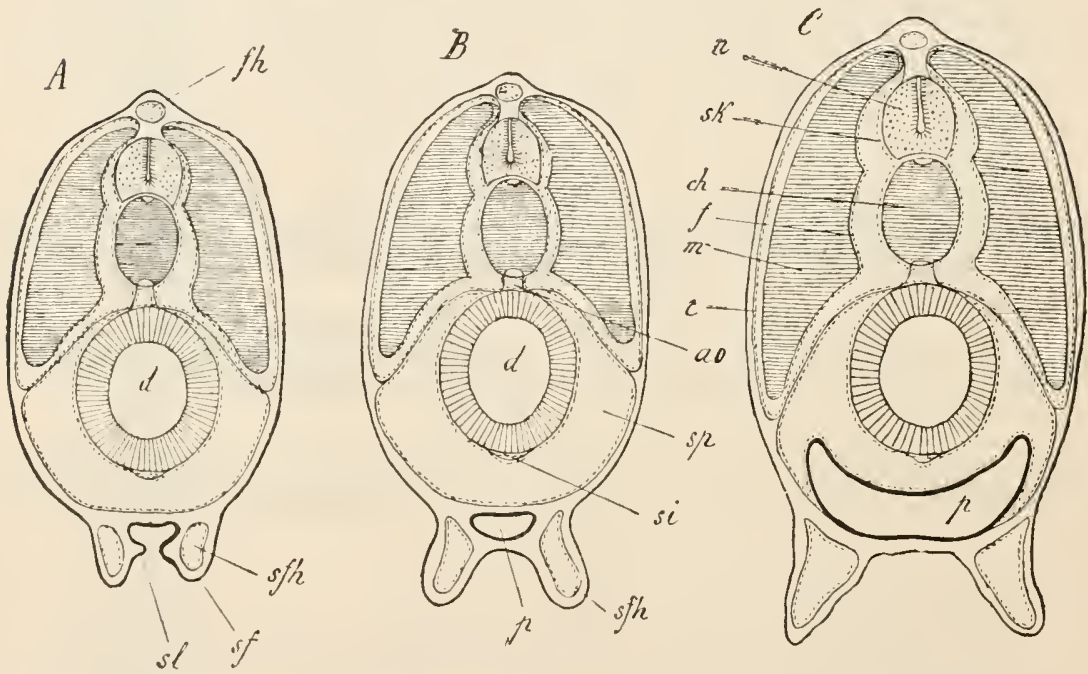

FIG. 765.-Amphioxus lanceolatus. Diagrammatic transverse sections of three larva to slow the development of the atrium. ao. aorta; $c$. dermis ; $d$. intestiue $; f$ fascia (layer of connective-tissue on inner surface of myomere); $f h$. cavity for dorsal fin-ray ; $m$. myomere; $n$. neuron; $p$. atrium : $s f$. metapleural folds; $s f h$. metapleural lymph-space; si. sub-intestinal vein; $s k$. sheath of notochord and neuron; sl. sub-atrial ridge ; $s p$. colome. (From Korschelt and Heider, after Lankester and Willey.)

permanently pelagic habit. It differs from Amphioxus in the absence of oral cirri and of an atrial cavity, the branchial slits opening directly on the exterior in an unpaired ventral row. No fully mature specimens have yet been found, but the larger specimens have a single row of gonads.

Distinctive Characters.-The Acrania may be defined as Euchorda in which the notochord extends to the anterior end of the snout, in advance of the central nervous system. There is no skull, and no trace of limbs. The ectoderm consists of a single layer of cells which may be ciliated. The pharynx is of immense 
size, perforated by very numerous gill-slits, and surrounded by an atrium. The liver is a hollow pouch of the intestine. There is no heart, and the blood is almost colourless. The nephridia remain distinct and open into the atrium. The brain is very imperfectly differentiated; there are only two pairs of cerebral nerves; and the dorsal and ventral spinal nerves do not unite. There are no paired eyes, but there is a median pigment-spot in the wall of the brain, and many others in the spinal cord; an auditory organ is absent. The gonads are metamerically arranged and have no ducts. There is a typical invaginate gastrula, and the mesoderm arises in the form of metameric cœlomic pouches. The cœlome is an enterocœle.

Affinities.-Amphioxus has had a somewhat chequered zoological history. Its first discoverer placed it among the Gastropoda, considering it to be a Slug. When its vertebrate character was made out, it was for a long time placed definitely among Fishes, as the type of a distinct order of that class; but it became obvious, from a full consideration of the case, that an animal with neither skull, brain, heart, auditory organs, nor paired eyes, with colourless blood, with no kidneys in the ordinary sense of the word, and with its pharynx surrounded by an atrium, was more widely separated from the lowest Fish than the lowest Fish from a Bird or Mammal.

There was still, however, no suspicion of any connection between Amphioxus and the Urochorda until the development of both was worked out, and it was shown that in many fundamental points, notably in the formation of the nervous system and the notochord, there was the closest resemblance between the two. The likeness was further emphasised by the presence in both forms of an endostyle, an epipharyngeal groove (dorsal lamina) and peripharyngeal bands, and of an atrium, and by the obvious homology of the stigmata or gill-slits of Tunicates with those of Amphioxus. The Urochorda being obviously a degenerate group, it was suggested that the peculiarities of the adult Amphioxus might also be due to a retrogressive metamorphosis. Of this, however, there is not sufficient evidence, and most recent investigations have tended to bring the Acrania nearer to the Craniate Vertebrata, and to remove them further from the lower Chordata.

\section{SECTION II.-CRANIATA (VERTEBRATA).}

The group of the Craniata (Vertebrata) includes all those animals known as Fishes, Amphibians, Reptiles, Birds, and Mammals, or, in other words, Vertebrata having a skull, a highly complex brain, a heart of three or four chambers, and red blood-corpuscles.

In spite of the obvious and striking diversity of organisation obtaining among Craniata-between, for instance, a Lamprey, a Pigeon, and a Dog-there is a fundamental unity of plan running 
through the whole group, both as to the general arrangement of the various systems of organs and the structure of the organs themselves - far greater than in any of the principal invertebrate groups. The range of variation in the whole of the six classes included in the division is, in fact, considerably less than in many single classes of Invertebrata - for instance, Hydrozoa or Crustacea. Hence, while the plan hitherto adopted of treating the group class by class will be followed, it will be found convenient to begin by devoting a considerable space to a preliminary account of the Craniata as a whole, since in this way much needless repetition will be avoided.

The Craniata include the following classes and sub-classes :-

Class I.-Cyclostomi,

Including the Lampreys and Hags.

Class II.-Pisces,

True Fishes, which are again divisible into

Sub-class 1.-Elasmobranchii,

Including the Sharks and Rays.

Sub-class 2.-Holocephali,

Including the Cat-fish (Chimora) and the Elephant-fish

(Callorhynchus).

Sub-class 3.-Teleostomi,

Including the bony Fishes, such as Perch, Cod, Trout, \&c., and the Sturgeons and their allies.

Sub-class 4.-Dipnoi, ${ }^{1}$

Mud-Fishes.

Class III.-Амphibia,

Including Frogs, Toads, Newts, and Salamanders.

Class IV.-Reptilia,

Including Lizards. Snakes, Crocodiles, Turtles, and Tortoises.

Class V.-Aves,

Birds.

1 'The animals included in Classes I. and II. are all "Fishes" in the broad sense of the word. 


\section{Class Vl. Nammalia,}

Including Hairy Quadrupeds, Seals, Whales, Bats, Monkeys, and IIan.

External Characters.-The body of Craniata (Fig. 766) is bilaterally symmetrical, elongated in an antero-posterior direction, and usually more or less cylindrical. It is divisible into three regions: the head, which contains the brain, the chief sensory organs, and the mouth and pharynx; the trunk, to which the coelome is confined, and which contains the principal digestive and circulatory as well as the excretory and reproductive organs; and the tail, or region situated posteriorly to the cœlome and anus, and containing none of the more essential organs. Between the head and trunk there is frequently a narrow region or neck, into which the coelome does not extend. In aquatic Vertebrates the tail is of great size, not marked off externally from the trunk, and is the chief organ of locomotion: in terrestrial forms it usually becomes greatly reduced in diameter, and has the appearance of a mere unpaired posterior appendage.

The mouth $(m t h$.$) is a transverse aperture placed at or near the$ anterior end of the head. Near it, sometimes dorsal, sometimes ventral in position, are the paired nostrils or anterior nares (na.) or in Cyclostomi the single nostril-leading to the organs of smell. Farther back, on the sides of the head, are the large paired eyes (e.), and on the dorsal surface there is sometimes more or less indication of a vestigial median or pineal sense-organ ( $p n$. e.), which may take the form of an eye. Posterior to the paired eyes are the auditory organs (au.), the position of which is indicated in the higher forms by an auditory aperture.

On the sides of the head, behind the mouth, are a series of openings, the gill-slits or external branchial apertures (e. br. a. $1-7)$ : they are rarely more than seven in number, $(C f$. p. 139$)$ and in air-breathing forms disappear more or less completely in the adult. In the higher Fishes a fold called the operculum (Fig. 780 , op.) springs from the side of the head immediately in front of the first gill-slit and extends backwards, covering the branchial apertures.

On the ventral surface at the junction of the trunk and tail is the anus (an.). Distinct urinary and genital apertures, or a single urino-genital aperture, are sometimes found either in front of or behind the anus, but more commonly the urinary and genital ducts open into the termination of the enteric canal, or cloaca, so that there is only a single egestive opening, known as the cloucal aperture. On either side of this there may be a small abdominal porc $(a b . p$.$) leading into the coelome.$

In Fishes and some Amphibians, the trunk and tail are produced in the middle dorsal line into a vertical fold or median fin, which is 


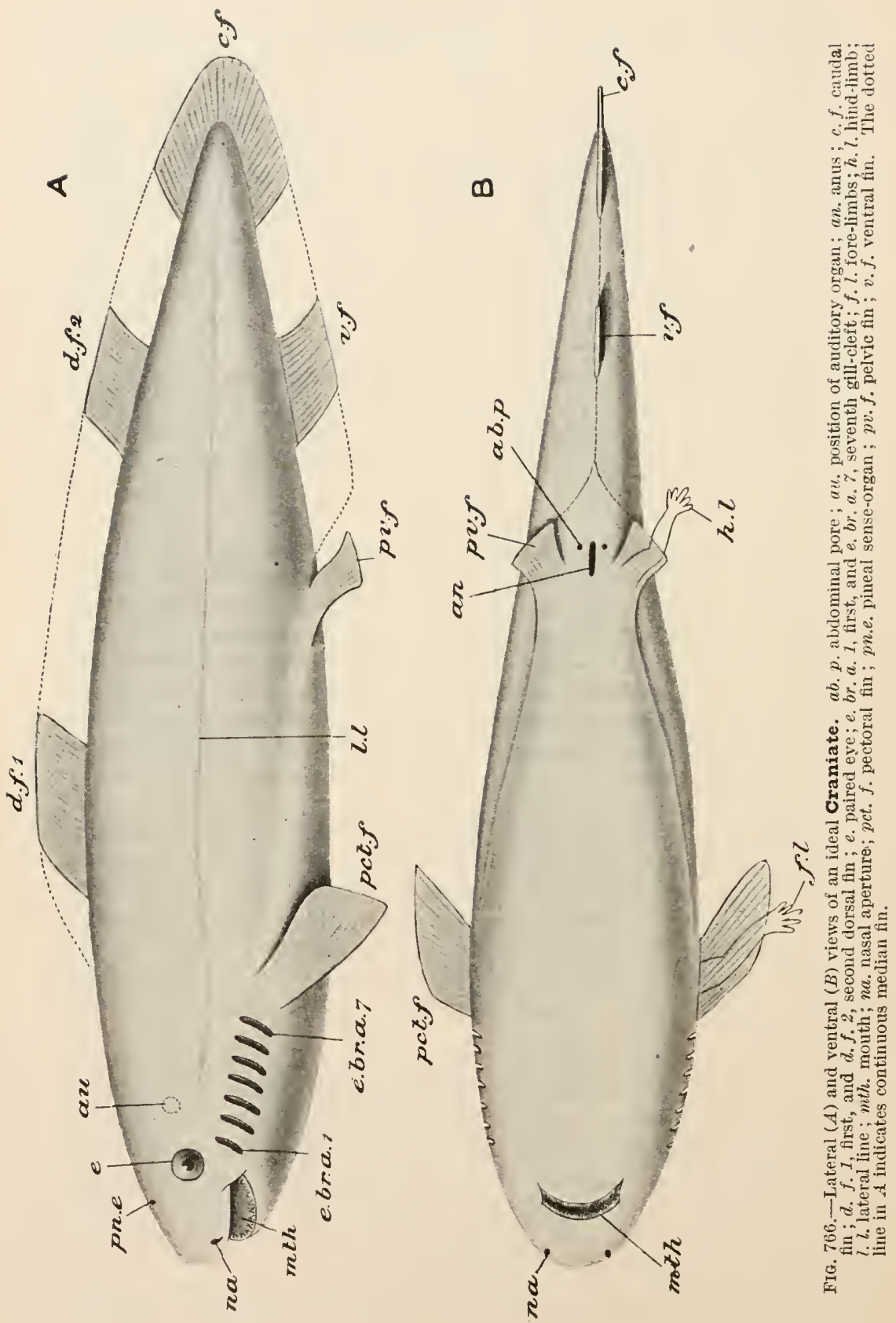


continued round the end of the tail and forwards in the middle line to the anus. Frequently this continuous fin becomes broken up into distinct dorsal (d.f. 1 and 2$)$, ventral $(v . f$.$) , and caudal (cd.f.)$ fins, which may assume very various forms: in the higher classes all trace of median fins disappears ( $c f$., however, the Cetacea).

Fishes also possess paired fins. Immediately posterior to the last gill-slit is a more or less horizontal outgrowth, the pectoral fin (pct.f.), while a similar but smaller structure, the pelvic fin $(p v . f$.$) ,$ arises at the side of the anus.

In all Craniata above Fishes, i.e., from Amp hibia upwards, the paired fins are replaced by fore- and hind-limbs (f.l., h.l.), each consisting of three divisions--upperarm, fore-arm, and hand in the one case; thigh, shank, and foot in the other. Both hand and foot normally terminate in five fingers or digits, and the pentadactyle limb thus formed is very characteristic of all the higher Vertebrata. The paired fins, or limbs, as the case may be, are the only lateral appendages possessed by Vertebrates.

Body-wall and Internal Cavities. - The body is covered externally by a skin consisting of two layers, an outer or epithelial layer, the

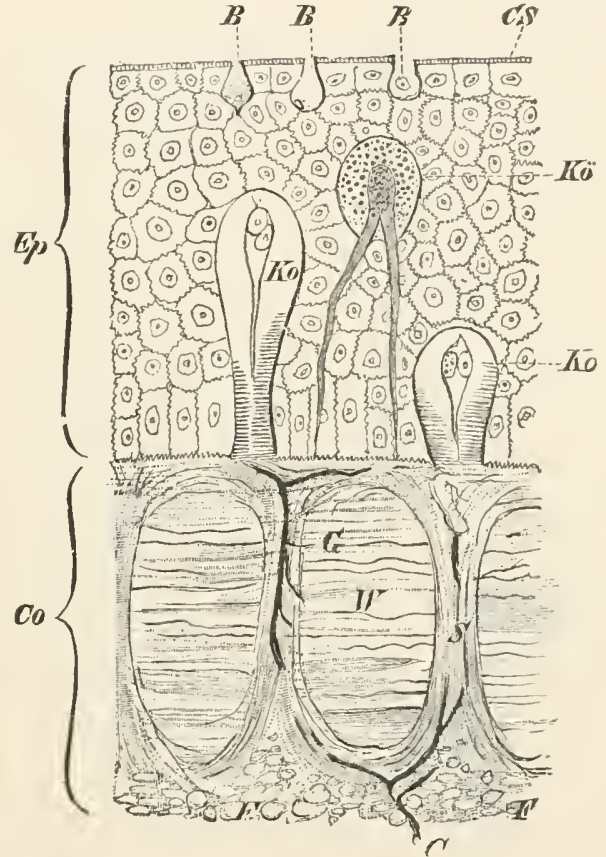

FIG. 767.-Diagrammatic vertical section of the shin of a Fish. $B$, unicellular mucous glands; $C O$, derm; $C S$, cuticular margin; $E p$, epiderm; $F$, fat ; $G$, blood: vessels ; Ko, goblet-cells ; $K \bar{o}$, granule-cells ; $S$, vertical, and $W$, horizontal bundles of connectivetissue. (From Wiedersheim's Vertelratu.)

epidermis (Fig. 767, Ep.), derived from the ectoderm of the embryo, and an inner or connective-tissue layer, the dermis $(\mathrm{Co})$, of mesodermal origin. The epidermis is always many-layered, the cells of the lower layers, forming the stratum Malpighii, being protoplasmic and capable of active multiplication, while those of the superficial layers often become flattened and horny, and constitute the stratum corneum. Glands are frequently present in the skin in the form of tubular or flask-shaped in-pushings of the epidermis or of isolated gland-cells $(B)$.

Beneath the skin comes the muscular layer. This is always 
highly developed, and, in the lower Craniata, has the same general arrangement as in Amphioxus, i.e., consists of zig-zag musclesegments or myomeres (Fig. 768, mym.), separated from one another by partitions of connective-tissue, or myocommas (myc.), and formed of longitudinally disposed muscle-fibres. The myomeres are not

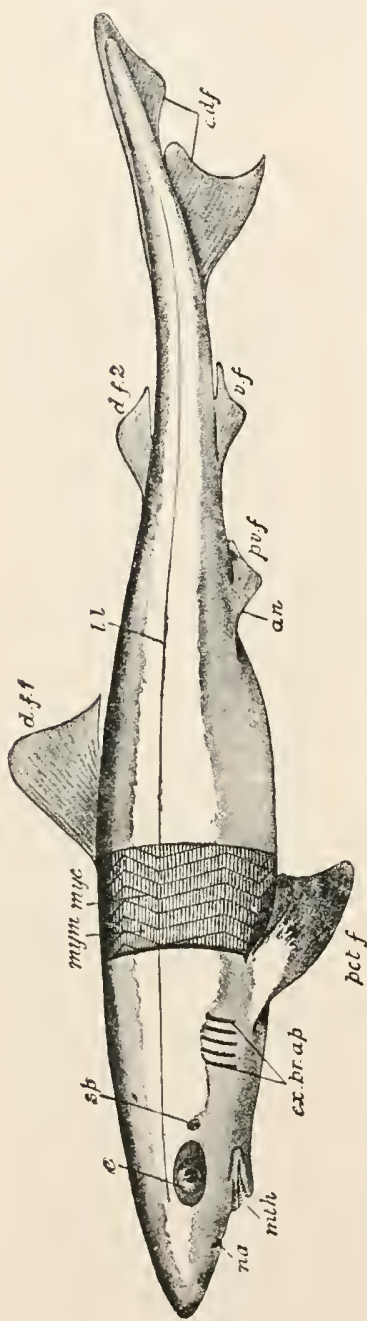
placed at right angles to the long च. axis of the body, but are directed from the median vertical plane outwards and backwards, and are at the same time convex in front Q

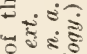
a cone-in-cone arrangement (Fig. $769, C)$. Each myomere, moreover, 웡 is divisible into a dorsal $(d . m$.) . In the higher groups this segmental 知 . 政 the myomeres becoming con今 E .

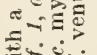

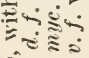

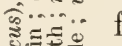

In the trunk, as shown by a section of that region, the muscles form a definite layer beneath the skin and enclosing the colome (Fig. 769, $A$ and $C$, coel.). The muscular layer, as in Amphioxus, is not of even diameter throughout, but is greatly thickened dorsally, so that the cœlome is, as it were, thrown towards the ventral side. Its dorsal portion, moreover, is excavated by a canal, the neural or cerebro-spinal cavity (c.s. c.), in which the central nervous system is contained, and the anterior portion of which is always dilated, as the cranial cavity, for the brain. Thus a transverse section of the trunk has the form of a double tube. In the head, neck, and tail $(B, D)$, the coelome is absent in the adult, and the muscles occupy practically the whole of the interval between the skin and the skeleton, presently to be referred to: in the tail, however, there is found a hocmal canal (h. c.) containing connective-tissue, and representing a virtual backward extension of the colome. The 


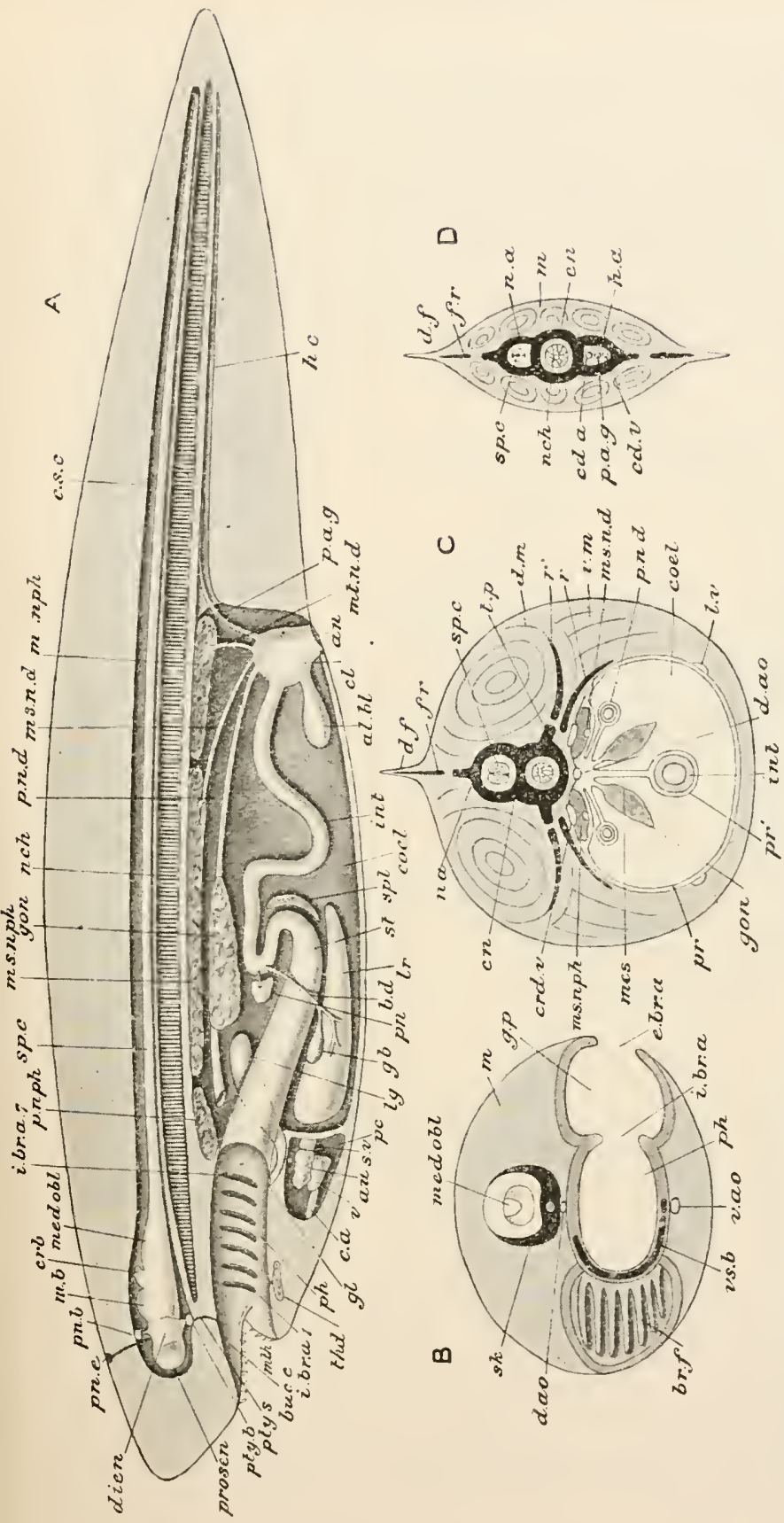

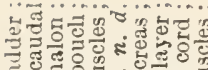

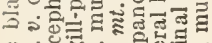
०खंड …ㄹ.

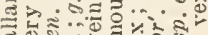

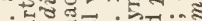
3...

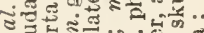
क्षु

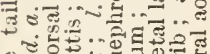
\% 4 은. $\therefore$

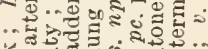
舟 至

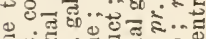
\&. ‥ a...

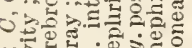

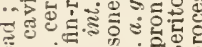
更 $0 . . .0$

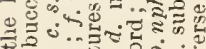
o 0 . 药 .... की o 0.

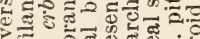

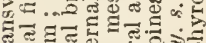
可.

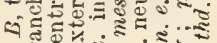
.

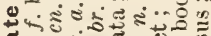
สํ.s. (....... ชै

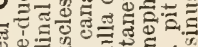
을 पें $\Rightarrow$ $00^{\circ}$ on

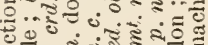

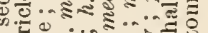

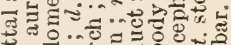
क力口

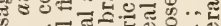

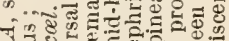
1를 कि 
fins, or fore- and hind-limbs, are moved by longitudinal muscles derived from those of the trunk. All the voluntary or body-muscles of Craniata are of the striated kind.

The colome is lined by peritoneum $(C, p r$.), a membrane consisting of an outer layer of connective-tissue, next the muscles, and an inner layer of colomic epithelium bounding the cavity, and thus forming the innermost layer of the body-wall. In Fishes the cœlome is divided into two chambers, a large abdominal cavity containing the chief viscera, and a small forwardly-placed pericardial cavity $(A, p c$.) containing the heart, and lined by a detached portion

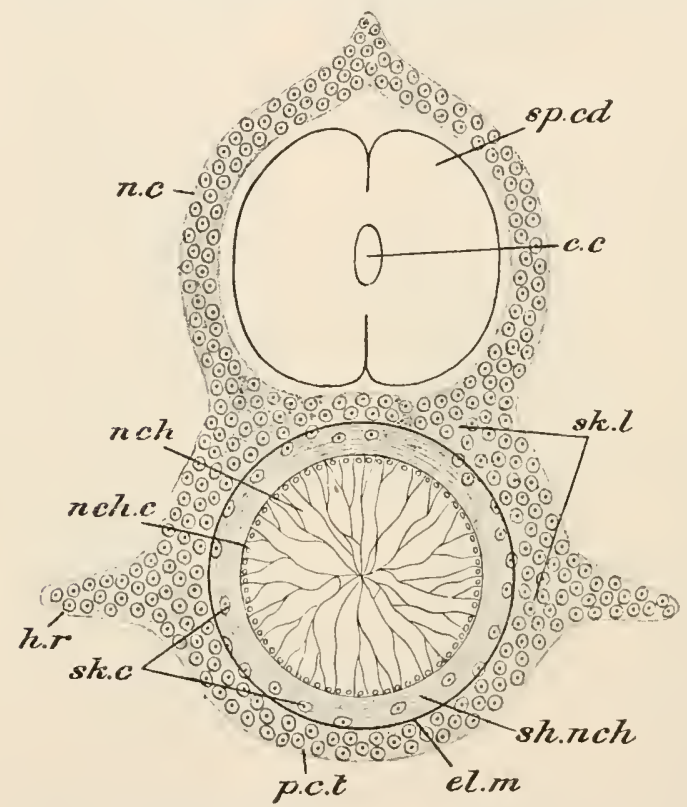

FI't 770.-Semi-diagrammatic transverse section of the vertebral column of a Craniate embryo. $c$. $c$. central canal ; el. $m$. ex ternal elastic membrane $; h . r$. hemal ridges $; n . c$. neural tube ; $n c h$. notochord; nch. $c$. notochordal cells ; $p . c$. $t$. perichordal tube; sh. nch. sheath of notochord; $s k$. $c$. skeletogenous cells migrating into notochordal sheath; sk. $l$. skeletogenous layer ; $s p$. $c d$. spinal cord. (Modified from Balfour and Gadow.) of peritoneum known as the pericardium. In Mammals there is a vertical muscular partition, the diaphragm, dividing the cœlome into an anterior chamber or thorax, containing the heart and lungs, and a posterior chamber or abdomen containing the remaining viscera.

Skeleton. - The hard parts or supporting structures of Craniata fall into two categories - the exoskeleton and the endoskeleton. The exoskeleton consists of bony or horny deposits in the skin, and may be either epidermalor dermal, or both, but is never, like the armour of an Arthropod or the shell of a Mollusc, cuticular. The epidermal exoskeleton is always formed by the cornification or conversion into horn of epidermal cells, and may take the form of scales-as in Reptiles, feathers, hairs, claws, nails, horns, and hoofs. The dermal exoskeleton occurs in the form of either bony or hornlike deposits in the derm, such as the scales and dermal fin-rays of Fishes, and the bony armour of the Strnrgeon, Crocodile, or Armadillo. The endoskeleton, or "skeleton" in the ordinary sense of the 
word, forms one of the most complex portions of the body, and presents an immense range of variation in the different classes and orders. As in Amphioxus, the axis of the entire skeletal system is formed by the notochord (Fig. 769, nch.), an elastic rod made of peculiar vacuolated cells (Fig. $770, n c h$.), resembling the pith of plants, and covered by a laminated sheath (sh.nch.), with an external elastic membrane (el. m.) around it. The whole sheath is, in the Craniata, a cuticular product of the superficial notochordal cells (nch. c.), i.e., is developed as a secretion from their outer or free surfaces. The notochord lies in the middle line of the dorsal bodywall between the cerebro-spinal cavity above and the colome below : it is usually developed, as in the lower Chordata, from a median longitudinal outgrowth of the dorsal wall of the gut. Posteriorly it extends to the end of the tail, but in front it always stops short of the anterior end of the head, ending near the middle of the brain i mmediately behind a peculiar organ, the pituitary body (Fig. $769, A$, pty. b.), which will be referred to a ga in in treating of the digestive organs and of the nervous system. The extension of the nervous system in front

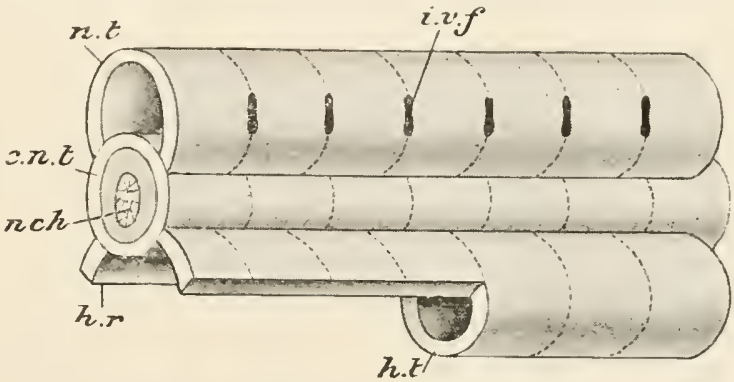

FIG. 771.-Diagram illustrating the segmentation of the vertebra column. c. n. $t$. perichordal tube; $h, r$. hemal risge; $h$. $t$. hæma tube; i. v. $f$. iutervertebral foranen; $n$. $t$. neural tube; $n c h$. notochord. The dotted lines indicate the segmentation into vertebra.

of the notochord is one of the most striking difierences between the Craniata and Amphioxus, in which, it will be remembered, the notochord is prolonged to a considerable distance beyond the anterior end of the nerve-tube.

In the majority of Craniata the notochord is a purely embryonic structure, and all but the anterior end of it is replaced in the adult by the vertebral column. The cells of mesoderm surrounding the notochord become concentrated around the sheath and give rise to the skeletogenous layer (Fig. 770, sk. l.), some of the cells of which $(s k . c$.$) may migrate through the elastic membrane into the sheath$ itself. In this way the notochord becomes surrounded by a cellular investment which soon takes on the structure of cartilage, and may be called the perichordal tube (Fig. 770, p.c.t., and Fig. 771, c.n.t.). The skeletogenous layer also grows upwards, and gives rise to an inverted tunnel of cartilage, the neural tube (n.c., n.t.), enclosing the cerebro-spinal cavity and comected below with the perichordal tube; and to paired hoemal ridges (h.r.) of cartilage standing out 
from the sides of the perichordal tube into the muscles: in the region of the tail these unite below to enclose the howal canal (h.t.) already referred to. Actually, however, the vertebral colunin thus constituted is from the first more or less broken up into seginents, and in the higher forms is replaced by a chain of bones called vertebra which follow one another from before backwards, beginning a short distance behind the anterior end of the notochord and extending to the extremity of the tail.

A vertebra consists essentially of the following parts: (1) a centrum or body (Fig. $769, C$, cn.) lying below the spinal canal in the position formerly occupied by the notochord and perichordal tube, and arising either in the skeletogenous layer proper, or in the notochordal sheath after its invasion by skeletogenous cells; (2) a neural arch (n. a.) which springs from the dorsal surface of the centrum and encircles the spinal canal, representing a segment of the neural tube; and (3) a pair of transverse processes ( $t . p$. which extend outward from the centrum among the muscles and represent segments of the hæmal ridges : to them are often attached ribs which extend downwards in the body-wall, sometimes between the dorsal and ventral muscles $\left(r^{\prime}\right)$, sometimes immediately external to the peritoneum $(r$.$) . In the anterior part of the ventral body-$ wall a cartilaginous or bony sternum or breast-bone may be developed: in the Amphibia it is an independent structure; in the higher classes it is formed by the fusion of some of the anterior ribs in the middle ventral line. In this way the anterior or thoracic region of the colome is enclosed in an articulated bony framework formed of the vertebral column above, the ribs at the sides, and the sternum below. The ribs under these circumstances become segmented each into two parts, a dorsal vertebral rib, articulating with a vertebra, and a ventral sternal rib with the sternum. In the tail there is frequently a homal arch (Fig. $769, D, h$. a.) springing from the ventral aspect of the centrum and enclosing the hæmal canal. Thus the line of centra in the fully-formed vertebral column occupies the precise position of the notochord; the neural arches encircle the spinal portion of the cerebro-spinal cavity; the transverse processes, ribs, and sternum encircle the colome; and the hæmal arches similarly surround the hæmal canal or vestigial colome of the tail. As we ascend the series of Craniata we find every gradation from the persistent notochord of the Cyclostomata, through the imperfectly differentiated vertebræ of Sharks and Rays, to the complete bony vertebral column of the higher forms.

The vertebræ are equal in number to the nyomeres, but are arranged alternately with them, the fibrous partition between two myomeres abutting against the middle of a vertebra, so that each muscle-segment acts upon two adjacent vertebre. Thus, the nlyomeres being metameric or segmental structures, the vertebra are intersegmental. 
In connection with the anterior end of the notochord, where no vertebræ are formerl, there are developed certain elements of the skull or cephalic skeleton, a structure which is eminently characteristic of the whole craniate division, and to the possession of which it owes its name. The skull makes its first appearance in the embryo in the form of paired cartilaginous plates, the parachordals (Fig. $772, p c$ ), lying one on each side of the anterior end of the notochord (nch), and thus continuing forward the line of vertebral centra. In front of the parachordals are developed a pair of curved cartilaginous rods, the trabecula $(t r)$, which underlie the anterior part of the brain, as the parachordals underlie its posterior part: their hinder ends diverge so as to embrace the pituitary body ( $p t y)$ already referred to. Cartilaginous investments are also formed around the organs of the three higher senses: a pair of olfactory capsules round the organs of smell, one of optic capsules round the organs of sight, and one of auditory capsules (au. c.) round the organs of hearing. The optic capsule, which may be either cartilaginous or fibrous, remains free from the remaining elements of the skull in accordance with the mobility of the eye ; it constitutes, in fact, the sclerotic or outer coat of that organ. The olfactory capsules are usually formed in relation to the trabeculæ, and are continuous with those structures from an early stage. The

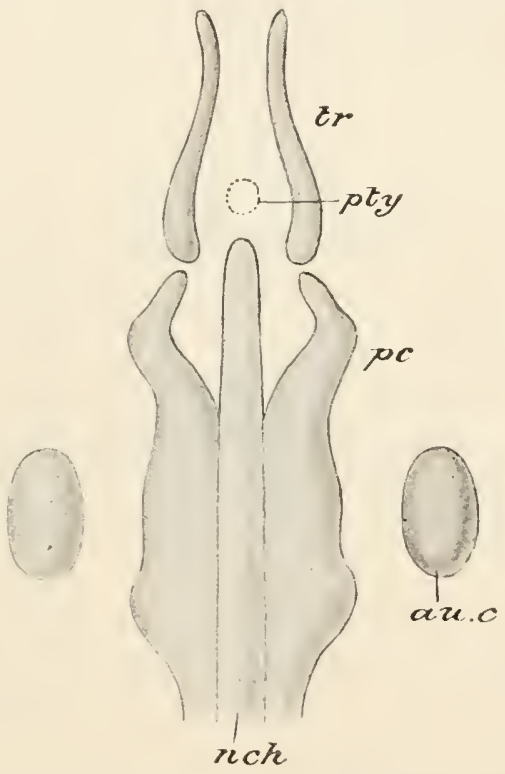

FIG. 772.-The elements of the cranium in an embryo Salmon, from above. au. c. auditory capsule ; nch. notochord ; pc. parachordal ; pty. position of pituitary body ; $t r$. trabeeula. (From a model by Ziegler.) auditory capsules in some cases arise as outgrowths of the parachordals, in others as independent cartilages, each of which, however, soon unites with the parachordal of its own side. As development goes on, the trabeculæ and parachordals become fused into a single basal plate (Fig. 773, $B, b$. cr.) underlying the brain : the skull-floor thus formed gives off vertical up-growths on each side which finally close in above to a greater or less extent, and so give rise to a more or less complete cranium or brain-case enclosing the brain and the organs of smell and hearing, and furnishing open cavities or orbits for the eyes.

In the continuous solid cranial box thus formed certain definite 
regions are to be distinguished: a posterior or occipital region, formed from the parachordals, united or articulated with the anterior end of the vertebral column, and presenting a large aperture, the foramen magnum (Fig. 773, B, for. mag.), through which the spinal cord becomes continuous with the brain; an auditory region formed by the two outstanding auditory capsules $(A, a u . c p$.$) ; and a trabecular region, including all the rest. The$ latter is again divisible into an interorbital region, between the orbits or eye-sockets; an olfactory region, constituted by the olfactory capsules (olf. $c p$.), and by a median vertical plate, the mesethmoid $(B, m$. eth.), which separates them from one another; and a pre-nasal region or rostrum $(r)$ extending forwards from the

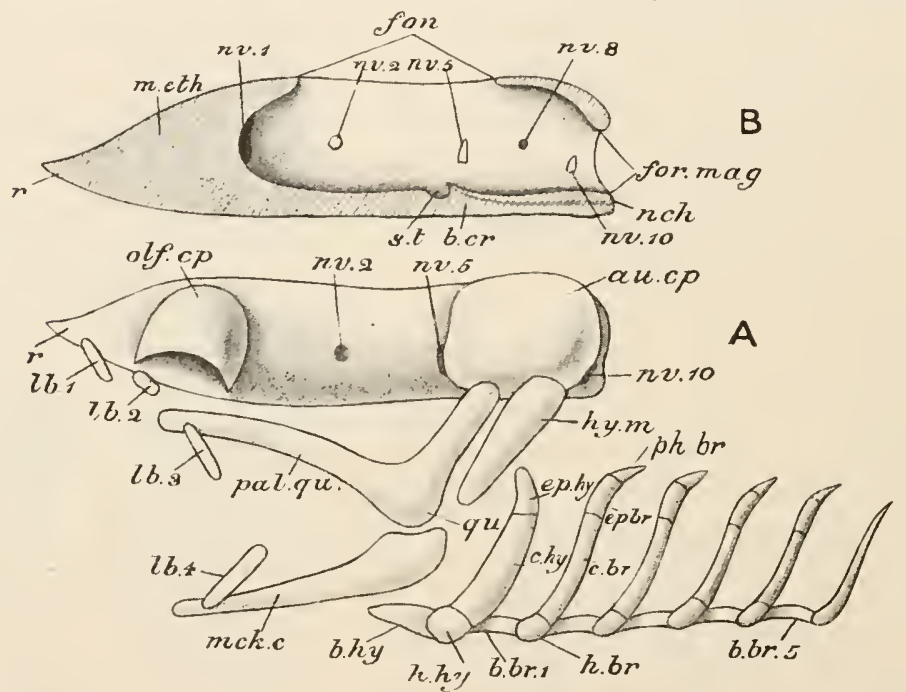

FIa. 773.- $A$, diagram of cartilaginous skull from the left side ; $B$, cranium in sagittal section. $a u$. $c p$. auditory capsule; $b . b r$. $I-5$, basi-branchials; $b$. cr. basis cranii ; $b$. hy. basi-hyal; c. br. cerato-branchial; $c$. hy. cerato-hyal; ep. br. epi-branchial ; ep. hy. epi-hyal ; fon. fontanelle; for. mag. foramen magnum; $h$. br. lyypo-branchial; $h$. hy. hypo-hyal; hy. $m$. hyomandibular; $l b .1-4$, labial cartilages ; mck. $c$. Meckel's cartilage ; $m$. eth. mesethmoid ; $n v .1-10$, foramina for cerebral nerves ; olf. cp. olfactory capsule ; pal. $2 u$. palato-quadrate; $p h$. br. pharyngo-branchial; $r$. rostrum; $s$. $t$. pituitary fossa or sella turcica.

mesethmoid and forming a more or less well-marked anterior prolongation of the cranium. The cavity for the brain $(B)$ extends from the foramen magnum behind to the olfactory region in front; its floor, formed from the basal plate of the embryo, is called the basis cranii $(b . c r$.$) : its roof is always incomplete, there being one or$ more apertures or fontanelles (fon.) closed only by membrane and due to the imperfect union above of the side-walls.

In the walls of the brain-case are apertures or furamina for the passage outwards of the cerebral nerves (vide p. 100). The most important of these are the olfactory foramina (nv. 1) for the nerves of smell, situated at the anterior end of the cerebral cavity, 
one on each side of the mesethnoid; the optic foramina (nv. 2) for the nerves of sight, in the interorbital region; the trigeminal foramina $(n v . ;)$ for the fifth nerves, just in front of the auditory capsule; the auditory foramina (nv. $S$ ) for the nerves of hearing, in the inner wall of the auditory capsules; and the vagus foramina (nv. 10) for the tenth nerves, immediately posterior to the auditory capsules.

In addition to the elements of the brain-case-parachordals, trabeculæ, and auditory capsules - there enter into the composition of the skull another set of elements called visceral bars. These are cartilaginous rods formed in the walls of the pharynx between the gill-slits, and thus encircling the pharynx like a series of paired half-hoops (Fig. 769, $B, v s$. b.). The corresponding right and left bars become united with one another below by an unpaired cartilage (Fig. $773, A, b . b r$.), forming a visceral arch, and the unpaired ventral pieces may unite successive arches with one another in the middle ventral line, thus giving rise to a more or less basket-like visceral skeleton. It will be noticed that the visceral skeleton has a segmental arrangement, being formed of parts arranged in an anteroposterior series, whereas in the cranium there is no clear indication of segmentation. There is, however, no exact correspondence between the segments of the visceral skeleton and the nietameres. The visceral arches vary in number from four to nine: the foremost of them is distinguished as the mandibular arch, and lies just behind the mouth; the second is called the hyoid arch, and the rest branchial arches, from the fact that they support the gills in water-breathing forms.

In all Craniata except the Cyclostomes the mandibular arch becomes modified into structures called jaws for the support of the mouth. Each mandibular bar divides into a dorsal and a ventral portion called respectively the palato-quadrate cartilage (Fig. 773, A, pal. qu.) and Meckel's cartilage (mck. c.) : the palato-quadrates grow forwards along the upper or anterior margin of the mouth, and unite with one another in the middle line, forming an upper jaw: Meckel's cartilages similarly extend along the lower or posterior niargin of the mouth and unite in the middle line, forming the lower jaw. The quadrate (qu.), or posterior end of the palato-quadrate, furnishes an articulation for the lower jaw, and often acquires a connection with the cranium, thus serving to suspend the jaws from the latter. Thus each jaw arises from the union of paired bars, the final result being two unpaired transverse structures, one lying in the anterior, the other in the posterior margin of the transversely elongated mouth, and moving in a vertical plane. The fundamental difference between the jaws of a Vertebrate and the structures called by the same name in an Arthropod or a Polychætous Worm will be obvious at once.

The hyoid bar usually becomes divided into two parts, a dorsal. 
the hyomandibular or pharyngo-hyal (hy.m.), and a ventral, the hyoid cornu, which is again divisible from above downwards into segments called respectively epi-hyal (ep.hy.), cerato-hyal (c.hy.), and hypo-hyal (h.hy.). The inedian ventral element of the arch, or basi-hyal (b.hy.), serves for the support of the tongue. In some Fishes the hyomandibular articulates above with the auditory region of the cranium, while the jaws are connected with its ventral end. We may thus distinguish two kinds of suspensorium or jaw-suspending apparatus - a mandibular suspensorium, furnished by the quadrate, and a hyoidean suspensorium, formed by the hyomandibular: in the former case the skull is said to be autostylic, i.e., having the jaw connected by means of its own arch; in the latter it is called hyostylic : in a few instances an amphistylic arrangement is produced by the presence both of a direct articulation between the palato-quadrate and the posterior region of the skull, and an indirect connection through the hyomandibular.

The branchial arches become divided transversely into dorsoventral segments called respactively pharyngo-branchial ( $p h . b r$.$) ,$ epi-branchial (ep.br.), cerato-branchial (c.br.), and hypo-branchial (h.br.), and the visceral skeleton thus acquires the character of an articulated framework which allows of the dilatation of the pharynx during swallowing and of its more or less complete closure at other times.

In connection with, and always superficial to, the rostrum, olfactory capsules, and jaws, are frequently found labial cartilages (lb. 1-4), which sometimes attain considerable dimensions.

In certain Fishes, such as Elasmobranchs, the cartilages of the skull become more or less encrusted by a supsrficial granular deposit of lime-salts, giving rise, as in the vertebral column of these Fishes, to calcified cartilage; but in all the higher forms true ossification takes place, the cartilaginous skull becoming complicated, and to a greater or less extent replaced, by distinct bones. Of these there are two kinds, replacing or "cartilage"-bones and investing or "membrane"-bones. Replacing bones may begin by the deposition of patches of bony matter in the cartilage itself (endochondral ossification). As development proceeds, these may be replaced by ossification starting within the perichondrium, or layer of connective-tissue surrounding the cartilage, and gradually invading the latter. More usually the bone is formed from the outset by the deposition of layers invading the cartilage from the perichondrium (or periosteum) inwards (perichondral or periosteal ossification). But in either case the bones in question are usually said to be preformed in cartilage, i.e., they replace originally cartilaginous parts. In the case of investing bones centres of ossification also appear, in constant positions, in the fibrous tissue outside the cartilage: they may remain quite independent of the original cartilaginous skull and its replacing bones, so as to be 
readily removable by boiling or maceration ; or they may eventually become, as it were, grafted on to the cartilage, in which case all distinction between investing and replacing bones is lost in the adult. 'The investing bones are to be looked upon as portions of the exoskeleton which have retreated from the surface and acquired intimate relations with the endoskeleton.

The replacing bones have a very definite relation to the regions of the cartilaginous cranium. In the occipital region four bones are formed, smrrounding the foramen magnum : a median ventral basi-occipital (Fig. 77t, $A$ and B, B. OC.), paired lateral ex-occipitals (EX. OC.), and a median dorsal supra-occipital (S. OC.). In each auditory capsule three ossifications commonly appear: a pro-otic
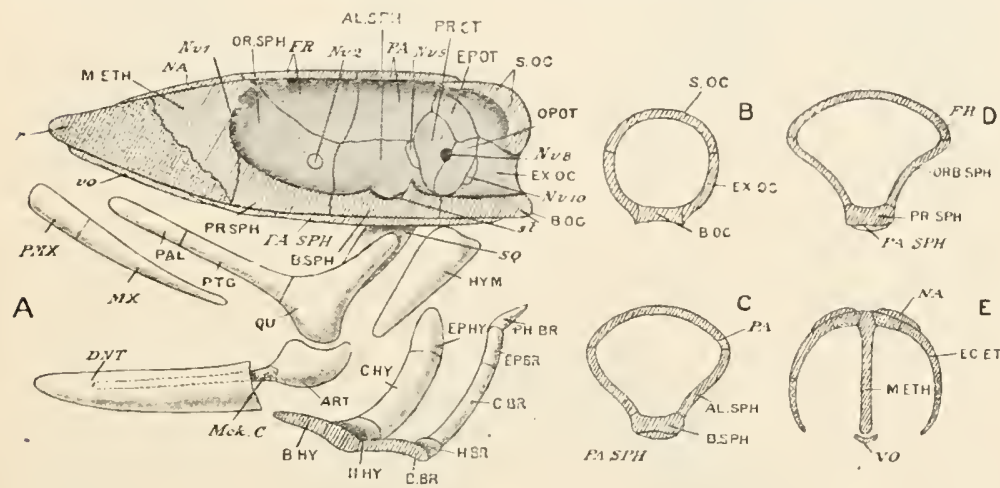

FIG. $774 .-A$, diagram of bony skull in sagittal section ; $B$, transverse section of occipital region ; $C$, of parietal region ; $D$, of frontal region; $k$, of ethmoirlal rcgion. Cartilaginous parts are dotted; replacing bones are marked in thick type, investing bones in italics. Mck. C. Meckel's cartilage; $N v .1$ - 10 , foramina for cerebral nerves ; $r$. rostrum ; $s$. $t$. sella tiurcica or pit uitary fossa, Replacing bones-AI.SPII. alisphenoid ; ART. articular; B.BR. basi-branchial : B.FY. bas-hyal; B. OC. hasi-ocripital; B.SPI. basi-sphenoid; C. BR. cerato-bran-

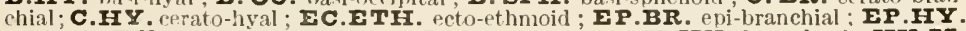
epi-hyal ; $\mathbf{E X . O C . ~ e x - o c c i v i t a l ~ ; ~ H . B R . ~ h y p o - t r a n c h i a l ; ~ H . H Y . ~ h y p o - h y a l ; ~ H Y . M . ~}$ hyomandibular: M. E I I. mesethmoid; OP.OT. opisthotic; OR. SPI. orbito-sphenoid ; PAT. Dalatine; Pr. BR. pharyngo-branchial: PR. OT. prootic; PR. SPY. pre-sphenoid: PTG. pterygoil : OU. quariate ; $\mathbf{S}$. OC. supra-occipital. Investing bones $-D N T$. dentary; FR. irontal; $M X$. maxilla; $N A$. nasal ; PA. parietal ; PA.SPH. parasphenoid; P.ILY. premavilla; SQ. squamosal: T'O., v.o. vomer.

(A, PR. OT.) in front, an opisthotic (OP. OT.) behind, and an epi-otic (EP. OT.) over the arch of the posterior semicircular canal of the ear (vide infra). In front of the basi-occipital a bone called the basi-sphenoid ( $A$ and $C, \mathbf{B}$. SPH.) is formed in the floor of the skull : it appears in the position of the posterior ends of the trabecnlæ, and bears on its upper or cranial surface a depression, the sella turcica (s.t), for the reception of the pituitary body. Connected on either side with the basi-sphenoid are paired bones, the ali-sphenoids (AL. SPH.), which help to furnish the side-walls of the interorbital region. The basi-sphenoid is continued forwards by another median bone, the pre-sphenoid ( $A$ and $D, \mathbf{P}$. SPH.), with which paired ossifications, the orbito-sphenoids (ORB. SPH), 
are connected and complete the side-walls of the interorbital region. The basi-occipital, basi-sphenoid, and pre-sphenoid together form the basis cranii of the bony skull. A vertical plate of bone, the mesethmoid (M. ETH.), appears in the posterior portion of the cartilage of the same name, and the outer walls of the olfactory capsules may be ossified by paired ecto-ethmoids (E, EC. ETH.).

So far, it will be seen, the cranial cavity has its hinder region alone roofed over by bone, viz. by the supra-occipital : for the rest of it the replacing bones furnish floor and side-walls only. This deficiency is made good by two pairs of investing bones, the parietals $(P A)$, formed immediately in front of the supra-occipital and usually articulating below with the ali-sphenoids, and the frontals $(F R)$, placed in front of the parietals, and often connected below with the orbito-sphenoids. A pair of nasals $(N A)$ are developed above the olfactory capsules and immediately in advance of the frontals; and below the base of the skull two important investing bones make their appearance, the vomer (vo)-which may be double-in front, and the para-sphenoid (PA.SPH) behind.

The result of the peculiar arrangement of replacing and investing bones just described is that the brain-case, in becoming ossified, acquires a kind of secondary segmentation, being clearly divisible in the higher groups, and especially in the Mammalia, into three quasi-segments. These are the occipital segment $(B)$ formed by the basi-occipital below, the ex-occipitals at the sides, and the supra-occipital above ${ }^{1}$; the parietal segment $(C)$, formed by the basi-sphenoid below, the ali-sphenoids laterally, and the parietals above; and the frontal segment $(D)$ constituted by the pre-sphenoid below, the orbito-sphenoids on either side, and the frontals above. It must be observed that this segmentation of the cranium is quite independent of the primary segmentation of the head, which is determined by the presence of myomeres and by the relations of the cerebral nerves.

The cranial bones have constant relations to the cerebral nerves. The olfactory nerves $(A, N v .1)$ pass out one on either side of the mesethmoid, the optic nerves $(N v .2)$ through or immediately behind the orbito-sphenoids, the fifth nerves $(N v .5)$ through or immediately behind the ali-sphenoids, and the tenth nerves $(N v, 10)$ through or immediately in front of the ex-occipitals.

It will be seen that a clear distinction can be drawn between the primary cranium or chondrocranium, formed by the fusion of the parachordals, auditory capsules, and trabeculæ, and consisting of an undivided mass of cartilage more or less replaced by bones, and the secondary cranium or osteocranium, modified by the superaddition of investing bones.

1 With the occipital segment in many Fishes are amalgamated one or several of the most anterior vertebra. 
A similar distinction may be drawn between the primary and secondary jaws. The primary upper jaw, or palato-quadrate, becomes ossified by three chief replacing bones on each side, the palatine (A, PAL.) in front, then the pterygoid (PTG.), and the quadrate (QU.) behind, the latter furnishing the articulation for the lower jaw or mandible. In the higher classes the primary upper jaw does not appear as a distinct cartilaginous structure, and the palatine and pterygoid are developed as investing bones. The
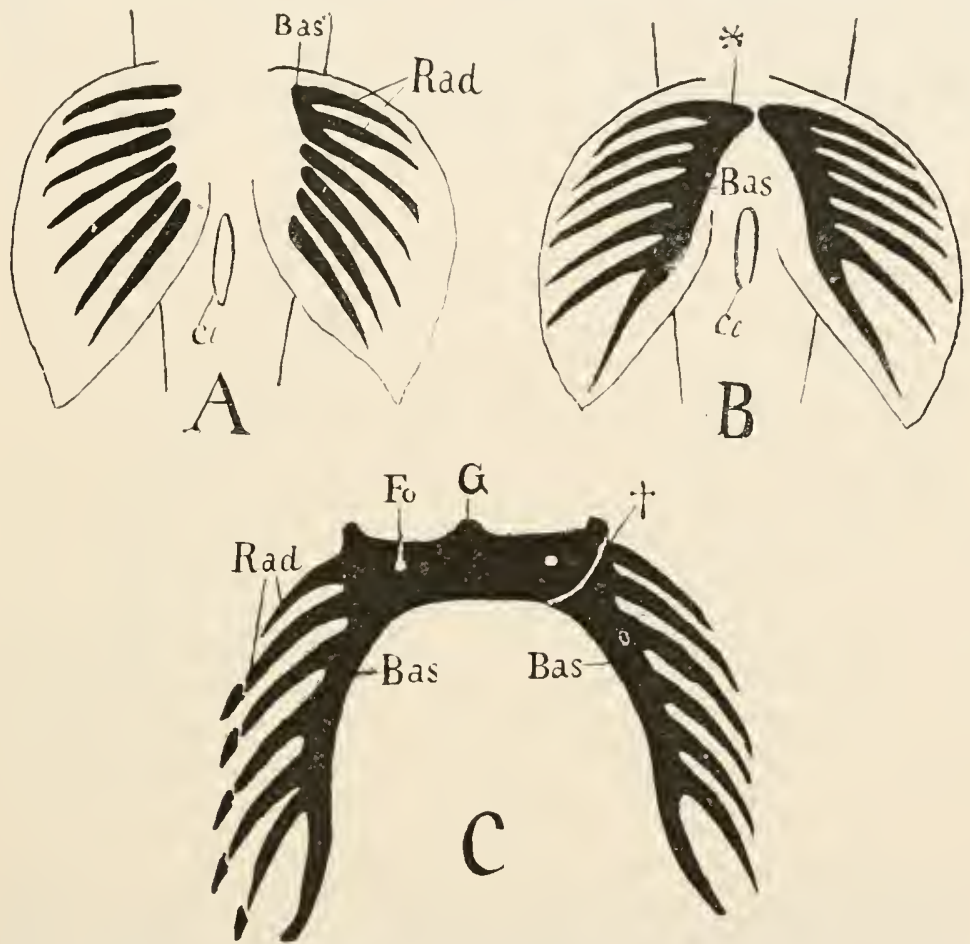

FId. 775.-Diagram of three stages in the development of the pelvic fins. In $A$, the anterior pterygiophores on the right side ( $R a d$ ) have united to form a basal cartilage ( $B a s$.$) ; in B$ the basalia (Bas) are fully formed and are uniting at * to form the pelvic girdle ; in $C$ the pervic girdle $(G)$ is fully constituted, and at $\dagger$ has segmented from the basale on the right sice. Cl. cloacal aperture. (From Wiedersheim's Comparative Anatomy.)

secondary upper jaw is constituted by two pairs of investing bones, the pre-maxilla $(P . M X)$ and the maxilla $(M X)$, which in bony skulls furnish the actual anterior boundary of the mouth, the primary jaw becoming altogether shut out of the gape. The proximal end of the primary lower jaw ossifies to form a replacing bone, the articular (ART.), by which the mandible is hinged: the rest of it remains as a slender, unossified Meckel's cartilage (Mck. C), which may disappear entirely in the adult. The secondary lower jaw is formed by a variable number of investing bones, the most important of 
which is the dentary (DNT). In Mammalia the dentary forms the entire mandible, and articulates, not with the quadrate, but with a large investing bone formed external to the latter, and known as the squamosal (SQ).

In the hyoid arch a replacing bone, the hyo-mundibular (HY. M), appears in the cartilage of the same name, and ossifications are also formed in the various segments of the hyoid cornua (EP. HY, C. HY, H. HY, B. HY) and of the branchial arches (PH. BR, EP. BR, C. BR, H. BR, B. BR). In the air-breathing forms both hyoid and branchial arches undergo more or less complete atrophy, the whole gill-bearing apparatus becoming reduced mainly to a small hyoid bone serving for the support of the tongue.
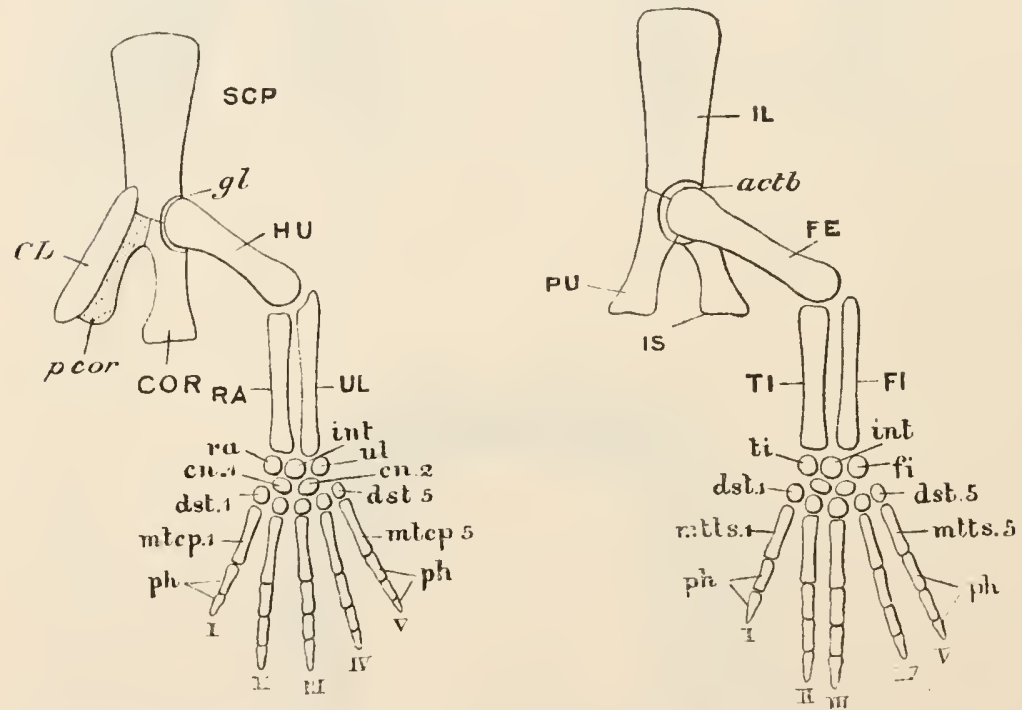

Fias, 776 and 777 - Diagra'ns of the fore- and hind-limbs with the limb-girdles, actb. acetabulum; $g l$. glenoid cavity; $p$. cor. procoracoid ; $l-V$, digits. Keplacing bones-cn.1, cn.2, centralia: COR. coracoid ; dst. $\mathbf{1}-\mathbf{5}$ distalia ; $\mathbf{F}$. femur ; $\mathbf{F}:$ fibula ; fi. fibulare ; HU. humerus: IL. ilium; int. intermedium; IS. ischium; mt $=\mathbf{p} .1$ - 5 metacarpals; mtts. $\mathbf{1} \mathbf{5}$, metatarsals; ph. phalanges ; $\mathbf{P U}$. pubis; $\mathbf{R A}$. radiu; $\mathbf{r} \mathbf{a}$. radiale; $\mathbf{S C P}$. scapula; Tr. tibia ; ti. tibiale; UL. ulna; ul. ulnare. Investiag bir-CL. clavicle.

The skeleton of the median fins is formed of a single row of cartilaginous rays or pterygiophores (Fig. $769, C$ and $D, f . r$. ), lying in the median plane, and more numerous than the vertebræ. They may ossify, and may be supplemented by dermal fin-rays, of varying composition, developed in the derm towards the free margin of the fin. The latter are clearly exoskeletal structures.

Both pectoral and pelvic fins are supported by pterygiophores or radialia (Fig. 775, Rud.), the basal or proximal ends of which are articulated with stout eartilages, the basalia (Bxs), often replaced by bones, which serve to strengthen the fin at its point of union with the trunk. 
In all classes above Fishes the paired fins are, as we have seen, replaced by five-toed or pentadactylc limbs. These are supported by bones, probably to be looked upon as greatly. modified pterygiophores, and obviously serially homologous in the fore- and hind-limbs. In the proximal division of each limb there is a single rod-like bone, the humerus (Fig. 776, HU), or upper-arm-bone, in the fore-limb, the fermur (Fig. 777, FE), or thigh-bone, in the hind-limb. In the middle division there are two elongated bones, an anterior, the radius (RA), and a posterior, the ulna (UL), in the fore-limb; an anterior, the tibia (TI), and a posterior, the fibula (FI), in the hindlimb. Next follow the bones of the hand and foot, which are again divisible into three sets : carpals or wrist-bones, metacarpals (mtcp) or hand-bones, the phalanges (ph) or finger-bones, in the forelimb; tarsals or ankle-bones, metatarsals (mtts) or foot-bones, and phalanges $(\mathrm{ph})$ or toe-bones, in the hind-limb. The carpals and tarsals consist typically of three rows of small nodules of bone or cartilage, the proximal row containing three, the middle two, and the distal five elements. The three proximal carpals are called respectively radiale (ra), intermodium (int), and ulnare (ul), those of the middle row the first and second centralia (cn. 1, cn. 2), those of the third row the five distalia (d.st. 1-5), the separate elements being distinguished by numbers, counting from the anterior or radial edge of the limb. In the tarsus the bones of the first row are known respectively as tibiale (ti), intermedium (int), and fibut.ure (fi), those of the second row as centralia (cn. 1, cn. 2), and those of the third as distalia (dst. 1-5). The metacarpals (mtcp. 1-5) and metatarsals (mtts. 1-5) are five rod-like bones, one articulating with each distale: they are followed by the phalanges (ph), of which each digit may have from one to five. The first digit of the fore-limb (Fig. 776, I) is distinguished as the pollex or thumb, that of the hind-limb (Fig. 777, I) as the hallux or great toe; the fifth digit of each $\operatorname{limb}(V)$ is the minimus.

In connection with the paired appendages are formed supporting structures called the limb-girdles; they occur in the portions of the trunk adjacent to the appendages and serve for the articulation of the latter. In the embryonic condition they are continuous with the basalia and are probably to be looked upon as ingrowths of the primitive fin-skeleton (Fig. 775). The shoulder-girdle or pectoral arch has primarily the form of paired bars, which may unite in the middle ventral line so as to form an inverted arch. Each bari.e., each half of the arch-furnishes a concave or convex glcnoid surface (Fig. 776, gl.) for the articulation of the pectoral fin or forelimb, and is thereby divided into two portions-a dorsal or scapular region, above the glenoid surface, and a ventral or coracoid region below it. The coracoid region is again divisible, in all classes above Fishes, into two portions: an anterior, the pro-coracoid $(p . c o r$.$) , and a posterior, the coracoid proper. Each of these regions$ 
commonly ossifies - a replacing bone, the scapula (SCP), appearing in the scapular region, another, the coracoid (COR), in the coracoid region, while in relation with the pro-coracoid is formed a bone, the clavicle $(C L)$, largely or entirely developed independently of pre-existing cartilage.

The constitution of the hip-girdle, or pelvic arch, is very similar. It consists originally of paired bars, which may unite in the middle ventral line, and are divided by the acetabulum (Fig. 777, actb.), the articular surface for the pelvic fin or hind-limb, into a dorsal or iliac region, and a ventral or pubo-ischial region, the latter being again divisible, in all classes above Fishes, into an anterior portion, or pubis, and a posterior portion, or ischium. Each region is replaced in the higher forms by a bone, the pelvic girdle thus consisting of a dorsal ilium (IL) serially homologous with the scapula, an antero-ventral pubis (PU) with the pro-coracoid and clavicle, and a postero-ventral ischium (IS) with the coracoid. The long bones of the limbs are divisible each into a shaft, and proximal and distal extremities. When ossification takes place the shaft is converted into a tubular bone, the cartilaginous axis of which is absorbed and replaced by a vascular fatty tissue called marrow. The extremities become simply calcified in the lower forms, but in the higher a distinct centre of ossification may appear in each, forming the epiphysis, which finally becomes ankylosed to the shaft.

Digestive Organs.-The enteric canal is divisible into buccal cavity (Fig. 769, A, buc. c.), pharynx (ph.), gullet, stomach (st.), and intestine (int.), the latter often communicating with the exterior by a cloaca $(c l$.$) , which receives the urinary and genital$ ducts. The buccal cavity is developed from the stomodæum of the embryo: the proctodæum gives rise to a very small area in the neighbourhood of the anus, or, when a cloaca is present, to the external portion of the latter; all the rest of the canal is formed from the mesenteron, and is therefore lined by an epithelium of endodermal origin. The pharynx communicates with the exterior, in Fishes and in the embryos of the higher forms, by the gill-slits (i. br. $a, 1-7)$; it communicates with the stomach by the gullet. The stomach (st.) is usually bent upon itself in the form of a $U$; the intestine (int.) is generally more or less convoluted; hence the stomach and intestine are together considerably longer than the enclosing abdominal cavity. In the embryo the intestine is sometimes continued backwards into the hæmal canal by an extension called the post-anal gut $(p . a . g$.$) , which may perhaps indicate that$ the anus has shifted forwards in the course of evolution.

The epithelium of the buccal cavity is usually many-layered, like that of the skin, of which it is developmentally an in-turned portion; the pharynx and gullet have also a laminated epithelium, 
but the rest of the canal is lined by a single layer of cells underlaid by a layer of connective-tissue, the deeper part of which is called the $s u b$-mucosa; epithelium and connective-tissue together constitute the mucous membrane. The mucous membrane of the stomach and sometimes of the intestine usually contains close-set tubular glands; those of the stomach, the gastric glands, secrete gastric juice, which acts upon the proteid portions of the food only; the secretion of the intestinal glands digests proteids, starch, and fats. Outside the mucous membrane are layers of unstriped muscle, usually an internal circular and an external longitudinal layer. Externally the intra-cœlomic portion of the canal is invested by peritoneum formed of a layer of connective-

$\mathrm{B}$
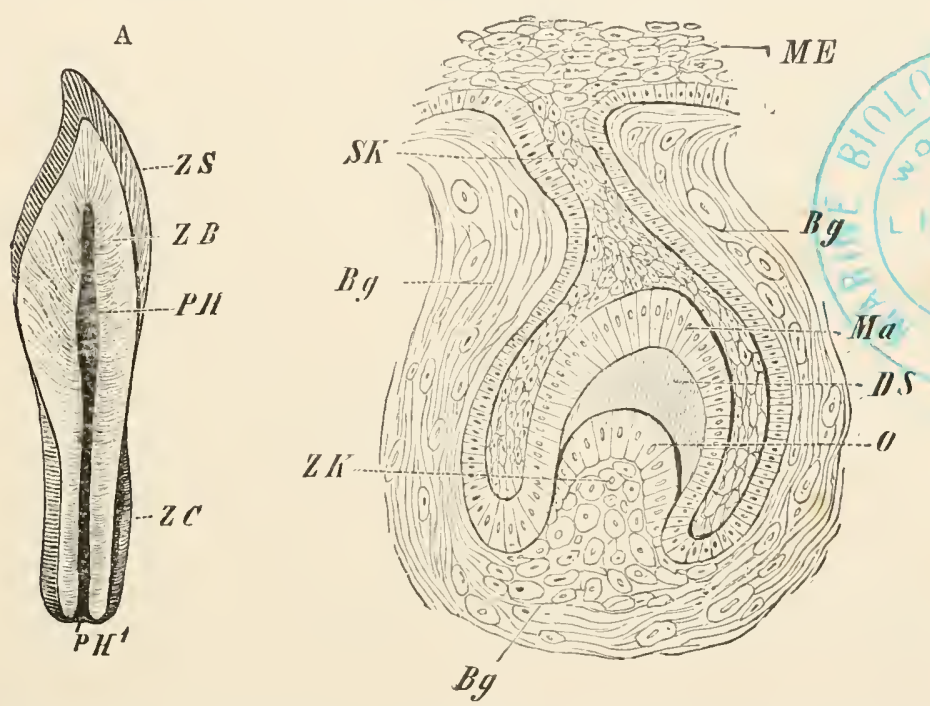

FIG. 778.-A, longitudinal seetion of a tooth, semi-diagrammatic. $P H$, pulp-eavity ; $P^{\prime} H^{\prime}$, opening of same; $Z B$, dentine; $Z C$, cement; $Z S$, enamel. $\mathbf{B}$, longitudinal section of developing tooth. $B g$, submueosa ; $l S S$, dentine ; $I f a$, invaginated layer of enamel-organ ; $M E$, epithelium of mouth; $O$, odontoblasts ; $S K$, stalk of enamel-organ ; $Z K$, tooth-papilla. (From Wiedersheim's Vertebrata.)

tissue next the gut and a single-layered colomic epithelium facing the body-cavity.

In connection with the enteric canal certain very characteristic structures are developed. In the mucous membrane of the mouth calcifications in most cases appear and form the teeth, which usually occur in a row along the ridge of each jaw, but may be developed on the roof of the mouth, on the tongue, and even in the pharynx. A tooth is usually formed of three tissues-dentine, enamel, and cement. The main bulk of the tooth is made up of dentine (Fig. 778, A, $Z B)$, which occurs under three forms. Hard dentine consists of a matrix of animal matter strongly impregnated with lime-salts and permeated by delicate, more or less parallel, tubules containing 
organic fibrits. Vaso-dentine is permeated with blood-vessels, and consequently appears red and moist in the fresh condition. Osteodentine approaches bone in its structure and mode of development. The free surface of the tooth is usually capped by a layer of enamel $(Z S)$, a dense substance, either structureless or presenting a delicate fibrillation, containing not more than 3 to 5 per cent. of animal matter, and being, therefore, the hardest tissue in the body. The cement $(Z C)$ coats that portion of the tooth which is embedded in the tissues of the jaw, and sometimes forms a thin layer over the enamel ; it has practically the structure of bone. At the inner end of the tooth there is frequently an aperture $\left(P H^{\prime}\right)$ leading into a cavity $(P H)$ filled in the fresh condition by the tooth-pulp, a sort of connective-tissue plug abundantly supplied with nerves and bloodvessels.

In the development of a tooth (Fig. 778, B) the deep layer of the buccal epithelium becomes invaginated and grows inwards into the sub-mucosa in the form of a narrow cord, the enamel-organ $(S K)$. The distal end of this enlarges into a flask-like form, and the bottom of the flask becomes invaginated $(M a)$ by the growth of a conical process of the sub-mucosa, the dental papilla $(Z K)$. Mesoderm cells accumulate on the free surface of the papilla and form a distinct layer of cells called odontoblasts $(O)$. From these the dentine is formed in successive layers, which gradually accumulate between the layer of odontoblasts and the inner or invaginated layer of the enamel-organ. The lower, or proximal, part of the papilla remains uncalcified and forms the tooth-pulp. The enamel is formed by the deposition of successive layers of calcific matter from the inner or invaginated layer of the enamel-organ, the cement by the ossification of the tissue immediately surrounding the papilla. Thus the tooth is partly of ectodermal, partly of mesodermal origin.

In some Fishes the scales or elements of the dermal exoskeleton pass insensibly into the teeth over the ridges of the jaws, and agree with them in structure, so that there can be no doubt as to the homology of the two. Teeth are, in fact, to be looked upon as portions of the exoskeleton which have migrated from the skin into the buccal cavity, and even into the pharynx, and have there increased in size and assumed special functions.

The tongue is a muscular elevation of the floor of the mouth, supported by the basi-hyal, and usually more or less protrusible. The roof of the buccal cavity in the embryo sends off a pouch, the pituitary diverticulum (Fig. 769, A, ply. s.), which grows upwards, and, losing its connection with the mouth, becomes attached to the ventral surface of the brain as the pituitary body (pty. b.). It may correspond with the neural gland of Urochorda.

In terrestrial Craniata buccal glands are present, opening by ducts into the mouth: the most important of these are the racemose salivary glands which secrete a digestive fluid-saliva, capable of 
converting starch into sugar. There are also two large and highly characteristic digestive glands in the abdominal cavity, both developed as outpushings of the intestine, but differing greatly from one another in their fully developed state, both in outward appearance and in histological structure: these are the liver and the pancreas.

The liver (Fig. $769, A, l r$. ) is a dark-red organ of relatively immense size : it not only secretes a digestive juice, the bile, which has the function of emulsifying fats, but also forms an amyloid substance called glycogen or animal starch, which, after being stored up in the liver-cells, is restored to the blood in the form of sugar. The liver is formed of a mass of polyhedral cells (Fig. 779, l.) with -minute intercellular spaces which receive the bile secreted from the cells and from which it passes to the ducts $(b)$. The pancreas (Fig. $769, A, p n$.) is a racemose gland, and secretes pancreatic juice which acts upon proteids, starch, and fats. The ducts of both glands usually open into the anterior end of the intestine : that of the liver $(b . d$.) generally gives off a blind offshoot ending in a capacious dilatation, the gall-bladder $(g . b$.$) in which the$ bile is stored. We thus have one or more hepatic ducts Fig. 779.-Diagram of structure of liver. $b$. a small branch of conveying the bile lepatic duct; $b^{\prime}$. its ultimate termination in the intercellular from the liver and

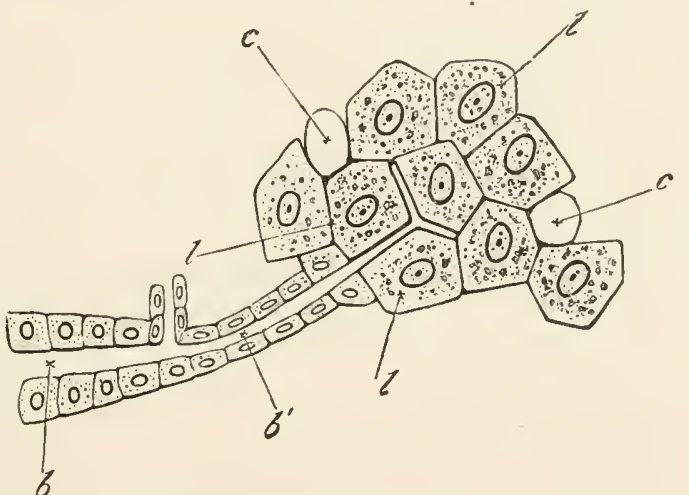
meeting with a cystic duct from the gall-bladder, while from the junction a common bile-duct leads into the intestine.

Another important and characteristic organ in the abdomen of Craniata is the spleen $(s p l$.), a gland-like organ of variable size and shape, attached to the stomach by a fold of peritoneum, but having no duct. It is formed of a pulpy substance containing numerous red blood-corpuscles, many of them in process of disintegration: dispersed through the pulp are masses of leucocytes which multiply and pass into the veins.

Two other ductless glands are formed in connection with the enteric canal. The thyroid (thd.) is developed as an outpushing of the floor of the pharynx which becomes shut off, and forms, in the adult, a gland-like organ of considerable size. Its final position varies considerably in the different classes. It has been compared with the endostyle of Tunicata and of Amphioxus, which, as 
will be remembered, is an open groove on the ventral side of the pharynx. This view is supported by the condition of the parts in the larval Lamprey (see Cyclostomata).

The thymus is developed from the epithelium of the dorsal ends of the gill-clefts : in the adult it may take the form of a number of separate gland-like bodies lying above the gills, or may be situated in the neck or even in the thorax. The thymus and thyroid, by virtue of internal secretions which they produce, and which mingle with the blood, control and modify the physiological condition of various organs and tissues with which they have no immediate anatomical connection.

The whole intra-abdominal portion of the enteric canal, as well as the liver, pancreas, spleen, and, indeed, all the abdominal viscera, are supported by folds of peritoneum, called by the general name

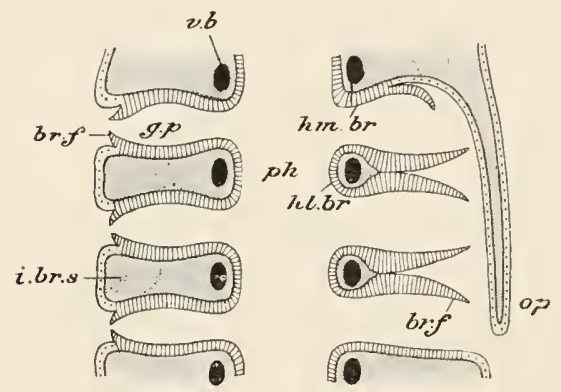

11G. 780.-Diagrammatic horizontal section of the pharyngeal region of a Craniate : on the left are shown three gill-pouches (g.p.) with fixed branchial tilaments $(b r . f$.$) and separated by inter-branchial$ septa (i.br. s.); on the right one hemibranch ( $h \mathrm{~m}$. $b r$.$) and two holobranchs ( h l$. br.) with free filaments, covered by an operculum $(o p)$. Ectoderm dotted, endoderm striated, mesoderm evenly shaded, visceral bars ( $v . b$. ) black; $p h$, pharynx. of mesentery (Fig. 769, C, mes.) and having the usual relation to the parietal and visceral layers of the peritoneum.

Two kinds of respiratory organs are found in Craniata : water-breathing organs or gills, and airbreathing organs or lungs.

Gills arise as a series of paired pouches of the pharynx which extend outwards, or towards the surface of the body, and finally open on the exterior by the gill-slits already noticed. Each gill-pouch thus communicates with the pharynx by an internal (Fig. $769, B, i . b r . a$ ), with the outside water by an external branchial aperture (e. br. a), and is separated from its predecessor and from its successor in the series by stout fibrous partitions, the interbranchial septa (Fig. $780, i . b r . s$ ). The mucous membrane forming the anterior and posterior walls of the pouches is raised up into a number of horizontal ridges, the branchial flaments (br.f.), which are abundantly supplied with blood. A current of water entering at the mouth passes into the pharynx, thence by the internal gill-slits into the gill-pouches, and finally makes its way out by the external gill-slits, bathing the branchial filaments as it goes. The exchange of carbonic acid for oxygen takes place in the bloodvessels of the branchial filaments, which are, therefore, the actual organs of respiration. It will be noticed that the respiratory 
epithelium is endodermal, being derived from that of the pharynx, which, as we have seen, is a portion of the mesenteron.

As already mentioned, the walls of the pharynx are supported by the visceral arches, which surround it like a series of incomplete hoops, each half-arch or visceral bar being embedded in the inner or pharyngeal side of an interbranchial septum. Thus the visceral arches $(v . b$. ) alternate with the gill-pouches, each being related to the posterior set of filaments of one pouch and the anterior set of the next. In the higher Fishes, such as the Trout or Cod, the interbranchial septa become reduced to narrow bars enclosing the visceral arches (right side of Fig. 780), with the result that a double set of free branchial filaments springs from each visceral bar and constitutes what is called a single gill. Thus an entire gill or holobranch ( $h l . b r$.$) is the morphological equivalent of two half-gills-$ hemibranchs ( $h m . b r$.$) , or sets of branchial filaments belonging to$ the adjacent sides of two consecutive gill-pouches. On the other hand, a gill-pouch is equivalent to the posterior hemibranch of one gill and the anterior hemibranch of its immediate successor.

In some Amphibia water-breathing organs of a different kind are found. These are the external gills: they are developed as branched outgrowths of the body-wall in immediate relation with the gill-slits, and differ from the internal gills just described in having an ectodermal epithelium. They are probably, however, of the same essential character as the endodermal gills ( $c f$. also under Pisces).

Lungs (Fig. 769, $A, l g$ ) are found in all Craniata from the Dipnoi upwards. They are developed as a hollow outpushing from the ventral wall of the embryonic fore-gut or anterior part of the enteric canal; this passes backwards and upwards, usually dividing into right and left divisions, and finally coming to lie in the dorsal region of the colome. The inner surface of the single or double lung thus formed is raised into a more or less complex network of ridges so as to increase the surface of blood exposed to the action of the air; and, in the higher forms, the ridges, increasing in number and complexity, and uniting with one another across the lumen of the lung, convert it into a sponge-like structure. The respiratory epithelium is, of course, endodermal. Since the lungs are blind sacs, some contrivance is necessary for renewing the air contained in them : this is done either by a process analogous to swallowing, or by the contraction and relaxation of the muscles of the trunk.

In some Fishes there occurs, in the position occupied in airbreathers by the lungs, a structure called the air-bladder, which contains gas, and serves as an organ of flotation. Like the lungs, it is developed as an outgrowth of the fore-gut, but, except in four instances, from its dorsal instead of its ventral side. In many cases the air-bladder loses its connection with the pharynx and becomes a closed sac. 
The blood-vascular system attains a far higher degree of complexity than in any of

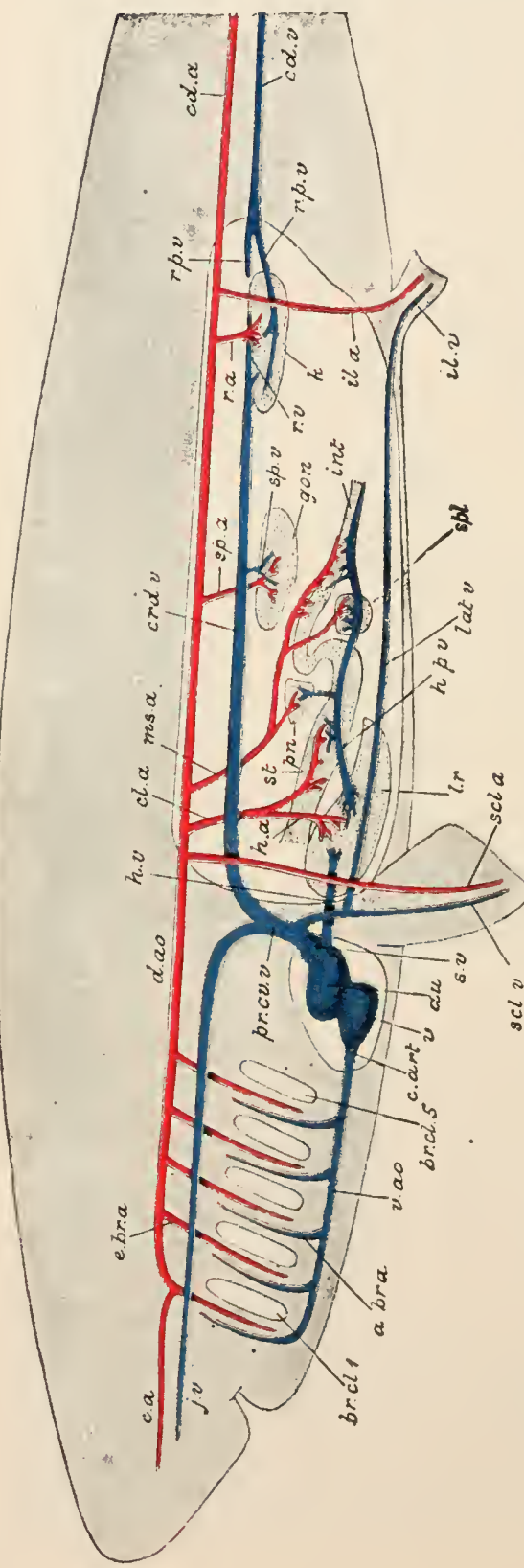

the groups previously studied: its essential features will be best understood by a general description of the circulatory organs of Fishes.

The heart (Figs. 769 and 781) is a muscular organ contained in the pericardial cavity and composed of three chambers, the sinus venosus $(s . v$.), the auricle (au.), and the ventricle (v.), which form a single longitudina l series, the hindmost, the sinus venosus, opening into the auricle, and the auricle into the ventricle. They do not, however, lie in a straight line, but in a zigzag fashion, so that the sinus and auricle are dorsal in position, the ventricle ventral. Usually a fourth chamber, the conus arteriosus (c. art.), is added in front of the ventricle. The various chambers are separated 
from one another by valvular apertures (Fig. 782) which allow of the flow of blood in one direction only, viz. from behind forwardsthat is, from sinus to auricle, auricle to ventricle, and ventricle to conus. The heart is made of striped muscle of a special kind - the only involuntary muscle in the body having this histological cliaracter-which is particularly thick and strong in the ventricle. It is lined internally by epithelium and covered externally by the visceral layer of the pericardium.

Springing from the ventricle, or from the conus when that chamber is present, and passing directly forwards in the middle line below the gills, is a large, thick-walled, elastic blood-vessel, the ventral aorta (Figs. $769, B$, and 781. v. ao.). At its origin, which may be dilated to form a bulbus aorto, are valves so

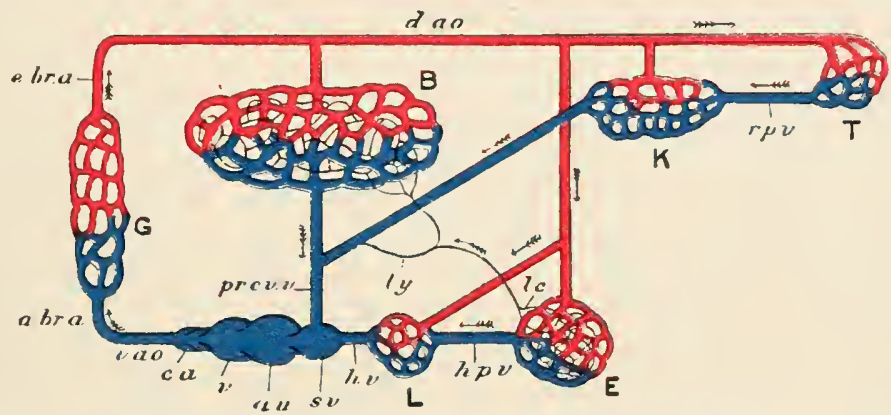

FlG. 782.- Diagram iliustratiug the course of the circulation i.1 a Fish. Vessels containing aërated bloorl red, those containing non-aërated blood blue, lymplatics black. $B$, capillaries of the body generally ; $E$, of the enteric canal ; $G$, of the gills ; $K$, of the kidneys ; $L$, of the liver ; $T$, of the tail. a. br. $a$. afferent branchial arteries; au. auricle; c. $a$. conus arteriosus; $\boldsymbol{d} . a o$. dorsal aorta ; $e . b r . a$. efferent branchial arteries; $h . p . v$. hepatic portal vein; $h$. $v$. hepatic vein; $l c$. lacteals; $l y$. lymphatics $: p r . c v, v$. precaval veins; $r . p, v$. renal portal veins; $s . v$. sinus venosus; $v$. ventricle; $v$. ao. ventral aorta. The arrows show the direction of the current. (From Parker's Elementary Biology.)

disposed as to allow of the flow of blood in one direction only, viz. from the ventricle into the aorta. It gives off on each side a series of half-hoop-like vessels, the afferent branchial arteries (a. br. a.), one to each gill. These vessels ramify extensively, and their ultimate branches open into a network of microscopic tubes or capillaries (Fig. 782, G), having walls formed of a single layer of epithelial cells, which permeate the connective-tissue layer of the branchial filaments, and have therefore nothing between them and the surrounding water but the epithelium of the filaments. The blood, driven by the contractions of the heart into the ventral aorta, is pumped into these respiratory capillaries, and there exchanges its superfluous carbonic acid for oxygen. It then passes from the capillaries into another set of vessels which join with one another, like the tributaries of a river, into larger and larger trunks, finally uniting in each gill, into an efferent branchial artery $(e . b r . a$.$) . The efferent arteries of both sides pass upwards$ and discharge into a median longitudinal vessel, the dorsal aorta 
(d. ao.), situated immediately beneath the notochord or vertebral column. From this trunk, or from the efferent branchial arteries, numerous vessels, the systemic arteries, are given off to all parts of the body, the most important being the carotid arteries (Fig. 781, c. a.) to the head, the subclavian (scl. a.) to the pectoral fins, the caliac (cl.a.) and mesenteric (ms. a.) to the stomach, intestine, liver, spleen, and pancreas, the renal $(r, a$.) to the kidneys, the spermatic (sp. a.) or ovarian to the gonads, and the iliac (il. a.) to the pelvic fins. After giving off the last the aorta is continued as the caudal artery $(c d . a$.) to the end of the tail.

With the exception of the capillaries, all the vessels described in the preceding paragraph, including the dorsal and ventral aortæ, are arteries. They are firm, elastic tubes, do not collapse when empty, usually contain but little blood in the dead animal, and serve to carry the blood from the heart to the body generally.

The systemic arteries branch and branch again into smaller and smaller trunks, and finally pour their blood into a capillary network (Fig. 782, $B, K$, and $T$ ) with which all the tissues of the body, except epithelium and cartilage, are permeated. In these systemic capillaries the blood parts with its oxygen and nutrient constituents to the tissues, and receives from them the various products of destructive metabolism-carbonic acid, water, and nitrogenous waste. The systemic, like the respiratory, capillaries are microscopic, and their walls are formed of a single laver of epithelial cells.

We saw that the respiratory capillaries are in connection with two sets of vessels, afferent and efferent. The same applies to the systemic capillaries, with the important difference that their efferent vessels are not arteries, but thin-walled, non-elastic, collapsible tubes called veins. They receive the impure blood from the capillaries, and unite into larger and larger trunks, finally opening into one or other of the great veins, presently to be described, by which the blood is returned to the heart. As a general rule the vein of any part of the body runs parallel to its artery, from which it is at once distinguished by its wider calibre, by its dark colour-due to the contained bluish-purple blood seen through its thin walls, by being gorged with blood after death, by the complete collapse of its walls when empty, and by its usually containing valves. In some cases the veins become dilated into spacious cavities called sinuses; but sinuses without proper walls, such as occur in many Invertebrates, are never found in the Craniata.

The veins from the head join to form large, paired jugular veins (Fig. 781, j.v.) which pass backwards, one on each side of the head, and are joined by the cardinal veins (crd. v.) coming from the trunk, each jugular uniting with the corresponding cardinal to form a large precaval vein $(p r . c v . v$.$) which passes directly downwards and enters$ 
the sinus venosus. The blood from the tail returns by a caudal vein (cd. v.), lying immediately below the caudal artery in the hæmal canal of the caudal vertebræ (Fig. 769, D). 'On reaching the cœlome the caudal vein forks horizontally, and the two branches either become directly continuous with the cardinals or pass one to each kidney under the name of the renal portal veins (Fig. 781, r. p. v.). In the kidneys they break up into capillaries (Fig. 782, K), their blood mingling with that brought by the renal arteries and being finally discharged into the cardinals by the renal veins (Fig. 781, r.v.). Thus the blood from the tail may either return directly to the heart in the normal manner or may go by way of the capillaries of the kidneys. In the latter case there is said to be a renal portal system, the essential characteristic of which is that the kidney has a double blood-supply, one of pure blood from the renal artery, and one of impure blood from the renal portal vein; in other words, it has two afferent vessels, an artery and a vein, and the latter is further distinguished by the fact that it both begins and ends in capillaries instead of beginning in capillaries and ending in a vein of higher order.

The blood from the gonads is returned to the cardinals by veins called spermatic (sp. v.) in the male, ovarian in the female. That from the paired fins takes, in what appears to be the most typical case, a somewhat curious course. On each side of the body there is a lateral vein (lat. v.), running in the body-wall and following the course of the embryonic ridge between the pectoral and pelvic fins. It receives, anteriorly, a subclavian vein (scl. v.) from the pectoral fin, and posteriorly an iliac vein (il.v.) from the pelvic fin, and in front pours its blood into the precaval.

The veins from the stomach, intestine, spleen, and pancreas join to form a large hepatic portal vein $(h . p . v$.), which passes to the liver and there breaks up into capillaries, its blood mingling with that brought to the liver by the hepatic artery $(h . a$.$) , a branch of$ the coliac. Thus the liver has a double blood-supply, receiving oxygenated blood by the hepatic artery, and non-oxygenated but food-laden blood by the hepatic portal vein (Fig. 782, $L$ ). In this way we have a hepatic portal system resembling the renal portal system both in the double blood-supply, and in the fact that the afferent vein terminates, as it originates, in capillaries. After circulating through the liver the blood is poured, by hepatic veins $(h . v$.), into the sinus venosus. The hepatic, unlike the renal portal system, is of universal occurrence in the Craniata.

In the embryo there is a sub-intestinal vein, corresponding with that of Amphioxus, and lying beneath the intestine and the postanal gut. Its posterior portion becomes the caudal vein of the adult, its anterior portion one of the factors of the hepatic portal vein. 
To sum up :-The circulatory organs of the branchiate Craniata consist of $(a)$ a muscular organ of propulsion, the heart, provided with valves and driving the blood into $(b)$ a set of thick-walled, elastic, afferent vessels, the arteries, from which it passes into $(c)$ a

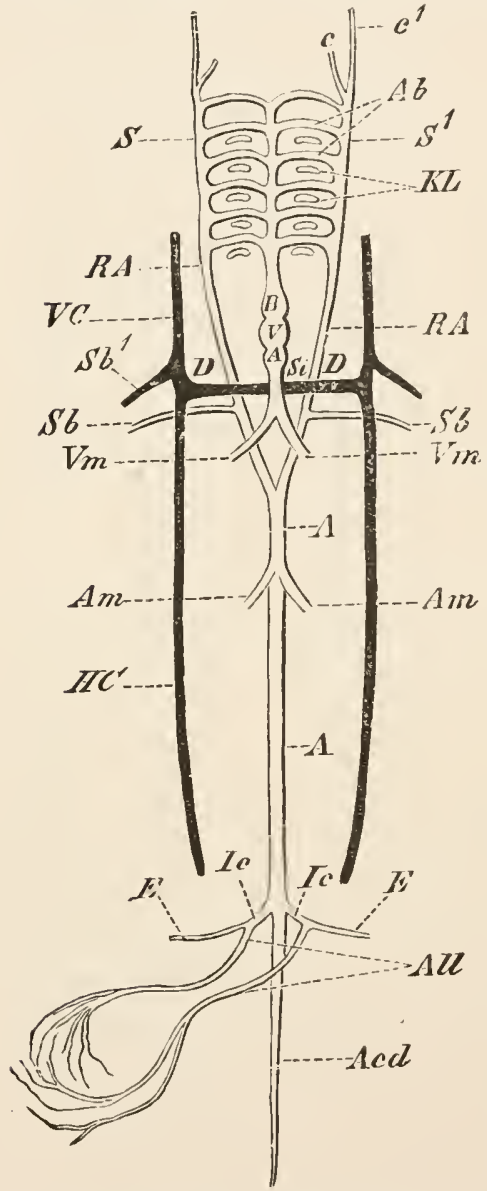

FIG. 783.-Diagram of the vascular system in the embryoof an air-breathing Craniate.

$A$. dorsal aorta and auricle; $A b$. aortic arches: $A c d$. caudal artery ; $A l l$. allantoic arteries ; $A m$. vitelline arteries ; $B$. ventral aorta $; c, c^{1}$. carotid arteries: $D$. precaval veins: $I c, E$. iliac arteries ; $H C$. cardinal veins ; $K L$. gillclefts : $R A, S, S 1$. roots of dorsal aorta $; S b$. subclavian arteries; $S b 1$. subclavian veins : $V$. ventricle ; $V C$. jugular vein ; $V m$. vitelline veins, (From Wiedersheim's Vertebrata.) network of microscopic vessels or capillaries which permeate the tissues, supplying them with oxygen and nutrient matters and receiving from them carbonic acid and other waste products : from the capillary network the blood is carried off by $(d)$ the veins, thin-walled, non-elastic tubes by which it is returned to the heart. Thus the general scheme of the circulation is simple: the arteries spring from the heart, or from arteries of a higher order, and end in capillaries; the veins begin in capillaries and end in vessels of a higher order or in the heart. Actually, however, the system is complicated $(a)$ by the interposition of the gills in the course of the outgoing current, as a result of which we have arteries serving as both afferent and efferent vessels of the respiratory capillaries, the efferent arteries taking their origin in those capillaries after the manner of veins ; and $(b)$ by the interposition of two important bloodpurifying organs, the liver and the kidney, in the course of the returning current, as a result of which we have veins acting as both afferent and efferent vessels of the hepatic and renal capillaries, the afferent vessels of both organs ending in capillaries after the fashion of arteries.

In the embryos of the higher, or air-breathing, Craniata, the circulatory organs agree in essentials with the above description, the most important difference being that, as no gills are present, the 
branches of the ventral aorta do not break up into capillaries, but pass directly into the dorsal aorta, forming the aortic arches (Fig. 783, $A b$.). With the appearance of the lungs, however, a very fundamental change occurs in the blood-system. The last aortic arch of each side gives off a pulmonary artery (Fig. 784, $A p$.) to the corresponding lung, and the blood, after circulating through the capillaries of that organ, is returned by a pulmonary vein (lr.), not into an ordinary systemic vein of higher order, but into the heart directly : there it enters the left side of the auricle, in which a vertical partition is developed, separating a left auricle $\left(A^{1}\right)$, which receives the aërated blood from the lungs, from a right auricle $(A)$, into which is poured the impure blood of the sinus venosus. Lastly, in Crocodiles, Birds, and Mammals (B) the ventricle also becomes divided into right and left chambers, and we get a four-chambered heart, having right and left auricles and right and left ventricles: at the same time the conus arteriosus and sinus
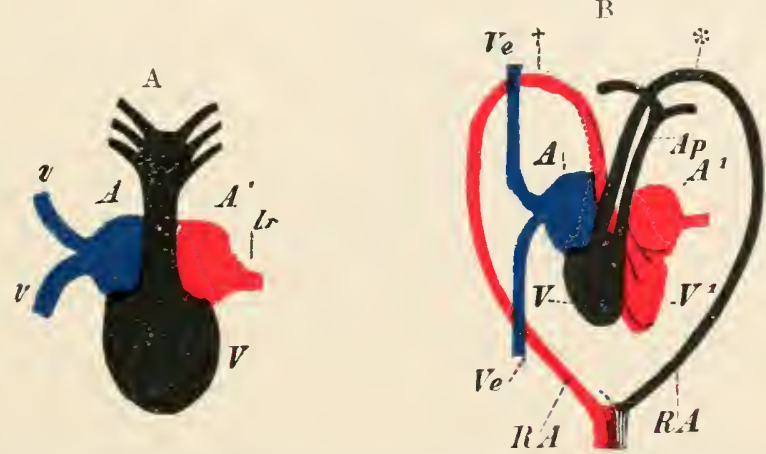

FIG. 784.-Diagram of the heart $\mathbf{A}$, in an $\mathbf{A m p h i b i a n ; ~} \mathbf{B}$, in a Crocodile. 4 , right auricle $A$. let auricle; $A p$.pulmonary artery $; l r$. pulmonary vein $; R A$. aortic arches $; V$. ventricle $V^{\prime}$. left ventricle; $c, t$, and $V e, V e$. pre-and post-cavals. (From Wiedersheim's Vertebrata.)

venosus cease to exist as distinct chambers. The left auricle receives aërated blood from the lungs and passes it into the left ventricle, whence it is propelled through the system : the right auricle receives impure blood from the system, and passes it into the right ventricle to be pumped into the lungs for aeration. Thus the four-chambered heart of the higher Vertebrata is quite a different thing from that of a Fish : in the latter the four chambers-sinus venosus, auricle, ventricle, and conus arteriosus-form a single longitudinal series, whereas in a Mammal, for instance, the four chambers constitute practically a double heart, there being no direct communication between the auricle and ventricle of the right side, or respiratory heart, and those of the left side, or systemic heart. The modifications undergone by the arteries and veins in the higher Tertebrata will be best considered under the various classes.

It will be noticed that there is a sort of rough correspondence vOL. II 
between the blood-vessels of Craniata and those of the higher Worms. The sub-intestinal vein, heart, and ventral aorta together form a ventral vessel, the dorsal aorta a dorsal vessel, and the aortic arches transverse or commissural vessels. The heart might thus be looked upon as a portion of an original ventral vessel, which has acquired strongly muscular walls, and performs the whole function of propelling the blood. But in making such a comparison it has to be borne in mind that the direction of the current of the blood in the Craniata is exactly the opposite of that in the Annulata.

The blood of Craniata is always red, and is specially distinguished by the fact that the hæmoglobin to which it owes its colour is not dissolved in the plasma as in most red-blooded Invertebrates, but is confined to certain cells called red blood-corpuscles (Fig. 785), which occur floating in the plasma in addition to, and in far greater numbers than, the leucocytes. They usually have the form of flat oval dises $(A)$, the centre bulged out by a large nucleus (nu.), but in Mammals $(B)$ they are bi-concave, non-nucleated, and usually circular. The red corpuscles do not perform amœeboid

A

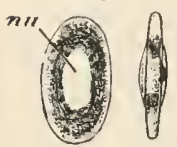

FIt. 785 . - Surface and edge views of red blond-corpuscles of Froo (d) and Man (B). nu. nucleus. (From Parker's Biology.) movements.

The colour of the blood varies with the amount of oxygen taken up by the hæmoglobin. When thoroughly aërated it is of a bright scarlet colour, but assumes a bluishpurple hue after giving up its oxygen to the tissues. Owing to the fact that oxygenated blood is usually found in arteries, it is often spoken of as arterial blood, while the non-oxygenated, purple blood, being usually found in veins, is called venous. But it must not be forgotten that an artery, e.g., the ventral aorta ог the pulmonary artery, may contain venous blood, and a vein, e.g., the pulmonary vein, arterial blood. The distinction between the two classes of vessels does not depend upon their contents, but upon their relations to the heart and the capillaries.

In addition to the blood-vessels the circulatory system of Craniata contains lymph-vessels or lymphatics (Fig. 782, ly.). In most of the tissues there is a network of lymph-capillaries, interwoven with, but quite independent of, the blood-capillaries. From this network lymphatic vessels pass off, and finally discharge their contents into one or other of the veins. Many of the lower Craniata possess spacious lymph-sinuses surrounding the blood-vessels; and there are communications between the lymphatics and the coelome by means of minute apertures or stomata. The lymphatics contain a fluid called lymph, which is to all intents and purposes blood minus its red corpuscles. The lymph-plasma 
consists of the drainage from the tissues: it makes its way into the lymph capillaries, and thence into the lymphatics, which are all efferent vessels, conveying the fluid from the capillaries to the veins. Lencocytes are added to the plasma in bodies called lymphatic glands, which occur in the course of the vessels. Valves may be present to prevent any flow of lymph towards the capillaries, and in some cases the flow of the fluid is assisted by lymph-hearts, muscular dilatations in the course of certain of the vessels. The lymphatics of the intestine have an important function in the absorption of fats, and are known as lacteals (lc.).

The nervous system attains a complexity, both anatomical and histological, unknown in the rest of the animal kingdom. It arises, as in other Chordata, from a dorsal medullary groove the edges of which unite and enclose a tube. From the ectoderm lining the tube the whole central nervous system, or neuron, is formed; its lumen forms the neurocole or characteristic axial cavity of the neuron. So far the agreement with the lower Chordata is complete, but a fundamental advance is seen in the fact that at an early period-

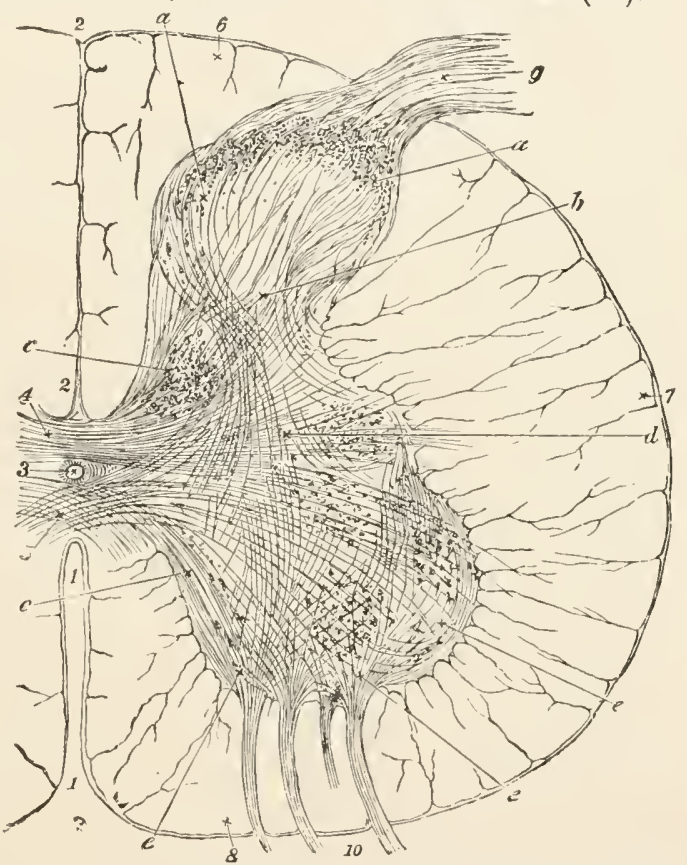

II I 786.-Transverse section of spinal cord. 1 , ventral fissure : 2, dorsal fissure ; 3 , central canal ; 4, 5, bridges connecting grey matter of right and left sides; $6,7,8$, white matter ; 9 , dorsal root of spinal nerve; 10 ventral root. $a, b$. dorsal horn of grey matter ; $c$. Clarke's column ; $e$. ventral horn. (From Huxley's Physiology.)

before the closure of the medullary groove-the anterior end of the neuron undergoes a marked dilatation and forms the rudiment of the brain, the rest becoming the spinal cord. Moreover, as growth goes on, a space appears in the mesoderm immediately surrounding the nervous system, and forms the neural or cerebro-spinal cavity already referred to (Fig. 769 , c.s.c.), so that the neuron, instead of being solidly imbedded in mesoderm, lies in a well-marked and often spacious tube enclosed by the neural arches of the vertebræ, and in front by the cranium (Fig. 769, $B-D$ ). 
The spinal cord (Fig. 786) is a thick-walled cylinder, continuous in front with the brain. It is traversed from end to end by a narrow central canal (3), lined by ciliated epithelium derived from the superficial layer of in-turned ectoderm cells. The dorsal surface of the cord is marked by a deep, narrow, longitudinal cleft, the dorsal fissure (2), the ventral surface is similarly scored by a groove, the ventral fissure (1); owing to the presence of these fissures a transverse section presents two almost semicircular halves with their straight edges applied to one another and joined in the middle by a narrow bridge $(4,5)$ in which the central canal lies.

The cord is made up of two kinds of tissue. Surrounding the central canal and having a somewhat butterfly-shaped transverse section, is the grey matter $(a, e)$ consisting of delicate, inter-twined, non-medullated nerve-fibres, amongst which are numerous nervecells. The superficial portion is composed of medullated nerve-fibres running longitudinally, and is called the white matter $(6,7,8)$. In both grey and white matter the nervous elements are supported by a non-nervous tissue called neuroglia, formed of branched cells.

From the cord the spinal nerves are given off. They arise in pairs from the sides of the cord, and agree in number with the myomeres. Each nerve arises from the cord by two roots, a dorsal and a ventral. The dorsal root (Fig. 788, d. r.) is distinguished by the presence of a ganglion ( $g n$. d.r.) containing nerve-cells, and its fibres are almost exclusively afferent, conveying impulses from the various parts and organs of the body to the central nervous system; the ventral root $(v . r$.$) is not ganglionated, and$ its fibres are efferent, conveying impulses from the neuron outwards. Each root arises from one of the horns of the grey matter, and the two mingle to form the trunk (sp. 1-3) of the nerve, which emerges from the spinal canal usually between the arches of adjacent vertebræ. Soon after its emergence it divides into two chief divisions, dorsal $(d$.$) and ventral (sp. 1, \&c.). The spinal$ nerves supply the muscles and skin of the trunk and limbs, and are therefore spoken of as somatic nerves. Frequently groups of nerves unite with one another to form more or less complex networks called plexuses.

Closely associated with the spinal are the sympathetic nerves (Fig. 788, sym.). They take the form of paired longitudinal cords with ganglia (sym. gn.) at intervals, lying one on each side of the aorta in the dorsal wall of the coelome. They contain both afferent and efierent fibres, the afferent derived from the dorsal, the efferent from the ventral roots of the spinal nerves, and both traceable, through those roots, into the grey matter of the cord. The sympathetic nerves supply the enteric canal and its glands, the heart, blood-vessels, \&c., and are therefore denominated splanchnic nerves. 
As already mentioned, the anterior end of the nervous system undergoes, at a very early period, a marked dilatation, and is distinguished as the brain (Fig. 787). Constrictions appear in the dilated part and divide it into three bulb-like swellings or vesicles, the fore-brain $(A, f . b$.), mid-brain (m. b.) and hind-brain (h.b.). Soon a hollow outpushing grows forwards from the first vesicle ( $B$, prs. en), and the third gives off a similar hollow outgrowth $(\mathrm{cblm}$.) from its dorsal surface. The brain now consists of five divisions : the prosencephalon (prs. en.) and the diencephalon (dien.), derived from the fore-brain: the mid-brain or mesencephalon $(m$. b.) which remains unaltered: the epencephalon or cerebellum $(c b l m$.$) , and the metencephalon or medulla oblongata (med. obl.),$ derived from the hind-brain. ${ }^{1}$ Additional constrictions appear in the medulla oblongata giving it a segmented appearance, but they disappear as development proceeds, and, whatever may be their significance, have nothing to do with the main divisions of the adult organ. The original cavity of the brain becomes correspondingly divided into a series of chambers or ventricles, all communicating with one another and called respectively the fore-ventricle or prosocoele, third ventricle or diacole, mid-ventricle or mesocole, cerebellar ventricle or epiccele, and fourth ventricle or metacole.

In some Fishes the brain consists throughout life of these five divisions only, but in most cases the prosencephalon grows out into paired lobes, the right and left cerebral hemispheres or parencephala $(I-L, c . h$.$) , each containing a cavity, the lateral$ ventricle or paracoele ( $p a$. coe.) which communicates with the diacœle (di. co.) by a narrow passage, the foramen of Monro $(f, m$.$) .$ Moreover, each hemisphere gives off a forward prolongation, the olfactory bulb or rhinencephalon (olf. l.), containing an olfactory ventricle or rhinoccele ( $r h . c \infty$.) : when there is an undivided prosencephalon, the olfactory bulbs $(C, D$, olf. $l$.) spring from it. In the embryo of some forms there is a median unpaired olfactory bulb, like that of Amphioxus. The part of the cerebral hemisphere with which the olfactory bulb is immediately related is the olfactory lobe.

The brain undergoes further complications by the unequal thickening of its walls. In the medulla oblongata the floor becomes greatly thickened $(D, H, K)$, while the roof remains thin, consisting of a single layer of epithelial cells, assuming the character therefore of a purely non-nervous epithelial layer (ependyme). In the cerebellum the thickening takes place to such an extent that the epicœle is usually obliterated altogether. In the mid-brain the ventral wall is thickened in the form of two longitudinal bands, the crura cerebri ( $c r . c r b$.$) , the dorsal wall in the form of$

1 The prosencephalon is sometimes called the telencephalon, the epencephalon the metencephalon, and the metencephalon the myelencephalon. 


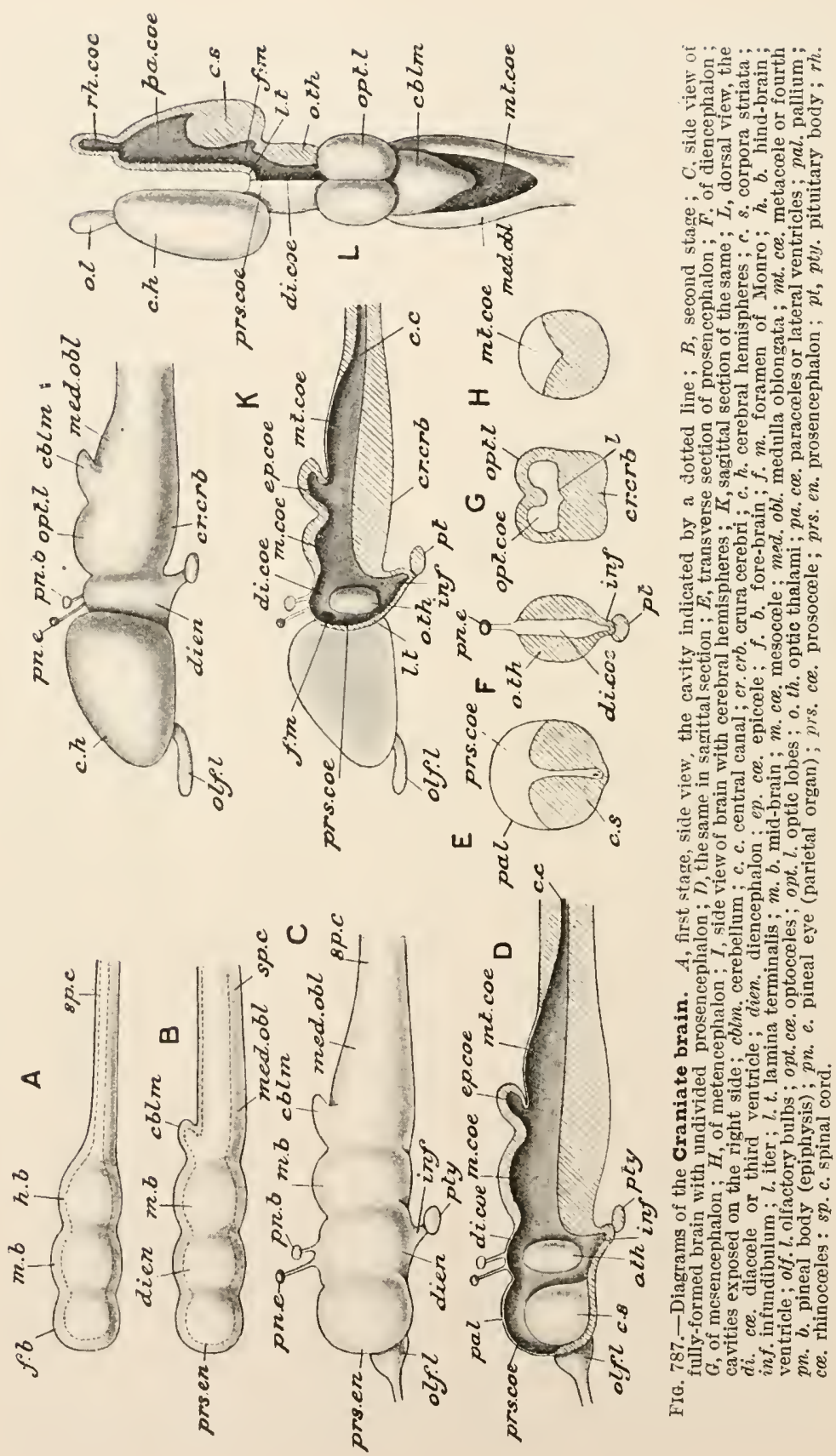


paired oval swellings, the optic lobes (opt. l.): extensions of the mesocoele into the latter form the optic ventricles or optocceles (G. opt. coe.): the median portion of the mesocœle is then called the iter $(l)$ or aqueduct of Sylvius. In the diencephalon the sides become thickened, forming paired masses, the optic thalami $(D$, $F, L, o$. th.), the roof remains for the most part in the condition of a thin membrane (ependyme) composed of a single layer of cells, but part of it gives rise to a very peculiar adjunct of the brain, the pineal apparatus. This originates as an outgrowth which consists typically of two narrow diverticula, one in front of the other, the anterior being the parietal organ, the posterior the pineal organ or epiphysis: these two parts may be developed independently, or the latter may originate by outgrowth from the former. The parietal organ in the Lampreys and some Reptiles develops an eye-like organ, the pineal eye ( $p n . e$.) at its extremity, but is vestigial or absent in most other Vertebrates. The epiphysis is eye-like (parapineal eye) only in the Lampreys; in other Vertebrates it is represented by a gland-like structure, the pineal body (pn. b.), connected by a hollow or solid stalk with the roof of the diencephalon. The term paraphysis is applied to a non-nervous outgrowth of the roof of the fore-brain developed in front of the epiphysis in the hinder region of the prosencephalon. ${ }^{1}$ The floor of the diencephalon grows downwards into a funnel-like prolongation, the infundibulum (inf.): with this the pituitary diverticulum of the pharynx (p. 84) comes into relation, and there is formed, partly from the dilated end of the diverticulum, partly from the extremity of the infundibulum, a gland-like structure, the pituitary body or hypophysis ( $p t$.$) , always situated immediately in front of the$ anterior extremity of the notochord and between the diverging posterior ends of the trabeculæ. The hypophysis in higher Craniates appears to be of the nature of a ductless, internally secreting gland. In lower Craniata it consists of two distinct glandular parts, the one (saccus vasculosus) situated more dorsally and formed as an outgrowth of the infundibulum, the other (hypophysis proper) ventral and arising from the pharyngeal diverticulum. In cases where cerebral hemispheres are not developed, the roof or pallium of the undivided fore-brain is reduced to a layer of epithelium. ( $D$ and $E$. pal.), its floor is thickened so as to form large paired masses, the corpora striata (c.s.). When hemispheres are developed the corpora striata form the floors of the two lateral ventricles $(L, c . s$.$) , and the roof (pallium) of each is formed of nervous tissue.$ In such cases the front wall of the diencephalon remains very thin, and is distinguished as the lamina terminalis $(l . t$.$) : this is the$ actual anterior extremity of the central nervous system, the cerebral hemispheres being lateral outgrowths.

In the preceding description the brain lias been described as if its

"The so-called "paraphysis" of Mammals is not homologous with this. 
parts were in one horizontal plane; but, as a matter of fact, at a very early period of development the anterior part becomes bent down over the end of the notochord, so that the whole organ assumes a retort-shape, the axis of the fore-brain being strongly inclined to that of the hind-brain. The bend is known as the cerebral flexure: it is really permanent, but, as the hemispheres grow forward parallel to the hind-brain and the floor of the midand hind-brain thickens, it becomes obscure, and is not noticeable in the adult.

The brain, like the spinal cord, is composed of grey and white matter, but the grey matter either forms a thin superficial layer or cortex, as in the hemispheres and cerebellum, or occurs as ganglionic masses surrounded by white matter.

The whole cerebro-spinal cavity is lined with a tough membrane, the dura mater, and both brain and spinal cord are covered by a more delicate investment, the pia mater: the space between the two contains a serous fluid. In the higher forms there is a delicate arachnoid membrane outside the pia, and in many cases the regions of the pia in immediate contact with the thin epithelial roofs of the diencephalon and medulla become greatly thickened and very vascular, forming in each case what is known as a choroid plexus.

From the brain are given off cerebral or cranial nerves: these, like the spinal nerves, are paired, but unlike them, are strictly limited in number, the number being constant, at least within very narrow limits: there are typically ten pairs in Fishes and Amphibians, twelve in Reptiles, Birds, and Mammals. ${ }^{1}$

The first or olfactory nerve (Fig. 788, I.) is rather a large number of fibres than a single nerve; it arises from the olfactory bulb, and supplies the organ of smell, i.e., the epithelium of the olfactory sac (see below). It is therefore a purcly sensory nerve.

The second or optic nerve (II.) arises from the ventral region of the diencephalon, just in front of the infundibulum. It differs from all the other nerves in being originally a hollow outpushing of the brain, containing a prolongation of the diacœle (see Fig. 795). It supplies the retina or actual organ of light, and is therefore a purely sensory nerve.

The third or oculomotor nerve (III.) arises from the crus cerebri or ventral region of the mid-brain. In its course is a ganglion, the oculomotor or ciliary ganglion (c.gn.). It supplies four out of the six muscles of the eye-ball (see below, Fig. 796), viz., the superior, inferior, and internal recti, and the inferior oblique, as well as the ciliary muscles and muscles of the iris in the interior of the eye. It is therefore a purely motor nerve.

1 In many Fishes a pair of very small nerves-the nervi terminales-are given off from the cerebral hemispheres and run forward to the olfactory sacs : they seem to be the nerves of ordinary sensatinn for these organs. Repre. sentatives of these nerves have also been deseribed in higher forms. 
The fourth or trochlear nerve (Figs. 788, IV, and 796, IV.) arises from the dorsal surface of the brain at the junction of the mid-brain with the medulla oblongata. It is a very small and purely motor nerve, supplying only the superior oblique muscle of the eye.

The fifth or trigeminal nerve (Fig. 788, V.) is of great size and wide distribution. It arises from the side of the medulla, frequently by two roots, a dorsal and a ventral, thus resembling in its origin a spinal nerve. Near its origin it enters a ganglion, the trigeminal or Gasserian ganglion ( $g . g n$.), which may be incompletely divided into two parts, an antero-dorsal and a postero-ventral. The trunk of the nerve early divides into two principal branches, the ophthalmic and the mandibular (V.md.) : the latter sends off a maxillary nerve (V.mx.), and we thus get the three divisions to which the name trigeminal is due. The ophthalmic nerve frequently

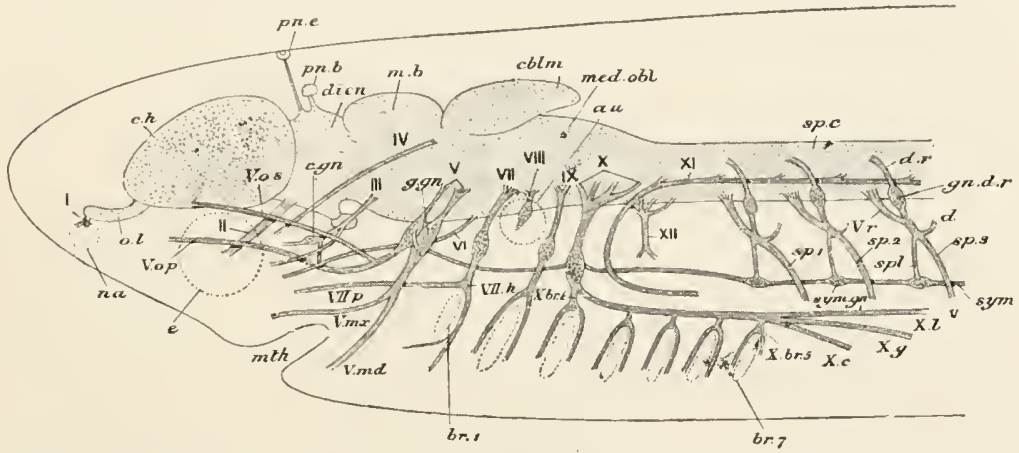

FIG. 788.-Diagram of the cerebral and anterior spinal nerves of a Craniate. I, olfactory nerve; II, optic ; III, oculomotor ; IV, trochlear ; V. trigeminal ; $\nabla .0 . s$. superficial ophthalmic branch $; V$. o. p. deep ophthalmic; VI, abducent; VII, facial ; $V I I$. $h$. hyomandibular branch; VII. p palatine branch; VIII, auditory ; IX, glossopharyngeal ; $\mathrm{X}$, vagus ; $\boldsymbol{X}$. br. $1-5$, branchiai branches ; $\boldsymbol{X} . c$, cardiac branch $; \dot{X} . g$, gastric branch $; \boldsymbol{X}$. $l$, lateral branch; $\mathrm{X} 1$, accessory ; X11, hypoglossal. au. auditory organ; $b r .1-7$, branchial clefts ; cblm. cerebellum; $c . g n$. ciliary ganglion; $c . h$. cerebral hemispheres ; $d$. dorsal branch of spinal nerve : $d$. $r$. dorsal root; $e$. eye ; $g n$. $d . r$. ganglion of dorsal root; $m$. $b$. mid-brain ; med. obl. medulla oblongata ; th . mouth; na. olfactory sac; $o$. $l$. olfactory bulb; $p n$. $b$. pineal body ; $p n$. $e$. pineal eye; $s p . c$. spinal cord ; $s p .1-3$, ventral branches of spinal nerves ; sym. sympathetic nerve; sym.gn. sympathetic ganglion; $\boldsymbol{V}$.r. ventral root.

divides into two branches, a superficial (V.o.s.) and a deep (V.o.p.), the former present in Fishes only: the latter in some Fishes a semi-independent nerve given off separately from the dorsal part of the trigeminal ganglion. The ophthalmic is purely sensory, and supplies the skin in the neighbourhood of the mouth and certain parts in the orbit. The maxillary nerve $(V . m x$.) is also sensory: it supplies the parts in relation with the upper jaw, including the teeth. The mandibular nerve $(V . m d$.) is partly sensory, partly motor : it supplies the muscles of the jaws, the skin and teeth of the lower jaw, and sends off a gustatory nerve or nerve of taste to the epithelium of the tongue in the higher forms. The ophthalmic nerve is connected by a branch with the ciliary ganglion.

The sixth or abducent (Figs. 788, VI, and 796, VI.) is a small motor 
nerve, arising from the ventral region of the medulla, and supplying the external rectus muscle of the eye. We thus have the remarkable fact that out of ten, or at the most twelve, cerebral nerves, three are devoted to the supply of the six small muscles by which the eye-ball is moved, and of those by which the accommodation of the eye for varying distances is effected.

The seventh or facial (Fig. 788, VII.) is, like the fifth, a mixed nerve in the lower Craniata, i.e., contains both sensory and motor fibres. It arises from the side of the medulla, a short distance behind the fifth, and is dilated near its origin into a facial ganglion. It has two chief branches, a palatine (VII. p.), which passes in front of the mandibulo-hyoid gill-cleft, and supplies the mucous membrane of the palate, and a hyomandibular $(V I I . h$.$) , which$ passes behind the same cleft and sends branches to the lower jaw and to the hyoid arch. In most aquatic Vertebrata an ophthalmic branch is given off from the trunk of the nerve, and usually accompanies the superficial ophthalmic division of the fifth. In the higher Vertebrata the seventh becomes a purely motor nerve, supplying the muscles of the face.

The eighth or auditory nerve (VIII.) arises immediately behind the seventh, with which it is intimately connected at its origin. It is a purely sensory nerve, supplying the organ of hearing, i.e., the epithelium of the membranous labyrinth presently to be described.

The ninth or glossopharyngeal (IX.) is a mixed nerve : it arises from the lateral region of the medulla, behind the organ of hearing, and is connected at its origin with the vagus ganglion (see below). Its trunk passes downwards and forks over the hyo-branchial gill-cleft, sending an anterior branch to the hyoid arch which bounds the cleft in front, and a posterior branch to the first branchial arch which bounds it posteriorly. Thus the entire nerve supplies the hyo-branchial gill-pouch, including both branchial filaments and muscles: its anterior branch goes to the posterior hemibranch of the hyoid arch, its posterior branch to the anterior hemibranch of the first branchial arch. In the air-breathing Vertebrata, in which gills are absent, the glossopharyngeal sends a gustatory nerve to the tongue and supplies the pharynix.

In Fishes a nerve known as the lateral $(X . l$.) takes its origin above the glossopharyngeal, sometimes in front of the latter, sometimes behind it. It usually joins the trunk of the following or tenth nerve, but becomes separate again and runs backwards, supplying the cutaneous sense-organs of the lateral line (see below).

The tenth nerve $(X$.$) , called the vagus or pneumogastric, is dis-$ tinguished by its wide distribution. It arises by numerous roots from the side of the medulla, the roots uniting into a stout trunk with a vagus ganglion at its origin. From the trunk are given off, in the first place, branchial nerves ( $X . b r .1-5)$, corresponding in number and position to the gill-slits from the third to the last 
inclusive. Each branchial nerve behaves in exactly the same way as the glossopharyngeal; it forks over the gill-pouch to which it belongs, sending one branch to the anterior, another to the posterior wall of the pouch. Thus each gill-pouch has its own nerve while each gill receives its supply from two sources; for instance, the gill of the second branchial arch has its anterior hemibranch innervated from the first, its posterior hemibranch from the second branchial branch of the vagus. The vagus also gives off a cardiac nerve $(X . c$.) to the heart, a gastric nerve $(X . g$.) to the stomach. In the air-breathing Craniata there are, of course, no branchial nerves; but the vagus still retains control of the respiratory organs by giving origin to pulmonary nerves to the lungs and laryngeal nerves to the larynx.

b

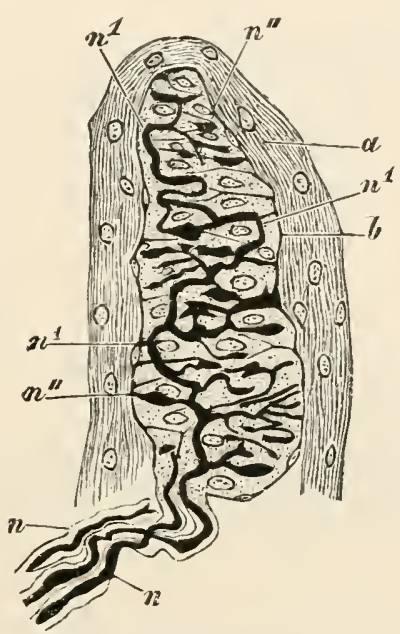

C

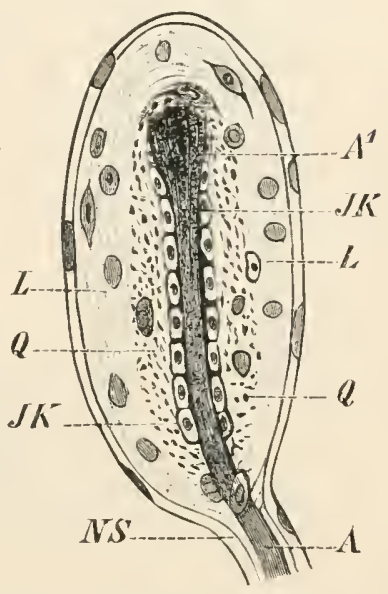

F'IG. 789.-A, tactile spot from skin of Frog. $a$. touch-cells ; $b$. epidermis ; $N$, nerve. B tactile corpuscle from dermal papilla of human hand. $a$. connective-tissue investment; $b$. touchcells ; $n, n^{\prime}, n^{\prime \prime}, n^{\prime \prime \prime}$, nerve. C, Pacinian corpuscle from back of Duck. $\boldsymbol{A}, \boldsymbol{A}^{1}$. neuraxis ; $J K$. central knob and surrounding cells : $L, Q$. investing layers; $N S$ medullary sheath of nerve-fibre. (From Wiedersheim's Vertebrata.)

The eleventh or accessory nerve (XI.), which is recognisable in some Fishes as a part of the vagus, is a distinct nerve in higher forms, and consists of cerebral and spinal portions, so that it occupies an intermediate position between the purely cerebral and the purely spinal nerves. It acts in higher Craniates mainly as the motor nerve for certain muscles of the shoulder.

The twelfth or hypoglossal (XII.) arises from the ventral aspect of the medulla oblongata, after the manner of the ventral root of a spinal nerve. It is purely motor, and supplies the muscles of the tongue and certain neck-muscles. In the Amphibia its place is taken by the first spinal nerve. and there is no doubt that it is 
to be looked upon as a spinal nerve which has become included in the cranial region: even in some Fishes it passes out through the skull.

The sympathetic nerve $(s y m$.$) is continued into the head and$ becomes connected with certain of the cerebral nerves.

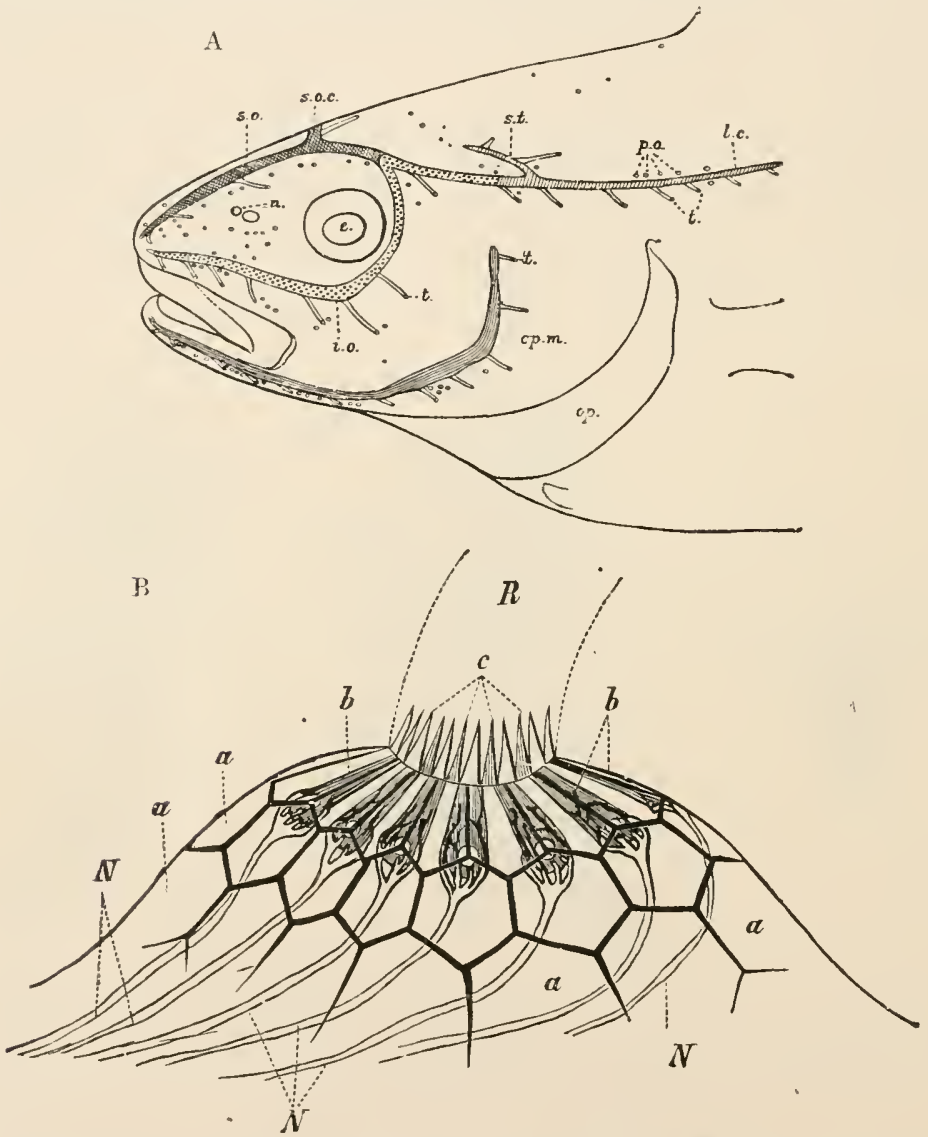

FIG. 790.-A, Sensory canals of the left side of the head of a Bony Fish, showing the supraorbital (s.o.), infra-orbital (i.o.), operculo-mandibular (op.m.), and lateral (l.c.) canals. (From the Cambridge $\mathbf{N}$ atural $H$ istory, after Cole.). $B$, organ of the lateral line (neuromast) in a tailed Amphibian (semi-diagrammatic). $a$. enidermic cells, through which are seen $b$, sensory cells ; $c$. sensory hairs; $N$. nerve ; $R$. liyaline tube. (From Wiedersheim's Vertebrata.)

Sensory Organs.-The whole surface of the body forms an organ of touch, but special tactile organs are more or less widely distributed. End-buds consist of ovoidal groups of sensory cells supplied by a special nerve : touch-cells (Fig. 789, A) are nerve-cells occurring in the dermis at the termination of a sensory nerve : touchcorpuscles (B) are formed of an ovoidal mass of connective-tissue containing a ramified nerve, the terminal branches of which end 
in touch-cells : Pacinian corpuscles (C) consist of at terminal nervebranch surrounded by a complex laminated sheath. Touchcorpuseles and Pacinian bodies are found only in the higher forms.

In Fishes, characteristic sense-organs are present, known as the neuromast-organs or organs of the lateral line. Extending along the sides of the trunk and tail is a longitudinal streak, due to the presence either of an open groove or of a tube sunk in the epidermis, and continued on to the head in the form of branching grooves or canals (Fig. 790, A). These organs and also certain others, in the form of pits or of unbranched canals, are lined with epithelium $(B)$, some of the cells of which are arranged in groups, the neuromasts, and have the form characteristic of sensory cells $(b)$ produced at their free ends into hair-like processes $(c)$ : they are innervated by the lateral nerve, and in the head, by the seventh and sometimes also the ninth nerve. At their first appearance in the embryo the organs of the lateral line are distinct, segmentally - arranged patches of sensory epithelium in intimate connection with the ganglia of the third, fifth,
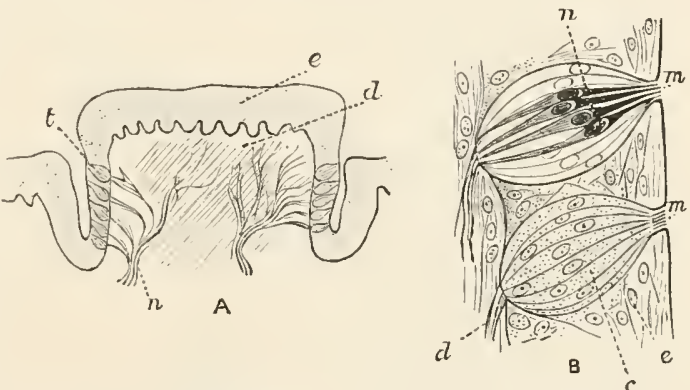

FIG. 791.-A, vertical section of one of the papilla of the tongue of a IMammal. $d$. submucosa ; $e$. epithelium; $n$. nerve-fibres; $t$. taste-buds. $B$, two taste-buds. $c$. covering cells shown in lower bud; $d$. sub-mucosa; $e$. epithelium of tongue ; $m$. sensory processes; $n$. internal sensory cells shown in upper bud. (From Foster and Shore's Physiology.)

seventh, ninth, and tenth nerves. Cutaneous sense-organs of the lateral-line system, having at first a metameric arrangement, also occur in the aquatic Amphibia.

The function of the neuromast-organs has been shown to be to enable the animal to detect vibrations in the water of too low a frequency to form a sound capable of perception by the ear.

The sense of taste has for its special organs taste-buds (Fig. 791), similar in general character to the end-buds in the skin, and composed of groups of narrow rod-shaped cells. In Fishes these* are widely distributed in the mouth and branchial cavities, also on the outer surface of the head, and in some Fishes over almost the whole surface of the body. In higher Craniates they are chiefly confined to the epithelium of the tongue and soft palate, and are supplied mainly by branches of the glossopharyngeal.

The olfactory organ is typically a sac-like invagination of the skin of the snout, anterior to the mouth, and communicating with the exterior by an aperture, the extemal nostril. It is paired in all Craniata, except Cyclostomes, in which there is a single olfactory 
sac, supplied, however, by paired olfactory nerves. The sac is lined by the olfactory mucous membrane or Schneiderian membrane, the epithelium of which contains peculiar, elongated sensory cells (Fig. 792), their free ends often produced into hair-like processes. In the Dipnoi and all higher groups the posterior end of each sac communicates with the cavity of the mouth by an aperture called the posterior nostril, and an analogous communication occurs in the case of the unpaired organ of the Hags (vide p. 135).

In many air-breathing Vertebrates there is formed an offshoot from the olfactory organ, which, becoming separated, forms a distinct sac lined with olfactory epithelium and opening into the

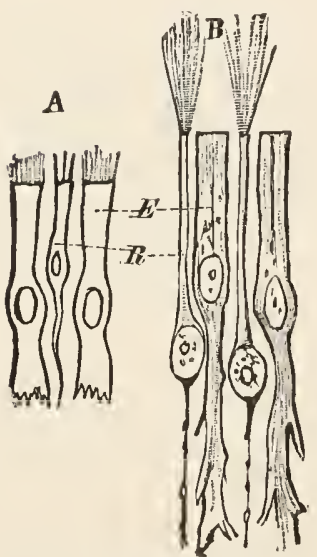

I'1G. 792.-Epithelial cells of olfactory mucous membrane. $A$, of Iamprey; $B$, of Salamander. $E$. interstitial cells; $R$. olfactory cells. (From Wiedersheim's Vertebrata.)

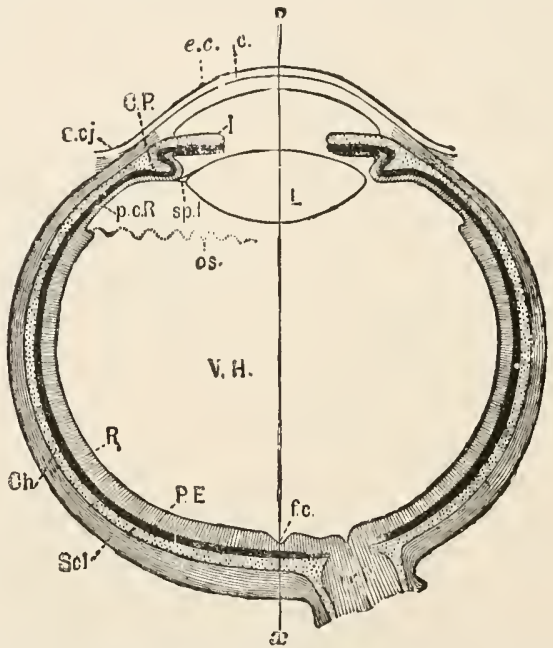

FIG. 793.-Diagrammatic horizontal section of the eye of Man. $c$. cornea ; $C h$. choroid (dotted); $C$. P. ciliary processes : $e . c$. epithelium of cornea; $e_{,} c j$. conjunctiva; $f$. o. yellow spot; $I$. iris ; $L$. lens $; O . N$. optic nerve; os. ora serrata; $0-x$, optic axis; p.c.R. anterior non-visual portion of retina; $P$. E. pigmented epithelium (black); $R$. retina; $s p$. l. suspensory ligament; Scl. sclerotic; $V . H$. vitreous chamber. (From Foster and Shore's Physiology.)

mouth. This is Jacobson's organ: it is supplied by the olfactory and trigeminal nerves.

The paired eye is a more or less globular structure, lying in the orbit, and covered externally by a thick coat of cartilage or of dense fibrous tissue, the optic capsule or sclerotic (Fig. 793, scl.). On the outer or exposed portion of the eye the sclerotic is replaced by a transparent membrane, the cornea $(c)$, formed of a peculiar variety of connective-tissue, and covered on both its outer and inner faces by a layer of epithelium. The whole external coat of the eye has thus the character of an opaque spherical case-the sclerotic, having a circular hole cut in one side of it and fitted with a transparent window, the cornea. The curvature of the cornea is 
not the same as that of the sclerotic; the former is almost flat in Fishes, but bulges outwards in terrestrial Vertebrates.

Lining the sclerotic is the second coat of the eye--the choroid (ch.) -formed of connective-tissue abundantly supplied with bloodvessels. At the junction of sclerotic and cornea, it becomes continuous with a circular membrane $(I)$, placed behind but at some distance from the cornea, and called the iris. This latter is strongly pigmented, the colour of the pigment varying greatly in different species, and giving, as seen through the transparent comea, the characteristic colour of the eve. The iris is perforated in the centre
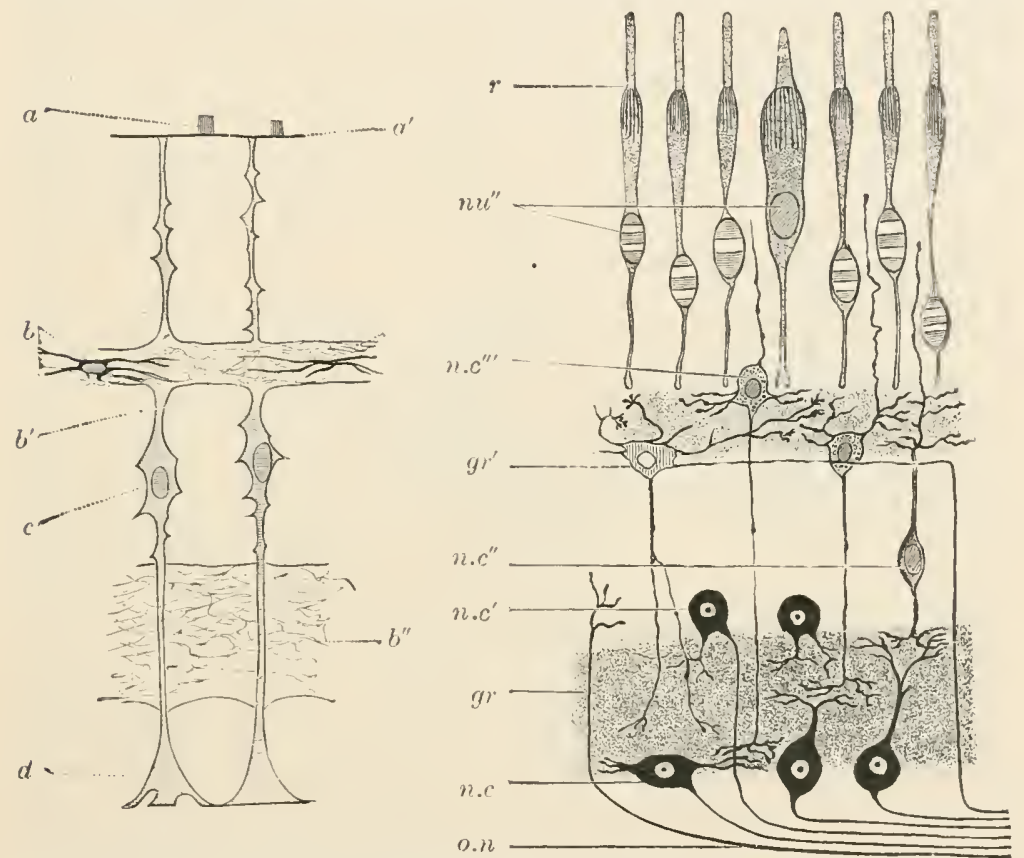

Fra. 794.-Diagram of the retina, the supporting structures to the left, the nervous and epithelial elements to the right. $a-d$, fibrous supporting structures ; $g r$. $g r^{\prime}$. granular lavers : $n, c_{.}, n, c^{\prime}$. $n . c^{\prime \prime} ., n . c^{\prime \prime}$. nerve-cells; $n u$. nuclear layer of rods and cones; $o$. $n$. fibres of optic nerve; $r$. rods and cones. (From Wiedersheim's Vertebrata, after Stöhr.)

by a circular or slit-like aperture, the pupil, which, in the entire eye, appears like a black spot in the middle of the coloured portion. Except in Fishes, the pupil can be enlarged by the action of a set of radiating unstriped muscle-fibres contained in the iris, and contracted by a set of circular fibres ; and the anterior or outer portion of the choroid, where it joins the iris, is thrown into radiating folds, the ciliary processes $(C . P$.$) , containing unstriped muscular fibres,$ the ciliary muscle.

Lining the choroid and forming the innermost coat of the eye is a delicate semi-transparent membrane, the retina $(R$.$) , covered on$ 
its outer or choroidal surface with a layer of black pigment $(P . E$.). It extends as far as the outer ends of the ciliary processes, where it appears to end in a wavy line, the ora serrata $(o . s$.$) : actually,$ however, it is continued as a very delicate membrane (p.c.R.) over the ciliary processes and the posterior face of the iris. The optic nerve $(O . N$.) pierces the sclerotic and choroid and becomes continuous with the retina, its fibres spreading over the inner surface of the latter. Microscopic examination shows that these fibres, which form the innermost layer of the retina (Fig. 794,o.n.), turn outwards and become connected with a layer of nerve-cells (n. c.). External to these come other layers of nerve-cells and granules, supported by a framework of delicate fibres, and finally, forming the outer surface of the retina proper, a layer of bodies which correspond to modified sensory cells and are called, from their shape, the rods and cones $(r$.$) . These are placed perpendicularly$ to the surface of the retina, and their outer ends are imbedded in a single layer of hexagonal pigment-cells, loaded with granules of the black pigment already referred to.

Immediately behind and in close contact with the iris is the transparent biconvex lens (Fig. 793, L.), formed of concentric layers of fibres, each derived from a single cell. The lens is enclosed in a delicate capsule, attached by a suspensory ligament (sp. l.) to the ciliary processes. The suspensory ligament exerts a pull upon the elastic lens so as to render it less convex than when left to itself ; when the ciliary muscles contract they draw the suspensory ligament towards the iris, relaxing the ligament and allowing the lens to assume, more or less completely, its normal curvature. It is in this way that the accommodation of the eye to near and distant objects is effected.

The space between the cornea in front and the iris and lens behind is called the aqueous chamber of the eye, and is filled by a watery fluid-the aqueous humour. The main cavity of the eye, bounded in front by the lens and the ciliary processes and for the rest of its extent by the retina, is called the vitreous chamber, and is filled by a gelatinous substance, the vitreous humour (V.H.).

The cornea, aqueous humour, lens, and vitreous humour together constitute the dioptric apparatus of the eye, and serve to focus the rays of light from external objects on the retina. The iris is the diaphragm by which the amount of light entering the eye is regulated. The percipient portion or actual organ of sight is the retina, or, more strictly, the layer of rods and cones. The great peculiarity of the vertebrate eye, as compared with that of a Cephalopod (Vol. I, p. 750), to which it bears a close superficial resemblance, is that the sensory cells form the outer instead of the inner layer of the retina, so that the rays of light have to penetrate the remaining layers before affecting them.

The mode of development of the eye is as characteristic as its 
structure. At an early stage of development a hollow outgrowththe optic vesicle (Fig. 795, A, opt. v.) - is given off from each side of the fore-brain (dien.). It extends towards the side of the head, where it meets with an inpushing of the ectoderm (inv. l.) which deepens and forms a pouch, and finally, separating from the ectoderm, a closed sac $(B, l$.) with a very small cavity and thick walls. This sac is the rudiment of the lens: as it enlarges it pushes against the optic vesicle, and causes it to become invaginated $(B)$; the single-layered optic vesicle thus becomes converted into a twolayered optic cup (opt. c., opt. $c^{\prime}$.), its cavity, originally continuous with the diacole, becoming obliterated. The invagination of the vesicle to form the cup does not take place symmetrically, but obliquely from the external (posterior) and ventral aspect of the vesicle, so that the optic cup is incomplete along one side where there is a cleft-the choroid fissure-afterwards more or less completely closed by the union of its edges. The outer layer of the optic cup becomes the pigmentary layer of the retina: from its inner layer the rest of that membrane, including the rods and cones, is formed. The stalk of the optic cup occupies, in the embryonic eye, the place of the optic
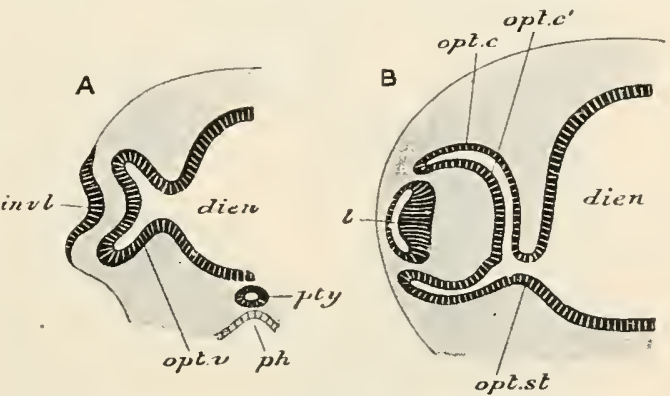

FIG. 795.-Farly (A) and later $(B)$ stages in the development of the eye of a Craniate. dien. diencephalon; inv. l. invagination of ectoderm to form lens; $l$. lens ; opt. c. outer, and $o p t$. $c^{\prime}$. inner layer of optic cup; opt. st. optic stalk; opt. $v$. optic vesicle; $p h$. pharynx; pty. pituitary body. (Altered from Marshall.)

nerve, but the actual fibres of the nerve are formed as backward growths from the nerve-cells of the retina to the brain.

During the formation of the lens, mesoderm grows in between the pouch from which it arises and the external ectoderm; from this the main substance of the cornea and its inner or posterior epithelium are formed, the adjacent ectoderm becoming the external epithelium. Mesoderm also makes its way into the optic cup, through the choroid fissure, and becomes the vitreous humour. Lastly, the mesoderm immediately surrounding the optic cup is differentiated to form the choroid, the iris, and the sclerotic.

Thus the paired eye of Vertebrates has a threefold origin : the sclerotic, choroid, iris, vitreous, and the greater part of the cornea are mesodermal: the lens and external epithelium of the cornea are derived from the ectoderm of the head: the retina and optic nerve are developed from a hollow pouch of the brain, and are therefore, in their ultimate origin, ectodermal. The sensory cells 
of the retina - the rods and cones, although not directly formed from the external ectoderm, as in Invertebrates, are ultimately traceable into the superficial layer of ectoderm, since they are developed from the inner layer of the optic vesicle, which is a prolongation of the inner layer of the brain, and the latter is continuous before the closure of the medullary groove with the ectoderm covering the general surface of the body.

The eye-ball is moved by six muscles (Fig. 796). Four of these arise from the inner wall of the orbit, and pass, diverging as they go, to their insertion round the equator of the eye. One of them is dorsal in position, and is called the superior rectus (s.r.), a second ventral, the inferior rectus (in. $r$.), a third anterior, the anterior or internal rectus (i.r.), and a fourth posterior, the posterior or external rectus (e. r.). The usual names (internal and external) of the two last-named muscles originate from their position in Man, where, owing to the eye looking forwards instead of outwards, its

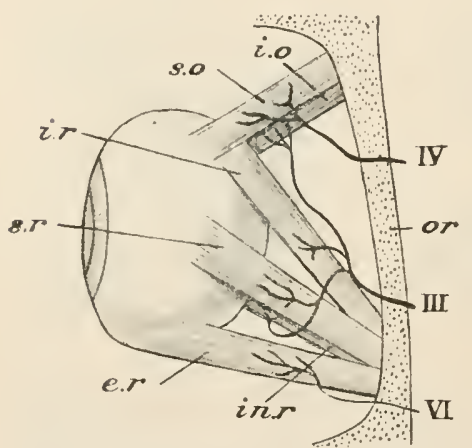

Fig. 796.-Muscles and nerves of the eyc of a Skate (semi-diagrammatic). III. oculomotor nerve; IV. trochlear; VI. abducent. e. r. posterior rectus; $i$. o. inferior oblique; $i n$. $r$. inferior rectus ; $i$. $r$. anterior rectus; or. wall of orbit; $s$. $o$. superior oblique; $s . r$. superior rectus. anterior surface becomes internal, its posterior surface external. The two remaining muscles usually arise from the anterior region of the orbit, and are inserted respectively into the dorsal and ventral surface of the eye-ball. They are the. superior $(s .0$.$) and$ inferior oblique $(i . o$.$) muscles.$

The median or pineal eye (Fig. 797 ) is formed, in certain cases, from the distal end of the parietal organ already mentioned. It has the form of a rounded capsule, the outer or anterior portion of the wall of which is a lens $(l$.) formed of elongated cells, while its posterior portion has the character of a retina $(M, r)$. The latter has a layer of nerve-fibres on its outer and one of rod-like visual elements $(r$.) on its inner surface : it thus agrees with the usual types of Invertebrate retina, and not with that of the paired eye.

The organ of hearing, like that of sight, presents quite peculiar features. It arises in the embryo as a paired invagination of the ectoderm in the region of the hind-brain, a shallow depression being formed which deepens and becomes flask-shaped, and finally, as a rule, loses its connection with the external ectoderm, forming a closed sac surrounded by mesoderm. At first simple, it soon becomes divided by a constriction into dorsal and ventral compartments. The dorsal compartment is differentiated into an irregular 
chamber, the utriculus (Fig. 798, u.), and, usually, thrce tubes, the semicircular canals. Of these two, the anterior (ca.) and posterior (cp.) canals, are vertical in position and have their adjaccnt limbs united, so that the two canals have only thrce opcnings between them into the utriculus: the third or external canal (ce.) is horizontal, and opens into the utriculus at either end. Each canal is dilated at one of its ends into an ampulla (aa., ae, ap.), placed anteriorly in the anterior and cxternal canals, posteriorly in the posterior canal.

The ventral compartment of the auditory sac is called the sacculus $(s$.$) : it gives off$ posteriorly a blind pouch, the cochlea (l.), which attains considerable dimensions in the higher classes; whilefromits inner face is given off a narrow tube, the endolymphatic duct (de.), which either ends blindly or opens on the dorsal surface of the head. The utricle and saccule are sometimes imperfectly differentiated, and are then spoken of together as the membranous vestibule.

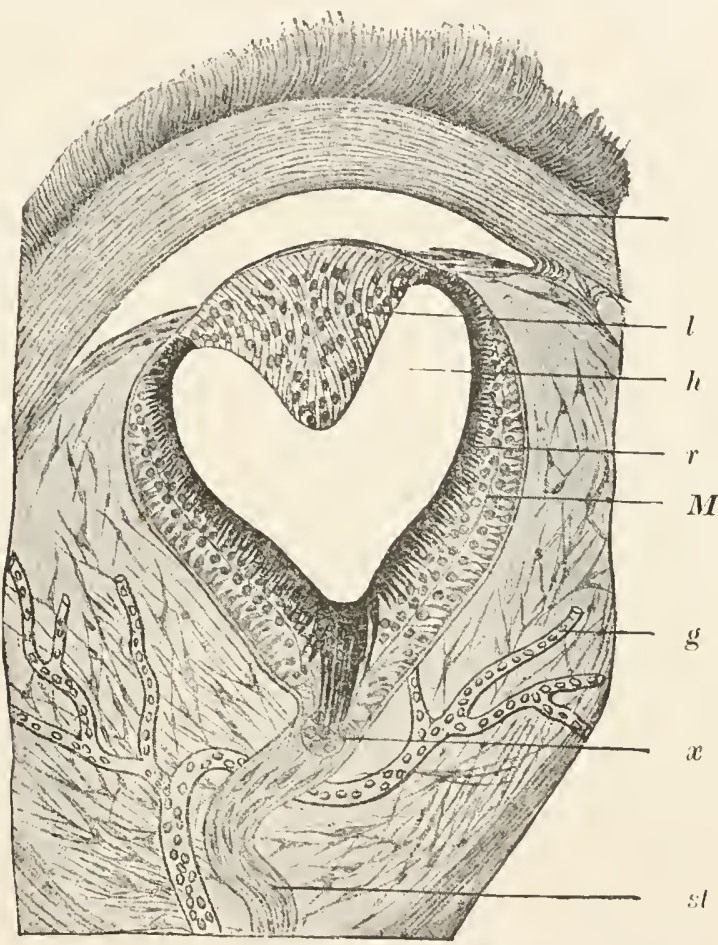

lif. 797.-Section of tre pineal eye of Sphenodon. $g$. blockvessel $; h$. cavity of eye, filled with fluid $; k$. connective-tissue capsule $; l$. lens $; M$. molecular layer of retina $; r$ layer of rods and cones; $s t$. nerve ; $\dot{x}$. cells in nerve. (From Wiedersheim's Tertebrata, after Baldwin Spencer.)

Patches of sensory cells (Fig. 799, ae.) - elongated cells produced into hair-like processes $(a . h$.$) -occur in the ampulla and in the$ utricle and saccule : they are known as maculce acusticce and cristce acustica (c. r.), and to them the fibres of the auditory nerve $(n$.) are distributed. A fluid, the endolymph, fills the whole of the auditory organ or membranous labyrinth, and in it are formed otoliths of varying size and number. There is every reason for thinking that the labyrinth, like the otocysts or statocysts in the lower animals, functions as an organ of equilibration as well as of hearing. 
As the membranous labyrinth develops in the embryo, it becomes surrounded "and enclosed by the auditory capsule, the cartilage of

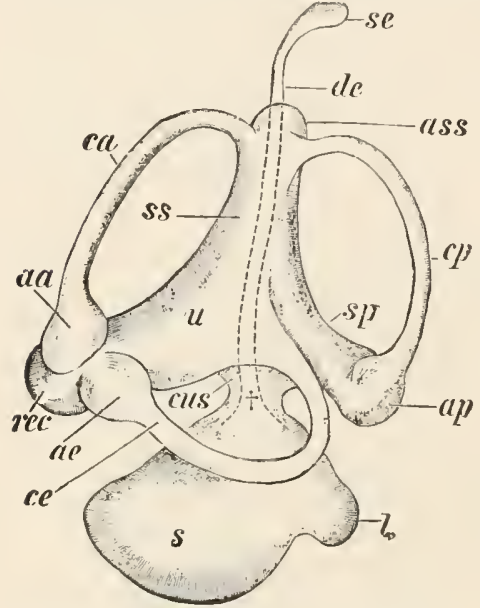

FIG. 798-External view of organ of liearing of Craniata (semi-diagrammatic). aa. ampulla of anterior canal; $a e$. of horizontal caual; ap. of posterior canal; ass. apex of superior utricular sinus; $c a$. anterior, $c e$. horizontal, $c p$. posterior semicircular canal ; cus. canal uniting sacculus with utriculus; de. endolymphatic duct: l. cochlea; rec. utricular recess ; $s$. sacculus ; se, endolymphatic sac ; $s p$. posterior utricular sinus; ss. superior utricular sinus; $u$. ntrieulus. (From Wiedersheim's Vertebrata.) which adapts itself to the form of the labyrinth, presenting a large excavation for the utricle and saccule and tunnel-like passages for the canals. The auditory organ does not, however, fit tightly into this system of cavities, but between it and the cartilage is a space, filled by a fluid called perilymph, which acts as a buffer to the delicate organ floating in it.

The early history of the auditory apparatus in the embryo shows that it belongs to the same series of structures as the lateral-line system, of which it may be regarded as a highly specialised part.

Nerve - components. - The nerve-fibres of which the nervescerebral, spinal, and sympathetic - are made up, the nerve-components as they are termed, are capable of being classified in accordance with the nature of the functions which they perform. A broad division into motor and sensory fibres has already been referred to. A more detailed classification is the following :-

Division I.Somatic sensory, comprising $(a)$ the fibres which have to do with general cutaneous or tactile sensations; $(b)$ th os e connected with the neuromast organs and with the auditory organs; (c) the fibres of the optic nerves.

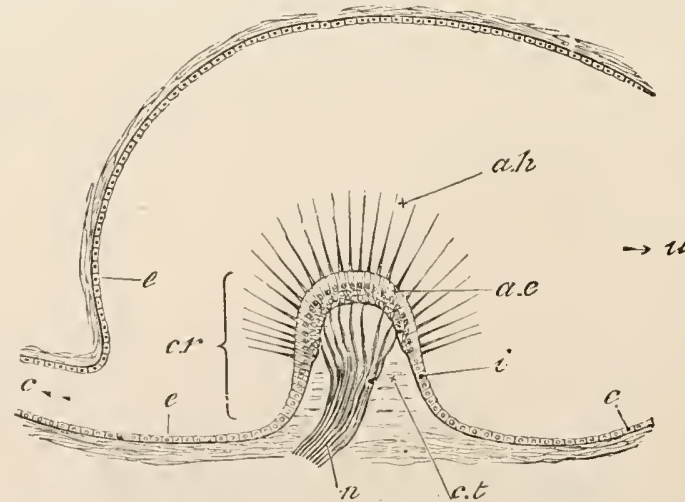

liG. 799.-Longitudinal section through an anpulla. $a$. $p$ auditory epithelium; $a$. $h$. auditory lıairs; $c$. part of semicircular canal ; $c r$. crista acustica ; $c t$. connective-tissue ; $e, i$. epithelium; $n$. nerve; $u$. junction witl utriculus. (From Foster and Shore's Physiology.) 
Division II.-Visceral sensory, comprising $(a)$ the fibres which end in visceral mucosæ and have to do with visceral sensations; $(b)$ the fibres ending in taste-buds; $(c)$ those which terminate in the olfactory epithelium.

Division III.-Somatic motor, consisting of components which terminate in somatic musculature.

Division IV.- Visceral motor, comprising all fibres which terminate in visceral musculature and have to do with visceral movements.

Urinogenital Organs.-In all Craniata there is so close a connection between the organs of renal excretion and those of reproduction that the two systems are conveniently considered together as the urinogenital organs.

Speaking generally, the excretory organ consists of three parts, all paired and situated along the dorsal wall of the coelome; the fore-kidney or pronephros (Fig. 801, A, p. nph.), the mid-kidney or mesonephros (ms. $n p h$.), and the hind-kidney or metanephros (mt. nph.). Each of these is provided with a duct, the pro- $(p n$. d.), meso(msn. d.), or meta-nephric (mt. n. d.) duct, which opens into the cloaca. The gonads (gon.) lie in the cœlome suspended to its dorsal wall by a fold of peritoneum : in some cases their products are discharged into the colome and make their exit by genital pores, but more usually the pronephric duct in the female assumes the functions of an oviduct and the mesonephric duct in the male those of a spermiduct $(c f$. p. 116). The pronephros is almost always functionless in the adult, and usually disappears altogether. The mesonephros is generally the functional kidney in the lower Craniata, in which, as a rule, no metanephros is developed, and the mesonephric duct, in addition to carrying the seminal fluid of the male, acts as a ureter. In the higher forms the mesonephros atrophies, and the metanephros is the functional kidney, the metanephric duct becoming the ureter.

The kidney-meso- or meta-nephros-of the adult is a massive gland of a deep red colour made up of convoluted urinary tubules (Fig. 800), separated from one another by connective-tissue containing an abundant supply of blood-vessels. The tubules are lined by a single layer of glandular epithelial cells $(B, C)$, and each ends blindly in a globular dilatation, the Malpighian capsule $(A, g l$.), lined with squamous epithelium. In many of the lower Craniata, a branch goes off from the tubule, near the Malpighian capsule, and, passing to the ventral surface of the kidney, ends in a ciliated funnel-like body (Fig. 801, nst.), resembling the nephrostome of a worm, and, like it, opening into the coelome. At their opposite ends the tubules join with one another, and finally discharge into the ureter.

The renal arteries branch extensively in the kidney, and give off to each Malpighian capsule a minute afferent artery (Fig. $800, A, v a$.) : this pushes the wall of the capsule before it, and breaks up into a 
bunch of looped capillaries, called the glomerulus, suspended in the interior of the capsule. The blood is carried off from the glomerulus by an efferent vessel (ve.), which joins the general capillary system of the kidneys, forming a network over the urinary tubules : finally, the blood is returned from this network to the renal vein. The watery constituents of the urine are separated from the blood in traversing the glomerulus, and, flowing down the tubule, take up and dissolve the remaining constituents-urea, uric acid, \&c.which are secreted by the cells of the tubules.

The development of the kidney reveals a resemblance to the coelomoducts of Annulata which would hardly be suspected from its adult structure. The pronephros (Fig. 801, $A, p$. nph.) originates as a small number of coiled tubes formed from mesoderm in the body-wall at the anterior end of the colome; they are arranged

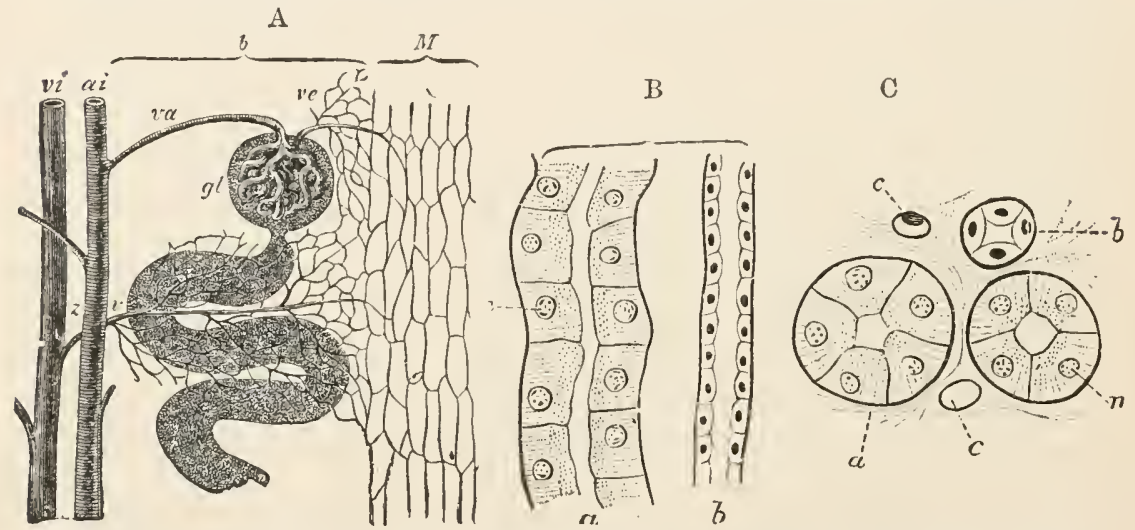

FIG. $800 .-A$, part of a urinary tubule with blood-vessels. $a i$, artery ; gl. Malpighian capsule containing glomerulus ; $v$. veinlet returning blood from capillary network (to the right) to vein $v i . ; v a$. afferent vessel of glomerulus; $v e$. efferent vessel. $B$, longitudinal, and $C$, transverse sections of urinary tubules. $a$. secreting part of tubules; $b$. conducting part of tubules; $c$. capillaries; $n$. nuclei. (From Foster and Shore's Physiology.)

metamerically, and each opens into the cœlome by a ciliated funnel (nst.). Obviously such tubes are colomoducts: their chief peculiarity is that their outer ends do not open directly on the exterior, but into a longitudinal tube, the pronephric or segmental duct (sg. d.), which passes backwards and discharges into the cloaca. It seems probable that this arrangement is to be explained by supposing that the coelomoducts originally opened externally into a longitudinal groove, which, by the apposition of its edges, was converted into a tube. All the tubules of the pronephros open, by their ciliated funnels, into the narrow anterior end of the colome, into which projects a branch of the aorta ending in a single large glomerulus.

The pronephros soon degenerates, its tubules losing their connection with the pronephric duct $(B)$, but in the meantime fresh tubules 
appear in the segments posterior to the pronephros, and together constitute the mesonephros or Wolffian body (B, ms. nph.), from which the permanent kidney is formed in most of the lower Craniata.
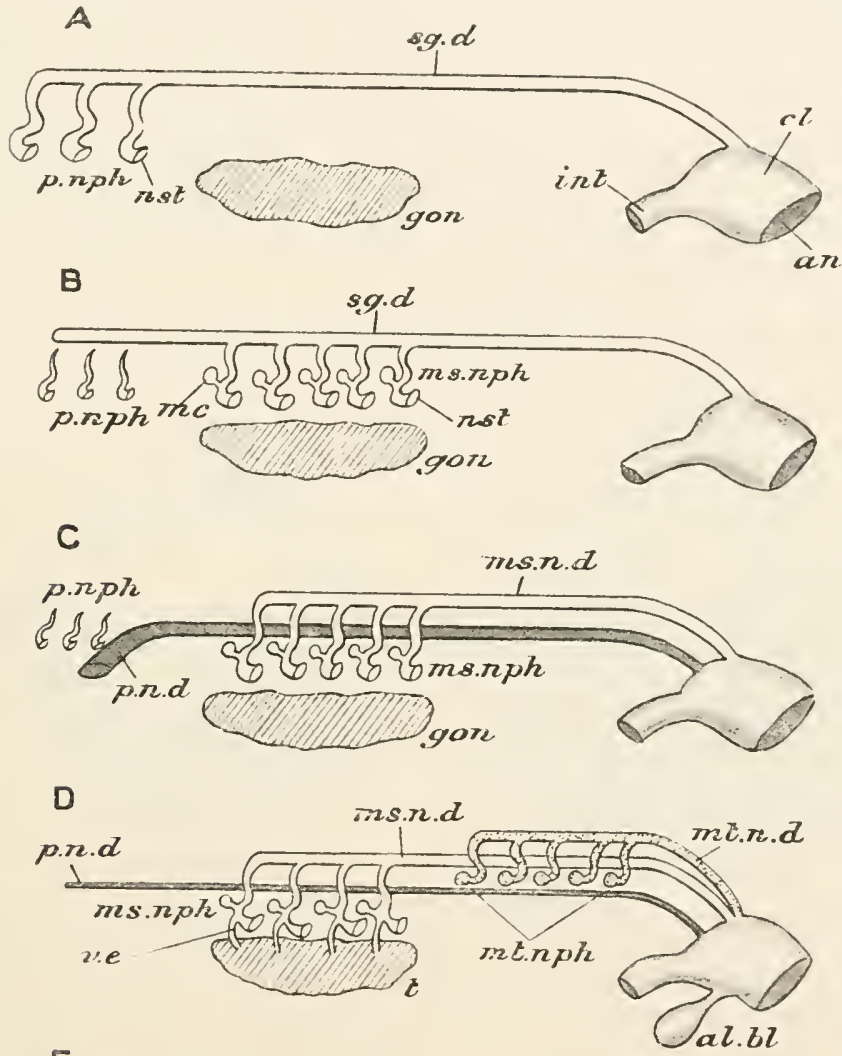

$E$

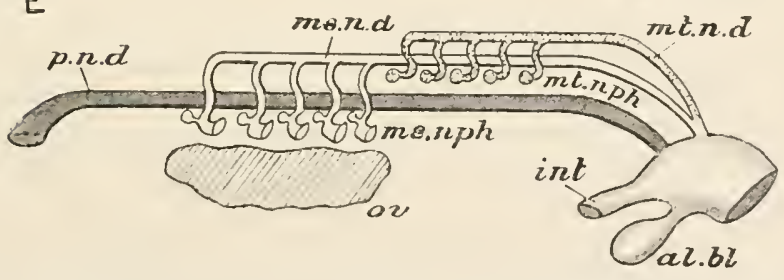

Frg. 801.-Diagrams illustrating the development of the urinogenital organs of Craniata. $A$, development of pronephros and pronephric duct; $B$, atrophy of pronephros, development of mesonephros; $C$, differentiation of pro-and mesonephric ducts ; $D$, development of metanephros, male type ; $E$, female type. al. bl. allantoic bladder; $a n$. anus; $c l$. cloaca ; gon. gonad ; int. intestine; $m$. c. Malpighian capsule; $m s, n$. $d$. mesonephric duct; $m s, n p h$. mesonephros ; mt. $n$. d. metanephric duct; $m t$. $n p h$. metanephros ; nst. nephrostome ; ov. ovary ; $p . n$. $d$. and $s g$. $d$. pronephric duct ; $p$. $n p h$. pronephros ; $t$. testis ; $v$. $e$. vasa efferentia.

The mesonephric tubules open at one end into the pronephric duct $(s g . d$.$) , at the other by ciliated funnels (nst.), into the cœlome; a$ 
short distance from the funnel each gives off a blind pouch, which dilates at the end and forms a Malpighian capsule (m. c.), and a branch from the aorta entering it gives rise to a glomerulus.

In some forms the pronephric duct now becomes divided by a longitudinal partition into two tubes: one retains its connection with the mesonephros and is known as the mesonephric or Wolffian duct $(C, m s . n . d$.$) : the other has no connection with the tubules, but$ opens into the colome in the region of the vanishing pronephros, and is called the Müllerian duct $(p . n . d$.). In some Craniata the Miillerian appears quite independently of the Wolffian duct: the latter is then simply the pronephric duct after the union with it of the mesonephric tubules.

In the higher Vertebrata, from Reptiles to Mammals, a diverticulum $(D, E, m t . n . d$.$) is given off from the posterior end of the$ Wolffian duct, which grows forwards and becomes connected with the hindmost tubules. In this way is formed a metanephros (mt. nph.), which forms the permanent kidney, and a metanephric duct $(m t . n . d$.), which gives rise to the ureter. The Wolffian body ceases to discharge a renal function, and becomes a purely vestigial organ.

In many Fishes there is a dilatation of the ureter, the urinary bladder, which serves as a receptacle for the urine. In the higher Craniata the ventral wall of the cloaca sends off a pouch, the allantoic bladder $(a l . b l$.), which serves the same purpose, although morphologically an entirely different structure.

The gonads (gon.) are developed as ridges growing from the dorsal wall of the coelome and covered by colomic epithelium, from the cells of which, as in so many of the lower animals, the ova and sperms are derived. The testis consists of crypts or tubules, lined with epithelium, and usually discharging their products through delicate vasa efferentia $(D, v$. e.) into the Wolffian duct, but in some groups into the cœlome. The sperms are always motile. The ovary is formed of a basis of connectivetissue or stroma, covered by epithelium, certain of the cells of which become enlarged to form ova. In the majority of cases the ova are discharged from the surface of the ovary into the coelome and thus into the open ends of the Müllerian ducts $(E, p . n . d$.$) ,$ which thus function simply as oviducts, having no connection in the adult with the urinary system. In some groups the ova, like the sperms, are shed into the coelome and escape by the genital pores, and in many bony Fishes the ovary is a hollow organ, as in Arthropoda, discharging its ova into an internal cavity, whence they are carried off by a duct continuous with the gonad.

A few Craniata are normally hermaphrodite, but the vast majority are dicecious, hermaphroditism occurring, however, occasionally as an abnormality.

In close topographical relation with the urinogenital organs are 
found certain "ductless glands," the adrenals or inter- and suprurenal bodies. They are developed partly from ridges of the dorsal wall of the colome-i.e., from mesoderm, partly from the sympathetic ganglia. There may be numerous adrenals segmentally arranged, or a single pair. Like other ductless glands the adrenals produce an internal secretion, which mingles with the blood and produces physiological effects on other parts.

Development.- The ova of Craniata are usually telolecithal, but the amount of food-yolk varies within wide limits. When it is small in quantity, segmentation is complete but usually unequal, when abundant, incomplete and discoidal. In the latter case the embryo proper is formed, as in Cephalopods, from a comparatively small portion of the oosperm, the rest giving rise to a large yolk-sac.
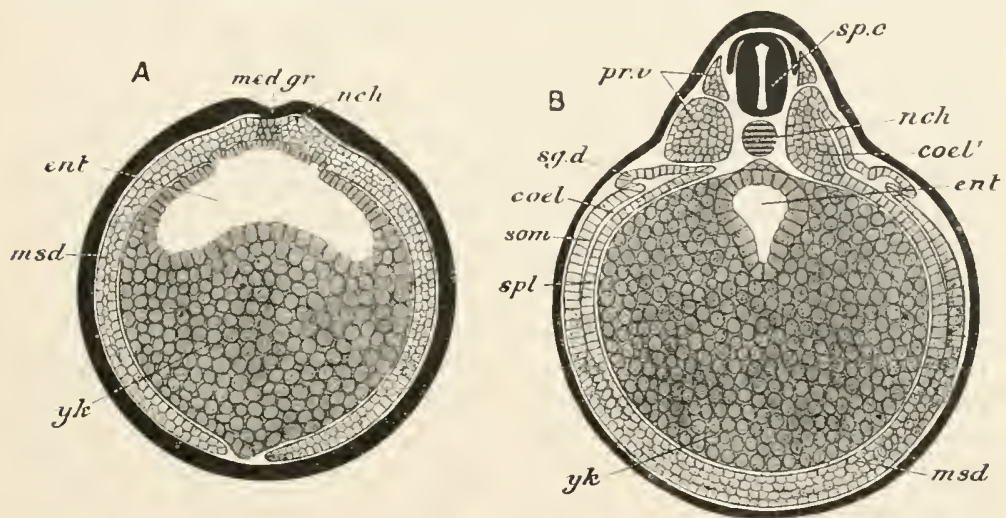

FIG, 802.-Transverse section of earlier $(A)$ and later $(B)$ embryos of Frog. coel, coelome "; coll". prolongation of calome into protovertebra ; ent. mesenteron; med. gr. medullary groove; $m s d$. mesoderm ; nch. notochord; $p r$. $v$. protovertebra; $s g$. d. segmental duet; som. somatic layer of mesoderm; $s p$. c. spinal corl; spl. splanchnic layer of mesoderm; $y k$. yolk-cells. (After Marchall.)

There is never a typical invaginate gastrula, as in Amphioxus, but in some of the lower Craniata a gastrula stage is formed by a combination of inpushing and overgrowth: details will be given in the sections on the various groups. In the higher forms a gastrula cannot be recognised with absolute certainty.

The mode of development of the mesoderm and of the coelome differs strikingly from the process we are familiar with in Amphioxus. At an early stage the mesoderm is found in the form of paired longitudinal bands (Fig. 802, $A$, msd.) lying one on each side of the middle line, where they are separated from one another by the medullary groove ( $m d . g r$.$) and the notochord ( \mathrm{ch}$.), and completely filling the space between the ectoderm and the endoderm. In all probability the mesoderm is derived from both of the primitive germ-layers. Each mesoderm-band becomes differentiated into a dorsal portion, the vertebral plate, bounding the nervous 
system and notochord, and a ventral portion, the lateral plate, bounding the mesenteron. The vertebral plate undergoes metameric segmentation, becoming divided into a row of squarish masses, the protovertebrce or mesodermal segments $(B, p r . v$.$) : the$ lateral plate splits into two layers, a somatic (som.), adherent to the ectoderm, a splanchnic ( $s p l$.), to the endoderm. The space between the two is the colome (coel.), which is thus a schizocoele, or cavity hollowed out of the mesoderm, and is, except in the head-region in the Lampreys (p. 132), at no stage in communication with the mesenteron, like the cœlomic pouches of Amphioxus. The dorsal portion of the cœelome assumes the character of a series of paired diverticula of the main ventral part, each situated in the interior of a protovertebra; but such an arrangement is temporary, and these protovertebral cavities early disappear. From the dorsal portions of the protovertebræ the myomeres are formed, from their ventral portions the vertebræ.

The development of the principal organs has been described, in general terms, in the preceding account of the organs themselves: it will be convenient to defer further consideration of this subject until we come to deal with the development of the various types of Craniata, and with the embryological characteristics of the classes and sub-classes.

Metamerism.-A tendency, more or less strongly marked, to a serial repetition of parts is to be observed in a number of different systems of organs. Instances of this have already been pointed out in the skeleton, and the muscular, nervous, and excretory systems. This phenomenon seems to lead to the conclusion that the structure of the Craniata can be understood only when they are regarded as metamerically-segmented animals. The phase of metamerism presented by the Craniata is, however, widely different from that which prevails in the segmented Invertebrates. In the latter the segmentation is usually quite distinctly pronounced externally, and it may involve a metameric division extending to the colome as well as to the various systems of internal organs. In the Craniata, on the other hand, segmentation is never visible on the exterior, and in the adult condition the colome never shares in the division. Even in the case of the organs which present metameric characters, the metamerism often appears indefinite and uncertain: thus, as already pointed out, the segmentation of the spinal column, which in the adult is the most pronounced of all, does not coincide with the segmentation of the muscular and nervous systems. Yet when we take the phenomena of embryonic development into account, it becomes sufficiently clear that in the Craniata we have to do witl animals possessing a metameric segmentation of the same general type as that possessed by Amphioxus, and that the apparent anomalies are due to processes of secondary modification. 
It is in the trunk region that the metamerism is most strongly pronounced, and that more particularly in the lower groups. In the head there is great specialisation in ec-ordination with the presence in this region of the brain, the chief organs of special sense, and the mouth and jaws; so that, though there are indications of metamerism of various parts, it is only by the study of development that it is possible to interpret the structurc of the head in terms of a metameric segmentation which becomes much modified and disguised in the adult animal. When the development is followed out, it becomes evident that, as in the Arthropoda, the head in Craniata is formed as a result of a process of fusion between a number of metameres, the individuality of which is quite evident in early stages, more particularly among lower forms, being most pronounced in the region behind the auditory capsules.

Distinctive Characters.-The Craniata may be defined a Euchorda in which the notochord is not continued to the end of the snout, but stops short beneath the fore-brain, some distance from its anterior end. A skull is always present, and there are usually paired limbs. The ectoderm is many-layered and is never ciliated in the adult, and only rarely in the larva. The pharynx is of moderate dimensions, and is perforated by not more than seven pairs of gill-slits (except in some Cyclostomes). The gill-pouches do not open into an atrium. The liver is large, massive, and not obviously tubular. There is a muscular, chambered heart, and the blood contains red corpuscles. The renal tubules unite to form large paired kidneys and open into ducts which discharge into or near the posterior end of the intestine. The brain is complex, and there are at least ten pairs of cerebral nerves: the spinal nerves are, except in Cyclostomes, formed by the union of dorsal and ventral roots. Paired eyes of great complexity, derived in part from the brain, are present; and there is a pair of auditory organs. There is typically a single pair of gonads, and the reproductive products are usually discharged by ducts derived from the renal system. There is never a typical invaginate gastrula, and the mesoderm arises in the form of paired longitudinal bands which subsequently become segmented. The colome is nearly alwaye developed as a schizocole.

\section{CLASS I-CYCLOSTOMATA.}

The Cyclostomata, or Lampreys and Hags, are eel-like animals, distinguished from all other Craniata by the possession of a suctorial mouth devoid of functional jaws, by the single olfactory organ, and by the absence of lateral appendages, or paired fins.

\section{Example of the Class.-The Lamprey (Petromyzon).}

Three species of Lamprey are common in the Northern Hemisphere: the Sea-lamprey ( $P$. marinus), which attains a length of a 
metre; the Isampern, or common fresh-water Lanprey ( $P$. fluviatitis), which may reach a length of about $90 \mathrm{~cm}$; ; and the Sandpride, or lesser fresh-water Lamprey ( $P$. planeri), not exceeding 45 cm. in length. In the Southern Hemispliere the Lampreys belong to two genera: Mordacia, found on the coasts of Chili and Tasmania, and Geotria, in the rivers of Chili, Australia, and New Zealand. Both genera differ from Petromyzon in minor details only.

External Characters.-The head and trunk (Fig. 803) are nearly cylindrical, the tail-region compressed or flattened from side to side. At the anterior end, and directed downwards, is a large basin-like depression, the buccal funnel (buc.f.), surrounded with
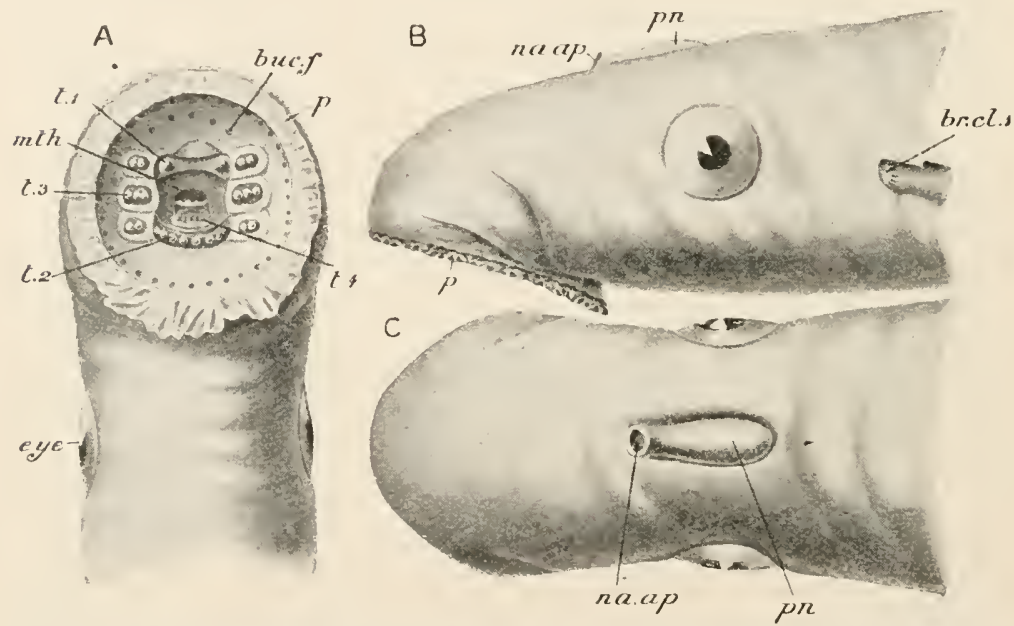

Fia, 803,-Petromyzon fluviatilis. Ventral $(A)$, lateral $(B)$, and dorsal $(C)$ views of the head. $b r . c l$. 1, first gill-cleft ; bur. $f$. buccal funnel ; eye, eye ; mth. mouth ; $m a$. ap. nasal aperture ; $p$. papille; $p n$. pineal area; $t 1 . t^{2} . t 3$. teeth of buccal funnel; $t 4$. teeth of tongue. (After

W. $\bar{K}$. Parker.)

papillæe $(p)$ and beset internally with yellow, hormy leeth $(t 1-t 3)$. At the bottom of the funnel projects a prominence, the so-called "tongue" ( $t$ 4), also bearing horny teeth, and having immediately above it the narrow mouth (mth.). On the dorsal surface of the head is the single median nostril (na. ap.), and immediately behind if a transparent area of skin $(p n$.$) indicates the position of the pineal$ organ. The paired eyes have no eyelids, but are covered by a transparent area of skin. The gill-slits $(b r . c l .1)$ are seven pairs of small apertures on the sides of the head, the first a little behind the eyes. On the ventral surface, marking the junction between trunk and tail, is the very small anus (Fig. 812,a.), lying in a slight depression, and having immediately behind it a small papilla pierced at its extremity by the urinogenital aperture $(z$.$) . There is no trace$ 
of paired appendages, and the only organs of locomotion are the unpaired fins. Two dorsal fins of approximately equal dimensions, separated by a noteh, and a caudal fin are present, the second dorsal being continuous with the caudal.

Lampreys prey upon Fishes, attaching themselves to the bodies of the latter by the sucker-like mouth, and rasping off their flesh with the armed tongue. They are often found holding on to stones by the buccal funnel, and under these cireumstances perform regular respiratory movements, the branchial region expanding and contracting like the thorax of a Mammal. The reason of this is that when the animal is adhering by the mouth the respiratory current cannot take its usual course - entering at the mouth and leaving by the gill-slits-but is pumped by muscular action both into and out of the branchial apertures.

The stin is soft and slimy, mottled greenish-brown in P. marinus, hluish above and silvery on the sides in the fresh-water species.

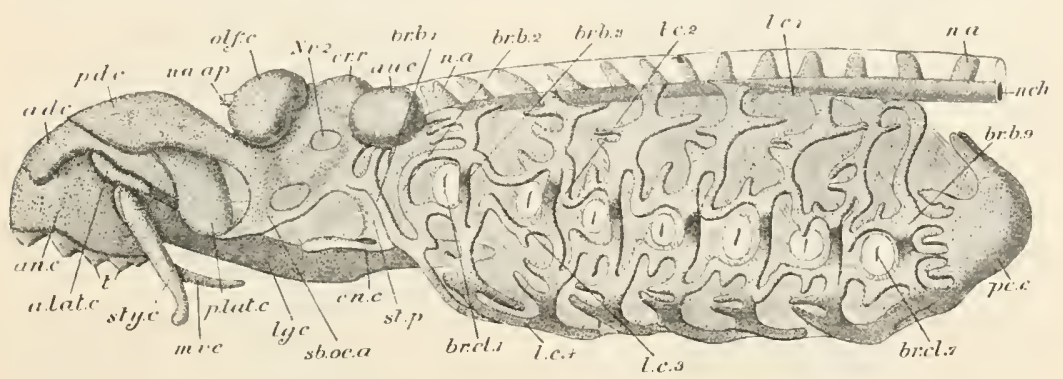

F'IG. 804.-Petromyzon marinus. Skull, with branchial basket and anterior part of rertebral column. The cartilaginous parts are dotted. a.d.c. anterior dorsal cartilage; $a$. lat. $c$. anterior lateral cartilage; an. $c$. annular cartilage; $a u$. $c$. auditory capsule; $b r . b .1-7$, vertical bars of branchial basket; $b r . c l .1-7$, external branchial clefts; $c n$. $c$. cornual cartilage; $c r . r$. cranial roof ; $l$. $c$. $1-4$, longitudinal bars of branchial basket; $l g . c$. lingual cartilage $; m$. v. $c$. median ventral cartilage; $n$. $a$. neural arch; na. ap. nasal aperture; $n c h$. notochord: $N v$. 2, foramen for optic nerve; olf. $c$. olfactory capsule ; $p c$. $c$. pericardial cartilage; $p . d . c$. posterior dorsal cartilage; $p$. lat. c. posterior lateral cartilage ; $s b . c c . a$. subocular arch; st. $p$. styloid process; sty. c. styliform cartilage; $t$. teeth. (After W. $K$. Parker.)

The epiderm contains unicellular glands, the secretion of which gives its slimy character to the skin. The segmental sense-organs take the form of a lateral line which is superficial, not enclosed in a canal, and of minute pits on the head. There is no trace of exoskeleton.

Skeleton.-The axial skeleton of the trunk is very simple. There is a persistent notochord (Fig. $804, n c h$.) with a tough sheath composed of an inner fibrous and an outer elastic layer. Attached to the sides of the notochord are little vertical rods of cartilage (n.a.), arranged segmentally, bounding the spinal canal on each side, and corresponding to rudimentary neural and interneural arches: in the caudal region these fuse into a single plate perforated by foramina for the spinal nerves and sending off processes to the base 
of the fin. For the rest of its extent the spinal canal is enclosed only by tough, pigmented connective-tissue. Slender rods of cartilage support the median fins.

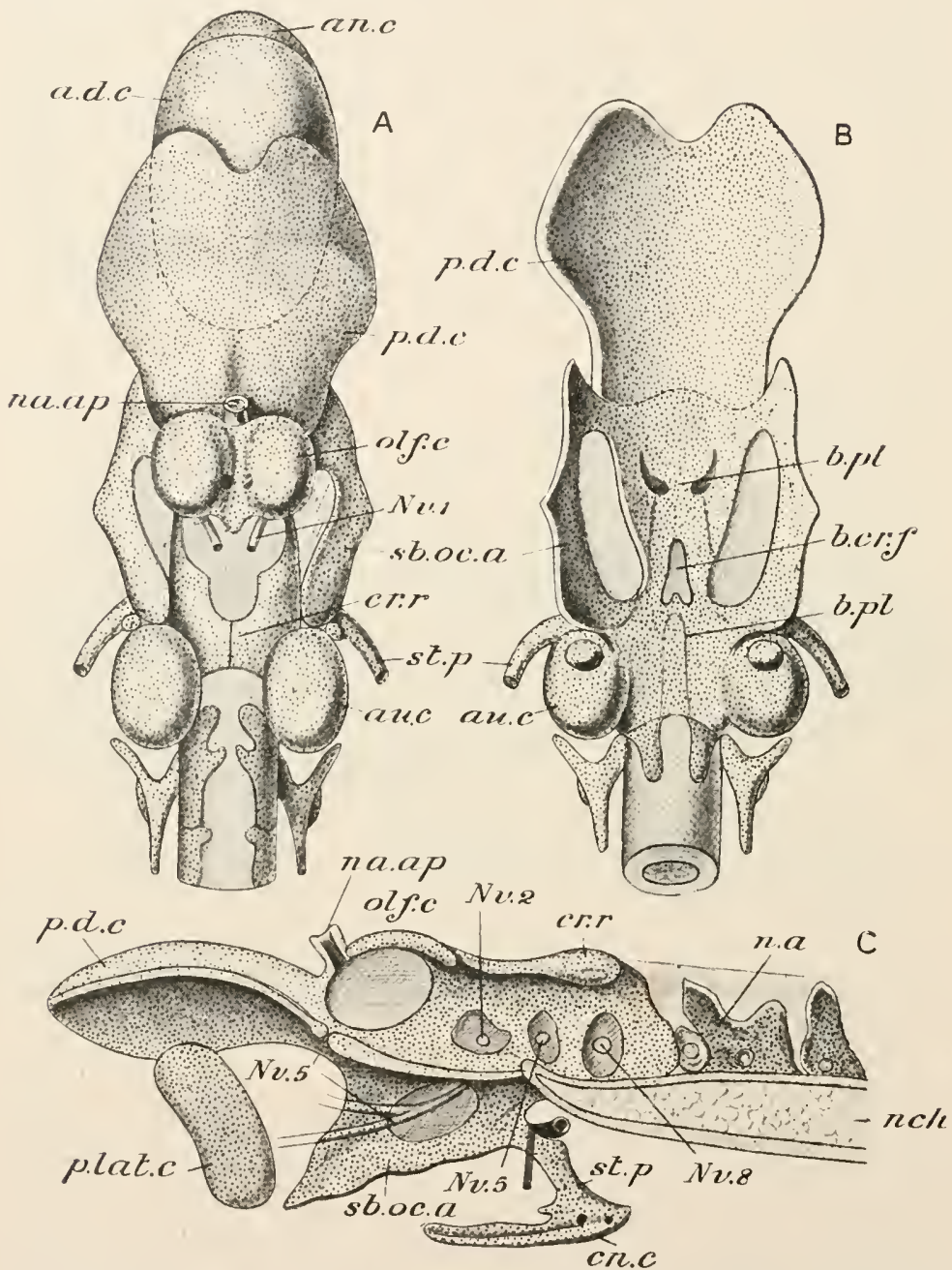

FIG. 805.-Petromyzon maxinus. Dorsal $(A)$, ventral $(B)$, and sectional $(C)$ views of skull. The cartilagino us parts are dotted. a.d.c. anterior dorsal cartilage; an. c. annular cartilage ; $a u$. c. auditory capsule; $b$. $c r . f$. basicranial fontanelle; $b . p l$. basal plate; $c n$. $c$. cornual cartilage; $c r . r$. cranial roof; $n$. $a$. neural arch; na. ap. nasal aperture; nch. notochord ; $N v .1$, olfactory nerve; $N v, 2,5$, and $\delta$, foramina for the optic, trigeminal, and auditory nerves; $N v .5^{\prime}$, fifth nerve; olf. $c$, olfactory capsule; $p . d . c$. posterior dorsal cartilage: p. lat. $c$. posterior lateral cartilage; sb. oc. a. sub-ocular arch; st. $p$. styloid process. (After W. K. Parker.)

The cranium also exhibits a very primitive type of structure. Its floor is formed by a basal plate (Fig. $805, b . p l$.), made by the 
union of the parachordals and trabeculæ, and surrounding posteriorly the fore-end of the notochord. Immediately in front of the termination of the notochord is a large aperture, the basi-cranial fontanelle $(b . c r . f$.$) , due to the non-union of the posterior ends of$ the trabeculæ; through it passes the pituitary pouch, presently to be referred to (Fig. 808), on its way from the olfactory sac to the ventral surface of the notochord. Lateral walls extend upwards from each side of the basal plate, but the roof of the cranium is formed by membrane except at one point, where a narrow transverse bar $(c r . r$.) extends across between the side-walls and furnishes a rudimentary roof. United with the posterior end of the basal plate are the auditory capsules (au.c.), and the side-walls are pierced with apertures for the cerebral nerves $(N v .2, N v .5, N v .8$.)

So far the skull is thoroughly typical, though in an extremely simple or embryonic condition; the remaining parts of it differ a good deal from the ordinary structure as described in the preceding section, and are in many cases very difficult of interpretation.

The olfactory capsule (olf. c.) is an unpaired concavo-convex plate which supports the posterior wall of the olfactory sac and is pierced by paired apertures for the olfactory nerves. It is unique in being united to the cranium by fibrous tissue only.

Extending outwards and downwards from each side of the basal plate is an inverted arch of cartilage, called the sub-ocular arch (Figs. 804 and $805, s b . o c . a$.) from the fact that it affords a support to the eye. From its posterior end a slender styloid process (st. p.) passes directly downwards and is connected at its lower end with a small cornual cartilage (cn. c.). Perhaps the sub-ocular arch answers to the palato-quadrate or primary upper jaw, the styloid and cornual cartilages to the main part of the hyoid arch. In close relation with the angle of the sub-ocular arch is an upwardly directed plate, the posterior lateral cartilage ( $p$. lat. c.).

Connected with the anterior end of the basal plate is the large bilobed posterior dorsal cartilage $(p . d . c$.$) ; it appears to be formed$ from the united anterior enids of the trabeculæ. Below and projecting in front of it is the anterior dorsal cartilage $(a . d . c$.$) , which$ is probably homologous with the upper labial cartilage of some Fishes and Amphibians (see below). Also belonging to the series of labial cartilages are the paired anterior lateral cartilages $(a . l . c$.) and the great ring-shaped annular cartilage (an.c.) which supports the edge of the buccal funnel.

The "tongue" is supported by a long unpaired lingual cartilage (Fig. 804, lg.c.), which may answer to the united Meckel's cartilages or ventral portion of the mandibular arch of other Craniata (see p. 75) ; it is tipped in front by a small median and a pair of still smaller lateral cartilages. Below it is a slender $\mathrm{T}$-shaped median ventral cartilage $(m . v . c$.), which may possibly be the median ventral element of the mandibular arch. Lastly, attached to each side of 
the annular cartilage and passing backwards and downwards are a pair of tapering, rod-like styliform cartilages (sty. c.).

The visceral skeleton also differs in a remarkable manner from the ordinary Craniate type, and is only doubtfully related to it. It consists of a branchial basket, formed, on each side, of nine irregularly curved vertical bars of cartilage (Fig. 804, br. b. 1-9), the first placed almost immediately posterior to the styloid cartilage, the second immediately in front of the first gill-cleft, the remaining seven just behind the seven gill-clefts. These bars are united together by four longitudinal rods (lc. $1-4)$, of which one lies alongside the notochord and is connected in front with the cranium, two others are placed respectively above and below the gill-clefts, while the fourth is situated close to the middle ventral line and is partly fused with its fellow of the opposite side. The posterior vertical bar is connected with a cup-like cartilage (pc. c.), which supports the posterior and lateral walls of the pericardium. The whole branchial basket lies external to the gill-pouches and branchial arteries, not, like typical visceral arches, in the walls of the pharynx.

The median fins are supported by the delicate cartilaginous rods already referred to, which are more numerous than the myomeres, and lie parallel to one another in the substance of the fin, extending downwards to the fibrous neural tube.

The structure of the cartilage is peculiar and varies in different parts; it has very little matrix.

The muscles of the trunk and tail are arranged in myomeres which take a zig-zag course. In the branchial region they are divided into dorsal and ventral bands, which pass respectively above and below the gill-slits; but in the trunk there is no division into dorsal and ventral parts. A great mass of radiating muscle is inserted into the buccal funnel, and the "tongue" has an extremely complex musculature which derives its nerve-supply from the trigeminal.

Digestive Organs.-The teeth are laminated horny cones: beneath them lie mesodermal papillæ covered with ectoderm which hear a superficial resemblance to the germs of true calcified teeth. When worn out they are succeeded by others developed at their bases. The mouth leads into a buccal cavity (Fig. 806, m.) formed from the stomodæum of the embryo, and communicating behind with two tubes placed one above the other: the dorsal of these is the gullet (œs.), the ventral the respiratory tube ( $r$. $t$., see below): guarding the entrance to the latter is a curtain-like fold, the velum (vl.). The gullet bends over the pericardium and enters the intestine (int.) by a valvular aperture. The intestine passes without convolutions to the anus; its anterior end is slightly dilated, and is the only representative of a stomach : its posterior end is widened to form the rectum (Fig. 812,r.). The whole of the intestine is formed from the mesenteron of the embryo, and the 
blastopore becomes the anus, there being no proctodxun. The

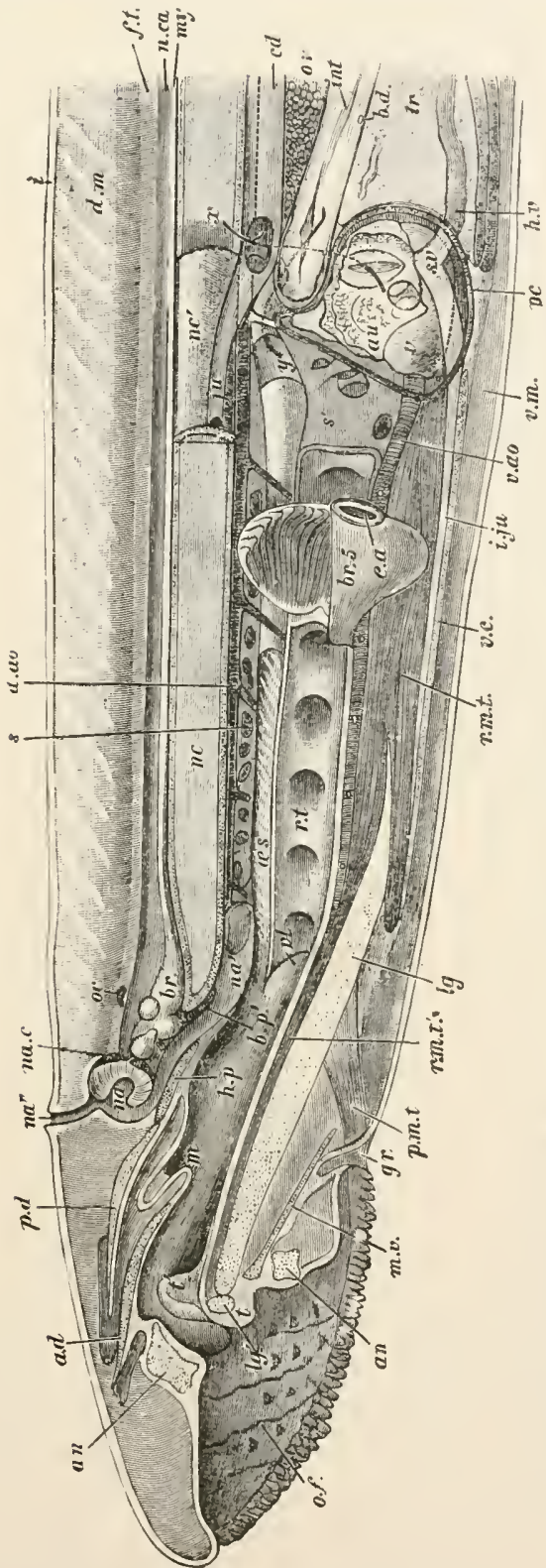

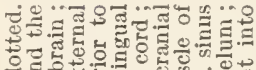

응

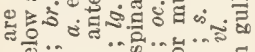

过

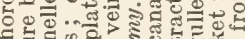

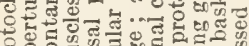

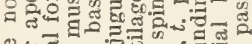
놇 फ

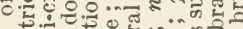

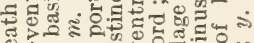
क⿺辶一 כ... \%

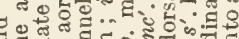

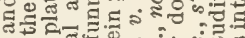

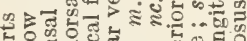

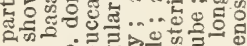

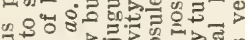

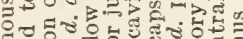
60.

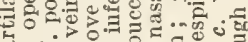
50. .

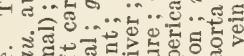
ส...

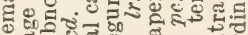

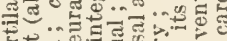
- 己ु

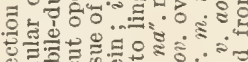

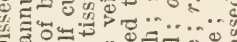
A मु

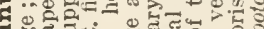
4

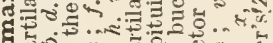
1

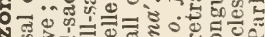
क 0 :

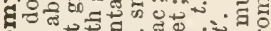

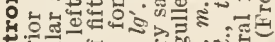
ป 4

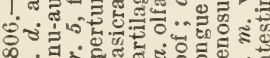
窟

lumen of the intestine is semilunar, owing to the presence of a typhlosole (Fig. 811, int.), which takes a somewhat spiral course 
and is hence known as the spiral valve. There is no continuous mesentery, but a number of narrow supporting bands.

The liver (Fig. 806, lr.) is a large bilobed organ, and is peculiar from the fact that there is neither gall-bladder nor bile-duct in the adult, except as an individual variation, although both are present in the larva. There are a few follicles on the surface of the liver, which may represent a pancreas: the spleen is absent. Paired glands imbedded in the muscles of the head, and opening into the mouth, are known as "salivary glands."

Respiratory Organs.-The Lampreys differ from all other Vertebrata in the fact that the gills do not open directly into the enteric canal in the adult, but into a respiratory tube (Fig. 806, r. t.) lying below the gullet. This is a wide tube opening in front into the buccal cavity, and ending blindly a short distance in front of the heart: in the larva it communicates behind with the intestine, and is, in fact, the pharynx, the gullet of the adult being not yet developed; but at the time of metamorphosis it loses its connection with the intestine, and the gullet is developed as a forward extension of the latter-an entirely new formation. The respiratory organs are typical gill-pouches $(b r .5)$ : they have the form of biconvex lenses, with numerous gill-lamellæ developed on the inner surfaces, and are separated from one another by wide interbranchial septa. In the larva an additional cleft has been found in front of the first of the adult series.

Circulatory System.-The auricle (au.) lies to the left of the ventricle $(v$.) and receives blood from a small sinus venosus (s.v.). There is no conus arteriosus, but the proximal end of the ventral aorta presents a slight dilatation or bulbus aortce. Both afferent and efferent branchial arteries supply each the posterior hemibranch of one gill-pouch and the anterior hemibranch of the next: they are thus related to the gills, not to the gill-pouches. In addition to the paired jugulars $(j u$.) there is a median ventral inferior jugular vein $(i . j u$.) returning the blood from the lower parts of the head. There is no renal-portal system, the two branches of the caudal vein being continued directly into the cardinals $(c d$.$) . The left precaval disappears in the adult, so that$ the jugulars and cardinals of both sides open into the right precaval. The red blood-corpuscles are circular, nucleated discs. There is a large system of lymphatic sinuses.

Nervous System.-In the brain the small size of the cerebellum (Fig. 807, crb.) is remarkable: it is a mere transverse band roofing over the anterior end of the metacœle. The optic lobes (opt. l.) are very imperfectly differentiated, and the central region of the roof of the mid-brain is formed merely of a layer of epithelium, so that when the membranes of the brain are removed, an aperture is left which is covered in the entire organ by a vascular thickening of the pia, or choroid plexus (ch. pl. 2). On the dorsal border of 
the lateral wall of the diencephalon are the two ganglia habenulce, the right $(r . g n . h b$.) much larger than the left $(l . g n . h b$.$) : they are$ connected with the pineal apparatus. Below the diencephalon is a small flattened pituitary body (Fig. 808, pty. b.). In front of the diencephalon are paired bean-like masses, each consisting of a small posterior portion, the olfactory lobe $(c r b . h$.$) , and a larger anterior$

A

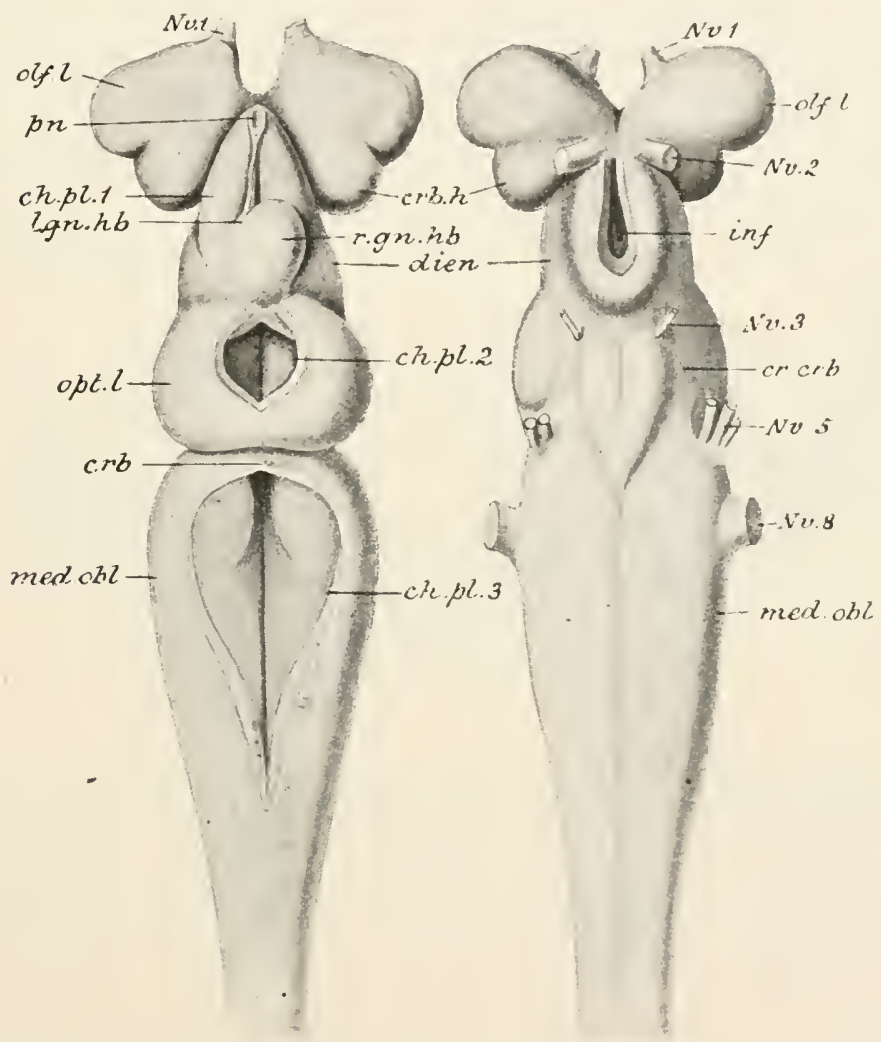

B

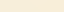


a rhinocœle going directly forwards into the olfactory bulb, and a paracœle backwards into the olfactory lobe.

The pineal apparatus consists of two vesicles placed in a vertical series : the dorsal-most of these is the vestigial pineal eye (Fig. 808, $p n$.e.): it has a pigmented retina, a flat and imperfectly formed lens, and is connected with the right ganglion habenulæ. The lower vesicle (parapineal organ, pn.), of the same nature as the upper but more imperfectly developed, is in connection with the small left ganglion habenulæ. The pineal eye is not an organ capable, like the paired eyes, of forming definite images of objects, but probably is capable of distinguishing differences in the intensity of the light. The optic nerves differ from those of most of the higher classes in the fact that the chiasma is not visible externally-the intercrossing of the fibres taking place beneath the surface.

The spinal cord (Figs. 806 and 811, my.) is flattened and band-like.

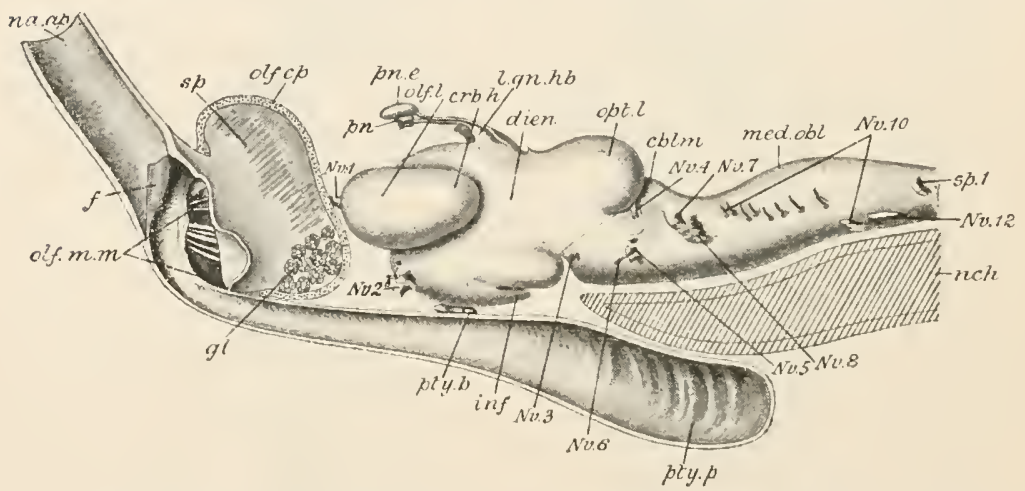

FIa. 808.--Petromyzon. Side view of brain with olfactory and pituitary sacs, in section. cblm. cerebellum; $c r b$. $h$. olfactory lobe; dien. diencephalon; $f$. fold in nasal tube; $a l$. nasal glands; inf. infundibulum; $l . g n$. $h b$. left ganglion habenulæ; med. obl. medulla

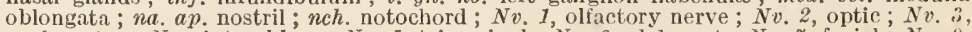
oculomotor; $N v .4$, trochlear ; $N v .5$, trigeminal ; $N v .6$, abducent ; $N v$. 7 , facial ; $N v . s$, auditory ; $N v .10$, vagus ; $N v$. 12 , hypoglossal ; olf. $\mathrm{cp}$. olfactory capsule ; olf. l. olfactory bulb; olf. m. m. olfactory mucous membrane; opt. l. optic lobe; $p n$. parapineal organ; $p n$. $e$. pineal eye ; pty. $b$. pituitary body; $p t y$. $p$. pituitary ponch; $s p$. median septum of olfactory sac; $s p$. 1, dorsal root of first spinal nerve. (Combined from figures by Ahlborn and Kaenische.)

The dorsal roots of the spinal nerves alternate with the ventral roots, and do not unite with them to form a trunk: the dorsal roots are opposite the myocommas, the ventral opposite the myomeres. A sympathetic is represented. The hypoglossal is the first spinal nerve.

Sensory Organs.-The external nostril (Fig. 806, na", Fig. 808, na. ap.) leads by a short passage into a rounded olfactory sac (Fig. 806, na, Fig. 808) placed just in front of the brain and having its posterior wall raised into ridges covered by the olfactory mucous membrane (Fig. 808, olf. m. m.). From the bottom of the sac is given off a large pituitary pouch (Fig. 806, na', Fig. 808, pty. p.) 
which extends downwards and backwards, between the brain and the skull-floor, passes through the basicranial fontanelle, and ends blindly below the anterior end of the notochord.

The relations between the olfactory sac, the pituitary pouch, and the pituitary body are very remarkable. In the embryo, before the stomodæum (Fig. 809, $A$, stdm.) communicates with the mesen-
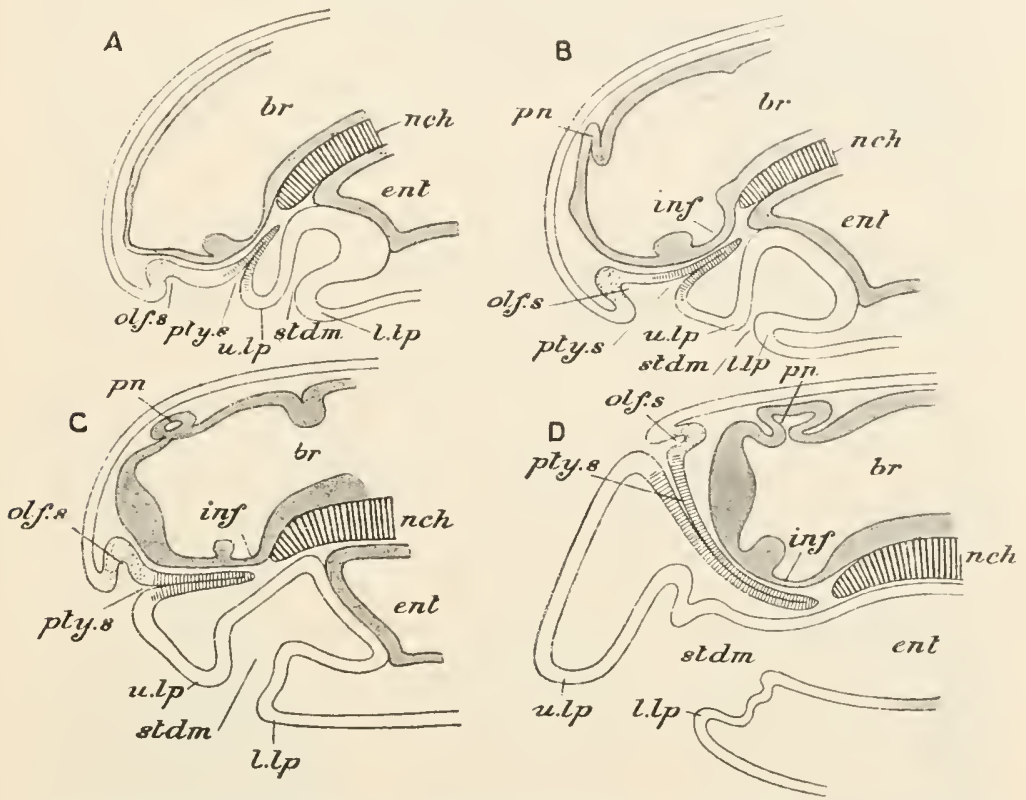

FIt. 809.-Petromyzon. Diagrams of four stages in the development of the olfactory and pituitary sacs. br. brain; ent. mesenteron; inf. infundibulum ; $l$. $l p$. lower lip; nch, notochord; olf. $s$. olfactory sac; $p n$. pineal body; $p t y . s$. pituitary sac; stdm. stomodieum u. $l p$. upper lip. (Altered from Dohrn.)

teron, two unpaired ectodermal invaginations appear in front of the mouth. The foremost of these is the rudiment of the olfactory sac (olf. s.). The other, which is situated between the olfactory sac and the mouth, is the pituitary sac (pty. s.), which in this case opens just outside the stomodæum instead of within it as in other Craniata : its inner or blind end extends to the ventral surface of the fore-brain and terminates just below the infundibulum (inf.). As development goes on, the olfactory and pituitary invaginations become sunk in a common pit $(B)$, which, by the growth of the immense upper lip (up.l.), is gradually shifted to the top of the head $(C, D)$, the process being accompanied by elongation of the pituitary sac, into which the olfactory sac opens posteriorly. Where the pituitary sac comes in contact with the infundibulum it gives off numerous small follicles which become separated off and give rise to the pituitary body (Fig. $808, p t y . b$.). Thus the entire nasal 
passage of the Lamprey, including its blind pouch, is a persistent pituitary sac into which the single olfactory organ opens. Moreover, owing to the extraordinary displacement undergone during development, the pituitary sac perforates the skull-floor from above

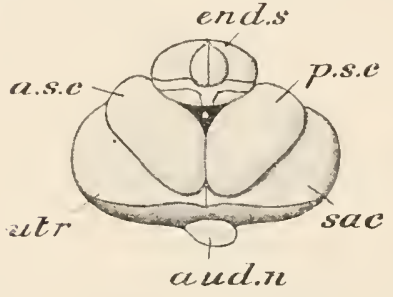

FIG. 810.-Auditory sac of Petromyzon. a. $s$. c. anterior semicircular canal; aud. $n$. auditory nerve : end. s, endolymphatic sac ; p.s.c. posterior canal ; sac. sacculus ; utr. utriculus. (After Retzius.) instead of from below, as in all other Craniata.

The auditory organ (Fig. 810) is remarkable for having only two semicircular canals, corresponding to the anterior (a.s.c.) and posterior (p.s.c.) of the typical organ.

Organs of taste are present on the wall of the pharynx between the gill-sacs, and neuromast- or lateral line-organs are present on the head and trunk.

Urinogenital Organs.-The kidneys (Figs. 811 and 812, k) are long strap-shaped bodies developed from the mesonephros of the embryo. The tubules have no nephrostomes. Each kidney is attached along one edge to the dorsal wall of the body-cavity by a sheet of peritoneum; along the other or free edge runs the ureter (ur.), which is the undivided pronephric duct. The ureters open posteriorly into a small urinogenital sinus (Fig. 812 , u.g.s.), placed just behind the rectum, and opening, by a urinogenital papilla (u.g.p.), into a pit in which the anus (a.) also lies. The side-walls of the sinus are pierced by a pair of small apertures, the genital pores $(y)$, which place its cavity in communication with the colome.

The gonad (Fig. 806, ov, Fig. 811 $t s$ ) is a large unpaired organ occupying the greater part of the abdominal cavity and suspended by a sheet of peritoneum. The sexes are separate, but ova have been found in the testis of the male The reproductive products are shed into the cœlome and make their way by the genital pores

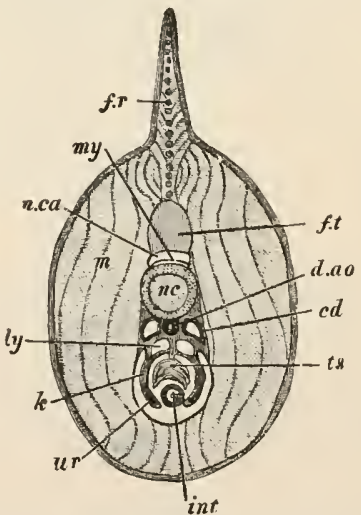

FIG. 811.-Petromyzon marinus. Transverse section of trumk. $c d$. cardinal veins; $d$. ao dorsal aorta: $f$. $r$. flin-r ays; $f$. $t$. fibrous tissue of spinal canal ; int. intestine, the line pointing to the spiral valve; $k$, kilneys; $l y$. subvertebral lymph-sinus; $m$. bodymuscles; my. spinal cord; nc. notochord; n. ca. spinal canal ; ts. testis ; ur. ureter. (From Parker's Zootomy.) into the urinogenital sinus, and so to the surrounding water, where impregnation takes place.

Development.-The oosperm is telolecithal, having a considerable accumulation of yolk in one hemisphere; in correspon- 
dence with this, segmentation is complete bnt unequal, the morula consisting of an upper hemisphere of small cells or micromeres (Fig. 813, mi. m.), free from yolk, and of a lower hemisphere of large cells or megameres ( $m g . m$.$) , containing much yolk. In the$ blastula stage $(D)$ the segmentation-cavity or blastocoele (blcl.) is situated nearer to the upper than to the lower pole. A transverse semilunar groove appears which is bounded by a prominent rim towards the future dorsal and anterior side: this is the blastopore

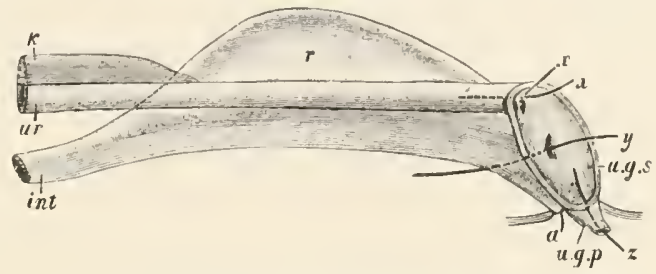

l'I(Y. 81 . - Petromyzon marinus. 'l'he urinogenital sinus with posterior end of intestine and part of leit kidney. $a$. anus; int. intestine; $k$. left kidney; $r$. rectum u.g.p. urinogenital papilla; u.g.s. urinogenital sinus; ur. left ureter; $x, x^{\prime}$, apertures of ureters into urinogenital sinus; $y$, bristle passed into right genital pore ; $z$, bristle passed from urinogenital aperture into sinus. (From Parker's Zootomy.)

$\left(b t_{p .}\right)$. The megameres become gradually enclosed by the micromeres as a result of a process which is partly invagination, partly epiboly. During this process the segmentation-cavity becomes displaced by the archenteron. The dorsal and ventral
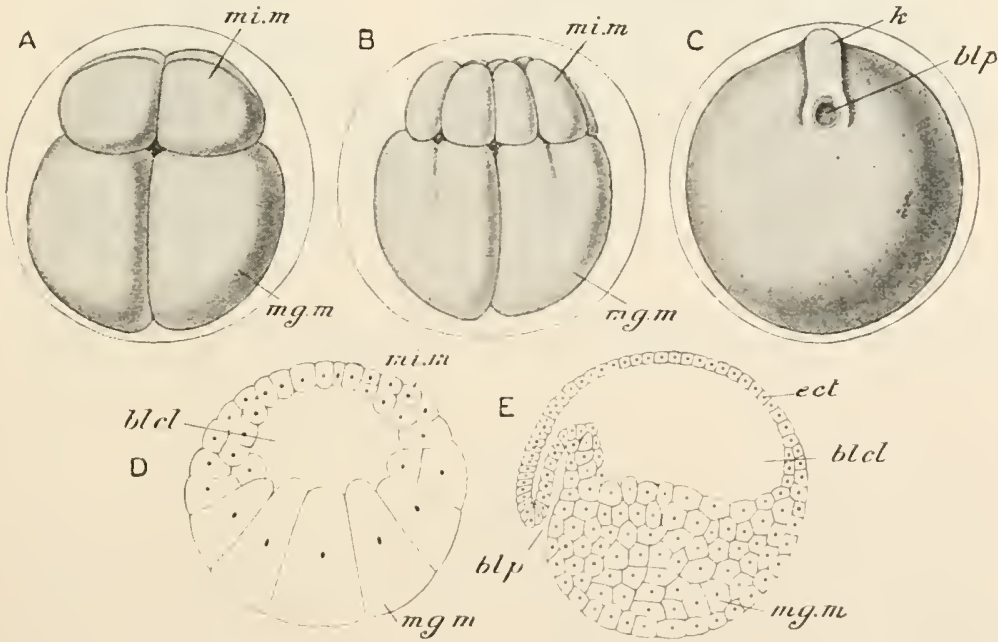

FIG. 813.-Petromyzon. $A$ and $B$, two stages in segmentation ; $C$, early embryo from the posterior aspect; $D$, section of blastula stage; $E$, section of gastrula stage. $b l p$. blastopore; blcl. blastocole or segmentation-cavity; $k$. lieel; $m g . m$. megameres; mi. m. micromeres. (After Shipley and Kuptfer.)

walls of the latter, unlike those of the archenteron of Amphioxus, differ widely from one another, the ventral wall being composed of a thick mass of yolk-cells (megameres), while the roof is comparatively thin and consists of two or three layers of rounded 
cells. The lumen is a narrow, dorso-ventrally compressed cleft. When the process of gastrulation is completed, the blastopore takes up a position at the postero-dorsal end. The development of the central nervous system differs widely from the corresponding process in Amphioxus, and is only approached among the Craniata by the Teleostomi or Bony Fishes. The dorsal surface becomes flattened along a narrow longitudinal area, and along this a groove appears, which stops short just in front of the blastopore. The area along which the groove runs soon becomes raised up above the general surface so as to form a narrow longitudinal elevation. Sections of this stage slrow that the ectoderm has developed a thickening along the course of the longitudinal groove, and this comes to grow downwards towards the archenteron as a solid longitudinal medullary keel (Fig. 813, $C, k$; Fig. 814, $A, m k$ ). This is the rudiment of the central nervous system. Subsequently the keel becomes separated off from the surface ectoderm, and lies below

A

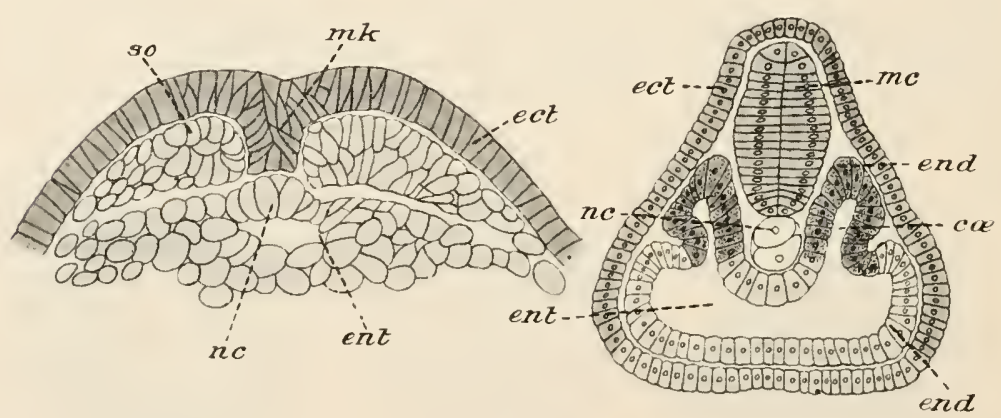

B
FIG. 814.- Petromyzon. Sections of embryos. A, transverse section of the trunk-region. $B$, transverse section of the head-region. co. colomic sacs ; ect. ectoderm; end. endoderm: cnt. enteric cavity; so. mesoderm-strand; m.c., $m k$. medullary cord and medullary kcel ; nc. notochord. (From $O$. Hertwig; $A$, after Goette, $B$, after Kupfier.)

it as a solid cord. It is only at a considerably later period that a lumen appears in this cord, and gives rise to the ventricles of the brain and the central canal of the spinal cord. During the formation of the medullary keel the rudiment of the notochord is developed from the underlying endoderm very much as in Amphioxus (p. 57). On each side of the medullary cord and notochord is a group of cells arranged as a longitudinal strand-the mesoderm plates. In the head-region (Fig. 814, B) a number of diverticula from the archenteron-colomic diverticula - are given off into these strands : in the trunk region $(A)$ these are absent. The inner portion of the mesoderm on each side becomes divided up into a series of mesodermal somites or protovertebræ, the lateral part remaining undivided and forming the lateral plate. In this restriction of somite-formation to the part of the mesoderm immediately adjacent 
to the middle line, the Lamprey differs from Amphioxus and resembles all the rest of the Craniata. The blastopore does not close up, but is converted into the anus, so that there is no proctodæum. The dorsal lip of the blastopore, very prominent from the first, becomes produced to give rise to the rudiment of the tail region. The mouth is developed later than the anus by the formation of a stomodxal invagination.

The young is hatched as a peculiar larval form called Ammocotes (Fig. 815), which differs from the adult in several respects. The median fin is continuous. There is a semicircular, hood-shaped upper lip $(u . l$.) instead of the suctorial buccal funnel of the adult, and teeth are absent. The buccal cavity is separated from the pharynx by a velum. A ciliated peripharyngeal groove encircles the pharynx in front and is continued backwards on the ventral side as a median groove opening behind into the thyroid gland, which is thus proved to be a special development of a structure corresponding to the endostyle of Amphioxus (p. 46) and the Tunicata. The eyes are rudimentary and hidden beneath the skin; the brain is of far greater proportional size than in the adult; and, as already mentioned, the

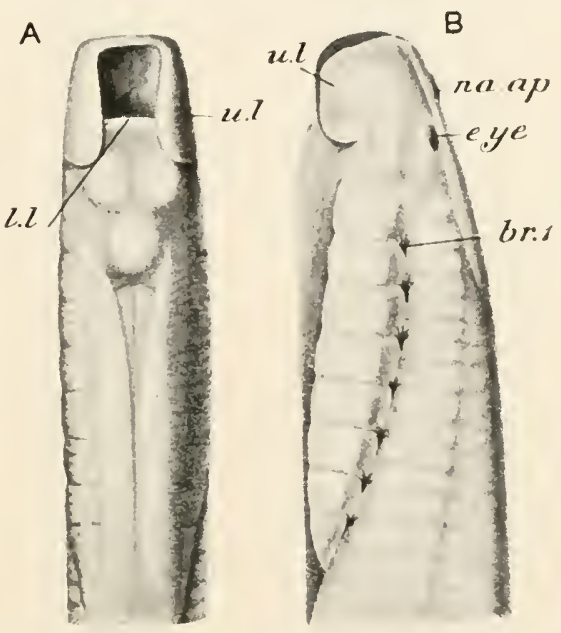

FIf. 815.-Petromyzon fluviatilis. Head of larva $A$, from beneath; $B$, from the side. $b r$. 1 , first branchial aperture; eye, eye; $l . l$. lower lip; na. ap. nostril ; $u$. l. upper lip. (After W. K. Parker.)! at gill-pouches open into the pharynx in the normal manner.

\section{Distinctive Characters and Classification.}

The Cyclostomata are Craniata in which the mouth lies at the bottom of a sucker-like buccal fumnel, or in a depression edged with tentacles, and there are no jaws. Horny teeth are borne on the interior of the buccal funnel and on the large "tongue." Paired fins are absent. There is no exoskeleton; the skin is glandular. The vertebral column consists of a persistent notochord with a fibrous neural tube, in which rudimentary neural arches may be developed. The skull is largely or wholly roofed by membrane, and there is an extensive development of labial cartilages. The segments of the post-auditory region of the head are more distinct than in the rest of the Craniata. The enteric canal is straight, and there is no 
cloaca. The respiratory organs are six to fourteen pairs of gillpouches. There is no conus arteriosus and no renal portal system. There are large olfactory lobes, which may be either hollow or solid; the cerebellum is very small. The olfactory organ is single and median, but is supplied by paired olfactory nerves ; it opens into a large persistent pituitary sac which perforates the basis cranii from above. The auditory organ has one or two semicircular canals. The kidney is a mesonephros, the ureter a pronephric duct. The gonad is unpaired, and there are no gonoducts, the genital products making their exit by genital pores.

The Class is divided into two Orders.

\section{Order 1.-Petronyzontes.}

Cyclostomata in which there is a well-developed dorsal fin and a complete branchial basket; the pituitary sac terminates posteriorly in a blind pouch; the gills open into a respiratory tube below the gullet. This order includes the Lampreys, which belong to the genera Petromyzon, Mordacia, Geotria, and Ichthyomyzon.

\section{ORDER 2.-MYYXINOIDEI.}

Cyclostomata in which the dorsal fin is absent or feebly developed; the branchial basket is reduced; the pituitary sac opens posteriorly into the mouth; the gills open into the pharynx in the normal manner.

This order includes the Hags or Slime-eels, belonging to the genera Myxine, Paramyxine, and Bdellostoma.

\section{3.-Comparison of the Myxinoids with the Laniprey.}

The organisation of the Lampreys is so uniform that all that will be necessary in the present section is to indicate the principal points in which the Hags differ from them.

Myxine is about the size of a fresh-water Lamprey-i.e. some forty-five cm. long: Bdellostoma is fully a metre in length. Both are remarkable for the immense quantities of slime they are capable of exuding from the general surface and from the segmentally arranged mucus-glands of the skin. It is said that two specimens of Myxine thrown into a bucket of water are capable of gelatinising the whole with their secretion. The slime-glands of Myxine contain peculiar "thread-cells" containing a muchcoiled thread which unwinds either before or after the discharge of the cell from the gland.

Myxine approaches most nearly to the condition of an internal parasite of any Vertebrate; it is said to attach itself to living Fishes and gradually to bore its way into the colome, devouring the flesh as it goes. 
There is no true buccal funnel: the space on which the inouth opens is edged with tentacles (Fig. 816) supported by cartilages; there is a single median tooth above the oral aperture, and two rows of smaller teeth on the tongue. The papillæ beneath the cone-like horny teeth bear a still closer superficial resemblance to rudiments (or vestiges) of true calcified teeth than is the case in the Lamprey; but it appears that no odontoblasts and no calcified substance of any kind are formed in connection with them. A velum separates the buccal cavity from the pharynx. The nostril ( $n a$. ap.) is a large unpaired aperture situated in the dorsal margin of the buccal space, and is continued into a passage, the pituitary sac, which opens into the pharynx by an aperture which appears in late embryonic life. Myxine commonly lives nearly buried in mud, and the respiratory current passes through this passage to the gills.

The only fin is a narrow caudal surrounding the end of the tail. The respira-. tory organs present striking differences in the two genera. In Bdellostoma there are in different species six to fourteen very small external branchial aper. tures (br. cl. 1) on each side, each of which communicates by a short tube with one of the gill-pouches, which is again connected with the pharnyx by

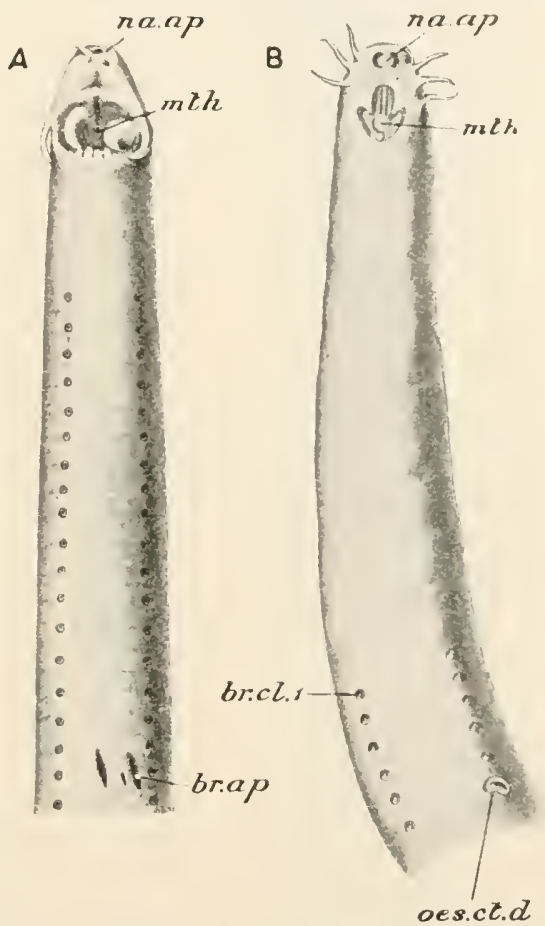

Fis. 816--Head of Myxine glutinosa (A) and of Bdellostoma forsteri $(B)$, from beneath. br. $a p$. branchial aperture: $b r . c l$. 1, first branchial cleft; mth. mouth: na. ap. nasal aperture; as. ct. $l$. ossophageo-cutaneous duct. The smaller openings in $A$ are those of the mucus-glands. (After $\mathrm{I}$. K. Parker.) another tube. Behind and close to the last gill-slit, on the left side, is an aperture leading into a tube, the osophageo-cutrneous duct (os. ct. d.), which opens directly into the pharynx. In Myxine (Fig. 817) the tubes leading outwards from the gill-pouches all unite together before opening on the exterior, so that there is only a single external branchial aperture (br. ap.) on each side; into the left common tube $(c . b r . t$.) the cesophageo-cutaneous duct (aes. ct. d.) opens. In both genera the internal branchial apertures 


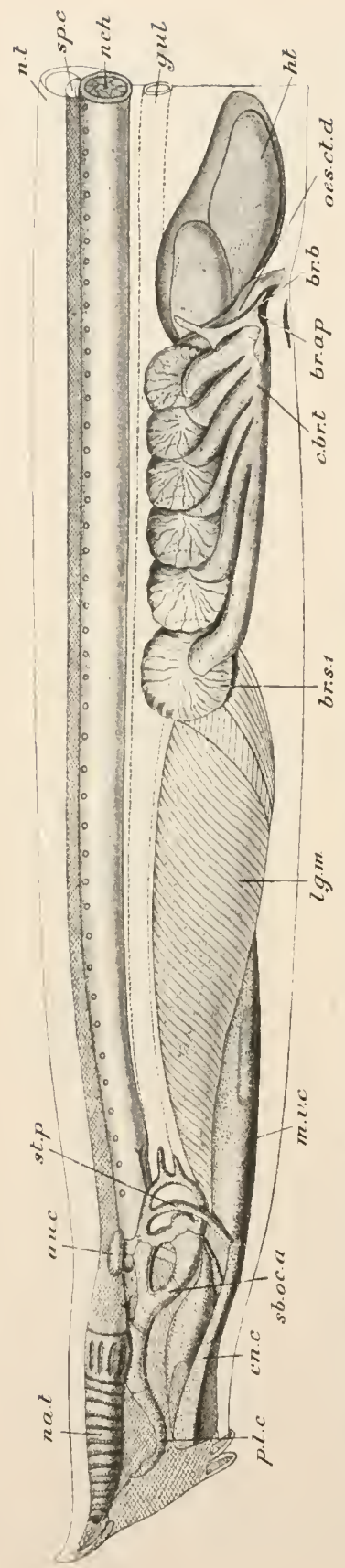

communicate directly with the pharynx; there is no respiratory tube.

The neural canal is over-arched merely by fibrous tissue (Fig. 817 , n.t.) ; there is no trace of neural arches in the trunk, but in the posterior part of the caudal region both neural canal and notochord are enclosed in a continuous cartilaginous plate. Similarly the roof of the skull is entirely membranous. The nasal passage $(n a . t$.$) is strengthened by rings$ of cartilage, and the buccal tentacles are supported by rods of the same tissue. Behind the styloid cartilage or hyoid bar (st.p.) is a rod connected below with the subocular arch; it probably represents the first branchial bar. The "tongue" is supported by an immense cartilage (m.v.c.), in part corresponding to the lingual cartilage of the Lamprey. The branchial basket is rudimentary, being represented only by certain small irregular cartilages, such as one in the walls of the œsophageo-cutaneous duct, and, in Myxine (Fig. 817, br.b.), one on the right side supporting the common external gill-tube.

The myotomes of one side alternate with those of the other.

The intestine is very wide. The liver consists of two separate portions, the ducts of which open separately into the gallbladder. A pancreas-like gland is present in both Myxine and Bdellostoma. The brain differs considerably from that of the Lamprey, especially in the larger olfactory lobes and the reduced ventricles, and smaller mid-brain. The dorsal and ventral roots of 
the spinal nerves unite instead of remaining separate. The eyes are vestigial and sunk beneath the skin, and the auditory organ (Fig. 818) has only a single semicircular canal, which, having an ampulla at each end, probably represents both anterior and posterior canals.

Bdellostoma has a persistent pronephros in the form of a paired irregularly ovoidal body situlated just above the heart and consisting of a large number of tubules richly branched peripherally : the nephrostomes open into

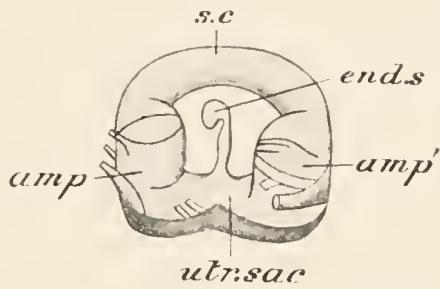

FIG. 818.-Auditory organ of Myxine. $a m p ., a m p^{\prime}$. ampulla; end. s. endolymphatic sac; s. e. semicircular canal; utr. sac. utriculo-sacculus. (After Retzius.)

the pericardinm. The tubules do not communicate with the

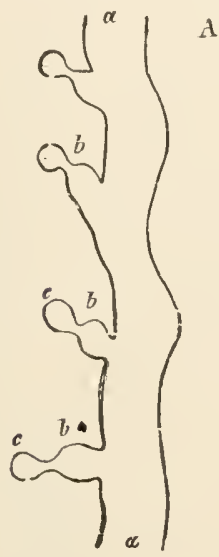

B

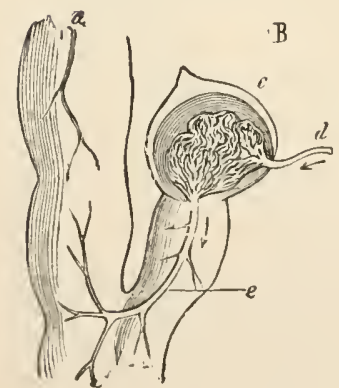

WIG. $819 .-A$, portion of kidney of Bdellostoma. $B$, segment of same, highly magnified. $a$, ureter; $b$, urinary tubule ; $c$, Malpighian capsule ; $d$, afferent artery; e, efferent artery. (From Gegenbaur's Compara tive Anatomy.) pronephric duct in Myxine, but end blindly: in Bdellostoma they open into an incomplete longitudinal duct, which does not communicate with the permanent kidneyduct. The functional kidney is the mesonephros, and is specially interesting from the fact that in Myxinoids it retains in the adult its primitive segmental arrangement. The ureter (pronephric duct, Fig. 819, a) sends off in each segment a coiled tubule (b) with a single Malpighian capsule (c), into which a branch from the aorta $(d)$ enters and forms a glomerulus.

Myxine is hermaphrodite, the anterior part of the gonad being ovary, the posterior testis: in some the ovary is mature and the testis rudimentary, in others the opposite condition holds good, so that, while hermaphrodite, each individual is either predominantly female or predominantly male. The eggs of both genera are of great proportional size, and those of Myxine are enclosed when laid in a horny shell bearing numerous hooked processes at each pole; by means of these the eggs are entangled together, and probably also attached to seaweed.

In Bdellostoma stouti, the only Myxinoid of which the development is known, the eggs are elongated and cylindrical, and contain a large quantity of food-yolk. The segmentation is meroblastic, being 
confined to a germinal disc situated at one end of the elongated egg. The blastoderm thus formed extends gradually over the surface of the yolk, which it only completely encloses at a late stage, when the gill-clefts are all formed. Bdellostoma differs from Petromyzon and resembles the majority of the Craniata in the mode of development of the central nervous system, which is formed, not from a solid ectodermal keel, but from an open medullary groove the lips of which bend inwards and unite to form a medullary canal.

\section{4.-General Remarks.}

The Lampreys and Hags are undoubtedly the lowest of craniate Vertebrata, but are in many respects so highly specialised that it is a matter of great difficulty to determine their affinities with the remaining classes. The structure of the vertebral column and of the cranium are undoubtedly primitive in the extreme; but in the development of what may be called the accessory portions of the skull, such as labial cartilages, they show a singularly high degree of specialisation. The branchial basket is quite sui generis, the theory that its vertical bars are true branchial arches, displaced outwards during development, being quite unproved. The absence of functional jaws is very remarkable, seeing that in the remaining Craniata these structures always bound the mouth at a period when the skull is in the stage of development in which it remains permanently in Cyclostomes : it is quite possible that their functionless condition may be due to degeneration accompanying the evolution of a suctorial mouth. The brain, in spite of its small size, is in some respects of a more advanced type than that of some of the true Fishes. The circumstance that the pituitary pouch perforates the skull-floor from above and becomes early associated with the olfactory sac is unique among the Vertebrata. The kidney of Bdellostoma is of the most primitive type, and the presence of a large pronephros is a 
significant archaic character. 'Tlie total absence of limbs may be a result of degeneration.

The geographical distribution of the class is interesting from the fact that each order contains some genera which are mainly northern, others which are exclusively southern. Petromyzon is found on the coasts and in the rivers of Europe, North America, Japan, and West Africa; it is therefore mainly Holarctic. Ichtliyoinyzon is found on the western coasts of North America, Mordacia in Tasmania and Chili, Geotria in the rivers of Chili, Australia, and New Zealand. Myxine occurs in the North Atlantic and on the Pacific Coast of South Amierica; Paramyxine in the Pacific; Bdellostoma on the coasts of South Africa, New Zealand, and Chili.

No undoubted fossil remains of Cyclostomes are known, but there is some reason to believe that a little fossil, Palcospondylus gunni (Fig. 820), discovered in the Devonian rocks of Scotland, may be referable to this class. It is about an inch long, and shows two regions, the cranium and the vertebral column; there is no trace of exoskeleton or teeth. The vertebral column is composed of calcified centra with neural arches; hæmal arches are present in the caudal region; the structure of this part of the skeleton is thus of a distinctly higher type than in recent Cyclostomes, and this perhaps lends support to the view that the latter are degenerate. There is a caudal fin supported by slender, sometimes forked, rays. The cranium consists of an anterior, probably trabecular, region $(t . p$.$) , and of a posterior region (p.a.), wlich seems to answer to$ the parachordals and auditory capsules. Just in advance of the anterior region is a ring-shaped opening surrounded by cirri $(c$.$) :$ this may be either the nasal aperture or the mouth. There are vestiges of upper and lower jaws, and about four branchial arches. The posterior region of the skull gives off paired plates $(x)$ which may perhaps represent pectoral fins.

\section{CLASS II.-PISCES.}

The Pisces, including the cartilaginous Fishes, the bony Fishes, and the Dipnoi, are Craniata which have the organs both of respiration and of locomotion adapted for an aquatic mode of life. The chief, and in the majority the only, organs of respiration are the gills, which are in the form of series of vascular processes attached to the branchial arches and persisting throughout life. The organs of locomotion are the paired pectoral and pelvic fins, and the unpaired dorsal, ventral, and caudal; these are all supported by fin-rays of dermal origin. A dermal exoskeleton is usually present. In the endoskeleton the notochord is usually more or less completely replaced by vertebræ; there is a well-developed skull. and a system of well-formed visceral arches, of which the first forms upper and lower jaws, the latter movably articulating with the skull, and both nearly always bearing teeth. There is frequently 
an air-bladder, which in certain exceptional cases acquires the function of a lung or chamber for breathing air. The hypophysis is not in any way connected with the nasal chambers and lies within the cranial cavity. There is a pair of nasal chambers which only exceptionally communicate internally with the mouth cavity. The auditory labyrinth contains the three typical semicircular canals. The kidney is a persistent mesonephros.

The first two sub-classes are nearly related to one another and are frequently regarded as sections of a single sub-class-the Chondrichthyes or Cartilaginous Fishes.

\section{Sub-Class I.-Elasmobranchii.}

The sub-class Elasmobranchii comprises the Sharks, Dog-fishes, and Rays. The skeleton of these fishes, like that of the Cyclostomes, is composed essentially of cartilage, and, though there may be calcification of the substance of the cartilage, true bony tissue, such as is found in all higher groups, is not present. The dermal fin-rays, supported on the cartilaginous skeleton of the fin, are of horn-like constitution. There is never (in recent forms) an operculum or gill-cover. A cloaca is present, the external opening of which serves as a common outlet for the rectum and the renal and reproductive ducts. Among some of the fossil representatives of this group are to be found the most primitive of all known Fishes.

\section{1.-Example of the Sub-Class: The Dog-Fish (Scyllium canicula or Hemiscyllium modestum).}

General External Features.-The general shape of the body (Fig. 821) may be roughly described as fusiform; at the anterior, or head-end it is broader and depressed; posteriorly it tapers gradually and is compressed from side to side. The head terminates anteriorly in a short, blunt snout. The tail is narrow and bent upwards towards the extremity. The colour is grey with brown markings, or dark-brown above, lighter underneath. The entire surface is covered closely with very minute hard placoid scales or dermal teeth, rather larger on the upper surface than on the lower. These are pointed, with the points directed somewhat backwards, so that the surface appears rougher when the hand is passed over it forwards than when it is passed in the opposite direction. When examined closely each scale is found to be a minute spine situated on a broader base. The spine consists of dentine covered with a layer of enamel ; the base is composed of bone-like substance, and the whole scale has thus the same essential structure as a tooth. Along each side of the head and body runs a faint depressed longitudinal line or slight narrow groove-the lateral line, marking the position of the lateral line canal, which contains integumentary sense-organs. 
As in Fishes in general, two sets of fins are to be recognised-the unpaired or median fins, and the paired or lateral. These are all flap-like outgrowths, running vertically and longitudinally in the case of the median fins, nearly horizontally in the case of the lateral : they are flexible, but stiffish, particularly towards the base, owing to the presence of a supporting framework of cartilage. Of the median fins two-the dorsal-are situated, as the name indicates, on the dorsal surface: they are of triangular shape; the anterior, which is the larger, is situated at about the middle of the length of the body, the other a little further back. The caudal fin fringes the tail : it consists of a narrower dorsal portion and a broader ventral, continuous with one another round the extremity of the tail, the latter divided by a notch into a larger, anterior, and a smaller, posterior lobe. The tail is heterocercal, i.e., the posterior extremity of the spinal column is bent upwards and lies in the dorsal portion of the caudal fin. The ventral or so-called anal fin is situated on the ventral surface, in Scyllium opposite the interval between the anterior and posterior dorsals, in Hemiscyllium behind the latter; it resembles the latter in size and shape.

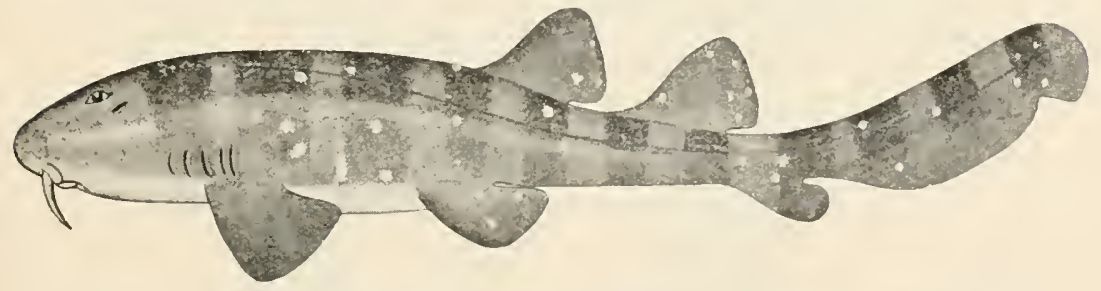

FIG. 821.-Dog-Fish (Hemiscyllium modestum). Lateral view. (After Waite.)

Of the lateral fins there are two pairs, the pectoral and the pelvic. The pectoral are situated at the sides of the body, just behind the head. The pelvic, which are the smaller, are placed on the ventral surface, close together, about the middle of the body. In the males the bases of the pelvic fins are united together in the middle line, and each has connected with it a clasper or copulatory organ. The latter is a stiff rod, on the inner and dorsal aspect of which is a groove leading forwards into a pouch-like depression in the base of the fin.

The mouth $-\mathrm{a}$ transverse, somewhat crescentic opening-is situated on the ventral surface of the head, near its anterior end. In front and behind it is bounded by the upper and lower jaws, each bearing several rows of teeth with sharp points directed backwards. The nostrils are situated one in front of each angle of the mouth, with which each is connected by a wide groove-the nasobuccal groove. In Hemiscyllium the outer edge of the groove is prolonged into a narrow subcylindrical appendage-the barbel. A small rounded aperture, the spiracle,-placed just behind the eye- 
leads into the large pharynx. Five pairs of slits running vertically on each side of the ncck-the branchial slits-also lead internally into the pharynx. A large median opening on the ventral surface at the root of the tail, between the pelvic fins, is the opening leading into the cloaca, or chamber forming the common outlct for the intestine and the renal and reproductive organs. A pair of small depressions, the abdominal pores, situated behind the cloacal opening, lead into narrow passages opening into the abdominal cavity.

The skeleton is composed entirely of cartilage, with, in ccrtain places, depositions of calcareous salts. As in Vertebrates in general, we distinguish two sets of elements in the skeleton-the axial set and the appendicular, the former comprising the skull and spinal column, the latter the limbs and their arches.
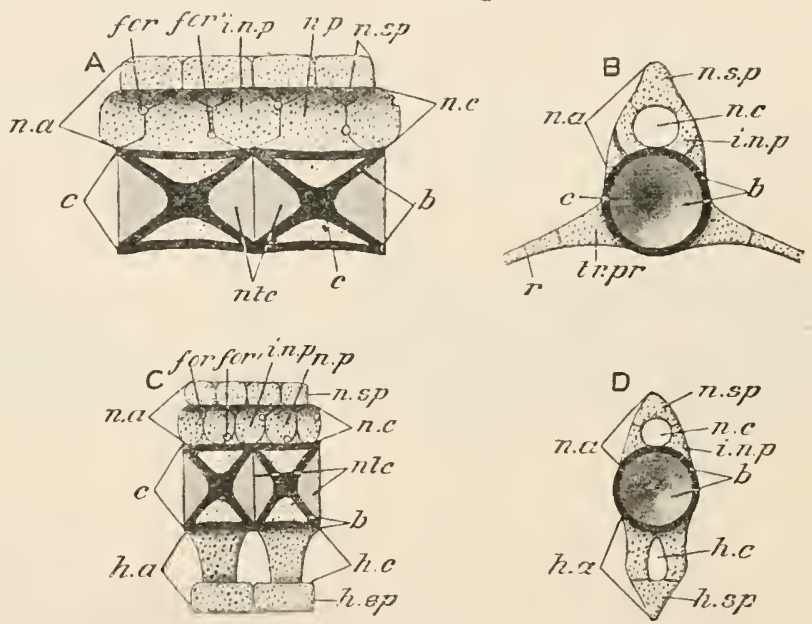

1'IG. 822.-Portions of the vertebral column of Scyllium canicula. $A$ and $B$, from the trunk ; $C$ and $D$, from the middle of the tail ; $A$ and $C$, two vertebre in longitudinal section; $B$ and $D$, single vertebrie viewed from one end. $b$, ealcified portion of centrum; $c$. cent rmm; for. foramen for dorsal, and for'. for ventral loot of spinal nerve; $h$. a. hiemal arch (basiventral); h.c. hemal canal; $h . s p$. lizenal spine; i.n.p. interealary piece (interdorsal, or interneural plate); n.a. neural arch; n.c. neural eanal ; n.p. neural plate (basi-(lorsal); $n . s p$. neural spine ; $n$ te. intervertehral substance (remains of notuchord) ; $r$. wroximal portion of rib; tr.pr. transverse process (basal stump). (From l'arker's Practical Zoology.)

The spinal colum is distinguishable into two regions-the region of the trunk and the region of the tail. In the trunk-region each vertebra (Fig. 822, $A$ and $B$ ) consists of a centrum (c.), neural arch (n.a.), and transverse processes (tr.pr). In the caudal region therc are no transverse processes, but inferior or hamal arches $(C, D, h . a$.$) take their place. The centra of all the vertebræ are$ deeply biconcave or amphicoelous, having deep conical concavities on their anterior and posterior surfaces. Through the series of centra runs the notochord (ntc.), greatly constricted in the centrum itself, and dilated in the large spaces formed by the apposition of the amphicœlous centra of adjoining vertebræ, where it forms a 
pulpy mass. The concave anterior and posterior surfaces of the centra are covered by a dense calcified layer, and in Hemiscyllium eight radiating lamellæ of calcified tissue run longitudinally through the substance of the centrum itself. The centra, unlike those of the higher forms, are developed as chondrifications of the sheath of the notochord into which cells of the skeletogenous layer have migrated (p. 71). On the dorsal side of the row of centra the spinal column is represented by the series of neural arches which support the walls of the spinal canal. Owing to the presence of a series of intercalary cartilages the neural arches appear to be twice as numerous as the centra. Each neural arch consists on each side of a process, the neural process, given off from the centrum, and

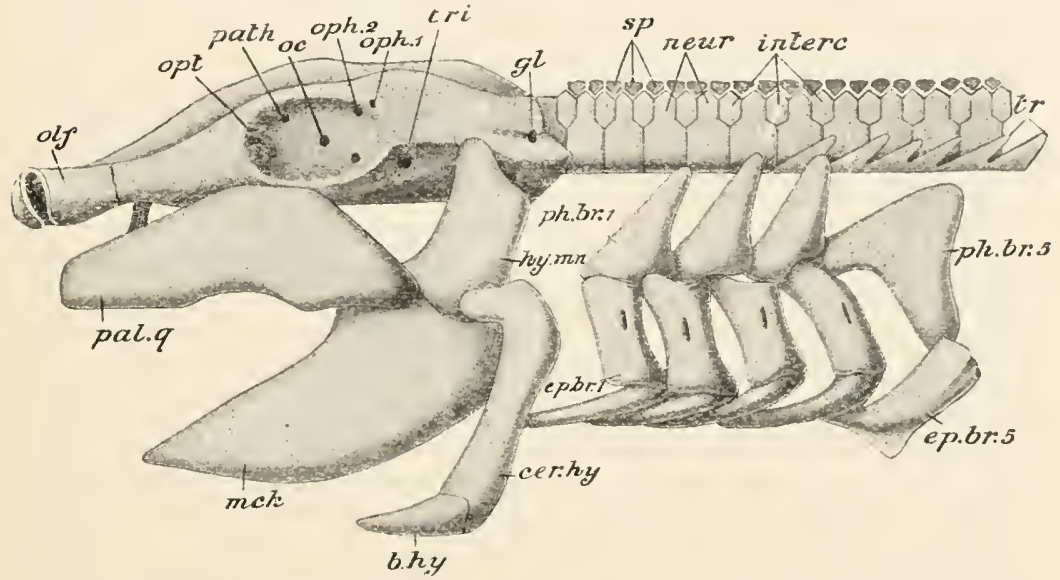

FIG. 823.-Femiscyllium, lateral view of skull with visceral arches and anterior part of spinal column; the branchial rays are not represented. The skull and hyoid arch are somewhat drawn down wards, so that the hyoid and first branchial arch are not exactly in their natural relations; cer. hy. ceratohyal; ep. br. 1, ep. br. 5 , first and fifth epibranchials ; gl. aperture for glossopharyngeal nerve; $b$. hy. basilhyal; $h y . m n$. hyomandibular ; interc. intercalary (interdorsal) plates; nirk. Meckcl's cartilage ; neur. neural processes; olf. olfactory capsule: $o c$. foramen for oculomotor; oph. 1, foramen for ophthalmic division of facial nerve; $o p h$. 2, foramen for ophthalmic division of trigeminal; opt. optic foramen ; pal. $q$. palatoquadrate; path. foramen for 4 th nerve; $p h$. br. 1 and $p h . b r .5$, first and fifth pharyngobranchial; $s p$. neural spines; tr. transverse processes and ribs; tri. foramell for trigeminal nerve.

of a small cartilage, the neural plate (basi-dorsal), which becomes completely fused with the neural process in the adult. Between successive neural plates, the width of each of which is only about half the length of the contrum, are intcrposed a series of plates of very similar shape, the interdorsal or interneural plates. Small median cartilages, the neural spines, fit in betwecn both neural and interneural plates of opposite sides and form keystones completing the arches.

The transverse processes are very short : connected with each of them is a rudimentary cartilaginous rib $(r$.) about half an inch in length.

The cranium (Fig. 823) is a cartilaginous case, the wall of which 
is continuous throughout, and not composed, like the skulls of higher Vertebrates, of a number of distinet bony elements fitting in together. At the anterior end is a rostrum, consisting in Scyllium of three cartilaginous rods converging as they extend forwards and the lateral ones meeting anteriorly. At the sides of the base of this are the olfactory capsules (olf.) - thin rounded cartilaginous sacs opening widely below, the cavities of the two capsules being separated from one another by a thin septum. The part of the roof of the cranial cavity behind and between the olfactory capsules is formed not of cartilage, but of a tough fibrous membrane, and the space thus filled in is termed the anterior fontunelle: in contact with the lower surface of the membrane is the pineal body, to be afterwards mentioned in the account of the brain. Each side-wall of this part of the skull presents a deep concavity-the orbit-over which is a ridge-like prominence, the supra-orbital crest, terminating anteriorly and posteriorly in obscure processes termed respectively the pre-orbital and post-orbital processes. Below the orbit is a longitudinal infra-orbital ridge.

Behind the orbit is the auditory region of the skull-a mass of cartilage in which the parts of the membranous labyrinth of the internal ear are embedded. On the upper surface of this posterior portion of the skull are two small apertures situated in a mesial depression. These are the openings of the aqueduct $\hat{u s}$ vestibuli (endolymphatic ducts), leading into the vestibule of the membranous labyrinth. Behind this again is the occipital region, forming the posterior boundary of the cranial cavity, and having in the middle a large rounded aperture - the foramen magnum - through which the spinal cord, contained in the neural canal and protected by the neural arches of the vertebræ, becomes continuous with the brain, lodged in the cranial cavity. Below this, on either side is an articular surface - the occipital condyle - for articulation with the spinal column, and between the two condyles is a concavity, like that of the vertebral centra, containing notochordal tissue.

A number of smaller apertures, or foramina, chiefly for the passage of nerves, perforate the wall of the skull. Behind and to the outer side of the anterior fontanelle are apertures $(o p h . \Omega, 1)$ through which the ophthalmic branches of the fifth and seventh "nerves leave the skull. Piercing the inner wall of the orbit are foramina through which the optic or second pair of cerebral nerves (opt.), the oculomotor (oc.), or third, the pathetic, or fourth (path.), the trigeminal, or fifth (tri.), the abducent, or sixth, and the facial, or seventh, gain an exit from the interior of the cramial cavity. Just behind the auditory region is the foramen for the glossopharyngeal $(g l$.$) , and in the posterior wall of the skull, near the$ foramen magnum, is the foramen for the vagus.

In close connection with the cranium are a number of cartilages composing the visceral arches (Figs. 823 and 824). These are in- 
complete hoops of cartilage, mostly segmented, which lie in the sides and floor of the mouth-cavity or pharynx. The first of these forms the upper and lower jaws. The upper jaw, or palatoquadrate (pal.q.), consists of two stout rods of cartilage firmly bound together in the middle line and bearing the upper series of teeth. The lower jaw, or Meckel's cartilage (mck.), likewise consists of two stout tooth-bearing cartilaginous rods firmly united together in the middle line, the union being termed the symphysis. At their outer ends the upper and lower jaws articulate with one another by a movable joint. In front the upper jaw is connected by a ligament with the base of the skull.

Immediately behind the lower jaw is the hyoid arch. This consists of two cartilages on each side, and a mesial one below. The uppermost cartilage is the hyomandibular (hy. mn): this articulates by its proximal end with a distinct articular facet on the auditory region of the skull: distally it is connected by ligamentous fibres with the outer ends of the palatoquadrate and Meckel's cartilage. The lower lateral cartilage is the ceratohyal (cer. hy.). Both the hyomandibular and ceratohyal bear a number of slender cartilaginous rods-the branchial rays of the hyoid arch. The mesial element, or basihyal (b.hy.), lies in the floor of the pharynx. Behind the hyoid arch follow the branchial arches, which are five in number. Each branchial arch, with exceptions to be presently noted, consists of four cartilages. The uppermost of these-pharyngobranchial (ph.br.1-ph.br.5)-lie in the dorsal wall of the pharynx, not far from the spinal column; the pharyngobranchials of the last two arches are fused together. The next in order-the epibranchials (ep. br.) -with the exception of those of the last arch, bear a number of slender cartilaginous rods-the branchial rays-which support the walls of the gill-sacs ; and the next-the ceratobranchials (cer. br.) - are, with the same exception, similarly provided. The hypobranchials (hyp. br.), which succeed these, are absent in the case of the first and fifth arches. In the middle line on the floor of the pharyngeal cavity is a mesial cartilage-the basibranchial (Fig. 824, b. br.) -which is connected with the ventral ends of the third, fourth, and fifth arches. A series of slender curved rods-the extrabranchials-lie superficial to the branchial arches, along the borders of the corresponding external branchial clefts.

Two pairs of delicate labial cartilages are present at the sides of the mouth, and a couple at the margins of the openings of the olfactory capsules.

The skeleton of all the fins-paired and unpaired-presents a considerable degree of uniformity. The main part of the expanse of the fin is supported by a series of flattened segmented rods, the pterygiophores or cartilaginous fin-rays, which lie in close apposition : in the case of the dorsal fins these may be partly calcified. At 
the outer ends of these are one or more rows of polygonal plates of cartilage. On each side of the rays and polygonal cartilages are a number of slender "horny" rays or ceratotrichia of dermal origin. ${ }^{1}$ In the smaller median fins there may be an elongated rod of cartilage constituting the skeleton, or cartilage may be entirely absent. In the pectoral fin (Fig. 825) the fin-rays are supported on three basal cartilages articulating with the pectoral arch. The latter (pect.) is a strong hoop of cartilage incomplete dorsally, situated immediately behind the last of the branchial arches.

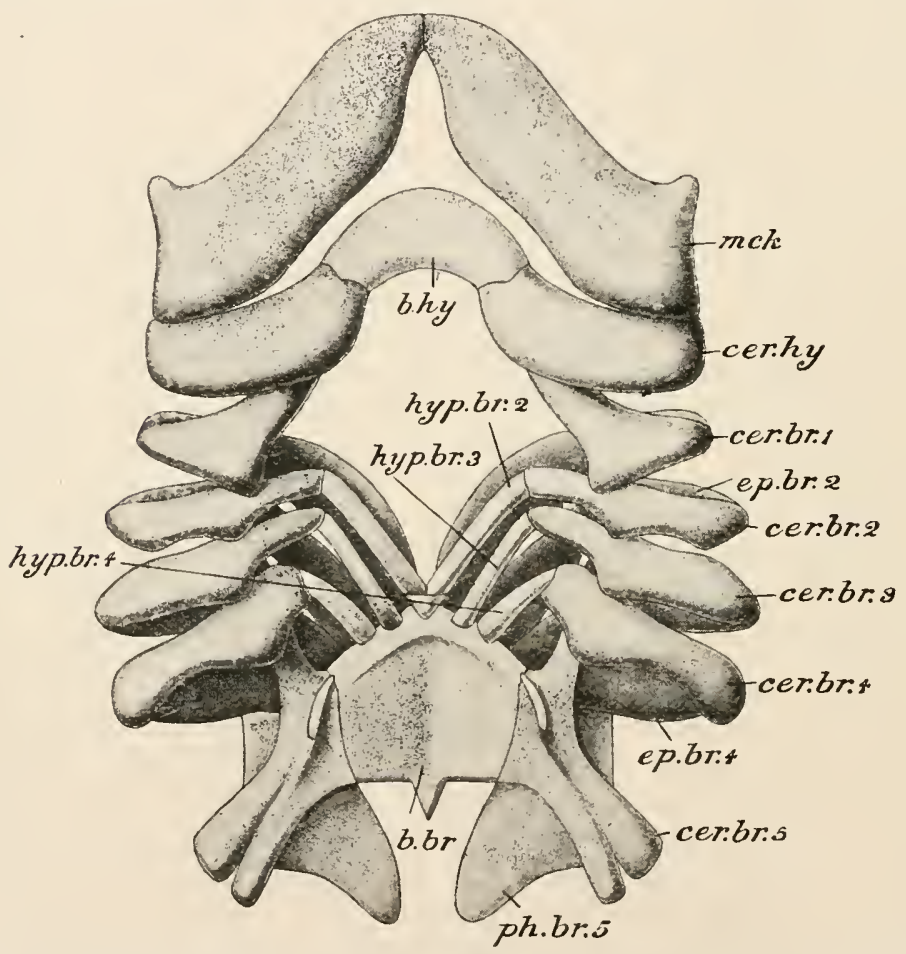

FIG. 82t- Femiscyllium, ventral view of the visceral arches. Letters as in precerling figure. In adclition-b. br. basibranchial plate; eer. br. ceratobranchials; hyp. br. hypobranchials.

It consists of a dorsal, or scapular, and a ventral, or coracoid portion, the coracoid portions of opposite sides being completely continuous across the middle line, while the scapular are separated by a wide gap in which the spinal column lies. Between the two portions are the three articular surfaces for the three basal cartilages. The

1 Though, on account of their appearance and hom-like consistency, these structures are commonly referred to as horny, they do not consist of true hom (which is always epidermal in origin), but of a substance called elastin, characteristic of elastic connective-tissue fibres. 
coracoid portions are produced forwards in the middle line into a flattened process supporting the floor of the pericardial cavity in which the heart is lodged. The three basal cartilages of the fin are named, respectively, the anterior, propterygium (pro.), the middle, mesopterygium (meso.), and the posterior, me. tapterygium (meta.). Of these the first is the smallest and the last the largest : the first bears only one large ray; the other two bear twelve or more rays, differently arranged in the two genera.

The pelvic fin

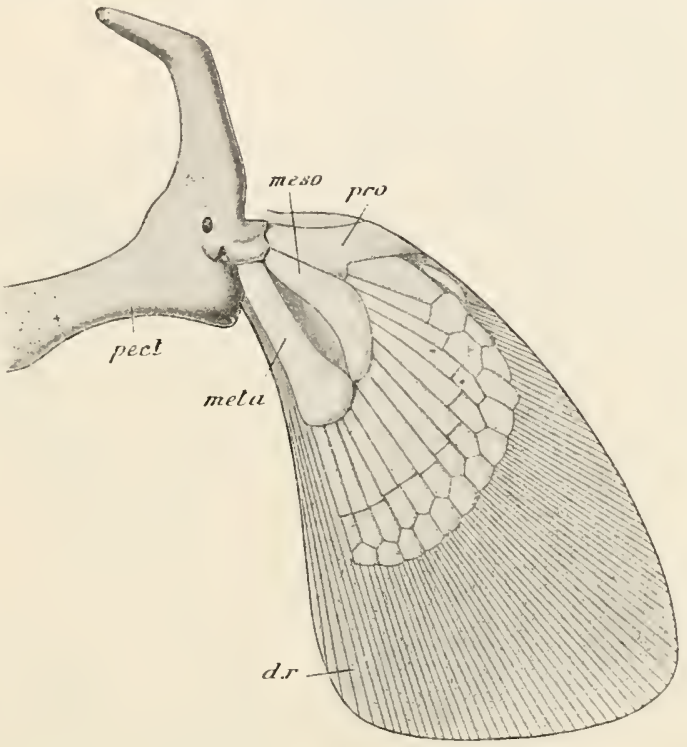
(Fig. 826) has only a single basal cartilage (meta.) articu-

FIG. 825.- Femiscyllium, pectoral arch and fin. $d . r$. dermal liorny rays; meso. mesopterygium; meta. netapterygium; pect. pectoral arch: pro. propterygium.

lating with the pelvic arch, with which also one or two of the

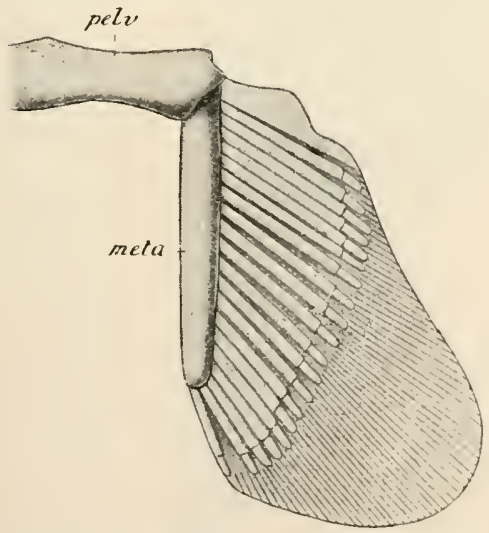

FIf. 8ะ6,- Hemiscyllium, pelvic arch ant pelvic fin. meta. metapterygium; pele. pelvic arch. fin-rays articulate directly. The pelvic arch (pelv.) is a nearly straight bar of cartilage which runs transversely across the ventral surface of the body, just in front of the cloacal opening.

Enteric Canal (Fig. 827).The mouth leads into a very wide cavity, the pharynx, into which open at the sides the internal apertures of the branchial clefts and of the spiracle. From this runs backwards a short wide tube-the osophagus (os.)which passes behind into the stomach. The stomach is a $U$ shaped organ, with a long left limb continuous with the œsophagus, and a short right one passing into the intestine. At the pylorus (pyl.) - the point where

K 2 


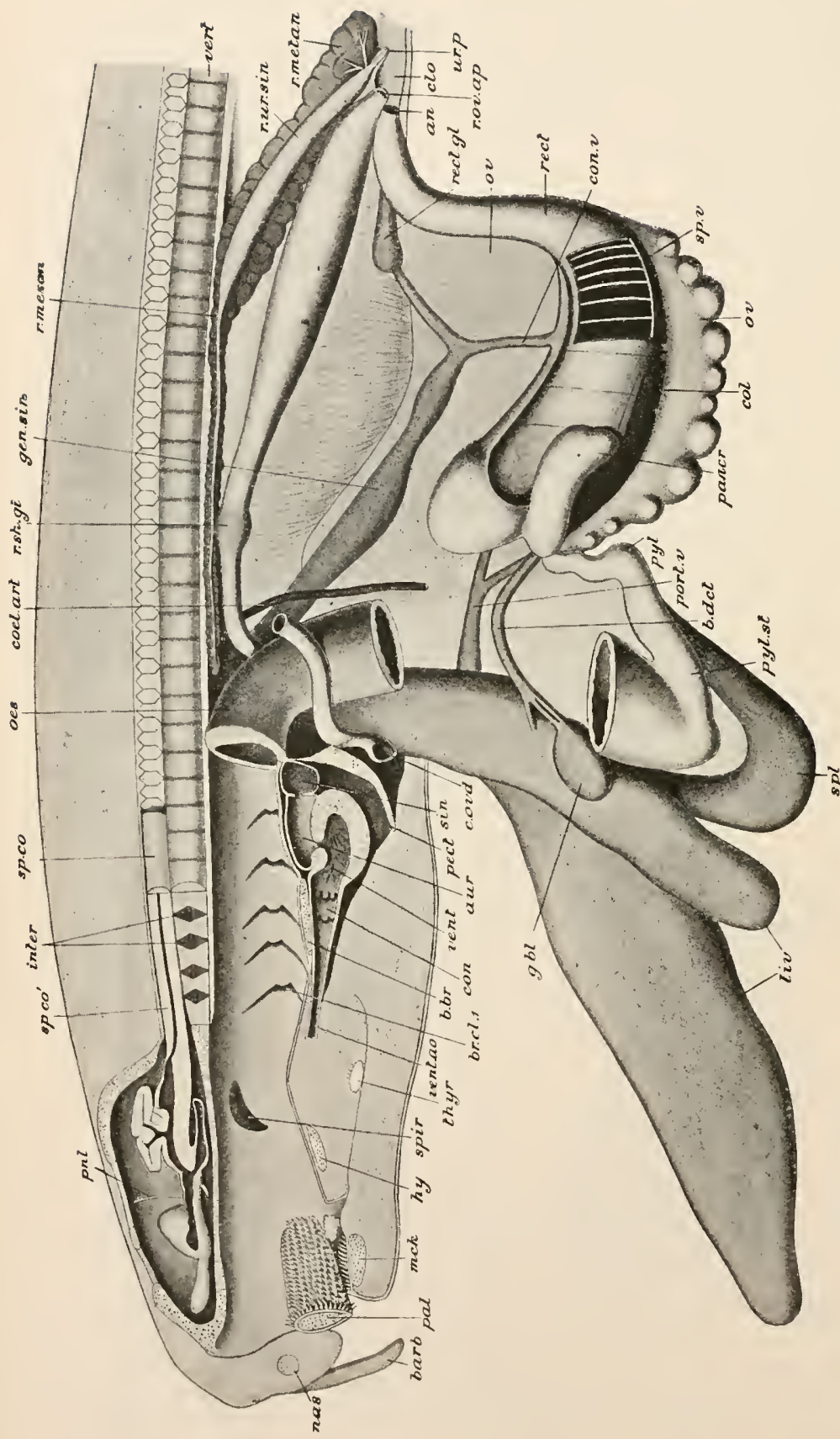

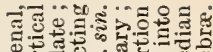

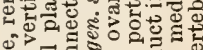

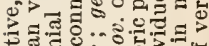
o

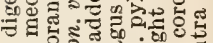
․․․

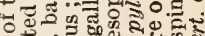

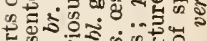
to

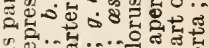
o 울

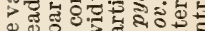
\%

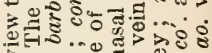

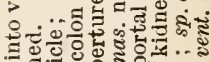

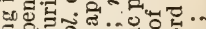

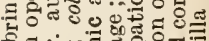

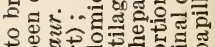
S \&

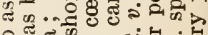
象 =

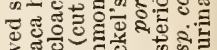

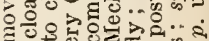
讨 I...

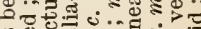
of of

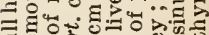

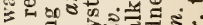
可. o 90 ... 今 स्र 웡

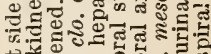

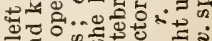
व

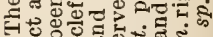
สี घ. \% 政的 o ฮี ॠ 5..4

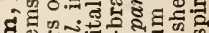
300.1 .1 .50 s.

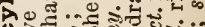
००

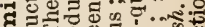
of मे

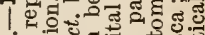

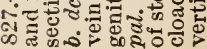
密 
the stomach passes into the intestine-is a slight constriction, followed by a thickening. The intestine consists of two parts-small intestine or duodenum, and large intestine. The former is very short, only an inch or two in length. The latter is longer and very wide ; it is divisible into two portions - the colon (col.) in front and the rectum (rect.) behind. The former is very wide and is characterised by the presence in its interior of a spiral valve, a fold of the mucous membrane which runs spirally round its interior, and both retards the too rapid passage of the food and affords a more extensive surface for absorption. The rectum differs from the colon in being narrower and in the absence of the spiral valve ; it opens behind into the cloaca.

There is a large liver (liv.) consisting of two elongated lobes. A rounded sac-the gall-bladder $(g . b l$. $)$-lies embedded in the left lobe at its anterior end. The duct of the liver-the bile-duct (b.dct.) -runs from the liver to the intestine. Proximally it is connected with the gall-bladder, and by branch-ducts with the right and left lobes of the liver. It opens near the commencement of the colon.

The pancreas (pancr.) is a light-coloured compressed gland consisting of two main lobes with a broad connecting isthmus, lying in the angle between the right-hand limb of the stomach and the small intestine. Its duct enters the wall of the small intestine and runs in it for about half an inch, opening eventually at the point where the small intestine passes into the colon.

Connected with the rectum on its dorsal aspect is an oval gland-the rectal gland

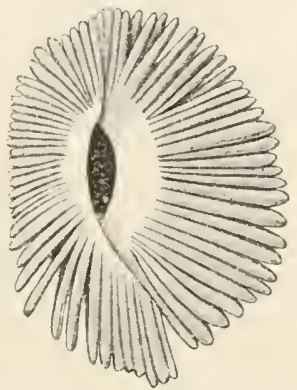

FIG. 828.-Femiscyllium. Branchial sac exposed from the outside. (rect. gl.)-about three-quarters of an inch in length.

The spleen ( $s p l$.) is a dark-red or purple body attached to the convexity of the $U$-shaped stomach and sending a narrow lobe along the right-hand limb.

The organs of respiration in the Dog-fish are the gills, situated in the five gill-pouches. Each gill-pouch (Fig. 828) is an anteroposteriorly compressed cavity opening internally into the pharynx and externally by the corresponding gill-slit. The walls of the pouches are supported by the branchial and hyoid arches with their rays, the first pouch being situated between the hyoid and first branchial arches, the last between the fourth and fifth branchial arches. On the anterior and posterior walls of the pouches are the gills, each hemibranch consisting of a series of close-set parallel folds or plaits of highly vascular mucous membrane. Separating adjoining gill-pouches, and supporting the gills, are a series of broad interbranchial septa, each containing the corresponding branchial arch with its connected branchial rays. The most anterior hemi- 
branch is borne on the posterior surface of the hyoid arch. The last gill-pouch differs from the rest in having gill-plaits on its anterior wall only. On the anterior wall of the spiracle is a vestigial gill - the pseudobranch or spiracular gill - in the form of a few slight ridges.

Blood-system.-The heart is situated in the pericardial cavity, on the ventral aspect of the body, in front of the pectoral arch, and between the two series of branchial pouches. The dorsal wall of the pericardial cavity is supported by the basibranchial cartilage. Placing it in communication with the abdominal cavity is a canal-the pericardio-peritoneal canal. The heart (Fig. 827) consists of four chambers-sinus venosus (sin.), auricle (aur.), ventricle (vent.), and conus arteriosus (con.), through which the blood passes in the order given. The sinus

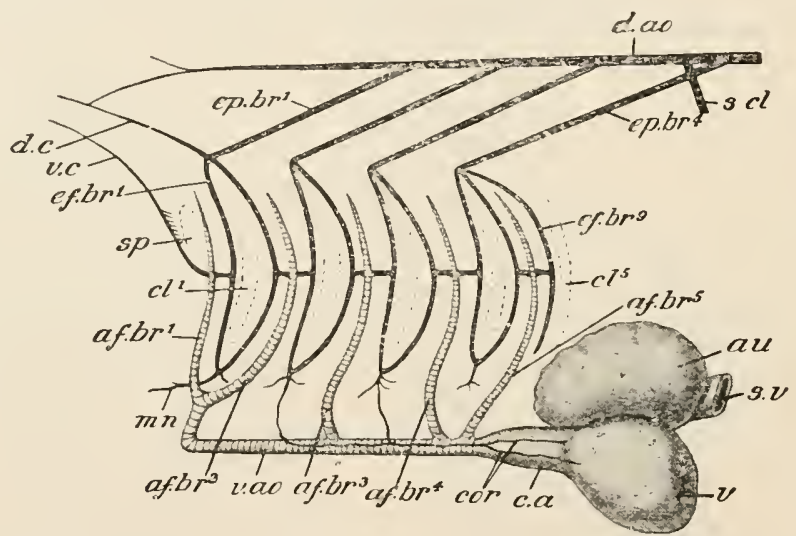

$F^{*} I G .829$ - The heart and branchial arteries of Scyllium, from the side. $a f$. br. $1-5$, afferent branchial arterics ; $a u$. auricle ; $c$. $a$. conus arteriosus ; $c l .1-5$, branchial clefts ; cor. coronary artery ; $d$. ao. dorsal aorta: $d$. $c$. dorsal carotid artery; $e f$. br. 1 -9, efferent branchial arteries ; $e p . b r .1-4$, epibranchial arteries; $m n$. mandibular artery ; $s p$. spiracle ; $s$. $c l$. subclavian artery;s.v. sinus venosus; $v$. ventricle; $v$. ao. ventral aorta; $v$. $c$. ventral carotid artery. (From Parker's Practical Zoology.)

venosus is a thin-walled, transverse, tubular chamber, into the ends of which the great veins open. It communicates with the auricle by an aperture, the sinu-auricular aperture. The auricle is a large, three-cornered, thin-walled chamber, situated in front of the sinus venosus and dorsal to the ventricle. Its apex is directed forwards, and its lateral angles project at the sides of the ventricle : it communicates with the ventricle by a slit-like aperture guarded by a twolipped valve. The ventricle is a thick-walled, globular chamber, forming the most conspicuous part of the heart when looked at from the ventral surface. From it the conus arteriosus runs forwards as a median stout tube to the anterior end of the pericardial cavity, where it gives off the ventral aorta. It contains two transverse rows of valves, anterior and posterior, the former 
eonsisting of three, the latter of three or four. The vcntral aorta (Fig. 829) gives origin to a series of paired affcrent branchial arterics (af. br.), one for eaeh branchial poueh. In Seyllium the two most posterior arise elose together near the beginning of the ventral aorta, the third pair a little further forwards. The ventral aorta then runs forwards a little distanee and bifureates to form the two innominatc arteries, right and left, eaeh of whieh in turn bifureates to form the first and seeond afferent vessels (af. $b r^{1}$., af. $b r .^{2}$ ) of its side. In Hemiseyllium (Fig. 830) the arrangement is somewhat different.

From the gills the blood passes by means of the cffercnt branchial arteries. These efferent vessels (Fig. 829, ef. br.) form a series of loops, one rumning around the margin of each of the first four internal branchial elefts: a single vessel runs along the anterior border of the fifth branehial eleft and opens into the fourth loop. The four main efferent branehial vessels (epibranchials, ep. br.) run inwards and baekwards from the loops under eover of the mucous membrane of the roof of the pharynx to unite in a large median trunk-the dorsal aorta (d. ao.). A dorsal carotid artery (d.c.) is given off from the first efferent branehial. A braneh (hyoidean) given off from the same efferent vessel supplies the pseudobranch, and the blood from the latter is taken up by the vcntral carotid (v. c.). Both earotids run forwards to supply the head.

The dorsal aorta (Fig. 829, d. ao.) runs baekwards throughout the length of the body-cavity, giving off numerous branehes, and is eontinued as the caudal artcry, whieh runs in the eanal enelosed by the inferior arehes of the caudal vertebræ. The first pair of branehes are the subclavians $(s . c l$.), for the supply of the peetoral fins; these are given off between the third and fourth pairs of epibranehial arteries. The next large braneh is the unpaired coliac (Fig. 827, coel.): this runs in the mesentery and divides into branehes for the supply of the stomaeh and liver, the first part of the intestine, and the panereas. The anterior mesenteric artery, also median, supplies the rest of the intestine and gives off branehes to the reproduetive organs. The lienogastric supplies part of the stomaeh, the spleen, and part of the panereas. The posterior mesenteric is a small vessel mainly supplying the reetal gland. Small renal arteries carry a small quantity of arterial blood to the kidneys, and a pair of iliac arteries, likewise of small size, pass to the pelvic fins. In addition to these a number of small arteries, the parietal, supplying the wall of the body, are given off throughout the length of the aorta.

The vcins are very thin-walled, and the larger trunks are remarkable for their dilated eharaeter, from whieh they have obtained the name of sinuscs, though they are true vessels and not sinuses in the sense in whieh the word is used in dealing with the Invertebrates ( $f$. p. 90). 
The venous blood is brought back from the head by a pair of jugular or anterior cardinal sinuses (Fig. $830, j u g . v$.), and from the trunk by a pair of posterior cardinal sinuses (r. and l.card.s.). At

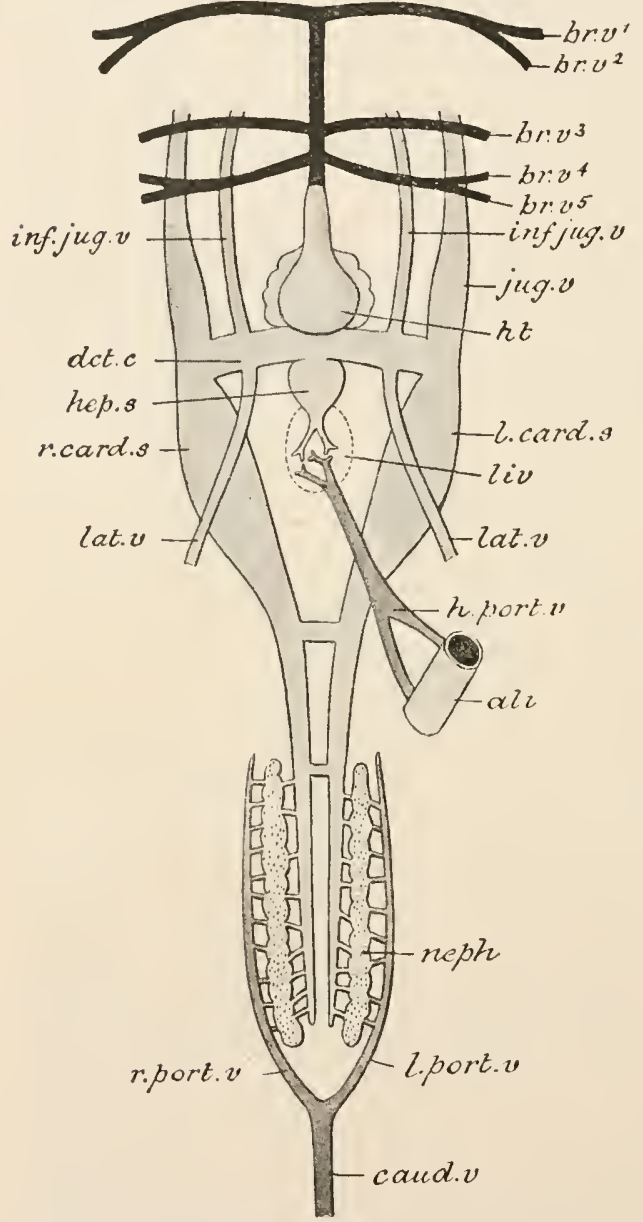

Fig. S30.-Hemiscyllium. Diagrammatie representation of the ventral aorta and afferent branchial arteries, and of the ehief veins. ali. alimentary canal; br.v.1-br.v.5 afferent branchial arteries; caud. $v$, eaudal vein; dct. $c$. preeaval sinus; ht. heart; h.port. $v$. hepatic portal vein; hep. s. hepatie sinus; inf. jug. v. inferior jugular vein or sinus; jug. $v$. jugular vein or sinus; lat. $v$. lateral vein ; liv. liver ; l. card. s. left carlinal sinus; $l$. port. $v$. left renal portal vein : $n e p h$. kidney; $r$. card. $s$. right eardinal sinus; $r$. port. $v$. right renal portal vein. the level of the sinus venosus the anterior and posterior cardinals of each side unite to form a short, nearly transverse sinus, the precaval sinus or ductus Cuvieri (Fig. 830 , dct. c.), which is continued into the lateral extremity of the sinus venosus. Into the precaval sinus, about its middle, opens an inferior jugular sinus (inf. jug. v.) which brings back the blood from the floor of the mouth and about the branchial region of the ventral surface. The two posterior cardinal sinuses extend backwards throughout the length of the bodycavity; in front they are enormously dilated, behind they lie between the kidneys. Anteriorly each receives the corresponding subclavian vein bringing the blood from the pectoral fin and adjacent parts of the body-wall. The lateral vein $(l . v$.$) , in-$ stead of joining with the subclavian (p. 91), opens separately into the precaval.
erior cardinal sinus.

The genital sinus discharges into the posterior cardinal sinus.

There are two portal systems of veins, the renal (r. and l.port.v.) and the hepatic portal (hep.port.v.), by which the kidneys and liver, 
respectively, are supplied with venous blood. The caudal vein, which brings back the blood from the tail, running, along with the caudal artery through the inferior arches of the vertebræ, divides on entering the abdominal cavity into right and left renal portal veins, which end in a number of afferent renal veins supplying the kidneys.

The hepatic portal vein (h. port. v.) is formed by the confluence of veins derived from the intestine, stomach, pancreas, and spleen, and runs forwards to enter the liver a little to the right of the middle line. In Hemiscyllium a large branch connects the genital sinus with the intestinal tributaries of the hepatic portal system: the blood from the liver enters the sinus venosus by two hepatic sinuses placed close together.

Nervous System.-The fore-brain consists of a rounded smooth prosencephalon (Fig. 831, V.H.) divided into two lateral parts by a very shallow median longitudinal groove. From its antero-lateral region each half gives off a thick cord, which dilates into a large mass of nerve-matter, the olfactory bulb $(L$. ol.), closely applied to the posterior surface of the corresponding olfactory capsule. The diencephalon $(Z H)$ is comparatively small; its roof is very thin, while the lateral walls are composed of two thickish masses-the optic thalami. Attached to the roof is a slender tube, the epiphysis cerebri or pineal organ $(G p$.), which runs forwards and terminates in a slightly dilated extremity fixed to the membranous part of the roof of the skull. Projecting downwards from its floor are two rounded bodies, the lobi inferiores $(U L)$, which are dilated portions of the infundibulum. Behind these give off a thin-walled vascular outgrowth-the saccus vasculosus (Sv.). Attached to the infundibulum and extending backwards from it is a thin-walled sac-the pituitary body or hypophysis cerebri $(H S)$, having on its ventral surface a median tubular body attached at its posterior end to the floor of the skull. In front of the infundibulum, and also on the lower surface of the diencephalon, is the optic chiasma, formed by the decussation of the fibres of the two optic nerves. The mid-brain $(M H)$ consists of a pair of oval optic lobes dorsally, and ventrally of a band of longitudinal nerve-fibres corresponding to the crura cerebri of the higher vertebrate brain. The cerebellum $(H H)$ is elongated in the antero-posterior direction, its anterior portion overlapping the optic lobes, and its posterior the medulla oblongata. Its surface is marked with a few fine grooves. The medulla oblongata $(N H)$, broad in front, narrows posteriorly to pass into the spinal cord. The fourth ventricle or fossa rhomboidalis ( $F$. rho.) is a shallow space on the dorsal aspect of the medulla oblongata covered over only by a thin vascular membrane, the choroid plexus : it is wide in front and gradually narrows posteriorly. At the sides of the anterior part of the fourth ventricle are a pair of folded ear-shaped lobes, the corpora restiformia. 
The fourth ventricle or metacoele (Fig. 832, meta.) is continuous behind with the central canal of the spinal cord. It gives off an epicœle above, and in front is continuous with a narrow passage,

A.

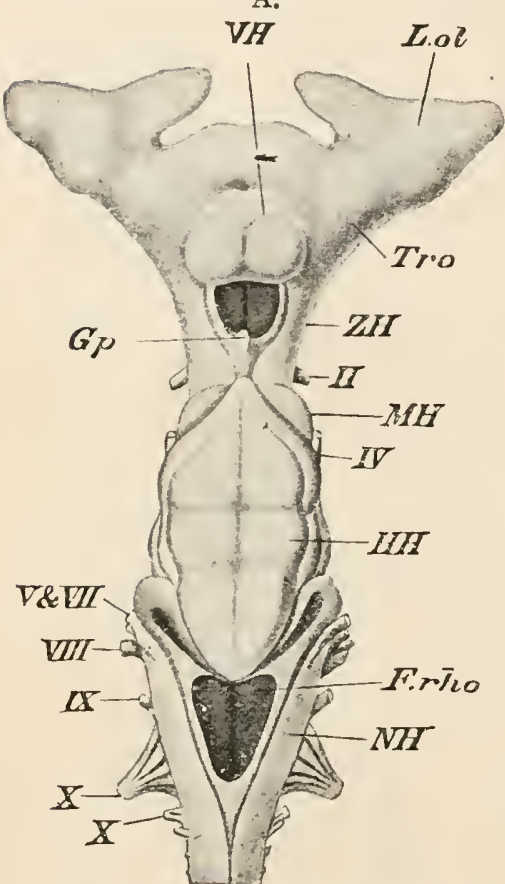

B.

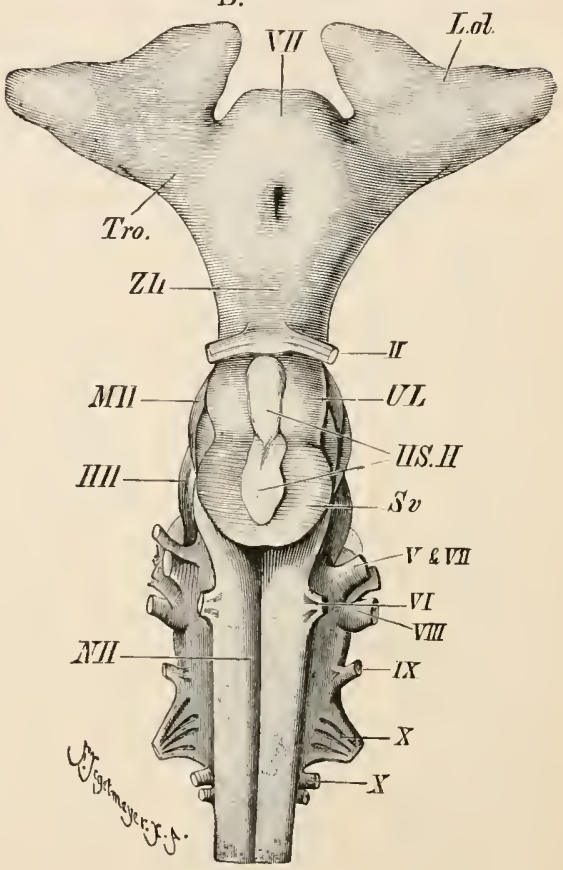

c.

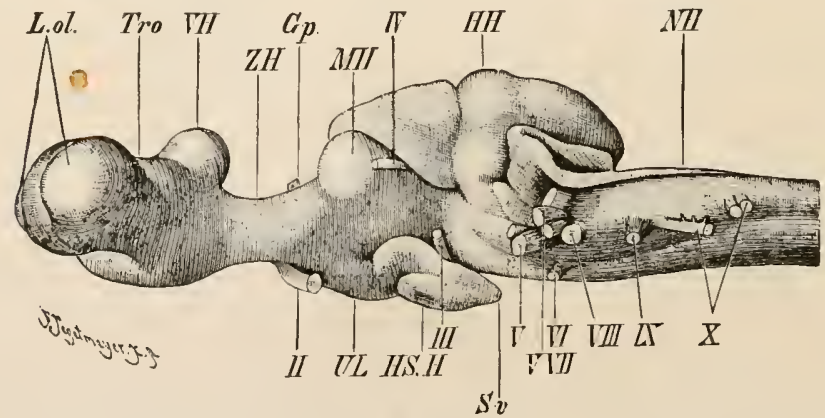

FIG. 831.-Brain of Scyllium canicula. A, dorsal view ; $\mathbf{B}$, ventral view ; $\mathbf{C}$, lateral view. $F$, rho, fossa rhomboidalis (fourth ventricle); $G p$, epiphysis : $I H$, cerebellun ; $H S . H$, hypophysis; $L$. ol. olfactory bulb; $I I I$, mid-brain ; $N I I$, medulla oblongata ; $S ?$, saccus vasculosus; Tro, olfactory peduncle; $U L$, lobi inferiores; $V I I$, prosencephalon ; $Z H$, diencephalon; $I I$, optic nerves $I I I$, oculomotor ; $I V$, pathetic; $V$, trigeminal ; $V I$, abducent ; $V I I$, facial ; $V I I I$, auditory ; $I X$, glossopharyngea] ; $X$, vagus. (From Wiedersheim's Comp. Anatomy.)

the iter or mesoccele (iter.), which opens anteriorly into a wider space, the diacoele or third ventricle (dia.), occupying the interior of 
the diencephalon. From this opens in front a median prosocole, which gives off a pair of paracoles (para.) extending into the two lateral portions of the prosencephalon.

Fron the anterior enlargements of the olfactory bulbs already mentioned spring numerous fibres which constitute the first pair of cerebral nerves and enter the olfactory capsules. Between the two olfactory lobes two small nerves, the terminal or pre-olfactory, arise from the prosencephalon: they are the nerves of ordinary sensation for the interior of the olfactory sacs. From the optic chiasma the two optic nerves (Figs. 831, 833, 834, II) run outwards through the optic foramina into the orbits, each perforating the sclerotic of the corresponding eye and terminating in the retina. The third, fourth, and sixth pairs of nerves have the general origin and distribution which have already been described as universal in the Craniata (p. 100).

The trigeminal (Figs. 831, 833, 831, V) arises in close relation to the facial. As it passes into the orbit it swells into a ganglion-the Gasserian. Its chief, branches are three in number. The first given off is the superficial ophthalmic (Fig. 833, oph. V ; Fig. 834, $V$ op.), which runs forwards through the orbit above the origin of the recti muscles, and in very close relation with the ophthalmic branch of the facial. Anteriorly it breaks up into branches distributed to the integument of the dorsal surface of the snout. ${ }^{1}$ The main trunk of the nerve then runs forwards and outwards across the floor of the orbit, and divides into two branches, the maxillary and mandibular, or second and third divisions of the trigeminal. The former $(m x . V)$

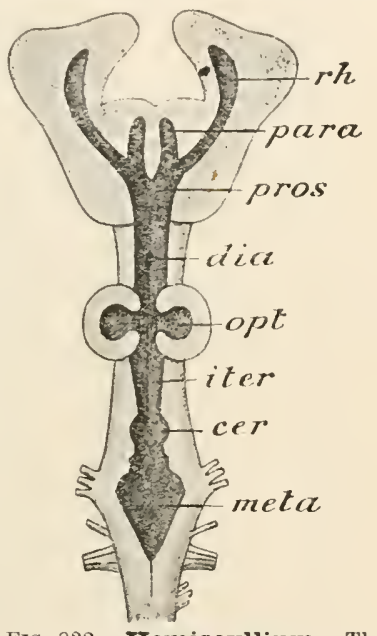

FIG. 832, Iemiscyllium. The brain viewed from the dorsal side, the roofs of the various vcntricles removed so as to show the relations of the cavities (semi-diagrammatic). cer, dilatation from which the epiccele is given off ; dia, diaccele, pointing to the opening leading into the infundibulum; iter. iter or mesocole; meta. metacorle ; opt. optocœele; para. paracole ; pros. prosoccle ; $r h$. rbinoccle. supplies the skin of the ventral surface of the snout, the latter (mnd. V) the skin and muscles of the lower jaw.

Of the branches of the facial, the ophthalmic runs through the

1 In most Elasmobranchs a nerve of considerable size-the ophthalmicus profundus (Fig. 788) -arises from the dorsal and anterior part of the Gasscrian ganglion, and is usually regarded as a branch of the trigeminal. It runs forwards over the posterior rectus muscle and under the superior rectus, and perforates the pre-orbital process to end in the integument of the snout. Among other branches it gives off ciliary branches to the iris: these are joined by the ciliary branches of the oculomotor. An ophthalmicus profundus is not present in Scyllium in the adult condition. 
orbit in close relation to the superficial ophthalmic branch of the trigeminal, and is distributed to the lateral line and ampullary canals of the snout region; the buccal runs forwards in intimate relation with the maxillary division of the trigeminal, and breaks up into branches which are distributed to the sensory canals and ampullæ of the region of the snout; the palatine ( $p l$. VII, VIIp.) passes to the roof of the mouth; the main body of the nervehyomandibular nerve (hy. mnd. VII, VIIhy.) - then runs outwards close to the edge of the hyomandibular cartilage and behind the spiracle, eventually becoming distributed to the muscles between

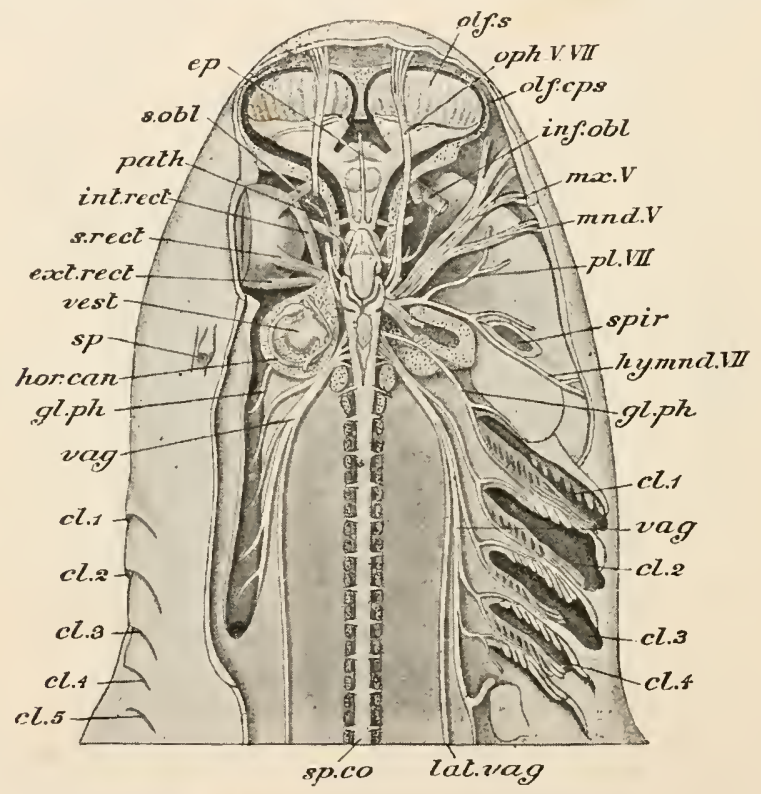

FIa. 833.-Scyllium catulus. Dissection of the brain and spinal nerves from the dorsal surface. The right eye has been removed. The cut surfaces of the cartilaginous skull and spinal column are dotted. The buccal branch of the facial is not represented. $c l .1-c l .5$, branchial clefts; $e p$. epiphysis ; ex. rect. posterior rcetus muscle of the eye-ball ; $g l . p h$. glossopharyngeal ; hor. can. horizontal semicircular canal; $h y$. mnd. VII. hyomandibular portion of the facial; inf. obl. inferior oblique muscle ; int. rect. anterior rectus muscle ; lat. vag. lateral branch of vagus; $m x . \boldsymbol{V}$. maxillary division of the trigeminal ; olf. cps. olfactory capsule; olf. $s$, olfactory sac; oph. $V . V I I$. superficial ophthalmic branches of trigeminal and facial; path. fourth nerve ; $p l$. $V I I$. palatine branch of facial ; $s p$. co. spinal cord; sp, spir. spiracle; s. rect. superior rectus muscle; $s$. olb. superior oblique ; vag. vagus ; vest. vestibule. (From Marshall and Hurst.)

the spiracle and the first branchial cleft; a small external mandibular branch (VII e.m.) comes off from it and goes to the lateral line and ampullary canals of the lower jaw.

The eighth or auditory nerve passes directly into the internal ear, and breaks up into branches for the supply of its various parts. The glossopharyngeal ( $g l$. ph., $I X)$ perforates the posterior part of the auditory region of the skull, and, after it reaches the 
exterior, passes to the first branchial cleft, where it bifurcates, one branch going to the anterior, and the other to the posterior wall of the cleft. The last nerve of the series-the pneumogastric or vagus (vag., $X$ )-is a large nerve which emerges from the skull by an aperture situated between the auditory region and the foramen magnum. It first gives off a series of four $b r a n c h i a l$ branches, each of which bifurcates to supply the anterior and posterior borders of the last four branchial clefts. The lateralis nerve (lat. vag., X.l.) is frequently referred to as a branch of the vagus since it runs in intimate connection with the trunk of that nerve for some distance, but it has a distinct origin in the medulla: after becoming separated from the vagus trunk it runs along beneath the peri-

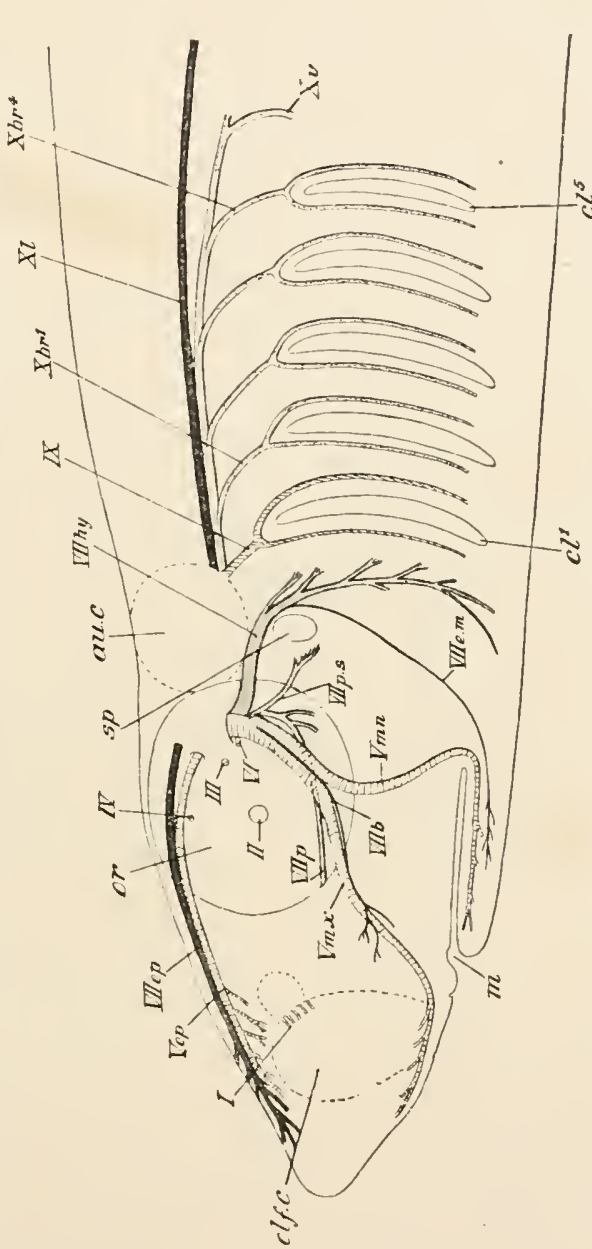

toneum opposite the lateral line, which it supplies, to the posterior end of the body. The rest of the vagus runs backwards to divide into cardiac branches for the heart and gastric branches for the stomach.

It will be observed that the system of neuromast organs (lateral line and ampullary organs) are supplied by nerve-fibres which pass out in various branches of the facial and in the lateralis: 
all these fibres originate in a centre in the medulla, the acusticolateral centre, common to them and the fibres of the auditory nerve.

The spinal cord is a cylindrical cord which extends from the foramen magnum, where it is continuous with the hind-brain, backwards throughout the length of the neural canal, enclosed by the neural arches of the vertebræ. As in the Craniata in general (see p. 96), it has dorsal and ventral longitudinal fissures and a narrow central canal, and gives origin to a large number of paired spinal nerves, each arising from it by two roots.

Organs of Special Sense.-The olfactory organs are rounded chambers enclosed by the cartilage of the olfactory capsules of the skull, and opening on the exterior by the nostrils on the ventral surface of the head. The interior has its lining membrane raised up into a number of close-set ridges running out from a median septum. The fibres of the olfactory nerves terminate in cells of the epithelium covering the surface of these ridges.

The eye has the general structure already described as characterising the Craniata in general (p. 106). The sclerotic is cartilaginous, the choroid has a shining metallic internal layer or tapetum cellulosum, and the lens is spherical. There are the usual eye-muscles, the two obliques situated anteriorly, the four recti posteriorly, not embracing the optic nerve. The eyelids are represented by stiff folds.

The ear consists only of the membranous labyrinth (Fig. 798), equivalent to the internal ear of higher Craniata, the middle and outer ear being absent. The membranous labyrinth consists of the vestibule and three semicircular canals. The former, which is divided into two parts by a constriction, communicates by a narrow passage - the endolymphatic duct or aqueductus vestibuli - with the exterior, in the position already mentioned. Of the three semicircular canals, the anterior and posterior are vertical and the external horizontal, as in Craniata in general. Each has an ampulla, that of the anterior and external canals situated at their anterior ends, and that of the posterior canal, which is the largest of the three and forms an almost complete circle, at its posterior end. In the fluid (endolymph) in the interior of the vestibule are suspended, in a mass of gelatinous connective-tissue, numerous minute calcareous particles or otoliths, giving it a milky character.

The sensory canals of the integument running along the lateral line and over the head contain special nerve-endings (neuromasts), and doubtless function as organs of some special sense (see p. 105). The same probably holds good of a number of unbranched canals (ampullary canals) arranged in groups situated on the anterior portion of the trunk and on the head, and being particularly numerous in the neighbourhood of the snout. These are dilated internally into vesicles, the ampulla, provided with special nerve-endings.

Urinogenital Organs. - In the female there is a single ovary (Figs. $\left.827,835^{\circ} \mathrm{ov}.\right)$, an elongated soft, lobulated body, lying a little to 
the right of the middle line of the abdominal cavity, attached by a fold of peritoneum, the mesoarium. On its surface are rounded elevations or follicles of various sizes, each containing an ovum of a bright yellow colour. There are two oviducts (Müllerian ducts) entirely unconnected with the ovaries. Each oviduct (Figs. 827 and 835 , ovd.) is a greatly elongated tube extending throughout the entire length of the abdominal cavity. In front the two unite behind the pericardium to open into the abdominal cavity by a wide median aperture $\left(o v d^{\prime}\right.$.). At about the point of junction of the middle and anterior thirds is a swelling marking the position of the shell-gland (sh. gl.). The posterior part dilates to form a wide uterine chamber, and in Scyllium the two unite to open into the cloaca by a common aperture situated just behind the opening of the rectum, while in Hemiscyllium they remain distinct and have separate cloacal openings. Each kidney consists of two parts, anterior and posterior. The former (Fig. 827, r. meson, Fig. $835, k^{\prime}$ ) is a long narrow ribbon of soft reddish substance, which runs along throughout a great part of the body-cavity at the side of the vertebral column, covered by the peritoneum. The posterior portion ( $r$. metan, $k$ ) is a compact, lobulated, dark-red body, lying at the side of the cloaca, continuous with the anterior portion; like the latter, it is covered over by the peritoneum. Both portions have their ducts. Those of the anterior are narrow tubes, which run over its ventral surface and become dilated behind to form a pair of elongated chambers, the urinary sinuses (Fig. 836, ur. sin.), uniting behind into a median sinus (med.ur. sin.), opening into the cloaca by a median aperture situated on a papilla, the urinary papilla. The ducts of the posterior portion, the ureters, which are usually from four to six in number, open into the urinary sinuses.

In the male (Fig. 835, $A$ ) there are two elongated, soft, lobulated testes, each attached to the wall of the abdominal cavity by a fold of peritoneum-the mesorchium. From each testis anteriorly, a small number of efferent ducts $(e f . d)$ pass to the anterior end of a long, narrow, strap-shaped body, which corresponds to the vestigial anterior portion of the kidney in the female. This is the epididymis ; the duct, spermiduct or vas deferens, runs along the entire length of the non-renal part of the kidney, or "Leydig's gland," and, where it leaves the latter posteriorly, becomes a wide tube, which opens into the urinogenital sinus (u.g. s.), a median chamber projecting into the cloaca. Posteriorly the spermiduct dilates to form a wide thin-walled sac, the vesicula seminalis. Closely applied to the latter is a thin-walled elongated sac, the sperm-sac. Anteriorly the sperm-sac narrows to a blind extremity ; posteriorly the right and left sperm-sacs combine to form the urinogenital sinus. The posterior part of the kidney has the same character as in the female; its ducts, usually five in number on each side, open into 


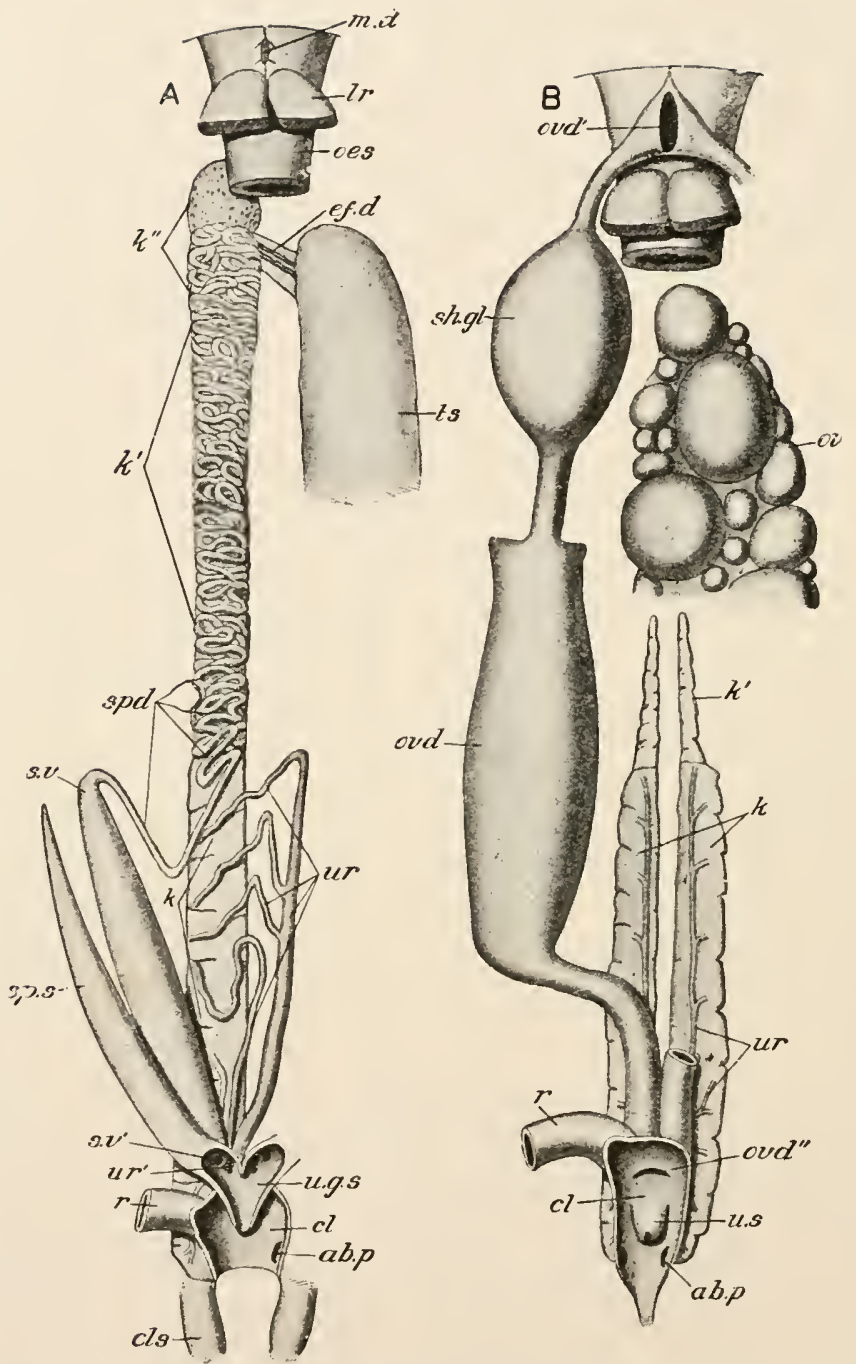

FIG. 835.-The urinogenital organs of Scyllium canicula from the ventral side, $A$, male, and $B$, female. Only the anterior end of the gonad is represented in each figure, and except that in $B$ both kidneys are shown, the organs of the right side only are drawn. In $A$ the seminal vesicle and sperm-sac are dissected away from the kidneys and displaced outwards, and the ureters inwards, $a b . p$. depression into which the abdominal pore opens ; $c l$. cloaca; $c l s$. clasper ; $\ell f$. $d$. efferent ducts of spermary $; k$. kidney $; k^{\prime}, k^{\prime \prime}$. anterior non-renal portion of the kidney, forming in the male the so-called " Leydig's gland," which, together with the coiled spermiduct, constitutes the epididymis ; $l r$. anterior portion of liver $; m$. $d$. vestigial Müllerian duct in the male ; oes. gullet ; ov. ovary ; ovd. oviduct ; ovd'. its coelomic aperture ; ond". the common aperture of the oviducts into the cloaca $: r$. rectum; $s h$. $g l$. shell-gland ; $s p d$. spermiduct; $s p$. s. sperm-sac;s. v. seminal vesicle; $s$. $v^{\prime}$. its aperture into the urinogenital sinus; $t s$. spermary (testis); $u$. g. s. urinogenital sinus; ur. ureters; $u r^{\prime}$. their a pertures into the urinogenital sinus; u.s. urinary sinus. (From Parker's Practical Zoology.) 
the urinogenital sinus, some of the most anterior first uniting to form a common tube. The sinus has a median aperture into the general cavity of the cloaca situated on the summit of a prominent urinogenital papilla. The oviducts (Miillerian ducts) of the female are represented in the male by vestiges of their anterior portions (m.d.). The entire kidney is sometimes regarded as a mesonephros, but the posterior portion, developed entirely behind the portion which, in the male, takes part in forming the epididymis, and having

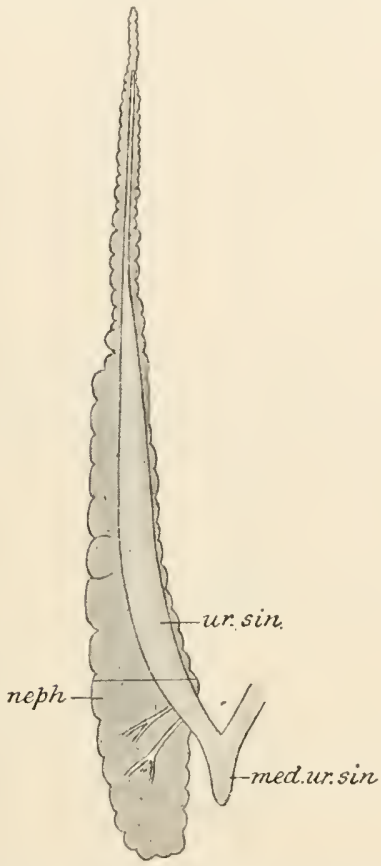

FIG. 836.-Hemiscyllium. Right kidney and urinary sinus of female. med.ur. sinus, median urinary sinus; neph. kidney; ur. sinus, right urinary sinus.

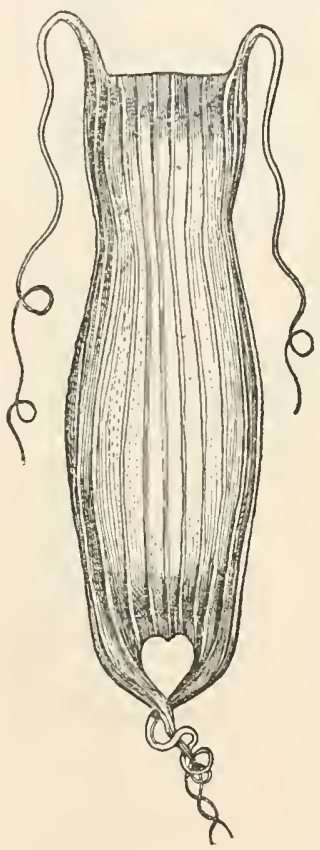

Fik. 837.-Dog-fish, egg-case. (After

its own ducts, is sometimes looked upon as foreshadowing the metanephros of the higher Vertebrates.

The ripe ovum, rupturing the wall of its follicle, escapes into the abdominal cavity, whence it reaches the interior of one of the oviducts; there it is fertilised by sperms received from the male in the act of copulation, and then becomes enclosed in a chitinoid case or shell (Fig. 837) secreted by the shell-gland.

\section{2.-Distinctive Characters and Classification.}

The Elasmobranchii are Pisces in which the cartilaginous cranium is never ossified by replacing-bones, and in which investing-bones 
are not developed in connection either with the cranium or the pectoral arch. The skull is hyostylic, except in some of the Protoselachii, in which it is amphistylic (p. 76). The dermal fin-rays are "horny"; at their bases the fins are supported by cartilaginous pterygiophores which are never very numerous. The pelvic arch is a distinct cartilage. There is nearly always an exoskeleton which is of the placoid type. The intestine has a spiral or a scroll-like valve. A cloaca is present into which both the rectum and the ducts of the urinary and reproductive systems open. There is never an operculum in recent Elasmobranchs, and only rarely in fossil forms. The inter-branchial septa are of considerable breadth, and the gill-filaments are attached to them throughout their entire extent. A mandibular spiracular gill is only exceptionally present as a fully developed organ ; it is represented usually by a vestige (pseudobranch). A conus arteriosus is

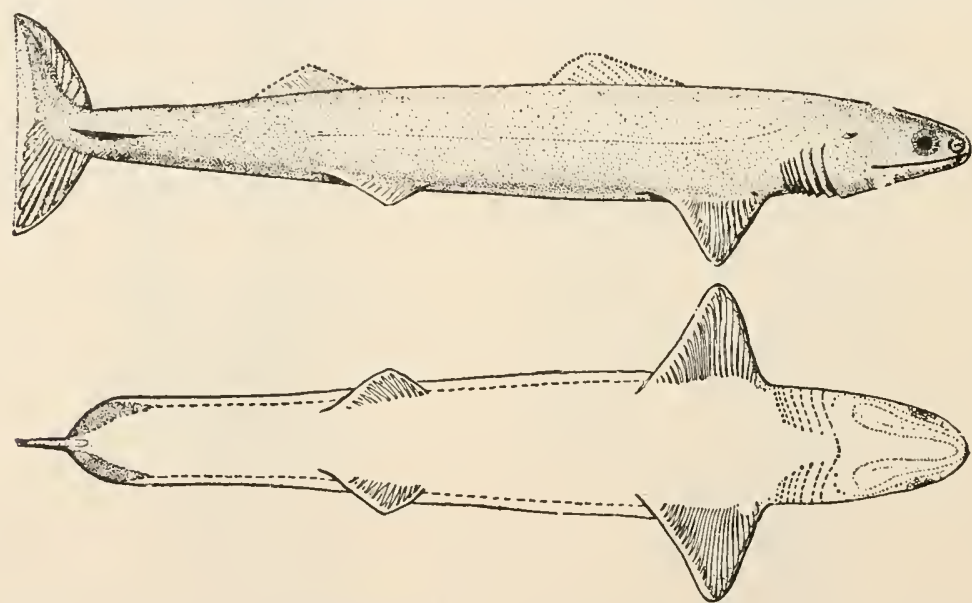

FIG. 838.-Restoration of Cladoselache fyleri, Iateral and ventral views. (After Dean.) always developed ; it is rhythmically contractile, and in its interior are several transverse rows of valves. The optic nerves form a chiasma. The ova are very large; with one possible exception they are always fertilised internally. The oviducts are not continuous with the ovaries, but open by wide mouths into the bodycavity.

\section{Order 1.-Cladoselachil (Pleuropterygii).}

Extinct Shark-like Elasmobranchs in which both pectoral and pelvic fins had much wider bases of attachment than in existing forms. There is an exoskeleton of small denticles. The notochord was persistent: there are calcified neural and hæmal arches, but no intercalary cartilages. The caudal fin is heterocercal. Claspers are absent. The gill-openings were apparently protected by a 
fold of skin. The teeth are of the nature of placoid denticles. The lateral line was represented by an open groove.

This order comprises only one known representative-Cladoselache (Fig. 838)-from the lower Carboniferous rocks of America.

\section{Order 2.-Pleuracanthei (Іентнуотомі).}

Extinct Shark-like Elasmobranchs in which the skeleton of the pectoral fin was constructed on the type of the so-called archipterygium, i.e., consisted of an elongated, segmented central axis bearing two rows of jointed rays. The notochord was persistent; intercalary cartilages were present in addition to neural and hæmal arches. The caudal fin is diphycercal. Claspers were present. There was no opercular fold, and the teeth resemble those of other Elasmobranchs. Placoid scales are not known to have been present, but the skull is protected by roofing dermal ossifications.

This order, like the last, includes only one satisfactorily known genus - Pleuracanthus (Fig. 839) -of Carboniferous and Permian age.

\section{ORdER 3.-ACANTHODEI.}

Extinct Elasmobranchs (Fig. 840) having the anterior margin of each fin supported by a stout spine. The tail is heterocercal. There were no claspers. There is a placoid exoskeleton of small denticles. An operculum was not present. The notochord was persistent, with neural and hæmal arches. Calcified plates are present in relation to the jaws

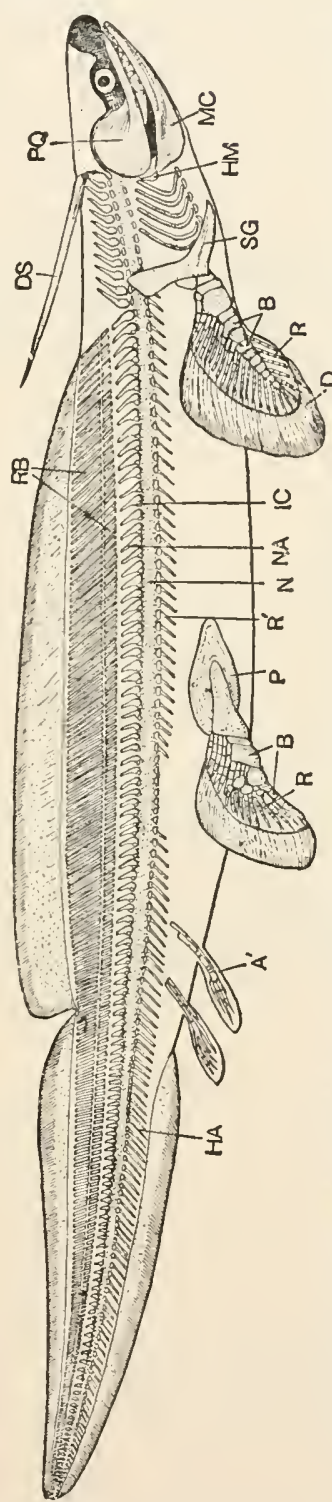
and to the roof of the skull. The teeth are few and large, numerous and minute, or altogether absent. The lateral line was in the form of an open groove. 


\section{Order 4.-Selachit.}

Living and extinct Elasmobranchs in which the skeleton of the paired fins is never of the nature of an "archipterygium." The notochord is more or less completely replaced by vertebræ, and there is a series of intercalary cartilages. The caudal fin is nearly always heterocercal. Claspers are always developed. A distinct opercular fold is never present.

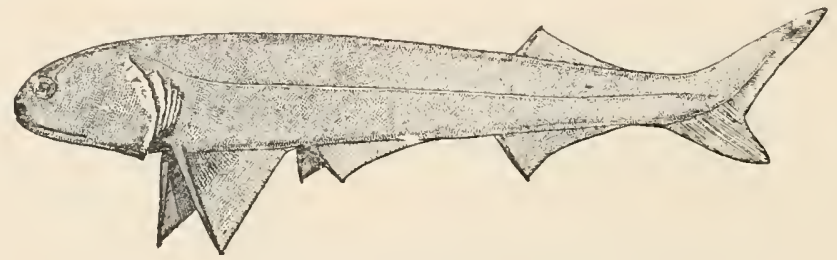

FiG. 840.-Acanthodes wardi. (Restored, after Dean.)

Sub-Order a.-Prolosclachii.

Selachii in which the spinal column is uncalcified, and the centra are very imperfectly developed; there are more than five branchial arches. Except in Chlamydoselachus, the palatoquadrate develops a process by which it articulates with the post-orbital region of the skull.

This sub-order includes the Notidanidee (Hcxanchus and Heptanchus), and Chlamydosclachus (Fig. 841), as well as, probably, many fossil forms.

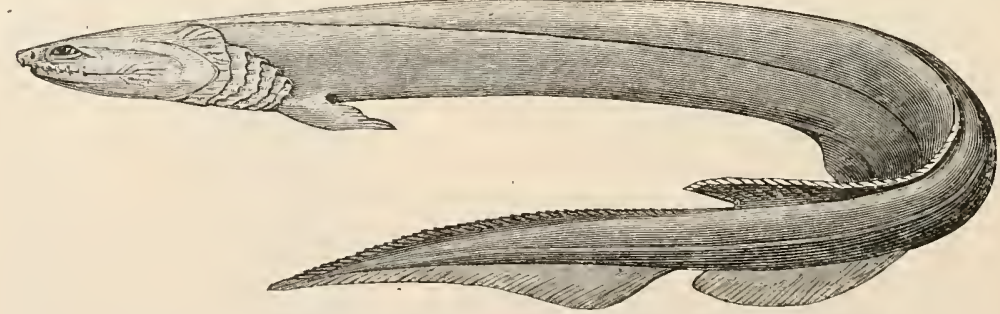

FIG. 841.-Chlamydoselachus anguineus. (From the Cambridge Natura III istory, after (iinther.)

\section{Sub-Order b.-Eusclachii.}

Selachii in which the spinal column is partly or completel calcified. There are only five branchial arches. The palatoquad rate has no post-orbital articulation with the skull.

\section{Scction a.-Squalida.}

Euselachii with fusiform body and well-developed caudal fir. The pectorals are of moderate size. A ventral fin is present. The vertebræ of the anterior part of the spinal column are not fused 
together. The branchial apertures and the spiracle are situated laterally.

This section comprises all the recent Sharks and Dog-fishes, with the exception of the Protoselachii.

\section{Section B.-Rajida.}

Euselachii with dorso-ventrally compressed body, and, usually, feebly developed caudal fin. The pectorals are of great size, the pelvies generally small. A ventral fin is usually absent. The vertebræ of the anterior region are fused together. The branchial apertures are ventral, the spiracles dorsal.

This section comprises all the recent and extinct Rays (Skates, Thorn-backs, Sting-Rays, Electric Rays, Saw-fish Rays).

\section{3.-General Organisation.}

External characters.-In general shape most Sharks (Fig. 842) are somewhat fusiform and slightly compressed laterally. In the Rays (Fig. 843), on the other hand, there is great dorsoventral compression. The head is in many cases produced forwards

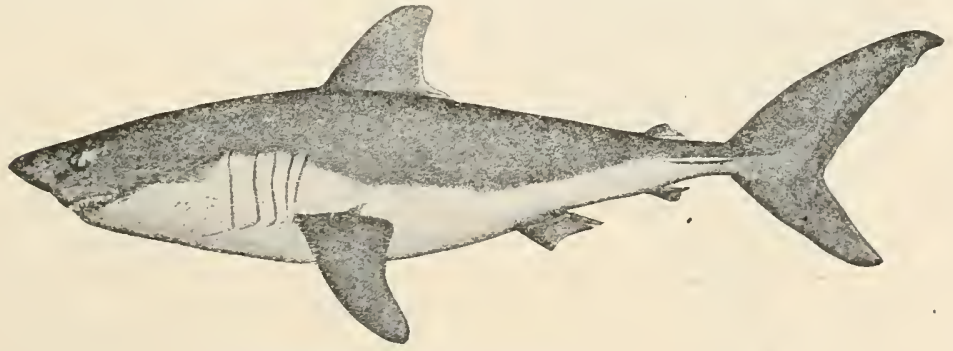

FiG. 812.-Porbeagle Shark (Lamna cornubica). (From Dean's Fishes.)

into a long rostrum, which is of immense length and bordered with triangular teeth in the Saw-fish Shark (Pristiophorus) and Saw-fish Ray (Pristis). In the Hammerhead Shark (Sphyrna or Zygana) the anterior part of the head is elongated transversely.

There are well-developed median and paired fins. The caudal fin is large, and, as a rule, strongly heterocercal in the Sharks and shark-like Rays, reduced in most of the latter group. The dorsal and ventral fins are large in the Sharks, the former completely divided into two : in the Rays the dorsal fin is usually small, and the ventral absent. The paired fins differ widely in the two groups. In the Sharks both pairs are well developed, the pectoral being the larger. In the Rays the pectoral fins are extremely large, very much larger than the pelvic, fringing the greater part of the length of the flattened body, and becoming prolonged forwards on either side and even in front of the head, so that the animal presents the appearance of a broad fleshy leaf. 
In all recent Elasmobranchs the male has, connected with the pelvic fins, a pair of grooved appendages-the claspers or pterygopodia-which subserve copulation.

The mouth is situated on the ventral surface of the head, usually a considerable distance from the anterior extremity. In front of each angle of the mouth on the ventral surface is the opening of one of the olfactory sacs, each of which is frequently connected by a groove-the naso-buccal groove-with the mouth-cavity. Behind the mouth, on the dorsal surface in the Rays, and at the side in the Sharks, is the spiracle. Along the sides of the neck in the Sharks, and on the ventral surface in the Rays, there is on either side a row of slit-like apertures - the branchial slits or branchial

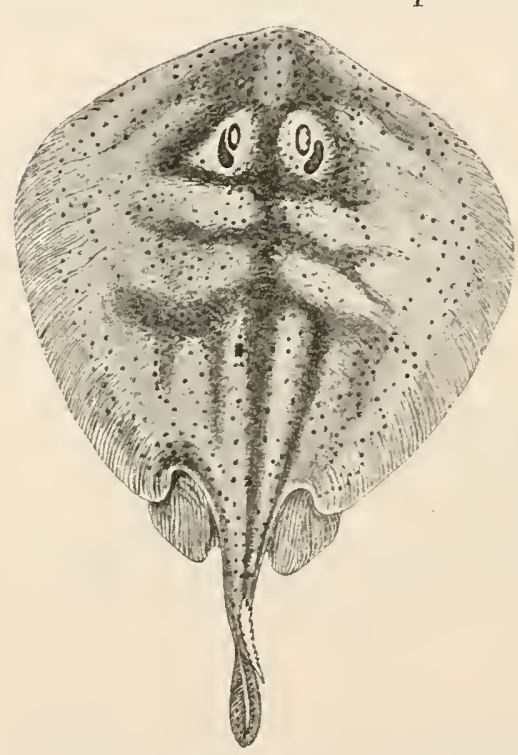

FIG. 843.--Sting-Ray (Urolophus cruciatus). (After Ginther.) clefts. These are usually five in number on each side; but in Hexanchus and Chlamydoselachus there are six, and in Heptanchus seven. In Chlamydoselachus (Fig. 841) a fold comparable to a rudimentary operculum extends back over the first branchial cleft, and is continuous across the middle line ventrally; in the remainder of the sub-class no such structure is represented. A large cloacal opening is situated just in front of the root of the tail, and in most members of the sub-class a pair of small openings placed close to it-the abdominal pores -lead into the abdominal cavity.

When the integument develops any hard parts, as is the case in the majority of the Elasmobranchs, they take the form, not of regular scales, as in most other fishes, but of numerous hard bodies (Fig. 844) which vary greatly in shape, are usually extremely minute, but are in some cases developed, in certain parts of the surface, into prominent tubercles or spines. When these hard bodies are, as is commonly the case, small and set closely together in the skin, they give the surface very much the character of a fine file; and the skin so beset, known as "shagreen," was formerly used for various polishing purposes in the arts. This is the placoid form of exoskeleton, to which reference has been already made. Each of the hard bodies has the same structure as a tooth, being composed of dentine, capped with an enamel-like layer, and supported on a base of a sub- 
stance somewhat resembling the bony cement or crusta petrosa of the tooth.

The skeleton is composed of cartilage, with, in many cases, deposition of calcareous matter in special places-notably in the jaws and the vertebral column. The entire spinal column may be nearly completely cartilaginous (Hexanchus and Heptanchus), but usually the centra are strengthened by radiating or concentric lamellæ of calcified tissue; or they may be completely calcified. They are deeply amphicœlous, the remains of the notochord persisting in the large inter-central spaces. Intercalary pieces (Fig. 845, Ic.) are interposed between both superior and inferior arches. In the Rays (Fig. 846) the anterior part of the spinal column becomes converted into a continuous solid cartilaginous and calcified mass-the anterior vertebral plate $(a . v . p$.). As in Fishes in general, two regions are distinguishable in the spinal column-the precaudal and the caudal, the latter being characterised by the possession of inferior or hæmal arches. In the pre-

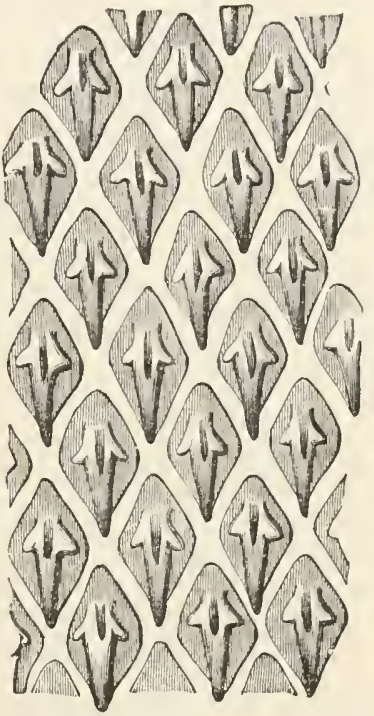

Fig. 814.-Dermal denticles of Centro. phorus calceus, slightly magnitied. (From Gegenbaur's Comparative Anatomy.) caudal region short ribs may be developed, but these are sometimes rudimentary or entirely absent. In the Sharks pterygiophores, sometimes jointed, fused at their bases with the hæmal spines,

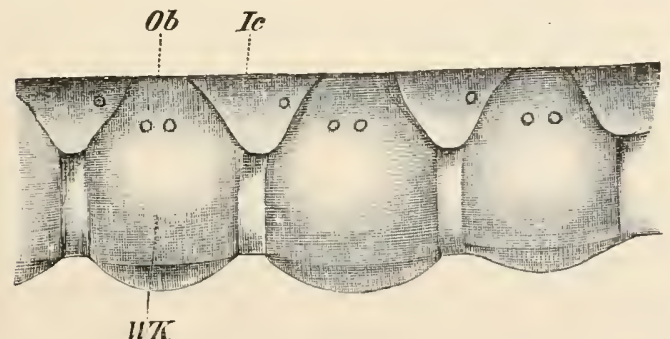
EIG. 845.-Portion of the spinal column of Scymnus. Ic.
intercalary cartilages: $O b$, neural arches; $W K$, centra. (From Wiedersheim's Vertebrata.)

The dorsal and ventral fins are sometimes supported by similar pterygiophores; but in many cases the cartilaginous supports of these fins consist, in whole or in part, of expanded plates of cartilage. The marginal portions of the unpaired fins beyond the limits 
of the endoskeleton are supported by dermal fibre-like structures (ceratotrichia) composed of elastin.

The skull is an undivided mass of cartilage, hardened, in many cases, by deposition of calcareous matter, but not containing any true bony tissue. It consists of a cartilaginous case for the protection of the brain and the organs of special sense. The structure of this cartilaginous brain-case as it occurs in the Dog-fish has already been described. The main differences observable in the different families are connected with the size and form of the rostrum. In the Rays the lower lip of the foramen magnum is deeply excavated for the reception of a short process, the so-called odontoid process, which projects forwards from the anterior vertebral plate, and on either side of this is an articular surface-the occipital condylefor articulation with corresponding surfaces on that plate. In the Sharks the skull is not so definitely marked off from the spinal column. The apertures of the aqueductûs vestibuli in the Rays are not situated in a median depression such as is observable in the Dog-fish and in all the Sharks. The articular surface in the auditory region for the hyomandibular is sometimes borne on a projecting process, sometimes on the general level of the lateral surface. Sometimes in the Rays there is a smaller articulation behind for the first branchial arch.

The upper and lower jaws - the palatoquadrate and Meckel's cartilage-are connected with the skull through the intermediation of a hyomandibular cartilage (Fig. 823, hy. mn.; Fig. 846, h. m.). The skull is thus of the hyostylic type as regards the mode of suspension of the jaws. In the Sharks the palatoquadrate has a process (absent in the Rays) for articulation with the base of the skull in the pre-orbital region. In Hexanchus and Heptanchus (Fig. 847) there is in addition to this a prominent postorbital process of the palatoquadrate for articulation with the post-orbital region of the skull (amphistylic arrangement). Cestracion is also in a sense amphistylic; the palatoquadrate is firmly united with the skull, articulating with a groove on the base, and the hyomandibular takes only a small share in the suspension of the jaws. At the sides of the mouth in all Elasmobranchs are a series of labial cartilages, usually two pairs above and one pair below. Attached to the hyomandibular is a thin plate of cartilage-the spiracular (Fig. 846, sp.)-which supports the anterior wall of the spiracle.

The hyoid arch proper is in most of the Elasmobranchs connected at its dorsal end with the hyomandibular-sometimes at its distal extremity, sometimes near its articulation with the skull; but in some Rays it is not so related, but articulates separately and independently with the skull behind the hyomandibular, and in the genera Hypnos and Trygonorhina it articulates with the dorsal portion of the first branchial arch. In the 
Sharks the hyoid is usually relatively massive; in the Rays it is smaller, and in most cases closely resembles the branchial arches, and bears similar cartilaginous rays; a larger or smaller median element, or basihyal, is present in all cases.

There are always five pairs of branchial arches except in Hexanchus and Chlamydoselachus, which have six, and Heptanchus, in which there are seven. Their dorsal ends are free in the Sharks,

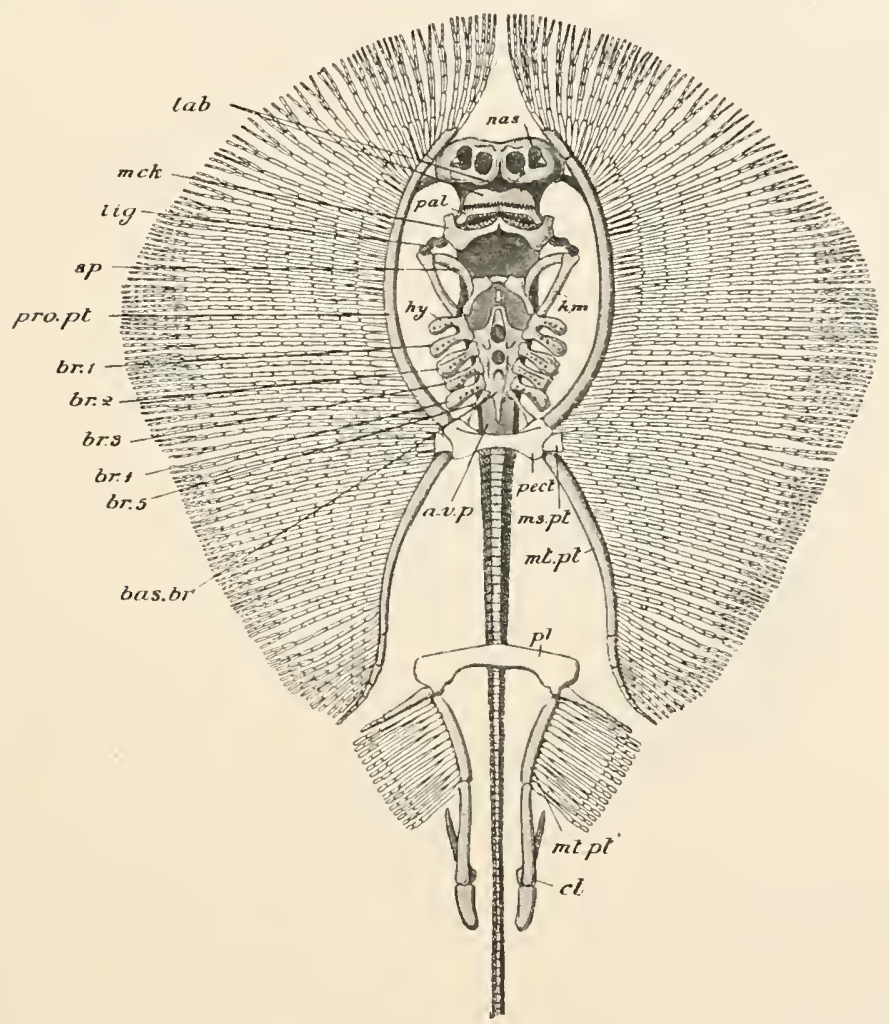

FIG. 846.-Skeleton of Sting-Ray (Urolophus), ventral view, $a . v . p$. anterior vertebral plate; bas. br. basibranchial plate; br.1-br.5 branchial arches. (The branchial rays are not represented, the round dots indicating their articulations with the arches.) $\mathrm{cl}$. skeleton of clasper; $h . m$. liyomandibular; $h y$. hyoid arch ; lab. labial cartilage; lig. ligament connecting the hyomandibular with the palatoquadrate and Meckel's cartilage ; $m c h$. Meckel's cartilage ; $m s$. pt. mesopterygium, and $m t$. $p t$. metapterygium of pectora! fin ; mt. $p t^{\prime}$. metapterygium of pelvic fin; nas. nasal cartilage; pal. palatofuadrate; pect. pectoral arch; $p l$. pelvic arch; pro. pt. propterygium; $s p$. spiracular cartilage.

articulated with the anterior vertebral plate of the spinal column in most Rays. Externally they bear a series of slender cartilaginous branchial rays. The median ventral elements of the branchial arches are usually more or less reduced, and in some cases are represented by a single basi-branchial plate (Fig. 846 bas. br.). In the Rays the fifth branchial arch articulates with the pectoral arch, a connec- 
tion which is absent in the Sharks. A series of slender cartilages, probably modified branchial rays - the extra-branchial cartilagesabsent as such in some Dog-fishes and Rays, support the branchial apertures.

The pectoral arch (Figs. 825, 846, pect.) consists of a single cartilage, with, however, in most of the Sharks, a mesial flexible portion by which it is divided into right and left halves. Each lateral half consists of a dorsal scapular and a ventral coracoid part, the two being separated by the articular surfaces for the basal cartilages of the fin. In the Rays, but not in the Sharks, the dorsal ends of the pectoral arch are connected with the spinal column (anterior vertebral plate) by a distinct articulation, the portion of the arch on which the articular surface is situated sometimes forming an independent cartilage (supra-scapula). In Hep-

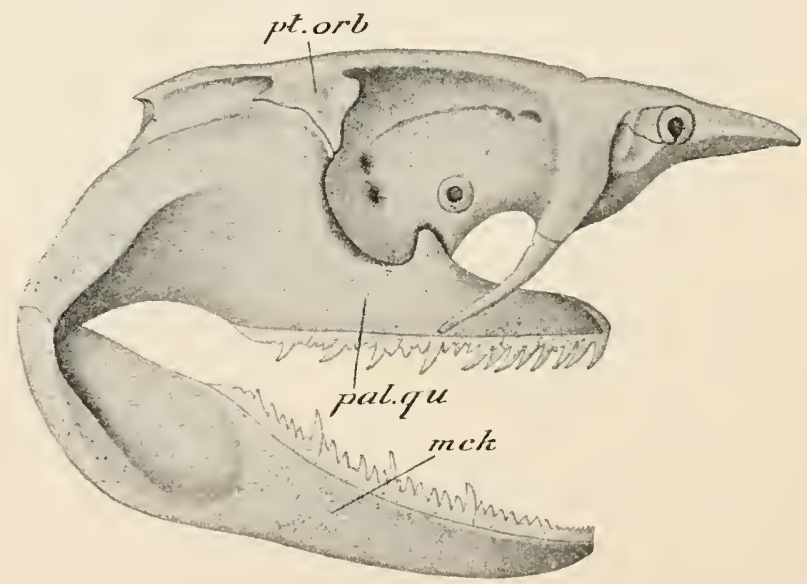

FIG. 847.-Lateral view of the skull of Heptanchus. mck. Meckel's cartilage; pal. qu. palatoquadrate; $p t$. orb. post-orbital process of the cranium, with which the palatoquadrate articulates. (After Gegenbaur.)

tanchus a small median ventral element may represent the sternal apparatus of the Amphibia.

The basal pterygiophores of the pectoral fin are typically three, pro-, meso-, and meta-pterygium (Figs. 825 and 846), but there are sometimes four, and the number may be reduced to two. The pro- and meta-pterygia are divided in the Rays (Fig. 846) into several segments, and the former articulates, through the intermediation of a cartilage termed the antorbital, with the olfactory region of the skull.

The pelvic $\operatorname{arch}(p l$.$) is usually, like the pectoral, a single cartilage,$ but in some exceptional cases it consists of two lateral portions. In some cases a median epipubic process projects forwards from the pelvic arch, and frequently there is on each side a prepubic 
process. A lateral iliac process, which becomes highly developed in the Holocephali, is sometimes represented, and may attain considerable dimensions. The pelvic fin has usually two basal cartilages, representing the pro- and meta-pterygia, but the former is often absent. In the male special cartilages attached to the metapterygia support the claspers. With the basal cartilages of both pectoral and pelvic fins are connected a number of jointed cartilaginous fin-rays supporting the expanse of the fin.

The arrangement of the muscles is simple. The trunk-muscles are divided into a pair of dorsal and a pair of ventral divisions, each composed of many myomeres with intercalated myocommata (Fig. 768, p. 68), following a metameric arrangement. The ventral part, where it forms the muscles of the wall of the abdominal cavity, is composed externally of obliquely running fibres, and represents one of the two oblique muscles of the abdomen of higher forms. Mesially this passes into a median band of longitudinally running fibres corresponding to a primitive rectus. The muscles of the limbs are distinguishable into two main sets-those inserted into the limb-arch and those inserted into the free part of the appendage. The latter, according to their insertion, act as elevators, depressors, or adductors. A series of circular muscles pass between the cartilages of the visceral arches and, when they contract, have the effect of contracting the pharynx and constricting the apertures. A set of muscles pass between the various arches and act so as to approximate them; and a broad sheet of longitudinal fibres divided into myomeres extends forwards from the shoulder-girdle to the visceral arches.

Electric organs-organs in which electricity is formed and stored up, to be discharged at the will of the Fish-occur in several Elasmobranchs. They are best developed in the Electric Rays (Torpedo and Hypnos, Fig. 848), in which they form a pair of large masses running through the entire thickness of the body, between the head and the margin of the pectoral fin. A network of strands of fibrous tissue forms the support for a number of vertical prisms, each divided by transverse partitions into a large number of compartments or cells. Numerous nerve-fibres pass to the various parts of the organ. These are derived mainly from four nerves, which originate from an electric lobe of the medulla oblongata, with a branch from the trigeminal. By means of the electric shocks which they are able to administer at will to animals in their immediate neighbourhood, the Torpedo-Rays are able to ward off the attacks of enemies and to kill or paralyse their prey. In the other Rays in which the electric organs are developed they are comparatively small organs situated at the sides of the root of the tail. In all cases the cells are formed from metamorphosed muscular fibres.

Luminous organs by the agency of which a phosphorescent 
light is produced occur on the surface of a few pelagic Elasmobranchs.

Digestive System.-Teeth are developed on the palatoquadrate and on Meckel's cartilage. They are arranged in several parallel rows, and are developed from a groove within the margin of the jaw, successive rows coming to the front, and, as they are worn out, falling off and being replaced by others. In the Sharks the

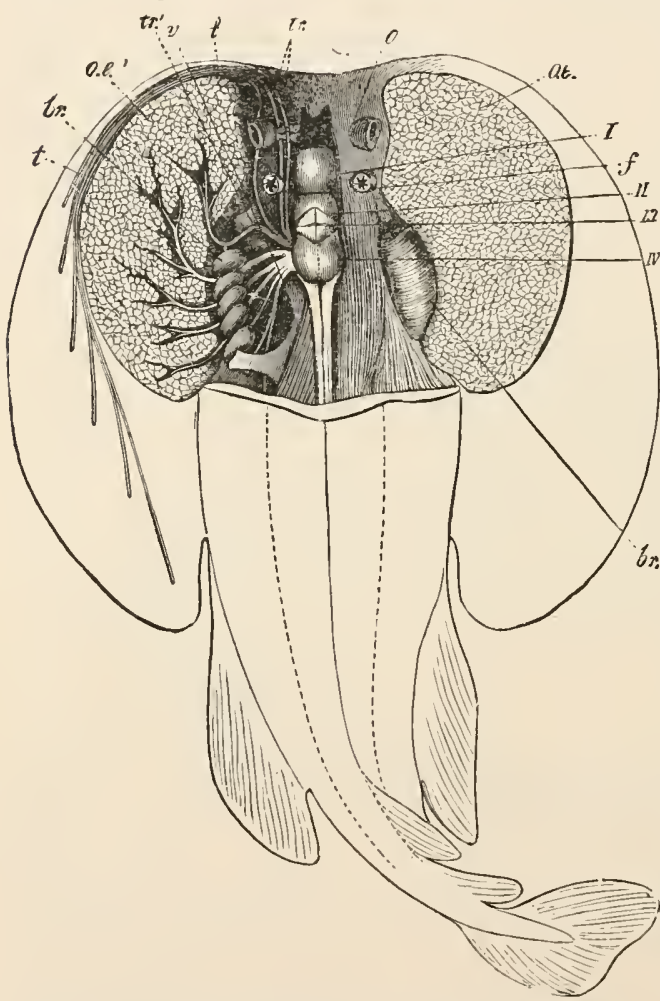

FIa. 84.,-A Torpedo-Ray with the electric organs dissected out. On the right the surface only of the electric organ $(O . E$.$) is shown, on the left the nerves passing$ to the organ are shown. The roof of the skull is removed to bring the brain into view. br. gills ; $f$. spiracle; $o$. eyes ; $t r$. trigeminal ; $t r^{\prime}$. its electric branch ; $v$, vagus; $I$, fore-brain; $I I$, midbrain; $I I I$, cerebellum; $I V$, electric lobe. (From Gegenbaur.) teeth are usually large, and may be long, narrow, and pointed, or triangular with serrated edges, or made up of several sharp cusps; in the Rays, however, the teeth are more or less obtuse, sometimes, as in the Eagle-Rays, forming a continuous pavement of smooth plates covered with enamel, adapted to crushing b: food consisting of such objects as Shell-fish and the like. The Sharks have a prominent tongue supported by the median basihyal; this is entirely or almost entirely absent in the Rays. The various divisions of the enteric canal are similar in all the members of the class to what has already been described in the case of the Dog-fish. A spiral valve is always present in the large intes-

tine, though its arrangement varies considerably in the different families. In some cases (e.g. Carcharias), the fold is not a spiral one, but, attached by one edge in a nearly longitudinal line to the intestinal wall, is rolled up in the shape of a scroll. A pair of pyloric cæca occur in Lamargus. Appended dorsally to the rectum is a median glandular cæcum, the rectal gland. The rectum always terminates in a cloaca, into which the urinary and genital 
ducts also lead. There is always a voluminous liver and a welldeveloped pancreas.

A thyroid lies in the middle line behind the lower jaw. A representative of the thymus lies on either side, a little below the upper angles of the branchial clefts.

The respiratory organs of the Elasmobranchii always have the general structure and arrangement already described in the case of the Dog-fish. In the Rays the water of respiration is taken in mainly through the spiracles; in the Sharks through the mouth.

In addition to the gills supported on the hyoid and branchial arches there is also in the Notidanidæ a gill on the anterior side of the spiracular cleft-the spiracular gill-represented in many others by a rete mirabile or network of blood-vessels (pseudobranch). In Selache (the Basking Shark) there are a series of slender rods, the gill-rakers, which impede the passage outwards through the branchial clefts of the small animals on which those Sharks feed.

Blood-system.-The heart has, in all essential respects, the same structure throughout the group. The conus arteriosus is always contractile, and contains several rows of valves. The general course of the circulation is the same in all (see p. 88), with some variation in the precise arrangement of the vessels. In some of the Rays the ventral aorta and the roots of the afferent vessels are partly enclosed in the cartilage of the basi-branchial plate.

The brain attains a much higher stage of development than in the Cyclostomata. The fore-brain greatly exceeds the other divisions in size. In Scymnus there are two widely-separated parencephalic lobes or cerebral hemispheres containing large lateral ventricles. In other genera there is at most, as in the Dog-fish, a median depression of greater or less depth, indicating a division into two lateral portions. In Scyllium, as already pointed out, there is a median prosocole which gives rise anteriorly to two lateral ventricles, or paracœles, and the same holds good of Rhina and Acanthias. In most Rays there is only a very small prosocœle without anterior prolongations; in Myliobatis this is absent. The olfactory bulbs are of great size, in some cases with short and thick, in others longer and narrower, stalks. In Scyllium, Rhina, and Acanthias, as well as in Scymnus, they contain ventricles (rhinocoles) continuous with the paracoeles; in the Rays they are solid.

The diencephalon is of moderate extent. On its lower aspect are a pair of rounded lobi inferiores, which are of the nature of dilatations of the infundibulum, and a saccus vasculosus, which is a diverticulum of the infundibulum; directly below the saccus vasculosus lies the hypophysis. The epiphysis is long and narrow.

In the hind-brain the cerebellum is relatively greatly elongated and overlaps the optic lobes and sometimes also the diencephalon in front, while behind it extends over the anterior part of the medulla oblongata. It usually contains a cerebellar ventricle or 
epicoele. The medulla is elongated in the Sharks, shorter and more triangular in the Rays. The Electric Rays are characterised by the presence of the electric lobes, rounded elevations of the floor of the fourth ventricle.

Organs of Sense.-Integumentary sense-organs (neuromasts, p. 105) are highly developed in the Elasmobranchs. They are supplied, as already mentioned, by branches of the nerves of what is known as the lateral system, comprising, in addition to the lateralis, nerves in relation with the facial and sometimes the glossopharyngeal. These integumentary sense-organs occur in the interior of a continuous system of closed tubes, the sensory tubes, more rarely of open grooves. The chief canals of this system are a lateral-line canal, running along the middle of each side of the body, which is continuous with certain canals in the head: these communicate with the exterior at intervals by small pores. In addition to the canals of the lateral-line system there are a number of isolated canals, the ampullary canals, with neuromasts contained in terminal enlargements or ampulloe; these, which are peculiar to the Elasmobranchs, are most numerous about the snout region. Of similar essential character are the vesicles of Savi which occur in the Electric Rays.

The olfactory organs are a pair of cavities opening on the lower surface of the head, a little distance in front of the mouth, and enclosed by the cartilaginous olfactory capsules of the skull. Their inner surface is raised up into a number of ridges on which the fibres of the olfactory nerves are distributed. The eye has a cartilaginous sclerotic, and is in most cases attached to the inner wall of the orbit by means of a cartilaginous stalk. There appears to be no mechanism providing for accommodation. A fold of the conjunctiva resembling the nictitating membrane, or third eyelid of higher Vertebrates, occurs in some Sharks. The ear consists of the membranous vestibule, which is partly divided into two (utriculus and sacculus), from which arise the three semicircular canals with their ampullæ, and also the aqueductus vestibuli or endolymphatic duct-which opens to the exterior on the dorsal surface of the head. In the Rays the semicircular canals form almost complete circles and open separately into the vestibule by narrow ducts.

Urinogenital Organs.-The kidneys, as already noticed in the account given of the Dog-fish, differ somewhat in their relations in the two sexes. In the male the anterior portion persists in the epididymis, and its duct becomes the spermiduct, while the posterior portion, which is the functional kidney, has a duct or ducts-the ureter or ureters - of its own. In the female there is no direct connection between the reproductive and renal organs; the anterior portion of the kidney may be functional, and its duct persists, opening along with those of the posterior portion. In the male the ureters open into a median chamber-the urinogenital sinus- 
which extends into the cloaca, and receives also the spermiducts: it communicates with the general cavity of the cloaca by a median opening situated on a papilla-the urinogenital papilla. In the female there is a median urinary sinus, into which the ureters open, or the latter may open separately into the cloaca.

Save in certain exceptional cases (e.g. Scyllium), there are two ovaries, varying considerably in form, but always characterised towards the breeding season by the great size of the follicles enclosing the mature ova. The oviducts (Müllerian ducts) are quite separate from the ovaries. The right and left oviducts come into close relationship anteriorly, being united in the middle on the ventral surface of the œsophagus, where each opens by a wide orifice into the abdominal cavity, or both open by a single median aperture. The following part of the oviduct is very narrow ; at one point it exhibits a thickening, due to the presence in its walls of the follicles of the shell-gland. Behind this is a dilated portion which acts as a uterus, and this communicates with the cloaca through a wide vagina. A considerable number of the Elasmobranchii are viviparous, and in these the inner surface of the uterus is beset with numerous vascular villi, while the shell-gland is small or vestigial.

The testes are oval or elongate: the convoluted epididymis is connected with the anterior end by efferent ducts, and from it arises the vas deferens. The latter is dilated near its opening into the urinogenital sinus to form an ovoid sac-the vesicula seminalis. A sperm-sac is sometimes present, opening close to the aperture of the vas deferens. The Müllerian ducts are vestigial in the male.

Impregnation is internal in all the Elasmobranchs with the possible exception of Læmargus (the Greenland Shark), the claspers acting as intromittent organs by whose agency the semen is transmitted into the interior of the oviducts.

In all the Elasmobranchs the ova are very large, consisting of a large mass of yolk-spherules held together by means of a network of protoplasmic threads, with, on one side, a disc of protoplasmthe germinal disc. The process of maturation is similar to that observable in holoblastic ova; one polar body is thrown off in the ovary, the other apparently at impregnation. The ripe ovum ruptures the wall of the enclosing follicle and so passes into the abdominal cavity to enter one of the oviducts through the wide abdominal opening. Impregnation takes place in the oviduct, and the impregnated ovum in the oviparous forms becomes surrounded by a layer of semi-fluid albumen and enclosed in a shell of keratin secreted by the shell-gland. The shell varies in shape somewhat in the different groups: most commonly, as in many Dog-fishes (Fig. 837), it is four-cornered, with twisted filamentous appendages at the angles, by means of which it becomes attached to sea-weeds and the like. In the Skates the filaments are absent. In the Port Jackson Sharks (Cestracion, Fig. 849) it is an ovoid body, the wall 
of which presents a broad, spiral flange. The young Elasmobranch goes through its development enclosed in the shell, until it is fully formed, when it eseapes by rupturing the latter. In the viviparous forms the ovum undergoes its development in the uterus, in which in most cases it lies free-except in some Mustelidx and Carchariidæ, in which there is a close connection between the yolksac of the embryo and the wall of the uterus, folds of the former interdigitating with folds of the latter, and nourishment being thus conveyed from the vascular system of the mother to that of the fotus by diffusion. In some of the viviparous forms a distinct

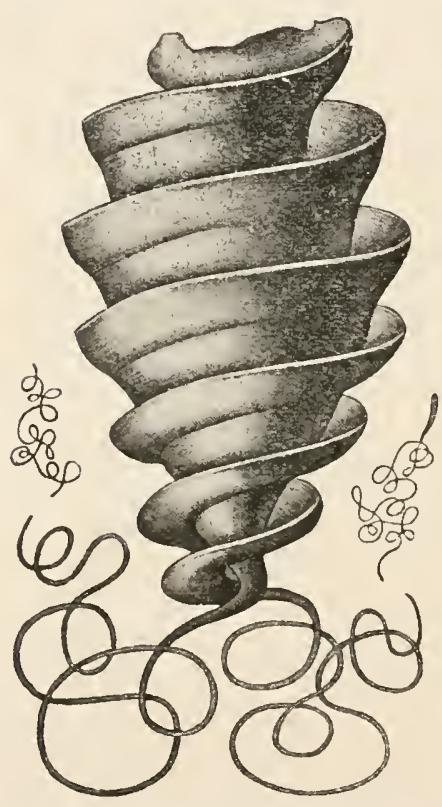

Fit. 849.-Egg-case of Cestracion galeatus. (After Waite.) though very delicate shell, sometimes having rudiments of the filaments, is formed, and is thrown off in the uterus. In the genera Rhinobatus and Trygonorhina, which are both viviparous, each shell encloses not one egg, but three or four. Læmargus is said to differ from all the rest of the Elasmobranchii in having the ova fertilised after they have been deposited, as well as in the small size of the ova.

Development.- Segmentation is meroblastic, ${ }^{1}$ being confined to the germinal dise, which, before dividing, exhibits anœeboid movements. While segmentation is going on in the germinal dise there appear a number of nuclei, the source of which is not certain, in the substance of the yolk. When segmentation is complete, the blastoderm appears as a lens-shaped disc, thicker at one end-the embryonic end. It is found to consist of two layers of cells -an upper layer in a single stratum, and a lower layer several cells deep. A segmentation-cavity appears early among the cells of the lower layer; the lower-layer cells afterwards disappear from the floor of this, the cavity then coming to rest directly on the yolk.

An in-folding (Fig. 850) now begins at the thickened embryonic edge of the blastoderm, which here becomes continuous with the cells of the lower layer. The cavity $(a l)$, at first very small, formed below this in-folding is the rudiment of the archenteron, and the cells lining this cavity above, which form a definite layer, partly derived from the in-folded ectoderm, partly from the cells 
of the lower layer, are the beginning of the definite endoderm. The edge of the in-folding, entitled the embryonic rim, is obviously the equivalent of the dorsal lip of the blastopore in Amphioxus. The endoderm and its underlying cavity soon grow forwards towards the segmentation-cavity. Under the latter appears a floor of lower-layer cells, but the cavity soon becomes obliterated as the archenteron develops.

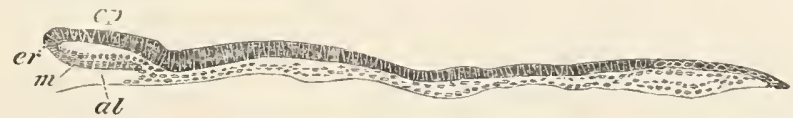

FIG. 850.-Longitudinal section through the blastoderm of a Pristiurus embryo before the medullary groove has become formed, showing the beginuing of the process of in-folding or invagination. al. archenteron; $c p$. ectoderm; $c r$. embryonic rim; $m$. mesoderm. (After Balfour.)

After the formation of the embryonic rim a shield-like embryonic area is distinguishable in front of it, with two folds bounding a groove-the medullary groove. The mesoderm becomes established at about the same time. It is formed from two separate and distinct sources (Fig. 851). Along the edge of the embryonic rim appears a horizontal groove-like depression : this - the external

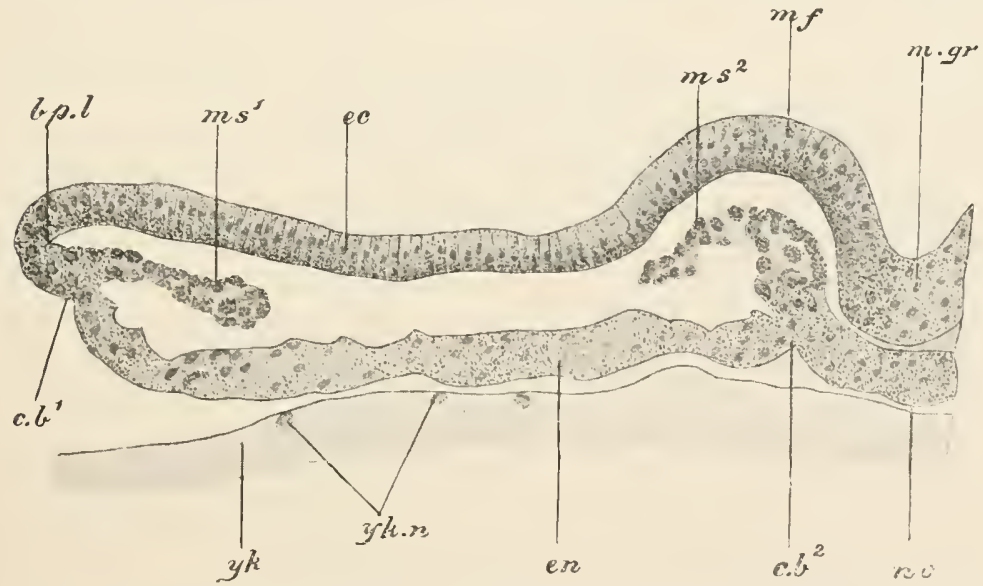

FIG. R51.- Pristiurus, transverse section of blastoderm, showing the formation of the meso. derm. $b p . l$. dorsal lip of blastopore; $c . b .1$ external colomic bay ; $c . b .^{2}$ internal culomic bay ; ec. ectoderm ; en. endoderm ; $m . f$. medullary fold $; m$. gr. medullary groove $m m^{1}{ }^{1}$ external rudiment of mesoderm; $m s .2$ internal rudiment of mesoderm; $n c$. notochord ; $y k$. yolk; $y k$. n. yolk nuclei. (From O. Hertwig, after Rabl.)

coelomic bay $\left(c . b .{ }^{1}\right)$ - marks the line of origin of the peripheral part of the mesoderm $\left(m . s^{1}\right)$, which grows inwards from it as a plate of cells between the ectoderm and the endoderm. The central part of the embryonal mesoderm $\left(m . .^{.}{ }^{2}\right)$ is developed from the endoderm at a point immediately external to the rudiment of the notochord: here also a slight groove-the internal colomic bay 
$\left(c . b .{ }^{2}\right)$-is distinguishable, and from this a plate of mesoderm cells grows outwards. Eventually the peripheral and central plates of mesoderm come into contact and coalesce to form a continnous sheet on each side of the middle line. Though the mesodermal rudiments, peripheral and central, contain no cavities, the grooves (colomic bays) from which their development takes its origin may represent the cavities of the cœlomic sacs of Amphioxus.

As the blastoderm extends over the yolk, the edge forms a ridge
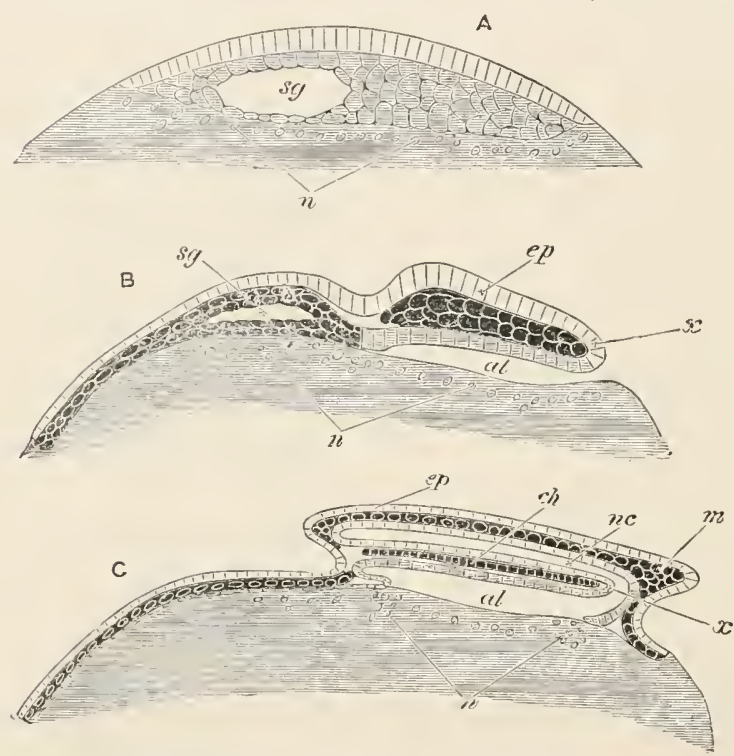

FIG. 85\%-Diagrammatic longitudinal sections of an Elasmobranch embryo. A, seetion of the young blastoderm with seginentation-cavity enclosed in the lower layer cells; $B$, older blastoderm with enbryo in which endoderm and mesoderm are distinctly formed, and in which the alimentary shit has appeared. The scgmentation-cavity is still representerl as being present, thouglı by this stage it has in rfality disappeared. $C$, older blastoderm with embryo in which the neural canal has become formed and is continuous posteriorly with the alimentary canal. Ectoderm without shading; mesoderm and also notochord black with elear outlines to the cells; endoderm and lower layer cells with simple shading. al. alimentary cavity; $c h$. notochord; ep. ectoderm ; $m$. mesoderm; n. nuclei of yolk; $n c$. neurocoele; $s g$. segmentation-cavity ; $x$, point where ectoderm and endoderm become continuous at the posterior end of the embryo. (From Balfour.)

temporary passage of communication between the medullary canal and the archenteron-the neurenteric passige.

The ectoderm gives rise, as in Vertebrates in general, not only to the epidermis and the central nervous system, but also to the peripheral nervous system, the lining membrane of the olfactory sacs, the lens of the eye, and the lining membrane of the auditory labyrinth of the mouth, and of the outer portions of the cloaca and gill-clefts.

continuous with

the embryonic rim. The latter assimes the form of two prominent caudal swellings (Fig. 853, cd.). The medullary groove meanwhile deepens, and its edges grow over so as to form a canal (Fig. 852, C; Fig. 854). The union takes place first in the middle, the anterior and posterior parts (Fig. 85t, neur.) remaining open for a while. When the posterior part closes, it does so in such a way that it encloses the blastopore, and there is thus formed, as in Amphioxus and Ascidians, a 
The notochord (Fig. 852, ch.) is developed as a cord of cells derived from the lower layer.

Each lateral sheet of mesoderm, soon after its formation by the coalescence of the peripheral and central rudiments, becomes divided by the development of a horizontally directed cleft-like space in its interior. The inner part of each sheet then separates from the outer by the formation of a longitudinal fissure. The former, which is known as the vertebral plate, becomes divided by transverse fissures into a number of squarish masses, the protovertebrce or mesodermal somites. The outer part forms a broad plate, the lateral

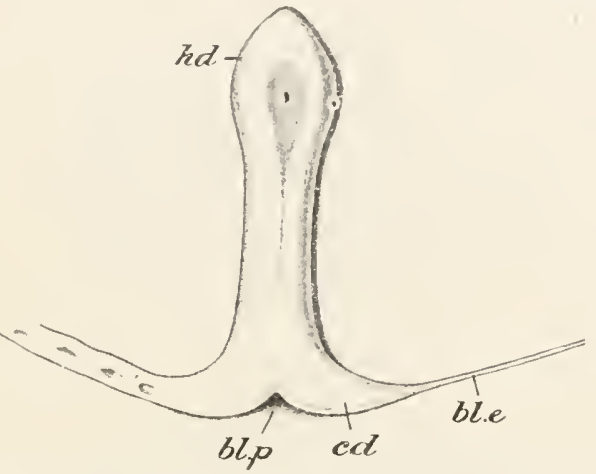

FIG. 853.-Embryo of Scyllium canicula with the tailswellings well marked and the medullary groove just beginning. $u l$. $e$. edge of blastoderm; $b l . p$. blastopore; cd. caudal swellings; hd. head. (After Sedgwick.)

plate. The lateral plate consists of two layers, a dorsal or somatic, and a ventral or splanchnic, and the cavity between them is the beginning of the coelome. The protovertebræ send off cells round the notochord to form the bodies of the vertebræ, the remainder

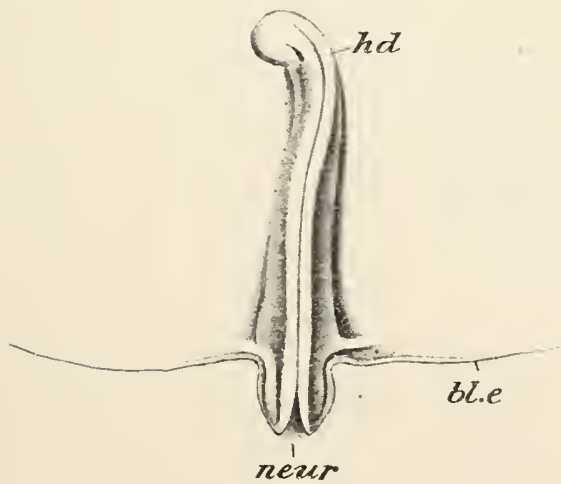

FiG. 854--Embryo of a Ray with the medullary groove closed except at the hind end. The notcherl embryonic part of the blastoderm has grown faster than yolk. bl. e. edge of blastoderm; $h d$. head ; neur. unenclosed part of the neurocœe. (After sedgwick.) the rest and come to project over the surface of the giving rise to the muscles of the voluntary system. An isthmus of mesoderm cells (nephrotome), which still connects each protovertebra with the lateral plate and contains a prolongation of the cavity, gives rise to the pronephric duct and tubules. The lateral plates eventually unite ventrally, and their cavities coalesce to form the body - cavity. The parts derived from the mesoderm are the system of voluntary muscles, the dermis, the inter-muscular connective-tissue, the endoskeleton, the muscular and connective-tissue layers of the alimentary canal, the vascular system, and the generative organs. The segmentation of the mesoderm does not at first extend into 
the head, but, on the formation of the gill-clefts, a series of mesodermal segments appear, the cells of which give rise to the cartilages and muscles of the branchial, hyoid, and mandibular arches, and probably also of the palatoquadrate and the eye.

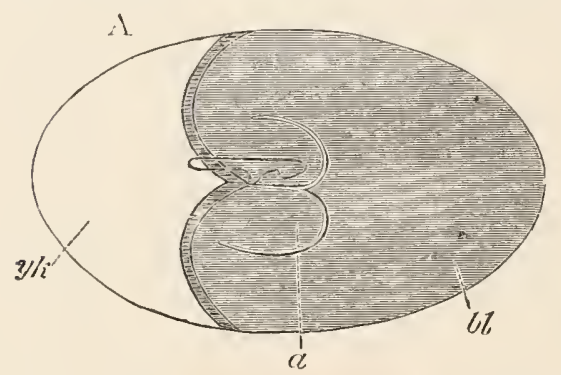

By degrees the body of the young Fish becomes moulded on the blastoderm. This is effected by the formation of a system of folds, anterior, posterior, and lateral, which grow inwards in such a way as to separate off the body of the embryo from the rest of the blastoderm enclosing

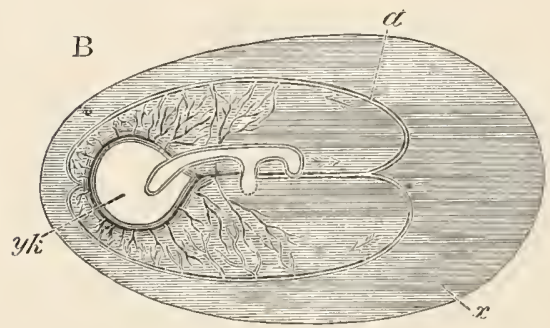
the yolk. As the folds approach one another in the middle, underneath the embryo, they come to form a constriction connecting the body of the embryo with the yolk enclosed in the extra-embryonic part of the blastoderm. 'The process may be imitated if $\mathbb{C}$ we pinch off a portion of a ball of clay, leaving only a narrow neck connecting the pinched-off portion with the rest. The body of the embryo is thus gradually folded off from the yolk-sac and comes to be connected with it only by a narrow neck or yolkstalk (Fig. 855). The head and tail of the young Fish soon undergo differentiation and a series of involutions at the sides of the neck (Fig. 856) form the branchial clefts and spiracle. A number of very delicate

Three views of the developing egg the vessels of the yolk-sac. The part (bl.) is the blastoderm, the white part the uncovered yolk. A, young stage with the embryo still attached at the edge of the blastoderm; $B$, older stage with the yolk not auite enclosed by the blastodernı ; $C$, stage after the complete closure of the yolk. $a$. arterial trunks of yolk-sac; $b l$. blastoderm; $v$. venous trunks of volk-sac; $y$. point of closure of the yolk-blastopore; $x$, portion of the blastoderm outside tlie arterial sinus terminalis. (From Balfour.)

filaments (Figs. 856, 857) grow out from these apertures and become greatly elongated; these are the provisional gills, which atrophy as the development approaches completion, their bases alone persisting to give rise to the permanent gills. The great 
derelopment of these gill-filaments in the embryos of some viviparous forms suggests that, in addition to their respiratory functions, they may also serve as organs for the absorption of nutrient fluids secreted by the villi of the uterine wall. ${ }^{1}$ The fins, both paired and

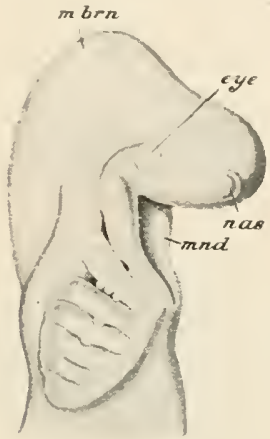

FIG. 856.--Side view of head of embryo of Scyllium canicula, with the rudiments of the gills on the first and second branchial arches, eye, eye m. brn. nid-brain; mnd. mandible; nas. nasal sac. (After Sedgwick.)

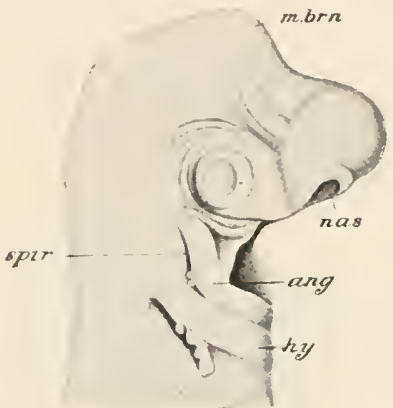

FIG. 857 .- Side view of the head of Scyulum canicula at a somewhat later stage. The gill-filaments have increased in number and are present on the mandibular arch. ang. angle of the jaw: hy. hyoid: $m$. brn. midhrain : nas. nasal sac; spir. spiracle. (After sedgwick.

unpaired, appear as longitudinal ridges of the ectoderm enclosing mesoderm. In some Elasmobranchs the paired fins are at first represented on each side by a continuous ridge or fold, which only subsequently becomes divided into anterior and posterior portions - the rudiments respectively of the pectoral and pelvic fins. Into these folds penetrate a series of buds from the protovertebræ: these, the muscle-buds, give rise to the fin-muscles; at first, from their mode of origin, they present a metameric arrangement, but this is in great measure lost during development.

Ethology and Distribution.-The habits of the active, fierce, and voracious Sharks, which live in the surface-waters of the sea, waging war on all and sundry, contrast strongly with those of the more sluggish Rays, which live habitually on the bottom. usually in shallow water, and feed chiefly on Crustaceans and Molluses, with the addition of such small Fishes as they can capture. As a group, the Elasmobranchs, more particularly the Sharks, are distinguished by their muscular strength, the activity of their movements, and also by the acuteness of their senses of sight and smell.

Nearly all are marine: some ascend rivers: a very few live habitually in fresh water. The only deep-water Elasmobranch

1 In a spscies of Trygon a number of the villi of the uterus project into the pharynx of the fnetus through the spiracles, and nourishment is probably. received by this means.

VOL. II 
known is a species of Ray, which extends to a depth of over 600 fathoms.

None of the Elasmobranchs are of very small size, and comprised among them are the largest of living Fishes: the harmless Basking Sharks (Selache) sometimes attain a length of 35 feet or more, the formidable Great Blue Shark (Carcharodon) sometimes reaches 40 feet, and some of the Rays also attain colossal dimensions. In this respect, however, recent Sharks and Rays are far behind some of the fossil forms, certain of which, if their general dimensions were in proportion to the size of their teeth, must have reached a length of as much as 60 feet.

The earliest fossil remains of Elasmobranch Fishes that have been found occur in rocks belonging to the Upper Silurian period. Throughout the Palæozoic epoch the Elasmobranchs constituted a very important section of the fauna-a large proportion of the fishremains that have been found in Palæozoic formations being the remains of Elasmobranchs, mainly in the form of spines and teeth. Most of the Palæozoic Elasmobranchs were characterised by a great development of the exoskeleton. The teeth differ from those of existing forms in being provided with broad bases by means of which they articulated together, and in various groups there is a union of the teeth by the coalescence of their bases so as to form broad crushing plates. A similar union is not uncommon between the parts of the general exoskeleton, a good many Palæozoic Sharks having been encased in an armour of solid plates formed by such a coalescence. In the endoskeleton there is to be observed among the fossil Elasmobranchs a gradual advance in the degree of calcification of the spinal column from the Palæozoic forms onwards, the Protoselachii alone among existing forms representing in this respect the condition which seems to have prevailed in the most ancient nembers of the class.

The group (Cestracionts) now represented by two or three species of Port Jackson Sharks seems to have been very abundant in Palæozoic times. Mitsukurina with long rostrum, occurring off the coasts of Japan and Australia, dates from the Cretaceous (Scapanorhynchus).

The extinct Pleuracanthea, together with Cladoselachus, which, as briefly stated in the sketch of the classification, differ from the other known members of the class in the structure of the fins and other points, range from the Devonian to the Permian, and are perhaps also represented in the Trias.

\section{Sub-Class II.-Holocephali.}

The existing representatives of the Holocephali are included under the single family Chimaride, containing three generaChimara, Callorhynchus, and Harriotta. Even taking in fossil forms, the group is a very small one; it agrees in many funda- 
mental characteristics with the Elasmobranchii, and is sometimes included in that sub-class. Of the recent genera, Chimæra, the so-called "King of the Herrings" (Fig. 858, A), is found on the coasts of Europe, Japan, and Australia, the west coast of North America, and at the Cape of Good Hope; Callorhynchus $(B)$ is tolerably abundant in the South Temperate seas; Harriotta is a deep-sea form.

External Characters. - The general form of the body is Shark-like, but the large, compressed head and small mouth are

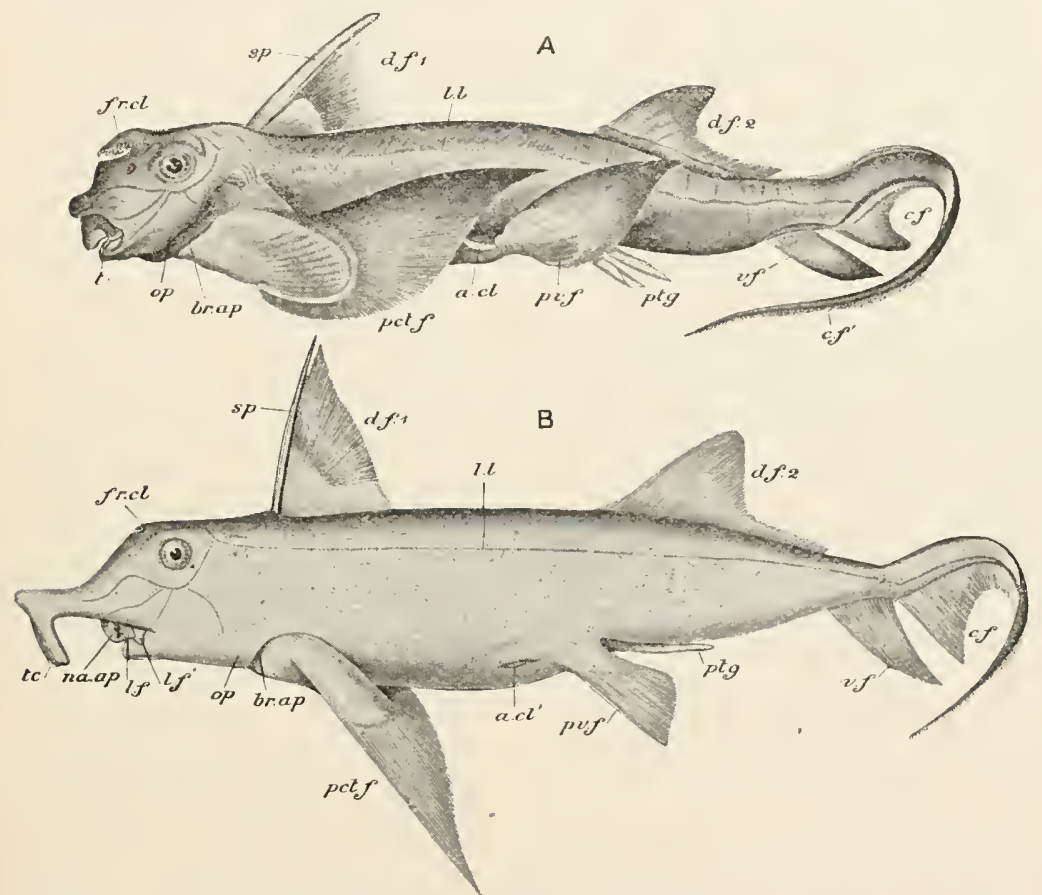

FIG, 858.-A, Chimara monstrosa ; $B$, Callorhynchus antarcticus. a. $c l$, anterior

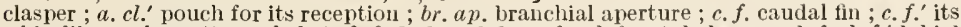
whip-like prolongation ; $d . f .1, d . f .2$, dorsal fins ; $f r . c l$. frontal clasper ; $l . f ., l . f$. ' labial folds ; $l$. $l$. lateral line ; na. ap. nasal aperture : op. operculum; pct. $f$. pectoral fin ; $p t g$. pterygopodia; $p v . f$. pelvic fin; $t$. teeth; $t c$. tactile tlap; $v$. $f$. ventral fin. ( $A$ after Cuvier.)

strikingly different from the depressed, shovel-shaped head and wide mouth of most Selachians. The mouth is bounded by lip-like folds, two of which $\left(B, l . f ., l . f .^{\prime}\right)$, placed laterally and supported by labial cartilages, resemble the folds in which the premaxillæ and maxillæ of many Bony Fishes are enclosed : a third fold, external to and concentric with the mandible, is also supported by labial cartilages and has the appearance of a second or external lower jaw. In Chimæra the snout is blunt, in Harriotta long and pointed : in Callorhynchus it is produced into a rostrum, from the end of which 
depends a large cutaneous flap $(B, t c)$ abundantly supplied with nerves and evidently serving as an important tactile organ.

A still more important difference from Elasmobranchs is the possession of only a single external branchial aperture (br. ap.), owing to the fact that a fold of skin, the operculum (op.), extends backwards from the region of the hyoid arch and covers the true gill-slits, which thus come to open into a common chamber situated beneath the operculum and communicating with the exterior by a single secondary branchial aperture placed just anterior to the shoulder-girdle: there is no spiracle. Equally characteristic is the circumstance that the urinogenital aperture is distinct from and behind the anus, there being no cloaca.

There are two large dorsal fins (d.f. $1, d . f .2)$ and a small ventral $(v . f$.$) ; the caudal fin (c . f$.$) is of the ordinary heterocercal type$ in the adult Callorhynchus, but in the young (Fig. 864) the extremity of the tail proper is not upturned, and the fin-rays are arranged symmetrically above and below it, producing the form of tail-fin called diphycercal. In Chimæra the tail may be produced into a long whip-like filament $\left(c . f^{\prime}\right.$.). The pectoral (pct.f.) and pelvic $(p v . f$.$) fins are both large, especially the former.$

In the male there is a horizontal slit $\left(B, a . c l^{\prime}\right.$.) situated a little in front of the pelvic fins; it leads into a shallow glandular pouch, from which can be protruded a peculiar and indeed unique apparatus, the anterior clasper $(A, a . c l$.$) , consisting of a plate$ covered with recurved dermal teeth, to which is added, in Callorhynchus, a plate rolled upon itself to form an incomplete tube. The use of this apparatus is not known. A rudiment of the pouch occurs in the female, although the clasper itself is absent. The male possesses, in addition, a pair of the ordinary pterygopodia or posterior claspers (pig.), and is further distinguished by the presence of a little knocker-like structure, the frontal clasper ( $f r . c l$.$) ,$ on the dorsal surface of the head. In Harriotta the paired claspers are poorly developed, and the frontal clasper is absent.

The lateral line $(l . l$. $)$ is an open groove in Chimæra, a closed tube in Callorhynchus, and there are numerous sensory pits, arranged in curved lines, on the head. The skin is smooth and silvery, and bears for the most part no exoskeletal structures. There are, however, delicate, recurved dermal teeth on the anterior and frontal claspers, and the first dorsal fin is supported by an immense bony spine or dermal defence $(s p$.$) . In the young, moreover, there is$ a double row of small dermal teeth along the back.

Endoskeleton.-The vertebral column consists of a persistent notochord with cartilaginous arches. In Chimæra, but not in Callorhynchus, there are calcified rings (Fig. 859, c. r.) embedded in the sheath of the notochord. The anterior neural arches are fused to form a high, compressed, vertical plate, to which the first dorsal fin is articulated. The cranium (Figs. 860 and 861) has a 
very characteristic form, largely owing to the compression of the region between and in front of the large orbits, which are separated from the cranial cavity only by membrane in Callorhynchus (Fig. 861, or.) ; in Chimæra they lie above the level of the cranial cavity and are separated from one another by a median vertical partition of fibrous tissue (Fig. 860, $i . o . s$ ). At first sight the palatoquadrate, or primary upper jaw, appears to be absent, but a little consideration shows it to be represented by a triangular plate ( $p a l$. qu.) which extends downwards and outwards from each side of the cranium and presents at its apex a facet for the articulation of the mandible. The palatoquadrate is therefore fused with the cranium and furnishes the sole support for the lower jaw; in a word the skull is autostylic. The pituitary fossa (Fig. 861, s.t.) is very deep and inclined backwards; on the rentral surface of the basis cranii is a pit $(p t$.) for the extra-cranial portion of the pituitary body. The posterior portion of the cranial cavity is very high ; the anterior part - containing most of the fore-brain-is low and tunnel-like, and has above it a cavity of almost equal size $\left(N v \cdot j o^{\prime}.\right)$ for the ophthalnic branches of the fifth nerves. The greater part of the membranous labyrinth is lodged in a series of pits on the side-walls of the cranium (a.s.c., p.s.c.), and is separated from the brain by

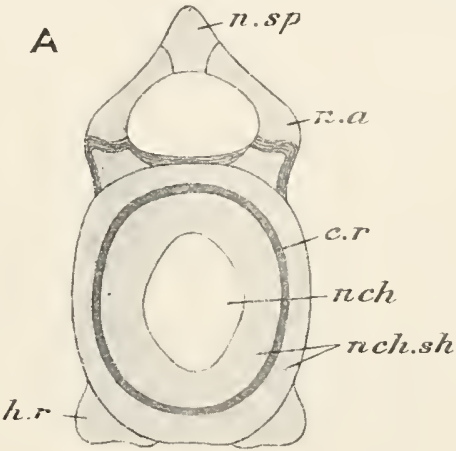

B

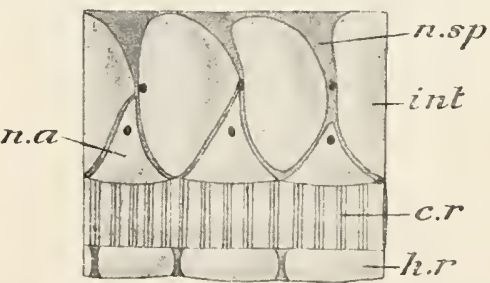

FiG. 859.-Chimæra monstrosa. A, transverse section of the vertebral columm ; $B$, lateral view of the same. $c . r$. calcified ring; $h$. r. hem: 1 ridge ; int. intercalary piece; $n$. a. neural arch; $n c h$. position of notochordal tissue; $n c h$. sh? sheath of notochord; $n$. sp. neural spine. (After Hasse.) membrane only. The occipital region articulates with the vertebral column by a single saddle-shaped surface or condyle (oc. cn.). There is a great development of labial cartilages, particularly noticeable being a large plate which, in Callorhynchus, lies just externally to the mandible, nearly equalling it in size and having the appearance of a secondary or external jaw. In Callorhynchus the snout is supported by three cartilaginous rods growing forward from the cranium, of which one $(r$.) is median and dorsal and represents the rostrum; these, as well as the great lower labial, are represented by comparatively small structures in Chimæra (Fig. $860,16.3$ ). 
The hyoid resembles the branchial arches in form and is little superior to them in size. Above the epihyal (Fig. 860, e. hy.) is a small cartilage $(p h . h y$.), evidently serially homologous with the pharyngobranchials, and therefore to be considered as a pharyngohyal. It represents the hyomandibular of Elasmobranchs, but, having no function to perform in the support of the jaws, it is no larger than the corresponding segments in the succeeding arches. Long cartilaginous rays (op.r.) for the support of the operculum are attached to the ceratohyal.

The first dorsal fin is remarkable for having all its pterygiophores fused into a single plate, which articulates with the coalesced

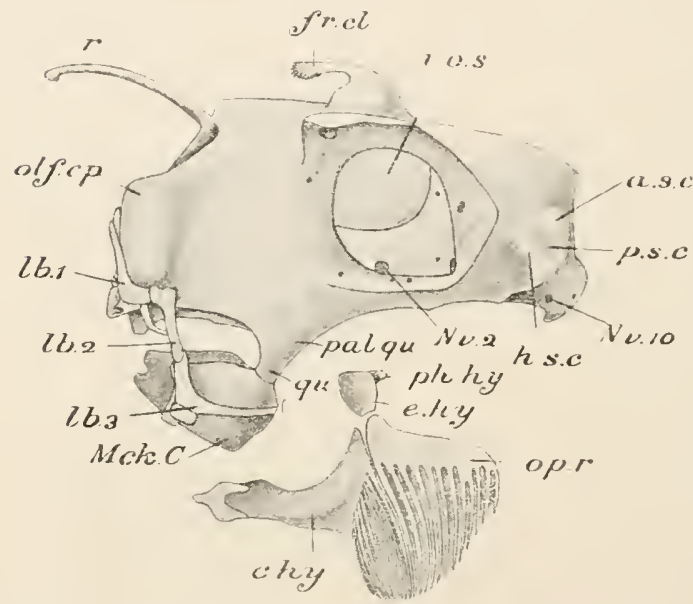

FIG, 860.-Chimara monstrosa, lateral view of skull. a. s. c. position of anterior semicircular canal: $c$. $h y$. ceratohyal; $\rho$. hy. epihyal; $f r$. cl. fontal clasper ; $h$. $s . c$. position of horizontal semicircular canal ; $i .0 .8$. interorbital septum ; $l b .1, l b, 2, l b .3$, labial cartilages ; $M c k$. C. mandible : Nv. 2, optic foramen; $N v$. 10 , vagus foramen; olf.ep. olfactory capsule; op. $r$. opereular rays; $p a l$. qu. palatocuadrate; $p h$. hy. pharyngohyal; $p . s$. $c$. position of posterior semirircular ranal; $q u$. quadrate region; $r$. rostrum. (After Hubrecht.)

The skeleton of the anterior clasper articulates with the pubic region.

Digestive Organs.-The teeth (Fig. 861) are very characteristic, having the form of strong plates with an irregular surface and a sharp cutting edge. In the upper jaw there is a pair of small vomerine teeth (vo.t.) in front, immediately behind them a pair of large palatine teeth ( $p a l . t$.), and in the lower jaw a single pair of large mandibular teeth (mnd. t.). They are composed of vasodentine, and each palatine and mandibular tooth has its surface slightly raised into a rounded elevation of a specially hard substance, of whiter colour than the rest of the tooth, and known as a tritor $(t r$.$) . The stomach is almost obsolete, the enteric canal$ 
passing in a straight line from gullet to anus; there is a welldeveloped spiral valve in the intestine.

Respiratory Organs. - There are three pairs of holobranchs or complete gills borne on the first three branchial arches, and two hemibranchs or half-gills, one on the posterior face of the hyoid, the other on the anterior face of the fourth branchial arch. The fifth branchial arch is, as usual, gill-less, and there is no cleft between it and its predecessor.

The small heart resembles that of the Dog-fish in all essential respects, being formed of sinus venosus, auricle, ventricle, and conus arteriosus, the last with three rows of valves.

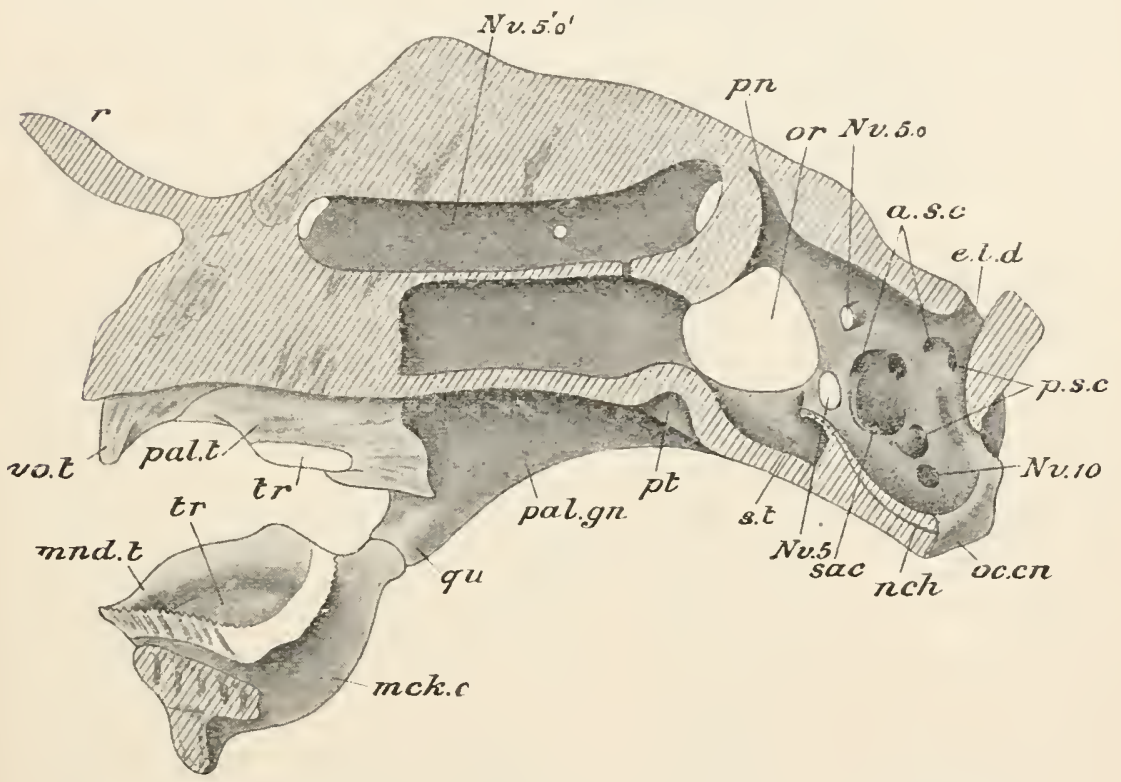

F1G. 861.-Callorhynchus antarcticus, sagittal section of skull ; the labial cartilages are removed. a.s.c. apertures through which the anterior semicircular canal passes irom the cranial cavity into the auditory capsule $; e . l$. $d$. aperture for elldolymphatic duct; $m c k$. $c$. Meckel's cartilage ; mnd. $t$. mandibular tooth ; $n c h$. notochord ; $N v .5$, trigeminal foramen ; $N v, 5, o$, foramen for exit of ophthalmic nerves ; $N v, 5 . o^{\prime}$, canal for ophthalmic nerves with apcrtures of entrance and exit; $N v$. 10 , vagus foramen; oc. cn. occipital condyle; or. fenestra separating cranial cavity from orbit; pal. gn. palatoquadrate; pal. $t$. palatine tooth; $p n$. position of pineal body; $p t$. pit for extra-cranial portion of pituitary body ; p.s.c. apertures through which the posterior semicircular canal passes into the auditory capsule ; $q u$. quadrate region of palatoquadrate; $r$. rostrum; sac. depression for sacculus; $s . t$. sella turcica ; tr. tritor ; vo. t. vomerine teeth.

The brain (Fig. 862), on the other hand, is very unlike that of Scyllium, but presents a fairly close resemblance to that of Scymnus. The medulla oblongata (med. obl.) is produced laterally into large frill-like restiform bodies ( $c p$. rst.), which bound the hinder half of the cerebellum $(\mathrm{cblm}$.$) . The diencephalon (dien.) is extremely$ long, trough-shaped, and very thin-walled, without pronounced optic thalami; it is continued without change of diameter into a 
distinct prosencephalon, which gives off the cerebral hemispheres (crb. h.) right and left. The combined diacole and prosocole (di. coe.) are widely open above in a brain from which the membranes have been removed $(A),{ }^{5}$ but in the entire organ $(B)$ are roofed over by a conical, tent-like choroid plexus (ch. plx. 1). The cavities of the small, spindle-shaped hemispheres (crb. h.), some-
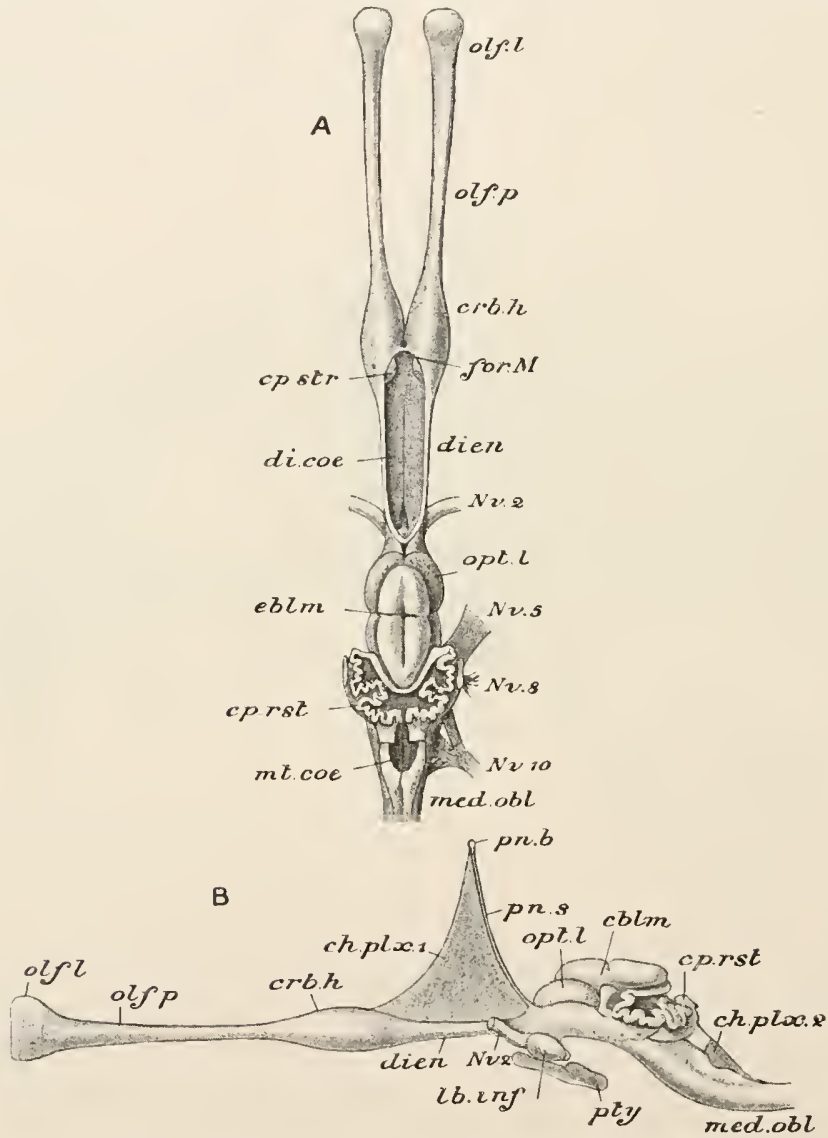

FIG. 862-Callorhynchus antarcticus. A, dorsal view of brain after removal of the membranes; $B$, side view with the membranes in place. cblm. cerebellum; ch. plx. 1 , choroid plexus of fore-brain, and $c h . p l x .2$, of hind-brain; $c p . r s t$. corpus restiforme ; $c p$. str. corpus striatum ; $c r b . h$. cerebral hemisphere; di. coe. diacole ; dien. diencephalon ; for. $M$. foramen of Monro: $l b$. inf. lobus inferior; med. obl. medulla oblongata; mt. coe. metacole; $N v .2$, optic nerve; $N v$. 5 , trigeminal; $N v .8$, aurlitory; $N v$. 10 , vagus; olf. $l$. olfactory bulb; olf. $p$. olfactory peduncle; opt. $l$. optic lobe; $p n$. $b$. pineal bory; $p n$. $s$. pineal stalk; pty. pituitary body.

times regarded as corresponding to olfactory lobes only, communicate with the third ventricle by wide foramina of Monro (for. M.), partly blocked up by hemispherical corpora striata (cp. str.). 
Fach hemisphere is continued in front into a slender thin-walled tube, the olfactory peduncle (olf. $p$.), bearing at its extremity a compressed olfactory bulb (olf. l.).

The optic nerves $\left.(N v .)^{\prime}\right)$ form a chiasma. The pineal body (pn.b.) is a small rounded vesicle borne on a hollow stalk $(p n . s$.) which runs just outside the posterior wall of the tent-like choroid plexus. The pituitary body ( $p l y$. consists of intra- and extra-cranial portions, the former lodged in the sella turcica, the latter in the pit already noticed on the ventral or external face of the skull-floor (Fig. $861, p l$.$) . In advanced em-$ bryos the two are united by a delicate strand of tissue.

Urinogenital Organs.-The kidneys (Fig. 863, kd.) are lobed, deep-red bodies, like those of the Dog-fish, but shorter and stouter. In the male they are much longer than in the female; the anterior portion is massive, and consists mainly of a mass of true renal tubules; it is indistinctly divided into segments: the posterior portion is narrower and also indistinctly segmented; from both parts arise a number of ducts (mesonephric ducts) the majority of which open into the vas defereus, while the last six open into the urinogenital sinus. In the female the ducts all open into a rounded median urinary bladder or urinary sinus, situated between the two oviducal apertures. The female reproductive organs are also constructed on the Elasmobranch pattern, and are chiefly noticeable for the immense size of the shell-glands and of the uteri. But the male organs present certain quite unique characters. The testes

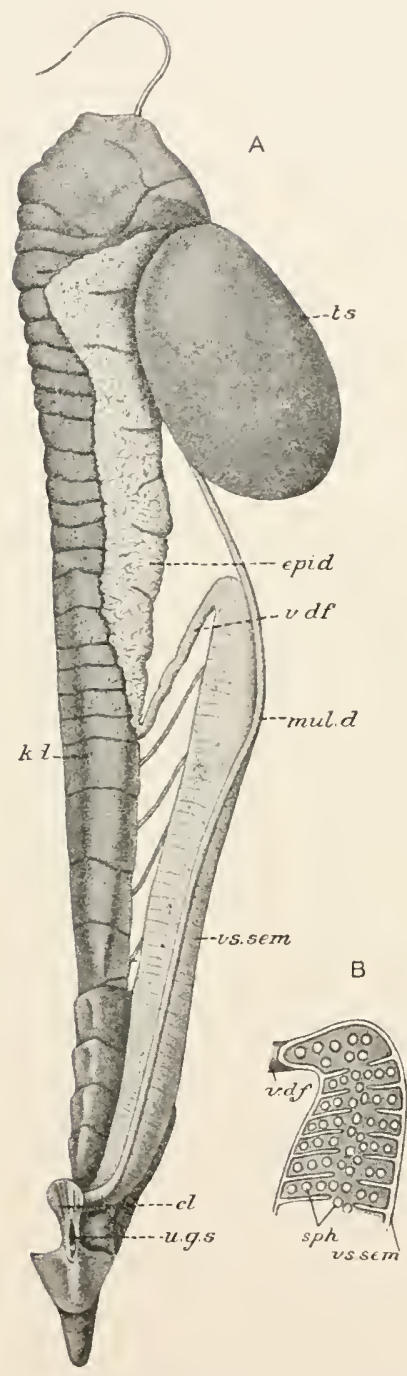

FIG. 863.-Callorhynchus antarcticus. $A$, male urinogenital organs of left sideventral aspect ; $B$, anterior part of vesicula seminalis in section. cl. cloaea ; epir. epididymis; $k d$. kidney ; mul. $d$. II illerian duct; sph. spermatophores; $t s$. testis : u. g. s. opening of urinogenital sinus; $v$ df. vas deferens; vs. sem. resicula seminalis. ( $A$ after Redeke.) 
$(t s$.$) are large ovoid bodies the tubules of which apparently$ do not contain fully-developed sperms, but only immature spermcells. These latter are probably passed through vasa efferentia into the vas deferens, which is coiled in a highly complicated manner to form a body of considerable size, commonly termed the epididymis, closely applied to the surface of the anterior part of the kidney. In this the sperms become aggregated into spermatophores in the form of small ovoidal capsules surrounded by a resistant membrane and full of a gelatinous substance in which bundles of sperms are imbedded. The lower end of the vas deferens $(v . d f$. ) is dilated to form a large cylindrical vesicula seminalis (v. sem.) imperfectly divided into compartments by transverse partitions $(B)$ and filled with a greenish jelly. The spermatophores (sph.) are passed into these compartments and finally make their way through the central passage into the urinogenital sinus $(u . g . s$.$) . The vestigial$ Muillerian ducts (mul. d.) are much more fully developed than in the Dog-fish : they are complete, though narrow, tubes opening in front by a large common aperture into the cœlome, and behind connected with the urinogenital sinus.

Development.-Internal impregnation takes place, and the oosperm becomes surrounded, as in the Dog-fish, by a horny eggshell secreted by the shell-glands. The egg-shell of Callorhynchus (Fig. 864) is of extraordinary size-about $25 \mathrm{~cm}$. in length, or fully five-sixths as long as the abdominal cavity-and the elongated chamber for the embryo is surrounded by a broad, flat expansion covered on one side with yellow hair-like processes, and giving the shell a close resemblance, doubtless protective, to a piece of kelp. The early development resembles that of the Elasmobranchs ; but the yolk becomes divided into nucleated masses which divide into smaller segments, and the smallest break away and become dissolved in a milky nutrient liquid which fills the spaces of the shell: the advanced embryo has elongated gill-filaments $(b r . f$. projecting through the branchial aperture (and probably serving to absorb the nutriment derived from the yolk), a diphycercal tail, and a curiously lobed and nearly sessile yolk-sac $(y k . s$.).

Fossil remains of Holocephali are known from the lower Jurassic rocks upwards. As might be expected, they consist mostly of teeth and of dorsal fin-spines, but in some cases, and notably in Squaloraja, practically the whole of the skeleton is preserved.

\section{Sub-Class III. -Teleostomi.}

In this sub-class are included all the commonest and most familiar Fishes, such as the Perch, Pike, Mackerel, Cod, Sole, Herring, Eel, Salmon, etc., as well as the so-called "Ganoid" Fishes, such as the Sturgeon, Bony Pike (Lepidosteus), and Bow-fin (Amia) of North America, and the Polypterus of the Nile. They 


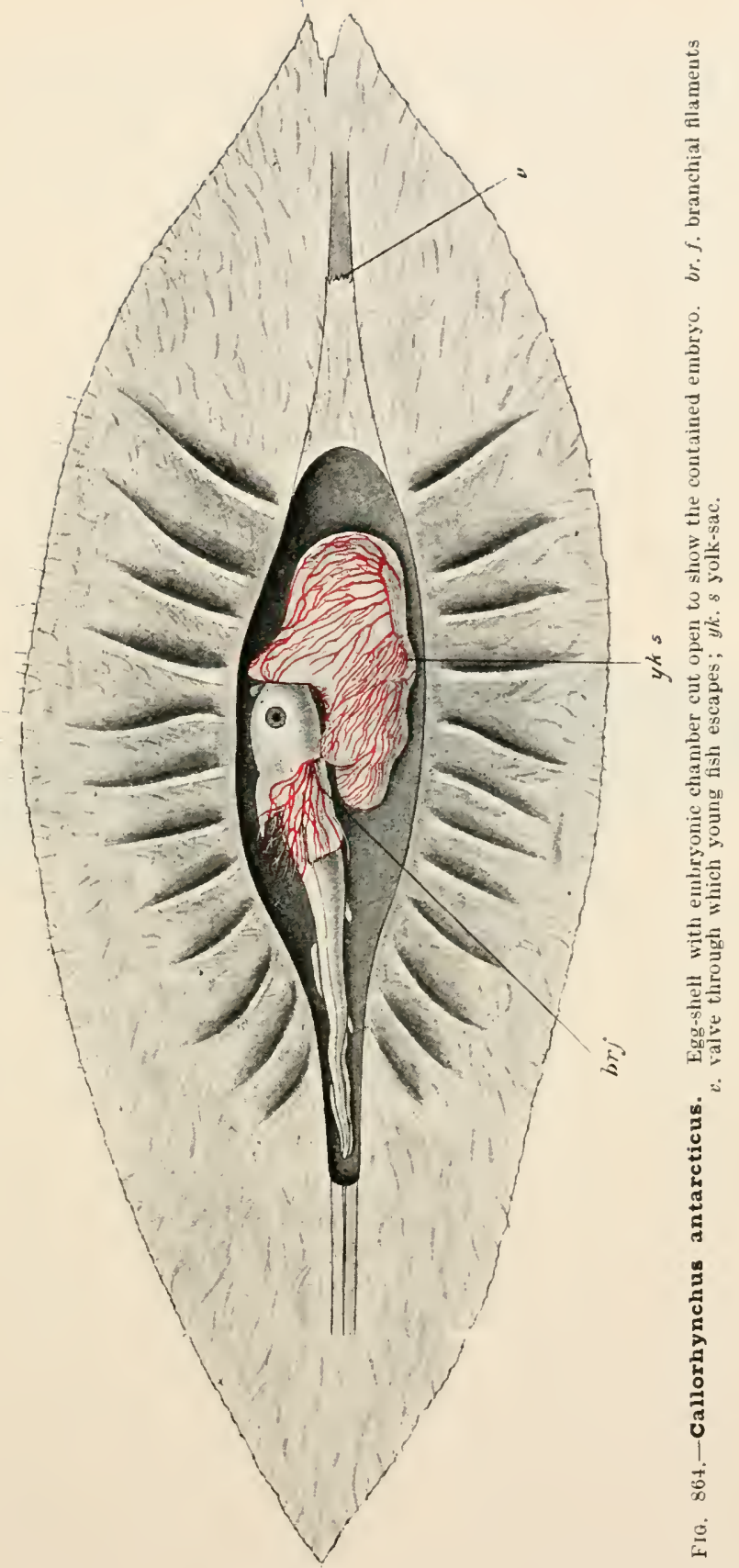


are distinguished from Elasmobranchs and Holocephali by having the primary skull and shoulder-girdle complicated by the addition of investing bones, and by possessing bony instead of horn-like fin-rays. The gills are covered by an operculum; the anus is distinct from the urinary and genital apertures; and the brain has in most cases no cerebral hemispheres, but an undivided prosencephalon.

\section{Example of the Sub-class.-The Brown Trout (Salmo fario).}

The Brown Trout is common in the rivers and streams of Europe, and has been acclimatised in other parts of the world, notably in New Zealand. It varies greatly in size according to the abundance of food and the extent of the water in which it lives : it may attain sexual maturity, and therefore be looked upon as adult, at a length of $18-20 \mathrm{~cm}$. (seven or eight inches), but in large lakes it may

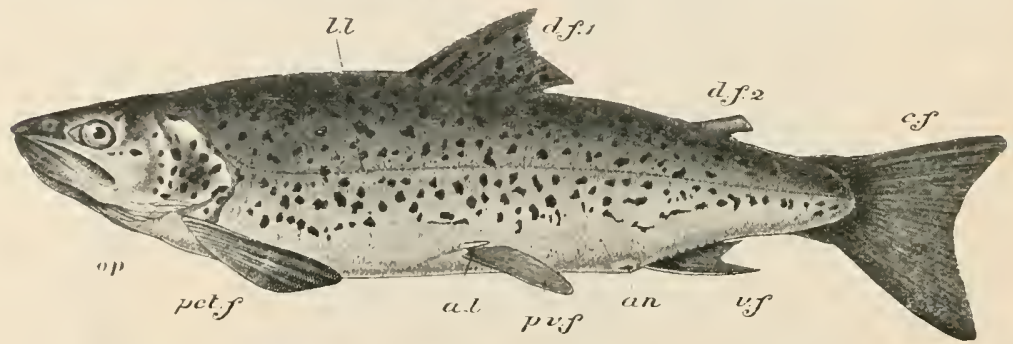

FIG. 865.-Salmo fario, a. $l$. adipose lobe of pelvic fin; an anus; $c . f$. caudal fin; $d . f .1$, first dorsal fin : d.f. 2 , second dorsal or adipose fin ; l.l. lateral line; op. operculum; $p c t . f$. pectoral fin: $p v . f$. pelvic fin; $r$. $f$. ventral fin. (After Jardine.)

grow to nearly a metre in length. Other. species of Salmo, such as the Salmon (S. salar), the Lake Trout (S. ferox), the American Brook Trout (S. fontinalis), are common in the Northern Hemisphere and differ only in details from $S$. fario.

External Characters.-The body (Fig. 865) is elongated, compressed, thickest in the middle, and tapering both to the head and tail. The mouth is terminal and very large; the upper jaw is supported by two freely movable bones, the premaxilla (Fig. 866, pmx.) in front and the maxilla $(m x$.) behind, both bearing sharp curved teeth arranged in a single row. When the mouth is opened a row of palatine teeth is seen internal and parallel to those of the maxilla, and in the middle line of the roof of the mouth is a double row of vomerine teeth. The lower jaw ( $m d$.) is mainly supported by a bone called the dentary and bears a row of teeth : on the throat each ramus of the mandible is bounded mesially by a deep groove. The floor of the mouth is produced into a prominent tongue $(t$. $)$ bearing a double row of teeth. In old males the apex of the lower jaw becomes curved upwards like a hook. 
The large eyes have no eyelids, but the flat cornea is covered by a transparent layer of skin. A short distance in front of the eye is the double nostril ( $n a 1$, na2), each olfactory sac having two external apertures, the anterior one ( $\mathrm{nal}$ ) provided with a flaplike valve. There is no external indication of the ear.

On each side of the posterior region of the head is the operculum (Fig. 865, op.) or gill-cover, a large flap which, when raised, displays the gills; between it and the flank is the large crescentic gillopening, from which the respiratory current makes its exit. The operculum is not a mere fold of skin, as in Holocephali, but is supported by four thin bones the outlines of which can be made out through the skin; they are the opercular (Fig. 866, op.), pre-opercular ( $p$. op.), sub-opercular (s. op.), and inter-opercular (i.op.); the last is attached to the angle of the mandible. The ventral portion of the operculum is produced into a thin membranous extension, the branchiostegal membrane (br. m.), supported by twelve flat, overlapping bones, the branchiostegal rays. The narrow area on the ventral surface of the throat which

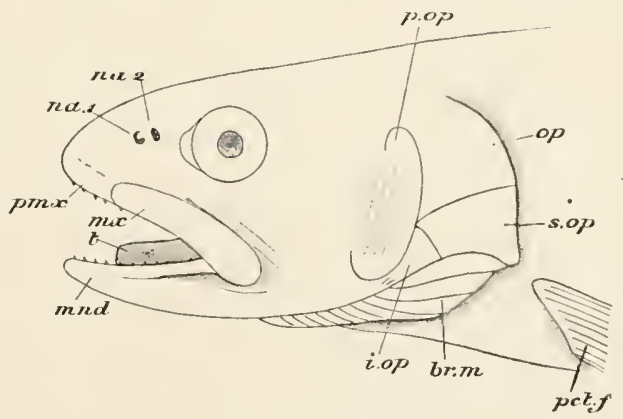

FIG. 866.-Heal of female salmo fario. br. $m$. branchiostegal membrane: $i$. op. interopercular; mnd. mandible; $m x$. maxilla; na1, anterior, and $n a 2$, posterior external nostril; op. opercular; pct. $f$. pectoral fin ; pmx. premaxilla ; $p$. op. preopercular ; s. op. subopercular ; $t$. tongue.

separates the two gill-openings from one another is called the isthmus. The gills, seen by lifting up the operculum, are four red comb-like organs, each having a double row of free gill-filaments ; alternating with the gills are the five vertically elongated gill-slits, opening into the mouth.

The Trout breathes by the drawing in of water through the mouth and its passage outwards through the gill-slits. The inspiration or inward movement of the water is effected by the opercula being moved outwards, the space internal to them thus being widened, and water flowing in through the open mouth to fill the vacuum, the branchiostegal membrane at the same time closing the gill-opening and thus preventing the water from flowing in from behind. Expiration is brought about by the opercula moving inwards and forcing the water out. Owing to the action of a pair of transversely directed membranous folds, the respiratory valves, one attached to the roof, the other to the floor of the mouth, which are so directed as to become expanded and block the passage when water presses on them 
from behind, the water is compelled to make its exit through the gill-slits.

On the ventral surface of the body, at about two-thirds of the distance from the snout to the end of the tail, is the anus (Fig. 865, an.) ; behind it is the urinogenital aperture, of almost equal size and leading into the urinogenital sinus, into which both urinary and genital products are discharged.

The region from the snout to the posterior edge of the operculum is counted as the head; the trunk extends from the operculum to the anus; the post-anal region is the tail.

There are two dorsal fins: the anterior dorsal (Fig. 865, d. f. 1) is large and triangular, and is supported by thirteen bony fin-rays ; the posterior dorsal $(d . f . ?)$ is small and thick, and is devoid of bony supports: it is distinguished as an adipose fin. The caudal fin (c.f.) is the chief organ of locomotion; it differs markedly from that of most Elasmobranchs in being, as far as its external appearance is concerned, quite symmetrical, being supported by fin-rays which radiate regularly from the rounded end of the tail proper; such outwardly symmetrical tail-fins are called homocercal. There is a single large ventral fin $(v . f$.) supported by eleven rays. The pcctoral fin (pct.f.) has fourteen rays and is situated, in the normal position, close behind the gill-opening, but the pelvic fin $(p v . f$. has shifted its position and lies some distance in front of the vent: it is supported by ten rays, and has a small process or adipose lobe $(a . l$.) springing from its outer edge near the base.

The body is covered by a soft, slimy skin through which, in the trunk and tail, the outlines of the scales can be seen; on the head and fins the skin is smooth and devoid of scales. A well-marked lateral line $(l$. l.) extends along each side from head to tail, and is continued into branching lines on the head. The skin is grey

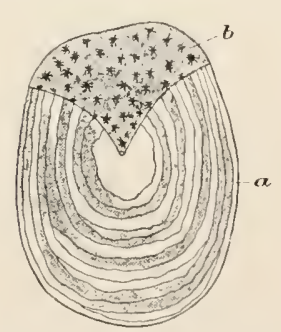

Fia.867.-Scale of Salmo fario. $a$. anterior portion covered by overlap of preceding scales; $b$. free portion covered only by pigmented epidermis. above, shading into yellowish below, and is covered with minute black pigmentspots which, on the sides and back, are aggregated to form round spots two or three millimetres in diameter. In young specimens orange-coloured spots are also present.

Skin and Exoskeleton.-The epidermis contains unicellular glands, from which the mucus covering the body is secreted, and pigment-cells, to which the colours of the animal are due. The scales (Fig. 867) are lodged in pouches of the dermis and have the form of flat, nearly circular plates of bone marked with concentric lines, but having no Haversian canals, lacunæ, or canaliculi. They have an imbricating arrangement, overlapping one another from before backwards, like the tiles of 
a house, in such a way that a small three-sided portion $(b)$ of each scale comes to lie immediately beneath the epidermis, while the rest $(a)$ is hidden beneath the scales immediately anterior to it. Besides the scales, the fin-rays belong to the exoskeleton, but will be most conveniently considered in comnection with the endoskeleton.

Endoskeleton.-The vertebral column shows a great advance on that of the two previous classes in being thoroughly differentiated into distinct bony vertebre. It is divisible into an anterior or trunk region and a posterior or caudal region, each containing about twenty-eight vertebræ.

A typical trunk vertebra consists of a dice-box-shaped centrum (Fig. 868, CN) with deeply concave anterior and posterior faces, and perforated in the centre by a small hole. The edges of the centra are united by ligament and the biconvex spaces between them are filled by the remains of the notochord; there are also articulations between the arches by means of little bony processes,

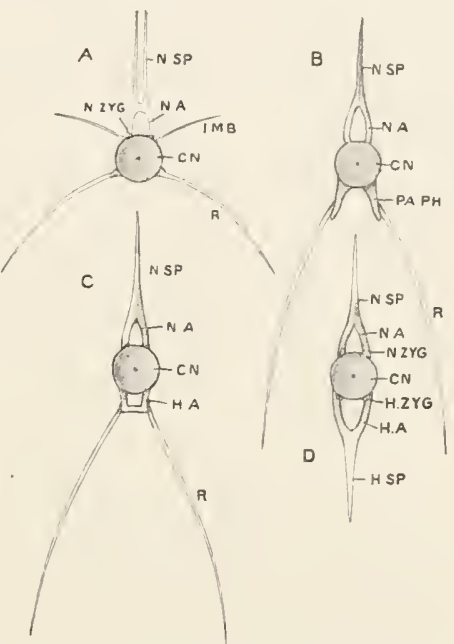

FIG. 868.-Salmo fario. $A$, one of the anterior, and $B$, one of the posterior trunk vertebre; $C$, one of the anterior, and $D$, one of the posterior caudal vertebre. CIN. centrum; IMB. intermuscular bone; HA. hæmal arch; $\mathbf{H}$. SP. hæmal spine; Ir. ZYG. hæmal zygapophysis ; N. A. neural arch; $\mathbf{N}$. SP. neural spine N. ZYG. neural zygapophysis; PA. PH. parapophysis ; R. pleural rib.

the zygapophyses (N. ZYG., H. ZYG.). To the dorsal surface of the centrum is attached, by ligaments in the anterior vertebræ, by ankylosis or actual bony union in the posterior, a low neural arch (N. A.), which consists in the anterior vertebræ of distinct right and left moieties, and is continued above into a long, slender, double neural spine (N. SP.), directed upwards and backwards. To the ventro-lateral region of the vertebra are attached by ligament a pair of long, slender pleural ribs (R.) with dilated heads, which curve downwards and backwards between the muscles and the peritoneum, thus encircling the abdominal cavity. In the first two vertebræ they are attached directly to the centrum, in the rest to short downwardly directed bones, the parapophyses (PA. PH.), immovably articulated by broad surfaces to the centrum. At the junction of the neural arch with the centrum are attached, also by fibrous union, a pair of delicate inter-muscular bones (I. IM. B.), which extend outwards and backwards in the fibrous septa between the myomeres. The first and second abdominal vertebræ bear no ribs. In the last three the neural spines $(B, \mathbf{N}$. SP.) are single. 
In the caudal vertebræ the outgrowths corresponding to the parapophyses are fused with the centrum and unite in the middle ventral line, forming a homal arch $(C, \mathbf{H}$. A.), through which the caudal artery and vein run. In the first six caudals each hæmal arch bears a pair of ribs (R.); in the rest the arch is produced downwards and backwards into a homal spine (D, H. SP.).

The centra as well as the arches of the vertebræ are formed entirely from the skeletogenous layer, and not from the sheath of the notochord as in Elasmobranchs (see pp. 71 and 143).

The posterior end of the caudal region is curiously modified for the support of the tail-fin. The hindmost centra (Fig. 869, CN.) have their axes not horizontal, but deflected upwards, and following the last undoubted centrum is a rod-like structure, the urostyle (UST.), consisting of the partly ossified end of the notochord, which has thus precisely the same upward flexure as in the Dog-fish.

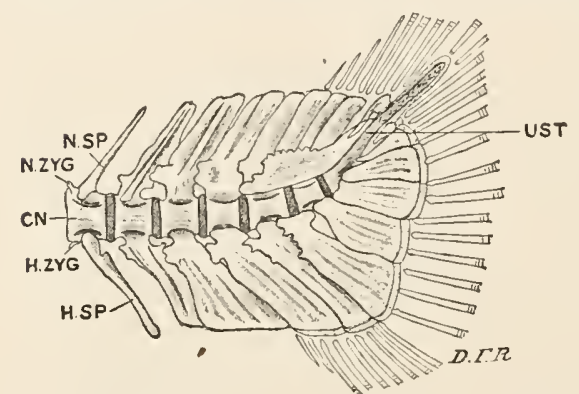

Fia. 869.-Salmo fario, caudal end of vertebral column. CN. centrum; $D . F . R$. dermal fin-rays; H. SP. hrmal spine; $\mathbf{I}$. $\mathbf{Z} \mathbf{Y G}$. hæmal zygapophysis ; IN. S.P. neural spine; IV. ZYYG. neural zygapophysis; UST. urostyle.

The neural and hæmal spines (N. SP., H. SP.) of the last five vertebræ are very broad and closely connected with one another, and are more numerous than the centra; and three or four hæmal arches are attached to the urostyle. In this way a firm vertical plate of bone is formed, to the edge of which the caudal fin-rays (D.F.R.) are attached fanwise in a symmetrical manner. It will be obvious, however, that this homocercal tail-fin is really quite as unsymmetrical as the heterocercal fin of the Dog-fish, since, its morphological axis being constituted by the notochord, nearly the whole of its rays are, in strictness, ventral.

The skull (Fig. 870) is an extremely complex structure, composed of mingled bone and cartilage. The cartilage has no superficial mosaic of lime-salts such as we find in many Elasmobranchs, but certain portions of it are replaced by bones, and there are in addition numerous investing bones developed in the surrounding connective-tissue. As in the Dog-fish, the skull may be divided into cranium, upper and lower jaws, with their suspensory apparatus, and hyoid and branchial arches.

The cranium (Fig. 871) is a somewhat wedge-shaped structure, its apex being directed forwards. At first sight the distinction between replacing and investing bones is not obvious, but after maceration or boiling certain flat bones (the paired parietals, $P A$., frontals, $F R$., and nasals, $N A$., and the unpaired supra-ethmoid, 
produced downwards into paired longitudinal ridges, enclosing between them a groove which is converted into a canal by the apposition of the parasphenoid bone and serves for the origin of the eye-muscles. In front of the auditory region the cranium is excavated on each side by a large orbit, a vertical plate or inter-orbital septum (OR. SPH.) separating the two cavities from one another. In front of the orbital region the cranium broadens out to form the olfactory capsules, each excavated by a deep pit (olf. s.) for the olfactory sac, and anterior to these is a blunt snout or rostrum. The occipital region is formed as usual from the parachordals of the embryonic skull, the auditory region from the auditory capsules, and the rest of the cranium from the trabeculæ.

The replacing bones, formed as ossifications in the chondrocranium, correspond in essentials with the typical arrangement already described (p. 77). In the occipital region are four bones; the basi-occipital (B. OC.), forming the greater part of the occipital condyle and the hinder region of the basis cranii or skull-floor : the ex-occipitals (EX. OC.), placed one on each side of the foramen magnum and meeting both above and below it; and the supraoccipital (S. OC.), forming the occipital crest already noticed. Each auditory capsule is ossified by five bones-i.e., two more than the typical number (p. 77 ) : the pro-otic (PR. OT.), in the anterior region of the capsule, uniting with its fellow of the opposite side in the floor of the brain-case, just in front of the basi-occipital; the opisthotic, in the posterior part of the capsule, external to the ex-occipital; the sphenotic (SPH. OT.), above the pro-otic and forming part of the boundary of the orbit; the pterotic (PT. OT.), above the ex-occipital and opisthotic, forming a distinct lateral ridge and produced behind into a prominent pterotic process; and the epiotic (EP. OT.), a small bone, wedged in between the supraand ex-occipitals and pterotic, and produced into a short epiotic process. On the external face of the auditory capsule, at the junction of the pro-, sphen-, and pter-otics, is an elongated facet $(h . m$.$) covered with cartilage and serving for the articulation of$ the hyomandibular.

The trabecular region of the cranium contains six bones. Immediately in front of the conjoined pro-otics, and forming the anterior end of the basis cranii, is a small unpaired $\mathrm{Y}$-shaped bone, the basisphenoid (B. SPH.). Above it, and forming the anterior parts of the side-walls of the brain-case, are the large paired alisphenoids (AL. SPH.). In the inter-orbital septum is a median vertical bone, representing fused orbitosphenoids (OR. SPH.). Lastly, in the posterior region of each olfactory capsule, and forming part of the boundary of the orbit, is the ecto-ethmoid (EC. ETH.).

The investing bones already referred to are closely applied to the roof and floor of the chondrocranium, and modify its form 
considerably by projecting beyond the cartilaginous part, and concealing apertures and cavities. The great frontals ( $F R$.) cover the greater part of the roof of the skull, concealing the fontanelles, and furnishing roofs to the orbits. Immediately behind the frontals is a pair of very small parietals $(P A$.$) ; in front of them is an unpaired$ supra-ethmoid (S. ETH.), to the sides of which are attached a pair of small nasals $(N A$.). On the ventral surface is the large para-

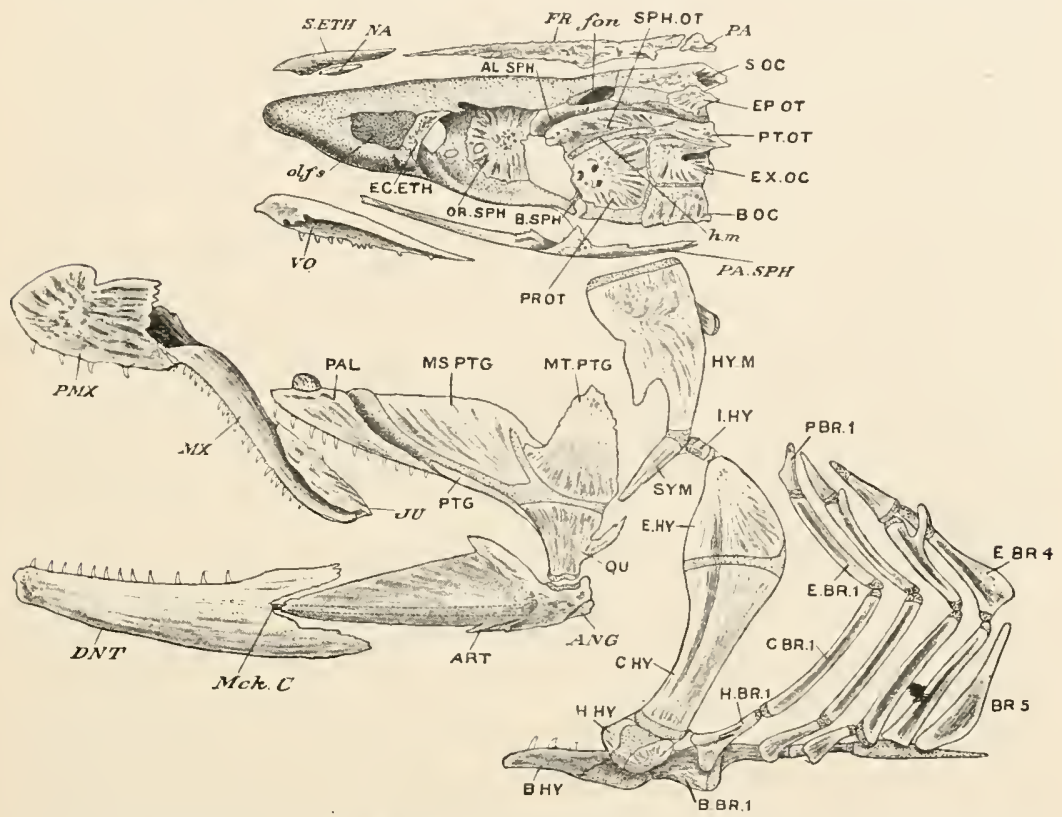

FIG. 871.-Salmo fario. Disarticulated skull with many of the investing bones removed. The cartilaginous parts are dotted. fon. fontanelle; $h$. $m$. articular facet for hyomandibular ; Mck. C. Meckel's cartilage; olf. $s$. hollow for olfactory sac. Replacing bonesAI. SPI. alisphenoid; ART. articular; B. BR. 1, first basibranchial; B. $\mathbf{Y} \mathbf{Y}$. basihyal ; B. OC. basioccipital ; BR. 5, fifth branchial arch; B. SPH. basisphenoid ; C. BR. 1, first ceratobranchial; C. HY. ceratoliyal; EC. ETr. ecto-ethmoid 耳. BR. 1, first epibranchial; $\mathbf{E}$. IIY. epi-lyal ; r. OT. epiotic ; EX. OC. ex-occipital ; I. BR. 1, first hypobranchial; II. HY. Iypohyal : FY. M. hyomandibular ; I. IX. interhyal ; MS. PTG. mesopterygoil ; MT. PTG. metapterygoid; OR. 'SPF. orbitosphenoid; PAI. palatine; BR. 1, first pharyngobranchial ; PTG. pterygoid; PT.OT. pterotic ; QU. quadrate; S. OC. supraoccipital ; SPF. OT. sphenotic; SYYMr. symplectic. Investing bones-ANG. angular; INT. dentary; FR. frontal ; $J U$. jugal ; $M X$. maxilla ; $N A$. nasal ; $P A$. parietal ; $P A$. SPH. paraspluenoid; P.H.Y. premaxilla; VO. vomer.

sphenoid (PA. SPH.), which forms a kind of clamp to the whole cartilaginous skull-floor; and in front of and below the parasphenoid is the toothed vomer (VO.). Encircling the orbit is a ring of scalelike bones, the sub-orbitals (Fig. 870, o.).

In the jaws, as in the cranium, we may distinguish between primary and secondary structures. The primary upper jaw or palatoquadrate is homologous with the upper jaw of the Dog-fish, but instead of remaining cartilaginous, it is ossified by five replacing 
bones: the toothed palatine (PAL.) in front, articulating with the olfactory capsule ; then the plerygoid (PTG.) on the ventral, and the mesopterygoid (IMS. PTG.) on the dorsal edge of the original cartilaginous bar; the quadrate (QU.) at the posterior end of the latter, furnishing a convex condyle for the articulation of the lower jaw ; and projecting upwards from the quadrate the metapterygoid (IMT. PTG.). These bones do not, however, enter into the gape, and do not therefore constitute the actual upper jaw of the adult fish: external to them are two large investing bones, the premaxilla $(P M X$.) and the maxilla (MX.), which together form the actual or secondary upper jaw : they both bear teeth. A small scale-like bone, the jugal (JU.), is attached to the posterior end of the maxilla.

The lower jaw is similarly modified. Articulating with the quadrate is a large bone, the articular (ART.), continued forwards by a narrow pointed rod of cartilage : the latter is the unossified distal end of the primary lower jaw or Meckel's cartilage; the articular is its ossified proximal end, and therefore a replacing bone. Ensheathing Meckel's cartilage and forming the main part of the secondary lower jaw is a large toothed investing bone, the dentary $(D N T$.), and a small investing bone, the angular $(A N G$.), is attached to the lower and hinder end of the articular.

The connection of the upper jaw with the cranium is effected partly by the articulation of the palatine with the olfactory region, partly by means of a suspensorium formed of two bones separated by a cartilaginous interval : the larger, usually called the hyomandibular (HY. IM.), articulates with the auditory capsule by the facet already noticed, and the small, pointed symplectic (SYMI.) fits into a groove in the quadrate. Both bones are attached by fibrous tissue to the quadrate and metapterygoid, and in this way the suspensorium and palatoquadrate together form an inverted arch, freely articulated in front with the olfactory and behind with the auditory capsule, and thus giving rise to an extremely mobile upper jaw. As its name implies, the hyomandibular (together with the symplectic) is commonly held to be the upper end of the hyoid arch and the homologue of the hyomandibular of Elasmobranchs, but there is some reason for thinking that it really belongs to the mandibular arch, and corresponds with the dorsal and posterior part of the triangular palatoquadrate of Holocephali : a perforation in the latter would convert it into an inverted arch having the same general relations as the upper jaw plus suspensorium of the Trout, but fused, instead of articulated, with the cranium at either extremity.

The hyoid cornu is articulated to the cartilaginous interval between the hyomandibular and symplectic through the intermediation of a small rod-like bone, the interhyal (I. HY.), which perhaps represents the hyomandibular of Elasmobranchs. It is 
ossified by three bones: an epithyal (E. HY.) above, then a large ceratohyal (C. HY.), and below a small double hypohyal (H. HY.). The right and left hyoid bars are connected by a keystone-piece, the unpaired, toothed basihyal (B. HY.), which supports the tongue.

Connected with the hyomandibular and hyoid cornu are certain investing bones serving for the support of the operculum. The opercular (Fig. 870, op.) is articulated with a backward process of the hyomandibular; the pre-opercular (praop.) lies outside the posterior border of the hyomandibular and quadrate, and clamps them together; the sub-opercular (subop.) is below and internal to the opercular; and the inter-opercular (intop.) fits between the lower portions of the three preceding bones, and is attached by ligament to the angle of the mandible. The twelve sabre-shaped branchiostegal rays (branchiost.) are attached along the posterior border of the epi- and cerato-hyal, and below the basi-hyal is an unpaired bone, the basibranchiostegal or urohyal.

There are five branchial arches, diminishing in size from before backwards (Fig. 871). The first three present the same segments as in the Dog-fish : pharyngobranchial (PH. BR.) above, then epibranchial (E. BR.), then a large ceratobranchial (C. BR.), and a small hypobranchial (H. BR.) below. The right and left hypobranchials of each arch are connected by an unpaired basibranchial (B. BR.). All these segments are ossified by replacing bones, and the basibranchials are connected with one another and with the basihyal by cartilage, so as to form a median ventral bar in the floor of the pharynx. In the fourth arch the pharyngobranchial is unossified, and the hypobranchial absent, and the fifth arch (BR. 5) is reduced to a single bone on each side. Small spine-like ossifications are attached in a single or double row along the inner aspect of each of the first four arches: these are the gill-rakers; they serve as a sieve to prevent the escape of food by the gill-slits.

The comparison of this singularly complex skull with the comparatively simple one of the Dog-fish is much facilitated by the examination of the skull of a young Trout or Salmon. In the latter, at about the second week after hatching, the only ossifications present are a few investing bones; when these are removed we get a purely cartilaginous skull (Fig. 872), exactly comparable with that of an Elasmobranch. There is a cranium devoid of replacing bones and divisible only into regions ; the upper jaw is an unossified palatoquadrate (Pl. Pt., M. Pt.,Qu.) and the lower jaw (Mck.) a large Meckel's cartilage; the suspensorium is an undivided hyomandibular $(H M$.$) , and the hyoid and branchial arches are$ unsegmented.

The first dorsal and the ventral fins are supported each by a triple set of pterygiophores, so that the fin-skeleton is multiserial, as in the Dog-fish. The proximal series consists of slender bony raysthe interspinous bones (Fig. 876, PTG.; Fig. 873, PTG. 1), lying 
in the median plane, between the muscles of the right and left

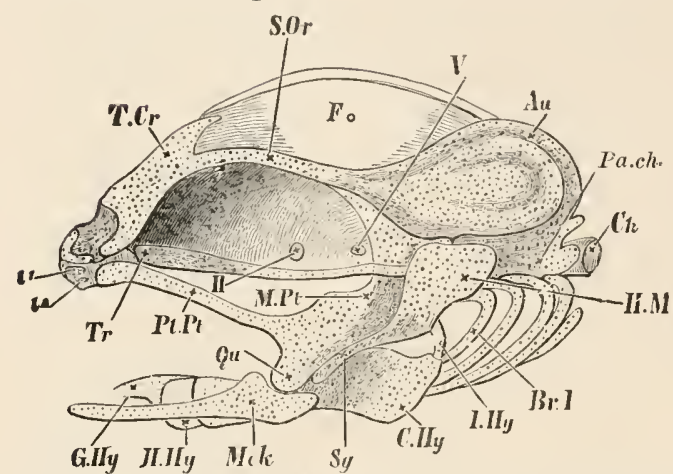

FIG. 872.-Skull of young Salmon, second week after hatching; the investing bones removed. Au. auditory capsule; $B r$, 1, first branchial arch: $C h$. notochord ; $C$. Hy. hyoid cornu ; Fo. fontanelle ; $G$. $I I y$. basiliyal ; $H$. $H y$. hypohyal : $H$. $\boldsymbol{M}$. hyomandibular; 1 . $H y$. interhyal ; $l^{\mathrm{i}}, l^{2}$, labial cartilages ; Mck. Meckel's cartilage; $M$. $P t$. metaptcrygoid region of primary upper jaw; $P \alpha$. ch. parachordal; $P l$. $P t$. palatopterygoid region; Qu. quadrate region; S.Or. supraorbital region of cranium; S\%. symplectic region of suspensorium; $T$. Cr. cranial roof ; $T r$. trabecula; $I I$, optic foramen: $V$, trigeminal foramen. (From Parker and Bettany's Morphology of the Skull.) sides, and more numerous than the myomeres of the regions in which they occur. Their distal ends are broadened, and with them are connected the second series (PTG. 2) in the form of small dicebox-shaped bones; to these, finally, are attached small nodules of cartilage (ptg. 3) forming the third series of radials. The dermal fin-rays or lepidotrichia (D.F.R.), which lie in the substance of the fin itself, are slender bones, jointed like the antennæ of an Arthropod, and mostly branched in the sagittal plane (Fig. 876, D.F.R.). Each is formed of distinct right and left pieces (Fig. 873), in close contact for the most part, but diverging below to form a forked and dilated end, which fits over one of the cartilaginous nodules ( $p t g .3)$. In the caudal fin (Fig. 869) the dermal rays (D.F.R.) are similarly seated on the broad hæmal arches of the posterior caudal vertebræ. The second dorsal or adipose fin has, as already noticed, no bony support.

The shoulder-girdle (Fig. 874), like the skull, consists of a primary shoulder-girdle, homologous with that of a Dog-fish, and of several investing bones. The primary shouldergirdle in the young fish is formed of distinct right and left bars of cartilage, which do not unite with one another ventrally. In the adult each bar is ossified by three bones, a scapula (SCP.), situated dorsally to the glenoid facets, and developed partly as a replacing, partly as an investing bone; a coracoid (COR.), situated ventrally to the glenoid facet, and a meso-coracoirl (MS.

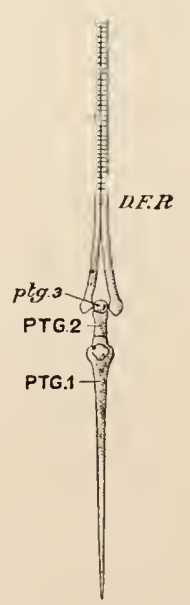

FIG. 873.-Salmo fario. A dermal fin-ray with its supports. D.F.R. dermal fin-ray; PTG. $\mathbf{I}$, proximal pterygiophore (i n t e rspinous bone); PTG. $\mathbf{2}$, middle pterygiophore ; ptg. 3, distal pterygiophore (cartilaginous). 
COR.), situated above the coracoid and anterior to the scapula. Externally to these is found a very large investing bone, the clavicle $(C L$.$) , extending downwards under the throat: its dorsal end is$ connected by means of a supra-clavicle (S.CL.) to a forked bone, the post-temporal (P.TM.), one branch of which articulates with the epiotic, the other with the pterotic process. To the inner surface of the clavicle are attached two flat scales of bone $\left(P . C L^{\prime}.\right)$, with a slender rod-like post-clavicle $(P . C L$.) passing backwards and downwards among the muscles.

The structure of the pectoral fin is very simple. Articulated to the posterior border of the scapula and coracoid are four dice-boxshaped bones, the proximal pterygiophores or radials (PTG. 1), followed by a row of small nodules of cartilage ( $p t g .2)$ representing distal pteryg io phores. The main body of the fin is supported by dermal fin-rays, which resemble those of the median fins, and have their forked ends seated upon the distal pterygiophores: the first ray, however, is larger than the rest, and articulates directly with the scapula.

There is no pelvic girdle, its

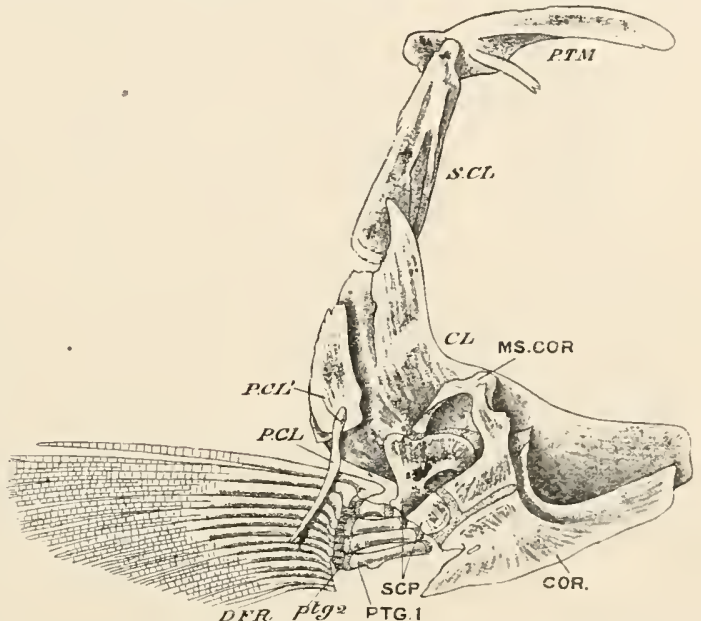

FIg, 874-Salmo fario. Left half of shoulder-girdle and pectoral fin, from the inner surface. CL. clavicle; COR. coracoid ; $D . F^{\prime} . R$. dermal fin-rays; MS. CoR. mesocoracoid P.CL., P.CL', post-clavicles; PTG.1, proximal, and ptg. 2 distal pterygiophores; $P$. TH. post-temporal ; S. CL. supraclavicle; SCP. scapula.

place being taken by a large, flat triangular bone, the basipterygium (Fig. 875, B. PTG.), probably representing fused proximal pterygiophores: to its posterior border are attached three partly ossified nodules; the distal pterygiophores (PTG.), and with these the dermal fin-rays are articulated. The adipose lobe of the pelvic fin is supported by a small scale-like bone.

The muscles of the trunk and tail are arranged, as in the Dog-fish, in zigzag myomeres: there are small muscles for the fins, and the head has a complex musculature for the movement of the jaws, hyoid, operculum, and branchial arches.

The cœlome is divisible into a large abdomen (Fig. 876) containing the chief viscera, and a small pericardial cavity, situated below the branchial arches, and containing the heart. 
Digestive Organs.-The mouth (Figs. 865 and 876) is very large and has numerous small, recurved, conical teeth, borne, as

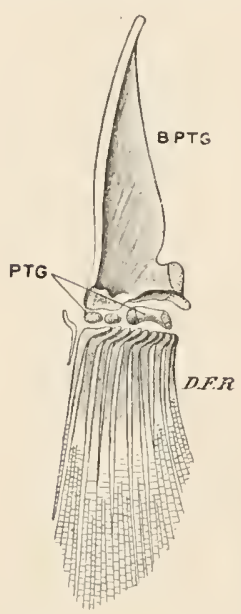

FIG. 875.- Salmo fario. Skeleton of left pelvic fin, dorsal aspect.

B. PTG. basipterygium; $D . F, R$. dermal fin-rays; PTG. distal pterygiophores. already mentioned, on the premaxillæ, maxillæ, palatines, vomer, dentaries and basihyal. They obviously serve merely to prevent the escape of the slippery animals used as food and are of no use for either rending or chewing. The pharynx $(p h$.$) is perforated on each side$ by four vertically elongated gill-slits, fringed by the bony tooth-like gill-rakers. Each gillslit is V-shaped, the epihyal being bent upon the ceratohyal so that the dorsal and ventral moieties of the branchial arches touch one another when the mouth is closed.

The pharynx leads by a short gullet (gul.) into a $U$-shaped stomach (st.) consisting of a wide cardiac and a narrow pyloric division: between the latter and the intestine is a ringshaped pyloric valve. The intestine passes at first forwards as the duodenum (du.), then becomes bent upon itself (int.) and passes backwards, without convolution, to the anus (an.). Its posterior portion has the inucous membrane raised into prominent annular ridges which simulate a spiral valve.

The liver $(l r$.$) is imperfectly divided into right and left lobes, and$ there is a large gall-bladder $(g . b l$. $)$. Opening into the duodenum are about forty blind glandular tubes, the pyloric caca (py. c.). There is a large spleen ( $s p l$.) attached by peritoneum to the fundus of the stomach. The stomach, duodenum, and pyloric cæca are surrounded by loose folds of peritoneum loaded with fat.

Lying below the kidneys and extending the whole length of the abdominal cavity is the air-bladder (a.bl.), a thin-walled sac serving as an organ of flotation. Anteriorly its ventral wall presents a small aperture leading, by a short pncumatic duct $(p n$. d.), into the oesophagus on the dorsal side somewhat to the right of the middle line.

Respiratory Organs.--There are four pairs of gills each with a double row of branchial filaments, united proximally but having their distal ends free: interbranchial septa are practically obsolete (see Fig. 780). The gills are borne on the first four branchial arches, the fifth arch bearing no gill. On the inner surface of the operculum is a comb-like body, the pseudo-branchia, formed of a single row of branchial filaments, and representing the vestigial gill (hemibranch) of the hyoid arch.

Circulatory Organs.-The heart (Fig. 876) consists of sinus venosus, auricle $(a u$.), and ventricle $(v$.$) . There is no conus arteriosus,$ 


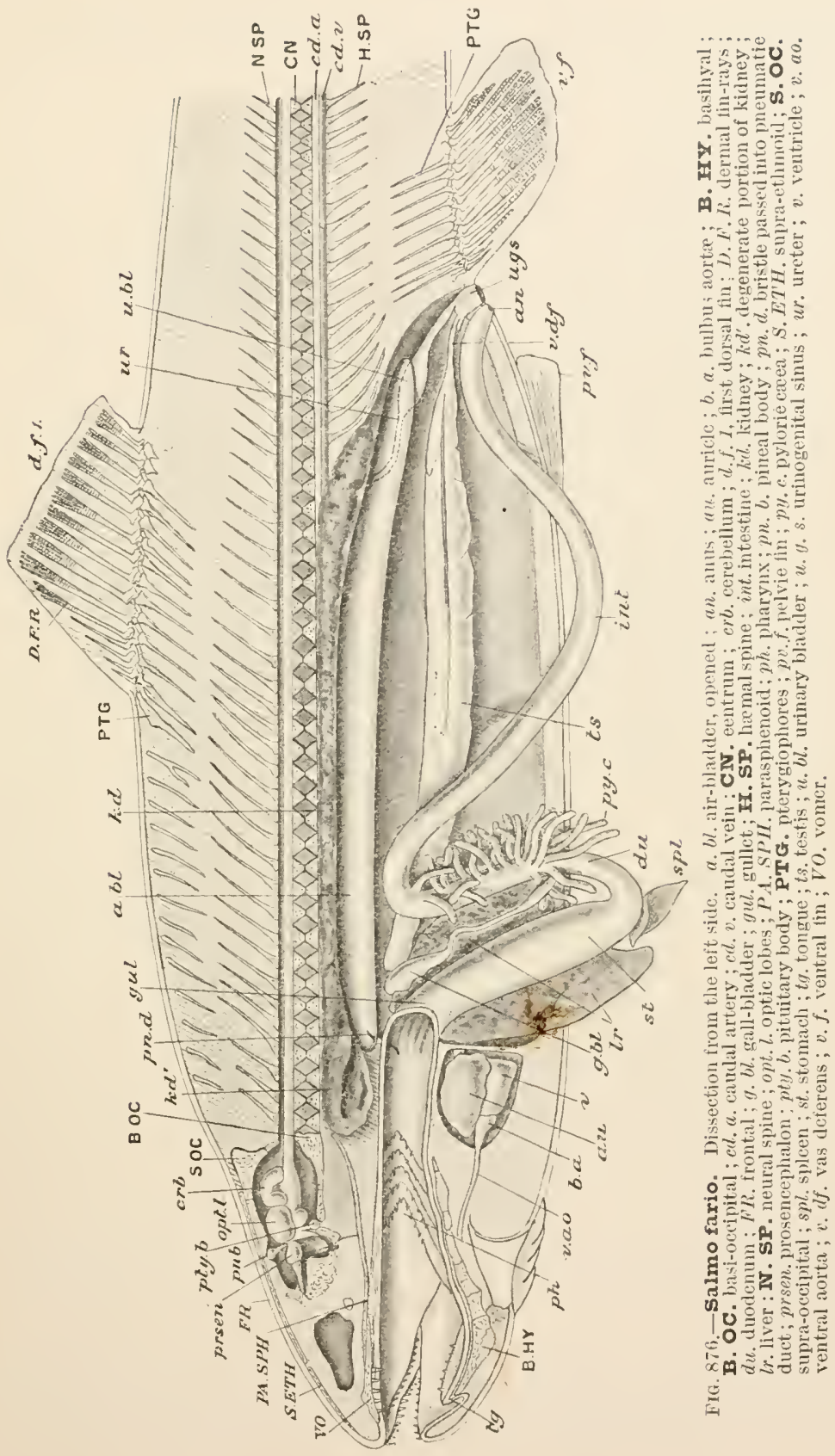


but the proximal end of the ventral aorta is dilated to form a bulbus aortce $(b . a$.$) , a structure which differs from a conus in being part$ of the aorta, and not of the heart ; its walls do not contain striped muscle, and are not rhythmically contractile.

In accordance with the atrophy of the hyoid gill there is no afferent branchial artery to that arch, but a hyoidean artery springs from the ventral end of the first efferent branchial and passes to the pseudobranch. The right branch of the caudal vein is continued directly into the corresponding cardinal, the left breaks up in the kidney, forming a renal-portal system. There are no lateral veins, but the blood from the paired fins is returned to the cardinals. The red blood-corpuscles are, as in other fishes, oval nucleated discs.

Nervous System.-The brain (Fig. 877) is very different from that of Elasmobranchs, and is in many respects of a distinctly lower type. The cerebellum (H.H.) is very large, and bent upon itself. The optic lobes (M.H.) are also of great size, and on the ventral surface are large bean-shaped lobi inferiores (U.L.). The diencephalon is much reduced, and, indeed, is indicated dorsally only as the place of origin of the pineal body $(G . p$.$) : ventrally it is pro-$ duced into the lobi inferiores with the infundibulum between them giving attachment to the pituitary body (Hyp.). Hence, seen from above, the small undivided prosencephalon (V.H.) comes immediately in front of the mid-brain : it has a non-nervous roof (Pall.) and its floor is raised into prominent corpora striata (BG., Bas. G.). The olfactory bulbs, situated in close apposition with the prosencephalon without intervening olfactory peduncles or olfactory tracts such as are present in Scyllium (L.ol.), are nearly as large as the corpora striata, and each contains a small cavity or rhinocole in communication with the undivided prosocœle. Three transverse bands of fibres connect the right and left halves of the fore-brain, an anterior commissure joining the corpora striata, a posterior commissure situated just behind the origin of the pineal body, and an inferior commissure in front of the infundibulum. The pineal body (G.p.) is rounded and placed at the end of a hollow stalk : a shorter offshoot of the roof of the diencephalon may perhaps represent a rudimentary pineal eye. Behind the pituitary body is a saccus vasculosus (s. v.). The optic nerves do not form a chiasma, but simply cross one another, or decussate $(C h$.), on leaving the brain, the right nerve going to the left, and the left nerve to the right eye.

Sensory Organs.-The most distinctive feature of the olfactory $s a c$ is the possession of two small apertures, the anterior provided with a valve.

The eye (Fig. 878) has a very flat cornea (cn.) with which the globular lens $(l$.$) is almost in contact, so that the aqueous chamber$ of the eye is extremely small. Between the cartilaginous sclerotic $(s c l$.$) and the vascular choroid (ch.) is a silvery layer or argentea$ 
(arg.), which owes its colour to minute crystals in the cells of which it is composed. There are no choroid processes. In the posterior part of the eye, between the choroid and the argentea, is a thickened

A

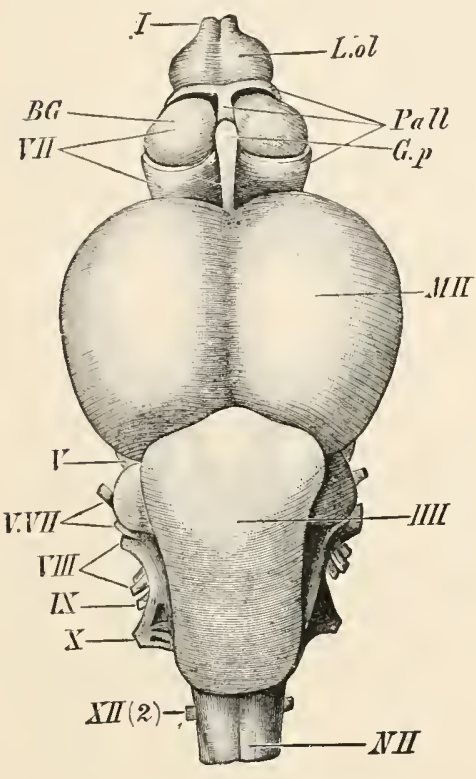

B

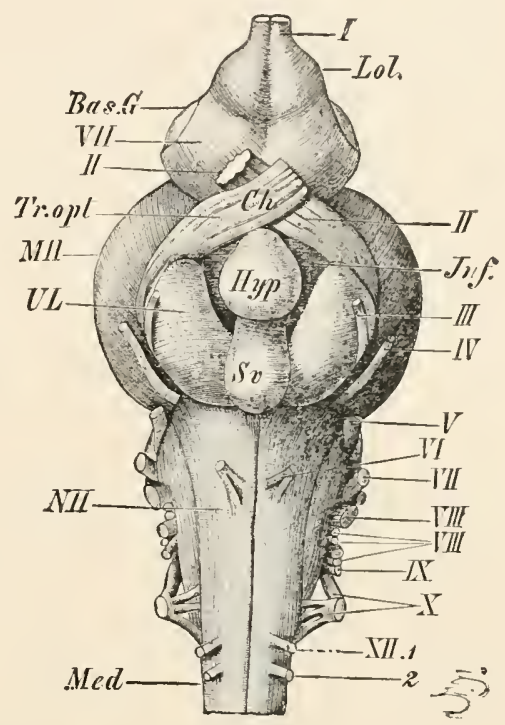

C

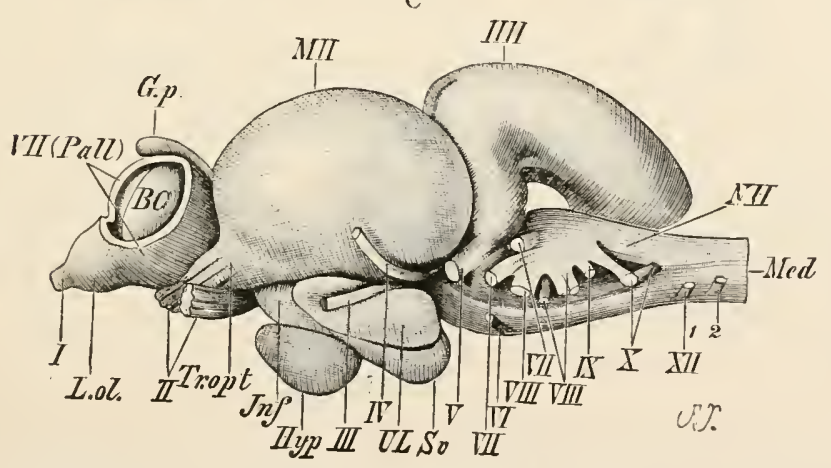

FIG. 877.-Salmo fario. Dorsal $(A)$, ventral $(B)$, and lateral $(C)$ views of brain. BG., Bas. $G$. corpora striata; $c h$, crossing of optic nerves; $G$. $p$, pineal body; $H H$. cerebellum: $H y p$, pituitary body ; Inf. infundibulum; $L$. ol. olfactory bulbs; $M e d$, spinal cord ; $M H$. optic lobes; NH. medulla oblongata; Pall. non-nervous roof of prosenceplalon: $\mathrm{Se}$. saccus vasculosus; $T r$. Opt. optic tracts; $U L$. lobi inferiores; VII, prosencephalon; $I-X$, cerebral nerves ; $Y I I, 1$, first spinal (hypoglossal) nerve; 2 , second spinal nerve. (From Wiedersheim's Vertebrata.)

ring-shaped structure (ch. gld.) surrounding the optic nerve, and called the choroid gland: it is not glandular, but is a complex network of blood-vessels, or rete mirabile. It is supplied with blood by the 
efferent artery of the pseudobranch. Close to the entrance of the optic nerve a vascular fold of the choroid, the falciform process ( $p r . f l$.$) , pierces the retina, and is continued to the back of the lens,$ where it ends in a knob, the campanula Halleri (cp. hal.), which

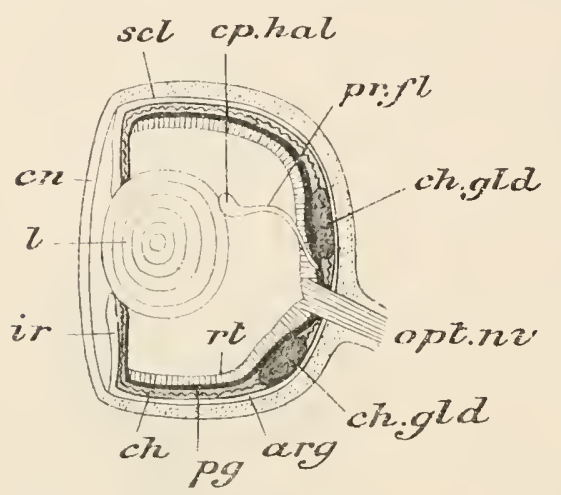

FIG. 878.-Salmo fario. Vertical section of eye (semi-diagrammatic). arg. argentea ; ch. choroid ; ch. gld choroid gland; $c n$. cornea; $c n$. hal. campanula Halleri : ir. iris; 7 . lens; opt. nv. optic nerve; $p g$. pigmentary layer; $p r$. $f$. processus falciformis; scl. sclerotic (dotted). contains smooth muscular fibres. The falciform process with the campanula Halleri takes an important part in the process of accommodation by which the eye becomes adapted to forming and receiving images of objects at various distances. Accommodation in the Bony Fish is effected, not by an alteration in the curvature of the lens as in higher Vertebrates, but by changes in its position, by which it becomes more approximated towards, or further withdrawn from, the retina. In bringing about these changes of position the structures in question appear to play the principal part.

The auditory organ (Fig. 879) is chiefly remarkable for the large size of the otoliths (ot. 1). They are three in number; one, called the sagitta (ot. 1 ). is fully $6 \mathrm{~mm}$. in length, and almost fills the sacculus : another, the astcriscus (ot.2), is a small granule lying in the lagena or rudimentary cochlea : the third, the lapillus (ot.3), is placed in the utriculus close to the ampullæ of the anterior and horizontal canals.

Urinogenital Organs.-The kidneys (Fig. 876, kd., and Fig. $880, R)$ are of great size, extending the whole length of the dorsal wall of the abdomen, above the air-bladder, and partly fused together in the middle line. They are derived from the mesonephros of the embryo. Their anterior ends (Fig. 876, kd, Fig. $880, R)$ are much dilated and consist in the adult of lymphatic tissue, thus ceasing to discharge a renal function. The ureters (mesonephric ducts, ur.) unite into a single tube, which is dilated to form a urinary bladder (Fig. 876, u. bl., Fig. 880, v.), and discharges into the urinogenital sinus.

The gonads are of great size in the sexually mature fish. The testes (Fig. 876, ts.) are long, smooth, pinkish paired organs, extending the whole length of the abdominal cavity; each is continued posteriorly into a duct $(v$. df.) which opens into the urinogenital sinus, and the homology of which with the ducts of the primitive 
nephridial system is still uncertain. The ovaries are also of the full length of the abdominal cavity and are much wider than the testes: they are covered with peritoneum on their inner or mesial faces only, and the numerous ova, which are abont $4 \mathrm{~mm}$. in diameter, are discharged when ripe from their onter faces into the colome. There are no oviducts, but the anterior wall of the urinogenital sinus is pierced by a pair of genital pores through which the ova make their way to the exterior. There is reason for thinking that these pores are to be looked upon as degenerate oviducts, and in no way homologous with the abdominal pores of Elasmobranchs.

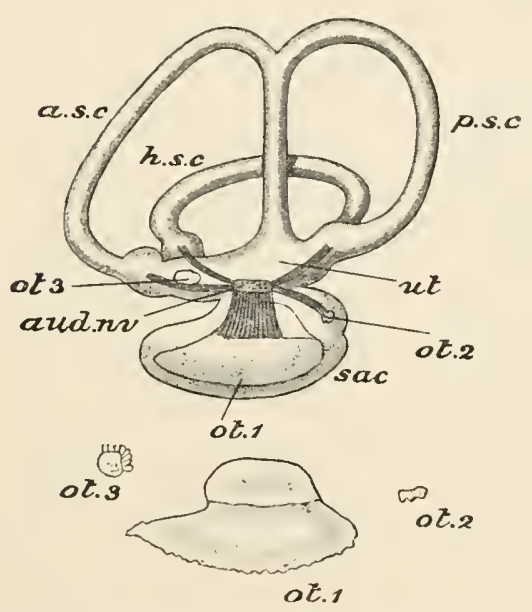

FIG. 879.-Salmo fario. The right auditory organ, from the inner side; the otoliths are shown separately below. $a . s$. $c$. anterior semicircular canal; aud. $n v$. auditory nerve; $h$. s. $c$. horizontal canal; ot. $1-3$, otoliths ; $p . s . c$. posterior canal ; sac. saceulus ; ut. utriculus.

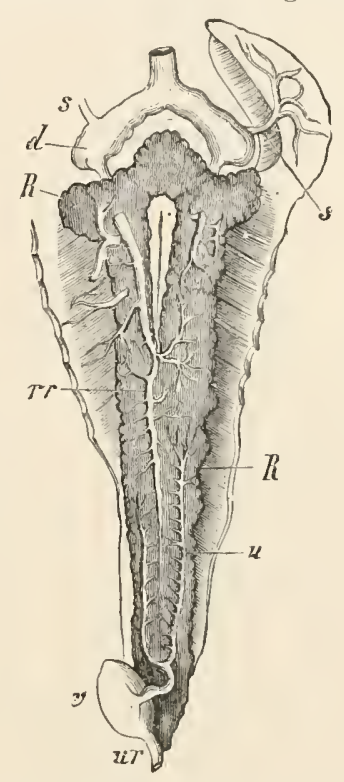

FIG. 880.- Salmo fario. The lidneys and adjacent parts. $d$, precaval vein ; $R$ (to the right), kidney ; $R$ (to the left), degenerate anterior portion of kiclney; $r r$, efferent renal vein; $s$. subclavian vein ; $u$, ur, ureter; $r$, bladder. (From Gegenbaur's Comparative Anatomy.)

Development.-Impregnation is external, the male shedding his milt or seminal fluid on the newly-laid eggs. The ovnm is covered by a thick membrane, the zona radiata, perforated by an aperture, the micropyle, through which the sperms find access : it is formed of a superficial layer of protoplasm surrounding a mass of transparent fluid yolk of a pale yellow colour. At one pole the protoplasm accumulates to form an elevated area or germinal disc, in which segmentation takes place (Fig. 881, $A, B$ ) in much the same way as in Elasmobranchs, except that, owing to the smaller proportion of yolk, the resulting blastoderm (bl.) and the embryo formed therefrom are proportionally much larger, and the yolk-sac 
(y.s.) correspondingly smaller, than in the two previous classes.

A
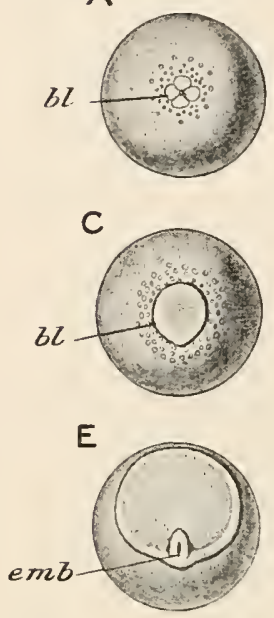

$\mathrm{G}$
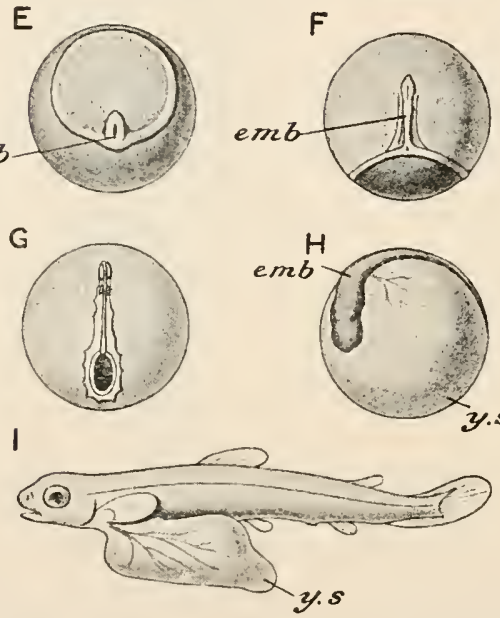

FIG. 881.-Nine stages in the development of Salmo fario. $A-H$, before hatching; $I$, shortly after hatching. $b l$. blastoderm; emb. enbryo; $r$. thickened edge of blastoderm; $y, s$, yolk-sae. $(A-G$ after Henneguy.)

tail become free from the yolk, and at the yolk-sac $(I, y s)$ is a shoe-shaped body sessile upon the rentral surface of the transparent embryo.

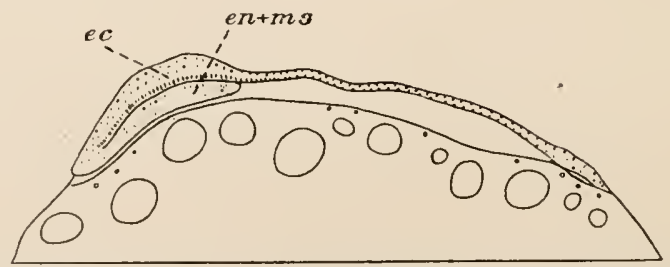

FIG. 882.-Longitudinal section of blastoderm of SaImo, at about the stage represented in $D$ of Fig. 881. ec. ectoderm; $e n+m s$, infolding giving rise to endoderm and mesoderm. (After O. Hertwig.) 


\section{2.-Distinctive Characters and Classification.}

The Teleostomi are Pisces in which the primary cranium is always complicated by the addition of investing bones, of which a pair of parietals and one of frontals above, and unpaired vomer and parasphenoid below, are the most constant. The chondrocranium is always more or less ossified by replacing bones, including a supra-occipital, and the upper and lower jaws are both bounded by investing bones. The jaws are connected with the cranium through the internediation of a hyomandibular, which is probably not homologous with the similarly named element of Elasmobranchs. The dermal fin-rays are formed of bones, and are supported by pterygiophores which may be either cartilaginous or bony, but which always show a great reduction in number as compared with the homologous structures in Elasmobranchs. The primary shoulder-girdle is complicated by the addition of investing bones, of which a large clavicle is the most constant. The pelvic girdle is vestigial or absent. The pelvic fins usually undergo a forward displacement, their position being either abdominal, i.e., between the anus and the pectoral region, or thoracic, i.e., in the pectoral region, or jugular, i.e., under the throat. A dermal exoskeleton is usually present. The intestine may or may not have a spiral valve ; the anus is distinct from, and placed in front of, the urinary and genital apertures. The gills are covered by an operculum supported by investing bones, and the interbranchial septa are reduced or absent, so that the gill-filaments are partially or wholly free; the hyoidean gill is reduced or absent. The conus arteriosus is sometimes present, sometimes absent; when it is absent there is a large bulbus aortæ formed as a dilatation of the ventral aorta. The prosencephalon has usually a non-nervous roof; the optic nerves either form a chiasma or simply decussate. The ova are small; the gonoducts are either continuous with the gonads, or open anteriorly into the colonre, or are absent: in the latter case the sexual products pass out by genital pores; true abdominal pores may be present in addition. Segmentation of the egg is either entire or discoidal; development is sometimes accompanied by a metamorpliosis.

The Teleostomi are classified as follows :-

\section{Order 1.-Crossopterygit.}

Teleostomi in which the pectoral fin consists of a rounded basal lobe supported by endoskeletal structures and fringed by dermal rays. There are no branchiostegal rays. The vertebral columm is well ossified, and the caudal fin is diphycercal. The pelvic fins, when present, are abdominal. A spiral valve and a conus arteriosus are present, and the optic nerves form a chiasma. 
The only existing members of this order are several species of

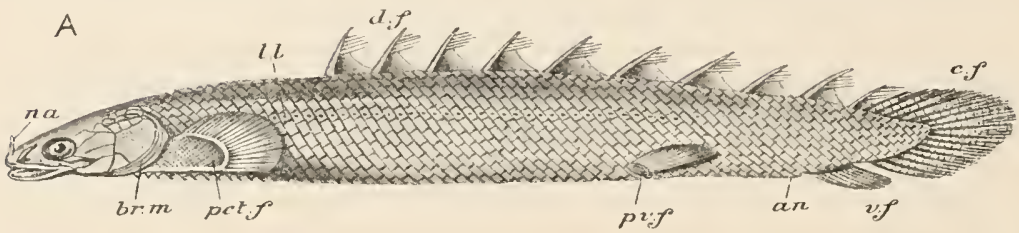

FIG. 883,-Polypterus bichir. A, entire animal ; $B$, ventral view of throat. an. anus; $b r . m$. branchiostegal membrane ; $c . f$. caudal fin; $d$. $f$. dorsal finlets ; jug. $p l$. jugular plates; na. nostril ; pet. $f$. pectoral fin; $p v . f$. pelvic fin ; $v . f$. ventral fin. (After Cuvier.)

B

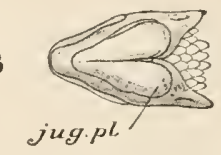

Polypterus (Fig. 883) from the Congo and Upper Nile, and Calamoichthys calabaricus from Old Calabar.

\section{Order 2.-Chondrostei.}

Teleostomi in which the paired fins have no basal lobe, but their whole free portion is supported by dermal rays. There are few replacing bones in the skull, and the primary shoulder-girdle is unossified. The vertebral column is represented by a persistent notochord with cartilaginous arches, and its anterior end is fused with the cranium. Branchiostegal rays are few or absent. The tail

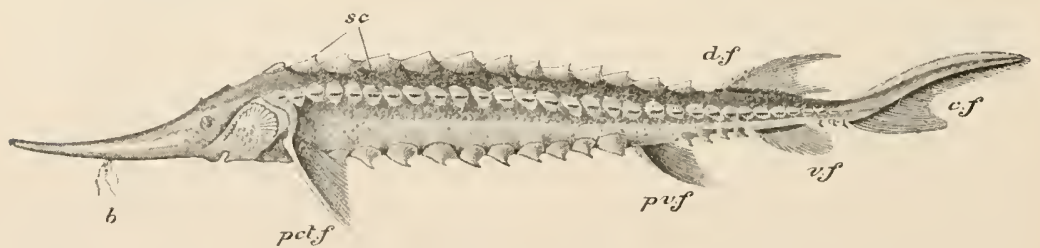

FIG. 884.-Acipenser ruthenus (Sturgeon). b. barbels; $e . f$. caudal fin; $d . f$. dorsal fin; pet. $f$. pectoral fin; $p v . f$. pelvic tin; sc. scutes; $v$. $f$. ventral fin. (After Cuvier.)

is heterocercal. The pelvic fins are abdominal. A spiral valve, conus arteriosus, and optic chiasma are present.

This order includes the Sturgeons (Acipenser, Fig. 884, and Scaphirhynchus) found in the rivers of Europe, Asia, and North America; the curious spoon-billed Polyodon, from the Mississippi ; and Psephurus from the rivers of China.

\section{Order 3.-Holostei.}

Teleostomi in which the paired fins have no basal lobe. The chondrocranium is well ossified by replacing bones and investing bones: branchiostegal rays are present. The vertebral column consists of bony vertebræ, and the tail is heterocercal or nearly 
homocercal. The pelvic fins are abdominal. A reduced spiral valve, a conus arteriosus, and an optic chiasma are present.

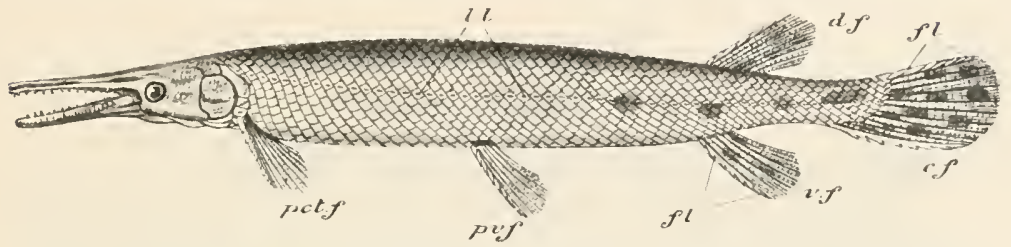

FIG. 885.- Lepidosteus platystomus (Bony like). $c . f$. caudal fin ; $d$. $f$. dorsal fin; $f$. fulcra; $l . l$. lateral line; pet. $f$. pectoral fin; $p v$. $f$. pelvic fin; $v$. $f$. veutral fin. (After Cuvier.)

This order includes the Gar-pike or Bony Pike (Lepidosteus, Fig. 885), from the fresh waters of North and Central America and Cuba, and the Bowfin or Mud-fish (Amia calva, Fig. 886) from the rivers of the United States.

Orders $1-3$ are frequently grouped together as the subclass Ganoidei, and, although such a group is an artificial one, it will often be convenient to refer to these fishes as

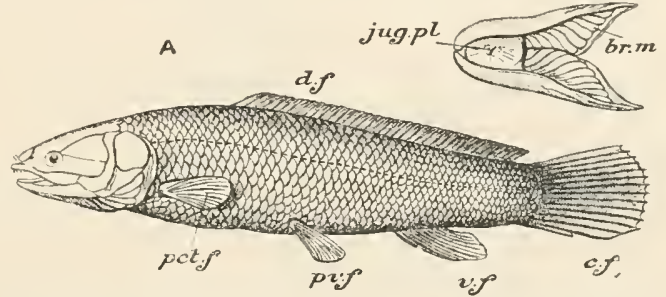

FIG. 886--Àmia calva (Bow-fin). $A$, the entire animal ; $B$, ventral view of throat. $b r . m$. branchiostegal membrane; $c . f$. caudal fin; $d . f$. dorsal fin; jug. pl. jugular plate ; pet. $f$. pectoral fin; $p v$. $f$. pelvic fin ; $v, f$. ventral fin. (After Günther.)

"Ganoids." They are all small and numerically insignificant groups at the present day, but formed the whole of the Teleostomian fauna in the Palæozoic and the greater part of the Mesozoic epoch.

\section{Order 4.-Teleostei.}

Teleostomi in which the paired fins have no basal lobe. The skull is well ossified both by replacing and investing bones : branchiostegal rays are present. The vertebral column is well ossified: the tail is homo- or diphycercal. There is no spiral valve except as a vestige in one genus. The conus arteriosus is absent except as a vestige in a small number of genera: a large bulbus aortæ is present. The optic nerves never form a chiasma and usually simply decussate.

The vast majority of existing Teleostomi are included in this order, which is divided into six sub-orders as follows :-

\section{Sub-order a.-Physostomi.}

Teleostei in which the air-bladder, when present, has an open pnemmatic duct. All the fin-rays are jointed, or the dorsal and

VOL. II 
the pectoral are armed each with an anterior ossified spine, and the pelvic fins, when present, are abdominal in position.

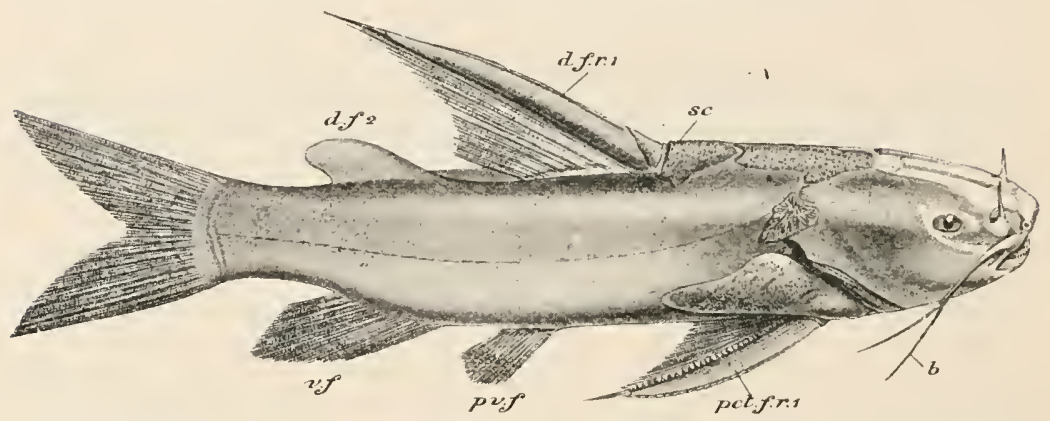

FIG. 887.-Rita buchanani, one of the Silurolds. $b$. barbel ; $d . f . r .1$, first dorsal fin-ray ; d. $f$. 2 , adipose fin; pct. $f$. $r$. 1 , first pectoral fin-ray; $p v$. $f$. pelvic fin; $v$. $f$. ventral tin. (After Day.)

Including the Cat-fishes or Siluroids (Fig. 887), Carp, Gudgeon, Loach, Pike, Salmon and Trout (Fig. 865), Smelt, Grayling, Herring, Anchory, Eel, \&c.

\section{Sub-order b.-Anacunthini.}

Teleostei in which the air-bladder, when present, has, except in one species, no pneumatic duct. The rays of the unpaired and of the pelvic fins are all jointed, and the pelvic fins are either thoracic

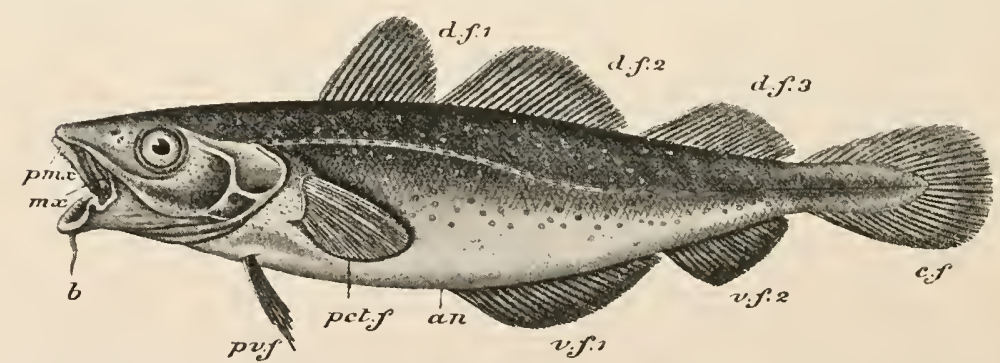

FIG. 888.-Gadus morrhua (Cod). an. anus ; $c$. $f$. caudal fin ; $d . f$. 1 - 3 , dorsal fins ; $m x$. maxilla ; pet. $f$. pectoral fin ; $p m x$. premaxilla ; $p v$. $f$. pelvic fin ; $v . f .1$ and 2 , ventral fins. (After Cuvier.)

or jugular. Including the Cod (Fig. 888), Haddock, Whiting, Hake, Ling, and the Pleuronectidæ or Flat-fishes (Fig. 893), such as the Sole, Flounder, Turbot, \&c.

\section{Sub-order c.-Acanthopteri.}

Teleostei in which the air-bladder, when present, has usually no pneumatic duct. More or fewer of the rays of the dorsal, ventral, and pelvic fins are unjointed, and have the form of strong spines. 
The right and left bars of the fifth branchial arch are usually not fused.

This immense group includes the greater number of marine

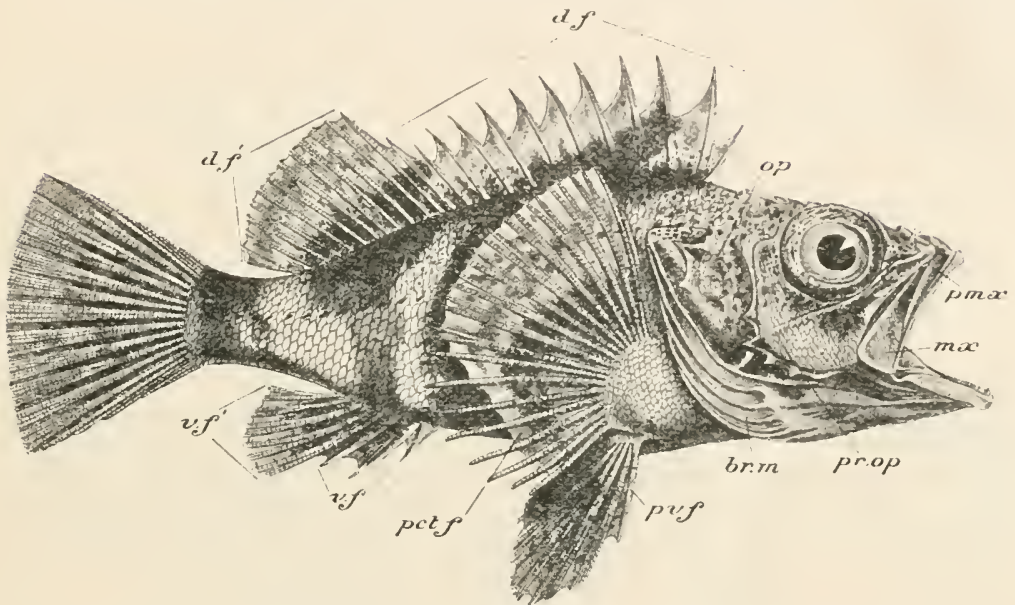

Fli. 839.-Sobastes parcoides. br. m. branchiostegal membrane ; $d$. $f$. spiny portion of dorsal fin ; $l$. $f^{\prime}$. Solt portion ; me. muxilla; op. opercular; pet. $f$. pectoral fin ; $p . m r$. premaxilla; $p r . o p$. pre-opercular; $p v . f$. pelvic fin; $v$. $f$. spiny portion of ventral fiu; $v$. $f$; soft portion. (After Richardson.)

fishes (Fig. 889), as well as many fresh-water forms: the Perch, Stickleback, Sea-bream, Mullet, Mackerel, and Gurnard may be specially mentioned.

\section{Sub-order d.-Pharyngognathi.}

Teleostei in which the right and left bars of the fifth branchial

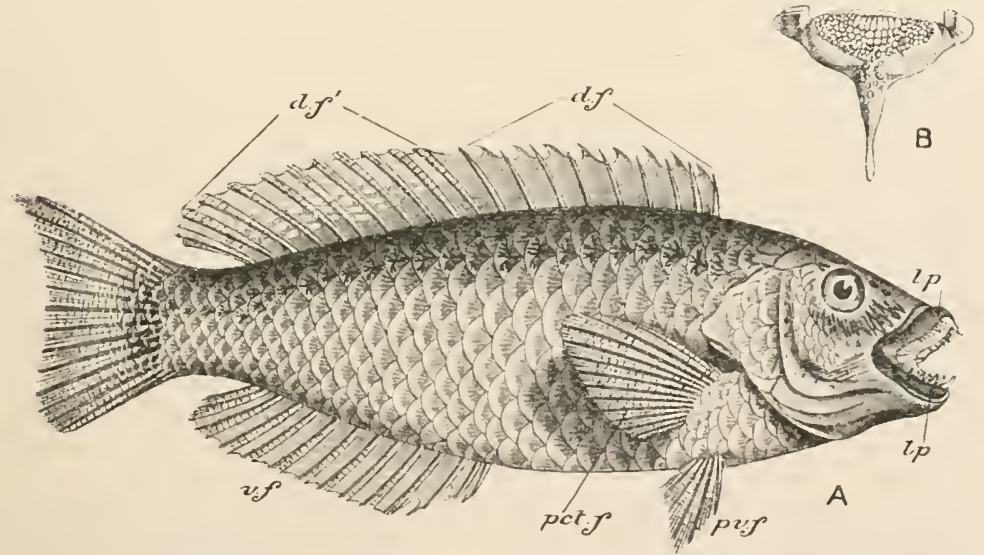

FIG. 890.-Labrichthys psittacula (Wrasse). $d . f$. hard lorsal fin ; $d . f^{\prime}$. soft dor sal, $l p$. lips; pct. $f$. pectoral fin; $p v . f$. pelvic fin; $v . f$. ventral fin. $B$, inferior pharyngea! boue of Labrichtiys. ( $A$, after lichardson; $B$, after owen.) 
arch are fused to form a single bone in the floor of the month (Fig. $890, B)$. The remaining characters are as in Acanthopteri.

Including the Wrasses (Fig. 890) and their allies.

\section{Sub-order e.-Plectognathi.}

Teleostei having no pneumatic duct. The exoskeleton, when present, takes the form of bony plates or spines. The gill-opening

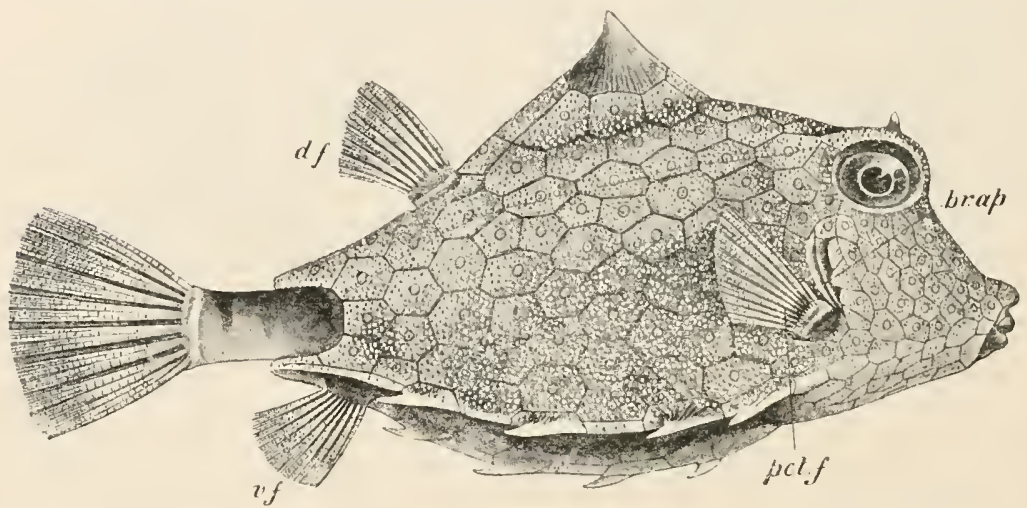

FIG. 891.-Ostracion (Coffer-fish). br. a.p. branchial aperture; $d . f$. dorsal fin ; pet. $f$. pectoral fin; $v$. ventral fin. (After loay.)

is very narrow. The mouth is very small, and the premaxilla and maxilla are united. The pelvic fins are absent or represented by spines.

This is a small sub-order, including the File-fishes, Globe-fishes, Sun-fishes and Coffer-fishes (Fig. 891).

\section{Sub-order f.-Lophobranchii.}

Teleostei having no pnemmatic dnct. The gills are not comblike, but have their filaments arranged in tufts (Fig. 892, $B$ ). The branchial aperture is very small. The exoskeleton consists of bony plates arranged segmentally.

This is also a very small sub-order, including only the Seahorses (Fig. 892), Pipe-fishes and their allies.

Sub-orders $b-f$ are frequently gromped together as Physoclisti, distingnished from Physostomi by the closed air-bladder.

\section{Systematic Position of the Example.}

Salmo fario is one of several species of the genus Salmo, belonging to the family Salmonidke, of the snb-order Physostomi and the order Teleostei.

The absence of a spiral valve and of a conns arteriosus, the 
presence of a bulbus aortæ, and the decussation of the optic nerves indicate its position among the Teleostei. It belongs to the

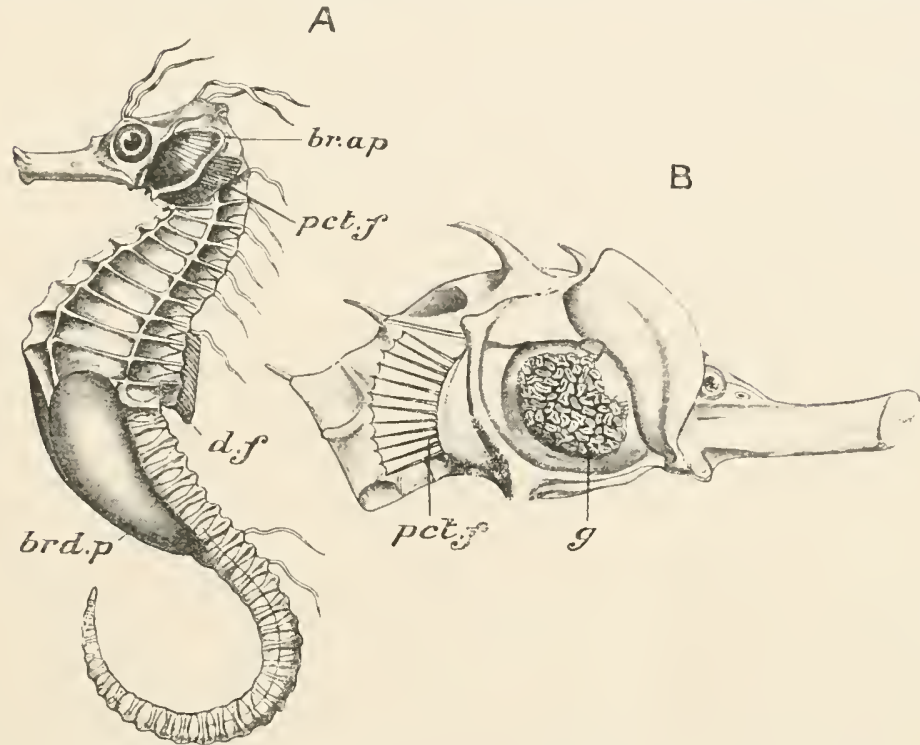

FIG. 892,- Irippocampus (Sea-horse). In $B$ the operculum is removed to show the gills. $b r$. ap. branchial a perture ; brd. $p$. brood-pouch ; $d$. $f$. doraal fin ; $g$. gills ; pct. $f$. pectoral fin. (From Claus and Günther.)

Physostomi in virtue of possessing a pneumatic duct, none but jointed fin-rays, and abdominal ventral fins. The characters which place it among the Salmonidx are the presence of an adipose fin and of pseudobranchir, the absence of oviducts, and the fact that the maxilla enters into the gape of the mouth. The genus Salmo is distinguished by its small scales, well-developed conical teeth, absent on the pterygoids, a short ventral fin with fewer than fourteen rays, numerous pyloric appendages, and comparatively large ova. The distinctive characters of the various species of Salmo depend upon comparatively minute points, such as the relative proportions of various parts, and are often difficult of determination owing to individual variations correlated with different enviromments. In S. fario the posterior margin of the operculum is evenly curved, the maxilla is longer than the snout, and the vomerine teeth are in a double series and persist throughont life.

\section{General Organisation.}

External Form.-The typical form of the Teleostomi is very fairly represented by that of the Trout (Fig. 865) - a long, compressed body, nearly half of which is formed by the tail, pointed anterior and posterior ends, a large vertical tail-fin, a head of 
moderate size, and a terminal mouth. Such a form is eminently fitted for rapid progression through the water. But from this characteristic fish-form there are many striking deviations. The body may be greatly elongated and almost cylindrical, as in the Eels; or of great length and strongly flattened from side to side, as in the Ribbon-fishes; or the head may be of immense proportional size and strongly depressed, as in certain shore-fishes, such as the "Fishing-frog"; or, as in the beautiful Reef-fishes, the whole body may be as high as it is long. The mouth sometimes has a ventral position, as in Elasmobranchs, with the snout prolonged over it. This is the case, for example, in the Sturgeons (Fig. 884); in the allied Polyodon the snout takes the form of a horizontally flattened shovel-like structure, about one-fourth the length of the body. On the other hand, in the ground-feeding "Star-gazers" and some other Acanthopteri the lower jaw is underlung like that of a bull-dog, and the mouth becomes dorsal in position. A beak may be produced by the prolongation of the upper jaw, as in the Sword-fish, or of the lower jaw, as in the Half-beak or Gar-fish, or of both jaws, as in the Bony Pike (Fig. 885). Such a projection is not to be confounded with the snout of the Sturgeon or Polyodon, being formed by the elongation of the bones of the jaws (premaxilla, maxilla, dentary, \&c.), whereas in the two Chondrostean forms referred to it is the anterior region of the cranium which is prolonged. Still another form of "snout" is produced in many Teleostei by the great mobility of the jaws, allowing of their protrusion in the form of a short tube. In the Wrasses or "lip-fishes" the mouth is bounded by fleshy lips (Fig. 890,lp.).

Tactile processes or barbels sometimes arise from the head; the most familiar example is that on the chin of the Cod and Haddock (Figs. 884 and $888, b$.). An operculum is always present, and is supported by a variable number of investing bones; it is continued below into a branchiostegal membrane (Fig. 866, br. m.), which, except in Crossopterygii and the Sturgeons, is supported by bony rays. In Polypterus a pair of bony jugular plates (Fig. $883, B, j u g . p l$.) are placed at the lower end of the branchiostegal membrane, between the rami of the mandible: Amia has a single plate (Fig. 886, B, jug. pl.) in the same position. Spiracles are present only in Polypterus (Fig. 898) and some Sturgeons.

The commonest number of median fins is two dorsals, one caudal, and one ventral, but this number may be increased or diminished (Figs. 888 and 890), or there may be a continuous median fin extending along the back and round the end of the tail to the anus. The dorsal fin is sometimes partly or wholly represented by a series of small finlets (Fig. 883). The tail fin may be diphycercal, heterocercal, or homocercal, and is usually the chief organ of progression. But in the Sea-horse (Fig. 892) there is no caudal fin, 
and the tail is prehensile, being used in the position of rest to coil, in the vertical plane, round sea-weeds, \&c. : when swimming it hangs downwards, having no lateral movement, and locomotion is effected by the vibration of the dorsal fin.

The dermal-rays of the caudal fin are always jointed, as in the Trout, but in most of the Acanthopteri and Pharyngognathi more or fewer of the foremost rays of the dorsal, ventral, and pelvic fins are unjointed, forming spines (Figs. 889 and 890, d.f.), sometimes large and strong enough to recall the dermal defences of some Sharks and of Holocephali (Fig. 858, d.f.r. 1, pct f. r. l). In Polypterus (Fig. 883) each finlet is supported along its anterior edge by a strong spine, to which the soft rays are attached.

The anterior dorsal fin may attain an immense size, and is subject to some curious variations. In the Fishing-frog or Angler (Lophius) its foremost rays are elongated and bear lobes or lures by which small fishes are attracted as to the bait on a fishing-line.

In the Sucking-fish (Echeneis) the anterior dorsal fin is modified into an adhesive disc by means of which the fish attaches itself to the bodies of Sharks and Turtles.

The portion of the paired fins visible externally is usually very thin, and supported entirely by dermal rays. But in the Crossopterygii (Fig. 883) the rays form a fringe round a thick basal lobe, which is supported by endoskeletal structures (vide infra). This condition of things forms an approach to the structure met with in Elasmobranchii and Holocephali. The pectorals vary considerably in size, and in the Flying-fishes (Exocotus, Dactylopterus) form large, wing-like expansions, capable of sustaining the animal in its long flying leaps into the air. In the Butterfly-fish (Gastrochisma) the pelvic fins are similarly modified. In many Fishes the pelvics are reduced to filaments or scales, and in some cases a sucking-disc is developed in connection with them. The pectorals always retain their normal position, just behind the gill-clefts, but the pelvics often become more or less shifted forwards from the typical position beside the vent. The change in position is least in the three "ganoid" orders (Figs. 883-886) and in the Physostomi (Figs. 865 and 866), in which they are usually between the middle of the abdomen and the anus, and are said to be abdominal in position; but in a large proportion of the fishes in the remaining orders of Teleostei they come to be placed almost beneath the pectorals (Fig. 890, pv.f.), when their position is called thoracic, or on the throat (Fig. 888), when they are said to be jugular in position.

A very remarkable deviation from the typical form occurs in the Flat-fishes (Pleuronectidæ), a family of Anacanthini. The body (Fig. 893) is very deep and strongly compressed : the fish habitually rests on the bottom, in some species on the right, in others on the left side, partly covering itself with sand, and occasionally swimming with a curious undulating movement. The under side is usually . 
pure white, the upper side dark. The eyes (r.e, l.e.) are both on the upper or dark-coloured side, and the skull is distorted so as to adapt the orbits to this change of position. The abdominal cavity is very small, the anus placed far forward, and the dorsal and ventral fins are sometimes continuous. Young Flat-fishes swim in the ordinary vertical position, but after a time they lie on one side and assume the adult peculiarities, the eye on the lower side gradually rotating until it reaches the upper surface.

Many Shore-fishes exhibit protective characters, the tints and markings of the skin being harmonised with those of the rocks, sea-weeds, \&c., among which they live. The effect may be heightened by fringes and lobes of skin, resembling sea-weed, and often giving the fish a most grotesque appearance. The colours are often adaptable: Trout, for instance, alter their colour by the

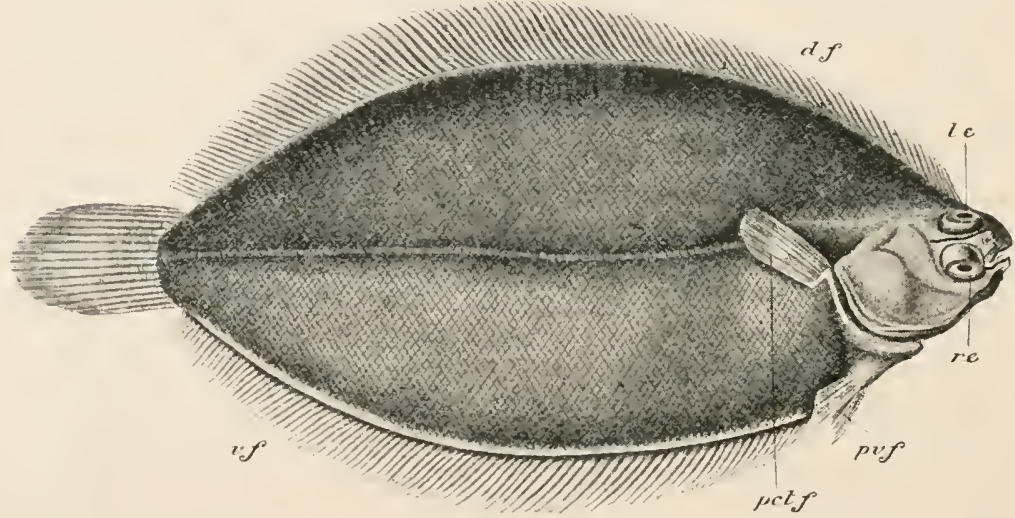

FIt. 893,-Pleuronectes cynoglossus (Craig-fluke), from the right side. $d . f$. dorsal fin ; l. $e$. left eye; pet. $f$. pectoral fin; $p v . f$. pelvic fin; r. $e$. right eye; $v$. $f$. ventral fin. (After ('uvier.)

contraction or expansion of their pigment-cells, according to whether the streams in which they live have a muddy or a sandy bottom. In some Shore-fishes, such as those of the coral reefs, the colours are of the most brilliant description; vivid reds, blues, and yellows, spots or stripes of gold or silver, are common, and although the combination of tints may sometimes seem to our eye rather crude and glaring, they appear to be distinctly protective. harmonising with the brilliant hues of the Coral Polypes and other members of the reef fauna. Pelagic fishes, such as the Mackerel and Herring, are usually steely-blue above, white beneath.

Many deep-sea Teleostei are phosphorescent: in some of these definite luminous organs (Fig. 894) are arranged in longitudinal rows along the body, each provided with a lens and other accessory parts, like those of the eye, the whole organ having the character of a minute bull's-eye lantern. Some species of the same order, 
such as the Weaver (Trachinus), possess poison-glands, opening either on one of the dorsal spines, or on a spinous process of the operculum, or, as in the Cat-fishes (Siluridæ), on the spine of the pectoral fin.

Exoskeleton.-- In many Teleostomi, suclı as Polyodon and many Eels, the skin is devoid of hard parts, but in most cases a

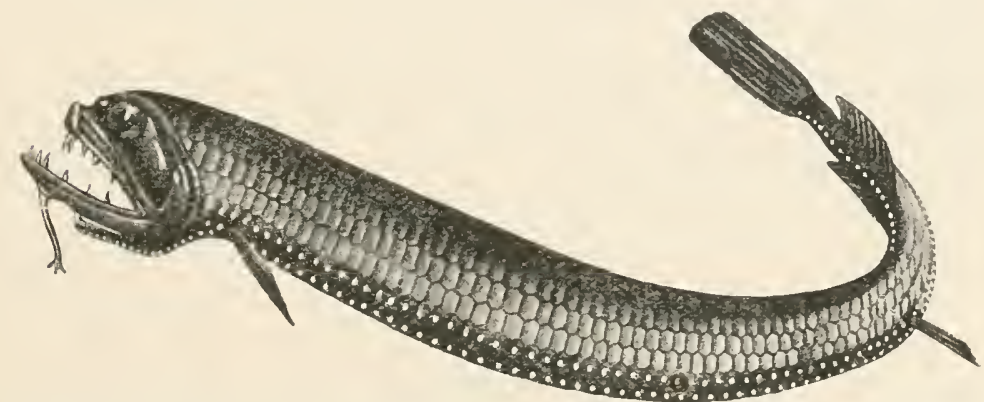

FIG. 894.-Stomias boa. The white dots are the luminous organs. (From Hickson, after Filhol.)

dermal exoskeleton is present. In Amia and in the majority of Teleostei this takes the form, as in the Trout, of scales, rounded plates of bone imbedded in pouches of the derm and overlapping one another from behind forwards. When the free border of the scales presents an even curve, as in Amia and most Physostomi and Anacanthini, they are called cycloid scales (Fig. 867); when, as in most Acanthopteri, the free edge is produced into small spines (Fig. 895, $A$ ), they are distinguished as ctenoid scales. Usually the integument is continued as a thin layer over the surface of the scales, but in a good many cases this investment is absent. In exceptional cases the scales may be so large and strong as to form a rigid armour. In the Sturgeon (Fig. 881) there is a strong armour, formed of stout bony plates, or scutes, produced into enamelled spines and articula-

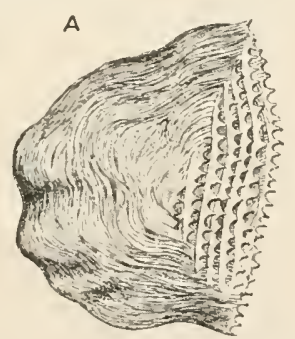

FIG. 895.-A, ctenoil scake ; $B$, ganoild scales. (After (iinther.)

ting with one another by suture. Seutes are also found in many Siluroids (Fig. 887) and in Lopho branchii (Fig. 892) and some Plectognathi (Fig. 891); while in other Plectognathi the exoskeleton takes the form, as in the File-fishes, of minute spines like the shagreen of Sharks, or, as in many Globe-fishes, of long. outstanding, bony spines. Lastly, in Polypterus and Lepidosteus are found rhomboid or ganoid scales (Fig. 895, B), in the form 
of thick, close-set, rhomboidal plates formed of bone, covered externally by a layer of enamel-like material (ganoin) and joined together by pegs and sockets. In many Ganoids the anterior fin-rays of both median and paired fins bear a row of spine-like scales called fulera (Fig. 885, $f$.). True dermal teeth similar to those of the Elasmobranchs occur scattered over the scales and lepidotrichia in some Teleostomi (e.g. Lepidosteus, Polypterus): these may be fixed or movable (Siluroids).

Endoskeleton.-In the Sturgeons the vertebral column (Fig. 897, WS.) consists of a persistent notochord with cartilaginous arches, and is fused anteriorly with the cranium. In the remaining orders bony vertebræ are present; the centra are biconcave, except

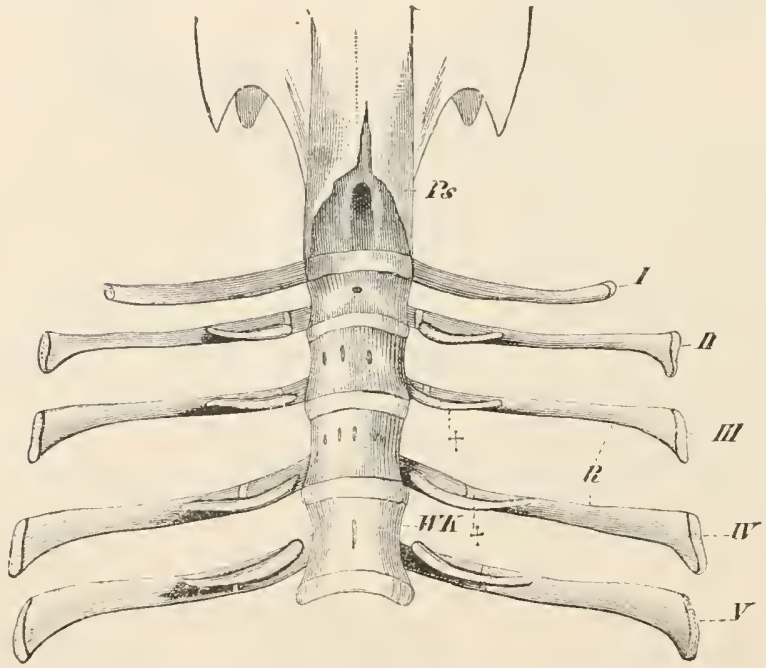

Fra. 896.-Anterior end of vertebral column of Polypterus. PS. parasphenoid ; $R . I-\mathrm{J}^{\top}$, dorsal ribs; $W K$, centra; $\dagger$, ventral (pleural) ribs. (From Wiedersheim's Comparative Anatomy.) in some Eels, in which the anterior face is flat or even convex, and in Lepidosteus, in which the anterior face is distinctly convex. Vertebræ of this form, i.e. having the centrum convex in front and concave behind, are called opisthoecelous. Ribs are usually present : in Polypterus each vertebra has two pairs, a dorsal pair (Fig. 896, $R, I-V$ ) of considerable length, running between the dorsal and ventral muscles, and a short ventral pair $(\dagger)$ between the muscles and the peritoneum: the former answer to the ribs of Elasmobranchs, the latter to the ribs (pleural ribs) of the remaining Teleostomi, which are always placed immediately beneath the peritoneum. There may be one or more sets of intermuscular bones, attached either to the neural arch (epineurals), to the centrum (epieentrals), or to the ribs (epipleurals), not preformed in cartilage, but developed as ossifications of the intermuscular septa. The posterior end of the vertebral column is turned up in the Sturgeons, Lepidosteus, and Amia, resulting in a heterocercal tail-fin: in Amia, however, the fin-rays are so disposed that the fin appears almost symmetrical. Among Teleostei the tail-fin is very usually homocercal, as in the 
Trout, with a more or less disguised asymmetry: in many cases in the adult the development of the large, fan-shaped, posterior hæmal arches completely hides the upturned end of the notochord, and in some the spinal column ends simply in a somewhat compressed centrum around which the fin-rays are symmetrically disposed; such truly symmetrical tail-fins are called diphycercal.

In the structure of the skull the Chondrostei make the nearest approach to Elasmobranchs. The cranium (Fig. 897) is an undivided mass of cartilage with a few isolated replacing bones. The roofing investing bones lie in the dermis, so as to be practically superficial, and behind pass insensibly into the scutes covering the trunk: the fact that these bones (parietals, frontals, \&c.) are exoskeletal structures is here perfectly obvious. The same is

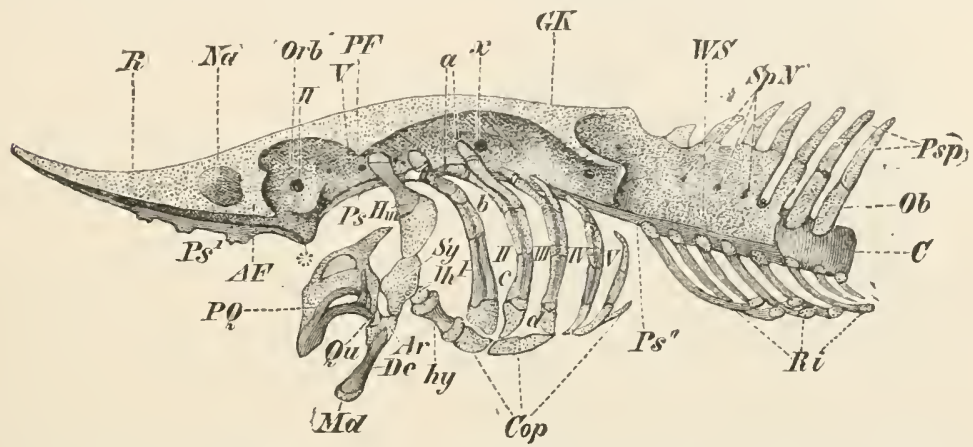

Fig. 897.-Skull of Sturgeon, with the investing bones removed. $a$, pharyngo-branchials ; $A F$, antorbital process; $A K$, articular ; $b$. epibranchial ; $c$. ceratubranchial ; $C$, notochord ; $C o p$. basibranchials ; $d$, hy pobranchial ; $D e$. dentary $; G K$, auditory capsule ; $H M$. hyoman dibular; $h y$. hyoid cornu ; $I h$. interhyal; $M d$. mandible; $N a$. nasal capsule ; $O b$. neural arches ; $P F$, post-orbital process ; $P Q$. palatoquadrate ; $P s$. $P s^{\prime} . \boldsymbol{P s}^{\prime \prime}$. parasphenoid ; $P s p$. neural spines; $Q u$. quadrate; $R$. rostrum; $R i$. ribs; $S p$. $N$. foramina for spinal nerves ; Sy. symplectic; WS, vertebral column; $x$. vagus foramen; $I-V$, branchial arches. (From Wiedersheim's Comparative Anatomy.)

the case in Polypterus (Fig. 898), in, which, however the replacing bones are better developed. In Lepidosteus and Amia, and especially the latter, the skull resembles that of the Trout in all essential respects, the main differences consisting in the absence of certain bones, such as the supra-occipital, and in the presence of additional investing bones. Among Teleostei it is only in the Physostomi that the investing bones remain separable from the chondrocranium in the adult; in the remaining orders, e.g. in the Cod, Haddock, or Perch, they become grafted on to the chondrocranium and so closely united with the replacing bones that they can be removed only by pulling the whole skull to pieces; most of the original cartilage frequently disappears in the adult and the cranium thus becomes a firm bony mass in which no distinction between replacing and investing bones is discernible.

The varying size of the gape, which is so noticeable a feature in the Teleostomi, depends upon the inclination of the suspensorium : 
in wide-mouthed Fishes (Fig. 888) the axis of the hyomandibular and suspensorium is nearly vertical or even inclined backwards; in small-mouthed forms (Fig. 891) it is strongly inclined forwards, and the length of the jaws is proportionately reduced. In the branchial arches the pharyngobranchials of each side are very commonly fused, and constitute wlat are called the superior

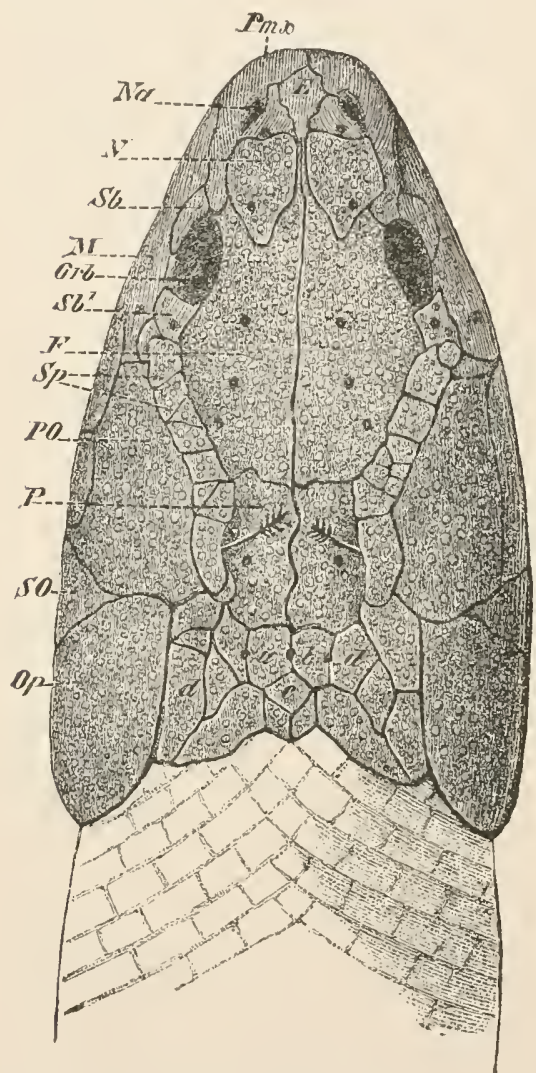

FIf. 898. - Skull of Polypterus, from above. $F$, frontal; $\boldsymbol{M}$. maxilla ; $\boldsymbol{N}$. nasal; $\boldsymbol{T}$ a. nostril; Op. opercular; Orb orbit; $l$ ' parietal 'The remaining letters point to less important investing bones. The arrow is passed into the spiracle. (From Wiedersheim's Comparative Anatomy.) pharyngeal bones: the reduced fifth branchial bars, or inferior pharyngeal bones, bite against them. The Pharyngognathi are distinguished by having the inferior pharyngeal bones united into a single bony mass of characteristic form (Fig. 890, $B)$. The gill-rakers are often very highly developed, and may form a mesh capable of retaining even microscopic organisms.

In the shoulder-girdle, as in the skiull, the Chondrostei approach the Elasmobranchs. There is a primary shouldergirdle consisting of large paired cartilages, not united in the middle ventral line, and unossified: each is covered externally by a large scute-like investing bone, the clavicle. In the remaining Ganoids and in Teleostei the primary shoulder-girdle is reduced in size and is usually ossified by two bones, a dorsal scapula and a ventral coracoid: sometimes, as in the Trout, there may be an additional ossification, the mesocoracoid. Additional investing bones-supra-clavicle, post-clavicle, \&c.-are added, and one of them, the post-temporal, serves to articulate the shoulder-girdle with the skull (Fig. 874).

In the skeleton of the pectoral fin it is the Crossopterygii which approach most nearly to Elasmobranchs. In Polypterus (Fig. 899) the basal lobe of the fin is supported by a rod-like ossified propterygium $(P r)$, a broad cartilaginous, partly ossified, meso- 
pterygium $(M S)$, and an ossified metapterygium $(M T)$; to these, two rows of elongated radials ( $R a$, $R\left(a^{1}\right)$ are articulated fanwise, and these in their turn give attachment to the fin-rays $(F S)$. In all the remaining orders the basalia (pro-, meso-, and meta-pterygium) are absent, and the endoskeleton of the fin consists only of a single or double row of radials (Fig. 874).

In Polypterus there is a vestigial pelvic girdle (Fig. 900, BP) in the form of a small rhomboidal cartilage to which the anterior ends of the basalia $\left(B a s^{1}\right)$ are attached: thus in the structure of the posterior extremities also, the Crossopterygii are the most primitive of the Teleostomi. In all the remaining orders the pelvic girdle appears to be atrophied. The pelvic fin is supported by a single bone of variable form (Fig. 875, B. PTG) and apparently arising from the fusion of proximal pterygiophores. Between its posterior end and the dermal rays irregular

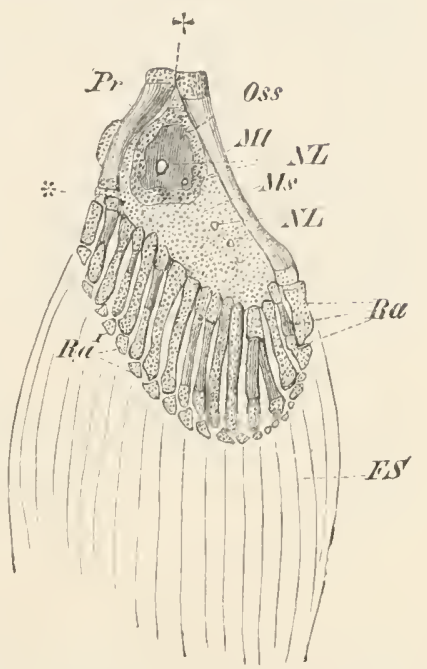

Fig. 899.-Pectoral fin of Polypterus. FS, dermal rays; $M S$, mesopterygium; $\boldsymbol{M T}$. metapterygium; $N L$, nerve-foramina; Oss. ossification in mesopterygium ; $P r$. propterygium ; $R a$. first radials; $R a$. second radials. At * the bony marginal rays meet and shut off the middle region from the shoulder-girdle. (From Wiedershein's Comparative Anatomy.) nodules, representing radials, may be interposed.

The distinction between laard or unjointed fin-rays, or spines, and

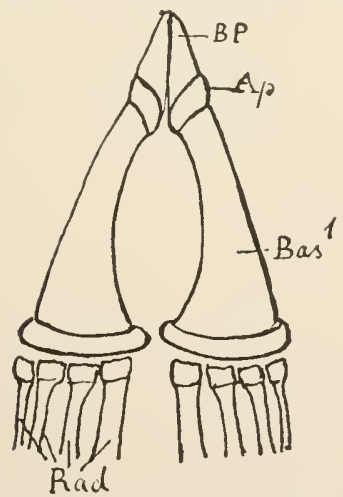

Fig. 900.-Pelvic fin of young Poly. pterus. Ap. part of basale ; Bas 1 . basale; $B T$. pelvic cartilages' (fused in adult); Rad. radiass. (From Wiedersheim.)

fishes (Pegutecus and Truchypter soft or jointed fin-rays has already been referred to. The first ray of the dorsal and pectoral fins sometimes, e.g. in Siluroids (Fig. 887), has the form of a very strong spine articulated by a bolt-and-shackle joint, i.e. by the interlocking of two rings. In some cases the first dor'sal spine springs from the skull.

The texture of the bones is subject to wide variation: in some Acanthopteri they are very thick and strong, in some places almost like ivory; while in the Lumpfish (Cyclopterus), the huge Sunfish (Orthagoriscus), and in many deepsea forms, such as the Ribbon- 
is so small that the bones are easily cut with a knife and weigh astonishingly little when dry.

Electric Organs.-Three genera of Teleostomi possess electric organs, the Electric Cat-fish (Malapterurus), one of the Siluridæ, found in the fresh waters of tropical Africa, the Electric Eel (Gymnotus), a Physostome occurring in Brazil and the Guianas, and an American Star-gazer of the genus Astroscopus. In Malapterurus the electric organ extends over the whole body, beneath

A
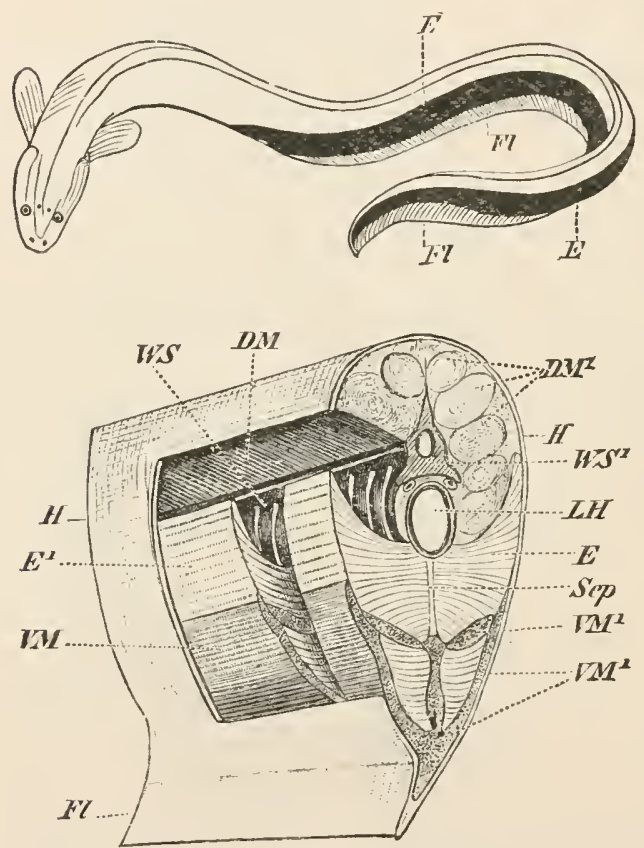

Fig. 901.-Gymnotus electricus, $A$, showing the extent of the electric organ $(E)$. $F l$, ventral fin. B, small portion of tail, in section. DM. DM.' dorsal muscles; $E$. $E$ ' electric organ ; $F l$, ventral fin ; $H$, skin ; $L H$ caudal canal; Sep. fibrous septum ; VM. VMI.' ventral muscles; $W S$, $W S^{\prime}$, vertebral column, with spinal nerves. (From Wiedersheim's Comparative Anatomy.) the skin; in Gymnotus (Fig. 901) there is a pair of batteries in the ventral half of the greatly elongated tail ; in Astroscopus the electric organs are situated on the upper surface of the head just behind the eyes. As in the Elasmobranchs, the electric organs are formed by modification of muscular tissue.

\section{Digestive Organs.} - Some Teleostomi are toothless; but in most instances teeth are present, and may be developed on the premaxilla, maxilla, palatine, pterygoid, vomer, parasphenoid, dentary, basihyal, and bones of the branchial arches. It is characteristic of most Teleostei, with the exception of Physostomi, that the maxilla is edentulous and does not enter into the gape (Fig. 889). In a large majority of species the teeth are small, conical, and recurved, suitable for preventing the struggling prey from slipping out of the mouth, but quite unfitted for either tearing or crushing. In some Fishes, such as the Pike, the teeth are hinged backwards so as to offer no resistance to the passage of the prey towards the gullet, but effectually barring any movement in the other direction. In many deep-sea Fishes (Fig. 894) the teeth are of immense size and constitute a very formidable armature to the jaws. Many 
instances occur in which there is a marked differentiation of the teeth, those in the front of the jaws (Fig. 902) being pointed or chisel-edged, and adapted for seizing, while the back teeth have spherical surfaces adapted for crushing. In the Wrasses (Fig. 890, B) strong crushing teeth are developed on the pharyngeal bones. In the Globe-fishes the teeth are apparently reduced to one or two in each jaw, but each " tooth" in this case really consists of numerous calcified plates fused together. The teeth may be either simply embedded in the mucous membrane so as to be detached when the bones are macerated or boiled, or they may be implanted in sockets of the bone, or ankylosed to it. They are formed of some variety of dentine,

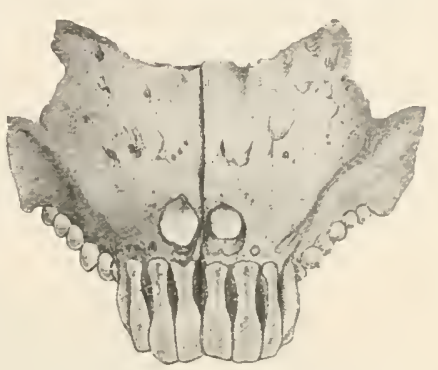

LFIG. 902.-Premaxillie of Sargus, showing teeth. (After Owen.) and are often capped with enamel. Their succession is perpetual, i.c. injured or worn-out teeth are replaced at all ages.

In some species the enteric canal shows little differentiation into regions, but, as a rule, gullet, stomach, duodenum, ileum, and rectum are more or less clearly distinguishable. The stomach is generally $\mathbf{V}$-shaped, but its cardiac region may be prolonged into a blind pouch; it is often very distensible, allowing some of the deep-sea Teleostei to swallow Fishes as large as themselves. In the Globe-fishes the animal can inflate the gullet with air, when it floats upside down on the surface of the water. The Ganoids have a spiral valve in the intestine, which is very well developed in Polypterus and the Sturgeon, vestigial in Lepidosteus (Fig. 904, sp. v.) and Amia : it is absent in all Teleostei, except possibly in Chirocentrus, one of the Physostomi. The liver is usually large; a pancreas may be present as a compact gland, as in Elasmobranchs, or may be widely diffused between the layers of the mesentery, or in part surrounded by the liver. Pyloric caeca are commonly present, and vary in number from a single one to two hundred. The anus is always distinct from, and in front of, the urinogenital aperture.

Respiratory Organs.-The gills are usually comb-like, as in the Trout, the branchial filaments being free, owing to the atrophy of the interbranchial septa. In the Sturgeon, however, the septa are fairly well developed, reaching halfway up the filaments, so that the latter are free only in their distal portions; this arrangement is obviously intermediate between the Elasmobranch and Teleostean conditions. The most striking deviation from the normal structure occurs in Lophobranchii, in which the gillfilaments are replaced by curious tufted processes (Fig. 892, $B, g$.). As a rule gills (holobranchs) are developed on the first four branchial arches, but the fourth is frequently reduced to a hemibranch, 
and further reduction takes place in some cases. The pseudobranch or vestigial hyoidean gill may either retain the characteristic comb-like structure, as in the Trout, or may be reduced, as in the Cod, to a gland-like organ formed of a plexus of blood-vessels and called a vaso-ganglion or rete mirabile. In most Teleostomi the mechanism of respiration is similar to what has already been described in the case of the Trout, and respiratory valves are developed in the mouth-cavity. But there are considerable differences in details, more especially as regards the relative importance of the opercula and the branchiostegal membranes in earrying on the movements of inspiration and expiration.

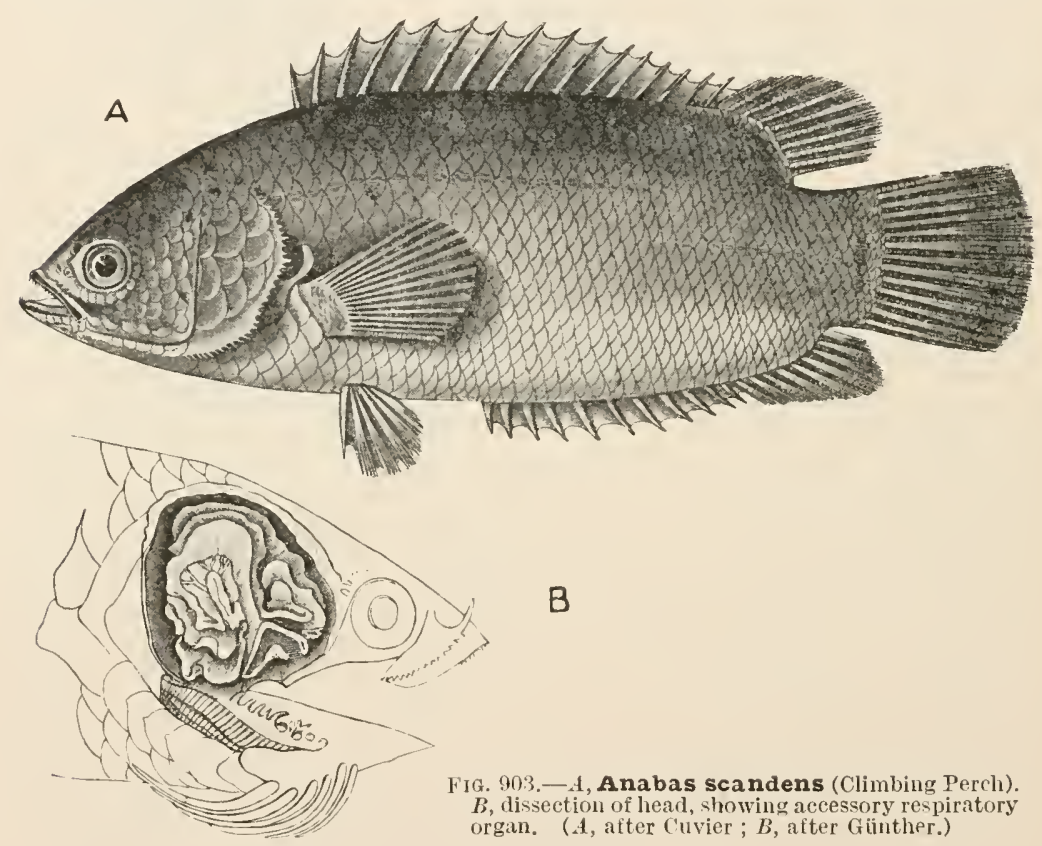

In addition to the gills some Teleostei possess accessory organs of respiration. In Amphipnous, an Indian Physostome, the gills are poorly developed and are functionally replaced by a vascular sac occurring on each side of the body and opening in front into the first (hyobranchial) gill-cleft. Such sacs are physiologically, though not morphologically, lungs. In the Climbing Perch (Anabas) of the Oriental Region (Fig. 903) the superior pharyngeal bones are developed into folded plates $(B)$ covered with vascular mucous membrane and capable of retaining water for a considerable period: the Fish is able to traverse the land, and is even said to climb trees, holding on alternately by the spines of its pre-operculum and of its ventral fins. It has become so thoroughly a land-animal 
that it is drowned if immersed in water. Siluroid Callichthys anal respiration takes place, air being drawn into and cxpelled from the rectum. Lastly, in the curious little goggle-eyed Periophthalmus of the Indian and Pacific Oceans the tail-fin scems to scrve as a respiratory organ, being kept in the water while the Fish perches on a rock.

The air-bladder retains its connection with the gullet (rarely with the stomath) in Ganoids and Pliysostomes; in the other Teleostei the pneunatic duct atrophies in the adult and the bladder becomes a shut sac. In Polypterus it consists of two lobes, a large left and a smaller right. The pneumatic duct is always connected with the dorsal wall of the gullet or stomach, except in Polypterus, in which the aperture is ventral. The bladder is sometimes divided into compartments or produced into lateral offshoots : in Amia and Lepidosteus (Fig. 904, a. b.) its wall is sacculated or raised into anastomosing ridges, enclosing more or less well-marked chambers and thus resembling a lung. In Polypterus its lung-like character is enhanced by the ventral position of the opening and by the blood being conveyed to it (as is also the case in Amia) by a pair of pulmonary arteries given off from the last pair of epibranchial arteries, as in the Dipnoi.

The air-bladder seems to be capable of acting as a sort of accessory respiratory organ; it has been found that in a Percl, asphyxiated in stagnant water, the oxygen in the bladder, which normally amounts to 20 or 25 per cent., is entirely absorbed and replaced by nitrogen and carbonic acid. Its normal function, however, is hydrostatic, i.e. it serves to keep the Fish of the same specific gravity as the water. The specific gravity of the Fish as a whole, rising or falling as it must on account of the increase or decrease of pressure at various depths as the Fish
In the little armourcd

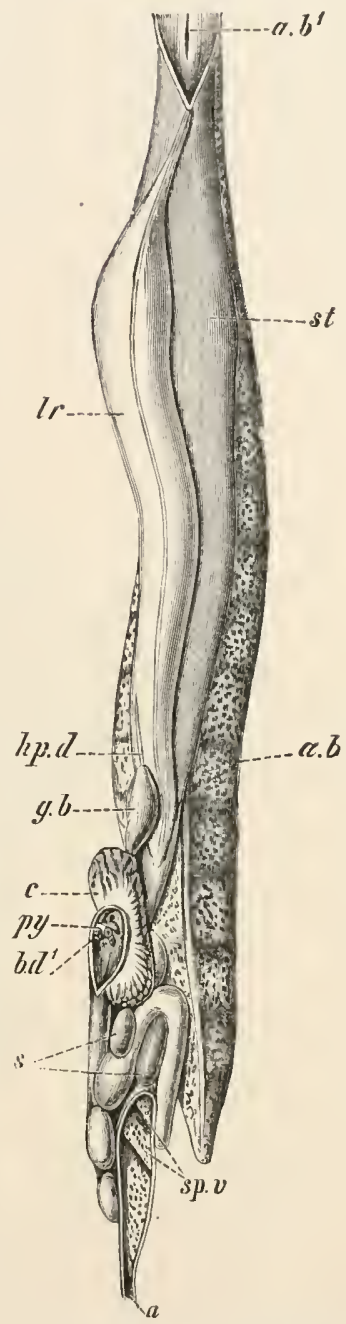

Fig. 904.-Digestive organs and air-bladder of Lepidosteus. $a$. anus; $a$. b. alr-bladder ; $a$. $b^{\prime}$. its aperture in the pharynx ; $b, d^{\prime}$. aperture of bile. duct; $c$. pyloric cæea ; $g$. $b$. gall-bladder; $h p, d$. hepatic duct; $l r$. liver; $p y$. pyloric valve; s. spleen'; sp. v. spiral valve ; st. stomach. (From Wiedersheim's Comparative Anatomy, after Balfour and Parker.) 
causing greater or less compression of the gases in the air-bladder, can be brought to approximate to that of the surrounding water by increase or decrease in the quantity of the contained gas. This is brought about by secretion or absorption, often by means of vaso-ganglia or red glands (Fig. 905, vs. gn). These are elevations of the wall of the bladder, abundantly supplied with blood, and containing tubular glands which open into the cavity of the bladder. In Fishes with a pneumatic duct the red glands are absent, but

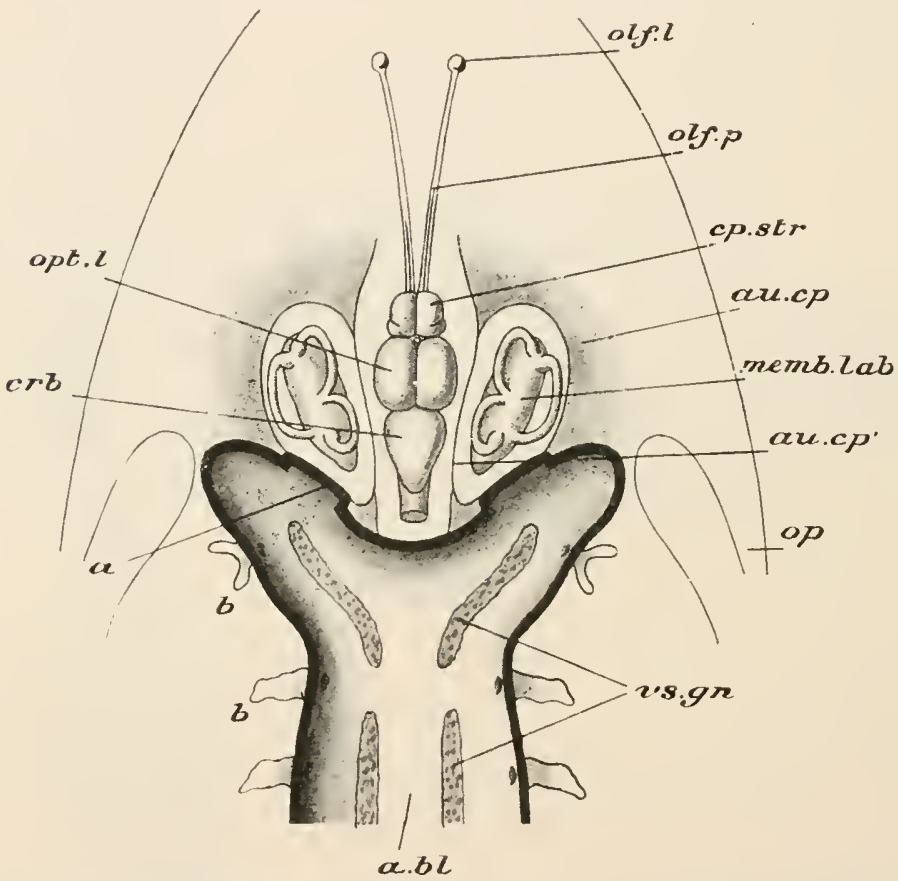

FIG. 905.- Horizontal section of posterior portion of head and anterior end of air-bladder in Pseudophycis bachus, one of the Gadida or Cods (semi-diagrammatic). $a$. thickened portion of air-bladder fitting into fenestra in posterior wall of auditory capsule; $a$. $b$. airblalder; $a u$. cp. outer wall of auditory capsule ; $a u . c p^{\prime}$. inner (membranous) wall ; $b$. hollow offshoots of air-bladder; $c n$. sir. corpora striata; $c r b$. cerebellum: memb. lab. membranous labyrinth; olf. $l$. olfactory bullos : olf. $p$. olfactory peduncles (olfactory tracts); op. operculum : opt. l. optic lobes; $v s . g n$. vaso-ganglia.

in the Eels and others their place is taken by red bodies of similar appearance, but with non-glandular epithelium. In some forms with closed air-bladder the anterior end of the organ is forked, and each branch (Fig. 905, a) fits closely against a membranous space in the posterior wall of the auditory capsule, while laterally it extends outwards in the region of the shoulder-girdle, and comes to lie inmediately beneath the skin; in this way varying pressures on the surface of the body are transmitted through the air in the bladder to the auditory organ. In the Carps and Siluroids a 
chain of bones connects the air-bladder with the auditory organ, forming the Weberian apparalus, the function of which, as of the simpler arrangement described above, is probably " to bring directly" to the consciousness of the Fish the varying tensions of the gaseous contents of the air-bladder, due to the incidence of varying liydrostatic pressures."

The structure of the heart forms one of the most striking differences betwcen the three Ganoid orders and the Teleostei. In Ganoids there is a muscular conus arteriosus with rows of valves, as in Elasmobranchs; in Teleostei a vestige of the conus containing two rows of valves has been found in Albula (Physostomi), and similar vestiges occur in several other genera of the same sub-order, but in all the rest of the order it is entirely unrepresented. On the other hand, Teleostei always have a large bulbus aortæ, formed as a dilatation of the base of the ventral aorta.

In the brain the cerebellum and optic lobes are usually large; the diencephalon is well developed in Ganoids, almost obsolete in Teleostei. In the Teleostei and Ganoidei the prosencephalon has the general features which have been described in the account of the brain of the Trout: it is not divided into hemispheres and has a roof which, except in Amia, is completely non-nervous; its floor consists of a pair of massive corpora striata (Fig. 906, prs., and Fig. 877, BG.). In most instances the olfactory bulbs are in close apposition with the olfactory region of the prosencephalon without the intervention of olfactory stalks or tracts; but in some cases, as in the Cod (Fig. 905, olf. p.), they are borne on long olfactory peduncles or

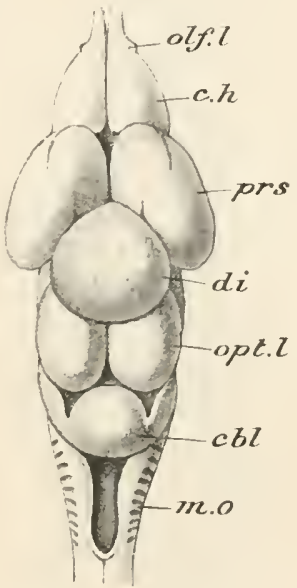

Fli. 906 - I Irain of Lepidosteus, dorial view. cbl. cerebellum ; $c$. $h$. olfartory part of prosencephalon ; $d$. dienceplablon ; $m$. o. medulla oblongata: olf. $l$. olfactory bulbs; opt. l. optic lobes; prs. corpora striata. (After Baltour and Parker.)

olfuctory tracts. The Ganoids agree with Elasmobranchs in the fact that the optic nerves form a chiasma, while in Teleostei they simply cross one another or decussate. Here also, however, the distinction is not quite absolute, since in the Herring and some other Physostomes one nerve passes through a slit in the other. In some Plectognaths the spinal cord undergocs a remarkable shortening : in a Sun-fish $2 \frac{1}{2}$ metres in length and weighing a ton and a half the cord is only 15 millimetres long, being actually shorter than the brain.

Urinogenital Organs.-The kidney (Fig. 876, kid) is formed from the mesonephros of the embryo, and usually attains a great size; the pronephros usually atrophies. The ureter (ur.) is the 
undivided pronephric duct : it unites with its fellow of the opposite side before opening either directly on to the exterior or into a urinogenital sinus. A urinary bladder is formed as a single or double dilatation of the ureter. The right and left kidneys undergo more or less fusion, and their anterior ends are usually converted into adenoid or lymphatic tissue $\left(k d .^{\prime}\right)$, so that, while resembling

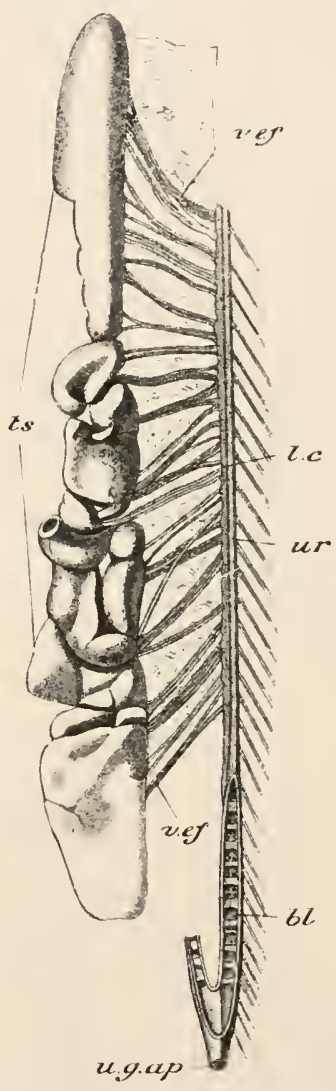

F19. 907.-Male organs of Lepi dosteus. $b l$. bladder; $l$. $c$. longitudinal canal ; $t s$. testis ; u.g. ap. urinogenital aperture ; ur. ureter ; $v$.ef. vasa efferentia. (After Balfour and Parker.) the rest of the organ in external appearance, they do not discharge a renal function.

The male organs of Lepidosteus may be taken as an example of those of Ganoids. The testis (Fig. 907, ts.) is a paired, lobulated organ, the secretion of which is carried by a large number of vasa efferentia $(v$. ef.) into a longitudinal canal (l.c.) lying alongside the ureter (ur.). From this canal tubes are given off which communicate with the urinary tubules of the kidney or open directly into the ureter, so that the seminal fluid has to traverse the latter in order to reach the urinary bladder $(b l$.$) and make its escape$ by the common urinogenital aperture (u.g. ap.). In Teleostei there are no vasa efferentia, but the posterior end of the testis is directly continued into a duct (Fig. $876, v . d$.) which unites with its fellow of the opposite side and opens either into a urinogenital sinus, as in the Trout, or, as in the Cod, directly on the exterior, between the anus and the urinary aperture. In the Eels the seminal fluid escapes into the cœlome and is discharged by genital pores.

In most Ganoids the oviducts (Fig. 908, $B$, ovd.) have funnel-like anterior ends (ovd.") opening into the cœlome, while posteriorly (ovd.') they discharge into the dilated ureters $(b l$.$) . A similar arrange-$ ment occurs in the Smelt, one of the Physostomi (Salmonidæ), in which the eggs are discharged from the outer or lateral face of the ovary into the open end of the oviduct. But in most Teleostei and in Lepidosteus (Fig. 908, A) the ovary (ovy.) is a hollow sac continued posteriorly into the oviduct (ovd.) : the eggs are set free into its cavity from the folds into which its inner surface is produced, and so pass directly into the oviduct without previously entering the 
colome. An ovary of this kind reminds us of the state of things in Arthropods, in which also the ovary is a hollow organ discharging its products into its internal cavity, whence they pass directly into the continuous oviduct. It was pointed out that the lumen of the ovary in this case was to be looked upon as a shut-off portion of the coelome: this is certainly the case in Lepidosteus and the Teleostei. In the embryo a longitudinal fold grows from the ventral edge of the then solid ovary, and turns upwards along, the lateral face of the organ : it is met by a descending fold of peritoneum from the dorsal wall of the abdomen, and by the union of the two folds a cavity is enclosed, which is the lumen of the ovary. The oviduct is developed as a backward continuation of these folds of peritoneum, and appears to be quite unconnected with the embryonic renal system, and therefore not to be homologous with the oviducts of Elasmobranchs and Holocephali, which, as we have seen, are Müllerian ducts. In the Salmonidæe and the Fels oviducts are absent, and the ova are discharged by

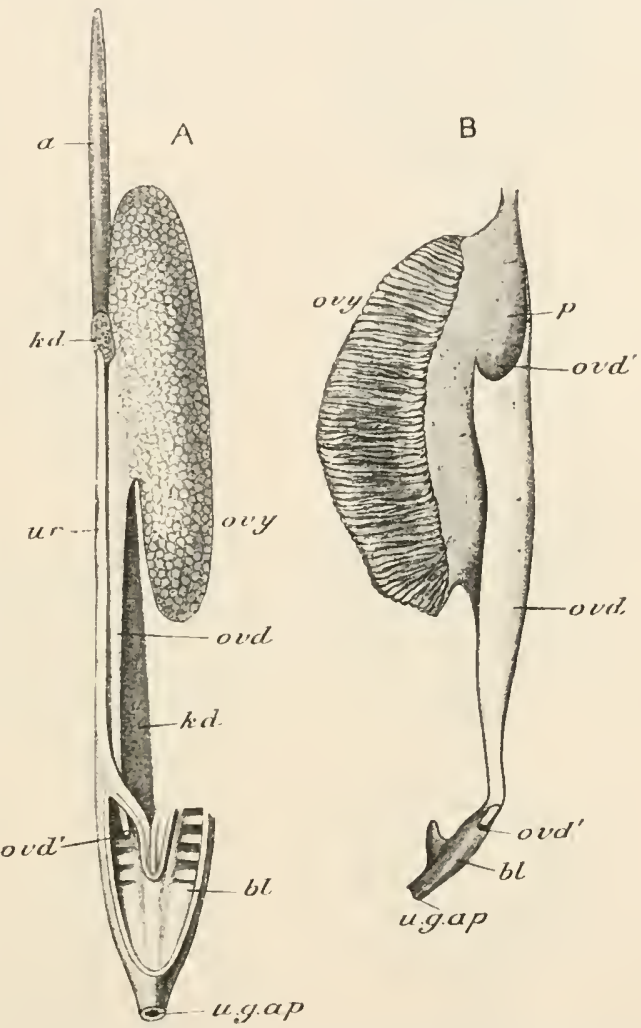

Fid. 908.-Female organs of Lepidosteus ( $A$ ) anil Amia (b). a, degenerate anterior portion of kidney; $b l$. bladder : kd. kidney ; ovd. oviduct; ovd.' a perture of ovidurt into bladder ; ovd." peritoneal aperture ; ovy. ovary ; $p$. peritoneum; u.g. ap. urinogenital aperture; ur. ureter. ( $A$, after Balfour and Parker ; $B$, after Huxley.)

genital pores, which are probably to be looked upon as degenerate oviducts. True abdominal pores are present in Ganoids and in some Physostomi. Most Teleostomi are diœcious, but Serranus, one of the Perch family, is hermaphrodite and self-impregnating; Chrysophrys is hermaphrodite and successively male and female; and there are many well-known species, such as the Cod and the Herring, which exhibit the hermaphrodite condition as an occasional variation. 
Reproduction and Development.-Most Teleostomi are oviparous, the eggs being impregnated after they are laid, but in some Teleostei, such as the Viviparous Blenny (Zoarees), internal impregnation takes place; the young are developed in the hollow

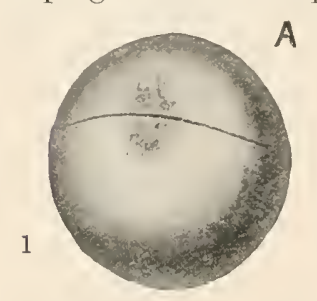
ovary and are brought forth alive. Many instances of parental care of the young are known, the most familiar being that of the male Stickleback (Gasterosteus), which constructs a nest of weeds, fastened together by a glutinous secretion of the kidneys, and
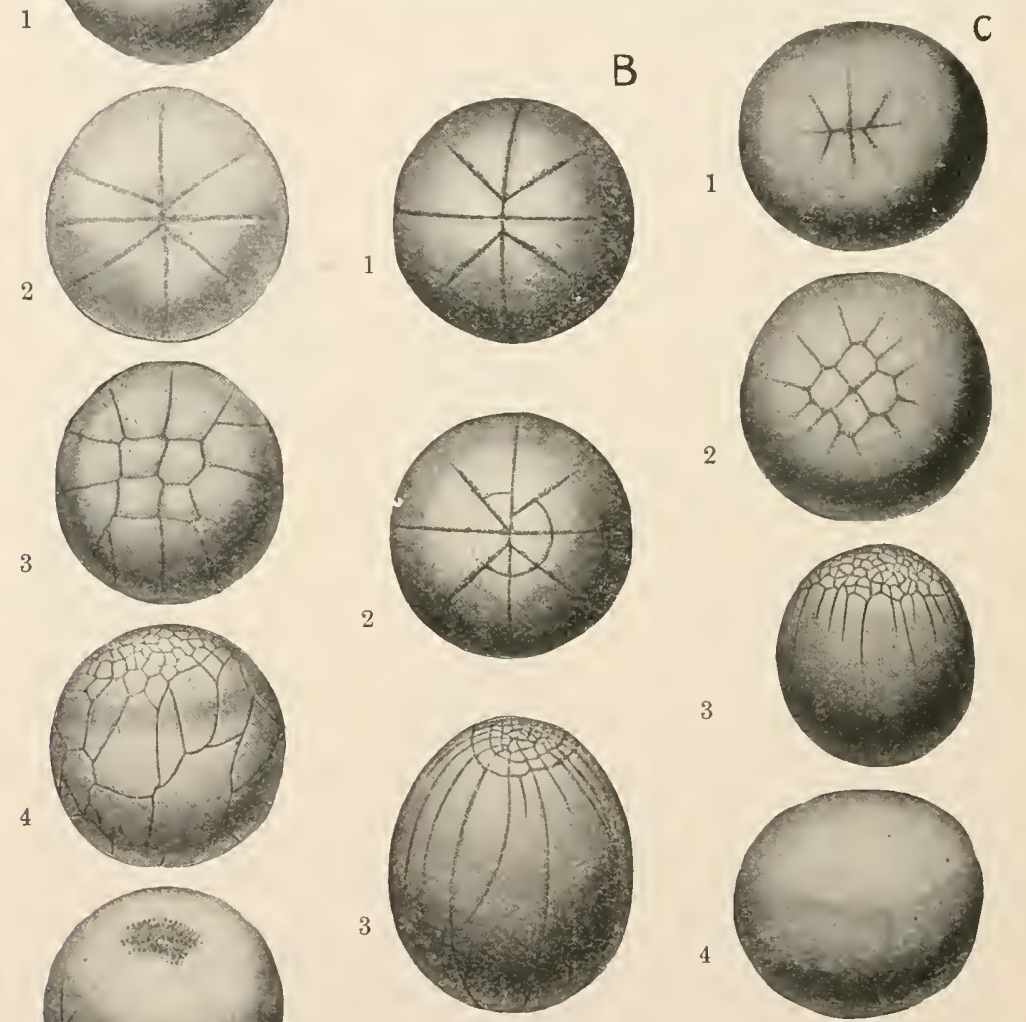

3

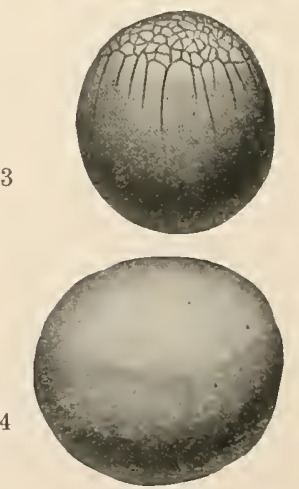

Hit. 909.-Segmentation in Acipenser (A), Amia (B), and Lepidosteus $(C) . A 1,2$, and $3, B^{\prime} 1$ and 2 , $C 1$ and 2 are views of the apical pole; the remaining figures are side views. (lrom Graham Kerr, after Dean, Whitman, and Eycleshymer.)

jealously guards the developing young. In the Sea-horse (Hippocampus) and the Pipe-fish (Syngnathus) the young are developed in a pouch (Fig. 892, brd. p.) on the abdomen of the malc. In the Siluroid Aspredo the eggs are pressed into the soft spongy skin of the belly and thus carried about by the parent. The ova are 
always small as compared with those of Elasmobranchs, never exceeding 5 to $10 \mathrm{~mm}$. in diameter, and being usually much smaller. 'They are rarely protected by an egg-shell. They are produced in immense numbers, a single female sometimes laying several millions: in such cases the mortality among the unprotected embryos and young is immense. The eggs may be pelagic, i.e. so light as to float when laid, as in the Cod, Haddock, Turbot, Sole, \&c. ; or demersal, i.e. so heavy as to sink to the bottom, as in the Herring, Salmon, Trout, \&c. In some cases (Chilobranchus) they become cenrented to the surface of a rock.

In all the Ganoids hitherto investigated, with the exception of Lepidosteus, segmentation is complete. In Acipenser and Amia (Fig. 909, $A$ and $B$ ) it is very unequal, the megameres being immense as compared with the micromeres: in Polypterus it is subequal at first, becoming unequal later: the process may be said to be intermediate between the holoblastic and meroblastic types. In Lepidosteus (Fig. 909, C) the segmentation is meroblastic, the fissures not extending much beyond the equator of the egg. In Teleostei segmentation is always partial and discoid a l. $\mathrm{Th}$ e general features of development are much the same as in the Trout, except that in the Sturgeon and

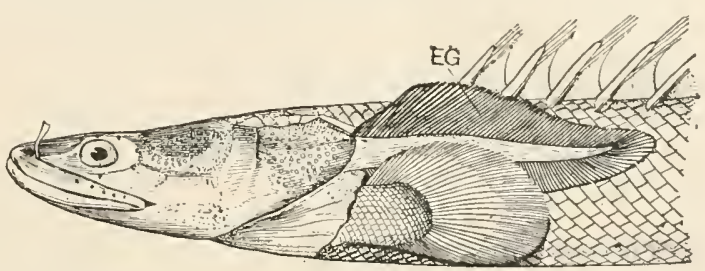

Fig. 910.- Polypterus bichir. Head of advanced larva. EG. external gill. (From Dean, after steindachner.)

Polypterus, as in Craniates in general, there is an open medullary groove which becomes closed in to form a medullary canal. There is frequently a metamorphosis: in Lepidosteus, for instance, the newly-hatched young is provided with a sucking-disc, and the proportions of the head are quite different from those of the adult. In the larval Sturgeon provisional teeth are present, and in many Teleostei the young differ from the adult in the presence of large spines, which probably, like the spines in the zoæa-stage of some Crustacea, serve a defensive purpose. The pelagic larva of Eels are strongly compressed, perfectly transparent, and have colourless blood. They are sometimes known as "Glass-fish," and were formerly placed in the genus Leptocephalus, their real nature being unknown. The young of the Crossopterygii (or at least Polypterus, Fig. 910) have external gills, as in Dipnoi and Amphibia (vide infra), and the same holds good of Cobitis, Heterotis, and Gymnarchus among the Teleostei.

The Geographical Distribution of the Ganoid Teleostomi is curiously limited: they are all essentially fresh-water formsalthough some Sturgeons are found in the sea-and are almost 
exclusively inhabitants of the Northern Hemisphere, and especially of the Holarctic Region. The Chondrostei occur in the rivers of Europe, Asia, and North America: one genus of Sturgeons (Scaphirhynchus) lives in the Mississippi and in the rivers of Central Asia, but not in the intermediate regions : in the same way Polyodon is found only in the Mississippi, while the closely-allied Psephurus is found in the Yangtse-kiang and Hoangho-a striking instance of discontinuous distribution. Amia is found in the fresh waters of the United States; Lepidosteus extends also into Central America and Cuba. Polypterus lives in the Upper Nile and some other tropical African rivers; Calamoichthys in the Old Calabar River.

A
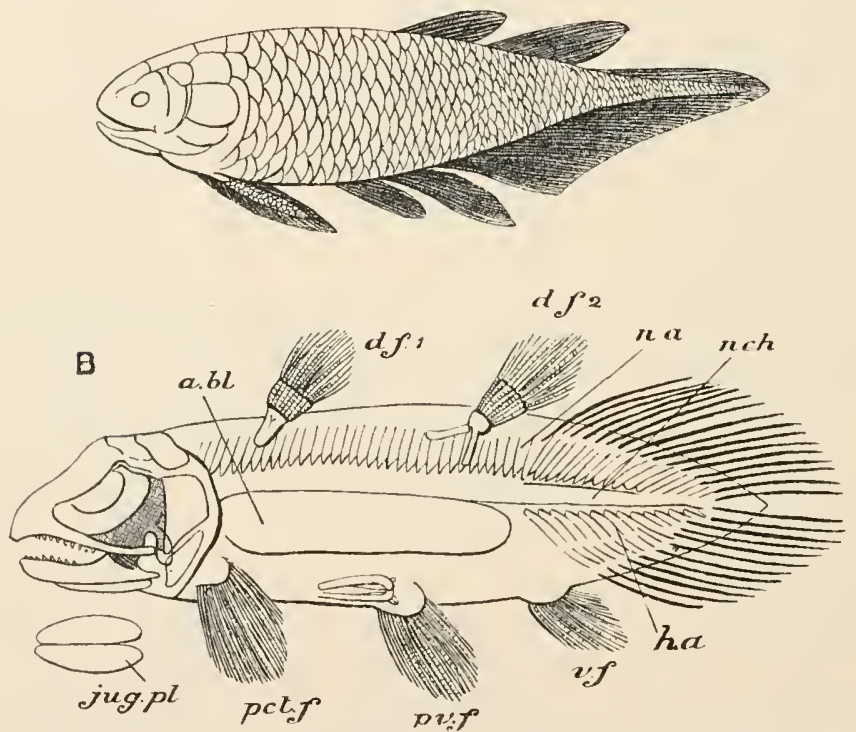

FIG. 911.-A, restoration of Glyptolepis (Devonian) ; B, Macropoma mantelli (Uretaceous). $a . b l$. ossified air-bladder; $d . f .1, d . f .2$, dorsal fins ; $h$. $a$. hæmal arches ; $j u g . p l$. jugular plates : $n$. $a$. neural arches; $n s h$. position of notochord; pct. $f$. pectoral fin ; $p v . f$. pelvic fin; $v$. f. ventral fin. (From Nicholson and Lydekker.)

Among Teleostei the Physostomi are largely, though uot exclusively, fresh-water Fish; the Carps, Eels, Salmonoids, and Siluroids are important examples. The Acanthopteri, Pharyngognathi, and Anacanthini are mostly marine, some being inhabitants of the shores, some pelagic, some abyssal, extending to a depth of nearly 3,000 fathoms. As we have seen, many species are practically terrestrial. All the sub-orders are universally distributed, so that we have to descend to families before meeting with any important facts in geographical distribution.

The Distribution in Time of the Teleostomi is interesting as showing the gradual replacement of the lower or more generalised 
members of a group by the higher or more specialised forms. During the whole of the Palæozoic and the greater part of the Mesozoic era the three orders of Ganoids, to-day small and isolated groups, formed the whole of the Teleostomian fauna, and it is not until the Cretaceous period that the Teleostei, the present dominant order, make their appearance. From the Cretaceous onwards the Ganoids undergo a progressive diminution in numbers, genus after genus and family after family becoming extinct, while a corresponding increase takes place in all the sub-orders of Teleostei.

The Crossopterygii make their first appearance in the Devonian period, and, between that period and the Cretaceous, include six families and a large number of genera and species. They exhibit (Fig. 911) a very considerable range of variation in external and

A
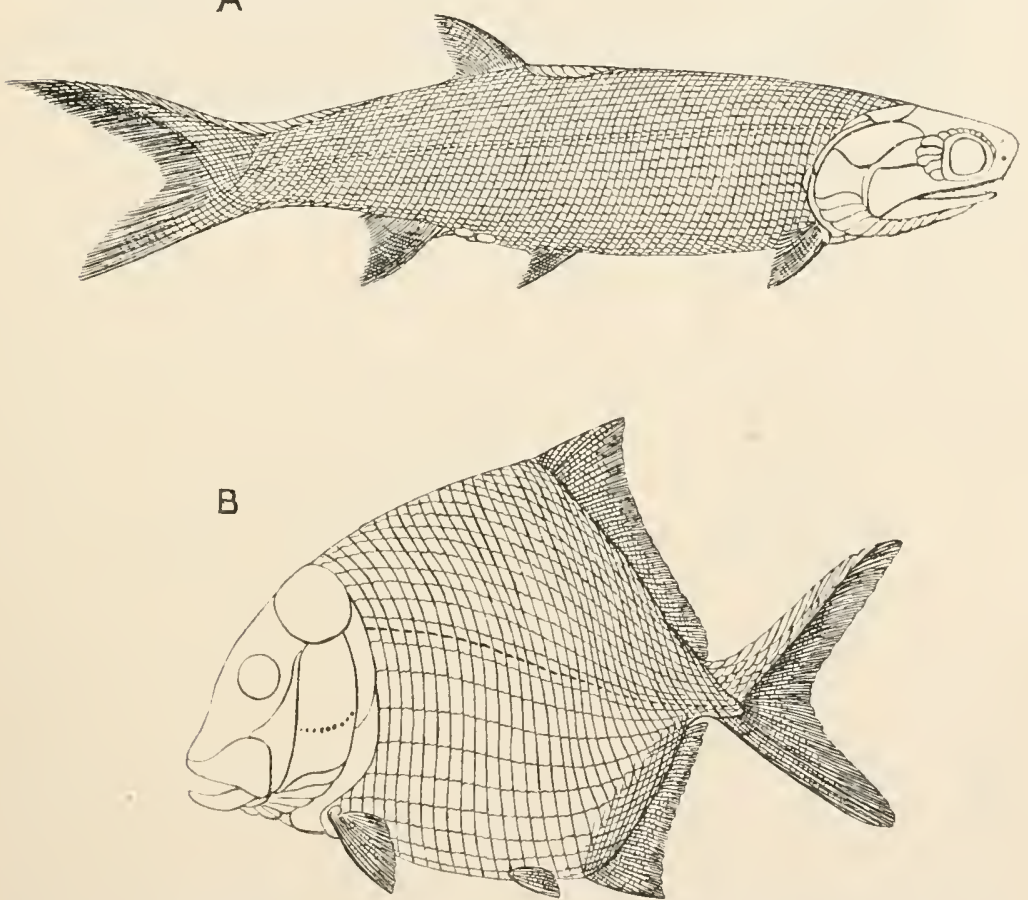

FIG. 912,- $A$, Falæoniscus macropomus (Permian); $B$. Platysomus striatus (Permian). (From Nicholson and Lydekker.)

internal characters. There are usually two dorsal fins, the tail may be diphycercal or heterocercal, the scales rhomboid or cycloid. In some genera, also, there was a persistent notochord (B. nch.), the fossils showing well-preserved neural and hæmal arches, but no signs of centra. In many cases the interspinous bones or proximal pterygiophores of the dorsal fins are fused into a single 
basal bone. All agree in the possession of lobed fins; the basal lobe is sometimes so long as to approach the type of structure we shall find to characterise the Dipnoi (vide infra).

The Chrondrostei are also largely represented, from the Devonian upwards, and include a great variety of forms, many of which, apart from the heterocercal tail, have a strong external resemblance to Teleostei (Fig. 912). Some have the characteristic spindle-form of strong-swimming Fishes $(A)$, others the high, compressed form of such shore-fishes as the Reef-fishes $(B)$. Scutes are present in
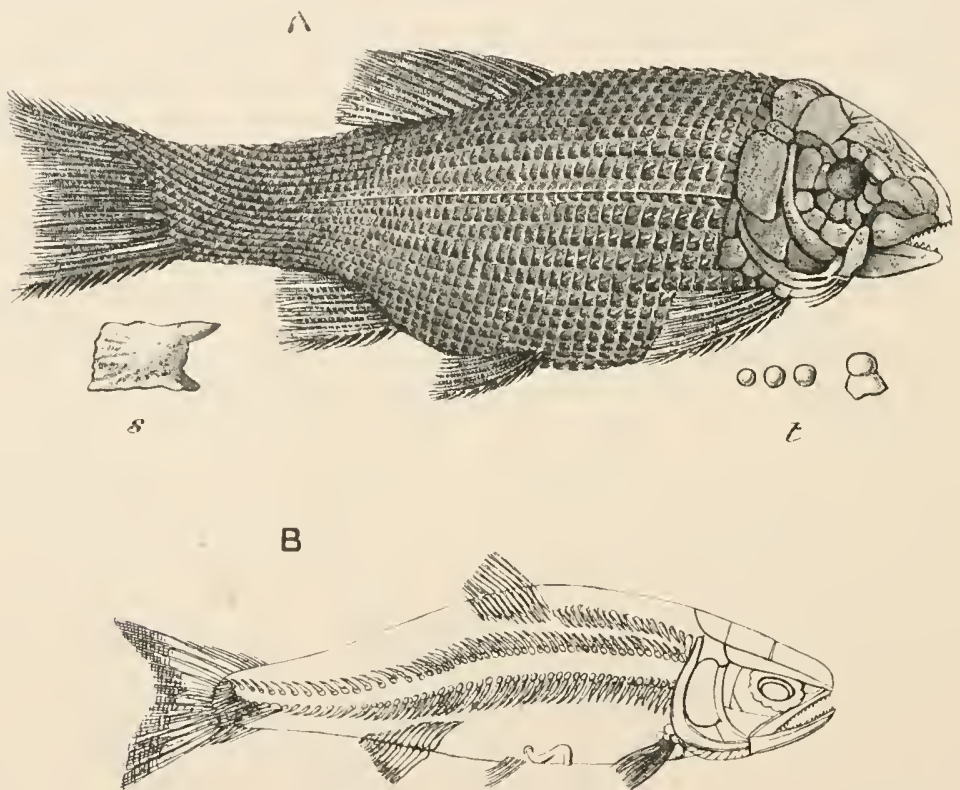

F'IG. 913. - A, Lepidotus maximus (Jurassic). $s$. scale ; $t$. teeth. $B$, Caturus furcatus (Jurassic). (From Nicholson and Lydekker.)

some species, rhomboid scales in others, and in one genus the greater part of the body is covered by cycloid scales, while rhomboid scales occur in the upper part of the tail.

The Holostei first make their appearance in the Triassic rocks and are abundant in Secondary and Lower Tertiary strata. They also (Fig. 913) show a wide diversity in form and structure. The body may be spindle-shaped or high and compressed; the scales may be rhomboid or cycloid, or may exhibit every gradation from rhomboid to cycloid in passing from the trunk to the tail of one and the same Fish; the teeth may be sharp and conical, or blunt, rounded, and adapted for crushing. A persistent notochord is present in some species, a well-ossified vertebral column in others.

We see, then, that all the orders of Ganoids, during the period 
of their prime, branched out into diverse forms, adapted to different environments, and often resembling, in a remarkable manner, the divergent forms of Teleostei which fill similar positions at the present day.

The Teleostei first appear in the Cretaceous rocks, where many existing families are represented. From this period onwards the three Ganoid orders undergo a progressive diminution in the number of families, genera, and species, their places being taken by the more highly differentiated Teleostei, until, at the present day, as we have seen, they are reduced to a few scattered forms, mostly confined to fresh waters.

\section{Sub-class IV.--The Dipnoi.}

The Dipnoi or Lung-fishes, comprising as their living representatives only the Queensland Ceratodus or "Burnett Salmon," and the Mud-fishes (Protopterus and Lepidosiren) of certain South African and South American rivers, are fishes of such well-marked and special features that by some zoologists they are separated from the true Fishes and regarded as constituting a separate class of Vertebrates. One of their peculiar features is indicated by the name Dipnoi. Not only do these animals breathe by means of gills, like ordinary Fishes, but they have a highly-developed apparatus for the respiration of air-a lung or lungs-with an arrangement of the circulation co-ordinated with this, such as is indicated in Polypterus and Amia only among the Teleostomi. They have bony scales and dermal fin-rays, but the paired fins, unlike those of any other Fishes, with the exception of certain extinct Elasmobranchs, are constructed on the biserial type ("archipterygium," see p. 163).

\section{Example of the Class - Ceratodus (Neoceratodus or Epiceratodus) forsteri.}

The Ceratodus or "Burnett Salmon" (Fig. 914) is the largest of the Dipnoi, attaining a length sometimes of four or five feet. It occurs at the present day only in the Burnett and Mary Rivers in Queensland, but fossil teeth referred to the same or nearly related genera have been found in abundance in Palæozoic and Mesozoic beds in Europe, America, the East Indies, Africa, and Australia. Ceratodus forsteri lives in still pools in which the water in the dry season becomes extremely stagnant and overladen with decomposing vegetable matter; and at that season it is only by rising to the surface occasionally, and taking air into its lung, that it is enabled to obtain sufficient oxygen for purposes of respiration. Its food consists of such small anmals as live among the waterplants and decaying leaves, and in order to obtain a sufficient amount of such food, it swallows relatively large quantities of vegetable 
matter, which passes with little or no alteration through its enteric

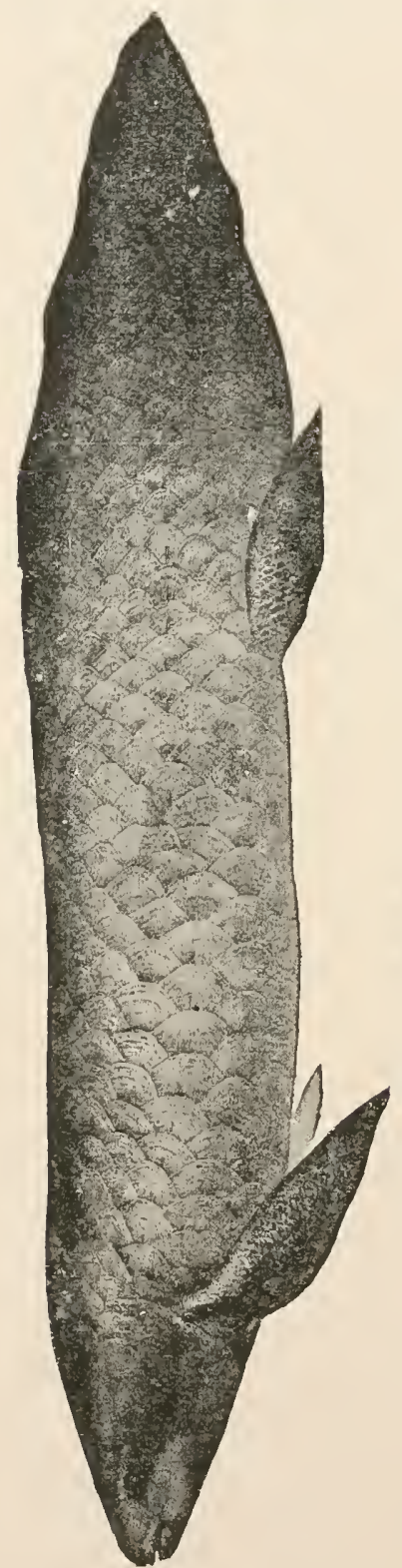

canal. Its movements are for the most part very sluggish, and are chiefly effected by the agency of the tail-fin. The paired fins are employed in steering and balancing and in the ascending and descending movements : owing to their great flexibility they are entirely incapable of supporting the body when the fish is removed from the water, but the pectoral fins may be employed as props when it lies in a resting condition at the bottom.

External Characters. - The body is fish-like (Fig. 914) with a diphycercal candal fin. The surface is covered with thin, bony, imbricated cycloid scales, very large on the head and trunk, somewhat smaller towards the tail end, and very much smaller over the fins and the posterior part of the operculum.

The limbs have a characteristic shape, being in the form of two pairs of elongated, leaf-like, pointed paddles. The marginal parts of the paired fins and the whole extent of the unpaired or caudal fin are supported by a double series of slender fibre-like unjointed, partly ossified, dermal rays (camptotrichia), which are much more numerous than the endoskeletal rays and which are covered by small surfacescales.

The mouth is situated on the ventral surface of the head, close to the anterior extremity of the snout. The external nares differ from those of other Vertebrates in being situated immediately outside the aperture of the mouth, enclosed within the upper lip. A pair of internal nares opens not far behind them into the anterior part of the mouth-cavity. At the root of the tail is the cloacal aperture with an abdominal pore on either side of it. There is an operculum similar to that of the 
Teleostomi, with a single slit-like branchial aperture behind it. There are no spiracles. There is a well-marked lateral line.

Endoskeleton.-The spinal column (Fig. 915) is represented by a persistent notochord, enclosed in a thick fibrous sheath, together with neural and hæmal arches.

A series of neural or basidorsal cartilages form the bases of the neural arches, and hæmal or basiventral cartilages are similarly related below to a series of pleural ribs in the precaudal region, and to a series of hemal arches in the caudal. These two sets of hasal rartilages are not precisely opposite thronghout, and regularly altermate for some distance in front. 'They are embedded in the sheath of the notochord, but no centra are formed, and the notochord, though pressed upon above and below by the series of basal cartilages, is not constricted in the isual annular manner. At the posterior end it becomes surrounded by cartilage. 'The neural and

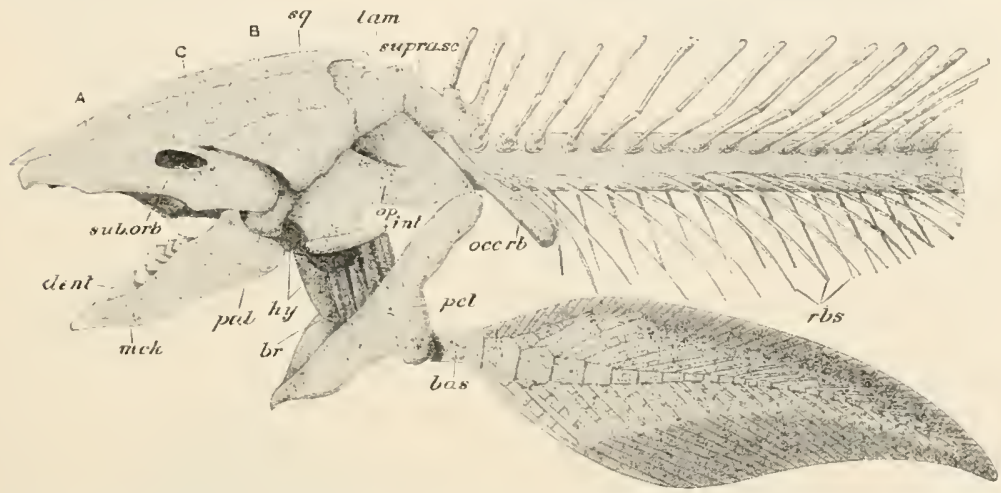

FIG. 915.-Ceratodus forsteri. Lateral view of the anterior portion of the skeleton. $A$, anterior median investing bone of the roof of the skull; $B$, posterior median investing bone ; $C$, inner lateral investing bone ; bas. basal cartilage of the pectoral fin ; br. branchial arches; dent. tooth of lower jaw : hy. hyoid arch; int. interoperculum; lam. plate over hanging branchial region; mck. Meckel's cartilage; occ. $r b$. occipital rib; op. operculum ; pal. palatoquadrate; pct. pectoral arch; rbs. ribs; sub. orb. sub-orbital bones; sq. so-called squamosal ; supra. sc. post-temporal.

hæmal arches are ossified; each is surmounted by a rod-like neural or hæmal spine which forms part of a continuous three-jointed ossified rod, the proximal segment being the spine, and the two others radials. 'The pleural ribs are curved bony rods extending downwards and somewhat backwards in the body-wall immediately outside the peritoneal membrane, like the pleural ribs of the Teleostomes. The first pair-the occipital ribs-(Fig. 915, occ. rb.), thicker and straighter than the rest, are connected with the skull in its vertebral portion.

The skull (Figs. 915, 916, and 917) consists of an undivided mass of cartilage, devoid of fontanelles, narrowest between the orbits, and broadening before and behind; posteriorly it is prolonged into a plate (lam.) overhanging the branchial region. Embedded in the 


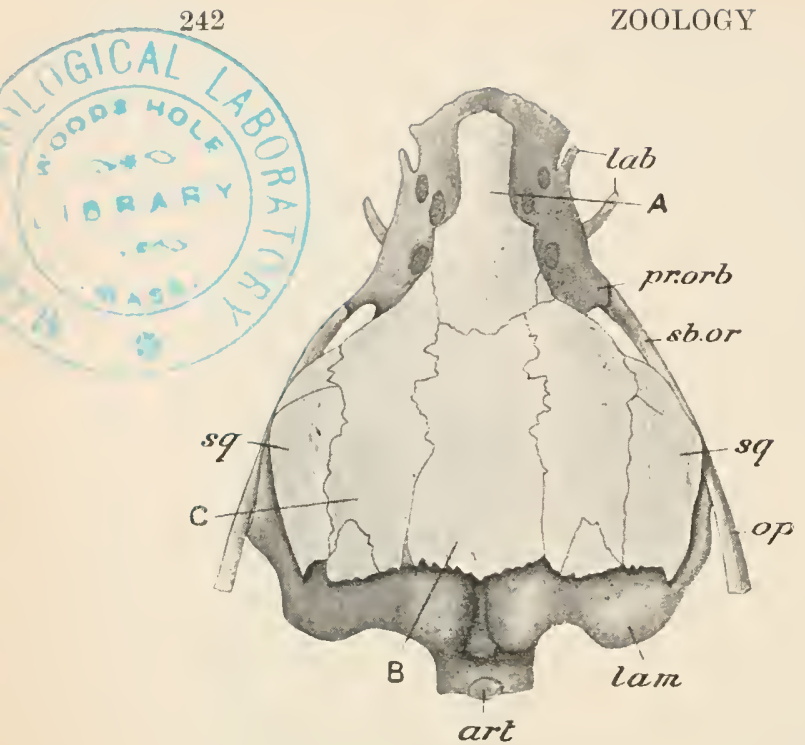

Fio. 916-Ceratodus forsteri. Dorsal vicw of the skull. $A$, anterior median investing bone ; art. articular surface for second fin-ray ; $\boldsymbol{B}$, posterior median investinc bone; $\boldsymbol{C}$, inner lateral investing bone; $l a b$. labial cartilages; lam. process projecting over gills; op. operculum ; pr. orb pre-orbital process of chondrocranium ; sb. or. sub-orbital bones; sq. outer lateral investing bone. (After Huxley.) cartilage of the posterior part are a pair of small replacing bones which apjear to represent the most anterior of the spinal elements fused with the skull. On the ipper surface are two unpaired ( $A$ and $B$ ) and four paired ( $C$ and $s q$. investing bones, the homologies of all of which are undetermined. Premaxillæ, maxillæ, and nasals are absent. On the ventral surface is a large investing bone (Fig. 917, P.Sph.) representing the parasphenoid of the Teleostomi. In front is a pair of sinall upper labial or nasal cartilages. A palatoquadrate cartilage (Fig. 915 , pal.), firmly fixed to the side-wall of the cranium, gives attachment to the mandible, so that the skull is autostylic; the quadrate element is distinct in the larva and independently developed. In front the palatoquadrate contains a palatopterygoid ossification which forms the support for the large composite tooth of the upper jaw. The mandible consists of Meckel's cartilage with an angular bone behind, and a large splenial, which bears the tooth, in front. The dentary is vestigial. The hyomandibular is only represented by a small vestige. Opercular (op.) and interopercular (int.) bones support the operculum. The hyoid (hy.) and branchial arches (br.) are cartilaginons. Of the latter, four are completely developed, and a fifth is represented by a vestige.

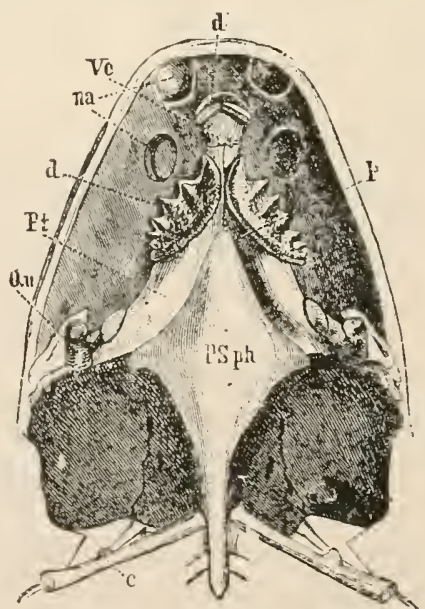

FIG. 917.-Ceratodus forsteri. Ventral view of the skull. $c$, occipital rib; $d$, palatine teeth; $d^{\prime}$, romerine teeth; na. anterior and posterior nares; $P$. palatine region of palatopterygoid; $P$. S S ph. parasphenoid : $I$ 't. pterygoid; $Q u$. quadrate region; Vo. vomer. (From Dean, after (iuntlier.) 
There are no branchial rays, but the branchial arches bear a series of gill-rakers with cartilaginous supports.

The pectoral arch (Fig. 915, pct.) is a stout cartilage with two pairs of investing bones, the cluvicles on the coracoid, and the cleithra on the scapular regions. The latter are connected with the skull by post-temporals. The skeleton of the pectoral fin consists of a stout basal cartilage (bas.), an elongated, tapering, central axis made up of a number of short cartilaginous segments, and two rows of jointed cartilaginous rays extending out on either side of the axis so as to support the middle part of the expanse of the fin. The pelvic arch is a single cartilage, produced forwards into an elongated rod-like epipubic process (Fig. 918). The skeleton of the pelvic fin is similar to that of the pectoral.

Digestive Organs. - The teeth (Fig. 917) are of a remarkable and characteristic shape. There are two pairs of large compound

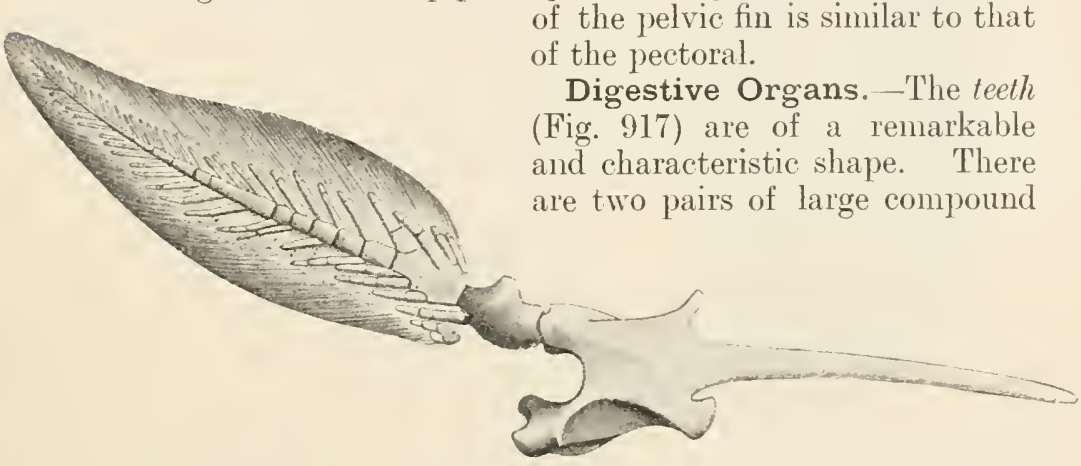

FIG. 918.-Ceratodus forsteri. Pelvic arch and skeleton of pelvic fin. (After fünther.)

teeth of similar character, one pair (the palatine, $d$ ) on the roof of the mouth (palatopterygoid bone) and the other (splenial) on the lower jaw. Each is a curved plate with the convex border, which is directed inwards and somewhat backwards, entire; while the concave border presents a series of six or seven vertical, ridgelike projections or cusps. In addition to these, there are, in front of the palatine pair, a pair of much smaller, simple, somewhat chisel-like vomerine teeth $\left(d^{\prime}\right)$ placed close together and directed vertically. In the embryo each tooth is represented by a number of separate denticles which subsequently coalesce.

In the enteric canal the chief feature of special interest is the presence, throughout the length of the intestine, of a spiral valve similar to that of the Elasmobranchs and Ganoids. The rectum opens into a small cloaca. A pair of abdominal pores open just behind this.

Organs of Respiration.-Ceratodus combines aquatic respiration by means of gills similar to those of ordinary fishes, with aërial respiration by means of a lung.

There are four pairs of gills, each consisting of a double row of gill-filaments. supported on the branchial arches. A rudimentary 
hyoidean gill or pseudobranch is present as well. The lung (Fig. 919 ) is an elongated median sac connected by a pneumatic duct with a muscular chamber or vestibule opening into the œsophagus

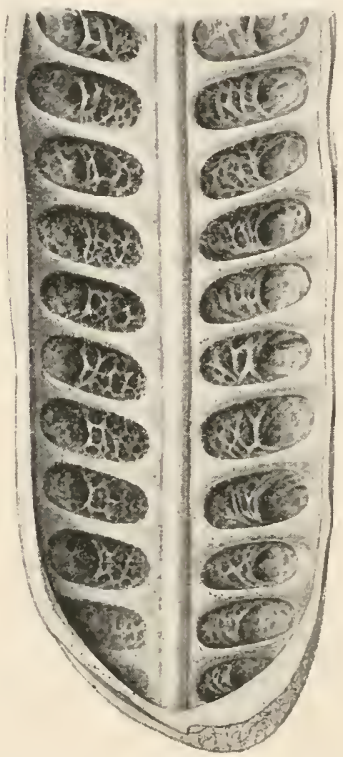

FIG. 919.-Ceratodus forsteri. Posterior half of the lung with the ventral wall slit up so as to show the interior. (After Gïnther.) on its ventral side by a slit-like aperture or glottis. The internal surface of the lung is sacculated, and a regularly-arranged series of blind pouches opens out of the main central cavity. 'T'his lung of C'eratodus corresponds morphologically to the airbladder of Ganoids and Teleosts, but differs from it in its blood-supply and consequently in its function, being supplied with blood by a special paired pulmonary artery (as is also the case in Polypterus) and acting as an important organ of respiration.

Blood-Vascular System. - Coordinated with the existence of a lung and distinct pulmonary circulation, is a complication in the structure of the heart. The sinus venosus is imperfectly divided into two parts, and the cavity of the auricle is divided into two by an incomplete septum in the form of a ridge. The venous blood enters the right-hand division of the sinus venosus and passes thence through the right-hand division of the auricle to the ventricle; the pulmonary vein, by which the blood is returned from the lung, passes through the sinus, and its blood reaches the ventricle through the left-hand division of the auricle. There are no auriculoventricular valves guarding the opening between the auricle and the ventricle. A contractile conus arteriosus is present, and has a remarkable spirally-twisted form; in its interior are four longitudinal rows of valves, one of which is modified to form an incomplete longitudinal septum. The channel on the left side of this septum, which receives the blood of the pulmonary vein, is in communication in front with the first two aortic arches (afferent branchials), that on the right with the last two.

The blood-vessels (Fig. 920) present an arrangement which is intermediate in some respects between that which has been already described as observable in the Elasmobranchs and that which will be found to characterise the Amphibia. The four afferent branchial arteries (aff.) take their origin close together, immediately in front of the conus, so that a ventral aorta can hardly be said to exist. Each branchial arch has two efferent branchial arteries. A hyoid 
artery (hy. art.) is connected with the most anterior of these. The eight efferent vessels unite in pairs to form four epibranchial arteries (epi.). The latter unite dorsally to form a main trunk, which combines with the corresponding trunk of the opposite side to form the median dorsal aorta $(d . a$.$) . The head is supplied by carotid$

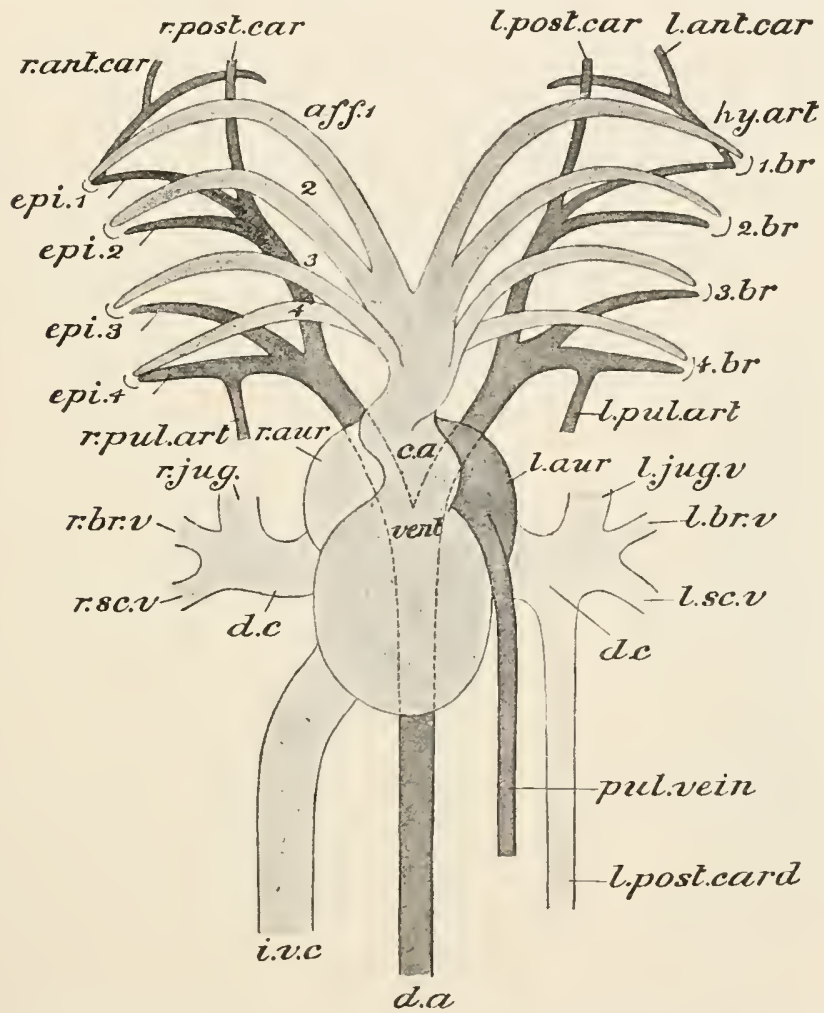

FIG. 920.-Ceratodus forsteri. Diagrammatic view of the heart and main blood-vessels, as seen from the ventral suriace. aff. $1,: 2,3,4$, afferent vessels ; 1 br, : br, $3 b r, 4 b r$, position of gills; $c$. $a$. conus arteriosus ; $l$. $a$. dorsal aorta; $d$. $c$. precaval vein ; epi. 1 , cpi. 2, epi. 3, epi. 4, epibranchial arteries; hy. art. hyoidean artery ; $i$. v. c. postcaval vein ; $l$. ant. car. left anterior carotid artery; $l$. aur. left auricle; $l$. br. $v$. left brachial vein ; $l$. jug. $v$. left jugular vein ; $l$. post. car. left posterior carotid artery ; $l$. post. carl. left cardinal vein ; $l$. $p u l$. art. left pulmonary artery; $l$. sc. $v$. left subscapular vein; $r$. ant. car. right anterior carotid artery; $r$. aur. right auricle; $r$. $b r$. $v$. right brachial veiu ; $r$. jug. right jugular vein; $r$. post. car. right posterior carotil ; $r$. pul. art. right pulmonary artery ; $r$. sc. v. right subscapular vein; vent. ventricle. (After Baldwin Spencer.)

branches given off" from the first epibranchial (l. post. car. and $r$. post. car.) and from the hyoidean arteries (l. ant. car. and $r$. ant. car.), and the latter also gives off a lingual artery to the tongue. From the last (fourth) epibranchial artery arises the pulmonary artery (l. pul. art. and r. pul. art.), carrying blood to the lung.

There are two precavals or ductûs Cuvieri (d. c.), as in the DogVOL. II 
fish (p. 152). The right is formed by the union of jugular (l. jug. v. and $r . j u g . v$.$) , brachial (l.br.v. and r. br.v.), and subscapular veins$ (l.sc. v. and r. sc. v.). The left receives in addition a left cardinal vein (l. post. card.). A large lateral cutaneous vein, running superficially along the side of the body, opens into the subscapular.

A large postcaval vein $(i . v . c$.) brings back the greater portion of the blood from the posterior parts of the body; it is situated somewhat to the right of the middle line, and opens into the sinus venosus between the two hepatic veins. A postcaval occurs in the Dipnoi alone amongst Fishes, but is universal in all the higher classes. Posteriorly the cardinal and the postcaval are formed by the bifurcation of a median caudal vein; close to its origin each receives the efferent renal veins bringing back the blood from the kidney. The blood from the pelvic fin is brought back by an iliac

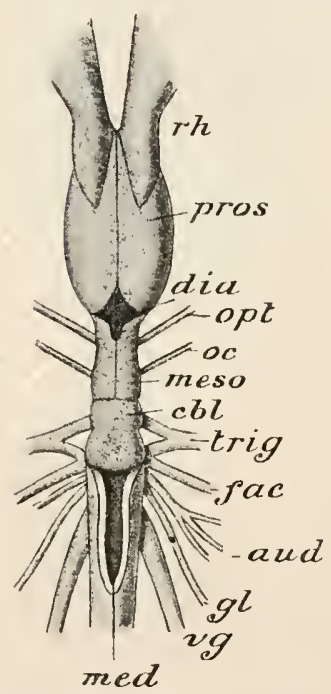

FIG. 921.-Brain of Ceratodus forsteri, dorsal view. aud. auditory nerve; $c b l$. cerebellum: dia. diencephalon; fac. facial nerve ; $g l$. glossopharyngeal ; med. medulla oblongata; meso. mesencephalon; oc oculomotor nerve; opt. optic nerve; pros. prosencephalon ; $r h$, rhinencephalon (olfactory lobe with olfactory tract and bulb); $v g$. vagus nerve. (Chiefly after Sanders.) commissure. The contained cavity is divided into two by the prolongation of the choroid plexus already referred to. The nervous wall of the hemisphere (pallium) is very thin and is

' How far this arrangement combines Fish-like and Amphibian characters will be best understood at a later stage. 
incomplete dorsally and internally: basally it forms a massive tuberculum olfactorium from which the olfactory nerve-fibres are derived. There is a pair of large olfactory lobes $(r h$.), each with its cavity, and each prolonged into an olfactory peduncular tract, which ends in front in an olfactory bulb in close apposition with the nasal capsule.

The pineal body is situated on the summit of a conical membranous cap on the roof of the third ventricle. The infundibulum develops a pair of lobi inferiores. The mesencephalon (meso.) is bilobed, but the division is not strongly pronounced. The cerebellum $(c b l$.$) is very small, being little more than a transverse$ bridge of nerve-matter over the anterior end of the fourth ventricle. The medulla $(e d$.$) is of relatively large size.$

Urinogenital Organs.-The kidneys are short, being confined to the posterior portion of the body-cavity, and are firmly attached to the ovaris or testes. Each has a thick-walled ureter which joins its fellow, the passages, however, remaining distinct to near the opening into the urinogenital division of the cloaca, when the right opens into the left.

There are two elongated ovaries (Fig. 922, l. ov., r. ov.), which remain distinct throughout. The oviducts $(l$.ovd. and $r$.ovd.) are a pair of thick-walled, greatly convoluted tubes which extend along the whole length of the body-cavity, into which they open in front (coel. ap.) ; posteriorly they coalesce immediately before opening into the cloaca. The testes are long, compressed bodies which remain distinct from one another throughout their length. The efferent ducts from the testes open into certain of the tubules of the mesonephros, and the sperms are thus enabled to pass out through the mesonephric duct. The Müllerian ducts in the male are remarkably well developed.

In the early stages of its development (Fig. 923) Ceratodus exhibits resemblances, on the one hand, to Petromyzon (p. 130), and on the other to the next class to be studied-the Amphibia. The ova become enclosed, while passing down the oviduct, in a gelatinous envelope which swells up considerably when it comes in contact with the water. At what stage fertilisation takes place is not exactly known. Segmentation is complete and unequal, and results in the formation of a lens-shaped blastula $(A)$ with smaller cells on one of the convex surfaces (the future dorsal), and larger on the other (the future ventral). A blastopore (bl. p.) first appears on the ventral surface as a short transverse slit, which grows into a semicircle $(B)$ or a horse-shoe. The free ends of this grow in towards one another and unite to enclose an irregularly circular or elliptical space filled in by a mass of large cells-the yolk-plug $(C, y k$. $p l$. $)$. Soon, however, this wide aperture becomes narrowed to a small longitudinal slit, the lips of the anterior part of which then unite, only the most posterior part 


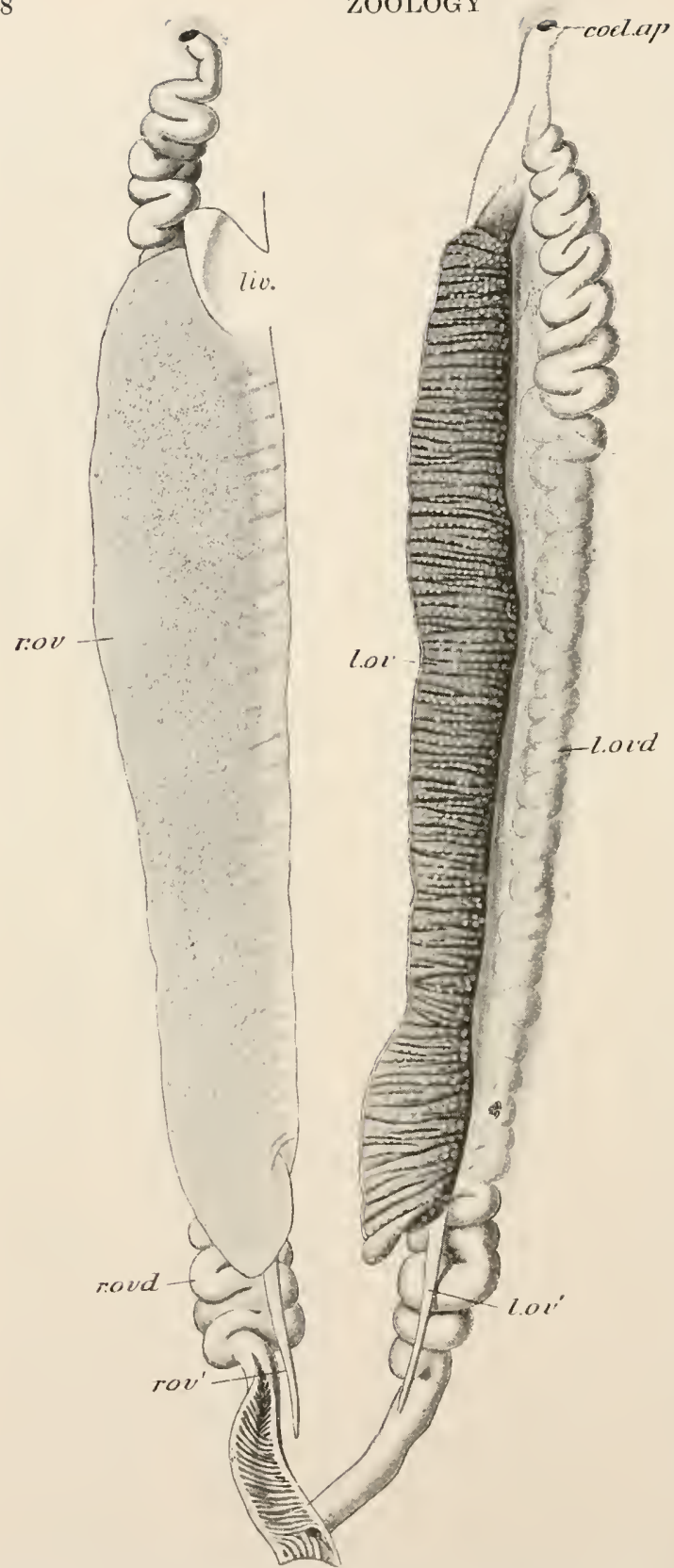

FIG. 922. Ceratodus forsteri. Reproductive organs of female; the inmer surface of the right and the outer surface of the left ovary shown. coel. ap. coelomic aperture of oviduct; liv. portion of the liver; $l$. $o v$. left ovary; $l . o v$. Its posterior termination; l. ord, left oviduct: $r$. ov. right ovary; $r$ ord. right oviduct : $r$. $o v^{\prime}$. its posterior termination. (After Günther.) remaining open (D) and subsequently giving rise to the anus. During its increase in size the blastopore has been g row ing over towards the dorsal side, and when its lips become united, it extends along the greater part of the dorsal surface. A narrow medullary groove $(E, b l p$. $s u t$.) a p pears along the dorsal surface, and a pair of medullary folds are seen at the sides of this $(E)$ and are coalescent in front of it. From the medullary folds and the groove between them th e neurocole, and subsequently the entire nervous system, are developed as in Cralliata in general (s e e p. 95). The portion of the blastoderm destined to give rise to the embryo becomes to a slight extent folded off from the rest, which forms an ill- 
defined rounded mass, or yolk-sac, to be subsequently absorbed as development proceeds. The most important features in the later
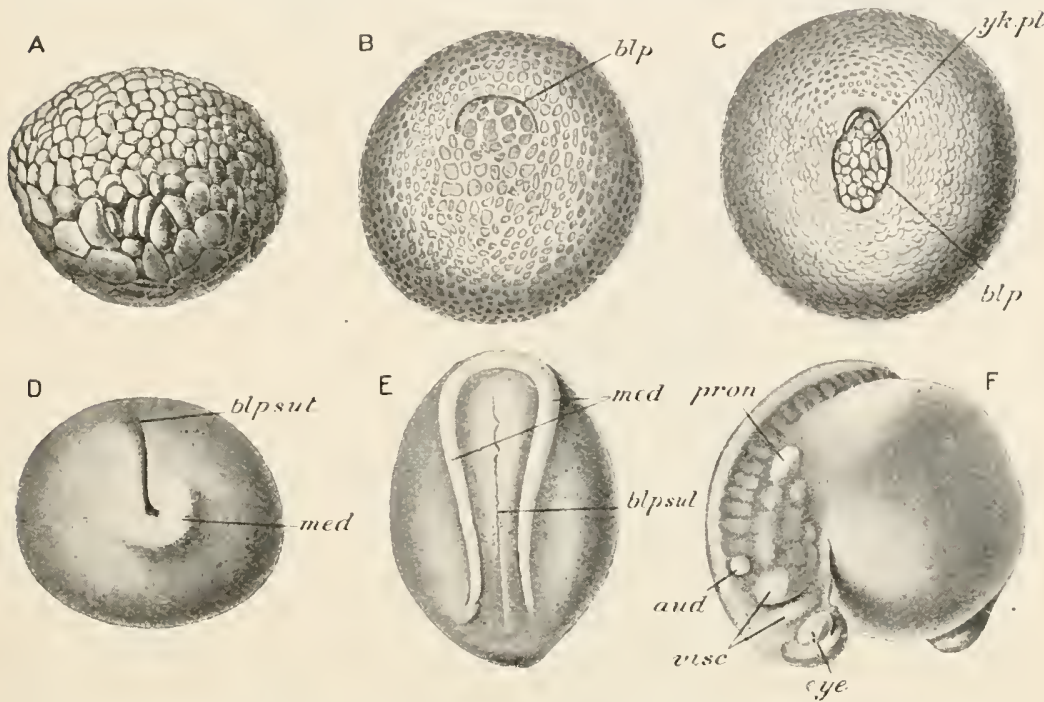

Fic. 923.-Ceratodus forsteri. Stages in the development. $A$, lens-shaped blastula ; $B$, stage with semicircular blastopore $\left(b l . p_{*}\right) ; C$, later stages in which the blastopore $\left(b l . p_{.}\right)$ has taken the form of a ring-like groove enclosing the yolk-plug (ylk. $p l$.); $D$, stage in which the narrow medullary groove $(b / p$. sut.) has appeared with the rudiment of the medullary folds $($ menl.) $; E$, stage in which the medullary folds (med.) have become well developed; $F$, later stage with well-formed head with two visceral arches (vise.) and rudiments of eye (eye) and ear (aud.) ; pron. mesonephros. (After Semon.)

stages $(F)$ are the negative ones of the absence of the external gills (to be referred to below and in the account of the Amphibia), and the absence of horny jaws.

\section{Distinctive Characters and Classification.}

The Dipnoi are Pisces in which the notochord is persistent, there are no vertebral centra, and the primary cranium persists with little ossification, but has added to it a number of investing bones. The skull is autostylic, the lower jaw articulating with a palatoquadrate process which is immovably fixed to each side of the skull. There are four to six cartilaginous branchial arches. The dermal fin-rays are slender more or less ossified fibres, and are supported by numerous cartilaginous or ossified pterygiophores. The caudal fin is diphycercal. The paired fins are of the character of " archipterygia." The pectoral arch is a single cartilage with a pair of superficial investing bones on each side. The pelvic arch is well-developed and cartilaginous. There are gills attached to the branchial arches, and in addition a single or double lung opening into the œsophagus by a ventral aperture. The gills are covered over by an operculum. There is a dermal skeleton in the form of 
overlapping cycloid scales. There is a distinct cloaca. The intestine contains a spiral valve. The auricle and the sinus venosus are each imperfectly divided into two parts. There is a contractile conus arteriosus, which has a spirally-twisted form, and is partly or completely divided internally by a longitudinal septum. The afferent branchial vessels take their origin close together immediately in front of the conus. A pulmonary artery is given off from the afferent branchial system on either side; a pulmonary vein opens into the left division of the auricle. The optic nerves form a chiasma. The oviducts open anteriorly into the colome. The ova are of moderate size ; segmentation is entire.

The Dipnoi are classified as follows :-

\section{Order 1.-MoNopneumona.}

Dipnoi in which the lung is single, and the lateral jointed rays of the "archipterygium" are well developed.

This order comprises only the Australian Ceratodus.

\section{Order 2.-Dipneumona.}

Dipnoi in which the lung is double, and the lateral rays of the " archipterygium" are vestigial or absent.

This order includes Protopterus (Fig. 924) of South Africa, and Lepidosiren of South America.

\section{General Remarks.}

The three genera of living Dipnoi are closely allied in all the most essential features of their structure, and it will only be necessary now to mention the principal points in which Protopterus and Lepidosiren differ from Ceratodus.

The limbs (Fig. 924) are long and very narrow, and the limbskeleton is correspondingly modified, consisting of a slender, jointed axis without, or with only vestiges of, the lateral rows of rays. A blind dorsal diverticulum of the cloaca, derived developmentally from the urinogenital sinus, is present, and perhaps corresponds to the sperm-sacs of the Elasmobranchs. There are two lungs, the anterior portions of which are united to form a median chamber, to which the presence of numerous trabeculæ gives a spongy character. There are five (or six) reduced rod-like branchial arches, of which the last three bear the internal gills ; in addition there is a series of external gills in the larva, vestiges of which persist in the adult Protopterus (Fig. 925, K). In the males of Lepidosiren, vascular filaments, which may be accessory respiratory organs, are developed on the paired fins during the breeding season. The conus arteriosus is completely divided by a longitudinal septum. The pulmonary artery is given off from the point of union of the 
epibranchial arteries into a single lateral trunk. In Protopterus there is usually a single abdominal pore opening on the dorsal wall of the cloaca; this leads into a cavity into which the true abdominal pores, which are very minute, lead. In Lepidosiren abdominal pores are absent.

The brain in both Lepidosiren and Protopterus, as well as in Ceratodus, differs from that of Fishes in general, and resembles that of Amphibia (see Section $\mathrm{XIV}$ ) in the presence of long and relatively narrow cerebral hemispheres. In the two former these have a pallium or nervous roof with a stratified layer of nerve-cells. In all the Dipnoi the part of the hemisphere (olfactory lobe and tuberculum) from which the olfactory nervefibres pass to the bulbus olfactorius is of relatively large size, but smaller in Protopterus and Lepidosiren. In Protopterus and Lepidosiren the olfactory tract is not distinguishable, bulbus and lobus being in immediate apposition instead of being widely separated as they are in Ceratodus. In both these genera the dorsal part of the mid-brain is undivided. In both genera the kidneys are relatively more elongated than in Ceratodus; in Protopterus the posterior portions are fused together; in Lepidosiren they remain separate throughout. In both genera the elongated testes are distinguishable into two regions-an anterior longer, sperm-producing part, and a

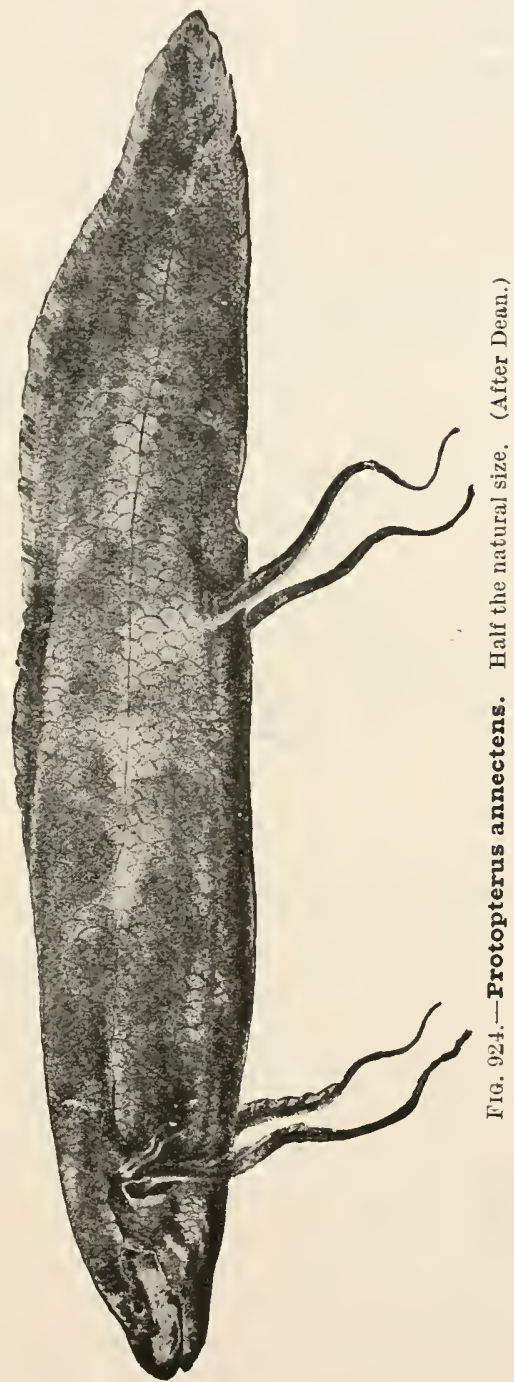
posterior shorter part which serves as a duct and a vesicula seminalis. In Lepidosiren about six vasa efferentia arise from this posterior region and enter the Malpighian capsules of the mesonephros: in Protopterus there is only a single vas efferens. The ducts of the two kidneys open by a single aperture (Protopterus) 
or two separate apertures on the summit of a urinogenital papilla into the cloaca at the base of the cloacal cxecum referred to above. Many of the cellular elements, such as the blood-corpuscles, are of comparatively large size. There is holoblastic, but unequal, segmentation, as in Ceratodus, followed by a true invagination. A pair of medullary folds are developed, and between them is formed a median solid ectodermal keel in which a neurocœle only appears subsequently. The larva has well-developed external gills.

The Dipnoi are a very ancient race. The genus Ceratodus itself

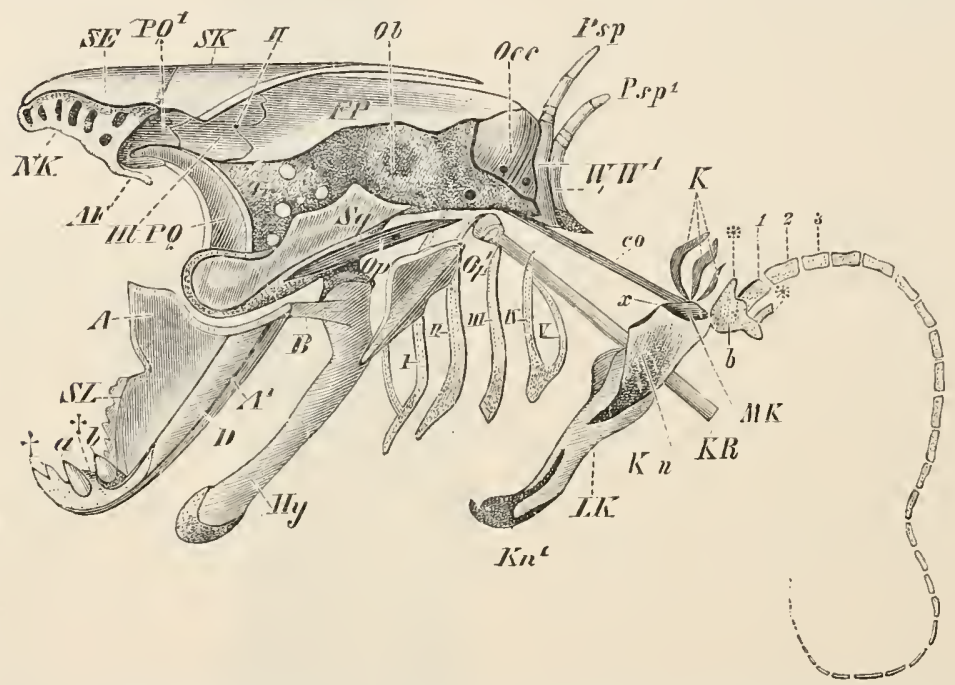

F19. 925.--Protopterus. Skull, shoulder-girdle, and skeleton of fore-limb. $A A^{1}$, splenial: $A F$, pre-orluital process; $a$ and $b$ (on lower jaw), and $S . L$ teeth $\cdot b$, basal cartilage of pectoral fin ; $B$, ligamentous band connecting the mandible with the hyoill ; co. liganentous band connecting the dorsal end of the pectoral arch with the skull ; $D$, angular $F P$, frontoparietal ; $H t$, membranous fenestra perforated by the foramen for the optic nerve $(I I)$; $H y$. hyoid ; $K$, external gills ; $K n$, $K_{n} n^{1}$, cartilage of the pectoral arch ; $K R$, occipital rib; $L K$ and $M K$, investing bones of the pectoral arch; $N K$, olfactory capsule; $O b$, auditory capsule; Occ. supra-occipital; $O p$. and $O p^{2}$. rudimentary opercular bones ; $P Q$. palatoquadrate; $P s p . P s p^{1}$. spinous processes of the anterior vertebre; $S E$. supra-ethmoid bone, $S K$, roofing investing bones: $T r$. palatoquadrate cartilage; $W W$, anterior vertebre coalescent with the skull; $\boldsymbol{I}-\boldsymbol{V}$, branchial arches (that marked $I$ is forked and the anterior bar may represent the first, in which case there are six brarchial arches) ; 1,2 , 3, segments of axis of pectoral fin ; *, *, vestigial lateral rays of pectoral fin. (From Wierdersheim.)

extends back to the early Mesozoic, and the remains of allied forms (Dipterus and other genera) are found in Devonian and Carboniferous formations. But if, as is conjectured, the Arthrodira are to be regarded as Dipnoi, then the group dates back as far as the Silurian. The evidence for this conclusion is, however, by no means complete, as our knowledge of the structure of the extinct Fishes in question is necessarily meagre. They had the head and anterior part of both dorsal and ventral surfaces (Fig. 926) protected by bony plates, the system of head-plates being connected with those on the trunk by a well-developed movable joint. The notochord was persistent, with partly calcified neural and hæmal arches, and the cranium was 
apparently cartilaginous; the mandible was probably autostylic. There were composite cutting dental plates. The pectoral fins are unknown; the rays of the small pelvic (VR) were supported on a flattened plate $(V B)$.

With some special features of their own the Dipnoi combine characteristics in which they resemble now one, now another, of the other groups of Fishes, together with a few in which they approach the next class of Vertebrates to be dealt with, viz., the Amphibia and even the higher Vertebrates. The brain and the heart are quite peculiar: the former in its undivided, or almost undivided, mid-brain; the latter in its imperfectly divided auricle, and spirally-twisted conus. The pallium of the cerebral hemispheres

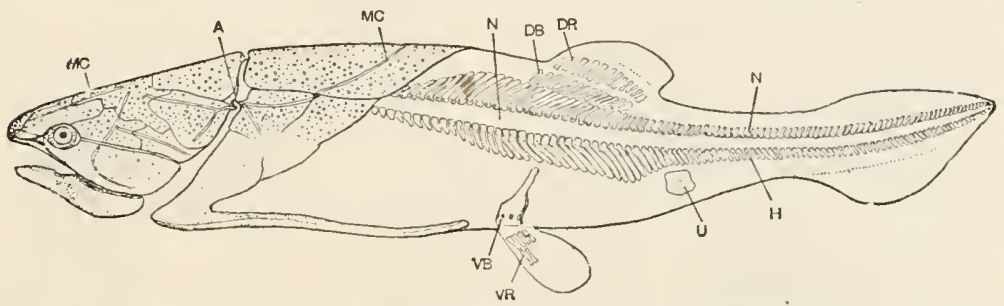

F1G. 926-Cocccsteus decipiens. Side view, restored. A, articulation of head with trunk $D B$, cartilaginous basals of dorsal fin ; $D T$, cartilaginous radials of dorsal fin; $H$, hrmal arches and spines; $M C$. mucous canals; $N$, neural arches and spines, and position of notochord: $U$, median inpaired plate (?) of hinder ventral region; $V B$, basals of pelvic fin; $V R$, radials of Felvic fin. (From Dean, after Smith Woodward.)

in the Dipneumona with its layers of nerve-cells has no parallel among the lower Vertebrates. In the limbs the Dipnoi are only closely approached by certain extinct Elasmobranchs (p. 163). In the presence of a cloaca and a spiral valve they also approach that sub-class, as well as in the contractile conus-the last two features being also shared with the Ganoid Teleostomi. The operculum with its supporting bones connects them with the Teleostomi. The Amphibian features will be referred to at a later stage. On the whole, though in some respects more primitive than the members of the other sub-classes of Pisces, the Dipnoi tend to establish a connection between that class and the higher Vertebrates.

\section{APPENDIX TO PISCES.}

\section{THE OSTRACODERMI.}

The Ostracodermi are a group of Palreozoic Fishes of uncertain affinity. characterised by the extraordinary devclopment of the cxoskeleton of the head and trunk, and the absence, in all the fossil remains hitherto found, of endoskeleton, including jaws. It may therefore be assumed that there was a persistent notochord, and that the rest of the skeleton was unossified. It is uncertain whether the group should be considered the equivalent of a Class or of a Sub-class : it is divisible into three orciers, which are best considered. separately. 


\section{Order 1.-Heterostraci.}

This order includes four families, the Pteraspide, the Cololepido, the Drepanaspidce, and the Psammosteidae. Of the first Pteraspis (Fig. 927) may

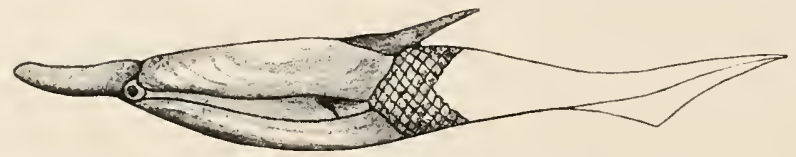

FIG. 927.-Pteraspis rostrata (Devonian). (From the Brit. Mus. Cat. of Fossil Fishes.)

be taken as an cxample. The body is elongated, and divided into an anterior region, representing the head and fore-part of the trunk, and covered by strong calcified plates or scutes, and a posterior or caudal region covered by rhomboidal scales. In the anterior region there are seven scutes abovc, constituting the dorsal shield, while below there is a single ventral shield. The dorsal shield is produced into a rostrum, and is hollowed by a pair of lateral orbits, between which is a pit, on the inner surface of the shicld, probably marking the position of the pineal

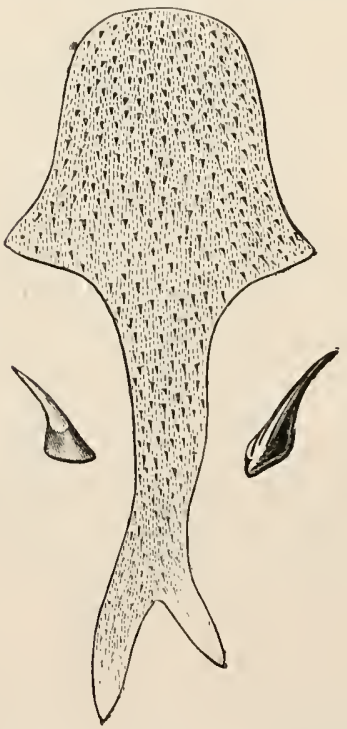

FIG. 928.-Restored outline of Lanarkia spinosa, in the position in which it occurs as a fossil, the hoad heing Hattened and the tail twisted round so as to appear in profile. On each side a much enlarged dermal denticle is shown. (From the Cambridge Natural History, after Traquair.)

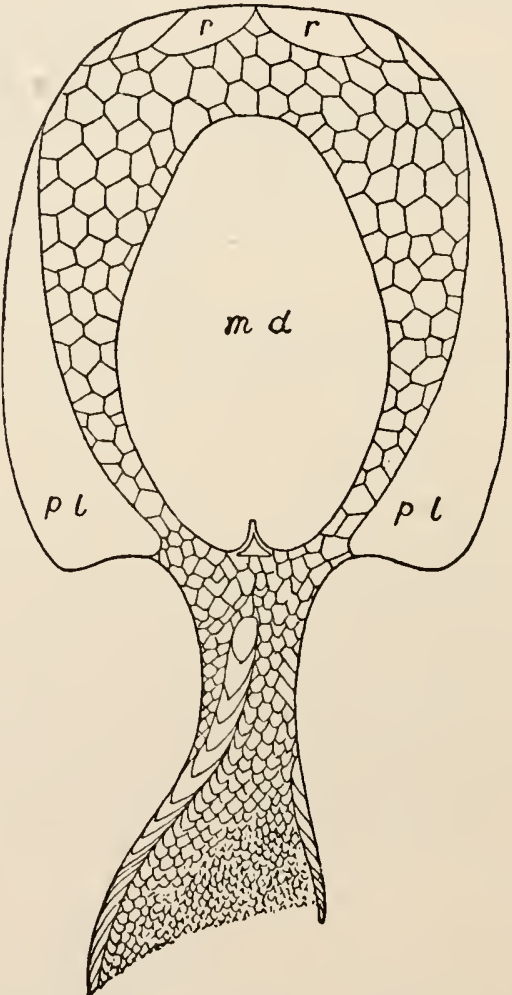

F1G. 929.-Restored outline of the dorsal surface of Drepanaspis gemundenensis. The tail appears in protile. $m . d$. median dorsal plate; $p$. $l$. postero-lateral plate: $r$ rostral plates. (From the Cambridge Natural II istory, after Traquair.)

body. The scutes contain no lacunæ or canaliculi, and have not, therefore, the structure of bone: they are lined by a nacreous layer, and are covered 
externally with a layer of vaso-dentine. The tail appears to have been heterocercal. A pair of longitudinal ridges may represent paired fins.

The Coelolepidoe (Fig. 928) have the head and anterior trunk region flattened and expanded, with postero-lateral lobes which may represent paired fins. There is a heterocercal tail-fin. Mouth, orbits, and branchial apertures have not been detected. The exoskeleton takes the form of numerous uniform, hollow, pointed spines, or tubercles, composed of dentine coated with ganoin. Two genera are known of Silurian and Devonian age.

The Drepanaspidae (Fig. 929) have a somewhat similar shape, but with the head and trunk expanded into a broad shield, which is sharply marked off from the tail. The exoskeleton consists of seales and fulcra (see p. 222), replaced in the middle of the dorsal surfacc by a large dorsal plate_(m. $\left.d_{-}\right)$'and

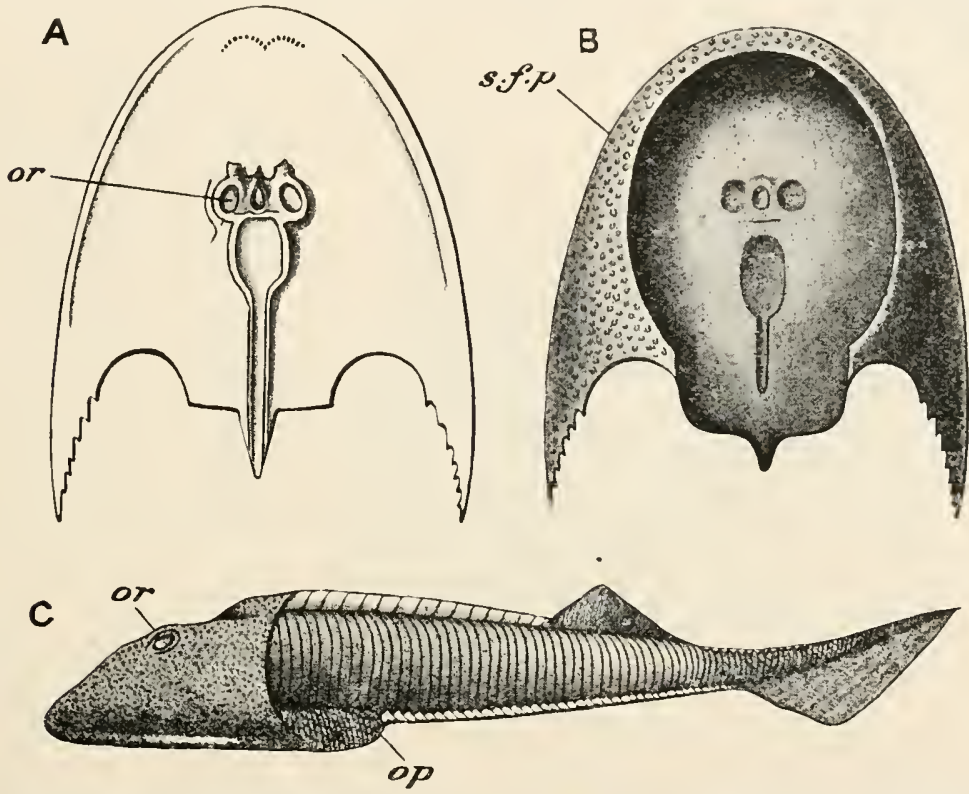

FIG. 930.-A, restoration of shield of Cephalaspis lyelli, dorsal aspect; $B$, idiagram of ventra aspect of shield of Cephalaspis; $C$, restoration of Cephalaspis murchisoni (Devonian). op. opercular plate or pectoral fin ; or. orbit ; s. f. p. sub-frontal plate. (From the Brit. Mus. Cat. of Fossil Fishes.)

at the sides by postero-lateral plates ( $p . l$.). A similar combination of large plates and small scales occurs on the ventral surface. The sole known representative of the family is of Lower Devonian age.

The family Psammosteid.e has been formed for the reception of certain fragmentary remains in the form of dermal plates which elosely resemble those of the Drepanaspidæ.

\section{ORDER 2.-OSTEOSTRACI.}

Cephalaspis (Fig. 930) may be taken as an example of the five genera included in this order. The head is covered with a calcified shield, which has a curious resemblance to the cephalic shield of Limulus or of a Trilohite, being gently curved above, produced behind into spines, continued ventrally into a sub-frontal plate $(B, s, f . p$.$) , and having a pair of orbits (A$, or $)$ for the eyes 
near the middle of the dorsal surface. Behind the shield, towards the ventral surface, is a plate which perhaps supported the operculum $(C, o p$. ), but may represent the pectoral fin. The scutes contain some lacunæ, and thercforc

A
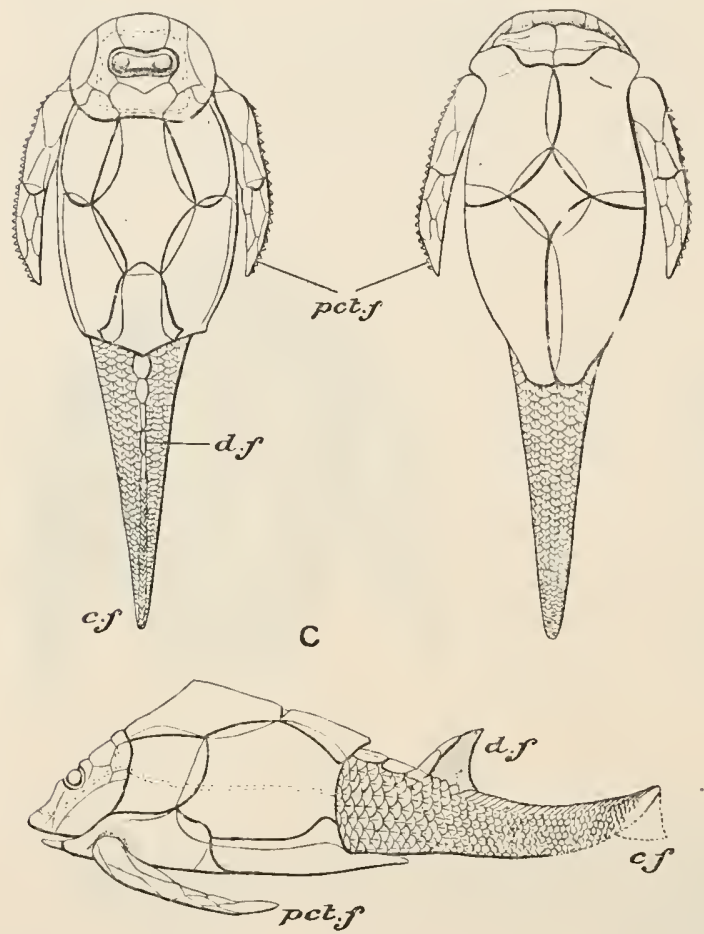

FIG. 931- - Pterichthys testudinarius. $A$ dorsal $P$ ventral, $C$, lateral aspect. $c, f$. caulal fin ; $d . f$. dorsal fin; pet. $f$. pectoral fin. (From the Brit. Mus. Cat. of Fossil Fishes.) approach in structure to bone. The posterior portion of the body is covered by deep, narrow scales; therc is a single dorsal fin and a heterocercal caudal.

\section{Order 3.-Anti- ARCHA.}

This group contains five genera, of which Pterichthys (Fig. 931) may be taken as an example. It presents a broad and high anterior region, covered by articulated plates which have the structure of bone and are covered by a laycr of enamel, and a caudal region covered by rounded or hexagonal scales. The orbits arc placed close together on the top of the head, and between them is a plate pitted on its inner surface, apparently for the pineal body. There is a pair of large pectoral fins (pct.f.) of a very remarkable character, covered by strong scutes and divided into two parts by a joint towards the middile; a single dorsal fin $(d . f$.$) with$ fulcra, but apparently no fin-rays; and a heterocercal tail-fin (c. $f$.).

\section{CLASS IV.-AMPHIBIA.}

The Amphibia are distinguished from Fishes by the possession of pentadactyle limbs instead of paired fins, and by the absence of fin-rays in the median fins. They nearly all breathe by gills in the larval condition, and many of them retain those organs throughout life; lungs are, however, usually present in the adult. The class includes the Frogs, Toads, Newts and Salamanders, as well as the peculiar snake-like Cxcilians, and the extinct Stegocephala or Labyrinthodonts. 
1. Example of the Class.-The Common Frog (Rana temporaria), OR THE EDIBLe Frog (Rana esculenta).

Rana temporaria is the common British species of Frog, found in ponds and damp situations all over the country, and occurring also in America; $R$. esculenta is the large green edible Frog found on the continent of Europe and occasionally in England; $R$. pipiens is the commonest North American species of the genus. Other species of the same genus occur in all parts of the world except New Zealand, the southern part of South America, and the various oceanic islands.

External Characters.-The trunk is short and stout, and is continued, without the intermediation of a neck, into the broad, depressed head. There is no trace of a tail, the anus being terminal. The mouth also is terminal, and is characterised by its extra ordin a r $y$ width, the gape extending considerably behind the eye. On the dorsal surface of the snout are the small nostrils; the eyes are large and prominent, and each is provided with an upper eyelid in the form of a thick fold

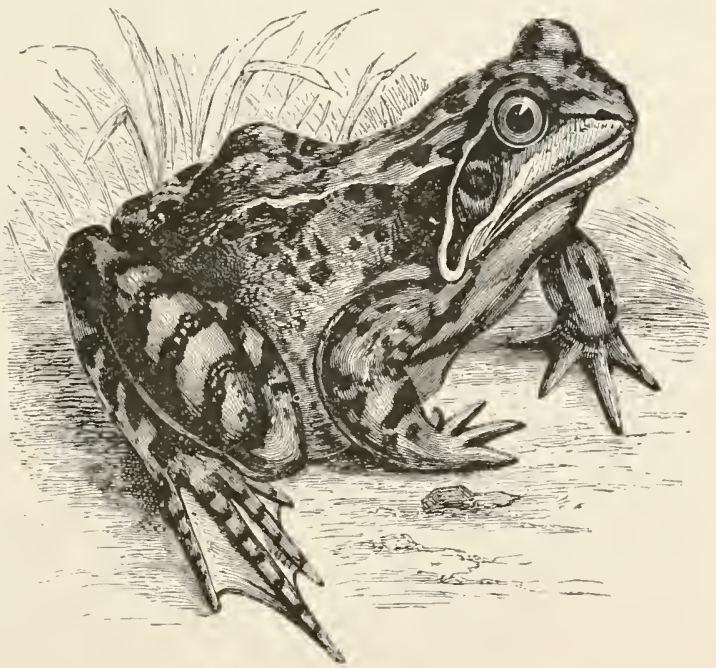

FIG. 932.-Rana temporaria. (From Mivart.) of skin, and a nictitating membrane, a much thimner fold, which arises from the lower margin of the eye and can be drawn up over it. Close behind the eye is a circular area of tenscly-stretched skin, the tympanic membrane, a structure not met with in any Fish : as we shall see, it is an accessory part of the auditory organ. There is no trace of branchial apertures.

The back has a peculiar bend or hump, in the sitting posture, marking the position of the sacral vertebra. The limbs are of very unequal size. The fore-limbs are short, and each consists of an upper arm, which, in the ordinary position, is directed backwards and downwards from the shoulder-joint; a fore-arm, directed downwards and forwards from the elbow; and a hand, ending in four short, tapering digits, directed forwards. The hind-limb is of 
great size; in the usual squatting posture the thigh is directed downwards, outwards, and forwards from the thigh-joint, the shank inwards, backwards, and upwards from the knee. The foot consists of two parts, a tarsal region directed downwards from the heel-joint, and five long, slender digits united by thin folds of skin or webs. Thus the limbs are placed in such a way that the elbow and knee face one another, and the first digit-that of the hand probably representing the index-finger, that of the foot, the hallux or great toe-is turned inwards or towards the median plane of the body.

The skin is greyish-brown in $R$. temporaria, greenish in $R$. esculenta, and is mottled, in both species, with dark brown or black; in R. temporaria there is a large black patch over the tympanic region. Sexual differences occur in both species; in $\mathrm{R}$. temporaria there is a large, black, glandular swelling on the inner side of the hand of the male, and in R. esculenta the male has, at each angle of the mouth, a loose fold of skin, the vocal sac, which can be inflated from the mouth into a globular form. The skin is soft and slimy owing to the secretion of mucous glands; there is no trace of exoskeleton.

Endoskeleton.-The vertebral column (Fig. 933) is remarkable for its extreme shortness; it consists of only nine vertebræ (V. 1-V. 9), the last followed by a slender, bony rod, the urostyle (U. ST.). The second to the seventh vertebræ have similar characters. The centrum $(\mathrm{B}, \mathrm{cn})$ is somewhat depressed and has a concave anterior and a convex posterior face-a form known as procoelous. Each half of the neural arch consists of two parts, a pillar-like pedicle $(p d)$ springing from the centrum and extending vertically upwards, and a flat, nearly horizontal lamina $(\mathrm{lm})$, forming, with its fellow, the roof of the neural canal. When the vertebræ are in position, wide gaps are left between successive pedicles; these are the intervertebral foramina and serve for the transmission of the spinal nerves. The zygapophyses $(a . z y g)$ or yoking processes are far better developed than in any Fish; they spring from the junction of pedicle and lamina, the anterior zygapophysis having a distinct articular facet on its dorsal, the posterior on its ventral surface. Thus when the vertebræ are in position the posterior zygapophyses of each overlap the anterior zygapophyses of its immediate successor. Laterally the neural arch gives off on each side a large outstanding transverse process $(t r . p r)$; its crown is produced into a very small and inconspicuous neural spine ( $n . s p)$.

The first or cervical vertebra (V.1) has a very small centrum and no transverse processes. There are no anterior zygapophyses, but at the junction of centrum and arch there occurs on each side a large oval concave facet for articulation with one of the condyles of the skull (vide infra). The eighth vertebra has a biconcave 


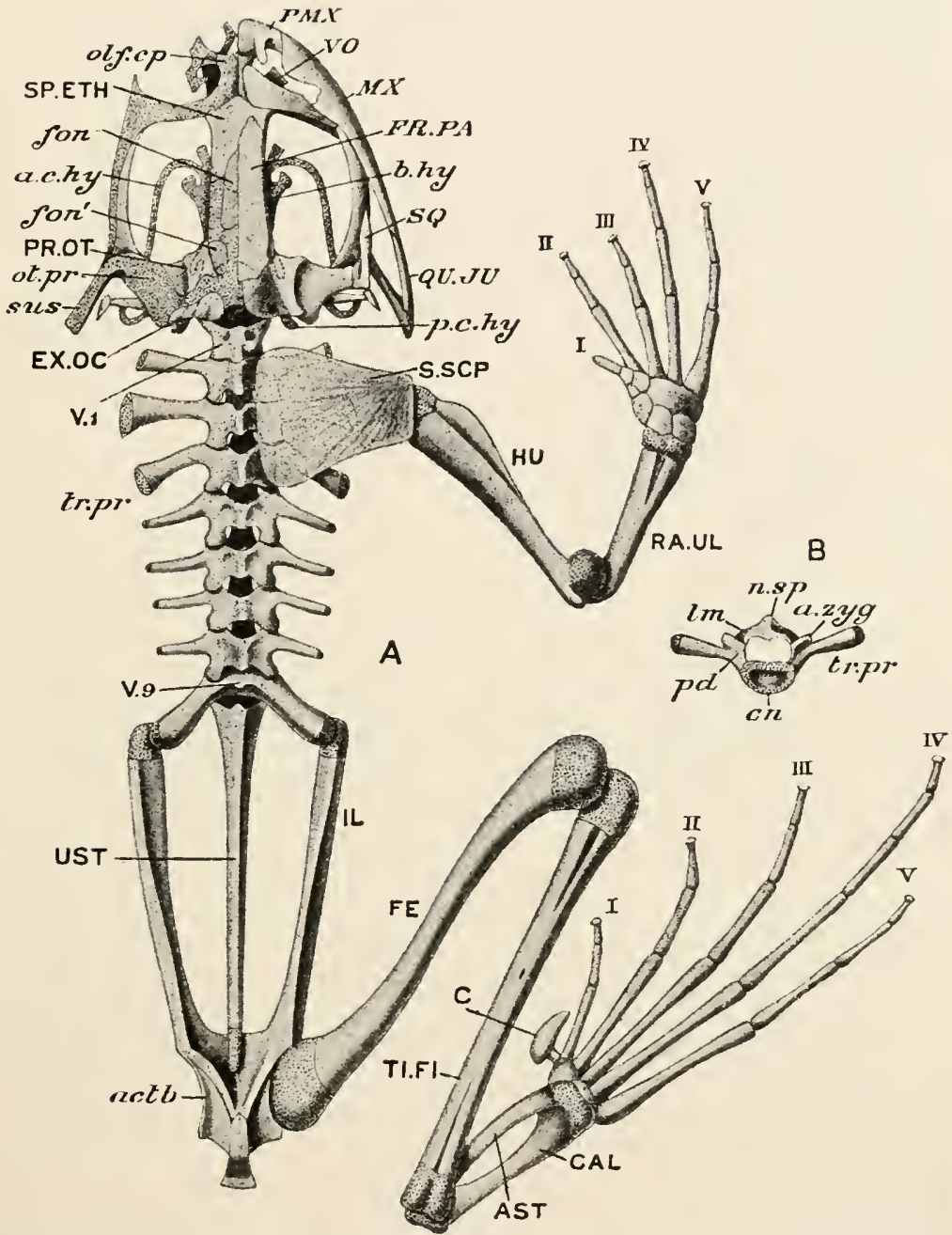

FIG. 933.-Rana temporaria. A, the skeleton from the dorsal aspect; the left half of the shoulder-girdle and the left fore-and hind-limbs are removed, as also are the investing bones on the left side of the skull. Cartilaginous parts dotted. Names of replacing bones in thick, those of investing bones in italic capitals, other references in small italics, $a, c . h y$. anterior cornu of hyoid ; $a c t b$. acetabulum ; A ST. astragalus ; $b$. hy. basi-hyal ; C. calcar ; CAI. calcaneum; rXX. OC. exoccipital ; r. femur ; fon. fon'. fontanelles ; $F R$. $P A$. fronto-parietal ; IUU. humerus ; II. ilium; $M \vec{X}$. maxilla ; olf. $c p$. olfactory capsule ; ot. $p r$. otic process; $p . c . h y$. posterior cornu of hyoid; $P M X$. premaxilla; PR. OT. pro-otic ; $Q U . J U$. quadrato-jugal; RA.UI. radio-ulna; SP.TTI. sphenethmoid; $S Q$. paraquadrate; S.SCP. supra-scapula; sus, suspensorium; TI.FI. tibio-fibula ; $t r$. pr. trans-

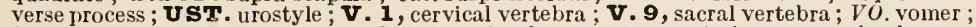
$I-V$, digits. $B$, the fourth vertebra, anterior face. a. zyg. anterior zygapophysis; cn. centrum ; lm. lamina; $n . s p$. neural spine; $p d$. pedicle ; $t r . p r$. transverse process. (After Howes, slightly altered.) 
centrum; that of the ninth or sacral vertebra (V. 9) is convex in front and presents posteriorly a double convexity articulating with a double concavity on the anterior end of the urostyle. The latter (U. ST.) is formed by the ossification of the perichordal tube (see p. 71), which, in this region of the vertebral column, does not become segmented into vertebræ.

The skull (Figs. 933 and 934) consists of a narrow brain-case, produced behind into great outstanding auditory cxpsules, and in front into large olfactory capsules. The whole of the bones of the upper jaw are immovably fixed to the cranium, so that the only free parts are the lower jaw and a small plate of mingled bone and cartilage, the hyoid apparatus, which lies in the floor of the mouth and is the sole representative in the skull of the entire hyobranchial or gill-bearing skeleton of Fishes.

As in the Trout, a number of investing bones can be removed from the skull without injury to the underlying chondrocranium. The latter, however, is not, as in the Trout, the primary cranium alone, but, as in Holocephali and Dipnoi, the primary cranium plus the palatoquadrate or primary upper jaw. The cranium in the strict sense includes the brain-case and the auditory and olfactory capsules : the palatoquadrate $(p a l . q u)$ is not a solid mass fused throughout its length with the cranium, as in Holocephali and Dipnoi, but rather resembles the subocular arch of the Lamprey (p. 123), being a slender rod attached to the cranium at either end, but free in the middle. It is divisible into three regions, a posterior quadrate-region or suspensorium (sus), an intermediate pterygoid region, and an anterior palatine region. The suspensorium extends backwards, outwards, and downwards from the auditory region of the cranium, to which it is immovably united by its forked proximal end, one branch of the fork-the otic process (Fig. 934, ot. $p r$-being fused with the auditory capsule, the other-the pedicle $(p e d)$ - with the trabecular region immediately anterior to the auditory capsule. Ventrally the suspensorium furnishes an articular facet for the mandible, and is connected with the delicate rod-like pterygoid region; this passes forwards and joins the palatine region, which is a transverse bar fused at its inner end with the olfactory capsule.

The occipital region of the cranium contains only two bones, the exoccipitals (EX. OC), which lie one on each side of the foramen magnum (for. mag) and meet above and below it: there is no trace of supra- or basi-occipital. Below the foramen magnum are a pair of oval projections, the occipital condyles (oc. $c n$ ), furnished by the exoccipitals and articulating with the cervical vertebra.

Each auditory capsule is ossified by a single bone, the pro-otic (PR. OT); there are no other ossifications of the auditory region (p. 77). In the adult the pro-otic fuses with the exoccipital: it presents on its outer surface, behind the otic process 
of the suspensorium, a small aperture, the fenestra ovalis, closed in the entire animal by membrane, and, when the latter is removed, leading into the cavity of the auditory capsule, containing the membranous labvrinth.

In front of the auditory capsules a considerable part of the cranial wall is formed of cartilage, and presents above a single large and a pair of small fontanelles (Fig. 933, fon., fon'), but anteriorly it is ossified by the sphenethmoid, or girdle-bone (SP. ETH), a short bony tube divided by a transverse partition into an anterior compartment which lodges the hinder ends of the olfactory sacs, and a posterior compartment which contains the olfactory bulbs. The

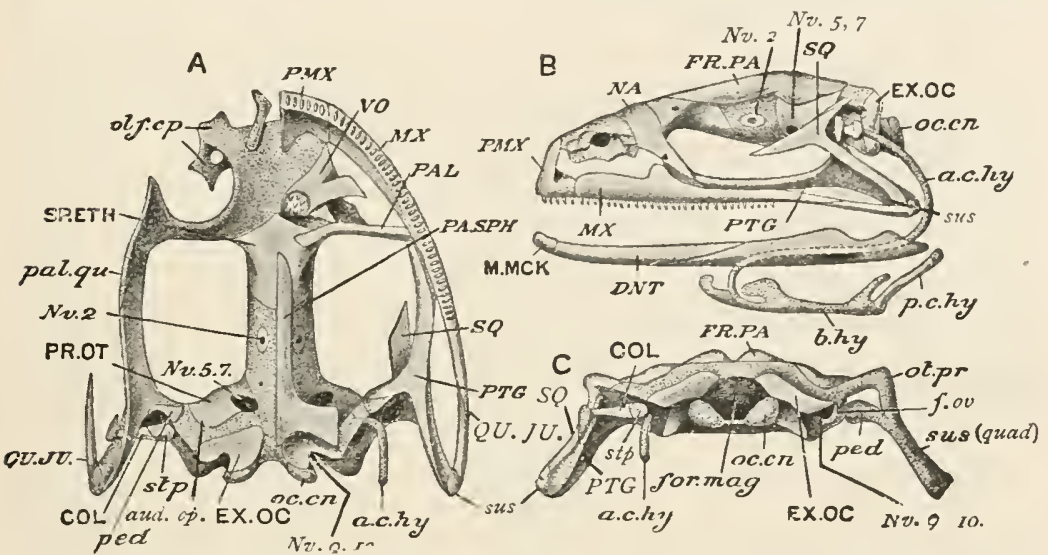

FIG. 931.-Rana temporaria. The skull. A, from beneath, with the investing bones removed on the right side (left of flgure); $\mathrm{B}$, from the left side, with mandible and hyoid C, from behind, the investing bones removed at sus. a. c. hy. anterior cornu of hyoid; aud.ep. auditory capsule; $b$. hy. body of hyoid ; COL. columella; DNT dentary; EX. OC. exoccipital; for. mag. foramen magnum; $f$. ov fenestra ovalis ; FR.PA. fronto-parietal;

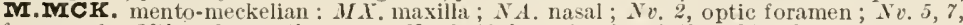
foramen for fifth and seventh nerves; $N v .9,10$, foramina for ninth and tenth nerves; $o c . c n$. occipital condyle; olf. $c p$. olfactory capsule; ot. $p r$. otic process; $P A L$. palatine ; pal. $q u$. palato-quadrate ; $P A . S P H$. parasphenoid ; $p . c . h y$. posterior cornu of hyoid ; ped, pedicle

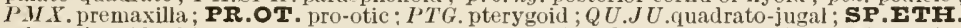
splienethmoid; $S Q$, paraquadrate ; stp. stapes; sus (quad) suspensorium (quadrate); $\mathbf{V} O$. vomer. (After Howes, slightly altered.) A minute investing bone, the septo-maxillary, which is present above the maxilla, close to the nostril, is not here represented.

anterior compartment is again divided by a vertical partition which separates the olfactory sacs from one another, and the transverse partition is perforated for the olfactory nerves. This very peculiar and characteristic bone may be taken to represent meso- and ecto-ethmoids and pre- and orbito-sphenoids all united together.

'The olfactory capsules (Figs. 933, 934, olf. cp) have a delicate cartilaginous roof and floor produced into irregular processes which help to support the olfactory sac. They are separated from one another by a vertical plate of cartilage, continuous behind with the girdle-bone and representing the unossified part of the mesethmoid ; and the anterior wall of each is produced into a little curved, rod- 
like rhinal process. The whole of the primary palatoquadrate arch is unossified.

To this partly ossified chondrocranium the usual investing bones are applied above and below. Covering the roof of the brain-case is a single pair of bones, the fronto-parietals (FR.PA), each formed by the fusion of a frontal and a parietal, distinct in the young Frog. Over the olfactory capsules are paired triangular nasals $(N A)$, and applied to their ventral surfaces small paired vomers $(V O)$. On the ventral surface of the skull is a large T-shaped parasphenoid (PA.SPH), its stem underlying the basis cranii, while its two arms extend outwards beneath the auditory capsules.

In the Trout, it will be remembered, the palatine and pterygoid are replacing bones, formed as ossifications of the palatoquadrate cartilage. In the Frog this cartilage is, as we have seen, unossified, but to its ventral face two investing bones are applied, a small rod-like palatine (PAL), and a three-rayed pterygoid (PTG) having an anterior arm extending forwards to the palatine, an inner arm applied to the pedicle of the suspensorium, and an outer arm extending along the whole inner face of the suspensorium. It will thus be seen that bones originally preformed in cartilage may give place to investing bones, developed in corresponding situations, but altogether independent of the cartilage, the latter remaining unossified.

The suspensorium, as we have seen, is strengthened on its inner face by the outer arm of the pterygoid; extermally it is similarly supported by a hammer-shaped investing bone, the paraquadrate, often known as the squamosal (SQ). The upper jaw is formed by three investing bones, the small premaxilla $(P M X)$ in front, then the long, narrow maxilla $(M X)$, and finally the short quadratojugal $(Q U$. JU), which is connected posteriorly with the quadrate.

The mandible contains a persistent Meckel's cartilage, as a sort of core, outside which are formed two bones, a long angulo-splenial on its inner face, and a sliort dentary $(D N T)$ on the outer face of its distal half. The actual distal end of Meckel's cartilage is ossified as a small replacing bone, the mento-meckelian (M. MCK), not represented in Fishes.

The hyoid apparatus consists of a shield-shaped plate of cartilage, the body of the hyoid $(b . h y)$, produced at its anterior angles into slender rods, the anterior cornua (a.c. hy), which curve upwards and are fused with the auditory capsules, and at its posterior angles into partly ossified rods, the posterior cornua $(p . c . h y)$, which extend backwards, embracing the glottis.

Two other cranial structures remain to be noticed. External to the paraquadrate is a ring of cartilage, the annulus tympanicus (Fig. 947, an. tymp.), which supports the tympanic membrane as 
the frame of a tambourine supports the parchment. Inserted into the fenestra ovalis is a nodule of cartilage, the stapes (stp.), to which is attached the inner end of a small hammer-shaped structure, the columella (COL), the handle of which is ossified, while its cartilaginous head, or extracolumella, is fixed to the inner surface of the tympanic membrane.

The comparison of the Frog's skull with those of Fishes is facilitated by a study of its development. In the tadpole or larval Frog there is a cartilaginous cranium (Fig. 935) connected on each side with a stout

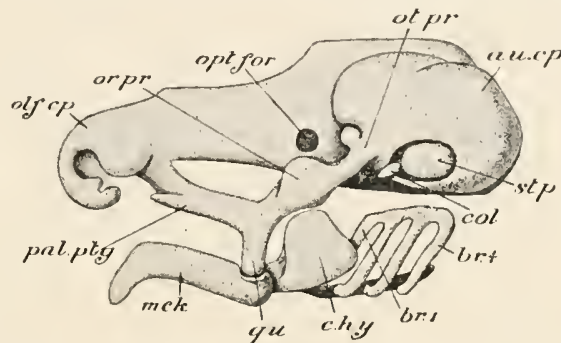

Fig. 935.-Skull of Tadpole. au. $c p$, auditory capsule br. I-4, branchial arches; $c$. hy. ceratohyal; col. columella : $m c k$. Meckel's cartilage ; olf. $c p$. olfactory capsule ; opt. for. optic foramen ; or. pr. orbital process of suspensorium; ot. pr. otic process; pal.ptg. ralato-pterygoid bar; qu. quadrate; stp. stapes. (After Marshall, slightly altered.)

inverted arch, like the subocular arch of the Lamprey or the palatoquadrate of Chimæra or Ceratodus, and, like them, developed from the dorsal region of the mandibular arch. The

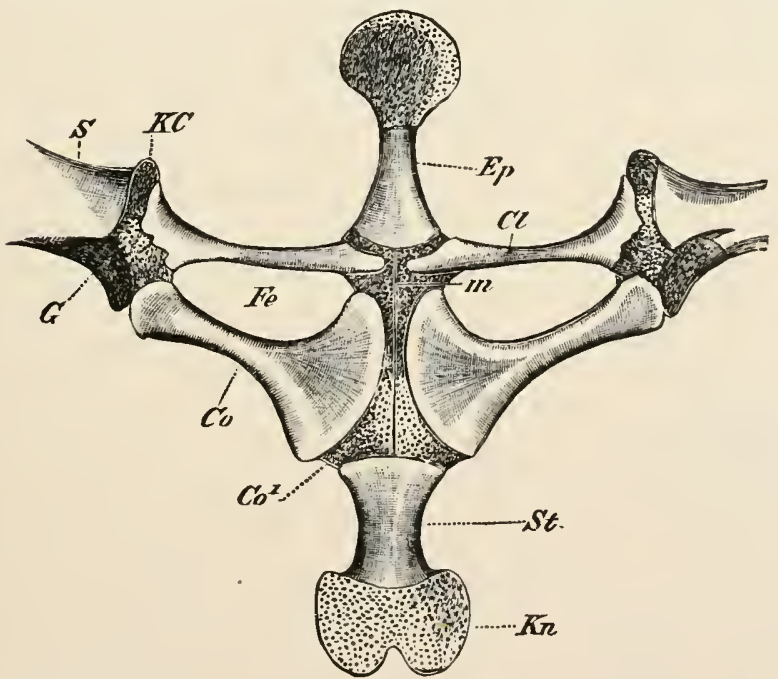

Fig. 936.- Rana esculenta. The shoulder-girdle from the ventral aspect. Cartilage dotted. Co. coracoid ; $\mathrm{Co}^{\perp}$. epicoracoid ; $\mathrm{Cl}$. clavicle; $\mathrm{Ep}$, omosternum; $\mathrm{G}$. glenoid cavity ; $\mathrm{F}^{\prime}$. fenestra between clavicle and coracoid; $K C$. cartilage separating scapula and clavicle; $K n$. xiphisternum; $m$. junction of epicoracoids; $S$. scapula; $S t$. sternum. (From Wiedersheim's Comparatice Anatomy.)

quadrate region $(q u)$ of this primary upper jaw is well in front of the eye, the axis of the suspensorium being inclined for- 
wards and the mandible very short, in correspondence with the small size of the tadpole's mouth. The quadrate is fused by its pedicle with the trabecular region, the otic process (ot. pr) which unites it with the auditory capsule being formed later. Behind the suspensorium are distinct hyoid (c.hy) and branchial (br. 1-4)

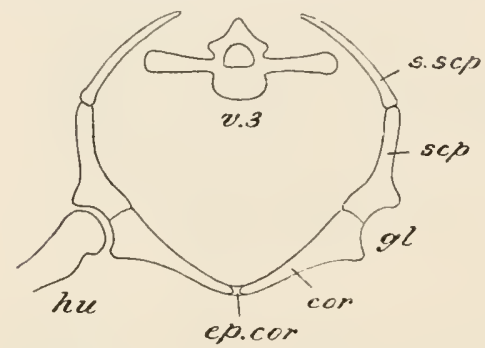

Fig. 937 -Rana. Jiagrammatic transverse section through the shoulder-girdle. cor. coracoid ; ep. cor. epicoracoid; $g l$. glenoid cavity; hu. humerus; scp. scapula; $s$. scp. supra-scapula; v. 3, third vertebra. (From Parker's Practical Zoology.) arches supporting the gills by which the tadpole breathes. As development goes on, the axis of the suspensorium is rotated backwards, producing the wide gape of the adult, and the stout palatopterygoid region of the subocular arch ( $p a l . p t g$ ) gradually assumes the slender proportions it has in the adult. The greater part of the hyoid arch gives rise to the anterior cornua of the adult hyoid-apparatus, the body of which is formed from the basi-hyal and basibranchials, and its posterior cornua probably from the fourth branchial arch. The columella is developed independently, but may perhaps represent a pharyngo-hyal or dorsal segment of the hyoid arch. The stapes is a detached portion of the outer wall of the auditory capsule. Thus, with the assumption of purely aërial respiration, the complex branchial skeleton is reduced to a simple structure for the support of the tongue.

The shoulder-girdlc has essentially the structure already described (p. 81) in general terms as characteristic of the pentadactyle Craniata. The scapula (Fig. 936, S, Fig. 937, scp) is ossified, and is connected by its dorsal edge with a suprascapula (Fig. 933, $\mathbf{S}$. SCP, Fig. 937, s. scp) formed partly of bone, partly of calcified cartilage, and developed from the dorsal region of the embryonic shoulder-girdle. The coracoid (Fig. 936, Co., Fig. 937, cor.) is also ossified, but the procoracoid is represented by a bar of cartilage having an investing bone, the clavicle $(\mathrm{Cl})$, closely applied to it. The suprascapula overlaps the anterior vertebræ; the coracoid and procoracoid are connected ventrally by a cartilage, the epicoracoid (Fig. 936, Co ${ }^{1}$, Fig. 937, ep. cor), which is in close contact with its fellow of the opposite side in the middle ventral line, so that the entire shoulder-girdle (Fig. 937), like that of the Dog-fish, forms a single inverted arch.

Passing forwards from the anterior ends of the united epicoracoids is a rod of bone, the omosternum (Fig. 936, $E p$ ), tipped by a rounded plate of cartilage, and passing backwards from their posterior ends is a similar but larger bony rod, the sternum $(S t)$, also tipped by a cartilaginous plate, to which the name xiphistermum $(K n)$ is applied. 
These two structures are the first indication of a sternum we have yet met with, with the possible exception of the median ventral element of the shoulder-girdle of Heptanchus (p. 170). The omosternum is developed as paired forward extensions of the epicoracoids which undergo fusion : the sternum and xiphisternum arise as paired rods lying posterior to the epicoracoids, and subsequently uniting with one another. This sternal apparatus of the Frog (and of the Amphibia in general) differs developmentally from the structures in the higher Vertebrates to which the same name is applied-the latter being formed from separated-off portions of embryonic ribs (costal sternum).

The fore-limbs deviate from the typical structure (p. 81) chiefly in the fusion of the radius and ulna into a single radio-ulna (Fig. 933, RA. UL), and in the presence of only four complete digits with a vestigial one on the radial side. In all probability the last represents the pollex, and the complete digits are the second to the fifth of the typical hand. Six carpals only are present, the third, fourth, and fifth digits articulating with a single bone which has apparently arisen by the fusion of the third, fourth and fifth distalia and of at least one centrale.

The pelvic girdle (Fig. 938) is very peculiarly modified; it resembles in form a Bird's "merrythought," consisting of two long, curved bars articulating in front with the transverse processes of the sacral vertebra (Fig. 933) and uniting posteriorly in an irregular vertical disc of mingled bone and cartilage which bears on each side a deep, hemispherical acetabulum $(G)$ for the articulation of the thigh-bone. The curved rods are the ilia $(I l ., P)$; they expand posteriorly and unite with one another in the median plane to form the dorsal portion of the disc and about one-half of the acetabulum. The posterior portions of the disc and acetabulum are furnished by the ischia $(I s)$, fused with one another in the sagittal plane, their ventral portions by the similarly united pubes $(K n)$. The ilium and ischium are formed of true bone, the pubis of calcified cartilage ; the union of the elements in the median plane is called the symphysis. In the larva the ilium is vertical, but during development

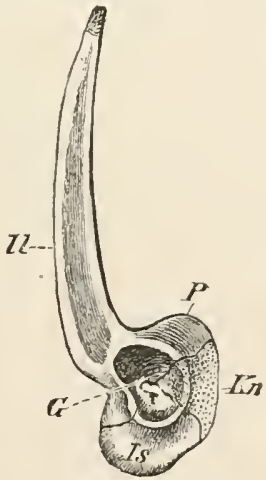

FIG. 938.-Rana esculenta. Pelvic girdle from the right side. G, acetabulum; $I l, P$ ilium; Is. ischium; $k n$, pubis. (From Wiedersheim's Comparative Anatomy.)

it becomes lengthened and at the same time rotated backwards, thus bringing the articulation of the hind-limbs as far back as, possible.

In the hind-limb the tibia and fibula are fused to form a single tibio-fibula (Fig. 933, TI. FI), and the two bones in the proximal 
row of the tarsus - the tibiale or astragalus (AST) and the fibulare or calcaneum (CAL) - are greatly elongated and provide the leg with an additional segment. There are three tarsals in the distal row, one of which appears to represent the centrale, another the first distale, and the third the fused second and third distalia. There are five well-developed digits, and on the tibial side of the first is a spur-like structure or calcar $(c)$, formed of three bones, a metatarsal and two phalanges: such an additional digit is called a pre-hallux.

All the long bones of the limbs consist of the shaft formed of true bone and of extremities of calcified cartilage. The distinction is a very obvious one, both in the freshly-prepared and in the dried skeleton.

The muscular system has undergone great modifications in correspondence with the complex movements performed by the limbs. The dorsal muscles of the trunk are no longer divisible into myomeres, but take the form of longitudinal or oblique bands (extensores dorsi, \&c.), lying partly above the vertebræ, partly between the transverse processes, partly between the ilia and the urostyle. The ventral muscles are differentiated into a paired median band, the rectus abdominis (Fig. 939, rct. abd), with longitudinal fibres, and a double layer of oblique fibres-obliquus externus (obl. ext) and internus (obl. int)-extending from the vertebral column to the recti. Both the extensor dorsi and the rectus abdominis are traversed at intervals by transverse bands of fibrous tissue, the inscriptiones tendinea (ins. ten), but the segments thus formed do not correspond with the embryonic myomeres. The right and left recti are united by a longitudinal band of tendon, the linea alba $(l$. alb).

The muscles of the limbs are numerous and complex, each segment having its own set of muscles by which the various movements of which it is capable are performed. There are muscles passing from the trunk to the limb-girdles; from the trunk or the limb-girdles to the humerus and femur; from the humerus and femur to the radio-ulna and tibio-fibula ; from the fore-arm or shank to the digits; and from one segment of a digit to another. For the most part the limb-muscles are elongated and more or less spindle-shaped, presenting a muscular portion or belly which passes at either end into a tendon of strong fibrous tissue serving to fix the muscle to the bones upon which it acts. The relatively fixed end of a muscle is called its origin, the relatively movable end its insertion, e.g. in the gastrocnemius muscle of the calf of the leg (gstr) the proximal end attached to the femur is the origin, the distal end attached to the foot the insertion. According to their action muscles are divided into flexors which bend, and extensors which straighten, one part upon another; adductors which draw towards, and abductors which draw away from, the middle line; 
elevators which raise, and depressors which lower, a part, such as the lower jaw. The names of the muscles may have reference to their position, e.g. pectoralis (pct.), the principal muscle of the chest ;

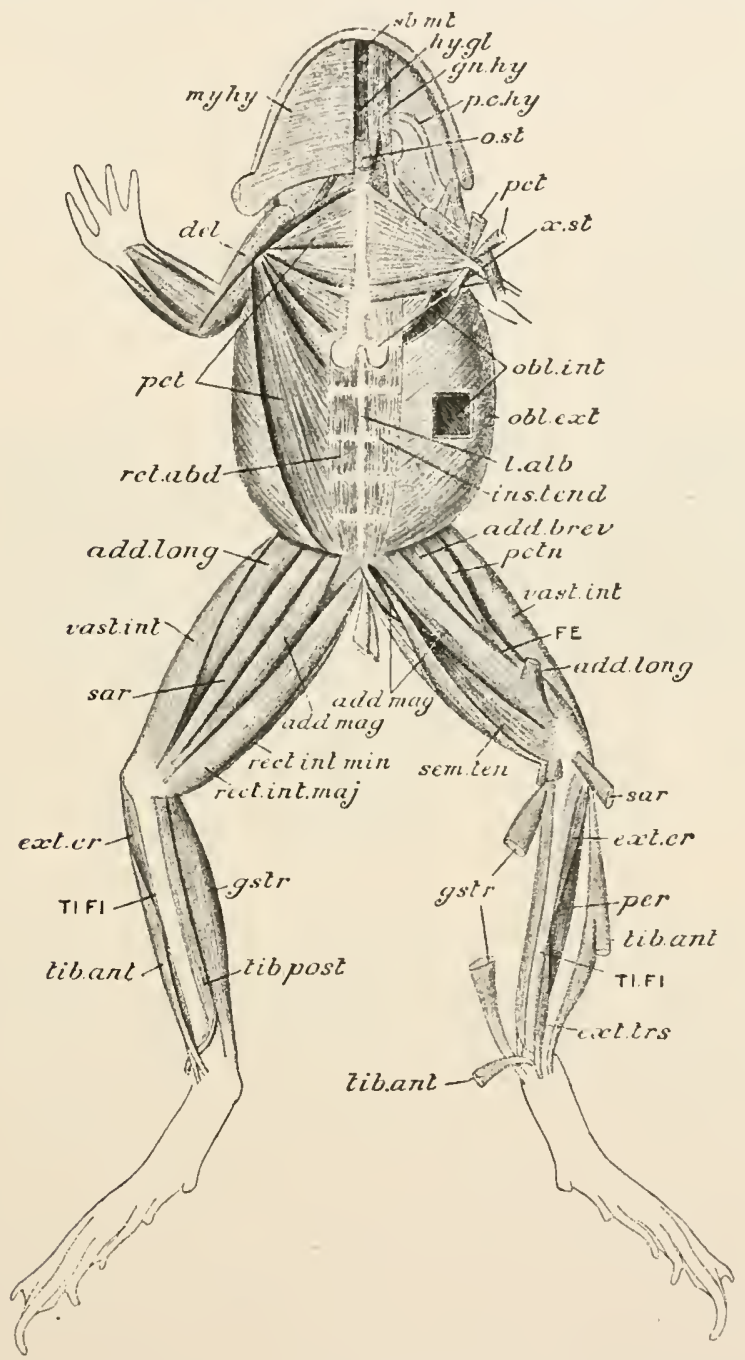

FIG, 939.- Rana esculenta. The muscles from the ventral aspect. On the left side (right of figure) many of the superficial muscles have been cut and reflected to show the deep layer. add. brev, adductor brevis ; add. long. adductor longus ; arld. mag. adductor magnus ; del. dedtoid ; ext cr. extensor cruris ; ext. trs. extensor tarsi : FE. femur ; $g n$. hy. genio-hyoid ; gstr. gastromemius; $h y$. gl. hyoglossus; ins. ten. inscriptio tendinea; $l$. alb. linea alba; my. hy my lo hyoid ; obl. int. obliquus internus; obl. ext. obliquus externus ; o.st. omosternum ret abd. rectus abdominis ; rcct int. maj. rectus internus major; rect. int. min. rect us internus ret. anticus ; $t i \dot{b}$. post. tibialis posticus; TI. FI. tibiofibula; vast. int. vast us internus; $x$. $s t$. xiphisternum, 
or to their form, e.g. biceps, the two-headed muscle; or to their action, e.g. flexor tarsi; or to their origin and insertion, e.g. coracohumeralis.

Digestive Organs. - The mouth leads into a wide buccal cavity having in its roof the internal or posterior nares (Fig. 940, p. na.), a pair of projections due to the downward bulging of the large eyes, and the openings of the Eustachian tubes (eus. t., vide infra). On its floor is the large tongue (tng.), attached in front and free behind, where it ends in a double point; by means of its muscles it can be suddenly projected, point foremost, from the mouth, and is used in the capture of Insects. Immediately behind the tongue is the glottis $(g l$.$) . Teeth are arranged in a single series round the$ edge of the upper jaw, attached to the premaxillæ and maxillæ;

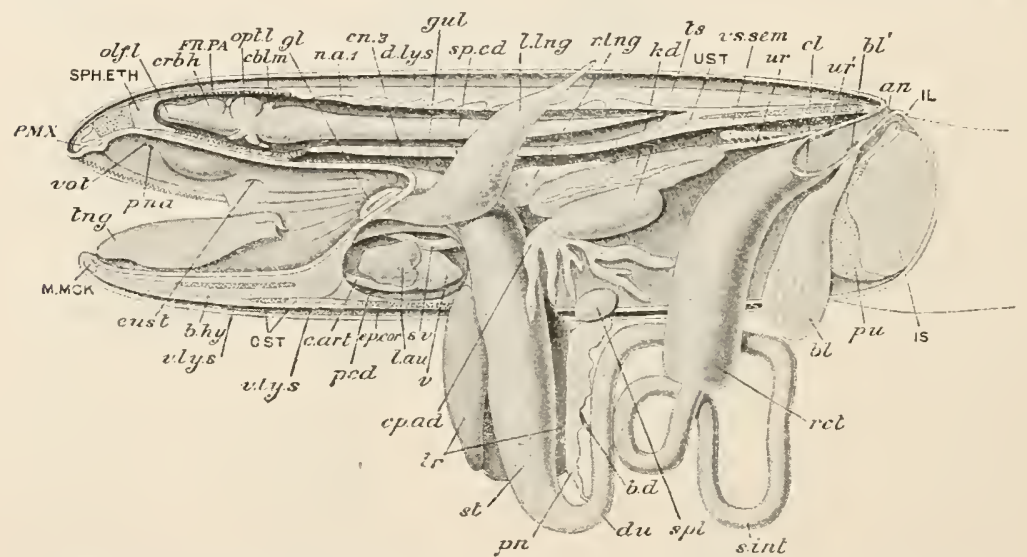

FIG. 940.-Rana temporaria. Dissection from the left side; the viscera somewhat displaced. $a n$. anus; $b . d$. bile-duct; $b$. $h y$. body of hyoid; $b l$. urinary bladder; $b l^{\prime}$. its opening into the cloaca ; $c$. art. conus arteriosus; cblm. cerebellum; $c 7$. cloaca; $c n$. 3 , centrum of third vertebra ; $c p$. ad. corpus adiposum; $c r b . h$. cerebral hemisplrere ; $d$. $l l . s$. dorsal lymph sinus; $d u$. duodenum ; ep. cor. epicoracoid; cus. $t$. Eustachian tube ; FR. PA. fronto-parietal; gl. glottis; gul. gullet; IL. ilium; Is. ischium; $k d$. kidney ; $l$. au. left auricle; $l$. Ing. left lung; Ir. liver; M. MCK. mento-meckelian; $n . a$, neural arch of first vertebra; olf. $l$. olfactory bulb; opt. l. optic lobe ; o. ST. omosternum; pcd. pericardium; PMX. premaxilla; pn. pancreas; p. na. posterior naris; pu. pubis; rct. rectum; r. ing. right lung; s. int. ileum ; sp. $c d$. spinal cord ; sPH. ETH. sphenethmoid; spl. spleen : st. stomach; s. v. sinus venosus ; $t h g$. tongue ; $t s$. testis ; ur. ureter : ur'. its aperture into the cloaca ; usT. urostyle ; $v$. ventricle; $v$. $l y . s$. ventral lymph sinus ; vo. $t$. vomerine teetl ; vs. sem. vesicula seminalis.

there is also a small patch of teeth (vo. t.) on each vomer just internal to the posterior nostril. The teeth are small conical bodies, their bases ankylosed to the bones: their only use is to prevent the polished or slimy bodies of the prey-Insects and Worms-from slipping out of the mouth.

The buccal cavity narrows towards the pharynx, which leads by a short gullet ( $g u l$.$) into a stomach (st.) consisting of a wide cardiac$ and a short, narrow, pyloric division. The duodenum $(d u)$, or first portion of the small intestine, passes forwards parallel with the stomach; the rest of the small intestine (ileum) is twisted into a 
coil. The large intestine or rectum (ret.) is very wide and short, and passes without change of diameter into the cloaca (cl.).

The liver $(l r$.$) is two-lobed; between the right and left lobes$ lies a large gall-bladder (Fig. 941, G). The pancreas $(P$.$) is an$ irregular gland surrounding the bileduct, into which it pours its secretion; the spleen (Fig. 940, spl.) is a small, red globular body attached near the anterior end of the rectum. The thyroids are small paired organs lying below the floor of the mouth in front of the glottis. The $t h y m u s$ is a lso paired, and is situated behind and below the tympanic membrane.

Respiratory Organs. - The lungs (l. lng., r. lng.) are elastic sacs lying in the anterior part of the coelome above the heart and liver; their size and appearance vary greatly according to their state of

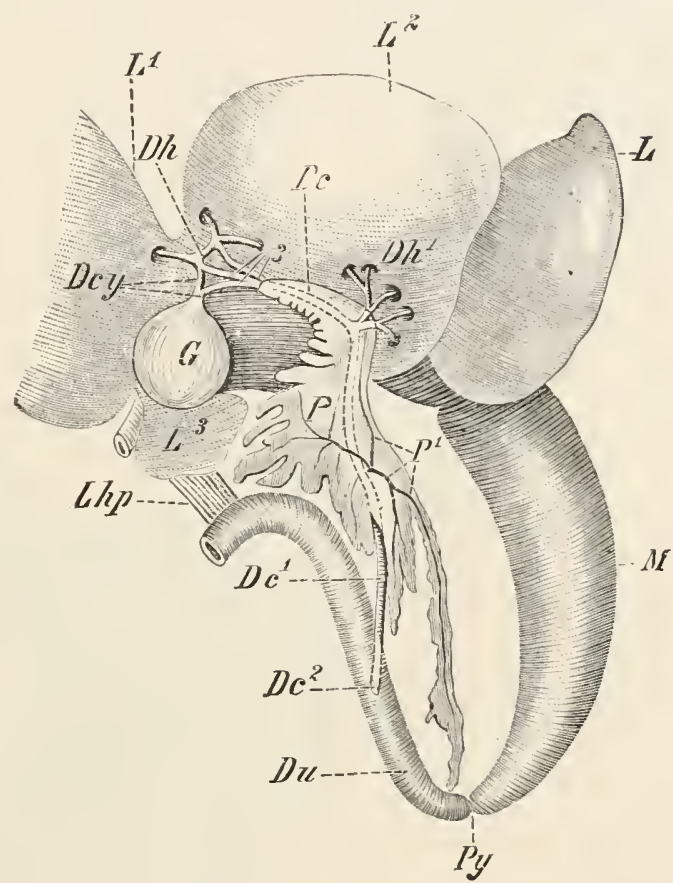

FIG, 941.-Rana esculenta. Stomach and duodenum with liver and pancreas. Dc., $D c .1$ common bile-duct; $D c .{ }^{2}$ its opening into the duodenum ; $D . c y$. cystic ducts: $D h$., Dh. 1 hepatic ducts: $D u$. duodenum; $\boldsymbol{G}$. gall-bladder ; $L, L^{1}, L^{2}, L^{3}$, lobes of liver, turned forwards ; $L h p$. duodenohepatic omentum, a sheet of peritoneum connecting the liver with the duodenum ; $\boldsymbol{M}$, stomach $P$. pancreas ; $P \mathbf{l}$, pancreatic duct: Py. pylorus. (From Wiedersheim's Comparatioc Anatomy.)

distension. Each contains a spacious cavity and has its walls raised into a complex network of ridges abundantly supplied with blood-vessels. The two lungs open anteriorly into a small laryngo-tracheal chamber which communicates with the mouth by the narrow slit-like gloltis. The walls of the laryngo-tracheal chamber are supported by a cartilaginous framework, and its mucous membrane is raised into a pair of horizontal folds, the vocal chords, by the vibration of which the croak of the Frog is produced.

In breathing, the Frog keeps its mouth closed, and by depressing the floor of the mouth, draws air into the buccal cavity through the nostrils. The floor of the mouth is then raised, the nostrils, 
which.are valvular, are closed, and the air is forced through the glottis into the lungs. The skin also is an important respiratory organ.

Circulatory Organs.-The pericardium (Fig. 940, pcd.) is not situated in front of the general cœlome, as in Fishes, but lies in the cœlomic cavity between the gullet above and the epicoracoids below ; it consists, as usual, of a visceral layer closely adherent to the heart, and a loose parietal layer, the two being continuous at the bases of the great vessels and separated by a small quantity of pericardial fluid.

The heart consists of a sinus venosus (Figs. 940 and $944, s . v$.), right and left auricles $(r . a u ., l$. au.), a ventricle $(v ., v t$.$) , and a conus$

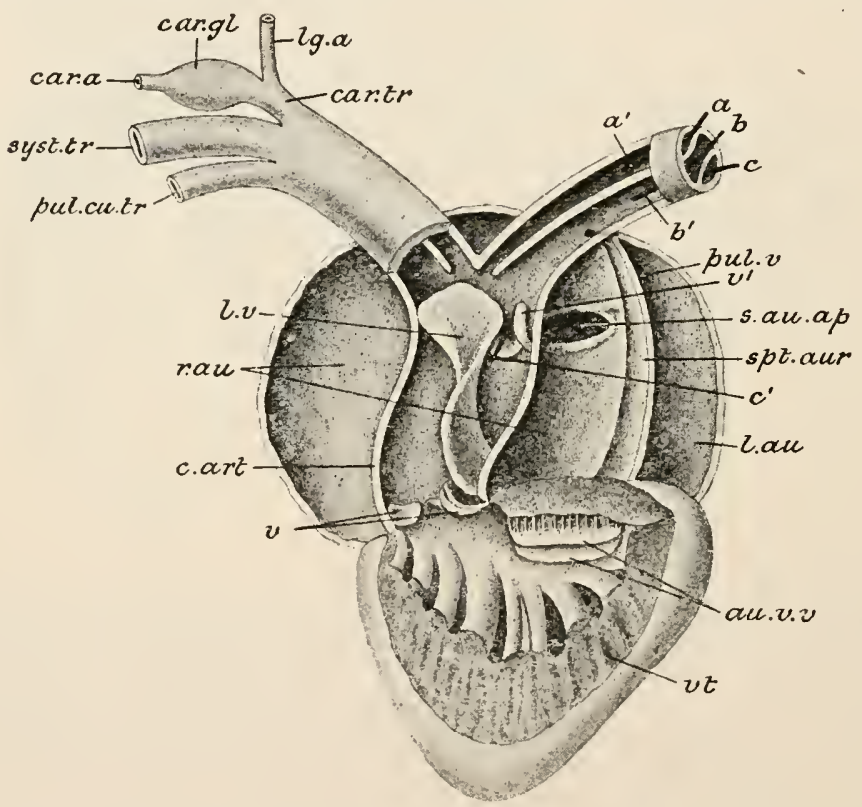

FIG. 942.-Rana temporaria. The heart from the ventral aspect with the cavities laid open. $a, a^{\prime}$, bristle in left carotid trunk; $a u, v, v$, auriculo-ventricular valves ; $b . b^{\prime}$, bristle in left systemic trunk; $c, c^{\prime}$, bristle in left pulmo-cutaneous trunk; car. a. carotid artery ; car. $g l$. carotid labyrinth; $c$. art, conus arteriosus ; car. tr. carotid trunk; $l$. au. left auricle ; $l q . a$. lingual artery ; $l . v$. longitudinal valve ; $p u l . c u$. $t r$. pulmo-cutaneous trunk; $p u l . v$. aperture of pulmonary veins; $r$. au. right auricle; $s$. au. ap. sinu-auricular aperture; spt. aur. septum auricularum ; $v, v^{\prime}$, valves ; $v t$. ventricle.

arteriosus (c. art.). The sinus venosus opens into the right auricle, the pulmonary veins into the left: a striking advance on the Dipnoi is seen in the greatly increased size of the left auricle and its separation by a complete partition, the septum auricularum (Fig. 942, spt. aur.), from the right. The two auricles open by a common auriculo-ventricular aperture, guarded by a pair of valves $(a u . v . v$.), into the single ventricle. The latter has a transversely 
elongated cavity, and its dorsal and ventral walls are raised up into muscular ridges or trabeculæ with interstices between them. The conus springs from the right side of the base of the ventricle; it is separated from the latter by three small semilunar valves $(v$.), and is traversed obliquely along its whole length by a large flap-like longitudinal valve $(l . v$. $)$ which springs from its dorsal wall and is free ventrally. The conus passes without change of diameter into a bulbus aorto, the two being separated by a semilunar valve $\left(v^{\prime}\right)$ and by the free end of the longitudinal valve. The bulbus gives off two branches, right and left, each of them divided by two longitudinal partitions into three vessels, an inner or anterior, the carotid trunk (car. tr.), a middle, the systemic trunk or aortic arch, and an outer or posterior, the pulmo-cutaneous trunk (pul. cu. tr.). The carotid and systemic trunks communicate separately with the bulbus; the two pulmo-cutaneous trunks communicate with the anterior end of the conus by a single valvular aperture placed just behind the free end of the longitudinal valve $\left(c^{\prime}\right)$.

After being bound together in the way described for a short distance, the carotid, systemic, and pulmo-cutaneous trunks separate from one another. The carotid trunk divides into carotid (Figs. 942 and 943, car.) and lingual ( $\mathrm{lg}$.) arteries for the supply of the head, the former having at its base a small swelling, the carotid "gland" or labyrinth (car. gl.), with a spongy interior containing numerous cavities. The systemic trunks curve round the gullet and unite with one another above it to form the dorsal aorta $(d . a o$.), from which, or from one of the systemic trunks themselves, the arteries to all parts of the body, except the head, the lungs, and the skin, are given off. The pulmo-cutaneous trunk divides into two, a pulmonary artery (pul.) to the lung, and a cutaneous artery (cu.) to the skin.

In the tadpole there are four aortic arches, each consisting of an afferent and an efferent branchial artery connected by the capillaries of the gills. As the water-breathing larva undergoes metamorphosis into the air-breathing adult the-gills disappear; the first aortic arch loses its connection with the dorsal aorta and becomes the carotid trunk; the second enlarges, retains its connection with the dorsal aorta, and becomes the systemic trunk; the third disappears; and the fourth sends off branches to the lungs and skin, loses its connection with the dorsal aorta, and becomes the pulmo-cutaneous trunk.

The blood from each side of the head is returned by internal (Fig. 944, int. ju.) and external (ext. ju.) jugular veins into the precaval vein ( $p r . v$.), which also receives the brachial vein (br.) from the fore-limb, and the musculo-cutaneous vein (ms. cu.) from the skin and muscles of the side and back, and part of the head: the two precavals open separately into the sinus venosus.

The course of the blood from the posterior part of the body 
is very different from what we have met with in Fishes, the differences being due partly to the abs ence of a tail, partly to a peculiar modifica-
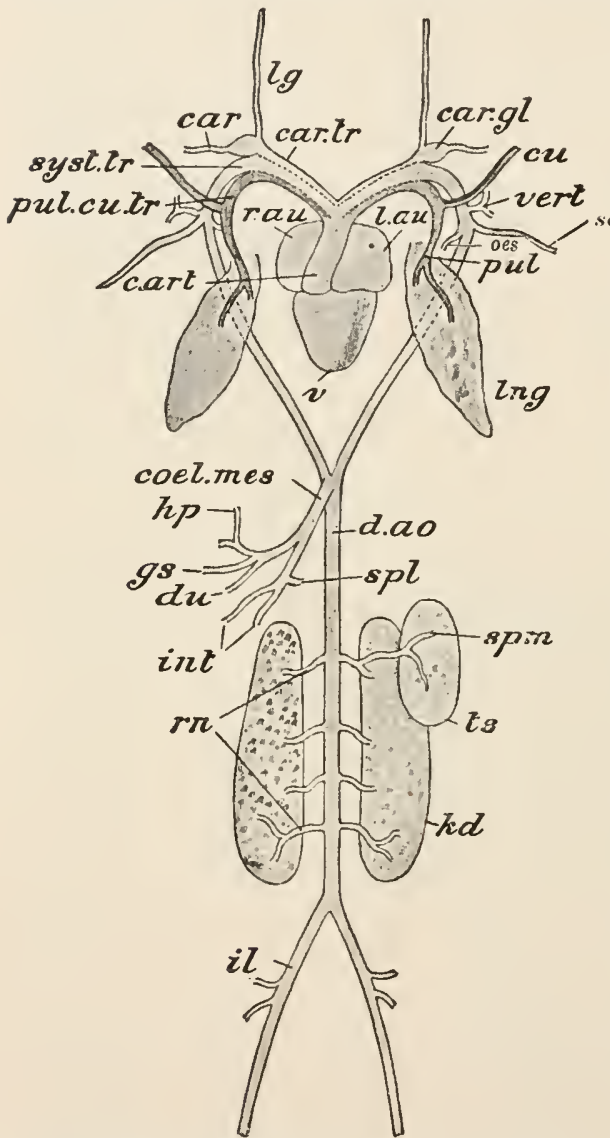

FIG. 943.-Rana temporaria. The arterial system, with the heart, lungs, kidneys, and left testis, from the ventral aspect, car. carotid artery ; car. $g l$, carotid labyrinth; c. art conus arteriosus; car. tr. carotid trunk; col. mes. coliaco-mesenteric artery; cu. cutaneous artery; $d$. ao. dorsal aorta ; $d u$. duodenal artery ; $g s$. gastric artery; $h p$. hepatic artery ; $i l$. iliac artery ; int. intestinal arteries $; k d$. kidney ; $l$. au. left auricle; $l g$. lingual artery; lng. lung; os. cesophageal artery ; pul. pulmonary artery; pul. cu. tr. pulmocutaneous trunk; $r$, au. right auricle; $r n$. renal arteries; $s c l$. subclavian artery; $s p l$. splenic artery; syst. $t r$. systemic trunk; spm. spermatic artery; $t s$. test is ; $v$. ventricle; vert. verteloral artery.

vein also receives vesical veins (vcs.) from the urinary bladder, and a small cardiac vein from the heart $(c d$.). It represents the lateral veins of Elasmobranchs united in the middle ventral line: the pelvic veins are their posterior free portions. and partly to the replacement of the cardinals by a postcaval vein, found among Fishes only in the Dipnoi.

The blood from the front part of the hind-leg is brought back by a femoral vein $(\mathrm{fm}$.$) , which,$ on reaching the colome, divides into two branches, a dorsal and a ventral. The dorsal branch is the renal portal vein (rn.pt.): it receives the sciatic vein (sc.) from the back of the leg and passes to the kidney, in which it breaks up into capillaries. The ventral branch is the pelvic vein ( $p v$.) : it unites with its fellow of the opposite side to form the abdominal vein (abd.) which passes forwards in the ventral body-wall, between the linea alba and the peritoneum, to the level of the sternum, where it turns inwards and divides into two branches, both breaking up into capillaries in the liver. Just as it enters the liver it is joined by the hcpatic portal vein (kp. pt.), bringing the blood from the stomach, intestine, spleen, and pancreas. The abdominal

tion of the lateral veins, 
The blood is collected from the kidneys by the renal veins ( $r n$.), which unite to form the large unpaired postcaval vein (pt.cv.). This passes forwards through a notch in the liver, receives the hepatic veins (h.p.) from that organ, and finally opens into the sinus venosus. Thus the blood from the hind-limbs has to pass through

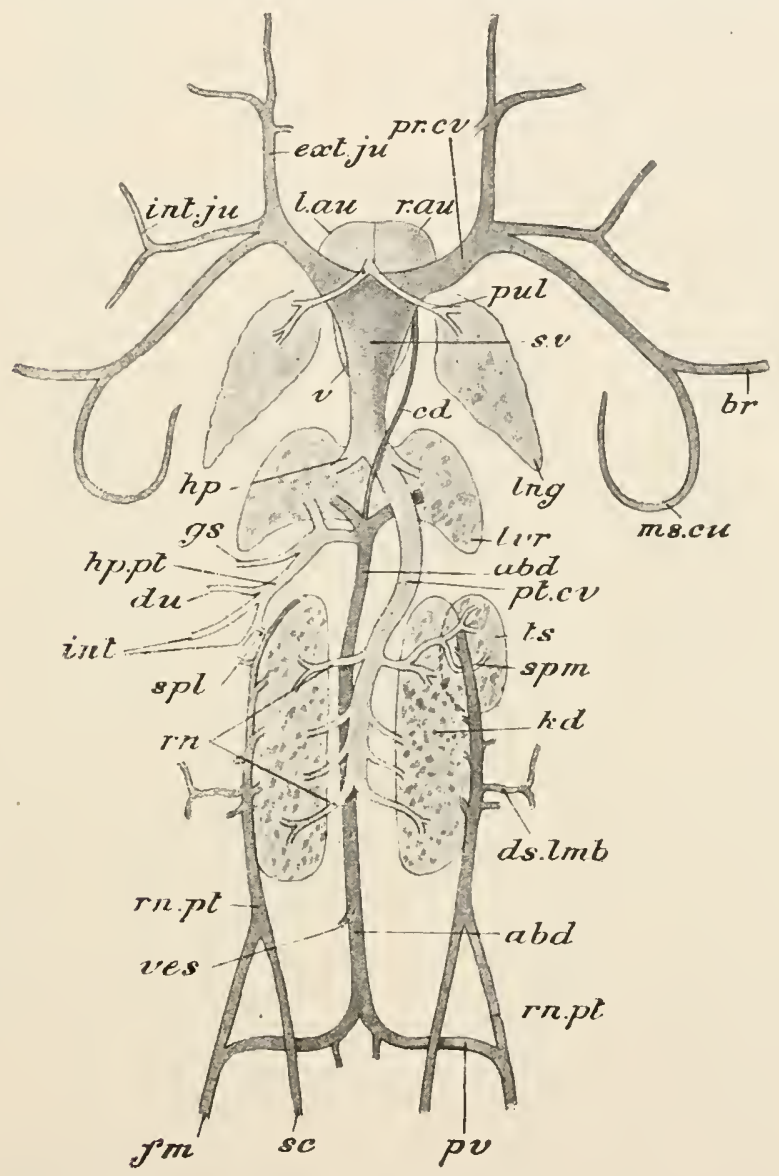

FIG. 944--Rana temporaria. The venous system with the heart, lungs, liver, kidneys, and right testis, from the dorsal aspect. abd. abdominal vein; $b r$. brachial vein ; $c d$. carrliac vein ; $d s$, lmb. dorso-lumbar vein; $d u$, duodenal vein; ext, ju. extermal jugular vein; fm. femoral vein; gs. gastric vein; $h p$. hepatic vein; $h p . p t$. hepatic portal vein ; int. intestinal veins; int. $j u$. internal jugular vein; $k l$. kidney; $l$. au. left auricle; lng. lung; lvr. liver ; $m s$. cu. musculo-cutaneous vein ; pr. cv. precaval vein; pt. cv. postcaval vein; pul. pulmonary vein $; p v$. pelvic vein ; $r$. $a u$. right auricle $r n$. renal veins ; $r n$. $p t$. renal portal vein ; $s c$. sciatic vein; spl. splenic vein; spm. spermatic vein; s. $v$. sinus venosus; $t s$. testis; ves. vesical reins.

one of the two portal systems on its way back to the heart: part of it goes by the renal portal veins to the kidneys, and thence by the renal veins to the postcaval, part by the pelvic and abdominal 
veins to the liver, and thence by the hepatic veins to the postcaval. Lastly, the blood which has been purified in the lungs is returned by the pulmonary veins ( $p u l$.) directly to the left auricle.

It will be seen that there is no trace of cardinal veins in the Frog. But in the larva both anterior and posterior cardinal veins are present: during the metamorphosis the ductûs Cuvieri, in which, as in Fishes, they unite, become converted into the precavals, while the posterior portions of the posterior cardinals contribute to the formation of the postcaval, and the anterior portions disappear.

It will be perceived that the blood poured into the right auricle is mostly impure or venous, that poured into the left fully aërated or arterial. When the auricles contract, which they do simultaneously, each passes its blood into the corresponding part of the ventricle, which then instantly contracts, before the venous and arterial bloods, kept separate as they are to some extent by the muscular trabeculæ acting as incomplete partitions, have time to mix. Since the conus arteriosus springs from the right side of the ventricle, it will at first receive only venous blood, which, on the contraction of the conus, might pass either into the bulbus aortæ or into the aperture of the pulmo-cutaneous trunks. But the carotid and systemic trunks are connected with a much more extensive capillary system than the pulmo-cutaneous, and the pressure in them is proportionally great, so that it is easier for the blood to enter the pulmo-cutaneous trunks than to force aside the valves between the conus and the bulbus. A fraction of a second is, however, enough to get up the pressure in the pulmonary and cutaneous arteries, and in the meantime the pressure in the arteries of the head, trunk, \&c., is constantly diminishing, owing to the continual flow of blood towards the capillaries. Very soon, therefore, the blood forces the valves aside and makes its way into the bulbus aortæ. Here again the course taken is that of least resistance: owing to the presence of the carotid labyrinth, the passage of blood into the carotid trunks is less free than into the wide, elastic, systemic trunks. These will therefore receive the next portion of blood, which, the venous blood having been mostly driven to the lungs, will be a mixture of venous and arterial. Finally, as the pressure rises in the systemic trunks, the last portion of blood from the ventricle, which, coming from the left side, is arterial, will pass into the carotids and so supply the head.

The red blood-corpuscles are, like those of Fishes, oval, nucleated discs. The lymphatic system (Fig. 945) is very well developed, and is remarkable for the dilatation of many of its vessels into immense lymph-sinuses. Between the skin and muscle are large subcutaneous sinuses (Fig. 940, v. ly. s.), separated from one another by fibrous partitions, and the dorsal aorta is surrounded by a spacious subvertebral sinus. The lymph is pumped into the veins 
by two pairs of lymph-hearts, one situated beneath the suprascapulæ, the other beside the posterior end of the urostyle.
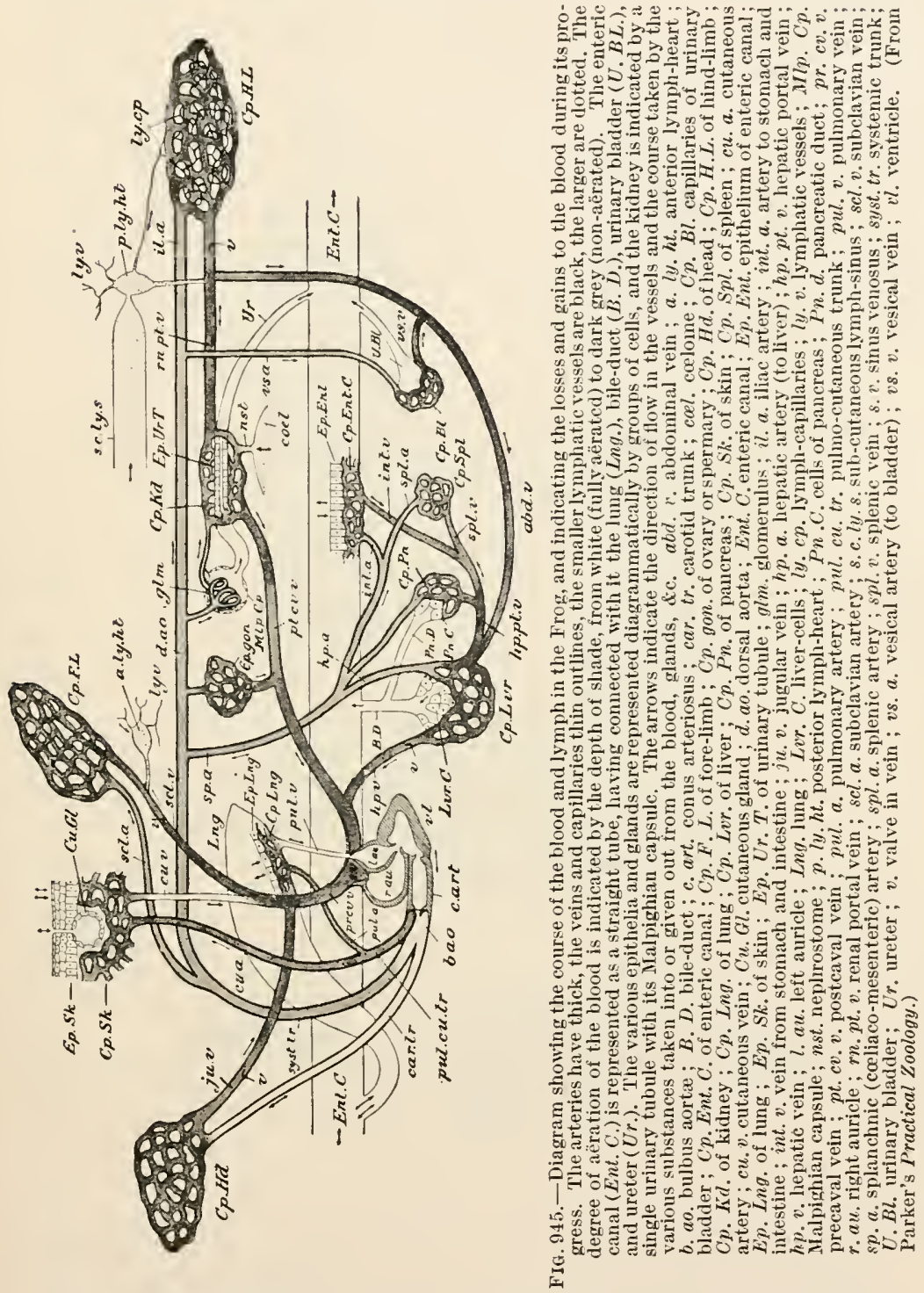

Nervous System.-The brain (Fig. 946) has a very small cerebellum, large optic lobes, a well-developed diencephalon, and large hemispheres and olfactory bulbs, the latter fused in the median 
plane. The corpora striata, or basal ganglia of the cerebral hemispheres, are connected together, as in all Vertebrates, by an anterior commissure ( $D$, com, below, lower line), above which is another com-
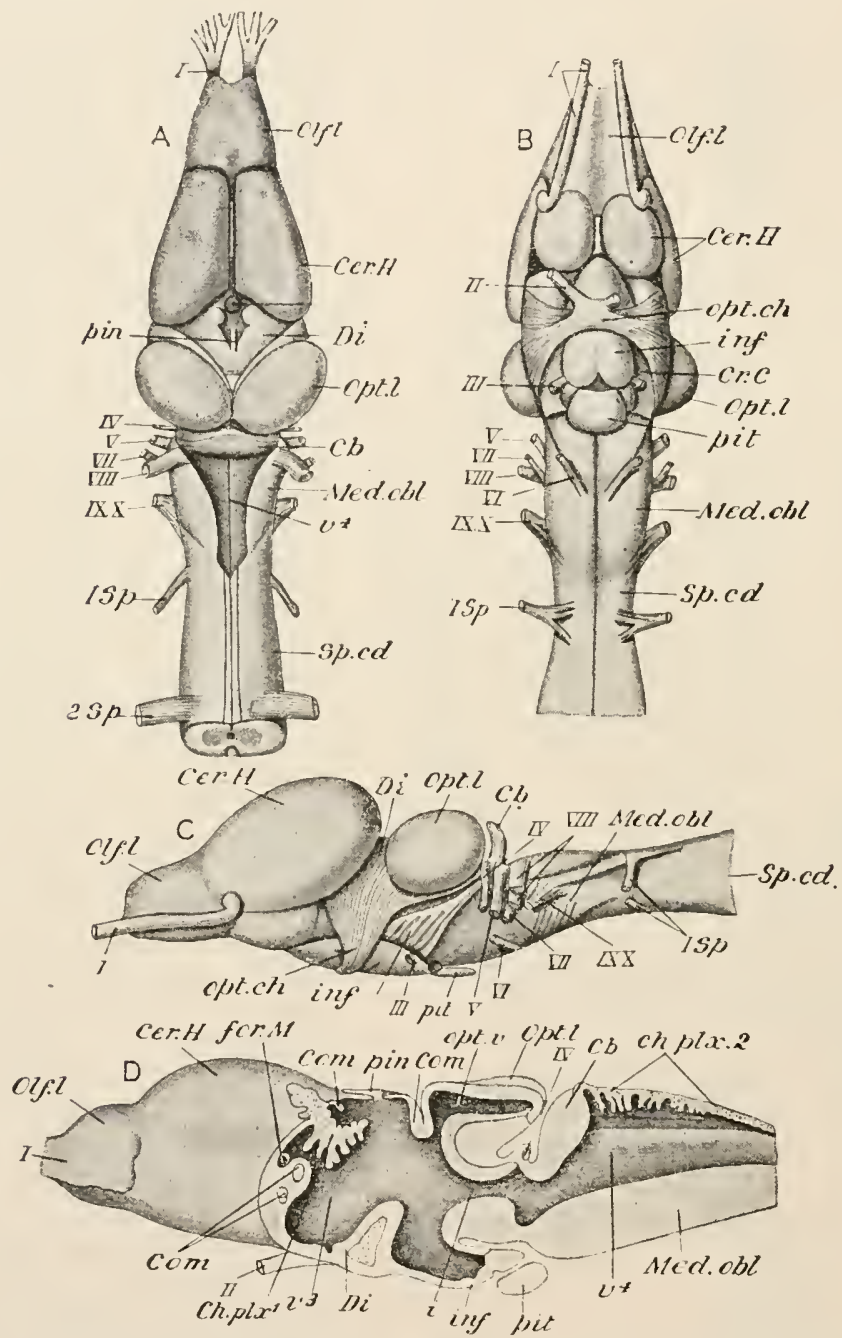

FIa. 946.-Brain of Rana. A, from above ; $B$, from below ; $C$, from the side ; $D$, in longitudinal vertical section. $C b$, cerebellum; Cer. 11 , cerebral hemispheres; $c h . p l x^{1}$, anterior, and ch. pl $x^{2}$, posterior choroid plexus (removed in A); com, commissures, the two in fronit the anterior and hippocampal, the two above the superior or habenular and the posterior; $\mathrm{Cr}$. $\mathrm{C}$, crura cerebri ; $\mathbf{L}$, diencephalon ; for. $M$, foramen of Monro ; $i$, iter, or aqueduet of Sylvius ; inf, infundibulum; Hed. obl, inedulla oblongata; Olf. l, olfactory bulb; opt. ch, optic chiasma; Opt. $l$, optic lohe ; opt. $v$, optic ventricle; pin, stalk of pineal body ; pit, pituitary body ; Sp. cd, spinal cord ; $v^{3}$, third ventricle; $v^{t}$, fonrth ventricle; $I-I$, cerebral nerves ; ISp. ¿Sp. spinal nerves. (From l'arker's I'ractical Zoology. A-C, after Gaupp; $D$, from Wiedersheim's Comparative A natomy, after Osborm.) 
missure (com, below, upper line) partly representing the hippocampal commissure of the biain of Reptiles and Manmals. T'He metacole is covered by a thick choroid plexus; the mesocoele is divisible into a median passage or iter (i.), and paired optocoles $(o p t . v$.) in the optic lobes: the paracoeles are large cavities each comnunicating with a rhinocole in the corresponding olfactory bulb. 'The pineal bodv is vestigial in the adult, a lobe of the anterior choroid plexus, with a restige of the stalk ( $p$ in), taking the position which it usually occupies: in the larva it is found outside the skull and immediately beneath the skin.

The first spinal nerve performs the function of the hypoglossal (Fig. 946, 1Sp.), supplying the muscles of the tongue: it passes out between the first and second vertebre. The spinal cord is short and ends in a delicate filament, the filum terminale. In corre-

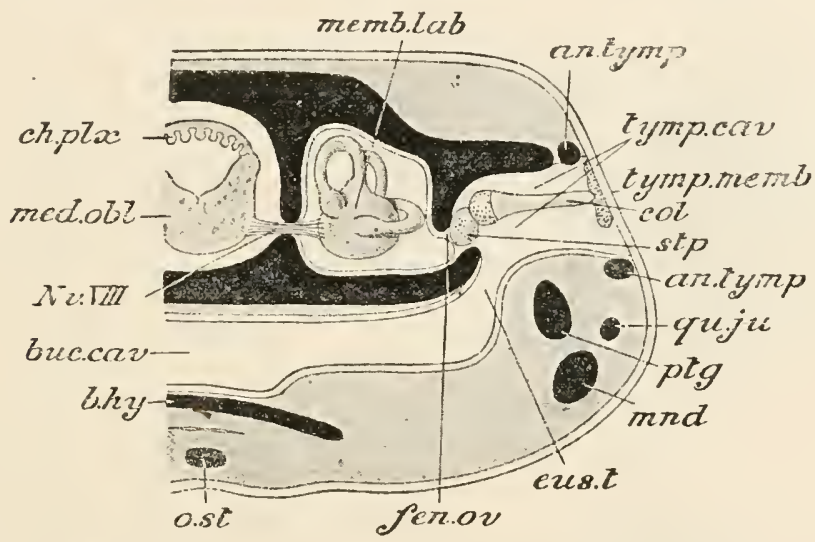

FIQ. 947.-Transverse section of head of Frog to show the relations of the accessory auditory apparatus (diagrammatic). Skeletal structures black, with the exception of the columelli1. an. tymp. annulus tympanicus; $b$. hy. body of hyod ; buc. cav. cavity of pharynx; $c h$. $p / x$. (horoid plexus; col. columella; eus. $t$. Eustachian tube ; fen. ov, fenestra oralis ; med. uh. Inedulla oblongata ; memb. lab. membranous labyrinth; mnd. mandible; ${ }^{\top} v$. $V I I I$. auditory nerve; o. st. omosternum; $p t_{\eta}$. pterygoid; qu. $j u$. quadrato-jugal; stp. stapes ; tymp. cae. tymuanic eavity ; tymp. m. tympanic membrane.

spondence with the number of vertebræ there are only ten pairs of spinal nerves, of which the second and third unite to form a brachial plexus giving off the nerves to the fore-limb, while the seventh to the tenth join to form a lumbo-sacral plexus giving off the nerves to the hind-limb.

Sensory Organs.--The olfactory sacs have each two openings: the anterior naris or external nostril and the posterior naris (Fig. 940, p. na.) or internal nostril, which opens into the mouth immediately external to the vomer.

The eye and the audilory organ have the usual structure, but in connection with the latter there is an important accessory organ of hearing not hitherto met with. Bounded externally by the tym- 
panic membrane and internally by the outer wall of the auditory capsule is a considerable space, the tympanic cavity (Fig. 947, tymp. cav.), which communicates with the pharynx by the short Eustachian tube (eus.t.) already noticed (Fig. 940, eus. t.), so that a probe thrinst through the tympanic membrane from outside passes directly into the pharynx. In the roof of the tympanic cavity lies the cohnmella $(\mathrm{col}$.$) , its head, or extra-columella, attached to the inner$ snrface of the tympanic membrane, its handle united to the stapes $(s t p$.$) , which is fixed in the membrane of the fenestra ovalis (fen.ov.).$ Sonorous vibrations striking the tympanic membrane are com-

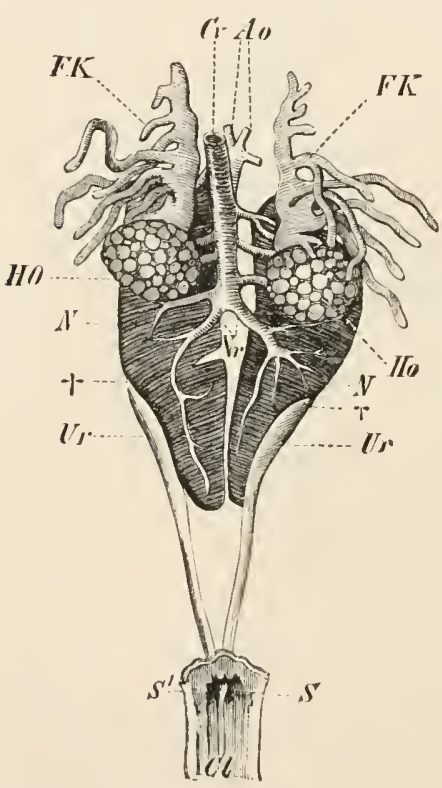

FIG. 948.-Rana esculenta. Urinogenital organs of the male. Ao. dorsal aorta ; $\mathrm{Cl}$. cloaca ; $\mathrm{C} v$. postcaval vein ; $F K$, fat-bodies ; $H O$, testes ; $N$, kidneys : $\mathrm{S}, \mathrm{s}^{\prime}$, apertures of ureters into cloaca ; Ur. ureters. (From Wiedersheim's Comparative Anatomy.) venous system. The ureters (Ur.) pass backwards from the outer border's of the kidneys and open into the dorsal wall of the cloaca $(C l$.). The kidney is developed from the mesonephros of the embryo, the ureter from the mesonephric duct. In the larva a large pronephros is present and is, for a time, the functional kidney.

Opening into the cloaca on its ventral side is an organ (Fig. 940, bl.) mentioned in the general account of the Craniata (p. 116), but here actually met with for the first time. It is a bilobed, thin-walled, and very delicate sac into which the urine 
passes by gravitation from the cloaca when the anus is closed. The sac is a urinary bladder, bnt, as it is quite different morphologically from the organ of the same name in Fishes, which is a dilatation of the ureter, it is distinguished as the allantoic bladder.

The testes $(H O)$ are white ovoid bodies lying immediately ventral to the anterior ends of the kidneys, to which they are attacher by folds of peritoneum. From the inner edge of each pass a number of delicate vas efferentia which enter the kidney and become connected with the urinary tubules. The spermatic fluid is thus passed into the urinary tubules and carried off by the ureter, which is therefore a urinogenital duct in the male Frog. A vesicula seminalis (Fig. 940, vs. sem.) opens by numerous small ducts into the outer side of the ureter. Attached to the testis are lobed bodies of a bright yellow colour, the fat-bodies $(F K)$.

The ovaries (Fig. 949, Ov.) are large folded sacs on the surface of which the black-and-white ova project. A fat-body is attached to each. The oviducts $(O d$.) are greatly convoluted tubes, the narrow anterior ends of which open into the cœlome by small apertures $(O t$. $)$ placed close to the bases of the lungs. Their posterior ends are wide and thin-walled ( $U t$.), and open into the cloaca

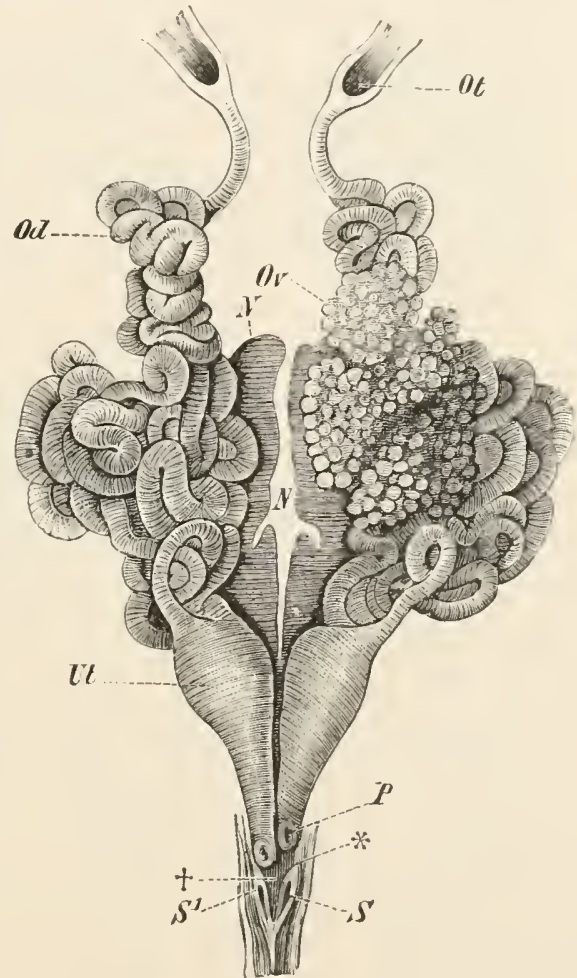

FIG.949.-Rana esculenta. Urinogenital organs of the female. $N$, tidneys; $O d$. oviduct; $O t$, its coelomIc aperture; $O v$. left ovary (the right is removed); $P$, cloacal aperture of oviduct; $S, S^{\prime}$, cloacal apertures of ureters; $U t$, uterine dilatation of oviduct. (From Wiedersheim's Comparative Anatomy.)

$(P)$. The ova break loose from the surface of the ovary and enter the cœelomic apertures of the oviducts, the walls of which are glandular and secrete an albuminous fluid having the property of swelling up in water. The eggs receive a coating of this substance as they pass down the oviducts and are finally stored up in the thin-walled posterior portions of those tubes, which, in the breeding season, become immensely dilated and serve as uteri.

Development.- The eggs are laid in water in large masses; 
each has a black and a white hemisphere, the former always directed upwards, and is surrounded by a sphere of jelly. The egg is telolecithal, the protoplasm being mainly accumulated on the pigmented hemisphere, while the white hemisphere is loaded with yolk. During oviposition the male sheds his spermatic fluid over the eggs, and the sperms make their way through the jelly and impregnate them. In a short time the jelly swells up and becomes thereafter impermeable to the sperms.

Segmentation begins by a vertical furrow dividing the oosperm into two cells (Fig. 950, $A$ ), and soon followed by a second vertical furrow at right angles to the first $(B)$, and then by an equatorial furrow placed nearer the black than the white pole $(C)$. Thus the eight-celled embryo consists of four smaller black cells and four larger white cells. Further divisions take place $(D)$, the black cells dividing rapidly into micromeres $(m i$.), the white, more slowly, into megameres ( $\mathrm{mg}$.$) : as in previous cases, the presence of yolk$ hinders the process of segmentation. The pigmented micromeres $(D-F, m i$.$) give rise to the ectoderm, which is many-layered: the$ megameres $(m g$.$) contribute to all three layers and are commonly$ called yolk-cells. During the process of segmentation a blastocoele $(E, b l . c c e l$.$) or segmentation-cavity appears in the upper hemisphere.$

The black now begins to encroach on the white hemisphere; cells, budded from the yolk-cclls, take on the character of ectoderm, acquire pigment, and gradually extend the black area until it covers the whole embryo except a small patch, known as the yolkplug $(G, H, y k . p l$.$) , at what will become the posterior end. This$ process is obviously one of epiboly : the margin of ectoderm cells surrounding the yolk-plug represents the blastopore.

The archenteron $(I$, ent.) arises by a split taking place among the yolk-cells, beginning at the edges of the blastopore and gradually extending forwards: the process is probably supplemented by a limited amount of invagination of the ectoderm. 'The archenteron is at first a very narrow cleft, but soon widens considerably : for a long time it does not actually communicate with the exterior, the blastopore being filled up with the yolk-plug. As the archenteron extends forwards the blastocole gradually disappears. The yolk-cells soon become differentiated into a layer of endoderm cells $(I$, end.) immediately surrounding the archenteron, and several layers of mesoderm cells (mes.). Ventrally, however, a large mass of yolk-cells $(K, y k$.) remains undifferentiated and serves as nutriment to the growing embryo.

The edges of the lower margin of the blastopore now begin to approach one another, and, uniting in the median plane, give rise to a vertical groove, the primitive groove. In the meantime medullary folds $(H, m d . f$.$) appear and mark the dorsal surface : they$ are at first widely separated, but gradually approach one another and close over the medullary groove (md. gr.), thus giving rise to 


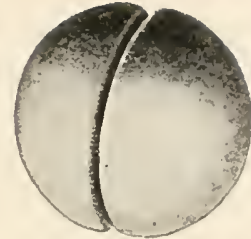

A

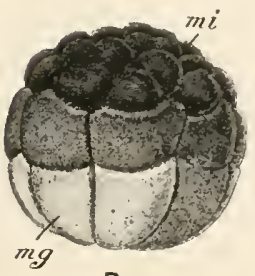

D

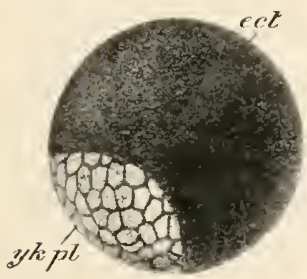

G

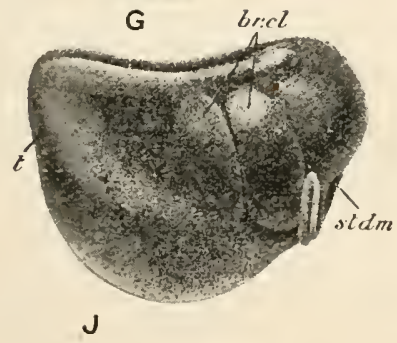

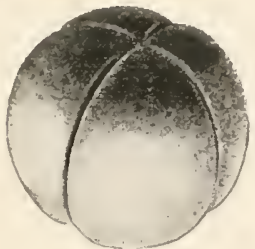

B

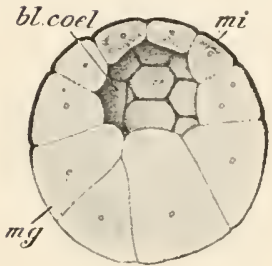

E

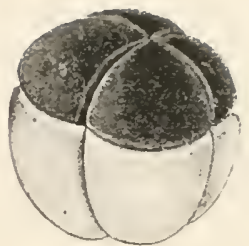

C

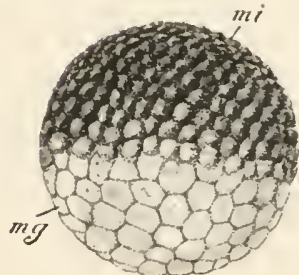

$\mathbf{F}$
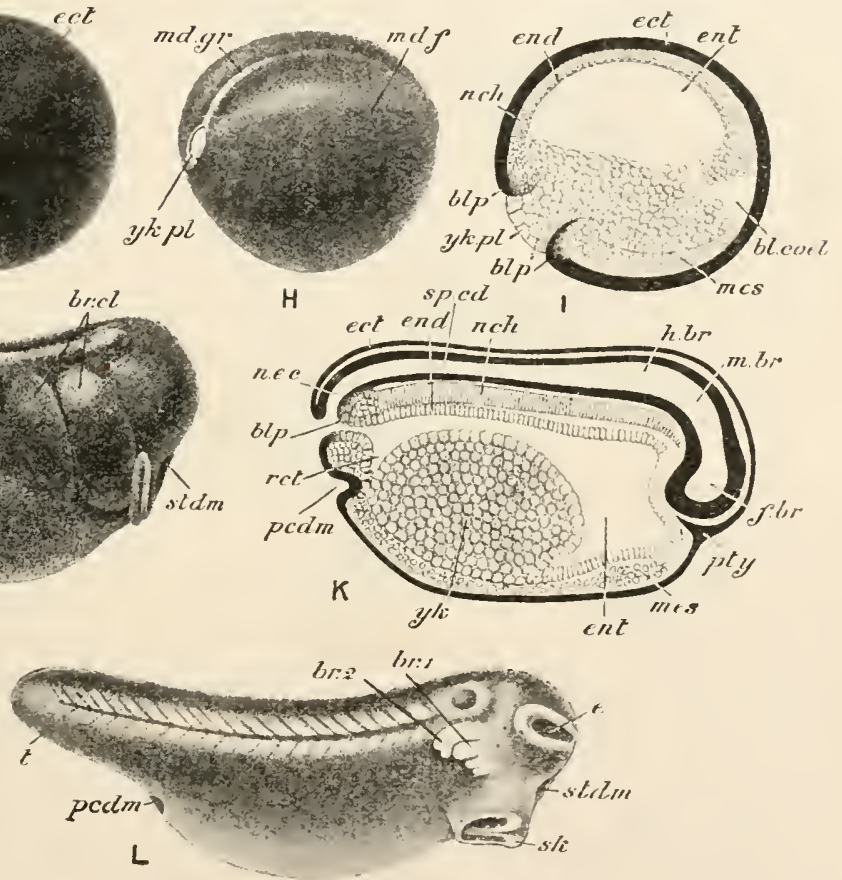

FIG. 950.-Development of the Frog. $A-F$. segmentation ; $G$, overgrowth of eetoderm ; $I, I$, establishment of germinal layers; $, J, K$, assumption of tadpole-form and establishment of nervous system, notochord, and enteric canal ; $L$, newly-hatched tarlpole. bl. col. blasto-

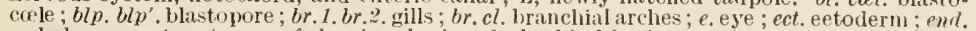
endoderm ; ent. enteron ; $f$. br. fore-brain ; h. br. hind-brain; $m . b r$. mid-brain ; md. $f$. medullary fold ; mil. gr. medullary groove ; mes. mesoderm ; mi. megameres; mi. micro. meres; $n c h$. notochord; $n$. e. $c$. neurenteric canal; pedm. proctorleum ; pty. pituitary invagination ; rct. commencement of rectum ; sk. sucker ; $s p$. crl. spinal cord ; st.dm. stomodæum ; $t$. tail ; $y k$. yolk-cells; $y k$. $p l$. yolk lug. $(A-D, H-H$, and $J$ from Ziegler's models ; $E, I, L$, and $L$ after Marshall.) 
the central nervous system. Posteriorly they are continuous with the lips of the blastopore, so that when the neural groove becomes closed in behind, the archenteron, as in Amphioxus, communicates with the neurocole by a neurenteric canal $(K, n . e . c$.$) .$

The embryo soon begins to elongate; one end is broad, and, becoming separated by a slight constriction, is marked out as the head: the other end is bluntly pointed and is the rudiment of the tail $(t$.$) . On the ventral surface of the root of the tail a procto-$ doum (pcdm.) appears and communicates with the archenteron.

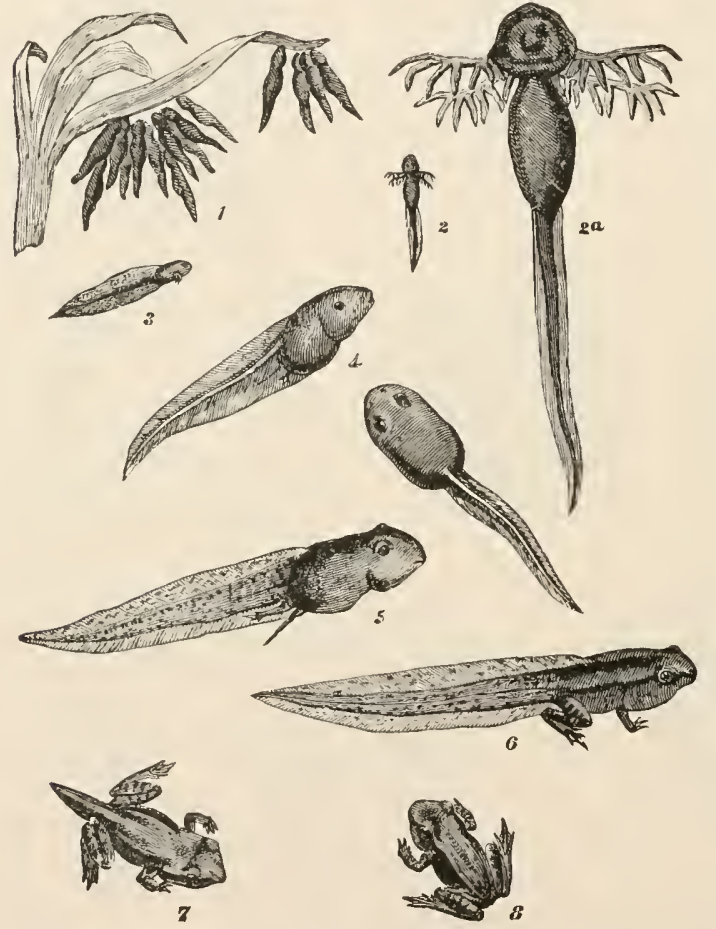

Fia. 951.-Rana temporaria. Stages in the life-history, from the newly-hatcher 'Tadpoles $(1)$ to the young Frog $(\delta)$. $2 a$ is a magnitied view of 2 . (From Mivart.)

The head and tail become more distinctly marked off from the trunk. A pit-the stomodoeum $(J-L$, st. $d m$.) - ap pears on the anteroventral surface of the head, and, immediately behind it, a semilunar area with raised edges, the sucker (sk.). At e a ch side of the head two branched processes appear: they are the $e x$ ternal gills (br.l., br...), and the regions from which they arise mark the positions of the first and second bran chial arches.

The embryos are now hatched as tadpoles. They swim freely in the water or adhere to weeds by means of their suckers (Fig. $951,1)$. They are still blind and mouthless, the stomodæum not having yet communicated with the archenteron. Soon a third pair of external gills appears on the third branchial arch, and the first two pairs increase greatly in size $(2,2 a)$ : the stomodæum joins the archenteron, gill-slits (branchial clefts) are formed between the branchial arches, and the eyes appear. The mouth is small, bounded by lips beset with horny papillæ and provided with a 
pair of horny jaws. The enteric canal grows to a great length and is coiled like a watch-spring, and the tadpole browses upon the water-weeds which form its staple food.

Soon the external gills show signs of shrivelling, and at the same time internal gills, like those of Fishes, are developed in the branchial clefts. A fold of skin, the operculum, appears on each side, in front of the gills, growing from the region of the hyoid arch, and extending backwards until the gill-slits and external gills are covered and there is only a single small external branchial aperture on each side, as in Holocephali $(3,4)$. On the right side the operculum soon unites with the body-wall so as to close the branchial aperture, but on the left side the opening remains for a considerable time as the sole means of exit of the water. At this time the tadpole is to all intents and purposes a Fish.

The lungs now appear, and the larva is for a time truly amphibious, rising periodically to the surface to breathe air: the single branchial aperture, however, soon closes, and henceforth respiration is purely aërial.

In the meantime the limbs are developed. The hind-limbs appear as little rounded buds, one on each side of the root of the tail (5). The fore-limbs arise beneath the operculum and are therefore hidden at first; soon, however, they emerge by forcing their way through the operculum. As the limbs increase in size the tail undergoes a progressive shrinking $(6-\$)$. The mouth widens by the backward rotation of the suspensorium, the intestine undergoes a relative diminution in length, and vegetable is exchanged for animal diet. The little, tailed Frog can now leave the water and hop about upon land; its tail is soon completely absorbed, and the metamorphosis is complete.

\section{Distinctive Characters and Classification.}

The Amphibia are Craniata which, in nearly all cases, possess gills either in the larval state only or throughout life, and which usually breathe by lungs in the adult condition. The skin is glandular, and there may or may not be a bony dermal exoskeleton. When unpaired fins are present, they are never supported by fin-rays. The paired appendages, when present, are pentadactyle limbs: the digits are usually devoid of claws. The skull is autostylic and is articulated with the first vertebra by paired occipital condyles borne on the exoccipitals. The basi-occipital and supraoccipital are usually, and the basisphenoid is always, absent: there is a large parasphenoid, and there are well-developed paraquadrates (squamosals). In the branchiate forms large hyoid and branchial arches persist throughout life: in the non-branchiate species these structures undergo more or less degeneration and give rise to the hyoid-cartilage. The heart has a sinus venosus, 
right and left auricles, a single ventricle, and a conus arteriosus; the aortic arches arise from a bulbus aortæ or abbreviated ventral aorta. The cardinal veins undergo more or less degeneration and are practically replaced by an unpaired postcaval vein. There is a renal portal system, part of the returning blood from the posterior parts of the body going through it, the rest through the hepatic portal system by an abdominal vein which represents fused lateral veins. The red corpuscles are oval and nucleated and are often of unusual size. The lymphatic svstem is well developed. In the brain the small size of the cerebellum is noticeable. The olfactory sacs open into the mouth by posterior nares. The outer wall of the auditory capsule is pierced by a fenestra ovalis into which is inserter a cartilaginous stapes: the stapes may be connected by a columella with a tympanic membrane. The efferent ducts of the testis open into the mimary tubules, and the mesonepliric duct of the male is a urinogenital duct. In the female the mesonephric ducts become the ureters, and the oviducts are pronephric ducts with colomic apertures. The pronephros is the functional kidney in the larva, the mesonephros in the adult. There is an allantoic bladder. Development is usually accompanied by a metamorphosis, the young being hatched in the form of a branchiate larva.

The Amphibia are classified as follows:-

\section{Order 1.-Urodela.}

Amphibia which retain the tail throughout life. There are usually two pairs of limbs of approximately equal size.

The order is conveniently divided into -

a. Peremibranchiata, which retain the gills throughout life: including the American Necturus, the blind Proteus of the underground caves of Carniola in Dalmatia, and the Eel-like Siren of North America.

b. Derotremata, in which the gills are lost in the adult, but there is usually a persistent gill-cleft : including the Newt-like Cryptobranchus and the Eel-like Amphiuma from North America, and the Giant Salamander, Megalobatrachus, of China and Japan.

c. Myctodera, the Salamanders and Newts, in which the gills are lost and the gill-clefts closed in the adult: including the common Newts or Efts (Molge), the Spotted and Black Salamanders (Salamandra) of the European Continent, and the American Amblystoma, the sexually mature larva of which is the well-known Axolotl.

\section{Order 2.-Anura.}

Amphibia having no tail in the adult condition. The trunk is short and broad, and the hind-limbs greatly exceed the fore-limbs in size. Gills and gill-slits are never present in the adult.

Including the Frogs and Toads. 
Order 3.-Gyinophiona (A poda).

Snake-like Amphibia having neither limbs nor tail. A dermal exoskeleton is present. There are no gills or gill-slits in the adult.

Inchuding the Cacilians (Cacilia, Epicrium, \&c.).

\section{Order 4.-Stegocephala.}

Extinct tailed Amphibia, often of great size, having usually two pairs of limbs and a well-developed dermal exoskeleton. The group ranges from the Permian to the Trias.

\section{Systematic Position of the Example.}

The genus Rana belongs to the family Ranida, which with three other families constitutes the series Firmisternia, of the sub-order Phaneroglossa and order Anura.

The absence of a tail and the presence of two pairs of limbs, of which the posterior are larger than the anterior, place the genus among the Anura. The presence of a tongue and of distinct paired Eustachian tubes separates the Phaneroglossa from the Aglossa (Pipe and Xenopes), a small group of Toads in which the tongue is absent and the Eustachian tubes have a common median opening. The Firmisternia are distinguished by having the coracoids joined by a common epicoracoid cartilage in contradistinction to the Arcifera (Tree-frogs, Toads, \&c.), in which the epicoracoids overlap one another. The Ranidæ are distinguished from the other families of Firmisternia by having teeth in the upper jaw and the transverse processes of the sacral vertebræ not dilated. $R$. temporaria is distinguished from $R$. esculenta by its smaller size and brown colour, by the large black patch in the tympanic region. and by the absence of external vocal sacs in the male.

\section{General Organisation.}

The Amphibia are specially interesting as illustrating the transition from the water-breathing to the air-breathing type of Craniate structure. The lower forms retain their gills throughout life, but possess lungs in addition : in the higher the gills occur only in the larval state, and the adult breathes exclusively by the lungs and skin, becoming transformed from an aquatic into a terrestrial animal. At the same time further adaptations to landlife take place, the most important being the modification of the blood-vessels consequent on the disappearance of the gills, the loss of median fins, and the strengthening of the limbs to support the weight of the body.

External Characters.-An excellent example of the lower Urodela with persistent gills is afforded by the great North American Water-newt, Necturus maculatus (Fig. 952). The animal attains a length of $30 \mathrm{~cm}$. (more than a foot); the elongated trunk is 
separated by a slight constriction from the depressed head, and passes insensibly into the compressed tail, which is bordered by a continuous median fin unsupported by fin-rays. The limbs are
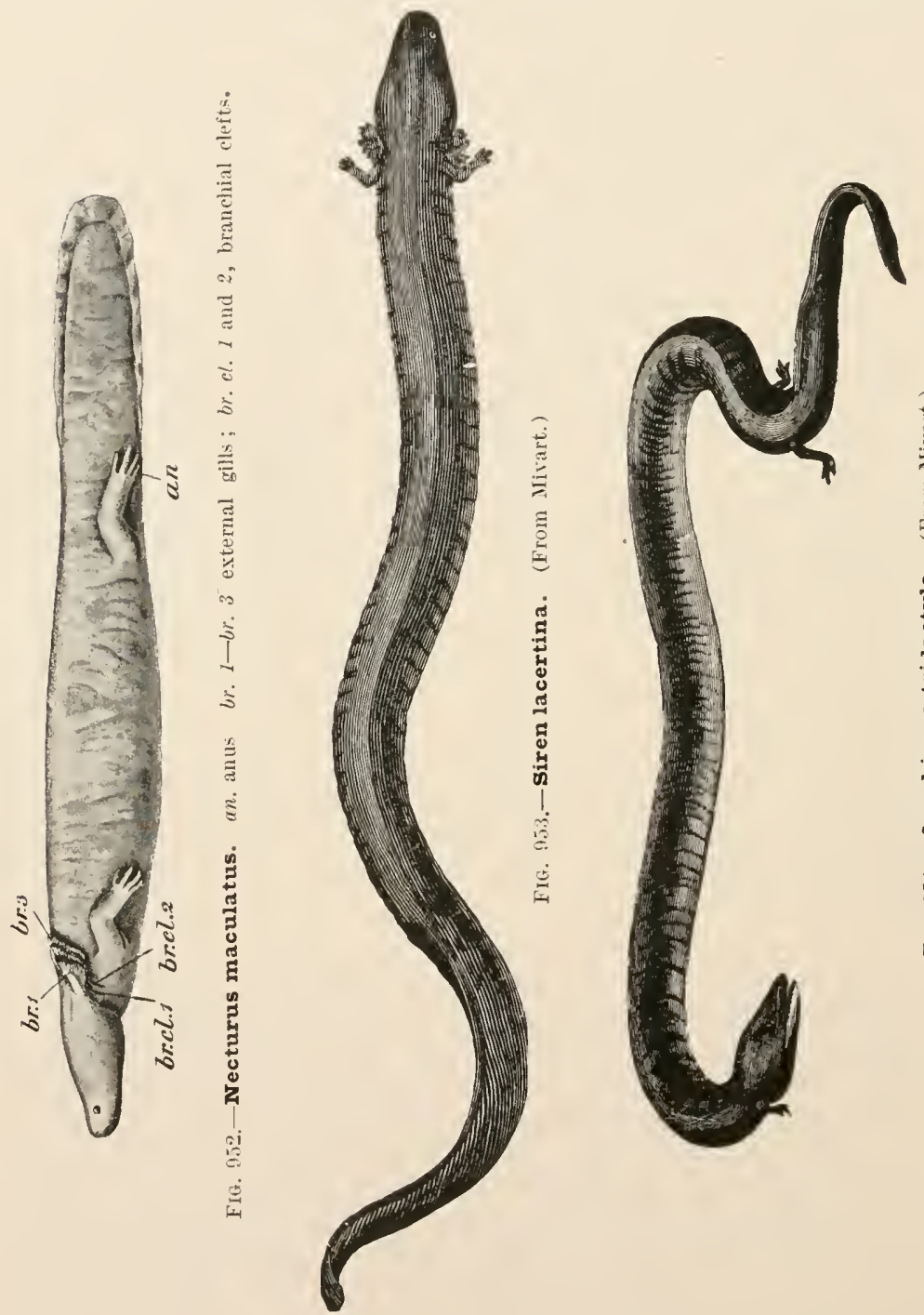

ב



small and weak in proportion to the size of the body, and in the ordinary swimming attitude are directed backwards, more or less parallel to the sagittal plane, the upper arm and thigh taking a direction backwards and slightly upwards, the fore-arm and 
hand and the shank and foot extending backwards and downwards. Fach limb thus presents an external or dorsal and an internal or ventral surface, an anterior or pre-axial border which terminates in the first digit, and a posterior or post-axial border which terminates in the last digit. The eyes are small and have no eyelids, there is no tympanic membrane, and the mouth is wide and bordered by thick lips. On each side of the neck are two gill-slits (br. cl. 1, br. cl. 2) leading into the pharynx, the first between the first and second branchial arches, the other between the second and third. From the dorsal end of each of the three branchial

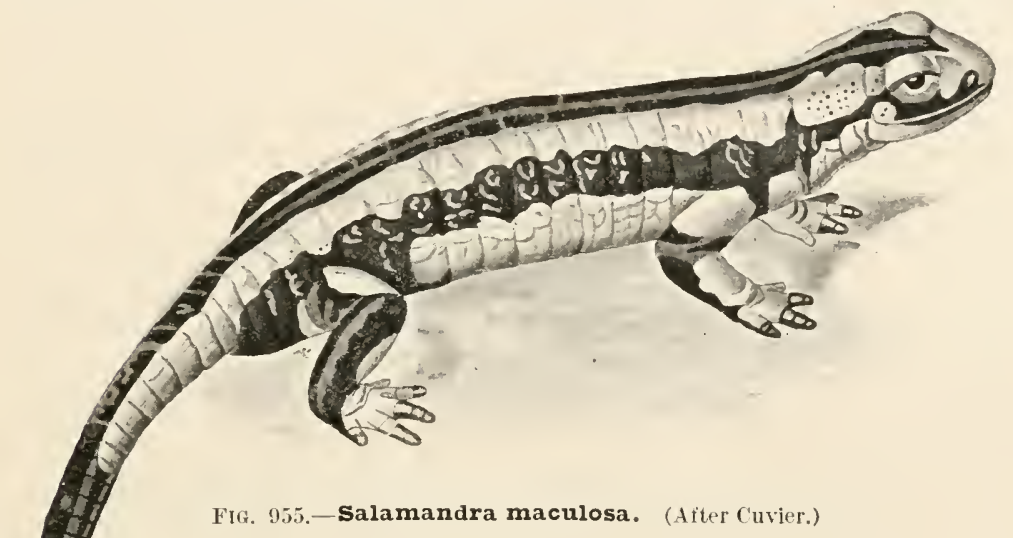

arches springs a branched external gill (br. 1-br. 3). Very similar in its external characters is the blind, cavedwelling Proteus; and Siren (Fig. 953) differs mainly in its elongated eel-like body and in the absence of hindlimbs. All three genera are perennibranchiate or persistentgilled.

The remaining Urodela are often called caducibranchiate or deciduous-gilled, and furnish a complete series of transitions from derotrematous forms which, while losing the gills, retain the gill-clefts, to salamandrine forms in which all trace of branchiate organisation disappears in the adult. In Amphiuma (Fig. 954) the body is eel-like and the limbs are extremely small : there are no gills in the adult, but two pairs of gillopenings are retained throughout life. In Cryptobranchus there is a single branchial aperture, sometimes present on the left side only; but, as in the previously mentioned genera, four branchial arches are retained. In Megalobatrachus, the Giant Salamander of Japan and China, all trace of gill-slits disappears, but two branchial arches persist. Lastly, in the Salamanders, such as the spotted Salamander (Salamandra maculosa, Fig. 955) of Europe, and the common British Newts (Molge), the adult has no trace either of gills or gill-slits, 
and the branchial arches are nuch reduced. The limbs, also, in the terrestrial Salamanders stand out from the trunk, and have the soles of the feet and hands applied to the ground with the toes directed forwards, so as to support the weight of the body. Moreover, all trace of the median fin disappears, the tail becoming nearly cylindrical.

In the Anura the body is always Frog-like, the head being large and depressed, with a very wide mouth and large tympanic membranes, the trunk short, the tail absent, and the hind-much larger than the fore-limbs. In the Toads, such as the common British Bufo vulgaris, and most Tree-frogs, the webs between the hind-toes are reduced or absent, and in many species of Hyla the toes end in rounded sucking-dises.

In the Gymnophiona (Fig. 956) the body is greatly elongated and snake-like, the head is small and not depressed, and the limbs are

A

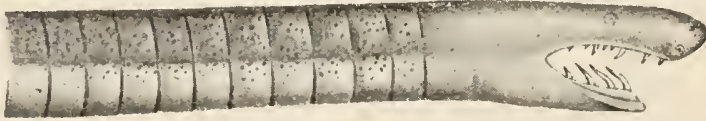

B

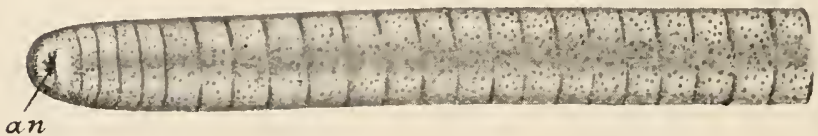

FI, 956.-Cocilia pachynema. $A$, anterior extremity from the right side; $B$, posterioI extremity from heneath. an. anus. (After Boulenger.)

absent. There is no tail, the anus (an.) being at the posterior end of the body on the ventral surface. The Stegocephala, or Labyrinthodonts as they are frequently called, were mostly salamander-like, having long tails and well-developed limbs: some, however, were snake-like and limbless, and probably retained their external gills throughout life. They varied in length from 10 centimetres to several metres.

The skin of Amphibia is soft and usually slimy owing to the secretion of the cutaneous glands, which is sometimes poisonous. In some forms, such as Bufo and Salamandra, there are large swellings on the sides of the head, formed of aggregated glands and called parotoids. In the larvæ of both Urodela and Anura, and in the adult aquatic Urodeles, lateral sense-organs are present, and impressions on the cranial bones show these organs to have been well developed in the Stegocephala. The colour of the skin is often very brilliant: the Spotted Salamander is yellow and black, and many Frogs are green and gold, scarlet and black, and so on. The green colour of Tree-frogs is protective, serving to conceal them 
among the foliage of the plants on which they live. The brilliant and strongly contrasted hues of the spotted Salamander and of some frogs are instances of "warning colour's"; the animals are inedible owing to the acrid secretion of their cutaneous glands, and their conspicuous colours serve to warn off the Birds and other animals which would otherwise devour them. A red and blue Nicaraguan Frog is said to show no sign of fear of the Frog-eating Birds, while the edible and more plainly coloured species are in constant danger. In many Tree-frogs the brightness of the coloration varies with changes in the intensity of the light and in the surroundings. In many Toads the skin is dry and covered with warts.

An exoskeleton is present in many Gymnophiona in the form of small dermal scales, and in some Anura in the form of bony plates beneath the skin of the back. In the Stegocephala a very conplete armour of bony scutes was present, sometimes covering the whole body, sometimes confined to the ventral surface. In a Urodele, Onychodactylus, and in the South African Toad, Xenopus, small pointed horny claws are present on the digits. With these exceptions the skin is devoid of hard parts.

Endoskeleton.-The vertebral column is usually divisible into a cervical region, containing a single vertebra devoid of transverse processes; an abdominal or thoraco-lumbar region, containing a variable number of vertebræ with transverse processes and often with ribs; a sacral region, containing usually a single vertebra, the large transverse processes - or the ribs - of which give attachment to the ilia; and a caudal region, forming the skeleton of the tail. In the Gymmophiona the caudal region is very short, and there is no sacrum: in the Anura the candal region is represented by a single rod-shaped bone, the urostyle. The total number of vertebræ may reach 250 in Urodela and Gymnophiona: in Anura there are only nine vertebræ and a urostyle.

In the lower Urodela (Fig. 957, $A$ and $B$ ) the centra are biconcave, as in Fishes: they consist of dice-box-shaped shells of bone, lined at either end by cartilage $(J v k)$, which is continuous between adjacent vertebræ. The bony shell is developed before the cartilage appears, so that the vertebre are, in strictness, investing bones. The neural arches, on the other hand, are far more perfectly developed than in any Fish, and have well-formed zygapophyses, which articulate with one another by synovial joints.

The Gymnophiona also have biconcave vertebræ, but in the higher Urodela (Fig. 957, $C$ and $D$ ) and the Anura absorption of cartilage takes place between adjacent centra in such a way that the convex end of one fits into the concave end of the next, forming a cup-andball joint. In the higher Urodela the convexity is on the anterior, the concavity on the posterior face of each centrum $(D)$, and the vertebræ are said to be ophisthocalous: in the Anura they are usually, as in the Frog, procolous. In the Stegocephala there is 
great diversity in the structure of the vertebral column. There may be well-developed dice-box-shaped centra, or the neural arches may be simply perched upon a persistent notochord surrounded by incomplete hoops of bone, twice as numerous as the arches, and alternately dorsal and ventral in position: the former represent centra, the latter intercentra, or ossifications alternating with the centra on the ventral region of the notochord.
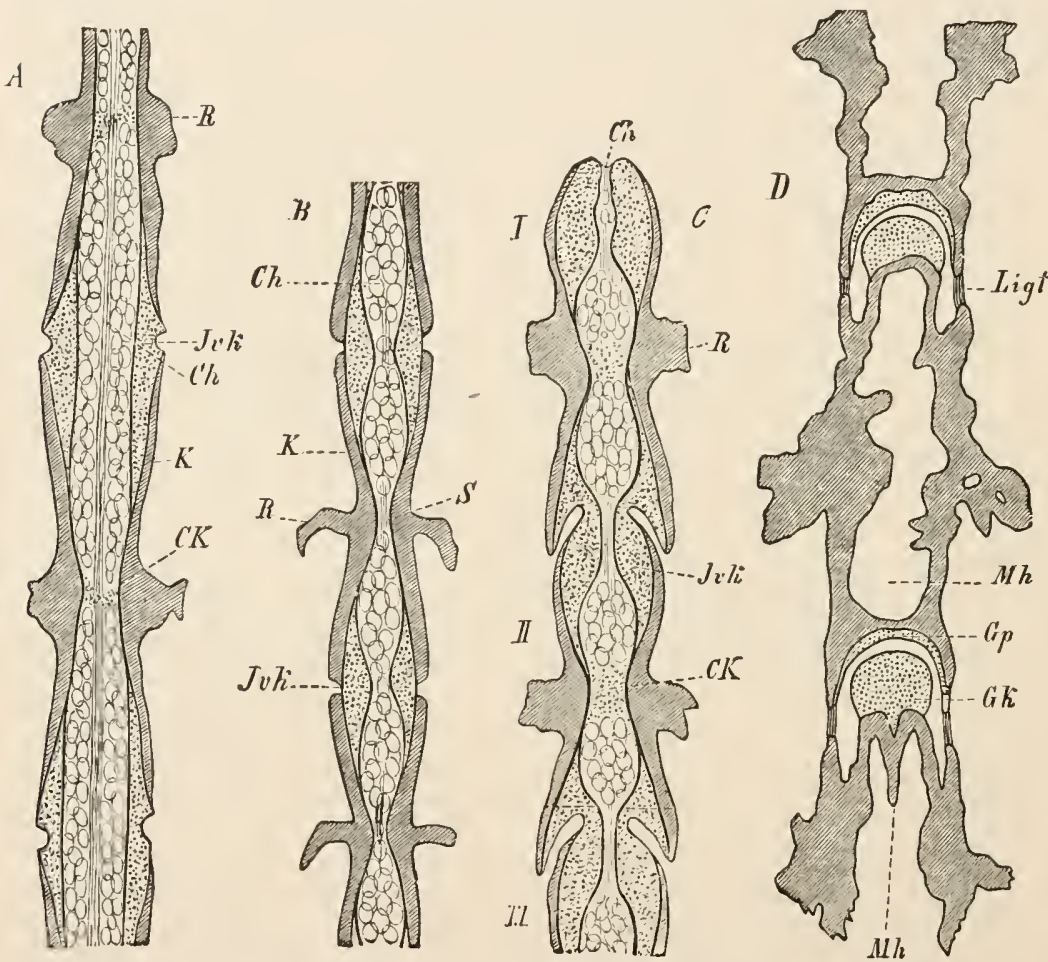

FIG. 957.-Longitudinal sections of vertebral centra of A, Ranidens ; $B$. Amblystoma ; $C$, spelerpes ; and $D$, Salamandrina. $C h$. notochord: $C K$, intra-vertebral cartilage and fat-cells ; $G k$, convex anterior face of centrum ; $G p$. concave posterior face ; $J i k$; intervertebral cartilage ; $\boldsymbol{K}$, superficial bone of centrum ; Ligt. inter-vertebral ligament; $\boldsymbol{M} h$, marrow-cavity ; $R$, transverse process ; $S$, intra-vertebral constriction. (From Wieders. heim's Comparative Anatomy.)

The first or cervical vertebra bears paired articular surfaces for the condyles of the skull, and between them the anterior face of the centrum gives off, in Urodela, a projection called the odontoid process. The Urodela, moreover, have ribs articulating with the transverse processes of the abdominal and sacral vertebræ: they are short bones, forked proximally, and the compressed transverse processes are correspondingly divided. The sacral ribs of Urodeles give attachment to the ilia, and the caudal vertebræ bear hæmal arches. 
The skull of Urodela differs from that of the Frog in many important respects, the most striking of which is the fact that the trabeculæ do not meet either below the brain to form a basis cranii or above it to form a cranial roof. Thus, when the investing bones are removed, the cranium (Fig. 958) is completed above and below in the parachordal or occipital region only: anterior to this it has side walls, but no roof or floor, there being above a huge superior cranial fontanelle, and below an equally large basi-cranial fontanelle, the former covered, in the entire skull, by the parietals and frontals, the latter by the parasphenoid. In the perennibranchiate forms Necturus and Proteus the trabeculæ remain, even in the adult, as narrow cartilaginous bars, and the chon drocranium is actually of a lower or more embryonic type than that of any other Craniata. with the possible exception of Cyclostomata.

In the Urodela, moreover, the parietals (Fig. 959, $P$ ) and frontals $(F)$ are separate, the parasphenoid $(P s)$ is not $\mathrm{T}$ shaped, the palatine and vomer are sometimes represented by a single bone, the vomeropalatine $(V o p)$, bearing teeth. The suspensorium is inclined forwards, as in the tadpole, not backwards, as in the adult Frog. The lyoid arch is large, and its dorsal end may be separated as a hyomandibular. There are three or four branchial arches which are large in the perenni-

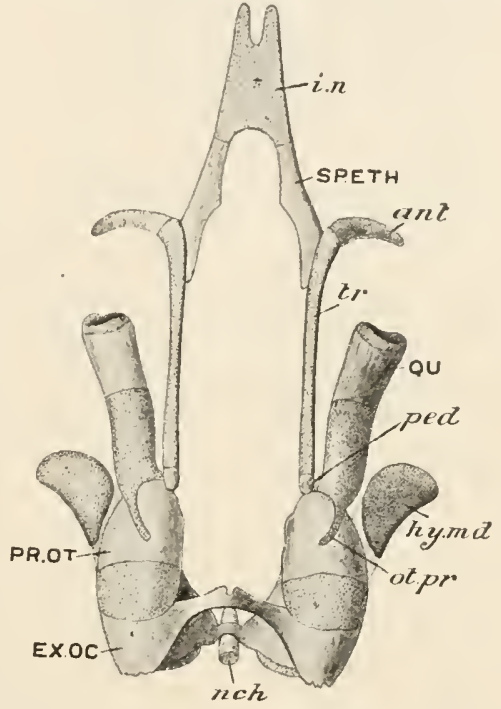

FI\%. 958.- Proteus anguinus. The chondrocranium from above. ant. antorbital process; EX.OC. exoccipital and epiotic; hy.md. hyomandibular ; $i . n$. inter-nasal plate ; $n c h$. notochord; ot.pr. otic process ; ped. pedicle; PR.OT.pro-otic; QU.quadrate; SP.ETH. sphenethmoid. (Aiter IV. K. Parker.) branchiate forms, but undergo more or less reduction in caducibranch species, never, however, forming such a simple structure as that seen in the Frog. The stapes has no columella attached to it, and, in correspondence with this, there is no tympanic cavity or membrane.

In the Anura there is a very wide range of variation in the skull. Among the most important points are the presence, in a few species, of small supra- and basi-occipitals, and the fact that in others the roofing investing bones are curiously sculptured and so strongly developed as to give the skull a singularly robust appearance.

In the Gymnophiona (Fig. 960) very little of the original cartilage remains in the adult state, but the investing bones are 

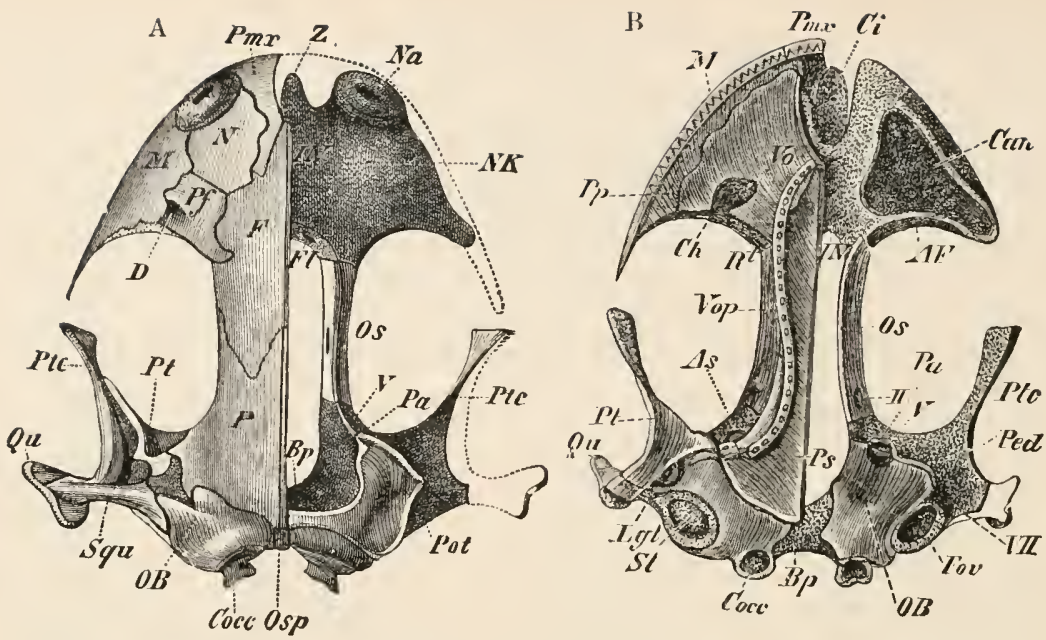

F 19. 959.--Salamandra atra. The skull. $A$, from above ; $B$, from below. In both the investing bones are removed on the riglit side of the figure. Af, antorbital process; $A s$, alisphenoid region ; $B p$, basal plate ; Can. nasal cavity ; $C h$, posterior natres ; $C i$, process of internasal plate; Cocc occinital colidyles; $F$. frontal; $F l$, olfactory foramen: $F o v$. fenestra ovalis ; $I N$. internasal plate ; $L g !$. ligament connecting stapes with suspencorium ; $M$. maxilla ; $N$. nasal : Na.nasal aperture : $N K$. olfactory capsule ; $O B$, auditory capsule ; os, sphenethmoid (orbitosphenoid); $O s p$, supra-occipital region ; $P$, parietal ; $P a$, ascending process of suspensorium; ped. pedicle: Pf. prefrontal; I'mx. premaxilla; Pot. otic process of suspensorium;' Pp. palatine process of maxilla ; Ps. parasphenoid ; $I t$. pterygoid bones; $P t c$, pterygoid cartilage; $R t$, foramen for ophthalmic branch of trigeminal : Qu. quadrate; Squ. paraquadrate (squamosal); St. stapes; V'o. vomer ; Vop. vomernpalatine; $Z$, process of internasal plate $I I$, optic foramen ; $T$, trigeminal foramen; III facia! foramen. (From Wiedersheim's Comparative Anatomy.)
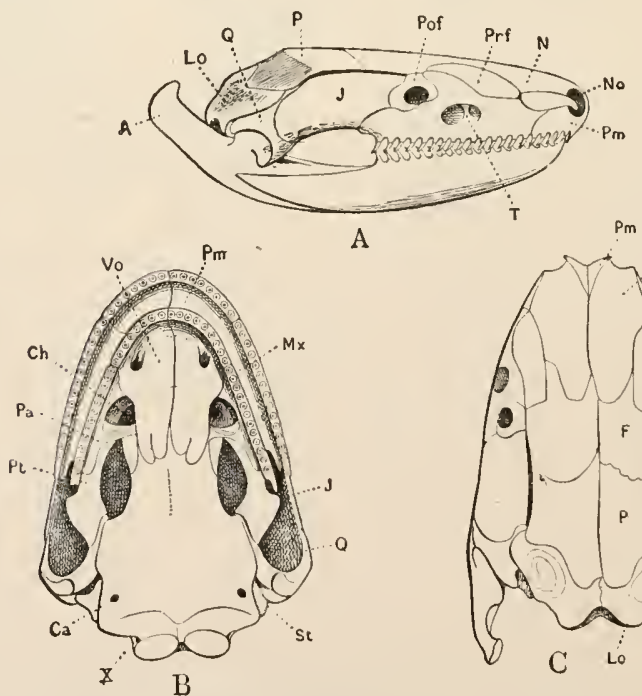

A

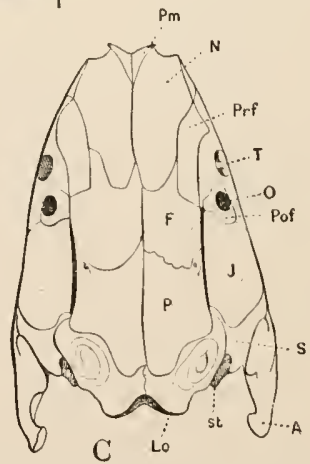

FIG, 960.--Skull of Ichthyophis glutinosa, $\times 3$. A, Lateral ; $B$, Ventral ; $C$, Dorsa jview $A$, posterior process of the os articulare ; C'a. carotid foramen; Ch. Choana or posterior nasal opening; $F$. frontal : $J$. jugal ; $L O$. exoccipital ; $M x$. maxilla ; $N$. nasal ; $N o$. nostril $O$ orbit; $P$. parietal ; $P a$. palatine ; Pm. premaxilla ; Pof. nostfrontal : Prf. preirontal $I t$. pterygoid ; $Q$. quadrate ; S. parayuadrate (syuamosal) ; $S t$. sta pes ; T. tentacular gloove; Vo. vomer; $\boldsymbol{X}$. exit of vagus nerve. (After Sarasin.) 
very large and form an extrenely complete and substantial structure, especially remarkable for the way in which the small orbit $(O)$ is completely surrounded by bones. In the Stegocephala (Fig. 961) the skull is broad and flattened, the supra-occipital (s. occ.) double, and the parietals $(P)$ and flontals $(F)$ are separate. Between the parietals is an apertule, the parictal foramen $(F p)$, which probably lodged a pineal eye. The eyes were sometimes sitrrounded by a ring of bonv sclerolic plates $(O c)$. Gill-arches have been found in many species.

The shoulder-girdle of Urodela (Fig. 962) is chiefly remarkable for the great size of the mossified coracoids ( $A, C o ., B, C$.) which overlay one another on the ventral body-wall. The procoracoid (cl) is also large, and there is no clavicle. The sternum (St) is usually a more or less rhomboid plate of cartilage between the posterior ends of the coracoids, and there is no omostermum. In Nec-

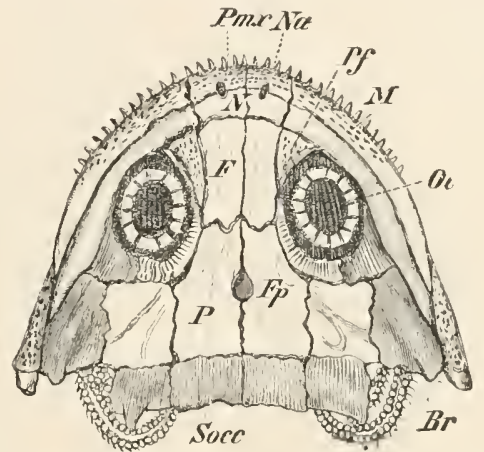

FIG. 961.-Skull of Frotriton, one of the smaller stegocephata, magnitied. Lr. branchial arches ; $F$. frontal ; $F p$, parietal foramen; $\boldsymbol{M}$. maxilla : $\boldsymbol{N}$. nasal ; $\boldsymbol{V} a$. nostril; Oc. sclerotic plates; $P$. parietal; $P f$. preirontal: I'mex. premaxilla; soce. supra-occilutal. (From Wielersheim, after Fritsch.) turus, however, the sternum presents a very interesting structure: it is a narrow, irregular, median bar, sending off branches riglit and left into the mvocommas, a condition of things which suggests its origin by the fusion of abdominal vibs, or supporting structures developed between the ventral portions of the myomeres, just as the true ribs are formed between their dorsal portions. In the Anura the epicoracoids either simply meet one another in the niddle ventral line, as in Rana, or overlap, as in the Fire-toad (Bombinutor) and the Tree-frogs $(I y / a)$. The overlapping of the coracoids, in Anura as in Urodela, is sometimes correlated with the absence of an omosternum. In the Stegocephala there is a median ventral investing bone, the inter-clavicle, which is connected on each side with the clavicle, and extends backwards ventral to the stermum. There is also, on each side, a bone called the cleithrum, connected with the corresponding clavicle: there is some reason for thinking this to be homologous with the bone usmally called clavicle in Teleostomi.

In the pelvic girlle of the Urodela the combined pubic and ischiatic regions (Fig. 963, $P, I s$ ) of the right and left sides are united to form an elongated cartilaginous plate which gives off on each side, above the acetabulum $(G)$, a slender vertical rod, the ilium $(I l)$. Ossifications are formed in the iliac and ischiatic regions, but the pubic region remains cartilaginons. The resem- 
blance of the pelvis of the lower Urodela, and especially of Necturus, to that of Polypterus (p. 225) and of the Dipnoi (p. 243) is noteworthy. In Anura the pelvic girdle resembles that of the Frog.

Attached to the anterior border of the pubic region there occurs

A

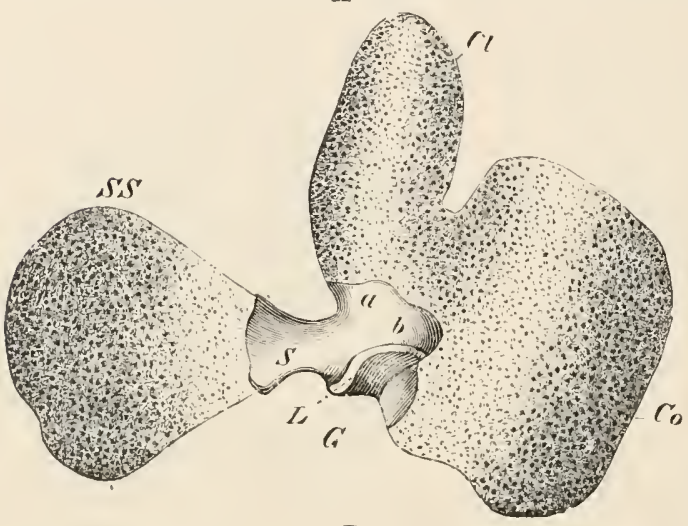

B

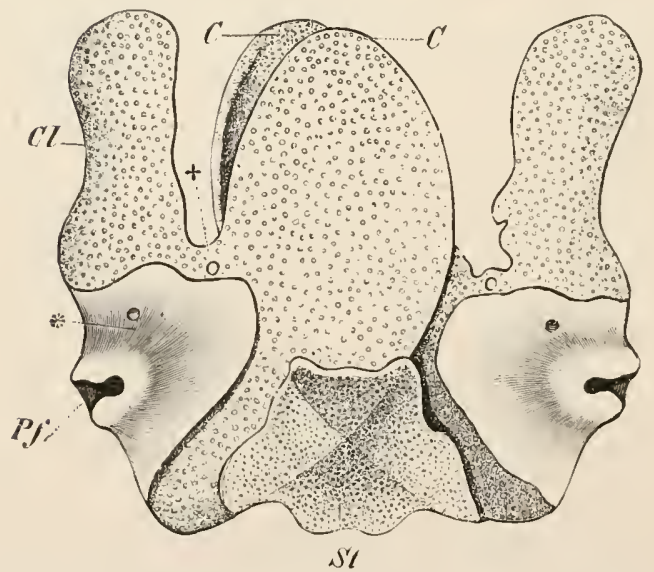

FIG. 962- - $A$, right side of shoulder-girdle of Salamandra $\cdot B$. shoulder-girlle and sternum of Amblystoma (Axolotl) from the ventral aspect. $a, b$, processes of scapula; $C$. (in $B$ ), coracoid : $C l$. procoracoid; $C o$. (in $A$ ), coracoid'; $G$. (in $A$ ),

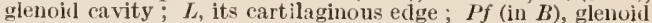
cavity; $S$. scapula ; SS. supra-scapula; st. sternum; * $†$. nerve foramina. (From Wiedersheim's Comparatice Anatomy.) in many Urodela and in Xenopus a rod of cartilage, forked in front, the epipubis $(E p)$. It is developed independently of the pelvis, and its relations to that structure are very similar to those of the sternum to the shoulder-girdle; it has, in fact, been proposed to call it a pelvi-sternum.

The limbs of Urodela differ from the typical structure already described only in details : there are usually four digits in the fore-limb and five in the h ind-limb. In Anura the limbs are modified by the fusion of the radius and ulna and of the tibia and fibula, and by the great elongation of the two proximal tarsals. A pre-hallux is frequently present.

Myology.- I n

the lower Urodela the muscles of the trunk and tail occur in the form of typical myomeres like those of Fishes. In the higher forms the myomeres become converted into longitudinal dorsal bands-the extensors of the back, paired ventral bands-the recti abdominis, and a double layer of oblique muscles, covering the flanks. 
Digestive Organs.-The teeth are always small and ankylosed to the bones: they may be singly or doubly pointed. They occur most commonly on the premaxillæ, maxillæ, and vomers, but may also be developed on the dentaries, palatines, and, in one instance, on the parasphenoid. In many Anura, such as the Common Toad, teeth are altogether absent. In some of the Stegocephala, such as Mastodonsaurus, the teeth are extraordinarily complex in structure, the tissues being folded in such a way as to produce in section a complex tree-like pattern. It is from this circumstance that the term Labyrinthodont, often applied to the Stegocephala, is derived.

The enteric canal is divisible

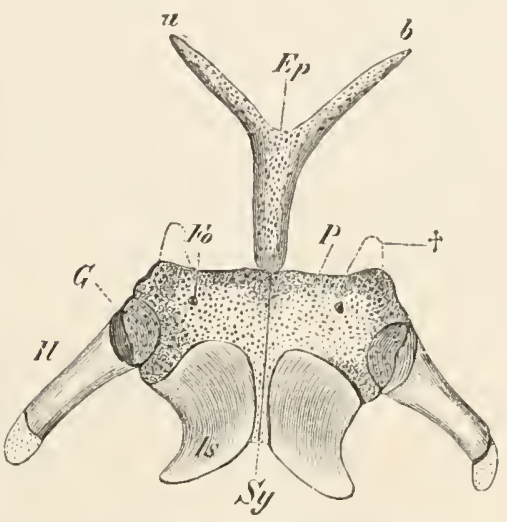

FIG. 963,--Pelvic girdle of Salamandra. $a, b$, processes of epipulis; Ep. epipubis: Fo. obturator foramen; $G$. acetabulum; $i l$. ilium : Is. ischium; $P$.' pubis; Sy. pubo-ischiatic symphysis; $t$, processes of pubis present in some Ũrodeles. (From Wiedersheim.) into buccal cavity, pharynx, gullet, stomach, small intestine, rectum and cloaca. The stomach and duodenum together form a U-shaped loop in which the pancreas lies. The tongue in many Urodeles is fixed and immovable, like that of a Fish: in most Anura it is free behind, as in the Frog; but in Xenopus and Pipa (hence called Aglossa) it is absent.

Respiratory Organs.--With very few exceptions Amphibia possess external gills in the larval state, and, in the perennibranchiate Urodela, these organs are retained throughout life. They are branched structures, abundantly supplied with blood, and springing from the dorsal ends of the first three branchial arches. The epithelium covering them is ectodermal, so that they are cutaneous and not pharyngeal gills, and are of a totally different nature from the so-called external gills of the embryos of Elasmobranchii and Holocephali, which are only the filaments of the internal gills prolonged through the branchial apertures.

Internal gills are developed only in the larvæ of Anura. They appear as papillæ on the outer borders of the branchial arches below the external gills. They closely resemble the internal gills of Fishes and appear to be homologous with them, although it seems probable that their epithelium is ectodermal.

In most adult Amphibia lungs are formed as outgrowths of the ventral wall of the œsophagus. The right and left lungs communicate with a common laryngo-tracheal chamber, supported by the cartilages of the larynx and opening into the mouth by a longitudinal slit, the glottis. In the more elongated forms, such 
as Siren, Amphiuma, and the Gymnophiona, the laryngo-tracheal chamber is prolonged into a distinct trachea or wind-pipe, supported by cartilages. In many species of Salamanders the lungs are absent, and respiration is exclusively cutaneous and pharyngeal.

Circulatory Organs.-The heart always consists of a sinus venosus, right and left auricles, ventricle, and conus arteriosus. The sinus venosus opens into the right auricle, the pulmonary veins enter the left, and the two are separated by a septum anricularum which forms a complete partition in Anura, but in Urodela and Gymnophiona is more or less fenestrated, i.e. formed of a network of muscular strands with intervening spaces. The
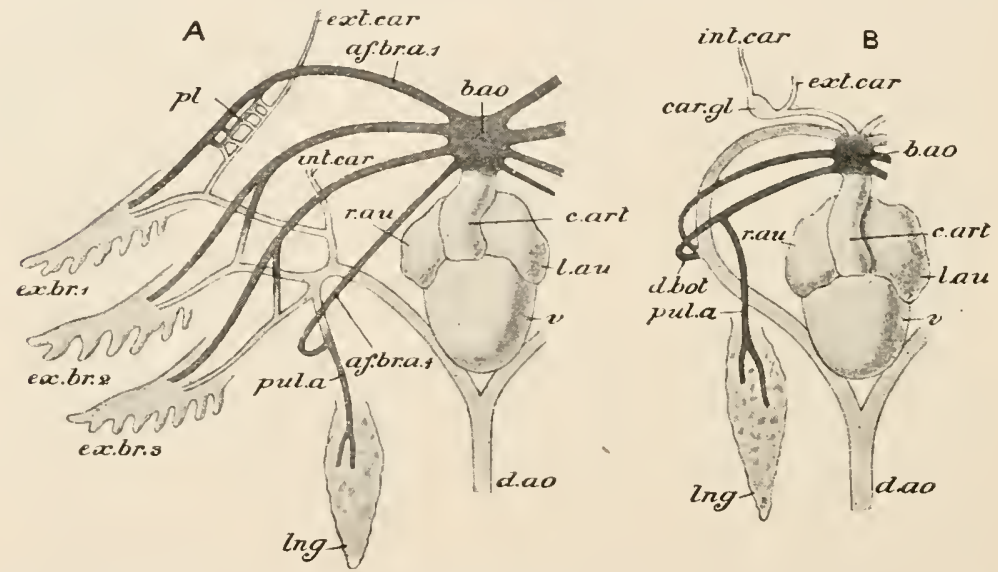

FIG. 964.- Heart and chief arteries of Salamandra. A. larva; $B$, adult. af. br.a. 1-4, afferent branchial arteries; bao bulbus aorte: car.gl carotid labyrinth ; $c$ art. couns arteriosus ; $d$. ao. dorsal aorta ; $d$. bot. ductus lotalii ; ex. br. 1 -3, external gills ; ext. car. external carotid ; int. car. internal carotid ; $l$. au. left auricle; lng. lung ; $p l$. plexus, giving rise to carotid labyrinth; $v u l$. $a$. pulmonary artery; $r$, $a u$, right auricle; v, ventricle. (Altered from Boas.)

conus arteriosus has no longitudinal valve in the lower Urodela and the Gymnophiona, but is separated both from the ventricle and from the bulbus aortæ by transverse rows of valves.

In the perennibranchiate Urodela and in the larvie of the airbreathing forms the circulation is essentially like that of a Fish. The bulbus aortæ (Fig. $964, A, b$. ao.), which represents an abbreviated ventral aorta, gives off four afferent branchial arteries (af. br. a. 1-4), three to the external gills, and a fourth which curves round the gullet and joins the dorsal aorta directly. From each gill an efferent branchial artery brings back the purified blood, and the efferent arteries unite, in a somewhat irregular way, to form the dorsal aorta $(d . a o$.). Each afferent with the corresponding efferent artery constitutes an aortic arch. Short connecting branches unite the afferent and efferent arteries of each gill, carotids (ext. car., int. car.) arise from the first efferent artery, and, when the 


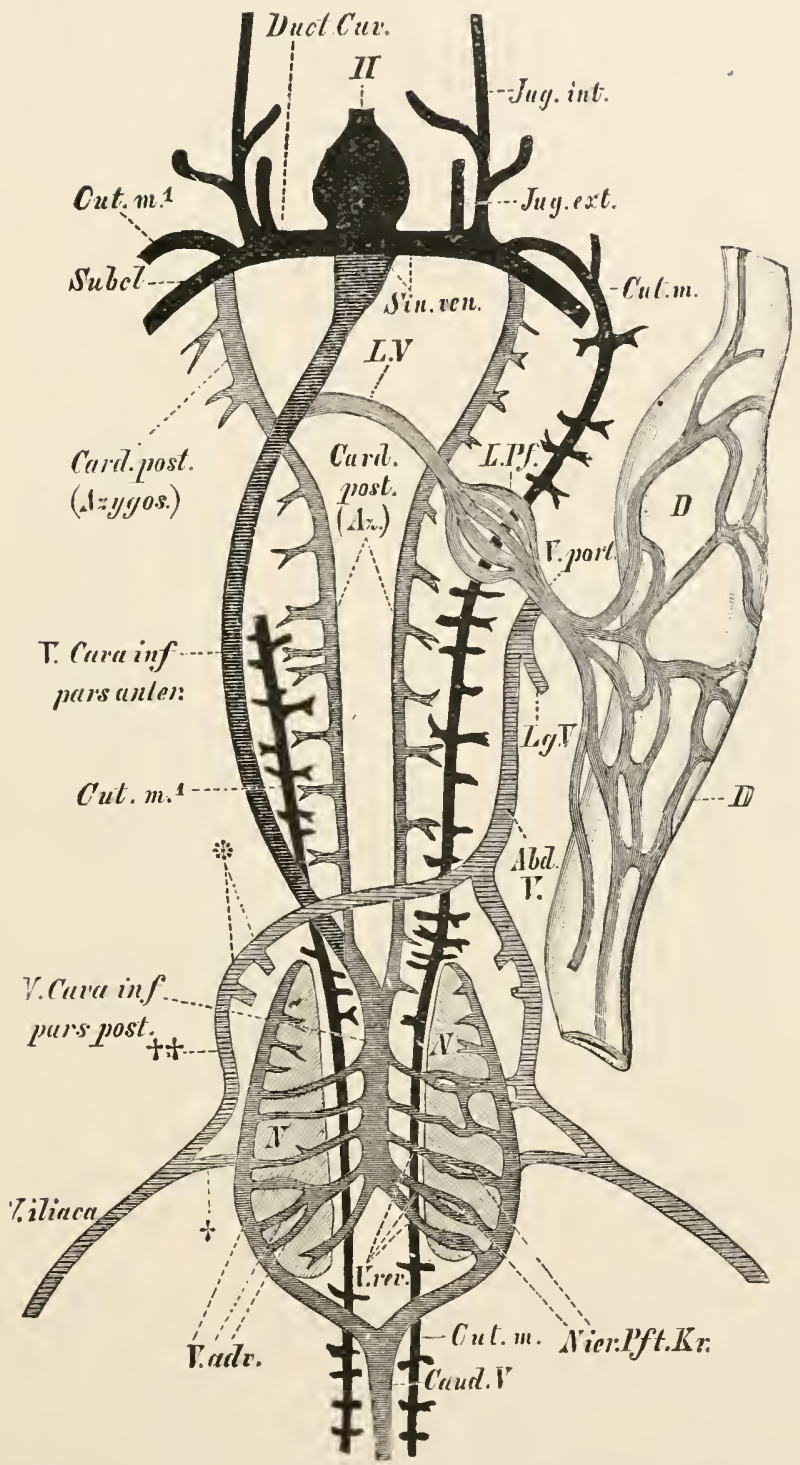

FIG. 94is.-Salamandra maculosa. Venous system, diagrammatic, from the vent ral aspect. Abd. I. ablominal vein; Card. post. (Az.), axygos vein ; Caud. I. caudal vein ; Cut. m, left musculo-cutaneous vein: Cut. $m$, the same on the right side (partly removed); $D$, intestine; $D u c t$. $C u v$. precaval vein ; $H$. lieart; $\boldsymbol{J} u g$. ext. external jugular ; $\boldsymbol{J} u g$. int. interual jugular; $L g . V$. mesenteric vein; $L$. $P f$. hepatic portal system; $L . I$. hepitic vein; $N$, kidney; Nier. Pft. Ir r. renal portal system; Sin. ven. sinus venosus; Subcl. subclavian vein; $I$.ade. branches of renal portal vein; $I$ t Caca inf. posteaval ; V. iliaca, iliac vein : I'. rev. renal veins; *, cloacal veins; $t$, branch of iliac to renal portal vein; $\uparrow \uparrow$, lateral vein, (From Wiedersheim's Comparative Anatomy.) 
lungs appear, a pulmonary artery (pul. a.) is given off from the dorsal portion of the fourth aortic arch of each side. In those Urodela which in the adult condition are devoid of gills, when the latter atrophy $(B)$ the first aortic arch loses its connection with the dorsal aorta, and becomes the carotid trunk; the second increases in size, forming the main factor of the dorsal aorta, and becomes the systemic trunk; the third undergoes great reduction, and the fourth becomes the pulmonary artery, its dorsal portion retaining its connection with the systemic trunk in the form of a small connecting branch, the ductus Botalli (d. bot.). In the Anura, as we have seen (p. 271), the third arch vanishes completely and there is no ductus Botalli.

As to the venous systcm, the Urodela exhibit very clearly the transition from the Fish-type to the condition already described in the Frog. The blood from the tail is brought back by a caudal vcin (Fig. 965, Caud. V.), which, on reaching the coelome, divides into two renal portal vcins, one going to each kidney. From the kidney the blood is taken, in the larva, into paired cardinal veins, each of which joins with the corresponding jugular to form a precaval vein. In the adult the anterior portions of the cardinals undergo partial atrophy, becoming reduced to two small azygos veins (Card. post.) which receive the blood from the region of the back : their posterior portions unite and are continued forwards by a new unpaired vein, the postcaval ( $V$. cava inf.), which, joined by the hepatic veins, pours its blood into the sinus venosus. The iliac vein from the hind-leg divides into two branches: one joins the renal portal, the other, representing the lateral vein of Elasmobranchs, unites with its fellow in the middle ventral line to form the abdominal vein (Abd. V.) and joins the hepatic portal, its blood, after traversing the capillaries of the liver, being returned by the hepatic vein into the post-caval.

The red corpuscles are oval and nucleated, and are remarkable for their unusual size. Those of Amphiuma are the largest known, being about ${ }_{14}^{1} \mathrm{~mm}$. in diameter, or eight times that of a human red corpuscle.

Nervous System and Sense-Organs.-The brain of Urodela differs from that of the Frog in its more elongated and slender form, in the comparatively small size of the optic lobes, and in the non-union of the olfactory bulbs. The olfactory sacs always open into the mouth by posterior nares situated behind or external to the vomers. The eye has no lids in the lower forms and is degenerate in the cave-dwelling Proteus and in some Gymnophiona. The Urodela, the Gymnophiona, and some Anura have no tympanic cavity or membrane, and no columella; there is, however, a stapes (Figs. 959, 960) in the form of a nodule of cartilage inserted in the fenestra ovalis. In the perennibranchiate Urodeles and in the larvæ of the air-breathing forms lateral-line sense-organs are 
present. 'There was an extensive lateral-line systein, leaving its impress on the bones of the skull, in the Stegocephala.

Urinogenital Organs.-- In the Urodela the kidneys (Fig. 966, $N$ ) are much elongated and are divided into two portions, a broad posterior part, the functional kidney $(G N)$, and a narrow anterior

A

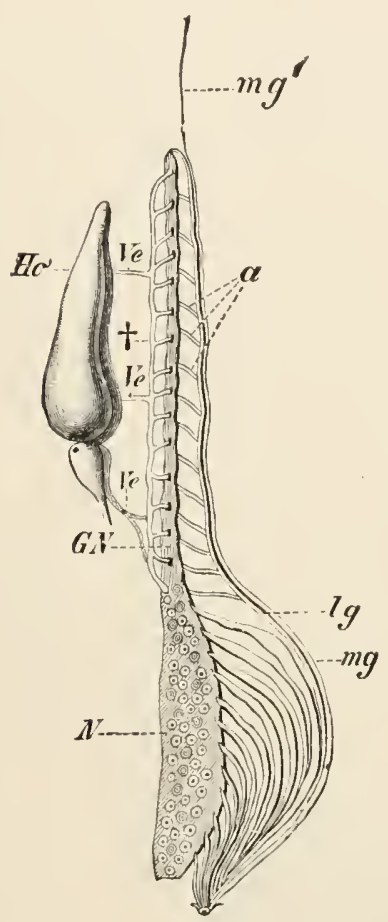

13

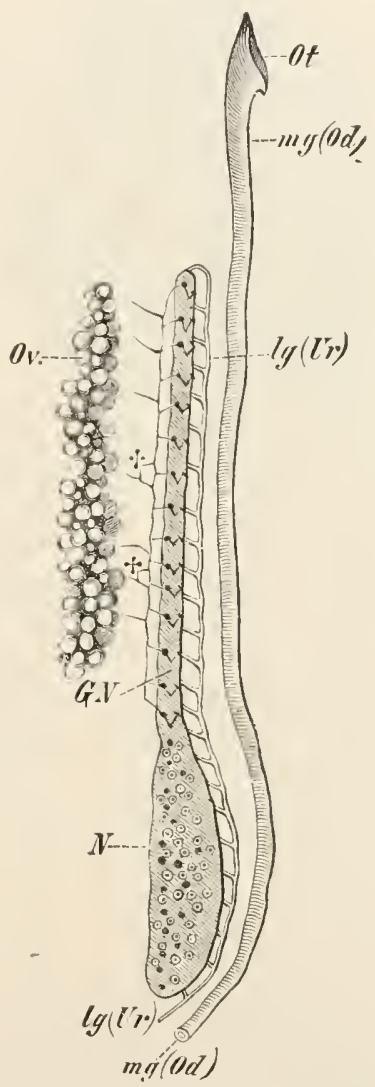

FIG. 966.--Diagrams of urinogenital organs of male $(A)$ and female $(B)$ Urodele. $\boldsymbol{u}$, collecting

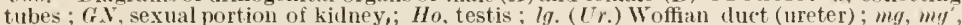
vestigial Müllerian duct of male; $m g$. $(O d)$, oviduet; $N$, non-sexual portion of kidney ; Ov. ovary ; Ie, vasa efferentia; $f$, longitudinal canal. (From Wiedersheim's Comparative Anatomy, after spengel.)

sexual part connected in the male with the efferent ducts of the testis. Numerous ducts leave the kidney and open into the Wolffian (mesonephric) duct $[\mathrm{lg} .(U r)$.$] , which thus acts as a ureter$ in the female, as a urinogenital duct in the male. The oviduct $[m g .(O d)$.$] is developed from the Miillerian duct, a rudiment of$ which ( $m g ., m g^{\prime}$.) occurs in the male. In the Gymnophiona the kidneys extend the whole length of the cœlome, and in the young 
condition are formed of segmentally arranged portions, each with a nephrostome and a glomerulus, as in Myxinoids (see p. 137). A pronephros is present in the larva, but disappears in the adult.

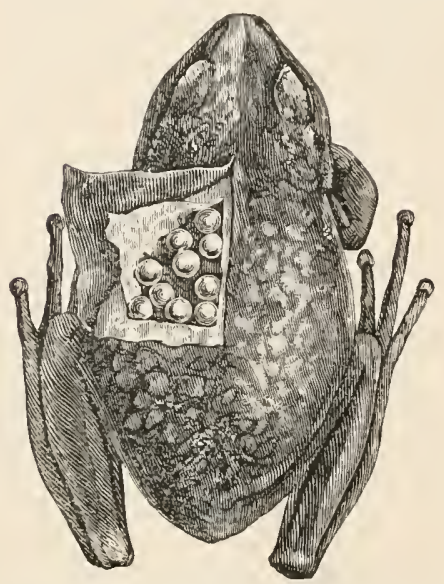

1'I(. 967 - Nototrema marsupiatum. Fentule, with pouch opened. (From Mivirt.)

A urinary bladder is almost always present, opening into the cloaca and having no connection with the ureters. In some Gymnophiona the cloaca can be protruded and acts as a penis.

Reproduction and Development. - External impregnation takes place in Anura, but in many Urodela the sperms are aggregated into spermatophores by glands in the wall of the cloaca, and these, being deposited on the body of the female, are taken into the cloaca and effect internal impregnation.

Several curious instances of parental care are known. A number of different speciess of Frogs and Toads construct nests or shelters of leaves or other materials in which the eggs are deposited and in which the young are developed. In the Obstetric Toad (Alyles obstetricans) of Europe the male winds the strings of egas-formed by the adhesion of their gelatinous investmentround his body and thighs, where they are retained until the tadpoles are ready to be hate he d. In Rhinoderma dar. wini, a little South American Frog, the eggs are transferred by the male to his immense vocal sacs, which extend over the whole ventral surfare, and there hatched. In another Anuran,

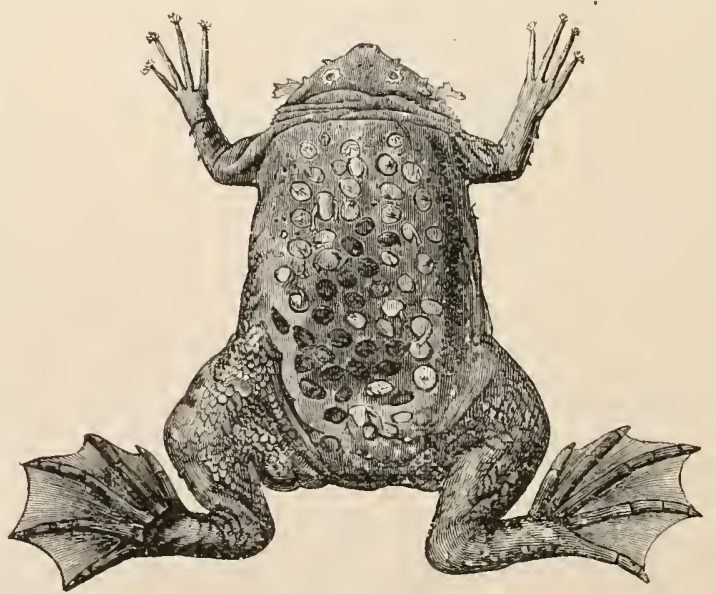

FIG. 968.-Fipa americana. Female. (From Mivart.)

Nototrema (Fig. 967), there is a pouch on the back of the female in which the eggs are stored, the young being hatched in some species as tadpoles, in others in the adult or Frog form. In the Surinam 
Toad (Pipa americana, Fig. 968) the skin on the back of the female becomes soft and spongy during the breeding season : the eggs are placed on it by the male, and each sinks into a little pouch of skin
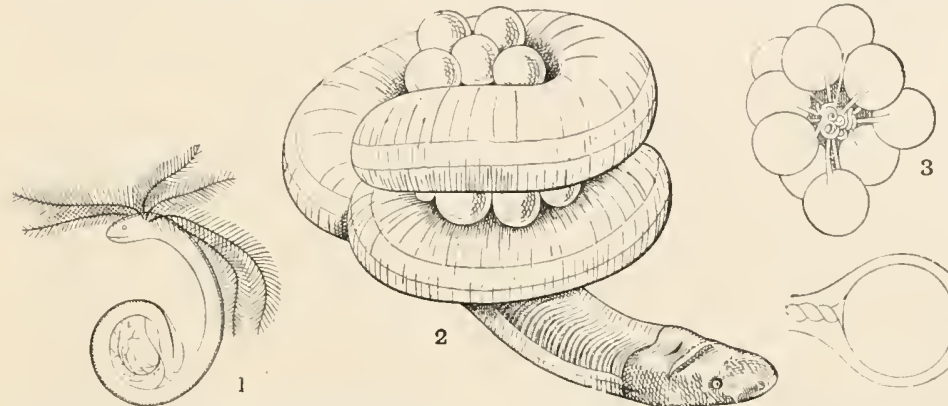

FIG, 969 - Ich thyophis glutinosa, $\times 1,1$ a ncarly ripe embryo, with gills, tail-till, aul st ill with a considerable amount of yolk; 2, female gnarding her eggs, coiled up in a lole underground : 3, a bunch of newly-laid eggs ; 4 , a single egg, enlarged, schematised to sliuw the twisted albuninous strings or chalazae within the outer nembrane, which surrounds the white of the egg. (After P. and F. Sarasin.)

covered by a gelatinous film. The embryos, which have a large yolksac, develop in these pouches; they never possess external gills, and are latched insthe adult form. In the case of several species the tadpoles are carried about by the female, adhering to her dorsal surface by suckers or by a viscid secretion. Another Anuran, Pseudis paradoxa, is remarkable for the fact that the tadpole is many times larger than the adult.

Some Salamanders ( $S$. maculosa and $S$. atra) and a species of Cacilia are viviparous. In the Black Salamander (S. atra), though many eggs are developed, only two larva survive, one in each oviduct, these being nourished in later stages by means of the remainder of the eggs. The larva in this species possesses long plume-like external gills during its existence in the oviduct, shedding them before birth. If, however, the unborn young is removed from the oviduct and placed in water, it swims about like an ordinary aquatic larva, losing its long gills and developing a new and shorter set. Most Gym-

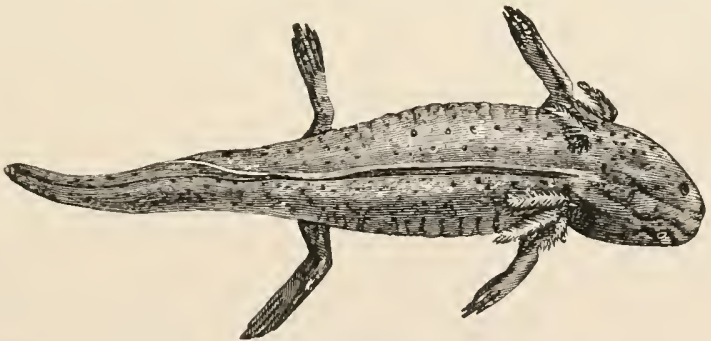

FIt*. 9711.-Amblystoma tigrinum. Lirvial or Axol., stige. (From llivart.)

nophiona lay their eggs in burrows, but the larva in some cases lead an aquatic life for a time, and during this period possess, like tadpoles, a tail with a tail-fin which afterwards undergoes absorption. The larvæ of most Gymnophiona have long external gills (Fig. 969). 
A very interesting case of padogenesis is furnished by the Axolotl (Amblystoma ligrinum). This animal frequently undergoes no metamorphosis, but breeds in the gilled or larval state (Fig. 970). But under certain circumstances the gills are lost, the gill-slits close, and a terrestrial salamandrine form is assumed. It is to the branchiate stage that the name Axolotl properly applies; before the metamorphosis was discovered its connection with Amblystoma was not suspected, and it was placed in a distinct genus, Siredon, among the Perennibranchiata.

Segmentation of the egg in the Anura and Urodela is always complete but unequal. In Pipa and Alytes there is a large quantity of food-yolk, and the developing embryo lies on the surface of a large yolk-sac. In the Gymnophiona the eggs, which are singularly like those of a Bird, are of large size and segmentation is partial, the formation of segments at the pole of the egg opposite that at which the formation of the embryo begins only taking place at the stage of gastrulation: the embryo is coiled over the surface of the yolk as in the Trout.

Distribution.-The Urodela are almost exclusively Palæarctic and Nearetic forms, occurring in North America, Europe, Asia, and North Africa : a few species extend southwards into the Neotropical and Oriental regions. The Gymmophiona, on the other hand, are mainly southern, occurring in the Neotropical, Ethiopian, and Oriental regions, but are absent in Australasia and the Pacific Islands. The Anura are almost universally distributed, and are abundant in all the greater zoo-geographical regions: they are, however, very meagrely represented in New Zealand and are absent in most Oceanic islands, a fact due to the fatal effects of salt water upon the eggs and embryos of Amphibia as well as upon the adults.

Remains of Stegocephala are found in considerable abundance from the Carboniferous to the Trias, and one genus extends into the Lower Jurassic, after which period the order apparently became extinct. The Urodela and Anura are not known until the Eocene, and no fossil remains of Gymnophiona have been found.

Mutual Relationships.-The perennibranchiate Urodela are undoubtedly the lowest of existing Amphibia; they lead up, through such forms as Amphiuma, with persistent gill-slits but deciduous gills, to the Land Salamanders, in which a purely terrestrial form is assumed. The Stegocephala exhibit a parallel series of modifications, some of them being perennibranchiate, others caducibranchiate. Their skull is more complex than that of the Urodela, but their vertebral colımn never reaches the same degree of specialisation as that of the Land Salamanders, and in some cases shows a lower grade of organisation than in any existing Amphibia. Both in their skeleton and in the distribution of their lateral sense-organs they show some affinity with the Crossopterygii. The Anura 
are a very specialised group: their development indicates their derivation from branchiate tailed forms, but there is no palæontological evidence on this point.

\section{CLASS IV.-REPTILIA.}

Reptiles, Birds, and Mammals are associated togrether as having in common certain features in which they differ from lower Vertebrates. The most important of these is the occurrence in all three classes of certain embryonic membranes termed the ammion and the allantois, to be described subsequently. The term Ammiota is, accordingly, frequently used for the group formed by these three highest classes of the Vertebrata.

The classes Reptilia and Aves are much more closely allied with one another than either of them is with the Mammalia; and the first two are sometimes associated together under the title of Sauropsida. The following are some of the most salient features of the Sauropsida when compared with the other Vertebrates:-

The integument always gives rise to important and characteristic exoskeletal structures in the form of scales or feathers; the dermis may or may not take part in the formation of an exoskeleton. The skull is well ossified: it rarely in the adult state contains a distinct parasphenoid. There is a single occipital condyle borne on the basi-occipital. The basi-sphenoid is a well-developed bone. The mandible articulates with the skull through the intermediation of a quadrate, and consists of five or six bones on each side. The ankle-joint is an articulation between the proximal and distal divisions of the tarsus. As in the Amphibia, there is a cloaca into which the rectum and the renal and reproductive ducts open. The heart consists of two auricles and a ventricle which is sometimes incompletely, sometimes completely, divided into two parts, Branchix are never present at any stage. The mesonephros is never the functional renal organ of the adult, but is always replaced by a metanephros. Both an amnion and an allantois are present in the embryo, the latter becoming highly vascular and acting as a temporary embryonic organ of respiration.

The class Reptilia comprises four orders having living representatives, in addition to a number of extinct groups. In the Mesozoic period the class reached its maximum both in the number of its representatives and the size which many of them attained; at that period they were very ummistakably the dominant class of the Animal Kingdom. In the Tertiary period they underwent a decline, while the Birds, and, in a yet higher degree, the Mammals, were gaining a preponderance over them. The living Reptiles are the Lizards and Chamæleons, the Tuataras, the Snakes, Tortoises and Turtles, and the Crocodiles and Alligators. Though horny scales are not by any means present in all the Reptiles, their occur- 
rence as a complete covering is characteristic of the group and almost peculiar to it. When scales are not present, the epidermis is always hardened and cornified so as to form plates of horny material, such as the horny plates of the Tortoises, which protect the underlying parts from injury and desiccation. Bony plates are frequently present as well. In most respects the internal structure of the Reptilia shows a very decided advance on that of the Amphibia. The skull and the pectoral and pelvic arches are more completely ossified, and both vascular and nervous systems show a higher grade of organisation.

\section{Example of the Classs.-A lizard (Lacerta).}

The most striking external differences between the Lizard (Fig. 971) and the Frog are the covering of scales, the comparative smallness of the head, and the presence of a distinct neck, the great

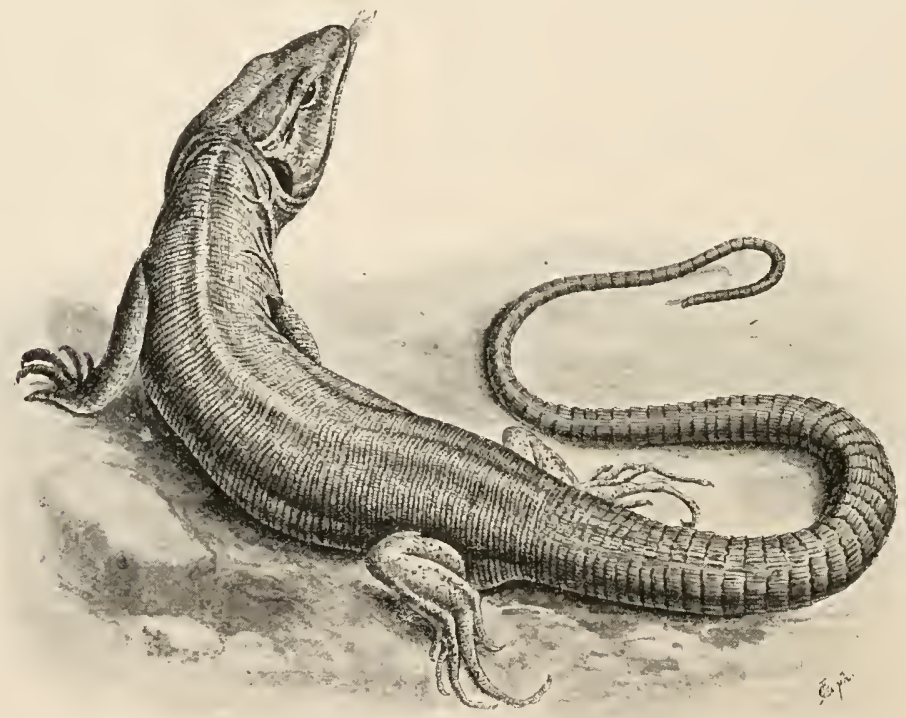

Fig. 971.-Lacerta viridis. (After Brehm.)

length of the candal region, the shortness of the limbs, and the approximate equality in length of the anterior and posterior pairs. The anterior limbs are situated just behind the neck, springing from the trunk towards the ventral surface. The fore-limb, like that of the Frog, is divided into three parts, the upper-arm or brachium, the fore-arm or anti-branchium, and the hand or manus; there are five digits provided with horny claws, the first digit or pollex being the smallest. The hind-limbs arise from the posterior 
end of the trunk towards the ventral aspect; each, like that of the Frog, consists of three divisions - thigh or femur, shank or crus, and foot or pes. The pes, like the manus, terminates in five clawed digits, of which the first or hallux is the smallest. The head is somewhat pyramidal, slightly depressed: the openings of the external nares are situated above the anterior extremity. The mouth is a wide slit-like aperture rumning round the anterior border of the head. At the sides are the eyes, each provided with upper and lower opaque, movable eyelids and with a transparent third eyelid or nictituting membrane, which, when withdrawn, lies in the anterior angle of the orbit. Behind the eye is a circular brown patch of skin-the tympanic membranc-corresponding closely to that of the Frog, but somewhat sunk below the general level of the skin. The trunk is elongated, strongly convex dorsally, flatter at the sides and ventrally. At the root of the tail on the ventral surface is a slit-like transverse aperture the amus or cloacal aperture. The tail is cylindrical, thick in front, gradually tapering to a narrow posterior extremity; it is nearly twice as long as the head and trunk together.

There is an exoskeleton of horny scales covering all parts. These are formed from folds of the dermis, each covered with a thick horny epidermal layer. In size they differ in different positions. On the dorsal surface of the trunk they are small, hexagonal, and indistinctly keeled. On the ventral surface they are larger and are arranged in eight longitudinal rows. Immediately in front of the cloacal aperture is a large pre-anal plate. A collar-like ridge of larger scales surrounds the throat. On the tail the scales are elongated, keeled, and arranged in regular transverse (annular) rows, giving the tail a ringed appearance. On the surface of the limbs the scales of the pre-axial (radial or tibial) side are larger than those of the post-axial (ulnar or fibular). The scales on the upper surface of the head (head-shields) are large, and have a regular and characteristic arrangement.

Endoskeleton.-The vertebral column is of great length and made up of a large number of vertebra. It is distinctly marked out into regions, a cervical of eight vertebræ, a thoraco-lumbar of twenty-two, a sacral of two, and a caudal of a considerable but indefinite number. A vertebra from the anterior thoracic region (Fig. 972, $A, B$ ) presents the following leading features. The centrum (cent.) is elongated and strongly procolous, i.e. the anterior surface is concave, the posterior convex; the neural arch bears a short neural spine $(s p$.$) . There are pre- and post-zygapophyses$ $(p r . z y, p t . z y)$, the former with their articular surfaces directed upwards, the latter downwards. On each side at the junction of centrum and neural arch is a facet-the capitular facet-for the articulation of a rib. The cervical vertebræ in general are similar in essential respects to those of the trunk, but are somewhat shorter. 
The first two, however, differ greatly from the others. The first is the atlas $(C, D)$. It has no distinct centrum, but is in the form of a ring; ventrally on its anterior face it bears a smooth articular facet for the occipital condyle of the skull. It consists of three distinct ossifications, one ventral, the others dorso-lateral: the

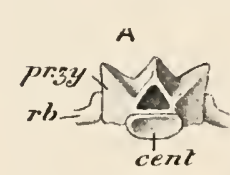

D
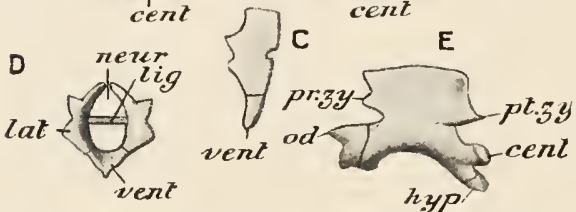

FIG. 972.-Vertebræ of Lizard. $A$, anterior, $B$, posterior view of a thoracic vertebra; $C$, lateral, $D$, anterior view of atlas vertebra; $F^{\prime}$, lateral view of axis. cent. centrum; hyp. liypapophysis of axis; lat. lateral piece of atlas; lig. ligamentous band dividing the ring of the atlas into two; neur. neural arch of atlas: od. odontoid process ; $p r$. $z y$. pre-zygapophysis ; $p t$. $z y$. post-zygapophysis : $r b$. rib ; sp. spine ; vent. ventral piece of atlas. latter do not quite meet dorsally, being separated by a space bridged over by membrane. The second or axis $(E)$ has a short conical process- the odontoid process (od)projecting forwards from its centrum. In the natural position of the parts the odontoid process-which is a part of the centrum of the atlas, and is not actually fused with, though firmly fixed to, the axis-lies in the lower or ventral part of the opening of the atlas, separated by a ligamentous band from the upper portion, which corresponds to the neural arch, and lodges the anterior end of the spinal cord. On the ventral surface of the axis and of each of the following five or six vertebræ is a distinct bony nodule, sometimes termed the intercentrum or hypapophysis (hyp). The sacral vertebræ have short centra and strong expanded processes-the transverse processes-which abut against the ilia; these are separately ossified, and are to be looked upon as sacral ribs. The anterior caudal vertebræ are like the sacral, but have the centra longer, the transverse processes more slender, and the neural spines longer. The posterior caudal vertebræ become gradually smaller as we pass backwards, and the various processes reduced in prominence, until, at the posterior end of the tail, the whole vertebra is represented merely by a rodlike centrum. Attached to the ventral faces of the centra of a number of the anterior caudal vertebræ are $Y$-shaped bones-the chevron bones - the upper limbs of the $Y$ articulating with the vertebra, while the lower limb extends downwards and backwards. In nearly all the caudal vertebræ the centrum is crossed by a narrow transverse unossified zone through which the vertebra readily breaks. The ribs are slender curved rods, the vertebral ends of which articulate only with the capitular facets of the corresponding vertebræ, there being no direct articulation with the transverse processes. The ribs of the five anterior thoracic vertebræ are connected by means of cartilaginous sternal ribs with the sternum. 
The posterior thoracic ribs do not reach the sternum, the sternal ribs being very short, and free at their ventral ends. The cervical ribs, which are present on all the cervical vertebræ with the exception of the first three, are all shorter than the thoracic ribs, and none of them are connected with the sternum. Thus, as regards the structure of the vertebræ themselves, there is nothing to distinguish the posterior cervical from the anterior thoracic ; but, for convenience of description, the first thoracic is defined as the first vertebra having ribs connected with the sternum.

The sternum (Fig. 974, st) is a rhomboidal plate of cartilage with a small central space, or fontanelle, completed by membrane. Posteriorly it is produced into two slender flattened processes. On its antero-lateral borders are articular surfaces for the bones of the pectoral arch, and on its postero-lateral borders and the processes are small facets for the sternal ribs.

In the skull (Fig. 973) the chondrocranium, though persistent, is replaced by bones to a much greater extent than in the Frog, and the number of investing bones is much greater. On the dorsal and lateral surface are a large number of dermal roofing bones. At the posterior end the rounded aperture of the foramen magnum (for. mag.) is surrounded by four bones-a basioccipital (bas. oc.) below, exoccipitals (ex.oc.) at the sides, and a supraoccipital (supr.oc.) above. The basioccipital forms the floor of the most posterior portion of the cranial cavity ; posteriorly it bears a rounded prominence, the occipital condyle (oc. cond). In front of it, forming the middle portion of the floor of the cranial cavity, is the basisphenoid (bas. sph), not represented in the Frog, in front of which again is an investing bone, the parasphenoid (para), corresponding to the bone of the same name in the Frog and Trout, but here much reduced in size and importance and ankylosed with the basisphenoid.

In the wall of the auditory capsule are three ossificationspro-otic, epiotic, and opisthotic (op.ot). The first remains distinct, the second becomes merged in the supraoccipital, and the third in the exoccipital. The exoccipital and opisthotic are produced outwards as a pair of prominent horizontal processes, the parotic processes.

The large orbits are closely approximated, being separated only by a thin vertical interorbital septum. The cranial cavity is roofed over by the parietals (par) and frontals $(f r)$. The former are united together; in the middle is a small rounded aperturethe parietal foramen (par. $f$ ). The frontals remain separated from one another by a median frontal suture: between them and the united parietals is a transverse coronal suture. The nasal cavities are roofed over by a pair of nasals (nas). A small pre-frontal ( $p r . f r$.) lies in front of the frontal, and helps to bound the orbit anteriorly, and another small bone-the lacrymal (lcr) - perforated by an aperture for the lacrymal duct, lies at the anterior extremity of the orbit, 
just within its border. A row of small bones-the supra-orbitals (s. orb) bounds the orbit above, and behind is a post-orbital or lateral post-frontal ( $p t$. orb.) articulating with the frontal. Just
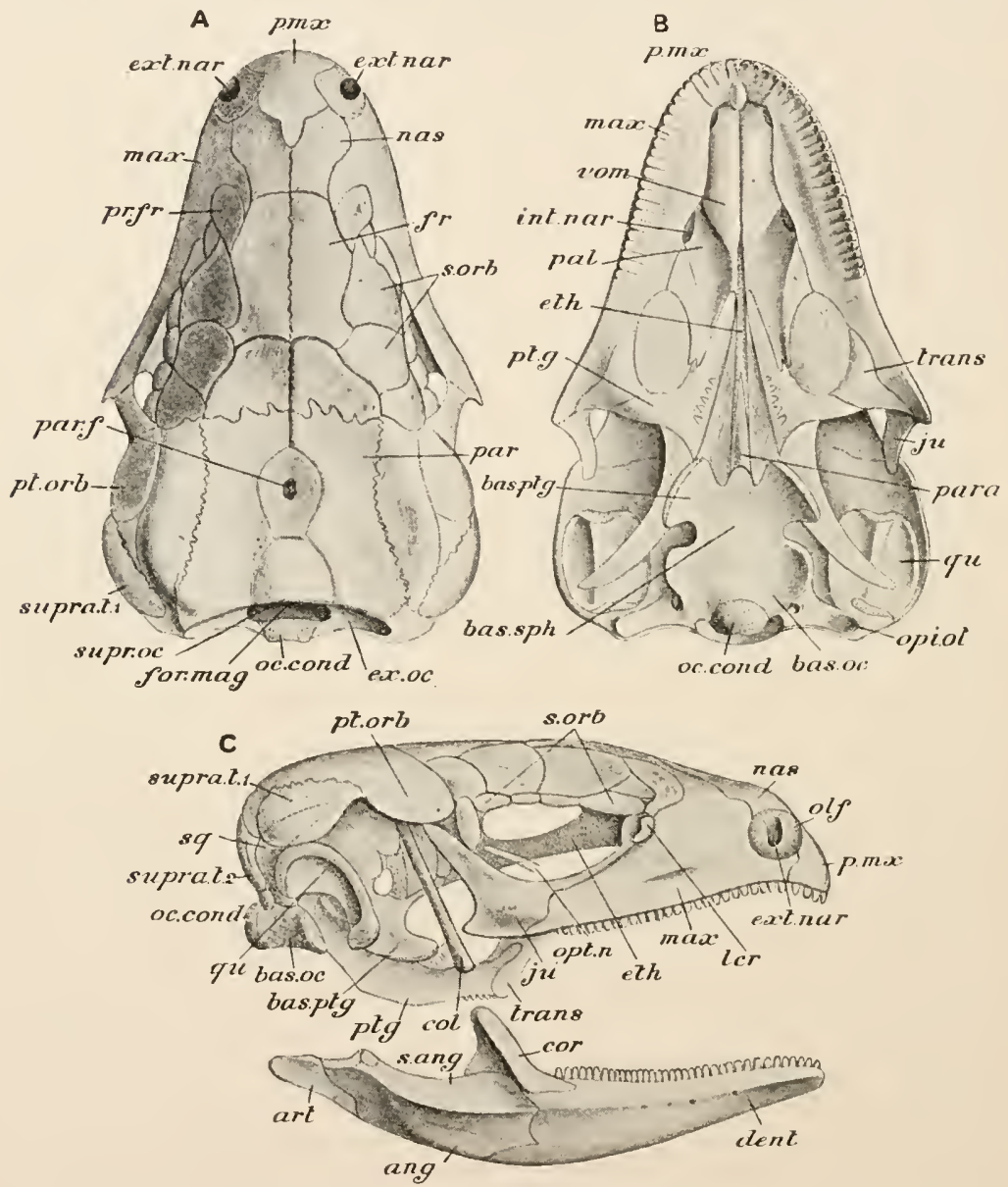

FIA. 973,- Skull of Lacerta agilis. A, from above; $B$, from below ; $C$, from the side. any. angular; art. articular; las. oc. basi-occipital; bas. pty. basipterygoid processes; bas. sph. basisphenoid ; col. epipterygoid ; cor. coronary; dent. dentary : eth. ethmoid ; ex. oc exoccipital ; ext. nar. external nares; for. mag. foramen magnum; fr. frolital; int. nar. internal nares; $j u$. jugal ; ler. lacrymal; max. maxilla ; nas. nasal; oc. cond occipital con. dyle; olf. olfactory capsule; opi. ot. opisthotic; opt. $n$. optic nerve; pal. palatine; par. parietal ; para. paras phenoid; par. $f$. parietal foramen : $p . m x$. premaxille ; $p r$. $f r$. pre-frontal ; $p t g$. pterygoid; $p t$. orb. post-orbital or lateral vost-frontal; qu. quadrate; $s$. anq. sujraangular; $s$ orb. supra-orbitals; $s q$. varaqualrate ; supra t.1. supratemporal 1 ; supra t.:. squamosal; trans. transverse; supr. oc. supra-occipital: rom. vomer. The unlettered bone internal to $p t$, orb. in $A$ is the post-irontal. The transverse line behind $f r$. is a superficial mark, lsot a suture. (After W. K. Parker.)

behind the post-orbital is a supra-temporal bone (supra t.1), in close relation to which are the paraquadrate $(s q)$ and squamosal (supra.t.:), 
the former bending forwards and upwards to form with the postorbital the superior temporal arch. At the anterior extremity of the snout is a median bone formed by the coalescence of the two premaxillce $(p . m x)$; this bears the four anterior teeth of each side. On each side behind the premaxilla is the maxilla (max), consisting of two portions, an alveolar bearing all the rest of the teeth, and a palatine extending inwards on the roof of the mouth, together with an ascending process articulating with the nasal and pre-frontal above. Articulating behind with each maxilla is a jugal $(j u)$, which forms the posterior half of the ventral boundary of the orbit. The quadrate $(q u)$ articulates movably with the parotic process, and bears at its distal end the articular surface for the mandible.

In the anterior portion of the roof of the mouth, articulating in front with the premaxillæ and maxillæ, are the vomers (vom). Behind and embracing them posteriorly are the flat palatines $(\mathrm{pal})$. The elongated pterygoids $(\mathrm{ptg})$ articulate in front with the posterior extremities of the palatines: behind each articulates with the corresponding basi-pterygoid process (bas. ptg) of the basisphenoid, and sends back a process which becomes applied to the inner face of the quadrate. A stout bone which extends between the maxilla externally and the pterygoid internally is termed the transverse bone or ecto-pterygoid (trans). Extending nearly vertically downwards from the pro-otic to the pterygoid is a slender rod of bone, the epi-pterygoid (col).

The columella is a small rod partly composed of cartilage and partly of bone, the outer end of which is fixed into the inner surface of the tympanic membrane, while the inner is attached to a small aperture, the fenestra ovalis, in the outer wall of the auditory capsule between the pro-otic and the opisthotic.

Certain depressions or fossæ and apertures or foramina are to be observed in the skull. The foramen magnum, the parietal foramen, and the orbits have been already mentioned. The posterior temporal fossa is situated on either side of and above the foramen magnum, bounded above and externally by the roofing bones, and on the inner side by the bones of the occipital region. The inferior temporal fossa is bounded internally by the pterygoid, and is separated from the palatine foramen by the transverse bone. The lateral temporal fossa is the wide space in the side wall of the skull behind the orbit; the bony bar which limits it above is the superior temporal arch; a bony inferior temporal or quadratojugal arch is here absent. The tympano-eustachian fossa, situated in the auditory region, is bounded by the bones of that region together with the quadrate. The posterior or internal nares are bounded posteriorly by the palatines. The anterior or external nasal aperture is situated at the anterior extremity of the skull bounded by the nasals and premaxillæ.

Each ramus of the mandible consists of six bony elements in VOI. II 
addition to the slender persistent Meckel's cartilage. The proximal element is the articular (art) which bears the articular surface for the quadrate, and is produced backwards into the angular process. The angular $(\mathrm{ang})$ is a splint-like bone covering the ventral edge and the lower half of the outer surface of the articular. The supraangular ( $s$. ang) overlies the dorsal edge and upper half of the outer surface of the same bone. The dentary (dent) forms the main part of the distal portion of the mandible, and bears all the mandibular teeth. The splenial is a flat splint applied to the inner face of the dentary. The coronary (cor), a small, somewhat conical bone, forms the upwardly directed coronoid process immediately behind the last tooth. All these, with the exception of the articular, are investing bones.

The hyoid apparatus (vide Fig. 979, b. hy) consists (1) of a median cartilaginous rod, the basi-hyal, (2) of the anterior cornua, elongated cartilaginous rods which, connected ventrally with the basi-hyal, curve round the gullet and end in close relation with the ventral surface of the auditory capsule, (3) of the middle cornua, rods of cartilage ossified at their proximal ends, and (4) of the posterior cornua, cartilaginous rods arising from the posterior edge of the basi-hyal and passing backwards and outwards. The middle cornua are vestiges of the first, the posterior of the second branchial arch.

In the pectoral arch (Fig. 974) the coracoids are flat bones articulating with the antero-lateral border of the sternum, and bearing the ventral half of the glenoid cavity (glen) for the head of the humerus; a cartilaginous epicoracoid (ep. cor.) element lies on the inner side of the procoracoid and coracoid; a large gap or fenestra divides each coracoid into a narrow anterior portion-the procoracoid $(\mathrm{pr} . \mathrm{cor})$, and a broader posterior portion, the coracoid proper $(c o r)$. The scapulce $(s c)$ articulate with the outer ends of the coracoids, and each bears the dorsal half of the glenoid cavity. Dorsally the scapulæ become expanded, and each has connected with it a thin plate of partly calcified cartilage-the suprascapula (supra. sc), which extends inwards towards the spinal column on the dorsal aspect of the body. An element not hitherto met with, except in the Stegocephala (p. 293), is the interclavicle or episternum (epist), a cross-shaped investing bone, the stem of which is longitudinal and is in the posterior portion of its extent closely applied to the ventral surface of the anterior part of the sternum, while the cross-piece is situated a little in front of the scapula. The clavicles $(\mathrm{cl})$ are flat curved bones articulating with one another in the middle line and also with the anterior end of the interclavicle. The bones of the fore-limb consist of a proximal bone or humerus, a middle division composed of two bones-the radius and ulna, and a distal division or manus. In the natural position of the parts the humerus is directed, from the glenoid cavity with which it articulates, backwards, upwards and outwards; the radius and ulna pass 
from their articulation with the humerus downwards and slightly forwards, while the manus has the digits directed forwards and outwards. When the limb is extended at right angles to the long axis of the trunk, it presents, like that of the Frog, dorsal and ventral surfaces, and pre-axial and postaxial borders, In this position the radius is seen to be pre-axial, the ulna post-axial. In the natural position the pre-axial border of the humerus is external, and the distal end of the forearm is rotated in such a way that, while the pre-axial border looks forwards and outwards at the proximal end,

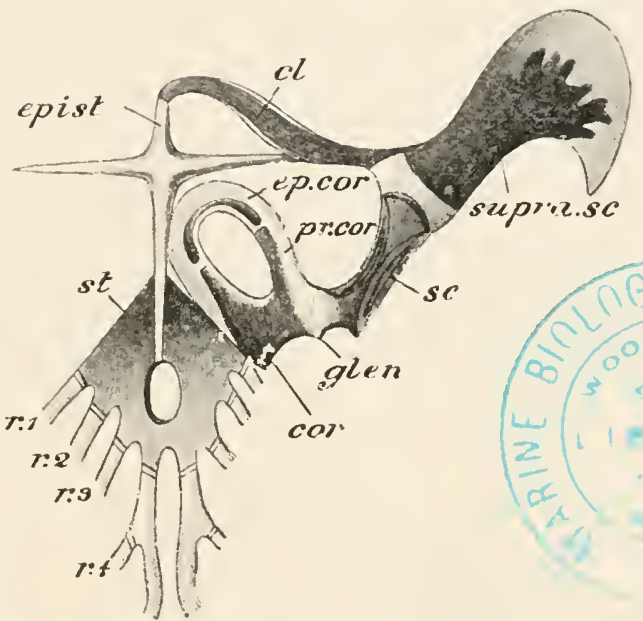

Fig. 974.- Pectoral arch and sternum of Lacerta agilis. cl. clavicle ; cor. coracoid ; ep. cor. epicoracoid; epist. e fisternum; glen. glenoid cavity for head of lumerus ; $p r$. cor. procoracoid ; r.1-r.t first to fourth sternal ribs; sc. seapula; st. sternum; supru. sc. suprascapula. (After Hoffmann.)

it faces directly inwards at its distal end, the manus being rotated so that its pre-axial border looks inwards.

The humerus is a long bone consisting of a shaft and two ex-

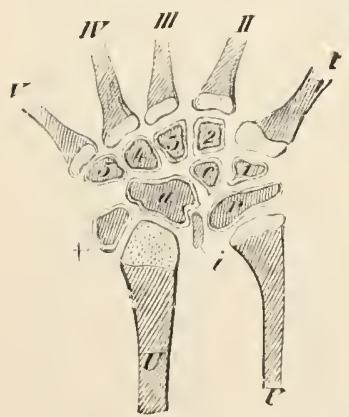

FIG. 975.- Carpus of Iacerta agilis, (left) from above. $l i$. radius; $U$.ulna; $c$. centrale ; $i$. intermedium ; $r$. radiale; $u$. ulnare : $1-5$, the five distal carpals ; $\uparrow$, pisiform ; $I-V$, the five metacarpals. (From itiedersheim's Comparative Anatomy.)

ulnare $(u)$, and intermedium $(i)$, of a centrale $(c)$, and of a distal row of five $(1-5)$; with an accessory or pisiform $(\dagger)$ bone attached of an epiphysis of calcified cartilage, the proximal rounded, the distal (trochlea) pulley-like, with two articular surfaces, one for the radius and the other for the ulna. The radius is a slender bone consisting, like the humerus, of a shaft and two epiphyses; the distal extremity has a concave articular surface for the carpus, and is produced pre-axially into a radial styloid process. The proximal end of the ulna is produced into an upwardly directed process - the olecranon: the distal end bears a convex articular surface for the carpus. The carpus (Fig. 975) is composed of ten small polyhedral or rounded carpal bones. These consist of a proximal row containing three, viz., the radiale $(r)$, tremities, each of the latter being formed 
to the distal epiphysis of the ulna on its post-axial side. The first digit or pollex consists of a metacarpal and two phalanges, the second of a metacarpal and three phalanges, the third of a metacarpal and four phalanges, the fourth of a metacarpal and five phalanges, and the fifth of a metacarpal and three phalanges. The number of phalanges in the first four digits is, therefore, one more than the serial number of the digit.

The pelvic arch (Fig. 976) consists of two triradiate bones, the ossa innominata, each ray being a separate bone. On the outer side at the point from which the rays diverge is a concave articular surface-the acetabulum $(A c)$-for the head of the femur. From the region of the acetabulum one of the rays, the ilium (I), a compressed rod, passes upwards and backwards to articulate with

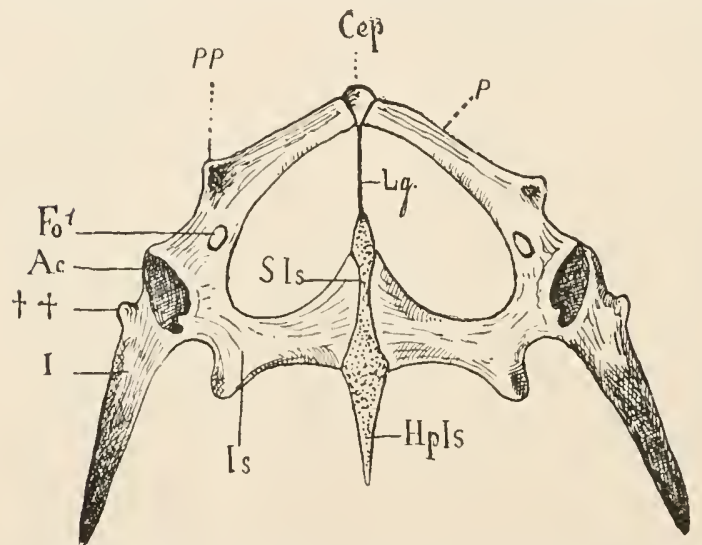

FiG. 976.- Pelvis of Lacerta vivipara, from the ventral side. Ac. acetabulum; Cep. epipubis. $F^{\prime} O^{\prime}$. foramen for obturator nerve ; $I P . I s$, hypoischium ; $I$. ilium ; $\dagger \dagger$, process representing the pre-acetabular part of the ilium; Lg. ligament; Is. ischium; $p$. pubis; $p p$. prepubis; $S$. Is. ischiatic symphysis. (From Wiedersheim's Comparative Anatomy.) the sacral region of the spinal column. A second ray-the pubis $(p)$ - passes downwards and forwards to meet its fellow in the middle line, the articulation being termed the pubic symphysis. In the middle line in front, between the anterior ends of the pubes, is a sinall nodule of calcified cartilage, the epipubis (Cep). The third ray or ischium $(I s)$ runs downwards and

backwards, and articulates with its fellow in the ischiatic symphysis, the ventral ends of the two bones being separated by a plate of calcified cartilage $(S . I s)$. Between the pubes and ischia is a wide space, the obturator foramen, divided by a median ligament $(l g)$ into a pair of apertures, and a smaller aperture in each pubis $\left(F^{\prime} o^{\prime}\right)$ transmits the obturator nerve. A small rod of bone, the os cloaca, or hypoischium (Hp. Is), passes backwards from the ischiatic symphysis and supports the ventral wall of the cloaca.

The hind-limb consists, like the fore-limb, of three divisions; these are termed respectively the proximal or femur, the middle or crus, and the distal or pes. The proximal division consists of one bone, the femur; the middle division of two, the tibia and fibula; the distal of the tarsal and metatarsal bones and the phalanges. When the limb is extended at right angles with the 
trunk, the tibia is pre-axial and the fibula post-axial: in the natural position of the parts the pre-axial border is internal in all three divisions of the limb. The femur is a stout bone consisting of a shaft and two epiphyses. The proximal epiphysis develops a rounded head which fits into the acetabulum; near it on the pre-axial side is a prominence, the lesser trochanter, and a nearly obsolete prominence on the post-axial side represents the greater trochanter. The distal extremity is pulley-shaped, with internal and external prominences or condyles for articulation with the tibia; immediately above the external condyle is a prominence or tuberosity for articulation with the fibula. The tibia is a stout, curved bone, along the anterior (dorsal) edge of which runs a longitudinal ridge, the cnemial ridge: the proximal extremity presents two articular surfaces for the condyles of the femur. The fibula is a slender bone, the proximal end articulating witl the external tuberosity of the femur, the distal with the tarsus.

The tarsus (Fig. 977) comprises only three bones in the adult, one large proximal bone, the tibio-fibulare $(t b . f b)$, and two smaller distal (tars. dist). Each digit consists of a metatarsal bone and phalanges, the number of the latter being respectively two, three, four, five, and three. The first and second metatarsals articulate with the tibial side of the tibio-fibulare, the rest with the distal tarsals.

Digestive System.-The upper and lower jaws, forming the boundary of

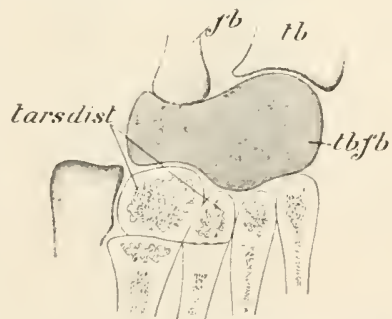

Fig. 977.-Tarsus of Lacerta agilis. $f b$. fibula; $t b$, tibia; $t b$. $f b$, tibiofibulare; tars. dist. 'distal tarsals. (After Gegenbaur.)

the aperture of the mouth, are each provided with a single row of small conical teeth, and there is a patch of similar teeth (palatine teeth) on the palatine. On the floor of the mouth-cavity is the tongue, a narrow elongated flesliy organ, bifid in front.

The stomach (Fig. 978, M, Fig. 979, St) is a cylindrical organ but little wider than the œsophagus, and with thick muscular walls. At the point where the small intestine joins the large intestine or rectum, the latter is produced into a short caecum (Fig. 980, coec). The liver $(l r)$ is divided into right and left lobes, and a gall-bladder (Fig. 978, GB.; Fig. 979, g.b; Fig. 980, g.bl) lies at the lower margin of the right lobe. The pancreas $(\mathrm{pn})$ is situated in the loop between the stomach and first part of the small intestine or duodenum $(d u)$. The stomach is attached to the body-wall by a fold of peritoneum, the mesogaster, the small intestine by a fold termed the mesentery, the rectum by a mesorectum. From the dorsal surface of the liver to the stomach extends a thin fold, the gastro-hepatic omentum; and this is continued backwards as the duodeno-hepatic omentum, connecting the liver with the first portion of the small intestine. 
Vascular System.-The heart is enclosed, like that of the Frog, in a thin transparent membrane, the pericardium. It consists of a sinus venosus, right and left auricles, and an incompletely divided ventricle. The sinus venosus (Fig. 979, s. v.), into which the large

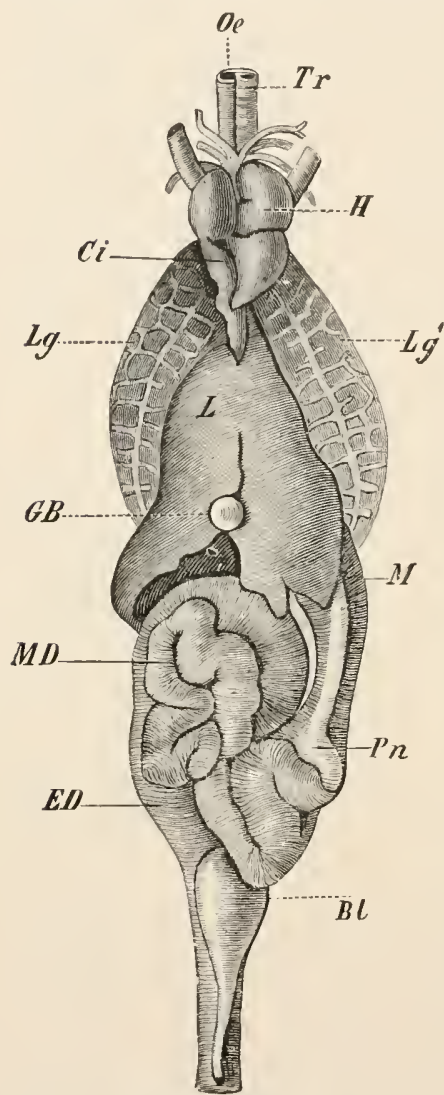

FIG. 978,- Lacerta agilis. General relations, $B l$. urinary bladder ; $C i$. postcaval vein; $E D$, rectum; $G B$, gallbladder; $H$. heart; $L$. liver: $L g, I g^{\prime}$. the lungs; $M$, stomach ; $M D$, small in. testine; $O e$. cesophagus; $P n$. pancreas; Tr. trachea. (From Wiedersheim's Comparative Anatomy.) view of the viscera in their natural

veins open, is thin-walled, and has a smooth inner surface. From it a sinu-auricular aperture, guarded by a two-lipped valve, leads to the right auricle. The auricles have their inner surfaces raised up into a network of muscular ridges, the musculi pectinati. Both auricles open into the cavity of the ventricle, the aperture of communication, or auriculo-ventricular aperture, being divided into two by the auricular septum, and guarded by the auriculo-ventricular valve, consisting of two semilunar flaps. The ventricle (Fig. 979, v. ; Fig. 980, vent.) has very thick spongy walls and a small cavity divided into two parts by an incomplete muscular partition. From the part of the veutricular cavity to the right of the partition arises the pulmonary artery; from the part to the left are given off the right and left aortic arches. When the two auricles contract, the blood from the right auricle (venous blood) tends to run more to the right-hand portion of the cavity of the ventricle, while that from the left auricle (arterial) occupies the left-hand portion. When the ventricle begins to contract, its walls come in contact with the dorsal and ventral edges of the ventricular partition, thus completing the separation of the right-hand part of the cavity, containing venous blood, from the left-hand part, containing arterial and mixed blood; and the further contraction results in the driving of the venous blood through the pulmonary artery to the lungs and of the rest through the aortic arches to the head and body. (Vide Fig. 1013.)

From the right aorta arise the carotid arteries (Fig. 979, cr.; Fig. 980, car. art.), and each runs for some distance parallel with 
the corresponding aortic arch, with which it anastomoses distally (the connecting part being termed the ductus Botalli), having previously given off the carotid artery proper, by means of which

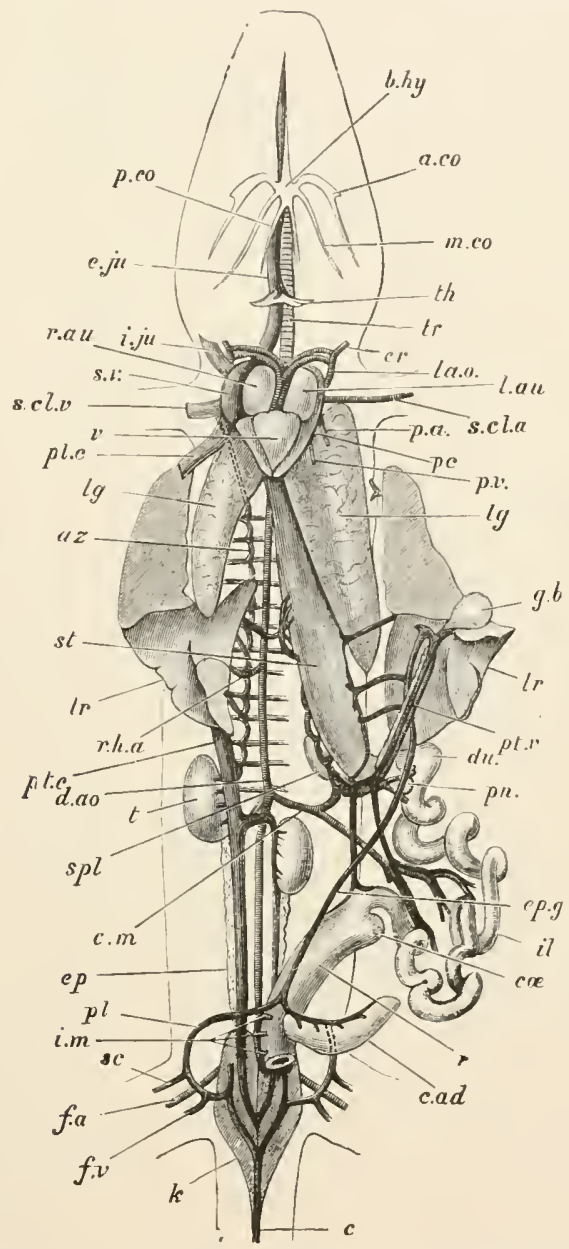

FIG. 979.- Lacerta viridis. Dissection from the ventral aspect showing the alimentary circulatory, respiratory and urinogenital organs (nat. size). The liver $(l r$.) is divided longitudinally and its two halves displaced outwards; the alimentary canal is drawn out to the animal's left ; the cloaca with the urinary bladder and posterior ends of the vasa deferentia is removed, as also is the right adipose body. $a, c o$. anterior cornu of hyoid ; $a z$. azygos or cardinal vein; $b . h y$. body of hyoid ; $c$. caudal vein; $c$. ad. adipose body; $c$. $m$. coliacomesenteric artery ; cœ. cecum ; $c r$. carotid artery ; $d$. ao. dorsal aorta ; $d u$. duodenum ; $e . j u$. external jugular vcin ; ep. epididymis; $e p . g$. epigastric vein ; $f$. $a$. femoral artery ; $f$. $v$. femoral vein ; $g . b$. gall-bladder ; $i$. ju. internal jugular vein; $i l$. ileum; $i . m$. posterior mesenteric arteries; $k$. kidney; $l a$. $o$. left aortic arch; $l$. $a u$. left auricle; lq. lungs ; lr. liver; $m . c o$. middle cornu of hyoid $; p$. $a$. pulmonary artery ; $p c$. pericardium : $p$. co. posterior corn $u$ of hyoid ; $p n$. pancreas; $p l$. pelvic vein ; $p t$. $c$. posteaval vein ; $p t$. $r$. hepatic portal vein; $p . v$. pulmonary vein; $r$. rectum ; $r$. au. right auricle; $r . h$. a. right hepatic artery ; sc. sciatic vein ; scl. $a$. subclavian artery ; scl. $v$. subclavian vein ; spl. spleen ; st. stomach; $s$. $v$. sinus venosus th. thyroid gland; tr. trachea; $t$. testis $v$, ventricle. (From Parker's Zootomy.) 
the blood is carried to the head. The two aortic arches curve backwards round the cosophagus, one on the right hand and the other on the left, and meet in the middle line dorsally to form the median dorsal aorta (Fig. 979, d. ao. ; Fig. 980, dors, aort.). From the right arch, just in front of the junction, arise the two subclavian arteries (Fig. 979, s. cl. a.), right and left, each running outwards to the corresponding fore-limb. From the dorsal aorta the first important branch given off is the coliaco-mesenteric (c. m.). This shortly divides into two trunks, a coeliac (Fig. 980, coel. a.) supplying the stomach, spleen, pancreas, duodenum, and left lobe of the liver, and an anterior mesenteric supplying the posterior part of the small intestine. Three small posterior mesenteric arteries given off further back supply the large intestine. Posteriorly, after giving off renal and genital branches, and a pair of large iliacs to the hindlimb, the dorsal aorta is continued along the tail as the caudal artery (Fig. 980, caud. art.). Throughout its length, in addition to the larger branches mentioned, the dorsal aorta gives origin to a regularly-arranged series of pairs of small vessels, the intercostal and lumbar arteries, giving off branches that enter the neural canal and others that supply the muscles and integument.

The venous blood from the tail is brought back by means of a caudal vein (Fig. 979, c.). This bifurcates at the base of the tail to form the two pelvic (lateral) veins $(p l$.$) ; these unite to form the$ median epigastric or abdominal (ep. g.), which eventually enters the left lobe of the liver. Entering the pelvic veins are the femoral and sciatic veins from the hind-limb. Arising from the pelvic are the renal portal veins distributed to the substance of the kidneys. The efferent renal veins, carrying the blood from the kidneys, combine to form a pair of large trunks, which soon unite to form the median postcaval. The postcaval runs forwards towards the heart, and, after receiving the wide hepatic vein from the liver, enters the sinus venosus.

Two precavals, right and left, carry the blood from the anterior extremities and the head to the sinus venosus. The right precaval is formed by the union of the internal and external jugular and the subclavian. On the left side the precaval is formed by the union of internal jugular and subclavian, the left external jugular being absent.

The liver is supplied, as is other Vertebrates, by a hepatic portal system of vessels, blood being carried to it by a portal vein, formed by the union of gastric, pancreatic, splenic, and mesenteric veins.

The adipose bodies (Fig. 979, c. ad.) are two masses of fat of somewhat semilunar shape in the posterior part of the abdominal cavity, between the peritoneum and the muscles of the bodywall.

The thyroid is a whitish, transversely-elongated body on the ventral wall of the trachea, a short distance in front of the heart. 


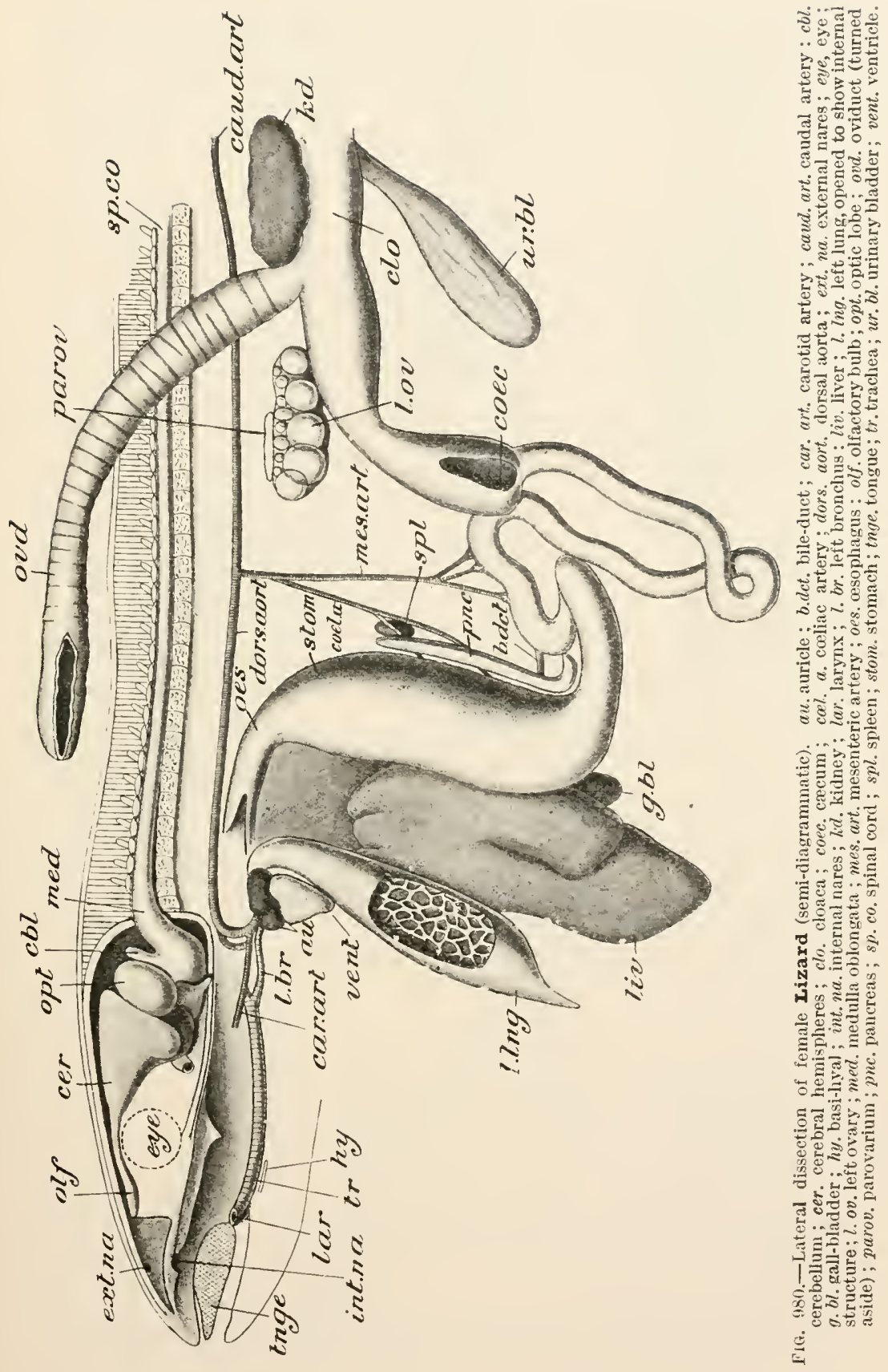


The spleen (Figs. 979 and $980, s p l$.) is a small red body lying in
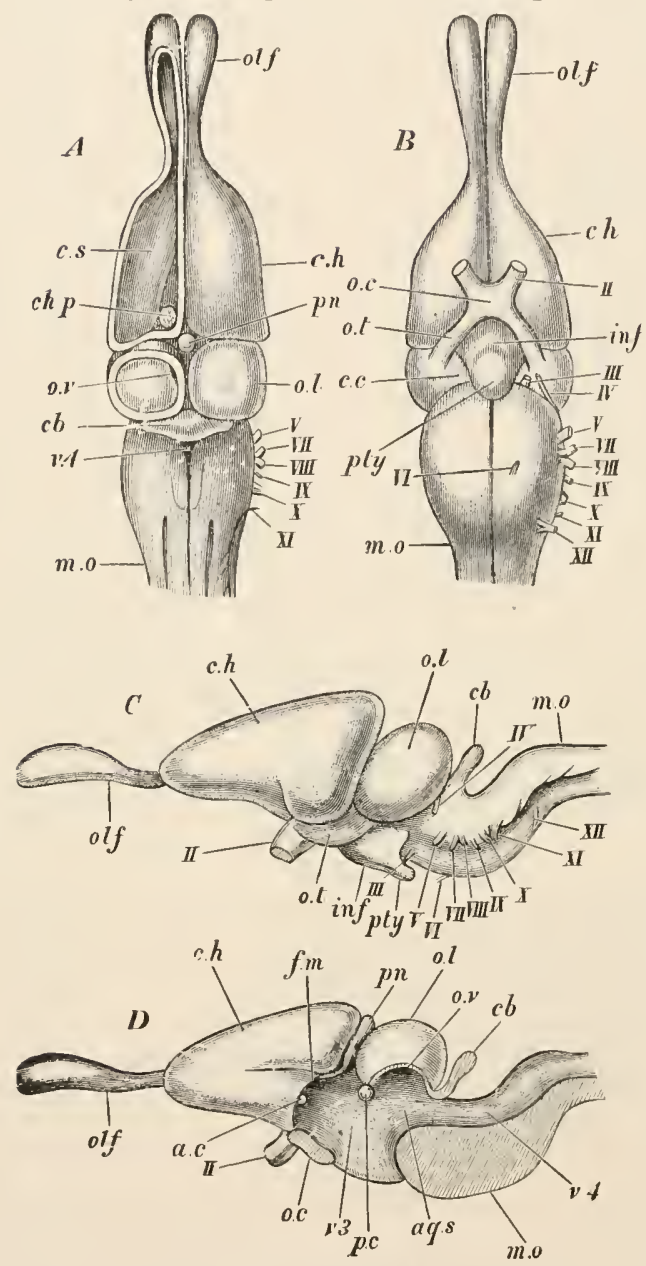

FIG. 981,-Brain of Lacerta viridis. $A$, from above, with the left hemisphere $(c . h$.$) and optic lobe (o . l$.$) opened.$ $B$, from beneath. $C$, from the left side. $D$, in longitudinal vertical section. $a$. $c$. anterior commissure; $a q$. $s$, aqueduct of Sylvius ; $c b$. cerebellum ; $c$. $c$. crura; cerebri ; $c . h$. cerebral hemispheres; $c h, p$, choroid plexus; $c$. $s$. corpus striatum ; f.m. foramen of Monro; inf. infundibulum; m. o. medulla oblongata: o, c. optic chiasma; $0 . l$. optic lobes ; olf, olfactory bulbs with their peduncles or tracts ; $o$. $t$. optic tracts; $o$. $v$. aperture between aqueduct of Sylvius and optic ventricle; $p$. $c$. posterior commissure ; pn. pineal a pparatus; pty. pituitary body : v3, diacoele $v 4$, metacole ; $\boldsymbol{I}-\boldsymbol{I} I \boldsymbol{I}$, cranial nerves. (From Parker's Zootomy.)

minor modifications. The two cerebral hemispheres (parencephala) (Fig. 981, c.h.) are oval bodies, somewhat narrower in front than

the mesogaster, near the posterior end of the stomach.

Organs of Respiration.-A slit-like aperture, the glottis, situated behind the tongue, leads into a short chamber, the larynx, the wall of which is supported by cricoid and arytenoid cartilages. From the larynx an elongated cylindrical tube, the trachea, passes backwards on the ventral side of the neck. Its wall is supported by a large number of small rings of cartilage, the tracheal rings. Posteriorly the trachea bifurcates to form two similar but narrower tubes, the bronchi, one entering each lung. The lung (Fig. 979, $l g$ ) is a fusiform sac, the inner lining of which is raised up into a network of delicate ridges, having the appearance of a honeycomb; these ridges are much closer and more numerous towards the anterior than towards the posterior end of the lung.

The brain (Figs. 981 and 982) presents all the parts that have been described in the brain of the Frog (p. 275), with some 
behind, closely applied together. Each is prolonged anteriorly into the corresponding olfactory peduncle or tract, somewhat dilated in front to form the olfactory bulb (olf.) from which the olfactory nerve arises. In the interior of each is a cavity, the lateral ventricle or paracole, sending a prolongation forwards into the
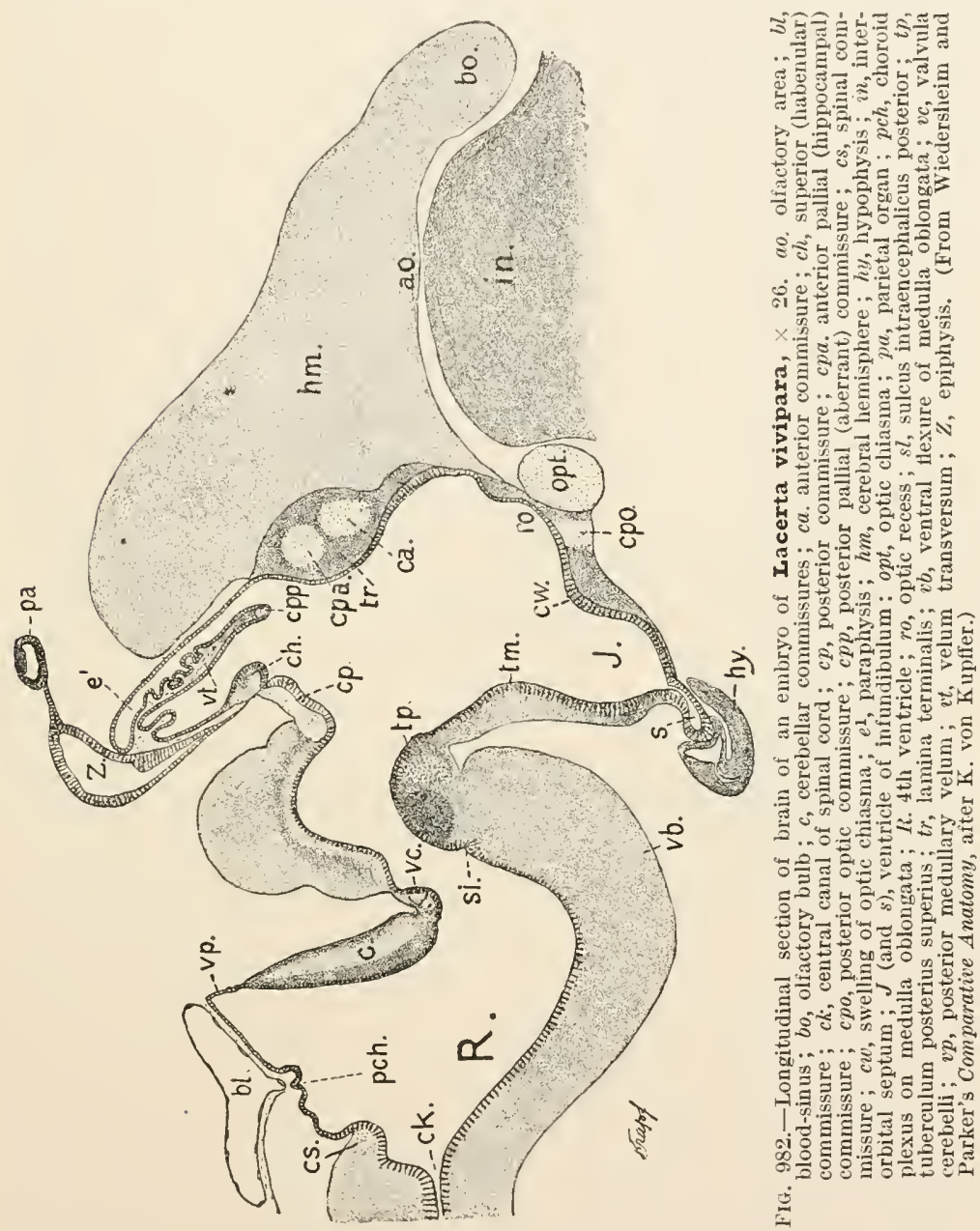

olfactory bulb, and communicating behind by a small aperture, the foramen of Monro $(D, f . m$.$) , with the diacole (v .3)$. Through the foramen of Monro there passes into each paracœle a vascular process of pia mater, the choroid plexus (ch. p.): immediately above and behind this is a hippocampal commissure (c.p.a.) connecting together two areas known as hippocampi, one on the mesial surface 
of each hemisphere. On the floor of each paracœle is a thickened mass of nerve-matter, the corpus striatum (c.s.), and between them passes a transverse band of nerve-fibres, the anterior commissure (a. c.). The diencephalon is a small rounded lobe between the paracoles and the mid-brain, containing a laterally compressed cavity, the diacole $(v .3)$. Its roof is extremely thin. Its lateral walls are formed of two thickenings, the optic thatami, behind which passes a transverse band, the posterior commissure $(p . c$.$) . Behind$ and below the thalami are the optic tracts (o.t.) continued into the optic nerves. Behind the optic tracts the floor is produced downwards into a tubular process, the infundibulum (inf.), ending below in a rounded body, the pituitary body or hypophysis (pty.). The roof is produced into a median outgrowth, the pineal apparatus (Fig. 981, D, pn ; Fig. 982, Z), which is divided into two parts, one of which has connected with its distal extremity an eye-like structure, the parietal organ or pineal eye (Fig. 982, pa), lying in the parietal foramen, while the other is the pineal organ or epiphysis. In front of the epiphysis, in the velum transversum (v.t.), a transverse fold of the thin roof of the brain marking the anterior limit of the diencephalon, is another commissure, the aberrant commissure (c.p.p.), which connects together the posterior and dorsal parts of the parencephala; this is not represented either in the Frog or in higher Vertebrates. The mid-brain consists dorsally of two oval optic lobes (corpora bigemina, Fig. 981, o. l.) and ventrally

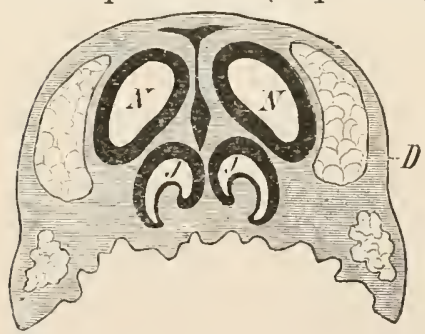

FIG. 983.-Transverse section of the nasal region of the head of Lacerta to show the relations of Jacobson's organs. $D$, nasal glands; $J, J$ Jacolson's organs: $N, \hat{N}$, nasal cavities. (From Wiedershein's Comparative Anatomy.) of a mass of longitudinal nervefibres, the crura cerebri (c. c.), passing forwards to the fore-brain. Each optic lobe contains a cavity (optococle) communicating with the iter, a narrow passage leading from the diacœle to the metacœle. The cerebellum $(c b)$ is, like that of the Frog, of small size, being a small antero-posteriorly flattened lobe overlapping the anterior portion of the metacole. The metencephalon (medulla oblongata, $m$. o.), broad in front, tapers behind to where it passes into the anterior portion of the spinal cord. The metacole is a shallow space on the dorsal aspect of the medulla oblongata, overlapped in front for a short distance by the cerebellum, and behind covered only by the pia mater, containing a network of vessels, the choroid plexus of the metacœle (Fig. 982, pch.). At the point where medulla oblongata and spinal cord meet is a strong ventral flexure.

The spinal cord is continued backwards throughout the length of the neural canal, becoming slightly dilated opposite the origins 
of the two pairs of limbs and tapering greatly towards the posterior end of the tail.

The cerebral nerves resemble those of the Frog as regards their origin and distribution in most respects, the principal difference being that a spinal accessory is intercalated in front of the hypoglossal, and that the hypoglossal arises from the medulla oblongata, not from the spinal cord, and is therefore a cerebral nerve.

The nasal cavities (Fig. 983) open at the extremity of the snout by the external nares, and into the cavity of the mouth by a pair of slit-like internal nares situated near the middle line of the palate. The external aperture opens into a sort of vestibule, beyond which is the nasal or olfactory cavity proper, containing a convoluted turbinal bone over which the mucous membrane extends. Opening into each nasal cavity, near the internal opening, is Jacobson's organ $(J, J$.$) , an oval sac with$ strongly pigmented walls supported by cartilage.

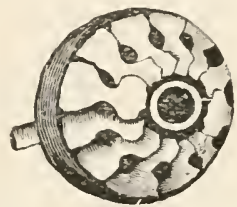

The eye has a cartilaginous sclerotic having a ring of small bones (Fig. 984) supporting it externally. There is a cushion-like pecten or vascular pigmented process similar to the structure of the same name occurring in Birds (see below, Class Aves), projecting into the inner chamber of the eye. In essential structure the rest of the

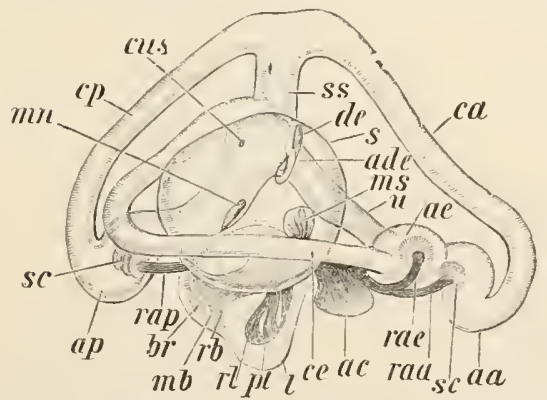

1IG. 985.-Membranous labyrinth of Iacerta viridis, viewed from the outer side. $a a$. anterior ampulla ; ac. auditory nerve; ade. opening of the ductus endolymphaticus; $a e$. external ampulla; $a p$. posterior ampulla ; br. basilar branch of nerve ; $c a$. anterior semicircular canal; $c e$. external semicircular canal; $c p$. posterior semicircular canal; eus. canal connecting utriculus and sacculus : de. ductus endolymphaticus; $l$. cochlea ; $m b$. basilar membrane; raa, rae, rap. $\urcorner$, branches of auditory nerve; $s$. sacculus ; ss. common canal of communication between anterior and posterior semicircular canals and utricle ; $u$. utriculus. (From Wiedersheim's Comparatice Anatomy, after Retzius.) eye agrees with that of the Craniata generally as already described. Two glands lie in the orbit, the lacrymal and the Harderian.

The ear consists of two principal parts, the internal ear or membranous labyrinth, and the middle ear or tympanum. The latter is closed externally by the tympanic membrane, the position of which has been already mentioned. It communicates with the cavity of the mouth by the Eustachian passage, which is narrower and longer than in the Frog. The inner wall of the tympanic cavity is formed by the bony wall of the auditory region of the skull, in which there are two fenestræthe fenestra ovalis and the fenestra rotunda. The columella stretches 
across the cavity from the tympanic membrane, and is fixed internally into the membrane covering over the fenestra ovalis.

The parts of the membranous labyrinth (Fig. 985) are enclosed by the bones of the auditory region: between the membranous wall of the labyrinth and the surrounding bone is a small space containing fluid, the perilymph. The labyrinth itself consists of the utriculus with the three semicircular canals and the sacculus with the cochlea (lagena). The utriculus $(u$.$) is a cylindrical tube, bent$ round at a sharp angle : the semicircular canals (ca., ce., cp.) are

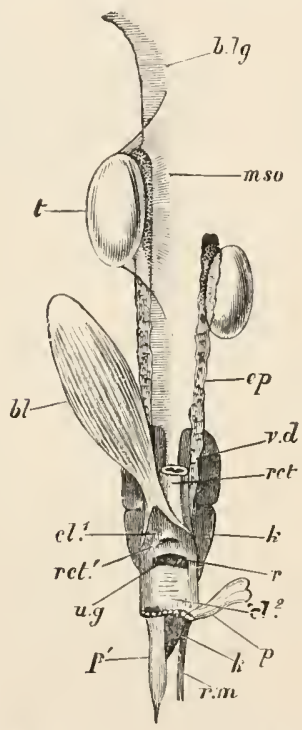

FIG. 986.-Male urinogenital organs of Lacerta viridis. The ventral wall of the cloaca is removed, the bladder is turned to the aninal's right, and the peritoneal covering of the left testis and epididymis is dissected away. $b l$. urinary bladder; $b . l g$. fold of peritoneum supporting epididymis; $c l^{\mathbf{1}}$. anterior and $\mathrm{cl}^{2}$. posterior divisions of the cloaca ; ep. epididymis ; $k$. kidney ; mso. nesorchium ; $p$. copulatory organs, of which the right is shown retracted $\left(p^{\prime}\right)$ and the leit everted $(p) ; r . m$. retractor nuscle of latter ; $r$. ridge separating anterior and posterior divisions of cloaca; ret. rectum; ret'. its opening into the cloaca ; $t$. testis; $u$. g. urinogenital papilla and aperture; $v$. $d$. vas deterens. (From Parker's Zootomy.)

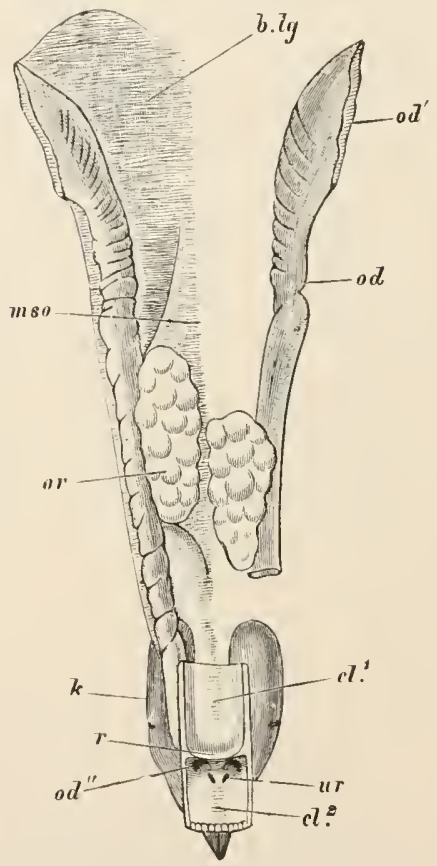

FIG. 987.-Female urinogenital organs of Lacerta viridis. The ventral wall of the cloaca, the urinary bladider, the posterior end of the left oviduct, and the peritoneal investment of the left ovary and oviduct are removed. $b$. $l g$. broad ligament; $c l^{1}$. anterior and $c l^{2}$. posterior divisions of the cloaca; $k$. lidney ; $m s o$. mesoarium ; od. left oviduct ; od'. its peritoneal aperture; od". aperture of right oviduct into the cloaca; $o v$. ovary; ur. aperture of ureter. (From 1'arker's Zootomy.)

arranged as in Vertebrates in general (p. 111). A narrow tube, the ductus endolymphaticus, leads upwards towards the roof of the skull and ends blindly in the dura mater. The sacculus is large and rounded. The cochlea $(l$.) forms a flattened, not very prominent, lobe, and is of simple form. 
Urinary and Reproductive Systems.-The kidneys (Figs. 986 and $987, k$.) are a pair of irregularly shaped, dark red bodies, each consisting of two lobes, anterior and posterior, situated in close contact with the dorsal wall of the posterior portion of the abdominal cavity, and covered with peritoneum on their ventral faces only. Their posterior portions, which are tapering, are in close contact with one another. Each has a delicate duct, the ureter, opening posteriorly into the cloaca. A urinary (allantoic) bladder (bl.), a thin-walled sac, opens into the cloaca on its ventral side.

In the male the testes (Fig. 986, t.) are two oval white bodies, that on the right side situated just posterior to the right lobe of the liver, that on the left somewhat further back. Each testis is attached to the body-wall by a fold of the peritoneum, the mesorchium (mso.). The epididymis (ep.) extends backwards from the inner side of each testis, and passes behind into a narrow convoluted tube, the vas deferens or spermiduct $(v . d$.), which opens into the terminal part of the corresponding ureter. A pair of vascular eversible copulatory sacs $\left(p, p^{\prime}\right.$.), which when everted are seen to be of cylindrical form with a dilated and bifid apex, open into the posterior part of the cloaca.

In the female the ovaries (Fig. 987, ov.) are a pair of irregularly oval bodies having their surfaces raised up into rounded elevations, marking the position of the ova. They are situated a little further back than the testes, and each is attached to the body-wall by a fold of the peritoneum, the mesoarium (mso.). The oviducts (od.) are thin-walled, wide, plaited tubes which open in front into the cavity of the body $\left(o d^{\prime}.\right)$, while behind they communicate with the posterior part of the cloaca, their opening ( $o d^{\prime \prime}$.) being distinct from, and a little in front of, those of the ureters. A fold of the peritoneum, the broad ligament $(b . l g$.$) , attaches the oviduct to the body-wall.$

\section{Distinctive Characters and Classification.}

The Reptilia are cold-blooded Sauropsida (p. 303), with a horny epidermal skeleton of scales, and frequently with an armour of dermal bony plates. The centra of the vertebræ have spheroidal articular surfaces. There are usually only two vertebræ in the sacral region. The episternum, when present, always remains distinct from the clavicles. The floor of the acetabulum is often completely ossified. The pubes and the ischia usually meet in ventral symphyses. The metatarsals do not become ankylosed. The mandible, as well as several bones of the upper jaw, very usually bear teeth. The optic lobes are situated on the dorsal aspect of the brain. The ventricle is rarely divided by a complete partition. There is always a paired aortic arch in the adult. 


\section{Order I.-Squamata.}

Reptilia in which the surface is covered with horny scales, sometimes with the addition of dermal ossifications. The opening of the cloaca is transverse in direction. There is a pair of eversible copulatory sacs in the male. The vertebræ are nearly always procœlous. The sacrum, absent in the Ophidia and some Pythonomorpha, consists of two vertebræ in the Lacertilia. The ribs have simple vertebral extremities. The quadrate is usually movably articulated with the skull. There is no inferior temporal arch. The nasal apertures of the skull are separate. The limbs, when present, are sometimes adapted for terrestrial locomotion (Lacertilia), sometimes for swimming (Pythonomorpha). The teeth are acrodont or pleurodont (see p. 345). The lungs are simple sacs. There is always a wide aperture of communication between the right and left divisions of the ventricular cavity. The optic lobes are approximated, and the cerebellum is extremely small.

\section{Sub-Order a.-Lacertilia.}

Squamata in which, as a rule, the limbs are present and are adapted for walking. The mouth is capable of being opened to only a moderate extent. The maxillæ, palatines, and pterygoids are incapable of free movement. The rami of the mandible are firmly united at the symphysis. There are nearly always movable eyelids and a tympanum. A sternum and an episternum are present.

Including all the Lizards, such as the Skincs, Geckos, Monitors, Iguanas, Amphisbænians, Chamæleons, and other groups.

\section{Sub-Order b.-Ophidia.}

Squamata with long narrow body, devoid of limbs. The mouth is capable of being opened to form a relatively very wide gape by divarication of the jaws. The maxillæ, palatines, and pterygoids are so articulated as to permit of free movement. The rami of the mandible are connected together only by elastic fibres at the symphysis, so that they are capable of being widely separated. There is no separate supra-temporal ossification. Sternum and episternum are absent. Movable eyelids and tympanum are absent.

Including all the Snakes-Vipers, Rattlesnakes, Sea-Snakes, Fresh-water Snakes, Tree-Snakes, Blind-Snakes, Pythons, and Boas.

\section{Sub-Order c.-Pythonomorpha.}

Extinct Squamata with elongated Snake-like body, provided with limbs which take the form of swimming-paddles. The skull resembles that of the Lacertilia; a supra-temporal helps to 
suspend the quadrate. The union of the rami of the mandible was ligamentous. There is, as a rule, no sacrum, the ilia not articulating with the spinal column.

\section{Order II.--Rhynchocephalia.}

Lizard-like, scaly Reptiles with well-developed pentadactyle limbs adapted for walking. The opening of the cloaca is transverse. There are no copulatory sacs. The vertebræ are amphicœlous, sometimes enclosing vestiges of the notochord. The sacrum consists of two vertebre. Numerous intercentra are present. The ribs have simple vertebral extremities, and are provided with uncinates (see p. 335). There is a system of abdominal ribs. The quadrate is immovably fixed to the other bones of the skull. There are both upper and lower temporal arches. The rami of the mandible are united by ligament. There is a sternum. The teeth are acrodont. The lungs, heart, and brain resemble those of the Squamata.

This order comprises only a single living genus, Sphenodon or Hatteria, together with a number of fossil forms.

\section{Order III.-Chelonia.}

Reptilia having the body enclosed in a shell of bony plates, consisting of a dorsal carapace and a ventral plastron, partly of dermal, partly of endoskeletal origin. There is usually on the surface an epidermal exoskeleton of horny plates. The vertebræ and ribs of the thoracic region are firmly fused with the bony carapace, into the composition of which they enter. The quadrate is immovably united with the skull. The nasal apertures in the skull coalesce into one. The limbs are sometimes terminated by clawed digits adapted for terrestrial locomotion, sometimes modified into the shape of flippers. There are no teeth, and the jaws have a horny investment. The lungs are compound sacs. In essentials the heart and brain resemble those of the Squamata. There are no copulatory sacs, but a median penis.

This order includes the Land Tortoises, Soft Tortoises, River and Mud Tortoises, and the Turtles, besides a number of fossil forms.

\section{Order IV.-Theromorpha.}

Extinct Reptiles with amphicœlous vertebræ sometimes enclosing remnants of the notochord, with a sacrum composed of from two to six vertebræ, and with ribs having bifid vertebral extremities. The quadrate is not movable. The limbs are adapted for walking. The pubes and ischia are united. The teeth, which are usually, though not always, present, are highly differentiated and lodged in sockets. 
This order comprises a large number of extinct Reptiles, which are grouped in the four sub-orders Anomodontia, Placodontia, Pareiosauria, and Theriodontia (Fig. 1022).

\section{Order V.-Crocodilia.}

Reptiles in which the dorsal surface, or both dorsal and ventral surfaces, are covered with rows of sculptured bony scutes supporting horny scales. The vertebral centra are either amphicœlous, flat at each end, or procclous. The anterior thoracic vertebræ have elongated and bifid transverse processes. The sacrum consists of two vertebræ. The ribs are bifid at their vertebral ends. The quadrate is immovable. A sternum is present, and there is a series of abdominal ribs. The limbs are adapted for walking. The teeth are lodged in sockets. The lungs are compound sacs. The ventricle of the heart is completely divided in recent forms. The opening of the cloaca is elongated in the direction of the long axis of the body. There is a median penis.

This order includes among living forms the true Crocodiles, the Gavials, the Alligators, and Caimans.

\section{Order VI.-Sauropterygia.}

Extinct aquatic Reptiles with elongated neck, small head, short tail, and usually flipper-like limbs. The centra are slightly amphicœlous or quite flat. The sacrum is composed of either one or two vertebræ. The cervical ribs are bifid, the thoracic simple. The quadrate bone is immovable. There is no sternum. The teeth are situated in sockets (Fig. 1023).

\section{ORder VII.-ICHTHYOPTERYGIA.}

Extinct aquatic Reptiles, with large head, without neck, and with elongated tail and completely flipper-like limbs. The centra are amphicolous, and there is no sacrum. The ribs are bifid at their vertebral ends. The quadrate is immovable. The premaxillæ are drawn out to form an elongated rostrum. There is no sternum, but there is a series of abdominal ribs. The teeth are lodged in a common groove. The integument is naked (Fig. 1026).

\section{Order Vili.-Dinosauria.}

Extinct terrestrial Reptiles with elongated limbs, having the surface sometimes naked, sometimes provided with a bony armour. The centra are usually amphicolous. The sacrum consists of from two to six vertebræ. The ribs are bifid. A sternum is present. The quadrate is fixed. The pelvis usually resembles that of a Bird, the ilium being extended fore and aft, and the pubis, as well as the ischium, directed backwards. The teeth 
are lodged in sockets, and usually have compressed crowns (Fig. 1027).

\section{Order IX.-Pterosauria.}

Extinct Reptiles, the structure of which is greatly modified in adaptation to a flying mode of locomotion. The vertebræ are procolous, the neck elongated. The sacrum contains three to six vertebræ. The anterior thoracic ribs are bifid. The skull resembles that of a bird in its general shape and in the obliteration of the sutures, but not in more essential features. There is a ring of sclerotic bones. The quadrate is immovable. There is a sternum. The fore-limbs are modified to act as wings by the great enlargement of the post-axial digit, for the support of a fold of skin. The posterior limbs are weak and have four or five digits. The teeth are implanted in sockets. In the brain the optic lobes were widely separated by the cerebellum, and the latter bore a pair of lateral processes or flocculi (Figs. 1029-1031).

\section{Systematic Position of the Example.}

'There are twenty known species of the genus Lacerta, occurring in Europe, Asia, Africa, and North America. Lacerta is a member of the sub-order Lacertilia of the order Squamata. The flattened and elongated tongue with notched apex places it in the section Leptoglossæ of that sub-order. Among the Leptoglossæ the family Lacertidæ, which comprises Lacerta and a number of other genera, is characterised by the presence of dermal bony supra-orbital and supra-temporal plates, by the presence of small granular or wedge-shaped scales, and of pleurodont conical teeth, excavated at the base. The chief distinctive marks of the genus Lacerta are the presence of comparatively large shields on the head and on the ventral surface, the arrangement of the scales of the trunk in transverse rows which become circular zones or rings on the tail, the development of a collar-like band of larger scales round the neck, and the laterally-compressed falciform claws, grooved on the lower surface.

\section{General Organisation of Recent Reptilia.}

External Features.-In external form, as in some other respects, certain of the Lacertilia exhibit the least specialised condition to be observed among the living Reptilia. Lacerta is such a central type, and the general account of that Lizard which has just been given applies in all the points of cardinal importance to a large proportion of the Lacertilia. Modifications take place, however, in a variety of different directions. Of such the following are a few of the chief. The tail region is usually, as in the example, extremely long and tapering; but in some groups of Lizards it is 
comparatively short and thick; and in others it is depressed and expanded into a leaf-like form. In the Chamæleons (Fig. 988) the long and tapering tail is used as a prehensile organ, the coiling of which round branches of the trees in which the animal lives aids in maintaining the balance of the body in climbing from branch to branch.

In the limbs there is likewise a considerable amount of variation in the different groups of the Lacertilia. Moderately long pentadactyle limbs lilke those of Lacerta are the rule. In the Chamæleons (Fig. 988) both fore- and hind-limbs become prehensile

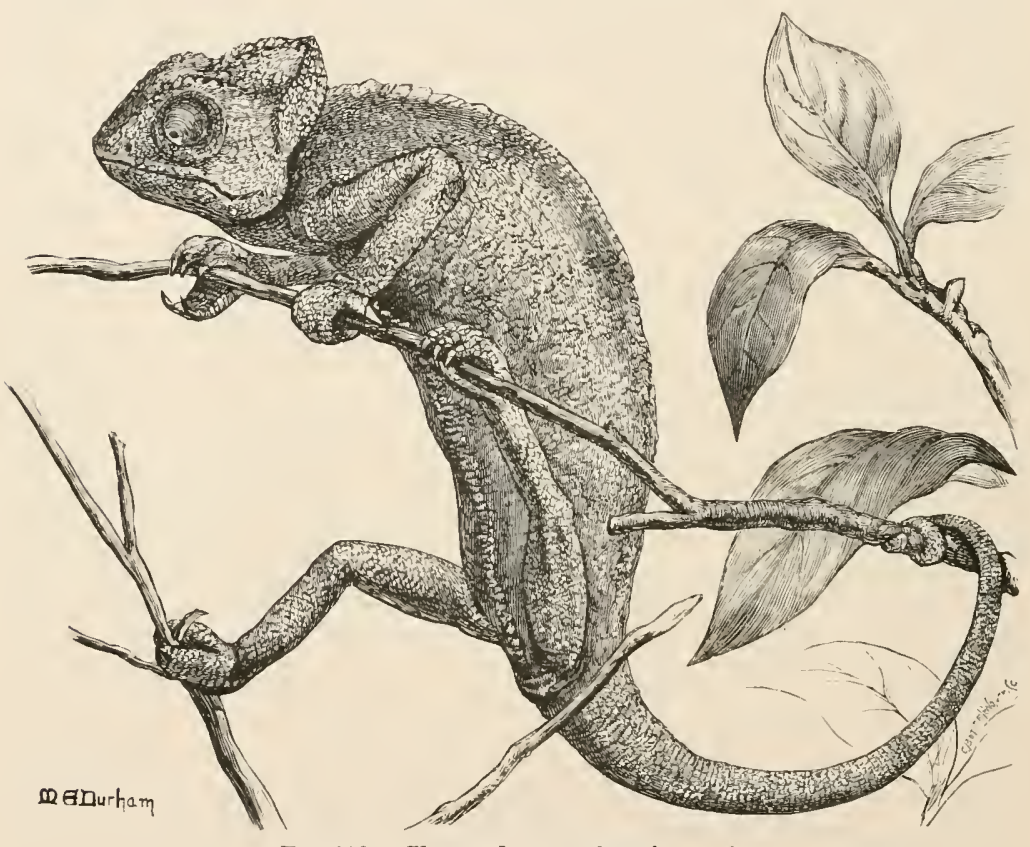

FIG. 988.-Chamæleon vulgaris, $\times \frac{2}{3}$.

(From the Cambridge Natural History.)

by a special modification in the arrangement and mode of articulation of the digits. In these remarkable arboreal Reptiles the three innermost digits of the manus are joined together throughout their length by a web of skin, and the two outer digits are similarly united: the two sets of digits are so articulated that they can be brought against one another with a grasping movement analogous to the grasping movenent of a Parrot's foot or of the hand of Man. A similar arrangement prevails in the pes, the only difference being that the two innermost and three outermost digits are united. In some groups of Lacertilia, on the other hand, such as the Blind-Worm (Anguis), limbs are entirely absent, or are 
represented only by mere vestiges; and numerous intermediate gradations exist between these and forms, such as Lacerta, with well-developed limbs. The limbless Lizards (Fig. 989) bear a very close resemblance to the Snakes, not only in the absence of the limbs, but also in the general form of the body and the mode of locomotion.

The body of a Snake is elongated, narrow and cylindrical, usually tapering towards the posterior end, sometimes with, more usually without, a constriction behind the head. In the absence of limbs the beginning of the short caudal region is only indicated by the position of the cloacal opening. The fore-limbs are never represented even by vestiges; in some Pythons there are inconspicuous vestiges of hind-limbs in the form of small claw-like processes. The mouth of the Snake is capable of being very widely opened by the free articulation of the lower jaw, and it is

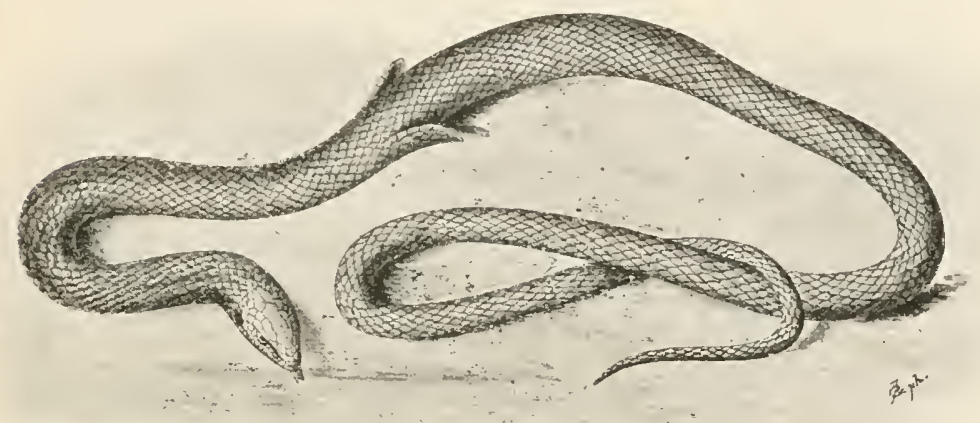

Fi木. 989.-Pygopus lepidopus, with scale-like vestiges of hind-limbs. (After Brehm.)

this mainly which distinguishes it from the snake-like Lizards. But other, less conspicuous, points of distinction are the absence of movable eyelids in the Snake, and also the want of a tympanum.

Sphenodon or Hatteria, the New Zealand Tuatara (Fig. 990), the only living representative of the Rhynchocephalia, is a Lizard-like Reptile with a well-developed laterally-compressed tail, and pentadactyle extremities, very sinilar to those of a typical Lizard. The upper surface is covered with small granular scales, and a crest of compressed spine-like scales runs along the middle of the dorsal surface. The lower surface is covered with transverse rows of large squarish plates.

In the Chelonia (Fig. 991) the body is short and broad, enclosed in a hard "shell" consisting of a dorsal part or carapace and a ventral part or plastron. These are in most cases firmly united, apertures being left between them for the head and neck, the tail and the limbs. The neck is long and mobile; the tail short. 


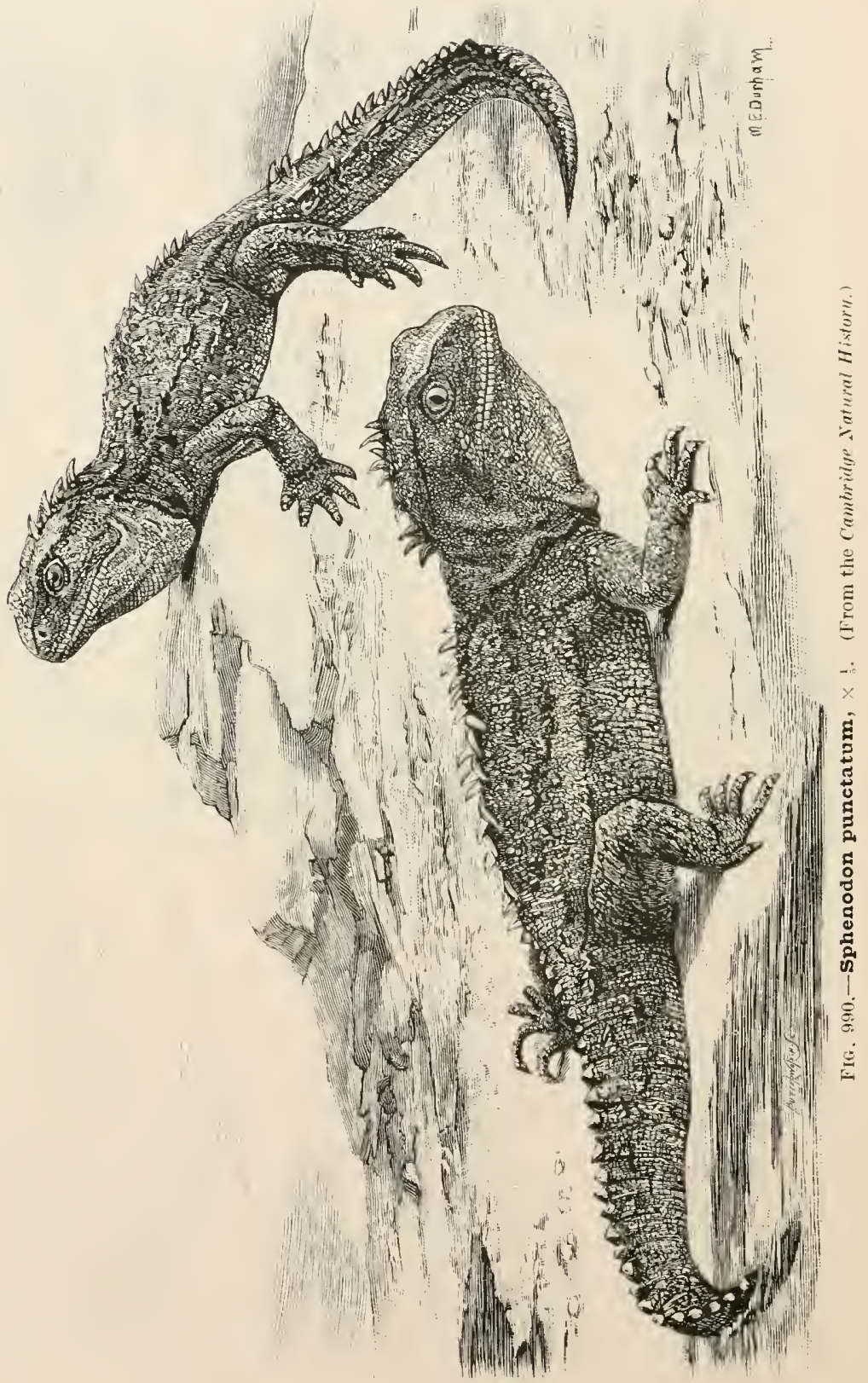


The limbs are fully developed though short. In some (land and fresh-water Tortoises) they are provided each with five free digits terminating in curved horny claws; in the Turtles the digits are closely united together, and the limb assumes the character of a "flipper" or swimming paddle. The cloacal aperture is longitudinal.

The Crocodilia, the largest of living Reptiles, have the trunk elongated and somewhat depressed, so that its breadth is much greater than its height. The snout is prolonged, the neck short, the tail longer than the body and compressed laterally. The limbs are relatively short and powerful, with five digits in the manus and four in the pes, those of the latter being partly or completely united by webs of skin. The eyes are very small;

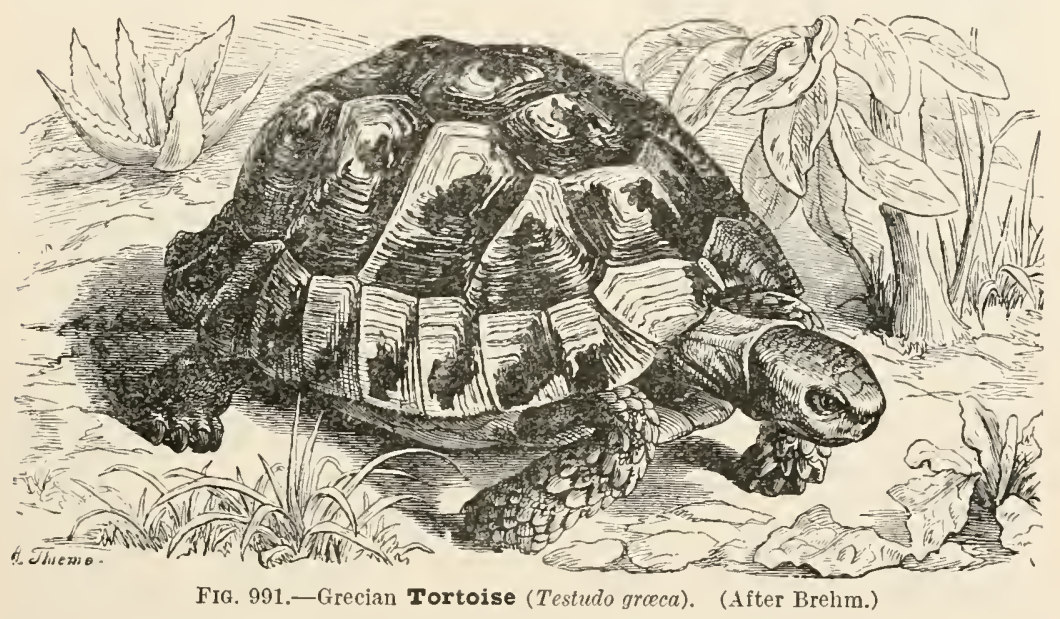

the nostrils placed close to the end of the snout and capable of being closed by a sphincter muscle. The cloacal aperture is a longitudinal slit. The dorsal and ventral surfaces are covered with thick, squarish horny scales, often pitted or ridged, those of the dorsal surface of the tail developed into a longitudinal crest.

Integument and Exoskeleton.-Characteristic of the Squamata is the development of horny plates which cover the entire surface, overlapping one another in an imbricating manner. These differ considerably in form and arrangement in different groups; sometimes they are smooth, sometimes sculptured or keeled. Sometimes they are similar in character over all parts of the surface; usually there are specially developed scales-the head shields-covering the upper surface of the head. In the majority of Snakes the ventral surface is covered with a row of large transversely elongated scales, the ventral shields. In certain Lizards 
(Chamæleons and Geckos) the scales are reduced and modified into the form of minute tubercles or granules. In some lizards special developments of the scales occur in the form of large tubercles or spines. Underlying the horny epidermal scales in some Lizards (Skincoids) are series of dermal bony plates. In the integument of the Geckos are numerous minute hard bodies which are intermediate in character between cartilage and bone.

In the Snake-like Amphisbænians there are no true scales, with the exception of the head shields, but the surface is marked out into annular bands of squarish areas.

In addition to the modification of the scales, the integument of the Chamæleons is remarkable for the changes of colour which it undergoes, these changes being due to the presence in the dermis of pigment-cells which contract or expand under the influence of the nervous system, reminding one of the integument of the Cephalopoda. Less conspicuous and rapid changes of colour take place in Anguis and in some Snakes.

In the Chelonia scales, when developed, are confined to the head and neck, the limbs and the tail ; but in all of them, with the exception of the Soft Tortoises, both dorsal and ventral surfaces are covered by a system of large horny plates. A series of horny head-shields usually cover the dorsal surface of the head. Beneath the horny plates of the dorsal and ventral surfaces are the bony carapace and plastron, partly composed of dermal bones, but so intimately united with elements derived from the endoskeleton that the entire structure is best described in connection with the latter (vide p. 335).

In the Crocodilia, the whole surface is covered with horny plates or scales, each usually marked with a pit-like depression about the centre, those on the dorsal surface ridged longitudinally. Underlying each of these, which are of epidermal derivation, is a thick pad of dermal connective-tissue which, in the case of the dorsal scales, is replaced by a bony scute. In the Caimans thin scutes also occur under the ventral scales.

A periodical ecdysis or casting and renewal of the outer layers of the horny epidermis takes place in all the Reptilia with the exception of the Crocodiles. Sometimes this occurs in a fragmentary manner; but in Snakes and many Lizards the whole comes away as a continuous slough.

Endoskeleton. - The vertebræ are always fully ossified. Among recent forms the Geckos and Sphenodon (Fig. 992) are exceptional in having the centra amphicœlous with remnants of the notochord in the intercentral spaces. The rest of the recent groups for the most part have the centra procolous. In many extinct forms the neural arches are not directly attached to the bodies by bone (temnospondyly) : in recent forms there is a bony union (stereospondyly) either through a suture or by fusion. 
Intercentra may be represented by intervertebral dises of fibrocartilage (Crocodilia) or by bony elements formed by ossification of the ventral portions of the dises (Geckos, Sphenodon). In Lizards in general and the Crocodiles there are inferior processes (hypapophyses), perhaps representing intercentra, situated below the centra in the anterior cervical region. Chevron bones (inferior arches) occur in the caudal region of many Reptiles (Sphenodon, Lacertilia, Crocodilia).

In the Snakes and in Iguanas, in addition to the ordinary articulating processes or zygapophyses, there are peculiar articular surfaces termed zygosphenes and zygantra (Fig. 993). The zygosphene is a wedge-like process projecting forwards from the anterior face of the neural arch of the vertebra, and fitting, when the vertebræ are in their natural positions, into a depression of corresponding

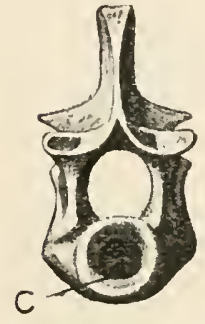

Fig. 992. - Vertebra of sphenodon, showing the amphicœlous centrum (C.). (After Headley.)
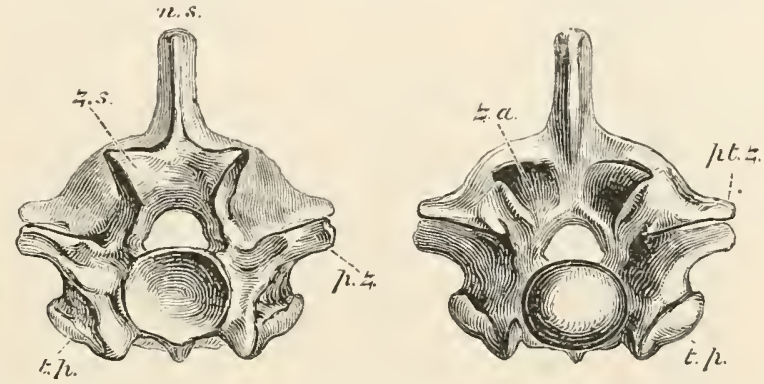

FIG. 993.-Vertebra of Python, anterior and posterior views. n. \&. neural spine; $p$. $z$. prezygapophyses ; $p t$. $z$. post-zygapophysis: t. $p$. transverse processes; z. la. zygantrum; z.s. zygosphene. (After Huxley.)

form-the zygantrum-on the posterior face of the neural arch of the vertebra in front. To this arrangement, as well as to the deeply concavo-convex centra, the extraordinary flexibility and strength of a Snake's back-bone are due.

The various regions of the spinal column are well marked in most of the Lizards, in the Rhynchocephalia, in the Chelonia, and in the Crocodilia (Fig. 994). In the Snakes and many of the snake-like Lizards only two regions are distinguishable-pre-caudal and caudal. In the others there is a sacral region comprising usually two vertebræ, both of which have strong processes (sacral ribs) for articulation with the ilia. The first and second vertebræ are always modified to form an atlas and axis: in the Lacertilia and Chelonia the latter has a distinct odontoid process. In Chamæleons, Sphenodon, and the Crocodiles there is a median bone, the pro-atlas (Fig. 996, O), intercalated between the atlas and the occipital region of the skull.

Ribs are developed in connection with all the vertebræ of the pre-sacral or pre-caudal region; in the caudal region they are 


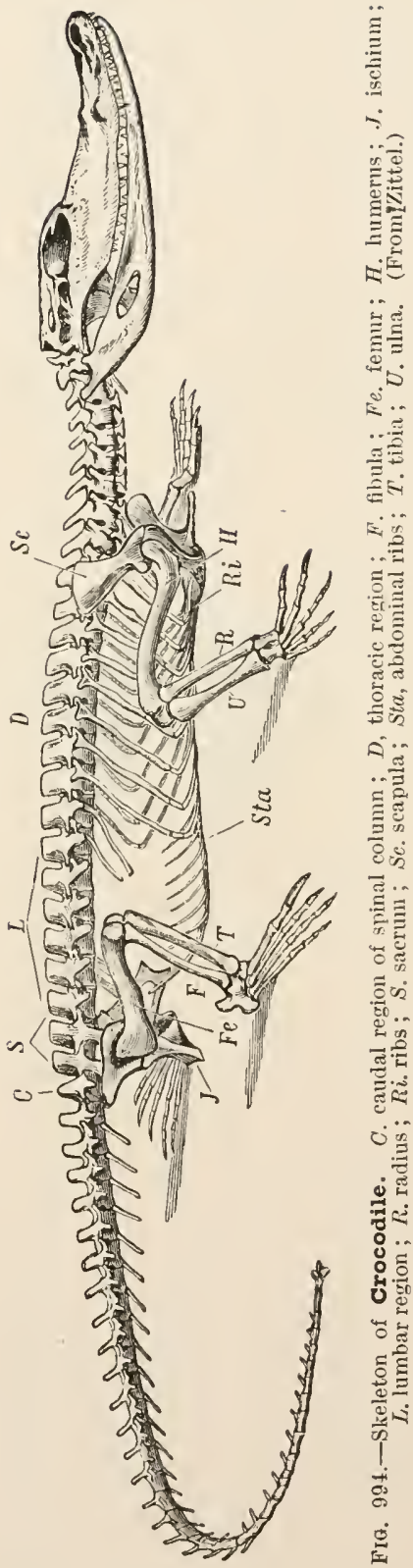

光管

i

4.

$\because 4 \bar{z}$

${ }_{00}^{4} \mathrm{O}$

星

150

คी

…

a

च苞

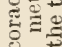

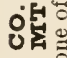

$\therefore$

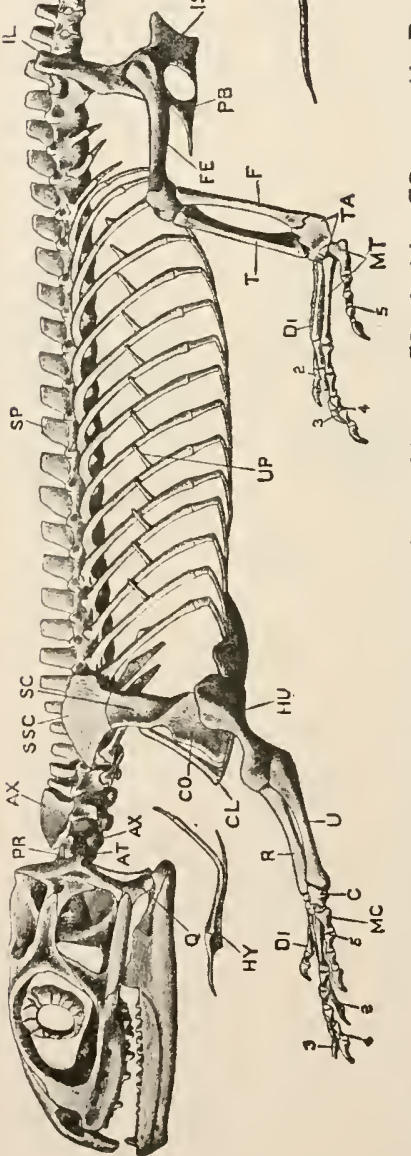

$\frac{\infty}{3}$

동

어ㅇㅛㅛ

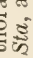

हี

…ㄹ.

పु以

空

:

$\dot{m g}$

:

ชั้

ธूँ

这密

$+$

棺

...ป

政影

巴를

0.

$\because n=$

....

远递

$4=\Phi$

건

造星

His

ह

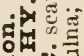
... 品

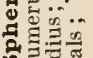
吃寻要 हैं E...

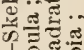

10 क्षिह s 
usually replaced by inferior arches; but Sphenodon, the Chelonia, and Crocodilia have caudal ribs which become fused with the vertebræ. In the Lacertilia only a small number (three or four) of the most anterior of the thoracic ribs are connected with the sternum by cartilaginous sternal ribs; the rest are free, or are connected together into continuous hoops across the middle line. In the so-called Flying Lizards (Draco) a number of the ribs are greatly produced, and support a pair of wide flaps of skin at the sides of the body, acting as wings, or rather as parachutes. In Sphenodon (Fig. 995) and Crocodilia (Fig. 991) each rib has connected with it posteriorly a flattened curved cartilage, the uncinate.

In the Chelonia (Fig. 997) the total number of vertebræ is always smaller than in the members of the other orders. The cervical ribs are small and fused with the vertebræ. The cervical and the caudal are the only regions in which the vertebræ are movable upon one another. The vertebræ of the trunk, usually ten in number, are immovably united with one another by means of fibro-cartilaginous intervertebral discs. Each of the neural spines, from the second to the ninth inclusively, is flattened and fused with a flat plate of dermal origin, the neural plate (Fig.

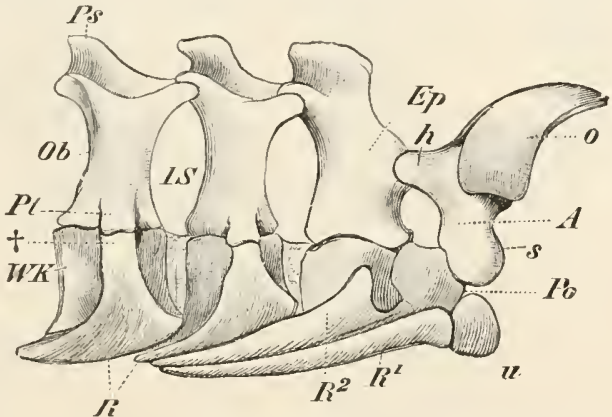

FIG. 996.-Anterior vertebræ of young Crocodile. $A$ atlas: $E p$ axis: $h$ articulation of atlas witul axis; $I S$. intervertebral dises; $o$. pro-atlas; $O b$. neural arches ; $P$ o. odontoid bone ; $P_{s}$. neural spines : $P t$. transverse processes; $R, R, 1, R,^{2}$ ribs; $s$, arch of atlas; $u$. median piece of atlas; IV $K$. centra. (From Wiedersheim's Comparative Anatomy.)

998), and the row of plates thus formed constitutes the median portion of the carapace. The ribs are likewise immovable; a short distance from its origin each passes into a large bony dermal costal plate, and the series of costal plates uniting by their edges form a large part of the carapace on either side of the row of neural plates. The carapace is made up of the neural and costal plates supplemented by a row of marginal plates (Figs. 997 and 998) running along the edge, and muchal and pygal plates situated respectively in front of and behind the row of neural plates. In some cases the neural plates (Chelodina) and even the costal plates and ribs (Testudo loveridgii) are absent.

The bony elements of the plastron of the Chelonia are an anterior and median plate (entoplastron) and four pairs of plates which are termed in their order from before backwards epiplastra, hyoplastra, hypoplastra, and xiphiplastra. The median element probably corresponds to the interclavicle or episternum of other 
Reptiles, the first pair (epiplastra) to the clavicles, the others probably being of the same character as the abdominal ribs of the

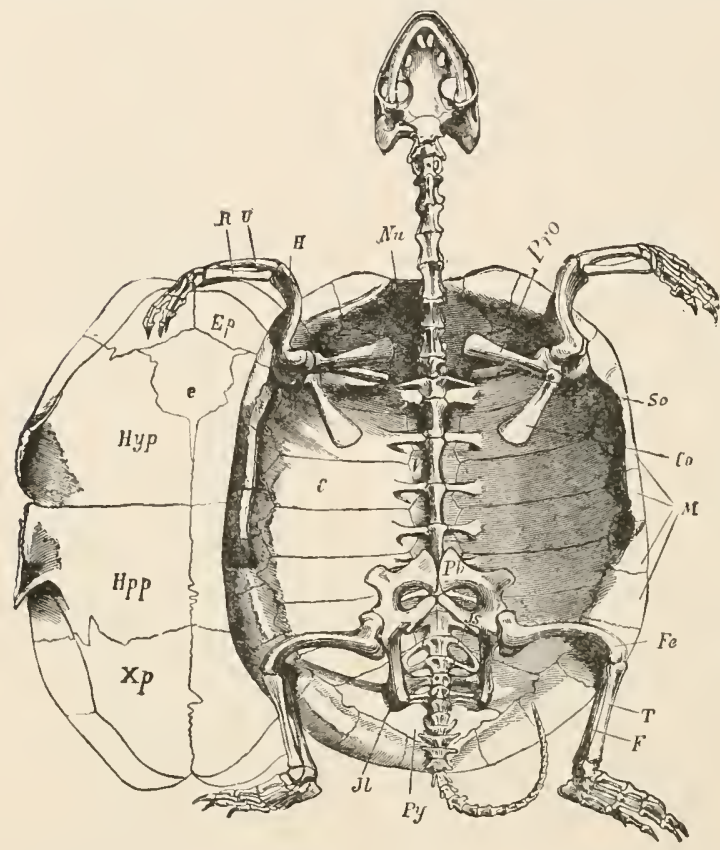

FIG. 997.-Cistudo lutaria. Skeleton seen from below; the plastron has been removed and is represented on one side. $C$. costal plate ; Co. coracoid ; e. entoplastron (episternum); $E p$. epiplastron (clavicle ?); $F$. fibula $; F e$. femur; $H$. humerus ; $H$ up . hyoplastron; $H p p$. hypoplastron ; $J l$. ilium ; $J_{S}$. ischium ; M. marginal plates: $N u$. nuchal plate; $P b$. pubis ; Pro. procoracoid or process of scapula; $P y$. pygal plates ; $R$. radius ; $S c$ scapula; $T$. tibia; $U$. ulna; $X p$. xiphiplastron. (From Zittel.)

Crocodilia.

The carapace of the Luth or Leather-backed Turtle (Dermatochelys or Sphargis) is distinguished from that of the rest of the order in being composed of numerous polygonal dises of bone firmly united together, and in not being connected with the endoskeleton; in the plastron the median bone is absent.

Carapace and plastron are firmly fixed together by bony union in most instances, but sometimes the connection is ligamentous.

The sternum in the Lacertilia is a plate of cartilage with a simple or bifid posterior continuation formed by the fusion of five or six pairs of ribs. In the Ophidia and Chelonia it is absent. In the Crocodilia it is a broad plate bearing the coracoids and two pairs of ribs with a posterior continuation which bifurcates behind.

A series of ossifications -the abdominal ribs-lie in the wall of the abdomen in the Crocodilia

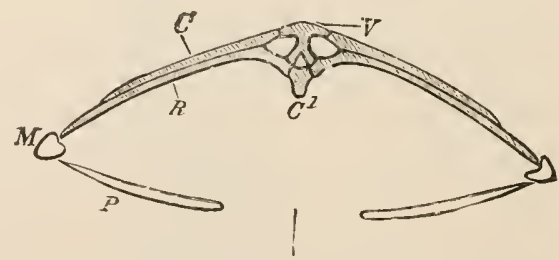

FIG. 993.-Chelone midas. Transverse section of skeleton. $C$. costal plate ; $C .^{1}$ centrum ; $M$. marginal plate; $P$. lateral element of plastron; $R$. rib $V$. expanded neural plate. (After Huxley.)

(Fig. 994, Sta), and similar ossifications occur also in the Monitors and in Sphenodon. As already noticed, the posterior 
elements of the plastron of the Chelonia are probably of a similar character.

In the skull ossification is much more complete than in the Amphibia, the primary chondrocranium persisting to a considerable extent only in some Lizards and in Sphenodon; and the number of bones is much greater. The parasphenoid is reduced, and its place is taken by the large basioccipital, basisphenoid, and presphenoid.

A fairly typical Lacertilian skull has been described in the case of Lacerta. Its principal characteristic features are the presence of
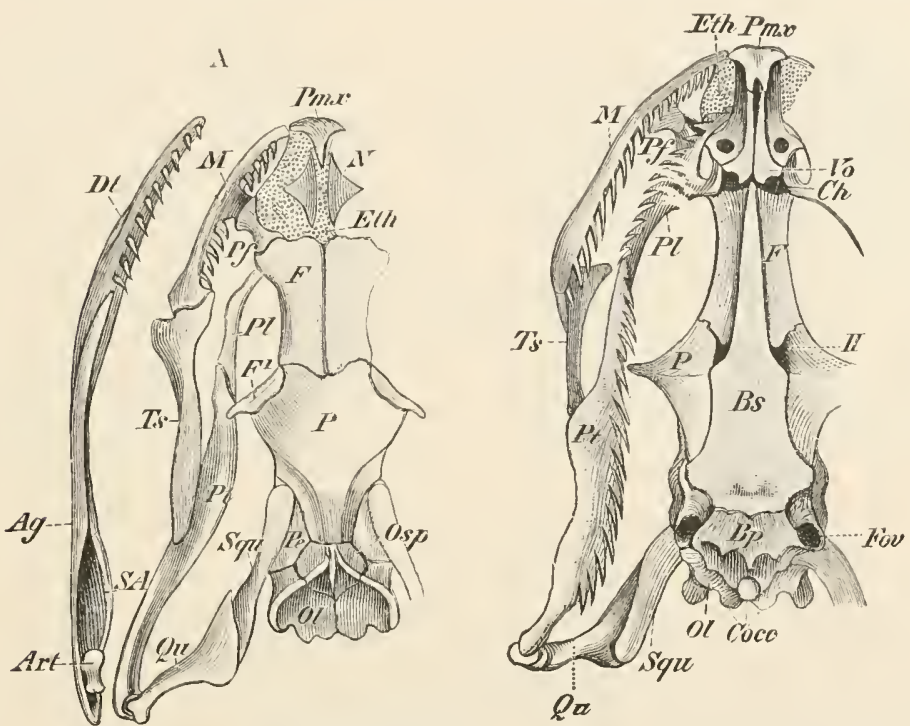

FIG. 999.-Skull of Colubrine Snake (Tropidonotus natrix). A, from above; $\mathbf{B}$, from below A $q$. angular ; $\boldsymbol{A} t$. articular; $B p$. basi-occipital ; Bs. basi-sphenoid ; $C h$. internal nares ; Cocc. occipital condyle ; $D t$. dentary ; Eth. ethmoid ; $F$. froutal: $F^{\prime}$. post-orbital ; Fov. fenestra ovalis ; $M$. maxilla ; $N$. nasal ; Ol. exoccipital; Osp. supra-occipital; $P$. parietal ; $P e$. periotic; $P$. $f$. pre-frontal ; $P l$. palatine ; $P m x$. premaxilla; $P t$. pterygoid; $Q u$. quadrate; SA. supra-angular; Squ. squamosal; Ts. transverse; Vo. vomer; $I I$, optic foramen. (From Wiedersheim's Comparative Anatomy.)

an inter-orbital septum, the presence of the epipterygoid, and the mobility of the quadrate. The last of these features it shares with that of the Ophidia. The epipterygoid is not universal in the Lacertilia, being absent in the Geckos, the Amphisbænians, and the Chamæleons. The quadrate is not always movable. The skull of the Chamæleons has a remarkable helmet-like appearance owing to the development of processes of the squamosal and occipital regions, which unite above the posterior part of the cranial roof. The skull of the Amphisbænians differs from that of other Lacertilia and approaches that of Snakes in the absence of an inter-orbital septum.

In the skull of the Ophidia (Fig. 999) orbitosphenoidal and 
alisphenoidal elements are absent, their places being taken by downward prolongations of the parietals and frontals. In the substance of the mesethmoid are two cartilaginous tracts (Fig. $1000, \mathbf{B}, T$ ) which are the persistent trabeculæ of the embryonic skull. The inter-orbital septum is absent, and the cranial cavity is prolonged forwards to the ethmoidal region. Neither upper nor lower temporal arches are present. The palatines $(P l)$ are movably articulated with the base of the skull; as in the Lizards, they are widely separated from one another, and do not develop palatine plates. They are movably articulated behind with the pterygoids $(P t)$, and the latter, through the intermediation of the slender

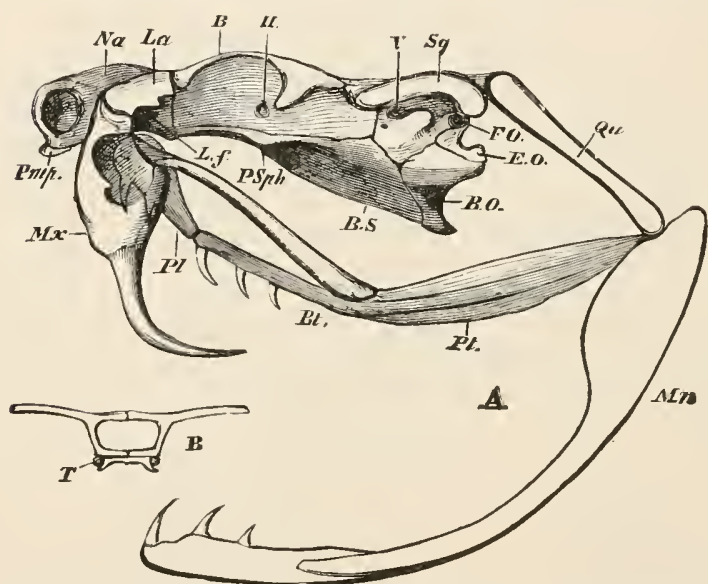

Fig. 1000,-A, lateral view of skull of Rattlesnake (Crotalus). $B$. O. basi-occipital ; $B . S$. basi-sphenoid ; $E$. O. exoccipital ; $F$. O. fenestra ovalis ; La. conjoined lacrymal and pre-frontal $L$. $f$. articulation between lacrymal and frontal ; $\boldsymbol{M} n$. mandible ; $M x$. maxilla ; $N a$. nasal ; $P l$. palatine ; $P m p$. premaxilla $P$. Sph. presphenoid ; $P t$. pterygoid ; $Q u$. quadrate; $S q$. squamosal; II, V, foramina of exit of the second and fifth cranial nerves. B, transverse section at point lettered $B$ in Fig. $\mathbf{A} ; T$. trabeculæ. (After Huxley.) transverse bones $(T s)$, with the maxillæ. The premaxillæ are very small (in some venomous Snakes entirely absent), and when present usually fused together. $\mathrm{Th} \mathrm{e}$ maxillæ $(M x)$, usually short, articulate by means of a movable hingepoint with the conjoined lacrymal and prefrontal $(L a)$, which, in turn, is movably connected with the frontal. The long and slender quadrate $(Q u)$ is freely articulated with the posterior end of the elongated squamosal. The rami of the mandible, likewise long and slender, are not united anteriorly in a symphysis, but are connected together merely by elastic ligamentous tissue, so that, when the mouth of the Snake is opened to allow of the entry of the relatively large prey, which it swallows whole, they are capable of being widely separated from one another. The Typhlopidæ differ from the rest of the Ophidia in having the maxillæ immobile, the quadrate more closely connected with the skull, and the rami of the mandible united by a fibro-cartilaginous symphysis.

The skull of Sphenodon (Fig. 1001) differs very considerably from that of the Lizards. There is a large supra-temporal fossa bounded by the parietal, post-orbital $(P t . f)$, and squamosal, and 
separated below by a bar of bone (superior temporal arch), formed of processes of the two last-mentioned bones and of the postfrontal, from a still larger space-the lateral temporal fossa. The latter is bounded below by a slender bony bar (the inferior temporal arch), formed of the long narrow jugal (Jug), with a small quad-
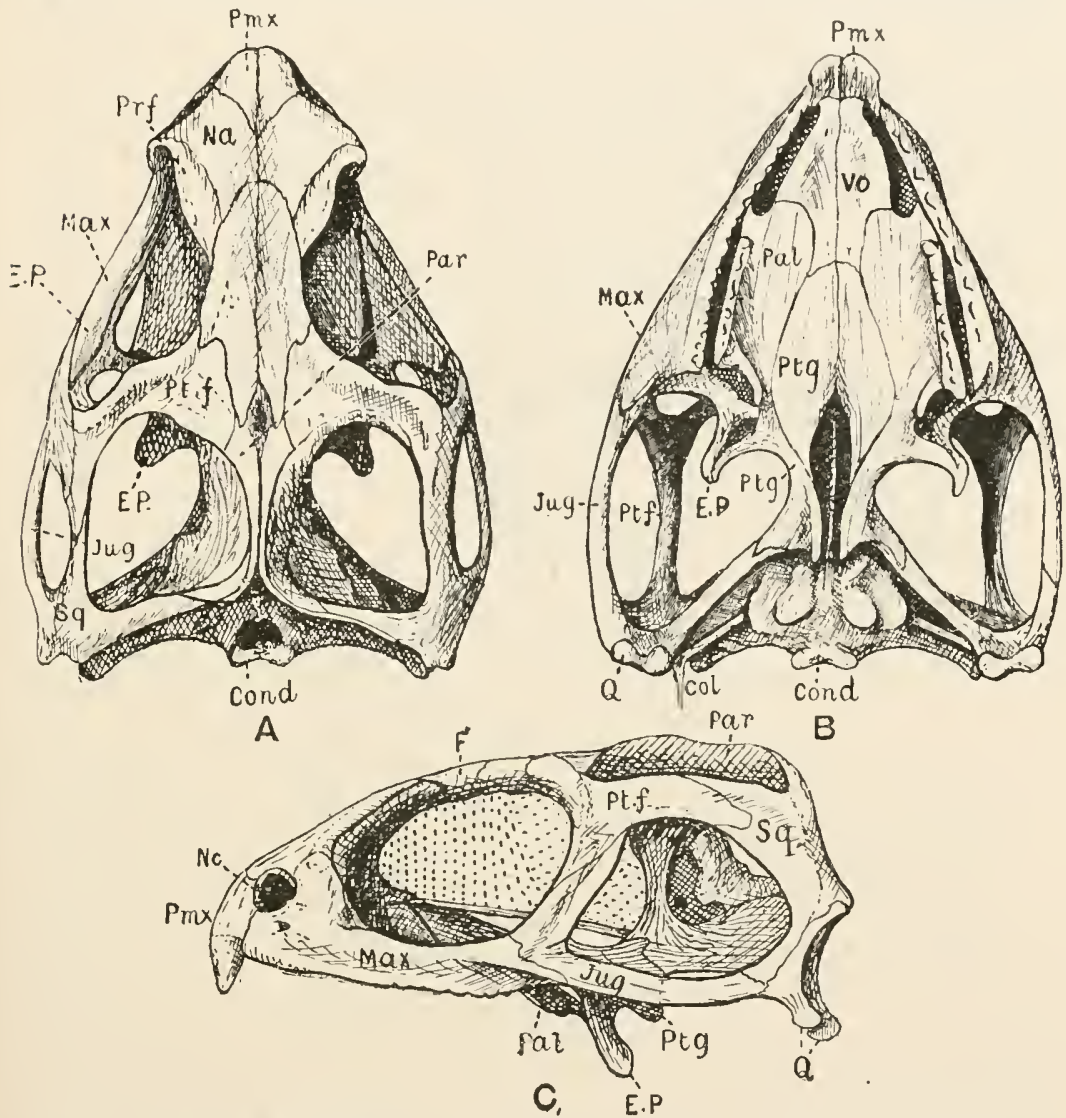

Fig. 1001.-Skull of Sphenodon. $A$, dorsal ; $B$, ventral ; $C$, left-sided riew of skull of Sphenodon, $\times 3$. Col Columella auris; Cond, occipital condyle ; $E$. $P$. ectopterygoid : F. frontal; Jug. jugal; Ma.x, maxilla; Na. nasal; Nc. anterior nasal opening; $P a l$. palatine; Par. parietal; Pmx. premaxilla ; Prf. pre-frontal ; Pt. $f$. post-frontal and postorbital ; Ptg. pterygoid or endopterygoid ; $Q$. quadrate and quadrato-jugal (paraquadrate); Sq. squamosal; Vo. vomer. (From the Cambridge Natural History.)

rato-jugal or paraquadrate, by which the jugal is connected with the quadrate $(Q)$. The lateral temporal fossa is separated from the orbit in front by a bar of bone formed of the jugal and postorbital, and is bounded behind by a posterior temporal arch formed of the parietal and squamosal. The quadrate $(Q u)$ is immovably fixed, wedged in by the quadrato-jugal, squamosal and pterygoid. The premaxillæ $(\dot{P} m x)$ are not fused together, but separated by 
a suture. There is a broad palate formed by the plate-like vomers, palatines, and pterygoids.

In the Chelonia (Figs. 1002, 1003) all the bones, including the quadrate, are solidly connected together. Transverse bones (ectopterygoids), lacrymals, orbitosphenoids and alisphenoids are absent. The place of alisphenoids is taken to a certain extent by vertical downward plate-like extensions of the parietals, the lower part of the plates perhaps representing the epipterygoids of Lizards. There may be open temporal fossæ, the inferior boundary of which (inferior temporal arch) may be incomplete owing to the absence of the quadrato-jugal (paraquadrate), or the entire temporal region may be covered over (Turtles, Fig. 1003) by a sort of false roof formed of expansions of the post-frontals $(p h)$, parietals $($ par $)$, and

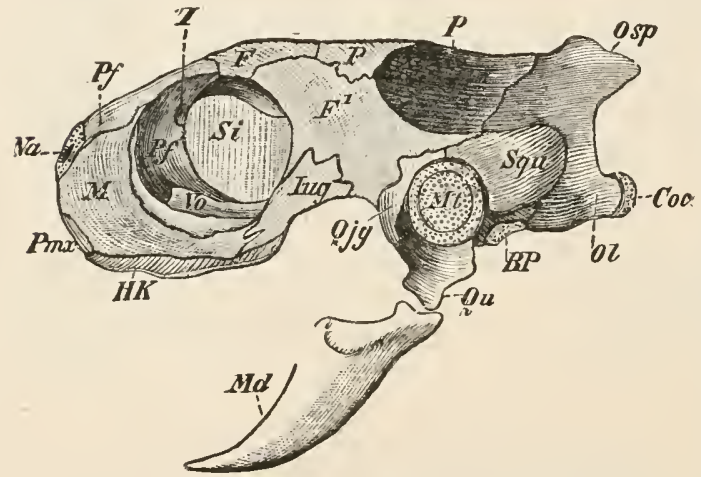

Fic. 1002.-Lateral view of skull of Emys europæa. Coc. occipital condyle; $F$. frontal ; $F^{l}$. post-frontal ; $I$, foramen by which the olfactory nerve enters the orbit; I ug. jugal; II. maxilla; $\boldsymbol{M} d$. mandible ; $\boldsymbol{M} t$. tympanic membrane ; $N a$. external nares ; $O l$. exoccipital ; Osp. supra-occipital ; $P$. parietal; $P f$. pre-frontal; $P m x$. pre-maxilla; $Q j g$. quadrato-jugal; $Q u$. quadrate ; $S i$. inter-orbital septum; Squ. squamosal; Vo. vomer. (From Wiedershein's Comparative Anatomy.)

plates, which for a short distance cut off a nasal passage from the cavity of the mouth. Nasals are usually absent as separate bones. The premaxillæ are very small. The rami of the mandibles are stout, and are firmly united together at the symphysis.

In the Crocodiles (Figs. 1004, 1005), as in the Chelonia, the quadrate $(Q u)$ is firmly united with the other bones of the skull. There is a membranous and cartilaginous inter-orbital septum. There are no distinct orbitosphenoids, but alisphenoids are well developed. The orbit is separated from the lateral temporal fossa by a stout bar situated somewhat below the surface, and formed of processes from the post-frontal, jugal and ectopterygoid. The lateral temporal fossa is bounded below, as in Sphenodon, by an inferior temporal arch composed of jugal and quadratojugal (paraquadrate). The frontals are early united into one, 
and the same holds good of the parietals. Both palatine $(P l)$ and pterygoid $(P t)$, as well as maxillæ, develop palatine plates in the roof of the mouth, cutting off a nasal passage of great length from the cavity of the mouth, the posterior nares $(c h)$ being situated far back towards the posterior end of the cranial base. The nature

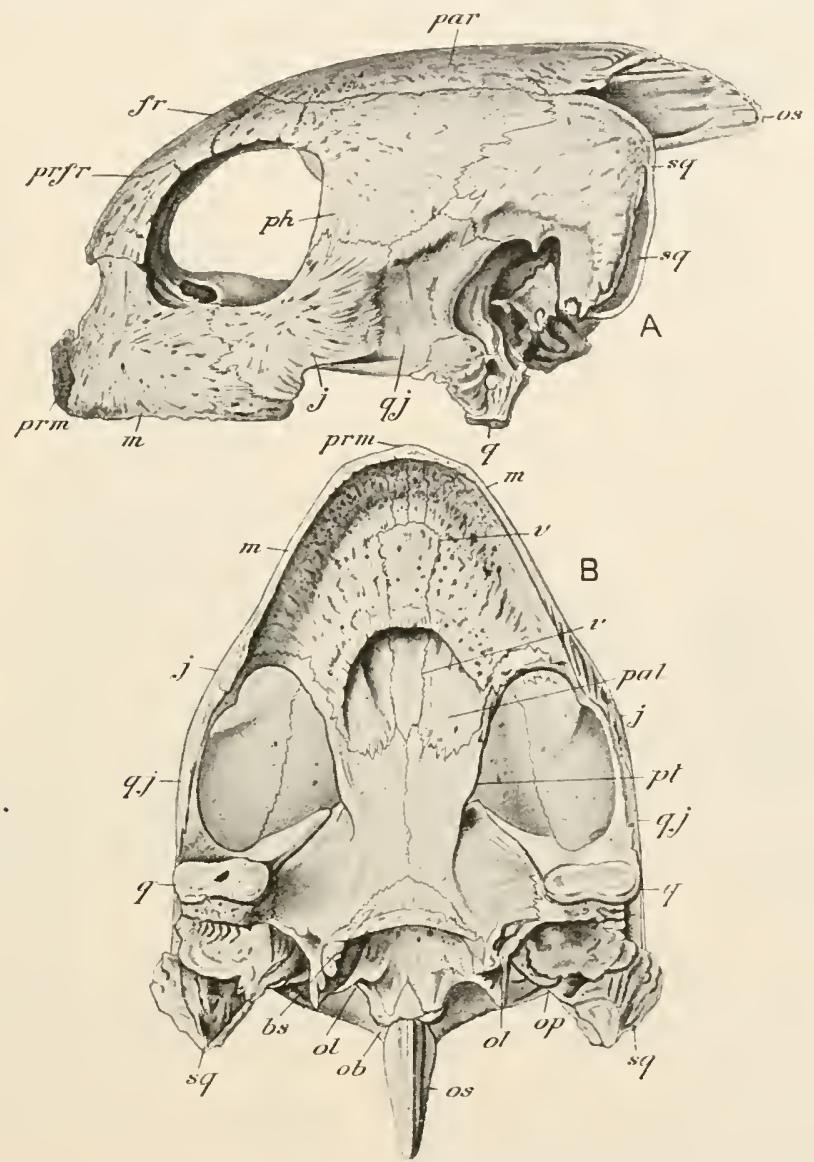

Fig. 1003-Ventral view of the skull of Chelone mydas. bs. basi-sphenoid; fr. frontal $j$.jugal ; m. maxilla ; ob. basi-occipital ; ol. exoccipital ; op opisthotic ; os. supra-occipital ; pal. palatine ; par. parietal; ph, post-frontal; prfr. pre-frontal; pt. pterygoid ; prm. preinaxilla ; $q$. quadrate ; $q j$. quadrato-jugal ; $s q$. squamosal ; $v$. vomer. (After Hoffmann.)

of the articulation between the mandible and the quadrate is such that movement is restricted to the vertical plane, and lateral displacement is further provided against by the development of a broad process of the pterygoid against which the inner surface of the mandibular ramus plays, an arrangement which occurs also in most Lacertilia. 
In accordance with their purely aërial mode of respiration, the visceral arches are much more reduced in the Reptilia than in the Amphibia in general. The only well-developed post-mandibular arch is the hyoid, and even this may undergo considerable reduction (Ophidia). The branchial arches are greatly reduced or aborted in the adult.

There is little variation in the structure of the limb-arches

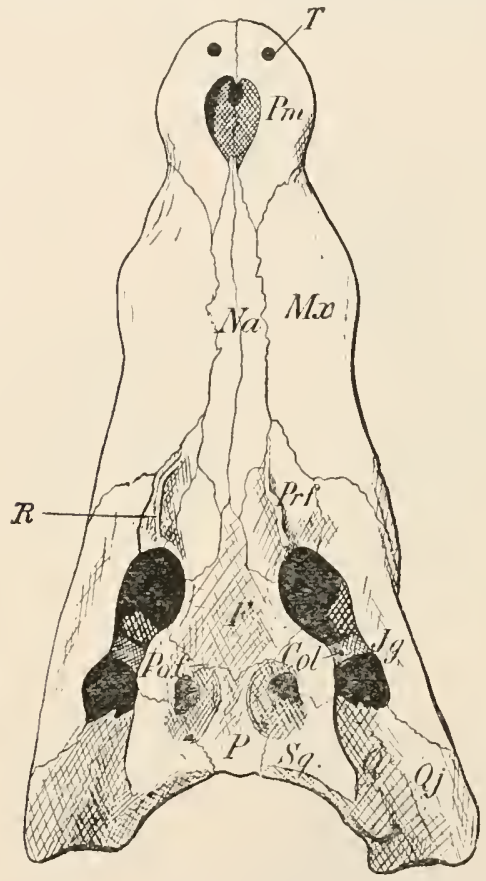

FiG. 1004.-Skull of Crocodilus porosus, dorsal view, $x$ about $\frac{1}{6}$. Col. buttress connecting the post-frontal with the jugal and ectoptery goid ; $F$. frontal ; Jg. jugal ; $M x$. maxilla; $N a$. nasal; $P$. parietal;

Pm. premaxilla; Po. f.' post-frontal ; Pr. f. pre-frontal; $Q$. quadrate ; $Q j$. quadrato-jugal; $R$. characteristic ridge on the pre-frontal bone ; $S q$. squamosal; $T$. perforation in the premaxilla caused by a pair of lower incisor teeth. (After Gadow.)

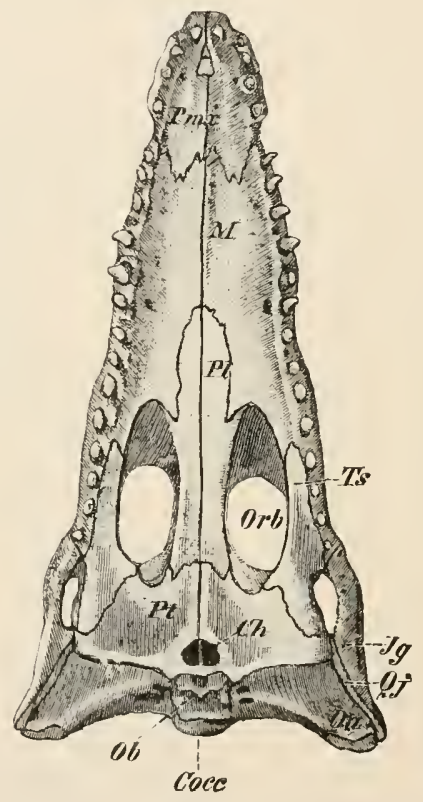

Fig. 1005.- Kentral view of the skull of young Crocodile. $C h$, posterior nares; Cocc. occipital condyle; $J g$. jugal ; $M$. maxilla (palatine process); $O b$. basi-occipital; Orb. orbit; $P l$. palatine; $P m x$. premaxillæ ; Pt. pterygoid ; $Q j$. quadrato-jugal: Qu. quadrate. (From Wiedersheim's Comparative Anatomy.)

and skeleton of the limbs in the different groups of Lacertilia. The pelvic arch is distinguished in the Lacertilia in general by its slender character; and the pubes and ischia are, as in fact is the case throughout the class, separated from one another by wide ischio-pubic foramina - a feature which markedly distinguishes the reptilian pelvis from that of the Amphibia. In limbless forms the pectoral arch may be present or may be absent. In the Ophidia all trace of limbs is, as a rule, absent; but in some 
Pythons vestiges of hind-limbs are to be detected in the form of two or three small bones which support a small horny claw.

In Sphenodon (Fig. 995) there is a foramen above the outer and one above the inmer condyle of the humerus. There are eleven carpal elements, of which there are four, including a pisiform, in the proximal row, two centrals, and five in the distal row. The pubes are united in a symphysis, in front of which is a cartilaginous epipubis. A large oval foramen intervenes between the ischium and the pubis. A cartilaginous hypo-ischium is attached to the ischia behind. In the tarsus the tibial and fibular elements are distinct, though firmly united. The intermedium and the

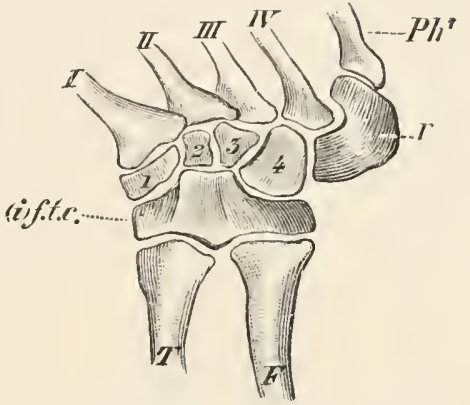

FiG. 1006.-Tarsus of Emys europaea (right side) from above. $F$. fibula; $T$. tibia; $(i) f .$.$t . c$. the united tarsals of the proximal row; $P h^{\prime}$. first phalanx of the fifth digit ; $1-4$, distal tarsals ; $I-V$, metatarsals. (From Wiederslieim's Comparative Anatomy.) centrale are firmly fixed to the tibiale. There are three distal tarsal bones.

In the Chelonia (Fig. 997) the interclavicle (episternum) and clavicles are absent, unless, as is probable, the former be represented by the median element of the plastron and the latter by the first

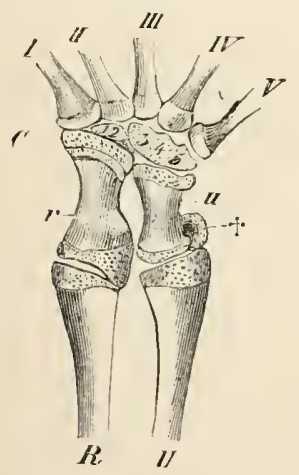

FIG. 1007.-Carpus of young Alligator. C. centrale (?); $R$. radius; $U$. ulna; $r$. radiale ; $u$. ulnare ; $1-5$, the five distal carpals (not yet ossified); 1 and 2 united into one, and also 3,4 , and 5 ; $\dagger$, pisiform; $I-V$, the five metacarpals. (From Wiedersheim's Comparative Anatomy.) lateral pair. The entire pectoral arch is a tri-radiate structure of which the most ventral and posterior ray, ending in a free extremity, is the coracoid; while the other two are the scapula and a process, sometimes regarded as representing the procoracoid, given off on the inner side of the scapula near its glenoid end. The bones of the carpus have nearly the typical arrangement, consisting, as in Lizards, of a proximal row of three, a distal row of five, and a centrale between the two. The pelvis resembles that of Lacertilia, except that it is broader and shorter. Both pubes and ischia meet in ventral symphyses, and epipubic and hypo-ischial cartilages may be present. In the tarsus (Fig. 1006) there is usually a single proximal bone and four distalia. There are never more than two phalanges in any of the digits.

In the Crocodilia also the clavicle is absent, but there is an episternum. The number of carpal elements is reduced, the largest being two proximal bones, the radiale and the ulnare (Fig. 1007, $r, u$ ). 
On the ulnar side of the latter is a small accessory bone (pisiform, $\dagger$ ). The pelvic arch (Fig. 1008) differs somewhat widely from that of other living Reptiles, and the parts have been variously interpreted. Two bones $(P)$, which are usually regarded as the pubes, extend from the region of the acetabula forwards and inwards, but, though they become closely approximated anteriorly, do not meet in a symphysis. Between and in front of their anterior extremities, which are tipped with cartilage, extends a membrane $(M)$ with which are connected in front the last pair of abdominal ribs $(B R)$. The posterior ends of the pubes are cut off from the acetabulum

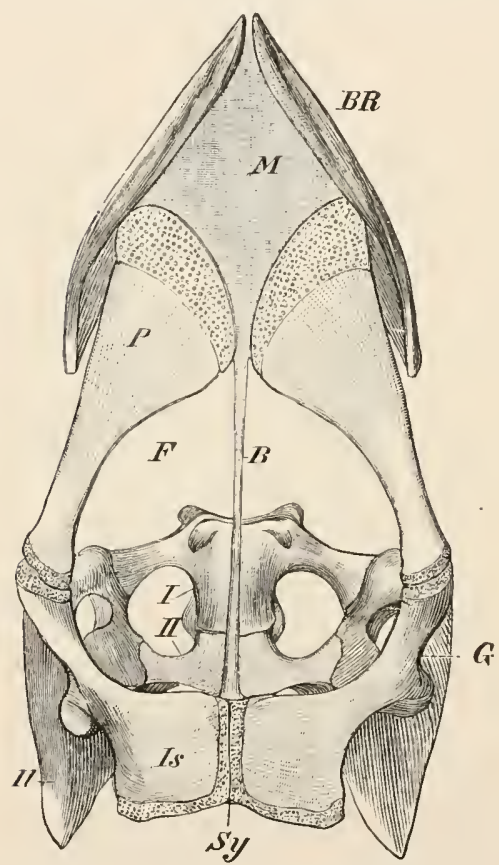

FIG. 1008.-Pelvis of young Alligator, ventral aspect. $B$, fibrous band passing between the pubic and ischiatic symphyses; $B R$. last pair of abdominal ribs; $F$. obturator foramen; $G$. acetabulum; $I l$. ilium ; Is. ischium; $M$. fibrous membrane between the anterior ends of the two innominate bones and the last pair of abdominal ribs; $P$. pubis; $S y$. ischiatic symphysis; $I, I I$, first and second sacral vertebræ. (From Wiedersheim's Comparative Anatomy.) by the interposition of a pair of bones which may be parts of the ilia, but are separately ossified. The ischia extend downwards and somewhat backwards from the acetabula and are fixed together ventrally (at $S y$. ), but there is no true symphysis, as their extremities remain cartilaginous. A hypoischium is not present. In the tarsus (Fig. 1009) there are two proximal bones - an

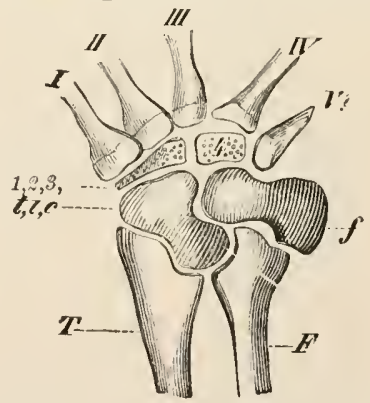

FIG. 1009.-Tarsus of Crocodile (righ side) from above. $\boldsymbol{F}$. fibula: $T$. tibia ; $t$. $i$. c. the astragalus, formed of the united tibiale, intermedium and centrale; $f$. fibulare (calcaneum) ; $1-3$, united first, second and third distal tarsals ; 4 , fourth tarsal ; $I-I V$, first to fourth metatarsals; $V$ ? fifth distal tarsal and fifth metatarsal. (From Wiedersheim's Comparative Anatomy.)

astragalo-scaphoid and a calcaneum-the latter laving a prominent calcaneal process, and two distal tarsal bones, together with a thin plate of cartilage supporting the first and second metatarsals. The missing fifth digit is represented by a rudimentary metatarsal. 
Digestive Organs. - The form and arrangement of the teeth already described in the account of Lacerta prevail in the majority of Lizards. In some of them the palatine teeth are absent. The teeth are sometimes fixed by their bases to the summit of the ridge of the jaw (acrodont forms), sometimes fixed by their sides to the lateral surface of the ridge (pleurodont); they are never embedded in sockets in any recent form. A Mlexican Lizard, Heloderma, differs from all the rest in having teeth which are grooved for the ducts of poison-glands. In the Snakes (Figs. 999, 1000) teeth are rarely developed on the premaxillæ, but are present on the maxillæ, palatines and pterygoids, as well as the dentary of the mandible. They may be of the same character throughout, solid, elongated, sharp-pointed teeth, which are usually strongly recurved, so that they have the character of sharp hooks, their function being to hold the prey and prevent it slipping from the mouth while being swallowed,-not to masticate it. Nonvenomous Snakes possess only teeth of this character. In the venomous Snakes more or fewer of the maxillary teeth assume the character of poison-fangs. These are usually much larger than the ordinary teeth and either grooved or perforated by a canal for the passage of the duct of the poison-gland. In the Vipers (Fig. 1000) there is a single large curved poison-fang with small reserve-fangs at its base, these being the only teeth borne by the maxilla, which is very short; in the venomous Colubrine Snakes the poison-fangs are either the most anterior or the most posterior of a considerable range of maxillary teeth. In the Vipers the large poison-fang is capable of being rotated through a considerable angle, and moved from a nearly horizontal position, in which it lies along the roof of the mouth embedded in folds of the mucous membrane, to a nearly vertical one, when the Snake opens its mouth to strike its prey. The rotation of the maxilla is brought about by the backward or forward movement of the pterygoid with the palatine and transverse. In Sphenodon (Fig. 1001) there are pointed, triangular, laterally-compressed teeth, arranged in two parallel rows, one along the maxilla, the other along the palatine. The teeth of the lower jaw, which are of similar character, bite in between these two upper rows, all the rows becoming worn down in the adult in such a way as to form continuous ridges. Each premaxilla bears a prominent, chisel-shaped incisor, represented in the young animal by two pointed teeth. In the young Hatteria a tooth has been found on each vomer-a condition exceptional among Reptiles. In the Chelonia, teeth are entirely absent, the jaws being invested in a horny layer in such a way as to form a structure like a Bird's beak. The Crocodilia have numerous teeth which are confined to the premaxillæ, the maxillæ, and the dentary. They are large, conical, hollow teeth devoid of roots, each lodged in its socket or alveolus (thecodont), and each 
becoming replaced, when worn out, by a successor developed on its inner side.

A bifid tongue like that of Lacerta occurs in several families of Lacertilia. Others have a thick, short tongue, undivided in front and often provided with two long appendages behind. The Monitors (Fig. 1010, A) have forked retractile tongues like those of Snakes. The tongue of the Chamæleons is an extremely remarkable organ; it is of sub-cylindrical form with an enlarged extremity, and is so extensile that it is capable of being darted out to a distance sometimes equalling, or even exceeding, the length of the trunk; this protrusion can be effected with lightninglike rapidity; and it is in this way that the animal catches the Insects which constitute its food. The tongue in Snakes is slender
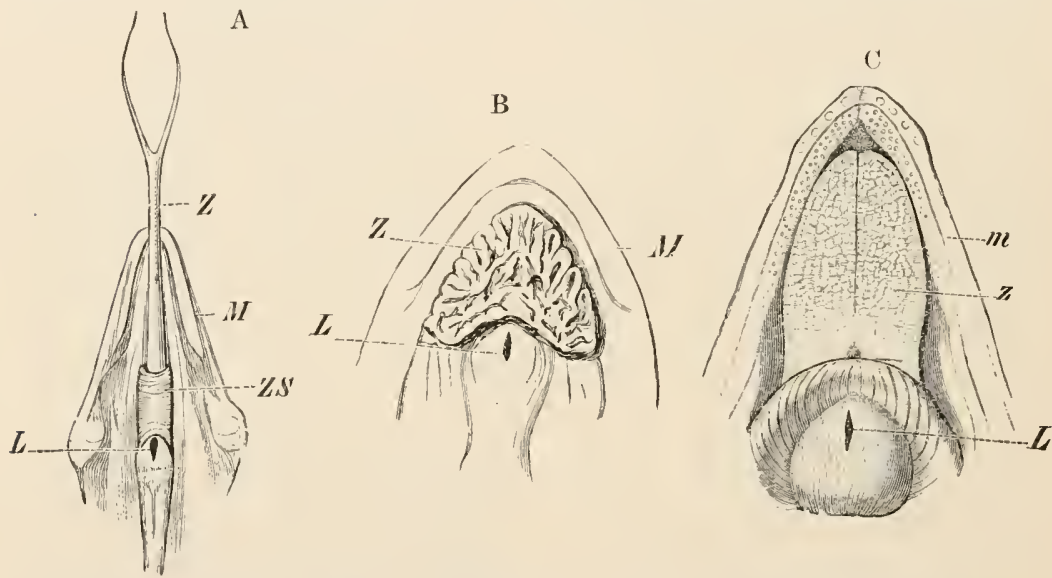

HIG. 1010.-A, tongue of Monitor indicus. B, tongue of Emys europæa. C, tongue of Alligator. $L$, glottis; $M$, mandible ; $Z$, tongue ; $Z S$, tongue-sheath. (From Wiedersheim's Comparative Anatomy.)

and bifid, capable of being retracted into a basal sheath, and highly sensitive, being used chiefly as a tactile organ. The tongue of the Crocodilia (C) is a thick, immobile mass extending between the rami of the mandible. In some of the Chelonia (B) the tongue is immobile; in others it is protrusible, sometimes bifid.

In the enteric canal of the Reptiles the principal special features to be noticed are the muscular gizzard-like stomach of the Crocodilia, the presence of a rudimentary cæcum at the junction of small and large intestines in most Lacertilia and in the Ophidia, and the presence of numerous large cornified papillæ in the œsophagus of the Turtles.

Organs of Respiration.-The Reptiles all have an elongated trachea, the wall of which is supported by numerous cartilaginous rings. The anterior part of this is dilated to form the larynx, the wall of which is supported by certain special cartilages-the 
cricoid and the arytenoids. The trachea bifurcates posteriorly to form two bronchi, right and left, one passing to each lung. The lungs of the Lacertilia and Ophidia are of the simple sac-like character already described in the case of the Lizard. In some the lung is incompletely divided internally into two portions - an anterior respiratory part with sacculated walls, and a posterior part with smooth, not highly vascular, walls, having mainly the function of a reservoir. The only additional complication to be specially noted is the presence in the Chamæleons (Fig. 1011) of a number of diverticula or air-sacs which are capable of being inflated, causing an increase in the bulk of the animal which doubtless has an effect on assailants. In the snakelike Lizards the right lung is larger than the left, and in the Amphisbænians the latter is entirely aborted. In the Snakes a similar reduction or abortion of the left lung is observable. In the Crocodilia and Chelonia the lungs are of a more complex character, being divided internally by septa into a number of chambers.

Organs of Circulation.-In the heart (Fig. 1012) the sinus venosus is always present, though not, except in Sphenodon, distinguishable externally; its aperture of communication with the right auricle is guarded by two valves. There are, as in the Amphibia, always two quite distinct auricles, the right receiving the venous blood from the body, the left the oxygenated blood brought from the lungs by the pulmonary veins. But a vital point of difference between the heart of the Reptile and that of

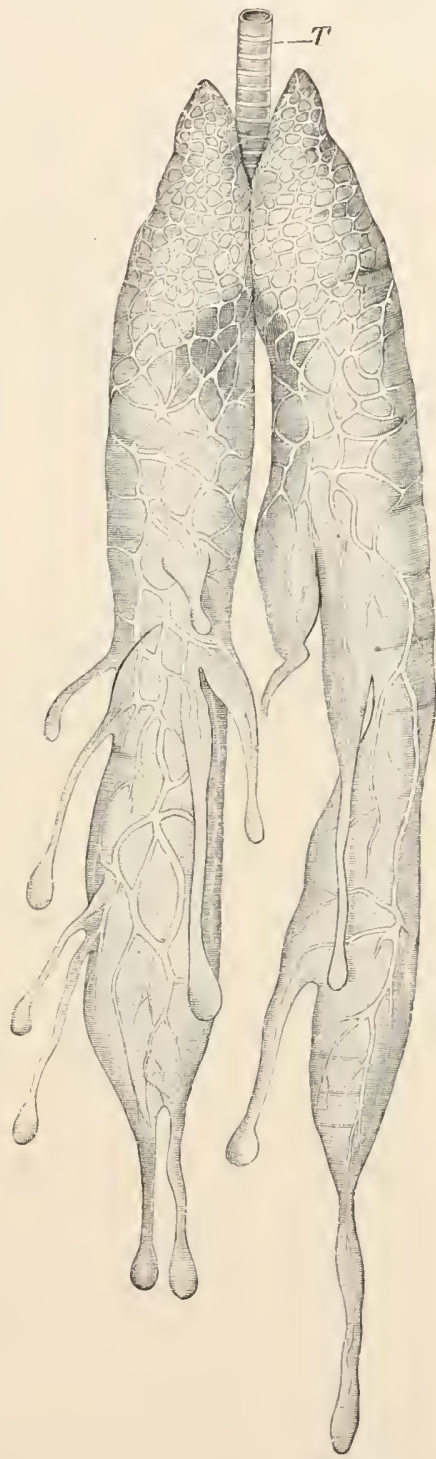

FIa. 1011.-Lungs of Chamæleon. T. trachea. (From Wiedersheim's Com. parative Anatomy.) the Amphibian is that in the former the ventricle is always more or less completely divided into right and left portions. In all 
the Lacertilia, Ophidia, and Chelonia (Fig. 1013) the structure is essentially what has been described in Lacerta, the ventricular septum being well-developed, but not completely closing off the

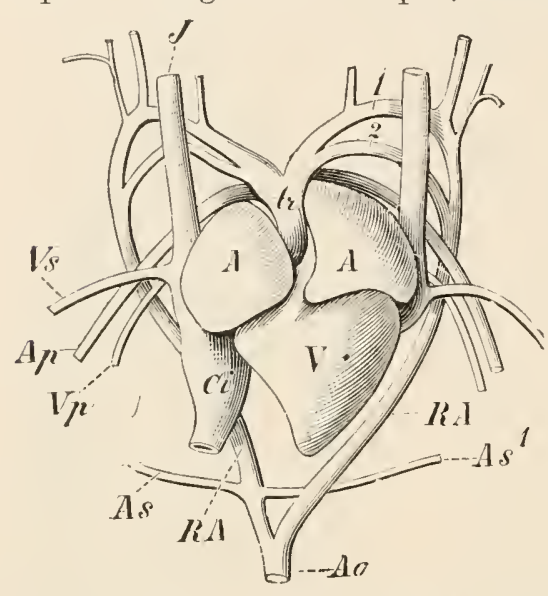

FIG. 1012.-Heart of Iacerta muralis, ventral vicw, $A, A$. auricles; $A p$, pulmonary artery ; $A s, A s^{\mathbf{1}}$, subclavian arteries ; $C i$, post-caval ; $J$. jugular vein; $R a$, aortic arches (made up on either side of two embryonic arches, 1 and 2$)$; $t r$, aortic root; $V$. ventricle; $V p$, pulmonary vein; Vs, subclavian vein. (From Wiedersheim's (omparative Anatomy.)

auricle, the cavum arteriosum with auricle; the cavum pulmonale becomes filled with venous blood which flows into it past the edges of the incomplete septum. When the ventricle contracts, its walls come in contact with the edge of the septum, and the cavum pulmonale is thus cut off from the rest of the ventricle. The further contraction consequently results in the venous blood of the cavum pulmonale being driven out through the pulmonary artery to the lungs, while the blood which remains in the ventricle (arterial and mixed) is compelled to pass out through the aorta. But in the Crocodilia (Fig. 1014) the cavity is completely divided, so that there we may speak of distinct right and left ventricles. left-hand portion of the cavity of the ventricle from the right (cavum pulmonale). The left-hand portion, which is much the larger, is further imperfectly divided into two parts-the cavum arteriosum on the left and the cavum venosum on the right - by the two elongated flaps of the auriculo-ventricular valve, which project freely into the cavity of the ventricle. From the cavum pulmonale arises the pulmonary artery, and from the cavum venosum the right and left aortic arches. When the auricles contract the cavum venosum becomes filled with venous blood from the right arterial blood from the left

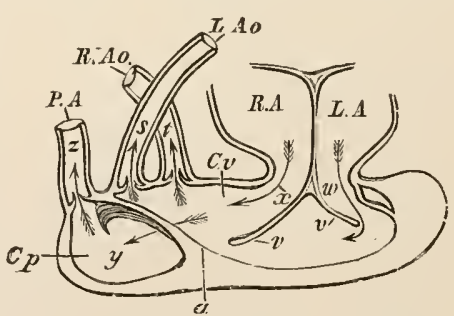

FIG. 1013.-Diagram of heart of Turtle. $a$, incomplete ventricular septum; $C . p$. cavum pulmonale; C.v. cavum venosum; $L$. $A$. left auricle ; $L$. Ao. left aortic arch; $P$. A. pulmonary artery ; $R$. $A$. right auricle ; $R$. Ao, right aortic arch $; s$, arrow showing the course of blood in left aorta; $t$, in right aorta; $v, v^{\prime}$. auriculo-ventricular valves ; $v$, arrow showing the course of blood in left auriculo-ventricular a perture ; $x$, in right; $y$, between cavum venosum and cavum pulmonale; $z$, in pulmonary artery. (After Huxley.)

From the right arises the pulmonary artery and the left aortic arch : from the left the right aortic arch only. The right and left 
arches cross one another, and where their walls are in contact is an aperture-the foramen Panizzce-placing their cavities in communication.

The brain of Reptiles is somewhat more highly organised than that of the Amphibia. The brain substance of the cerebral hemispheres exhibits a distinction into superficial grey layer or cortex containing pyramidal nerve-cells, and central white medulla, not observable in lower groups. The cerebral hemispheres are well developed in all, and there is a lippocampus (see below in the description of the brain of the Rabbit, and of that of the Mammals in general) in the shape of a specially modified region of the dorsal and mesial walls of each hemisphere, represented less distinctly in the Amphibia ; a commissure- the hippocampalconnects the hippocampi of opposite sides, and is dorsal to the chief cerebral commissure-the anterior commissure. The mid-brain consists dorsally usually of two closely-approximated oval optic lobes; rarely it is divided superficially into four. The cerebellum is always of small size, except in the Crocodilia (Fig. 1015), in which it is comparatively highly developed, and consists of a median and two lateral lobes.

\section{Sensory Organs.-} In most Lacertilia, but not in the Ophidia, the

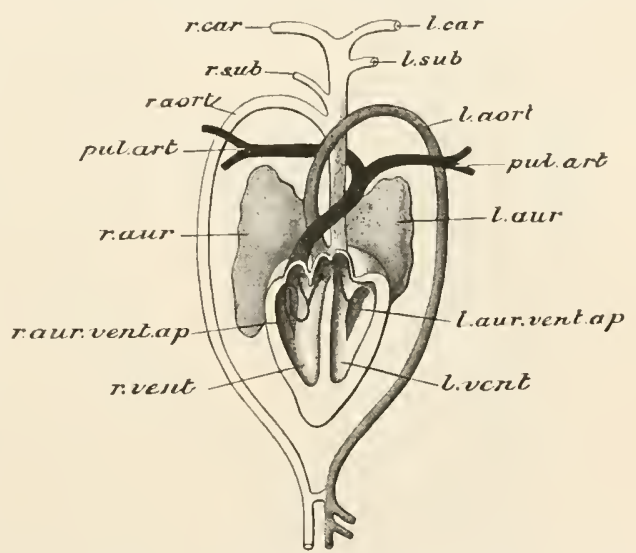

FIG. 1014,--Heart of Crocodile with the principal arteries (diagrammatic). The arrows show the direction of the arterial and venous currents. $l$. aort. left aortic arch; $l$. aur. left auricle; $l$. aur. vent. ap. left auriculo-ventricular aperture ; $l$. car. left carotid ; l. sub. left subclavian ; $l$. vent. left ventricle; pul. art. pulmonary artery; $r$. aort. right aortic arch; $r$. aur, right auricle ; r. aur. vent. ap. right auriculo-ventricular aperture ; $r$. car. right carotid ; $r$. sub. right subclavian; $r$. vent, right ventricle. (From Hertwig's Lehrbuch.)

nasal cavity consists of two parts-an outer or vestibule, and an inner or olfactory chamber-the latter having the sense-cells in its walls, and containing a turbinal bone. In the Turtles each nasal chamber is divided into two passages, an upper and a lower, and the same holds good of the hinder part of the elongated nasal chamber of the Crocodilia.

Jacobson's organs (Fig. 983) are present in Lizards and Snakes, absent in Chelonia and Crocodilia in the adult condition.

The eyes are relatively large, with a cartilaginous sclerotic in which a ring of bony plates (Fig. 984) is developed in some cases. The muscular fibres of the iris are striated. A pecten is present in most. Most Reptiles have both upper and lower eyelids and 
a nictitating membrane. The greater number of the Geckos and all the Snakes constitute exceptions, movable eyelids being absent in both of these groups; in the former the integument passes uninterruptedly over the cornea with a transparent spot for the admission of the light; in the Snakes there is a similar modification,

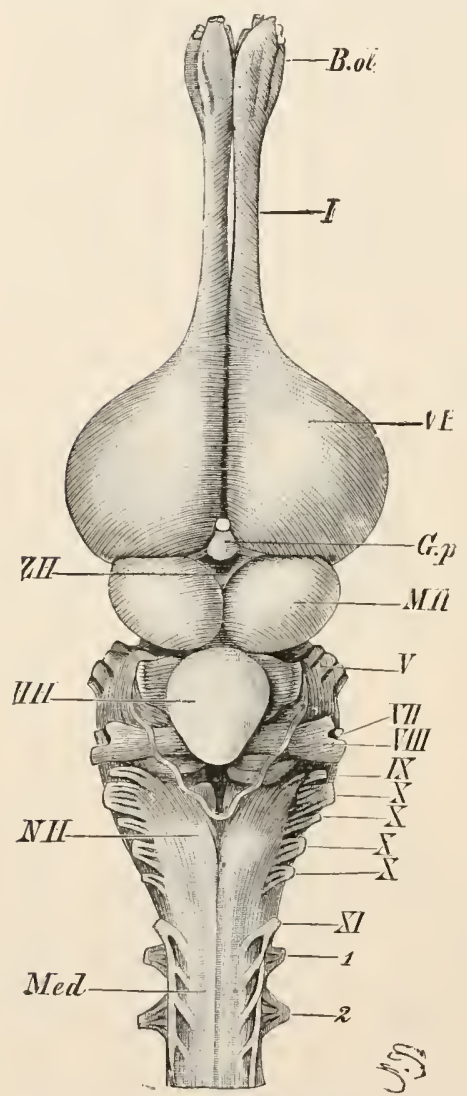

FIG. 1015.-Brain of Alligator, from above. $B$. ol. olfactory bulb; $G$. p. epiphysis ; $H H$, cerebellum; Med, spinal cord ; $M H$, optic lobes ; $N H$, medulla oblongata; $V H$, cerebral hemispheres $I-X I$, cerebral nerves; 1,2 , first and second spinal nerves. (From Wiedershein's Comparative Anatomy.) but the study of development shows that the transparent area is derived from the nictitating membrane which becomes drawn over the cornea and permanently fixed. In the Chamæleons there is a single circular eyelid with a central aperture.

The middle ear is absent in the Snakes, though a columella auris is present, embedded in muscular and fibrous tissue and attached externally, in some cases at least, to the middle of the quadrate.

Developed in close relation to the epiphysis there is in many Lizards (Lacerta, Varanus, Anguis, Amphibolurus and others), and in Sphenodon, a remarkable eye-like organ-the parietal organ or pineal eye (Fig. 1016), which is situated in the parietal foramen of the cranial roof immediately under the integument, and covered over by a specially modified, transparent scale. The pineal eye is developed from a hollow outgrowth of the roof of the diencephalon in front of the epiphysis; the distal end of this becomes constricted off as a hollow sphere, while the remainder is converted into a nerve. The wall of the hollow sphere becomes divergently modified on opposite sides; the distal side gives rise to a lens-like thickening $(l)$, the proximal forms a membrane several layers in thickness-the retina $(r$.$) : the whole is enclosed in a capsule of connective-tissue$ $(k)$. The nerve usually degenerates before the animal reaches maturity, so that the organ would appear-though evidently, from its structure, an organ of sight-to have now entirely or nearly lost its function. 
Reproductive Organs.-The description already given of the reproductive organs of the Lizard (p. 323) applies, so far as all the leading features are concerned, to all the Lacertilia and to the Ophidia ; in Hatteria the copulatory sacs are absent.

In the Crocodilia and Chelonia, instead of the copulatory sacs, there is a median solid penis attached to the wall of the cloaca, and a small process or clitoris occurs in a corresponding position in the female. Though fertilisation is always internal, most Reptilia are oviparous, laying eggs enclosed in a tough, parchmentlike or calcified shell. These are usually deposited in holes and left to hatch by the heat of the sun. In the Crocodiles they are iaid in a rough nest and guarded by the mother. In all cases development has only progressed to a very early stage when the deposition of the eggs takes place, and it is only after a more or less prolonged period of incubation that the young, fully formed in every respect, emerge from the shell and shift for themselves.

Many Li z a r ds, however, and most Snakes are viviparous, the ova undergoing develop-

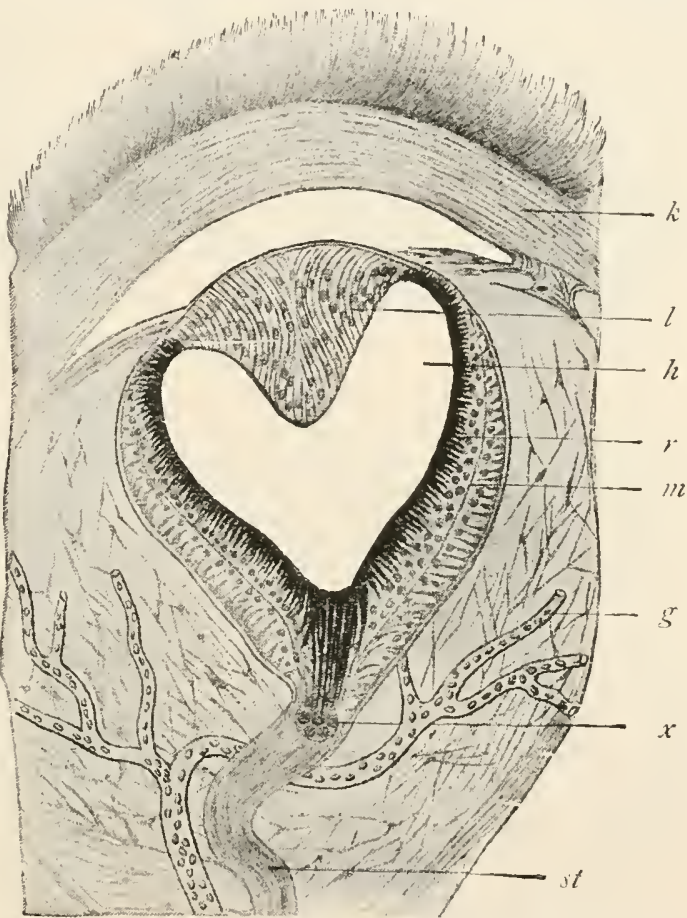

FIG. 1016.-Section of the pineal eye of Sphenodon punc. tatum. $g$, blood-vessels: $h$, cavity of the eye filled with fluid; $k$, capsule of connective-tissue ; $l$. lens ; $m$. molecular layer of the retina; $r$. retina ; st. stalk of the pineal eye; $x$, cells in the stalk. (From Wiedersheim's Comparative Anatomy, after Baldwin Spencer.)

ment in the interior of the oviduct, and the young reaching the exterior in the completely-formed condition.

Development.-In all the Reptilia the segmentation is meroblastic, being confined to a germinal disc of protoplasm situated on one side of the yolk. This divides to form a patch of cells which gradually extends as a two-layered sheet, the blastoderm, over the surface of the ovum. The upper of the two layers is the ectoderm, the lower the yolk-endoderm; the latter is the 
equivalent of the mass of yolk-cells of the Frog, and the shallow space between it and the yolk represents the segmentation-cavity. As the blastoderm extends (Fig. 1017), it becomes distinguishable into a central clearer area-arca pellucida $(a$. pel.) —and a peripheral

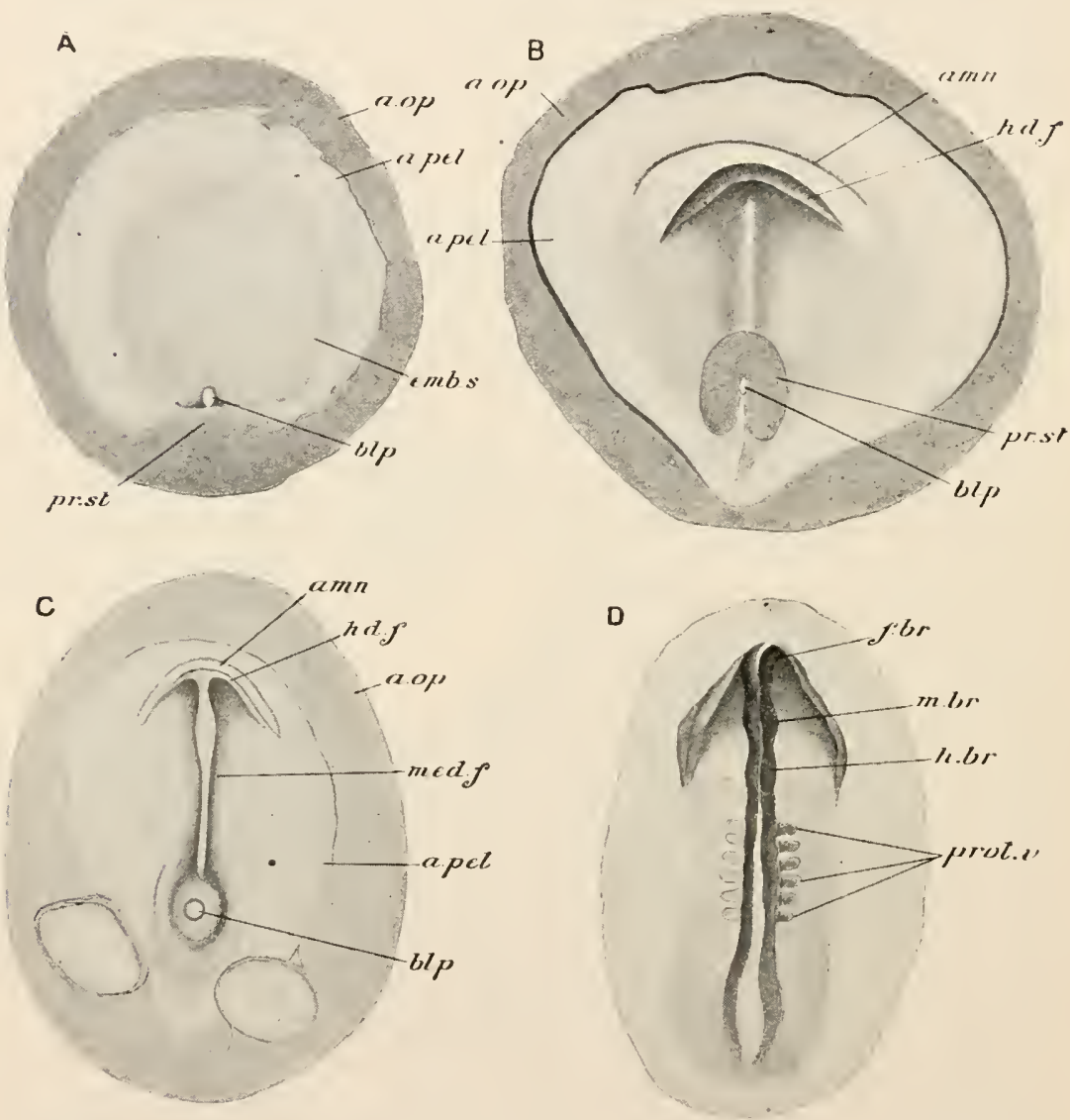

FIG. 1017.- $A-D$, early stages in the development of the Alligator. $A$, stage with embryonic shield, primitive knot and blastopore; $B$, considerably later stage in which the medullary groove has become formed, together with the head-fold of the embryo and the head-fold of the amnion ; $C$, somewhat later stage with well-developed medullary folds and medullary groove ; $D$, later stage in which the medullary groove has become partly closed in by the medullary folds and in which six pairs of protovertebro have become developed. amn amnion; $a . o p$, area opaca ; $a$, pel. area pellucida; $b l p$. blastopore ; emb. $s$, embryonic shield; $f$. $b r$. fore-brain; $h . b r$. hind-brain; $h d . f$. head-fold; m.br. mid-brain; med.f. medullary folds ; prot. $v$. protovertebræ; pr. st. primitive knot. (After S. F. Clarke.)

whitish zone--area opaca ( $a . o p$.). On the former now appears an elliptical thickened patch-the embryonic shield (emb. s.)-which is formed by the ectoderm cells in this region assuming a cylindrical form while remaining flat elsewhere. Behind the embryonic shield appears a thickening, due to a proliferation of the ectoderm 
cells-the so-called primitive knot or primitive plate ( $p r$. st.), and on this is formed an invagination opening on the surface by an aperture-the blastopore (blp.) - which subsequently takes the form of a narrow slit running in the direction of the long axis of
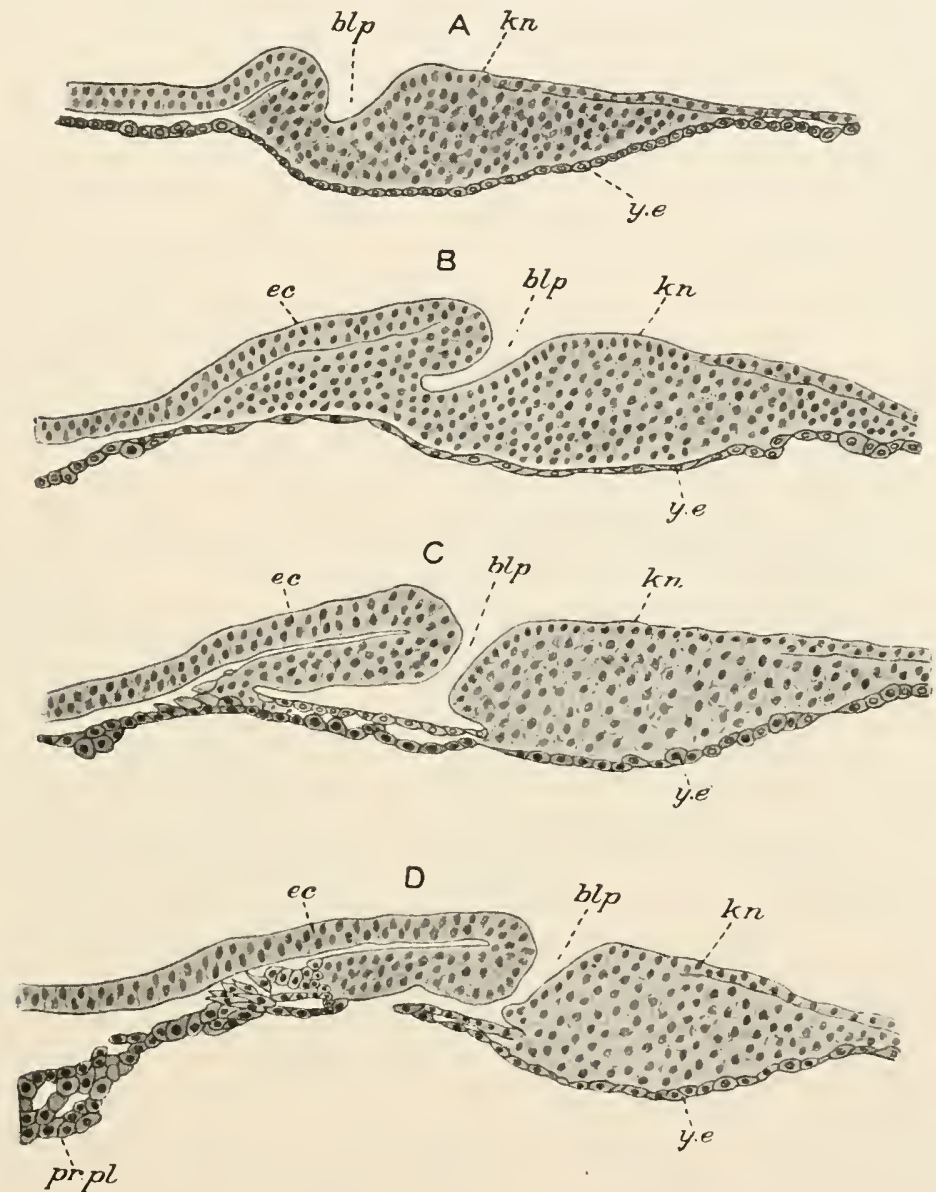

FIG. 1018.- Lacerta, longitudinal sections through the embryonic area of blastoderms illustrating successive stages in the formation of the invagination-cavity (archenteron) and its communication with the segmentation-cavity. blp. blastopore; ec. ectoderm of embryonic shield ; kn. primitive knot; $p r . p l$. protochordal plate; $y . e$. yolk-endoderm. In $D$ the opening below and in front of blp. points to aperture of communication established between the invagination-cavity and the underlying space. (Modified after Wenckebach.)

the future embryo. The cavity of the invagination corresponds to the archenteron of the Frog, and the cells lining it are the endoderm (primitive endoderm). The latter subsequently (Fig. 1018) coalesces with the yolk-endoderm below the floor of the archenteron, and in this position an aperture is formed through 
which the archenteron opens freely into the shallow space that lies between the yolk-endoderm and the yolk. It is from the common cavity thus formed that the lumen of the enteric canal is derived. At a somewhat earlier stage a thickening $(p r . p l$.) has appeared in the yolk-endoderm in the region which will give rise to the head of embryo. This is the protochordal plate; it enters into intimate relationship with the endoderm cells that roof over the archenteron, and, when the floor of the latter becomes opened out, forms with them a continuous plate. In this the notochord originates along the niddle line, and the mesoderm of all the region in front of the blastopore grows out from it at the sides. The aperture of invagination becomes narrowed, and is eventually closed by the approximation and coalescence of its edges. In the region in which the coalescence of the edges takes place there is for a time complete union of the layers, as in the region of the primitive streak of Birds and Mammals. The anterior part of the aperture, however, remains open for a time as the opening of the neurenteric canal.

In front of the blastopore a longitudinal depression bounded by a pair of longitudinal folds (Fig. 1017, med. $f$ ) is the beginning of the medullary groove. As this becomes closed, it encloses, in its posterior portion, the blastopore or dorsal opening of the neurenteric canal. At the sides of the medullary groove appear the protovertebræ (prot. v) : the general history of these parts has already been sketched in the section on the Craniata, and further details will be given in the account of the development of Birds, which agrees with that of Reptiles in most essential respects. Under the head of Birds also will be found an account of the formation of the characteristic fotal membranes, the amnion and the allantois, which applies in all essential respects to the Reptilia as well.

A species of the genus Chalcides or Seps, a Lizard with vestigial limbs, which is viviparous, is apparently exceptional in the formation of a structure closely homologous with the placenta of Mammals, a structure by means of which an intimate connection is established between the embryo and its membranes and the wall of the special compartment of the oviduct in which it lies. As in the case of the Mammal, the intimate union thus brought about facilitates the transmission of nourishment from the blood of the parent to that of the fœtus.

Ethology.-The Lizards are, for the most part, terrestrial animals, usually extremely active in their movements and endowed with keen senses. The majority readily ascend trees, and many kinds are habitually arboreal; but the Chamæleons are the only members of the group which have special modifications of their structure in adaptation to an arboreal mode of life. The Skinks and the Amphisbænians are swift and skilful burrowers. The Geckos are enabled by the aid of the sucker-like dises on the ends 
of their toes to run readily over vertical or overhanging smooth surfaces. A few, on the other hand (Water-Lizards), live habitually in fresh water. The Flying Lizards (Draco, Fig. 1019) are arboreal, and make use of their wings-or, to speak more accurately, aëroplane or parachute - to enable them to take short flights from branch to branch. Chlamydosaurus and Amphibolurus are exceptional in frequently running on the hind-feet, with the fore-feet entirely elevated from the ground. A tolerably high temperature is essential for the maintenance of the vital activities of Lizards, low temperatures bringing on an inert condition, which usually passes during the coldest part of the year into a state of suspended

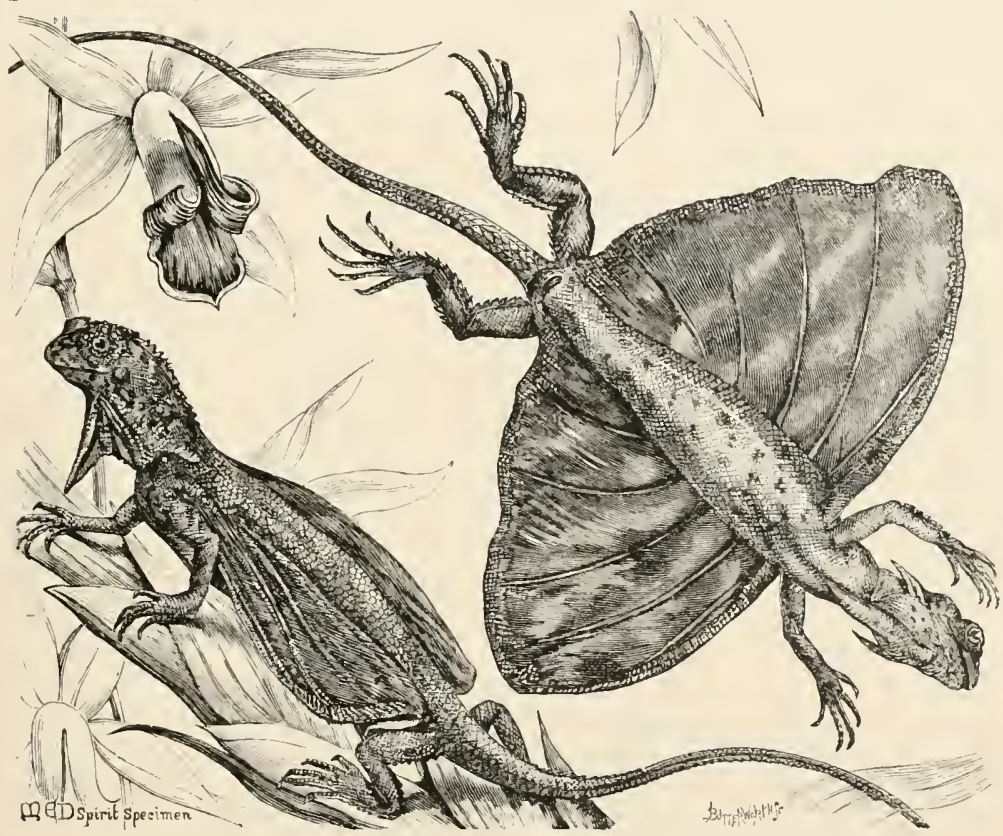

FIG. 1019.-Draco volans, $\times ?$. (From the Cambridge Natural History.)

animation or hibernation. The food of Lizards is entirely of an animal nature. The smaller kinds prey on Insects of all kinds, and on Worms. Chamæleons, also, feed on Insects, which they capture by darting out the extensile tongue covered with a viscid secretion. Other Lizards supplement their insect diet, when opportunity offers, with small Reptiles of various kinds, Frogs and Newts, small Birds and their eggs, and small Mammals, such as Mice and the like. The larger kinds, such as the Monitors and Iguanas, prey exclusively on other Vertebrates; some, on occasion, are carrion-feeders. Most Lizards lay eggs enclosed in a tough calcified shell. These they simply bury in the earth, leaving them 
to be hatched by the heat of the sun. Some, however, as already stated, are viviparous; in all cases the young are left to shift for themselves as soon as they are born.

Most of the Snakes also are extremely active and alert in their movements; and most are very intolerant of cold, undergoing a hibernation of greater or less duration during the winter season. Many live habitually on the surface of the ground-some kinds by preference in sandy places or among rocks, others among long herbage. Some (Tree-Snakes) live habitually among the branches of trees. Others (Fresh-water Snakes) inhabit fresh water ; others (Sea-Snakes) live in the sea. The mode of locomotion of Snakes on the ground is extremely characteristic, the reptile moving along by a series of horizontal undulations brought about by contractions of the muscles inserted into the ribs, any inequalities on the surface of the ground serving as fulcra against which the free posterior edges of the ventral shields (which are firmly connected with the ends of the ribs) are enabled to act. The burrowing Blind-Snakes and other families of small Snakes feed on Insects and Worms. All the rest prey on Vertebrates of various kinds, Fishes, Frogs, Lizards, Snakes, Birds and their eggs, and Mammals. The Pythons and Boas kill their prey by constriction, winding their body closely round it and drawing the coils tight till the victim is crushed or asphyxiated. Some other non-venomous Snakes kill with bites of their numerous sharp teeth. The venomous Snake sometimes, when the prey is a small and weak animal such as a Frog, swallow it alive; usually they first kill it with the venom of their poisonfangs.

When a venomous Snake strikes, the poison is pressed out from

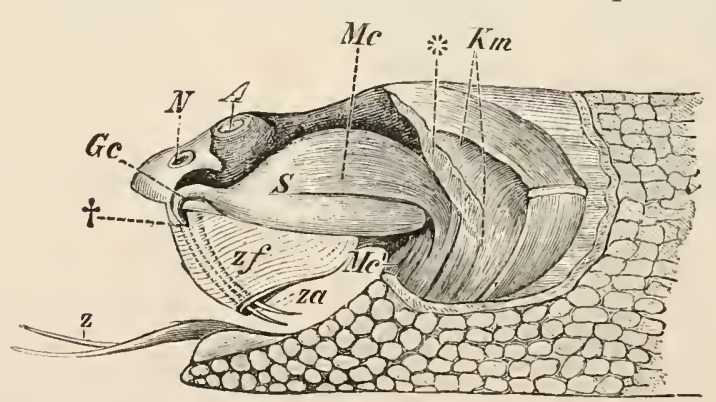

FIG. 1020.-Poison apparatus of Rattlesnake. A, eye ; $G$ c, poison-duct entering the poison-fang at $\dagger$; $\mathrm{I} m$, muscles of mastication partly cut through at $* ; M c$. constrictor (masseter) muscle; $\boldsymbol{M} \boldsymbol{e}^{\prime}$. continuation of the constrictor muscle to the lower jaw; $N$. nasal opening; $S$, fibrous poison sac; $z$, tongue; $z a$, opening of the poison-duct; $z f$, pouch of mucous membrane enclosing the poison-fangs. (From Wiedersheim's Comparative Anatomy.) the poison-gland by the contraction of the masseter (Fig. 1020, Mc), one of the muscles which raise the lower jaw; it is thus forced along the duct $(G c)$ to the aperture $(z a)$, and injected into the wound made by the fang. The effect is to produce acute pain with increasing lethargy and weakness, and in the case of the venom of some kinds of Snakes, paralysis. According to the amount of the poison injected (in 
relation to the size of the animal) and the degree of its virulence (which differs not only in different kinds of Snakes, but in the same Snake under different conditions) the symptoms may result in death, or the bitten animal may recover. The poison is a clear, slightly straw-coloured or greenish liquid; it preserves its venomous properties for an indefinite period, even if completely desiccated. The poisonous principles are certain proteids not to be distinguished chemically from other proteids which have no such poisonous properties. Immunity against the effects of the poison, and relief of the symptoms after a bite has been inflicted, have been found to be conferred by injections of the serum of animals which have been treated with injections of increasing doses of the poison.

The majority of Snakes are viviparous. Some, however, lay eggs, which, nearly always, like those of the oviparous Lizards, are left to be hatched by the heat of the sun, some of the Pythons being exceptional in incubating them among the folds of the body.

Sphenodon lives in burrows in company with a Bird-the Shearwater (Puffinus) - and feeds on Insects and small Birds. It lays eggs enclosed in a tough, parchment-like shell.

Of the Chelonia some (Land Tortoises) are terrestrial; others (Fresh-water Tortoises) inhabit streams and ponds, while the SeaTurtles and Luths inhabit the sea. Even among Reptiles they are remarkable for their tenacity of life, and will live for a long time after severe mutilations, even after the removal of the brain ; but they readily succumb to the effects of cold. Like most other Reptiles, the Land and Fresh-water Tortoises living in colder regions hibernate in the winter; in warmer latitudes they sometimes pass through a similar period of quiescence in the dry season. The food of the Green Turtle is exclusively vegetable; some of the Land Tortoises are also exclusively vegetable feeders; other Chelonia either live on plant food, together with Worms, Insects, and the like, or are completely carnivorous. All are oviparous, the number of eggs laid being usually very great (as many as 240 in the Sea-Turtles); these they lay in a burrow carefully prepared in the earth, or, in the case of the Sea-Turtles, in the sand of the sea-shore, and, having covered them over, leave them to hatch.

The Crocodiles and Alligators, the largest of living Reptiles, are in the main aquatic in their habits, inhabiting rivers and, in the case of some species, estuaries. Endowed with great muscular power, these Reptiles are able, by the movements of the powerful tail and the webbed hind-feet, to dart through the water with lightning-like rapidity. By lying in wait motionless, sometimes completely submerged with the exception of the extremity of the snout bearing the nostrils, they are often able by the suddenness and swiftness of their onset to seize the most watchful and timid animals. In the majority of cases the greater part, and in some the whole, of their food consists of Fishes; but all the larger and more 
powerful kinds prey also on Birds and Mammals of all kinds, which they seize unawares when they come down to drink or attempt to cross the stream. On land their movements are comparatively slow and awkward, and they are correspondingly more timid and helpless.

The Crocodilia, as already mentioned, are all oviparous, and the eggs, as large in some species as those of a Goose, are brought forth in great numbers (sometimes 100 or more) and either buried in the sand, or deposited in rough nests.

Geographical Distribution.-The order Lacertilia, the most numerous of the orders of Reptiles living at the present day, is of very wide distribution, occurring in all parts of the earth's surface except the circumpolar regions; but some of its larger sections are of limited range. The Geckos are numerous in all warm countries, their headquarters being Australia and the Oriental region. The snake-like Pygopidæ are entirely confined to the Australian region. The Agamidæ (a family which includes the Flying Lizards besides many others) are most abundantly represented in the Australian region, though extending to other regions of the Old World, except New Zealand and Madagascar. Of the Iguanas two genera occur in Madagascar and one in the Friendly Islands ; all the other members of this group, which is a large one, are confined to America. Three families occur exclusively in America - the Xenosauridæ, the Tejidæ, and the Helodermidæ, or poisonous Lizards. The Zonuridæ or Girdle-tailed Lizards are confined to Africa and Madagascar. The Anguidx or Blind-worm Yizards are mostly American, but are represented in Europe and Asia. The family of the Monitors is distributed in Africa, Southern Asia, Oceania, and the Australian region. The Snake-like Amphisbænians are most numerous in America, but are well represented in Africa, and occur also in the Mediterranean area. The Lacertidæ are most abundant in Africa, but occur in Europe and Asia. The family of the Skinks (Scincidæ) is of world-wide range, but is most abundant in Australia, Oceania, the Oriental region and Africa. Sphenodon is confined to the New Zealand region, and at the present day only occurs on certain small islands off the N.E. coast and in Cook's Straits. The Chamæleons are most abundant in Africa and Madagascar, but there are representatives in various other parts of the Old World; they do not occur in the Australian, New Zealand, or Polynesian regions, and are only represented in Europe by one species which occurs in Andalusia.

Chelonia are widely distributed over the surface of the earth, by far the greater number being natives of tropical and temperate zones. The Sea-turtles, including the Hawk's-bills and the Luths, are for the most part, but not entirely, confined to the tropical seas. Giant Land-tortoises occur, or occurred in historic times, on islands of the Galapagos and Mascarene groups.

Of the Crocodilia the Caimans are confined to Central and South 
America. The Alligators are represented in North America by one species and in China by another. The true Crocodiles occur widely distributed over Africa, Southern Asia, the northern parts of Australia and tropical America, while the Gavial occurs only in certain Indian and Burmese rivers.

Geological Distribution.-The Squamata are geologically the most recent of the existing orders of Peptiles. The earliest fossil rem a ins of Lizards have been found in beds belonging to the Jurassic and Cretaceous periods; but most of the families are not represented earlier than the Tertiary. All the known fossil $\mathrm{rem}$ a ins of Snakes, except one imperfectly known form from the Cretaceous, have been found in deposits of Tertiary age. The Rhynchocephalia are much more ancient, being represented in deposits as old as the Permian by a genusPalceohatteriawh ich, though differing in some

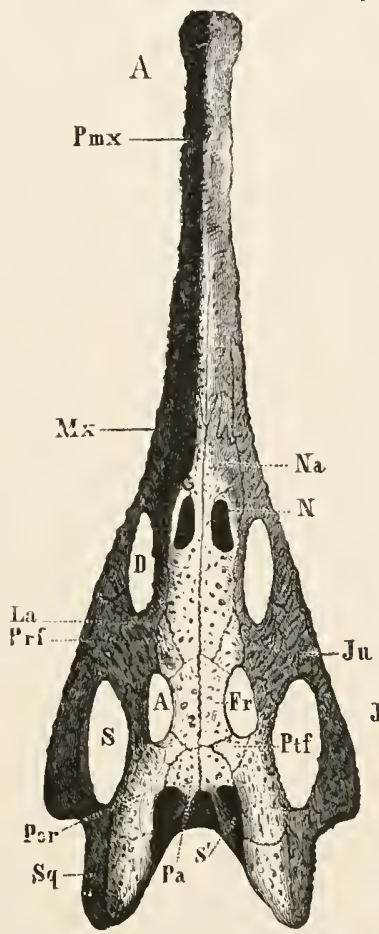

FIG. 1021.-Skull of Belodon. A, from above; $\mathbf{B}$, from below. $A$, orbit ; $B o$, basi-occipital ; $C h$, internal nares ; $D$, pre-orbital fossa ; Exo. exoccipital ; Fr. frontal ; Ju. jugal ; La. lacrymal ; $M x$. maxilla; $n$. external nares; $Y^{\prime} a$. nasal; $P$ a . parietal ; $P l$. palatine ; P $m x$, premaxilla ; Por. post-orbital ; Prf. pre-frontal $P t$. pterygoid; Qu. quadrate; $S$, lateral temporal fossa ; $S^{\prime}$, superior temporal fossa; Sq. squamosal; Vo. vomer. (From Zittel.)

respects from the living Hatteria, is sufficiently near it to be looked upon as a member of the same order : and other extinct Rhynchocephalians have been found in Triassic and in Tertiary strata. The order Chelonia was represented from the Triassic period onwards. Of the extinct forms one group--the Athecata-differs from the living Chelonia in having the carapace incompletely developed, entirely composed of dermal elements, and quite separate from the vertebræ and ribs. The Crocodilia date back as far as the Trias. The most primitive of the fossil forms (Fig. 1021) had the internal nares 
situated in front of the palatines, while the external nares were situated towards the middle of the snout. Later forms (post-Triassic) had palatine plates aeveloped from the premaxillæ, the maxillæ, and the palatines; and some resembled the living members of the order in having such plates developed also from the pterygoids; all had the external nares situated towards the end of the snout. Those in which the palatine plates of the pterygoids were absent had usually amphiccelous vertebræ. Some of the fossil Crocodiles reached an immense size.

\section{Extinct Groups of Reptiles.}

\section{Proreptilia.}

The Permian genera Eryops and Cricotus, formerly assigned to the Stegocephala (p. 285), have been proved, on the ground mainly of their vertebral structure, to be true Reptiles, and are regarded as the most primitive members of the class with strong Amphibian affinities.

\section{THEROMORPHA.}

The Theromorpha are a very extensive and varied group of fossil Reptiles which all have limbs adapted to terrestrial locomotion. The vertebra are amphicolous, and most of the ribs have distinct capitula and tubercula. A sternum is present, and also, in many cases at least, an episternum. The quadrate is firmly fixed. Palatine plates are developed comparable to those of Chelonia. There is a parietal foramen. The temporal region is in some covered over by flattened bones as in a Turtle, in others there is a wide lateral temporal fossa bounded above by a superior temporal arch; in the latter case no quadrato-jugal is developed. An arch corresponding with the zygoma of Mammals (see Section XV) is formed by the extension backwards of the jugal to meet an anterior process of the squamosal, both articulating with a downgrowth from the post-frontal. In the pectoral arch, clavicle, coracoid, pro-coracoid, and scapula are present. The pubes and ischia are closely united, with a common symphysis, as in Mammals ; and the obturator foramen is usually small. The teeth (Fig. 1022) (which are not present in all)

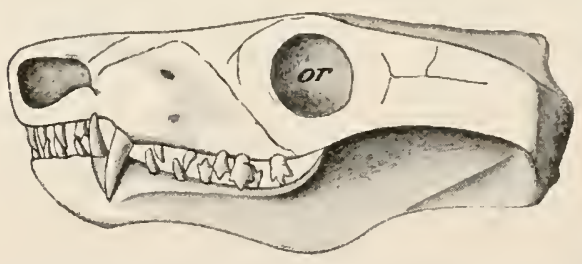

Fig. 1022.-Left lateral aspect of the skull of Galesaums planiceps. or. orbit. (After Nicholson and Lydekker.) are thecodont, and in the higher forms bear a considerable resemblance to those of Mammals in the regularity of their arrangement in sets, often with large canines or tusks. Palatine teeth are sometimes present. One order, the Placodontia, have remarkable broad crushing teeth on both upper and lower jaws and on the palate.

The Theromorpha mainly occur in beds between Permian and Triassic age, and have been found in South Africa and North America, as well as Europe and India. Among them have recently been found many transition forms which tend to bridge over the interval between the Reptilia and the Mammalia.

SAUROPTERYGIA.

The typical representatives of this order, such as Plesiosaurus (Fig. 1023), were aquatic Reptiles, sometimes of large size (up to 40 feet), though many 
were quite small. They had a lizard-like body, a very long neck, a relatively small head, and a short tail which supported a vertical caudal fin ; the limbs were modified to form swimming-paddles. In older and less specialiscd members of the group, however, the limbs were not paddle-like, but adapted for walking.

The spinal column of the Sauropterygia is characteriscd by the great

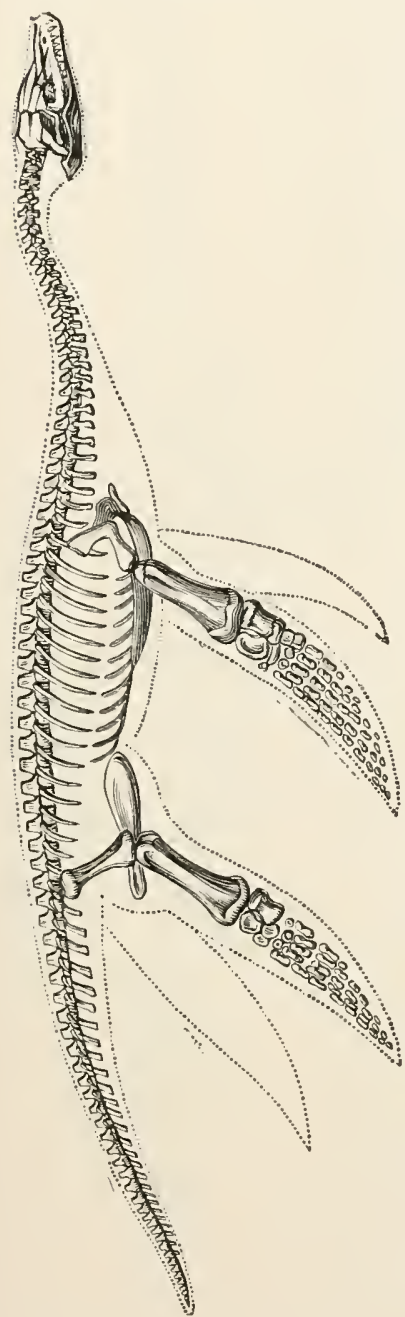
length of the cervical and the shortness of the caudal region. The vertebrie are usually amphicœlous. The sacrum consists of either one or two vertebræ. There is no sternum. In the skull there are large premaxillæ; a bony palate is absent; a transverse bone is present. One temporal arch only is developed. There is a wellmarked parietal foramen. The ring of bony plates (developed in the sclerotic)

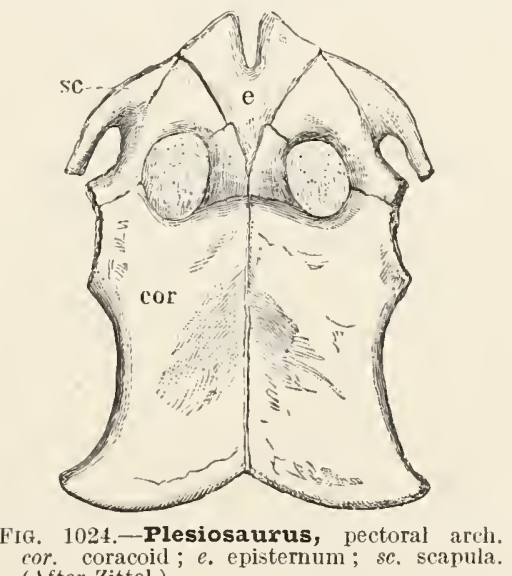

cor. coracoid ; $e$. episternum ; $s c$. scapula. (After Zitte!.)

found in the orbit of somc fossil Reptiles is not developed. The quadrate is not movable. The pectoral arch (Fig. 1024) presents some remarkable features. The coracoids always meet in a ventral symphysis, and the ventral portions (acromial processes) of the scapulæ may also ineet. In front there is, in most cases, an arch of bone, consisting of a riedian and two lateral portions, which probably represent the episternum and the clavicles: in some forms this arch is reduced or absent. In the pelvis the broad pubes and ischia meet in the middle line : the two symphyses may remain separate (Fig. 1025), or they may unite so as to divide the space into two separate obturator foramina. The teeth are implanted in distinct sockets.

The Sauropterygia date back to the Trias, and perhaps to the Permian, extending onwards to the Cretaceous. 


\section{ICHTHYOPTERYGIA.}

The Ichthyopterygia, including Ichthyosaurus (Fig. 1026) and its allies, were aquatic Reptiles, some of very large size (30 or 40 feet in length), with somewhat fish-like body, large head produced into an elongated snout, no neck, and an elongated tail, with a large vertical caudal fin, and with limbs in the form of swimmingpaddles. The vertebræ are amphicolous. A sacrum is absent, so that only precaudal and caudal regions are distinguishable. The ribs have two heads for articulation with the vertebræ: a sternum is absent, but there is a highly developed system of abdominal ribs. The skull is produced into an elongated rostrum, formed chiefly of the premaxillæ, and with small nostrils situated far back. The orbits are large and contain a ring of bones developed in the sclerotic. An epipterygoid is present as in Lizards, and there is a large parietal foramen. Both supcrior and inferior temporal arches are developed. The quadrate is immovably fixed to the

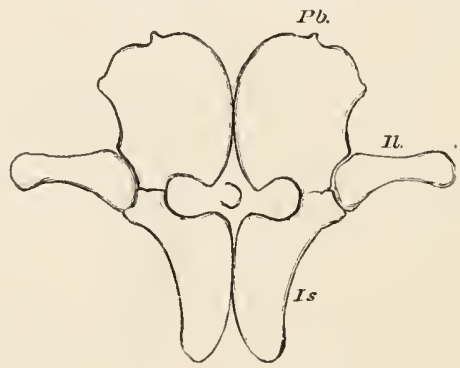

FIG. 1025.-Plesiosaurus, pelvic arch. $1 l$. ilium ; 1 s. ischium; $P b$. pubis. (After Huxley.)

skull. The pectoral arch consists of coracoid, scapula, and clavicle, the pro-coracoid being absent or very small. The coracoids are broad bones which meet ventrally for a short distance without overlapping. A T-shaped episternum is present. The bones of the pelvis are not strongly developed; the ilia are not connected with the spinal column; the pubes and ischia of opposite sides meet in ventral symphyses, but there is no obturator foramen. Humerus and femur are both short, and the rest of the bones of the limb are disc-like or polyhedral. The phalanges are numerous, and are usually in more, sometimes in fewer, than the usual five series. The teeth are not in separate sockets, but set in a continuous groove.

The Ichthyopterygia are of Mesozoic age, ranging from the Upper Trias to the Upper Cretaceous.
Geographically their remains have a very wide distribution, having been

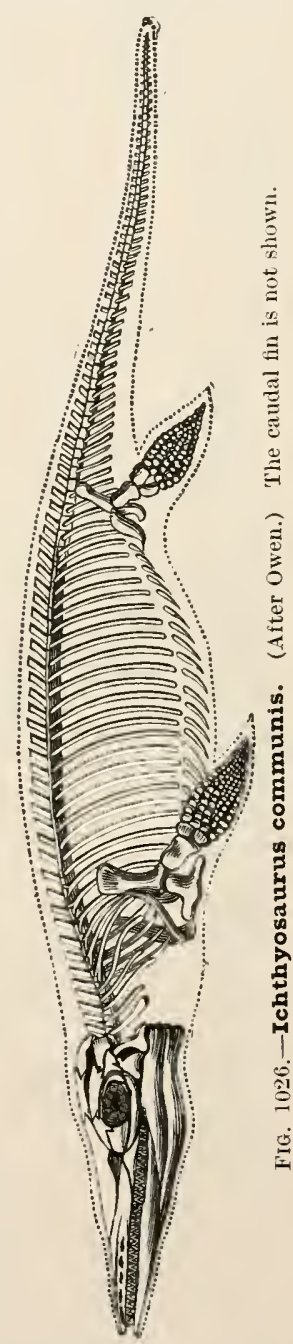
found not only in Europe and North America, but in the Arctic Regions, in India, Africa, Australia, and New Zealand.

\section{Dinosauria.}

This order comprises a vast number of terrestrial Reptiles, some of gigantic size (up to over 100 feet in length), of lizard-like or bird-like form, some 
approaching Birds in certain features of their structure, others coming nearer the earliest fossil Crocodiles. The surface was in some covered with a bony

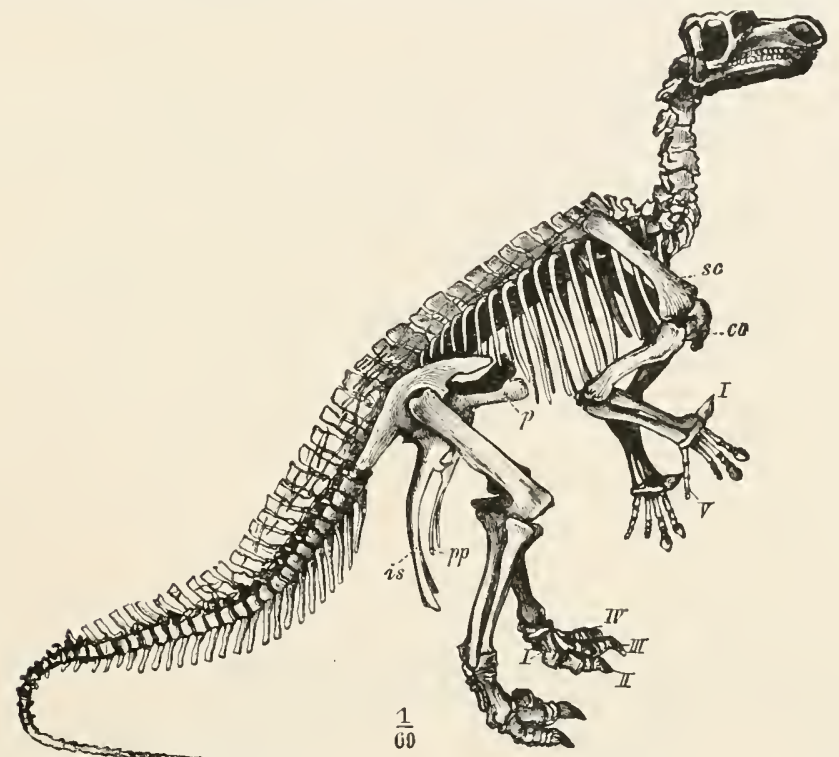

FIG. 1027.-Iguanodon bernissartensis. One sixtieth natural size. co. coracoid; is. ischium; $p$. pubis (pectineal process); $p p$. post-pubic process (pubis); $s c$. scapula $I-I, I-I V$, digits. (From Zittel, after Dollo.)

armour, sometimes armed with long spines. The forc- and hind-limbs were in some equally developed; in others the hind-limbs were much more powerful than the fore-limbs, and in many their structure appears adapted to a bipedal mode of progression (Fig. 1027).

The centra are in general amphicœlous, but vary greatly. The sacral region usually comprises 3 to 6 vertebræ. The thoracic ribs have double heads. Ab. dominal ribs are sometimes present. The sternum was incompletely ossified, and an episternum is absent. There is no parietal foramen. There are complete upper and lower temporal arches, and the fossa is divided into upper and lower

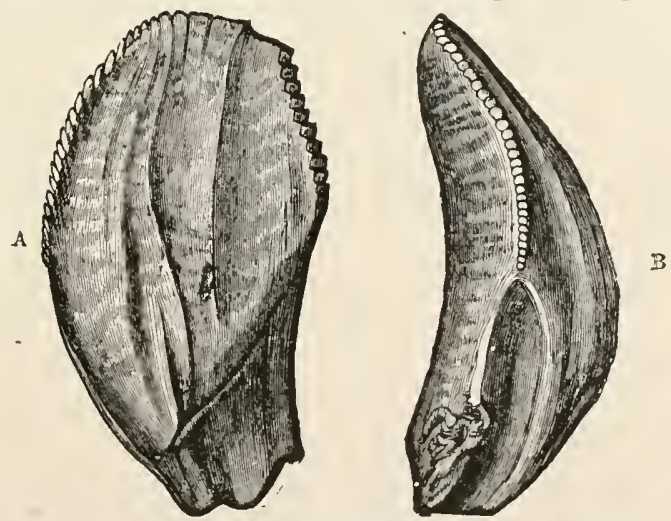

FI(i. 10:28.-Teeth of Iguanodon mantelli. $A$, from the inner, $B$, from the outer side. (From Zittel, after Mantell.) parts by a barformed from the post-frontal and squamosal. Ectopterygoids are present. The quadrate is firmly fixed. In the pectoral arch the scapula is very large, the coracoid small, and the pro-coracoid and clavicle absent. 
The pubis in some Dinosauria has a remarkable slender prolongation (Fig. $1027, p p$.) running downwards and backwards from the body of the bone parallel with the ischium, an arrangement not found elsewhere except in Birds; a pubic symphysis does not always occur. In certain points in the structure of the hind-limb itself some of the Dinosauria also bear a resemblance to Birds. The teeth, which are usually compressed and may have serrated edges, are sometimes placed in sockets, sometimes in grooves.

Iguanodon (Fig. 1027), one of the best-known genera, attains the length in the case of one species of over 30 feet. The limb-bones are hollow. The ischium and pubic process are long and slender, and inclined backwards and downwards parallel to one another. The hind-foot was digitigrade, i.e., the weight was supported on the phalanges of the digits, and the elongated metatarsals, which were immovably fixed, had a nearly vertical position as in Birds; the fore-limbs are relatively small, and fossil footprints that have been found indicate that the animal supported itself habitually in a halfcrect posture like a Kangaroo, with the fore-limbs raised from the ground. The teeth (Fig. 1028) are of a remarkable shape, flattened and with serrated edges, sometimes with vertical ridges which may be serrated. The Dinosauria range from the Trias to the Upper Cretaceous, and were most abundant in the Jurassic and Wealden.

\section{Pterosauria.}

The Pterosauria or Pterodactyles are perhaps even more remarkable modifications of the reptilian type than any of the orders that have been

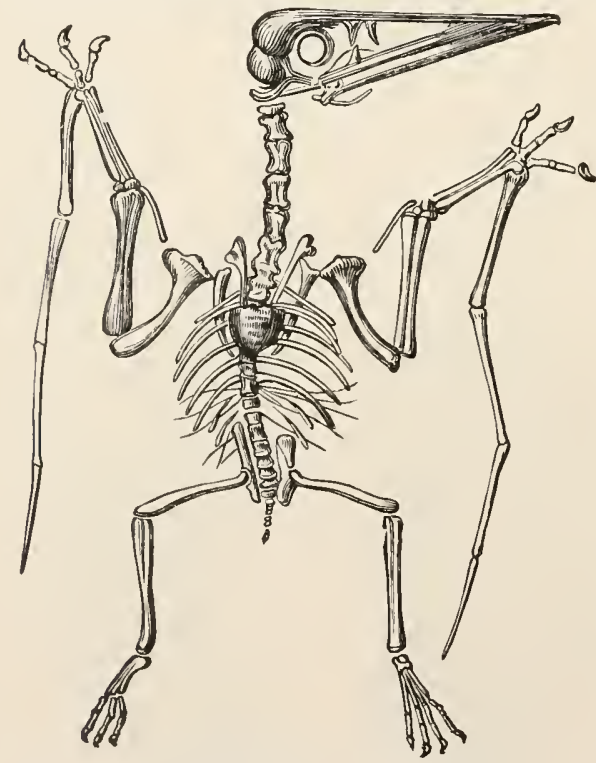

FIG. 1029.-Pterodactylus spectabilis. Threefourths of the natural size. (From Zittel, after II. v. Mayer.) hitherto alluded to. The chief peculiarities in the structure of these Reptiles were asso. ciated with a flying mode of locomotion, the organs of flight being, as in the Bird and the Bat, the fore-limbs. In the Pterodactyles (Fig. 1029) the last digit on the ulnar side of the manus is enormously prolonged and thickened, and supported a wcb of skin (Fig. 1031) which cxtended backwards to the hind-limbs and the tail. Most of the bones are hollow, and have pneumatic foramina as in Birds (q.v.). The vertebræ are procœlous, except the caudals, which are amphicolous. The cervical vertebræ are elongated and stout, the neck being of considerable length; there are three to six ankylosed sacrals. The anterior thoracic ribs are bifid at their vertebral ends. The sternum is broad, with a longitudinal keel. The skull (Fig. 1030), set on the neck at right angles as in a Bird, is of large size and superficially resembles that of a Bird in general shape, and particularly in the presence of an elongated, pointed rostrum; the orbits are large, and contain a ring of sclerotic ossifications, The sutures are largely obliterated, 
as in the skull of a Bird. 'The quadrate is immovably fixed to the skull. In the pectoral arch the scapula and coracoid are long and slender, like those of Birds : pro-coracoids and clavicles are absent. The pelvis and hind-

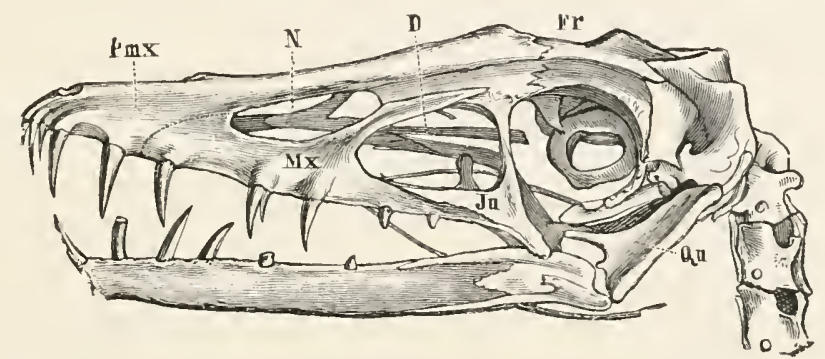

FiG. 1030.-Skull of Scaphognathus. D. pre-orbital aperture ; Fr. frontal ; $J u$. jugal. IX. maxilla; $N$. nasal opening; Pmx. premaxilla; Qu. quadrate. (After Zittel.)

limbs are weak as compared with the fore-limbs, and the pelvis does not exhibit any resemblance to that of Birds, but appears to come nearer to that of the Crocodiles. The astragalus sometimes unites with the tibia. There is no trace of any exoskeleton. The brain, as shown by casts of the interior

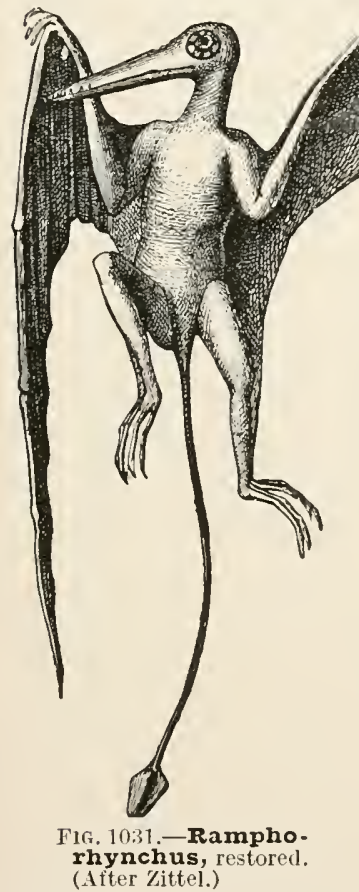

of the skull, bore interesting resemblances to that of Birds in the relations of the cerebellum and optic lobes, the latter being separated from one another by the approximation of the cerebellum to the fore-brain, instead of being in close apposition with one another as in existing Reptiles.

The Pterosauria are confined to formations of the Jurassic and Cretaceous periods.

\section{PythoNOMORPHA.}

The Pythonomorpha were large marine Reptiles with extremely elongated snake-like bodies, but with well-developed limbs, which were modified as swimming-paddles. The vertebræ, which are very numerous, are procœlous, sometimes with, sometimes without, zygosphenes and zygantra. The sacrum is absent as a rule. A sternum has been found in one genus. The skull resembles that of a Lizard, both in form and structure; the quadrate is mobile; there is a parietal foramen; the premaxillæ are united. There is no inferior temporal arch, the quadrato-jugal being absent. The quadrate is movable, articulating with the squamosal and epiotic. The rami of the mandible are united by ligament at the symphysis. The pectoral arch (Fig. 1032) comprises discoidal coracoids (c) which meet ventrally, and a 
scapula (sc.) which resembles that of the Rhynchocephalia: a clavicle is never present. In the pelvis the ilium, which usually does not articulate with the spinal column, is a rod-shaped bone: the ischium and pubis resemble those of the Lizards. The bones of both fore- and hind-limbs are sliort

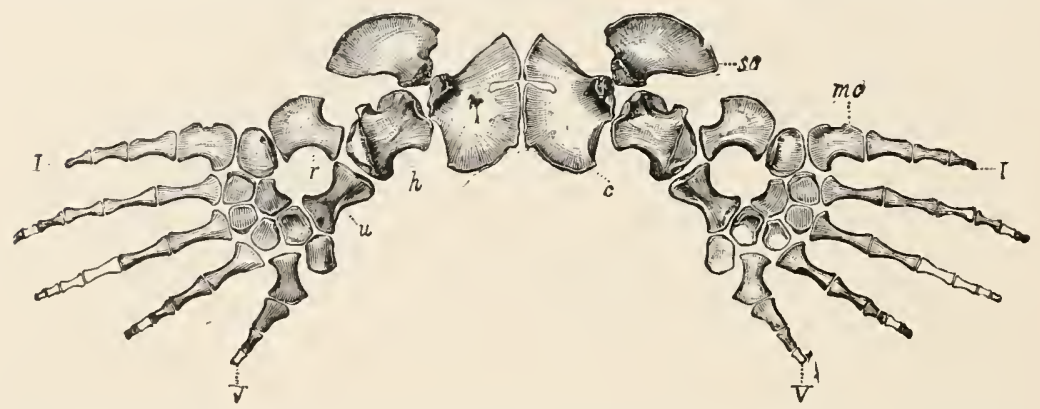

FIG. 1032.-Edestosaurus (Pythonomorpha). Pectoral arch and fore-limbs. c. coracoid with pro-coracoid; $h$. humerus; $m c$. metacarpus; $r$. radius; sc. scapula $u$. ulna; $I$, first digit; $V$, fifth digit. (From Zittel after Marsh.)

there are five digits in each. The teeth are conical, pointed, and ankylosed by expanded bases to the summits of the maxillæ and pterygoids. Dermal scutes have been observed in one genus.

The remains of Pythonomorpha have been found only in certain beds belonging to the Cretaceous period in Europe, North America, and New Zealand.

\section{CLASS V.-AVES.}

In many respects Birds are the most highly specialised of Craniata. As a class they are adapted for aërial life; and almost every part of their organisation is modified in accordance with the unusual environment. The non-conducting covering of feathers ; the modification of the fore-limbs as wings, of the sternum and shoulder-girdle to serve as origins of the great wing-muscles, and of the pelvic girdle and hind-limbs to enable them to support the entire weight of the body on the surface of the ground; the perfection of the respiratory system, producing a higher temperature than in any other animals: all these peculiarities are of the nature of adaptations to flight. Add to them the absence, in all existing Birds, of teeth, the loss of the left aortic arch, and of the right ovary and oviduct, the specialised character of the brain, the poorly developed olfactory organs, and the extraordinarily large and perfect eyes, and we have a series of strongly-marked characteristics such as distinguish hardly any other class. Moreover, the organisation of existing Birds is, in its essential features, singularly uniform, the entire class presenting less diversity of structure than many single orders of Fishes, Amphibians, and Reptiles. 


\section{Example of the Class.-The Common Pigeon (Columba livia, var. domestica).}

The Common or Domestic Pigeon is known under many varieties, which differ from one another in size, proportions, coloration, details in the arrangements of the feathers, and in many points of internal anatomy. The Pouters, Carriers, Fantails, and Tumblers may be mentioned as illustrating extreme forms. All these varieties liave, however, been produced by artificial selection, that is, by breeders selecting, generation after generation, the Birds which most nearly attained to some artificial standard of perfection, breeding from them alone, and killing off the inferior strains. The ancestral species from which the domestic breeds have in this way been evolved is the Rock Pigeon (Columba livia), which is widely distributed in the Palæarctic and Oriental regions. The following description refers especially to the common Dovecot Pigeon.

External Characters.-In the entire Bird (Fig. 1033) the plump trunk appears to be continued insensibly into the small, mobile head, with its rounded brain-case and prominent beak, formed of upper and lower jaws covered by horny sheaths. The head, neck, and trunk are invested in a close covering of feathers, all directed backwards and overlapping one another. Posteriorly the trunk gives origin to a number of outstanding feathers which constitute what is ordinarily called the tail. From the anterior region of the trunk spring the wings, also covered with feathers, and, in the position of rest, folded against the sides of the body. The legs spring from the hinder end of the trunk, but, owing to the thick covering of feathers, only the feet are to be seen in the living Bird, each covered with scales and terminating in four digits $\left(d g .1^{\prime}-d g .4^{\prime}\right)$, three directed forwards and one backwards.

In order to make a fair comparison of the outer form with that of other Craniate types, it is necessary to remove the feathers. When this is done, the Bird is seen to have a long, cylindrical, and very mobile neck, sharply separated both from head and trunk. The true tail is a short, conical projection of the trunk, known as the uropygium, and giving origin to the group of large feathers (rct.) to which the word "tail" is usually applied. On the dorsal surface of the uropygium is a papilla bearing on its summit the opening of a large gland, the oil-gland $(o . g l$.), the secretion of which is used for lubricating or "preening" the feathers.

The wings show the three typical divisions of the fore-limb, upper arm, fore-arm, and hand, but the parts of the hand are closely bound together by skin, and only three imperfectly-marked digits, the second $(d g .2)$ much larger than the first $(d g .1)$ and third $(d g .3)$, can be distinguished. In the position of rest the three divisions of the wing are bent upon one another in the form of a $\mathrm{Z}$; during flight they are straightened out and extended so that the 
axis of the entire wing is at right angles to that of the trunk. On the anterior or pre-axial border of the limb a fold of skin stretches between the upper arm and the fore-arm; this is the alar membrane or pre-patagium (pr. ptgm.). A similar but much smaller fold extends, post-axially, between the proximal portion of the upper arm and the trunk; this is the post-patagium (pt. ptgm.).

In the hind-limb the short thigh is closely bound to the trunk, not standing well out as in a Reptile, but directed downwards and

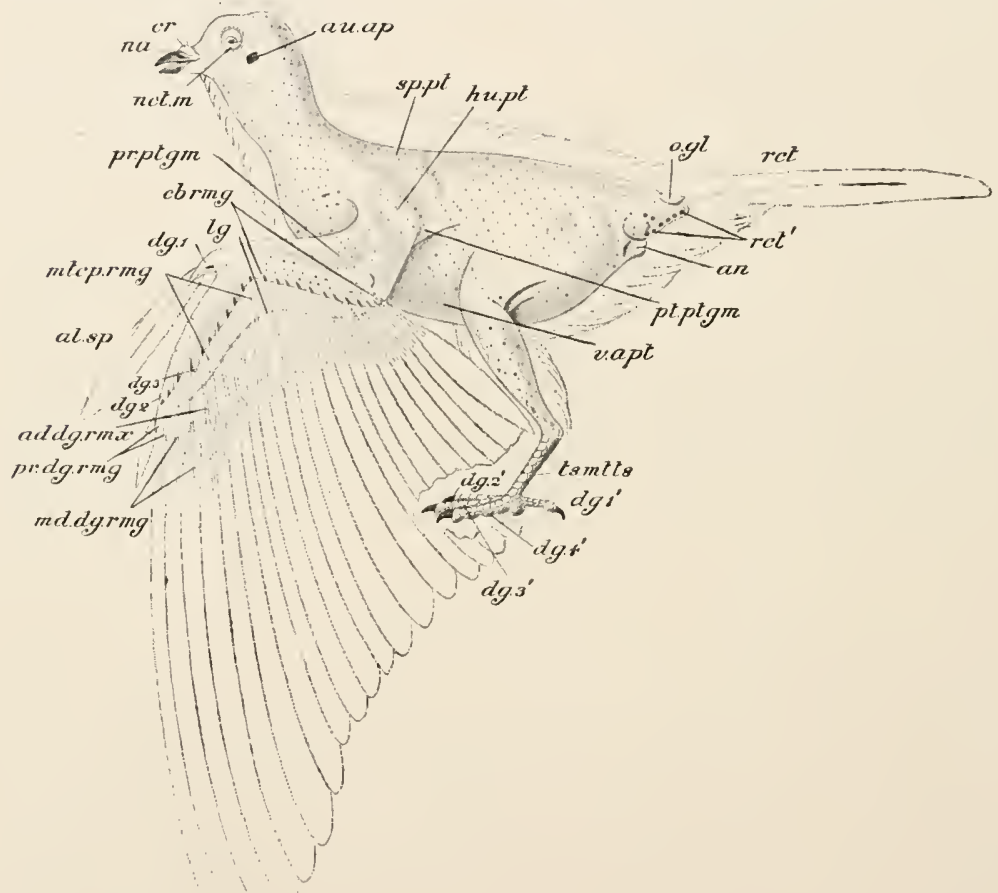

FIt. 1033.-Columba livia. The entire animal from the left side with most of the feathers removed. ad.dg.rmx. ad-digital remex; al. sp. ala spuria; an. anus; $a u$. ap. auditory a perture; $c b$. $r m g$. cubital remiges ; $c r$. cere; $d g .1,2,3$, digits of manus ; dg. $1^{\prime}, 2^{\prime}, 3^{\prime}, 4^{\prime}$, digits of pes; hu.pt. humeral pteryla; $l g$. ligament of remiges ; md.dg.rmg. mid-digital remices; na. nostril ; net.m. nictitating membrane; o.gl. oil-gland ; pr.dg.rmg. pre-digital remiges ; pr.ptgm. pre-patagium ; pt.ptgm. post-patagium ; rct. mesial rectrix of right side ; ret'. sacs of left rectrices; sp. pt. spinal pteryla; $t$.mtts. tarso-metatarsus ; v.apt. ventral apterium.

forwards; the long shank extends from the knee downwards and backwards ; and the foot is clearly divisible into a proximal portion, the tarso-metatarsus ( $t s$. mits.), and four digits, of which one, the hallux $\left(d g . I^{\prime}\right)$, is directed backwards, the others, the $2 \mathrm{nd}, 3 \mathrm{rd}$, and 4 th of the typical foot, forwards. The entire hind-limb is in a plane parallel with the sagittal plane of the trunk. 
The mouth is terminal, and is guarded by the elongated upper and lower beaks; it has, therefore, a very wide gape. On each side of the base of the upper beak is a swollen area of soft skin, the cere ( $c r$.$) , surrounding the nostril ( (n a$.$) , which has thus a remark-$ ably backward position. The eyes are very large, and each is guarded by an upper and lower eyelid and a transparent nictitating membrane (nct. m.). A short distance behind the eye is the auditory aperture (au. ap.), concealed by feathers in the entire Bird, and leading into a short external auditory meatus, closed below by the tympanic membrane. The anus or cloacal aperture (an.) is a large, transversely-elongated aperture placed on the ventral surface at the junction of the uropygium with the trunk.

Exoskeleton.-The exoskeleton is purely epidermal, like that of the Lizard, which it also resembles in consisting partly of horny scales. These cover the tarso-metatarsus and the digits of the foot, and are quite reptilian in a p pearance and structure. Each digit of the foot is terminated by a claw, which is also a horny product of the epidermis; and the beaks are of the same nature. The rest of the body, however, is covered by feathers, a unique type of epidermal product found nowhere outside the present class.

A feather (Fig. 1034) is an elongated structure consisting

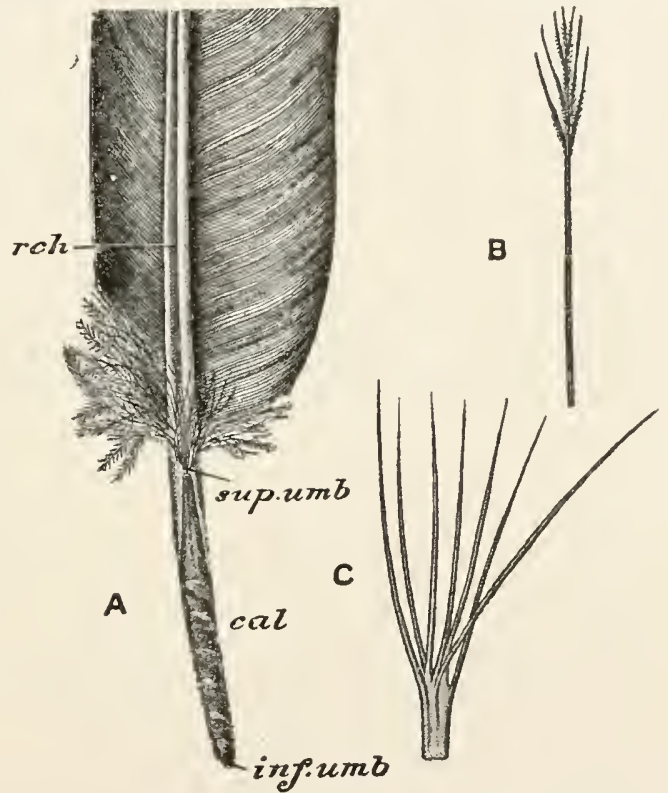

FIG. 1034-Columba livia. A, proximal portion of a remex. cal. calamus; inf. umb. inferior umbilicus; rch. rachis; sup. umb. superior umbilicus. $B$, filoplume. $C$, nestling-down. (C. from Bronn's Thierreich.) of a hollow stalk, the calamus or quill (cal.), and an expanded distal portion, the vexillum or vane. At the proximal end of the quill is a small aperture, the inferior umbilicus (inf. umb.), into which fits, in the entire Bird, a small conical prolongation of the skin, the feather papilla. A second, extremely minute aperture, the superior umbilicus (sup. umb.), occurs at the junction of the quill with the vane on the inner or ventral face of the feather, i.e., the face adjacent to the body. A small tuft of down in the neighbourhood of the 
superior umbilicus represents the after-shaft of many Birdsincluding some Pigeons (vide infra).

The vane has a longitudinal axis or rachis (rch.) continuous proximally with the quill, but differing from the latter in being solid. To each side of the rachis is attached a kind of membrane forming the expanded part of the feather and composed of barbsdelicate, thread-like structures which extend obliquely outwards from the rachis. In an uninjured feather the barbs are closely connected so as to form a continuous sheet, but a moderate amount of force separates them from one another, and it can readily be made out with the aid of a magnifying glass that they are bound

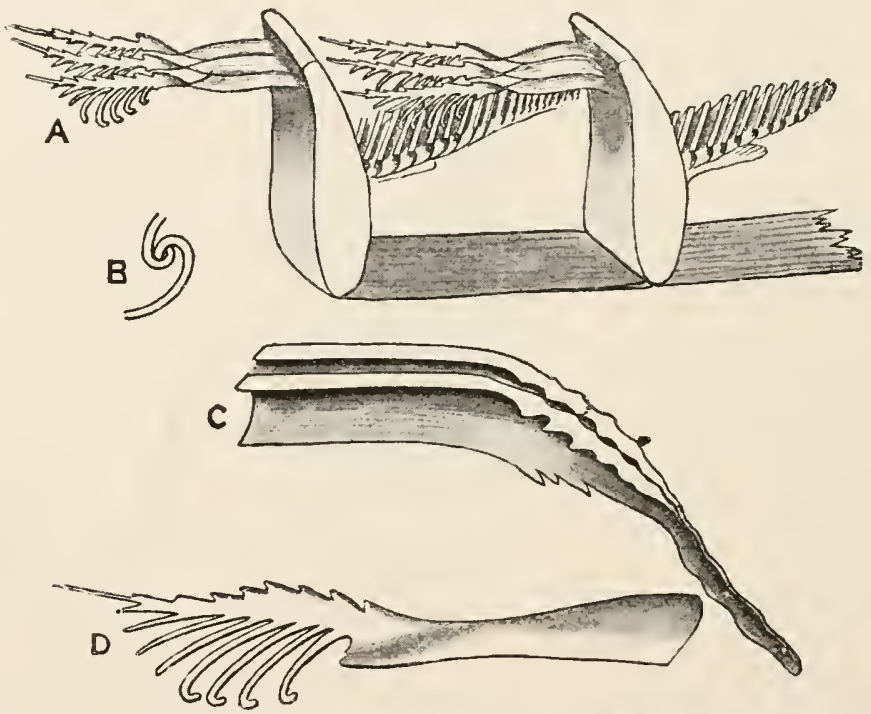

Fia. 1035.--Structure of Feather. A, small portion of feather with pieces of two barbs, each having to the left three distal barbules, and to the right a number of proximal barbules, many of them belonging to adjacent barbs. $B$, hooklet of distal barbule interlocking with tlange of proximal barbule. $C$, two adjacent proximal barbules. $D$, a distal barbule. (From Headley, after Pycraft.)

together by extremely delicate oblique filanents, the barbules, having the same general relation to the barbs as the barbs themselves to the rachis.

The precise mode of interlocking of the barbs can be made out only by microscopic examination. Each barb (Fig. 1035, A) is a very thin and long plate springing by a narrow base from the rachis, and pointed distally. From its upper edge-the edge furthest from the body of the Bird-spring two sets of barbules, a proximal set $(C)$ directed towards the base of the feather, and a distal set $(D)$ towards its tip. Owing to their oblique disposition the distal barbules of a given barb cross the proximal barbules of the next, each distal barbule being in contact with several proximal 
barbules of the barb immediately distal to it $(A)$. The lower edge of the distal barbule is produced into minute hooklets $(D)$ : in the entire feather the hooklets of each distal barbule hook over prominent flanges of the proximal barbules with which it is in contact $(A, B)$.

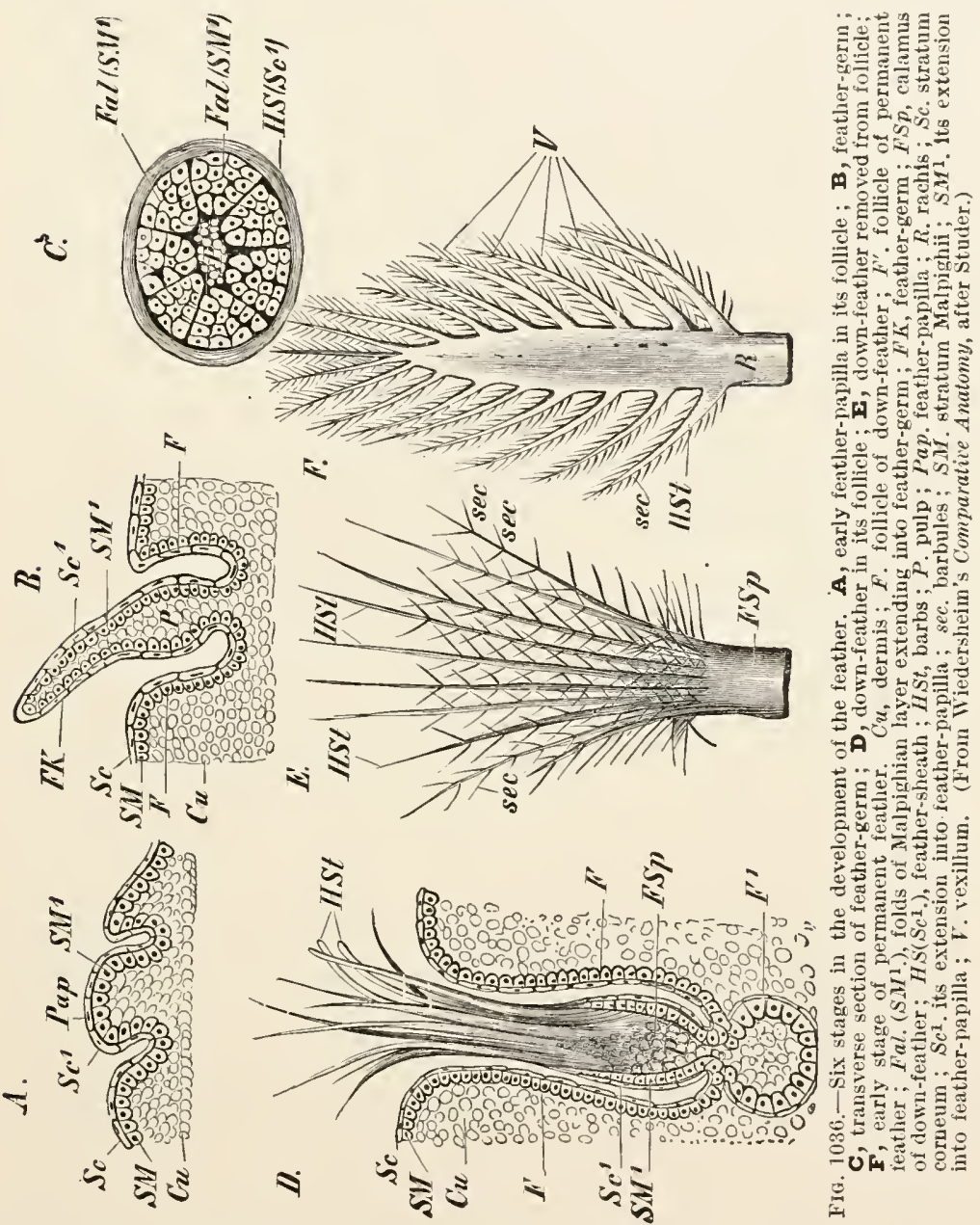

In this way the parts of the feather are so bound together that the entire structure offers great resistance to the air.

Among the contour feathers which form the main covering of the Bird and have the structure just described are found filoplumes (Fig. 1034, B), delicate, hair-like feathers having a long axis and a few barbs, devoid of locking apparatus, at the distal end. Nestling Pigeons are covered with a temporary investment of down-feathers 
$(C)$, in which also there is no interlocking of the barbs: when these first appear each is covered by a horny sheath like a glove-finger.

Feathers, like scales, arise in the embryo from papillæ of the skin (Fig. 1036, A, Pap.), formed of derm with an epidermal covering. The papilla becomes sunk in a sac, the feather-follicle $(\mathbf{B}, F)$, from which it subsequently protrudes as an elongated feather-germ $(F K)$, its vascular dermal interior being the feather-pulp $(P)$. The Malpighian layer of the distal part of the feather-germ proliferates in such a way' as to form a number of vertical radiating ridges (C, $\left.F a l\left(S M^{1}\right)\right)$ : its proximal part becomes uniformly thickened, and in this way is produced the rudiment of a down-feather, having a number of barbs springing, at the same level, from the distal end

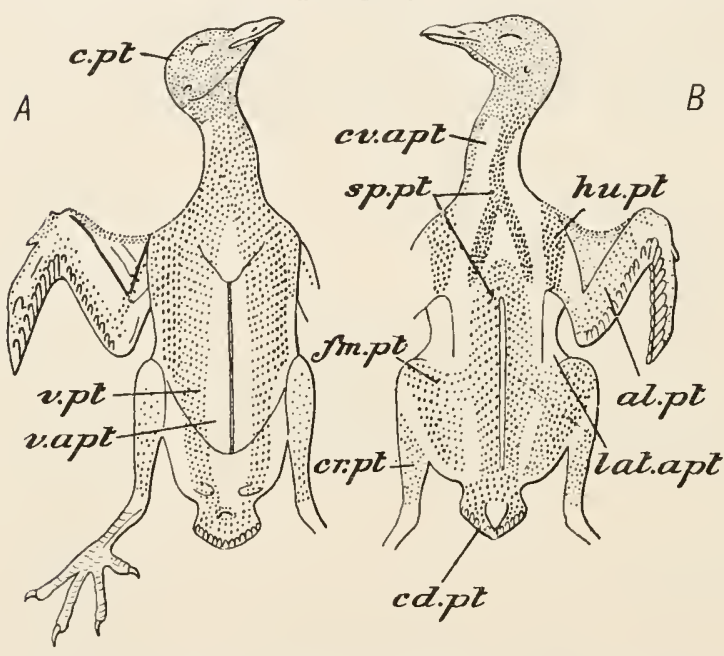

Fia, 1037,-Pterylosis of Columba livia. $A$, ventral; $B$, dorsal. al.pt. alar pteryla or wing-tract; c.pt. cephalic pteryla or headtract; cd.pt. caudal pteryla or tail-tract ; $c r . p t$. crural pteryla cv.apt. cervical apterium or neck-space ; fm.pt. femoral pteryla ; hu.pt. humeral pteryla; lat.apt. lateral apterium; sp.pt. spinal pteryla; $v$.apt. ventral apterium; $v$.pt. ventral pteryla. (After Nitzsch.)

one size, two adjacent ones outgrow the rest and become the rachis; as the latter elongates it carries up with it the remaining ridges, which become the barbs.

The feathers do not spring uniformly from the whole surface of the body, but from certain defined areas (Fig. 1037), the feather tracts or pterylce (sp. pt., hu. pt., \&c.), separated from one another by featherless spaces or apteria (v. apt., \&c.), from which only a few filoplumes grow. The feathers are, however, long enough to cover the apteria by their overlap, and the body is thus completely covered with a thick, very light, and non-conducting investment.

In the wings and tail certain special arrangements of the feathers are to be distinguished. When the wing is stretched out at right 
angles to the trunk, twenty-three large featliers (Fig. 1033) are seen to spring from its hinder or post-axial border: these are the remiges or wing-quills. Twelve of them are connected with the ulna and are called cubitals or seconduries (cb. rmy.). The rest are known as primaries: seven of these are attached to the metacarpal region, and are hence called metacarpals (mtcp. rmg.), the remaining four or digitals to the phalanges of the second and third digits. These are again distinguished into a single ad-digital (ad. dg. rmx.), connected with the single phalanx of the third digit (Fig. 1045, ph. 3), two mid-digitals (md. dg. rmg.) with the proximal phalanx of the second digit (Fig. 1045, ph. 2), and two pre-digitals (pr. dg. rmg.) with its distal phalanx (Fig. 1045, ph. $2^{\prime}$ ). A special tuft of feather's on the anterior border of the wing, arising from the pollex (Fig. 1045, ph. 1), forms the ala spuria (al. sp.). The spaces which would otherwise be left between the bases of the remiges are filled in, both above and below, by several rows of upper and under wingcoverts. In the tail there are twelve long rectrices (Fig. 1033, rct.) or tail-quills, springing in a semicircle from the uropygium; their bases are covered, as in the wing, by upper and under tail-coverts. The whole feather-arrangement is known as the pterylosis.

Endoskeleton.--The vertebral column is distinguished from that of most other Craniata by the great length and extreme mobility of the neck, the rigidity of the trunk-region, and the shortness of the tail. As in Reptilia, the cervical passes almost insensibly into the thoracic region, and the convention is again adopted of counting as the first thoracic (Fig. 1038, th. v. 1), the first vertebra having its ribs united with the sternum. There are fourteen cervical vertebræ, the last or last two of which have doubleheaded ribs $(c v . r$.$) , each having its proximal end divisible into the$ head proper articulating with the centrum of the vertebra, and a tubercle with the transverse process : their distal ends are free, not uniting with the sternum. In the third to the twelfth there are vestigial ribs (Fig. 1039, rb.), each having its head fused with the centrum, and its tubercle with the transverse process. The whole rib thus has the appearance of a short, backwardly-directed transverse process perforated at its base; the perforation transmits the vertebral artery, and is called the vertebrarterial foramen (vrb.f.).

The centra of the cervical vertebræ differ from those of all other Vertebrata in having saddle-shaped surfaces, the anterior face (Fig. 1039, A) being concave from side to side and convex from above downwards, the posterior face $(B)$ convex from side to side and concave from above downwards. Thus the centrum in sagittal section appears opisthocœlous, in horizontal section procolous. This peculiar form of vertebra is distinguished as heterocolous. The centra articulate with one another by synovial capsules, 'each traversed by a vertical plate of cartilage, the meniscus, with a 
central perforation through which a suspensory ligament passes from one centrum to the other.

The first two vertebræ, the atlas and axis, resemble those of the

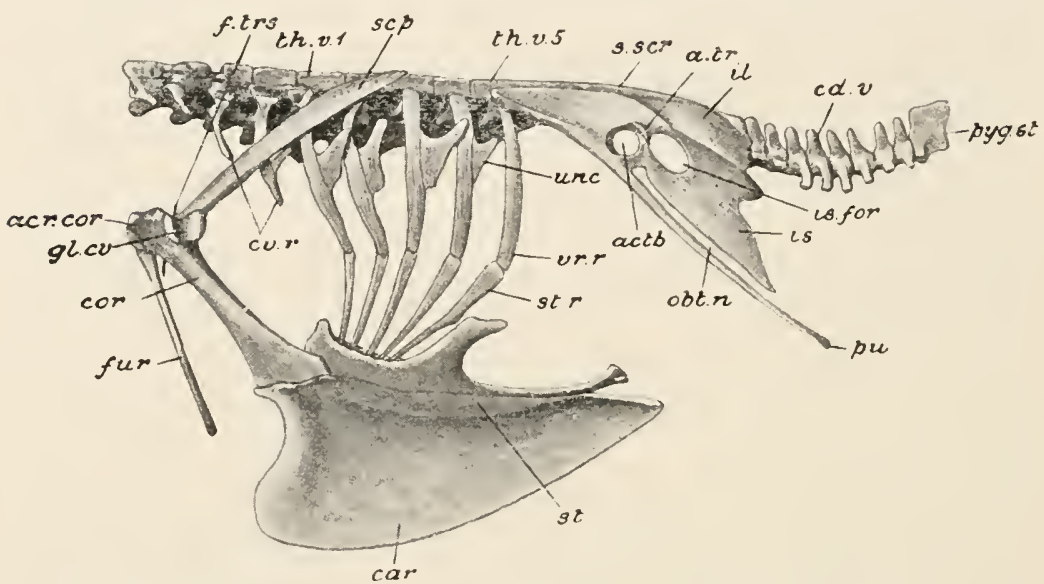

FIG. 1038.-Columba livia. The bones of the trunk. acr.cor. acrocoracoid; a.tr. antitrochanter; $a c t b$. acetabulum; $c a r$. carina sterni ; $c d$. v. caudal vertebre ; cor. coracoid : $c v . r$. cervical ribs; f.trs. probe passed into foramen triosseum; fur. furcula ; $g l . c v$. glenold cavity ; il. ilium ; is. ischium ; is.for. ischiadic foramen ; obt.n. obturator notch ; pu. pubis ; pyg.st. pygostyle; scp. scapula ; s.scr. syn-sacrum; st. sternum; st. r. sternal ribs; th. $v, 1$, first, and th. $v$. 5, last thoracic vertebra; unc. uncinates; vr.r. vertebral ribs.

Lizard, but have the various elements of which they are composed completely fused. The small size of the ring-like atlas is noticeable.

Between the last cervical vertebræ and the pelvic region come
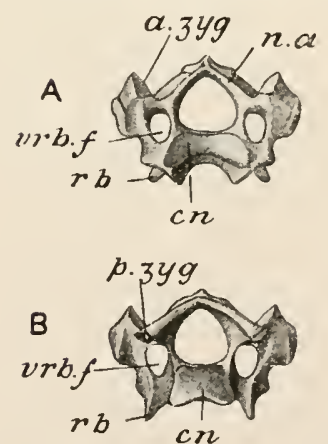

FIG. 1039-Columba livia. Cervical vertebra. $A$, anterior, $B$, posterior face. $a$. zyo. anterior zygapophysis ; $c n$. centrum; n.a. neural arch; p.zyg. posterior zygapophysis ; $r b$. rib; $2 r b . f$. vertebrarterial foramen. either four or five thoracic vertebræ (Fig. 1038)-the first three, when four only are present, the second, third, and fourth, when there are five, united into a single mass, the last free. The anterior thoracic as well as the posterior cervical vertebræ have the centrum produced below into a compressed plate, the hypapophysis, for the origin of the flexor muscles of the neck. They all bear ribs, each consisting of a vertebral (vr.r.) and a sternal (st.r.) portion, and articulating with the vertebra by a double head. The sternal, like the vertebral rib, is formed of true bone, not of calcified cartilage as in Reptiles, and articulates with the vertebral rib by a synovial joint. Springing from the posterior edge of the vertebral rib is an uncinate (unc.), resembling that of Sphenodon and the Crocodile, but formed of bone and ankylosed with the rib. 
Following upon the fourth or fifth thoracic are about twelve vertebræ, all fused into a single mass (Fig. 1038, s.scr), and giving attachment laterally to the immense pelvic girdle. The whole of this group of vertebræ has, therefore, the function of a sacrum, differing from that of a Reptile in the large number of vertebræ composing it. The first of them bears a pair of free ribs, and is, therefore, the fifth or sixth (last) thoracic (th.v.5). The next five or six have no free ribs, and may be looked upon as lumbar (Fig. 1040, l. ${ }^{1}-s^{3}$ ) : their transverse processes arise high up on the neural arch, and the ligament uniting them is ossified, so that the lumbar region presents dorsally a continuous plate of bone. Next come two sacral vertebræ $\left(c .^{1}\right)$ homologous with those of the Lizard: besides transverse processes springing from the neural arch, one or both of them bears a second or ventral outgrowth (c.r.) springing from each side of the centrum and abutting against the ilium just internal to the acetabulum. These distinctive processes are ossified independently and represent sacral ribs. The remaining five vertebræ of the pelvic region are caudal. Thus the mass of vertebræ supporting the pelvic girdle in the Pigeon is a compound sacrum, or syn-sacrum, formed by the fusion of the posterior thoracic, all the lumbar and sacral, and the anterior caudal vertebræ.

The syn-sacrum is followed by six free caudals, and the vertebral column ends posteriorly in an upturned, compressed bone, the pygostyle or ploughshare-bone

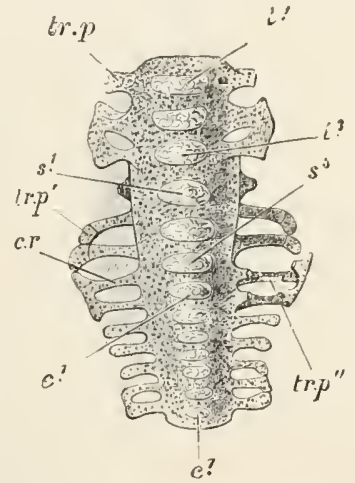

FIG. 1040.-Columba livia. Sacrum of a nestling (about fourteen days old), ventral aspect. $c^{1}$. centrum of first sacral vertebra; $c^{\tilde{T}}$. centrum of fifth caudal; c.r. flrst sacral rib $; l$. centrum of first lumbar ; $l^{3}$. of third lumbar : $s^{1}$, of fourth lumbar; $s^{3}$, of sixth lumbar; tr.p. trans. verse process of first lumbar ; tr. $p^{\prime}$. of fifth lumbar; tr.p". of first sacral. (From Parker's Zootomy.)

(Fig. 1038, pyg.st.), formed by the fusion of four or more of the hindmost caudal vertebræ.

Thus the composition of the vertebral column of the Pigeon may be expressed in a vertebral formula as follows :-

Syn-sacrum.

Pyg.

Cerv. 14. Thor. 4 or $5+1$ Lumb. 5 or 6 . Sacr. 2. Caud. $5+6 \div 4=43$.

The sternum (Fig. 1038, st.) is one of the most characteristic parts of the Bird's skeleton. It is a broad plate of bone produced ventrally, in the sagittal plane, into a deep keel or carina sterni (car.), formed, in the young Bird, from a separate centre of ossification. The posterior border of the sternum presents two pairs of notches, covered, in the recent state, by membrane; its anterior edge bears a pair of deep grooves for the articulation of the coracoids. 
The skull (Fig. 1041) is distinguished at once by its rounded brain-case, immense orbits, and long, pointed beak. 'The foramen magnum $(f . m$. ) looks downwards as well as backwards, so as to be visible in a ventral view, and on its anterior margin is a single, small, rounded occipital condyle (o.c.). Most of the bones, both of the cranial and facial regions, are firmly ankylosed in the adult,

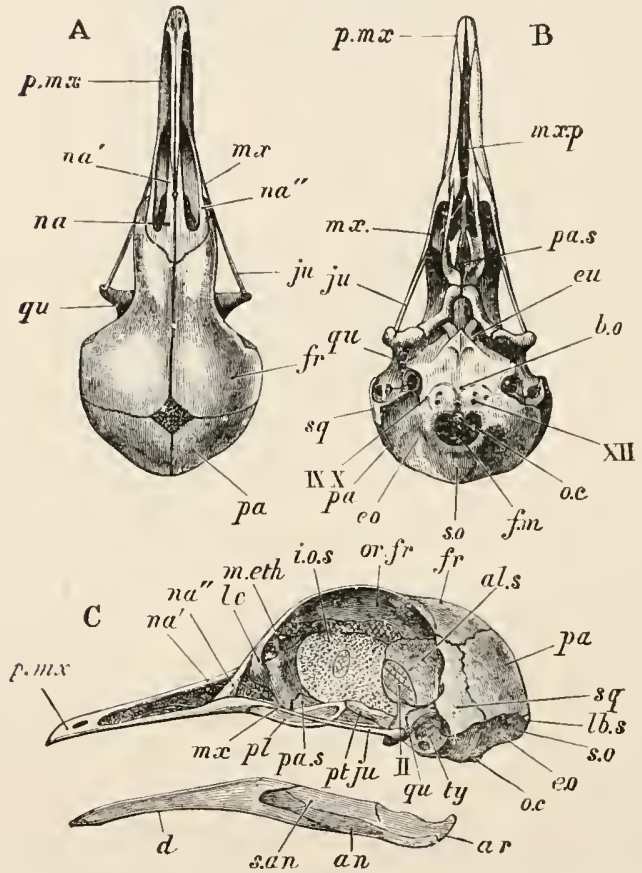

Fia. 1041-Columba livia. Skull of young specimen. $A$, dorsal ; $B$, ventral ; $C$, left side. al.s. alisphenoid; $a n$. angular; ar. articular; $b$. o. basi-occipital; $d$. dentary; e. o. exoccipital; eu. aperture of Eustachian tube; $f . m$. foramen magnum ; $f$. frontal ; $i . o . s$. inter-orbital septum ; $j u$. jugal ; lc. lacrymal ; $l b .8$. lambdoidal suture; $m$. eth. mesethmold ; $m x$. maxilla ; mx.p. maxillo-palatlne process; na. $n a^{\prime}$. na". nasal ; o.c. occipital condyle ; or.fr. orbital plate of frontal ; $p a$. parletal ; pa.8. parasphenoid (rostrum); $p l$. palatine ; $p . m x$. premaxilla ; $p t$. pterygold; qu. quadrate ; s. an. supra-angular; s. o. supra-ocelpital ; sq. squamosal ; ty. tympanic cavity; $I I-X I I$, foramina for cerebral nerves. (From Parker's Zootomy.)

posterior portion of the parasphenoid. The tympanic cavity is bounded by the squamosal (Fig. 1041, sq.), which is firmly united to the other cranial bones. The main part of the auditory capsule is ossified by a large pro-otic (Fig. 1042, PR.OT.) : the small opisthotic of the embryo early unites with the exoccipital, the epiotic with the supra-occipital. The parasphenoid and mesethmoid together form the inter-orbital septum (Fig. 1041, i.o.s.), a vertical partition, partly bony, partly cartilaginous, which separates the orbits from 
one another. It is very characteristic of the Bird's skull that the immense size of the eyes has produced a compression of this region of the skull. The ecto-ethmoids or turbinals are comparatively poorly developed, in correspondence with the small size of the olfactory organs. There are large lacrymals (Fig. 1041, lc., Fig. 1042, LCR.), and the nasals ( $n a, n a^{\prime}, n a^{\prime \prime}, N A$ ) are forked bones, each furnishing both an inner and an outer boundary to the corresponding nostril.

The premaxillæ $(p . m x ., P M X$.) are united into a large triradiate

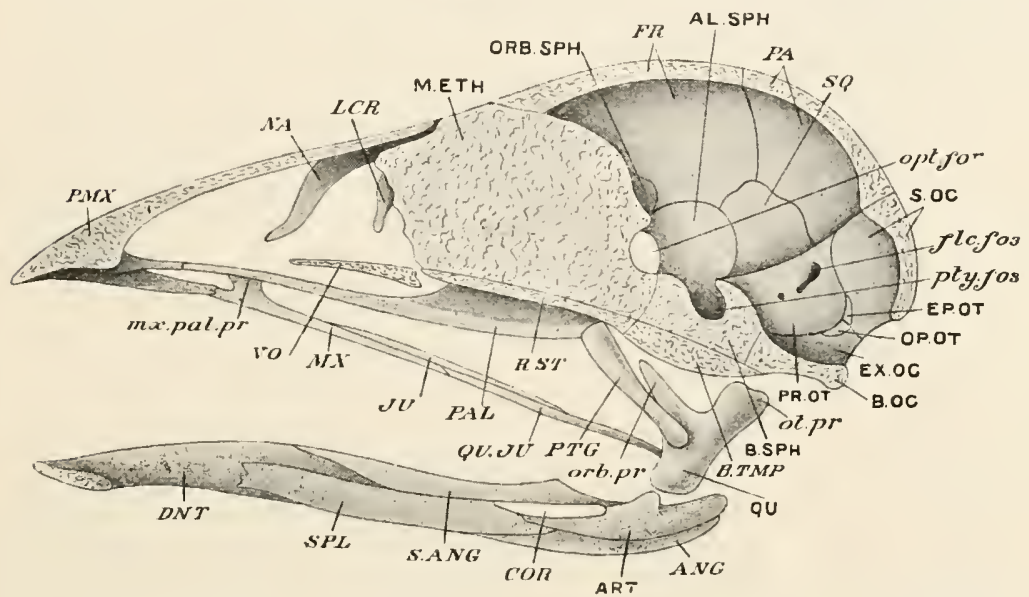

FIG. 1042.-Sagittal section of a Bird's skull (diagrammatic). Replacing Bones-AI.SPI. alisphenoid; ART. articular; B.OC. basi-occipital; B.SPF. basl-sphenoid; FP.OT. epiotic : EX.OC. exoccipital ; IM.FTI. mesethmoid; OP.OT. opisthotlc ; ORB.SPY. orbito-sphenoid; PR.OT. pro-otic ; QU. quadrate; S.OC. supraoccipital. Investing bones-A $N G$. angular; B. TMP. basi-temporal ; $C O R$. coronary ; $I N T$. dentary ; $F R$. frontal ; $J U$. jugal ; $L C R$. lacrymal; $M X$. maxilla; $N A$. nasal ; $P A$. parietal : $P A L$. palatine; $P M X$. premaxilla ; $P T G$. pterygoid : $Q U$. $J U$. quadratojugal ; RST. rostrum ; $S$. $A N G$. supra-angular ; $S P L$. splenial; $S Q$. squamosal ; VO. vomer; flc. fos. floccular fossa; $m x$. pal. pr. maxillo-palatlne process; opt. for. optlc foramen; orb. pr. orbltal process; ot. pr. otic process ; pty. fos. pituitary fossa.

bone which forms practically the whole of the upper beak. The maxillæ $(m x ., M X$.), on the other hand, are small, and have their anterior ends produced inwards into spongy maxillo-palatine processes (Fig. 1041, mx.p., Fig. 1042, mx.pal.pr.). The slender posterior end of the maxilla is continued backwards by an equally slender jugal $(j u ., J U$.$) and quadrato-jugal (Q U . J U$.$) to the$ quadrate. The latter (qu., QU.) is a stout, three-rayed bone articulating by two facets on its otic process (ot. pr.) with the roof of the tympanic cavity, sending off an orbital process (orb. pr.) from its anterior margin, and presenting below a candyle for articulation with the mandible; it is freely movable upon its tympanic articulation, so that the lower jaw has a double joint as in Lizards and Snakes.

The palatines ( $p l, P A L$.) have their slender anterior ends ankylosed with the maxilla, their scroll-like posterior ends articulating 
with the pterygoids and the rostrum. The pterygoids (pt., PTG.) are rod-shaped and set obliquely ; each articulates behind with the quadrate, and, at about the middle of its length, with the basipterygoid process, a small faceted projection of the base of the rostrum. There is no vomer in the Pigeon.

The mandible of the young Bird consists of a replacing bone, the articular (ar., ART.), and four investing bones, the angular (an., $A N G$.), supra-angular (s.an., S. $A N G$.), dentary (d., DNT.), and splenial $(S P L$.$) , all having the same general relations as in$ the Lizard. The hyoid apparatus (Fig. 1043) is of characteristic form, having an arrow-shaped body $(b$. hy.) with a short pair of anterior cornua (c. hy.) derived from the hyoid arch, and a

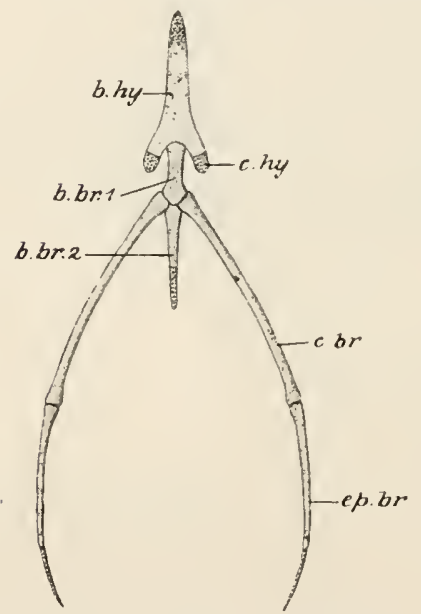

FIG. 1043.-Columba livia. Hyoid apparatus. The cartilaginous parts are dotted. b.br. 1, b.br. 2, basibranchials; b.hy. basi-hyal; c.br
cerato-branchial; c. hy. hyoid cornu; ep.or. epi-branchial. long pair of posterior cornua (c.br., ep.br.) from the first branchial. The columella (Fig. 1044) is a rod-shaped bone ankylosed to the stapes, and bearing at its outer end a three-rayed cartilage, the extra-columella (e.st.,

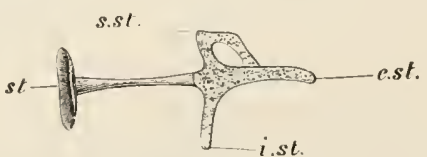

FIG. 1044.-Columba livia. The columella auris (magnified). The cartilaginous parts are dotted. $e$. st. extra-stapedial ; $i$. st. infra-stapedial ; $s$. st. supra-stapedlal; st. stapes. (From Parker's
$Z$ ootomy.)

i.st., s.st.), fixed to the tympanic membrane.

The shoulder-girdle (Fig. 1038) is quite unlike that of other Craniates. There is a pair of stout, pillar-like coracoids (cor.) articulating with deep facets on the anterior border of the sternum, and directed upwards, forwards, and outwards. The dorsal end of each is produced into an acro-coracoid process (acr. cor.), and below this, to the posterior aspect of the bone, is attached by ligament a sabre-shaped scapula (scp.) which extends backwards over the ribs, and includes, with the coracoid, an acute angle, the coraco-scapular angle. The glenoid cavity $(g l . c v$.$) is formed in$ equal proportion by the two bones; internal to it the scapula is produced-into an acromion process.

In front of the coracoids is a slender $\mathrm{V}$-shaped bone, the furcula (fur.) or " merrythought," the apex of which nearly reaches the stemum, while each of its extremities is attached by ligament to the acromion and acro-coracoid processes of the corresponding side in such a way that a large aperture, the foramen triosseum 
(f.trs.), is left between the three bones of the shoulder-girdle. The fureula is an investing bone and represents fused clavicles and interclaviele.

Equally characteristic is the skeleton of the fore-limb. The humerus (Fig. 1015, hu.) is a large, strong bone, with a greatly expanded head and a prominent ridge for the insertion of the pectoral muscle. In it, as in all the other long bones, the extremities as well as the shaft are formed of true bone. The radius (ra.) is slender and nearly straight, the ulna stouter and gently curved. There are two large free carpals, a radiale (ra.') and an ulnare (ul.'), and articulating with these is a bone called the carpometacarpus (cp.mlep.) consisting of two rods, that on the pre-axial side strong and nearly straight, that on the post-axial side slender and curved, fused with one another at both their proximal and distal ends; the proximal end is produced, preaxially, into an outstanding step-like process. The study of development shows that this bone is formed by the union of the distal carpals with three metacarpals (Fig. 1046), the second and third of which are the two rod-like portions of the bone, the first the step-like projection. Articulating with the first meta- FIG. 1045.-Columba livia. Skeleton of the ticulating with the left wing. cp.mtep. carpo-metacarpus; $h u$. earpal is a single pointed phalanx (Fig. 1045, ph. 1); the second metacarpal bears two phalanges, the proximal one

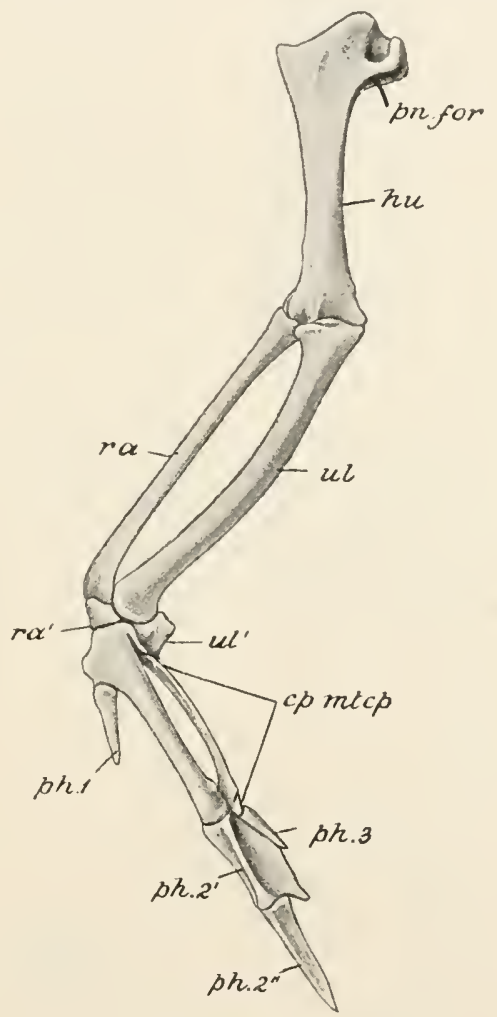
humerus ; $p h .1$, phalanx of first digit ; $p h .2^{\prime}$, $p h .2^{\prime \prime}$, phalanges of second digit; $p h .3$, phalanx of third digit; pn. for. pneumatic foramen. $r a$. radlus; $r a^{\prime}$. radiale ; $u l$. ulna ; $u l^{\prime}$. ulnare.

$\left(p h .2^{\prime}\right)$ produced post-axially into a flange, the distal one $\left(p h .2^{\prime \prime}\right)$ pointed; the third metacarpal bears a single pointed phalanx ( $p h .3)$.

The pelvic girdle (Fig. 1038) resembles that of no other Vertebrate with the exception of some Dinosaurs. The itium (il.) is an immense bone attached by fibrous union to the whole of the syn-sacrum and beeoming ankylosed with it in the adult. It is divisible into pre-acetabular and post-acelabular portions of approximately equal 
size. As usual it furnishes the dorsal portion of the acetabulum, and on the posterior edge of that cavity is produced into a process, the anti-trochanter (a.tr.), which works against the trochanter, a

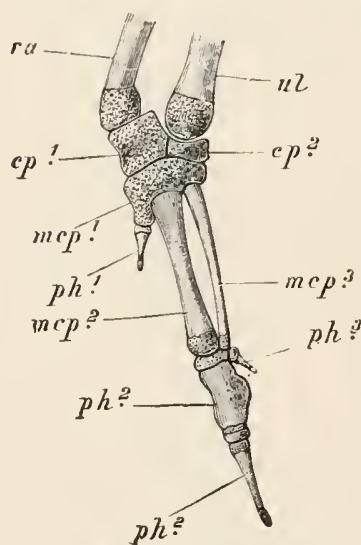

FIG. 1046.-Columba livia. Left manus of a nestling. The cartilaginous parts are dotted. $c p .1$, radiale ; $c p .2$, ulnare mcp. 1, 2, 3, metacarpals ; $p h$ 1 , phalanx of first digit ; $p h .2$, ph 2' phalanges of second digit ; $p h .3$, phalanx of third digit; ra. radius; $u$. ulna. (From Parker's Zootomy.) process of the femur. The ventral portion of the acetabulum is furnished in about equal proportions by the pubis and ischium (Fig. 1047) : it is not completely closed by bone, but is perforated by an aperture covered by membrane in the recent state. Both pubis and ischium are directed sharply backwards from their dorsal or acetabular ends. The ischium (is.) is a broad bone, ankylosed posteriorly with the ilium, and separated from it in front by an ischiatic foramen (Fig. 1038, is.for.; Fig. 1047, i.s.f.). The pubis - (pu.) is a slender, curved rod, parallel with the ventral edge of the ischium, and separated from it by an obturator notch (Fig. 1038, obt.n. ; Fig. 1047, ob.f.). Neither ischium nor pubis unites ventrally with its fellow to form a symphysis.

In the hind-limb the femur (Fig. 1048, $f e$.) is a comparatively short bone. Its proximal extremity bears a prominent trochanter $(t r$.$) and a rounded head (h d$.$) , the axis of which is at$ right angles to the shaft of the bone; so that the femur, and indeed the whole limb, lies in a plane parallel with the sagittal plane of the trunk, and is not directed outwards as in Reptiles. Its distal end is produced into pulley-like condyles. There is a small sesamoid bone (i.e., a bone developed in a tendon), the patella (pat.), on the extensor side of the kneejoint. Articulating with the femur is a very long bone, the tibio-tarsus (ti.ts.), produced on the anterior face of its proximal end into a large cnemial process (cn.pr.) for

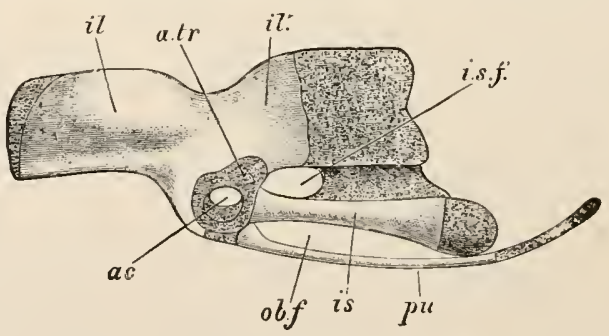

FIa, 1047.-Columba livia. Left innominate of a nestling. The cartilage is dotted. ac. acetabulum: a. $t r$. anti-trochanter; il. pre-acetabular, and $i l^{\prime}$. postacetabular portion of ilium; is. ischium; $i$. s. $f$. ischiadic foramen; $o b . f$. obturator notch ; $p u$. pubis. (From Parker's Zootomy.)

the insertion of the extensor muscle of the thigh. Its proximal articular surface is slightly hollowed for the condyle of the femur ; its distal end is pulley-like, not concave like the corresponding extremity of the tibia of other Amniota. The study of develop- 
ment shows that the pulley-like distal end of the bone (Fig. 1049, 1l.1) consists of the proximal tarsals-astragalus and calcaneum-

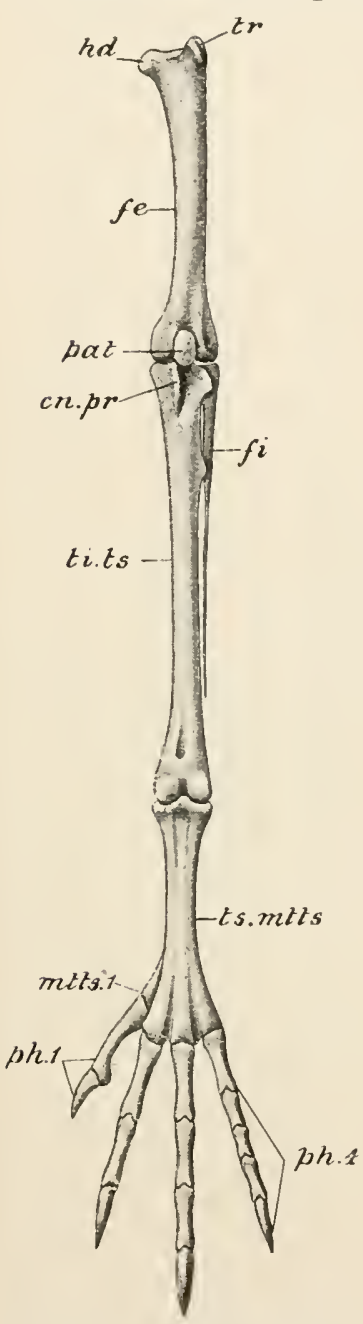

FIG. 1048. - Columba Iivia. cn.pr. cnemial process ; fe, femur ; fi. fibula; $h d$. head of femur; mits. 1, flrst metatarsal; pat. patella; ph.1, phalanges of first digit; $p h .4$, phalanges of fourth digit ; ti. ts. tibio-tarsus; $t s$. mits. tarso-metatarsus; tr. trochanter. Bones of the left hind-limb. which at an early period unite with the tibia and give rise to the compound shank-bone of the adult. The fibula $(f$.$) is very small, much shorter than$ the tibia, and tapers to a point at its distal end.

Following the tibio-tarsus is an elongated bone, the tarso-metatarsus (Fig. 1048, ts. mtts.), presenting at its proximal end a concave surface for the tibio-tarsus, and at its distal end three distinct pulleys for the articulation of the three forwardly-directed toes. In

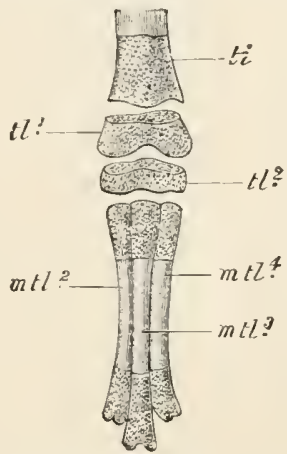

FIG, 1049.-Columba livia. Part of left foot of an unhatclied embryo (magnified). The cartilage is dotted. mtl.2, second, mtl.3, third, and mtl.4, fourth metatarsal; $t i$. tibia; $t l$. 1 , proximal tarsal cartilage; $t$. 2, distal tarsal cartilage. (From Parker's Zootomy.)

the young Bird the proximal end of this bone is a separate cartilage (Fig. $1049, \quad t l .2)$, representing the distal tarsals, and followed by three distinct metatarsals, belonging respectively to the second, third, and fourth digits. Thus the ankle-joint of the bird is a mesolarsal joint, occurring, as in the Lizard, between the proximal and distal tarsals, and not, as in Mammals (q.v.), between the tibia and the proximal tarsals. To the inner or pre-axial side of the tarso-metatarsus, near its distal end, is attached by fibrous tissue a small irregular bone, the first metatarsal (Fig. 1048, mtts.1). The digits have the same 
number of phalanges as in the Lizard, the backwardly-direeted hallux two, the seeond or inner toe three, the third or middle toe four, and the fourth or outer toe five. In all four digits the distal or ungual phalanx is pointed and eurved, and serves for the support of the horny elaw.

It will be observed that every part of the Bird's skeleton presents charaeteristic and indeed unique features. The vertebral eolumn, the skull, the sternum, the ribs, the limb-girdles, and the limbs themselves are all so highly speeialised that there is hardly a bone, exeept the phalanges of the toes and the free eaudal vertebræ, whieh eould possibly be assigned to any other Vertebrate elass.

A further peeuliarity is the fact that the larger proportion of the bones eontain no marrow, but are filled during life with air, and are therefore said to be pneumatic. The cavities of the various bones open externally in the dried skeleton by apertures ealled pneumatic foramina (Fig. 1045, pn.fr.), by whieh, in the entire bird, they eommunieate with the air-saes (vide p. 386). In the Pigeon the bones of the fore-arm and hand, and of the leg, are non-pneumatie.

Muscular System.-As might naturally be expected, the museles of the fore-limb are greatly modified. The powerful downstroke of the wing by which the bird rises into and propels itself through the air is performed by the pectoralis (Fig. 1050, pct.), an immense musele having about one-fifth the total weight of the body; it arises from the whole of the keel of the sternum (car. st.), from the posterior part of the body of that bone (cp.st.), and from the clavicle $(c l$.$) , filling nearly the whole of the wedge-shaped space$ between the body and the keel of the sternum and forming what is commonly ealled the "breast" of the Bird. Its fibres eonverge to their insertion (pct.") into the ventral aspeet of the humerus $\left(h u ., h u^{\prime}.\right)$ which it depresses. The elevation of the wing is performed, not, as might be expeeted, by a dorsally-plaeed muscle, but by the subclavius $(s b$. clv.), arising from the anterior part of the body of the sternum, dorsal to the peetoralis, and sending its tendon $\left(s b . c l v^{\prime}.\right)$ through the foramen triosseum to be inserted into the dorsal aspect of the humerus. In virtue of this arrangement, the foramen aeting like a pulley, the direetion of aetion of the musele is ehanged, the baekward pull of the tendon raising the humerus. There are three tensores patagii (tns. lg., tns. br., Ins. acc.), the aetion of which is to keep the pre-patagium tensely stretehed when the wing is extended. A similar musele (tns. m. p.) aets upon the post-patagium. The museles of the digits are naturally much redueed.

The muscles of the neek and tail are well developed; those of the baek are praetieally atrophied, in eorrespondenee with the immobility of that region. In the leg certain of the muscles are modified to form the perching mechanism. The toes are flexed 
by two sets of tendons, deep and superficial. The deep tendons of the three forwardly-directed digits are formed by the trifurcation of the tendon of a single muscle, the peronceus medius; that of the hallux is derived from a separate muscle, the flexor perforans, which is joined by a slip from the peronæus medius. Thus a pull upon one tendon flexes all the toes. When the leg is bent, as the bird settles to roost, the flexion of the tarso-metatarsus on the shank puts the flexor tendons on the stretch as they pass over the mesotarsal joint, and by the pull thus exerted the toes are automatically bent round the perch by the simple action of flexing the leg. They are kept in this position while the Bird

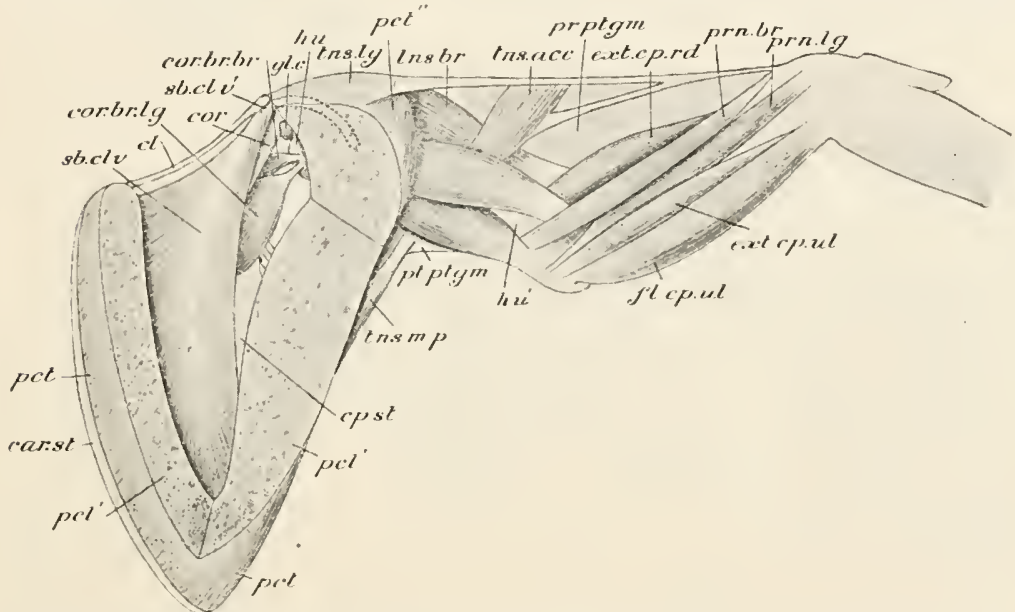

FIG. 1050.-Columba livia. The princinal muscles of the left wing; the greater part of the pectoralis (pct.) is removed. car. st. carina sterni : cl. furcula ; cor, coracoid ; cor. br. br. coraco-brachialis brevis; cor. br. la. coraco-brachialis longus; cp. st. corpus sterni; ext. $c p, r d$. extensor carpi radialis; $c x t, c p, u l$. extensor carpi ulnaris; $f l, c p . u l$. flexor carpi ulnaris; gl. c. glenoid cavity ; hu. head of humerus; $h u^{\prime}$. its distal end ; pct. pectoralis; pct'. its cut edge ; pct". its insertion ; prn. br. pronator brevis ; prn. $l g$. pronator longus ; pr. ptgm. pre-patagium; pt. ptgm. post-patagium; sb. clv. subclavius; $s b . c l v^{\prime}$. its tendon of insertion passing through the foramen triosseum, and dotted as it goes to the humerus; tns. acc. tensor accessorius; tns. br. tensor brevis; $t n s$. $l g$. tensor longus; $t n s, m$. $p$. tensor membranæ posterioris alæ.

is asleep by the mere weight of the body. The action is assisted by a small but characteristic muscle, the ambiens, which arises from the pubis, passes along the inner surface of the thigh, and is continued into a long tendon which comes round to the outer side of the knee, enclosed in a special sheath, and, continuing down the leg, joins the superficial flexors of the digits.

Digestive Organs.- The mouth (Fig. 1051) is bounded above and below by the horny beak, and there is no trace of teeth. The tongue (tng.) is large and pointed at the tip. The pharynx leads into a wide and distensible gullet ( $g u l$.) which soon dilates into an immense reservoir or crop (crp.) situated at the base of the neck, between the skin and the muscles, and immediately in 
front of the sternum. In this cavity the food, consisting of grain, undergoes a process of maceration before being passed into the stomach. From the crop the gullet is continued backwards into the stomach, which consists of two parts, the proventriculus (pron.) and the gizzard (giz.). The proventriculus appears externally like a slight dilatation of the gullet; but its mucous membrane is very thick, and contains numerous gastric glands so large as to be visible to the naked eye. The gizzard has the shape of a biconvex

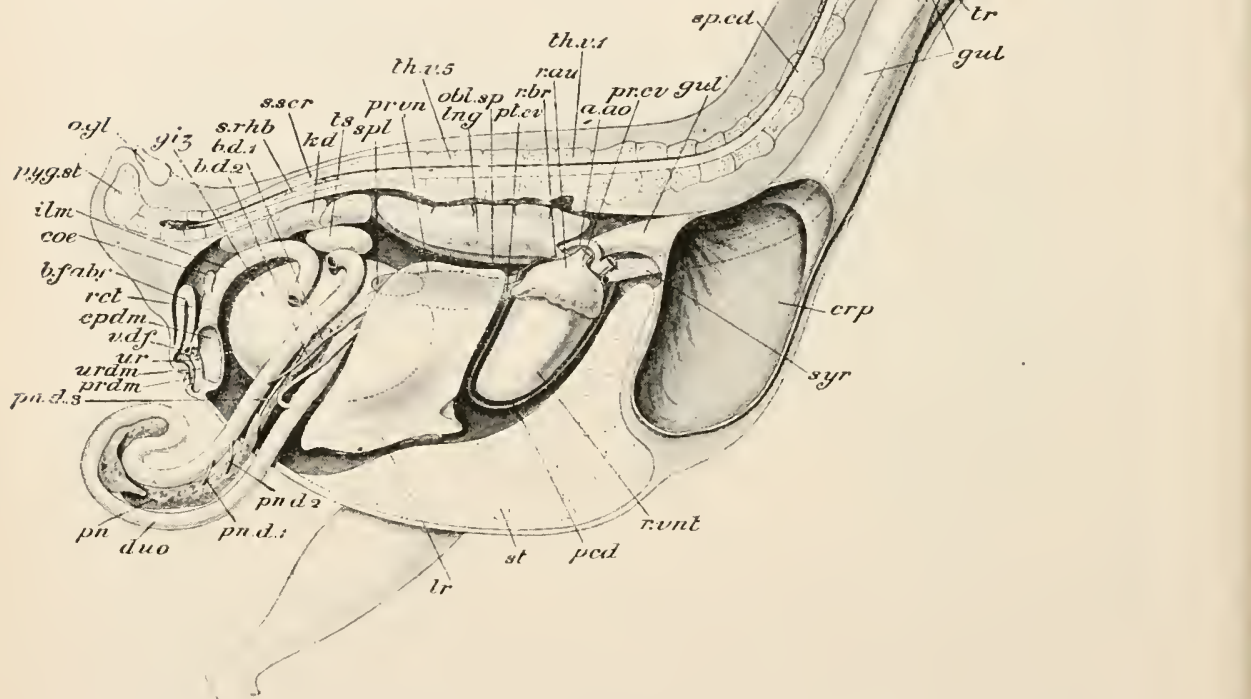

Fig, 1051.-Columba livia. Dissection from the right sidle. The body-wall, with the vertebral column, sternum, brain, \&c., are in sagittal section; portions of the gullet and crop are cut away and the cloaca is opened; nearly the whole of the ileum is removed, and the duodenum is displaced outwards. $a$. ao. aortic arch; $b d .1, b d$. 2 , bile-ducts; $b . f a b r$. bursa Fabricii ; cbl. cerebellum; coe. right cæcum; $c p d m$. coprodæum ; $c r$. cere; $c r b . h$. left cerebral hemisphere; $c r p$. crop; $c r . v$. 1 , first cervical vertebra; di.co. diacole; $d n t$. dentary : duo. duodenum : eus. ap. aperture of Eustachian tubes ; giz. gizzard (dotted lehind the liver); $g l$. glottis; $g u$ ?. gullet; $i l m$. ileum; $i$. orb. $s p$. inter-orbital septum ; lid. right kidney; ling. right lung; $l r$. liver (right lobe); na. bristle passed from nostril into mouth ; obl. sep. oblique septum ; $o . g l$. oil-gland ; pcd. pericardium ; pmx. premaxilla ; $p n$. pancreas; $p n$. $b$. pineal body; $p . n d$. $I-3$, pancreatic ducts; $p r$. $c v$. right pre-caval; prdm. proctodæum ; prin. proventrjculus (dotted behind liver); $p t$. cv. post-caval ; $p t y . b$. pituitary body ; pug. st. pygostyle ; $r$. $a u$. right auricle ; $r$. br. right bronchus ; rct. rectum ; $r$. vnt. right ventricle; $s p . c d$. spinal cord; $s p l$. spleen (dotted behind liver); $s$. $r h b$. sinus rhomboidalis ; s. ser. syn-sacrum ; st. carina sterni ; syr. syrinx ; th. $v$. 1 , first, and th. $v, 5$, fifth thoracic vertebra; $t n g$. tongue ; $t$. trachea ; $t s$. right test is ; ur. aperture of left ureter ; urdm. urodxum; $v$. $d f$. aperture of left vas deferens.

lens : its walls are very thick and its lumen small. The thickening is due mainly to the immense development of the muscles which radiate from two tendons, one on each of the convex surfaces. The epithelial lining of the gizzard is very thick and horny, and of a 
yellow or green colour: its cavity always contains small stones, which are swallowed by the Bird to aid the gizzard in grinding up the food.

The duodenum (duo.) leaves the gizzard quite close to the entrance of the proventriculus and forms a distinct loop enclosing the pancreas. The rest of the small intestine is called the ileum $(i \mathrm{~lm}$.) : it presents first a single loop; then follows its greater part coiled into a sort of spiral; and lastly comes a single loop which passes without change of diameter into the rectum (rct.), the junction between the two being marked only by a pair of small blind pouches or caca (сое.). The cloaca is a large chamber divided into three compartments, the coprodceum (cpdm.), which receives the rectum, the urodceum (urdm.), into which the urinary and genital ducts open, and the proctodceum ( $p r d m$.), which opens externally by the anus.

There are small buccal glands opening into the mouth, but none that can be called salivary. The liver $(l r$.$) is large, and is divisible$ into right and left lobes, each opening by its own duct $(b . d .1$, $b . d .2)$ into the duodenum : there is no gall-bladder. The pancreas $(p n$.$) is a compact reddish gland lying in the loop of the duodenum,$ into which it discharges its secretion by three ducts ( $p n . d .1-3)$. A thick-walled glandular pouch, the bursa Fabricii (b. fabr.), lies against the dorsal wall of the cloaca in young Birds and opens into the proctodæum : it atrophies in the adult.

Ductless Glands.-The spleen ( $\mathrm{spl}$.) is an ovoid red body, of unusually small proportional size, attached by peritoneum to the right side of the proventriculus. There are paired thyroids at the base of the neck; and, in young Pigeons, there is an elongated thymus on each side of the neck. The adrenais (Fig. 1060, adr.) are irregular yellow bodies placed at the anterior ends of the kidneys.

Respiratory and Vocal Organs.-The glottis (Fig. 1051, gl.) is situated just behind the root of the tongue, and leads into the larynx, which is supported by cartilages-a cricoid divided into four pieces, and paired arytenoids - but does not, as in other Vertebrates, function as the organ of voice. The anterior part of the trachea (tr.) has the usual position, ventral to the gullet; but further back it is displaced to the left by the crop, becoming ventral once more as it enters the body-cavity, where it divides into the right $(r . b r$. $)$ and left bronchi. The rings supporting the trachea are not cartilaginous but bony, as also is the first ring of each bronchus, those of the trachea completely surrounding the tube, those of the bronchi incomplete mesially.

At the junction of the trachea with the bronchi occurs the characteristic vocal organ, the syrinx (syr.), found in no other class. The last three or four rings of the trachea (Fig. 1052, tr.), and the first or bony half-ring of each bronchus $(b r$.$) , are modified to form$ a slightly dilated chamber, the tympanum, the mucous membrane 
of which forms a cushion-like thickening on each side. At the junction of the bronchi a bar of cartilage, the pessulus, extends dorso-ventrally and supports an inconspicuous fold of mucous membrane, the membrana semilunaris. The membranous inner walls of the bronchi form the internal tympaniform membranes. A pair of intrinsic syringeal muscles arise from the sides of the trachea and are inserted into the syrinx, and a pair of sternotracheal muscles arise from the sternum and are inserted into the trachea. The voice is produced by the vibration of the semilunar membrane : its pitch is altered by changes produced by the action of the muscles.

The lungs (Figs. 1051, lng., and 1052) are very small in com-

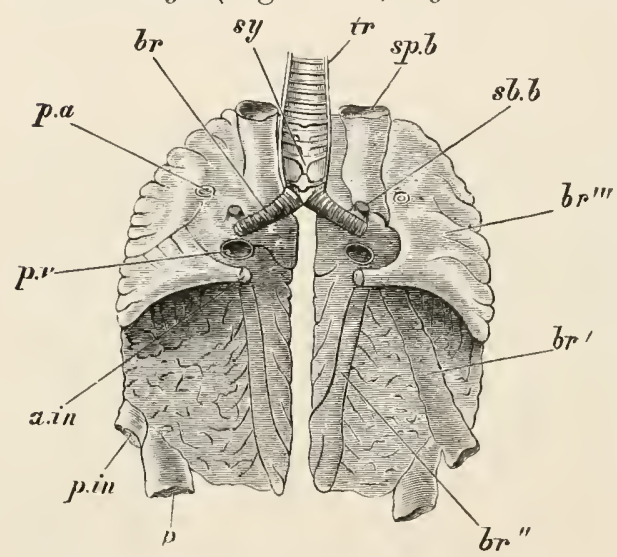

FIG. 1052-Columba livia. The lungs witl the posterior end of the trachea, ventral aspect. $a$. in. bronchus; $b r^{\prime}, b r^{\prime \prime}, b r^{\prime \prime \prime}$, secondary bronchi; $p$. aperture of abdominal air-sac; p.a. pulmonary artery entering lung; $p$. in. aperture of posterior thoracic air-sac; $p$. $v$. pulmonary vein leaving lung; $s b . b$. aperture of interclavicular air-sac; $s p . b$. aperture of cervical air-sac; sy. syrinx; $t r$. trachea. (From Parker's Zootomy.) aperture of anterior thoracic air-sac; br. principal

parison with the size of the Bird, and are but slightly distensible, being solid, spongy organs, not mere bags with sacculated walls as in Amphibia and many Reptiles. Their dorsal surfaces fit closely into the spaces between the ribs, and have no peritoneal covering: their ventral faces are covered by a strong sheet of fibrous tissue, the pulmonary aponeurosis or pleura (Fig. 1053, B, pul. ap.), a special development of the peritoneum. Into this membrane are inserted sinall fan-like costopulmonary muscles, which arise from the junction of the vertebral and sternal ribs.

The bronchus, on entering the lung, is continued to its posterior end (Figs. 1052 and 1053), dividing into two branches, each of which enters a bladder-like air-sac, formed as a dilatation of the mucous membrane of the bronchus. One of these, the abdominal air-sac (Fig. 1053, $A$, abd. a.s.), lies among the coils of the intestine; the other, or posterior thoracic air-sac (post. th. a. s.), is closely applied to the side-wall of the body. The bronchus also gives off, near its entrance into the lung, three short branches, one of which becomes connected with an anterior thoracic air-sac (ant. th. a. s.), situated just in front of the posterior thoracic; another with an interclavicular air-sac (int. clav.a. s.), which is median and unpaired, and connected with both lungs; the third enters a cervical air-sac 
(cere. a. s.) placed at the root of the neck. Each side of the interclavicular: gives off an axillary air-sac, lying in the arm-pit. All these sacs are paired except the interclavicular, which is formed by the fusion of right and left moieties. The sacs are in communication with the pneumatic cavities of the bones.

The ventral or free walls of the thoracic air-sacs of each side are covered by a sheet of fibrous tissue, the oblique septum (obl. sept.), which is continued forwards to the pericardium, and is united with its fellow of the opposite side in the middle dorsal line: it divides the cœlome into two compartments-one containing the lungs with the interclavicular and thoracic air-sacs, the other $(a b d . c a v$.$) the heart,$ liver, stomach, intestine, \&c., with the abdominal airsacs.

Besides the branches to the air-sacs, the main bronchus gives
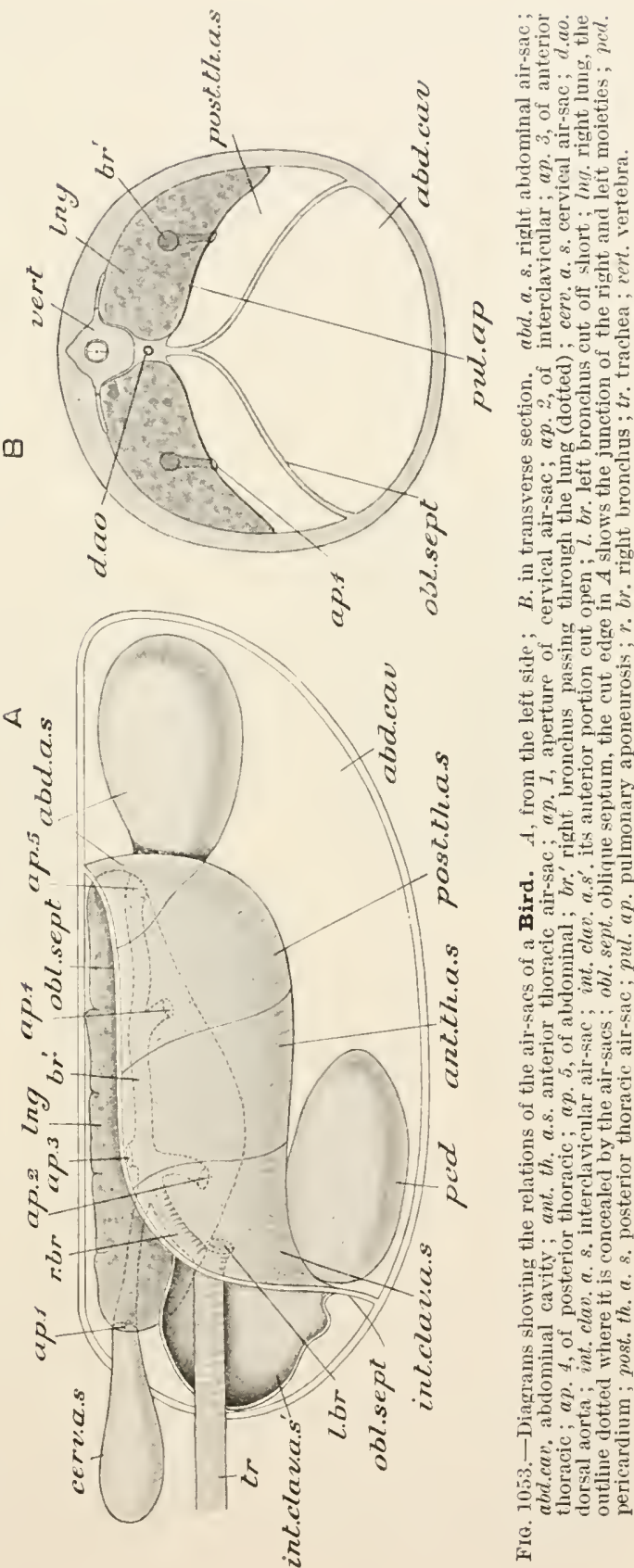
off secondary bronchi, and these branch again, sending off tubes which give rise to a system of fine branching and anastomosing tubules, the "lung-capillaries," which make up the main substance of the lung.

When the Pigeon is standing, the alternate elevation and depression of the sternum, produced partly by the abdominal, partly by the intercostal muscles, causes an alternate enlargement and diminution of the capacity of the colome, and thus pumps air in and out of the lungs. During flight, when the weight is supported by the wings, and the sternum is thus rendered relatively immovable, the same effect seems to be produced by the elevation and depression of the back. In either case the inspired air rushes through the lungs into the air-sacs and thence by diffusion into the pneumatic cavities of the bones. Thus, while in other animals a certain amount of unchanged or residual air is always left in the lungs after each expiration, in Birds the residual air is confined to the air-sacs and to the smaller branches of the bronchi, every respiratory movement drawing a current of fresh or tidal air through the lungs. As a result of this the aëration of the blood is very complete and its temperature correspondingly high. It is worthy of notice that Birds agree with Insects, the only other typically aërial class, in having the inspired air distributed all over the body, so that the aëration of the blood is not confined to the limited area of an ordinary respiratory organ.

Circulatory Organs.-. The heart (Fig. 1051) is of great proportional size, and, like that of the Crocodile, consists of four chambers-right and left auricles, and right and left ventricles. There is no sinus venosus, that chamber being, as it were, absorbed into the right auricle (Fig. 1054, $A, r$, au.). The right ventricle (Fig. 1054, B) partly encircles the left, the former having a crescentic, the latter a circular cavity in transverse sections. The left auriculo-ventricular valve has the usual membranous structure, consisting of two flaps connected with the wall of the ventricle by tendons, but the corresponding valve of the right side $(V$.$) is a$ large muscular fold, very characteristic of the class.

The right auricle receives the right and left precavals ( $r$. prc., $p c . v$.) and the postcaval ( $p t c$.$) ; the left four large pulmonary veins$ $(p . v$.). The left ventricle (Fig. 1055, l.vn.), as in the Crocodile, gives origin to the right aortic arch $(a . a o$.), but the right ventricle $(r . v n$.) gives off only one trunk, the pulmonary artery, which soon divides into two (r.p.a., l.p.a.). The left aortic arch is absent in the adult, and it is the right alone which is continued into the dorsal aorta. The result of this is that the systemic arteries receive pure arterial blood from the left side of the heart, and the only mingling of aërated and non-aërated blood is in the capillaries. This is perhaps the most important physiological advance made by Birds over Reptiles. 
The aortic arch curves over the right bronchus to reach the dorsal body-wall, and then pasises directly backwards as the dorsal aorta (d. ao.). Owing to the immense size of the pectoral minscles, the arteries supplying then are of corresponding dinensions, and the right and left innominate arleries (in. a.), from which the carotids (c. c.), subclavians $(b r . a$.$) , and pectorals (p c . a$.$) arise,$ are actually larger than the aorta itself beyond their origin. In correspondence with the position of the legs, the femoral $(f . a$. and sciatic $(s c . a$.$) arteries arise very far forward: the caudal$ artery $(c$.$) is naturally small.$

The most characteristic feature in the disposition of the circulatory organs is the almost complete disappearance of the

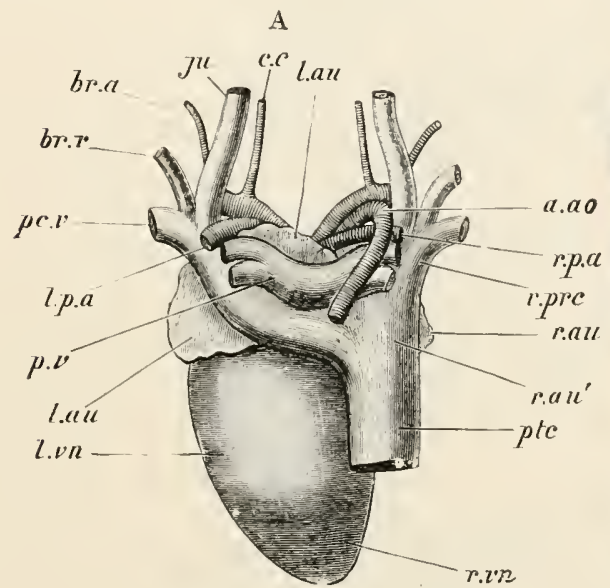

B

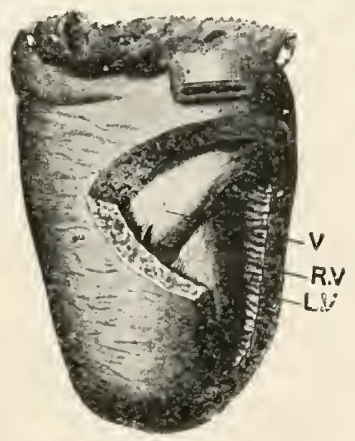

a

Fit. 1054.-A, heart of the Pigeon, dorsal aspect. a.to. arch of aorta; br. a. brachial artery ; $b r$. $v$. brachial vein ; c.c. common carotid ; $j u$. jugular; $l$. au. leit auricle; $l . p . a$. left pulmonary artery; l.vn. left ventricle; $p c . v$. left precaval; ptc. postcaval; $p$. v. pulmonary veins; r.au, r.au, right auricle; r.p.a. right pulmonary artery ; r.pr.c. right precaval ; r.vn. right ventricle. $B$ heart of a Bird with the right ventricle opened. $L, V$. septum ventriculorum; $R$. $V$. right ventricle; $V$. right auriculo-vent ricular valve, $(A ;$ from Parker's Zootomy; $B$, from Headley's Birils.)

renal portal system. There are two renal portal veins (r.p.) formed by the bifurcation of the caudal; but each, instead of breaking up into capillaries in the kidney, sends off only a few small branches $(a, r . v$.) which apparently carry blood to that organ, the main vein passing forwards, through the substance of the kidney, and joining the femoral vein $(f . v$.) from the leg to form the iliac vein $(i . v$.), which, uniting with its fellow of the opposite side, forms the post-caval ( $p t . c$.$) . Thus the main part, at any$ rate, of the blood from the caudal and pelvic regions is taken directly to the heart, and not through the renal capillaries as in most Fishes and all Amphibians and Reptiles.

At the point of bifurcation of the caudal veins a large coccygeomesenteric vein $(c . m . v$.) comes off, and, running parallel with the 

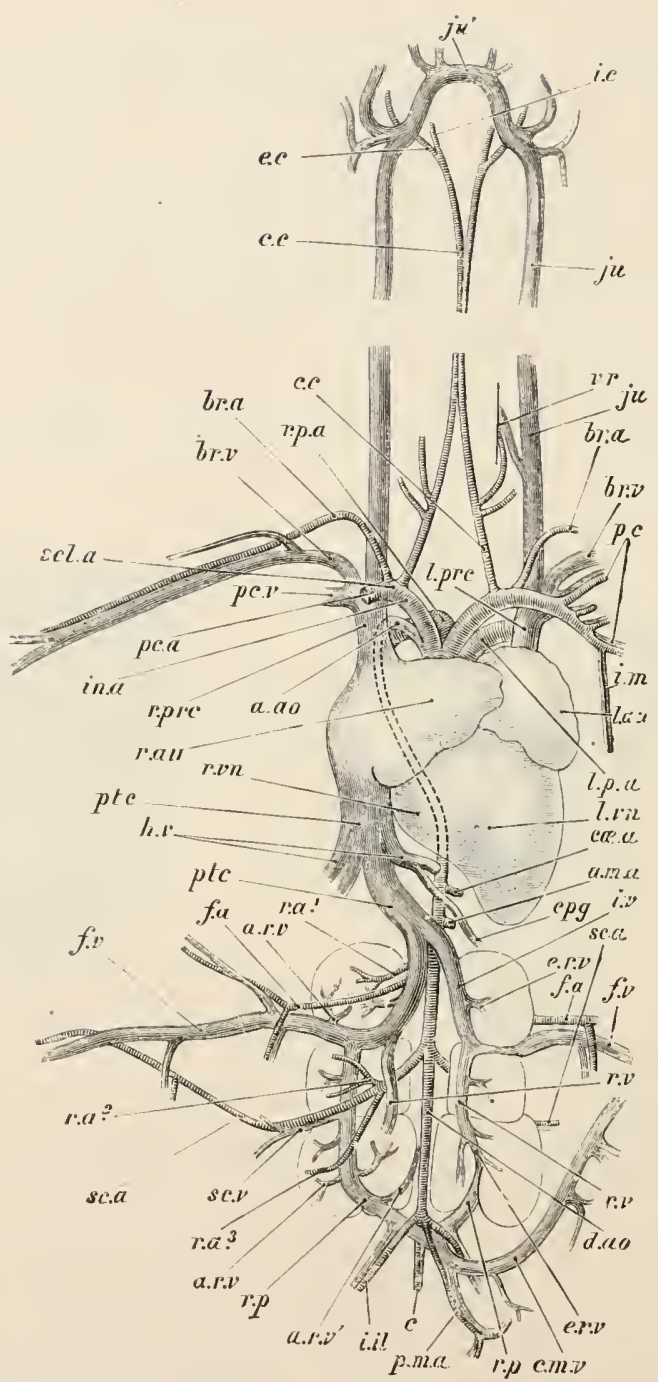

IfG. 1055-Columba livia. The heart and chief blood-vessels, ventral aspect. $a . a o$. arch of aorta ; $a . m . a$. anterior mesenteric artery : a.r.v. afferent renal veins; $a . r . v^{\prime}$. vein bringing blood from pelvis into renal portal system; br.a. brachial artery; br. $v$. brachial vein ; c. caudal artery and vein; c.c. common carotid artery; c.m.v. coccygeo-mesenteric vein, displaced to the right; co.a. coliac artery ; d.ao. dorsal aorta ; e.c. external carotid artery ; $c m$. epigastric vein; e.r.v. efferent renal vein; f.a. femoral artery; f.v. femoral vein ; $h . v$. hepatic vein; i.c. internal carotid artery; $i . i l$. internal iljac artery and vein ; $i . m$. internal mammary artery and vein; in. $\alpha$. innominate artery; $i . v$. iliac vein ; ju. jugular vein; $j u^{\prime}$. anastomosis of jugular veins; $l$. au. left auricle; $l . p . a$. left pulmonary artery ; l.mc. left pre-caval vein; l. $\mathrm{m}$. left ventricle; $p c$. left pectoral arteries and veins; $p c . a$. right pectoral artery; $p c . v$. right pectoral vein; $p . m . a$. posterior mesenteric artery; $p t c$. postcaval vein : $r a .^{1}, r a^{2}{ }^{2}, r a .^{3}$, renal arteries; $r . a u$. right auricle; $r . p$. renal portal vein, on the left side of the figure, supposed to be dissected so as to show its passage through the right kidney; r.p.a. right pulmonary artery; r.prc. right pre-caval vein; r.v. renal vein ; r.vn. right ventricle; $s c . a$. sciatic artery ; $s^{r}, v$. sciat ic vein ; $s c l . a$. subclavian artery ; rr. vertebral artery and vein. (From Parker's Zootomy.) 
rectum, from which it receives tributaries, joins the portal vein. The abdominal vein of Amphibians and Reptiles appears to be represented, in part at least, by the epigasiric vein (epy.), which returns the blood, not from the ventral body-wall, but from the great omentum, a fold of peritoneum, loaded with fat, lying ventral to the intestine and gizzard: the epigastric discharges into the hepatic vein.

The red blood-corpuscles are oval and nucleated. The temperature of the blood is unusually high-over $38^{\circ} \mathrm{C} .\left(100^{\circ} \mathrm{F}\right.$.).

Nervous System.-The brain (Fig. 1056) completely fills the cranial cavity, and is remarkable for its short, broad, rounded

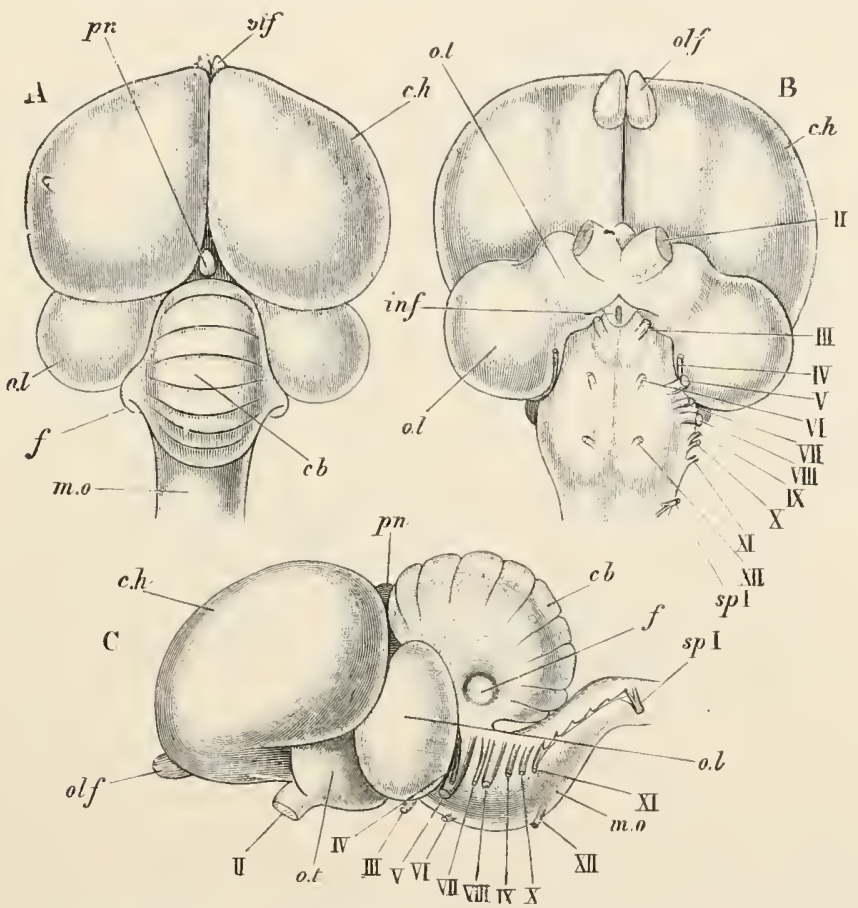

Fia. 1056.-Columba livia. The brain. $A$, from above; $B$, from below ; $C$, from the left side. $c b$. cerebellum; $c$. $h$. cerebral hemispheres; $f$. llocculıs; in $f$. infundibulum ; $m . o$. medulla oblongata; $o$. $l$. optic lobes; olf. olfactory bulbs: o.t. optic tricts; $m$. pineal body ; $I I-X I I$, cerebral nerves; sp. 1, first spinal nerve. (From Parker's Zootomy.)

form. The medulla oblongata $(m .0$.$) has a well-marked ventral$ flexure, as in the Lizard. The cerebellum $(c b$. $)$ is of great size, and has a large median portion and two small lateral lobes or flocculi $(f$.$) ; the surface of the middle lobe is marked by grooves$ passing inwards in a radiating manner and carrying with them the grey matter, the extent of which is thus greatly increased. The metacole (Fig. 1057, $v^{4}$.) is completely hidden by the cerebellum, 
and the latter is solid, having no epicoele. The hemispheres (c.h.) extend backwards to meet the cerebellum, and the optic lobes $(o, l$.) are thereby pressed outwards so as to take up a lateral instead of the usual dorsal position: these are of rounded form, and each contains an optocoele (Fig. 1057, o. v.) opening from a narrow passage, the iter, which represents the original cavity of the mid-brain. A further result of the extension of the hemispheres and cerebellum respectively backwards and forwards is that no part of the diencephalon (thc.) appears externally except on the

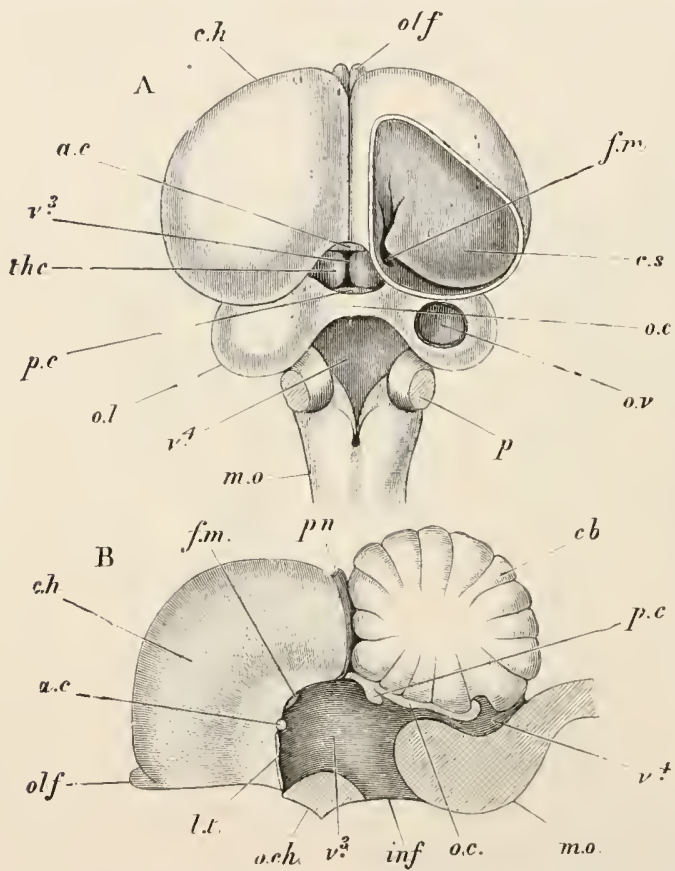

FIG. 1057.-Columba livia. The brain. $A$, with the cavities opened from above; $B$, in sagittal section. a.c. anterior commissure; $c b$. cerebellum; $c . h$. cerebral heni. spheres; $c . s$. corpus striatum : f.m. foramen of llonro; inf. infundibulum; $m . o$. medulla oblongata ; o.c. optic cominissure; o.ch. optic chiasma; o.l. optic lobes; olf. olfactory bulbs; o.v. optocole; $p$. peduncles of cerebellum; $p . c$. posterior commissure; $p n$. pineal body ; thc. dienceplalon ; $v^{3}$., diacœle; $v^{4}$, metacœle. (From Parker's Zootomy.) ventral surface : elsewhere it is seen only when the hemispheres are pressed aside. It contains a narrow vertical cavity, the diacoele $\left(v^{3}.\right)$, bounded laterally by the optic thalami, and communicating on each side by the foramina of Monro (f.m.) with the paracoles or cavities of the hemispheres. The corpora striata (c. s.) are of immense size, and form the great mass of the hemispheres: the dorsal portions of the latter, forming the roofs of the paracœles, are very thin. Hippocampi are absent. The anterior commissure is, as in lower Vertebrates, the chief commissure of the fore-brain. The olfactory bulbs

(olf.) are extremely small, in correspondence with the poorly developed olfactory organ : on the other hand the optic nerves and tracts are of unusual size.

The spinal cord (Fig. 1051, sp. cd.) presents large brachial and lumbar enlargements from which the nerves of the fore- and hind-limbs respectively are given off. In the lumbar enlargement there is a divergence of the dorsal columns of the cord converting the central canal into a wide, diamond-shaped cavity, the sinus 
rhomboidalis (s. $r h b$.), bounded above only by the membranes of the cord.

Sensory Organs.-The olfactory organs are paired chambers in the base of the beak, separated from one another by the mesethmoid and bounded externally by the ecto-ethmoid. The latter is produced inwards into three scroll-like processes, the turbinals, which greatly increase the surface of mucous membrane. The anterior portion of the cavity, including the anterior turbinal, is covered by laminated epithelium and serves as a vestibule; its posterior portion, including the middle and posterior turbinals, is invested by the one-layered epithelium of the Schneiderian membrane to which the fibres of the olfactory nerve are distributed.

The eye (Fig. 1058) is not even approximately globular, but has

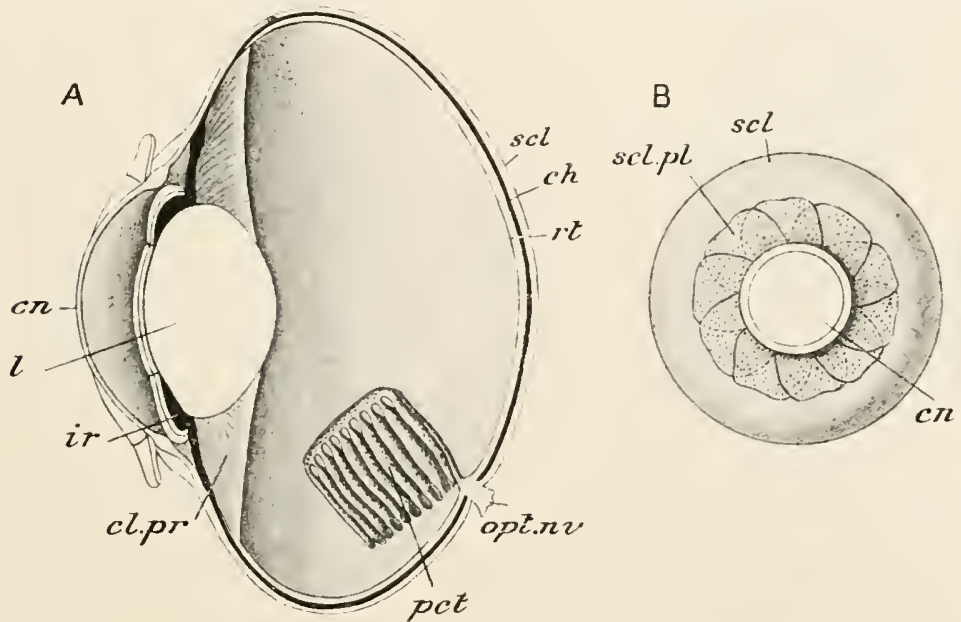

Fig. 1058.-Columba livia. The eye. $A$, in sagittal section : $B$, the entire organ, external aspect. cn. cornea; $c h$. choroid; cl.pr. ciliary processes; ir. iris; $l$. lens ; opt. nv. optic nerve; pct. pecten; rt. retina; scl. sclerotic; scl. $p l$. sclerotic plates. (After Vogt and Yung.)

the form of a biconvex lens. Sclerotic bony plates ( $B$, scl. $p l$.) are present, and there is a large pecten (pct.) in the form of a plaited and strongly pigmented membrane projecting into the cavity of the eye from the entrance of the optic nerve. The pecten is stated to be of nervous character, and is in all probability a sensory organ having some function connected with the process of accommodation.

The auditory organ (Fig. 1059) is chiefly distinguished from that of Reptiles by the great development of the cochlea (lag). The anterior canal $(S B)$ is of great size, and the whole membranous labyrinth is closely invested by a layer of dense ivory-like bone, which can be isolated by cutting away the surrounding spongy bone, and is then seen to form a sort of model of the contained 
organ, to which the name bony labyrinth is applied. The tympanic cavity and columella have the same arrangement as in the Lizard; the narrow Eustachian tubes open by a common aperture (Fig. 1051, eus. ap.) in the roof of the pharynx.

Urinogenital Organs.--The kidneys (Fig. 1051, kd, Figs. 1060 and $1061, k$ ) have a very characteristic form. Each is a flattened organ divided into three main lobes and fitted closely into the hollows of the pelvis. It is formed from the metanephros, the large mesonephros or Wolffian body, which forms the embryonic kidney, undergoing atrophy. The ureters (ur.) are narrow tubes

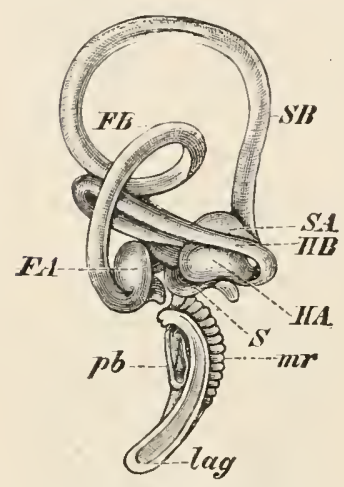

FiG. 10.59-Columba livia. The right membranous labyrinth, outer aspect. $F A$, anpulla of posterior canal; $F B$, posterior canal; $\boldsymbol{H A}$, ampulla of horizontal canal ; $H B$, horizontal canal; lag. cochlea or lagena; $m r$. membrane of Reissner; $p b$, basilar part of cochlea ; $S$, sacculus; $S A$, ampulla of anterior canal ; $S B$, anterior canal. (From wiedersheim, after Hasse.) passing directly backwards to open into the urodæum or middle compartment of the cloaca.

The testes (Figs. 1051 and 1060, ts.) are ovoid bodies, varying greatly in size according to the season, attached by peritoneum to the ventral surfaces of the anterior ends of the kidneys. From the inner border of each goes oft a convoluted vas deferens $(v d$.$) , which passes backwards,$ parallel with the ureter, to open into the urodrum on the extremity of a small papilla. The posterior end of the spermiduct is slightly enlarged to form a vesicula seminalis (v.s.). There is no copulatory organ.

The female organs (Fig. 1061) are remarkable for the more or less complete atrophy of the right ovary and oviduct. The left ovary (ov.) is a large organ in the adult Bird, its surface studded with follicles or ovisacs, varying in size from about $15 \mathrm{~mm}$. in diameter downwards, and each containing a single ovum. The left oviduct $(l$. od.) is long and convoluted; its anterior end is enlarged to form a wide membranous colomic fummel (l. od.") into which the ripe ova pass on their liberation from the orisacs; the rest of the tube has thick muscular walls, lined with glandular epithelium, and opens into the urodæum. A fair-sized vestige of the right oviduct $(r . o d$.$) is found in connection with the right side of the cloaca,$ and a more or less extensive vestige of the right ovary is frequently present.

Internal impregnation takes place. As the ova or "yolks" pass down the oviduct they are invested with the secretions of its various glands ; first with layers of albumen or "white," next with a parchment-like double shell membrane, and lastly with a white calcareous shell. They are laid, two at a time, in a rough nest, 
and are incubated or sat upon by the parents for fourteen days, the temperature being in this way kept at about $38^{\circ}$ to $40^{\circ} \mathrm{C}$. $\left(100^{\circ}\right.$ to $103^{\circ} \mathrm{F}$.). At the end of incubation the young Bird is

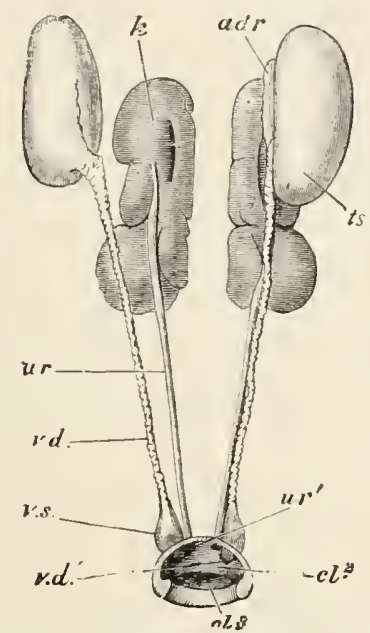

FIG. 1060.-Oolumba livia. Jlale urinogenital organs. alr. adrenal; $c l^{2}$., uro. dæum ; $c l^{3}$., proctodæum ; $k$. kidney ; $t s$. testis, that of the right side displaced ; $u r$. ureter ; $u r^{\prime}$. aperture of ureter ; $v l$. vas deferens; $v^{\prime}$. its cloacal aperture; $x$ s. vesicula seminalis. (From raker's Zootomy.)

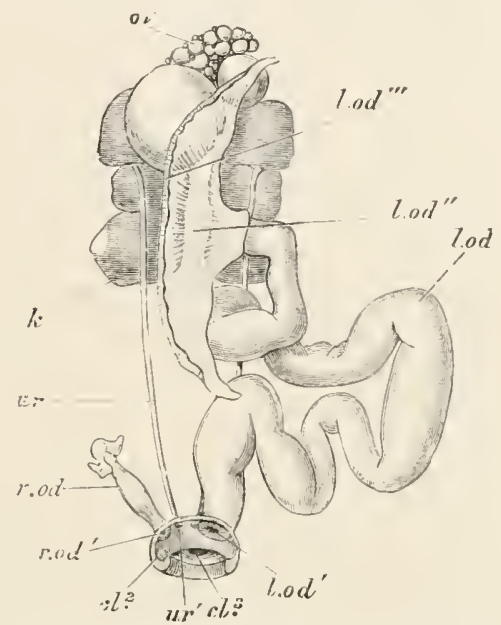

FIG. 1061.-Columba livia. Female urinogenital organs. $c l^{2}$, urodaum; $\mathrm{cl}^{3}$., proctodieum ; $k$. kidney; $l$. od . left oviduct ; l.orl'. its cloacal aperture: $l . o d^{\prime \prime}$. its coelomic funnel; $l . o l^{\prime \prime \prime}$. its colomic aperture ; ov. ovary ; $r$. orl. right ovidnet; $r$. or $d^{\prime}$. its cloacal apertine; ur. ureter; ur'. its cloacal aparture. (From Parker's Zootomy.)

sufficiently developed to break the shell and begin free life. It is at first covered with fine down, and is fed by the parents with a secretion from the crop, the so-called "Pigeon's milk."

\section{Distinctive ('haracters and Classifichtion.}

Aves are Craniata in which the epidermal exoskeleton takes the form of feathers over the greater part of the body, of a rhamphothecu or horny sheath to the beak, and of claws on the digits of the foot and sometimes of the hand. In the standing position the body is entirely supported on the hind-limbs, the articulations of which are thrown forward. The fore-limbs are modified to form wings, usually provided with large feathers for the support of the body during flight. The cervical and free thoracic vertebræ are usually heterocoelous, but may be procoelous or amphicoelous. The sacral vertebræ are fused with the lumbar and with more or fewer of the posterior thoracic and anterior caudal to form a syn-sacrum for the support of the ilia. The posterior caudal vertebræ are usually fused to form a pygostyle around which the tail-quills are arranged in a semicircle. The bones of the skull undergo early ankylosis. There is a single, rounded, occipital condyle; the 
united premaxillie form nearly the whole of the upper jaw; and the lower jaw is composed originally of five or six bones in each ramus, and is supported by a freely articulated quadrate. The vertebral ribs are double-headed, provided with bony uncinates, and articulate with the bony sternal ribs by synovial joints. The sternum is broad, and is typically produced into a longitudinal ventral keel, having a separate centre of ossification. The coracoid is usually more or less pillar-like, the scapula is sabre-shaped, and the clavicles and interclavicle unite to form a furcula. Except in one extinct species the distal carpals and the metacarpals are united to form a carpo-metacarpus. There are usually only three digits in the wing, which probably represent the first, second, and third of the typical hand. The ilium is of great size, having large pre- and post-acetabular portions. The acetabulum is perforated in the dry bone. The pubis and ischium are directed backwards and, except in one case of each, there is neither pubic nor ischiatic symphysis. The head of the femur is at right angles to the shaft. The proximal tarsals are fused with the tibia to form a tibio-tarsus; the fibula is much reduced. The distal tarsals are fused with the second, third, and fourth metatarsals to form a tarso-metatarsus; the first metatarsal is free. The fifth digit of the typical foot is absent.

In all tertiary and recent Birds teeth are absent. The gullet is frequently dilated into a crop and the stomach is usually divided into proventriculus and gizzard. The junction between the large and small intestines is marked by a pair of cæca. The lungs are spongy and non-distensible. The bronchi give off branches which open on the surface of the lung into thin-walled air-sacs, and these in their turn usually communicate with pneumatic cavities in more or fewer of the bones. The voice is produced in a syrinx situated at or near the junction of the trachea with the bronchi. The heart is four-chambered, the right auriculo-ventricular valve is muscular, and the right aortic arch alone is present in the adult. The renal portal system is vestigial. The red blood-corpuscles are oval and nucleated. The temperature of the blood is high (about $38^{\circ} \mathrm{C}$.). The optic lobes are displaced laterally owing to the meeting of the large cerebral hemispheres and cerebellum. The lumbar region of the spinal cord has a sinus rhomboidalis. The olfactory organ is usually poorly developed. The eye is usually large, and has sclerotic plates and a pecten. The auditory organ has a large curved cochlea. The kidney is three-lobed, and is developed from the metanephros, the mesonephros undergoing atrophy. There is no urinary bladder. The ovary and oviduct of the right side are more or less completely atrophied.

Birds are all oviparous, and the large ovum, containing much food-yolk, becomes invested with albumen, a double shell-membrane, and a calcareous shell, in its passage down the oviduct. The 
embryo has an ammion, an allantois, and a large yolk-sac. The newly-hatched young may be either well covered with down and able to run or swim and to obtain their own food, in which case they are said to be precocious ; or may be more or less naked and dependent for a time upon the parents for their food supply, when they are non-precocious.

There is no general agreement with regard to the classification of Birds. Owing to the singular uniformity of the class in essential matters of structure, the vast and bewildering diversity in detail, and the puzzling cross-relationships between group and group, the splitting up of the class into orders is a matter of great difficulty and one upon which hardly two ornithologists are agreed. The following scheme will probably answer the present purpose sufficiently well.

\section{Sub-Class I.-Archæornithes.}

Mesozoic Birds: have no ploughshare bone (pygostyle), but a long tail of many vertebræ, having the rectrices arranged in two rows, one on each side of it. The carpals and metacarpals are probably free, and the hand has three clawed digits. Teeth are present in both jaws.

Including the single genus Archaoptoryx with three species, known only from three specimens found in the Jurassic rocks of Bavaria.

\section{Sub-Class II.-Neornithes.}

Birds in which the greatly shortened tail usually ends in a pygostyle, around which the rectrices, when present, are arranged in a semicircle. Except in a few extinct forms there are no teeth. The metacarpals are fused with the distal carpals to form a carpometacarpus. Except in one instance, not more than two digits of the hand bear claws, and in nearly all cases claws are absent in the manus.

\section{Division A.-Ratitæ.}

Flightless Neornithes, usually of large size, having no hooked barbules to the feathers, so that the barbs are free. Apteria are usually absent in the adult. The rectrices are absent or irregularly arranged, and the pygostyle is small or undeveloped. The sternal keel is vestigial or absent. The coracoid and scapula are comparatively small and completely ankylosed; the acrocoracoid process is vestigial, and the coraco-scapular angle approaches two right angles. The wing is reduced in size and may be vestigial or absent. There are large basi-pterygoid processes developed from the basi-sphenoid. The vomer is large and broad. The quadrate 
articulates with the skull by a single or partially divided facet. The male has a penis. The young are precocious.

\section{Order 1.-Megistanes.}

Including the Emus (Dromous) and Cassowaries (Casuarius).

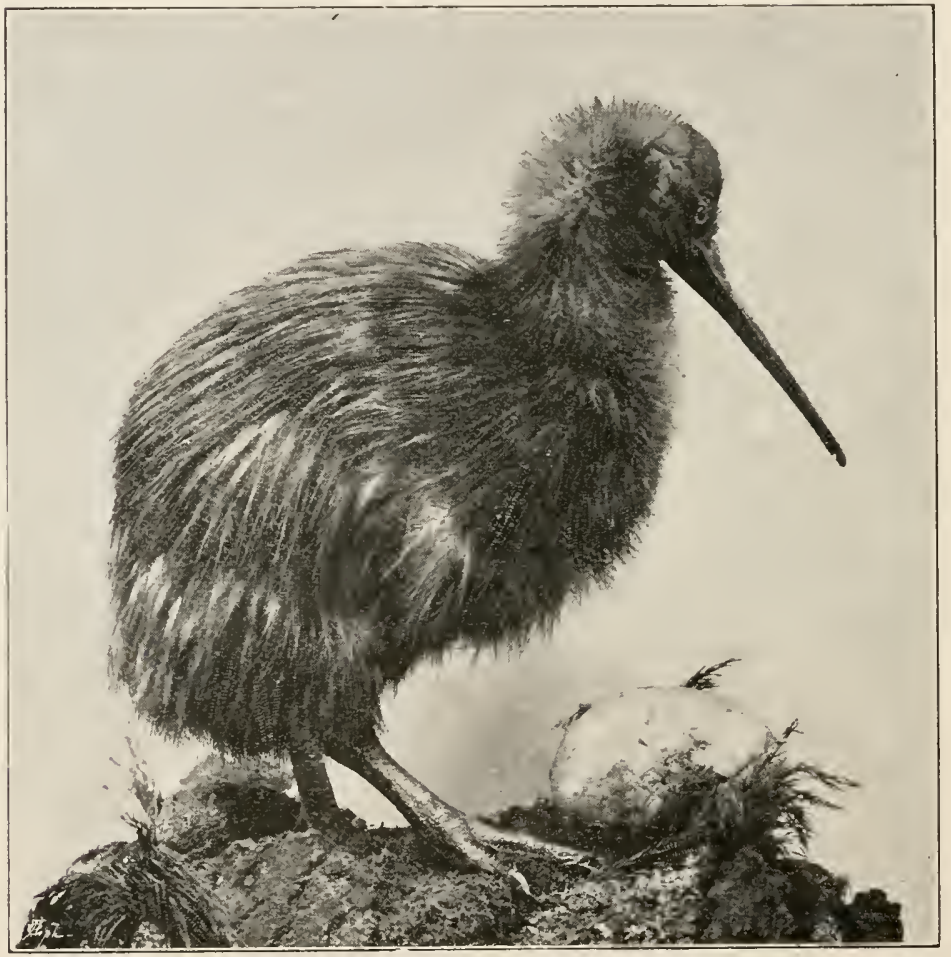

FIG. 1062,-Apteryx australis, with egg. (Froin a specimen in the Royal College of Surgeons, Lomilou.)

Order 2.-APTERyges.

Including only the Kiwis (Apteryx, Fig. 1062).

Order 3.- Dinornithes.

Including the Moas (Dinomithila, Fig. 1080).

$$
\text { Order 4.-Rheæ. }
$$

Including the South American Ostriches (Rhea). 


\section{Order 5.-STRUTHiones,}

Including the true Ostriches (Struthio).

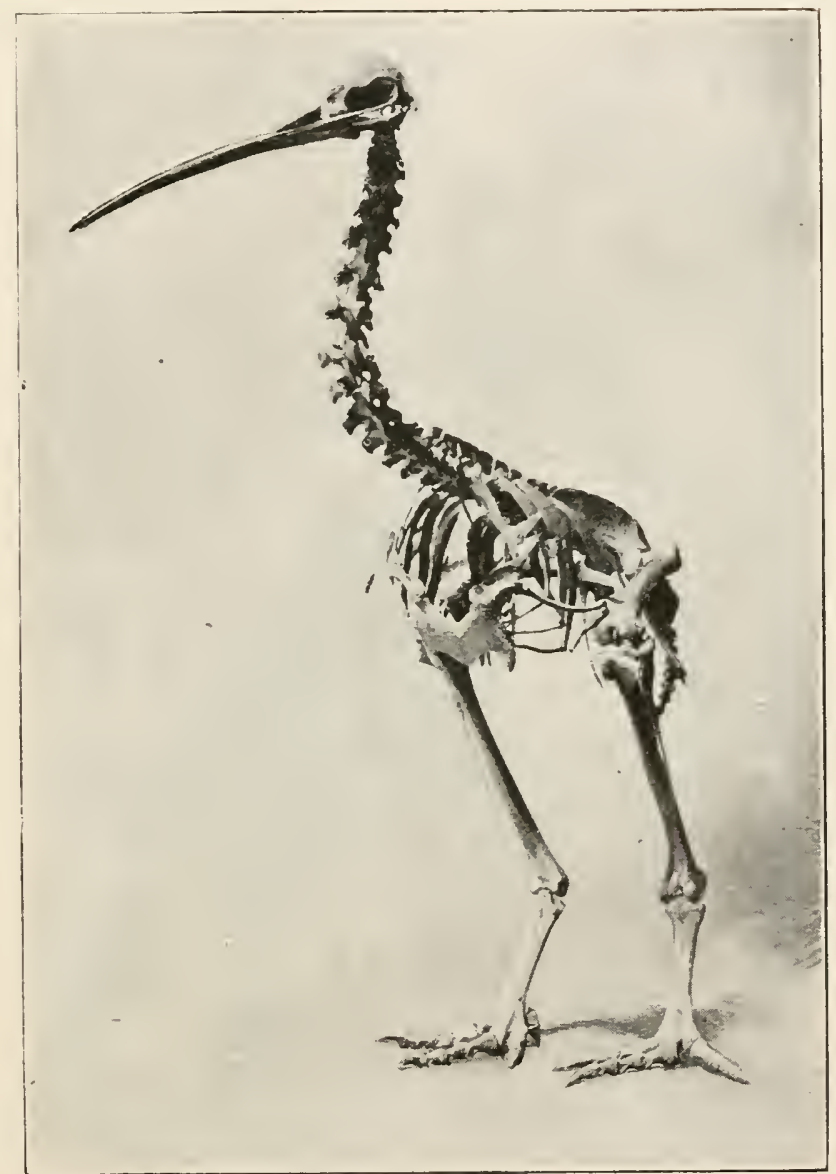

FIG. 1063.-Apteryx australis. Skeleton. (From a specimen in the British Museum, Natural History.)

Order 6.-Aipyornithes.

Including only the post-pliocene Madagascan genera Epyornis and Mullerornis.

Order 7.-Gastornithes.

Including Gastornis and other genera from the Eocene of Europe. 


\section{Division B.-Carinatæ.}

Neornithes in which, with the exception of some flightless species, the sternum has a kee], the coracoid and scapula are not ankylosed, the acrocoracoid and usually the furcula are well developed, and

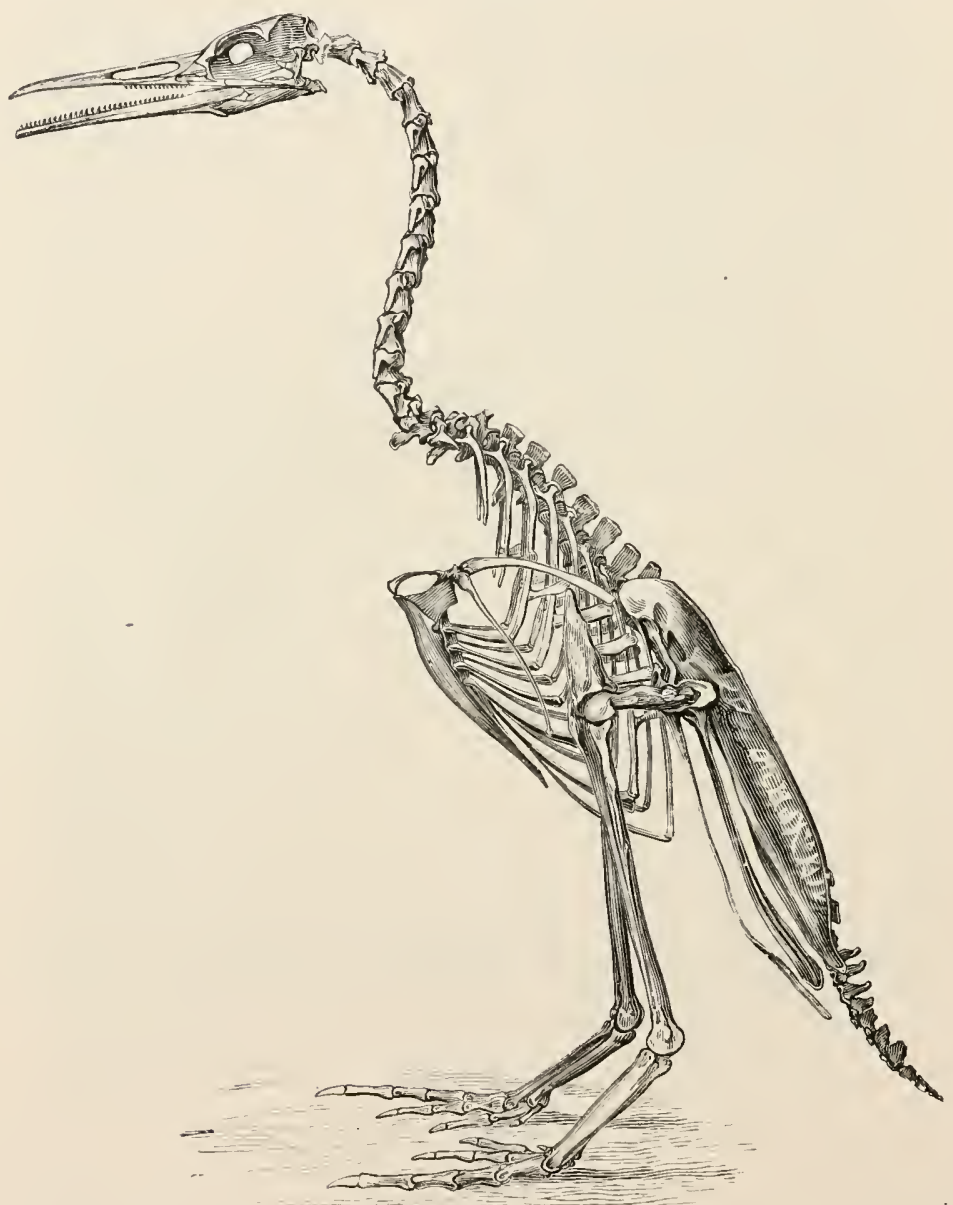

F'IG. 1064.- Hesperornis regalis. The restored skeleton. (After Marsh.)

the coraco-scapular angle is less than a right angle. There is a pygostyle around which the rectrices are arranged. The quadrate usually articulates with the skull by two facets. The barbs of the feathers have hooklets. ${ }^{1}$ 


\section{ORDER 1.-STEREORNITHES. ${ }^{1}$}

Including Phororhacos, Dryornis, and other genera from the Eocene of South America.

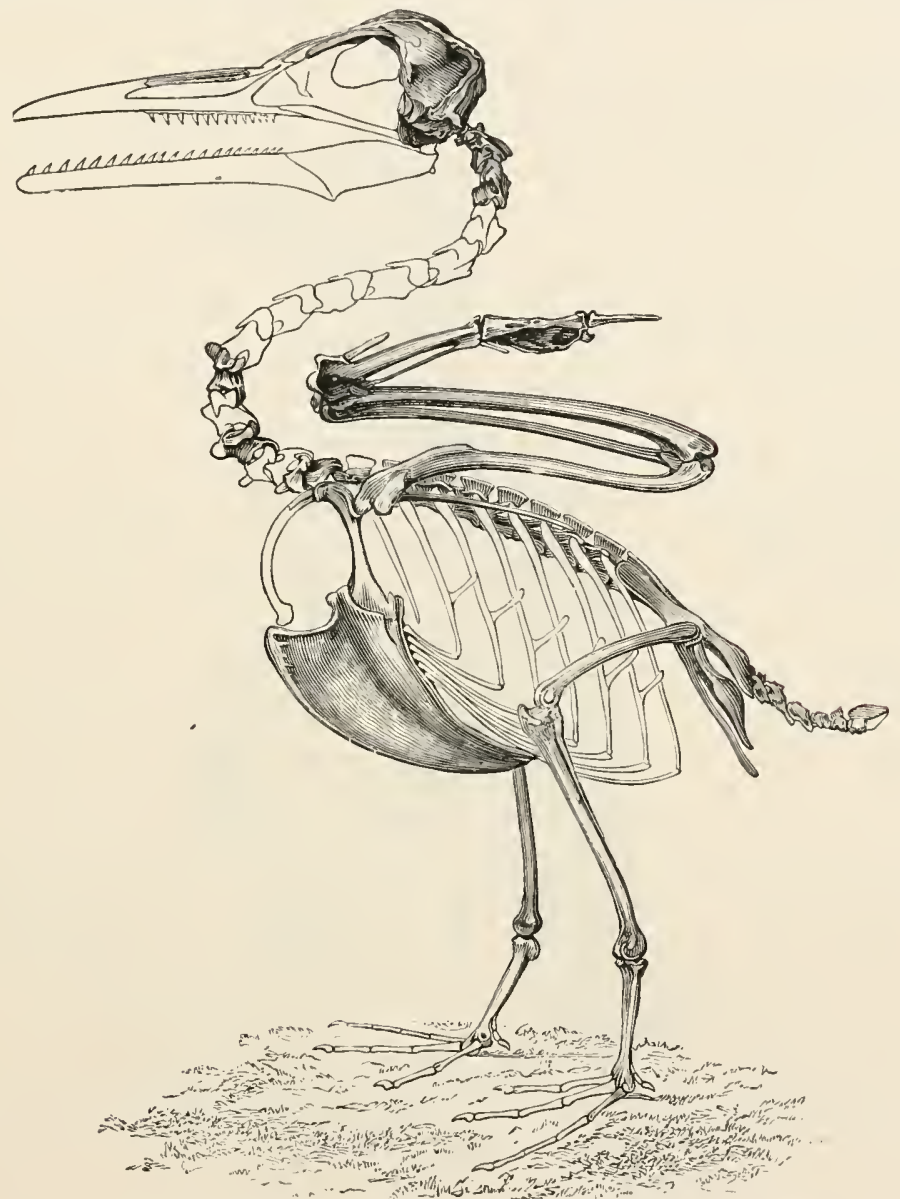

FtG, 1065.- Ichthyornis victor. The restored skeleton. (Aiter Marsh.)

\section{ORDER 2.-OdONTOLCE.}

Including Hesperornis ${ }^{2}$ (Fig. 1064), a large diving and swimming Bird from the Cretaceous of North America, and other less known genera.

1 Recent investigations indicate that this is not a natural group, but that its various genera will have to be distributed amongst various orders both of Ratit:e and of Carinatæ.

2 Hesperornis is perhaps more nearly related to the Ratitæ. 
Order 3.--ICHTHYORNithes.

Including Ichthyornis (Fig. 1065) and Apatornis. Tern-like Birds from the Cretaceous of North America.

\section{ORDER 4.-PYGOPODES.}

Including the Divers (Colymbus) and the Grebes (Podicipes).

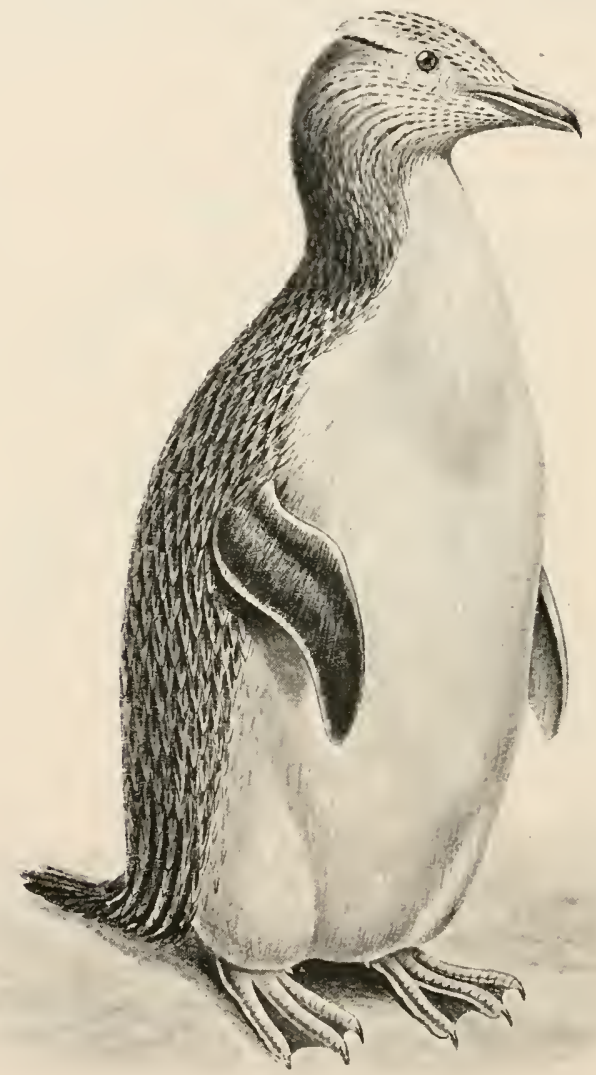

FIG. 1066.-Eudyptes antipodum. (After Buller.)

Order 5.-IMPENnes.

Including the Penguins (Aptenodytes, Eudyptes, \&c., Fig. 1066).

\section{Order 6.-TUBiNARES.}

Including the Petrels, such as the Albatrosses (Diomedea), Stormpetrels (Oceanites), Fulmars (Fulmans), Shearwaters (Puffinus), \&c. 


\section{Orber 7.-STEGANOPOdes.}

Including the Boatswain-bird (Phaëthon), Gannets (Sula), Cormorants or Shags (Phalacrocorax). Frigate-bird (Fregata), and Pelicans (Pelecumus).

\section{Order 8.-THerodiones.}

Inchuding the Herons (Ardea, \&c.), Storks (Ciconia, \&c.), Ibises (Ibis), Spoonbills (Platalea), and Flamingoes (Phanicopterus).

\section{Order 9.-ANSERES.}

Including the Ducks (Anas, \&c.), Geese (Anser), Swans (Cygnus), and Mergansers (Mergus); and the Screamers (Palamedea and Chauna).

\section{Order 10.-ACCIPITRES.}

Including the diurnal Birds of prey, such as the Eagles (Aquila), Falcons (Fulco), Vultures (Vultur, \&c.), and Secretary Bird (Gypoger(mus). The American Vultures or Turkey-buzzards (Cathartes) are sometimes placed in a distinct order.

\section{Order 11.-Crypturi.}

Including only the Tinamous (Tinamus, \&c.).

\section{Order 12.-GaLlinæ.}

Including the Fowls (Gallus), Pheasants (Phasianus), Grouse (Tetrao), and other Game Birds; Curassows (Crax), Brush-turkeys (Megapodius), Hemipodes or Button-quails (Turnix), and the Hoatzin (Opisthocomus).

\section{Order 13.-Gralt E.}

Including the Rails (Rallus, Ocydromus, \&c.), the flightless Giant Rail (Aptomis), the Cranes (Grus, \&c.), the Bustards (Otis), \&c.

\section{Order 14.-Gavie. ${ }^{1}$}

Including the Gulls (Larus) and Terns (Sternu), and the Auks (Alca and Fratercula).

\section{ORder 15.-LIMICOLF.}

Including the Plovers (Charadrius, \&c.), Oyster-catchers (Icrmatopus), Curlews (Limosa), Jacanas (Parra), etc.

1 Sometimes united with the next two orders under the designation Charadriformes. 
Order 16.-Pterocletes.

Including the Sand-grouse (Pterocles and Syrrhaptes).

\section{Order 17.-Columba.}

Including the Pigeons and Doves (Columba, Turtur, \&c.), Crowned Pigeons (Goura), and the extinct flightless Dodo (Didus) and Solitaire (Pezophaps).

\section{Order 18.-Pittaci. ${ }^{1}$}

Including the Parrots (Psittacus, \&c.), Parrakeets (Platycercus), Cockatoos (Cacatua), Lories (Lorius), and Macaws (Ara).

\section{ORDER 19.-STRIGES.}

Including the Owls (Strigida).

\section{Order 20.-Picarlæ.}

A somewhat heterogeneous group including the Cuckoos (Cuculida), Plantain-eaters (Musophagidae), Rollers (Coraciida), Motmots (Momotida), Kingfishers (Alcedinida), Bee-eaters (Meropida), Hoopoes (Upupidoe), Goat-suckers (Caprimulgi), Swifts (Cypselido), Humming Birds (Trochilidae), Colies (Colii), Trogons (Trogones), Woodpeckers and Hornbills (Pici), \&c.

\section{Order 21.-PASSERES.}

Including the Lyre-birds (Menura), Larks (Alaudida), Starlings (Sturnidce), Finches (Fringillida), Swallows (Hirundinidoe), Blackbirds and Thrushes (Turdidae), Birds of Paradise (Paradiseido), Crows (Corvidoe), \&c.

\section{Systematic Position of the Example.}

The numerous species of Columba belong to the family Columbida, of the order Columba.

The following are the chief characters of the Columbæ: There are eleven primary remiges, the first very small; the skull is schizognathous (see p. 415); the oil-gland has no tuft of feathers; the vomer is vestigial ; there is a large crop ; the cæca are vestigial ; and the young are non-precocious.

Of the two families of Columbæ the Columbida, or Doves and Pigeons, are distinguished from the Didida, including the Dodo and Solitaire, by the power of flight and the accompanying typical carinate characters of the sternum and shoulder-girdle.

In Columba there are twelve retrices ; the second primary remex

1 Sometimes combined with the Cuckoos. 
is longer than the sixth, and the proximal portion of the tarsometatarsus is feathered.

\section{General Organisation.}

In respect of range of structural variations, the entire class of Birds is hardly the equivalent of a single order of Reptiles. Among existing Birds, the Emu and the Raven, which may be said to stand at opposite ends of the series, present nothing like the anatomical differences to be found between a common Lizard and a Chamæleon, or between a Turtle and a Tortoise. Hence in dividing the class into orders we find none of those striking distinctive characters which separate the orders of Fishes, Amphibia, and Reptiles, but have to be content with characters which in other groups would be considered insignificant, such as details in the structure of the skull and sternum, in the arrangement of the muscles of the wing and leg, in the form of the foot, and in the peculiarities of the newly-hatched young. It is for this reason that in the preceding classification no diagnoses of the orders are given : to define them adequately would involve a degree of anatomical detail quite beyond the scope of the present work.

The differences between the two avian sub-classes, the Archæornithes and the Neornithes, are, however, of a far more fundamental nature ; and as Archæopteryx, the sole representative of the first of these groups, is a unique form, and perhaps the best example of an undoubted link between two classes-Reptiles and Birds-it will be convenient to deal with it separately.

\section{Sub-Class I.-Archæornithes.}

Only two specimens of Archcoopteryx lithographica have hitherto been found, both in the finely-grained lithographic limestone of Solenhofen, Bavaria, belonging to the Jurassic period. The Bird (Fig. 1067) was about the size of a Crow, and in the fossils not only are the bones preserved, but also many of the feathers.

The most striking feature in the organisation of this Bird is the fact that the tail is composed of about 18-20 free caudal vertebræ gradually tapering to the distal end as in a Lizard. The rectrices are arranged in two rows, one on each side of the caudal vertebræ, forming a long tail quite unlike that of any existing Bird. The centra probably had biconcave faces. In addition to cervical and thoracic ribs (which were apparently devoid of uncinates) there were abdominal ribs, like those of Sphenodon and Crocodiles.

The skull (Fig. 1068) is proportionately large, with rounded brain-case and strong jaws, in each of which is a series of conical teeth lodged in sockets. There is no trace of sternum in either 
specimen, and the coracoids (co.) are only partially visible: the scapula (sc.) are slender, curved bones, and there is a $\mathbf{U}$-shaped furcula $(c l$.$) .$

The bones of the upper and fore-arm are of the normal avian character: only one caipal is certainly known (Fig. 1069, c.) : it

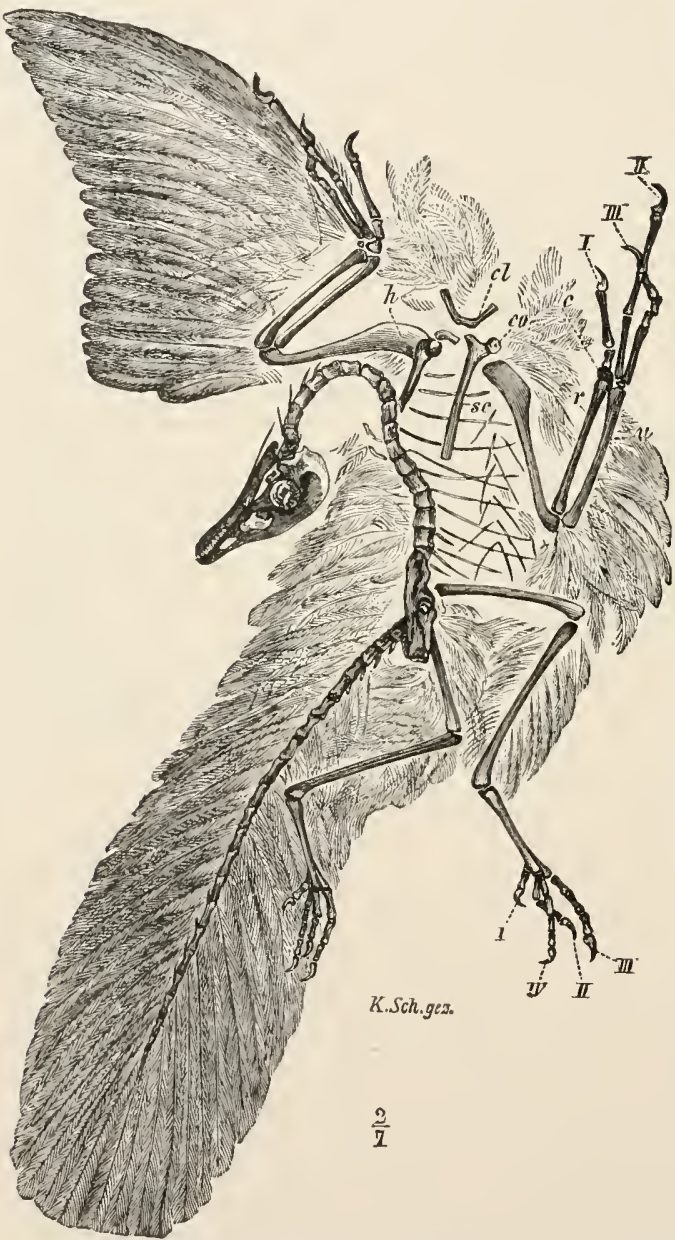

FIG, 1067.-Archxopteryx lithographica. From the Berlin specimen. $c$. carpal; $c l$ furcula; co. coracoid: $h$. humerus ; $r$. radius ; se. scapula ; $u$. ulna ; $I-I V$, digits. apparently belongs to the distal row, and is closely applied to the first and second metacarpals. Three digits $(d .1,2,3)$ are clearly visible in one of the specimensthat in the Berlin Museum-the metacarpals of which are usually stated to be all free, in which case there is no carpo-metacarpus as in other Birds, and the hand approaches the normal reptilian type. The number of phalanges follows the usual reptilian rule, two in the first digit, three in the second, and four in the third, and the ungual phalanx of all three digits is claw-shaped and doubtless supported a horny claw.

The remiges, like the rectrices, are in a wonderful state of preservation (Fig. 1067), and are divisible, as usual, into primaries or metacarpo-digitals, and secondaries or cubitals. The primaries were probably attached to the second or to the second and third of the digits just described.

The pelvis and the hind-limb have the usilal avian character. The tibia and fibula are separate. The foot consists of a slender 
tarso-metatarsus and four digits, the lrallux being small and directed backwards.

In addition to the wing and tail-quills already referred to, there are remains of contour feathers at the base of the neck and of wing-

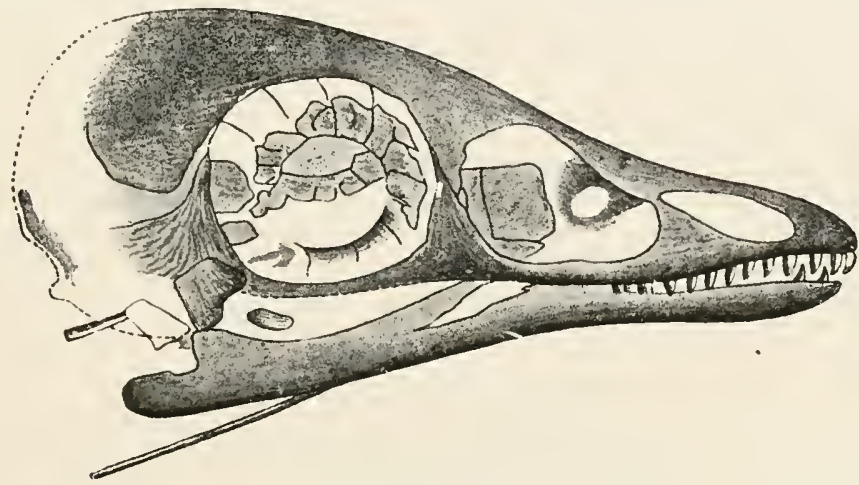

FIG. 1068.-Archaopteryx lithographica. The skull, showing teeth and sclerotic plates. (From Headley, after loames.)

coverts. Moreover the rectrices are continued forwards by a serjes of large feathers which extend for some distance along the sides of

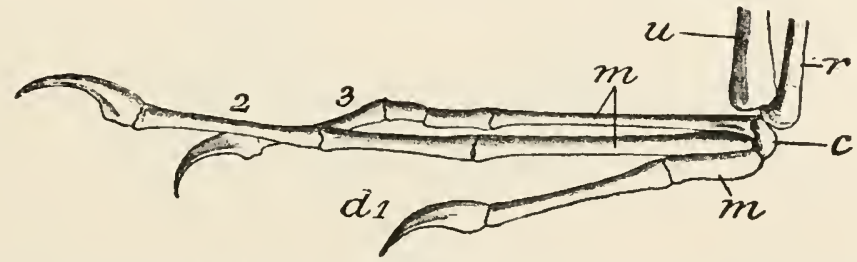

FIG. 1069.-Archaoptexyx lithographica. The left manus, $c$. carpal ; $d$, first digit, 2 , second digit; 3 , third digit ; $m$, m. metacarpals ; $r$. radius ; $u$. uhna. (From Headley after Dames.)

the body, and a row of similar but smaller feathers is attached along both anterior and posterior faces of the tibio-tarsus.

A second species of Archaopteryx, which has been named Archaopteryx siemensi, has been found more recently in the same locality.

\section{Sub-Class II,-Neornithes.}

External Characters.-In the general build of the body the Neornithes differ from Archæopteryx chiefly in the shorter and stouter trunk, and in the point of articulation of the hind-limbs being thrown forward, so as to be almost directly below the centre of gravity of the body : the animal is thus enabled without effort to support itself on the legs alone. In a word Birds are essentially bipedal, the only exception being the young of the Hoatzin (Opisthocomus), which uses its wings in climbing. 
The neck is always well developed, and is often, as in the Swan and Flamingo, of immense proportional length. The cranial portion of the head is usually not large, but the beak may attain extraordinary dimensions, and exhibits a wide range of form. It may be extremely short and wide for catching Moths and other flying Insects, as in Swifts and Goatsuckers ; short and conical for eating fruit, as in Finches; strongly hooked for tearing the bodies of animals, as in Birds of Prey, or for rending fruits of various kinds, as in Parrots ; long, conical, and of great strength, as in Storks ; slender and elongated, as in Swifts, Ibises, and Curlews; broad and flattened for feeding in mud, as in Ducks and Geese; expanded at the end, as in Spoonbills ; immensely enlarged, as in Hornbills and Toucans. It is most commonly bent downwards at the tip, but

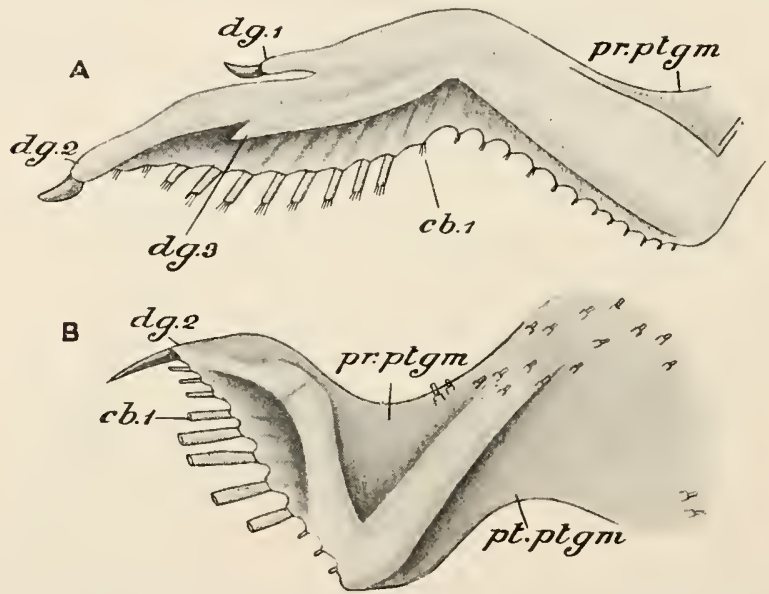

FIG, 1070.-A, Wing of nestling of Opisthocomus ; $B$, Wing of adult Apteryx ; both from the inner (ventral) aspect. $c b .1$, first cubital remex; $d g .1, d g .2, d g .3$, digits : pr. $p t y m$. pre-patagium; pt. ptgm. post-patagium. ( $A$, after Pycraft ; $B$, after T. J. Parker.)

may be straight or curved upwards, as in the Avocet, or bent to one side, as in the New Zealand Crook-billed Plover. It is sometimes, as in the Toucans, brilliantly coloured, and there may also be bright coloration of the cere, as in the Macaws, and of naked spaces on the head, as in the Cassowaries. In the latter the head is produced into a great horny prominence or "casque," supported by an elevation of the roof of the skull. The cere is frequently absent. The nostrils are placed at the base of the beak, except in Apteryx, in which they are at the tip.

The essential structure of the wing-apart from its feathers-is very uniform. As a rule all three digits are devoid of claws, as in the Pigeon, but the Ostrich has claws on all three digits; Rhea on the first and sometimes on the second and third ; the Cassowary, Emu, and Kiwi (Fig. 1070, B) on the second; the Crested Screamer 
(Chauna) and two other species, and, as a rare abnormality, the Common Fowl and Goose, on the first. With these exceptions the hand of the adult bird has lost all the characters of a fore-foot; but in the young of the Hoatzin (Opisthocomus) claws are present on the first two digits (Fig. 1070, $A$ ), which are sufficiently mobile to be used in climbing. Besides the true claws horny spurs are sometimes present on the carpo-metacarpus.

There is almost every gradation in the proportional length of the hind-limb, from Birds in which nothing but the foot projects beyond the contour feathers, and even the toes may be feathered, to the long-legged Storks and Cranes, in which the distal part of the tibio-tarsus is covered with scales as well as the foot. In aquatic forms a fold of skin or web is stretched between the toes, sometimes including all four digits, as in the Cormorants; sometimes leaving the hallux free, sometimes forming a separate fringe to each digit, as in the Coots and Grebes. As to the toes themselves, the commonest arrangement is for the hallux to be directed backwards, and Nos. 2, 3, and 4 forwards, but in the Owls No. 4 is reversible, i.e., can be turned in either direction, and in the Parrots, Woodpeckers, \&c., it, as well as the hallux, is permanently turned backwards. In the Swifts, on the other hand, all four toes turn forwards. The hallux is frequently vestigial or absent, and in the Ostrich No. 4 has also atrophied, producing the characteristic two-toed foot of that Bird.

Pterylosis.-With the exception of the Penguins, most Carinatæ have the feathers arranged in distinct feather-tracts or pterylæ, separated by apteria or featherless spaces. These are commonly much more distinct than in the Pigeon, and their form and arrangement are of importance in classification (Fig. 1071). In the Ratitæ, apteria are usually found only in the young, the adult having a uniform covering of feathers. The Ratitæ, also, have nothing more than the merest trace of hooklets on the barbules, so that the barbs do not interlock and the vanes of the feathers are downy or hair-like. In the Penguins the wing-feathers are degenerate and scale-like.

Many Birds are quite naked when hatched, but in most cases the body is more or less completely covered by a temporary crop of feathers, the nestling-downs, of various forms, but always having a short axis, soft loose barbs, devoid of interlocking apparatus, and, except in the Emu, having no after-shaft (vide p. 410). They are succeeded, as already described, by the permanent feathers.

Many Birds, such as the Swan, possess down-feathers or plumula throughout life, interspersed among and hidden by the contour feathers or penno. In the Heron and some other Carinatæ are found powder-down patches (Fig. 1071, B, p.d. p, p.d. $p^{\prime}$ ), areas of downs, the ends of which break off and make a fine dust. Semiplumes are downs with a well-developed axis: filoplumes, as we 
have seen (Fig. 1034, B), have an elongated axis and vestigial vexillum.

In many Birds there springs from the under side of the quill, near the superior umbilicus, a second vane, the after-shafi (Fig. 1072), usually smaller than the main shaft, but sometimes of equal size. Both among Carinatx and Ratitx we find genera with doubleshafted feathers and allied forms in which the after-shaft is rudimentary or absent.

The feathers are always shed or "moulted" at regular intervals,

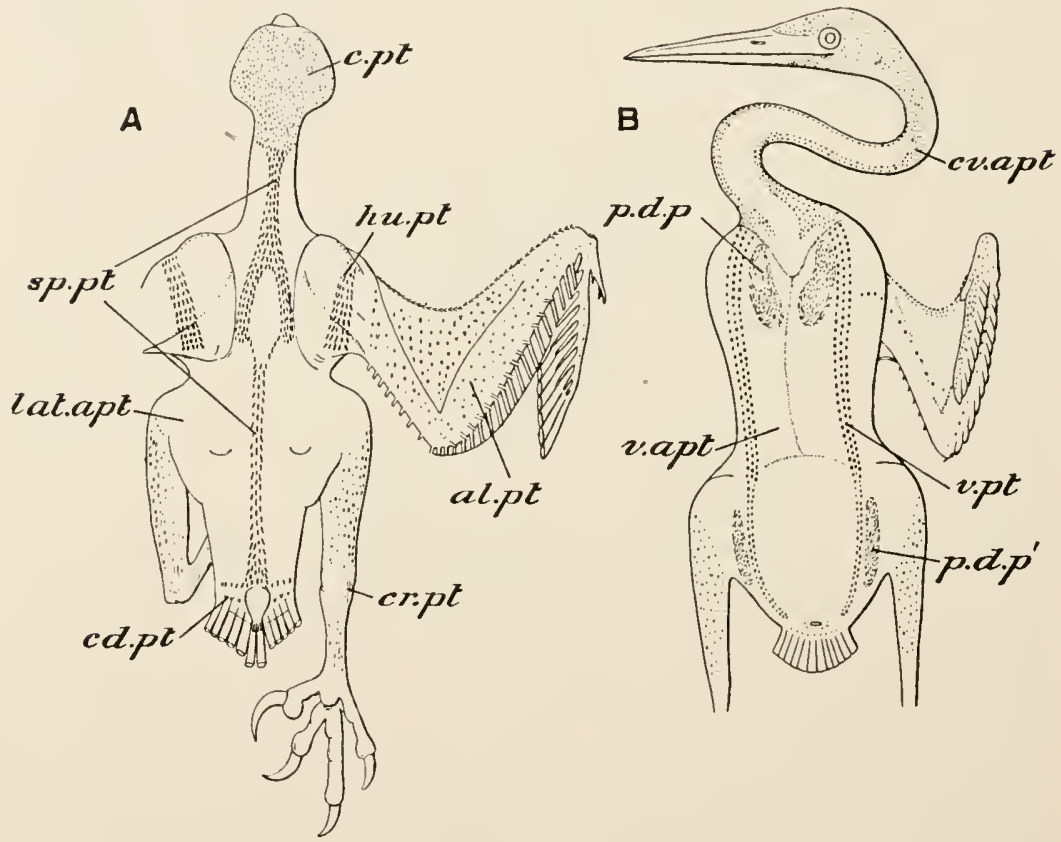

FiG. 1071.-A, pterylosis of Gypaëtus (Bearded Vulture); $B$, of Ardea (Heron). al. pt, wing-tract; $c . p t$, head-tract; $c d$. pt, caudal tract; $c r . p t$, crural tract; $c v$, apt. cervical space; hu. pt, humeral tract; lat. apt, lateral space ; $p . d . p ., p . d . p^{\prime}$, powder-down patches ; $s p$. $p t$, spinal tract; $v$. apt, ventral space; $v . p t$, vent.ral tract.

as a rule annually. The old feathers drop out and new ones are formed from the same pulps.

The colours of feathers present great variety. Black, brown, red, orange, and yellow colours are due to the presence of definite pigments, i.e. are absorption-colours. White, and in some cases yellow, is produced by the total reflection of light from the spongy air-containing substance of the feather, there being, as in nearly all other natural objects, no such thing as a white pigment. Blue, violet, and in some cases green are produced by the light from a brown pigment becoming broken up as it passes through the super- 
ficial layer of the feathers in its passage to the eye : no blue or violet pigments occur in feathers, and green pigments are very rare. The beautiful metallic tints of many birds are entirely the result of structure, owing their existence to a thin, transparent, superficial layer, which acts as a prism : in such feathers the colour changes according to the relative position of the Bird and of the eye of the observer with regard to the source of light.

There is also infinite variety in the general coloration of Birds. In many the colouring is distinctly protective, harmonising with the environment, and even changing with the latter-as in the Ptarmigan, which is greyishbrown in summer, white in winter, the former hue helping to conceal the Bird among herbage, the latter on snow. Frequently, as in Pheasants and Birds of Paradise, the female alone is protectively coloured, while the male presents the most varied and brilliant tints, enhanced by crests, plumes or tufts of feathers on the wings, elongated tail, \&c., \&c. These have been variously explained as "courtship colours" for attracting the female; as due simply to the exuberant vitality of the male Bird; or as helping to keep the number of males within proper limits by rendering them conspicuous to their enemies. Such ornaments as the bars and spots on the wings and tail of many gregarious birds, such as Plovers, fully exposed only during flight, and often widely different in closely allied species, have been explained as " recognition marks," serving to enable stragglers to distinguish between a flock of their own and of some other species.

Skeleton.-The vast majority of Birds have saddle-shaped or heterocoelous cervical and thoracic vertebræ, but the thoracic vertebræ are opisthocœlous in the Impennes (Penguins), the Gaviæ (Gulls), and the Limicolæ (Plovers, \&c.), while in the Ichthyornithes alone they are biconcave. The spaces between adjacent

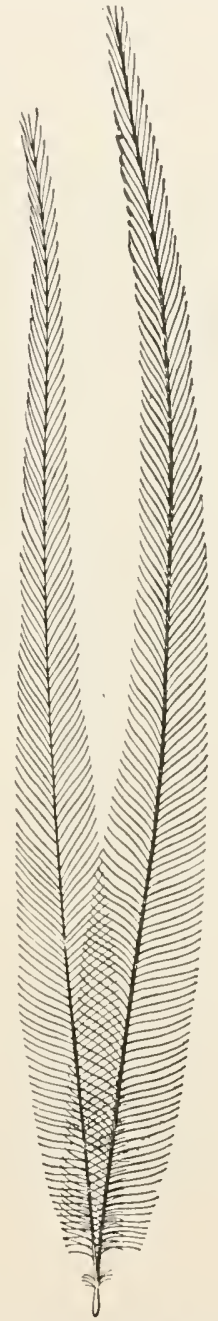

Fig. 1072.-Feather of Casuarius (Cassowary), showing aftershaft and disconnected barlos. (From llearley.) centra are traversed by a meniscus with a suspensory ligament, as in the Pigeon (p. 374). The number of vertebræ is very variable, especially in the cervical region, where it rises to twenty-five in the Swan and sinks to nime in some 
Song-birds. There is very commonly more or less fusion of the thoracic vertebræ, and the formation of a syn-sacrum by the concrescence of the posterior thoracic, lumbar, sacral, and anterior caudal vertebræ is universal. The posterior cervical and anterior thoracic vertebræ commonly bear strong hypapophyses or inferior processes for the origin of the great flexor muscles of the neck. The number of true sacral vertebræ varies from one to five. A pygostyle, formed by the fusion of more or fewer of the caudal vertebræ, is of general occurrence, but is small and insignificant or absent in the Ratitæ.

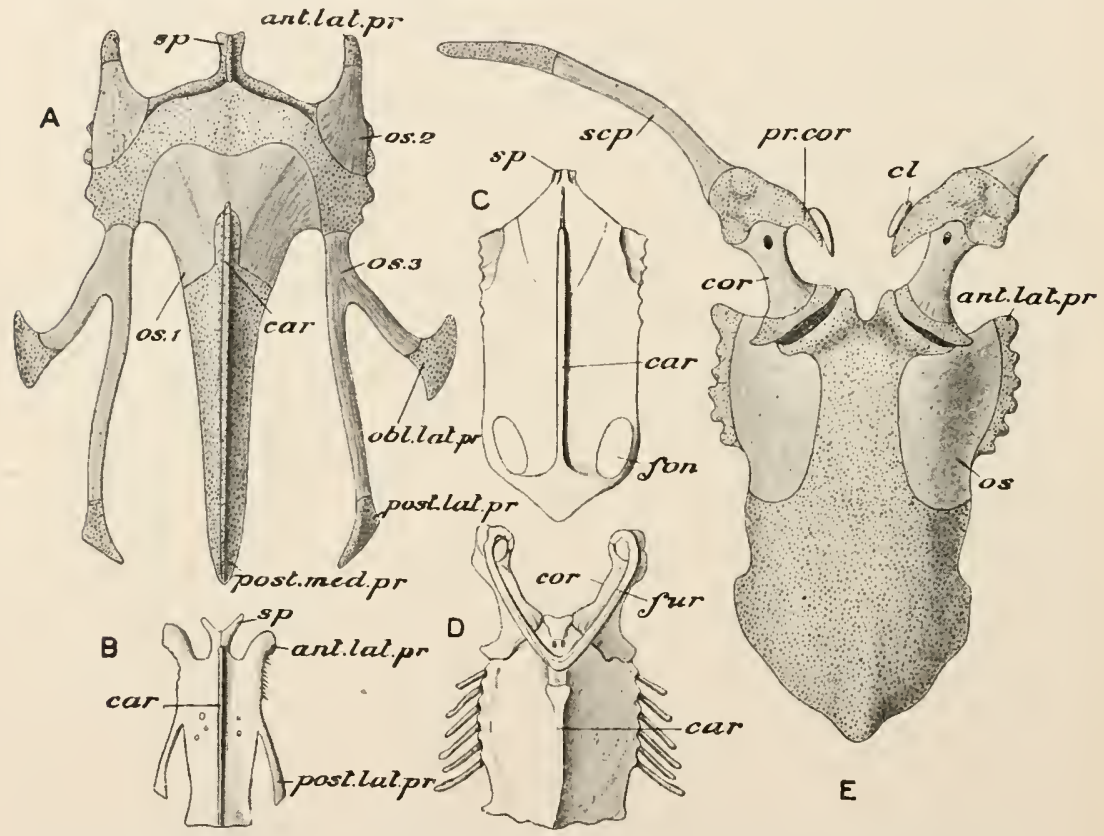

FIG. 1073.- Sterna of various Birds. $A$, Gallus (common Fowl, young); $B$, Turdus (Thrush); $C$, Vultur (Vulture); $D$, Procellaria (Petrel); $E$, Casuarius (C'assowary). ant. lat. pr. anterior lateral process; car. carina ; $c l$. clavicle ; cor. coracoid ; fon. fontanelle ; fur. furcula ; obl. lat. pr. oblique lateral process ; $0 s$. paired ossification of sternum in $E ;$; $0 s$. 1 , carinal ossification in $\boldsymbol{A}$; os. 2, os. 3, lateral ossifications; post. med. pr. posterior median process ; post. lat. pr. posterior lateral process : pr. cor. pro-coracoid; scp. scapula ; sp. spina sterni. ( $A$ and $E$, after W. K. Parker; $B, C$, and $D$, from Bronn's Thierreich.)

The ribs are always double-headed, the sternal ribs are ossified, not merely calcified, and are united with the vertebral ribs by synovial joints. Ossified uncinates are nearly always present, and usually become ankylosed to the vertebral ribs.

What may be considered as the normal type of sternum is a broad plate, concave dorsally from side to side, and produced ventrally into an antero-posterior keel which is ossified from a distinct centre (Fig. 1073, A, os. 1). The posterior edge of the bone is either entire $(D)$, or presents on each side of the keel one or two 
more or less deep notches $(A, B)$ or foramina $(C)$. In the Ratita $(E)$ the keel is either absent or reduced to the merest vestige, and there is no trace of the carinal ossification in the young. External to the coracoid grooves the anterior edge of the sternum is produced into larger or smaller antero-lateral processes (ant. lat. pr.); in the Emu these are of great size and are closely applied to the pericardium.

It was upon the characters of the raft-like sternum that the group Ratitæ was founded, but the difference between them and the Carinatx in this respect is not absolute, the ratite condition having been acquired by many Carinatæ which have lost the power of flight. The keel is very small in Ocydromus, Notornis, and Aptornis, three flightless Railsthe last extinctfrom New Zealand, and is practically absent in the Dodo (Didus) and Solitaire (Pezophaps), two gigantic extinct Pigeons from Mauritius and Rodriguez, in the $\mathrm{K}$ a k a p o o r Ground-parrot (Stringops) of New

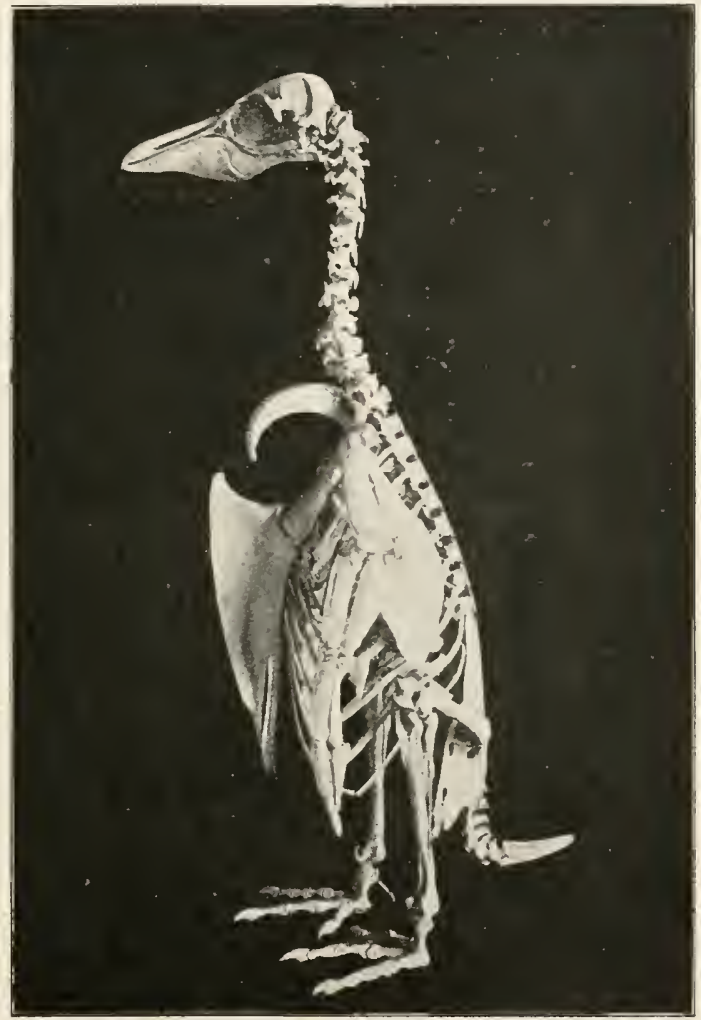

FIG. 1074.-Eudyptes pachyrhynchus (Penguin). Skeleton. (From a photograph by A. Hamilton.)

Zealand, in the extinct Giant-Goose (Cnemiornis) from the same country, and in Hesperornis. The absence of the carina may therefore be considered as an adaptive modification of no significance as indicating affinity.

The entire order of Penguins (Impennes) and the extinct Great Auk (Alca impennis) are also flightless, but their wings, instead of being functionless, are modified into powerful swimming paddles (Fig. 1074). There has therefore, in these cases, been no reduction either of the pectoral muscles or of the carina. 
The skull of Birds is generally remarkable for its huge orbits separated by a thin inter-orbital septum, and for the comparatively small size of the ethmoid bone and its turbinals. The most striking exception is afforded by the Kiwi (Apteryx), in which the orbits (Fig. 1075) are small and indistinct, while the olfactory chambers (Ec. Eth.) extend backwards between the eyes; the orbits being therefore separated from one another by the whole width of the organ of smell. The same thing occurs, to a less degree, in the Moas.

In its essential features the skull is remarkably uniform through-

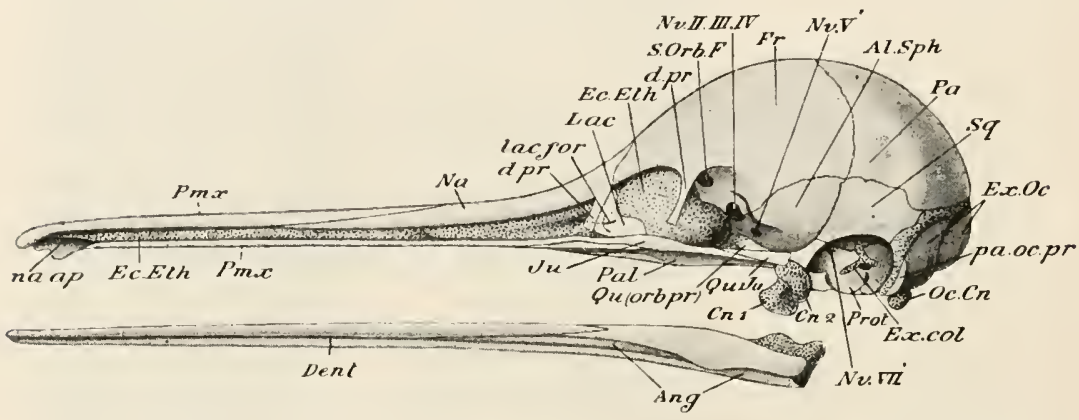

FIG. 1075.-Apteryx mantelli. Skull of a young specimen, sidc vicw. The cartilaginous

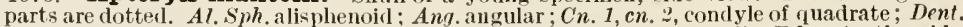
dentary; d. pr., d. pr. descending processes of nasal and frontal; Ec. Eth. ectoethmoid Ex. col. extra-columella ; Ex. Oc. ex-occipital ; Fr. frontal; Ju. jugal ; Lac. lacrymal ; lac. for. lacrymal foramen: $N a$. nasal ; na. ap. nasal aperture ; $N v$. $I I, I I I, I V$, optic foramen, transmitting also the 3rd and th nerves; $N v$. $V^{\prime}$, foramen for orbito-nasal nerve ; $N v . V I I$, for facial ; $P a$. parietal; Pal. palatine ; $p a$. oc. pr. par-occipital process ; Pmx. premaxilla ; Pr. ot pro-otic : Qu. Ju. quadrato-jugal : Qu. (orb. pr.) orbital process of quadrate; S. Orb. F. supra-orbital foramen; $S q$. squamosal. (After T. J. Parker.)

out the class. The rounded form of the brain-case, more or less concealed externally by ridges for the attachment of muscles; the upper beak, composed mainly of great triradiate premaxillæ; the single, small, rounded occipital condyle; the slender maxillo-jugal arch; the large parasphenoidal rostrum; the freely articulated quadrate, with its otic, orbital, and articular processes ; the absence of the reptilian post-frontals ; and the early ankylosis of the bonesall these characters are universal among Birds. There are, however, endless differences in detail, some of which, connected with the bones of the palate, are of importance in classification.

In the Ratitæ and the Tinamous (Crypturi) there are large basipterygoid processes (Fig. 1076, B, ptg. pr) springing, as in Lizards, from the basi-sphenoid, and articulating with the pterygoids near their posterior ends. The vomer $\left(V_{o}\right)$ is large and broad, and is usually connected posteriorly with the palatines $(\mathrm{Pal})$, which do not articulate with the rostrum. The maxillo-palatine processes are comparatively small, and do not unite with one another or with the vomer. This arrangement of the bones of the palate is called dromoognathous. 
In many Carinatx, e.g. the Pigeon and the Fowl, the basipterygoid processes are either absent or spring from the base of the rostrum. The vomer is small and pointed, or may be absent, and the palatines articulate posteriorly with the rostrum. The maxillo-palatines do not unite with one another. These peculiarities characterise the schizognathous arrangement. In the Passeres a similar arrangement obtains, but the vomer is broad and truncated instead of pointed in front. This gives the agithognathous arrangement. Lastly in the Storks, Birds of Prey, Ducks and Geese, \&c., the maxillopalatines (Fig. 1077, $m x . p$ ) fuse with one another in the middle line, often giving rise to a flat, spongy palate and producing the desmognathous arrangement.

The most specialised form of skull is found in the Parrots (Fig. 1078). In many Birds the nasals and the ascending process of the premaxilla are very thin and elastic where they join the skull, and there is an unossified space in the mesethmoid, so that the upper beak is capable of a considerable amount of movement in the vertical plane. In Parrots there is a true joint between the upper beak and the skull, allowing of that movement of the former which is so striking in the living Bird. When the mandible is depresser, the contraction of the digastric

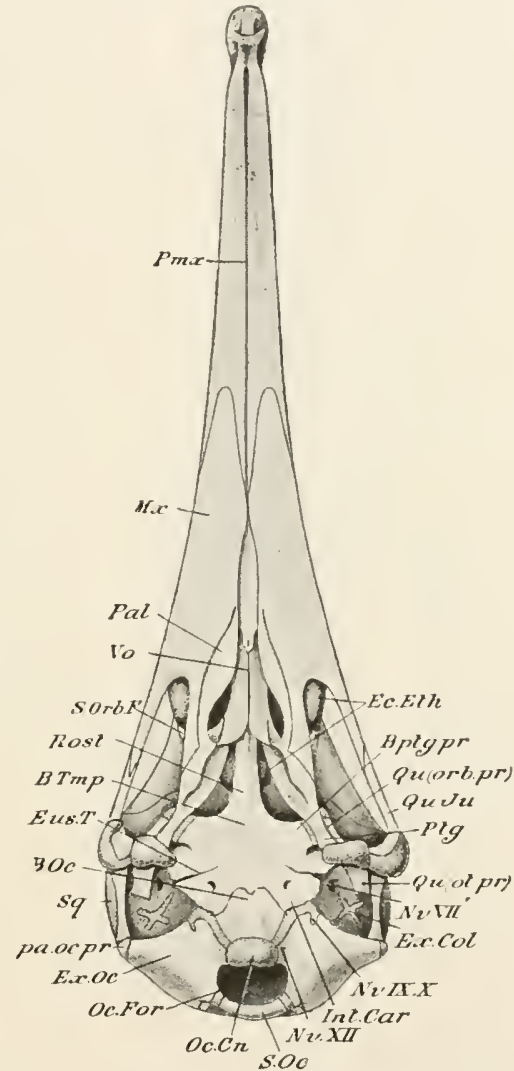

FIG. 1076.-Apteryx mantelli. Skull of young specimen, from below. The cartilaginous parts are lotted. B. Oc. basi-oreipital; B. ptg. pr. basi-pterygoid process; $B$. T $m$ p. basi-temporal Ec. Eth ecto-ethmoid : Eus. T. Eustachian tube: Ex. Col. extra-columella; Ex. Oc. ex-occipital; Int. Car, carotid foramen ; $I x$. maxilla ; $N v^{\prime}$. III', foramen for facial ; $i x . I . Y, X$, for tossopharyngeal and vagus: $N$ r. $X I I$, for hypoglossal ; Oc. Cn. occipital condyle; Oc. For. foramen magnum; Pal. palatine: pa. oc. pr. par-occipital process; $P m x$. premaxilla ; Pta. pterygoid ; $Q u$. (orb. $p r$.) orbital process of quadrate; Qu. (ot. pr.) otic process : Rost. rostrum : S. Oc. supra-oceipital ; s. Orb. $F$. supra-orbital foramen; sq. squamosal; vo. vomer. (Atter 'T'. J. Parker.) muscle causes a forward movement of the lower end of the quadrate, which pushes forwards the maxillo-jugal bar and the palatines and pterygoids, the latter sliding upon the rostrum. Both the maxillæ 


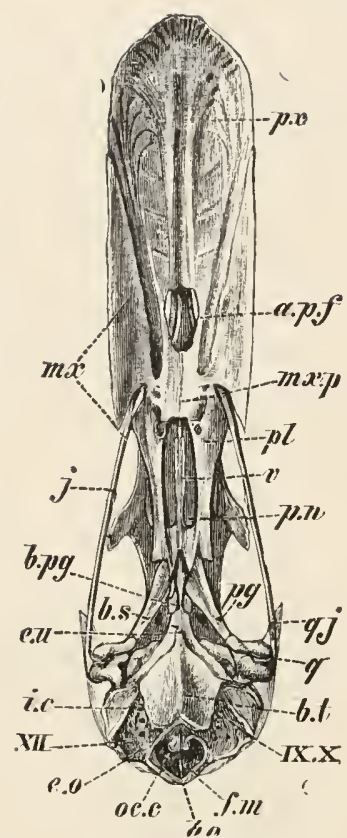

FItt. 1077,- Anas boschas (Duck). Ventral view of skull. $a, p . f$. anterior palatine foramen; $b .0$. basi-occipital ; b. pg. basiiterygoid process; $b . s$. basi-sphenoid ; $b . t$. basi-temporal ; e.o. ex-occipital ; eu. aperture of lustachian tube; $f, m$. foramen magnum ; i. c. internal carotid foramen ; $j$. jugal ; nix. maxilla ; m.x. p. maxillo. palatine process; oc. $c$. occipital condyle; w. palatine; $p$. $n$. posterior nares; $p x$. premaxilla; $q$. quadrate: $q j$. quadratojugal ; $v$. vomer; $I X, X$, foramen for ninth and tenth nerves; $X I 1$, for twelfth nerve. (From Wiedersheim's Vertebrata.) and the palatines are articulated in front with the premaxilla, and together push it upwards; in this way depression of the lower produces an automatic raising of the upper jaw. The great size and strength of both premaxilla and mandible are remarkable, as also is the fact that the orbit is completely surrounded by bone, a backward process of the lacrymal being joined beneath it by a forward process of the frontal.

The mandible contains in the young Bird the six bones on each side characteristic of Reptiles; the coronary is, however, often absent. As a rule the head of the quadrate articulates with the roof of the tympanic cavity by a single facet in Ratitæ, by a double facet in Carinatæ. The hyoid always agrees in essential respects with that of the Pigeon ; in the Woodpecker the posterior cornua are curved round the head and attached to the skull in the neighbourhood of the right nostril, a very flexible and protrusible tongue being produced.

The structure of the shoulder-

girdle furnishes one of the most fundamental distinctive characters between Ratitæ and Carinatæ, but, as with the sternum, the differences are adaptive and not of phylogenetic significance. In most Carinatæ both coracoid and scapula are large and united with one another by ligament; the coracoid has an acrocoracoid and the scapula an acromion process ; the coraco-

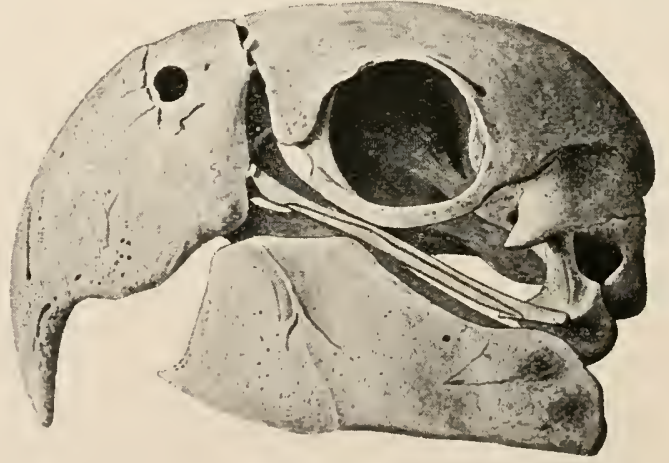
Fra. 1078.- Skull of Ara (Macaw). (From a photograph by A. Hamilton.) 
scapular angle is acute; and there is a furcula. In the Ratitix the coracoid (Fig. 1079, cor.) and scapula (scp.) are much reduced in proportional size and are ankylosed with one another; the acrocoracoid (acr. cor.) and acromion (acr.) processes are reduced or absent; the coraco-scapular angle approaches two right angles; and there is no furcula, although separate vestiges of clavicles are present in the Emu and Cassowary. In some of the Moas (Pachyornis, \&c.) the shoulder-girdle is wholly absent. But, as in the case of the sternum, the distinction is not absolute. In Hesperornis, the Dodo, the Solitaire, Aptornis, Notornis, Ocydromus, and Cnemiornis the bones of the shouldergirdle are proportionally small, the coraco-scapular angle exceeds $90^{\circ}$, and in some cases, such as certain Parrakeets and Owls, the furcula is feeble, or represented by paired vestiges, or absent. Curiously enough, considering that increase in the coraco-scapular angle is usually correlated with diminished powers of flight, it also slightly exceeds $90^{\circ}$ in the Albatross and some of its allies.

In most adult Birds the procoracoid is reduced to a process on the dorsal end of the coracoid, but in the Ostrich and in the

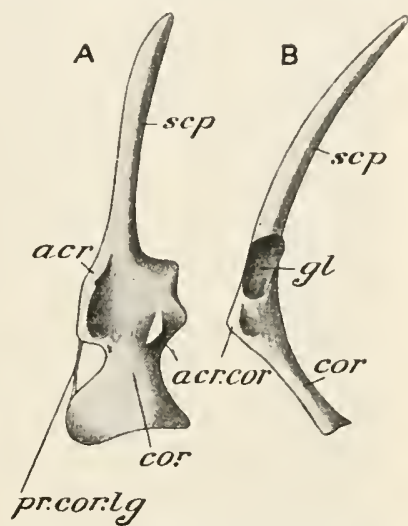

FIG. 1079.-Apteryx mantelli. The left shoulder-girdle. $A$, anterior ; $B$, lateral (outer) surface. acr. acromion; acr. cor. acrocoracold; cor. coracoid ; gl. glenoid cavity ; pr. cor. lg. procoracoid, reduced to a ligament; scp. scapula. (After T. J. Parker.)

embryo of Apteryx it is well developed and separated by a fenestra from the coracoid. A small bone, the accessory scapula, is sometimes found on the outer side of the shoulder-joint.

The variations in the structure of the wing are mostly matters of proportion, but a remarkable flattening of all the bones is very characteristic of Penguins (Fig. 1074), which are further distinguished by the presence of a sesamoid bone, the patella ulnaris, taking the place of the olecranon process. In the Emu and Kiwi the first and third digits of the normal wing have atrophied during development, the middle one alone remaining. In the Moas (Fig. 1080) no trace of a wing has been found, and in one species only is there even a trace of the glenoid cavity. In the embryos of several Birds an additional digit has been found on the ulnar or post-axial side (Fig. 1081, $d g$. 4) : this brings the total number of digits up to four, the fifth of the pentadactyle land alone being unrepresented.

The simplest type of pelvic girdle is found in Apteryx (Fig. 1082) and the Tinamous, in which both pubis and ischium are free along 


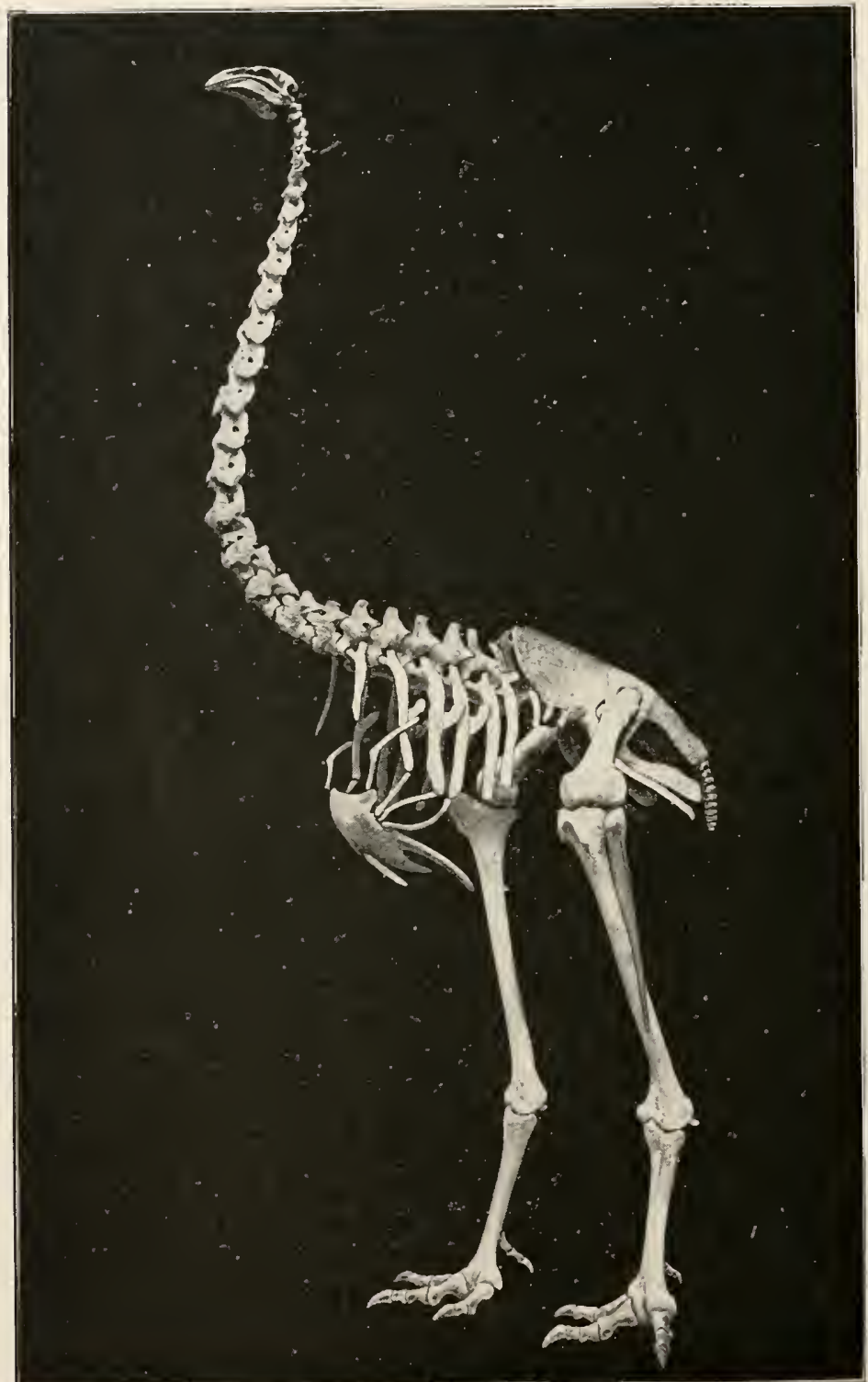

FIG. 1080.-Skeleton of Dinornis robustus, one of the Moas: actual height 9 ft. 6 in. (From a specimen at the Royal College of Surgeons, London.) 
their whole length, as in Dinosaurs. In the Emu and Cassowary the pubis and ischium unite by cartilage or bone at their posterior end with the ilium, and in most Birds the union between the two last is extensive, the deep ischiatic notch being replaced by a small foramen. In the embryonic condition (Fig. 1083) the ilium has a very small pre-acetabular portion, the pubis and ischium are nearly vertical, and there is a distinct pectineal process $(p p)$-retained in Apteryx (Fig. 1082, p.) - the whole pubis being singularly like that of a Dinosaur. In the Ostrich alone the pubes unite in the middle ventral line to form a symphysis: Rhea presents the unique peculiarity of a dorsal symphysis of the ischia, just below the vertebral column : in the Emu the posterior end of the pubis gives off a slender process, which extends forwards close

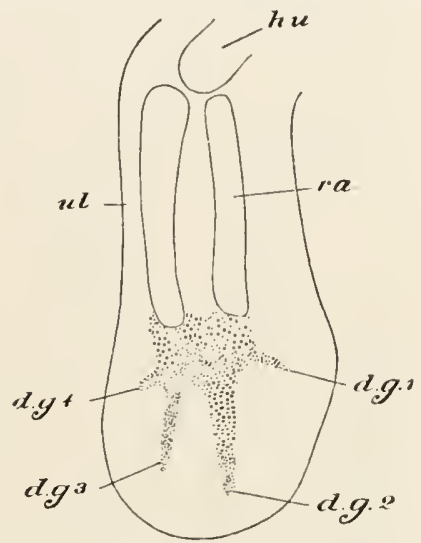

FIG. 1081.- Sterna wilsoni (Tern). Fore-limb of embryo. $d y .1-4$, digits; $h u$. humerus; $r a$. radius; $u l$. ulna. (Aiter Leighton.) to the ventral edge of that bone and probably represents the epi-pubis of Reptiles.

The bones of the hind-limb are very uniform throughout the class, but the form of the tarso-metatarsus of Penguins is worthy

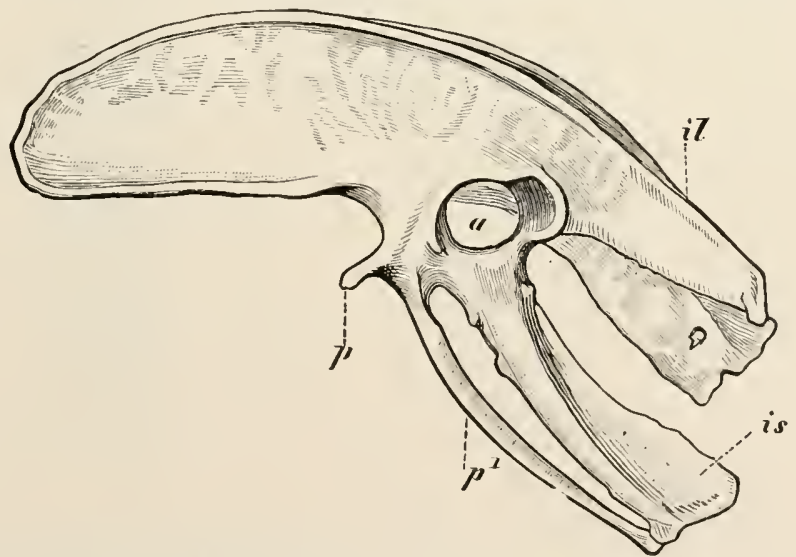

FIG. 1082.-Apteryx australis. Left innominate. $a$. acetabulum ; $i$. ilium; is. ischium ; $p$. pectineal process ; $p^{1}$. pubis. (From Wiedersheim, after llarsh.)

of notice. It is short and wide, its three constituent metatarsals, though fused, are clearly distinguishable throughout their whole length, and the resemblance to the homologous part in Iguanodon 
is very striking. In the embryo (Fig. 1084) a vestige of the fifth

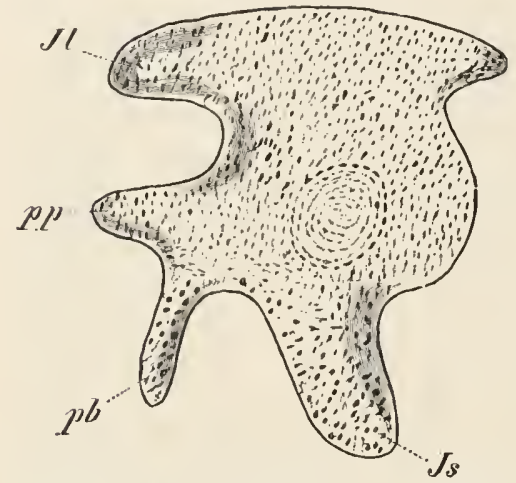

FIG. 1083 - Gallus bankiva (common Fowl). Innominate of a six days' embryo. Jl. ilium; $J \delta$. ischium ; $p b$. pubis ; $p p$. pectineal process. (From Wiedersheim, after Johnson.) digit $(m t . t s l .5)$ has been found in the form of a small rod of cartilage on the post-axial or fibular side. One or two free centralia may occur in the mesotarsal joint.

The skeleton is always more or less pneumatic, but there is no definite relation between pneumaticity and power of flight. A very usual arrangement is for all the bones to contain air except those of the fore-arm and hand, shank and foot. But in Apteryx, Penguins, and some Song-birds the skull alone is pneumatic,

while in the Hornbill every bone in the body contains air.

Myology.-As might be inferred from a study of the skeleton, the muscles of flight undergo a great reduction, often amounting to complete atrophy, in the Ratitæ ; and to a less degree in the flightless Carinatæ. The presence or absence of an ambiens and of certain other muscles in the leg and in the wing furnish characters of considerable classificatory importance.

Digestive Organs.-In all existing Neornithes the jaws are covered by a horny beak and there are no teeth. But that teeth were present in the more primitive Birds, and have gradually been lost during the evolution of the recent orders, seems certain from the fact that the cretaceous Birds were toothed. In Hesperornis (Fig. 1064) there are long conical teeth in both jaws, set in a continuous groove. In Ichthyornis (Fig. 1065) the teeth are thecodont, like those

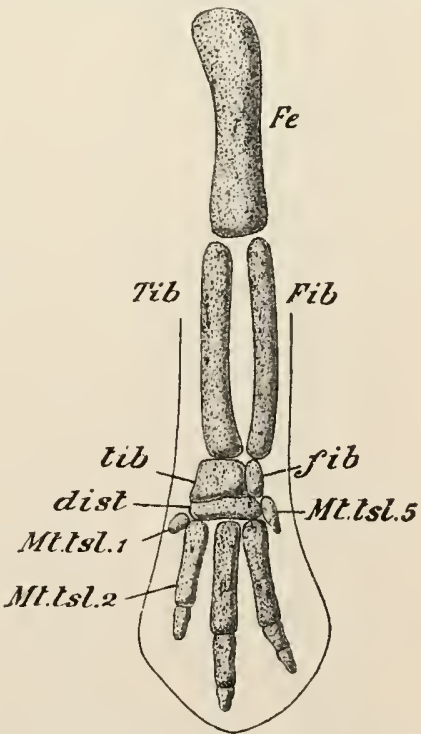

FIG, 1084.-Apteryx oweni. Lefthindlimb of embryo, dorsal aspect. dist. distale; $F e$. femur ; Fib. tibula ; $f b$. fibulare; $M$ t. $t s l$. $1-5$, metatarsals: Tib. tibia; tib. tibiale. (After T. J. Parker.) of the Crocodile, each being placed in a distinct socket. In Gastornis and in Odontopteryx, an extinct carinate form allied 
to the Anseres, the margins of the bony jaws are produced into strong, pointed, tooth-like prominences.

In the enteric canal the chief variations have to do with the size of the crop and of the cæca, with the gizzard, and with the coiling of the intestine. In grain-eating Birds the gizzard has thick muscular walls and is lined by a thickened horny epithelium, as in the Pigeon : in flesh-eaters, such as Gulls, Petrels, Hawks, and $\mathrm{Owls}$, it is thin-walled and lined with epithelium of the ordinary character. In the Common Fowl and many other Birds the cæca are of great length. A gall-bladder is usually present: the spleen is always small. The tongue may be pointed, as in the Pigeon; very long and protrusible, as in Woodpeckers; short and thick, as in Parrots ; or modified for honey-sucking by the tip being produced either into a brush-like organ or into paired sucking tubes. There are variously situated buccal glands, to some of which the name salivary is often applied.

Respiratory and Vocal Organs.-The rings of the trachea are always ossified: the tube is frequently deflected to one side by the crop, as in the Pigeon, and may undergo such an increase in length as to extend beneath the skin of the abdomen, or even into the keel of the sternum. The syrinx is either tracheo-bronchial, as in the Pigeon, i.e., formed by the distal end of the trachea and the proximal ends of the bronchi, or is exclusively tracheal or exclusively bronchial. In singing Birds it is complex, and is provided with numerous muscles-five or seven pairs.

The lungs are always firmly fixed to the dorsal body-wall by a pulmonary aponeurosis, and are but slightly distensible. The general arrangement of the air-sacs has been described in the Pigeon (p. 386) : in Apteryx the abdominal air-sacs are small, and are completely enclosed by the oblique septum, so as not to extend into the abdominal cavity among the viscera. The bronchi send off branches at right angles.

The Circulatory Organs agree in all essential respects with those of the Pigeon : their most characteristic features are the large size of the heart, the muscular right auriculo-ventricular valve, the atrophy of the left aortic arch, and the vestigial character of the renal portal system. The red blood-corpuscles are always oval and nucleated.

Nervous System and Sense Organs.-The brain is also very uniform in structure, being characterised by its short, rounded hemispheres, large folded cerebellum produced forwards to meet the hemispheres, and laterally placed optic lobes. In the embryo the optic lobes have the normal dorsal position, and the whole brain resembles that of a Reptile. In Apteryx, in correlation with the reduction of the eyes, the optic lobes are very small, and are situated on the under side of the brain. Above the anterior commissure is a small bundle of fibres which has been 
considered as the homologue of the hippocampal commissure of Mammals.

Apteryx is also distinguished by the high development of the olfactory chamber, which extends from the tip of the beak to the level of the optic foramina: the turbinals are large and complex, and there is a vestige of the cartilage of Jacobson's organ. The small eye differs from that of all other Birds in the absence of a pecten, although a vestige of that organ occurs in the embryo. The structure of the auditory organ is very uniform throughout the class.

Urinogenital Organs.-In these, also, the general agreement with the Pigeon is very close, the most characteristic feature being the more or less complete atrophy of the right ovary and oviduct. The Megistanes, Rheæ, Anseres, and some other Birds have a penis in the form of a thickening of the ventral wall of the cloaca : it has a groove on the dorsal surface acting as a sperm-channel, and its distal end is invaginated, in the position of rest, by an elastic ligament. In the Ostrich there is a solid penis, like that of Chelonia and Crocodiles: it can be retracted into a pouch of the cloaca.

Development.-The process of development in Birds has been most thoroughly worked out in the Common Fowl, but enough is known of the embryology of other Birds to show that the differences are comparatively unimportant.

The ovum is always large owing to the great quantity of food-yolk ; the protoplasm forms a small germinal disc at the upper pole. Impregnation is internal, and as the oosperm passes down the oviduct it is coated by successive secretions from the oviducal glands. It first receives a coat of thick, viscid albumen (Fig. 1085, alb.), which, as the egg rotates during its passage, becomes coiled at either end into a twisted cord, the chalaza (ch.). Next, more fluid albumen $\left(a l b^{\prime}\right)$ is deposited layer by layer, then a tough, parchment-like shell-membrane (sh. m.), and finally a calcareous shell (sh.). The shell-membrane is double, and, at the broad end of the egg, the two layers are separate and enclose an air-cavity $(a$.). The shell may be white or variously coloured by special pigments : it consists of three layers, and is traversed by vertical pore-canals, which are unbranched in the Carinatæ and in Apteryx, branched in the other Ratitæ.

The eggs may be laid on the bare ground or on the rocks by the sea-shore, as in Penguins and Auks, or on the ledges on inaccessible cliffs, as in the Sooty Albatross (Diomedea fuliginosa); but as a rule a nest is constructed for their reception by the parent Birds. This may simply be a hole in the sand, as in the Ostrich; a mere clearing on the hill-side surrounded by a low wall of earth, as in the Wandering Albatross (Diomedea exulans); or a cylinder with excavated top, built of grass, earth, and manure, as in the Mollymawks (Diomedea melanophrys, \&c.). It may take the form of a 
burrow, as in many Petrels, Kingfishers, and Sand-martins, or it may be more or less elaborately built or woven of sticks, moss, leaves, hair, or feathers, showing every stage of constructive skill, from the rude contrivance of sticks of the Pigeon and Eagle to the accurately constructed cap- or dome-shaped nests of many familiar Passeres. In the Tailor-Bird (Orthotomus) it is formed of leaves sewn together, the beak acting as needle: in a Malayan Swift (Collocalia) it is largely built of the secretion of the Bird's buccal glands.

The number of eggs laid varies from $15-18$ in the Partridge to a single one in many Sea-birds and in the Kiwi. As a rule the size of the eggs bears some proportion to that of the Bird, the smallest

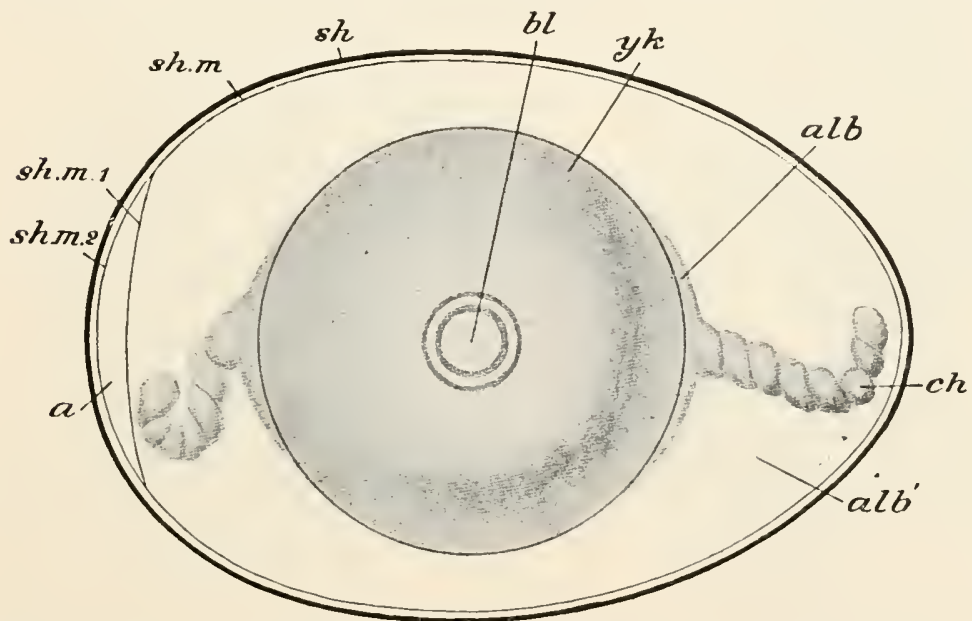

FIa. 1085.-Gallus bankiva (domestic Fow). Semi-dingrammatic view of the egg at the time of laying. a. air-space; alb. dense layer of albumen; alb' more fluid albumen; $b l$. blastoderm; $c h$. chalaza; $s h$. sliell; $s h . m$. shell-membrane; sh. m. 1, sh. m. 2, its two layers separated to enclose air-cavity. (From Marshall's Embryology, slightly altered.)

being those of Humming-birds, the largest those of the Moas and of Apyornis : but in Apteryx the egg is of disproportionate sizeas large as a Swan's or an Albatross's, the Kiwi itself being no larger than a barndoor Fowl.

Segmentation takes place during the passage of the egg down the oviduct, and results, as in Reptiles, in the formation of a blastoderm (Fig. 1085, bl.) occupying a small area on the upper pole of the yolk. After the egg is laid, the process of development is arrested unless the temperature is kept up to about $37^{\circ}$ to $40^{\circ} \mathrm{C}$. : this is usually done by the heat of the body of the parent Birds, one or both of which sit upon, or incubate, the eggs until the young are hatched; but in the Australian mound-makers (Megapodius) the eggs are buried in heaps of decaying vegetable matter, the decomposition of which generates the necessary heat. 
In the newly-laid egg the blastoderm is divisible, as in Reptiles, into two parts, a central, clear area pellucida (Fig. 1086, ar. pl.) and a peripheral area opaca (ar.op.), and is formed of a superficial ectoderm having below it a somewhat irregular aggregation of cells not yet forming a definite layer.

On the surface of the area pellucida, as in the Reptiles, appears an embryonic shield, the formation of which is due to the elongation of the ectoderm cells in a ventral direction. A primitive knot (p. 353) is absent as a distinct structure, and there is no invagination. In the posterior part of the area pellucida behind the embryonic shield appears a longitudinal opaque band, the primitive streak ( $p r . s t$.), and along the middle of this is formed a groove, the primitive groove. The latter represents the blastopore of the Reptiles, and there is no archenteric cavity. It is by active

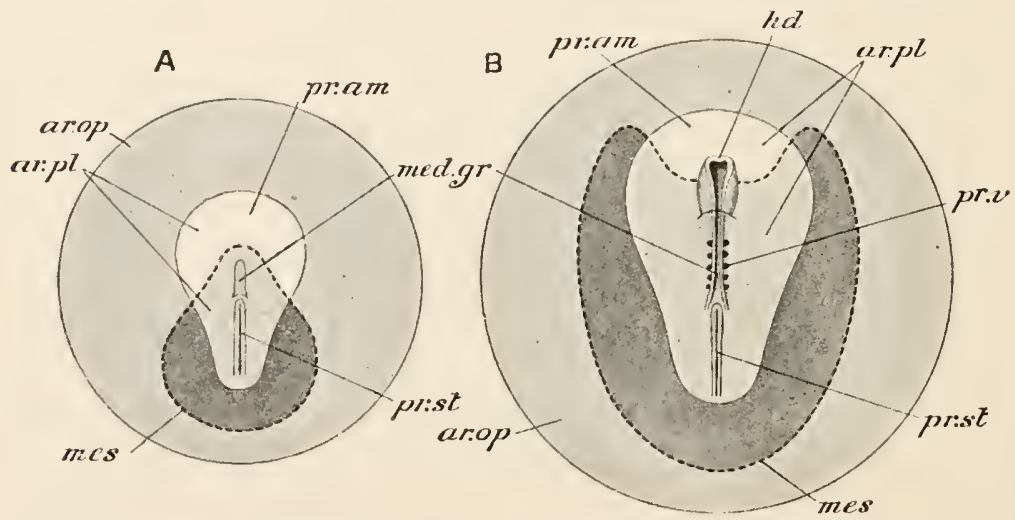

FIa. 1086.-Gallus bankiva. Two stagesi n the development of the blastoderm : diagrammatic. ar. op. area opaca ; $a r . p l$. area pellucida ; $h d$. head ; med. gr. medullary groove ; mes. mesoderm, indicated by dotted outline and deeper shade; pr. am. pro-amnion ; pr. st. primitive streak ; pr. v. protovertebre. (From Marshall's Embryology, in part after Duval.)

proliferation of cells along the course of the primitive streak, which represents the coalescent lips of the blastopore, leading to the formation of masses of new cells that grow out laterally and forwards into the space between the ectoderm and yolk-endoderm, that the foundations of the mesoderm are formed. In the anterior primitive streak-region the primitive knot of the Reptiles is represented by a close union, for a short space, of all three layers. In front the primitive streak becomes free from the ectoderm and unites below with the endoderm: this anterior extremity of the primitive streak is known as the head-process.

As there is no invagination in Birds in general, there is no primitive endoderm, and the definitive endoderm is formed solely from cells underlying the embryonic shield. The notochord is formed by an axial modification of the endoderm cells along the anterior primitive streak-region and the head-process. In the latter is formed the 
anterior or head-part of the notochord, and from it are derived also the mesoderm of the head and the endodermal lining of the headpart of the enteric canal.

Immediately in front of the primitive streak the medullary groove (med. gr.) appears, and the medullary folds which bound it on the right and left diverge posteriorly, so as to embraee the anterior end of the primitive streak, in just the same way as they embraee the blastopore in Amphioxus. In some Birds there is an invagination at the anterior end of the primitive groove, resulting in the formation of a neurenteric canal. Both primitive streak

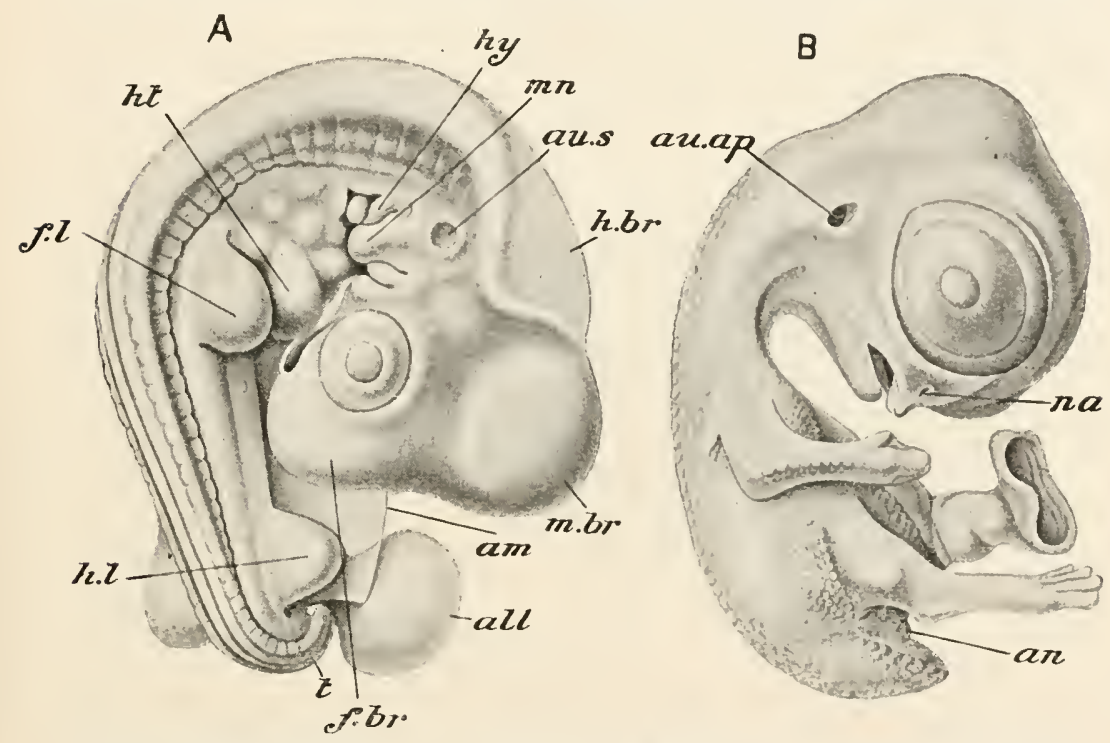

FIG. 1087.-Gallus bankiva. Two stages in the development of the embryo. all. allantois; $a m$. cut edge of amnion; an. anus; $a u$. $a p$. auditory a perture; au. $s$, auditory sac ; $f$. br. fore-brain; f.l. fore-limb; $h$.br. hind-brain; h.l. hind-limb; ht. heart; hy. hyoid arch; $m . b r$. mid-brain; $m n$. mandibular arch; na. nostril ; $t$. tail. (After Duval.)

and medullary groove lie at right angles to the long axis of the egg, the broad end of the latter being to the embryo's right.

The blastoderm gradually extends peripherally so as to cover the yolk, and thereby becomes divisible into an embryonic portion, from which the embryo is formed, and an extra-embryonic portion which invests the yolk-sac, and takes no direct share in the formation of the embryo. The extension of the eetoderm and endoderm takes plaee regularly and symmetrieally, but the extra-embryonic mesoderm, while extending equally in the lateral and posterior regions, grows forwards in the form of paired extensions, whieh afterwards unite, so that for a time there is an area of the blastoderm in front of the head of the embryo, formed of ectoderm and endoderm only: this is called the pro-amnion ( $p r . a m)$. 
At an early period the vertebral plate or dorsal portion of mesoderm bounding the medullary groove becomes segmented into protovertebræ (Fig. 1086, B, pr.v.), and the lateral plate or ventral portion of the same layer splits into somatic and splanchnic layers with the cœlome between (Fig. 1089, B).

Gradually the embryo becomes folded off from the yolk-sac, as in other large-yolked eggs; but, owing apparently to the confined space in which it is enclosed, it soon turns over so as to lie with its left side against the yolk, and its right side facing the shell (Fig. 1088). The body (Fig. 1087, A) becomes strongly flexed so as to bring the head and tail almost into contact, and the head soon acquires a proportionally immense size, with very large projecting eyes. At first the head is quite like that of one of the lower vertebrate embryos, with protuberant'brain-swellings $(f . b r ., m . b r ., h . b r$.$) ,$

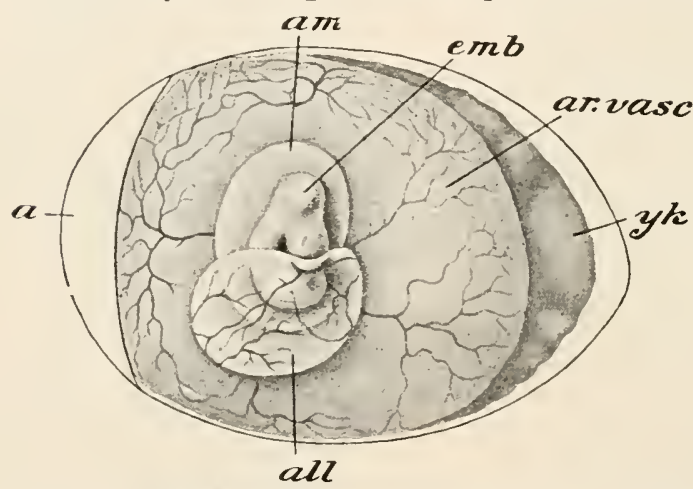

FIG. 1088.-Gallus bankiva. Egg with embryo and fetal appendages. a. air-space; all. allantois; am. amnion; ar.rase. area vasculosa; emb. embryo; $y k$. yolk-sac. (After Duval.)

clefts. Soon the margins of the mouth grow out into a beak (Fig. 1087, $B$ ), the clefts close, with the exception of the first, which gives rise to the tympano-eustachian passage, and the head becomes characteristically avian. The limbs are at first alike in form and size $(A, f . l ., h . l$.$) , and the hands and feet have the character of paws,$ the former with three, the latter with four digits; but gradually the second digit of the hand outgrows the first and third, producing the characteristic avian manus $(B)$, while the metatarsal region elongates and gives rise to the equally characteristic foot. At the same time feather-papillæ make their appearance, arranged in narrow and well-defined pterylæ.

At an early period capillaries appear in the extra-embryonic blastoderm between the opaque and pellucid areas, and give rise to a well-defined area vasculosa (Fig. 1088, ar. vasc.) : they are supplied by vitelline arteries from the dorsal aorta, and their blood is returned by vitelline veins which join the portal vein and take the 


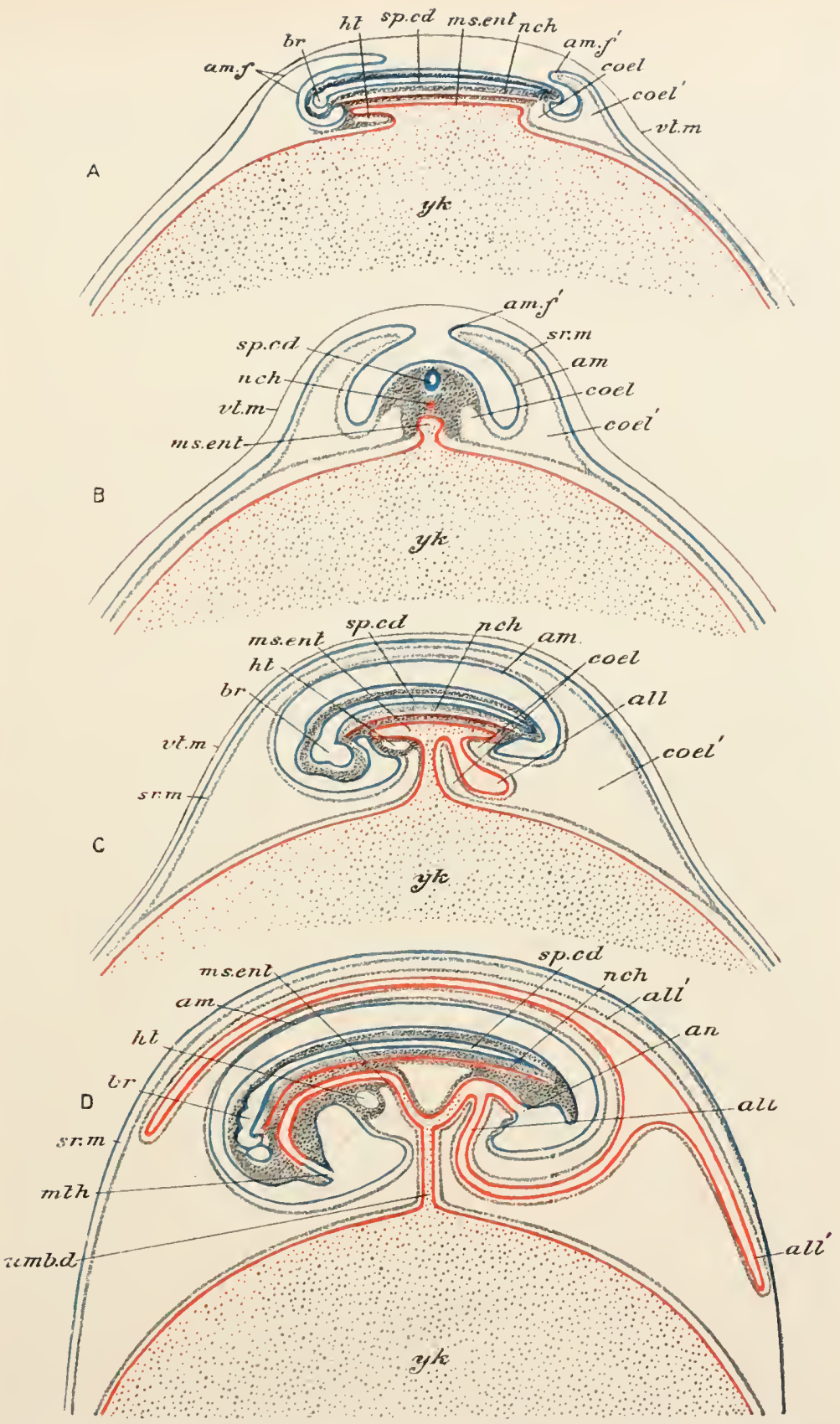

FIG. 1089.-Diagrams illustrating the development of the fœetal membranes of a Bird. A, early stagein the formation of the amnion, sagittal section; $B$, slightly later stage, transverse section; $C$, stage with completed amnion and commencing allantois; $D$, stage in which the allantois has begun to envelop the embryo and yolk-sac. The ectoderm is represented by a blue, the endoderm by a red line; the mesoderm is grey. all. allantois; a.ll'. the same growing round the embryo and yolk-sac; am. amnion ; am.f., am. $f^{\prime}$. amniotic fold ; an. anus ; br. brain ; coel. colome ; col'. extra-enbryonic cœlome; ht. heart; ms.ent. mesenteron; mth. mouth; nch. notochord; sp. ct. spinal cord; sr.m. serous membrane; $u m b . d$. umbilical duct; $v t . m$. vitelline membrane $; y k$. yolk-sac. 
blood, through the liver, to the heart. The vascular area gradually extends, until it covers the whole of the yolk-sac: its vessels take an important share in the absorption of the yolk by the embryo.

Before the embryo has begun to be folded off from the yolk the rudiment of one of the two characteristic embryonic membranes, the amnion, has appeared. A crescentic amniotic fold arises (Fig. 1089, $A$, am.f.), in front of the head-end of the embryo, from the region of the pro-amnion : it consists at first of ectoderm only, the mesoderm not having yet spread into the pro-amnion. The fold is soon continued backwards along the sides of the body $(B)$ and round the tail $(A)$, but in these regions ( $a m . f^{\prime}$.) it consists from the first of ectoderm plus the somatic layer of mesoderm, i.e., it is a fold of what may be called the extra-embryonic body-wall. The cavity is a prolongation of the space between the somatic and splanchnic layers of mesoderm, i.e., is an extension of the extraembryonic colome.

The entire amniotic fold gradually closes in above $(C)$, forming a double-layered dome over the embryo. Its inner layer, formed of ectoderm internally and mesoderm externally, is the amnion $(\mathrm{am}$.$) , the cavity of which becomes filled with a watery amniotic$ fluid, serving as a protective water-cushion to the enclosed embryo. Its outer layer, formed of ectoderm externally and mesoderm internally, is the serous membrane (sr.m.): it comes to lie just beneath the vitelline membrane, with which it subsequently fuses.

The second of the embryonic membranes, the allantois, is developed as an outpushing of the ventral wall of the mesenteron at its posterior end $(C$, all. $)$, and consists, therefore, of a layer of splanchnic mesoderm lined by endoderm. It has at first the form of a small ovoid sac having the precise anatomical relations of the urinary bladder of Amphibia (Fig. 1087, $A$, all.). It increases rapidly in size (Fig. 1088, all.), and makes its way, backwards and to the right, into the extra-embryonic colome, between the amnion and the serous membrane (Fig. 1089, C, D). Arteries pass to it from the dorsal aorta, and its veins, joining with those from the yolk-sac, take the blood through the liver to the heart. Next, the distal end of the sac spreads itself out and extends all round the embryo and yolksac $\left(D, a l l^{\prime}.\right)$, fusing, as it does so, with the serous and vitelline membranes, and so coming to lie immediately beneath the shellmembrane. It finally encloses the whole embryo and yolk-sac together with the remains of the albumen, which has by this time been largely absorbed. The allantois serves as the embryonic respiratory organ, gaseous exchange readily taking place through the porous shell; its cavity is an embryonic urinary bladder, excretory products being discharged into it from the kidneys.

At the end of incubation the embryo breaks the shell, usually by means of a little horny elevation or caruncle at the end of the beak. 
By this time the remainder of the yolk-sac has been drawn into the colome, and the ventral body-walls liave closed round it. On the shell being broken respiratory movements begin, the aperture is enlarged, and the young bird is hatched and begins a free life.

In the Ratitæ, Anseres, Gallinæ, and some other Birds the young when hatched are clothed with a complete covering of down or of feathers, and are able from the first to run about and feed themselves; such Birds are called Prococes or Nidifuga. In the higher types, such as the Rapacious Birds, Pigeons, and Passeres, the young are at first either quite naked, blind and helpless, or covered with mere patches of soft down, so that they require to be fed and kept warm by the parents; these forms are called Altrices or Nidicolce. In many Sea-birds, such as Petrels, Gulls, and Penguins, the young have a complete covering of woolly down, but remain in the nest for a prolonged period, sometimes until the full size is attained.

Distribution.-The Ratitæ furnish an interesting case of discontinuous distribution. Struthio occurs in Africa and Southwestern Asia, Rhea in South America, Dromæus in Australia, Casuarius in Australia, New Guinea, and some of the other AustroMalayan islands, and Apteryx in New Zealand. Thus, taking recent forms only, each of the great Southern land-masses contains one order of Ratitæ not found elsewhere; the Struthiones are Ethiopian, but extend also into the adjacent part of the Palæarctic region, the Rheæ Neotropical, and the Megistanes Australasian. Apyornis, the affinities of which appear to be with the Megistanes, occurs only in Madagascar, where it has become extinct within-geologically speaking-comparatively recent times. When we take the scattered distribution of the above-mentioned Ratitro into consideration, one of the most remarkable facts in distribution is the occurrence, in the limited area of New Zealand, of no fewer than six genera and between twenty and thirty species of Dinornithidæ or Moas, some of which became extinct so short a time ago that their skin, flesh, feathers, dung, and egg-shells are preserved.

Among the Carinatæ the Penguins are exclusively southern, occurring only in the South Temperate and Arctic Oceans. They may be said to be represented in the Northern Hemisphere by the Puffins and Auks, one of which, the Great Auk or Gare-fowl (Alca impennis), was actually impennate, its wings being converted, as in the Penguins, into paddles. The Crypturi (Tinamous) are exclusively Neo-tropical, the Humming-birds American, the Birds of Paradise and Bower-birds Australian and Austro-Malayan. Amongst negative facts, the Psittaci or Parrots are characteristically absent in the Palæarctic and most of the Nearctic region, the Finches in 
the Australasian region, as well as in New Zealand and Polynesia, and the Starlings in both regions of the New World.

Birds are comparatively rare in the fossil state: their powers of flight render them less liable to be swept away and drowned by floods and so imbedded in deposits at the mouths of rivers or in lakes. Up to the Cretaceous period, Archæopteryx, from the Jurassic, is the only Bird known. In the Cretaceous of North America toothed Birds of the orders Odontolcæ and Ichthyornithes make their appearance, while in the Eocene numerous interesting forms occur, including the Gastornithes and the Stereornithes.

Ethology.-It is impossible here to do more than allude, in the briefest way, to the immense and fascinating group of facts relating to the instincts, habits, and adaptations found in the present class. Their social instincts, their song, their courtship-customs, the wonderful advance in the parental instinct, leading to diminished mortality in the young, are all subjects for which the reader must be referred to the works on general Natural History mentioned in the appendix. The same applies to the puzzling subject of migration, which will be referred to in the Section on Distribution.

Phylogeny.-That Birds are descended from Reptilian ancestors, that they are, as it has been said, "glorified Reptiles," seems as certain as anything of the kind can well be. Apart from the direct evidence afforded by Archæopteryx and by the numerous avian characteristics of Dinosauria and Ornithosauria, the indirect evidence of anatomy and embryology is very strong. The single occipital condyle, the six bones to each mandibular ramus, the anklejoint between the proximal and distal tarsals, the number of phalanges in the digits of the foot, the epidermal exoskeleton partly taking the form of scales, the meroblastic egg with large food-yolk, the amnion, and the respiratory allantois, are all characters common to Birds and Reptiles and not found together-indeed for the most part not found at all-in any other class. For this reason Reptiles and Birds are often conveniently grouped together, as already stated (p. 303), as Sauropsida.

It seems probable that the earliest Birds could fly, and that their evolution from Reptilian ancestors was directly connected with the assumption of aërial habits. It is not unlikely that these ancestors possessed a patagium, like that of Ornithosauria, and that, as the scales of the fore-limb developed into feathers, this organ was gradually reduced to the small pre- and post-patagia of the existing Bird's wing. What was the nature of the Reptilian ancestor is a question as yet quite unsolved. It can hardly have been a Pterodactyle, since in that order the modification of the fore-limb has proceeded on entirely different lines from those which characterise Birds; it cannot well have been a Dinosaur, since we have 
no evidence that any member of that order was arboreal, or showed the least tendency on the part of the fore-limb to assume the wing-form. Nevertheless the skull and brain of Ornithosauria and the pelvis and hind-limb of many Dinosauria show such approximation to avian characters as can hardly be without significance.

Probably the earliest Birds were all, in the etymological sense, Carinatæ, i.e., had the sternum provided with a keel for the attachment of the pectoral muscles. Probably, also, they all possessed teeth, and had diverged into well-marked orders before those organs were lost. The Odontolcæ, for instance, have their nearest allies in the Divers (Pygopodes), while the Ichthyornithes resemble the Terns, members of the widely separated order Gaviæ.

In several existing types of Carinatæ the power of flight is wanting, and in all such cases it is practically certain that flightlessness is due to the degeneration of the wings: in other words, that the ancestors of the Penguins, Great Auk, Dodo, Weka (Ocydromus), Kakapo (Stringops), \&c., were ordinary flying Birds. In the Penguins and the Great Auk the wings have simply undergone a change of function, being converted into paddles, and consequently the only parts of them which have degenerated are the feathers; but in the other forms referred to the wing las become more or less functionless, and hence has diminished in size, while the partial atrophy of the muscles has resulted in a more or less complete reduction of the carina sterni and furculum and an increase of the coraco-scapular angle. Now it is by an exaggeration of these peculiarities that the Ratitæ are distinguished from the Carinatæ, and there is every reason for thinking that they also are the descendants of flying Birds, and that their distinctive characters-absence of locking apparatus in the feathers; flat sternum, wide coracoscapular angle, \&c.- - are all due to degeneration correlated with disuse of the wings. From the fact that the dromæognathous skull is more reptilian than any other type, it would seem that the Ratitæ diverged early from the carinate stock. From the fact that, in the structure of the skull and pelvis, the Ostrich and Rhea are widely separated both from one another and from the Australasian Ratitæ, it seems probable that the three orders of Ratitæ arose independently from primitive Carinatæ, and that the entire division is to be looked upon as a convergent or polyphyletic group, owing its distinctive characters, not to descent from a common ancestor, but to the independent acquisition of similar characters under the influence of like surroundings.

The question of the phylogeny of the orders of Carinatre is far too complex to be discussed here. Suffice it to say that the Ichthyornithes, Odontolcæ, Impennes, Pygopodes, and Crypturi are to be looked upon as the lowest or most generalised orders, while the highest or most specialised are the Psittaci, the Accipitres, the 
Striges, the Picarix, and especially the Passeres. Among the latter the Corvidæ (Crows) are probably to be looked upon as the most exalted members of the class (Fig. 1090).

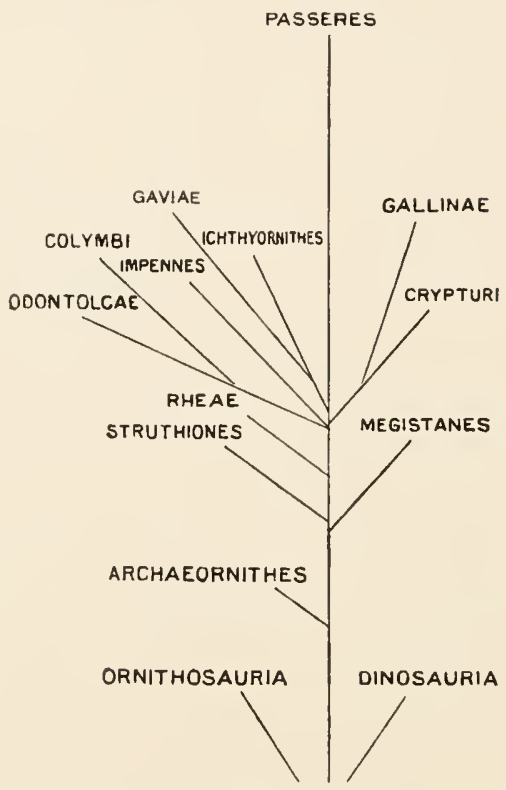

FIG. 1090.-Diagram illustrating the Relationships of the chief groups of Birds.

\section{CLASS VI. - MAMMALIA.}

The class Mammalia, the, highest of the Vertebrata, comprises the Monotremes and Marsupials, the Hoofed and Clawed Quadrupeds, the Whales and Porpoises and Sea-Cows, the Rodents, Bats and Insectivores, the Lemurs and Apes, and the Human Species. All Mammals, though many are aquatic, are air-breathers throughout life, lungs being, as in Reptiles and Birds, the sole organs of respiration. The blood of Mammals has a high temperature, resembling in that respect the blood of Birds and differing from that of Reptiles and Amphibia. The scales of Reptiles and the feathers of Birds are replaced in Mammals by peculiar epidermal structures, the hairs, usually developed in such quantities as to form a thick, soft covering or fur. The young are nourished after birth by the secretion of mammary or milk-glands.

1. Example of the Class-The Rabbit (Lepus cuniculus).

External Characters.-The Rabbit (Fig. 1091) is a fourfooted or quadrupedal animal, having the whole surface of its body covered with soft fur. The head bears below its anterior 
extremity the mouth, in the form of a transverse slit bounded by soft lips. The upper lip is divided by a longitudinal cleft, running backwards to the nostrils, and exposing the chisel-shaped incisor teeth. Behind the incisor teeth the hairy integument projects on each side into the cavity of the mouth. At the end of the snout, above the mouth, are the nostrils, in the shape of two oblique slits. The large eyes, situated at the sides of the head, have each three eyelids, an upper and a lower hairy lid, and an anterior hairless third eyelid or nictitating membrane, supported by a plate of cartilage. Vibrissa-very long stiff hairs-are scattered above and below the eyes and on the snout. Behind the eyes, and a little nearer the summit of the head, are a pair of very long flexible and movable external ears or pinno. These are somewhat spout-shaped, expanding distally, and are usually placed vertically with the concavity directed laterally and somewhat forwards, leading to the

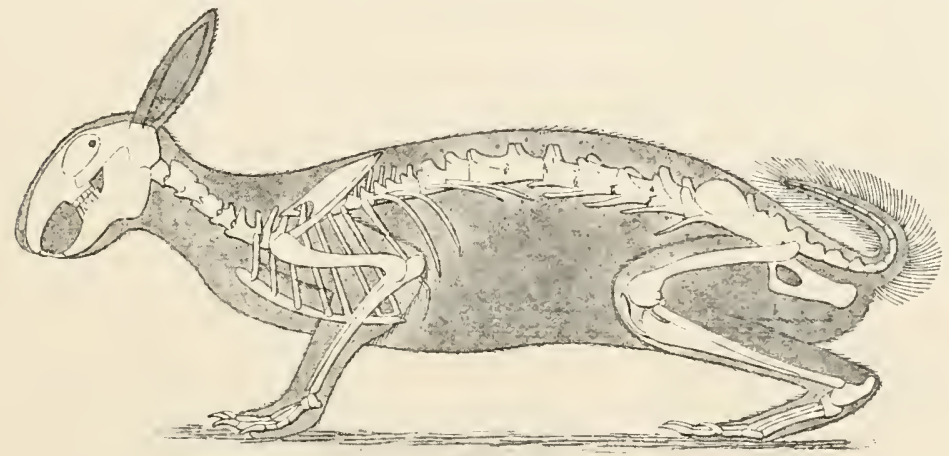

FIG. 1091.-Lepus cuniculus. Lateral view of skeleton with outline of body.

external auditory opening. The neck is a distinct constriction, but relatively short as compared with the neck of the Pigeon. The trunk is distinguishable into thorax in front and abdomen behind. On the ventral surface of the abdomen in the female are four or five pairs of little papillæ-the teats. At its posterior end, below the root of the tail, is the anal opening, and in front of this in the male is the penis, with a small terminal urinogenital aperture, and with the testes, each in a prominent scrotal sac, at the sides; and in the female the opening of the vulva. In the space (perinceum) between anus and penis or vulva are two bare, depressed areas of skin into which open the ducts of certain glands- the perinceal glands-with a secretion having a strong and characteristic odour. The tail is very short and covered with a tuft of fluffy fur.

The fore- and hind-limbs, both of which take part in locomotion and in supporting the weight of the animal, differ considerably in size-the fore-limbs being much shorter than the hind-limbs. Both have the same general divisions as in the Lizard. The upper 
arm is almost completely hidden by the skin, being applied closely against the side of the body. The manus is provided with five digits, each terminating in a horny claw. The thigh is also almost hidden by the skin; the pes has four digits only, all provided with claws.

Skeleton.-The spinal column of the Rabbit is divisible, like that of the Pigeon and the Lizard, into five regions-the cervical, the thoracic, the lumbar, the sacral, and the caudal. In the cervical region there are seven vertebræ; in the thoracic twelve, or sometimes thirteen, in the lumbar seven, or sometimes six, in the sacral four, and in the caudal about fifteen.

The centra of the vertebræ in a young Rabbit consist of three parts - a middle part which is the thickest, and two thin discs of bone-the epiphyses - anterior and posterior, applied respectively to the anterior and posterior faces of the middle part or centrum proper. Between successive centra in an unmacerated skeleton are thin disc-like plates of fibro-cartilage-the inter-vertebral discs.

The transverse processes of all the cervical vertebræ, except the seventh or last, are perforated by a canal, the vertebrarterial canal, for the passage of the vertebral artery. The first vertebra or atlas (Fig. 1092, A) resembles the corresponding vertebra of the Pigeon in being of the shape of a ring without any solid centrum like that

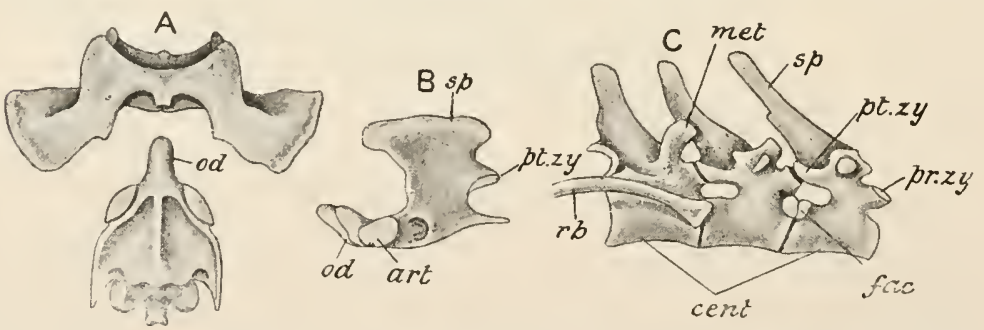

FIG. 1092.-Iepus cuniculus. A, atlas and axis, ventral aspect. od. odontoid process of axis. $B$, lateral view of axis. art. articular facet for atlas; od. odontoid process; $p t$. $z y$ : post-zygapophysis; $s p$. neural spine. $C$, thoracic vertebre, lateral view. cent. centrum; fac. facet for rib; met. metapophysis ; pr.zy. pre-zygapophysis ; $p t$. $z y$. post-zygapophysis ; rb. rib; sp. spinous process.

of the rest. On the anterior face of its lateral portions are two concave articular surfaces for the two condyles of the skull. The second vertebra or axis ( $A$ and $B$ ) bears on the anterior face of its centrum a peg-like process--the odontoid process (od.) -which fits into the ventral part of the ring of the atlas: it has a compressed spine ( $s p$.$) , produced in the antero-posterior direction ; its transverse$ processes are short and perforated by a canal for the vertebral artery. All the cervical vertebræ except the last have their transverse processes bifurcated into dorsal and ventral lamellæ. The seventh differs from the others in having a more elongated neural spine, in having its transverse processes simple and without perfora- 
tion for the vertebral artery, and in the presence on the posterior edge of the centrum of a little concave semi-lunar facet.

The thoracic vertebræ $(C)$ lave clongated spines which are mostly directed backwards as well as upwards. The transverse processes are short and stout; each bears near its extremity a small smooth articular surface or tubercular facet for the tubercle of a rib. On the anterior and posterior borders of each vertebra is a little semilunar facet, the capitular facet (fac.), situated at the junction of the centrum and the neural arch. The two contiguous semi-lunar facets of successive vertebræ form between them a little cup-like concavity into which the head or capitulum of a rib is received. The semi-lunar facet on the last cervical vertebra forms, with that on the anterior border of the first thoracic, the concavity for the head of the first rib.

In the lumbar region the spines are comparatively short, and both transverse processes and bodies are devoid of facets. From the centrum of each of the first two (or three) projects downwards a short flattened process - the hypapophysis. Certain accessory processes-the metapophyses (met.) and anapophyses - are welldeveloped, the former being extremely long in the posterior lumbar region. The metapophyses are situated in front, projecting forwards and outwards over the pre-zygapophyses; and the anapophyses arc situated below the post-zygapophyses and project backwards. The transverse processes are long, and are directed forwards and outwards; that of the last lumbar is bifurcated.

The sacral vertebræ are firmly ankylosed together to form a single composite bone, the sacrum. The vertebræ bear a close resemblance to those of the lumbar region, but the hypapophyses and anapophyses are wanting, and the metapophyses are comparatively small. The first and second bear great expanded lateral processes, or sacral ribs, with roughened external surfaces for articulation with the ilia. These are the only sacral vertebræ in the strict sense of the term, the following two being in reality anterior caudal.

Of the caudal vertebræ the more anterior resemble those of the sacral region, and have similar processes; but as we pass backwards in the caudal region all the processes gradually diminish in size, the most posterior vertebra being represented merely by nearly cylindrical centra.

There are twelve pairs of ribs, of which the first seven are known as true ribs, i.e. are connected by their cartilaginous sternal parts with the stcrnum; while the remaining five, the so-called false or floating ribs, are not directly connected with the sternum. All, except the last four, bear two articular facets, one on the vertebral extremity or capitulum, and the other on a little clevation "or tubercle situatcd at a little distance from this - the formsr for the bodies, the latter for the transveris prosesses of the vertebræ. 
The sternum consists of six segments or sternebra: the first, the manubrium sterni or presternum, is larger than the rest, and has a ventral keel. With the last is connected a rounded cartilaginous plate, the xiphisternum.

The skull (Figs. 1093, 1094), if we leave the jaws out of account, is not at all unlike that of the Pigeon in general shape. The length is great as compared with either the breadth or the depth ; the maxillary region, or region of the snout (corresponding to the beak of the Pigeon), is long in proportion to the rest, the orbits closely approximated, being separated only by a thin inter-orbital partition, and the optic foramina united into one. But certain important differences are to be recognised at once. One of these is in the mode of union of the constituent bones. In the Pigeon, as we have seen, long before maturity is attained, the bony elements of the skull, originally distinct, become completely fused together so that their limits are no longer distinguishable. In the Rabbit, on the other hand, such fusion between elements only takes place in a few instances, the majority of the bones remaining more or less distinct throughout life. The lines along which the edges of contiguous bones are united-the sutures as they are termed-are sometimes straight, sometimes wavy, sometimes zig-zagged serrations of the edges of the two bones interlocking; in some cases the edges of the bones are bevelled off and the bevelled edges overlap, forming what is termed a squamous suture.

Another conspicuous difference between the skull of the Rabbit and that of the Pigeon is in the mode of connection of the lower jaw, which in the former articulates directly with the skull-the quadrate, through which the union is effected in the Pigeon, being apparently absent. Certain large apertures which are distinguishable are readily identified with the large openings in the skull of the Pigeon. In the posterior wall of the skull is a large rounded opening, the foramen magnum, flanked with a pair of smooth rounded elevations or condyles for articulation with the first vertebra, these obviously corresponding to the single condyle situated in the middle below the foramen in the Pigeon. A large opening, situated at the end of the snout and looking forwards, obviously takes the place of the external nares of the Pigeon; and a large opening in the roof of the mouth, leading forward to the external nasal opening, plainly represents, though much wider and situated further back, the internal or posterior nares of the Pigeon; while the rounded tubular opening (aud. me.) situated at the side of the posterior part of the skull, some distance behind the orbit, is evidently the same as the auditory aperture of the Pigeon.

Surrounding the large opening of the foramen magnum are the bones of the occipital region of the skull, the supra-, ex- and basioccipitals. The first of these $(s . o c$. ) is a large plate of bone whose 

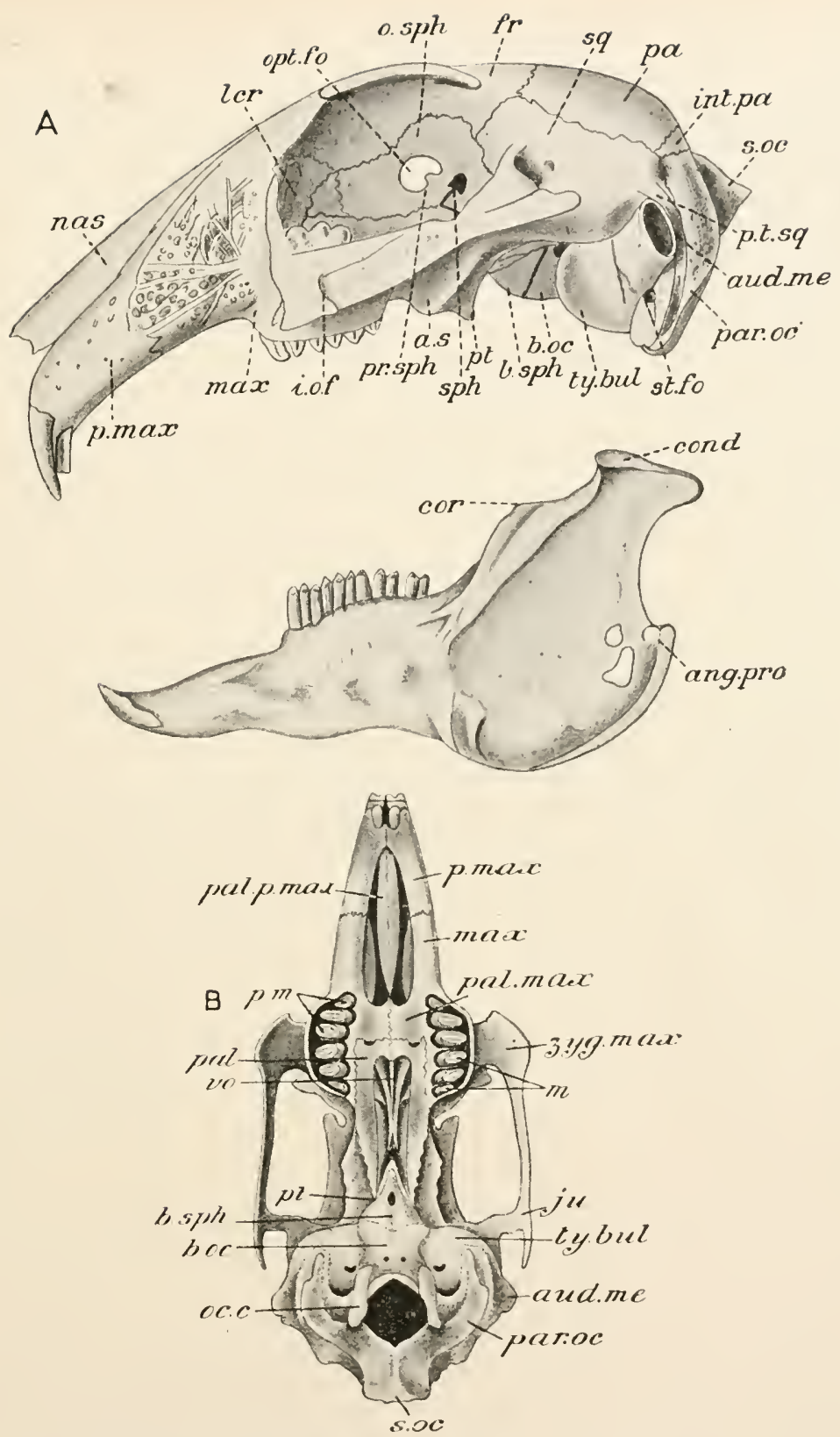

Fig. 1093.-Lepus cuniculus. Skull. $A$, lateral view; $B$, ventral view. ang. proc. angular process of mandible; a.s. alisphenoid (external pterygoid process); aud. me. external auditory meatus; $b$. oc. basi-occipital; $b . s p h$. basi-sphenoid; cond. condyle ; $f r$. frontal : int.pa. interparietal ; i.o.f. infra-orbital foramen; ju. jugal ; lcr. lacrymal ; $m$. molars ; max. maxilla; nas. nasal; opt. fo. optic foramen; $0 . s p h$. orbito-sphenold ; $p a$. parietal ; pal. palatine; pal. max. palatine plate of maxilla ; par.oc. paroccipital process ; pal. p. max. palatine process of pre-maxilla ; p.m. pre-molars; $p$. max. pre-maxilla ; $p r . s p h$. pre-sphenoid; $p t$. pterygoid ; $p . t$. sq. post-tympanic process of squamosal; s. oc. supraoccipital ; sph. points to position of sphenoidal fissure, not clearly visible in a lateral view ; sq. squamosal ; st.fo. stylomastoid foramen; ty.bul. tympanic bulla; vo. vomer; zyg. max. zygomatic process of maxilla. 
external surface is directed backwards and upwards, and elevated in the middle into a shield-shaped prominence. The cx-occipitals lie at the sides of the opening, and each bears the greater part of the somewhat oval prominence or condylc with which the corresponding surface of the atlas or first vertebra articulates. Each is produced below into a process called the par-occipital (par. oc.), closely applied to the tympanic bulla. At the end of this, imbedded in the tendon of a muscle, the styloglossus, is a small bony rod, the stylo-hyal. A small aperture, the condylar foramen, situated below the condyle, is for the passage of one of the cerebral nerves, the hypoglossal. The basi-occipital is a median plate of bone, almost horizontal in position, which forms the floor of the most posterior part of the cranial cavity ; it bears the lower third of the occipital condyles. All these four bones of the occipital region are in the adult Rabbit united together to form the single occipital bone. Articulating in front with the basi-occipital, but separated from it by a plate of cartilage, is a plate of bone, also horizontal in position, which forms the middle part of the floor of the cranial cavity. This is the basi-sphenoid; it is perforated at about its middle by an oval foramen-the pituitary foramen - and on its upper surface is a depression, the sella turcica, or pituitary fossa (Fig. 1094, s.t.), in which the pituitary body rests. In front of it is another median bone of laterally compressed form, the pre-sphenoid, with which it is connected by cartilage, the removal of which leaves a gap in the dried skull; the pre-sphenoid forms the lower boundary of the single large optic foramen (Fig. 1093, opt.fo.) Connected laterally with the basi-sphenoid and pre-sphenoid are two pairs of thin irregular plates, the ali-sphenoids (as.) behind and the orbito-sphenoids (o. sph.) in front. The alisphenoids are broad wing-like bones, each produced below into a bilaminate process, the pterygoid process. A large foramen, the sphenoidal fissure ( $s p h$.), situated between the basisphenoid and the alisphenoid of each side, transmits from the interior of the skull the third and fourth cerebral nerves, the first and second divisions of the fifth, and the sixth nerves.

The boundary of the anterior part of the brain-case is completed by a narrow plate of bone, the cribriform plate of the ethmoid (Fig. 1094, eth.), perforated by numerous small foramina for the passage of the olfactory nerves. This cribriform plate forms a part of a median vertical bone, the mesethmoid, the remainder of which, or lamina perpendicularis, forms the bony part of the partition (completed by cartilage in the unmacerated skull) between the nasal cavities. Fused with the mesethmoid are two lateral, thin, twisted bones, the ethmo-turbinals (e. $t b)$, and with its inferior edge articulates a long median bone, with a pair of delicate lateral wings, the vomer (vo.). None of these, with the exception of the cribriform plate, take any share in the bounding of the cavity 
of the cranium. Roofing over the part of the cranial cavity the walls and floor of which are formed by the sphenoid elements, is a pair of investing bones, the parietals (Fig. 1093, pa.), and further forward is another pair, the frontals $(f r$.$) . The parietals are plate-$ like bones, convex externally, concave internally, which articulate with the supra-occipital behind by a transverse serrated lambdoidal suture. The right and left parietals articulate together by means of a somewhat wavy suture, the sagittal; in front a transverse serrated suture, the coronal, connects them with the frontals. Between the supra-occipital and the parietals is a median ossification or inter-parietal (int. pa.). The frontals are intimately united along the middle line by means of the frontal suture. Laterally their orbital plates form an important part of the upper portion of the inner wall of the orbit; above this, over each orbit, is a curved, somewhat crescentic process, the supra-orbital process. Between the alisphenoid below, the parietal and frontal above, the frontal and orbito-sphenoid in front, and the parietal behind, is a broad bone (sq.), the superior margin of which is bevelled oft: this is the squamosal. It is produced in front into a strong zygomatic process, which curves outwards, then downwards, and finally forwards, to unite with the jugal in the formation of the zygomatic arch. Below the root of the process is a hollow, the glenoid fossa. Behind the squamosal gives off a slender process, the post-tympanic process $(p . t$. sq.), which becomes applied to the outer surface of the periotic.

Between the occipital and parietal bones, below and behind the squamosal, are the tympanic and periotic bones. The tympanic forms the bony part of the wall of the external auditory meatus; below it is dilated to form a process $(t y$. bul.) projecting on the under surface of the skull-the bulla tympani. The periotic is a bone of irregular shape, its internal (petrous) portion (Fig. 1094, peri) enclosing the parts of the membranous labyrinth of the internal ear, and externally presenting two small openings-the fenestra ovalis and fenestra rotunda-visible only when the tympanic is removed; internally it bears a depression, the floccular fossa, for the lodgment of the flocculus of the cerebellum. Part of the periotic (mastoid portion) is seen on the exterior of the skull between the tympanic and exoccipital. The periotic and tympanic are not ankylosed together, and are loosely connected with the surrounding bones, being held in position by the posttympanic processes of the squamosal. Between the tympanic and periotic are two foramina of importance-the stylomastoid, which transmits the seventh cerebral nerve, and the Eustachian aperture, at which the Eustachian tube opens.

Roofing over the olfactory cavities are two flat bones-the nasals (nas.) - each having on its inner surface a very thin hollow process, the naso-turbinal. In front of the nasals are the premaxillæ 
(p. max)-large bones which form the anterior part of the snout, bear the upper incisor teeth, and give off three processes - a nasal, a palatine (pal. p. max), and a maxillary. The maxilloe (max.), which form the greater part of the upper jaw, and bear the premolar and molar teeth, are large, irregularly-shaped bones, the outer surfaces of which are spongy. They give off internally horizontal processes - the palatine processes (pal. max)-which unite to form the anterior part of the bony palate. Between the premaxillæ and maxillæ and the palatines on the lower surface of the skull is a large triangular opening divided into two-the anterior palatine foramina-by the palatine processes of the premaxillæ. On the outer surface of each maxilla, above the first premolar tooth, is a foramen-the infra-orbital (i.o.f.)-through which the second division of the fifth nerve passes. A strong process which is given off from the outer face of each maxilla, and turns outwards and then backwards to unite with the zygomatic process of the squamosal and thus complete the zygomatic arch, is a separate bone in the young, the malar or jugal ( $j u$.$) .$

The maxillæ help to bound the nasal cavities externally, and with each is connected on its inner aspect a pair of thin scroll-like bones-the maxillo-turbinals (Fig. 1094, $m x . t b$.). The rest of the narrow bony palate, forming the roof of the mouth and the floor of the nasal cavities, is formed by the palatine plates of the palatine bones (pal.). The so-called pterygoids (pt.) are small irregular bones, each of which articulates with the palatine in front and with the pterygoid process of the alisphenoid behind: these are probably not the equivalents of the pterygoids of other Vertebrates, but of part of the parasphenoid. The lacrymals (lcr.) are small bones, one situated in the anterior wall of each orbit, perforated by a small aperture-the lacrymal foramen.

In the interior of the skull (Fig. 10今4) are three cavities, the two olfactory or nasal cavities, right and left, in front, and the cranial cavity behind. The former are separated from one another by a median partition or septum, partly cartilaginous, partly bony, formed, as above described, by the mesethmoid. Each contains the turbinals or turbinated bones of its side; it opens on the exterior by the large external nasal aperture, and behind it communicates with the cavity of the mouth by the posterior nasal aperture.

The cranial cavity has its walls moulded to a considerable extent on the surface of the contained brain, and, in consequence, there are to be recognised concavities in the former corresponding with the prominent portions of the latter. These concavities are termed the fossa, and they consist of the cerebellar fossa behind and the cerebral fossa in front, with the inconspicuous olfactory fossa in the frontal region.

The mandible, or lower jaw, consists of two lateral halves or rami, which are connected with one another in front by a rough articular 
surface or symphysis, while behind they diverge like the limbs of a letter V. In cach ramus is a horizontal portion (anterior), which bears the teeth, and a vertical or ascending portion, on which is the articular surface or condyle (cond.) for articulation with the glenoid cavity of the squamosal; in front of the condyle is the compressed coronoid process. The angle where the horizontal and ascending processes meet gives off an inward projection or angular process (ang. pro.).

The hyoid consists, in addition to the separate vestigial stylohyals already mentioned (p. 438), of a stout thick body or basi-hyal, a pair of small anterior cornua or cerato-hyals, and a pair of long backwardly directed cornua or thyro-hyals.

The auditory ossicles, contained in the cavity of the middlc ear,

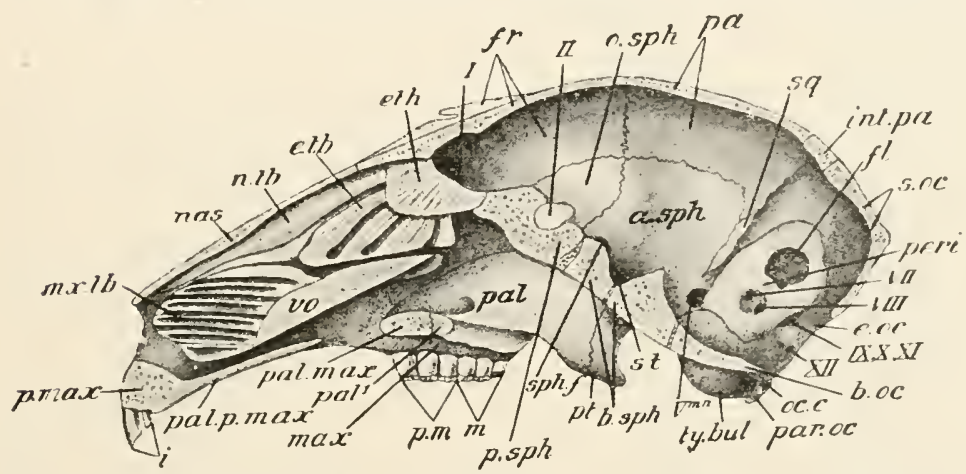

FIG. 1094.-Iepus cuniculus. Skull in longitudinal vertical section. The cartilaginous nasal septum is removed. a. $s p h$. alisphenoid; $e$. oc. exoccipital; $e$. tb. ethmo-turbinal; $e t h$. ethmoid ; $f$. fossa for flocculus of brain ; $i$. incisors ; $m x$. $t b$. maxillary turbinal ; $n$. $t b$. naso-turbinal ; plt' $^{\prime}$. palatine portion of the bony palate ; peri. periotic (petrous portion): p. $s p h$. pre-sphenoid ; sph.f. sphenoidal fissure ; s.t. sella turcica, or depression in which the pituitary body lies; $I$. point at which the olfactory nerves leave the skull; $I I$. optic foramen; $V . m n$. foramen for mandibular division of trigeminal; $V I I$. for facial nerve;

$V I I I$. for auditory nerve ; $I X, X, X I$, for glossopharyngeal, vagus, and spinal accessory;

$X I I$. for hypoglossal. Other letters as in Fig. 1093. (From Parker's Practical Zoology.)

and cut off from the exterior, in the unmacerated skull, by the tympanic membrane, are extremely small bones, which form a chain extending, like the columella auris of the Pigeon, from the tympanic membrane externally to the fenestra ovalis internally. There are three of these auditory ossicles-the stapes, which corresponds to the columella of the Pigeon; the incus, and the malleus, the latter with a slender process (processus gracilis): these are said to be derived respectively from the quadrate and articular elements (q.v.) of lower vertebrates. In addition there is a small disc-like bone, the orbicular, which is attached to the incus.

The elements of the pectoral arch are fewer than in the Lizard. There is a broad, thin, triangular scapula, the base or vertebral cdge of which has a thin strip of cartilage (the supra-scapular cartilage) continuous with it. Along the outer surface runs a ridge-the spinc; the spinc ends below in a long process-the acromion process 
-from which a branch process or metacromion is given off behind. The part of the outer surface of the scapula in front of the spine is the pre-spinous or pre-scapular fossa, the part behind is the post-spinous or post-scapular fossa. At the narrow lower end of the scapula is a concave surface-the glenoid cavity-into which the head of the humerus fits, and immediately in front of this is a small inwardly curved process-the coracoid process-which is represented by two separate ossifications in the young Rabbit. A slender rod-the clavicle-lies obliquely in the region between the pre-sternum and the scapula, but only extends a part of the distance between the two bones, and in the adult is only connected with them through the intermediation of fibrous tissue.

The skeleton of the fore-limb is more readily comparable with that of the Lizard than with that of the Bird; but there is a difference in the position of the parts owing to the rotation baekwards of the distal end of the humerus, all the segments being thus brought into a plane nearly parallel with the median vertical plane of the body, with the pre-axial border directed outwards, and the original dorsal surface backwards. The radius and ulna are fixed in the position of pronation, i.e., the distal end of the radius is rotated inwards, so that, while the proximal end is external to the ulna, the distal end becomes internal, and the digits of the manus are directed forwards.

At the proximal end of the humerus are to be recognised: (1) a rounded head for articulation with the glenoid cavity of the scapula; (2) externally a greater and (3) internally a lesser tuberosity for the insertion of muscles; (4) a groove, the bicipital groove, between the two tuberosities. On the anterior surface of the proximal portion of the shaft is a slight ridge, the deltoid ridge. At the distal end are two articular surfaces, one large and pulley-like-trochlea-for the ulna; the other smaller-capitellum -for the radius: laterally are two prominences or condyles, an internal and an external.

The radius and ulna are firmly fixed together so as to be incapable of movement, but are not actually ankylosed. The radius articulates proximally with the humerus, distally with the scaphoid and lunar bones of the carpus. The ulna presents on the anterior aspect of its proximal end a deep fossa, the greater sigmoid cavity, for the trochlea of the humerus; the prominent process on the proximal side of this is the olecranon process. Distally it articulates with the cuneiform.

The carpal bones (Fig. 1095), nine in number, are all small bones of irregular shape. Eight of these are arranged in two rows-a proximal and a distal; the ninth, centrale (cent.), lies between the two rows. The bones of the proximal row aretaken in order from the inner to the outer side-scaphoid (sc.), lunar (or semi-lunar) (lun.), cuneiform (cun.), and pisiform. Those 
of the distal row are, reckoned in the same order, trupezium (trpm.), trapezoid (trpz.), magnum (mag.), and unciform (unc.). ${ }^{1}$

The five metacarpals are all small but relatively narrow and elongated bones, the first being smaller than the rest. Each of the five digits has three phalanges, except the first, which has only two. The distal (ungual) phalanges are grooved dorsally for the attachment of the horny claw.

The pelvic arch (Fig. 1096) contains the same elements as in the Pigeon, but the union of the ilium with the sacrum is less intimate, the acetabulum is not perforated, and the pubes and ischia of opposite sides unite ventrally in a symphysis (sy.). The three bones of the pelvis-ilium, pubis, and ischium-are separate ossifications

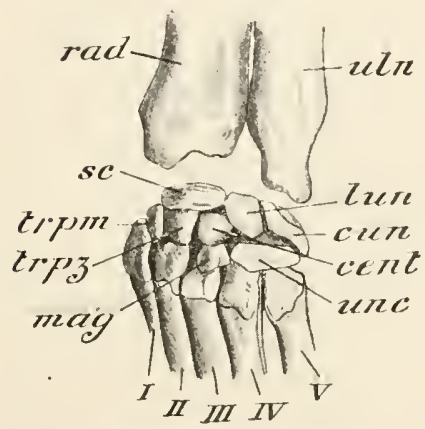

F1G. 1095.-Lepus cuniculus. Jistal end of fore-arm and carpus, dorsal view, the bones bent towards the dorsal side so as to be partly separated. cent. centrale; cun. cuneiform ; lun. lunar; mag. magnum; rad. radius; $s c$. scaphoid; trpz. trapezoid; trpm. trapezium; uln. ulna; unc. unciform ; $I-V$, bases of metacarpals. (After Krause.)

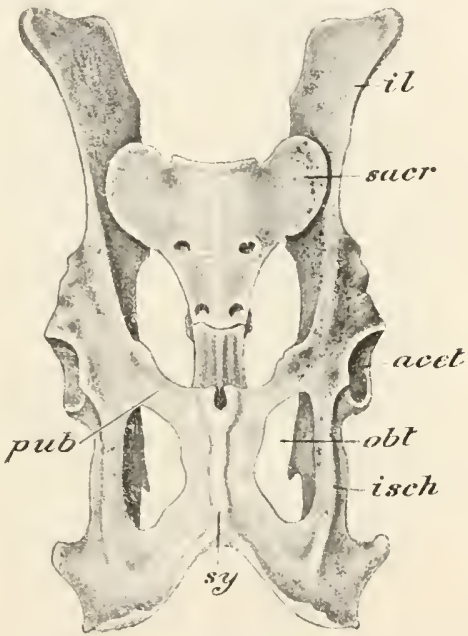

FIG. 1096.-Lepus cuniculus. Innominate bones and sacrum, ventral aspect. acet a cetabulum; il. ilium ; isch. ischium; obt. obturator foramen; pub. pubis; sacr, sacrum; sy. symphysis.

in the young Rabbit; but in an adult animal complete fusion takes place between the bones. The ilium and ischium meet in the acetabulum or articular cavity, which they contribute to form, for the head of the femur; but the remainder of the cavity is bounded, not by the pubis, but by a small intercalated ossification - the cotyloid bone. The ilium (il.) has a rough surface for articulation with the sacrum. Between the pubis $(p u b$.$) in front and$ the ischium (isch.) behind is a large aperture - the obturator foramen (obt.). The femur is rotated forwards when compared with that

1 The homologies of these bones are not quite certain, but are very probably as follows :- scaphoid $=$ radiale, lunar $=1$ st centrale, cuneiform $=$ intermedium, pisiform $=$ ulnare, centrale $=2$ nd centrale, trapezium $=1$ st distale, trapezoid $=2$ nd distalo, magnum $=3 \mathrm{rd}$ distale, unciform $=4$ th and 5 th distalia. 
of the Lizard, so that the limb is nearly in the same plane as the fore-limb, and the pre-axial border is internal and the originally dorsal surface anterior. The femur has at its proximal end a prominent head for articulation with the acetabulum, external to this a prominent process-the great trochanter, and internally a much smaller-the lesser trochanter, while a small process or third trochanter is situated on the outer border a little below the great trochanter. At its distal end are two prominences or condyles, with a depression between them. Two small sesamoids or fabellae are situated opposite the distal end on its posterior aspect; and

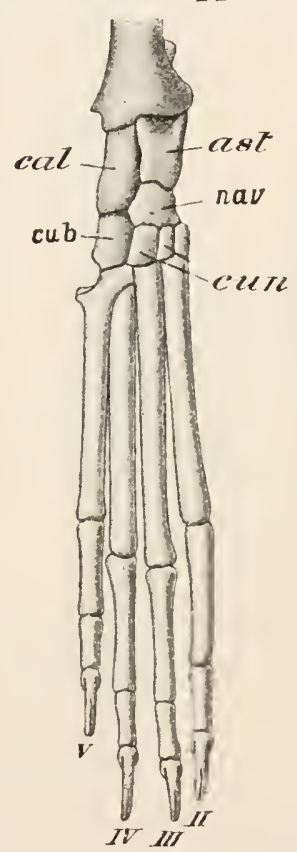

Fig. 1097.-Lepus cuniculus.

Skeleton of pes. ast. astragalus : cal. calcaneum ; $c u b$. galus a cal. calcaneum, cub: nav. navicular. opposite the knee-joint, or articulation between the femur and the tibia, is a larger bone of similar character-the knee-cap or patella. The tibia has at its proximal end two articular surfaces for the condyles of the femur; distally it has also two articular surfaces, one, internal, for the astragalus, the other for the calcaneum. The fibula is a slender bone which becomes completely fused distally with the tibia.

The tarsus (Fig. 1097) consists of six bones of irregular shape, arranged in two rows, one of the bones-the navicular (nav.)-being intercalated between the two rows. In the proximal row are two bones-the astragalus (ast.) and the calcaneum (cal.)-both articulating with the tibia; the calcaneum presents behind a long calcaneal process. The distal row contains three bones, the meso-cuneiform, ecto-cuneiform, and cuboid (cub.) ; the entocuneiform, which commonly forms the most internal member of this row in other Mammals, is not present as a separate bone. ${ }^{1}$

There are four metatarsals, the hallux or first digit being vestigial and fused with the second metatarsal in the adult. The proximal end of the second is produced into a process which articulates with the navicular. Each of the digits has three phalanges, which are similar in character to those of the manus.

The cœlome of the Rabbit differs from that of the Pigeon and

1 In all probability the homologies of these bones are as follows :-astragalus $=$ tibiale + intermedium, calcaneum $=$ fibulare, navicular $=$ centrale, ento-cuneiform $=1$ st distale, meso-cuneiform $=$-nd distale, ecto-cuneiform $=$ 3 rd distale, cuboid $=4$ th and 5 th distalia. 
Lizard in being divided into two parts by a transverse muscular partition, the diaphragm. The anterior part, or thorax, contains the heart and the roots of the great vessels, the lungs and bronchi, and the posterior part of the cesophagus. The posterior part, or abdomen, contains the stomach and intestine, the liver and pancreas, the spleen, the kidneys, ureters and urinary bladder, and the organs of reproduction.

Digestive Organs. - The teeth (Fig. 1093) are lodged in sockets or alveoli in the pre-maxillæ, the maxillæ, and the mandible. In the pre-maxillæ are situated four teeth-the four upper incisors. Of these the two anterior are very long, curved, chisel-shaped teeth, which are devoid of roots, growing throughout life from persistent pulps. Enamel is present, and forms a thick layer on the anterior convex surface, which accounts for the bevelled-off character of the distal end-the layer of enamel being much harder than the rest, which therefore wears more quickly away at the cutting extremity of the tooth. Along the anterior surface is a longitudinal g r oo ve. The second pair of incisors of the upper jaw are small teeth which are lodged just behind the larger pair. In the

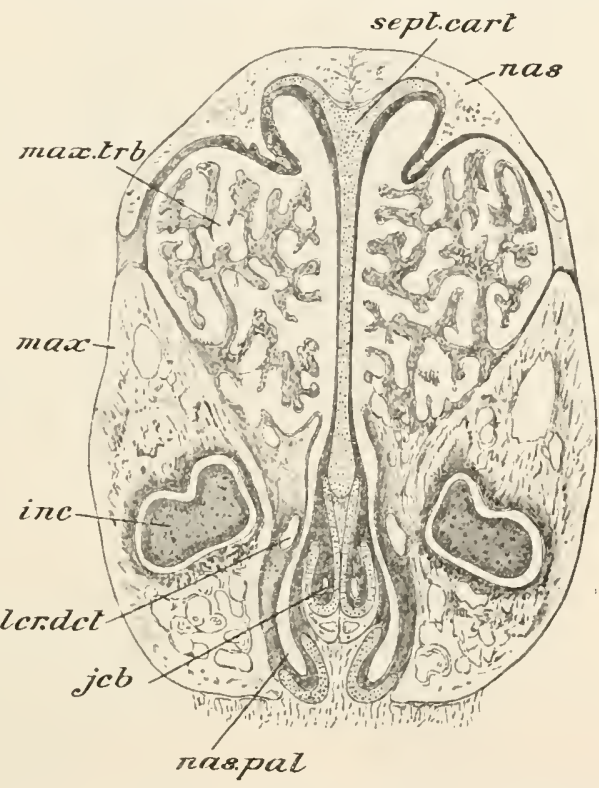

FIG. 1098.-Lepus cuniculus. Vertical section through the anterior part of the nasal region of the head. inc. section of larger incisor tooth: $j c b$. lumen of Jacobson's organ, surrounded by cartilage ; ler. det. lacrymal duct ; max. maxilla; max. trb. maxillary turbinals; nas, nasal bone; nas. pal. naso-palatine canal; scpt. cart. cartilaginous nasal septum. (After Krause.)

lower jaw are two incisors, which correspond in shape with the anterior pair of the upper jaw, the main.difference consisting in the absence of the longitudinal groove. The remaining teeth of the upper jaw are lodged in the maxillæ. Canines, present in most Mammals as a single tooth on each side, above and below, are here entirely absent, and there is a considerable space, or diastema, as it is termed, between the incisors and the teeth next in order-the pre-molars. Of these there are three in the upper jaw and two in the lower. They are long, curved teeth with persistent pulps like the incisors. The first of the upper jaw is smaller than the others 
and of simple shape, the rest have each a longitudinal groove on the outer side and a transverse ridge on the crown. The first pre-molar of the lower jaw has two grooves; the second is similar to those of the upper jaw. Behind the pre-molars are the molars, three on each side both in the upper and lower jaws.' 'These are similar to the upper pre-molars, except the last, which is small and of simple shape.

Opening into the cavity of the mouth, or buccal cavity, are the ducts of four pairs of salivary glands-the parotid, the infraorbital, the sub-maxillary (Fig. 1099, s. mx. gl.), and the sub-lingual (s.gl.). On the floor of the mouth is the muscular tongue, covered with a mucous membrane which is beset with many papillæ, on certain of which the taste-buds (p. 105) are situated. The roof of the mouth is formed by the hard palate, which is crossed by a series of tranverse ridges of its mucous membrane. Posteriorly the hard palate passes into the soft palate, which ends behind in a free pendulous flap in front of the opening of the posterior nares. At the anterior end of the palate is a pair of openings-the naso-palatine or anterior palatine canals, leading into the nasal chambers, and into them open a pair of tubular structures-the organs of Jacobson (Fig. $1098, j c b$.$) - enclosed in cartilage and situated on the floor of the$ nasal cavities. Behind the mouth or buccal cavity proper is the

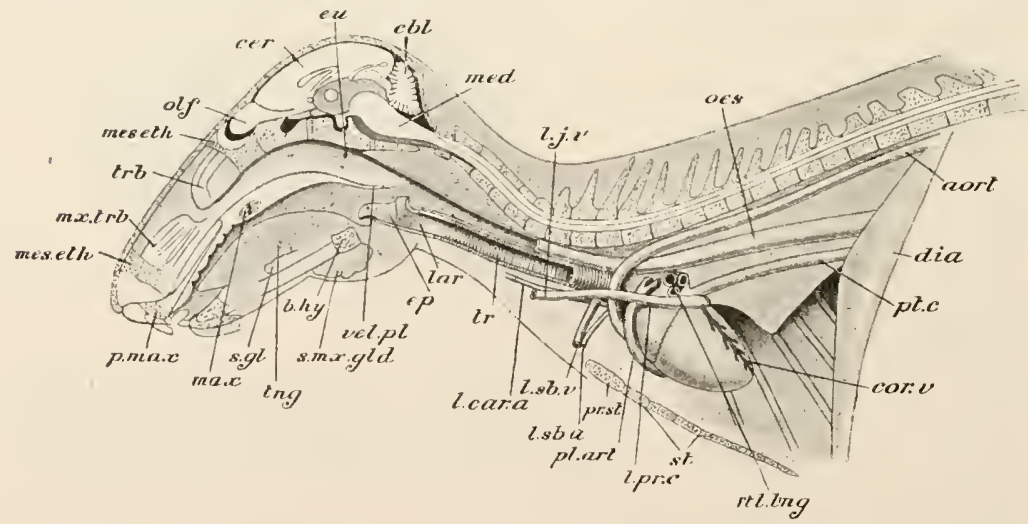

FIG. 1099.-Lepus cuniculus. Lateral dissection of the head, neck, and thorax. The heal and spinal column are represented in mesial vertical section; the left lung is removed ; the greater part of the nasal septum is removed so as to show the right nasal cavity with jts'; turbinals, aort, dorsal aorta ; b. hy. basi-hyal ; cbl. cerebellum; cer. cerebral hemispheres ; cor. $v$, coronary vein ; dia. diaphragin; ep. epiglottis; $e u$. opening of Eustachian tube into pharynx; lar. larynx; l.j.v. left jugular vein; l.sb.a. left subclavian artery; $l . s b . v$. left subclavian veil ; max. maxilla; med. medulla oblongata; mes.eth. mesethmoid; mx.trb. maxillo-turbinal; ces. cesophagus; olf. olfactory bulb; $p l$. art. pulmonary artery ; $p$. max. pre-maxilla; pr.st. presternum; pt.c. post-caval vein; rt.l.lng. root of left lung witl bronchus and pulmonary veins and artery cut across ; s.ol. sub-lingual salivary glands; s.mx.gld. sub-maxillary salivary gland; st. sternebre; $t n g$. tongue; tr. trachea; trb. ethmoturbinals; vel. $p l$. soft palate.

pharynx, which in the Rabbit is not sharply marked off from the buccal cavity, but begins where the hard palate ends. 'The pharynx 


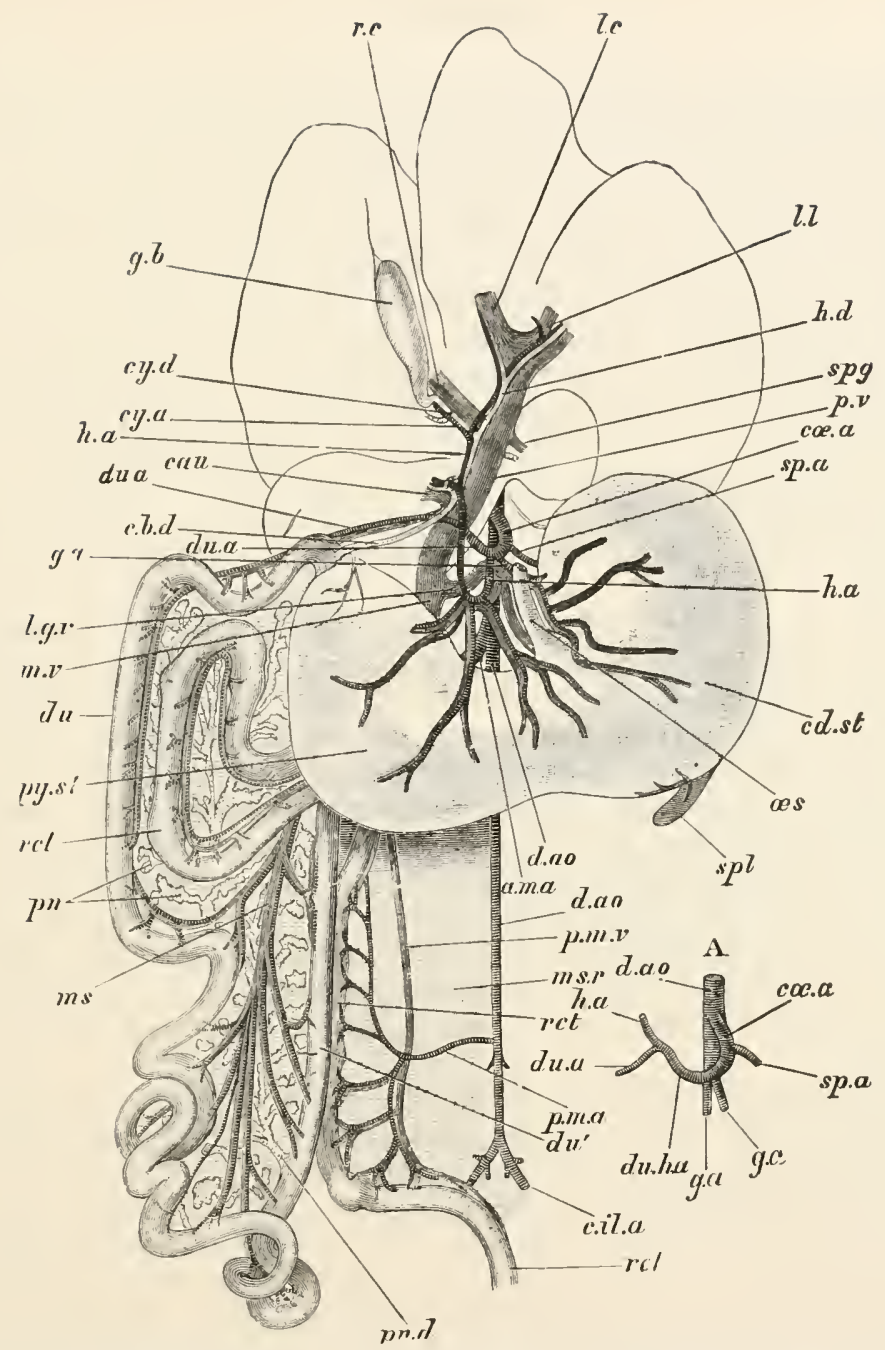

FIG. 1100,-Lepus cuniculus. The stomach, duodenum, posterior portion of rectum and liver (in outline) with their arteries, veins and ducts, $A$, the coliac artery of another specimen (both $\times{ }_{3}^{2}$ ). The gullet is cut through and the stomach somewhat displaced backwards to show the ramifications of the cœliac artery (ca.a.); the duodenum is spreal out to the right of the subject to show the pancreas $(p n$.$) ; the branches of the bile-duct$ $(c . b . d$.$) , portal vein (p, v)$, and hepatic artery $(h, a$.$) are supposed to be traced some dis-$ tance into the various lobes of the liver, $a . m$. $a$. anterior mesenteric artery; cau. caudate lobe of liver with its artery, vein and bile-duct; $c . b . d$. common bile-duct; $c d$. st. cardiac portion of stomach; $c$. il .a. common iliac artery ; cœ.a. cœliac artery ; $c y . a$. cystic artery $c y . d$. cystic duct; $d$. ao. dorsal aorta ; $d u$. proximal, and $d u^{\prime}$. distal limbs of duodenum ; du. a. duodenal artery; du.h.a. (in A), duodeno-hepatic artery; $q$. $a$. gastric artery and vein; g.b. gall-bladder; $h$. $a$. hepatic artery ; $h$.d. left hepatic duct ; $l$. $c$. left central lobe of liver, with its artery, vein and bile-duct; $l . g . v$. lieno-gastric vein; $l$. $l$. lateral lobe of liver with its artery, vein and bile-duct; $m s$. branch of mesenteric artery and vein to duodenum ; $m s . r$. mesorectum ; m.v. chief mesenteric vein ; os. asophagus ; p.m.a. posterior mesenteric artery ; p.m.v. posterior mesenteric vein ; pn. pancreas; pn.d. pancreatic duct ; p.v. portal vein ; py.st. pyloric portion of stomach ; rct. rectum; r.c. right central lobe of liver, with artery, vein and bile-duct; spg. Spigelian lobe of liver with its artery, vein and bile-duct ; $s p l$. spleen; $s p$. a. splenic artery. (From Parker's Zootomy.) 
is divided into two parts, an upper or nasal division and a lower or buccal division, by the soft palate. The passage of the posterior nares is continuous with the nasal division, at the sides of which are the openings of the Eustachian tubes. The nasal division is continuous with the buccal division round the posterior free edge of the soft palate. From the buccal division leads ventrally the slit-like opening of the glottis ${ }^{1}$ into the larynx and trachea; overhanging the glottis is a leaf-like movable flap (Fig. 1099, ep). formed of a plate of yellow elastic cartilage covered with mucous membrane; this is the epiglottis. Behind, the pharynx becomes continuous with the cesophagus or gullet (oss.). The latter is a narrow but dilatable muscular tube which runs backwards from the pharynx through the neck and thorax to enter the cavity of the abdomen through an aperture in the diaphragm, and opens into the stomach.

The stomach (Fig. 1100) is a wide sac, much wider at the cardiac end, at which the œsophagus enters, than at the opposite or pyloric end, where it passes into the small intestine. The small intestine is an elongated, narrow, greatly coiled tube, the first part of which, or duodenum $\left(d u\right.$ and $\left.d u^{\prime}\right)$, forms a U-shaped loop. The large intestine is a wide tube, the first and greater part of which, termed the colon, has its walls sacculated, and is continued into a narrow, smooth-walled posterior part or rectum (rct.). At the junction of the small with the large intestine is a very wide blind tube, the cocum, which is of considerable length and is marked by a spiral constriction, indicating the presence in its interior of a narrow spiral valve. At its extremity is a small, fleshy, finger-like vermiform appendix.

The intestine, like that of the Pigeon, is attached throughout its length to the dorsal wall of the abdominal cavity by a mesentery, or fold of the lining membrane or peritoneum.

The liver is attached to the diaphragm by a fold of the peritoneum. Its substance is partly divided by a series of fissures into five lobes. A thin-walled gall-bladder lies in a depression on its posterior surface. The common bile-duct (c.b.d.), formed by the union of the cystic duct from the gall-bladder and hepatic ducts from the various parts of the liver, runs to open into the duodenum near the pylorus.

The pancreas $(p n$.$) is a diffused gland in the fold of mesentery$ passing across the loop of the duodenum. Its single duct, the pancreatic duct $(p n . d$.), opens into the distal limb of the loop.

Circulatory Organs.-The heart (Fig. 1101) is situated in the cavity of the thorax, a little to the left of the middle line, and lies between the two pleural sacs enclosing the lungs. Between the

1 The term glottis is more strictly applied not to this slit, but to the slitlike aperture between two folds of the mucous membrane within the larynxthe vocal cords - which constitute the chief parts of the vocal apparatus, 
pleural sacs is a space, the mediastinum (Fig. 1104). This is divisible into four parts, the anterior, the dorsal, the middle, and the ventral. In the anterior part lie the posterior part of the trachea, the neighbouring parts of the cesophagus and of the thoracic duct of the lymphatic system, the roots of the great arteries and the veins of the precaval system, and the phrenic, pneumogastric, and other nerves. In the dorsal part are sitnated the posterior part of the csophagus, the thoracic part of the dorsal aorta, the pneumogastric nerve, the azygos vein, and the thoracic duct. The middle part is the widest, and lodges the heart and roots of the aorta and pulmonary artery enclosed in the pericardium, the posterior portion of the precaval veins, the phrenic nerves, the terminal part of the azygos vein, and the roots of the lungs. The ventral part contains only areolar tissue with the thymus gland. The pericardial membrane enclosing the heart consists of two layers, a parietal, forming the wall of the pericardial cavity, and a visceral, immediately investing the heart. Between the two is a narrow cavity containing a little fluid-the pericardial fluid. In general shape the heart resembles the heart of the Pigeon, with the apex directed backwards and slightly to the left, and the base forwards. Like that of the Pigeon, it contains right and left auricles and right and left ventricles, the

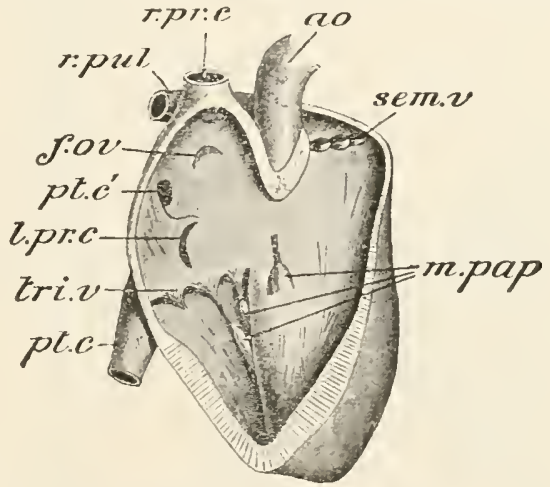

FIG. 1101.- Iepus cunieuius. Heart, seen from the right side, the walls of the riglit auricle and right ventricle partly removed so as to expose the cavities. ao. aorta ; $f$. ov. fossa ovalis ; l.pr.c. opening of left pre-caval; m.pap. musculi papillares; $p t$. c. post-caval; $p t$. $e^{\prime}$. opening of post-caval, with Eustachian valve below; r.pr.c. right precaval; r.pul. right pulmonary artery; sem. v. semilunar valves; tri. $v$. tricuspid valve.

right and left sides of the heart having their cavities completely separated off from one another by inter-auricular and interventricular partitions.

Into the right auricle open three large veins--the right and left precaval veins and the single postcaval - the first into the anterior part, the second into the left-hand side of the posterior portion, and the third into the dorsal surface (Fig. 1101). Projecting forwards from it is an ear-like auricular appendix, the inner surface of which is raised up into numerous cords of muscular fibres, the musculi pectinati. A membranous fold, the remnant of the fœtal Eustachian valve, extends from the opening of the postcaval forwards towards the auricular septum. The opening of the left precaval is bounded behind by a crescentic fold, the valve of Thebesius. On the septum is an oval area where the partition is thinner than elsewhere; this 
is the fossa ovalis ( $f . o v$. ) : it marks the position of an aperture, the foramen ovale, in the foetus. The crescentic anterior rim of the aperture is known as the annulus ovalis. The cavity of the right auricle communicates with that of the right ventricle by the wide right auriculo-ventricular opening. This is guarded by a valve, the tricuspid (tri. v.), composed of three membranous lobes or cusps, so arranged and attached that while they flap back against the walls of the ventricle to allow the passage of blood from the auricle to the ventricle, they meet together across the aperture so as to close the passage when the ventricle contracts. The lobes of the valve are attached to muscular processes of the wall of the ventricle, the musculi papillares ( $m$. pap.), by means of tendinous threads called the chordce tendinece. The right ventricle, much thicker than the auricle, forms the right side of the conical apical portion, but does not extend quite to the apex. Its walls are raised up into muscular ridges called columnce carnece. It gives off in front, at its left anterior angle, the pulmonary artery, the entrance to which is guarded by three pouch-like semilunar valves (sem.v.).

The left auricle, like the right, is provided with an auricular appendix. Into its cavity on its dorsal aspect open together the right and left pulmonary veins. A large left auriculo-ventricular opening leads from the cavity of the left auricle into that of the left ventricle : this is guarded by a valve, the mitral, consisting of two membranous lobes or cusps with chordæ tendineæ and musculi papillares. In the walls of the ventricle are columnæ carnæe, rather more strongly developed than in the right. At the basal (anterior) end of the left ventricle is the opening of the aorta, guarded by three semilunar valves similar to those at the entrance of the pulmonary artery. The coronary arteries, which supply the muscular substance of the heart, are given off from the aorta just beyond the semilunar valves. The corresponding vein opens into the terminal part of the left precaval. The pulmonary artery divides into two, a right and a left, each going to the corresponding lung.

The aorta gives origin to a system of arterial trunks by which the arterial blood is conveyed throughout the body. It first runs forwards from the base of the left ventricle, then bends round the left bronchus, forming the arch of the aorta (Fig. 1102), to run backwards through the thorax and abdomen, in close contact with the spinal column, as the dorsal aorta $(d . a o$.). From the arch of the aorta are given off two large arteries, the innominate (in.) and the left subclavian. The innominate divides to form the right subclavian (s. cl. a.) and the right (r. c. c.) and left (l. c. c.) common carotid arteries. The right subclavian passes to the fore-limb as the brachial artery, giving origin first to the vertebral artery, which, after passing up through the vertebrarterial canal, enters the cranial cavity, having first supplied branches to the spinal cord; and then to the anterior epigastric or internal mammary, which supplies the 

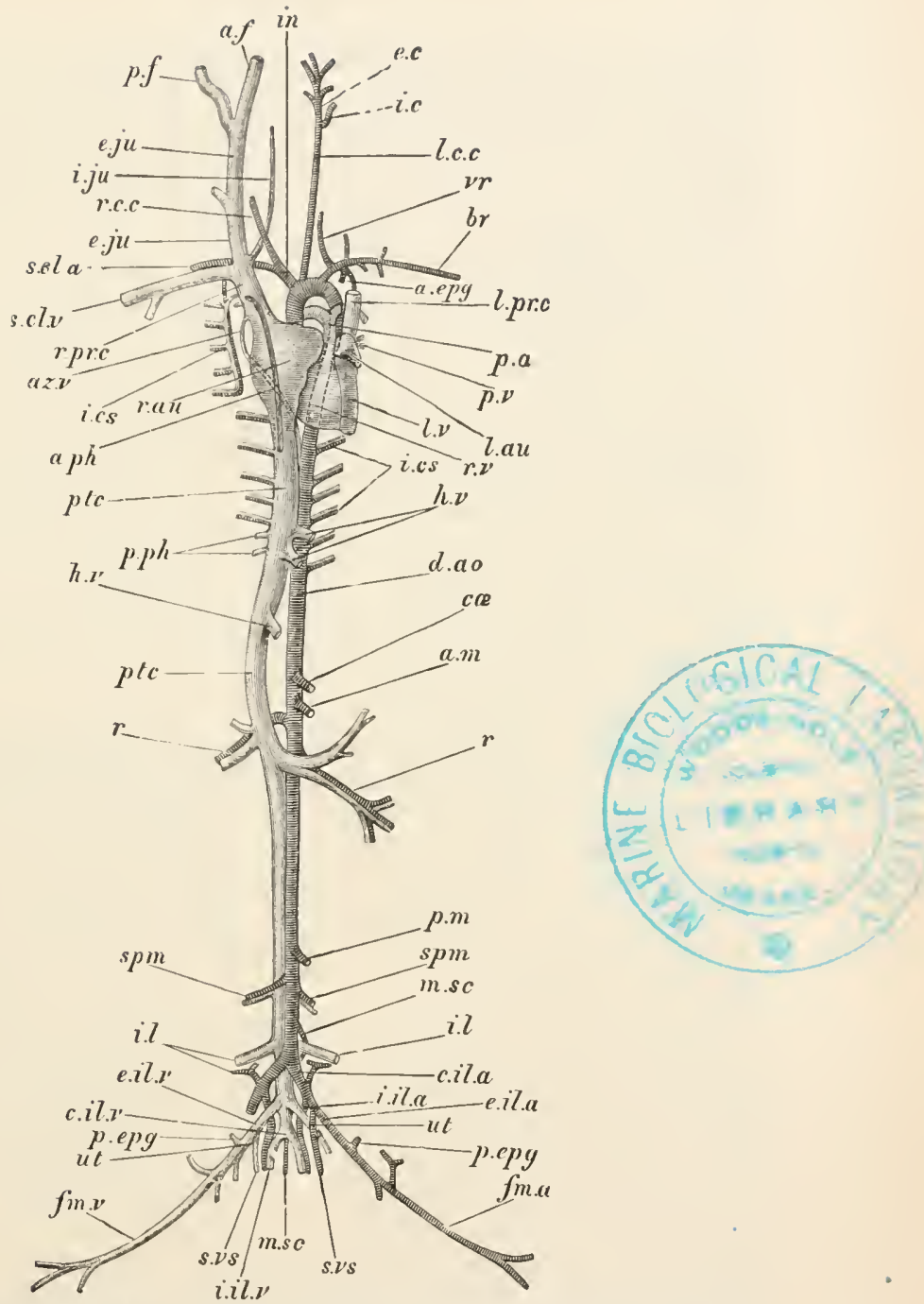

FIG. 1102,-Iepus cuniculus. The vascular system. The heart is somewhat displaced towards the left of the subject; the arteries of the right and the veins of the left side are in great measure removed. a.epg. internal mammary or anterior epigastric artery ; a.f. anterior facial vein; a.m. anterior mesenteric artery; $a . p h$. anterior phrenic vein; $a z . v$. azygos veln; br. brachial artery; c.il.a. common iliac artery; c.il.v. hinder end of postcaval; ca. coliac artery ; d.ao. dorsal aorta ; e.c. external carotid artery ; e.il.a. external iliac artery $e . i l . v$. external iliac vein ; e.ju, external jugular vein ; $f m . a$. femoral artery ; $f m . v$. femoral vein $; h . v$. hepatic veins; $i$. $c$. internal carotid artery $; i$. $c s$. intercostal vessels $; i$. $j u$. internal jugular vein ; $i$. l. ilio-lumbar artery and vein ; $i n$. innominate artery ; $l$. au. left auricle ; l.c.c. left common carotid artery; l.prc. left precaval vein; $l$. $v$. left ventricle; m.sc. inedian sacral artery; $p . a$. pulmonary artery ; p.epg. posterior epigastric artery and vein; $p . f$. posterior facial vein; $p . m$. posterior mesenteric artery ; $p . p h$. posterlor phrenic veins; pte. postcaval vein; $p . v$. pulmonary vein; r. renal artery and vein; $r$. au. rlght auricle; r.c.c. right common carotid artery; r.prc. right precaval vein; $r$. $v$. right ventricle; $s c l$. $\boldsymbol{a}$. right subclavian artery; $s c l . v$. subclavian vein; $s p m$. spermatic artery and vein; $s . v s$. superior vesical artery and vein ; $u t$. uterine artery and vein ; vr. vertebral artery. (From Parker's Zootomy.) 
side of the chest behind the root of the fore-limb. The right carotid divides opposite the angle of the jaw into internal and external carotids. The left carotid and left subclavian correspond in their distribution and branching to the right carotid and right subclavian respectively. The aorta, in passing through the thorax, gives off a series of small paired intercostal arteries $(i$. cs.). In the abdomen its first large branch is the coeliac artery (cœ.), which supplies the liver, stomach, and spleen. Behind this it gives origin to the anterior mesenteric $(a . m$.), which supplies the intestine and the pancreas. Opposite the kidneys it gives off the two renal arteries $(r$.) for the supply of these organs, and a good deal further back the spermatic (spm.) or ovarian arteries for the testes or ovaries as the case may be. Just in front of the origin of the spermatic arteries is given off a posterior mesenteric (p.m.), which supplies the hinder part of the rectum. A series of small lumbar arteries supply the side-walls of the abdominal cavity. Posteriorly the dorsal aorta divides to form the two common iliac arteries (c. il. a.) which supply the pelvic cavity and hind-limbs, a small median sacral (caudal) artery (ms. c.) passing backwards in the middle line to supply the caudal region.

The system of caval veins which open into the right auricle consists of the right and left precavals and of the single postcaval. The right precaval is formed by the union of the right jugular $(e . j u$.$) vein and$ right subclavian (scl. v.). The azygos vein (az. v.), the right anterior intercostal (i.cs.), and the right anterior epigastric or internal mammary also open into it. The left precaval receives a series of veins similar to those forming the right, except that there is no azygos on the left side (cf. p. 298).

The postcaval vein ( $p t$. c.) is formed in the hinder part of the abdominal cavity by the union of the internal iliacs $(i . i l . v$.) bringing the blood from the back of the thighs. Shortly after its origin it receives the two external itiacs (e. il. v.) coming from the hindlimb. In front of this a pair of ilio-lumbar (i.l.) veins join it; a little farther forward a pair of spermatic $(\mathrm{spm}$.) or ovarian veins ; and opposite the kidneys a pair of renal veins $(r$.$) . From$ the liver the blood is carried to the postcaval by the hepatic veins. A pair of small posterior phrenic veins ( $p . p h$.) bring the blood from the diaphragm and open into the postcaval as it passes through the substance of the latter.

The hepatic portal system consists, as in other Vertebrates, of a system of veins conveying blood from the various parts of the alimentary canal to the liver, the trunks of the system uniting to form the single large portal vein (Fig. $1100, p . v$.). The principal veins of the portal system are the lieno-gastric, duodenal, anterior mesenteric, and posterior mesenteric. There is no trace of a renal portal system. The red blood corpuscles are circular, bi-concave, non-nucleated dises. 
Respiratory Organs.-The larynx (Fig. 1103) is a chamber with walls supported by eartilage, lying below and somewhat behind the pharynx, with which it eommunicates through a slitlike aperture. The cartilages of the larynx are, in addition to the epiglottis, which has been already referred to (p. 448), the large thyroid (th.), which forms the ventral and lateral walls, the ring-like cricoid (cr.), the two small arytenoids (ary.), and a pair of small nodules, the cartilages of Santorini (sant.), situated at the apices of the

A

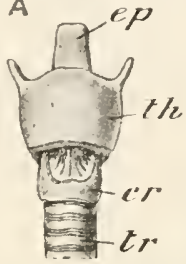

HIG. 1103.- Lepus cuniculus. Laryux. A. ventral view $; B$, dorsal view. ary. arytenoid: cr. cricoid; ep. epiglottis ; sant. cartilage of Santorini; th. thyroid; $t r$ traehea. (From Krause, after Schneider.)

arytenoids. The vocal cords extend across the eavity from the thyroid below to the arytenoids above. Leading baekwards from the larynx is the trachea or wind-pipe (Fig. 1099, tr.), a long tube

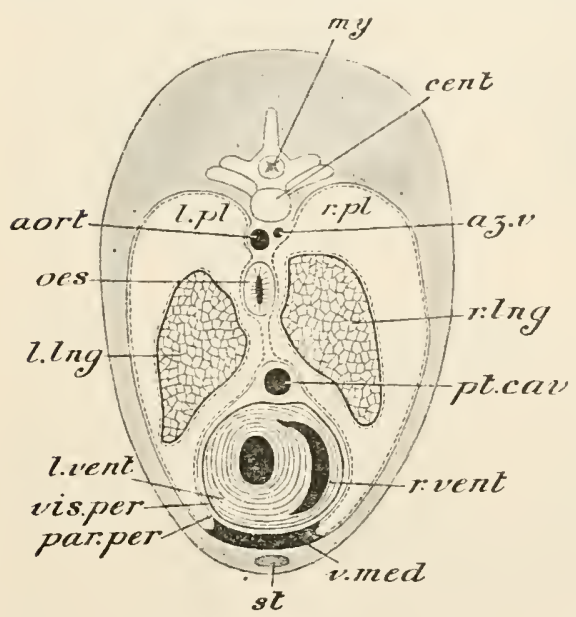

FIG. 1104-Lepus cuniculus. Diagram of a transverse seetion of the thorax in the region of the ventrieles to show the relations of the pleura, mediastinum, ete. The lungs are contraeted. aort, dorsal aorta; az.e. azygos vein ; cent. eentrum of thoraeic vertebra; i.lng. left lung; l.pl. left pleural sae; l.vent. left ventriele; my. spinal eord; ces. cesophagus; par. per. parietal layer of perieardium; $p t$. cav. post-eaval, elose to its entrance into right auricle ; r.lng. right lung; r.pl. right pleural eavity; r.vent. right ventricle; st. sternum; v.med. ventral mediastinum. the wall of which is supported by cartilaginous rings which are incomplete dorsally. The traehea enters the eavity of the thorax, and there divides into the two bronchi, one passing to the root of each lung.

The lungs (Fig. 1104) are enclosed in the lateral parts of the cavity of the thorax. Each lung lies in a eavity, the pleural sac, lined by a pleural membrane. The right and left pleural sacs are separated by a considerable interval owing to the development in the partition between them of a space, the mediastinum, in which, as already explained, lie the heart and other organs. The lung is attached only at its root, where the pleural membrane is reflected over it. In this respect it differs widely from the lung of the bird. It differs also in its minute structure. The bronchus, entering at the root, divides and subdivides to form a ramifying system of tubes, each of the 
ultimate branches of which, or terminal bronchiolcs, opens into a minute chamber or infundibulum, consisting of a central passage and a number of thin-walled air-vesicles or alveoli given off from it. A group of these infundibula, supplied by a single bronchiole, which divides within it to form the terminal bronchioles, is termed a lobule of the lung.

In shape the lung may be roughly described as conical, with the apex directed forwards. The base, which is concave, lies, when the lung is distended, in contact with the convex anterior surface of the diaphragm. The outer or costal surface is convex in adaptation to the form of the side-wall of the thorax; the internal surface is concave.

Ductless Glands.-The spleen is an elongated, compressed, dark-red body situated in the abdominal cavity in close contact with the stomach, to which it is bound by a fold of the peritoneum. The thymus, much larger in the young rabbit than in the adult, is a soft mass, resembling fat in appearance, situated in the ventral division of the mediastinal space below the base of the heart. The thyroid is a small, brownish, bilobed, glandular body situated in close contact with the ventral surface of the larynx.

Nervous System.-The neural cavity, as in the Pigeon, contains the central organs of the cerebro-spinal nervous systemthe brain and spinal cord. The brain (Figs. 1105-1107) of the Rabbit contains the same principal parts as that of the Pigeon, with certain differences, of which the following are the most important.

The surface of the cerebral hemispheres or parencephala (Fig. $1105, f . b$. . Fig. $1106, c . h$.), which are relatively long and narrow, presents certain depressions or sulci, which, though few and indistinct, yet mark out the surface into lobes or convolutions not distinguishable in the case of the Pigeon or the Lizard. A slight depression-the Sylvian fissure-at the side of the hemisphere separates off a lateral portion, or temporal lobe (Fig. 1107 c. $h^{2}$.), from the rest. There are very large club-shaped olfactory bulbs at the anterior extremities of the cerebral hemispheres, and behind each on the ventral surface of the hemisphere is the corresponding olfactory tract leading back to a slight rounded elevation, the tuberculum olfaclorium. Connecting together the two hemispheres is a commissural structure - the corpus callosum (Figs. 1106, 1107, $c p . c l$.) -not present in the Pigeon; this runs transversely above the level of the lateral ventricles. Examined in transverse section, i.e., in a longitudinal section of the brain (Fig. 1107), the corpus callosum is seen to bend slightly downwards, forming what is termed the genu; posteriorly it bends downwards and forwards, forming the splenium, which passes forwards and is united with the fornix. Below the corpus callosum is another characteristic structure of a commissural nature-the fornix (b. fo.)-a narrow median 
strand of longitudinal fibres, which bifurcates both anteriorly and posteriorly to form the so-called pillars of the fornix-anterior (Figs. 1106 and 1107, a. fo), and posterior (Fig. 1106, p. fo.). Below the corpus callosum, between it and the fornix, the thin inner walls of the hemispheres (septum lucidum, sp. lu.) enclose a small,
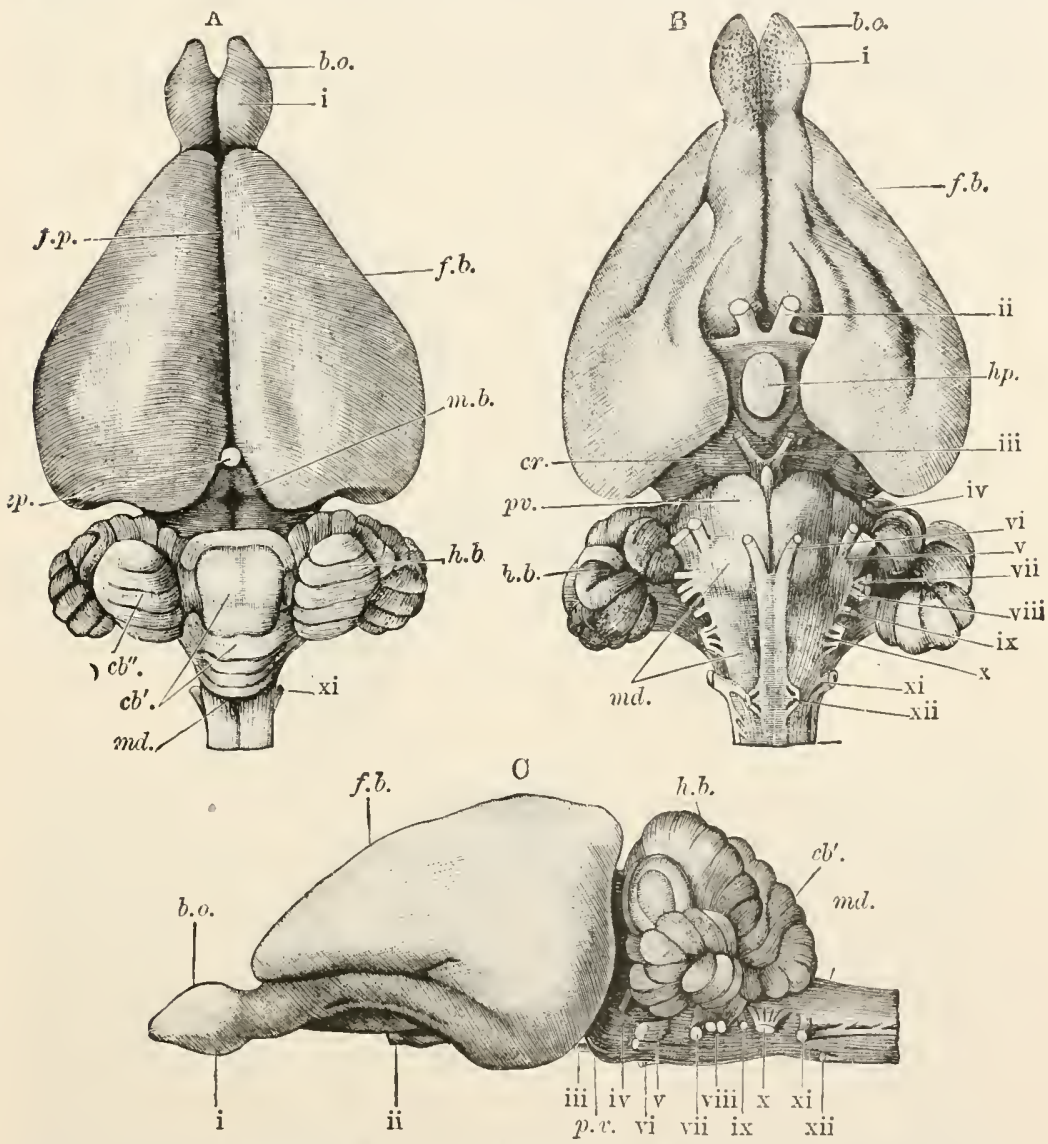

FIG 1105 - Iepus cuniculus. Brain. $A$, dorsal, $B$, ventral, $C$, lateral view, b.o. olfactory bulb; $c b^{\prime}$. median lobe of cerebellum (vermis); $c b^{\prime \prime}$. lateral lobe of cerebellum; $c r$. crura cerebri ; $e p$. epiphysis ; $f . b$. parencephala; $f . p$. longitudinal fissure ; $h . b$. cerebellum ; $h p$. hypophysis; $m . b$. mid-brain (corpora quadrigemina); md. medulla oblongata; $p v$. pons Varolii, the transverse fibres of which are here not indicated; $\mathrm{i}-\mathrm{xii}$, cerebral nerves. (From Wiedersheim.)

laterally compressed cavity, the so-called fifth ventricle or pseudoccele; this is not a true brain-ventricle, but merely a space between the closely-apposed hemispheres.

The lateral ventricles of the cerebral hemispheres are much more extensively developed than in the brain of the Pigeon, and of somewhat complex shape. Each consists of a middle portion or body 
roofed over by the corpus callosum, a narrow anterior prolongation, or anterior cornu, a posterior cornu, which runs backwards and inwards, and a descending cornu, which passes at first almost directly outwards, then downwards, and finally inwards and forwards. On the floor of the body of the ventricle, and continued along the whole extent of the descending cornu, is a prominent ridge of nearly semicircular transverse section-the hippocampus (hp. m.); this corresponds in position with a groove, the hippocampal sulcus, on the

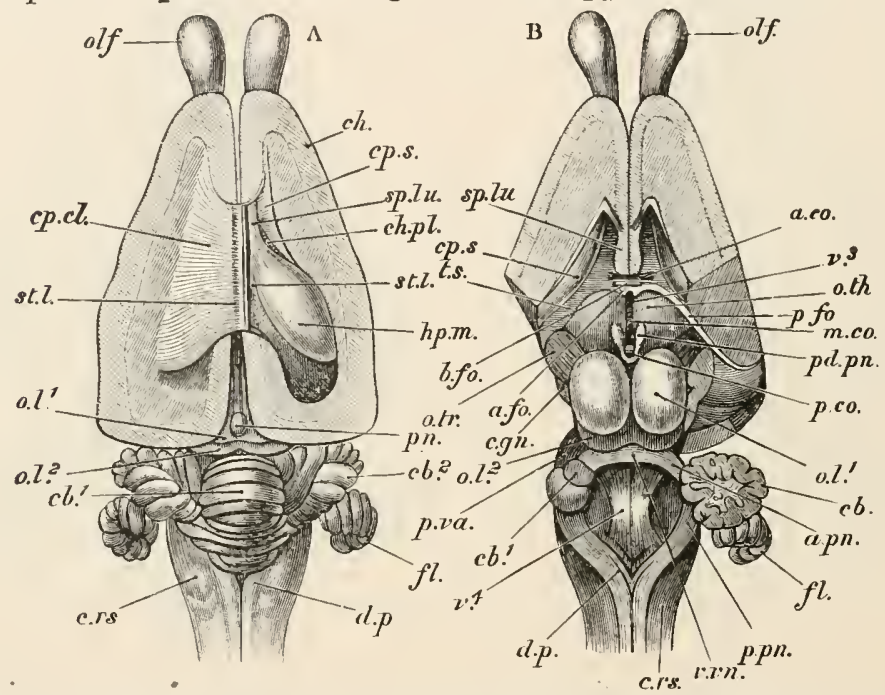

FIG. 1106.-Lepus cuniculus. Two dissections of the brain from above (nat. size). In $A$ the left parencephalon is dissected down te the level of the corpus callosum : on the right the lateral ventricle is exposed. In $B$ the cerebral hemisplieres are dissected to a little below the level of the genu of the corpus callosum; only the frontal lobe of the left hemisphere is retained; of the right a portion of the temporal lobe also is left; the velum interpositum and pineal body are removed, as well as the greater part of the body of the fornix, and the whole of the leit posterior pillar; the cerebellum is removed with the exception of a part of its right lateral lobe. a.co. anterior commissure ; $\alpha . f o$. anterior pillar of fornix ; $a . p n$. anterior peduncles of cerebelium; $b$.fo. body of fornix; $c b^{1}$. superior vermis of cerebellum; $c b^{2}$. its lateral lobe; $c . g n$. corpus geniculatum; $c . h$. cerebral hemisphere ; $c h . p l$. choroid plexus; $c p$. cl. corpus callosum; $c p$. s. corpus striatum; c.rs. corpus restiforme; d.p. dorsal pyramid; $f$. flocculus; $h p$. m. hippocampus; $m . c o$. middle commissure ; $0 . l^{1}$. anterior, and $0 . l^{2}$. posterior lobes of corpora quadrigemina ; olf. olfactory bulb; 0. th. optic thalamus; o.tr. optic tract; p.co. posterior commissure; p.fo. posterior pillar of fornix (tænia hippocampi); $p n$. pineal body; $p d . p n$. peduncle of pineal body; $p . p n$. posterior peduncles of cerebellum; $p . v a$. fibres of pons Varolifi forming middle peduncles of cerebellum ; $s p . l u$. septum lucidum; st. $l$. stria longitudinalis; $t$. $s$. tania semicircularis (narrow band of white matter between corpus striatum and optic thalamus); v.vn. valve of Vieussens; $v^{3}$, third ventricle; $v^{4}$, fourth ventricle. (From Parker's Zootomy.)

inner surface of the temporal lobe. Internally the two hippocampi merge in a median commissural area-the psalterium or lyra (ly.).

Running along the anterior edge of the hippocampus is a ridge of fibres - the tonia hippocampi or fimbria - which passes down into the descending cornu. The union of the two tæniæ forms a median longitudinal strand, the body of the fornix, which, as already explained, lies below the corpus callosum, continuous with the splenium of the latter behind, but diverging from it anteriorly by 
dipping down towards the base of the brain. In the angular space between the corpus callosum above and the fornix below is the septum lucidum with the "fifth ventricle." The trenix hippocampi are the posterior pillars of the fornix (Fig. 1106, p. fo.); the anterior pillars (a.fo.) are a pair of vertical bands which pass from the anterior end of the body downwards to the corpus mammillare (see below) at the base of the diencephalon.

Lying immediately in front of the hippocampus is a vascular membrane, the choroid plexus (ch. $p l$.); this passes inwards to join its fellow of the opposite side through a transverse passage, the foramen of Monro $(f . m$.), which opens behind into the diacœele. The floor of the anterior cornu is formed of an eminence of grey matter-the corpus striatum (cp. s.). The right and left corpora striata are connected together by a narrow transverse band of white fibres-the anterior commissure (a.co.)-situated in front of the anterior pillars of the fornix.

The diacœle $\left(v .^{3}\right)$ is a laterally compressed cavity, the roof of which is formed by a delicate vascular membrane, the velum interpositum $(v l$. ip.), in which there is a network of blood - vessels (choroid plexus

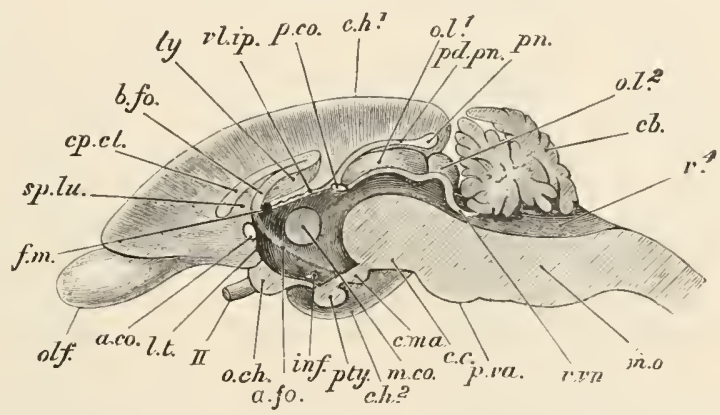

FIG. 110 \%.-Iepus cuniculus. Longitudina ]vertical section of the brain (nat size). Letters as in preceding figure; in aldition- $c b$. cerebellum, showing arbor vita ; $c$. $c$. crus cerebri ; $c$. $h^{1}$. parencephalon; $c . h^{2}$. temporal lobe; c. ma. corpus mammillare ; $f$. $m$. foramen of Nonro; inf. infundibulum $l y$. psalterium or lyra; $m$. o. medulla oblongata; o. ch. optic chiasma; olf. olfactory bulb; pty. pituitary body; $p$. va. pons Varolii; $v l$. $i p$. velum interpositum; $v$. $v n$. valve of Vieussens; $I I$, optic nerve. (From Parker's Zootomy.)

of the diacole) continuous with the choroid plexuses of the lateral ventricles. From the posterior part of the roof of the diacole rise the peduncles of the pineal body. The optic thalami (o. th.) are large masses of mixed grey and white matter forming the lateral portions of the diencephalon; they are connected together by a thick mass of grey matter, the middle or soft commissure $(m . c o$.$) , not represented in lower Vertebrates, passing across$ the diacole. A rounded elevation near the anterior end of the external surface of each thalamus is the corpus yeniculatum (c.gn.). The anterior boundary of the diacœele is a thin vertical lamina-the lamina terminalis - of which the septum lucidum is a mesial anterior prolongation. The floor of the diencephalon is produced downward into a mesial rounded process, the tuber cinereum or infundibulum (inf.), to which the pituitary body is attached. In front of this, on the ventral aspect of the brain, is a thick transverse 
band of nerve-fibres, the united optic tracts, from the anterior border of which the optic nerves are given off. Behind the tuber cinereum, and formed as a thickening of its posterior wall, is a rounded elevation, the corpus mammillare (c. ma.).

In the mid-brain the dorsal part is remarkable for the fact tliat each optic lobe is divided into two by a transverse furrow, so that two pairs of lobes $\left(o . l .^{1}, o . l . .^{2}\right)$, the corpora quadrigemina, are produced. Between the anterior lobes passes the delicate posterior commissure (p.co.). On the ventral region of the mid-brain the crura cerebri are far more prominent than in the lower groups. In the hind-brain the cerebellum (Fig. 1105, $c b^{\prime}, c b^{\prime \prime}$.) is very large; it consists of a central lobe or vermis and two lateral lobes, divided by very numerous fissures or sulci into a large number of small convolutions. Each lateral lobe bears an irregularly-shaped prominence, the flocculus. On section (Fig. 1107, cb.) the cerebellum exhibits a tree-like pattern (arbor vita) brought about by the arrangement of the white and grey matter. On the ventral aspect of the hind-brain a flat band of transverse fibres-the pons Varolii-connects together the lateral parts of the cerebellum. The cerebellum is attached to the other parts of the brain by three pairs of peduncles, the anterior, connecting it with the posterior optic lobes, the middle, passing on each side into the pons Varolii, and the posterior, connecting it with the dorsal portion of the medulla oblongata. Between the anterior peduncles extends a transverse band, the valve of Vieussens (Fig. 1107, v. vn.), connected by its anterior edge with the corpora quadrigemina. Behind this is a short tract of transverse fibres-the corpus trapezoideum - and behind this again is a slightly elevated area marking the position of the olivary body. The floor of the fourth ventricle presents a median groove which ends posteriorly in a pointed depression-calamus scriptorius-leading into the central canal of the spinal cord.

The Rabbit, like most other Vertebrates, possesses a sympathetic nervous system, consisting of a series of ganglia united together by commissural nerves, and giving off branches to the various internal organs. Two sympathetic ganglia are situated on each side in the neck-the anterior and posterior cervical ganglia. From the anterior nerve-branches pass forwards to enter the cranial cavity; from the posterior a nerve-cord passes backwards to the first thoracic ganglion. Of the thoracic ganglia there are twelve on each side. From one of the more posterior of these originates the splanchnic nerve which passes backwards into the abdomen, ending in a ganglion-the coliac-connected with an extensive nerve-plexus, the coliac plexus. In the abdomen there are, on each side, twelve ganglia, the chain ending behind in a single ganglion impar.

In the organs of special sense the following special features are to be seen when a comparison is made with the Pigeon or Lizard. In the eye, the sclerotic is composed entirely of dense 
fibrous tissue; the pecten is absent. In the internal ear the principal point of difference is in the special development of the cochlea. This part of the membranous labyrinth, instead of retaining the simple curved form which it presents in the Bird, is coiled on itself in a close spiral of two-and-a-half turns. The spiral channel in the substance of the bone, in which this cochlear spiral runs, contains three passages; the middle one, much the smallest, being the membranous cochlca, the uppermost the scala vestibuli, and the lowermost the scala tympani.

The special features of the middle ear with its auditory ossicles, and of the external ear, have been already referred to (pp. 433 and 441).

Urinogenital Organs.-The kidneys are of somewhat compressed oval shape, with a notch or hilus on the inner side. They

A

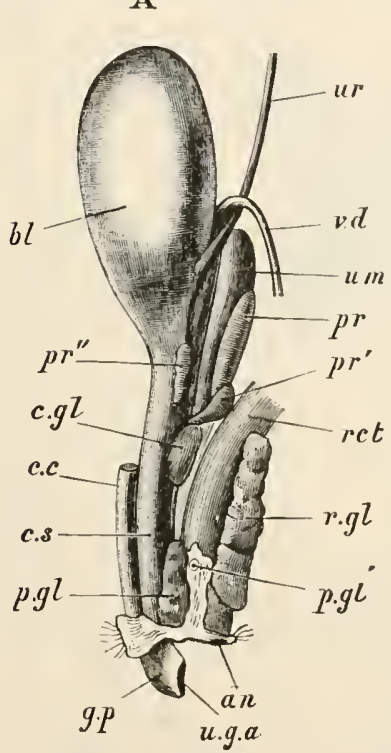

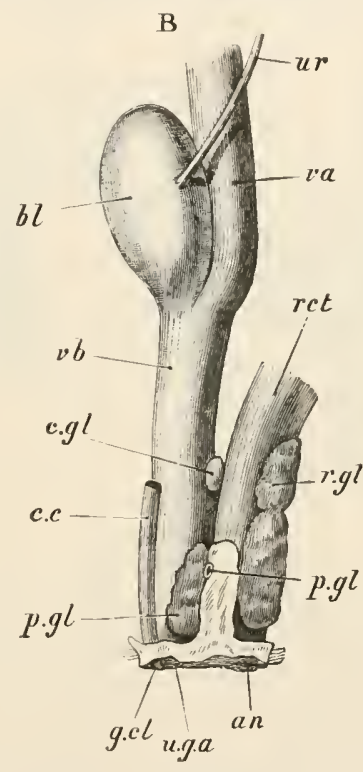

FIG. 1108.-Lepus cuniculus. The urinogenital organs ; $A$, of male ; $B$, of female ; from the left side (half nat. size). The kidneys and proximal ends of the ureters, in $A$ the testes, and in $B$ the ovaries, Fallopian tubes and uteri, are not shown. an. anus; $b l$. urinary bladder; c. c. corpus cavernosum; c. $s$. corpus spongiosum ; c. gl. Cowper's gland ; g. cl. apex of clitoris ; $g$. $p$. apex of penis; $p . g l$. perineal gland ; $p$. $g l$. a perture of its duet on the perineal space ; $p r$. anterior, $p r^{\prime}$. posterior, and $p r^{\prime \prime}$. lateral lobes of prostate ; rct. rectum ; $r$. gl. rectal gland; $u$. g. a. urinogenital aperture; $u$. m. uterus masculinus ; ur. ureter; $v a$. vagina; $v b$. vestibule ; $v$. $l$. vas deferens. (Fron Parker's Zootomy.)

are in close contact with the dorsal wall of the abdominal cavity, the right being somewhat in advance of the left. Towards the hilus the tubules of the kidney converge to open into a wide chamber-the pelvis-which forms the dilated commencement of the ureter. When the kidney is cut across, its substance is seen to be divided into a central mass or medulla and a peripheral portion or cortex. An adrenal (suprarenal) body lies in contact with the 
anterior end of each kidney. The ureter (Fig. 1108, ur.) runs backwards to open, not into a cloaca, but directly into the urinary bladder $(b l$.$) . The latter is a pyriform sac with muscular walls$ which vary in thickness according as the organ is dilated or contracted. In the male the openings of the ureters are situated nearer the posterior narrower end or neck than in the female.

In the male Rabbit the testes are oval bodies, which, though in the young animal occupying a similar position to that which they retain throughout life in the Pigeon, pass backwards and downwards as the animal approaches maturity, until they come to lie each in a scrolal sac situated at the side of the urinogenital opening. The cavity of each scrotal sac is in free communication with the cavity of the abdomen by an opening - the inguinal caral.

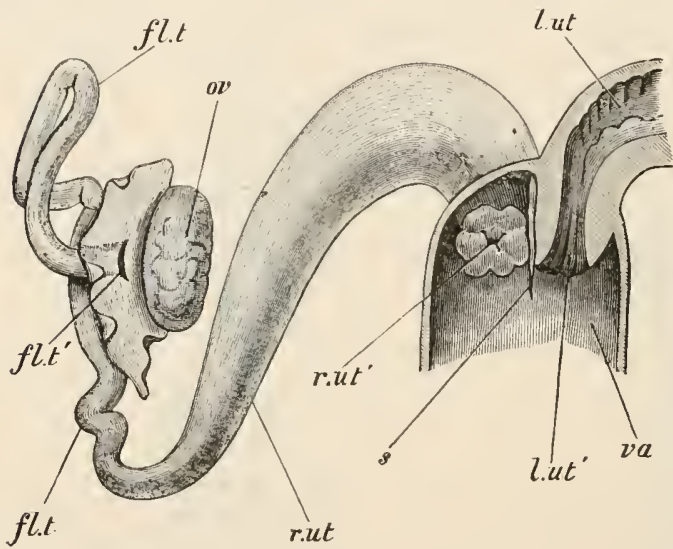

FIG. 1109.- Lepus cuniculus. The anterior end of the vagina, with the right uterus, Fallopian tube and ovary (nat. size). Part of the ventral wall of the vagina is removed, and the proximal end of the left uterus is shown in longitudinal section. $f$. $t$. Fallopian tube ; $f$. $t^{\prime}$. its peritoneal aperture ; $l$. $u t$. left uterus; $l$. $u t^{\prime}$. left os uteri ; ov. right ovary; $r$. $u t$. right uterus; $r$. $u t^{\prime}$. right os uteri; $s$. vaginal septum; va. vagina. (From Parker's Zootomy.)
The sperms have an oval compressed "head" $0.005 \mathrm{~mm}$. in length and a slender "tail" $0.045 \mathrm{~mm}$. long. A convoluted ep $i$ didymis, closely adherent to the testis, forms the proximal part of the vas deferens. The vasa deferentia (v.d.) terminate by opening into a urinogenital canal, or urethra, into which the neck of the urinary bladder is continued. A prostate gland ( $p r$.$) sur-$ rounds the commencement of the urethra, the neck of the bladder, and the terminal part of the vasa deferentia. A diverticulum of the urethra-the ulerus masculinus $(u . m$.)-lies embedded in the prostate gland close to the neck of the bladder. A small pair of ovoid glands, Cowper's glands $(c . g l$.$) , lie just behind the prostate close to the side of the$ urethra.

The terminal part of the urethra traverses a cord of vascular tissue, the corpus spongiosum (c. s.), which forms the dorsal portion of the penis. The greater part of the penis is formed of two closely approximated firm cores of vascular tissue - the corpora cavernosa (c.c.), which are attached proximally to the ischia, and terminate in a pointed apex (g.p.). A loose fold of skin, the prepuce, encloses the penis. A pair of glands with an odorous secretion, the perineal 
glands $(p . g l$.$) , open on the perineal space at the base of the penis :$ two similar glands, the rectal gland.s $(r . g l$.$) , lie on either side of the$ rectum.

In the female the ovaries (Fig. 1109, ov.) are small ovoid bodies attached to the dorsal wall of the abdomen behind the kidneys. The Graafian follicles enclosing the ova form only very small rounded projections on their outer surface.

The oviducts in the anterior part of their extent (Fallopian tubes, fl.t.) are very narrow and slightly convoluted. They open into the abdominal cavity by wide funnel-shaped openings ( $f t . t^{\prime}$.) with fimbriated or fringed margins. Posteriorly each passes into a thick-walled uterus $(r . u t$.). The two uteri open separately into a median tube, the vagina (va.). The vestibule (Fig. 1108, vb.), or urinogenital canal, is a wide median passage, into which the vagina and the bladder open. On its ventral wall is a small, hard, rod-like body, the clitoris (c. c.), with a pointed apex (g.cl.), corresponding to the penis of the male, and composed of two very short corpora cavernosa attached anteriorly to the ischia, and invested internally by a soft, grooved corpus spongiosum. The vulva, or external opening of the vestibule, is bounded laterally by two prominent folds-the labia majora.

Development. - The Rabbit is viviparous. The ovum, which is of relatively small size, after it has escaped from its Graafian follicle, passes

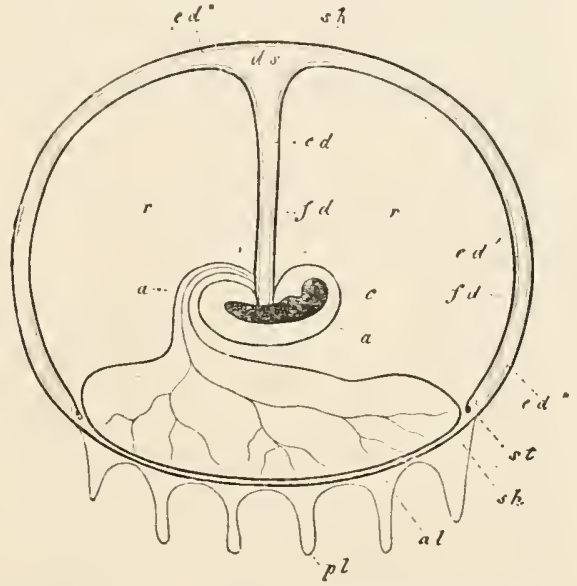

FIG. 1110.-Diagrammatic longitudinal section of a Kabbit's embryoat an advanced stage of pregnancy. $a$. amnion; $a$. stalk of allantois ; al. allantois with blood-vessels; $c$. embryo; ds. cavity of yolk-sac (umbilical vesicle); ed. endodermal layer of yolksac; ed'. inuer portion of endorlerm; ed'. outer portion of endoderm lining the compressed cavity of the yolk-sac; $f d$. vascular layer of yolk-sac: $p l$. placental villi ; $r$. space filled with fluid between the amnion, the allantois and the yolk-sac; sh. subzonal membrane; st. sinus terminalis. (From Foster and Balfour, after Bischoff.)

into the Fallopian tube, where it becomes fertilised, and then reaches the uterus, in which it develops into the foetus, as the intra-uterine embryo is termed. The young animal escapes from the uterus in a condition in which all the parts have become fully formed, except that the eyelids are closed and the hairy covering is not yet completed. As many as eight or ten young are produced at a birth, and the period of gestation, i.e., the time clapsing between the fertilisation of the ovum and the birth of the young animal, is thirty days. Fresh broods may be born once a month throughout 
a considerable part of the year, and as the young Rabbit may begin breeding at the age of three months, the rate of increase is very rapid.

The segmentation is of the holoblastic type. An amnion and an allantois are developed much as in the case of the Bird (p. 428). But the later history of these fotal membranes is widely different in the Rabbit, owing to the modifications which they undergo, in order to take part of the formation of the placenta - the structure by whose instrumentality the fœtus receives its nourishment from the walls of the uterus. The placenta is formed from the serous membrane or chorion - the outer layer of the amniotic folds-in a limited disc-shaped area, in which the distal portion of the allantois coalesces with it (Fig. 1110). The membrane thus formed develops vascular processes - the chorionic villi-which are received into depressions (the uterine crypts) in the mucous membrane of the uterus. The completed placenta with its villi is supplied with blood by the allantoic vessels. The placenta of the Rabbit is of the type termed deciduate, the villi of the placenta being intimately united with the uterine mucous membrane, and a part of the latter coming away with it at birth in form of a decidua, or after-birth.

\section{Distinctive Characters and Classification of Recent Mamialia. ${ }^{1}$}

The Mammalia are air-breathing Vertebrates, with warm blood, and with an epidermal covering in the form of hairs. The bodies of the vertebræ are in nearly all Mammals ossified each from three independent centres, one of which develops into the centrum proper, while the others give rise to thin discs of bone-the epiphyses. Also characteristic of the spinal column of Mammals are the discs of fibro-cartilage termed intervertebral-dises, which intervene between successive centra.

The skull has two condyles for connection with the atlas, instead of the single condyle of the Sauropsida; and the lower jaw, which consists of only a single bone on each side, articulates with the skull in the squamosal region without the intermediation of the separate quadrate element always present in that position in Birds and Reptiles.

Each of the long bones of the limbs is composed in the young condition of a central part or shaft and terminal epiphyses, the latter only becoming completely united with the shaft at an advanced stage.

In the pectoral arch the coracoid of the Birds and Reptiles is usually represented only by a vestige or vestiges, which unite with the scapula in the adult. The ankle-joint is always situated between the tibia and the tarsus.

1 Extinct groups are referred to in dealing with the distribution. 
Mammals are typically diphyodont, i.e., have two sets of teeth - a milk or deciduous set, and a permanent set: some are monophyodont, i.e., have only one set. The teeth are thecodont, i.e., the base of each tooth is embedded in a distinct socket or alveolus in the substance of the bone of the jaw; and nearly always the teeth in different parts of the jaw are clearly distinguishable by differences of shape into incisors, canines, and grinding teeth, i.e., are heterodont; in some instances the teeth are all alike (homodont). A cloaca is absent as a rule, except in the Prototheria.

A movable plate of cartilage-the epiglottis-represented only by a rudiment in some Amphibia and Sauropsida-overhangs the slit, commonly termed glottis, leading from the pharynx into the cavity of the larynx.

A partition of muscular fibres, usually with a tendinous centre, the diaphragm, divides the cavity of the body into two parts, an anterior, the thorax, containing the heart and lungs, and a posterior, the abdomen, containing the greater part of the alimentary canal with its associated glands - the liver and pancreas - and the renal and reproductive organs.

The lungs are freely suspended within the cavity of the thorax. The heart is completely divided into two halves - a right and a left-between which there is no aperture of communication. Each half consists of an auricle and a ventricle, opening into one another by a wide aperture, guarded by a valve composed of three membranous cusps on the right side, two on the left. The right ventricle gives off the pulmonary artery; from the left arises the single aortic arch, which passes over to the left side, turning round the left bronchus in order to run backwards as the dorsal aorta: it therefore represents the left aortic arch of Reptiles. The blood is warm. The red blood-corpuscles are non-nucleated and usually circular.

The two cerebral hemispheres, in all but the Monotremes and Marsupials, are connected together by a band of transverse fibresthe corpus callosum-not represented in the lower Vertebrates. The dorsal part of the mid-brain is divided into four optic lobes - the corpora quadrigemina. On the ventral side of the hind-brain is a transverse band of fibres-the pons Varoliiby which the lateral portions of the cerebellum are connected. An external ear, and a malleus and incus are characteristic.

The ureters, except in the Prototheria, open into the bladder.

With the exception of the Monotremes, Mammals are all viviparous. The foetus is nourished before birth from the bloodsystem of the parent through a special development of the foetal membranes and the lining membrane of the uterus, termed the placenta. After birth the young Mammal is nourished for a longer or shorter time by the milk or secretion of the mammary glands of the mother. 


\section{Sub-class I.-Prototheria.}

Mammals in which the mammary glands are devoid of teats; the oviducts are distinct throughout, and there is a cloaca into which the ureters and urinary bladder open separately. In the centra of the vertebræ the epiphyses are absent or imperfectly developed; the bones of the skull early coalesce by the obliteration of the sutures; there is a large coracoid articulating with the sternum, a T-shaped episternum, and a pair of epipubic (marsupial) bones. A corpus callosum is absent. The ova are meroblastic, and are discharged in an early stage of their development, enclosed in a tough shell.

This sub-class comprises a single living order, the Monotremata, including the Duck-Bill or Platypus (Ornithorhynchus) and the Spiny Ant-eater (Echidna).

\section{Sub-class II.-Theria.}

Mammals in which the mammary glands are provided with teats; the oviducts are united in a longer or shorter part of their extent; there is usually no trace of a cloaca; the ureters open into the base of the bladder. The centra of the vertebræ possess distinct epiphyses; the bones of the skull in most instances do not completely coalesce, most of the sutures remaining distinguishable throughout life; the coracoid is represented by vestiges, and an episternum is absent as a distinct bone. The ova are holoblastic, and the early development of the young takes place in the uterus.

\section{Section A.-Metatheria (Marsupialia).}

Theria in which the young, born in a comparatively rudimentary condition, are sheltered during their later development in an integumentary pouch-the marsupium. A common sphincter muscle surrounds anus and urinogenital aperture : the vaginæ are distinct. The tympanic cavity is partly bounded by the alisphenoid; the jugal furnishes a part of the glenoid cavity for the condyle of the mandible; therc are well-developed epipubic bones. There is no corpus callosum. A placenta is usually wanting; when present, it is functional only for a short period.

\section{Order 1.-Polyprotodontia.}

Marsupials with numerous small, sub-equal incisor teeth, and large canines; the molars provided with sharp cusps.

'This order includes the Opossums (Didelphyida), the Dasyures (Dasyurid(e), and the Bandicoots (Peramelidce). 


\section{Order 2.-DiprotonONTtA.}

Marsupials with not more than three incisors on each side in the upper jaw, and usually only one in the lower; the central incisors large, the canines usually small or absent; the molars blunt, with tubercles or transverse ridges.

This order includes the Wombats (Phascolomyidex), the Phalangers (Phalangeride), and the Kangaroos (Macropodidee).

\section{Section B.-Eutheria.}

Theria in which a marsupium is absent, and the young are always nourished in utero, for a considerable period, through the agency of a placeuta. The anus and urinogenital aperture are usually not surrounded by a common sphincter. The alisphenoid never contributes to the formation of the wall of the tympanic cavity; except in the Hyracoidea and some Rodents, the jugal takes no part in bounding the glenoid cavity; and there are no marsupial bones. A corpus callosum is present.

\section{Order 1.-Edentata.}

Eutheria in which the teeth are absent in the adult or the dentition is imperfect, incisors and canines being seldom represented; and, though there may be numerous premolars and molars, these never form roots and are devoid of enamel. All, with the exception of two genera, are monophyodont. The sacral vertebræ are frequently in excess of the number usual in other orders. The coracoid process is usually relatively larger than in other Eutheria, and does not become completely fused with the scapula. The brain is sometimes of low, sometimes of comparatively high organisation. The placenta is deciduate or non-deciduate, diffuse, zonary or discoidal (vide p. 576).

There are five families usually comprised in the order, each characterised by the presence of a number of remarkable and peculiar features: viz., the Sloths (Bradypodida), the American Ant-eaters (Myrmecophagidce), the Armadillos (Dasypodidce), the Scaly Anteaters (Manidos), and the Cape Ant-eaters (Orycteropodido $)$. The two last are probably not sufficiently ncarly related to the rest to be included with them in one order.

\section{Order 2.-Cetacen.}

Aquatic Eutheria with large head, fish-like fusiform body, devoid of hairy covering, with the pectoral limbs paddlc-like, the pelvic limbs absent, and with a horizontal caudal fin. A vertical dorsal fin is usually present. There is a long suout, and the nostrils open by two lateral external apertures or a single median one sitnated in all the recent forms far back towards the summit 
of the head. The cervical region of the spinal column is very short, and its vertebræ usually completely united together. Clavicles are absent. The humerus is freely movable at the shoulder, but all the other articulations of the limb are imperfect. The number of phalanges of the second and third digits always exceeds the number (three) normal in the Mammalia. The pelvis is represented by a pair of horizontally placed styliform vestiges of the ischia. Teeth may be absent and the mouth may be furnished with sheets of baleen or "whalebone"; when present they may be very numerous and homodont, or less numerous and heterodont, or reduced to a single pair. The epiglottis and arytenoids are prolonged, and embraced by the soft palate, so as to form a continuous tube for the passage of the air from the nasal cavities to the trachea. The brain is large, and the cerebral hemispheres are richly convoluted. The testes are abdominal. The teats are two, and are posterior in position. The uterus is two-horned, the placenta diffuse and non-deciduate (vide p. 576).

This order includes the Baleen Whales (Balcenida), SpermWhales (Physeter), Killers (Orca), Porpoises (Phoccena), and Dolphins (Delphinus).

\section{Sub-order a.-Mystacoceti.}

Cetacea in which plates of baleen are developed. Functional teeth are never present, and the premaxillæ are narrow and take only a small share in the formation of the rostrum. The nostrils are situated far back. The nasal cavities are roofed over by the nasals. The tympanic bones are scroll-like, and are fused with the periotics. The rami of the mandible are not united anteriorly.

This sub-order includes the Whalebone Whales (Balcena and others).

\section{Sub-order b.--Odontoceti.}

Cetacea in which the premaxillæ are narrow, and the nostrils far back, as in the Mystacoceti. The nasals are reduced and do not roof over the nasal cavities. The tympanic bones are not scroll-like, and do not become fused with the periotic. The rami of the mandible are united at the symphysis. Baleen plates are never present, and teeth are developed, and are usually very numerous and homodont. This sub-order comprises the Porpoises (Phoccena), Dolphins (Delphinus and others), and Killers (Orca); the Spermwhales (Physeter and Cogi $)$, the Bottle-nosed Whales (Hyperoödon), and Beaked Whales (Mesoplodon).

\section{Order 3.- Sirenia.}

Aquatic Eutheria with moderate-sized head and fish-like, depressed fusiform body, with the pectoral limbs paddle-like, the 
pelvic absent, and with a horizontally expanded tail fin. There is no vertical dorsal fin. There is a very thick wrinkled integument devoid of or with only a scattered covering of hairs. The snout is not greatly elongated, and the nostrils open by a pair of valvular apertures on its upper surface. The cervical vertebræ (of which there are only six in the Manatee) are not fused. A clavicle is absent. There is a distinct, though small, articulation between the humerus and the bones of the fore-arm. There are never more than three phalanges in any of the digits. The pelvis is represented by a pair of vertically situated vestiges. The anterior part of the palate and the symphysis of the mandible (which is prolonged) are covered with rugose horny plates. The epiglottis and arytenoids are not prolonged as they are in the Cetacea. The brain is comparatively small, and the convolutions are not highly developed. The testes are abdominal. The teats are two in number and pectoral in position. The uterus is two-horned. The placenta is non-deciduate and zonary.

This order includes among recent forms only the living Dugong (Halicore) and Manatee (Manatus), and the recently extinct Rhytina.

\section{Order 4.-Ungulata.}

Terrestrial, chiefly herbivorus, Eutheria, with the fur abundant or scanty, with the terminal phalanges, on which the weight of the body usually rests, nearly always invested in solid horny hoofs. The teeth are heterodont and diphyodont; the canines usually absent or small, and the premolars and molars well developed, with broad crowns having tuberculated or ridged surfaces. The clavicle is absent; the humerus has no foramen over the inner condyle : the scaphoid and lunar of the carpus are always distinct. The villi of the placenta are diffuse or gathered into patches-the cotyledons.

\section{Section 1.-Ungulata vera.}

Ungulata in which the feet are always unguligrade, with never more than four functional digits. The os magnum of the carpus articulates with the scaphoid. The testes are contained in a scrotum. The teats are usually four, and situated far back, never exclusively thoracic in position. The uterus is two-horned. The allantois is large, the placenta non-deciduate, and the villi diffuse or gathered into cotyledons.

This section comprises all the typical Ungulates.

\section{Sub-order a.-Perissodactyla.}

Ungulata vera in which the third toe of both manus and pes is larger than the others and symmetrical in itself, and in which there is a tendency to reduction of the others. The femur has a third 
trochanter. The tibial articular surface of the astragalus is pulleyshaped; the distal surface flat and more extensively related with the navicular than with the cuboid; the calcaneum does not articulatc with the fibula. The premolars and molars are complexly folded, and the posterior premolars usually resemble the molars in size and pattern. The stomach is simple; the cæcum large. There is never a gall-bladder. The teats are situated in the groin, and the placenta is diffuse.

This sub-order includes the Horses, Asses, and Zebras (Equida), the Tapirs (Tapirus), and the Rhinoceroses (Rhinoceros).

\section{Sub-order b.-Artiodactyla.}

Ungulata vera in which the third and fourth digits of both manus and pes form a symmetrical pair, and in which the others are usually absent or vestigial. The femur has no third trochanter. The tibial surface of the astragalus is flat, the distal surface articulates largely with the cuboid, and the calcancum has a flat articular surface for the fibula. The premolars are smaller than the molars. The stomach is almost always complex, and the cxcum is small. The teats are few and situated in the groin, or numerous and extending along the abdomen. The placenta is diffuse or cotyledonary.

This sub-order includes the Ruminants-such as the Camels (Camelida), Oxen (Bovida), Sheep (Ovis), Goats (Capra), Antelopes, Giraffes (Giraffa), and Deer (Cervida); and the Non-Ruminants, viz., the Pigs (Sus), Peccaries (Dicolyles), and Hippopotami (Hippopotamus).

\section{Section 2.-Subungulata.}

Ungulata in which the feet may be plantigrade, and thcre may be five functional digits. The magnum of the carpus does not articulate with the scaphoid, at least in living forms.

\section{Sub-order a.-Hyracoidca.}

Snall Subungulata with furry integument, with four completely formed digits in the fore-foot (the pollex being vestigial), and three in the hind-foot (the hallux bcing absent and the fifth digit vestigial). The ungual phalanges of the four complete digits of the fore-foot are small, somewhat conical and flattened; that of the second digit of the hind-foot is decply cleft, and has a long, curved claw; the rest of the digits of the hind-foot have broad nails. There arc no canines, and in the upper jaw in the adult there is only a single pair of incisors, which resemble those of the Rodents in their elongated curved form and in growing from persistent pulps. The thoracic and lumbar vertebræ are very numerous (28-30), twenty-onc or twcnty-two bearing ribs. The 
tail is very short. Clavicles are absent. There is a centrale in the carpus. The stomach is divided into two parts by a constriction. The large intestine has connected with it a pair of large supplementary cæca. 'There is no gall-bladder. 'The testes do not descend into a scrotum. There are six teats, four in the groin and two in the axillie. The villi surround the placenta in a broad band (zonary placenta).

This sub-order includes only a single family, the Hyracido, with two genera, Hyrax and Dendrohyrax.

\section{Sub-order b.-Proboscidea.}

Large Subungulata with greatly thickened integument scantily furnished with hair ; with massive limbs, each having five complete digits united by skin, but each terminating in a distinct hoof ; and with the nose produced into a long, flexible, and prehensile proboscis or trunk, at the end of which the external nares are situated. In existing forms only a single pair of incisors is present, situated in the upper jaw, and developed into enormous tusks. There are no canines, and the molars are large and transversely ridged. The stomach is simple. The testes do not descend into a scrotum. There are two teats, situated on the thorax. The uterus is two-horned, the placenta deciduate and zonary.

This sub-order includes among existing forms only the Elephants (Elephas).

\section{Order 5.-CARNIVORA.}

Mostly carnivorous Eutheria with furry integument, with never less than four well-developed digits in the manus and pes, all provided with claws, which are frequently more or less retractile. The pollex and hallux are never capable of being opposed to the other digits. The clavicle is frequently absent, and, when present, is never complete. There is often a foramen over the inner condyle of the humerus. The scaphoid and lunar of the carpus are always united, and there is never an os centrale.

They are diphyodont and heterodont, and the teeth are provided with roots. The incisors, usually three pairs in the upper and three in the lower jaw, are small and chisel-shaped. The canines are usually large, conical, curved, and pointed. The premolars and molars are usually compressed and trenchant, especially the most anterior. The stomach is simple; the cæcum, when present, is small. The brain is usually highly developed, and the cerebral hemispheres always convoluted. The teats are abdominal. The uterus is two-homed; the placenta deciduate and nearly always zonary.

\section{Sub-order a.-Carnivora vera.}

Carnivora which have the limbs nearly always adapted for a terrestrial existence, with all the digits usually provided with 
claws which may be retractile into a sheath. 'The first digit of the manus and the first and fifth of the pes are never longer than the others. One tooth on each side in each jaw-the last premolar in the upper jaw and the first molar in the lower-is always modified to form the carnassial or sectorial tooth with a cutting edge which bites against the edge of the opposed tooth.

This sub-order comprises the Cats (Felide), Civets (Viverrida), Hyænas (Hy(enidce), Dogs (Canidae), Bears (Ursidae), Weasels (Mustelidce), and Otters (Lutridce).

\section{Sub-order b.-Pinnipedia.}

Carnivora in which the limbs are adapted to an aquatic life, the proximal segments being short, the distal elongated and webbed between the digits; with five well-developed digits in each manus and pes, the first and fifth of the pes being larger than the others. The number of incisors is reduced, and there are no carnassials. The ccrcbral hemispheres are very richly convoluted.

This order includes the Eared Seals (Otariidre), the Earless Seals (Phocider), and Walruses (Trichechides).

\section{ORder 6.-RODENTIA.}

Vegetable-feeding Eutheria, mostly of small size, with furry (sometimes spiny) integument, clawed digits, and usually plantigrade limbs. A clavicle is usually present. The dentition is diphyodont; there are no canines, and there are never more than two incisors in the lower jaw and usually only two in the upperall elongated, chisel-like, and growing from persistent pulps; the premolars and molars are usually few, and in many cases grow from persistent pulps. There is a large cæcum. The cerebral hcmispheres have in most instances smooth surfaces, and do not much overlap the other parts of the brain. The testes are retaincd in the abdomen or descend to the groin. The uterus is two-horned or double. The placenta is deciduate and disc-shaped (discoidal).

This extensive order includes the Rats and Mice (Muridoc), Hares and Rabbits (Leporidoe), Squirrels (Sciurida), Jerboas (Dipodida), Beavers (Castorida), and Porcupines (Hystricidae).

\section{ORDER 7.--INSECTIVORA.}

Small insectivorous Eutheria with the nose usually produced into a short soft muzzle, with furry (sometimes spiny) integument, clawed digits, and usually pentadactyle plantigrade limbs. The dentition is diphyodont and complete, and all the teeth are rooted; the incisors are small; there are never fewer than two incisors on each side of the lower jaw; the molars are small and provided with pointed cusps. A clavicle is present. The brain is simple 
and devoid of convolutions. The testes are situated in the groin, and are not enclosed in a scrotum. The uterus is two-horned or double. The placenta is deciduate and discoidal.

Included in this order are the Moles (Talpide), Shrews (Soricide), and Hedgehogs (Erinaceida).

\section{Order 8.-Chiroptera.}

Eutheria in which the pectoral limbs are modified to form wings, the bones, more especially those of the second to the fifth digits, being greatly elongated so as to support a broad web of skin extending back to the hind-limbs. The sternum has a keel for the attachment of the pectoral muscles, which play an important part in bringing about the movements of flight. The ulna is vestigial ; the pollex is small, the remaining digits greatly elongated. The hind-limb is rotated outwards, so that the knee is directed backwards. There is a cartilaginous rod (calcar) attached to the inner side of the ankle-joint and helping to support a fold of skin (inter-femoral membrane) which extends from the hind-limbs to the tail or caudal region of the body. The cerebral hemispheres are smooth and do not overlap the cerebellum. The dentition is complete, heterodont and diphyodont. The penis is pendent; the testes abdominal or situated in the groin. The uterus is simple or two-horned (bicornuate); the placenta deciduous and discoidal.

\section{Sub-order a.-Megachiroptera.}

Large frugivorous Chiroptera with elongated snout, without foliaceous appendages to the nose and ears, the second digit of the manus terminating in a claw. The tail, when present, is not enclosed in the inter-femoral membrane, but lies below it. The crowns of the molar teeth are devoid of sharp cusps.

This sub-order comprises the so-called Flying Foxes (Pleropus) of tropical and sub-tropical parts of the Eastern Hemisphere.

\section{Sub-order b.--Microchiroptera.}

Small, mostly insectivorous, Chiroptera with short snout, frequently with foliaceous appendages of the nose and ears, the second digit of the manus never provided with a claw. The tail when present is enclosed in the inter-femoral membrane. The crowns of the molar teeth are provided with sharp cusps.

This sub-order includes all the ordinary Bats (Vespertitio and other genera).

\section{Order 9.- Primates.}

Eutheria nearly all adapted to an arboreal life, the limbs being prehensile owing to the pollex and hallux being more or less com- 
pletely opposable to the other digits. There are nearly always five digits, provided with flat nails in both manus and pes. The orbit is surrounded by a eomplete bony rim. The elavieles are in all cases well developed. There is no foramen above the inner eondyle of the humerus, and the femur rarely has a third troehanter. The stomaeh is generally simple. The testes descend into a serotum. There are nearly always two teats on the thoraeic region. The placenta may be non-deeiduate, or deeiduate and netadiseoidal.

\section{Sub-order a.--Prosimii.}

Ape-like, mostly noeturnal, arboreal Primates of eomparatively low organisation. All the digits of both feet are provided with flat nails, exeept the second of the hind-foot, which has a claw. Both the pollex and hallux are always well developed. The posterior, bony rim of the orbit is a narrow bar beneath whieh there is a free eommunieation between the orbit and the temporal fossa. The laerymal foramen is situated outside the margin of the orbit. In nearly all cases the inner pairs of incisors of the upper jaw are separated by a median spaee. The eerebral hemispheres are not very highly developed, and do not eompletely overlap the eerebellum. There may be an additional pair of teats on the abdomen. The uterus is two-horned and the placenta diffuse.

This sub-order comprises the Lemurs (Lemur, Tarsius, and other genera) and Aye-Ayes (Chiromys).

\section{Sub-order b.-Anthropoidea.}

Most highly organised Primates, chiefly modified for an arboreal life. The digits are all provided with flat nails, except in the Hapalidx, in whieh all exeept the hallux are provided with a elaw. The pollex is in some rudimentary or absent. The orbit is separated from the temporal fossa by a broad vertieal plate, and the laerymal foramen is situated within the margin of the orbit. The inner upper ineisors are in close contact. The eerebral hemispheres are usually richly eonvoluted, and eompletely, or nearly eompletely, eover over the eerebellum. The uterus has no horns. The plaeenta is deciduate and metadiseoidal.

\section{Family i.-Hapalidae.}

Anthropoidea with the pollex not opposable, all the digits exeept the hallux provided with curved pointed claws, without eheekpouches or ischial callosities, with a broad nasal septnm, without bony external auditory meatus, and with a non-prehensile tail. The dental formula (vide p. 545 ) is i. $\frac{2}{2}$, c. $\frac{1}{1}$, p. $\frac{3}{3}, \mathrm{~m} . \frac{2}{2}=32$.

This family includes the Marmosets (Hapale). 


\section{Family ii.-Ccbides.}

Anthropoidea with the pollex not opposable, all the digits provided with flat nails, without cheek-pouches or ischial callosities, with a broad nasal septum, and without bony external auditory meatus. The tail is sometimes prehensile. The dental formula is

$$
\text { i. } \frac{2}{2} \text {, c. } \frac{1}{1}, \text { p. } \frac{3}{3}, \text { m. } \frac{3}{3}=36 \text {. }
$$

This family includes the Ilowling Monkeys (Mycetes), Tee-Tees (Callithrix), Squirrel Monkeys (Chrysothrix), Spider-Monkeys (Ateles), and Capuchin Monkeys (Cebus).

\section{Family iii.-Cercopithecidae.}

Anthropoidea with the pollex, when present, opposable, with or without cheek-pouches, with ischial callosities, with a narrow nasal septum, and a bony external auditory meatus. The tail is not prehensile. The sternum is narrow. The dental formula is i. $\frac{2}{2}$, c. $\frac{1}{1}, p \cdot \frac{2}{2}, \mathrm{~m} \cdot \frac{3}{3}=32$. The cæcum is devoid of vermiform appendage.

This family includes the Baboons (Cynocephalus) and Nacaques (Macacus).

\section{Family iv.-Simiidce.}

Anthropoidea with the pollex opposable, without cheek-pouches, usually without ischial callosities, with a narrow nasal septum and a bony external auditory meatus. The pectoral limbs are much longer than the pelvic. The cxcum has a vermiform appendage. The centrale of the carpus is sometimes absent. The dental formula is the same as that of the preceding family.

This family includes the Gibbons (Hylobates), Orangs (Simia), Chimpanzees (Anthropopithecus), and Gorillas (Gorilla).

\section{Family v.-Hominida.}

Anthropoidea which differ from the Simiidro mainly in the more perfect assumption of the erect posture, co-ordinated with alteration of the curvature of the spine, and with the more complete adaptation of the hind-limbs to bearing the weight of the body ; in the absence of the power of opposition in the hallux, and its more complete development in the pollex; in the greater length of the hind-as compared with the fore-limbs; in the smaller size of the canine teeth; and the much greater size and complexity of the brain.

This family includes only the Human Species (Homo sapiens). 


\section{Systematic Position of the Example.}

The genus Lepus, to which the common Rabbit belongs, comprises a number of other species, the common Hare being among the number, distinguished from one another by slight differences in the proportions of the parts and in other general features. Lepus is the only genus of the family Leporida, which is associated with the family Lagomyidce or Picas under the designation Duplicidentata, owing to the presence in these two families, and in these two alone of the entire order Rodentia to which they belong, of a second pair of incisors in the upper jaw. The chief distinctive features of the family Leporidæ are the elongated hind-limbs, the short recurved tail, the long ears, and the incomplete clavicles.

\section{General Organisation.}

Integument and General External Features.--Nearly all Mammals are covered with hairs (Fig. 1111) developed in hair-

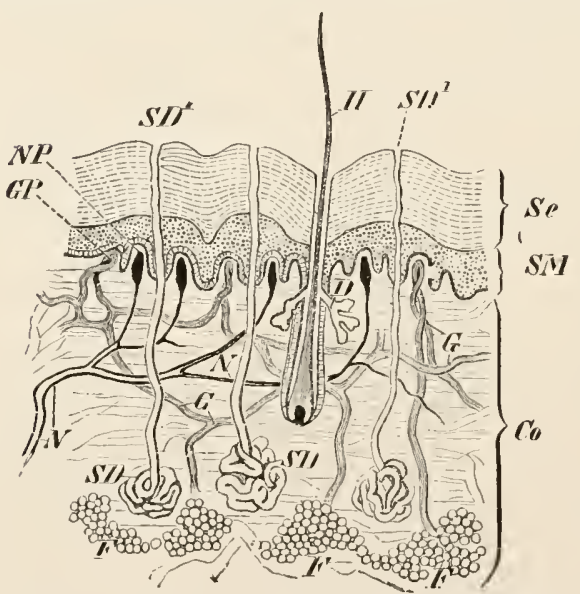

FIG. 1111.-Section of human skin. Co, dermis ; $D$, sebaceous glands ; $F$, fat in dermis ; $G$, vessols in dermis ; $G P$, vascular papillæ; $\| l$. hair ; $N$. nerves in dermis ; $N P$. nervous papillie; se, horny layer of epidernis ; $S D$, sweat-gland ; $S D^{1}$, duct of sweatgland ; sil, Malpighian layer. (From Wiedersheim's Comparative Anatomy.) follicles. Each hair (Fig. 1112 ) is a slender rod, and is composed of two parts, a central part or pith $(M)$ containing air, and an outer more solid part or cortex $(R)$ in which air does not occur ; its outermost layer may form a definite cuticle $(O)$. Commonly the cortical part presents transverse ridges so as to appear scaly. In one case only, viz., Sloths, is the hair fluted longitudinally. The presence of processes on the surface, by which the hairs when twisted together interlock firmly, gives a special quality to certain kinds of hair (wool) used for clothing-the felling quality as it is termed. A hair is usually. cylindrical; but there are many exceptions: in some it is compressed at the extremity, in others it is compressed throughout; the latter condition is observable in the hair of negroid races of men. The fur is usually composed entirely of one kind of hair ; but in some cases there are two kinds, the hairs of the one sort very numerous and forming the soft fur, and those of the other consisting of longer and coarser hairs scattered over the surface. Examples 
of a hairy covering of this kind are seen in the case of the Platypus and the Fur-Seals.

A hair, like a feather, is formed from the epidermis. The first rudiment of a developing hair (Fig. 1113) usually takes the form of a slight downwardly projecting outgrowth, the hair-germ (grm.), from the lower mucous layer of the epidermis, beneath which there is soon discernible a condensation of the dermal tissue to form the rudiment of a hair papilla $(p p$.$) . In some Mammals,$ however, the dermal papilla makes its appearance before the hair-germ. The hairgerm, which consists of a solid mass of epidermal cells, elongates, and soon its axial portion becomes condensed and cornified to form the shaft of the hair, while the more peripheral cells go to form the lining of the hairfollicle, becoming arranged in two layers, the inner and outer root-sheaths (sh.1, sh.2). The epidermal cells in immediate contact with the hair-papilla retain their protoplasmic character and form the hair-bulb (blb.), by the activity of which the further growth of the hair is effected. Soon the upper end of the hair-shaft grows out beyond the surface of the epidermis, and the projecting part eventually becomes much longer than that which lies embedded in the follicle. At the same time the follicle grows downwards into the

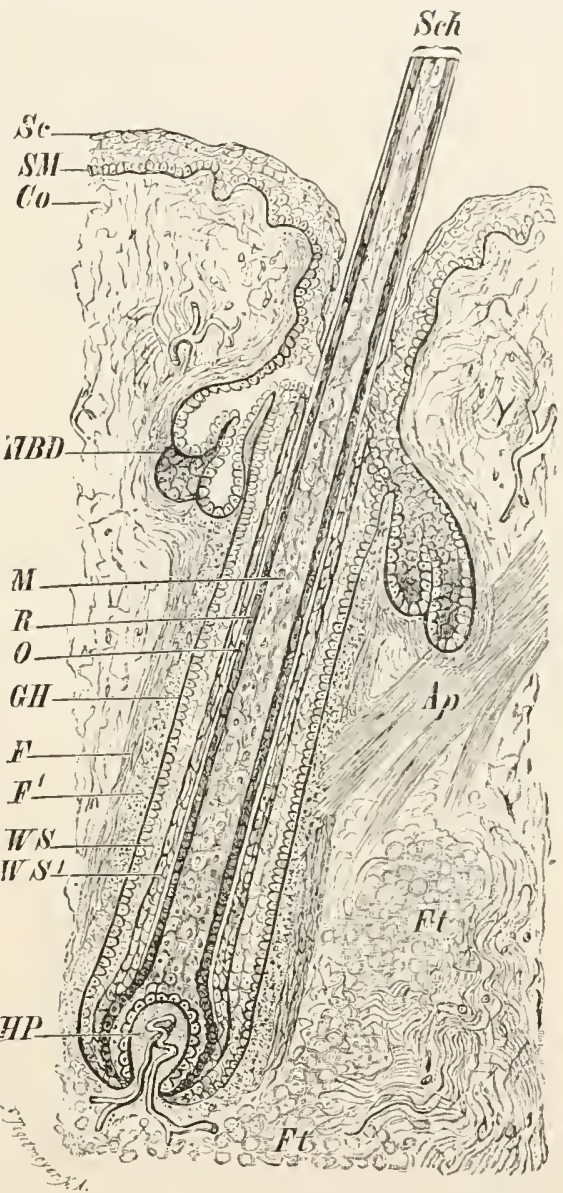

FIG. 1112,-Longitudinal section through a hair (cliagrammatic). A $p$, band of muscular fibres inserted into the hair-follicle; Co. dermis; $F$. external longitudinal, and $F^{\prime \prime}$. internal circular fibrous layer of follicle ; $F t$, fatty tissue in the dermis ; $G H$, hyaline membrane between the root-sheath and the follicle ; $H B D$, sebaceous gland ; $H P$. hair-papilla with vessels in its interior ; $\boldsymbol{M}$. medullary substance (pith) of the hair: $O$, cuticle ; $R$, cortical layer ; Sc, horny layer of epidermis ; SM, Malpighian layer of epidermis : $W S, W S^{\prime}$, outer and inner layers of root-sheath. (From Wiedersheim's Comparative Anatomy.) dermis. During its growth the hair is nourished by the bloodvessels in the dermal hair-papilla, which projects into its base. 
Modifications of the hairs are often found in certain parts. Such modified hairs are the elongated hairs of the tails of some Mammals, e.g., most Ungulates; the eye-lashes of the eye-lids, which are stronger than the ordinary hairs; and sensitive hairs or vibrissa about the snout. In some Mammals the hairs in part assume the form of spines, viz., in Echidna, the Hedgehogs, and the Porcupines.

The coating of hairs is scanty in some Mammals, and is virtually absent in the Cetacea and Sirenia. In such cases the skin is greatly

A

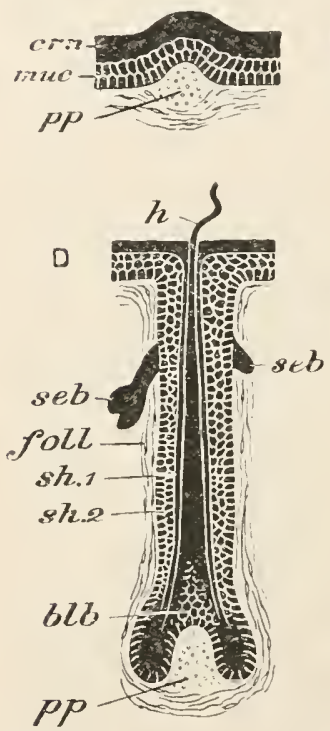

B
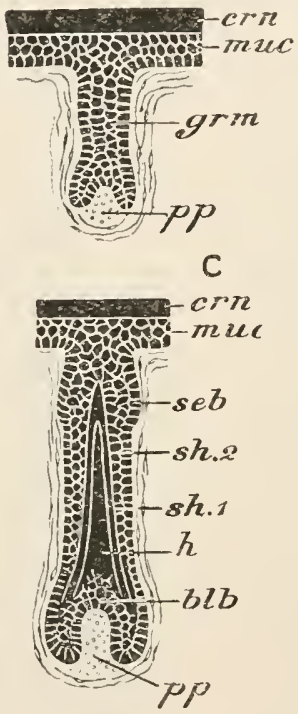

FIG. 1113.-Four diagrams of stages in the development of a hair. $A$, earliest stage in one of those Mlammals in which the dermal papilla appears first ; $B, C, D$, three stages in the development of the hair in the human embryo. blb. hair-bulb; crn. horny laver of the epidermis ; foll. hairfollicle ; $y \mathrm{rm}$. hair-germ : $h$. extremity of hair projecting on the surface in $D$; muc. Malpighian layer of epiderunis; $y p$. dernal papilla; seb. fleveloping sebaceous glands; sh. $1, s h .2$, inmer and outer root-sheatls. (A iter Hertwig.)

thickened, as in the Elephants, \&c. ; or, as in the Cetacea, an underlying layer of fat performs the function of the hair as a heatpreserving covering.

In Manis (Fig. 1126) the greater part of the surface is covered with large, rounded, overlapping horny scales of epidermal origin, similar in their mode of development to those of Reptiles. A similar phenomenon is seen in the integument of the tail of Anomalurus-a Flying Rodent. The Armadillo (Fig. 1125) is the only Mammal in which there occurs a bony dermal exoskeleton (vide infra).

Also epidermal in their origin are the horny structures in the

form of nails, claws, or hoofs, with which the terminations of the digits are provided in all the Mammalia except the Cetacea. And the same holds good of the horny portion of the horns of Ruminants. The horns of the Rhinoceros are also epidermal, and have the appearance of being formed by the agglutination of a number of hair-like horny fibres.

Cutaneous glands are very general in the Mammalia, the most constant being the sebaceous glands (Figs. 1111, D; 1112, HBD), which open into the hair-follicles, and the sweat glands (Fig. 1111, $S D$ ). In many Mammals there are, in addition, in various parts of the body, aggregations of special glands secreting an odorous matter. 
The mammary glands, by the secretion of which the young are nourished, are specially developed cutaneous glands. In the Prototheria they differ somewhat widely from those of the rest of the Mammalia in structure, and they also differ in the absence of teats. They consist of two groups of very large tubular follicles, the ducts of which open on the ventral surface. In Echidna (Fig. 1114) the two areas on which the ducts open become depressed towards the breeding season to give rise to a pair of pouches-the mammary pouches. A large brood-pouch or marsupium is subsequently formed, and the egg is deposited in this. When the

A

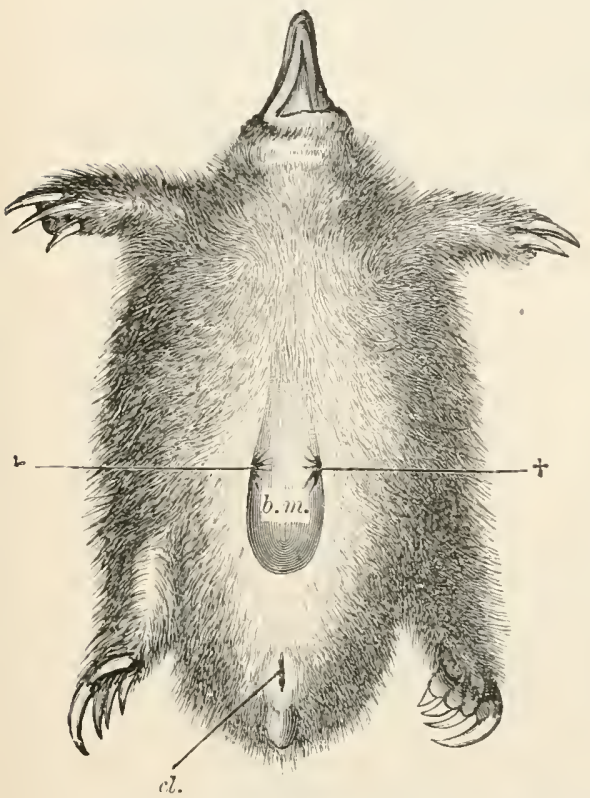

B

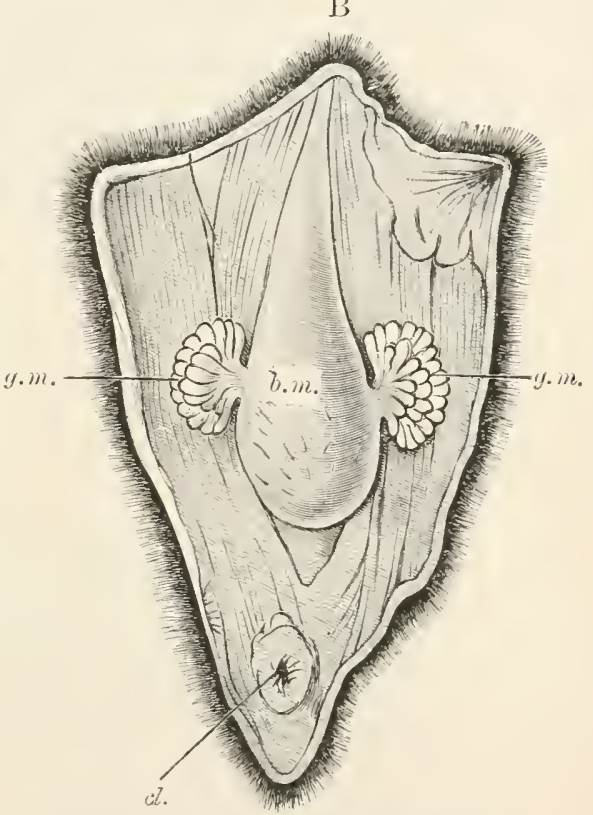

FIG. 1114.-Echidna aculeata. $A$, Iower surface of brooding female ; $B$, dissection showing a dorsal view of the marsupium and mammary glands; $t+$, the two tufts of hair projecting from the mammary pouches from which the secretion flows; $b . m$. brood-pouch or marsupium ; $\mathrm{cl}$. cloaca ; $g$. m. groups of mammary glands. (From Wiedersheim's Comparative Anatomy, after W. Haacke.)

young animal is hatched it is sheltered in the posterior deeper part of this marsupium, while in the shallower anterior part lie the mammary pouches. In Ornithorhynchus mammary pouches are indicated only by extremely shallow depressions, and no marsupium is developed.

In the higher Mammals, when the mammary glands are first developed (Fig. 1115), a depression (mammary pouch) is formed, from the floor of which branching cylindrical strands of epidermis grow inwards to give rise to the glands. At a later stage there is developed around the opening or openings of the mammary 
ducts a prominence, the teat (Fig. 1115), the wall of which may be formed of the manmary pouch area alone (Marsupials, Rodents, Primates), or, with greater or less reduction of the latter, mainly from the surrounding integument. In the latter case the teat may have a wide central canal. The number and situation of the teats vary in the different groups, and have been noticed in the synopsis of the characters of the orders and sub-orders (pp. 464 to 473$)$.

The two genera of the Prototheria, Ornithorhynchus and Echidna, differ somewhat widely from one another in general appearance. The former (Fig. 1116) has the surface covered with a close, soft fur, and has the upper jaw produced into a depressed muzzle, not unlike the beak of a duck, covered with a smooth, hairless
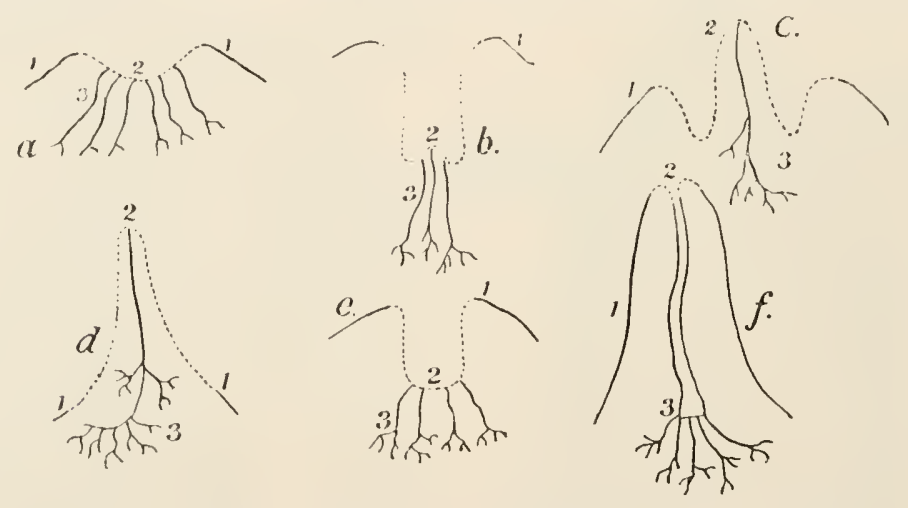

FIG. 1115,-Diagrams of the phylogentic development of teats. u, primitive condition corresponding to the condition in Echidna; $b$, Wallaby (Halmaturus) before lactation ; $c$, Opossum (Didelphys) before lactation; $d$, Opossum during lactation ; th is diagram stands also for the House and Man; $e$, embryonal, and $f$, full-grown cow. 1 , integumentary wall ; 2, manmary area, the broken line represents the mammary pouch ; 3, milk-ducts. (Aiter Max Weber.)

integument, which forms a free fold or flap at the base. The eyes are very small, and there is no auditory pinna. The legs are short, and the five digits end in strong claws, and are connected together by a web, so that the limbs are equally adapted for burrowing and for swimming. The tail is elongated and depressed, and is covered with fur. The male has a sharp-pointed, curved spur on the inner side of the foot, having the duct of a gland opening at its apex. Echidna (Fig. 1117) has the body covered above with strong, pointed spines, between which are coarse hairs; the lower surface is covered with hair only. The jaws are produced into a rostrum, which is much narrower than that of Ornithorhynchus. The eyes are small, and there is no auditory pinna. The limbs are short and powerful. There are five toes on each foot, each ending in a very strong claw, by means of which the Echidna is able to burrow with rapidity. There is a spur on 
the inner side of the hind foot, larger in the male than in the female. The tail is vestigial.

The Opossums (Didelphyida, Fig. 1118) are arboreal rat-like Marsupials, with elongated naked muzzle, with well-developed,

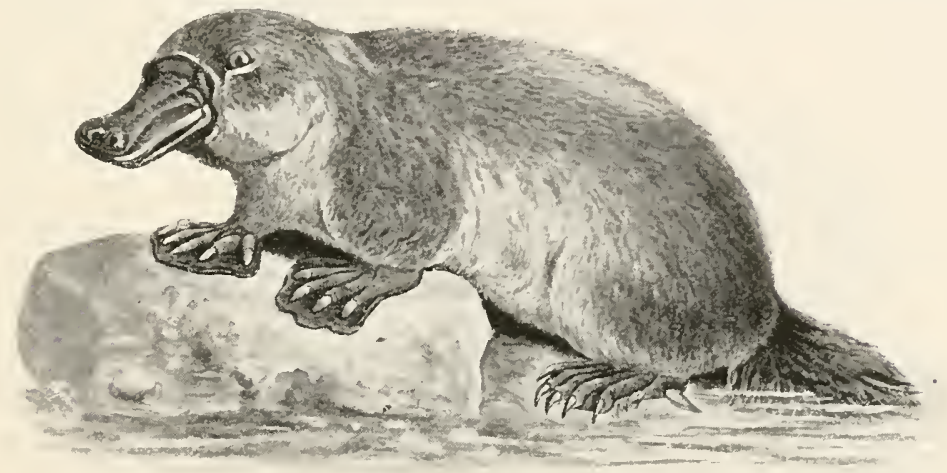

Fig. 1116.-Duck-Bill (Ornithorhynchus anatinus). (After Vogt and Specht.)

thongh nailless, opposable hallux, and elongated prehensile tail. A narsupium is sometimes present, but is absent or incomplete in the majority. One species - the Water Opossum-has the toes webbed. The Dasyuridx (Australian Native Cats, Tasmanian Devil, Thylacine, \&c.) often have the pollex rudimentary, the foot

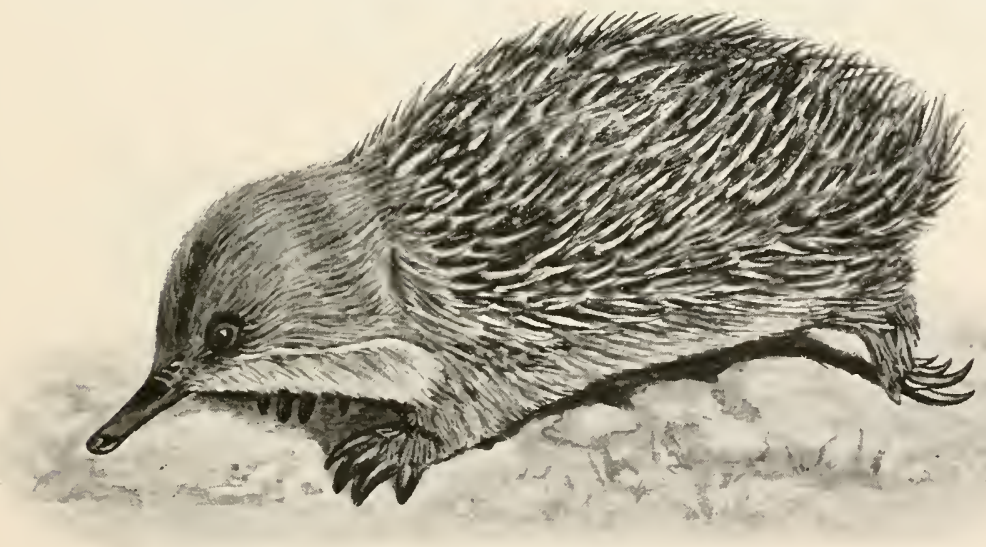

FIG. 1117.-Spiny Ant-eater (Echidna aculeata). (After Vogt and Specht.)

four-toed, the hallux, when present, small and clawless, and the tail .on-prehensile. There is a well-developed marsupium. The Native Cats (Fig. 1119) and their near allies are cat-like animals, the largest equal in size to a Domestic Cat, some no larger than Rats or Mice; 
the Tasmanian Devil has a more thickset body; the Thylacine has a remarkable resemblance in general shape, as well as size, to a Wolf. The Banded Ant-eater (Myrmecobius) is devoid of the marsupium. The Bandicoots (Peramelidee) are burrowing Marsupials, the

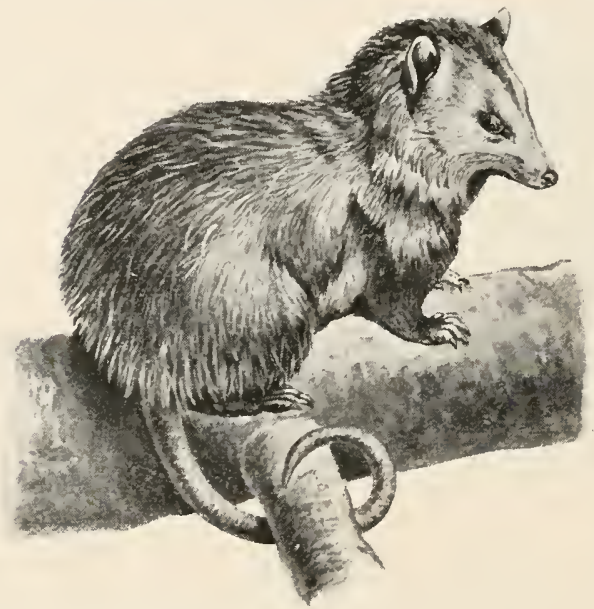

FIG. 1118,-Virginian Opossum (Didelphys virginiana). (After Vogt and Specht.)

size of which varies from that of a large Rat to that of a Rabbit. They $\mathrm{h}$ ave an elongated pointed muzzle, and, in some cases, large auditory pinnæ. The tail is usually short, sometimes long. The first and fifth digits of the fore-feet are vestigial or absent, the remaining three nearly equally developed. In the hindfoot the fourth toe is much longer and stouter than the others, while the second and third are small and slender, and united together by a web of skin, and the first is vestigial or absent. The marsupium has its opening directed backwards.

Notoryctes, the Marsupial Mole (Fig. 1121), is a small burrowing Marsupial, with short and powerful limbs, each with five toes,

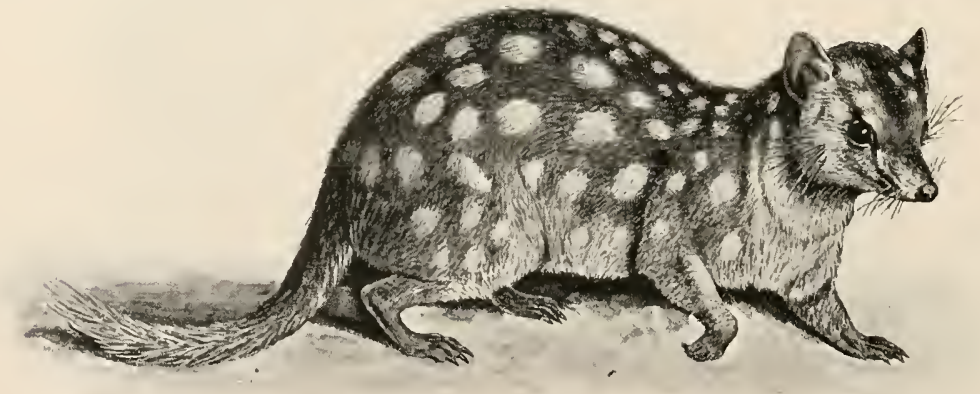

FIG. 1119.-Dasyure (Dasyurus viverrinus). (After Vogt and Specht.)

the third and fourth of the fore-foot provided with remarkable, large, flat, triangular claws. The tail is short and covered with bare skin. An auditory pinna is absent, and the eyes are vestigial. The pouch opens backwards, 
The Wombats (Phuscolomyida, Fig. 1122) are large, heavy, thiek-bodied, burrowing animals, with short, flattened heads, short,

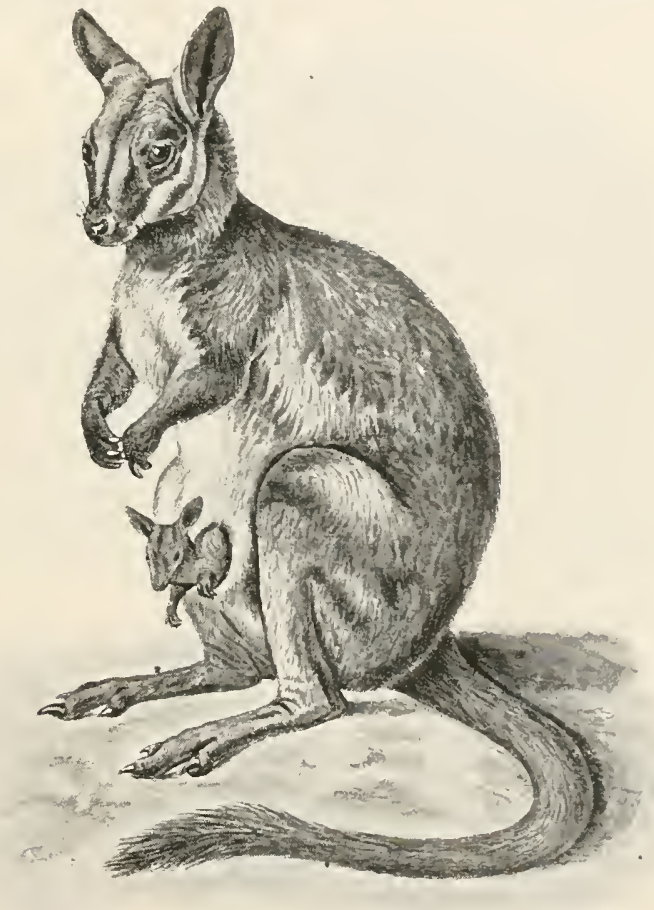

Fig. 1120,-Rock Wallaby (Petroyale xanthopus). (After Vogt and Specht.)

thiek limbs, provided with strong elaws on all the digits except the hallux, and with the seeond and third toes of the hind-

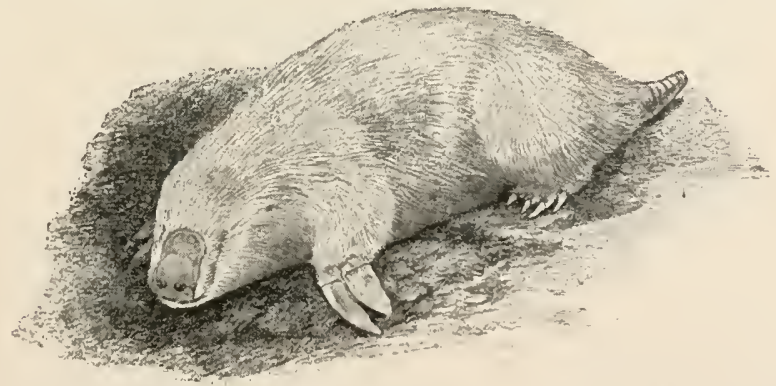

FIG. 1121.- Marsupial Mole (Notoryctes typhlops). (From the Cambrilye Natural History.

foot partly eonneeted together by skin. The tail is very short. The Kangaroos and their allies (Macropodidæ, Fig. 1120) are adapted, 
as regards their limbs, for swift terrestrial locomotion. They have a relatively small head and neck, the fore-limbs small, and - each provided with five digits; the hind-legs long and powerful;

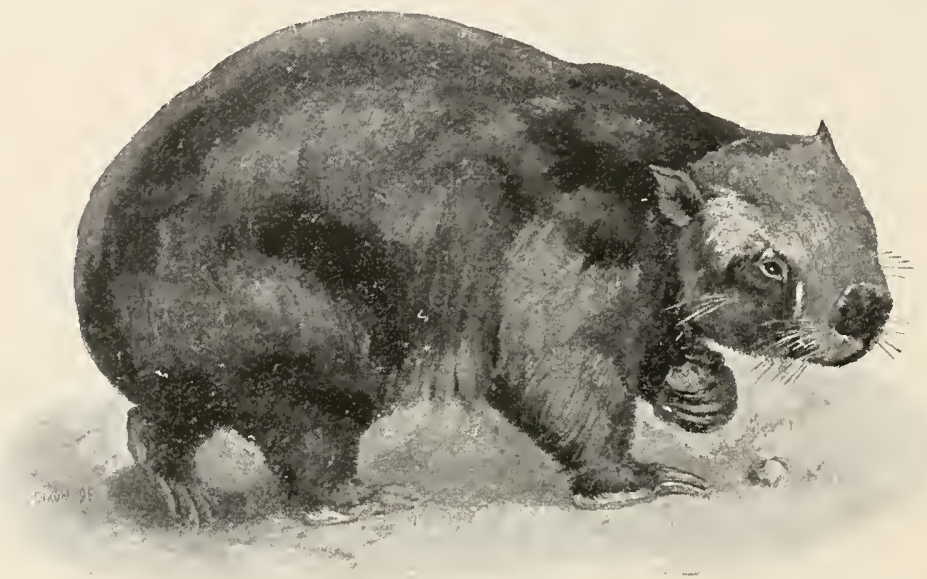

FIG. 1122.-Wombat (Phascolomys mitchelli). (From the Cambridge Natural IIistory.)

rapid progression is effected by great springing leaps, with the body inclined forwards and the fore-limbs clear of the ground. The foot is narrow and provided with four toes, the hallux being absent ; the two inner (second and third) small and united together

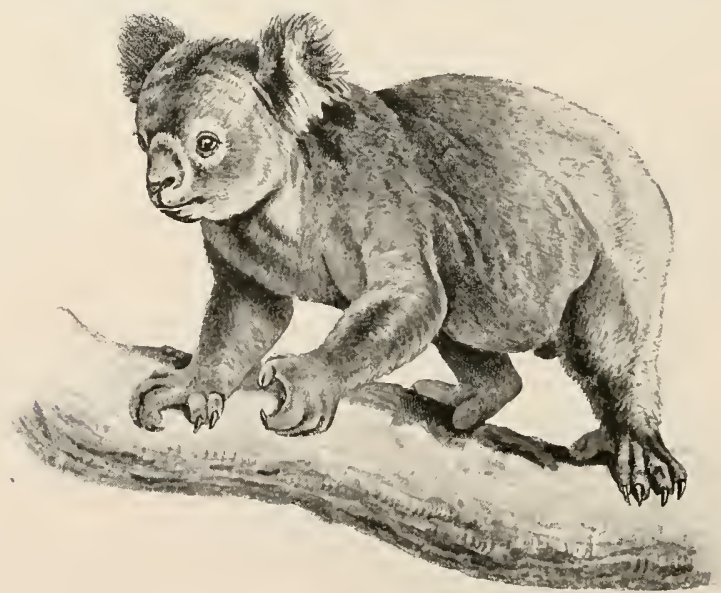

Fig. 1123.- Koala (Phascolarctos cinercus). (After Vogt and Specht.)

by integument, while the middle toe is very long and powerful. The tail is very long, and usually thick. There is a large marsupium. The Tree-Kangaroos differ from the ordinary Kangaroos in their 
shorter and thicker hind-limbs, in whieh the seeond and third toes are nearly as large as the fourth.

The Phalangers (Phalangerida) are climbing Marsupials which have both fore- and hind-feet prehensile; the second and third toes of the hind-foot slender and united by a web, as in the Kangaroo, but the hallux, which is nailless, opposable to them; the fourth and fifth nearly equal. The tail is well developed and prehensile. A number of Phal. angers (Flying Phalangers) are provided with lateral folds of skin extending from the fore- to the hind-limbs and, aeting as a parachute, enabling the animal, as in the Flying Squirrels, to perform flying leaps from tree to tree. The Koalas (Fig. 1123) differ from the Phalangers mainly in the relatively thieker body and the vestigial tail.

The Sloths (Bradypodida, Fig. 1124) are more completely adapted, in the structure of their limbs, to an ar-

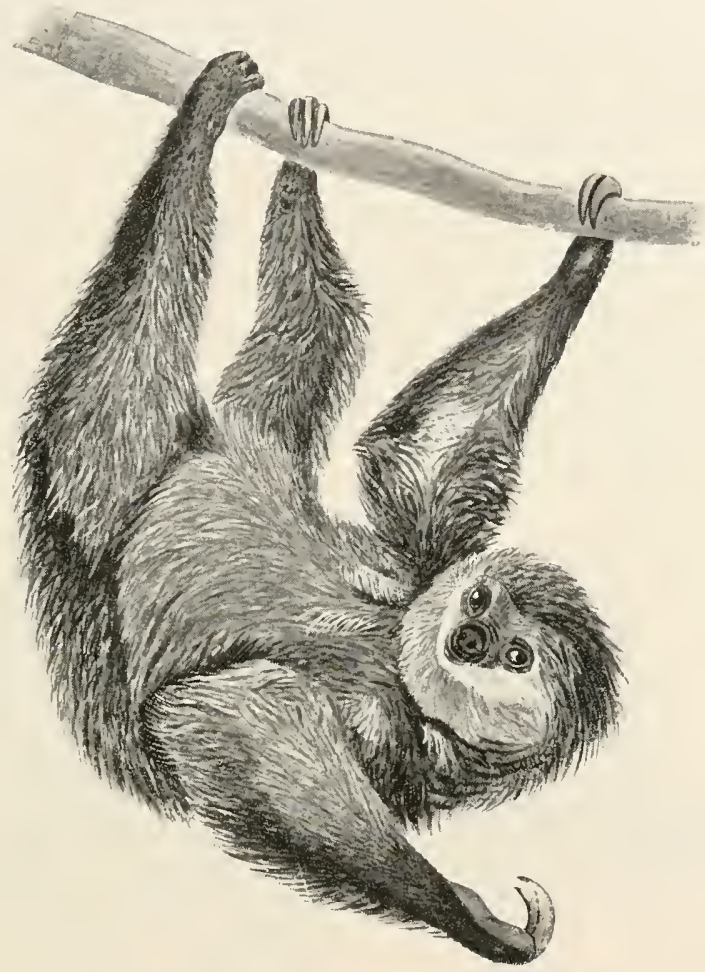

FIG. 1124.-Unau, or Two-toed Sloth (Cholopus diductylus). (Aiter Vogt and specht.)

boreal life than any other group of the Nammalia. They have a short, rounded head, with small pinnæ, and long, slender limbs, the anterior much longer than the posterior, with the digits, whieh are never more than three in number, long, curved, and hook-like, adapted for enabling the animal to hang and climb, body downwards, among the branches of trees. In the three-toed Sloth there are three toes in both manus and pes; in the two-toed Sloth there are only two in the manus, three in the pes. The tail is rudimentary. The body is covered with long, coarse hairs, which differ from those of other Mammals in being longitudinally fluted. On these hairs 
grows abundantly an alga, the presence of which gives a greenish tinge to the fur.

The ordinary Ant-eaters (Myrmecophagida) have a greatly elongated snout, with the mouth as a small aperture at its extremity, small eyes, and the auditory pinna sometimes small, sometimes well developed. There are five digits in the fore-foot, of which the third has always a very large curved and pointed claw, rendering the manus an efficient digging organ. The toes of the hind-foot, four or five in number, are sub-equal, and provided with moderatesized claws. In walking, the weight of the body rests on the dorsal surfaces of the second, third, and fourth digits of the manus and on a thick callous pad on the extremity of the fifth, and, in the pes, on the entire plantar surface. The tail is always very long, and is sometimes prehensile. The body is covered with long hair. In the Two-toed Ant-eater (Cycloturus) the muzzle is short; there are four toes in the manus, of which the second and third only have claws; that of the third being the longer ; the pes lias four sub-equal clawed toes, forming a hook not unlike the foot of the Sloths ; the tail is prehensile.

In the Armadillos (Dasypodide, Fig. 1125) the head is comparatively short, broad, and depressed. The number of complete

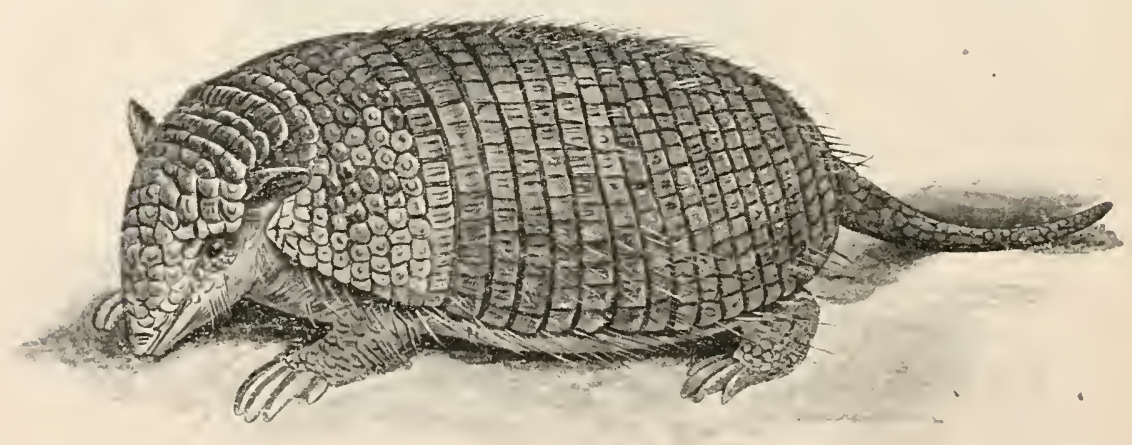

FIG. 1125.-Tatu Armadillo (Dasypus sexcinctus). (After Vogt and specht.)

digits of the fore-foot varies from three to five; these are provided with powerful claws, so as to form a very efficient digging organ. The hind-foot always has five digits with smaller claws. The tail is usually well developed. The most striking external feature of the Armadillos is the presence of an armour of bony dermal plates; this usually consists of a scapular shield of closelyunited plates covering the anterior part of the body, followed by a series of transverse bands separated from one another by hairy skin, and a posterior pelvic shield. In the genus Tolypeutes these bands are movable, so that the animal is enabled to roll itself up 
into a ball. The tail is also usually enclosed in rings of bony plates, and a number protect the upper surface of the head.

In the Scaly Ant-eaters (Manis, Fig. 1126) the head is produced

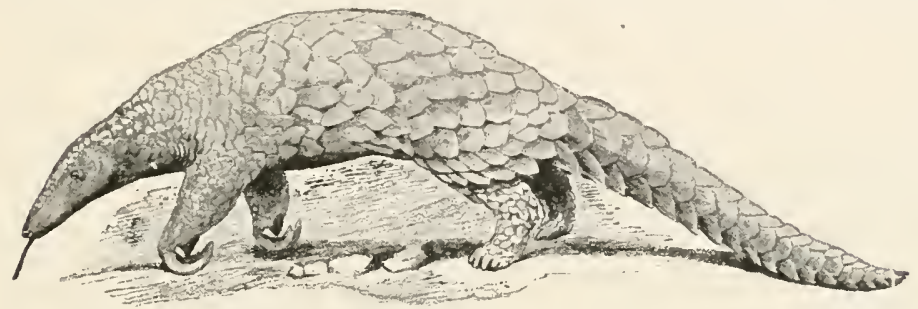

Fig. 1126.-Scaly Ant-eater (Hanis gigantea). (From the Cambridge Natural History.)

into a short, pointed muzzle. The limbs are short and strong, with five digits in each foot. The upper surface of the head and body, the sides of the latter, and the entire surface of the tail are covered with an investment of rounded, horny, epidermal scales. The lower surface is covered with hair, and there are a few coarse hairs between the scales. In walking, the weight rests on the upper and outer side of the fourth and fifth toes of the manus and on the sole of the pes.

The Aard-varks (Orycteropus, Fig. 1127) have a thick-set body,

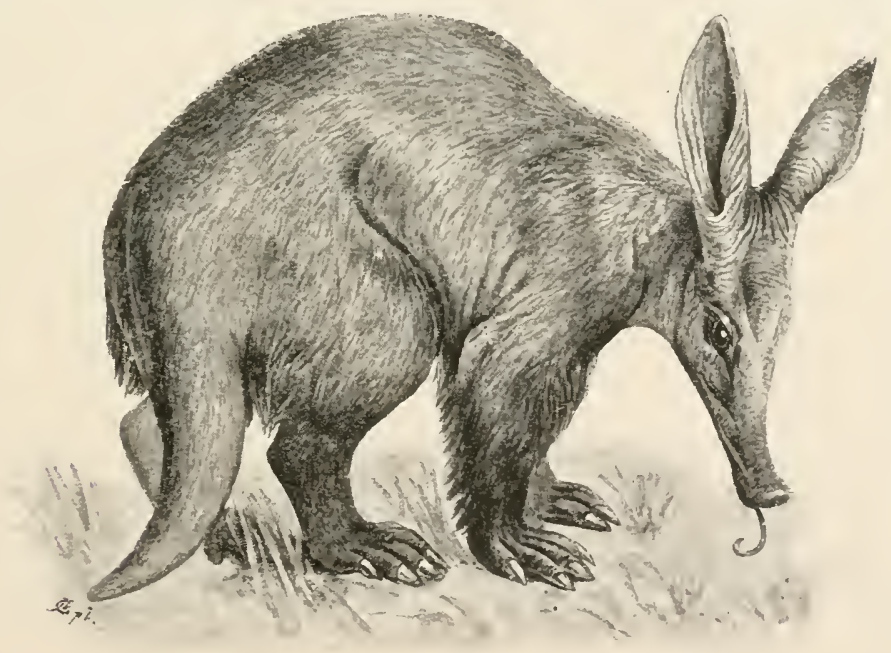

FiG. 1127.-Aard-vark (Orycteropus capensis). (After Vogt and Specht.)

the head produced into a long muzzle with a small tubular mouth, the pinnæ of great length, the tail long and thick. The fore-limbs are short and stout, with four toes, the palmar surfaces of which 
are placed on the ground in walking. The hind-limb is five-tocd. The surface is covered with thick skin with sparse hairs.

The Cetacea (Fig. 1128), among which are the largest of existing Mammals, some reaching a length of 80 or 90 feet, are characterised by the possession of a fusiform, fish-like body, tapering backwards

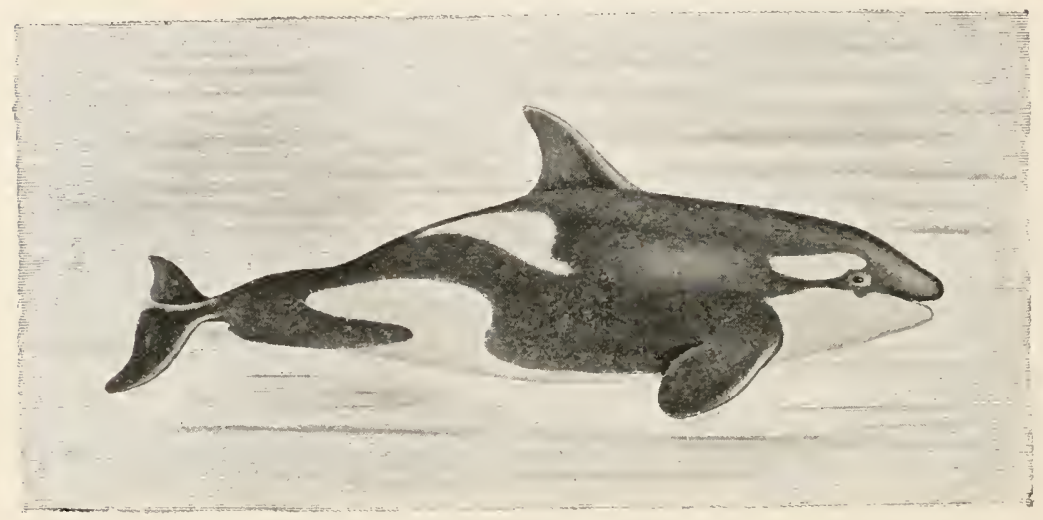

FIG. 1128.-Killer (Orca gladiator). (After True.)

to the tail, which is provided with a horizontally expanded caudal fin divided into two lobes or "flukes," and a relatively large head, not separated from the body by any distinct neck. A dorsal median fin is usually present. The fore-limbs take the form of flippers, with the digits covered over by a common integument, and devoid of claws; the hind-limbs are absent. The mouth is very wide; the nostrils are situated on the summit of the head, and the auditory pinna is absent. Hairs are completely absent, or are represented only by a few bristles about the mouth. In the Whalebone Whales the nostrils have two external slit-like apertures; in the toothed Whales, Porpoises, and Dolphins, on the other hand, the two nostrils unite to open by a single crescentic valvular aperture.

In the Sirenia also the body is fish-like, with a horizontal caudal fin, the fore-limbs flipper-like, the hind-limbs absent, and the integument almost hairless. But the body is distinctly depressed, and the head is by no means so large in proportion as in the Cetacea and has a tumid truncated muzzle, not far back from the extremity of which the nostrils are situated. There is no dorsal fin. The eyes are small, the pinnæ of the ears absent. The digits are in some cases provided with claws.

In the Ungulata vera the claws or nails are replaced by thick, solid masses, the hoofs, investing the ungual phalanges and bearing the weight of the body. The number of digits is more or less reduced, and the limbs as a whole are usually specially modified to act as organs of swift locomotion over the surface of the ground, their 
movements being restricted, by the nature of the articulations, to antero-posterior movcments of flexion and extension. The metacarpal and metatarsal regions are relatively very long. In the Artiodactyla the third and fourth digits of each foot form a symmetrical pair. In the Ruminants vestiges of the second and fifth digits are also commonly present; but these are usually not functional, never reaching the ground, though in the Reindeer they are better developed than in the others and have the effect of preventing the foot from so readily sinking in the snow. In the Camels the third and fourth digits alone are present. The Giraffes are distingnished from the other Ruminants by the enormous length of the neck. Characteristic of the Ruminants, though absent in the Camels and some others, are the cephalic appendages known as horns and antlers. The horns of the Hollow-horned Ruminants (Oxen, Sheep, Goats, Antelopes), sometimes developed in both sexes, sometimes only in the males, are horny sheaths supported by bony cores which are outgrowths of the frontal bones. In the Giraffe the horns, which are short and occur in both sexes, are bony structures covered with soft skin, and not at first attached by bony union to the skull, though subsequently becoming firmly fixed. Between them is a short rounded median bony protuberance on the frontal region of the skinll. The antlers of the Deer, which, except in the casc of the Rcindeer, are restricted to the male scx, are bony growths covered only while immature by a layer of skin, the "velvet," provided with very soft, short fur. Antlers are shed annually, and rencwed by the growth of fresh vascular bony tissue from the summit of a pair of short processes of the frontal bones, the pedicles. Eventually, when the antlers are full grown, a ring-like thickening of the bone, the "burr," appears round the base of the antler, and constricts the blood-vessels, so that the substance of the antler becomes converted into dry, dead bone; the skin shrivels and is peeled off. The antler is shed by the absorption of the bone immediately bencath the burr. The pinnæe of the ear of the Ruminants are well devcloped. The tail is sometimes elongated and provided with a terminal leash of long coarse hairs; sometimes short and bushy. The entire surface, with the exception of the end of the muzzle, which is naked, is always covered with a close coat of longer or shorter hairs.

In the Pigs the legs are relativcly short, and the two latcral toes of both manus and pes are fully developed, though scarcely reaching the ground. The surface is covered with a scanty coat of coarse bristles. There is a truncate, mobile snout, the anterior end of which is disc-shaped and free from hairs. The pinnæ are large; the tail is rather long, narrow, and cylindrical, provided with a terminal tuft of strong hairs. A remarkable feature of the males is the development of the caninc teeth of both jaws into large, upwardly-curved tisks. In the Peccaries, which resemble 
the Pigs in most of the features mentioned, the points of the upper tusks are directed downwards.

In the Hippopotami (Fig. 1129) the body is of great bulk,

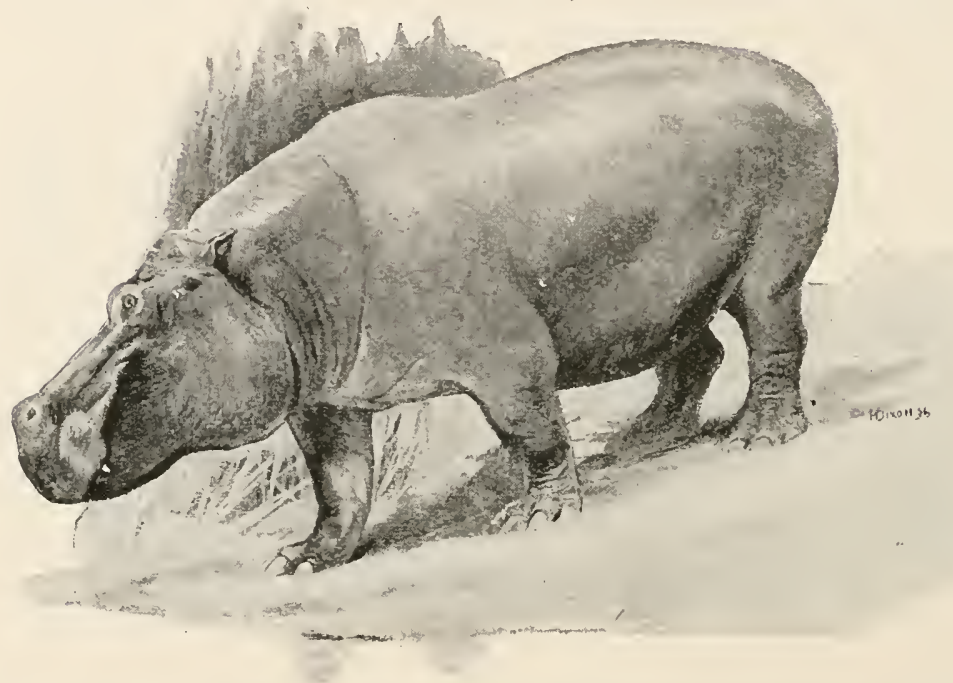

FIG. 1129.-Hippopotamus (Hippopotamus amphibius). (From the Cambridge Natural History.)

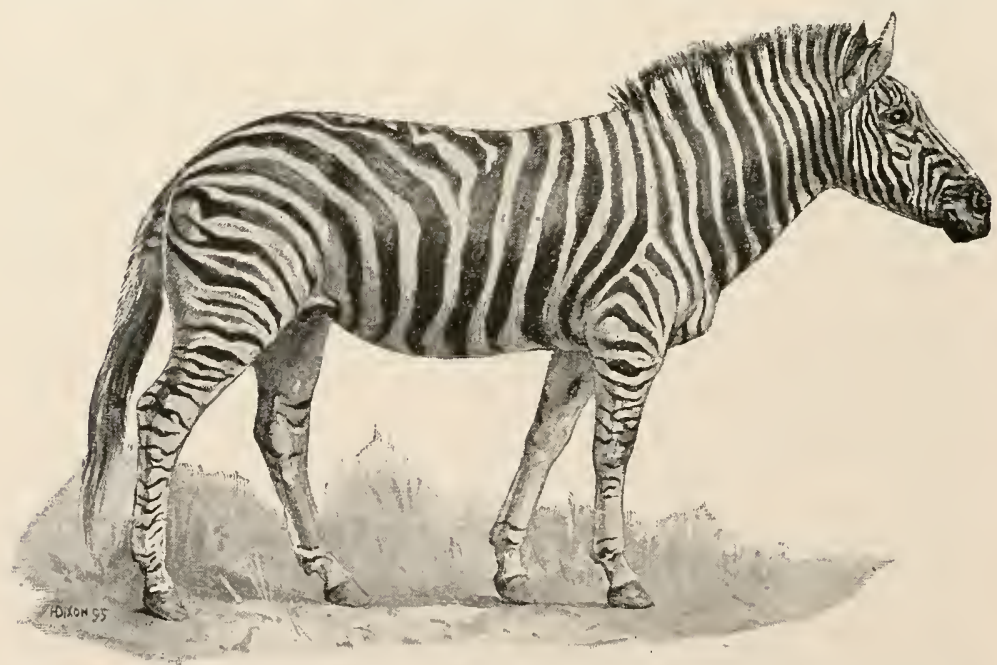

FIG. 1130.-Burchell's Zebra (Equus burchelli). (From the Cambridge Natural History.)

the limbs very short and thick, the head enormous, with a transversely expanded snout, prominent eyes, and small pinnæ. The tail is short and laterally compressed. The toes are four in each 
manus and pes, all reaching the ground. The surface is naked, with only a few hairs in certain positions; the skin is of great thickness.

In the Perissodactyles the third digit is either the only complete one in both fore- and hind-foot (Horses), or there are only three digits - second, third, and fourth-in each (Rhinoceroses), or there are four in the fore-foot and three in the hind (Tapirs). The "Horses" (Equidæ, Fig. 1130) have the distal divisions of the limbs slender, the metacarpals and metatarsals nearly vertical to the surface of the ground; the single hoof massive and with a broad lower surface. Though the head is elongated, the nasal region is not produced into a proboscis. The tail is short or moderately long, and is either beset throughout with a large number of very long, coarse hairs, or with a tuft of such specially developed hairs at the extremity. A mane of similar large hairs usually runs along the dorsal surface of the neck. There is a wart-like callosity above the wrist, and in the true Horses a second a little below the heel or "hock."

The Tapirs (Fig. 1131) have the body more massive than the Horses, and the limbs, especially the distal segments, shorter and

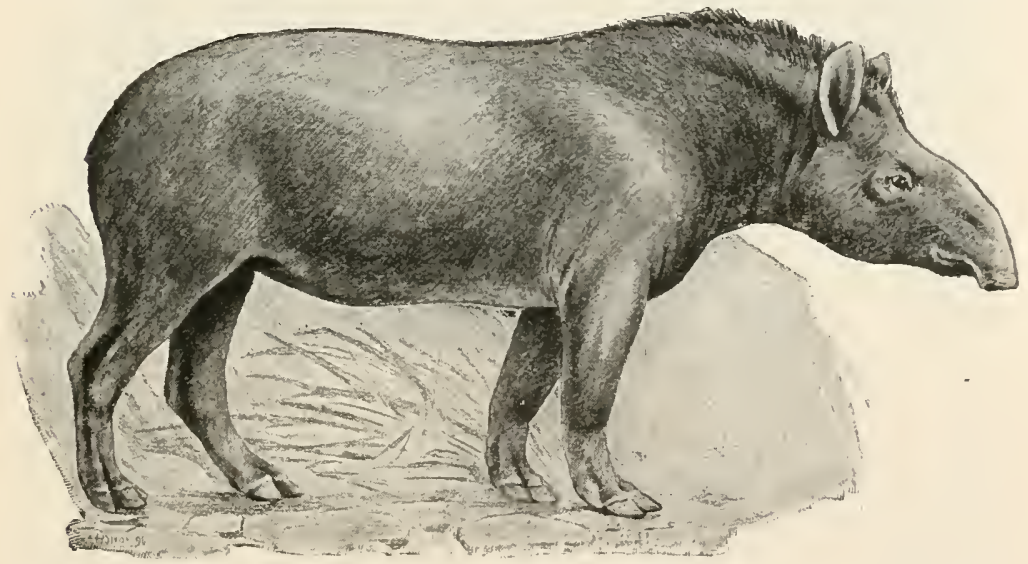

FIG. 1131.-American Tapir (Tapirus terrestris). (From the Cambridge Vatural History.)

stouter. The nasal region is produced into a short proboscis. The surface is beset with a scanty covering of hairs. The tail is vestigial.

In the Rhinoceroses (Fig. 1132) the body is extremely massive, the limbs short and stout, each digit provided with a hoof-like nail. There is a short, soft muzzle. Either one or two remarkable median horns are borne on the nasal region, not attached directly to the skull: these are epidermal structures which are formed of a dense aggregation of slender fibre-like elements. The eyes are 
small, the auditory pinnæ well developed. The surface is devoid, or nearly devoid, of hairs, and the skin is enormously thick and in some species thrown into deep folds. The tail is narrow and of moderate length.

The Hyraxes are small, somewhat Rabbit-like animals, with slender limbs and vestigial tail. There are four functional digits in the manus and three in the pes, all provided with short, flat nails, except the innermost of the pes, which has a curved claw. The body is covered with soft fur.

The Elephants, the largest of existing terrestrial Mammals, have the limbs much more typically developed than in the true Ungulates, there being five comparatively short digits, enclosed in a common integument in each foot, all of them in the fore- and

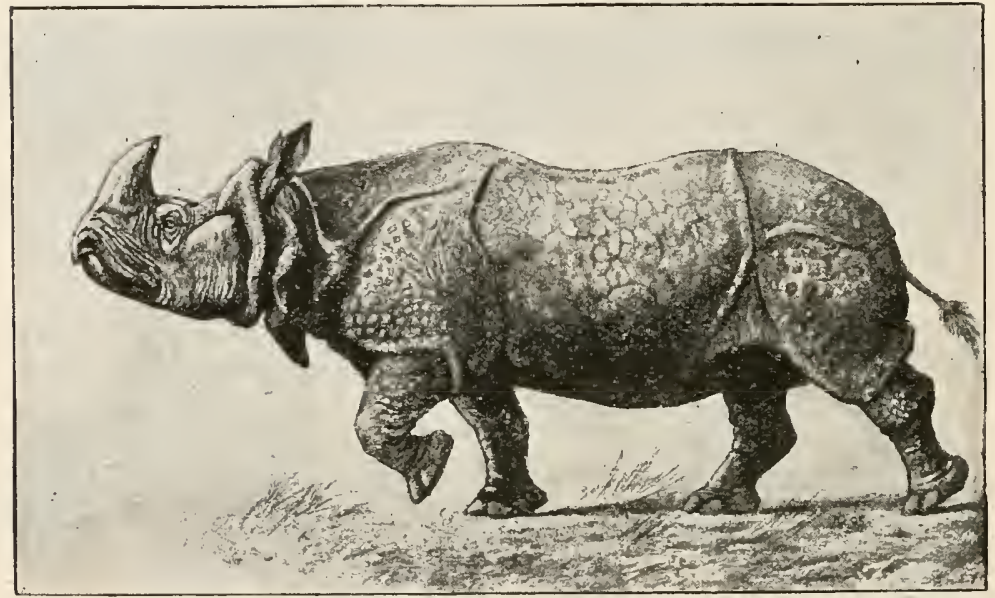

FIa. 1132.-Indian Rhinoceros (Rhinoceros indicus). (From the Cambridge Natural History.)

three or four in the hind-foot, terminating in a broad, flat nail or hoof. The weight of the body is borne on an integumentary pad forming the sole of the foot. The limbs are very stout and pillar-like, and the thigh and leg when at rest are in a straight line instead of being, as in the Ungulata vera, placed nearly at right angles to one another-a circumstance which gives a characteristic appearance to the hind-quarters. The nasal region is produced into a proboscis or "trunk," a mobile cylindrical appendage, longer than the rest of the head, at the extremity of which the nostrils are situated. There is in the male a pair of immense tusks-the incisors of the upper jaw. The eyes are small, the pinnæ of the ears enormous. The tail is small, and provided with a tuft of hairs at its extremity. The skin is very thick, and provided with only a scanty hairy covering. 
In the Carnivora vera the typical number of digits is sometimes present, or, more usually, there are five. in the fore- and four in the hind-foot, or four in each. The extremities of the digits are provided with compressed, curved claws, which may be very long and sharp, when they are capable, when not in use, of being retracted into a sheath of skin situated at their bases; or relatively short and blunt, when they are incompletely, or not at all, retractile. The Dogs (Canid $x)$ and Cats (Felid $x)$ are digitigrade, the Bears (Ursid $x$ ) and allied groups plantigrade (p. 501). The Otters (Lutra) differ from the rest in having short limbs with the toes connected by webs of skin.

The Pinnipedia (Fig. 1133) have the proximal segments of the limbs short, so that the arm and thigh and nearly all the fore-arm and leg are enclosed in the common integument of the trunk; the manus and pes are elongated. The Earless Seals (Phocidce) are much more completely adapted to an aquatic life than the Eared

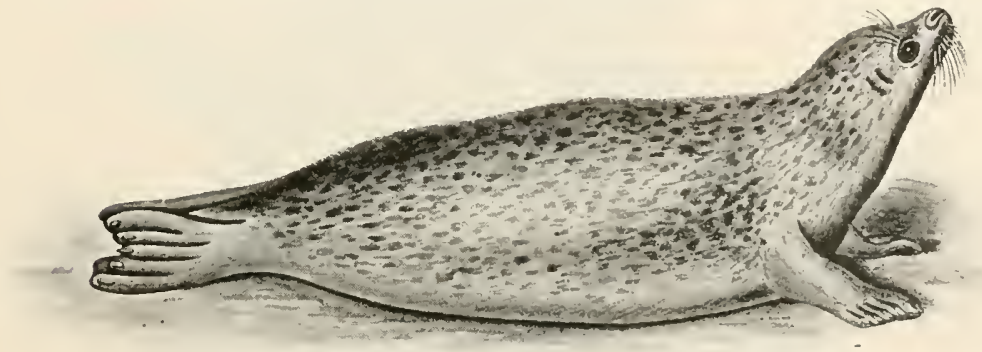

Frg, 1133.-Seal (Phoca ritulina).

Seals (Otariid $x$ ) and Walruses (Trichechides), being unable to flex the thigh forwards under the body so that the hind-limbs may aid in supporting the weight, and thus being only able to drag themselves along very awkwardly when on dry land. The pinna of the ear is absent in the Earless Seals and Walruses, well developed in the Eared Seals. The surface in all is covered with a thick, soft fur. In the Fur-Seals there are two kinds of hairs-those of the one kind being longer and coarser, and scattered through the more numerous shorter and finer hairs composing the fur proper. A remarkable feature of the Walruses is the presence of a pair of large tusks-the enlarged canine teeth-projecting downwards from the upper jaw.

Some of the Rodents (Beavers, Water-Voles) are aquatic, some (Squirrels and Tree-Porcupines) are arboreal, while others (the majority of the order) lead a terrestrial life, and are active burrowers. They are on the whole a very uniform group, and exhibit few such remarkable modifications as are to be observed in some of the other orders of Mammals. They are nearly all furry 
animals with five-toed plantigrade or semiplantigrade limbs. The tail is usually elongated, and may be naked or covered with fur ; but sometimes, as in the Rabbits and Hares, it is very short. A few special modifications, however, have to be noted in certain families of Rodents. The Flying Squirrels have on each side a fold of skin, the patagium, which serves as a parachute. The African Flying Squirrels (Anomalums) are remarkable also on account of the presence of a series of overlapping horny scales on the lower surface of the basal part of the tail. The Jerboas (Dipus)

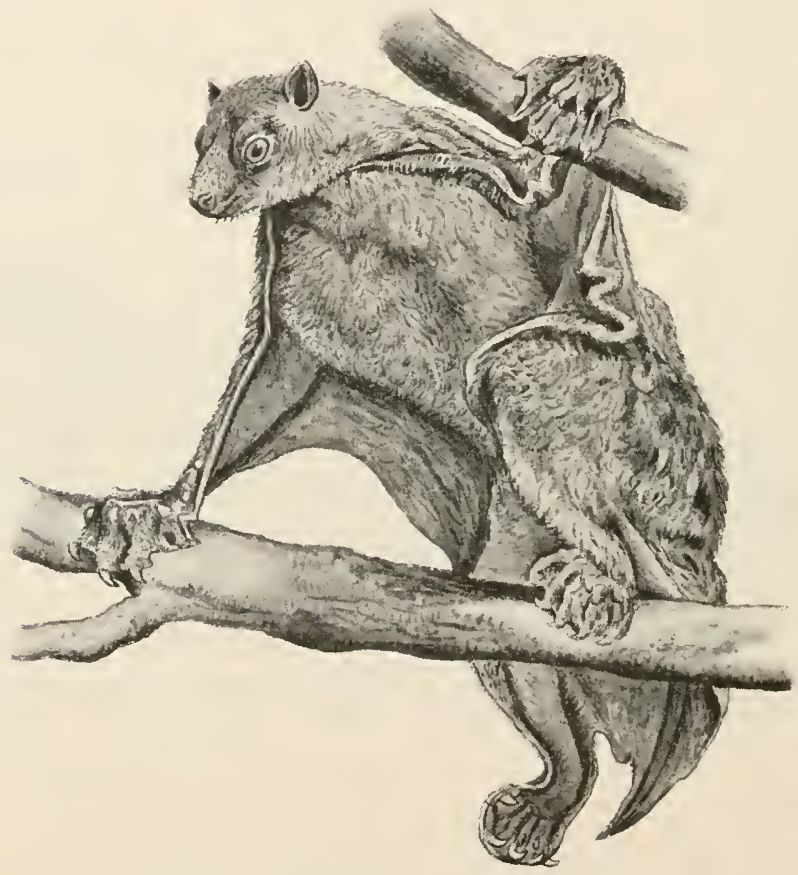

Fig. 1131.-Galeopithecus. (After Vogt and Specht.)

and their allies are characterised by the great relative length of the hind-limbs - the mode of locomotion of these remarkable Rodents being by a series of leaps not unlike those of the Kangaroo - and by the rednction of the number of the toes to three in some of them. The Porcupines (Hystricida) have numerous elongated spines or "quills" among the hairs of the dorsal surface, and some of them have prehensile tails. The Agoutis (Dasyprocta) and the Capybara (Hydrochcerus) have hoof-like claws, the latter having webs between the digits.

The Insectivora are, in general, small, furry, burrowing Mammals with plantigrade limbs and an elongated muzzle. But there is a 
considerable range of modification within the order in adaptation to different modes of life. The Colugos (Galeopithecus, Fig. 11:34) have a fold of skin (patagium) extending along each side of the neck and body and continued between the hind-legs, enclosing the tail ; the fore- and hind-feet are both webbed, and the tail is prehensile. The Hedgehog (Erinaceus) has the surface beset with pointed spines. The Moles (Talpa) and their allies, which are active burrowers, have the limbs very short and stont and provided with extremely strong claws. The jumping Shrews (Maeroscelida) have slender limbs adapted to progressing by leaps on the surface of the ground.

The Chiroptera (Fig. 1135) are the only Mammals which are capable of active flight. The fore-limbs have the segments greatly elongated, especially the fore-arm and the four ulnar digits, and these support a thin fold of the integument which stretches to the hind-limbs and constitutes the wing. $A$ fold (inter-femoral

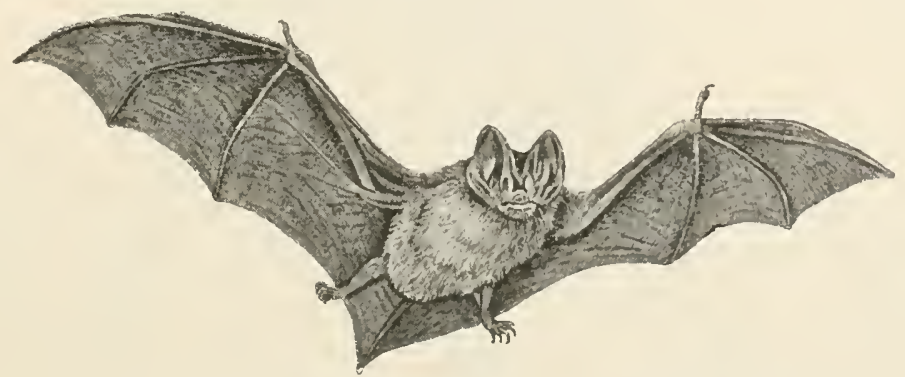

Fıa. 1135,-Bat (Synotus barbastellus). (After Vogt and specht.)

membrane) also extends between the liind-limbs, and may or may not involve the tail. The pollex is much shorter than the other digits, is directed forwards, and terminates in a well-developed curved claw ; in the Megachiroptera, but not in the Microchiroptera, the second digit also has a claw ; the other digits are always clawless. The position of the hind-limbs is peculiar, and the knee is directed backwards instead of forwards as in other Mammals; the five digits of the foot are all provided with claws. So complete is the adaptation of the limbs to the purpose of flight that Bats are only able to shuffle along with great difficulty on the ground ; though with the aid of their claws they are able to climb and to suspend themselves from branches of trees by the hind-feet. In the Megachiroptera the muzzle is nearly always elongated, and the pinna of the ear simple, while in the Microchiroptera the muzzle is short, the pinna usually complicated by the presence of an inner lobe or tragus, and often produced into remarkable arborescent appendages, and the nose also often provided with elaborate leaflike or arborescent lobes. The surface is usually covered with 
soft fur, except in one group of Microchiroptera in which the integument is practically naked. The tail is sometimes short, sometimes well developed ; in the latter case it may or may not be involved in the tail-membrane.

In the Lemurs and their allies (Prosimii) the body is slender, and the limbs adapted for an arboreal existence. The hallux is divergent from the other digits of the foot and opposable to them, and the same holds good, in some cases, of the pollex. In some, all the digits are provided with claws, in others all but the hallux. More commonly all the digits have flat nails, except the second of the pes, which always has a claw. The eyes are very large. The muzzle is sometimes elongated, sometimes short; the nostrils are slit-like. The tail is sometimes absent or short; more usually it is greatly elongated, but it is never prehensile. The surface is always covered with soft fur.

Of the Anthropoidea the Hapalidx or Marmosets are small squirrel-like animals with all the digits except the hallux provided with pointed claws, with the pollex incapable of opposition, the tail non-prehensile, and without cheek-pouches or callous patches over the ischia. The Cebidæ resemble the Hapalidæ in the negative characters of the absence of ischial callosities and of cheek-pouches, and of the power of opposition in the hallux. But the limbs are much longer, the digits are all provided with flat nails, and the tail is frequently prehensile. The Cercopithecidæ all have brightlycoloured, bare, callous patches of skin (callosities) over the ischia, and most of them have cheek-pouches for the storage of food. All the digits are provided with flat nails. The tail may be long, or short, or absent; when present it is never prehensile. The pollex, when developed, is always opposable to the other digits. In the Simiidæ or Man-like Apes (Fig. 1136) a tail is never developed, and there are no cheek-pouches; ischial callosities are present only in the Gibbons. The Gibbons can walk in an upright position, without the assistance of the fore-limbs; in the others, though, in progression on the surface of the ground, the body may be held in a semi-erect position with the weight resting on the hind-limbs, yet the assistance of the long fore-limbs acting as crutches is necessary to enable the animal to swing itself along.

Endoskeleton.-The spinal column of Mammals varies in the number of vertebræ which it contains, the differences being mainly due to differences in the length of the tail. The various regions are very definitely marked off. In the cervical region the first two vertebræ are modified to form the atlas and axis. Owing to the absence of distinct cervical ribs, the posterior cervical vertebræ are much more sharply marked off from the anterior thoracic than is the case in Reptiles and Birds. The vertebræ of the cervical region have double transverse processes (or a transverse process perforated at the base by a foramen) in all except the last. The 
lower portion of the transverse process in certain cases (e.g., seventh and sometimes some of the others in Man) arises from a separate ossification, and this is regarded as evidence that the lower part, even when not independently ossified, represents a cervical rib. Seven is the prevailing number of vertebre in the cervical region; there are only three exceptions to this-the Manatee, Hoffmann's Sloth, and the three-toed Sloth ( $f f . p .509)$. The number of thoracic and lumbar vertebræ is not so constant; usually there are between nineteen and twenty-three. Hyrax has a larger

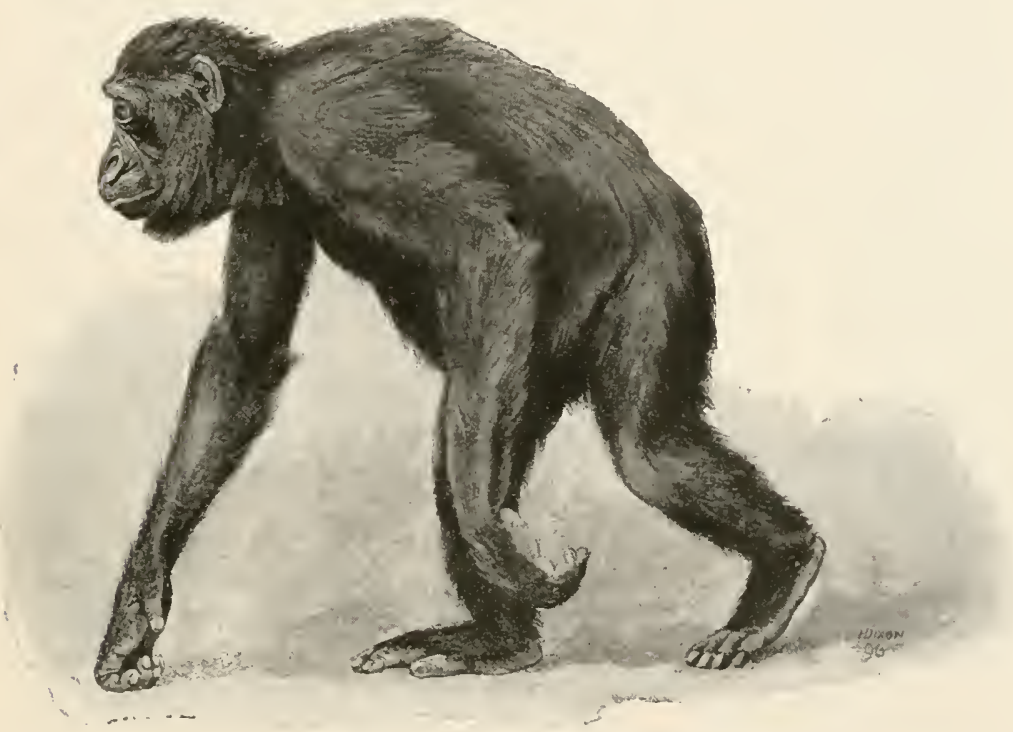

FIG. 1136.-Gorilla. (From the Cambridge Natural History.)

number of thoraco-humbar vertebræ than any other Mammal-from twenty-nine to thirty-one.

The thoracic vertebræ have ribs which are connected, either directly or by intermediate ribs, with the sternal ribs, and through them with the sternum. Each rib typically articulates with the spinal column by two articulations one articular surface being borne on the head and the other on the tubercle. The tubercle articulates with the transverse process, and the head usually with an articular surface furnished partly by the vertebra with which the tubercle is connected, and partly by that next in front; so that the head of the first thoracic rib partly articulates with the centrum of the last cervical vertebra.

In all the Mammalia in which the hind-limbs exist, that is to say, in all with the exception of the Sirenia and the Cetacea, there is a 
sacrum consisting of closely united vertebræ, the number of which varies in the different orders. The caudal region varies greatly as regards the degree of its development. In the caudal region of many long-tailed Mammals there are developed a series of chevron bones-V-shaped bones, which are situated opposite the intervertebral spaces.

The centrum of each vertebra ossifies from three centres ${ }^{1}-\mathrm{a}$ middle one, an anterior, and a posterior. The middle centre forms the centrum proper; the anterior and posterior form the epiphyses. The epiphyses are almost entirely absent in the Monotremes, and in the Dugong (Sirenia) have not been detected. Between successive centra are formed a series of discs of fibro-cartilage-the intervertebral discs - represented in lower Vertebrates only in Crocodiles and Birds. The anterior and posterior surfaces of the centra are nearly always flat.

The stermum consists of a number of segments - the presternum in front, the mesostermu, or corpus sterni, composed of a number of segments or sternebra, in the middle, and the xiphisternum behind. The sternum is formed in the fotus in great part by the separating off of the ventral ends of the ribs. Some of the Cetacea and the Sirenia are exceptional in having a sternum composed of a single piece of bone. The sternal ribs, by which the vertebral ribs are connected with the sternum, are usually cartilaginous, but frequently undergo calcification in old animals, and in some cases early become completely converted into bone.

The skull of a Mammal (Fig. 1137) contains the same chief elements and presents the same general regions as that of the Sauropsida, but exhibits certain special modifications. A number of the bones present in the skull of Sauropsida are not represented, or, at all events, not certainly known to be represented definitely by separate ossifications in the Mammalia. Such are the supraorbital, the pre-frontal, the post-orbital, the ecto-pterygoid and the quadrato-jugal. The bones of the skull, with the exception of the auditory ossicles, the lower jaw, and the hyoid, are all immovably united together by means of sutures.

The palatine bones develop palatine plates separating off a posterior nasal passage from the cavity of the mouth, a condition found among the Sauropsida only in the Crocodilia, and, to a less extent, in the Chelonia and some Lizards.

The zygomatic arch is a strong arch of bone formed partly of the squamosal, partly of the jugal, and partly of the maxilla: in position it represents the upper temporal arch of Amphibia and Sauropsida, but is differently constituted (see p. 339). The orbit in the skull of some Mammals is completely enclosed by bone, constituting a well-defined cavity ; in others it is not completely

1 Usually the two centres of ossification which form the neural arches also cuntribute to the formation of the bony centrum. 
surrounded by bone behind, and so communicates freely with the temporal fossa, which lies behind it.

The periotic bones (pro-otic, opisthotic, and epi-otic) are not separately represented in the skull of Mammals. Part of the periotic

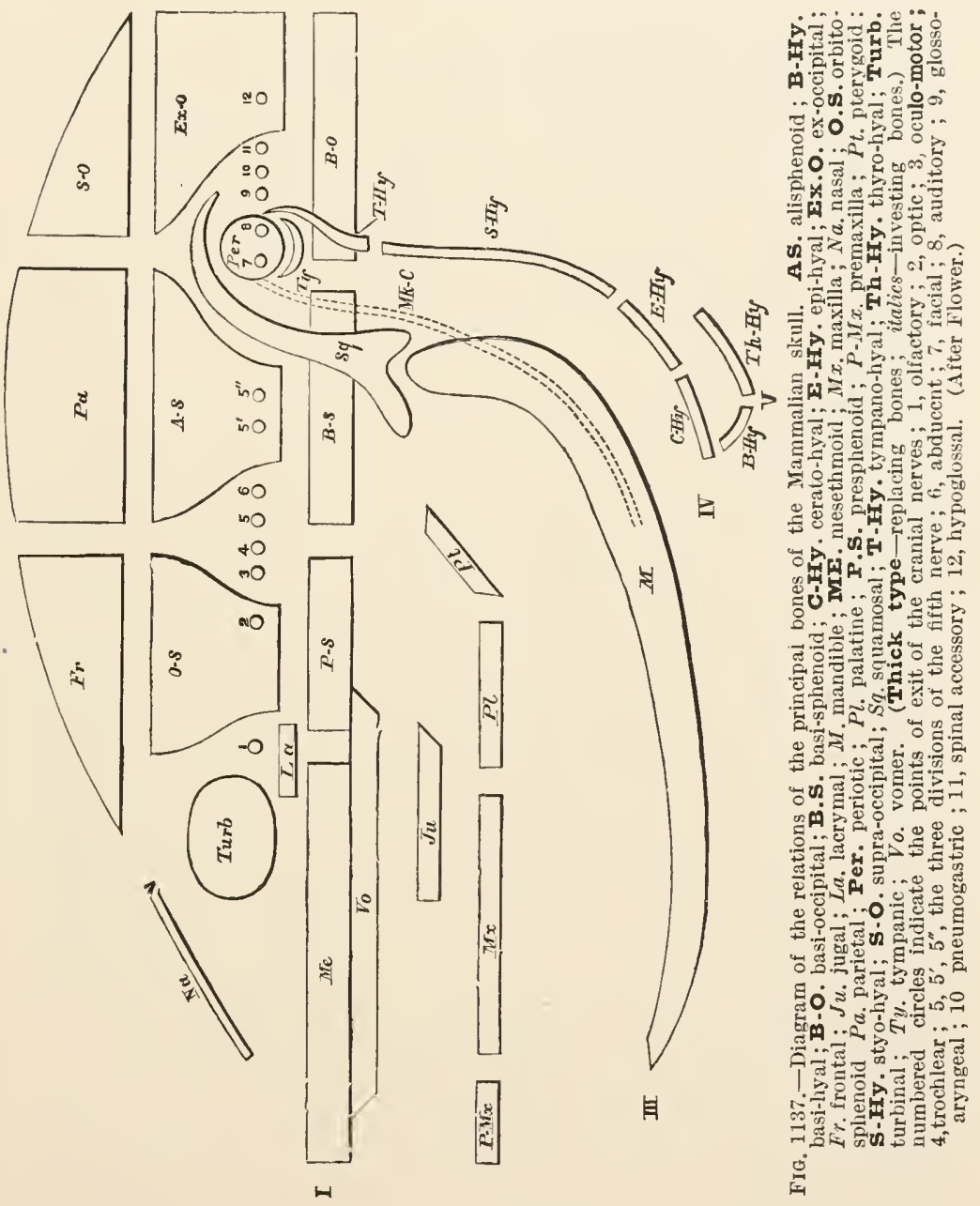

mass sometimes projects on the exterior at the hinder part of the lateral region of the skull, and is the mastoid portion; the rest is commonly called the petrous portion of the periotic, and encloses the parts of the internal ear-the mastoid portion containing only air-cells. The tympanic bone, which perhaps represents the quadrato-jugal of Sauropsida, sometimes only forms a long tube, vOL. II 
sometimes a mere ring of bone. In other cases it not only gives rise to a tube for the external auditory meatus, but also forms the bulla tympani, a dilated bony process containing a cavity.

The occipital region presents two condyles for the articulation of the atlas.

The mandible consists in the adult of one bone, the equivalent of the dentary of Sauropsida, on each side-the two rami, as they are called, being in most Mammals closely united at the symphysis. The mandible articulates with an articular surface formed for it by the squamosal bone, below the posterior root of the zygomatic arch.

The hyoid consists of a body and two pairs of cornua-anterior and posterior; of these the anterior pair are usually longer, and consist of several bones, the most important and most constant of which is the stylo-hyal, connected usually with the periotic region of the skull. The posterior cornua or thyro-hyals are usually much smaller.

The ratio borne by the capacity of the cranial cavity to the extent of the facial region varies greatly in the different orders. The greater development of the cerebral hemispheres in the higher groups necessitates a greater development of the corresponding cerebral fossa of the cranium. This is brought about by the bulging upwards, forwards, and backwards of the cranial roof, resulting in a great modification in the primitive relations of certain of the great planes and axes of the skull (Fig. 1138). Taking as a fixed base line the basi-cranial axis-an imaginary median line running through the basi-occipital, basi-sphenoid, and pre-sphenoid bones-we find that the great expansion of the cerebral fossa in the higher Mammals leads to a marked alteration in the relations to this axis (1) of the occipital plane or plane of the foramen magnum ; (2) of the tentorial plane or plane of the tentorium cerebelli (a transverse fold of the dura mater between the cerebral hemispheres and the cerebellum); and (3) of the ethmoidal plane or plane of the cribriform plate of the ethmoid. In the lower Mammals $(A)$ these are nearly at right angles to the basi-cranial axis. In the higher groups, by the bulging forwards and backwards of the cranial roof, the occipital and tentorial planes incline backwards and the ethmoidal forwards, until all three may become approximately horizontal. At the same time there is produced a change in the relations of the basi-cranial axis to the basi-facial axis-a line passing along the axis of the face between the mesethmoid and the vomer. In the lower forms the angle at which the basi-facial axis, when produced, meets the basi-cranial is an exceedingly open one; in the higher forms, owing to the downward inclination of the facial region, this angle decreases in size, though it is never reduced to less than a right angle.

The pectoral arch of the Theria has fewer distinct elements than that of the Sauropsida. The coracoid, which in the latter is a large 
bone, taking a share at its dorsal end in the bounding of the glenoid cavity, and at its ventral end articulating with the sternum, is
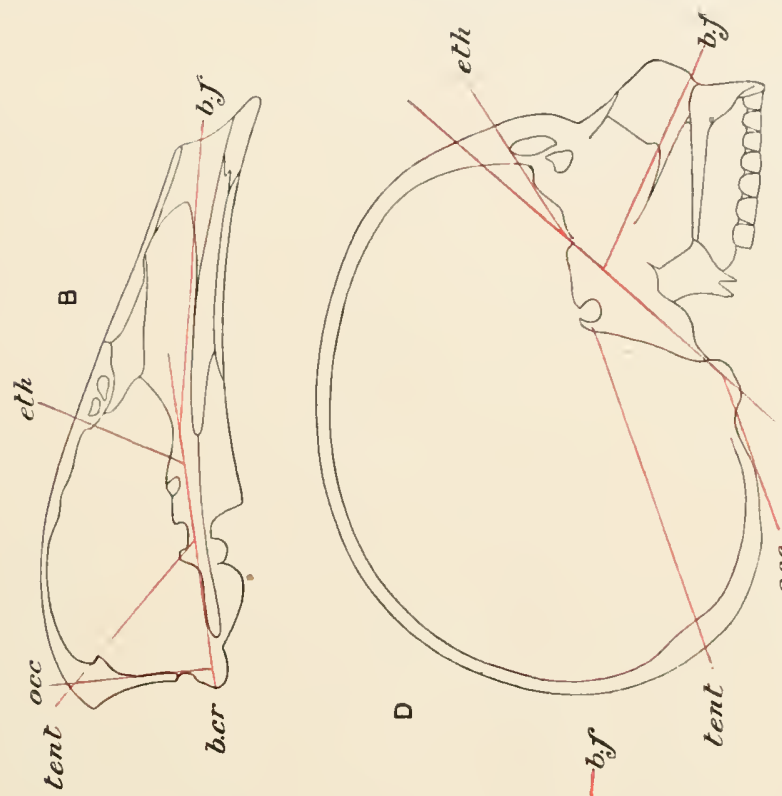

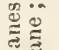
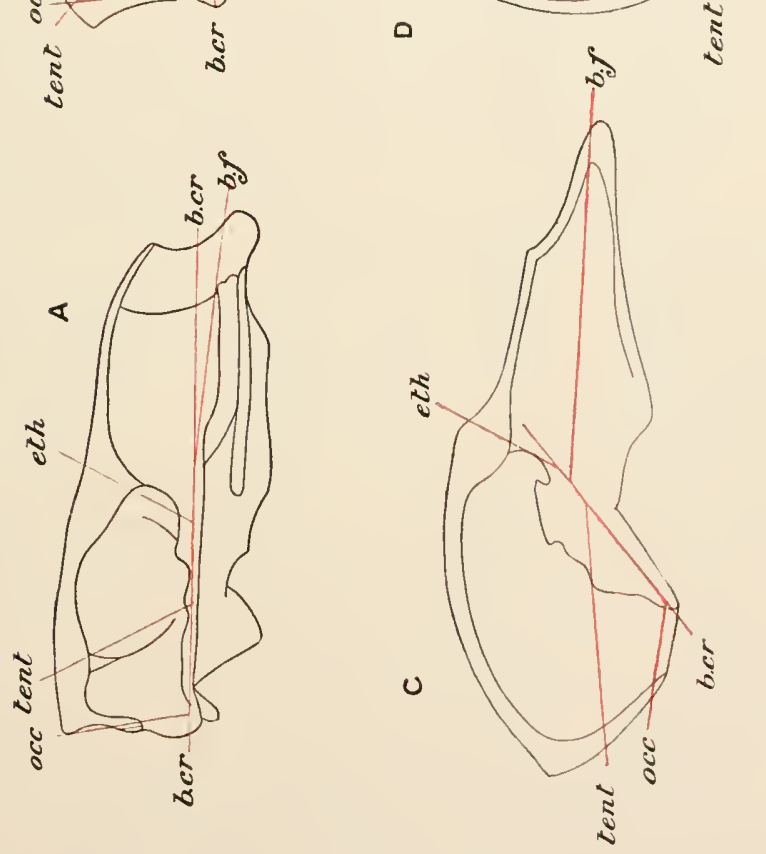

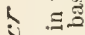

$\leqslant$ का

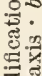

过

है.

$=\frac{1}{30}$

का एँ

$\frac{5}{3}$

要

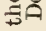

फิ

की $\cdots$

政

never present, in the adult, as a distinct bone. In the young of many Mammals it appears to be represented by a small ossification 
which enters into the glenoid facet; but this very soon coalesces with the scapula. The coracoid process, which is a separate ossification in the young Mammal, and, though in most instances completely fusing with the scapula and with the smaller coracoid element, is sometimes recognisable as a distinct element up to a late period (many Marsupials, Sloths), appears to correspond to the bone called epicoracoid in the Prototheria (vide p. 505). In fotal Marsupials the coracoid is represented by a well-developed cartilaginous element which extends inwards and meets the rudiment of the sternum.

In the scapula a spine is nearly always developed, and usually ends in a freely-projecting acromion-process. It is developed, unlike the main body of the scapula, without any antecedent formation of cartilage, and is perhaps to be compared with the cleithrum, an investing bone occurring in some Amphibia and Reptilia (p. 293). A clavicle is well developed in many Mammals, but is incomplete or absent in others ; its presence is characteristic of Mammals in which the fore-limbs are capable of great freedom of movement. In the embryo of the Theria there is, in the position of the clavicular bar, a bar of cartilage, which coalesces with its fellow in the middle line. The cartilaginous tract thus formed segments into five portions-a median, which coalesces with the pre-sternum, two small inner lateral, which unite with the clavicles or are converted into the sterno-clavicular ligaments, and two long outer lateral, which give rise to the clavicles. The median and inner lateral portions appear to correspond to the episternum of Reptiles and Prototheria. An additional small cartilage may represent the inner portion of the procoracoid of Amphibia. A piece of cartilage at the outer end of the clavicle proper is sometimes distinguishable - the meso-scapular segment.

In the carpus there are four proximal bones-scaphoid, lunar, cuneiform, and pisiform. The scaphoid corresponds to the radiale of the typical carpus (p. 81); the lunar perhaps represents a second centrale that occurs in some Amphibia; the cuneiform is probably the intermedium, and the pisiform the ulnare.

The centrale is present sometimes as a distinct ossification; the five distal carpals are represented by the trapezium, trapezoid, magnum, and unciform, the last being the equivalent of the fourth and fifth distalia. There are never more than five digits, and in many forms the number is greatly reduced; only in certain Cetacea does the number of phalanges in a digit ever exceed three.

The three elements of the pelvic arch unite to form a single bone, the innominate. The ilia unite by broad surfaces with the sacrum; the pubes, and sometimes the ischia, unite in a symphysis. All three may take a share in the formation of the acetabulum, but the pubis is usually shut out by a small cotyloid bone. In the shank the inner or tibial element is always the larger ; the fibula may be rudimentary. A large sesamoid bone-the 
patella-is almost universally formed in close relation to the kneejoint. In the tarsus there are two proximal bones, the astragalus and calcaneum, the latter undoubtedly corresponding to the fibulare of the carpus of lower Vertebrates, and the proximal part of the former to the intermedium and its distal portion to the proximal of the two central elements present in the tarsus of some Amphibia. The scaphoid or navicular represents the second central bone, and the distal tarsals are represented by the cuboid and the three cuneiforms.

The external form of the limbs and the mode of articulation of the bones vary in the various orders of the Mammalia, in accordance with the mode of locomotion. In most the habitual attitude is that which is termed the quadrupedal-the body being supported in a horizontal position by all four limbs. In quadrupedal Mammals the manus and pes sometimes rest on the ventral surfaces of the entire metacarpal and metatarsal regions as well as on the phalanges - when the limbs are said to be plantigrade; or on the ventral surfaces of the phalanges only (digitigrade); or on the hoofs developed on the terminal phalanges (unguligrade). Many of the quadrupeds have the extremities prehensile, the hand and foot being converted into grasping organs. This is most marked in quadrupeds that pass the greater part of their life among the branches of trees, and in the Sloths the modification goes so far that both hands and feet are converted into mere hooks by means of which the animal is enabled to suspend itself body downwards from the branches of trees.

Certain Mammals, again, have their limbs modified for locomotion through the air. The only truly flying Mammals are the Bats and the so-called "Flying Foxes," in which the digits of the forelimb are greatly extended so as to support a wide delicate fold of skin constituting the wing. In other so-called flying Mammals, such as the Flying Squirrels and Flying Phalangers, there is no active flight, and the limbs undergo no special modification; the flying organ, if it may be so termed, in these cases being merely a parachute or patagium in the form of lateral flaps of skin extending along the sides of the body between the fore- and hind-limbs.

Further, there are certain groups of swimming Mammals. Most Mammals, without any special modification of the limbs, are able to swim, and some of the quadrupeds, such as the Tapirs and Hippopotami, spend a great part of their life in the water. But there are certain Mammals in which the limbs are so specially modified to fit them for an aquatic existence-assuming the form of flippers or swimming paddles - that locomotion on land becomes almost, if not quite, impossible. Such are the Whales and Porpoises, the Dugongs and Manatees, and, in a less degree, the Seals and Walruses.

Skeleton of Prototheria.-In the Prototheria (Fig. 1139) the 


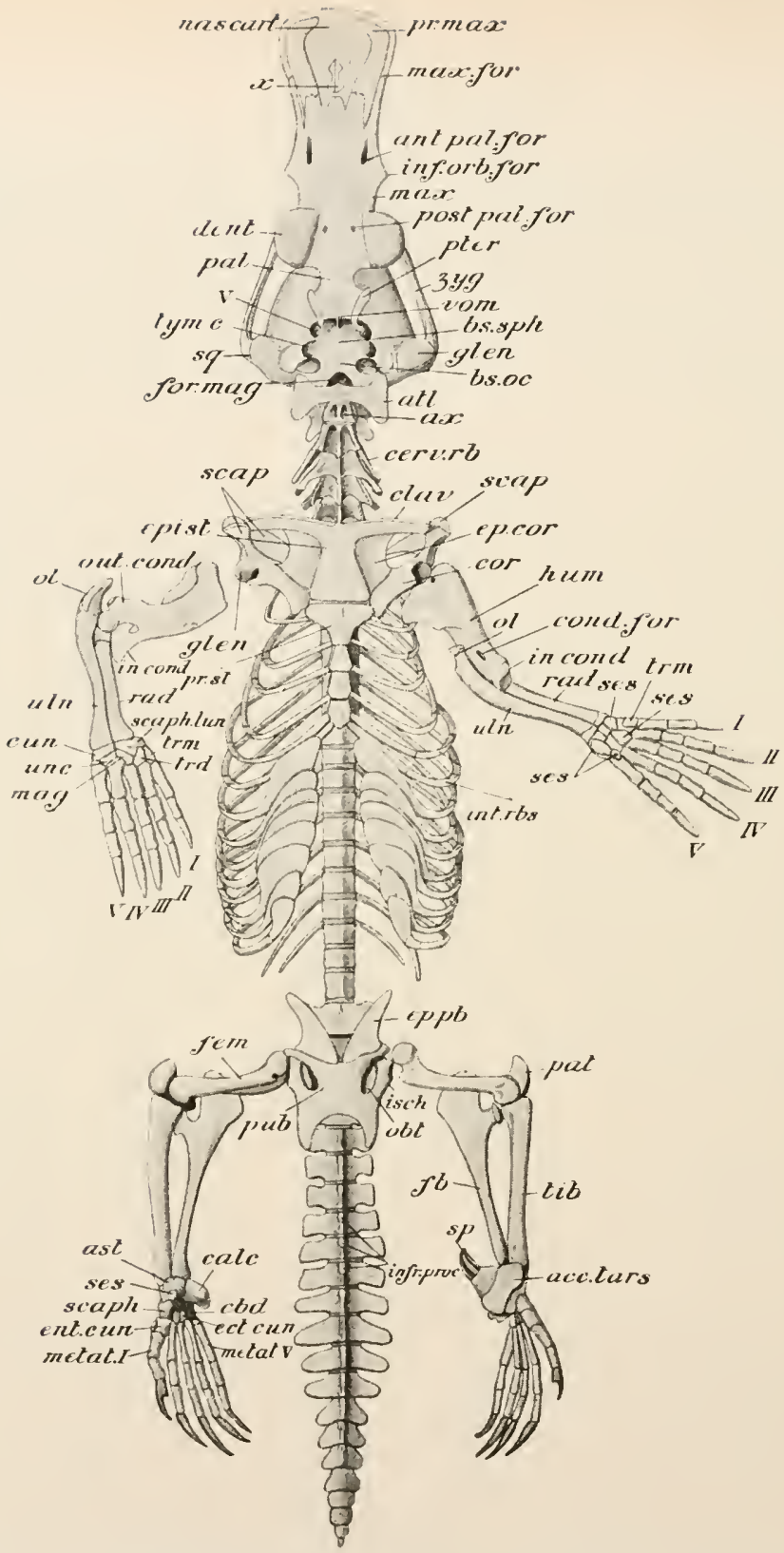

FiG. 1139.-Skeleton of male Ornithorhynchus. Ventral vicw. The right fore-limb has been separated an turned round so as to bring into view the dorsal surface of the manus: the lower jaw is removed. acc. tar accessory tarsal bone supporting the spur; ant. pal. for. anterior palatine foramen ; atl. atlas ; ast. astragalus ; ax axis; $b s . o c$. basi-occipital; bs. sph. basi-sphenoid; calc. calcaneum; $c b d$. cuboid ; cerv. $r b$. cervical rib ; $c l a v$. clavicle cond.for. foramen above inner condyle of humerus; cor. coracoid ; cun. cuneiform of carpus ; dent. position of horn teeth; cct. cun. ecto-cuneiform ; ent. cun. ento-cuneiform; ep. cor. epicoracoid ; epist. episternum; $e p$. pb. epipubis $\mathrm{fb}$. fibula ; fem. femur; for. mag. foramen magnum; glen. glenoid cavity of shoulder-joint and glenoid cavit for mandible ; hum. humerus; in. cond. inner condyle of humerus; inf. orb. for. points to position of infra-orbita foramen; infr. proc. inferior processes of caudal vertebre; int. rbs. intermediate ribs; isch. ischium; mas magnum of carpus ; max. maxilla ; max. for. maxillary foramen ; metat. $I$, first metatarsal ; metat. $V$, fifth`metata sal; nas. cart. nasal cartilagc; obt. obturator foramen; ol. olecranon; out. cond. outer condyle of humerus pal. palatine; pat. patella; post. pal. for. posterior palatine foramen; pr, max. pre-maxilla ; pr. st. presternum pter. pterygoid; $p u b$. pubis; rad. radius; scap. scapula ; scaph. scaphoid of tarsus; scaph. lun. scapho-lunar; se sesamoid bones of wrist and ankle; sp. tarsal horny spur; sq. squamosal; tib. tibia; trd. trapezoid; trm trapezium; tym. c. tympanic cavity; uln. ulna; unc. unciform; vom. vomer; $x$, dumb-bell-shaped bone; $z y$ 
epiphyses of the vertebre are not well developed in the Platypus, being represented only in the caudal region, and they appear to be absent in Echidna. In both genera there is the normal number of vertebræ in the cervical region. The odontoid process long remains separate from the centrum of the axis. The cervical transverse processes are separately ossified, and only completely unite with the vertebræ at a late period, sutures being traceable in all but very old animals. Zygapophyses are absent in the cervical region. There are nineteen thoraco-lumbar vertebræ in both genera. The transverse processes are short, and the ribs do not articulate with them, but only with the sides of the centra. In the sacrum of Echidna there are three or four, in that of Platypus two, united vertebræ. The caudal region differs considerably in its development in the two genera. In Echidna the tail is very short, the vertebræ depressed, with no inferior spines, but with about five subvertebral bones, which differ from ordinary chevron bones in being mere flat nodules. In the Platypus the tail is long, and the number of caudal vertebræ is twenty or twentyone. Each has a distinct inferior spinous process (infr. proc). The sternum consists of a pre-sternum and three keeled sternebræ: in Echidna but not in Platypus there is a xiphisternum. The most remarkable feature of the sternal apparatus in the Prototheria is the presence of a $\mathrm{T}$-shaped episternum (interclavicle, epist.) corresponding to that of Reptiles. The sternal ribs are ossified, and are connected with the vertebral ribs by imperfectly ossified intermediate ribs (int. rbs.).

The skull of the Monotremes differs widely from that of other Mammals. The bones early become fused together, so that it is difficult to trace their exact boundaries. The brain-case is larger and more rounded in Echidna than in the Platypus, in accordance with the larger size of the brain in the former genus. In both genera there is a pterygoid (investing) bone not separately represented in higher Mammals, corresponding to the pterygoid of lower Vertebrates. The parasphenoid represents the lateral parts of the parasphenoid of lower Vertebrates and the inner lamella of the pterygoid process (usually regarded as the pterygoid) of higher Mammals. Perforating the posterior root of the zygomatic arch is a canal, comparatively wide in the full-grown Ornithorhynchus, narrow in Echidna-the temporal canal-wlich is not present in higher Mammals, and apparently represents the post-temporal fossa of Reptiles.

In Echidna (Fig. 1140) the squamosal extends further forwards, and the posterior root of the zygomatic arch is more anterior than in Mammals in general. The zygoma is very narrow, and there is no rudiment of post-orbital processes: the jugal is absent as a separate ossification. The alveolar border of the maxilla (max.) is narrow and devoid of teeth. The nasal and premaxillary 
region of the skull is drawn out into a long, narrow rostrum. Near the anterior end of this is a rounded opening, the external nasal opening, which is entirely bounded by the premaxillæ- the nasals not extending so far forwards. An aperture in the nasal septum corresponds to an actual perforation by which the nasal cavities are in direct communication in the living animal. The pterygoids

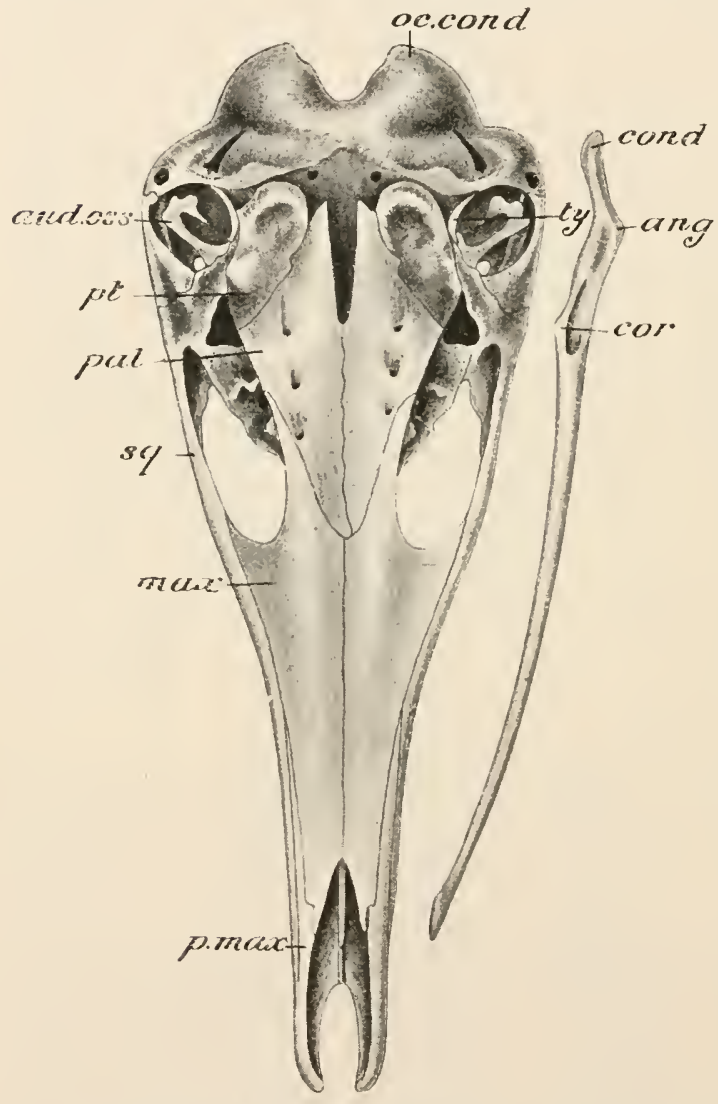

Fia. 1140.-Echidna aculeata. Ventral view of skull and right ramus of mandible. ang. angle of mandible; aud. oss. auditory ossicles; cond. condyle of mandible; cor. coronoid process ; max. maxilla ; oc. cond. occipital condyle; pal. palatine; $p$. max. pre-maxilla; $p t$. pterygoid ; $s q$. squamosal ; ty. tympanic ring.

than in Echidna, and there is a post-orbital process which is formed by the jugal. The maxillary root of the zygoma develops a process which supports the horny tooth (dent.) of the upper jaw. The nasal and premaxillary region is expanded into a rostrum which is much broader than in Echidna. The premaxillæ ( $p r$. max.) diverge from one another anteriorly, and then curve inwards again,

$(p t$.$) are in the$ form of flat plates continuous with the bony palate ; they extend back so as to form a part of the walls of the tympanic cavities. The tympanic $(t y$.$) is an$ imperfect $\mathrm{r}$ ing which does not become united with the periotic. The mandible consists of very narrow styliforn rami, which are not firmly united at the symphysis. The condyle (cond.) is narrow, rather more elongated anteroposteriorly than transversely. There are very slight rudiments of the angle and of the coronoid process (cor.).

In the Platypus (Fig. 1139) the zygoma is stouter 
partly enclosing a large space in which the nostrils are situated, and which is covered over in the recent state by the tough but sensitive hairless integument investing the cartilage of the rostrum, the latter being continuons with the nasal septum. In this space between the premaxillæ is situated a dumb-bell-shaped bone $(x)$ which appears to be of the nature of an anterior vomer. The pterygoid (pter.) is much smaller than in Echidna, and does not extend as far back as the tympanic cavity. The mandible has its rami stouter than in Echidna; they meet for a short distance anteriorly, and then again diverge slightly. The condyle is much larger than in Echidna, and is elongated transversely. In front of it is a broad process bearing the horny tooth.

It is in the shoulder-girdle that we find perhaps the most striking peculiarities of the skeleton of the Prototheria. There is a T-shaped episternum (epist.), as already stated, similar to that of Reptiles, the median limb articulating behind with the presternum and the crosspiece closely applied to the clavicles. There are two short and broad coracoids (cor.) articulating internally and behind with the presternum, and, externally, uniting with the scapula to form the glenoid cavity. In front of the coracoid is a flat plate, the epicoracoid (ep. cor.). The scapula (Fig. 1141 ) is very unlike that of other Mammals. There is a well-developed acromion process (acr.) with which the clavicle articulates; this terminates the anterior border, so that the latter would appear to correspond to the spine of the scapula of other Mammals : this is confirmed by the arrangement of the

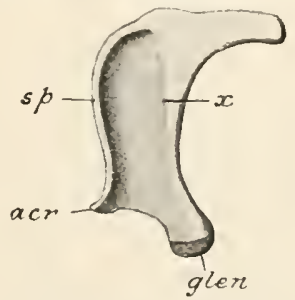

Fig. 1141.-Onter surface, left scapula of ornithorhynchus. acr. process corresponding to acromion glen, glenoid articular surface ; $s p$. anterior border, corresponding to the spine: $x$, slight ridge which bounds the surface of origin of the sub-scapularis muscle anteriorly. scapular muscles. The anterior part of the inner surface is in reality the pre-spinous fossa; the anterior portion of the outer surface the post-spinous fossa; and the part behind this, separated from it by a slight ridge, together with the posterior portion of the imner surface, is the sub-scapular fossa.

The humerus is of remarkable shape, with greatly expanded extremities-especially in Echidna-and prominent tuberosities and condyles. In the carpus the scaphoid and lunar are united; there is no separate centrale. There are a radial sesamoid and two very large palmar sesamoids, which are sometimes united.

In the pelvis there is a very long symphysis in which pubes and ischia take an almost equal share. The acetabulum is perforated in Echidna. With the anterior border of the pubes are articulated a pair of large epipubic or " marsupial " bones (Fig. 1139, ep. $p b$.). The femur has expanded extrenities with prominent external and internal trochanters. There is a large ossified patella 
(pat.). The fibula $(f b$.$) has at its proximal end a remarkable$ compressed process which ossifies from a separate centre, and resembles the olecranon of the ulna. In the tarsus there are the usual bones. In the Platypus the astragalus and calcaneum are firmly united, and an accessory ossification (acc. tars.) on the inner side in the male bears the tarsal spur. The metatarsals are short and broad, as are all the phalanges except the last.

Skeleton of Metatheria.-In the Marsupials the inferior arch of the atlas (Fig. 1142) is often incompletely ossified, a gap being

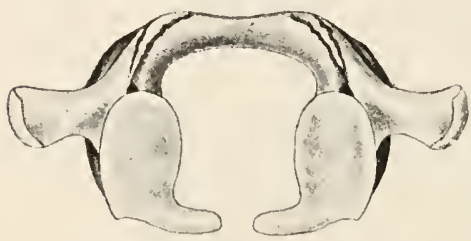

FiG. 1142.-Atlas of Kangaroo. left in the prepared skeleton; sometimes the gap becomes closed in by the ingrowths of the lateral parts of the areh, sometimes a small separate ossification is developed, filling up the opening. In the trunk there are always nineteen vertebræ. The transverse processes of the thoracic vertebræ are always well developed, and the ribs articulate with them as well as with the bodies. Prominent metapophyses and anapophyses are developed; these are largest in the lumbar region. Only one sacral vertebra is present in most Marsupials; in some a second is ankylosed with it. The caudal region varies greatly in length. It is short in the Koala and the Wombat, long in the Opossums, Dasyures, Phalangers and Kangaroos (Fig. 1143). Chevron bones are generally present, except in the Koala and the Wombat.

In the skull (Figs. 1144-1146) the brain-cavity is relatively small, with the cerebellar fossa entirely behind the cerebral. The pituitary fossa is not distinct, and there are no clinoid processes. The zygoma is complete, but the orbit is not completely bounded by bone behind. The jugal extends bencath the squamosal root of the zygoma to form part of the outer wall of the glenoid fossa. The lacrymal foramen is usually on the anterior margin of the orbit, sometimes on the face. The palate usually presents vacuities in its posterior portion. The pterygoid is always small. The alisphenoid is large, and forms the anterior boundary of the tympanic cavity ; in the Kangaroos (Fig. 1145, ali) it extends backwards so as to join the paroccipital process, which is greatly elongatcd. When an anditory bulla is developed, it is formed by this bone, the tympanic being always small, and never ankylosed to neighbouring bones. The internal carotid artery perforates the basisphenoid. The optic foramen is not separate from the sphenoidal fissure. In all except Tarsipes the angle of the mandible sends inwards a remarkable process (ang.), and is said to be inflected.

In the pectoral arch of the Marsupials the coracoid process is, as usual, developed from a special bony centre, and a distinct 
suture is often recognisable between it and the scapula until a comparatively late stage. In the young condition (when the fotus is attached to the teat) the coracoid is comparatively extensive and reaches the pre-sternum ventrally. A clavicle is always present, except in the Bandicoots, but may be incomplete. There is never

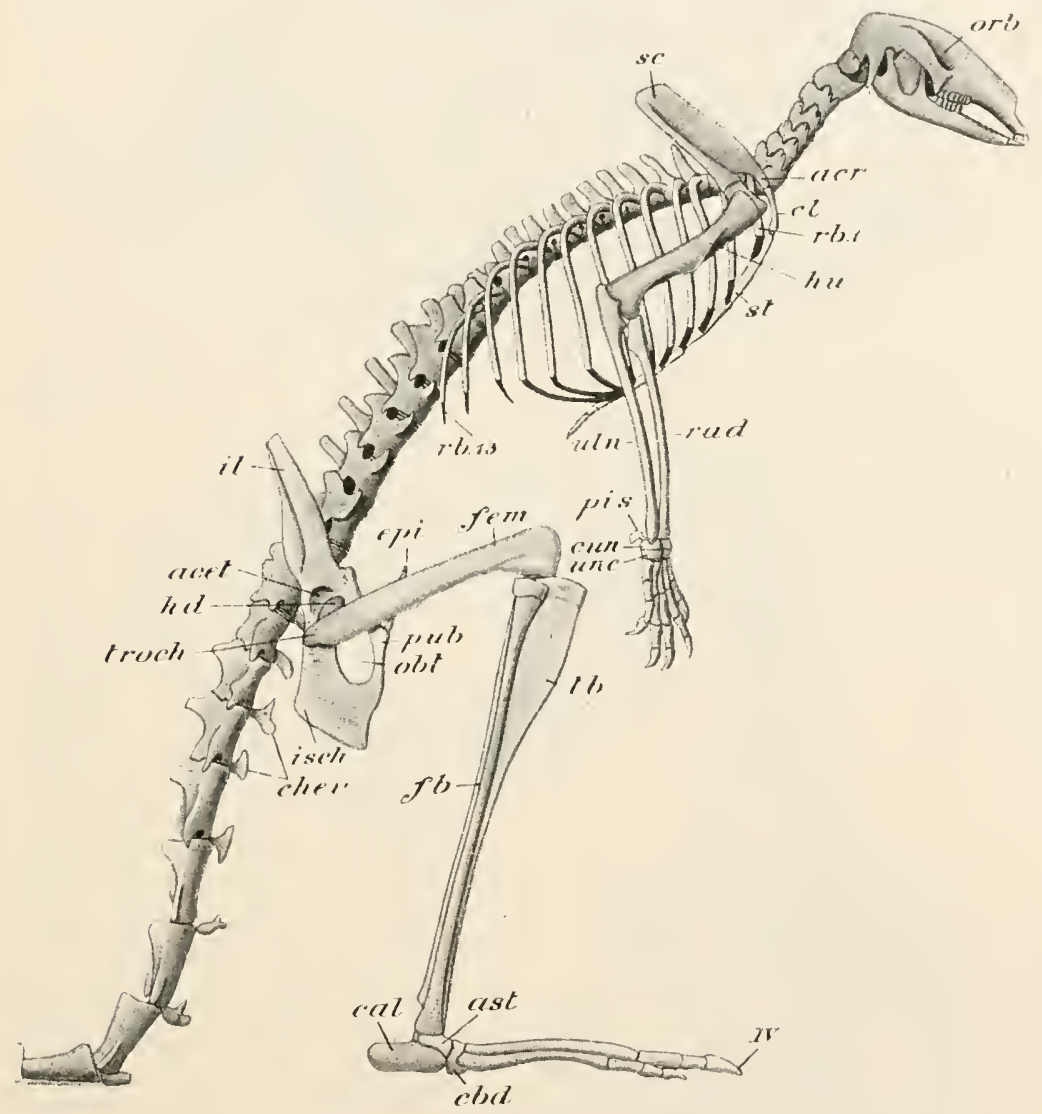

FIG. 1143.-Skeleton of Wallaby (IIalmainerus ualabatus). The scapula is represented as raised somewhat higher than it would be in the natural relations of the parts. The liead of the fenur has been separated from the acetabulum. acct. acetabulum; acr. acromion process ; ast. astragalus ; calc. calcaneum ; cbl. cuboid; chev. chevron bones ; $c l$. clavicle ; cın. cuneiform of carpus; epi. epipubis; $f b$. fibula; fem. femur ; $h d$. heal of femur; $h u$. humerus; $i l$. ilium ; isch. ischium ; obt. obturator foramen; orb. orbit ; pis, pisiform ; pub. pubis; rad. radius; $r b .1$, first rib; rb.13, last rib; $s c$. scapula; st. sternum; $t b$. tibia; troch. great trochanter of femur; uln. ulna ; unc. unciform ; $I V$. fourth toe.

a distinct centrale in the carpus. In the Opossums the ilium has the primitive form of a straight, three-sided rod. In the Kangaroos (Fig. 1143, il.) it is still simple and three-sided, but somewhat curved outwards; in the rest it is more or less compressed. In nearly all the Marsupials there is a pair of epipubic or marsupial 
bones (Fig. 1143, epi.) - elongated and compressed bones which articulate posteriorly with the anterior edge of the pubes: in the

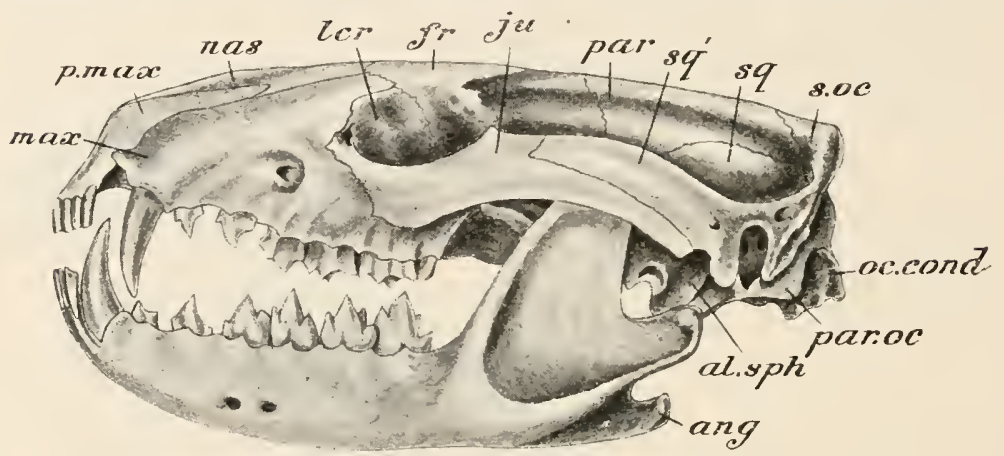

FIt. 1144.-Skull of Dasyurus (lateral view). al.sph. alisphenoid; ang. angular process o mandible; fr. frontal ; ju. jugal ; ler. lacrymal : max, maxilla ; nas. nasal ; oe. cond. occipital condyle ; par. varietal; par. oc. par-occipital process; p. max. pre-maxilla; $s$. oe. supraoccipital ; $s q$. squamosal ; $s q$;. zygomatic process of squamosal.

Thylacine they are represented only by small unossified fibrocartilages. In the leg the fibula is always well developed. In the

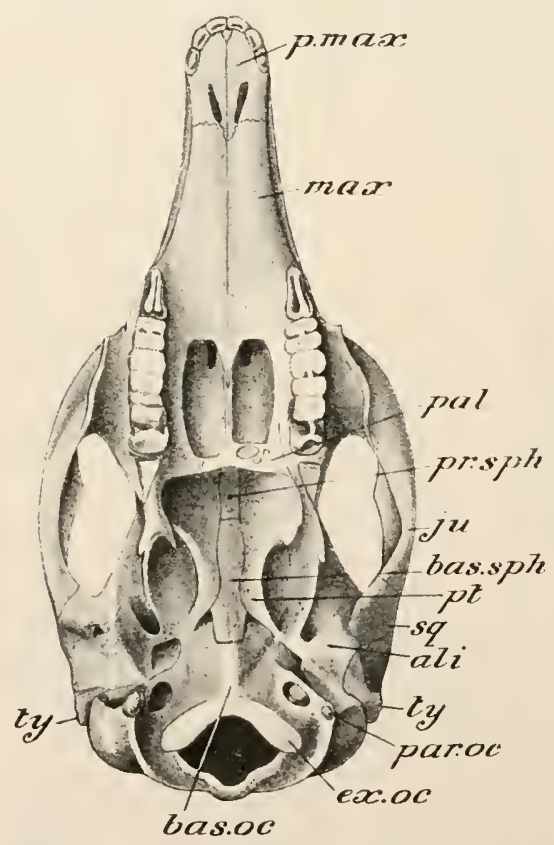

FIG. 1145.-Skull of Rock Wallaby (Petrogale penicillata) (ventral view). Letters as in Fig. 1144, except ali. alisphenoid. In addition, bas. oc. basi-occipital; bas. sph. basi-sphenoid; ex. oc. ex-occipital; pal. palatine; pt. ptervgoid; ty. tympanic. young condition of some Marsupials there is an accessory element situated outside the fibula at its proximal end: this apparently corresponds to a bone known as the parafibula which occurs in some Lacertilia. In the Phalangers (Fig. 1147) and the Koala there is always a considerable range of movement between the fibula and the tibia, comparable in some degree to the movements of pronation and supination of the radius and ulna. The foot (Figs. 1147, 1148), as already stated in the account of the external characters, presents a much greater range of modification than the manus.

Skeleton of Edentata.In the Armadillos more or fewer of the cervical vertebræ are ankylosed together 
both by their bodies and by their neural arches. In the lumbar region the metapophyses are greatly prolonged-longer than the transverse processes-and support the bony carapace. A remarkable peculiarity of the spinal column in the Armadillos is the fusion of a number of the anterior caudal vertebræ with the true sacrals to form the long sacrum, containing as many as ten vertebræ altogether (Fig. 1158). The caudal region is of moderate length ; there are numerous chevron bones. In Manis, Orycteropus, and Myrmecophaga none of the neck-vertebræ are united. In the posterior-thoracic and the lumbar regions of Myrmecophaga there are developed complex accessory articulations between the vertebræ: the sacrum contains, in addition to the true sacral vertebræ, a number derived from the caudal region, a condition which occurs also in Orycteropus.

In the Sloths none of the cervical vertebræ are ankylosed together ;

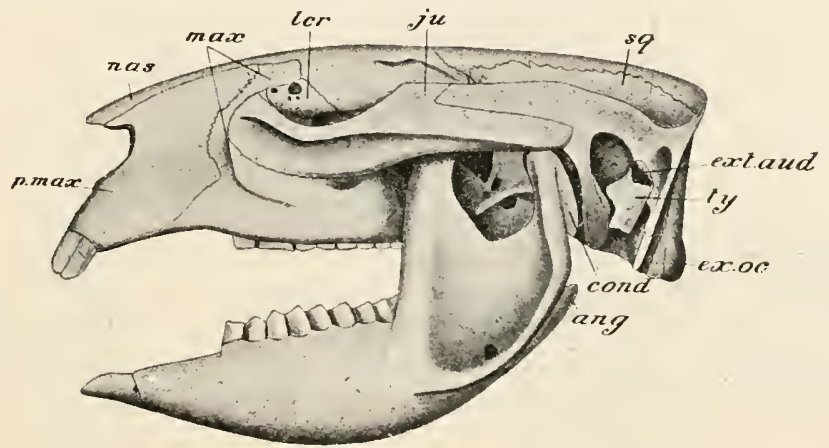

FIG. 1146. - Skull of Wombat (Phascolomys wombat) (lateral view). Letters as in Figs. 1144 and 1145 . In addition, ext. aud. opening of bony auditory meatus; cond. condyte of mandible

but in the three-toed Sloths there is an important divergence from ordinary Mammals in the number of vertebræ in the cervical region, there being nine or ten instead of seven; while in one species of two-toed Sloth (Cholopus hoffmanni) there are only six. The neural spines of all the vertebræ are very short. A number of the anterior caudal vertebræ are united firmly, though not quite fused, with one another and with the true sacrals.

In the Armadillos the sternal ribs, which are sub-bifid at their sternal ends, are ossified, and articulate with the sternum by means of well-developed synovial articulations. In the American Anteaters there are similar synovial joints, and the sternal ends of the sternal ribs are completely bifid. In the Sloths the sternum is long and narrow, and there are no synovial joints. In front the sternal ribs are ossified and completely united with the vertebral ribs, but behind they are separated from the latter by intermediate ribs which are less perfectly ossified. 
In the Armadillos the skull (Fig. 1149) is broad and flat, the facial region triangular. The tympanic (ty.) is in some developed into a bulla. The bony auditory meatus is in some cases elongated. The zygoma is complete. The pterygoids are small, and do not develop palatine plates. The mandible has a well-developed ramus

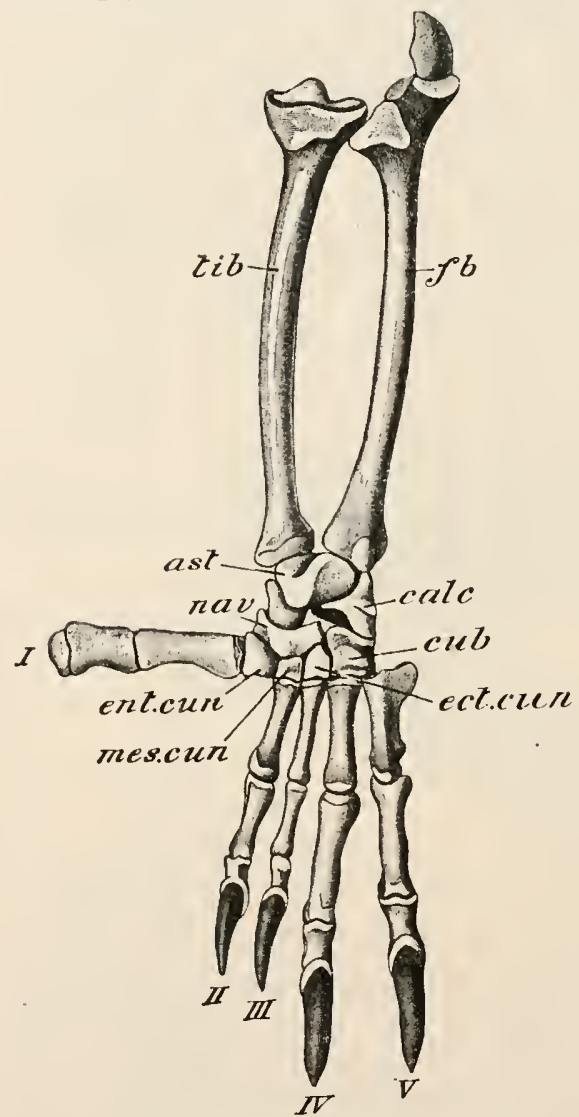

FIG. 1147.-Bones of leg and foot of Phalanger. ast. astragalus; calc, calcaneum; cub. cuboid ; ect. cun. ecto-cuneiform ; ent. cun. ento-cuneitorm ; $f b$. fibula : mes. cun. meso-cuneiform; nav. navicular; tib. tibia; $I-V$, digits. (After Owen.) with a prominent coronoid process and a wellmarked angular process.

In the American Anteaters (Figs. 1150 and 1151) the skull is extremely long and narrow - the facial region being drawn out into a long, narrow rostrum, with the external nares at its ex-

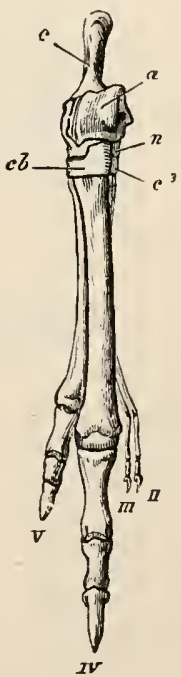

Fra. 1148.-Bones of right foot of Kangaroo (Macropus). a. astragalus ; $c$. calcaneum ; $c b$. cuboid ; $e^{3}$. ento-cuneiform; n. navicular; $I \dot{I}-V$, digits. (After Flower.)

tremity. The olfactory fossæ are greatly developed. The rostrum is composed of mesethmoid, vomer, maxillæ, and nasals-the premaxillæ being very small. The zygoma is incomplete, and the orbit is not closed behind by bone, the post-orbital processes of the frontal being entirely absent. The pterygoids (pter.), in all but Cycloturus, develop palatine plates. There is no bony anditory meatus. The mandible is entirely devoid of ascending ramus- 
consisting of two long and slender horizontal rami, with a very short symphysis.

In the Sloths (Fig. 1152) the cranial region is elevated and rounded, the facial short ; the frontal region is elevated, owing to the development of extensive frontal air-sinuses. The premaxillæ are small,

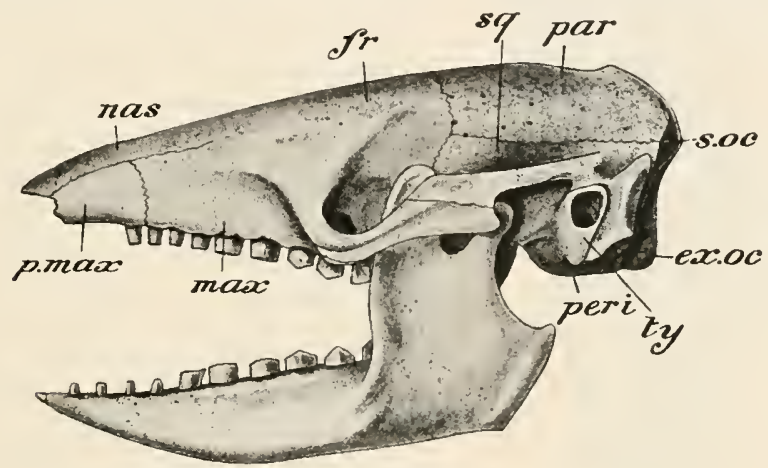

Fig. 1149.- Skull of Armadillo (Dasypus sexcinctus). Letters as in Figs. 1144-1146. In addition, peri. periotic.

and not firmly connected with the maxillæ, so that they are commonly lost in the macerated skull. The jugal ( $j u$.) develops a strong zygomatic process which bifurcates behind into two branches, neither of which is connected with the rudimentary zygomatic process of the squamosal, so that the zygomatic arch remains incomplete. There are, at most, the rudiments of post-orbital pro-

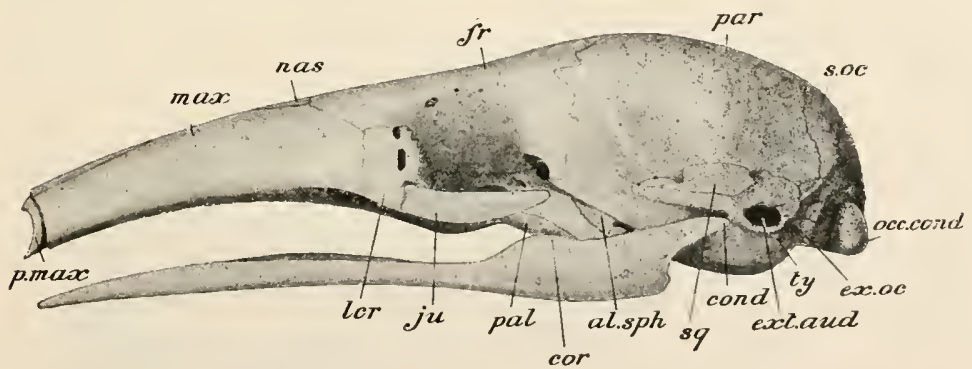

FIG. 1150.--Skull of Ant-eater (Myrmecophaga), lateral view, al.sph. alisphenoid; cond. condyle of mandible; cor. coronoid process of mandible ; ex.oc. ex-occipital; ext, aud. external auditory meatus ; fr. frontal ; ju. jugal ; ler. lacrymal ; max. maxilla ; nas. nasal ; occ. cond. occipital condyle; pal. palatine; par. parietal ; p.max. pre-maxilla; s.oc, supraoccipital ; sq. squamosal ; ty. tympanic.

cesses of the frontals. The pterygoids develop vertical laminæ and form no palatine plates. The ascending ramus and coronoid process of the mandible are both well developed.

In the American Ant-eaters and Armadillos the bones of the fore-limb are short and powerful. The scapula in the Ant-eaters 


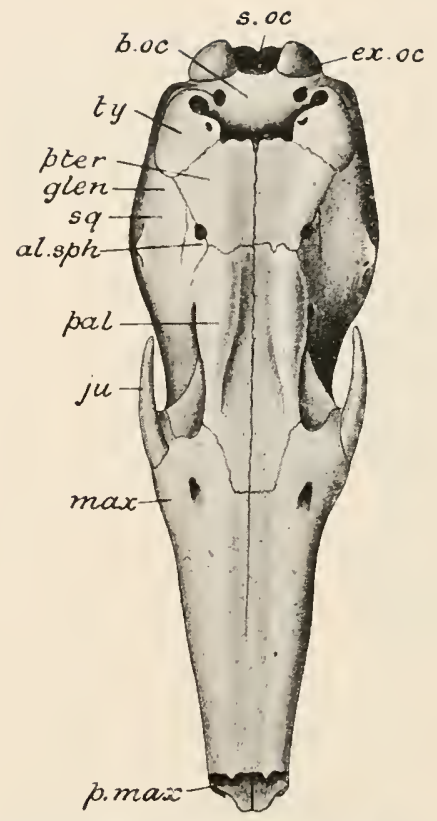

F10. 1151.-Skull of Ant-eater (Myrmecophaga), ventral view. Letters as in Fig. 1150 . In addition, b.oe. basioccipital; glen. glenoid surface for mandible; pter. pterygoid. is broad and rounded; the anterior border unites with the coracoid process so as to convert the coraco-scapular notch into a foramen. In the middle of the spine there is a triangular process: a ridge on the post-spinous fossa presents the appearance of a second spine. The fibres of origin of the sub-scapularis muscle extend on to the outer surface as far forward as this ridge, so that the part of the outer surface behind the ridge corresponds to a part of the subscapular fossa, which in other Theria is co-extensive with the inner surface. Except in Cycloturus the clavicles are rudimentary. All the carpal bones are distinct.

In the Armadillos the scapula (Fig. 1153) has an extremely prolonged acromion (acr.), sometimes articulating with the humerus. A ridge $\left(s p^{\prime}\right.$.) representing a second spine is present. The clavicle is well developed. The humerus is short and powerful, with well-developed processes and ridges, and

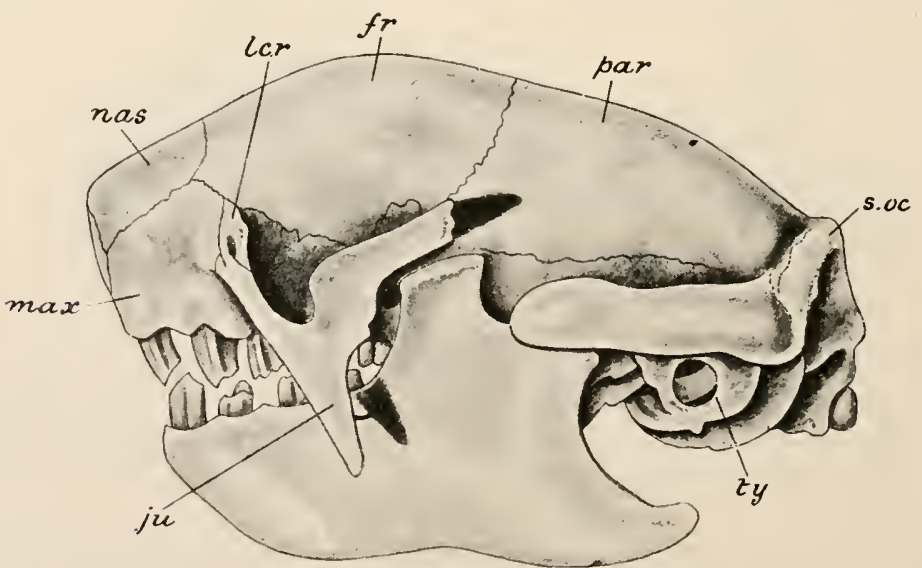

Fig. 1152.-Skull of Three-toed Sloth (Bradypus tridactylus), Letters as in Fig. 1150.

with a foramen above the inner condyle (entepicondylar foramen). The carpus consists of the ordinary eight bones. 
In the Sloths (Fig. 1154) the arm-bones are comparatively long and slender. A coraco-scapular foramen is formed as in the Ant-eaters. In the three-toed Sloths (Fig. 1155) the acromion (acr.) is at first connected with the coracoid process, but becomes reduced and loses the connection; in the two-toed Sloth the connection persists. The clavicle $(c l$. $)$ is not directly connected intemally with the sternum ; externally it is directly connected with the coracoid process - a condition observed in no other Mammal. The humerus is very long and slender; so are the radius and ulna, which are capable of a certain amount of movement in pronation and supination. In the carpus (Fig. 1156) the trapezoid and magnum are united in Bradypus, distinct in Cholopus: in the former the trapezium is usually fused with the rudimentary first metacarpal. The first and fifth metacarpals are represented only by rudiments. The proximal phalanges of the three digits are early ankylosed with the corresponding metacarpals, so that it might readily be supposed that one of the ordinary bones of each digit was absent.

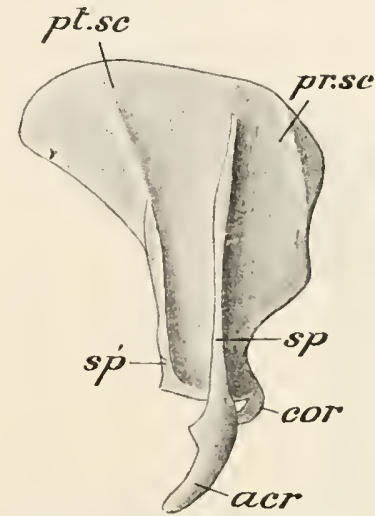

FIG. 1153. - Shoulder-girdle of Armadillo (Dasypus sexcinctus). acr. acromion; cor. coracoid process : pr.sc. pre-spinous fossa; $p t$. sc. postspinous fossa; $s p$. spine; $s p^{\prime}$. ridge probably marking the anterior limit of origin of the subscapularis muscle.

The pelvis of the American Ant-eaters is elongated, with a short symphysis pubis. The ischia unite with the spinal column. There is no third trochanter. The tibia and fibula are nearly straight, and parallel with one another. In Cycloturus the pes is modified to form a climbing organ.

In the Sloths the pelvis is short and wide; the spines of the ischia unite with the anterior caudal vertebræ so that a sacro-sciatic foramen is formed as in Ant-eaters. The femur is long and slender; it is devoid of third trochanter. The tibia and fibula are also long and slender. At its distal end (Fig. 1157) the fibula develops a peg-like process $(x)$ which fits into a depression in the outer face of the astragalus. The calcaneal process is extremely prolonged in Bradypus, in which there is a tendency to ankylosis between the tarsal bones, and the proximal phalanges ankylose with the metatarsals.

In the Armadillos the pelvis (Fig. 1158) is extremely long, and both ilia and ischia are firmly fused with the spinal column. The femur has a prominent third trochanter. The bones of the pes (Fig. 1159) are normal.

Skeleton of Cetacea.-In the Cetacea (Fig. 1160) the cervical 
region (cerv.) is always very short, and the constituent vertebræ are often completely fused together into a continuous bony mass, or the atlas alone may be separated from the rest; but

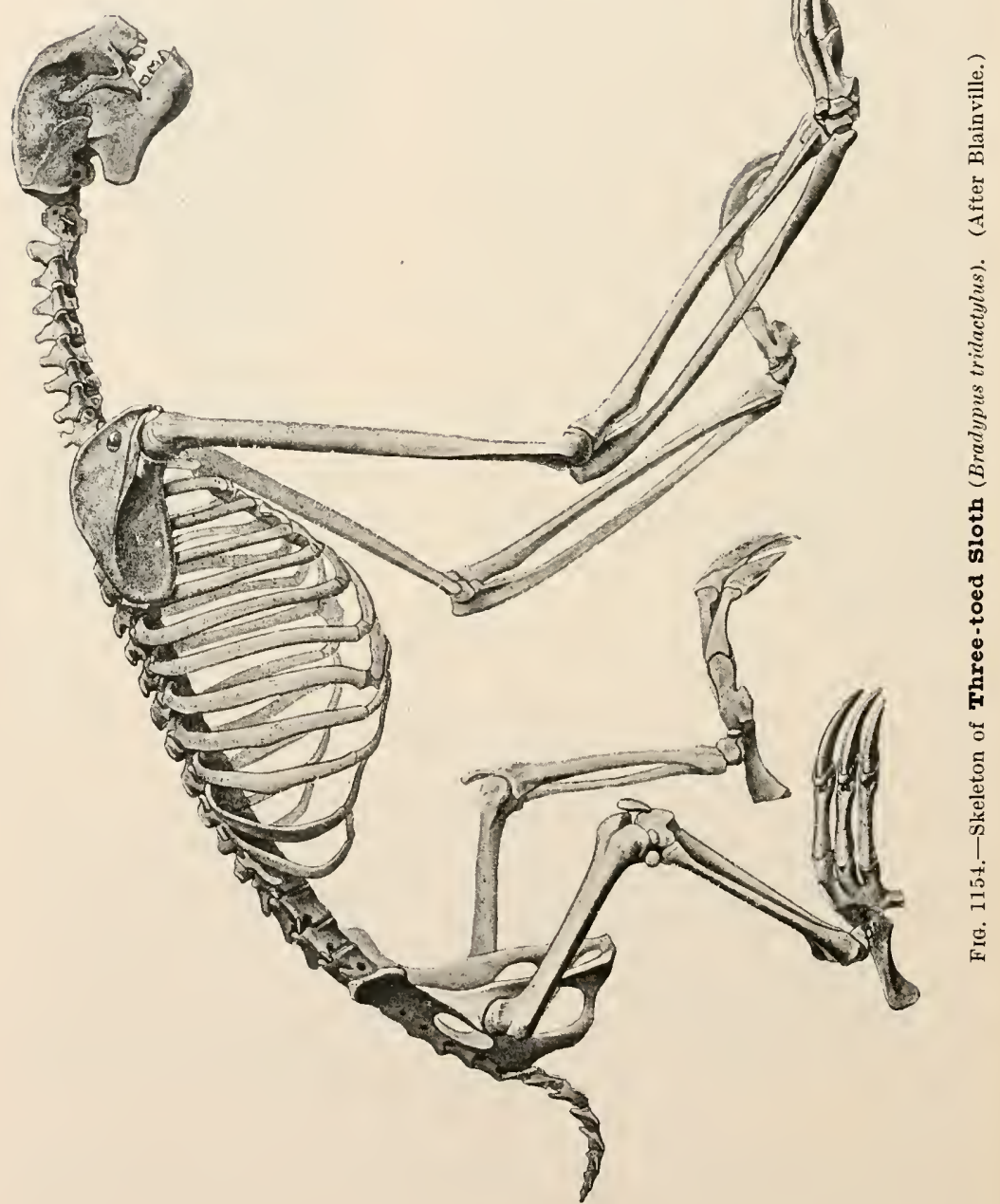

sometimes all the vertebræ are complete and separate. In the latter case they have small arches and long transverse processes 
consisting of two narrow bars with a wide space between them. The epiphyses are very distinct dises which often remain separate from the bodies up to a late period. The neural spines are well developed. The zygapophyses are not well developed, and are

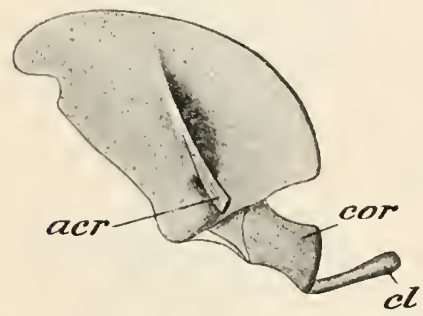

Fia. 1155.-Shoulder-girdle of Three. toed Sloth (Bradypus tridactylus). acr. acromion; $c l$. clavicle ; cor. coracoid.

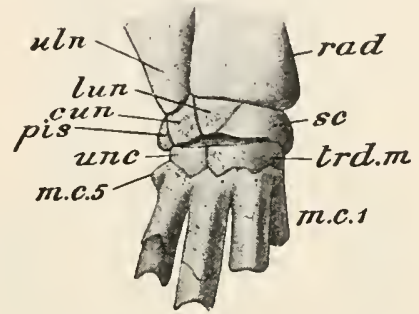

FIG. 1156.-Right manus of Three-toed Sloth. cun. cuneiform; lun. lunar; m.c.l, first metacarpal; m.c.5, rudiment of fifth metacarpal; pis. pisiform ; rad. radius; $s c$. scaphoid ; trd. $m$. trapezoid and magnum united ; uln. ulna ; unc. unciform.

absent in the posterior portion of the trunk. In the absence of hind limbs there is no sacral region. The caudal region consists of numerous vertebræ beneath which, opposite the intervertebral spaces, are a series of chevron bones (chev.).

In the Whalebone Whales orily one pair of ribs articulates with the sternum, and none articulate with the bodies of the vertebræ, but only with the transverse processes. In the Toothed Whales only a small number are connected with the sternum, sometimes through the intervention of intermediate ribs, and a few of the anterior only, in most cases, articulate with the bodies of the vertebræ; but in some a greater number articulate with both transverse processes and bodies by distinct tubercles and heads.

The sternum varies in shape. Sometimes it consists of a pre-

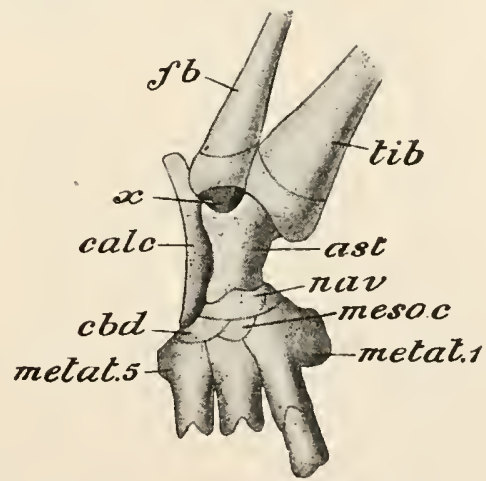

FIa. 1157-Pes of Three-toed Sloth. ast. astragalus ; calc. calcancum; cbcl. cuboid ; fb. fibula ; mesoc. mesocnneiform ; mctat.1, vestige of first metatarsal; metat.5, vestige of fifth metatarsal ; nav. navicular ; tib. tibia; $x$, peg-like process at distal end of fibula.

sternum and a series of several sternebræ without xiphisternum ; sometimes (Fig. 1161) it is a continuous plate of bone, occasionally with median notches or fontanelles.

In the skull (Fig. 1162) the brain-case is rounded, the jaws greatly elongated, often unsymmetrical, The parietals $(\mathrm{Pa})$ do 
not meet in the middle line above in most Cetacea, being separated by the supra-occipital (SO.) and an inter-parietal (IP.); there is

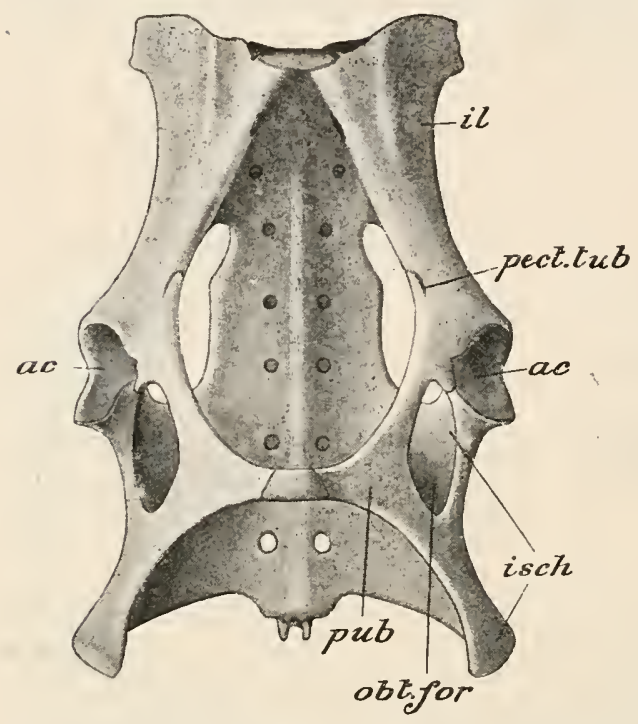

FIG. 1158.-Pelvis and sacrum of Armadillo (Dasypus sexcinctus). ac. acetabulum; $i l$. ilium; isch. ischium ; obt.for. obturator foramen; pect. tub. pectineal tubercle; pub. pubis. thus no sagittal suture. A large supra-orbital plate is developed from the frontal. There are large and stout zygo-

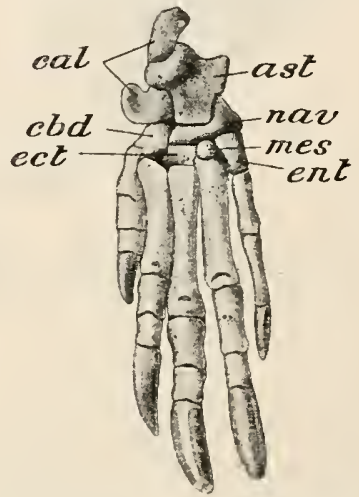

FIG. 1159.- Pes of Armadillo (Dasypus sexcinctus). ast, astragalus; cal. calcaneum: cbd. cuboid ; ect. ecto-cuneiform ; ent. ento-cuneiform ; mes. mesocuneiform; nav. navieular.

matic processes of the squamosal, but the jugals are extremely small. In all the recent forms the maxilla $(M x$.) is very large and extends backwards to overlap a good deal of the frontal, and forwards nearly to the extremity of the snout; while the premaxillæ $(P . M x$. $)$, which are long narrow bones, bound but a very small part of the oral border of the upper jaw. The nasals $(\mathrm{Na}$.) are very small. The tympanic bone is very large, and is sometimes fused with the periotic (Mystacoceti), sometimes not (Odontoceti). The lower jaw is remarkable for the absence of an ascending ramus.

The scapula in most of the Cetacea is very broad and flat, expanded into the shape of an open fan. The spine is usually situated close to the anterior border, sometimes coalescent with it. The acromion is curved and flat, the coracoid also compressed and parallel with the acromion. In some, both acromion and coracoid are absent. There is never any trace of a clavicle. The humerus is short and very stout; the head freely movable in the glenoid cavity ; the distal articulating surfaces are flat and oblique, meeting at an angle. The proximal ends of the radius and ulna are so firmly united with the humerus as to allow of very little movement; at the distal end there are no complete synovial membranes. The 


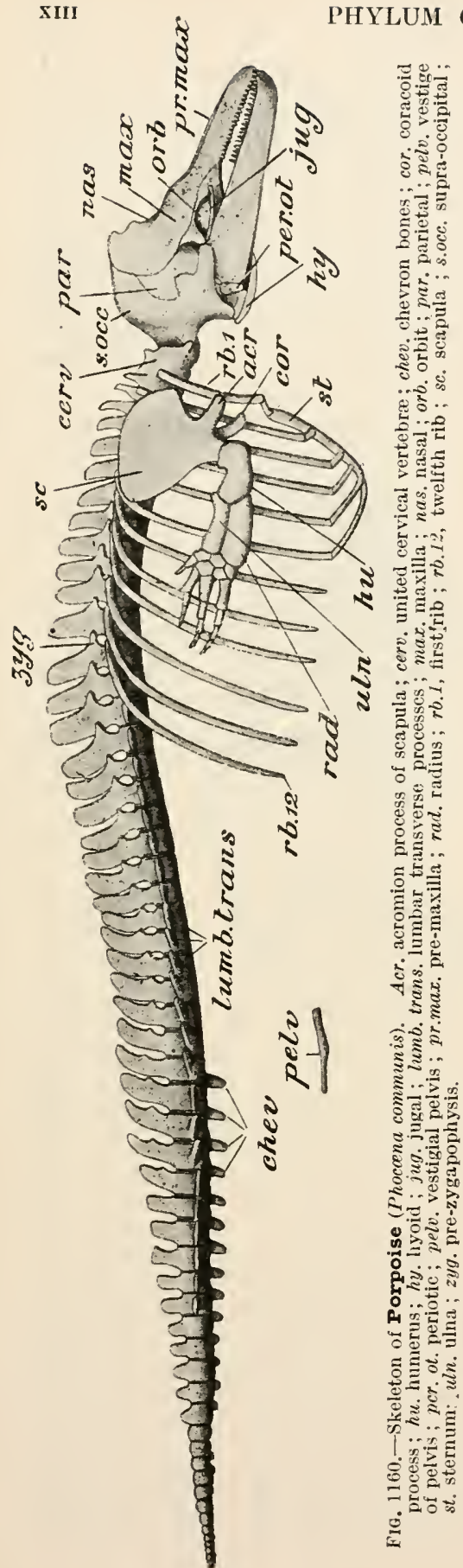

manus is extremely modified. There are no synovial joints; the carpus is in some (Whalebone Whales) almost entirely cartilaginous, as also are the metacarpals and phalanges - the cartilages being coalescent or separated by intervals of fibrous tissue : in some of the carpal elements bone is deposited. In the toothed Whales the carpals are completely ossified, and are of polygonal form: the phalanges are also ossified, with incomplete synovial articulations. In the Cetacea there are

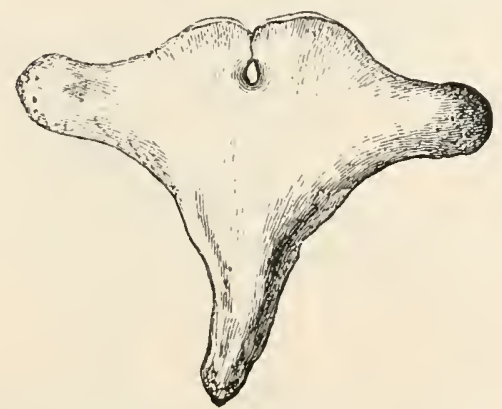

Fig. 1161.-Sternum of Rorqual (Balanop tera musculus). (After Flower.)

sometimes five digits, sometimes only four : more or fewer have considerably more than the normal number of phalangessometimes as many as fourteen. The second digit is usually the longest.

Vestiges of the pelvis are present in the form of a pair of long narrow bones (Fig. 1160, pelv.) which lie parallel with the spinal column some little distance below the region where the chevron bones begin: these appear to represent the ischia. A second pair of smaller bones which lie close to these in the 
Whalebone Whales are apparently vestiges of the femora, and there may be additional vestiges representing the tibiæ.

Skeleton of Sirenia.-In the Sirenia (Fig. 1163) the cervical vertebræ do not coalesce, with the exception of two of them in the Manatee. In the Manatee there are only six cervical vertebræ, and the neural arches are sometimes incomplete. In the trunk the thoracic vertebræ are numerous; all have well-developed facets for the heads of the ribs, and well developed zygapophyses. The caudal vertebræ are numerous, depressed, with wide transverse processes. The ribs are numerous, but few of them are connected

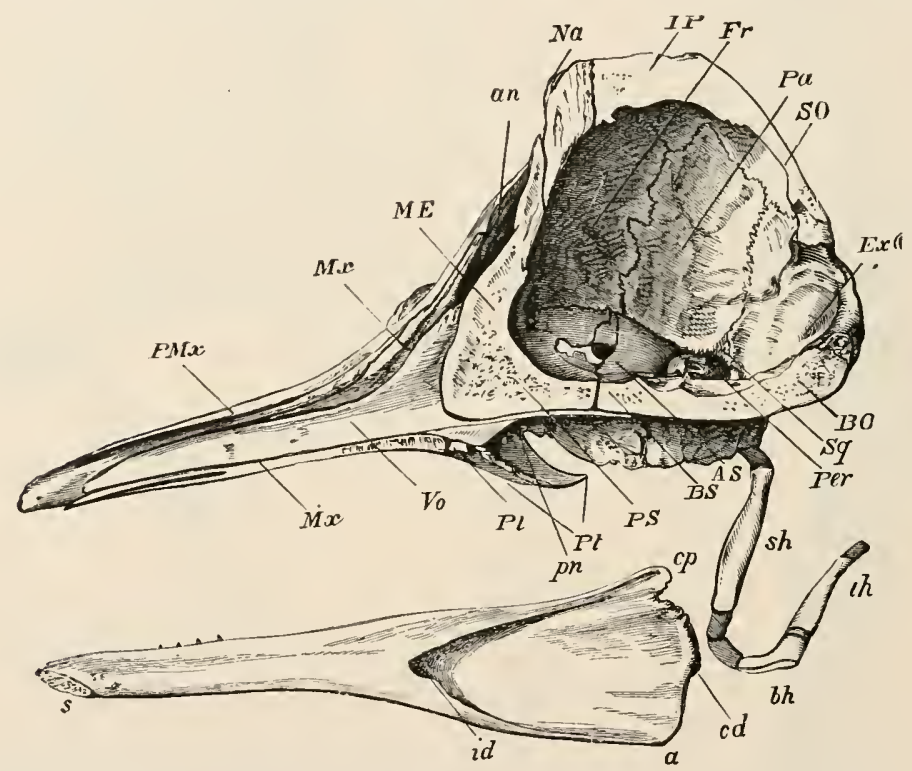

FIG. 1162.-Skull of Dolphin (Globiocephalus), sagittal section. a. angle of mandible; an. external nares; $A S$. alisphenoid ; $b h$. basi-hyal ; $B O$. basi-occipital ; $B S$. basi-sphenoid; $c d$. condyle of mandible ; $c p$. coronoid process ; Ex.O. ex-occipital ; F'r. frontal ; IP. interparietal ; ME . mesethmoid ; $M I x$. maxilla ; Na. nasal ; Pa. parietal ; Per. periotic ; $P l$. palatine ; $P . M x$. pre-maxilla ; $p n$. posterior nares; $P S$. presphenoid ; $P t$. pterygoid ; sh. stylohyal; SO. supra-occipital; Sq. squamosal; th. thyro-hyal; Vo. vomer. (After Flower.)

with the sternum. The sternum is a broad bone not composed of distinguishable segments.

The skull (Fig. 1164) is characterised by its extreme hardness. The cranial cavity is rather long and narrow as compared with that of the Cetacea. Although the supra-occipital (SO.) is produced forwards on the upper surface of the skull for a considerable distance, it does not separate the parietals $(P a$.) from one another. The frontals develop broad supra-orbital plates. The zygoma is stout. As in the Cetacea, the external nares are very wide, but they are relatively further forwards. The nasals are rudimentary. The tympanic and periotic are readily separable from the other 
bones. There are enormous premaxillæ in the Dugongs. The mandible has a well-developed ascending ramus and coronoid process (cp.).

The scapula of the Sirenia is much more like that of the terrestrial Mammals than is that of Cetacea, but is nearer that of the Seals; it is narrow and curved backwards. The spine is situated about the middle; the acromion is directed downwards. The coracoid is fairly well developed, and of a conical shape. The clavicle is absent, as in the Cetacea. The skeleton of the arm also departs less from the ordinary Mammalian type than in the Cetacea. The radius and ulna are ankylosed at their extremities. The carpus has seven bones in the Manatee : the pisiform is absent. In the Dugong coalescence takes place between the carpal bones, so that the number of ossifications is reduced in the adult. There are five digits, all of which possess the normal number of phalanges.

The pelvis is represented by a pair or more of vestiges widely separated from the spinal column, and having a vertical position : they probably represent the ilia.

Skeleton of the Ungulata. - In general, the centra of the Ungulata are more or less distinctly opisthocœlous. The odontoid process of the axis (Fig. $1165)$ has a peculiar spout-like form in the majority of the Ruminants, and in a less marked degree in the Horses and Tapirs : in the Chevrotains, the Pigs, and

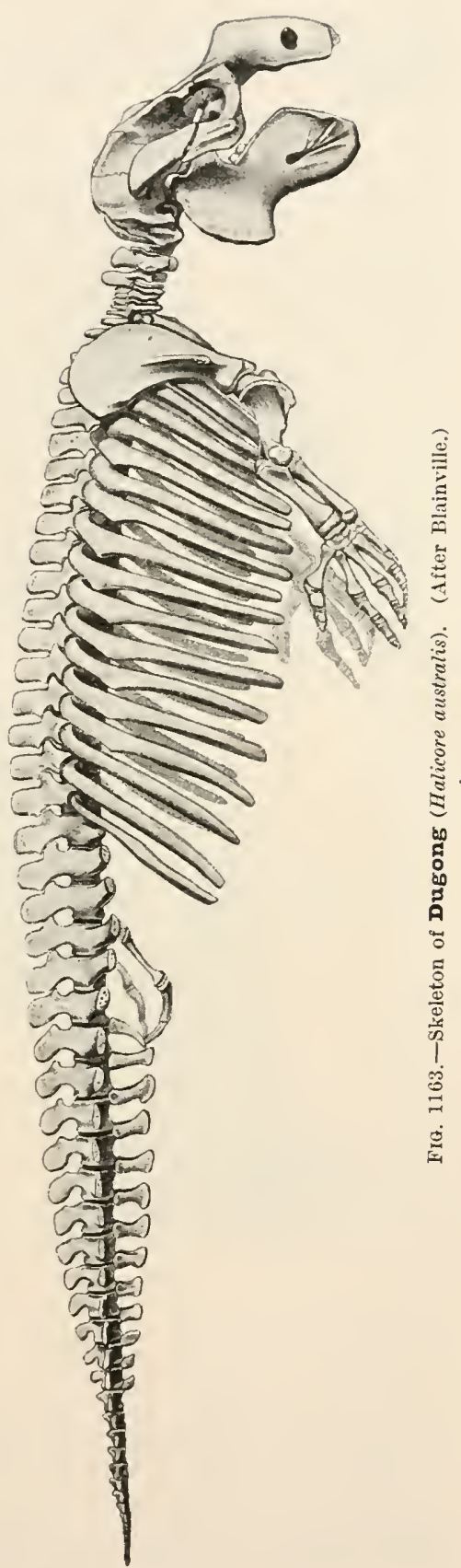


the Proboscidea it is conical. In the Ruminants the cervical vertebræ present a median keel below, produced in the posterior part of

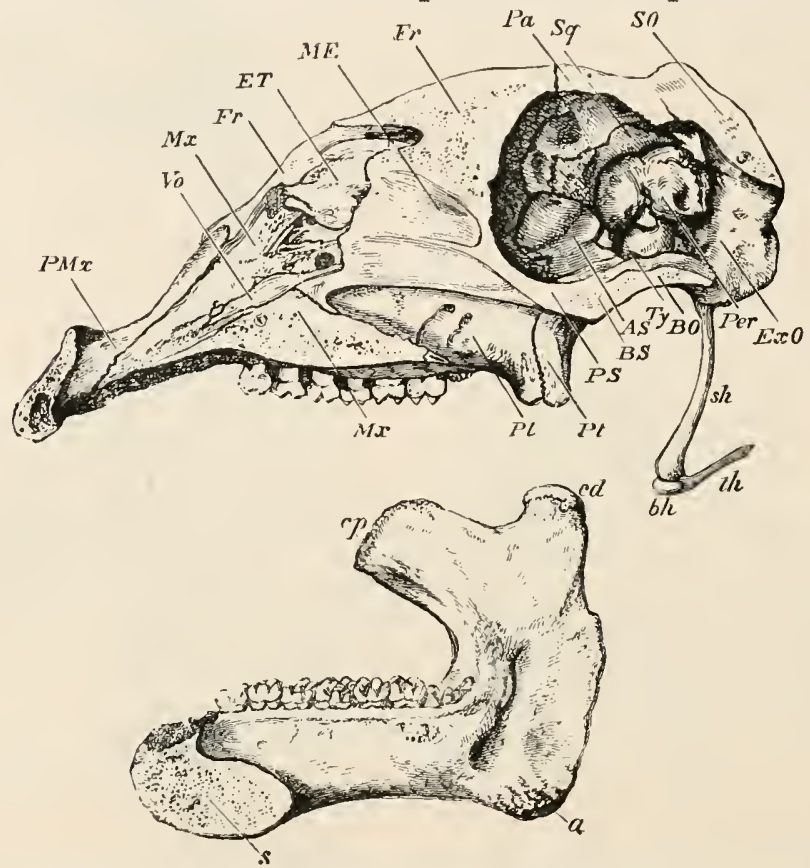

FIG. 1164.-Section of skull of Manatee (Manatus senegalensis). Letters as in Fig. 1162. In addition, $E T$. ethmo-turbinal ; Ty. tympanic. (After Flower.)
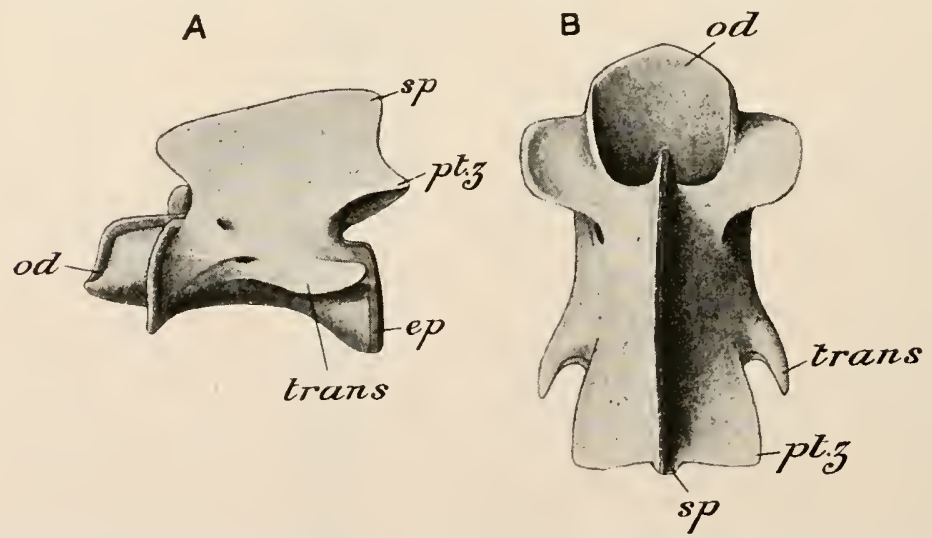

F1G. 1165.-Axis of Red Deer (Cervus elaphus). A, lateral view; $B$, dorsal view. en . epiphysis of centrum; od. odontoid process; $p t . z$. post-zygapophysis; $s p$. neural spine; trans. transverse process.

the region into a process. The development of the cervical neural spines varies : in most they are elongated and compressed ; but in 
the Horses they are almost completely absent, and in the Elephants they are all small, with the exception of the last. The number of thoraco-lumbar vertebræ is nearly always nineteen in the Artiodactyles, twenty-three in the Perissodactyles and in the Proboscidea. Hyrax has a larger number of trunk-vertebrætwenty-eight to thirty - than any other terrestrial Mammal. The transverse processes of the lumbar vertebræ are nearly always elongated, flattened, and directed outwards, or outwards and slightly forwards. Usually there is a single wide sacral vertebra united with the ilia, ankylosed with which behind are a varying number of narrow caudal vertebræ. There are never chevron bones in the caudal region of any existing Ungulate.

In all the Ungulata the sternebræ are distinct. As a general rule the presternum is narrow, sometimes (Horses and Tapirs) greatly compressed laterally, while the mesosternum is broad; but in the Rhinoceros the mesosternum is no broader than the presternum.

Among the Perissodactyle Ungulates the skull of the Horse (Fig. 1166) is elongated, especially in the facial region; the axis of the skull, or the line from the anterior margin of the pre-maxillæ

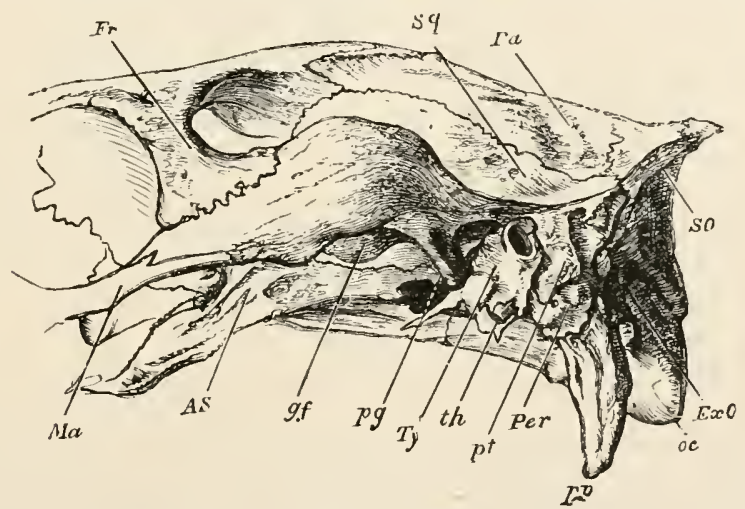

Fig. 1166.-Side view of posterior parts of skull of Horse (Equus caballus). AS. alisphenoid ; $E x O$. exoccipital ; Fr. frontal ; $g f$. glenoid fossa ; $\mathbf{M a}$, jugal ; oc. occipital condyle; $P a$. parietal ; pp par-occipital process ; $P$ er. periotic ; pg. post-glenoid process of squamosal; $p t$. post-tympanic process ; $S O$. supra-occipital ; $S q$. squamosal ; th. tympano-hyal ; $T y$. tympanic. (After Flower.)

to the lower edge of the foramen magnum, is nearly straight, and both the occipital plane and ethmoidal plane are nearly perpendicular to it. The supra-occipital (SO.) has a prominent transverse crest; and in front of this the temporal ridges, which limit the temporal fossa above, unite to form a median longitudinal sagittal crest, running along the course of the sagittal suture. The exoccipital develops a prominent, downwardly-directed, par-occipital process $(p p)$. The tympanic $(T y$.) is small and, with the periotic $(P e r$.$) , is only loosely connected with the neighbouring bones,$ 
being held in place mainly by a post-tympanic process developed from the squamosal. A considerable part of the periotic (mastoid portion) appears on the surface of the skull between this and the exoccipital. The tympanic forms a tubular auditory meatus, but is not expanded into a bulla. The glenoid fossa is extended transversely, and is bounded behind by a post-glenoid process. The orbit, which is relatively small, is completely surrounded by bone. The nasals are large, and are separated from the pre-maxillæ in a great part of their extent. The mandible has a large ascending ramus, and a coronoid process which rises high above the level of the condyle; the latter is elongated transversely in co-ordination with the form of the glenoid cavity.

The skull of the Rhinoceros differs from that of the Horse mainly in the presence of large air-cells in the supra-occipital and parietal bones, and in the orbit not being separated by bone from the temporal fossa, except in the two-horned Asiatic species. The post-glenoid process equals or exceeds the paroccipital; the mastoid does not appear on the surface, owing to the post-tympanic process of the squamosal extending backwards to articulate with the exoccipital and concealing it from view.

The skull of the Tapirs resembles that of the Rhinoceros in most respects. As in the latter, the orbits are not completely bounded by bone behind. The nasal openings are very large, and extend backwards above the orbits, separated from them only by a thin plate. The nasals are very prominent, and the inferior and lateral boundaries of the nasal apertures are formed entirely by the maxillæ. There are large post-glenoidal and post-tympanic processes; the latter is united with the paroccipital process. The mandible differs from that of the other Perissodactyles chiefly in the prominent incurved angle.

In the Ruminant Artiodactyles (Fig. 1167) the facial region is more or less bent downwards on the basi-cranial axis, and while the occipital plane is nearly perpendicular to the latter, the ethmoidal plane is nearly horizontal. There are prominent paroccipital processes $(p p)$. The tympanic $(T y)$, which may or may not be ankylosed with the periotic, forms a tubular auditory meatus and sometimes a distinct bulla. The mastoid appears for a short space on the surface, between the squamosal and the exoccipital. The frontals usually bear a pair of processes, more or less prominent, for the support of the horns, and between these a transverse ridge frequently extends. The orbit is completely encircled by bone, and has a prominent margin. The nasals are elongated and the premaxillæ slender. The condyle of the mandible is broad and flat; the horizontal ramus usually rather slender, and expanded in front for the lodgment of the incisors.

In the Pigs, as in the Ruminants, the facial region is bent downwards. There is a prominent transverse occipital crest at 
the junction of the supra-occipital and parietals ; but the temporal ridges do not meet in the middle to form a sagittal crest such as occurs in the skull of the Horse. There are prominent paroccipital processes. There is a large, but compressed, bulla tympani; the auditory meatus is very long, directed upwards and outwards, and is surrounded by the post-glenoidal and post-tympanic processes, which are in contact with one another. The mastoid is rudimentary, and does not appear on the outer surface of the skull. The frontal develops a short post-orbital process; but this does

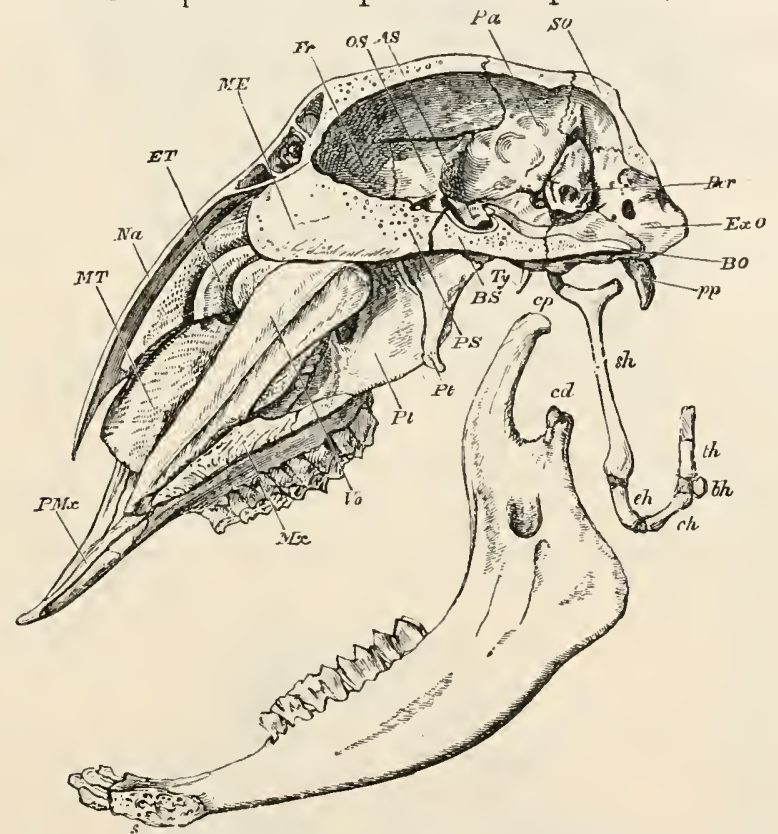

FIG. 1167.-Section of skull of Sheep (Ovis aries). $A S$, alisphenoid; $b h$. basi-hyal; $B$ J. basi-occipital ; $B S$. basi-sphenoid ; $c d$. condyle; $c h$. cerato-hyal; $c p$. coronoid process ; $e h$. epihyal ; $E O$. ex-ocelpital; $E T$. ethmo-turbinal ; $F r$. frontal ; $M E$. mesethmoid ; $M T$. maxillary turbinal; $\boldsymbol{M} x$. maxilla ; $\mathrm{Na}$. nasal; $O S$. orbito-sphenoid ; $P a$. parietal ; $P l$. palatine ; Per. periotic; $P . M x$. pre-maxilla ; $P . S$. pre-sphenoid ; $P t$. pterygoid ; $s . h$. stylo-hyal ; $S O$. supra-occipital (with inter-parietal); $p p$. par-occipital process; th. thyro: hyal; Ty. tympanic; Vo, vomer. (After Flower.)

not meet the zygoma, so that the bony margin of the orbit is incomplete behind. The facial region as a whole is elongated and laterally compressed. The nasals are long and narrow, and the premaxillæ send backwards long processes on each side of them. A peculiar bone-the pre-nasal-is developed in the nasal septum. The condyle of the mandible is transversely elongated ; the coronoid process very small.

The skull of the Hippopotamus differs from that of the Pig mainly in the proportions of the various parts. The cranial cavity is relatively small, and the face large. The orbits are almost 
tubular, and are almost, or quite, encircled by bone. The face is laterally contracted in front of the orbit and again expands anteriorly. The mandible is extremely massive; anteriorly the

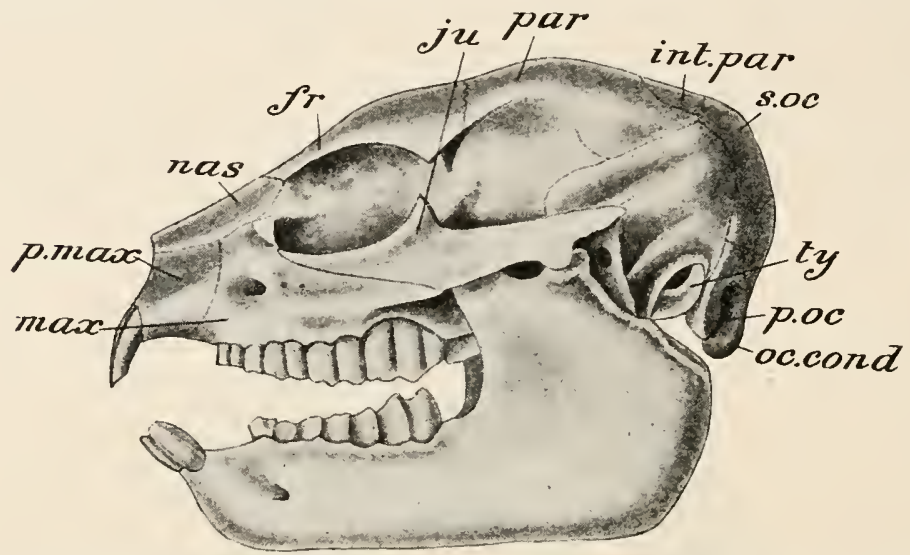

F16. 1168.-Skull of Fyrax. Letters as in Fig. 1144; in addition, int. par. inter-parieta l; $t y$. tympanic. The suture between the frontal and parietal has been by an error made $t o$ run behind the post-orbital process.

symphysial portion is greatly expanded to support the large incisor and canine teeth.

In the Hyracoidea (Fig. 1168) the skull shows affinities with Rodents and also with Perissodactyles. The zygomatic arch is

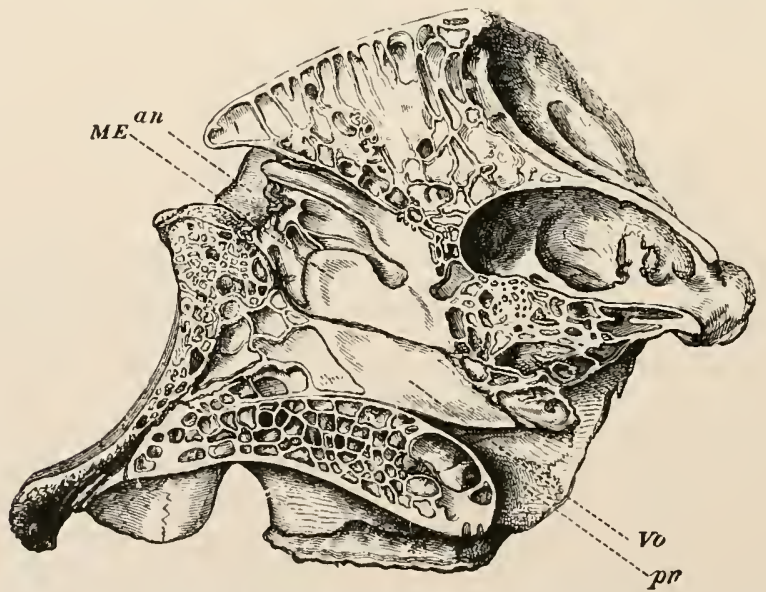

FIa. 1169.-Section of skull of African Elephant (Elephas africanus), to the left of the middle line. an. anterior nares; $M E$. mesethmoid; $p n$. posterior nares; Vo. vomer. (After Flower.)

stout: it is formed mainly by the jugal $(j u)$, which forms part of the glenoid fossa. The post-orbital processes meet in some to 
bound the orbit behind; the upper one is formed from the parietal (par). The facial region is comparatively short. The premaxillæ $(p . \max )$ are not greatly developed. There are distinct paroccipital processes $(p . o c$.$) . The periotic and tympanic are ankylosed$ together, but not to the squamosal. The tympanic $(t y$.$) forms a$ bulla with a spout-like prolongation.

In the Proboscidea (Fig. 1169) the bones of the skull are of enormous thickness, the inner and outer tables being separated by extensive air-cells. The sutures are early obliterated. Paroccipital and postglenoidal processes are absent. The tympanic forms a large, rounded auditory bulla; but the external auditory meatus is bounded chiefly by the post-tympanic process of the squamosal. The mastoid portion of the periotic does not appear on the surface. The orbit is not completely separated by bone from the temporal fossa. The nasal aperture is situated far back, and looks upwards and forwards almost as in the skull of some of the Cetacea. The chief characteristic of the mandible is its prolongation forwards into a spout-like process at the symphysis.

In the Ungulata vera the scapula (Fig. 1170 ) is never very broad; the spine is usually near the middle. Neither the acromion nor the coracoid process is very prominent; sometimes, as in the Horse, the former is absent. A clavicle is never present. In the Ruminants, as in some other Manmals, the vertebral portion of the scapula remains cartilaginous, forming the so-called supra-scapular cartilage $(s s)$. In Pigs and some Perissodactyles, though there is no acromion, there is a triangular process about the middle of the spine.

The humerus is short and stout, the radius always well developed, the ulna in some (Pigs, Hippopotami, Tapirs, and Rhinoceroses) well developed, in others (the Horses and the Ruminants) incomplete.

The first digit is always absent. There is never a centrale. The trapezoid and magnum unite in most of the Ruminants.

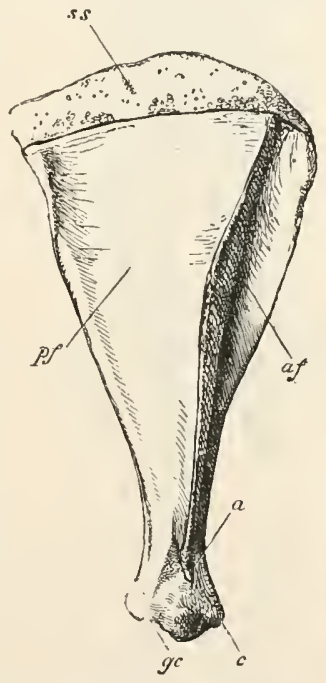

FIG. 1170.-Right scapula of Red Deer (C'ervus elaphus). $a$. acromion; $a f$. prescapular fossa ; $c$. vestigial coracoid process ; gc. glenoid cavity ; pf. post-scapular fossa; $s s$. imperfectly ossified suprascapular portion. (After Flower.)

In the Perissodactyla the third digit in both the fore- and hindfoot is symmetrical in itself. In the Rhinoceroses the second and fourth are also present, and in the Tapirs (Fig. 1171) the fifth of the fore-foot is developed as well. The Horses (Fig. 1172) present the greatest reduction in the number of the digits observable in any Mammal, the third being the only functional digit in each foot. Its elongated metacarpal or metatarsal (sometimes called 
the cannon bone) has in apposition with it laterally a pair of splintlike vestiges which represent the metacarpals or metatarsals of the second and fourth digits. In the Artiodactyla, on the other hand, the third and fourth digits form a symmetrical pair. In the Ruminant Artiodactyles (Fig. 1174) the metacarpals or metatarsals of these digits unite to form a single elongated bone, the cannon bone.

The pelvis of most Ungulata is greatly elongated. The ilia are wide transversely; the symphysis is very long, involving a part of

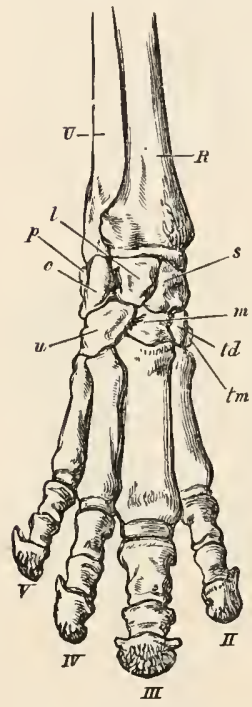

FIG. 1171.-Bones of the manus of Tapir (Tapirus indicuss). $c$ cuneiform ; $l$. lunar $m$. magnum ; $p$, pisiform ; $R$, radius : $s$ scaphoid ; $t d$. trapezoid ; $t m$. trapezium ; $U$. ulna ; $u$. unciform. (After Flower.)

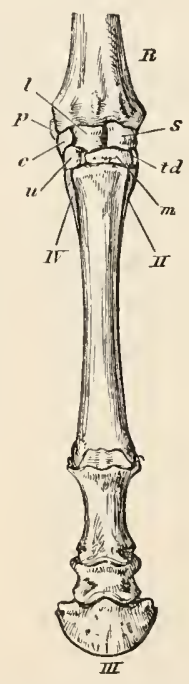

Fia. 1172.-Bones of the manus of Irorse (Equus caballus). c. cuneiform ; 7 . lunar ; $m$. magnum; $p$. pisiform ; $R$. radius ; $s$ scaphoid ; $t d$. trapezoid; $u$. unciform $I I, I V$, vestigial second and fourth metacarpals. (After Flower.)

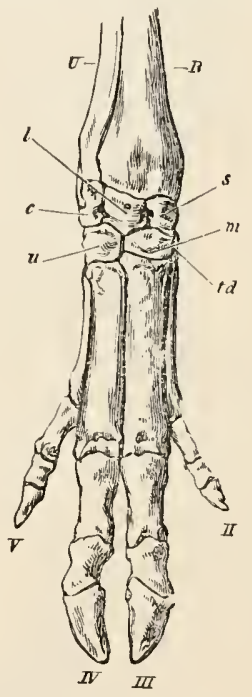

FIG, 1173,-Bones of manus of Pig (Sus scrofa). c. cuneiforın ; $l$. kunar ; $m$. magnum ; $R$, radius ; $s$, scaphoid: $t d$. trapezoid ; $U$. ulna; $u$. unciform. (After Flower.)

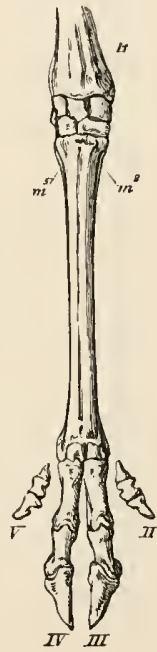

Fig, 117t.-Bones of manus of Red Deer (Cervus claphus). $m^{2} \cdot m^{5}$. vestigial second and tifth metacarpals ; $R$. radius, (After

Flower.)

the ischia as well as the pubes. In the Perissodactyla, but not in the Artiodactyla, there is a well-marked third trochanter.

In some Ungulates (Rhinoceroses, Tapirs, Pigs, Hippopotami) the fibula is distinct though slender. In the Horse it is represented by a vestige. In the Ruminants it is represented only by a small vestige, the malleolar bone, which articulates with the distal end of the tibia.

The structure of the foot exhibits a close parallelism to that of the manus. The tarsal bones are closely dovetailed together, and articulate with one another by flat surfaces. The hallux is 
never developed. In the Perissodactyla the third digit is symmetrical in itself. In the Rhinoceros and Tapirs the second and fourth digits are also completely developed; but in the Horses (Fig. 1175) they are represented only by splint-like vestiges of

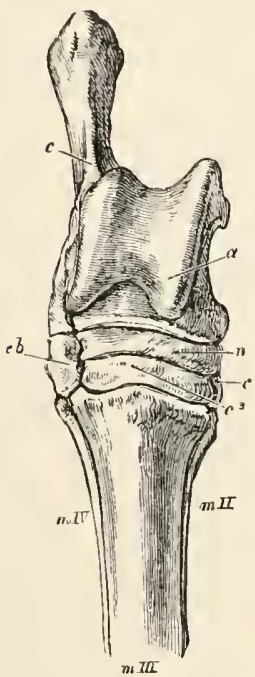

FIG. 1175.-Dorsal surface of right tarsus of Forse (Equus caballus). $a$. astragalus; $c$. calcaneum; $c b$. cuboid; $c$. united meso- and entocuneiform; $c^{3}$. ecto-cuneiform; $n$. navicular; $m I I$ $I Y$, vestigial second and fourtl metatarsals; III, third metatarsal. (After Flower.)

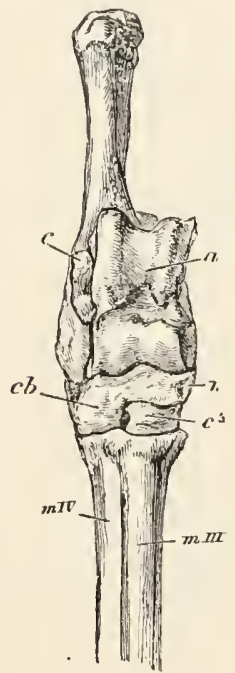

FIG. 1176.-Dorsal surface of right tarsus of Red Deer (Cervus elaphus). a. astragalus ; $c$. calcaneum; $c b$. cuboid ; $c^{3}$. conjoined ecto-' and meso-cuneiform: $m I I I, m I V$, third and fourth metatarsals; $n$. navicular. (After Flower.)

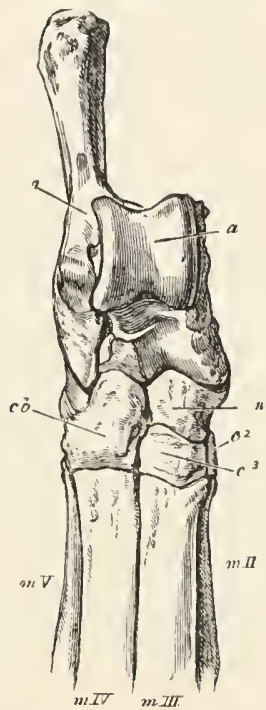

Fig. 1177.--Dorsal surface of right tarsus of Pig (Sus scrofa). a. astragalus ; $c$. calcaneum ; $c b$. cuboid ; $c^{3}$ ecto-cuneiform; $\quad c^{2}$ meso-cuneiform; $m I I$ - $r$, metatarsals; $n$. navicular. (Áfter Flower.)

their metatarsals, the metatarsal of the third digit forming an elongated "cannon bone," like the metacarpal of the third digit of the manus. In the Rhinoceroses and Tapirs all the usual tarsal bones are present; in the Horses the ento-cuneiform and meso-cuneiform are united. In the Artiodactyles the third and fourth digits form a symmetrical pair as in the manus; and in the Ruminants (Fig. 1176) their metatarsals unite to form a cannon bone. In most Ruminants there are no vestiges of the second and fifth digits. In the Pigs (Fig. 1177) all the tarsal bones are present. In most Ruminants the cuboid and navicular are united; in the Camels these bones are distinct, but the ento-cuneiform is wanting.

In the Hyracoidea the scapular is triangular, like that of the Ungulata vera, and the spine is moderately developed, most prominent in the middle. There is a large supra-trochlear foramen. The radius and una are complete, but often ankylosed. 
In the carpus there is a centrale between the scaphoid and the trapezoid. There are five digits, the first very small ; in some the last is represented only by a vestigial metacarpal.

In the femur an indistinct ridge-like elevation is to be regarded as representing the third trochanter. The foot resembles that of the Rhinoceros in having three digits developed; but there is a small bone representing the fifth metatarsal, and the ungual phalanx of the second is cleft.

In the Proboscidea the coracoid process is small. The acromion presents a recurved process or metacromion, as in Rodents. The clavicle is absent. The radius and ulna are permanently fixed in the prone condition. The manus is short and broad; the carpals are squarish, with flat articular surfaces. There is no centrale ; five digits are present. The pelvis has its long axis nearly vertical. The iliac crest is directed transversely, and is greatly expanded; the iliac and gluteal surfaces look almost directly forwards and backwards. The pubes and ischia are comparatively small. The femur is very long as compared with that of the Ungulata vera. There is no third trochanter. The fibula is complete. The foot is short and broad, somewhat smaller than the manus.

Skeleton of the Carnivora.-In the Carnivora the atlas is very large, with wing-like lateral processes. The neural spine of the axis is elongated and compressed, the odontoid conical.

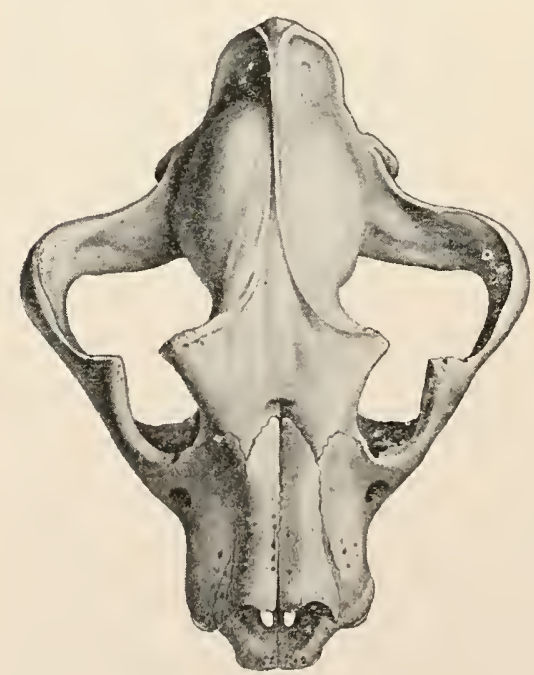

FIG. 1178.-Skull of Tiger (Felis tigris). (After Blainville.)

The other cervical vertebræ have small spines and large transverse processes. There are twenty or twenty-one thoraco-lumbar vertebræ. The most anterior thoracics have long, slender, backwardly-sloping spines. In the posterior thoracics large metapophyses and anapophyses are developed. The transverse processes of the lumbar vertebræ are extremely long and the spines short. The sternum is long and narrow, composed usually of eight or nine pieces. The sternal ribs are almost uncalcified.

In the skull of the Carnivora vera (Figs. 1178 and 1180) there are prominent sagittal and lambdoidal crests. The temporal foss $x$ are very deep; the orbits are not separated from them by bone. The relative development of the facial region varies in 
the different groups ; in the Bears and their allies, and in the Dogs, it is elongated ; in the Cats it is very short. The zygoma is strong and greatly arched outwards. The glenoid cavity is in the form of a transverse groove, to the shape of which the transversely elongated condyle is adapted. In the Cats there is a large rounded tympanic bulla (Fig. 1179), the cavity of which is divided into two parts - anterior and posterior-by a septum, the anterior containing the auditory ossicles and the opening of the Eustachian tube; the bony auditory meatus is short: the parocei-

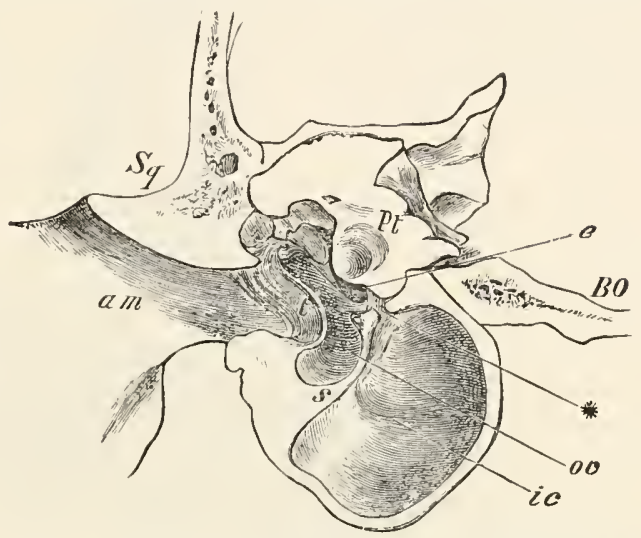

FIG. 1179. - Section of the left auditory bulla of Tiger (Felis tigris). * aperture of communication between the two chambers into which the cavity of the bulla is divided; $a$. $m$. external auditory meatus; $B O$. basi-occipital; $e$. Eustachian tube; ic. the inner chamber; oc. the outer chamber; $P t$. periotic; $s$. septum between the two chambers; Sq. squamosal. (After Flower.) pital is closely applied to the posterior surface of the tympanic bulla. In the Dogs the septum of the bulla is incomplete, the auditory meatus short, and the paroccipital process not applied

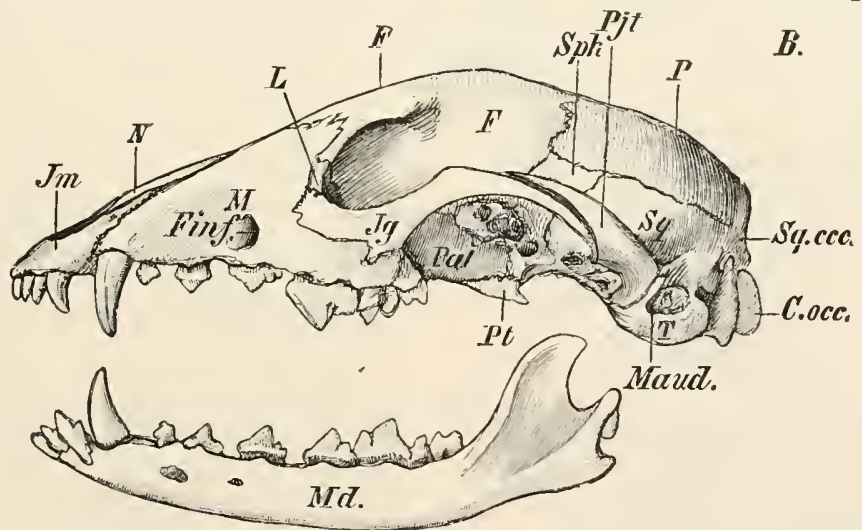

FıG. 1180.-Lateral view of skull of Dog (Canis familiaris). C. occ. occipital condyle ; $F$. frontal ; $F$.inf. infra-orbital foramen $J g$. jugal $; J m$. premaxilla $;$. lacrymal ; $M$. Maxilla Maud. external auditory meatus; $M d$. mandible; $N$. nasal; P. parietal ; Pal. palatine ; Pjt. zygomatic process of squamosal; Pt. pterygoid; $S p h$. ali-sphenoid; Sq. squamosal ; Sq. occ. supra-occiptial ; T. tympanic.' (From Wiedersheim's Comparative Anatomy.)

to the bulla. In the Bears and their allies (Fig. 1181) the bulla is usually less dilated, and the septum is absent or only represented by a ridge, while the bony auditory meatus is elongated. 
The cranium in the Pinnipedia (Fig. 1184) is broad and rounded, rather compressed from above downwards. The orbits are large and approach near to one another.

In the Carnivora vera the spine of the scapula is situated at about the middle of the outer surface of the bone. The acromion

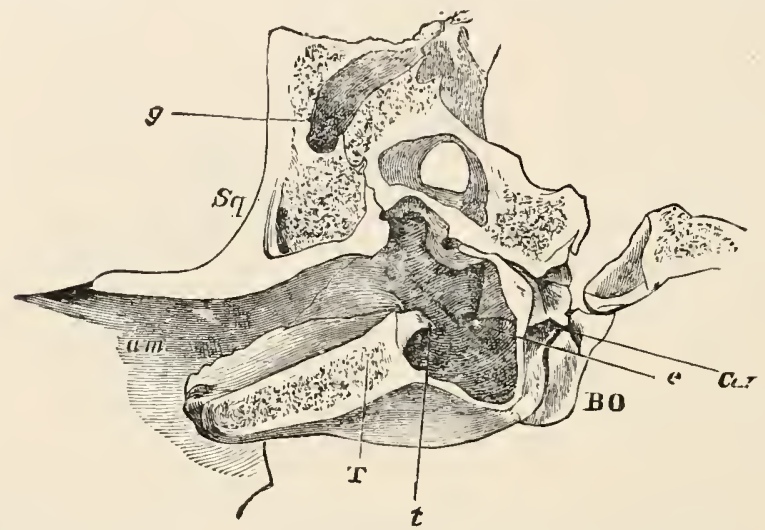

FIG. 118I.-Section of the left auditory bulla and surrounding bones of a Bear (Ursus ferox). a. m. external auditory meatus ; B.O. basi-occipital; Car. carotid canal ; $e$. eustachian canal ; $S q$. squamosal ; $T$. tympanic ; $t$. tympanic ring. (After Flower.)

is usually well developed, sometimes with a metacromion. The coracoid process is very small. The clavicle is never complete, sometimes entirely absent. There is a supra-condyloid foramen in the Cats and some of the other groups, not in the Dogs or Bears.

The scaphoid and lunar are united (Fig. 1182). There is no centrale. Usually a radial sesamoid is present. There are five

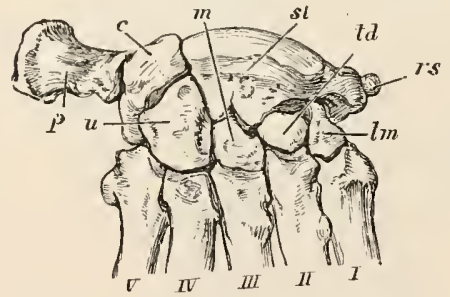

FIG. 1182.-Carpus of Bear (Ursus americanus). c. cuneiform; $m$. magnum ; $p$. pisiform; r.s. radial sesamoid ; sl. scapho-lunar; $t d$. trapezoid; $t m$. trapezium; $u$. unciform. (After Flower.)

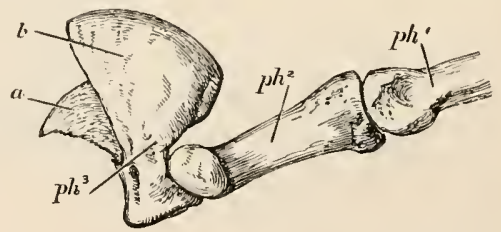

FIt. 1183.-The phalanges of the midlle digit of the manus of the Iion (Felis leo). $p h^{1}$. proximal phalanx; $p h^{2}$. middle phalanx; $p h^{3}$. ungual phalanx; $a$, the central portion forming the internal support to the horny claw $; b$, the bony lamina reflected around the base of the claw. (After Flower.)

digits, though the pollex may be reduced in size, as in the Dog, and it is vestigial in the Hyæna.

The pelvis is long and narrow. In the tarsus all the ordinary bones are developed. The hallux is fully formed in the Bears, etc., but shorter than the other digits. In the Cats and Dogs it is represented only by a vestige of the metatarsal. 
In the Pinnipedia (Fig. 1184) both acromion and coracoid are short, and the scapula is curved backwards; there is no clavicle. The bones of the fore-limb are short and stout; the humerus has a prominent deltoid crest; there is no foramen above the inner condyle. The ulna is greatly expanded at its proximal, the radius at its distal end. The manus is broad and expanded. The scaphoid and lunar are united to form a scapholunar. The ungual phalanges are nearly straight, slender, and pointed. The ilia are short; the symphysis pubis is short and without firm union of the bones. The femur is short, thick, and flattened. The fibula and tibia are commonly ankylosed proximally. The calcaneum is short and usually without a distinct calcaneal process; the lateral digits are usually the longest.

Skeleton of the Rodentia. - Among the Rodents the Jerboas are exceptional in having the cervical vertebræ ankylosed. Generally, as in the Rabbit, the transverse processes of the lumbar vertebræ are elongated. As in the Ungulata, the sacrum usually consists of one broad anterior vert bbra followed by several narrower ones. The caudal region varies in length in

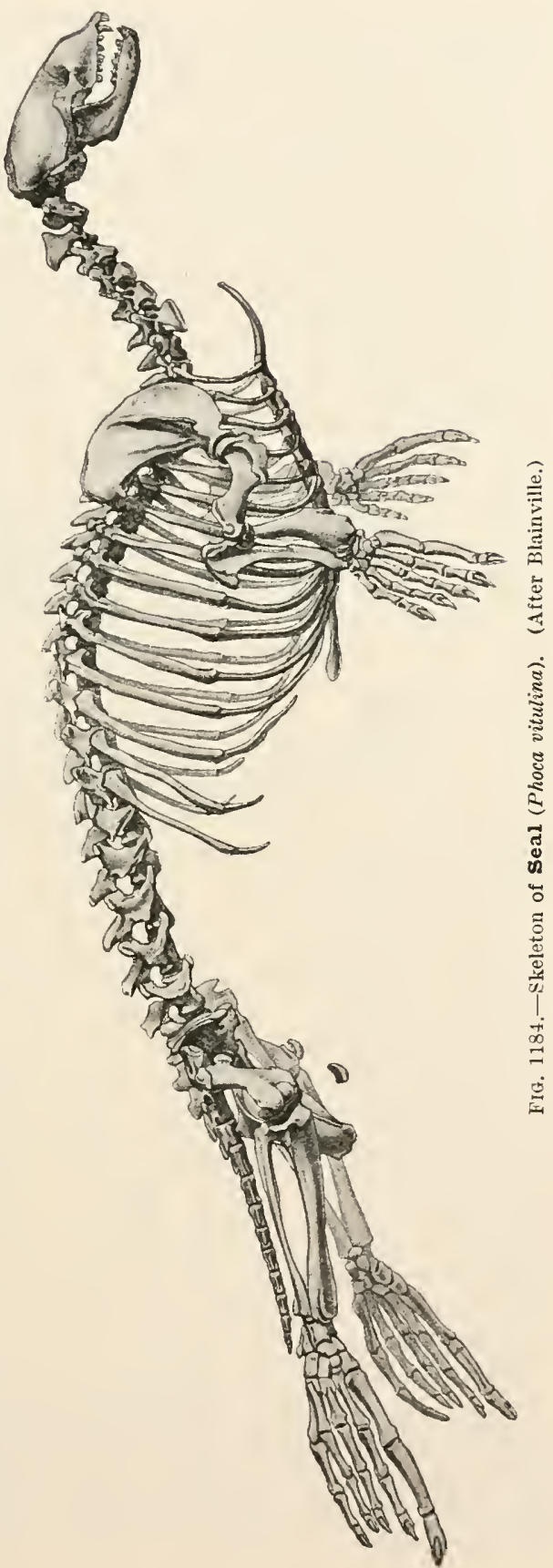


the different families; in some it is very short, but it is elongated in many (the Porcupines, Squirrels, and Beavers). The sternum of the Rodents has a long and narrow body; sometimes there is a broad presternum; the posterior end is always expanded into a cartilaginous xiphisternum.

The skull is elongated, narrow in front, broader and depressed behind. The nasal cavities are very large, especially in the Porcupines, with air sinuses in the upper part. In some the optic foramina fuse into one. An interparietal is often present. Paroccipital processes are developed. The orbit and the temporal fossa are always continuous. The nasal bones are large, and the nasal apertures are terminal or nearly so. The premaxillæ are always very large. A remarkable feature of the skull is the presence in many of a large opening corresponding to the infra-orbital foramen. The middle part of the zygoma is formed by the jugal; the latter often helps to bound the glenoid cavity, as in the Marsupials. The palate is short, and the anterior palatine foramina large. The periotic and tympanic may become ankylosed together, but not to the neighbouring bones. The coronoid process of the mandible is sometimes rudimentary or absent; the angle is often produced into a process.

The scapula of the Rodentia is generally long and narrow. The spine sometimes has a metacromion process and a long acromion. The coracoid process is small. The clavicle varies as regards its development. Vestiges of the sternal end of the coracoid are - sometimes distinguishable. There is considerable variation in the bones of the arm and fore-arm. The radius and ulna are in most instances distinct, though in close and firm apposition. The scaphoid and lunar are usually united; the centrale is sometimes present, sometimes absent. The pelvis and femur vary greatly. Sometimes there is a third trochanter. The fibula is sometimes distinct, sometimes fused with the tibia. In the Jerboa the metatarsals of the three digits are fused together.

Skeleton of the Insectivora.-The neural spine of the axis is usually well developed, that of the remaining cervical vertebræ small or obsolete. The number of trunk-vertebræ varies in the different families from eighteen to twenty-four, and there is also great variation in the development of the various processes. The caudal region varies in its length; frequently it has chevron bones. The presternum is expanded, the mesosternum composed of distinct, narrow sternebræ.

The skull (Fig. 1185) varies greatly in the different families, in the higher forms approaching that of the Lemurs, with comparatively large cerebral fossæ, large orbits with complete or nearly complete bony rims, well-developed zygoma, and a tympanic bulla and tubular auditory meatus. In the others the cranial capacity is less, and the orbits and temporal fossæ are completely 
continuous; the zygoma is incomplete, and the tympanic does not usually form a bulla.

The pectoral arch also varies a good deal in the different families of the Insectivora. In the true Moles and their allies there is a remarkable bone of cuboid shape articulating ventrally with the presternum and dorsally witl the humerus, and only connected by a ligamentous band with the scapula. Its mode of formation from a mass of cartilage-to the anterior face of which the clavicle, formed as usual in membrane, becomes applied-proves that this bone represents a pro-coracoid as well as a clavicle. In other Insectivora this bone is not developed, and the clavicle is a distinct, long and slender bone, but vestiges of the inner or ventral ends of the coracoid and pro-coracoid may be recognisable. Sometimes

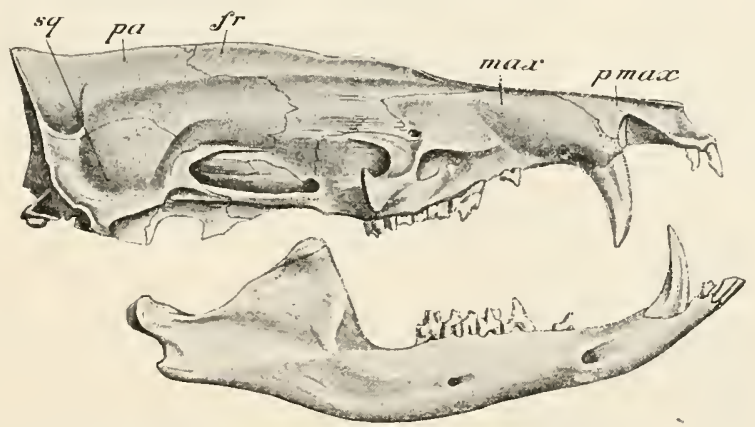

FIG. 1185.-Skull of Tenrec (Centetes ecaudatus). fr. frontal; max. maxilla ; pa. parietal ; p.max. premaxilla ; sq. squamosal. (After Dobson.)

the "mesoscapular segment" (p. 500) is represented by a distinct bone intervening between the outer end of the clavicle proper and the acromion process.

The humerus usually has a supracondylar foramen. In the Moles this is absent, and their humerus is remarkable in other respects, being short, greatly expanded at the extremities, with a prominent deltoid ridge, and with two synovial articular surfaces at the proximal end, one for the glenoid cavity of the scapula, the other for the coraco-clavicle. The radius and ulna are completely developed in all and are usually distinct, but sometimes fused distally. In the carpus the scaphoid and lunar sometimes coalesce, sometimes remain distinct; an os centrale is usually present. In the Moles the manus is extremely broad, the breadth being increased by the presence of a large, curved, radial sesamoid.

In the pelvis the symphysis pubis is in some cases elongated, in others short, and sometimes absent, the pubes remaining separated by a wide median ventral cleft. A third trochanter is sometimes represented by a ridge. The fibula usually, though not always, fuses distally with the tibia. 


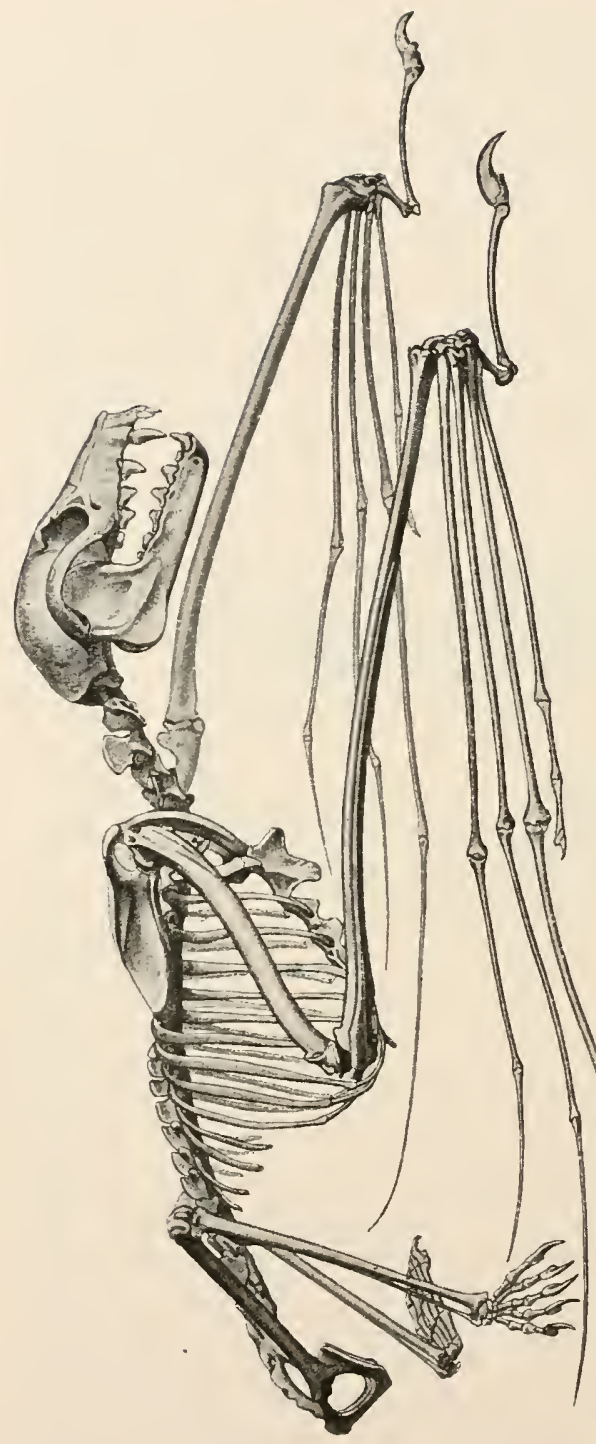

Skeleton of the Chiroptera (Fig. 1186).-The cervical region of the vertebral column is characterised by the absence of any distinct neural spines, and the same holds good to a less extent of the trunkvertebræ; the trans$\widehat{o}$ verse processes of the lumbar region are also I rudimentary. The tail * varies in development: when it is elongated the component verte$\dot{\infty}$ bræ are long, cylindrical centra without processes. Sagittal $\stackrel{\text { }}{\approx}$ and occipital crests are developed in the skull $\approx$ of some species. The i facial region is rather of elongated, especially in the Megachiroptera (Fig. 1187). Postorbital processes of the frontal are present or absent : the zygoma is long and slender : the malar is small and applied to the outer surface of the zygoma. The long and narrow nasals are in some cases united ; the premaxillæ are small. The mandible has an angular process in the Microchiroptera, not in the Megachiroptera. The segments of the sternum are sometimes distinct, sometimes united; the presternum has a mesial keel 
developed in co-ordination with the great size of the pectoral muscles. The sternal ribs are ossified.

The scapula is large and oval in shape: the spine is near the anterior margin : the post-scapula fossa has ridges for the origin of the muscular fibres: the spine has a well-developed acromion. The coracoid is elongated and in some cases bifurcated. The clavicle is long. The pro-coracoid is represented by a separate ossification; there are rudiments of the sternal end of the coracoid between the clavicle and the first rib. The humerus and radius are

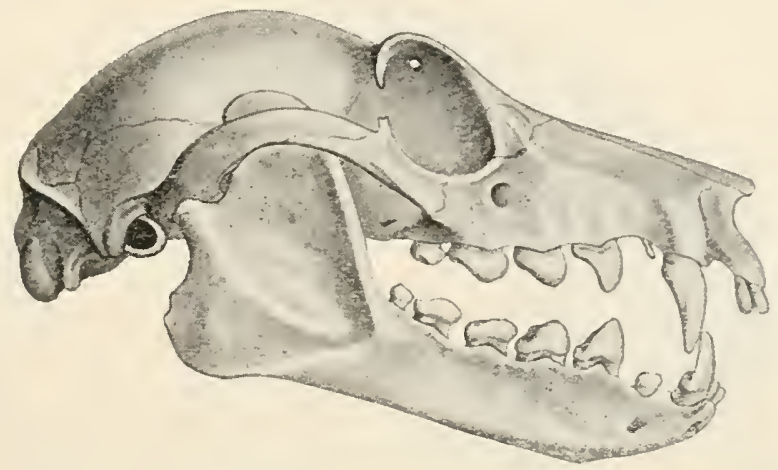

Fit. 1187.-Skull of Pteropus fuscus. (After Blainville.)

both elongated. The ulna is reduced, and is sometimes only represented by the proximal end, ankylosed with the radius. A large sesamoid is developed in the tendon of the triceps muscle near the olecranon process of the ulna. In the carpus the scaphoid and lunar are united: sometimes also the cuneiform is united with these: the pisiform is small. There is no centrale. The ungual phalanges are absent in the nailless digits. The pelvis is small, and the symphysis pubis often imperfect. The fibula is sometimes well-developed, sometimes rudimentary. The tuber calcanei is an inwardly curved process of the calcaneum, attached to which by means of ligamentous fibres is a slender rod of bone or cartilage, the calcar, which supports the inter-femoral membrane.

Skeleton of the Primates.-The atlas is ring-like, the odontoid sub-conical. The spines of the cervical vertebræ are usually well-developed and simple: in Man they are short-with the exception of the seventh-and bifid: in some they are trifid. The number of thoraco-lumbar vertebræ is usually nineteen, but only seventeen in Man, the Gorilla and Chimpanzee, sixteen in the Orang ; in some Lemurs it may be twenty-three or twenty-four. The number of sacral vertebræ varies from two to five. The sacral region of Man, which comprises five ankylosed vertebræ, differs from that of other Primates in its greater relative breadth and in its 
backward curvature; it forms a well-marked angle where it joins the lumbar region-the sacro-vertebral angle-scarcely recognisable in other Mammals. The number of caudal vertebræ varies with the length of the tail-from four to about thirty-three. In Man there are only four vestigial caudal vertebræ, ankylosed together to form the coccyx. In all those forms in which the tail is well developed chevron bones are present.

The human skull (Fig. 1188) presents a marked contrast in certain respects to that of other Mammals, but in many points is

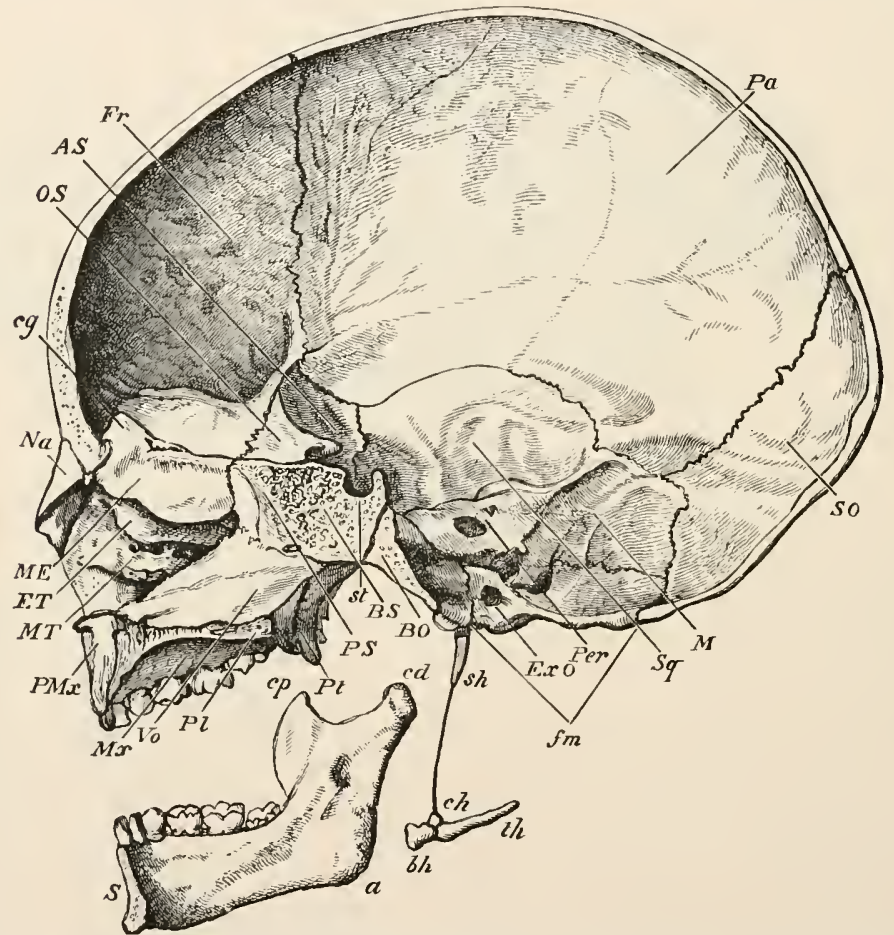

FIG, 1188,- Skull of Man. Letters as in Fig. 1167. In addition, $a$. angle of mandible; c.g. crista galli, a process of the mesethmoid; $\mathrm{fm}$. foramen magnum; $M$. mastoid; st. sella turcica. (After Flower.)

approached by that of the other Primates, more especially by that of the Simiidx. One of the most important characteristics of the human skull is the large size of the brain-case, the cubic content of the cranial cavity averaging 1500 cubic centimetres in the male of white races. This great development is most marked in that part of the cavity which lodges the cerebral hemispheres, in adaptation to the large dimensions of which the cranium bulges out both anteriorly and posteriorly to such an extent that the entire length of the cavity greatly exceeds that of the basi-cranial axis. A 
result of the posterior bulging of the brain-case is that the foramen magnum $(f . m)$ is no longer situated at the posterior extremity of the skull as in other Mammals, but assumes a position further forwards towards the middle of the base. The anterior expansion, causing a strong arching forwards of the frontal region, brings about an alteration in the position of the ethmoidal plane, which, instead of being perpendicular or inclined to the basi-cranial axis, becomes horizontal, and the cribriform plate forms the middle part of the floor of the anterior extension of the cranial cavity. The fossa for lodgment of the cerebellum lies entirely beneath the posterior portion of the cerebral fossa : the olfactory fossa is comparatively small. (See Fig. 1138, D.)

The outer surface is smooth and rounded, devoid of any prominent ridges or crests. The occipital crest of lower Mammals is represented merely by a rough raised line-the superior curved line of the occiput. The paroccipital processes are only represented by slight eminences-the jugular eminences. There is no auditory bulla; the mastoid portion of the periotic projects downwards as a prominent mastoid process. The periotic, tympanic, and squamosal early fuse into one bone-the temporal bone. The post-glenoid process is very slightly developed. The whole facial region is relatively small. The orbits, which are of moderate size, are directed forwards; the bony margin is complete, and a plate of bone, developed partly from the jugal, partly from the alisphenoid, almost completely cuts it off from the temporal fossa, leaving only a small aperture of communication-the spheno-maxillary fissure. The frontal suture usually early disappears. The nasals are rarely fused. The suture between the premaxillæ and the maxillæ becomes obliterated at an early stage, so that the entire upper jaw appears to consist of a single bone. A peculiar spine, the nasal spine, is developed in the middle line below the nasal opening. The most marked feature of the mandible is the presence of a prominence, the mental prominence, in the lower part of the symphysial region (S.). The stylo-hyal nearly always becomes fused-together with the tympano-hyal-to the periotic and tympanic, giving rise to a slender process-the styloid process (sh.) - projecting downwards from the base of the skull.

None of the other Primates have a cranial capacity approaching that of Man; and those modifications in the shape of the skull which are the concomitants of the great development of the brain in the human species are accordingly not recognisable, or are much less strongly marked. The various fossæ of the cranium, as a rule, occupy, however, the same relative positions as in Man ; the cerebellar fossa is entirely beneath the cerebral ; and the ethmoidal plane, and that of the foramen magnum (occipital plane), are usually both horizontal or nearly so. In all the Simiidæ, with the exception of the Orang, the frontals meet in the middle line below, over 
the pre-sphenoid. In many monkeys the outer surface of the cranium is smooth and free from prominent ridges; but in the Baboons, the Orangs, the Gorilla, and the Chimpanzee (Fig. 1189)

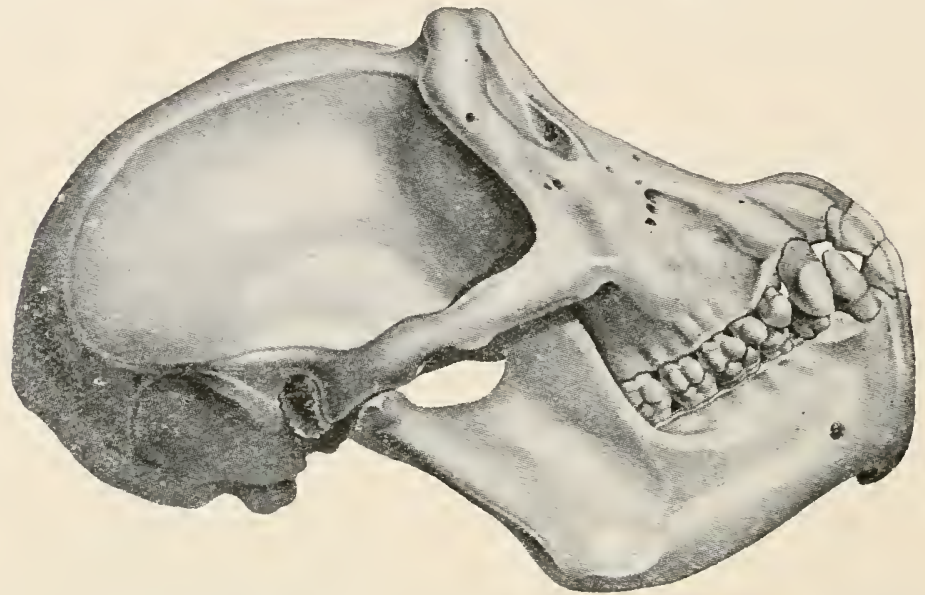

FIa. 1189.-Skull of Chimpanzee (Anthropopithecus trojlodytes). (After Blainville.

there are strongly developed occipital, sagittal, and supra-orbital ridges, usually much more prominent in the male than in the female, and increasing in size with age. The paroccipital processes are always rudimentary, but there are well-marked post-glenoid processes. The mastoid does not form a distinct mastoid process. In the Cebidæ and Hapalidæ alone is there a tympanic bulla. The entire facial region is relatively larger than in Man; the premaxillo-maxillary region is always more prominent, and in the Baboons projects forwards as a distinct muzzle. The orbit is separated from the temporal fossa as in Man. The nasals are usually ankylosed in the adult. The nasal spine is never developed. The suture between the premaxilla and the maxilla only becomes obliterated, if at all, in old individuals. The mental prominence of the mandible is never developed, the anterior surface of the symphysial region sloping backwards and downwards from the bases of the incisor teeth. The stylo-hyal never gives rise to an ossified styloid process.

In the skull, as in many other respects, the Lemurs occupy an intermediate position between the higher Primates and the lower orders of Manmals. The occipital and ethmoidal planes are usually vertical. The tympanic forms a large bulla. The orbits, which are large, are usually separated from the temporal fossæ only by a narrow rim of bone. The lacrymal foramen is situated on the face outside the margin of the orbit. The facial region is usually elongated, and may form a prominent muzzle.

In all the Primates the clavicle is present and complete, and 
in the scapula the spine, acromion, and coracoid process are well developed. In Man and the higher Apes the glenoid border of the

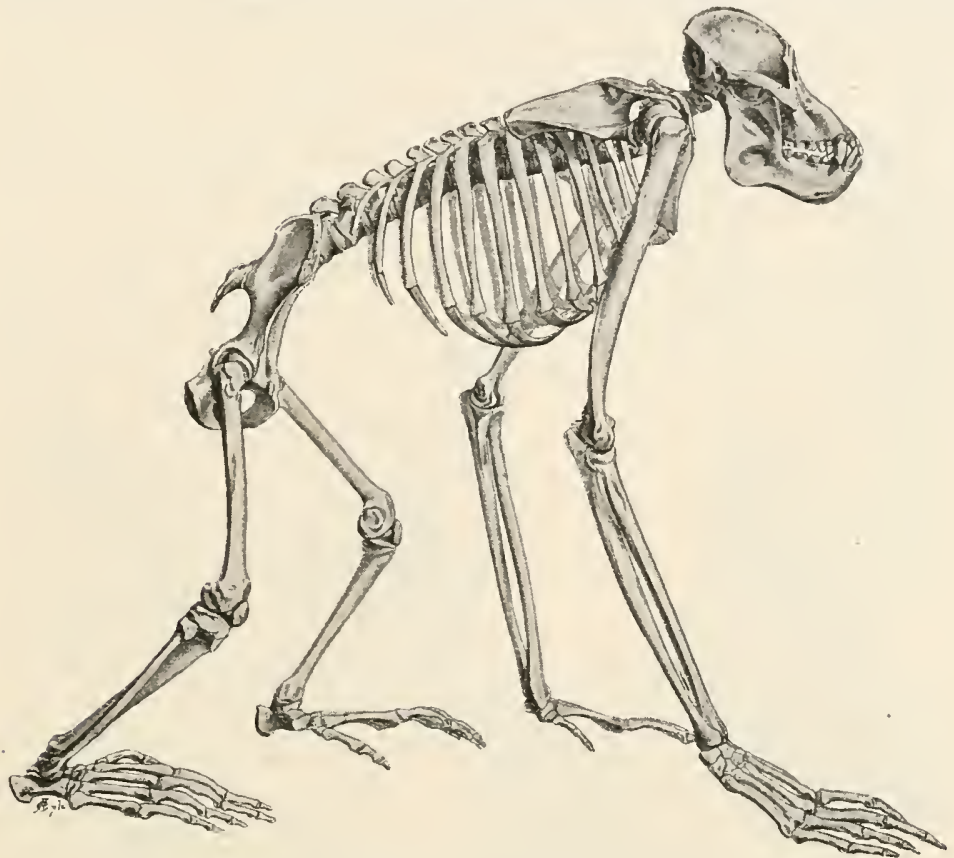

FIG. 1190.-Skeleton of Orang (Simia satyrus). (After Plainville.)

scapula is much longer than the coracoid border. In the lower Monkeys, on the other hand, these borders are nearly equal. The humerus is comparatively long and slender; the tuberosities and ridges are not, as a rule, very strongly developed. In Man and the Simiidre the bone is twisted around its long axis; in the lower forms this torsion is absent. In Man and the higher Apes the foramen above the inner condyle is absent; it is present in many of the American Monkeys and in most Lemurs. Characteristic of the ulna of Man and the higher Apes is the small upward extension of the olecranon process. The radius and ulna are distinct in all; in the higher forms the shafts of the two bones are bent outwards, so that there is a wide interosseous space, and there is considerable freedom of movement in pronation and

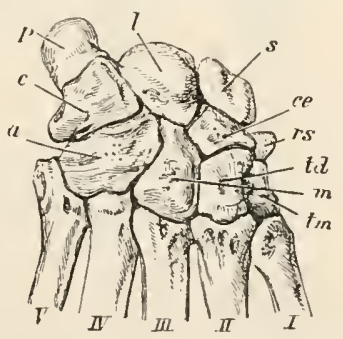

Fia. 1191.-Carpus of Baboon (Cynocephalus anubis). ce. centrale; $c$. cuneiform; $l$. lunare; $m$. magnum ; $p$. pisiform; rs. radial sesamoid ; $s$. scaphoid ; $t d$. trapezoid ; tm. trapezium ; $u$. unciform. (After Flower.) supination. In the carpus (Fig. 1191) the scaphoid and lunar are always distinct, and a centrale is present in all except some of the 
Lemurs, the Gorilla, Chimpanzee, and Man. A pisiform is present, and in most a radial sesamoid. As compared with that of the other Primates, the carpus of Man is short and broad; the trapezium has a saddle-shaped articular surface turned somewhat inwards. In Man, the Chimpanzee, Gorilla, and Orang the carpus articulates exclusively with the radius; in all the others it articulates also with the ulna. In Man the pollex has a remarkable and characteristic freedom of movement in opposition to the other digits.

The human pelvis is remarkable for its relative breadth, for the expanded form of the ilia and the deep concavity of their inner surfaces, and for the shortness of the pubic symphysis. In the higher Apes some of these features are recognisable, though less

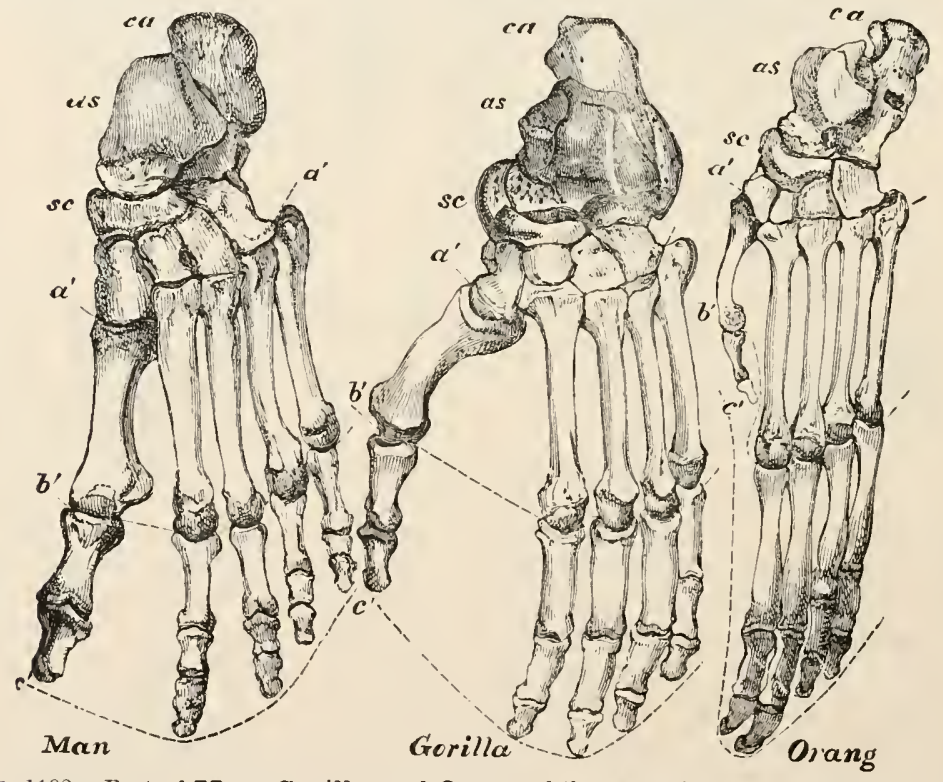

FIG. 1192.-Foot of Man, Gorilla, and Orang of the same absolute length, to show the difference in proportions. The line $a^{\prime} a^{\prime}$ indicates the boundary between tarsus and meta. tarsus ; $b^{\prime} b^{\prime}$, that between the latter and the proximal phalanges; and $c^{\prime} c^{\prime}$ bounds the ends of the distal phalanges; as. astragalus; $c a$. calcaneum; sc. scaphoid. (After Huxley.)

pronounced; but in the lower the ilia are long and narrow, and usually curved outwards ; in the Old-world Monkeys the tuberosities of the ischia are strongly everted and roughened for the attachment of the ischial callosities.

The tibia and fibula are well developed and distinct in all. In nearly all the hallux, owing to the form and direction of the articulation between it and the internal cuneiform, is opposable to the other digits, converting the foot into a grasping organ. The human foot (Fig. 1192) is distinguished from that of the other 
Primates by the absence of this power of opposition and by the relative length of the tarsus, which exceeds that of the metatarsus.

Digestive Organs. - Teeth are present in nearly all Mammals, but in some they are wanting in the adult condition (Whalebone Whales and Platypus). In Echidura teeth are not present even in the young. In some of the Ant-eaters teeth are developed in the fotus and are thrown off in utero - the adult animal being devoid of them.

Teeth, as already explained in the general account of the Craniata (p. 84), are developed partly from the epidermis and partly from the underlying dermis. In the Mammals each tooth is lodged in a socket or alveolus in the jaw. The part of the tooth developed from the epidermis is the enamel; the remainder of the toothdentine, cement and pulpbeing formed from the subjacent mesodermal tissue.

Along the oral surface of the jaw is formed a ridgelike ingrowth of the ectoderm-the dental lamina (Fig. 1194, lam.). The position of this is indicated externally by a groove - the dental groove ( $g r$.$) , and from$ it a bud is given off in the position to be occupied by each of the teeth. This becomes constricted oft as a conical cap of cells-the enamel-organ-which remains in continuity with the
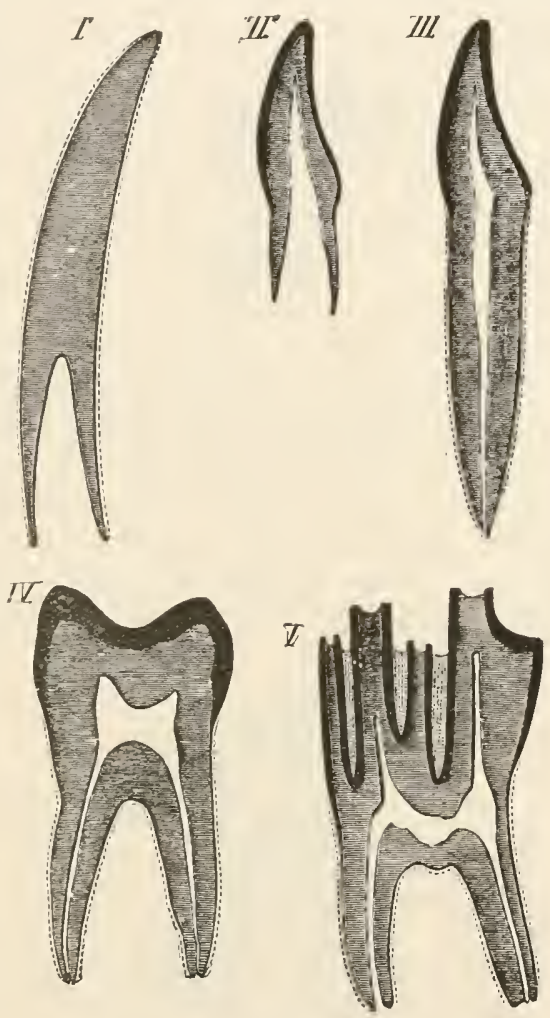

FIG. 1193.-Diagrammatic sections of various forms of teeth. $I$, incisor or tusk of Elephant with pulpcavity persistently open at base ; $I I$, human Incisor during development, with root imperfectly formed, and pulp-cavity widely open at base ; $I I I$, completely formed human incisor, with pulpcavity opening by a contracted aperture at base of root; $I V$, human molar with broad crown and two roots; $V$, molar of the $0 x$, with the enamel covering the crown deeply folded, and the depressions filled up with cement; the surface is worn by use, otherwise the enamel coating would be continuous at the top of the ridges. In all the figures the enamel is black, the pulp white, the dentine represented by horizontal lines, and the cement by dots. (After Flower and Lydekker.) dental ridge only by a narrow isthmus. This cap-like form is brought about by the development of a papilla of condensed dermal tissue-the dental papilla (pap.). which pushes upwards, against the enamel-organ. On the surface of this papilla, in contact with 
the enamel-organ, the cells (odontoblasts) become arranged into a layer having the appearance of an epithelium-the dentine-forming layer. The cells of the enamel-organ form two layers, of which that in contact with the dental papilla assumes the character of a

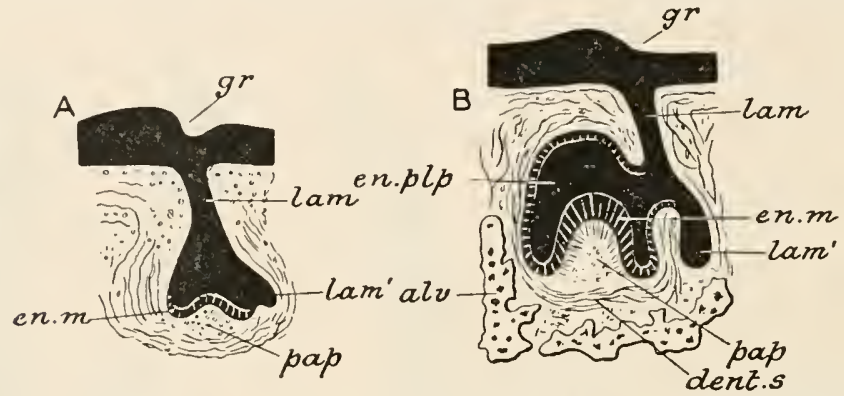

F10. 1194.-Two stages in the devclopment of the teeth of a Mammal (diagrammatic sections). alv. bone of alveolus; dent. $s$. dental sac ; en.m. enamel-membrane ; en. $p l p$. enamel-pulp; gr. dental groove; lam. dental lamina ; lam'. part of dental lamina which grows downwards below the tootl-germ; pap. dental papilla. (After O. Hertwig.)

layer of long cylindrical cells-the enamel-membrane (en. m.); the more superficial layer consists of cubical cells. Between the two the remaining cells of the enamel-organ become modified to form a kind of connective tissue-the enamel-pulp (en. plp.).

The connective tissue immediately surrounding the entire rudi-

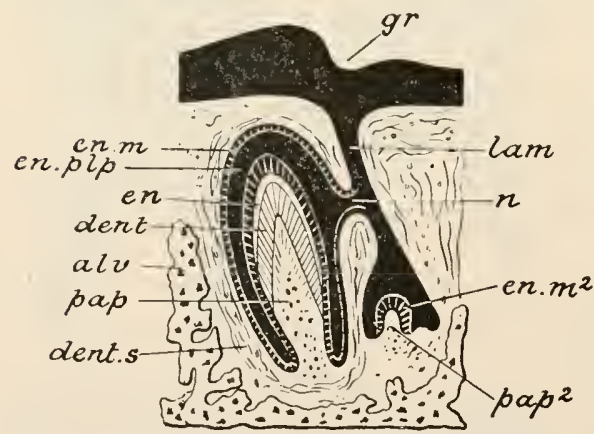

FIG. 1195.-Diagrammatic section showing the development of the milk- and permanent teeth of Mammals. alv. bone of alveolus; dent. dentine; dent. s. dental sac; en. layer of enamel ; en. $m$. enamel-membrane of milk-tooth; en. $\mathrm{m}^{2}$. enamel-membrane of permanent tooth ; en.plp. enamel-pulp of milk-tooth ; gr. dental groove; lam. dental lamina; n. neck connecting milk-tooth with lamina; pap. dental papilla of milk-tooth ; pap ${ }^{2}$. dental papilla of permanent tooth. (After O. Hertwig.)

of the dental papilla gives rise to the pulp. As the tooth substance of the dental papilla gives rise to the pulp. As the tooth elongates, it projects on the surface and eventually breaks through the mucous membrane of the gum, the remains of the enamel-organ becoming thrown oft. The cement is formed by the ossification of the connective-tissue of the dental sac.

ment of the tooth becomes vascular and forms a distinct investment - the dental sac (dent. s.); from this blood-vessels extend into the papilla.

Ossification begins by the formation of a cap of dentine (Fig. 1195, dent.), produced by the dentineforming cells, and of a layer of enamel (en.) on the surface of this, produced by the cells of the enamel membrane. To these additional layers are added until the crown of the tooth becomes fully developed. The substance 
In the teeth of most Mammals distinct roots are formed, each with a minute opening leading into the pulp-cavity (Fig. 1193, III-V); but in some there are no roots, the pulp-cavity being widely open below $(I)$, and the tooth constantly growing from the base as it becomes worn away at the crown; such teeth are said to have persistent pulps.

Usually Mammals have two distinct sets of teeth developed, the milk and permanent dentitions, but sometimes there is only one, and accordingly we distinguish diphyodont and monophyodont Manmals : in nearly all of the latter, however, another set are developed, though they early become absorbed or remain in the condition of functionless vestiges; and in a considerable number
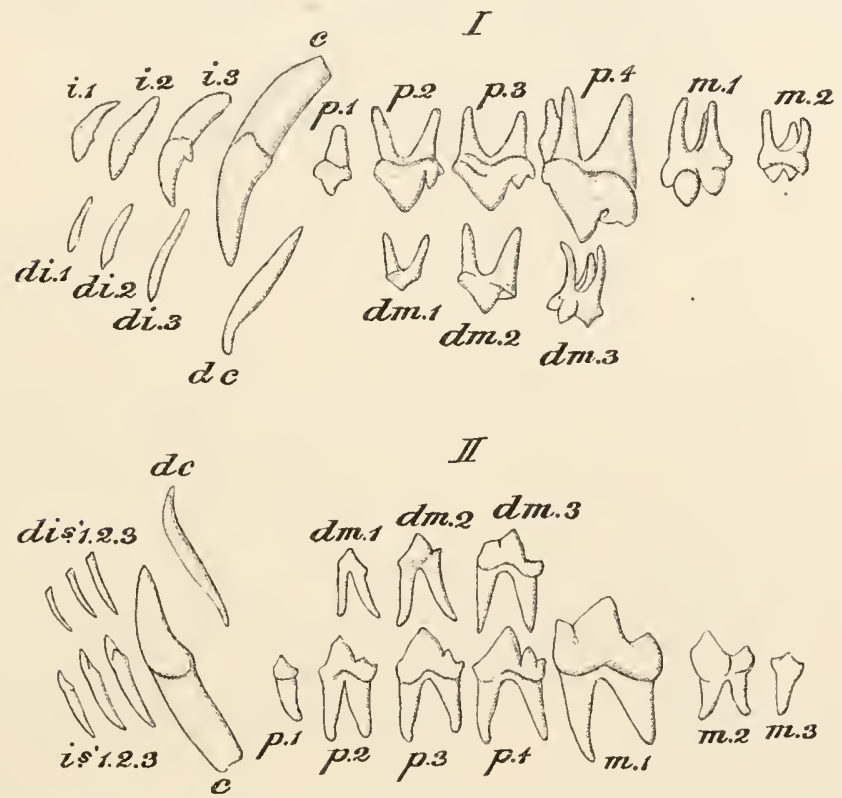

FiG. 1196.-Milk- and permanent dentition of upper $(I)$ and lower $(I I)$ jaws of the Dog (Canis familiaris), with the symbols by which the different teeth are commonly designated. (After Flower and Lydekker.)

of groups it has been found that more than two sets of teeth are formed, only one, or at most (in diphyodont forms) two, of these sets becoming fully developed. "The milk-teeth in Mammals with typical diphyodont dentition sometimes disappear at an early stage, and sometimes do not become replaced by the permanent teeth till long after birth. Some Mammals have the teeth almost indefinite in number, e.g., the Dolphins and Porpoises, in which they are all uniform (homodont) and not divided into sets (Fig. 1197). In the typical dentition there are forty-four teeth, viz., three incisors on each side, one canine, and seven premolars and molars above and below. The incisors (Fig. 1196,i.) of 
the upper jaw are to be distinguished as being the teeth that are lodged in the premaxillæ; the incisors of the lower jaw are the teeth that are placed opposite to these. The upper canine $(s$.$) is$ the most anterior tooth of the maxilla, situated on or immediately behind the pre-maxillo-maxillary suture, and has usually a characteristic shape. The lower canine is the tooth which bites in front of the upper canine. The pre-molars $(p$.$) are distinguished from$ the molars by having milk predecessors $(d . m$.$) , but the first pre-$

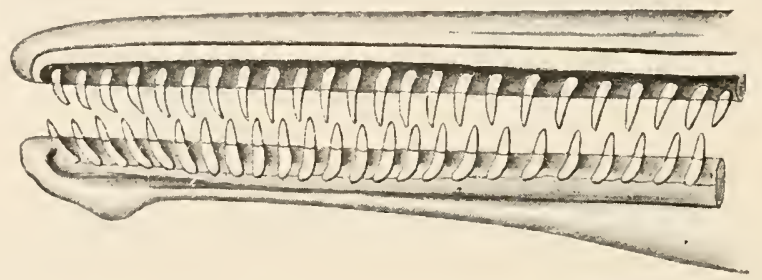

FIG. 1197.-Upper and lower teeth of one side of the mouth of a Dolphin (Lagenorhynchus) illustrating the homodont type of dentition in a Mammal. (After Flower and Lydekker.)

molar is, except in the Marsupials, nearly always a persistent milk-tooth; the molars $(m$.) have no teeth preceding them, and are sometimes looked upon as persistent teeth of the first set. The various sets of teeth are also usually distinguishable by their shape. As a rule the incisors have cutting edges; the canines are pointed and conical; the pre-molars and molars have broad surfaces with ridges and tubercles for crushing the food, and may have from two to four roots.

The simplest form of molar tooth (which occurs, however, only in

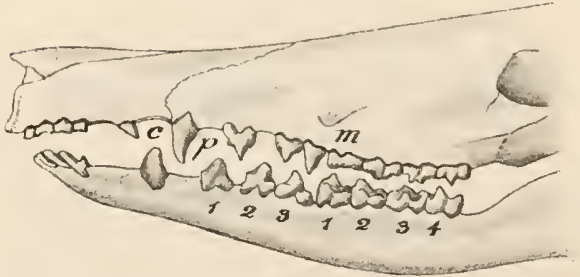

-FIG. 1198.-Teeth of Bandicoot (Perameles). (After Owen.) certain extinct forms) is that of a simple cone, or a cone with two small accessory processes or cusps. Almost as primitive is the type of tooth termed triconodont (likewise occurring only in a few extinct Mammals), in which there are three equal conical cusps set in a straight line, the upper teeth biting on the outer side of the lower. From the triconodont is derivable the trituberculate molar, in which the free surface of the tooth presents three cusps or tubercles arranged in a triangle, the apex of which is internal in the upper, external in the lower jaw. In the upper molar the inner cusp is termed the protocone, the antero-external the paracone, and the postero-external the metacone. These terms are modified in the case of the molars of the lower jaw, the equi- 
valent of the protocone, here external, being termed the protoconid, and the others paraconid and metaconid respectively. This trituberculate type of molar is usually complicated by various additions and modifications-accessory cusps being added, together with ridges or folds connecting the cusps together. The resulting complex tooth may be modified to act as a cutting (secodont) or a crushing (bunodont) molar. A modification of the bunodont molar is brought about by the cusps, instead of retaining their conical form, being drawn out into the shape of crescents (selenodont).

The number of the various sets of teeth in the jaws is conveniently expressed by a dental formula, in which the kind of tooth (incisor, canine, pre-molar, molar) is indicated by the initial letter $(i ., c ., p$. , $m$.), and the whole formula has the arrangement of four vulgar fractions, in each of which the numerator indicates the teeth of the upper, the denominator those of the lower jaw. Thus:

$$
\text { i. } \frac{3 \cdot 3}{3 \cdot 3}, c \cdot \frac{1 \cdot 1}{1 \cdot 1}, p \cdot \frac{4 \cdot 4}{4 \cdot 4}, m \cdot \frac{3 \cdot 3}{3 \cdot 3}=44 \text {; }
$$

or, in a simpler form, since the teeth of the right and left sides are always the same,

$$
i . \frac{3}{3}, c \cdot \frac{1}{1}, p \cdot \frac{4}{4}, m \cdot \frac{3}{3}=44 .
$$

Echidna has no teeth at any stage. In Ornithorhynchus teeth are present in the young and are functional for a time, but they are thrown off when the animal is about a year old: vestiges of an earlier dentition have been detected. The function of teeth is performed in the adult by broad. horny plates, one on each upper and one on each lower jaw.

The Marsupials have the milkdentition in a degenerate condition. Germs of milk-teeth are developed, but with the exception of one-the last pre-molarthese remain in an imperfect state of development, though they persist, as functionless vestiges, to a comparatively late stage.

In the adult dentition of the Marsupials the number of incisors in the upper and lower jaws is always dissimilar, except in Phas-

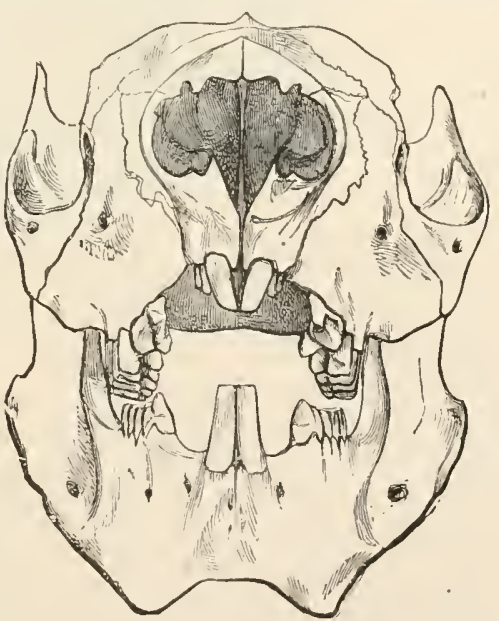

FIG. 1199.-Front view of skull of Koala (Phascolarctos cinereus), illustrating diprotodont and herbivorous dentition. (After Flower.) colomys. With regard to the arrangement of these teeth, the order falls into two series, termed respectively the diprotodont and the 
polyprotodont. In the former (Figs. 1199, 1200) the two anterior incisors are large and prominent, the rest of the incisors and the canines being smaller or absent. On the other hand, in the poly-

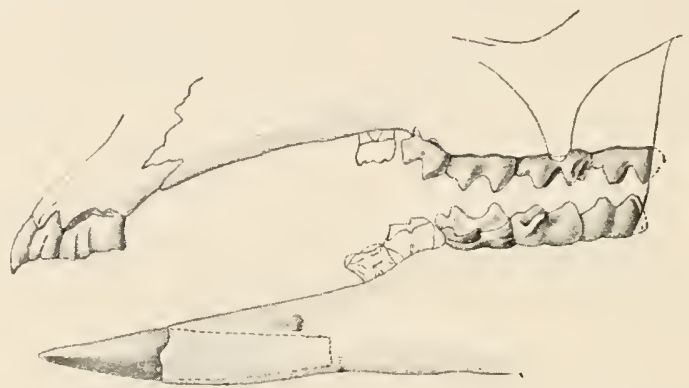

FIa. 1200.-Teeth of Great Kangaroo (Macropus major). (After Owen.)

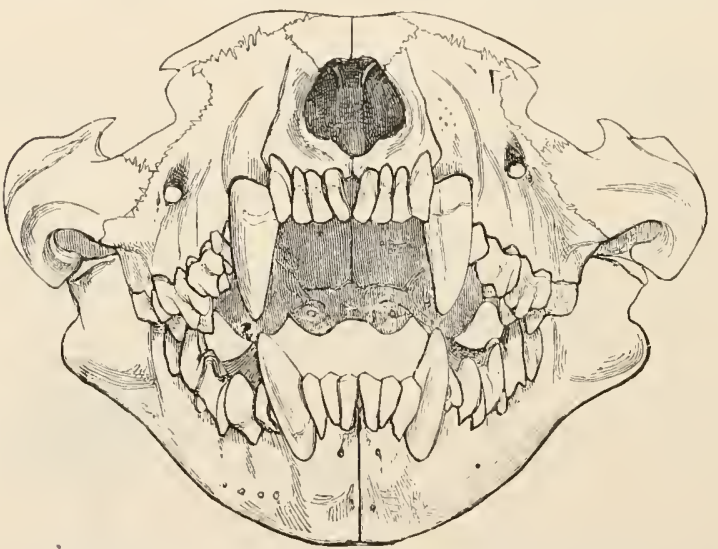

FIt. 1201.-Front view of the skull of Tasmanian Devil (Sarcophilus ursinus), showing polyprotodont and carnivorous dentition. (After Flower.)

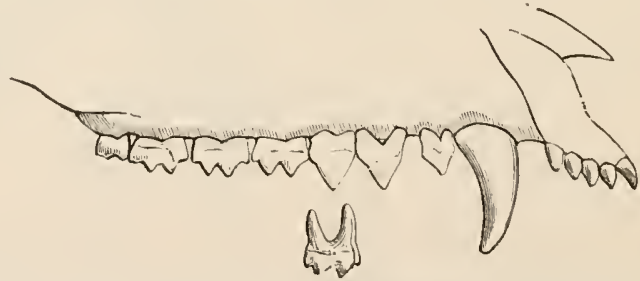

FIG. 1202,-Teeth of upper jaw of Opossum (Didelphys marsupialis), in all of which there is no succession except in the last pre-molar, the place of which is occupied in the young animal by a molariform tooth represented in the figure below the line of the other tcetl. (After Flower and Lydekker.)

protodont forms (Figs. 1201, 1202), which are all more or less carnivorous, the incisors are numerous and sub-equal and the canines large. There are typically three pre-molars and four 
molars. A good example of the diprotodont arrangement is the Kangaroo (Macropus, Fig. 1200), which lias the dental formula-

$$
i . \frac{3}{1}, c \cdot \frac{1}{0}, p \cdot \frac{2}{2}, m \cdot \frac{4}{4}=34 .
$$

The canine is very small and early lost. Of the polyprotodont forms the Australian Dasyure or Native Cat (Fig. 1144) has the formula-

$$
\text { i. } \frac{4}{3}, c . \frac{1}{1}, p \cdot \frac{2}{2}, m \cdot \frac{4}{4}=42 \text {; }
$$

and the American Opossum (Didelphys) (Fig. 1202)-

$$
i . \frac{5}{4}, c \cdot \frac{1}{1}, p \cdot \frac{3}{3}, m \cdot \frac{4}{4}=50 \text {. }
$$

The Edentata, as noticed in the outline of the classification, though not by any means all toothless, always have some defect in the dentition; when teeth are present in the adult the anterior series are absent and the teeth are imperfect, wanting roots and devoid of enamel. The tooth-characters differ widely in the

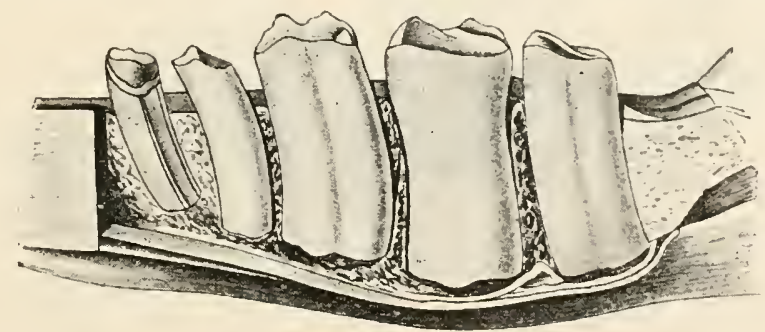

FIG. 1203.- Section of lower jaw and teeth of Orycteropus. (After Owen.

different groups. In the Sloths there are five teeth aboye and four below on each side; no second series is known. In the American Ant-eaters there are no teeth in the adult. In the Armadillos, on the other hand, the teeth are numerous, though simple and rootless, and, in one genus at least, two series occur. In the Scaly Ant-eaters there are no teeth. In the Cape Ant-eaters (Fig. 1203), again, there are numerous teeth which are heterodont and diphyodont, and have a peculiar structure, being perforated by numerous minute, parallel, vertical canals; the pulp of each tooth, entire at its base, is divided distally into a number of parallel columns.

In the Ungulata the dentition is heterodont and diphyodont, and the teeth are very rarely devoid of roots. In the Artiodactyla the pre-molars and molars differ from one another in pattern; the first upper pre-molar is almost always without a milk predecessor. 
The Pigs (Fig. 1204) are among the very few recent Mammalia which possess what has been referred to as a typical dentition: the formula of the completed dentition is-

$$
\text { i. } \frac{3}{3}, c \cdot \frac{1}{1}, p \cdot \frac{4}{4}, m \cdot \frac{3}{3}=44 \text {. }
$$

The incisors of the upper jaw are vertical, those of the lower greatly inclined forwards. The canines are greatly developed,

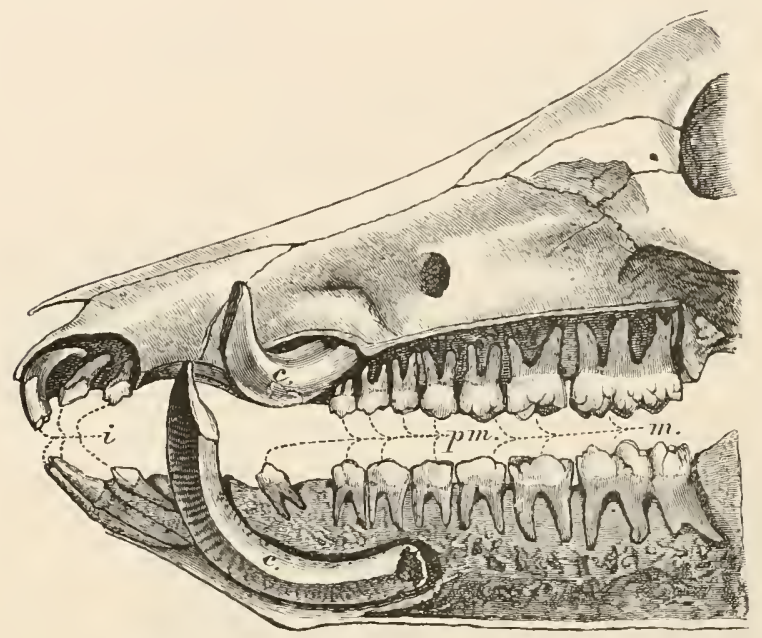

Fig.i1204,- Left lateralivieweof the dentition of the Boar (Sus scrofa), the roots of the teeth being exposed. (After Flower and Lydekker.) especially in the male, and grow from persistent pulps; both the upper and lower are bent upwards and outwards and work against one another in such a manner that the upper wears on its anterior and external surface, the lower at the extremity of the posterior surface. The premolars are compressed, with longitudinal cutting edges, and the molars are provided with numerous tubercles or cusps arranged for the most part in transverse rows (bunodont type). The first permanent pre-molar has no predecessor, the formula of the milk dentition being--

$$
\text { i. } \frac{3}{3}, c . \frac{1}{1}, m \cdot \frac{3}{3}=28 \text {. }
$$

In the typical Ruminants there are no teeth on the premaxillæ, the incisors of the lower jaw and the canines, which resemble them in shape, biting against a thickened callous pad on the opposed surface of the upper jaw, and the upper canines are also usually absent; there are three pre-molars and three molars in both upper and lower series, all characterised by the presence of column-like vertical folds of enamel, the interstices between which may be filled up with cement-the worn surface of the tooth presenting a pattern of the selenodont type (Fig. 1193, V). In the Camels there are a pair of upper incisors and a pair of large canines in each jaw. 
In the Perissodactyla the molars and pre-molars form a continuous series of large teeth with ridged or complexly-folded crowns, the posterior pre-molars often differing little in size and structure from the molars. In the Horse (Fig. 1205) the formula is-

$$
\text { i. } \frac{3}{3}, c \cdot \frac{1}{1}, p \cdot \frac{4}{4}, m \cdot \frac{3}{3}=44
$$

but the first pre-molar is a small tooth which soon becomes lost, and may belong to the milk-dentition. A fold of the enamel dips downwards (i.e. towards the root) from the extremity of the incisor teeth like the partly inverted finger of a glove; the canines are small in the female, and may not appear on the surface. There

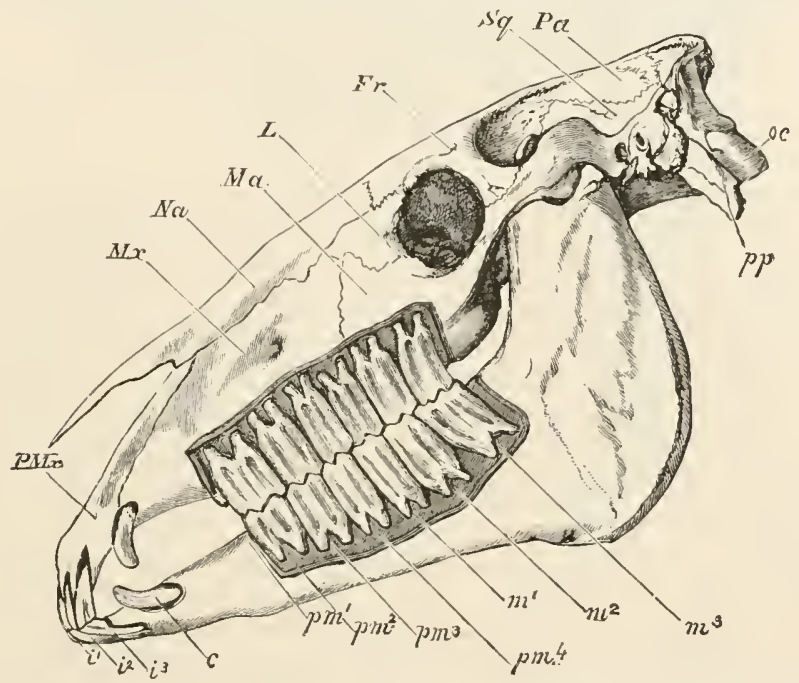

FIa. 1205.- Side view of skull of Horse with the bone removed so as to expose the whole of the teeth. $c$. canine ; $F r$. frontal ; $i^{1} . i^{* 2} \cdot i^{*}$. incisors ; $L$. lacrymal ; $M a$. jugal ; $M x$. maxilla ; $m^{1} \cdot m^{2} \cdot m^{3}$. molars; $N a$. nasal ; oc. occipital condyle ; $P a$. parietal ; $p . m^{1}$. situation of the vestigial first pre-molar, which has been lost in the lower, but is present in the upper jaw: $p m^{2}, p m^{3}$. $p m^{4}$. remaining pre-molars; $P M x$. pre-maxilla ; $p p$. par-occipital process ; $S q$. squamosal. (Aiter Flower and Lydekker.)

is a wide interval in both jaws between the canines and pre-molars. The pre-molar and molar teeth present a complicated pattern dne to folds of the enamel, which differ in their arrangement in the upper and lower jaws; their roots become completed only at a late period.

In the Hyracoidea the dental formula is-

$$
\text { i. } \frac{1}{2}, c . \frac{0}{0}, p \cdot \frac{4}{4}, m \cdot \frac{3}{3}=34 \text {. }
$$

The npper incisors are not unlike the larger pair of the Rabbit in shape, though prismatic and pointed, instead of compressed and chisel-like; they grow from persistent pulps. The outer incisors 
are elongated, inclined forwards, and trilobed at the extremities. The pre-molars and molars form a continuous series, separated by an interval from the incisors, and in pattern closely resemble those of some of the Perissodactyla.

The Elephants (Fig. 1206) have an extremely specialised dentition. There are no canines and no lower incisors. The single

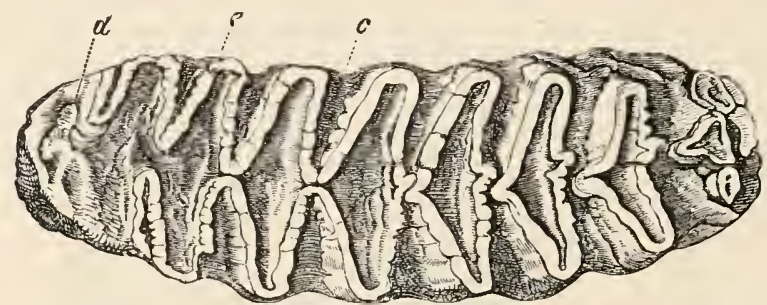

FIG. 1206. - Grinding surface of a partially worn"rightjupperimolar of the African Elephant (Elephas africanus). (After Ow்en.)

pair of upper incisors are developed into the enormous tusks (Fig. 1193, I), which grow continuously from persistent pulps throughout the life of the animal; they are of elongated conical form, and usually become curved. The tusks are composed of solid dentine, enamel occurring only on the apices, and becoming early worn away. The molars (Fig. 1206) are very large, and their worn surfaces are marked with prominent transverse ridges ; there are six molars altogether on each side, but only one or two are functional at once, the more posterior moving forward and taking the place of the more anterior as these become worn out.

When teeth are developed in the Cetacea they are nearly always. numerous, homodont, and monophyodont: in the Sperm-whales they are confined to the lower jaw. In the Whalebone Whales, though teeth are developed in the fœtal condition (Fig. 1207),

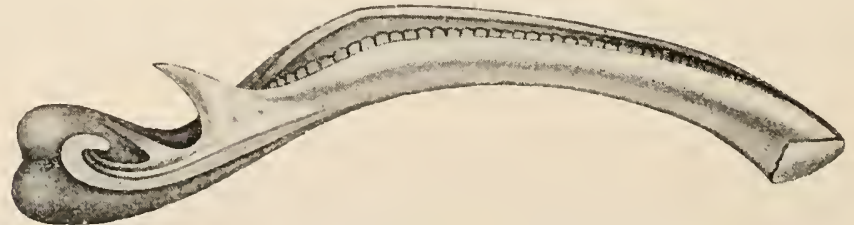

FIG. 1207.-Left lower jaw of foetus of Balænoptera rostrata, inner aspect, showing teeth natural size. (After Julin.)

they become lost either before or soon after birth, and they are succeeded in the adult by the plates of baleen or whalebone (Fig. 1208), which, in the form of numerous triangular plates, hang vertically downwards from the palate.

Of the Sirenia, the Dugong and Manatee have a heterodont dentition; in Rhytina teeth were absent. In the two former Sirenians there are incisors and molars with a wide diastema between them. 
In the Manatce there are two rudimentary incisors on each side, both in the upper and the lower jaw; these disappear beforc the adult condition is reached. There are altogether eleven molars on each side above and below, but not more than six of these are in use at once, the more anterior when worn out being succeeded by the more posterior. They have enamelled crowns with transvers ridges, and are preceded by milkteeth. In the Dugong there are no incisors in the mandible of the adult, and only one tusk-like pair in the upper jaw, large in the male - in which they grow from persistent pulps, little developed in the female and remaining concealed in their sockets. In the young there are rudimentary incisors in the mandible, and also a rudimentary second pair in the upper jaw. There are either five or six molars on cach side, both in the upper and lower jaws. These are cylindrical teeth, devoid of enamel, and with persistent pulps.

In the Carnivora vera (Fig. 1209) the dentition is complete, heterodont, and diphyodont, and all the teeth are provided with roots. The incisors are relatively small, chisel-shaped teeth; there are nearly always three of them on each side, in both upper and lower jaws. The canines are always large and pointed. The presence of carnassials, consisting of the last pre-molar in the upper and the first molar in

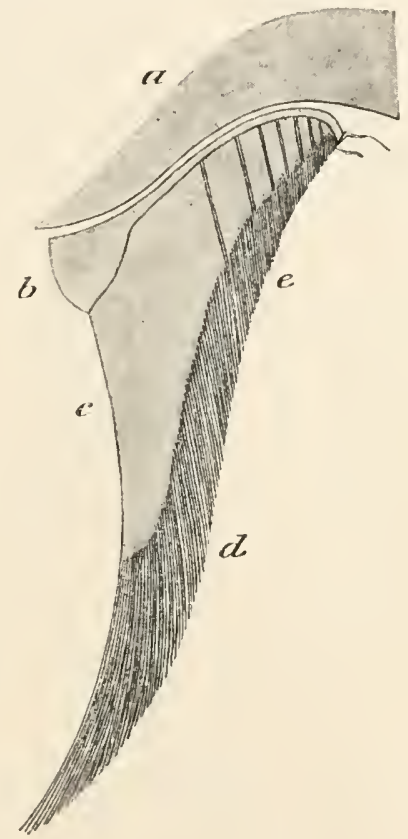

FIG. 1208.-Section of upper jaw of Balanoptera $(a)$, with baleenplates $(b, c)$ frayed out at their free edges $(d, e)$. (After Owen.)

the lower jaw, is universal. In front of the carnassial the teeth are compressed and pointed; behind it they have broad surfaces. In the Cat family (Felidce) the formula is-

$$
i . \frac{3}{3}, c . \frac{1}{1}, p . \frac{3}{2}, m \cdot \frac{1}{1}=30 .
$$

The lower carnassial is thus the last of the series. In the Dogs (Canidx) the formula is usually-

$$
i . \frac{3}{3}, c \cdot \frac{1}{1}, p \cdot \frac{4}{4}, m \cdot \frac{2}{3}=42,
$$

and in the Bears (Ursidæ) it is the same.

In the Pinnipedia there are always fewer than $\frac{3}{3}$ incisors, and carnassials are not developed. The pre-molars and molars have a 
compressed, conical, pointed form. The prevailing dental formula of the Seals is-

$$
i \cdot \frac{3}{2}, c . \frac{1}{1}, p \cdot \frac{4}{4}, m \cdot \frac{1}{1}=34 \text {. }
$$

In the Walrus the adult formula is-

$$
i . \frac{1}{0}, c \cdot \frac{1}{1}, p \cdot \frac{3}{3}, m \cdot \frac{0}{0}=18 \text {. }
$$

The upper canines take the form of large, nearly straight tusks.

In the large order of the Rodents the dentition is remarkably uniform, and, in all its general characters, resembles what has already been described in the Rabbit. But the second, smaller
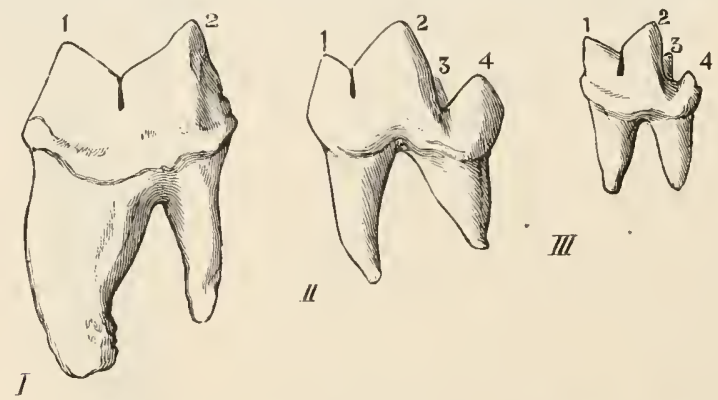

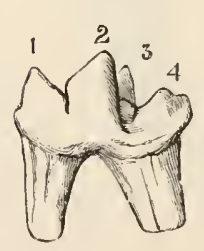

IV

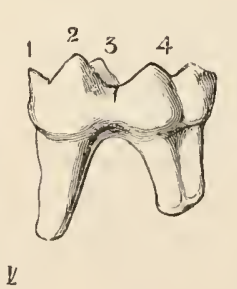

UI

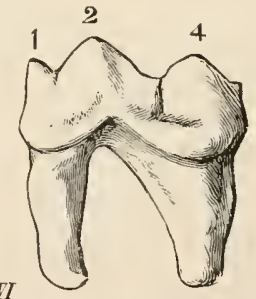

FIG. 1209,-Left lower carnassial teeth of Carnivora. I, Felis ; II, Can is ; III, Irerpestes ; $I V$, Lutra ; V, Meles ; VI, Ursus. 1, anterior lobe (paraconid) of blade ; 2, posterior lobe (protoconid) of blade; 3 , inner cusp (metaconid); 4 , talon (hypoconid). (After Hlower and Lydekker.)

pair of incisors of the upper jaw is present only in the Mares and Rabbits; the number of pre-molars and molars varies from-

$$
p \cdot \frac{0}{0}, m \cdot \frac{2}{2} \text { to } p \cdot \frac{3}{2}, m . \frac{3}{3},
$$

and they may develop roots.

In the Insectivora the dentition is heterodont, complete, and diphyodont. All the teeth are rooted. There are never fewer than two incisors on either side of the lower jaw. The canines are not of large size. The crowns of the molars are beset with pointed tubercles.

In the Chiroptera the dentition is complete, and the teeth are 
all rooted. There is a milk-series whieh differs entirely from the permanent teeth. In the inseetivorous Chiroptera (Bats) the molars are provided with pointed eusps, while in the frugivorous forms ("Flying Foxes") they are longitudinally grooved or exeavated.

In the Primates the teeth are heterodont and diphyodont, and always form roots. There are almost invariably two ineisors on each side in eaeh jaw, and, in all but the Hapalidæ, three molars. The dental formula of the various families have been given in the synopsis of the elassifieation. The dentition of Man differs from that of the rest of the order in the teeth forming a continuous series not interrupted by a diastema, and in the comparatively small size of the eanines.

The mouth in Mammals is bounded by fleshy lips. On the floor of the mouth is situated the tongue, which is usually well developed, but varies in size and shape in different orders. Its surfaee is eovered with papillæ of different forms, in assoeiation with certain of which are the special end-organs of the nerves of taste-the taste-buds. The roof of the mouth is formed in front by the hard palate, consisting of the horizontal palatine plates of the maxillary and palatine bones covered with mueous membrane. Behind the hard palate projects backwards the soft museular fold of the soft palate, also with taste-buds, which divides the eavity of the pharynx into two chambers, an upper and a lower. In front of the opening, leading from the lower division of the pharynx into the larynx, is a cartilaginous plate - the epiglottis - of which rudiments only are found in certain lower Vertebrates.

The oesophagus is always a simple straight tube. The stomach varies greatly in different orders, being sometimes simple, as in the majority of Mammals, sometimes divided into chambers, as in the Cetaee and the Ruminants.

In the majority of Mammals the stomach is a simple sac, as in the Rabbit (p. 448). But in certain groups it is complieated by the development of internal folds, and may be divided by eonstrictions into a number of different ehambers. The complieation of this organ reaches its extreme limit in the ruminant Ungulata and in the Cetacea. In a typical Ruminant (Fig. 1210, E, Fig. 1211), sueh as a sheep or an ox, the stomach is divided into four ehambers-the rumen or paunch, the reticulum, the psalterium, and the abomasum, or rennet stomach. The first of these (Fig. 1211, b) is much larger than the rest; its mucous membrane is beset with numerous short villi. The retieulum $(c)$, which is mueh smaller than the rumen, has its mucous membrane raised up into a number of anastomosing ridges, giving its wall the appearance of a honeycomb with shallow eells. From the aperture by which the reticulum communicates with the rumen to that with which it eommunicates with the psalterium, runs a groove bounded by a pair of muscular 
ridges, which are capable of closing together in such a way as to convert the groove into a canal. The mucous membrane of the psalterium $(d)$ is raised up into numerous longitudinal leaf-like folds. The abomasum (e), smaller than the rumen, but larger than the reticulum, has a smooth vascular and glandular mucous mem-
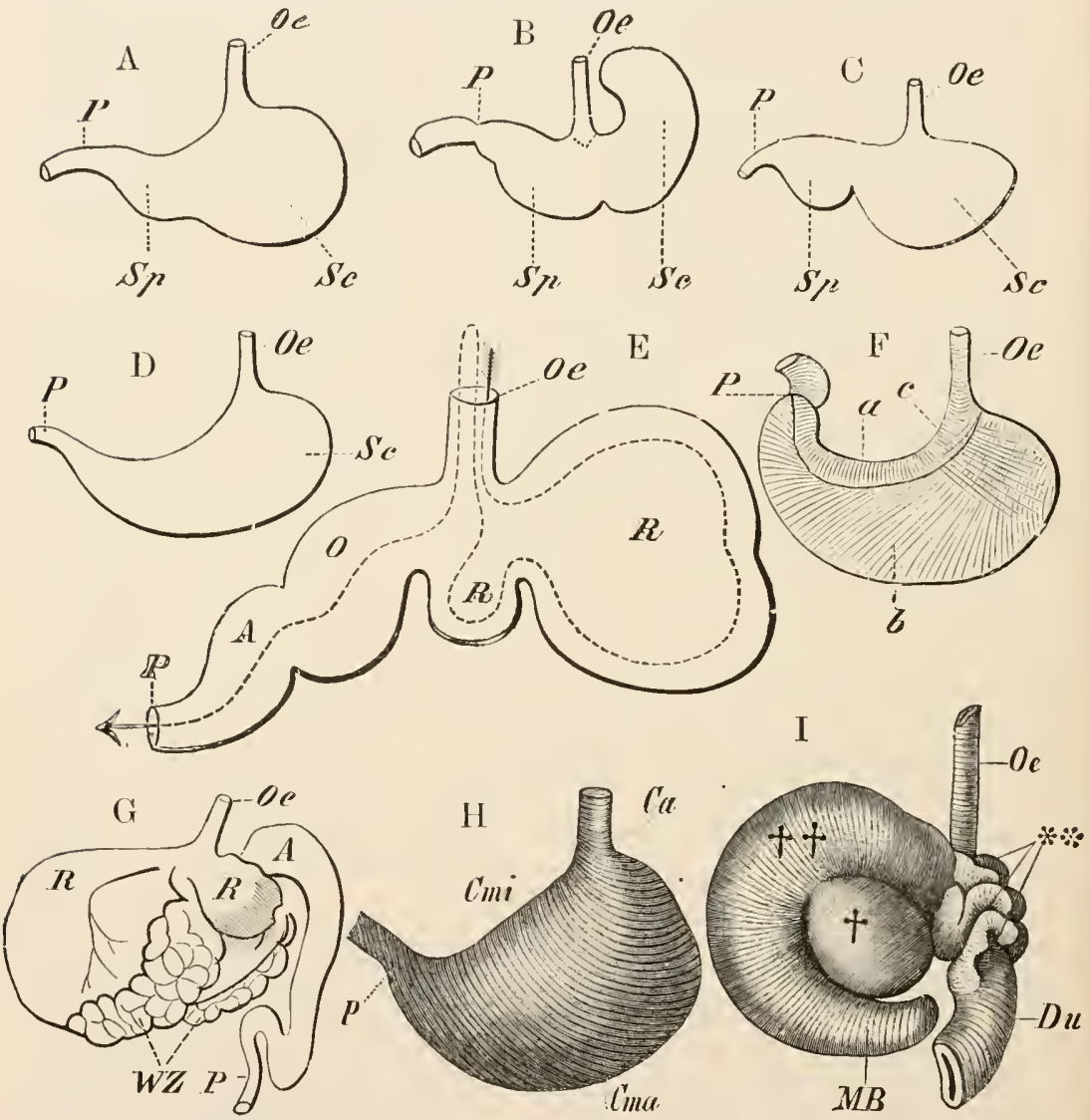

FIG. 1210.-Different forms of the stomach in Mammals. $A, \mathbf{D o g} ; B$, Mus decumanus ; $C$, Mus musculus ; $D$, Weasel; $E$, schene of the ruminant stomach, the arrow with the dotted line showing the course taken by the food; $F$, human stomach, $a, b, c$, muscles on inner side; $G$, Camel; $H$, Echidna aculeata ; $I$, Bradypus tridactylus. $A$. (in $E$ and $G$ ) abomasum; $C a$. cardiac end ; $C m a$, greater curvature ; $C m i$, lesser curvature; $D u$. duodenum; $I I B$, cæcum; $O$, psalterium; $O e$. cesophagus ; $P$. pylorus ; $R$. (to the right in Fig. $E$ ), $t \dagger$, rumen ; $R$ (to the left in Fig. $E$ ), $\dagger$, reticulum; Sc. cardiac division; $S p$, pyloric division; $W Z$, water-cells; ** duodenal pouches. (From Wiedersheim's Comparative Anatomy.)

brane. The œsophagus opens into the rumen close to its junction with the reticulum. The herbage on which the Ruminant feeds is swallowed without mastication, accompanied by copious saliva, and passes into the rumen and reticulum, where it lies until, having finished feeding, the animal begins ruminating or chewing the 
cud. In this process the sodden food is returned in rounded boluses from the rumen to the moutli, and there undergoes mastication. When fully masticated it is swallowed again in a

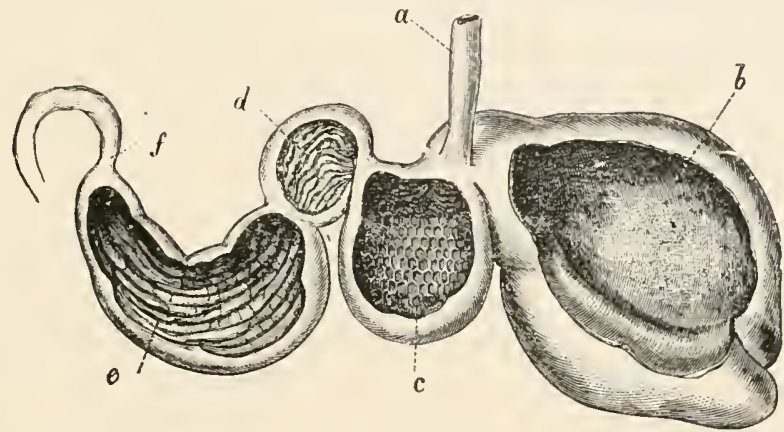

FIG. 1211.-Stomach of Ruminant opened to show the internal structure. $a$, œsophagus; $b$, rumen ; $c$, reticulum ; $d$, psalterium ; $c$, abomasum ; $f$, duodenum. (After Flower and Lydekker.)

semi-fluid condition, and passes along the groove into the reticulum, or over the unmasticated food contained in the latter chamber, to strain through betwecn the leaves of the psalterium and enter the abomasum, wherc the process of digestion goes on. In some Ruminants the psalterium is wanting. In the Camels (Fig. 1210, $G$ ) the stomach is not so complicated as in the more typical Ruminants, there being also no distinct psalterium, and the rumen being devoid of villi; both the rumen and the reticulum have connected with them a number of pouchlike diverticula

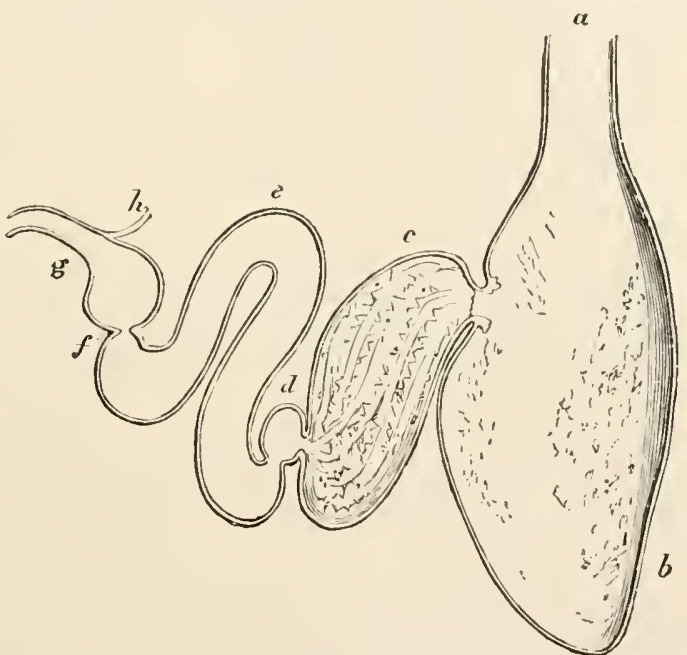

FIG. 1212. - Diagrammatic section of the stomach of the Porpoise. $a$, wesophagus; $b$, left or cardiac compartment; $c$, middle compartment ; $d$ and $e$, the two divisions of the right, or pyloric compartment $; f$, pylorus ; $g$, duodenum, dilated at its commencement; $h$, bile-duct.' (After Flower and Lydekker.)

$(w . z$.), the openings of which are capable of being closed, by sphincter muscles; in these water is stored. In the Cetacea the stomach is also divided into compartments. In the Porpoise 
(Fig. 1212) the osophagus $(a)$ opens into a spacious paunch (b), the cardiac compartment of the stomach, with a smooth, thick, mucous membrane. This is followed by a second chamber $(c)$, of considerably smaller dimensions, with a glandular mucous membrane, which is thrown into a number of complex folds. A long and narrow third, or pyloric, compartment $(d, e)$ follows upon this, terminating in a constricted pyloric aperture; beyond which the beginning of the intestine is dilated into a bulb.

A cæcum, situated at the junction of the large and small intestines, is usually present, but varies greatly in extent in the different orders and families. It is much larger in vegetable-feeding than in carnivorous forms, and among the former it is those that have a simple stomach, such as the Rabbit, that have the largest cæcum. Hyrax differs from all the rest of the class in having a pair of

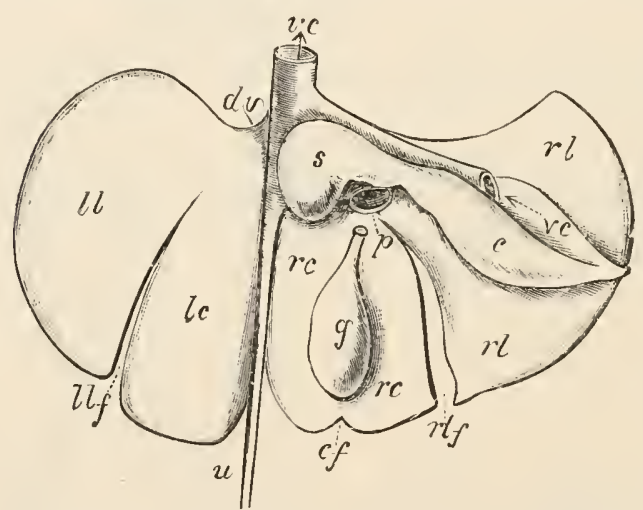

FIG. 1213.-Diagrammatic plan of the liver of a IMammal (posterior surface). $c$. caudate lobe; $c f$. cystic fissure $d v$. ductus veuosus; $g$. gall-bladder ; $i c$. left central lobe; $l l$. left lateral lobe; $u f$. left lateral fissure ; $p$. portal vein entering transverse fissure; $r c$. right central lobe; $r l$. right lateral lobe; $r l f$. right lateral fissure; $s$. Spigelian lobe ; $u$. umbilical vein; $v c$. nost-caval. (After Flower and Lydekker.) supplementary cæca situated some distance down the large intestine. A cæcum is absent in the Sloths, some Cetacea, and a few Carnivora.

The Prototheria resemble Reptiles, Birds, and Amphibia, and differ from other Mainmals, in the presence of a cloaca, into which not only the rectum but the urinary and genital ducts open. In the Marsupials a c om mon sphincter muscle surrounds both anal and urinogenital apertures and in the female there is a definite cloaca; in nearly all the Eutheria the apertures are distinct, and separated from one another by a considerable space-the perincum.

The liver (Fig. 1213) consists of two parts or main divisions, right and left, incompletely separated from one another by a fissure termed the umbilical, owing to its marking the position of the foetal umbilical vein. Typically each of these main divisions is divided by a fissure into two parts, so that right lateral $(r l$.) and right central (rc.) and left lateral (ll.) and left central (lc.) lobes are distinguishable. When a gall-bladder is present, as is the case in the majority of Mammals, it is attached to, or embedded in, the right central lobe. A fissure, the portal, through which the portal vein and hepatic artery pass into the substance of the liver, and the hepatic vein 
passes out, crosses the right central lobe neal the anterior borler. The postcaval lies in contact with, or embedded in, the riglit lateral lobe near its anterior border, and given off from this lobe, between the postcaval and the portal fissure, is a small lobe, of varying extent-the Spigelian. The term caudatc lobe is applied to a process of the right lateral lobe, of considerable extent in most Manmmals, having the postcaval vein in intimate relation to it, and often closely applied to the kidney. A gall-bladder is usually present, but is absent in the Cetacea, the Perissodactyle Ungulata, the Hyracoidea, and some Rodents.

Vascular System.-The blood of Mammals is warm, having a temperature always of from $35^{\circ}$ to $40^{\circ} \mathrm{C}$. The red corpuscles are non-nucleated: in form they are most usually biconeave dises, always circular in outline, except in the Camelidæ, in which most of them are elliptical. The lymphatic system of vessels is very highly developed, ramifying richly throughout all parts of the body. In the course of this system occur numerous lymphatic glands. The special part of the lymphatic system of vessels (lacteals) which ramify in the wall of the intestine and absorb the fatty matters of the food, combine with the lymphatic vessels from the hind-limbs and body to form a receptaele - the receptaculum chylifrom which a tube, the thoracic duct, which may be double, runs forward to open into the base of one of the great veins of the precaval system by a valvular aperture.

The general statements which have been given with regard to the heart of the Rabbit (p. 148) hold good for the Mammalia in general. The sinus venosus is never distinet from the right auricle ; of its valves, which are more completely retained in the Edentata than in the other orders, the right gives rise to the Eustachian valve a membranous fold, often fenestrated in the adult, extending from the right wall of the postcaval to the edge of the foramen ovale (annulus ovalis); while the left becomes merged in the auricular septum, helping to complete the annulus ovalis behind. Each auricle has an auricular appendix. The right auriculoventricular aperture has a three-lobed tricuspid valve, and the left a two-lobed bicuspid, or mitral, with chordæ tendineæ and musculi papillares. In all Mammals the openings of the pulmonary artery and aorta are provided with three-lobed semilunar valves.

The single aortic arch, situated in all Mammals on the left side, varies greatly in the way in which it gives off the main arterial trunks. Sometimes a single large trunk passes forward from the arch of the aorta and gives rise to botli carotids and both subclavians. Sometimes there are two main trunks-right and left innominatc arteries - each giving rise to the carotid and subclavian of its own side. Sometimes there is a right innominate giving off right carotid and right subclavian, the left carotid and left subclavian coming off separately from the arch of the aorta; or, 
as in the Rabbit, an innominate may give origin to the right subclavian and both carotids, the left subclavian coming off separately.

In Monotremes and Marsupials, in most Ungulates, and in the Rodentia, Insectivora, and Chiroptera, both right and left precavals persist; in the others the left aborts, its vestige giving rise to the coronary sinus. In the Monotremes the openings of all three cavals are provided with valves, only vestiges of which exist in the other groups. In the Monotremes all the pulmonary veins open by a common trunk. In the Metatheria and Eutheria the four veins sometimes open separately, sometimes the two veins of each side unite to form a single lateral trunk. In Echidna there is an abdominal vein corresponding to that of the Frog and Lizard (pp. 272 and 316).

The following are some of the principal variations in the structure of the heart which occur in the different groups of Mammals. In the Monotremes there is a deep fossa representing the fossa ovalis in the auricular septum. The auriculo-ventricular valves depart from the structure typical of Mammals and approach the corresponding valves in the heart of Birds. In the Marsupials the fossa ovalis and annulus ovalis are absent; in the uterine fotus of the Kangaroo the auricles communicate by a fissure, but all trace of this becomes lost before the adnlt stage is reached.

In the Cetacea, Eustachian and Thebesian valves are both absent. In some of the Cetacea the apices of the ventricles are separated by a slight depression. In the Sirenia there is a corresponding, but much deeper and wider, cleft, so that the apex of the heart is distinctly bifid.

In the Ungulata, Eustachian and coronary valves are both absent; in some there is a cartilage or a bone-the os cordisoften double, at the base of the heart. The Eustachian valve is absent in most of the Carnivora. In the Pinnipedia an aperture of communication between the auricles often persists in the adult.

The organs of respiration resemble those of the Rabbit in the general features mentioned on p. 453 .

In the Cetacea, the epiglottis and arytenoids are prolonged to form a tube, which extends into the nasal chambers and is embraced by the soft palate, so that a continuous passage is formed leading from the nasal chambers to the larynx, and giving rise to the condition of intra-narial epiglottis. In all the remaining orders a similar condition occasionally occurs - the epiglottis being produced upwards into the respiratory division of the pharynx behind the soft palate. In fotal Marsupials, in which the intra-narial condition is very complete, it is obviously associated with the passive absorption of the milk, while breathing is being carried on continuously through the nostrils. Some Cetacea and Artiodactyla, etc., are exceptional in having a third bronchus, which passes to the right lung anteriorly to the ordinary bronchus of that side 
and to the pulmonary artery. In connection with various parts of the respiratory system there are cavities containing air. The connection of the tympanic cavity with the pharynx by means of the Eustachian tubes has been already mentioned. Air-sinuses, connected with the nasal chambers, extend into the bones of the
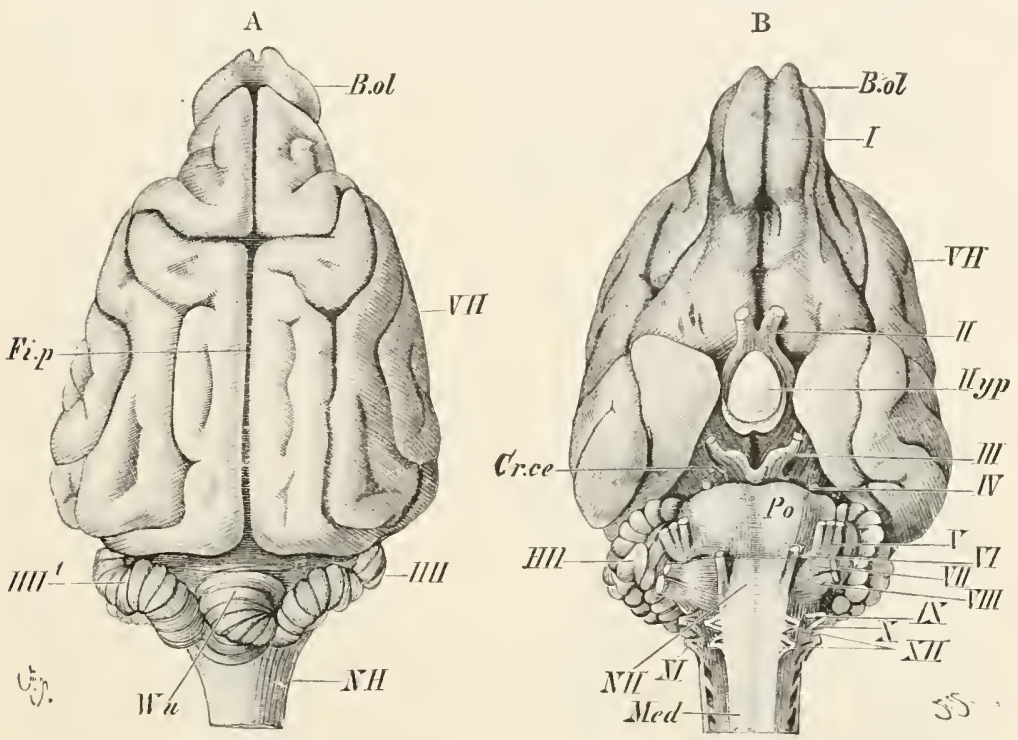

C

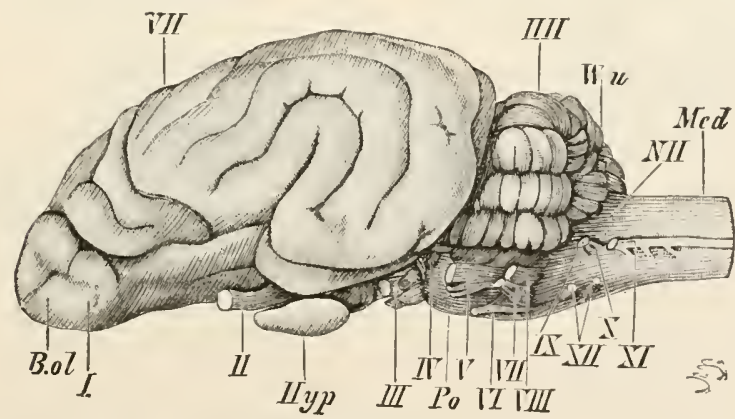

FIG. 1214.-Brain of Dog. $A$, dorsal ; $B$, ventral ; $C$, lateral aspect. $B$. ol. olfactory bulb ; $\mathrm{Cr}$. ce. crura cerebri ; $\mathrm{F}$. $p$. great longitudinal fissure; $H H, H H^{\prime}$, lateral lobes of cerebellum: Hyp. hypophysis ; Meil. spinal cord; $N H$, medulla oblongata; $P o$. pons Varolii ; $F H$. cerebral hemispheres; $I V u$, middle lobe (vermis) of cerebellum; $I-I I I$. cerebral nerves. (From Wiedersheim's Comparative Anatomy.)

skull, especially into the maxillæ and frontals, where they may reach large dimensions, and are known as the maxillary antra and frontal sinuses. Air-sacs are also developed in connection with the larynx in many of the Apes.

Nervous System.-The brain of Manmals (Fig. 1214) is 
distinguished by its relatively large size, and by the large size and complex structure of the cerebral hemisphercs of the fore-brain.

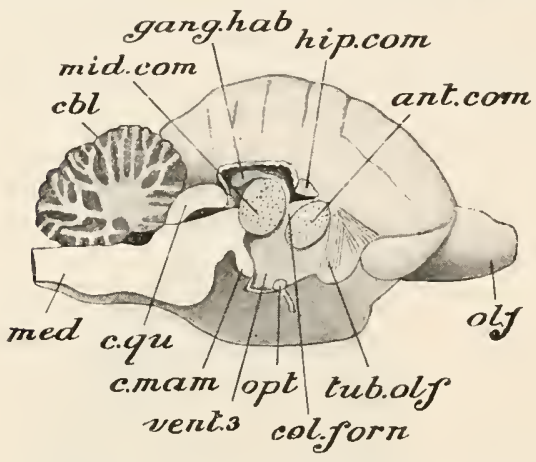

FIG. 1215. - Brain of Fchidna aculeata, sagittal scetion. ant. com. anterior commissure; cbl. cercbellum; $c$. mam. corpus mammillare; col. forn. column of the fornix : $c . q u$, corpora quadrigemina ; gany. hab. habenular ganglion; hip. com. hilpocampal commisiure; med. medulla ohlongata; miul. com. mirllle commissure ; olf. olfactory bulb ; opt. optic chiasma; tub. olf, tuberculum olfactorium; vent. 3 , third ventricle.

The optic lobes, which
arts, and are hence
he lateral parts of the cere Mammals, attains a high degree of development, is a transverse flattened band -the pons Varolii (Po.) - crossing the hind-brain on its ventral aspect.

In the Monotremes and Marsupials (Figs. $1215,1216)$ there is no corpus callesum, while the anterior commissure (ant. com.) is of relatively large size, and, unlike the corresponding

commissure in lower Vertebrates, contains fibres connecting together areas of the non-olfactory regions (neo-pallium) of the hemispheres.
The cerebral hemispheres of opposite sides are connected together across the middle line in all Mammals, except the Monotremes and Marsupials, by a band of nerve-tissue termed the corpus callosum-a structure not present in the Sauropsida. The hemispheres, in all but certain of the lower and smaller Mammals, are not smooth, but marked by a number of grooves or sulci separating winding ridges or convolutions. The lateral ventricles in the interior of the hemispheres are of large size and somewhat complex form. 
The hippocampi extend along the whole length of the lateral ventricles. The layer of ncrve-cells in each hippocampus gives origin, as in Euthcria, to mumerous fibres, which form a layer on the surface, the alveus, and become arranged in a band-the tonia hippocampi. In the Eutheria, as we have seen in the case of the Rabbit, the tæniæ unite mesially to form the body of the fornix (sec p. 456). In the Monotremes and Marsupials, on the other hand, there is no such minion; the fibres of the tænia run towards the foramen of Monro, where they become divided into several sets. Of these one set, constituting the great majority of the fibres, pass into the hippocampus of the opposite side, giving rise to a hippocampal commissure (hip. com., cf. Figs. 946 and 982), the great development of which readily leads to its being mistaken for a corpus callosum. The fibres cntering into the formation of this commissure correspond, however, not to the fibres of the corpus callosum, which

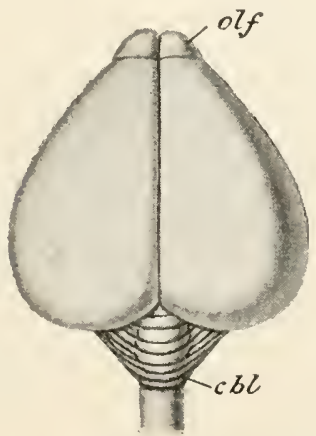

Fia. 1217.-Brain of Ornithorhynchus anatinus, dorsal view (natural size), cbl. cerebellum; olf. olfactory bulbs.

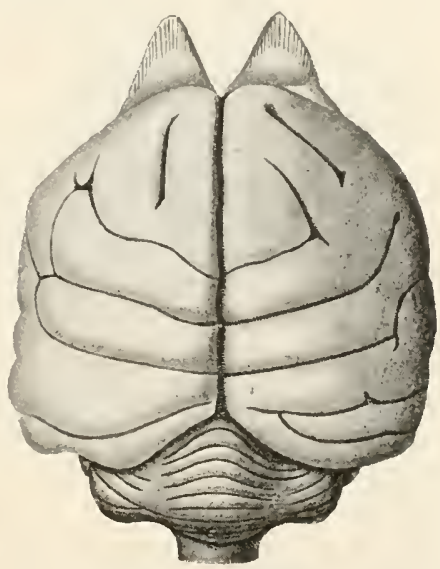

FIG, 1218.-Brain of Echidna aculeata, dorsal view (natural size).

is the commissure of the neo-pallium, but, as proved by thcir modc of origin, to the fibres of the fornix, and they connect together only the hippocampi, the fascice dentatce, or specialised lower borders of the hippocampi, and an arca of the hemisphere in front of the anterior commissure (pre-commissural area): they thus constitute an olfactory or archipallial commissure, since all these parts bclong to the olfactory region or archipallium of the hemispheres. In the Monotremes (Fig. 1215) the hippocampal commissure is only very slightly bent downwards at its posterior extremity. In most Marsupials (Fig. 1216) it bends sharply round posteriorly and runs forward agam, becoming thus folded into two layers, dorsal and ventral, continuons with one another at a posterior bend or splenium, similar to the splenium of the corpus callosum. The dorsal layer of the hipjocampal commissure becomes alnost completely replaced 
in the Eutheria by the fibres of the corpus callosum, and the ventral part persists in the shape of the psalterium or lyra.

In Ornithorhynchus (Fig. 1217) the hemispheres are smooth; in Echidna (Fig. 1218) they are tolerably richly convoluted. Both

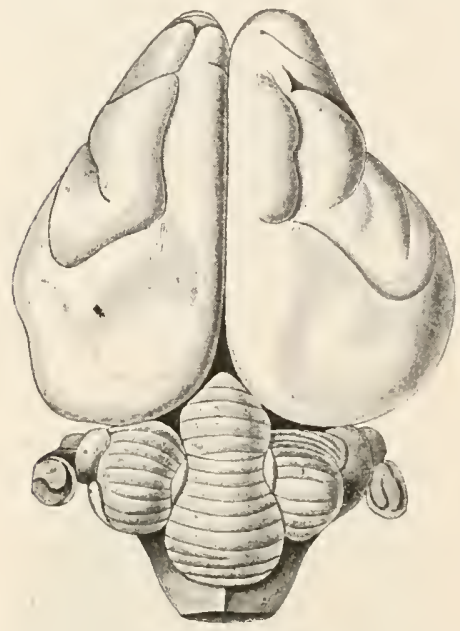

FIG.\{1219,-Brain of Kangaroo (Macropus major). (After Owen.)

genera, but more particularly Echidna, are characterised by the enormous development of the parts of the hemispheres (archipallium) connected with the olfactory sense. In the lower Marsupials there are no convolutions (Notoryctes, Koala, Phalangers), while in the higher the convolutions are numerous, though the sulci are not very deep (Macropus, Fig. 1219). Among the Eutheria there is a great range in the grade of development of the brain, from the Rodents and lower Insectivores to the higher Primates. In the lower types of Mammalian brain the cerebral hemispheres are relatively small, do not overlap the cerebellum, and have smooth, or nearly smooth, surfaces. In the higher types the relative development of the hemispheres is immense, and their backward extension sauses them to cover over all the rest of the brain, while the cortex is thrown into numerous complicated convolutions separated by deep sulci (Fig. 1220). This development of the cerebral hemispheres reaches its maximum in Man.

The organs of special sense have the same general structure and arrangement as in the Sauropsida. Jacobson's organs, which in the Sauropsida constitute such important

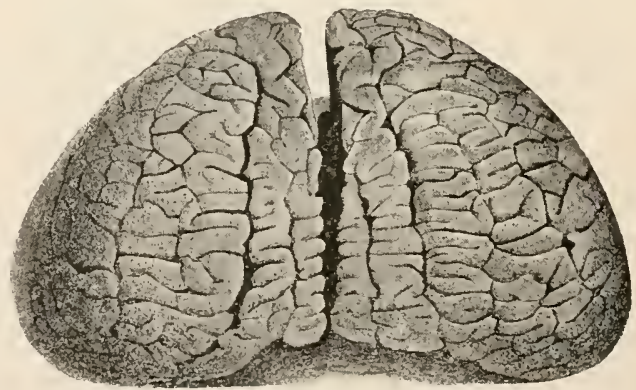

F16. 1220.-Dorsal view of brain of Gray's Whale (Cogin grayi). (Atter Haswell.) accessory parts to the olfactory apparatus, are well developed only in the lower groups of Mammals. The olfactory mucous membrane is of great extent, owing to the development of the convoluted ethmo-turbinal bones over which it extends. In the toothed Cetacea alone among 
Mammals do the nasal chambers lose their sensory functions-the olfactory nerves being vestigial or absent. The organs of taste are taste-buds in the mucous membrane covering certain of the papillæ on the surface of the tongue and in that of the soft palate.

In essential structure the eye of the Mammal resembles that of the Vertebrates in general (see p. 106). The sclerotic is composed of condensed fibrous tissue. The pecten of the eye of Birds and Reptiles is absent. In most Mammals there are three movable eyelids, two, upper and lower, opaque and usually covered with hair, and one anterior, translucent, and hairless - the nictilating membrane. The secretions of a lacrymal, a Harderian, and a series of Meibomian glands moisten and lubricate the outer surface of the eye-ball and its lids. In Moles, and certain other burrowing Insectivores and Rodents, and in Notoryctes among the Marsupials, the eyes are imperfectly developed and functionless.

The ear of a Mammal is more highly developed than that of other Vertebrates, both in respect of the greater complexity of the essential part-the membranous labyrinth-and in the greater development of the accessory parts. A large external auditory pinna, supported by cartilage, is almost invariably present, except in the Monotremata, Cetacea, and Sirenia. This is a widely open funnel, of a variety of shapes in different groups, having the function of collecting

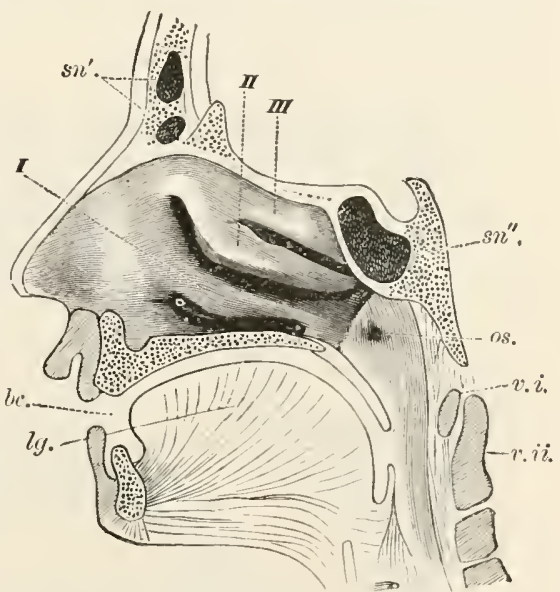

Fra. 1221.- Sagittal section through the nasal and buccal cavities of the human head. $I, I I, I I I$, the three olfactory ridges formed by the turbinals; $b c$, entrance to the mouth; ; lg; tongue : $o s$, opening of Eustachian tube; $s n^{\prime}$, frontal sinus; $s n^{\prime \prime}$, sphenoidal sinus ; $v$, athas vertebra : $v$. $i$, a avis vertebra. (After Wicdersheim.)

the waves of sound. By the action of a system of muscles it is usually capable of being turned about in different directions. Enclosed by its basal part is the opening of the external auditory passage (Fig. 1222, Ex.). This, the length of which varies, leads inwards to the tympanic membrane (M.), which separates it from the cavity of the middle ear or tympanic cavity. The wall of the external auditory passage is sometimes entirely membranous or cartilaginous, sometimes in part supported by a tubular part of the tympanic bone; in Echidna it is strengthened by a series of incomplete rings of cartilage. The tympanic cavity, enclosed by the periotic and tympanic bones, communicates with the upper or respiratory division of the pharrnx by a longer or shorter tubular 
passage-the Eustachian tube (E.). On its inner wall are the fenestrce ovalis and rotunda, and across its cavity, from the tympanic membrane to the fenestra ovalis, runs the irregular chain of auditory ossicles - the malleus $\left(O_{3}\right)$, the incus $\left(O_{2}\right)$ and the stapes $\left(O_{1}\right)$. These vary somewhat in form in different Mammals. The stapes is usually perforated by a considerable foramen, as in the Rabbit, but, in the Monotremes, certain Marsupials, and Manis among the Edentata, approximates more towards the rod-like shape which the columella presents in Amphibians, Reptiles, and Birds. The membranous labyrinth $\left(L_{.}\right)$of the internal ear of a Mammal is characterised by the special development of the cochlea (Cch.), which (except in the Monotremes) is coiled into a spiral like the shell of a Snail.

Urinogenital Organs.-The kidneys of Nammals are compact

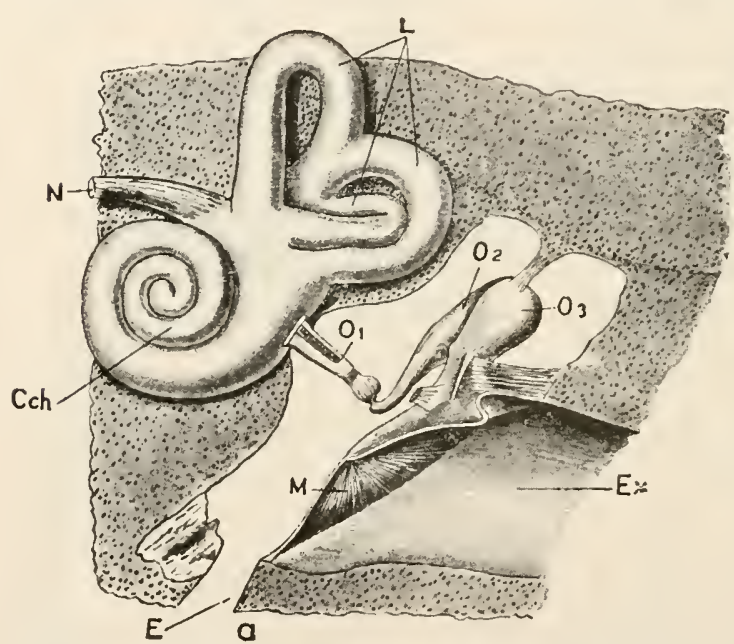

FId, 1222.-Parts of the Fuman ear(diagrammatic), Cch. cochlea ; $E$. Eustachian tube ; $E x$, outer opening of ear ; $L$. labyrinth; $M$. tympanic nembrane; $N$, entrance of auditory nerve; $O_{1}, O_{2}$, $\mathrm{O}_{3}$, the three auditory ossicles, stapes, incus, malleus. (After Headley.) by which the secretion is carried to the ureter. The ureter dilates as it enters the kidneys to form a chamber-the pelvis -into which the straight tubules of the medulla of the kidney open. The openings of the tubules are on the summits of papillæ, which are the apices of a series of pyramidal masses into which, in most cases, the substance of the kidney is incompletely divided. In many Mammals, however, there is no such division of the kidney substance, and all the ducts open on the surface of a single papilla. In others again (Ox, Bears, Seals, Cetacea) the division is carried so far that the kidney is divided externally into a number of distinctly separated lobules. 
The ureters in all the Theria open into a large median sac-the urinary bladder-situated in the posterior or pelvic part of the cavity of the abdomen. From this a median passage, the urinogenital passage or urethra -into which in the male the vasa deferentia open-leads to the exterior. Only in the Monotremes do the two ureters and the bladder all have separate openings into the urinogenital division of the cloaca.

The testes are oval bodies, which only exceptionally retain their original position in the abdominal cavity, descending in the majority of Nammals through a canal-the inguinal canal-in the posterior part of the abdominal wall to lie in the perinceum, or space between the urinogenital and anal apertures, or to be received into a pendulous pouch of skin, sometimes double-the scrotum. The penis, present in the males of all Mammalia, consists of two corpora cavernosa, firm strands of vascular tissue, attached proximately to the ischia except in the Monotremes, Marsupials, and some Edentata, and a central strand, the corpus spongiosum, perforated by the urethral canal and often dilated at the extremity to form the glans. The two vasa deferentia continued from the epididymes, which are in close relation to the testes, join the urethral canal near the neck of the bladder, each often having connected with it, near its distal end, a sacculated reservoir-the vesicula seminalis. A small diverticulum of the proximal part of the urethra - the uterus masculinus - may be a remnant of the Müllerian duct. Surrounding this part of the urethra is a glandular mass - the prostate gland; and the ducts of a pair of small glands-Cowper's glands-open into the urethra near the base of the penis.

The ovaries are compressed oval bodies which retain their primary position in the abdomen, or pass backwards into its posterior or pelvic part. In the Monotremes, large Graafian follicles project on the surface of the ovary, while in other Mammals the Graafian follicles are very small, and the surface of the ovary almost smooth.

The oviducts have dilated funnel-like abdominal openings, the edges of which, except in the Monotremes, are fimbriated or fringed. In the Monotremes the two oviducts are distinct throughout their length, and open separately into a urinogenital sinus. In nearly all the Theria more or less coalescence takes place. In the Marsupials this coalescence is confined to the proximal part of the vagina. In the Opossums (Fig. 1223, A) the two oviducts are merely in close apposition at one point behind the uteri, and there is no actual coalescence. In the rest of the Marsupials (B, C) the anterior portions of the oviduct in the region (vagina) behind the uteri unite to form a median chamber which may send backwards a median diverticulum (median vagina, $V g ., B$ ), and in this way communicate behind with the urinogenital passage. In the Eutheria there is a single median vagina (Fig. 1224, Vg.) formed by the union of the posterior parts of the two oviducts. In some cases the two uteri 


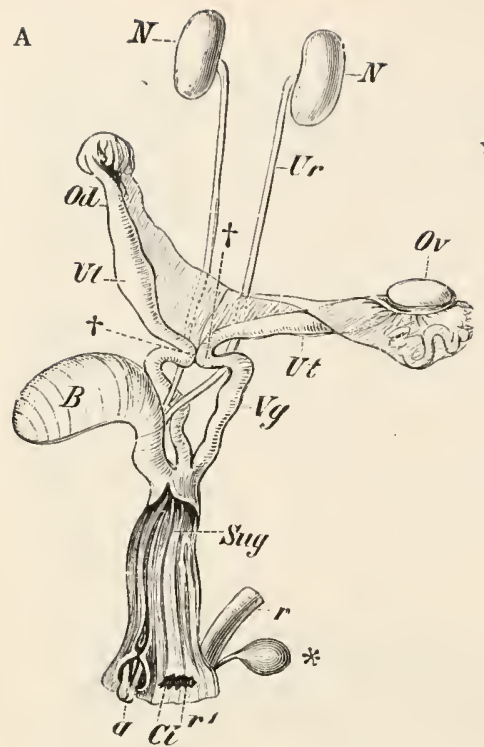

B
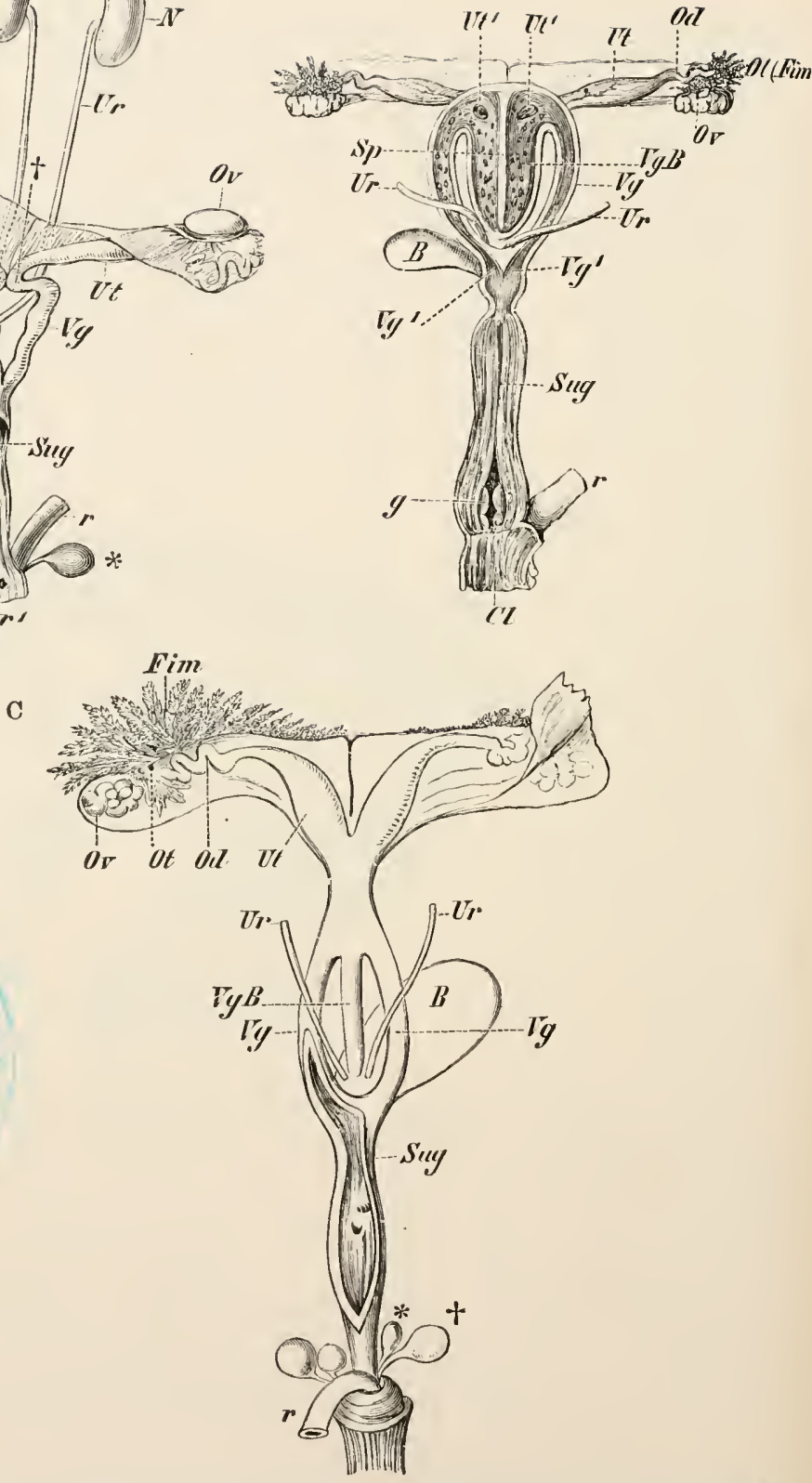

FIG. 1223.-Female urinogenital apparatus of various Marsupials. A, Didelphys dorsigera (young); B, Trichosurus ; C, Phascolomys wombat. B. urinary bladder ; Cl. cloaca ; Fim. fimbrix; $g$. clitoris ; $N$. kidney ; Od. Fallopian tube ; Ot. its aperture; Ov. ovary ; $r$. rectum ; $r^{1}$, its opening; Sug, urinogenital canal; Ur. ureter ; Ut. uterus ; $U t^{1}$. opening of the uterus into the median vagina $\left(V^{\prime} g . B.\right) ; V g$. lateral vagina ; $V g^{1}$. its opening into the urinogenital canal; $t^{*}$, rectal glands; $\dot{t}$, bend between uterus and vagina. (From Wiedersheim's Comparative Anatomy.) 
$(\mathrm{A}, u t$.$) remain distinct; in others their posterior portions coalesce$ $(B, C)$, the anterior parts remaining separate, so that there is formed a median corpus uteri with two horns or cormua. In Primates and some Edentates the coalescence goes still further, there being an undivided uterus (D) in addition to an undivided vagina, the only parts of the oviducts which remain distinct from one another being the narrow anterior parts or Fallopian tubes. In all Mammals there is, in the vestibule or urinogenital passage through which the vagina c o m municates with the exterior by the aperture of the vulva, a small body-the clitoris-the homologue of the penis, and sometimes perforated by the urethral canal.

Development. -The ova of Mammals (Fig. 1225), like those of Vertebrates in general, are developed from cer-

A

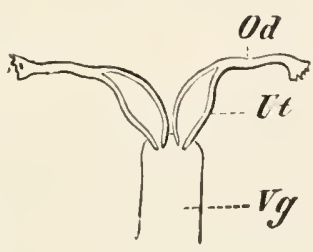

C
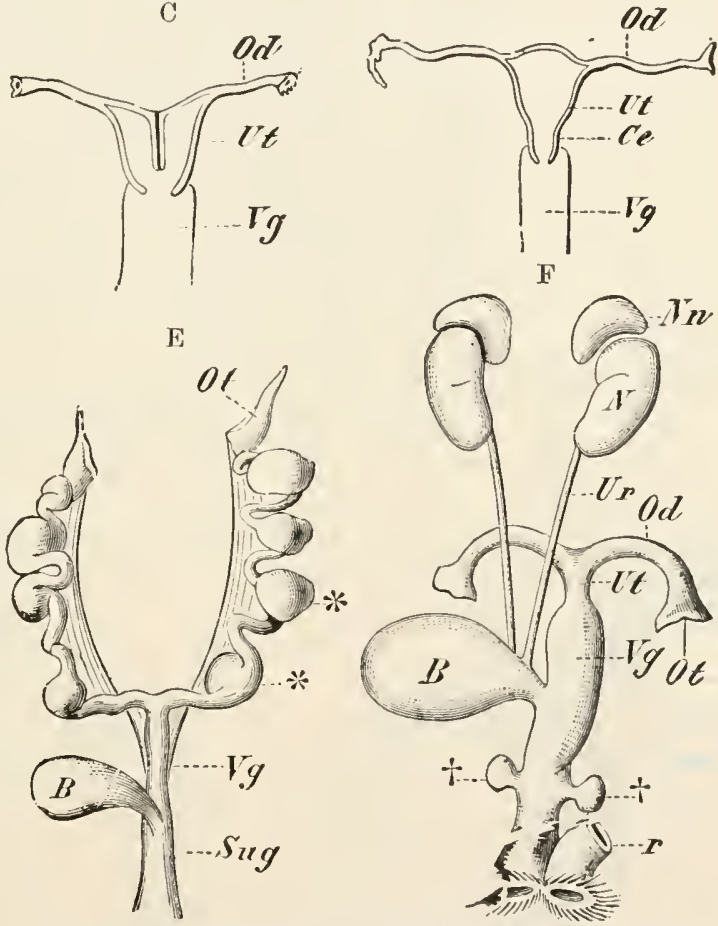

B

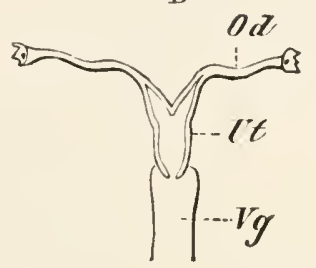

D

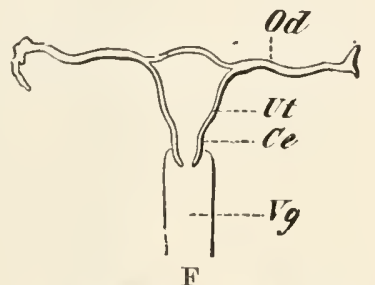

IG. 1224.- Various forms of uteri in Eutheria. A, B, C, D, diagrams illustrating the different degrees of coalescence of the oviducts. A, two distinct uteri. B, bicornuate uterus. $\mathrm{C}$, uterus with a median partition. D, complete? coalescence. E. female reproductive organs of one of the Mustelina with embryos $\left({ }^{* *}\right)$ in the uterus. $F$, female reproductive organs of the Hedgehog. $B$, urinary bladder; $C e$. cervix uteri (neck of uterus); $N, N n$, kidneys and adrenal bodies ; $O d$. Fallopian tube; Ot, ostium tube (abdominal opening of Fallopian tube; $r$. rectum : Suq. urinngenital canal ; Ur. ureter ; Ut. uterus ; Vo. vagina; †, accessory glands. (From Wiedersheim's Comparative Anatom?.) tain cells of the germinal epithelium, the primitive ova $(p r . o v$.). Each of these, surrounded by smaller unmodified cells of the epithelium, sinks 
into the stroma of the ovary, in which it becomes imbedded, the small cells forming a Graafian follicle (foll.) which encloses it. Soon spaces filled with fluid appear among the follicle cells (Fig. $1226, A, s p$.$) , and these eventually coalesce to form a single cavity.$

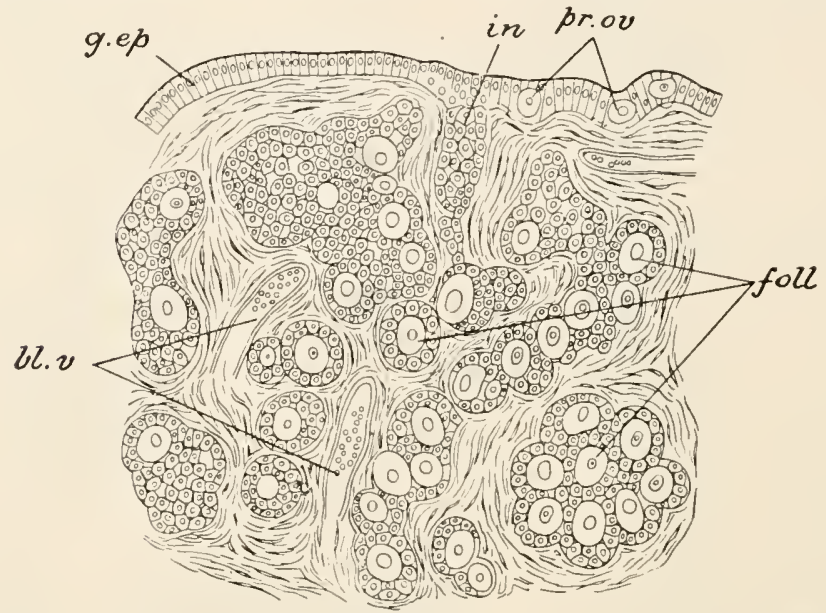

Fif. 1225. - Part of a sagittal section of an Ovary of a new-born child. $b l$. $v$, blood-vessels foll. strings and groups of cells derived from the germinal epithelium becoming developed into follicles ; $/$. ep. germinal epithelium; in. ingrowing cord of cells from the germinal epithelium ; pr. or. primitive ova. (From Hertwig, aiter Waldeyer.)

This cavity, which in some Mammals is crossed by strings of cells, separates an outer layer of the follicle cells-the membrana granulosa (mem.) - from the mass-cumulus proligerus (disc.)-
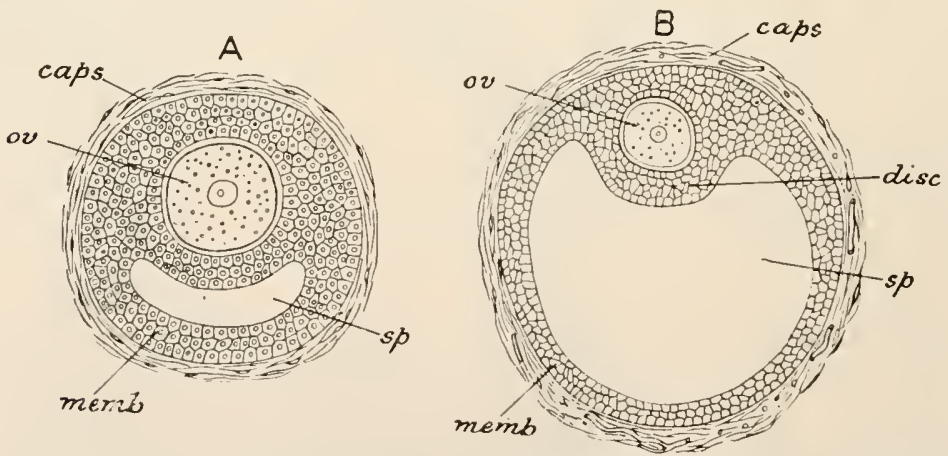

Frf. 1226. - Two stages in the development of the Graafian follicle. A, with the follicular fluid beginuing to appear; $B$, after the space has largely increased. caps. capsule ; lisc. cumulus proligerus; memb. nembrana grunulosa; ot. ovum; sp. suace containing fluid. (After Hertwig.)

surrounding the ovum, except on one side where they coalesce. A basement membrane is formed externally to the follicle cells, and the stroma around this becomes vascular, and forms 
a two-layered investment for the folliche. The cells immediately surrounding the ovum become arranged as a definite layer of cylindrical cells - the corona radiata. A thick membrane-the zona radiuta - perforated by numerous radially arranged pores, into which project processes from the cells of the corona, invests the ovum ; and in many, if not in all, there is beneath this a delicate vitelline membrane. As the ovum increases in size, granules of yolk become distinguishable among the protoplasm.

As the ovum approaches maturity the fluid-liquor folliculi-in the cavity of the follicle increases in quantity, so that the follicle becomes greatly distended. The follicle has meanwhile approached the surface of the ovary, on which it comes to project as a rounded prominence. Eventually the middle region of the projecting part of the wall of the follicle thins out and ruptures, setting free the ovum, which passes into the Fallopian tube. On the way along the Fallopian tube impregnation takes place, and, after becoming enclosed in an envelope of albumen (not always present), the ovum passes onwards to the uterus, there to undergo its development. In the place of the discharged ovum there is left a space which becomes filled with connective tissue to form a body known as the corpus luteum. If the ovum should become fertilised and proceed to develop in the uterus, the corpus luteum increases in size and persists for a considerable time : if no development takes place it disappears comparatively quickly.

With the absence of food-yolk are connected most of the differences observable between the early stages of the development of a higher Mammal (Fig. 1227) and the corresponding stages in the development of a Reptile or Bird. One of the most striking of these is in the mode of segmentation. In the case of the large ovum of the Bird, as we have seen, the segmentation is of the incomplete or meroblastic type, being confined to a small disc of protoplasm-the germinal disc on one side of the ovum. In the Mammals, on the other hand, except in the Monotremes, segmentation is complete or holoblastic, the entire ovum taking part in the process of segmentation. The segmentation is nearly or quite regular, the cells into which the ovum divides being of equal, or approximately equal, size. The result, in the Eutheria, is the formation of a sphere of cells, which soon become distinguishable into an outer layer and a central mass, the inner cell-mass or embryonal knot. In the Marsupials, so far as known, the stage of a solid cellular sphere or morula does not occur, a central cavity being present from the outset. In the Eutheria, by imbibition of liquid, a cavity, which is formed in the interior of the sphere, increases rapidly in size. The stage now reached is called the blastodermic vesicle. During the growth in size of the internal cavity the central mass of cells remains in contact with one side only of the outer layer, where it spreads out as a stratum several cells deep. From it are 
A

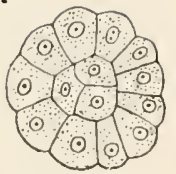

B

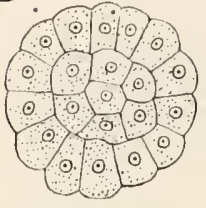

C
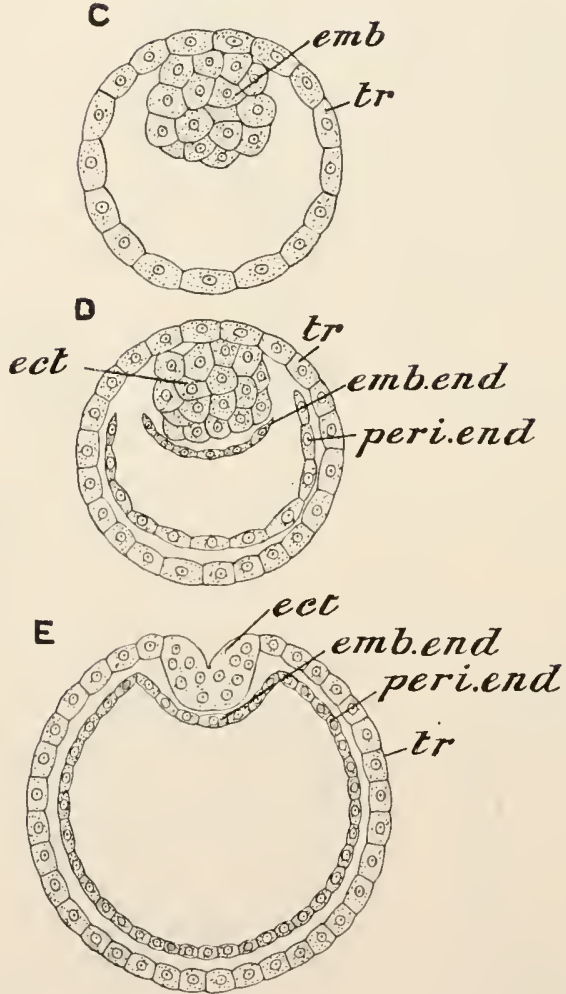

FIG. 1227.-Diagram representing sections of the embryo of a Mammal at successive stages in the segmentation and formation of the layers. $A$ and $B$, formation of enclosing layer (troploblast) and immer cell-mass destined to give rise to the embryo; $C$, blastodermic vesicle with embryonic cell-mass $(e m b$.$) separated from trophoblast (t r$.$) except on$ one side $; D$, blastodermic vesicle in which peripheral and embryonic portions of endoderm have become established : the break here represented on each side between the two does not nccur. $E$, stage in which the embryonic ectoderm has broken through the trophoblast and become joined to it peripherally. derived the embryonal ectoderm and the entire endoderm of the vesicle.

The outer layer is apparently the equivalent of the extraembryonal ectoderm of the Bird and Reptile, and has been termed the trophoblast or trophoblastic ectoderm, because of the part which it plays in the nutrition of the foetus. Immediately beneath it, throughout its extent, a thin layer of flattened cells appears - the peripheral endoderm: this is continuous with a similar layer formed on the inner surface of the embryonic cellmass-the embryonic endoderm-and is formed by outgrowth from it. The rest of the cell-mass gives rise to the embryonic ectoderm. The part of the trophoblast lying over this embryonic ectoderm, known as the covering layer or Rauber's layer, has a widely different fate in different Eutheria : it may thin out and disappear.

A primitive linot and embryonic shield are formed as in Reptiles. The primitive knot has simply the appearance of the somewhat 
enlarged anterior extremity of a primilive streak (Fig. 1228, pr.) which is developed very much in the same way as in the Bird, its formation being due to the same cause as in the Bird, viz., active proliferation of cells leading to the development of the beginnings of the mesoderm. A dark median streak, the hcad-process, appears in front of the primitive knot, and in some Mammals there is an invagination on the surface of the latter leading to the formation of a neurenteric canal and of a notochordal canal which gives rise to the rudiment of the posterior part of the notochord. In the region of the anterior part of the primitive streak, the primitive knot and the head-process, the mesoderm coalesces with the endoderm; but there does not appear to be any breaking through into the underlying space such as occurs in Reptiles (p. 353). A medullary groove $(r f)$ and canal are formed in front of the primitive streak, and a row of protovertebræ (Fig. 1229) make their appearance on each side of the former. The embryo becomes folded off from the blastoderm as in the Bird, and at length the body of the young Mammal is constricted off from the "yolk-sac" or umbilical vesicle, so that, ultimately, the

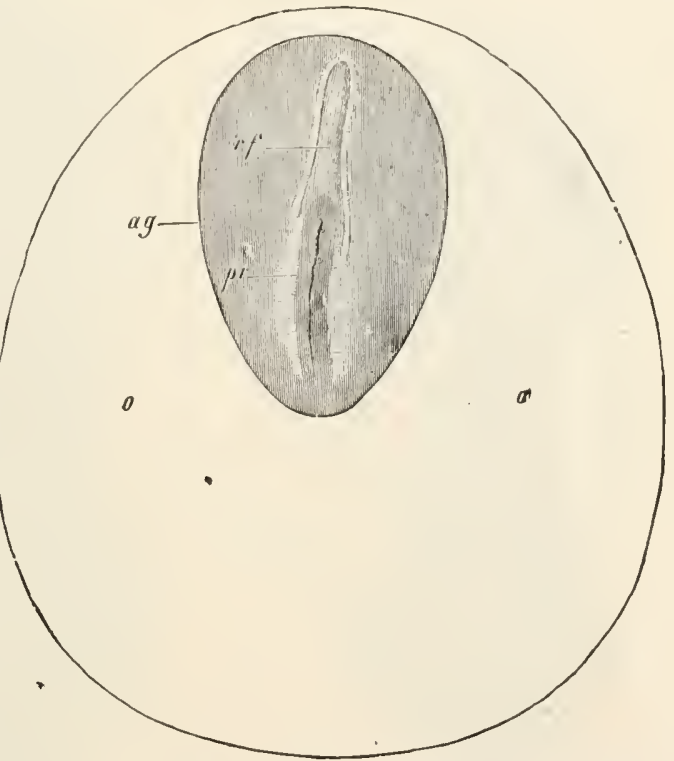

FIG. 1228. - Embryonic area of seven days' embryo Rabbit. $a g$, embryonic area ; $o$, place of future vascular area; $p r$, primitive streak ; if, medullary groove. (From Balfour, after Kölliker.)

two come to be connected only by a narrow yolk-stalk (Figs. 1230 and 1231): the yolk-sac is a thin-walled såc containing a coagulable fluid in place of yolk. A vascular area early becomes established around the embryo on the wall of the yolk-sac.

The most important of the points of difference between a Mammal and a Bird, as regards the latter part of the history of the development, are connected with the fate of the foctal membranes. The amnion is in many Mammals developed in the same way as in the Bird, viz., by the formation of a system of folds of the extra-embryonal somatopleure which arise from the blastoderm around the embryo, and grow upwards 
and inwards, eventually meeting in the middle over the body of the embryo, and uniting in such a way as to form two layers. Of the two layers thus formed the outer, consisting of trophoblastic ectoderm and somatic mesoderm, simply constitutes a part of the extra-embryonic somatopleure which forms a complete investment for the entire ovum, and is known as the chorion or sub-zonal membrane (Fig. 1230, 2 and 3). In the account of the development of the Bird it has been referred to as the false amnion or serous membrane. The inner layer or true amnion, as in the Bird,
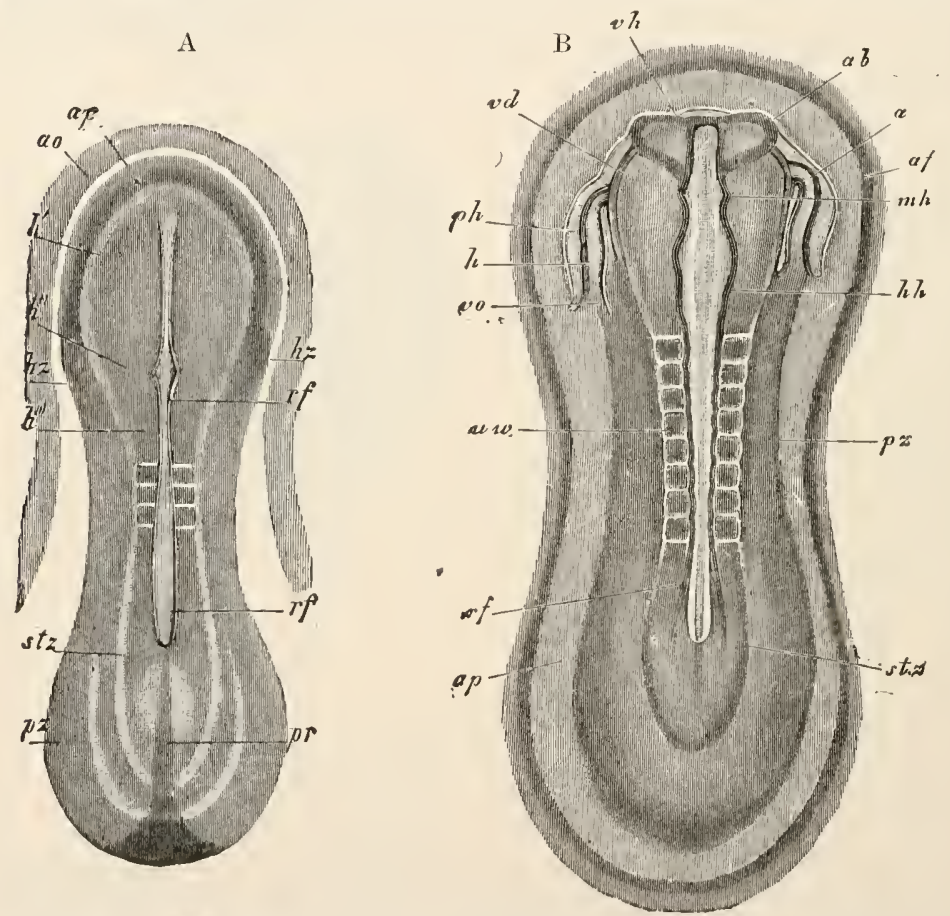

FIG. 1229.-Embryo Rabbit, of about nine days, from the dorsal side. a. aorta ; at, optic vesicle ; $a f$, fold of amnion; ao. area opaca; ap, area pellucida; $h, h z$, heart; $h^{\prime}, h^{\prime \prime}$, medullary plate in the region of the future fore-brain ; $h^{\prime \prime}$, medullary plate in the region of the future mid-brain; $h h$ and $h h^{\prime \prime \prime}$, hind-brain ; $m h$, mid-brain ; $p h$, pericardial section of body cavity; $p r$, primitive streak; $p z$, lateral zone ; $r f$, medullary groove ; $u w$, protovertebræ; stz, vertebral zone; $v d$, pharynx; vo, vitelline vein. (From Balfonr, after Kölliker.)

forms the wall of a cavity--the amniotic cavity (4 and 5, ah)-which becomes tensely filled with fluid (the liquor amnii) over the body of the embryo; this serves the purpose of protecting the delicate embryo from the effects of shocks. As in the case of the Bird, the folds giving rise to the amnion and serous membrane may consist from the first (except the head-fold, which, being formed from the pro-amnion, consists solely of ectoderm and endoderm) of somatic mesoderm as well as ectoderm (trophoblast): or mesoderm may 

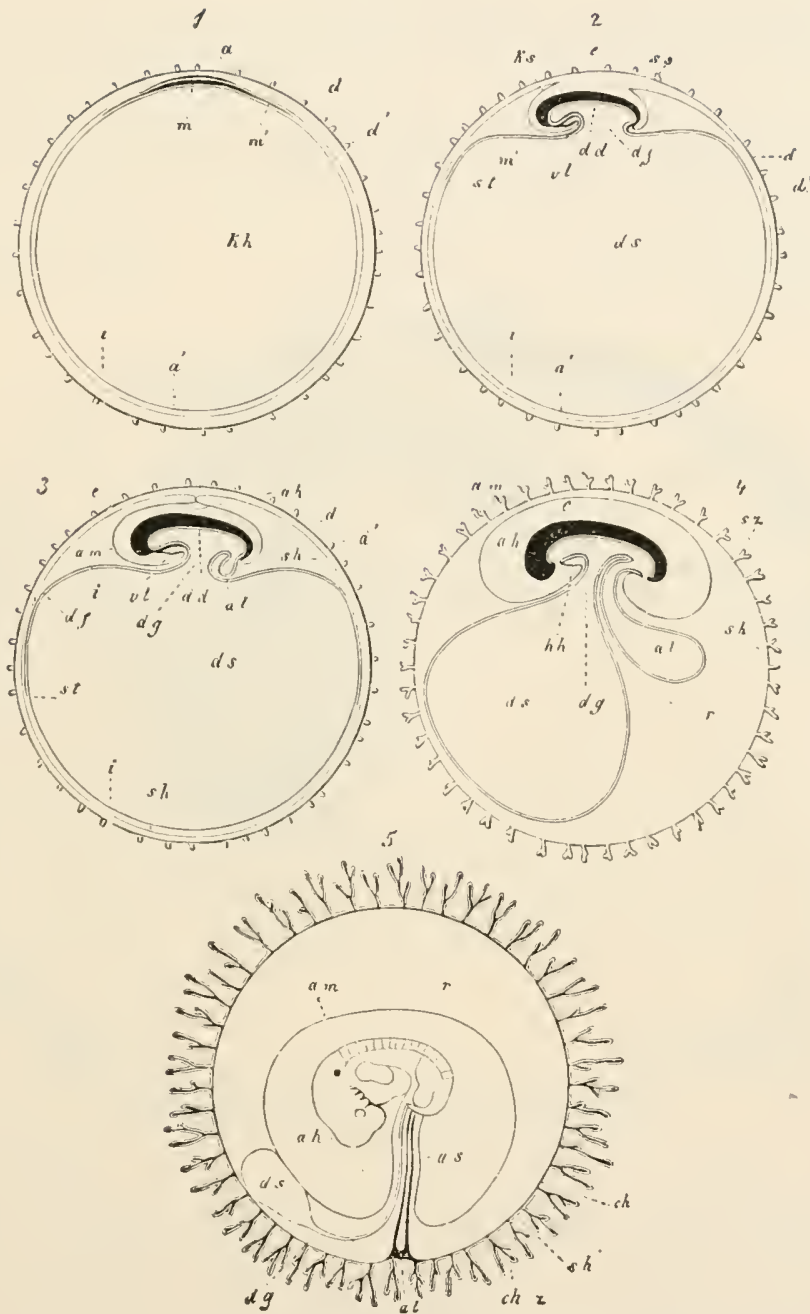

F'IG. 1230.--Five diagrammatic sections illustrating the formation of the fotal membranes of a Manmal. In $1,2,3,4$ the embryo is represented in longitudinal scetion. 1, Embryo with zona pellucida, blastodermic vesicle, and embryonic area; 2 , embryo with commencing formation of yolk-sac and amnion ; 3 , embryo with amnion about to close ; 4 , embryo with villous chorion, larger allantois, and mouth and anus; 5 , embryo in which the mesoderm of the allantois has extended round the inner suriace of the chorion and united with it to form the foetal part of the placenta; the cavity of the allantois is aborted. $a$, ectoderm of embryo; $a^{\prime}$, ectoderm of non-embryonic part of the blastodermic vesicle ; $a h$. amniotic cavity; al. allantois; am. amnion; $c h$. fotal part of placenta; $c h z$, placental vili ; $d$, in 1 zona radiata, in 2 and 3 chorion; $d$, processes of zona radiata and chorion; $d d$, embryonic endoderm; $d f$. area vasculosa; $d g$, stalk of umbilical vesicle; $d s$, cavity of umbilical vesicle; $e$. embryo; $h$, pericardial cavity ; $i$, non-embryonic endoderm ; $K h$, cavity of blastodernic vesicle ; $K s$. head-fold of amion; $m$. embryonic mesoderm; $m^{\prime}$, non-embryonic mesoderm; $r$, space between chorion and amnion; sh, subzonal membrane (chorion); $s s$, tail-fold of amnion; $s t$, sinus terminalis; $s z$, villi of chorion ; r. ventral body wall. (From Foster and Balfour, after Kölliker.)

VOL. II 
.extend into them later, so that, either from the first, or as a result of outgrowth which takes place subsequently, the chorion contains mesoderm as well as ectoderm. The ectodermal cells-trophoblast cells-of the chorion may enter into close relationship with the mucous membrane of the wall of the uterus, and send out processes or primary villi (Fig. 1231, EK) by means of which the ovum becomes intimately attached, and by means of which perhaps nourishment is absorbed.

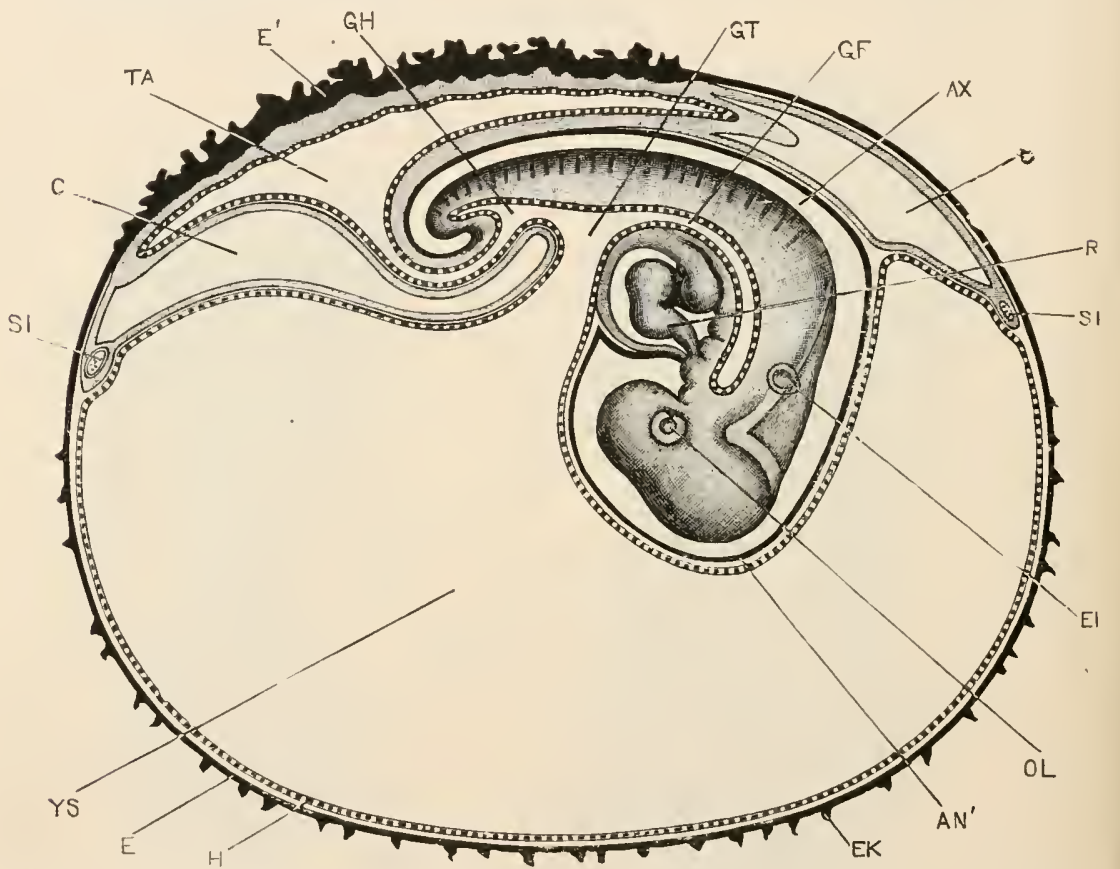

IG. 1231.-A Rabbit enbryo and blastodermic vesicle at the end of the tenth day. The 'mbryo is represented in surface view from the right side, the course of the alimentary canal being indicated by the broad dotted line; the blastodermic vesicle is slown in median longitudinal section. 'The greater part of the tail has been removed. $A N^{\prime}$. pro-amnion : $A X$. cavity of amnion ; $C$. extra-embryonic portion of colome $;$. ectoderm $: E^{\prime \prime}$, thickener ectoderm by which the vesicle is attached to the uterus and from which the foetal part of the placental is derived; $E I$. auditory vesicle; $E \boldsymbol{K}$, ectodermal villi; $G F$. fore-gut; $G H$ lind-gut; $G T$. mid-gut; $H$. endoderm; $O$, extra-embryonic coelome; $O L$. lens of eye ; L. heart; $S 1$. sinus-terminalis ; TA. allantoic cavity ; $Y S$. yolk-sac. (From Marshall, in part after Van Beneden and Julin.)

In certain Mammals the history of the amnion is very different from that above described. In the Hedgehog (Fig. 1232), for example, a cavity appears in the ectoderm of the embryonic area; this is destined to give rise to the cavity of the amnion. The ectoderm, which forms its roof, is entirely trophoblastic or chorionic ; that which forms its floor is partly destined to become amniotic ectoderm, partly embryonal ectoderm. After the mesoderm has begun to become differentiated, the margins of the amniotic part 
of this ectodermal floor $(B)$ begin to grow upwards, giving rise to a layer which extends over the roof on the inner side of the chorionic ectoderm and eventually $(C)$ forms a complete layer-the ectodermal layer of the amnion.

In the Mole (Talpa) spaces appear in the layer of ectoderm of the embryonal area, and these subsequently coalesce to form a
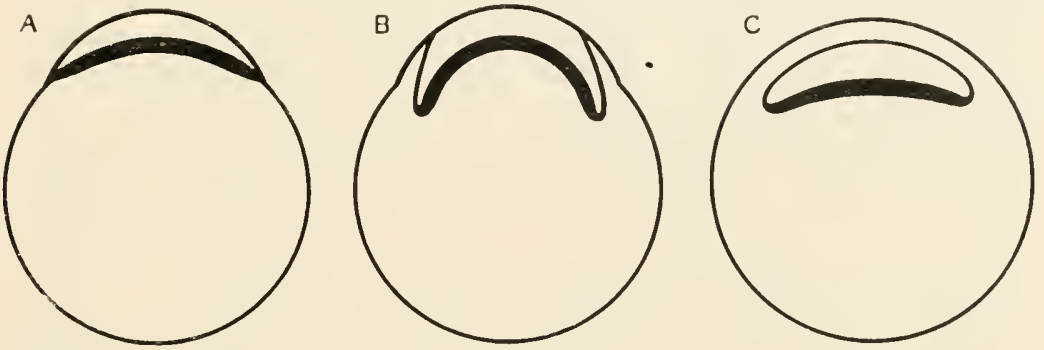

FIG, 1232.-A $-C$, diagran illustrating the formation of the amnion and trophoblast in the Hedgehog. Only the ectoderm is represented. A, early stage in which the amniotic cavity has appeared, roofed over by chorionic ectoderm; $B$, later stage in which the amniotic ectoderm is growing up betow the chorionis frnm the edges of the ectodermal floor; $C$, stage in which the amniotic ectolerin completely roofs over the cavity. (Atter Hubrecht.)

single cavity--the primitive amniotic cavity, but this has only a temporary existence, the amnion arising later by the formation of a series of folds. In Mus, Arvicola, and others (Fig. 1233, A), the amnion is developed from a series of folds of the ectoderm which arise beneath the trophoblast. In other Mammals $(B)$ the amnion arises in the manner already described, and the portion of the trophoblast immediately overlying the embryonic part of the ectoderm eventually disappears.

The allantois has, in all essential respects, the same mode of development as in the Bird, arising in most cases as a hollow outgrowth from the hinder part of
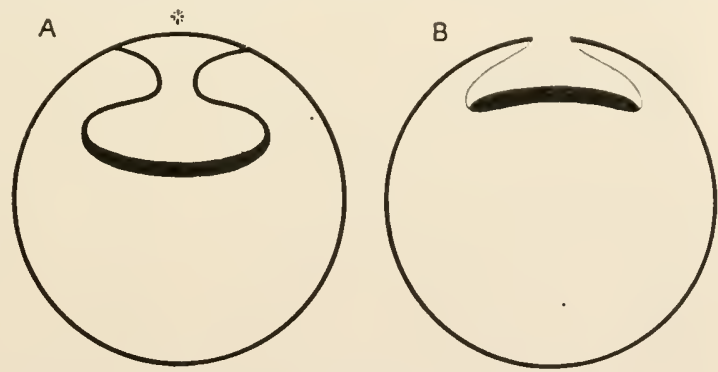

FIG. 1233.-Diagram illustrating the mode of formation of the amnion in various Mammals. $A$, commencing formation of the amnion in Mus, Arvicola, etc. The asterisk marks what corresponds to the portion of the trophoblast overlying the embryo in Fig. 1232, C. B, mode of formation of the aminion il many Manmals. The portion of the trophoblast indicated by the asterisk in $A$ disappears before the anmiotic folds make their appearance. (After Hubrecht.)

the alimentary canal : this, growing out into the space (extraembryonic coelome) between the chorion and the amnion, becomes in all the Eutheria applied to the former, and unites with it to contribute towards the formation of the placenta. But in 
some cases the allantois does not at first contain a cavity, and in some (Primates) the severance between the amnion and the chorion is not completed, and the allantois arises from the outset in continuity with the latter. Sometimes, as in the Rabbit (Fig. 1231), the union between the allantois $(T A)$ and the chorion is limited to a comparatively small part of the extent of the latter, but in most instances the allantois spreads over the entire inner surface of the chorion, and becomes united with it throughout its entire extent (Fig. 1230). Villi, into which mesoderm with blood-vessels penetrates, grow out from the surface of the chorion and are received into depressions or crypts in the mucous membrane of the uterus, which becomes profoundly modified. The villi branch and enter into intimate union with the uterine mucous membrane, so that a close connection becomes established between the vascular system of the foetus and that of the parent.

The term placenta is applied to the entire structure by means of which this connection is brought about; the parts derived from the embryo are termed the foetal placenta, those developed from the wall of the uterus the maternal placenta. In some Mammals the union between the two is not very close, so that at birth no part of the uterine mucous membrane is thrown off ; such a placenta is said to be non-deciduate (semi-placenta). In other Mammals the union is closer, and at birth a part of the hypertrophied mucous membrane is thrown off in the form of a decidua; such a placenta is termed deciduate (placenta vera). In the Mole and the Bandicoot not only is there no decidua thrown off, but the foetal placenta with the distal portion of the allantois does not pass out after the foetus, but remains, and is broken up or absorbed in the uterus. Such a condition has been termed contra-deciduate.

In one of the simplest forms of placenta-the discoidal-found in the Rabbit and other Rodents (Fig. 1231), the yolk-sac extends over the surface of the chorion and becomes fused with it, except in a small area on the dorsal side of the embryo. In this small area the allantois is applied to the chorion and coalesces with it, and from the membrane so formed vascular villi grow out, and are received into the uterine crypts. In most Mammals, however, as already stated, the allantois becomes applied to the chorion throughout its entire extent, and thus completely encloses the embryo. Villi may be developed from all parts except the poles : when this condition persists in the fully-formed placenta, the term diffuse is applied. Sometimes the diffuse condition is temporary, and the completed placenta has villi disposed in a broad band or zone (zonary placenta). Sometimes the villi are grouped together in patches or cotyledons (cotyledonary placenta). In Man and the Apes the villi become secondarily restricted to a disc-shaped area of the chorion situated on the ventral side of the embryo (metadiscoidal placenta). 
In many Mammals the yolk-sac, through the medium of the chorion, enters into a close relationship with the uterine wall, and a connection, the so-called yoll-sac placenta, is established through which nourishment can be conveyed to the embryo ; but this rarely persists after the true (allantoic) placenta has become established.

The stalk of the yolk-sac, with the corresponding narrowed part of the allantois and the vessels which it contains, forms the umbitical cord by which the foetus is connected at the umbilicus with the yolk-sac and placenta. This is enclosed in a sheath formed by the ventral portion of the amnion. The part of the allantois which remains within the cavity of the body develops into the urinary bladder, together with a cord - the urachus - connecting the bladder with the umbilicus.

In the Marsupials the ovum is comparatively large. After fertilisation it becomes enclosed in a thick shell-membrane with a layer of albumen. The first cleavage of the ovum (Didelphys), or the fourth (Dasyurus), involves a separation of the embryo-forming part of its substance from that destined to give rise only to the trophoblastic ectoderm. Further divisions take place in such a way as to give rise, not to a solid morula as in the Eutheria, but to a hollow blastodermic vesicle with a wall composed of a single layer, the cells on one side of which form the embryonic area. An allantoic placenta is not developed except in Perameles. The intra-uterine development of the foetus is abbreviated, and birth takes place when the young animal is still relatively very small and has many of the parts incompletely formed. In this helpless

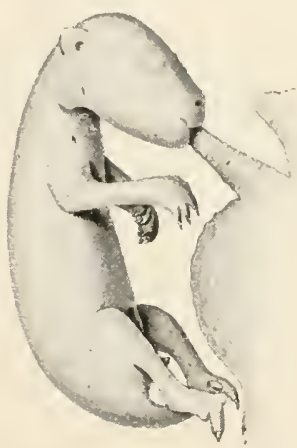

FIG. 1234.- Nammary fuetus of Kangaroo attached to the teat. (Natural size). condition the young Marsupial is placed by the mother in the marsupium, where it remains for a time as a mammary foetus (Fig. 1234), hanging passively to the teat, to which the mouth becomes firmly adherent. The milk is expressed from the mammary gland by the contraction of a muscle, the cremaster, and passes down the gullet of the foetus, which is enabled to breathe unobstructedly through the nostrils by the establishment of a continuous passage from the nasal cavities to the larynx, as already described (p. 558).

In all the Marsupials, so far as known, the embryo is covered over, except in a limited area, by the compressed and expanded yolk-sac. In the majority (Fig. 1235) the allantois (all.) is small, and is completely enclosed with the embryo in the yolk-sac. In the Koala, however (Fig. 1236), it stands out and becomes closely applied to the serous membrane over the small area not covered by the yolk-sac; but no vascular villi are developed. In the Native Cat (Dasyurus) there is a well-developed yolk-sac placenta. 
Only in the Bandicoots (Fig. 1237), so far as known, is the outgrowth of the allantois to the chorion followed by the establishment of an intimate relationship between the chorion and the uterine wall, with the formation of interlocking ridges and depressions, the

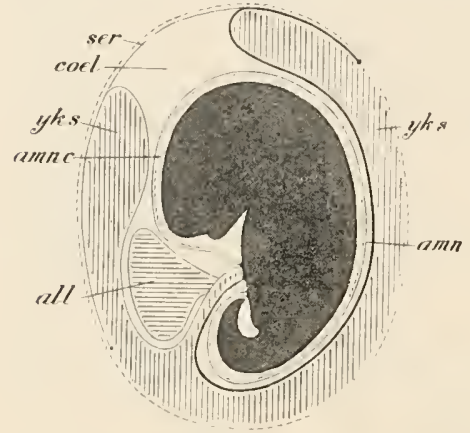

Fis, 1.3.35-Diagram of the embryo and fortal membranes of Iypsiprymnus rufescens. wil. allantoic cavity ; $a m n$. amnion; amn $c$. cavity of amnion; cal extra-embryonic coelome; ser. serous membrane (chorion); yk.s, yolk-sac. (After Semon.)

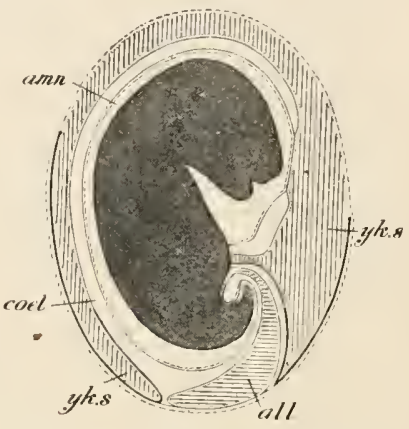

Fiti, 1236.-Diagram of the enturyo and fortal membranes of Phascolarctos cinereus. Letters as in Fir. 12:5. (After Semon.)

whole constituting a placenta of the same essential character as that of the Eutheria, though devoid of actual villi.

The Prototheria, unlike all the rest of the Mammalia, are oviparous. In Echidna only a single egg, as a general rule, is laid in

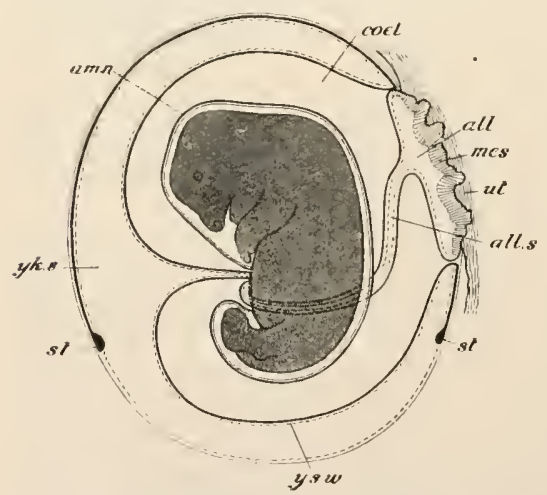

Fif. 1:37.- Hiagram of the embryo and placenta of Perameles obesula. Letters a-in Fig. 1235. In addition, all. s, allantoie stalk; mes. mesen(hyme of outer surface of allantois fused with mesenchyme of serous membrane; $s, t$. sinus terminalis; ut. uterine wall. (After J. P. Hill.) a season. This is placed in a temporary marsupium, formed as already described (p. 477) in the mammary region of the ventral surface. The young animal soon emerges from the egg, and remains enclosed in the marsupium till it reaches an advanced stage of development. Ornithorhynchus develops no marsupium, and the two" eggs which it produces are deposited in its burrow. In Echidna the eggshell is composed of keratin; in Ornithorhynchus it contains carbonate of lime. The

ova of the Prototheria (Fig. 1238) are very much larger than those of other Mammals, their greater dimensions being due to the presence of a large proportion of food-yolk. The segmentation, unlike that of all the Theria, is meroblastic, and the blastoderm 
eventually forms a complete investment, of two layers, to the yolk. An embryonic area is differentiated at one pole, and on it appears a primitive streak with a primitive knot and head-process.

Geographical Distribution.-The Monotremes are entirely confined to Australia, Tasmania, and New Guinea. The Marsupials are most abundantly represented in the Australian region, the greater. number of the Australian families and genera being restricted to the Australian continent and to Tasmania, though several genera extend to New Guinea and some of the neighbouring islands. The family Didelphyidæ, or Opossums, inhabits South America and extends into the southern part of North America; and a single genus, Canolestes, of a

\section{A}
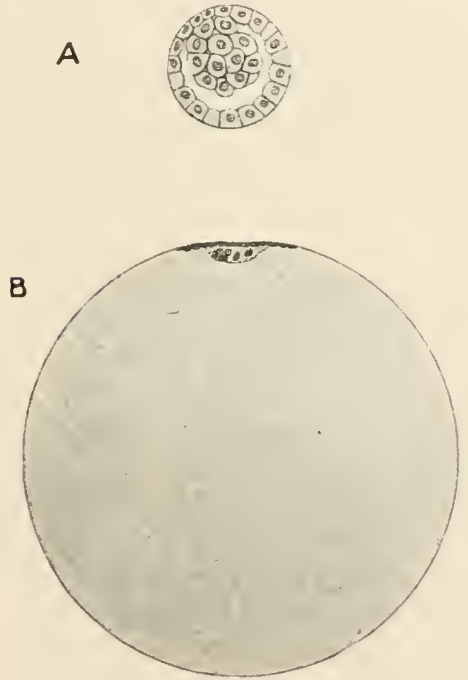

Fif. 1238, $-A$, blastula stage of one-of the Theria. $B$, transition stage between the morula and blastula in a Monotreme. Both represented in cliagrammatic section. (After Semon.) family at one time supposed to be related to the Australian Diprotodonts, but now more generally regarded as derived from Didelphyid (Polyprotodont) stock, has been comparatively recently found in South America.

The Edentates are most numerously represented in South and Central America, the true Anteaters, the Sloths, and the Armadillos being all inhabitants of that region. But the Scaly Anteaters and the Ard-varks (Cape Ant-eaters) are denizens of the Old World; the former inhabiting Southern Africa and South-Eastern Asia, the latter being confined to Africa.

The Cetacea are cosmopolitan in their distribution : the great majority are marine, but some ascend rivers, and a few are exclusively fluviatile, inhabiting the rivers of South America and South-Eastern Asia.

The distribution of the Sirenia is somewhat restricted. The recently extinct Rhytina inhabited Behring's Straits. The Manatee is confined to the Atlantic coasts of South America and of Africa, living chiefly in the larger rivers. The Dugong occurs on the east coast of Africa, in the Red Sea, the Indo-Malayan islands, and the northern coast of Australia.

The Ungulata occur in all the great regions, with the exception of the New Zealand, Polynesian, and Australian. Oxen are, with the exception of the American Bison, natives of the Palæarctic, Ethiopian, and Oriental regions. Wild sheep, 
with the exception of one African and one North American species, are confined to the Nearctic and Oriental regions. Goats are also mainly Nearctic and Oriental. Antelopes are confined to the Old World, and are by far more numerous in the Ethiopian than in other regions. The Prongbucks are Nearetic; the Giraffes exclusively Ethiopian. Deer are widely distributed in the Nearctic, Neotropical, Palæarctic, and Oriental regions, but are absent from the Ethiopian. The Camels are natives of the Old World; the Llamas of the Neotropical region. Wild species of Pigs are widely distributed in the Old World and are absent in the New; while the Peccaries are confined to the Nearctic and Neotropical regions. Hippopotami are confined to Africa. The Horses, including the Zebras and Asses, are restricted at the present day, as regards their natural distribution, to the Old World, though they abounded also in America in the Pleistocene period. Rhinoceroses are Oriental and Ethiopian. Tapirs have a singular distribution, one species occurring in the Malay Archipelago, and the rest in the Neotropical region. Hyraxes are confined to Africa, Arabia, and Syria. Of the Elephants, one species is confined to the Oriental, the other to the Ethiopian regions, but fossil remains prove that in Pleistocene times the range of the Elephants, and their gigantic extinct allies, the Mammoths, was very much wider, and extended over Northern Africa and the entire Palæarctic region. Only one fossil species has been found in America.

Carnivora, if we leave out of account the Australian Dingo or Native Dog, are absent in the Australian, Polynesian, and New Zealand regions, but range over all the other geographical provinces. The Cats and the Dogs are found in all parts of this extensive area : the Hyænas are restricted to the Western part of the Oriental region and the warmer parts of the Holaretic and the Ethiopian. The Civets are most abundant in Africa, Madagascar, and SouthEastern Asia, but occur also in the Southern parts of Europe; and many of the smaller groups have a yet more restricted range. Bears have a wide distribution, but are absent from the whole of the Ethiopian region.

The majority of the Pinnipedia are found in the Arctic and Antarctic regions, and in the temperate zones of both hemispheres, few ranging into the tropics. The Walruses are almost exclusively Northern, while the Eared Seals and Earless Seals are almost equally abundant in the Northern and Southern hemispheres.

The Rodents have a wider range than any others of the orders of land Mammals, and occur in all parts of the globe, though they are poorly represented in Australia and Madagascar. The Rodents reach their greatest development, as regards the number of families, in South America, in which region occur also the majority of the largest members of the order.

Insectivora are absent in the Australian, Polynesian, and New 
Zealand regions, and in South America, but occur in all the other provinces.

The Chiroptera are world-wide in distribution, occurring in greatest abundance in tropical and warm temperate zones. The Flying Foxes (Pteropidæ) are absent from the Nearetic and Neotropical regions, and the Vampire Bats occur exclusively in the latter.

The distribution of the Lemurs is remarkable; they occur only in Madagascar, a limited part of South Africa, Southern India and Ceylon, some of the islands of the Malay Archipelago, and the Philippines. The headquarters of the group is the island of Madagascar, of which they constitute one half of the entire Nammalian fauna.

Of the other groups of Primates, the Marmosets (Hapalide) and the Cebidx are exclusively American; the Cercopithecidæ Palæarctic, Oriental and Ethiopian, with a single species in Madagascar. Of the Simiide the Gibbons occur only in South-Eastern Asia and the Malay Peninsula; the Orangs only in Borneo and Sumatra; the Gorilla and Chimpanzee in certain parts of Western Equatorial Africa.

Geological Distribution. - The earliest fossil remains of Mammals have been found in strata of Upper Triassic and of Jurassic age in Europe and America. These remains consist almost exclusively of jaws and teeth, and, as the latter differ widely from those of existing Mammals, there is frequently great difficulty, in the absence of remains of the other hard parts, in determining the affinities of these Mesozoic forms. Some of the Triassic and Jurassic Mammalian molar teeth are constructed on the most primitive form of the triconodont type, which has already been referred to (p. 544) as being the primitive form in the class, having three cones or cusps in a longitudinal row. In Dromatherium and its allies each molar has a single main cusp with two smaller accessory cusps. There is no decisive evidence as to the affinities of these primitive triconodont Mammals, but they may be provisionally set down as allied to the Prototheria.

of the remainder of the Mesozoic Mammals, some were probably Prototheria, others Metatheria, while others again may have been Insectivores. Most of them fall into two main groups. The type of dentition presented by the members of one of these groups (Fig. 1239) is more nearly allied to that of the Polyprotodont Marsupials (p. 464) than to any other. In the other group (Multituberculata) (Fig. 1240) there is a superficial resemblance to the Diprotodont Marsupials ; a single chisel-shaped incisor is present on each side of the lower jaw, and one large, and sometimes one or two smaller, on each side of the upper. $\AA$ wide diastema separates these from the pre-molars. The molars present a number of variously arranged small tubercles. In some cases the pre-molars have a 
pattern similar to that exhibited by the molars, but in others they have a cutting edge which may be serrated or obliquely grooved. The fact that the vestigial molar teeth of Ornithorhynchus come nearer in pattern to those of certain of the Multituberculata than to those of any other known group is looked upon as evidence that the affinities of the latter are rather with the Prototheria than with the Metatheria. In the American Cretaceous beds in which these teeth are most abundant a number of limb-bones have also been

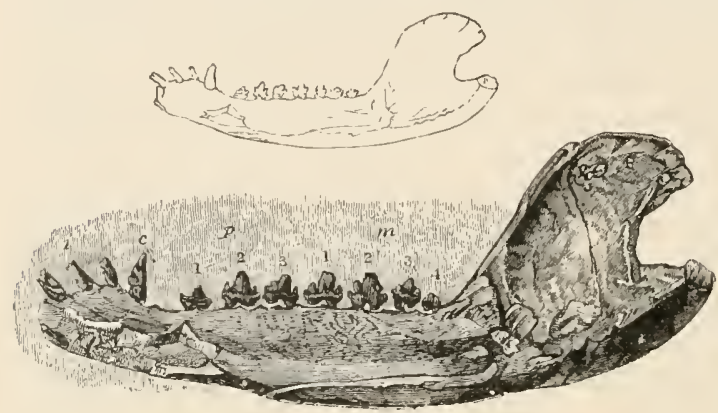

FIG. 1239- Thascolotherium bucklandi. Inner view of right ramus of mandible. (After Uwen.) found, some of which show evidence of Monotreme characteristics.

Fossil remains of Mammals be longing to the Cret a ceous age are known only from certain limited beds in North America. But in deposits of the succeeding Tertiary period there have been found the remains of an extensive and varied Mammalian fauna. The earlier Tertiary Mammals in many cases present features which enable us without hesitation to refer them to one or other of the existing orders; but when this is the case there is nearly always to be recognised an absence, or a less advanced development, of some of the more salient characteristics; in other words, the earlier Tertiary Mammals, when referable to existing orders, are less highly specialised than the living representatives of these orders. No less significant is the fact that these early Tertiary representatives of existing orders had the cavity of the brain-case nearly

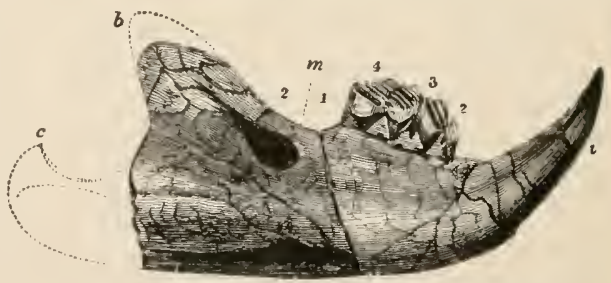

Hik. 1240.-Plagiaulax becklesi. Mandible with always much smaller in proportion to the other dimensions than in living forms. But many are not so readily referable to existing orders, sometimes owing to their possessing marked special features of their own, sometimes owing to their combining characteristic features of two or more living orders. Through the series of Tertiary and Post-tertiary formations it is possible to trace a gradual development from the early generalised to the existing specialised 
genera, and in some instances by such gradual transitions that the actual course of the evolution can be followed stage after stage. There is only space here for a very brief review of this extensive and remarkable Tertiary and Post-tertiary Mammalian fauna.

No remains of Prototheria are known from the Tertiary, and it is only when we come to Post-tertiary (Pleistocene) that we meet with fossil representatives of the group. These, which have been found only in Australia, differ little from the existing Echidna and Ornithorhynchus.

Of the Marsupials, the Opossums (Didelphyidæ) of America are represented not only in Cretaceous, Tertiary, and Pleistocene deposits in that continent, but in beds of Tertiary age in Europe. In addition, in certain European deposits of Eocene age, there occur teeth and jaws which may be Marsupial in character, but the affinities of

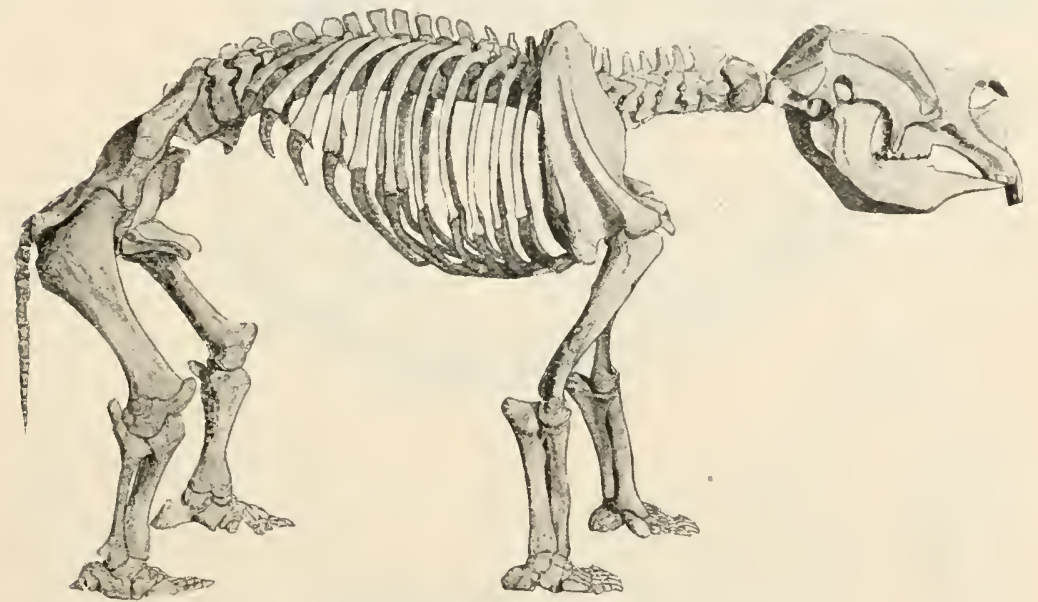

FIG. 1241.-Diprotodon australis. (From a restoration of the skeleton by Prof. E. r. stirling in the Adelaide Museum.)

which are uncertain; and in Tertiary deposits of South America have been found numerous remains of Marsupials belonging to a group represented by the single surviving genus, Canolestes. These South American Conolestoids appear, so far as is known, to have differed from the Australian Diprotodonts in the absence of the characteristic syndactylism of the latter. Another group of South American Tertiary Mammals, the Sparassodonts, are regarded as Polyprotodonts nearly related to the Tasmanian Thylacine. The remainder of the fossil Marsupials hitherto discovered are of Pleistocene age, and have nearly all been found in Australia. The Australian Pleistocene Marsupials are for the most part referable to existing families and even genera, representing both the Diprotodont and the Polyprotodont sections; but some differ widely from existing forms. One of these, Diprotodon (Fig. 1241), was the 
largest known Marsupial, having reached the dimensions of a Rhinoceros; it occupies a position intermediate between the Phalangers and the Kangaroos. Unlike the latter, Diprotodon had the limbs of approximately equal size, and adapted for walking:

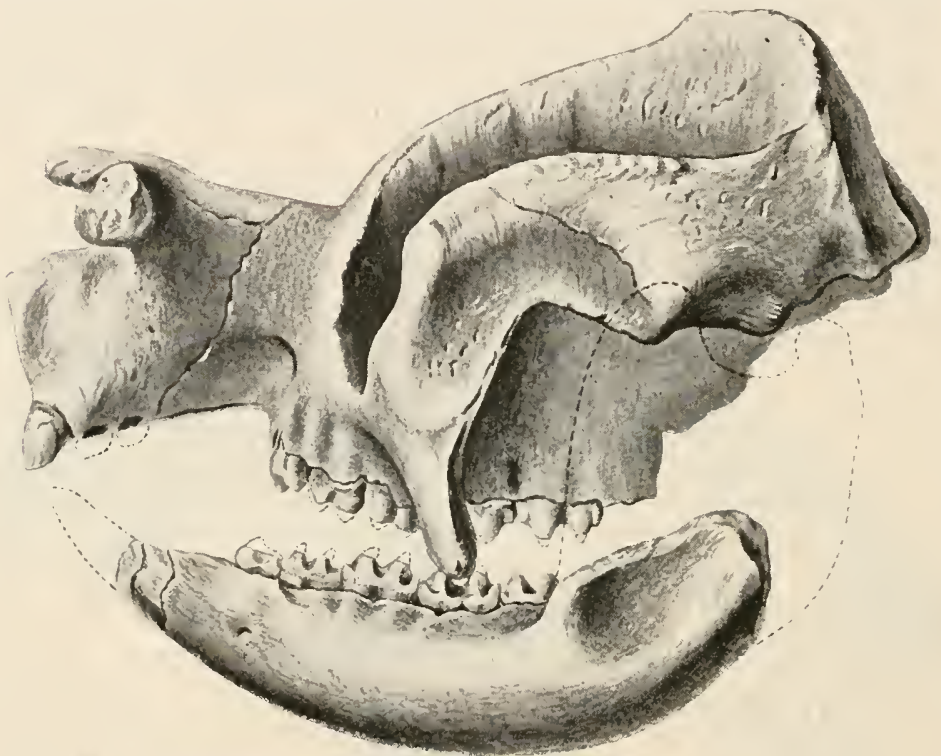

FIG. 1242.-Nototherium mitchelli. Side view of skull. (After Owen.)

both manus and pes were pentadactyle with very small sub-equal digits. Nototherium (Fig. 1242), somewhat smaller than Diprotodon, but also of large size, seems to connect together Diprotodon, the Wombats, and the Phalangers. Thylacoleo (Fig. 1243) is an extinct genus referable to the Phalanger family, and characterised by an extremely modified dentition, the only func. tional teeth being a single pair of large incisors in the middle in both upper and lower jaws, with a single elongated trenchant pre-molar on each side both above and below.

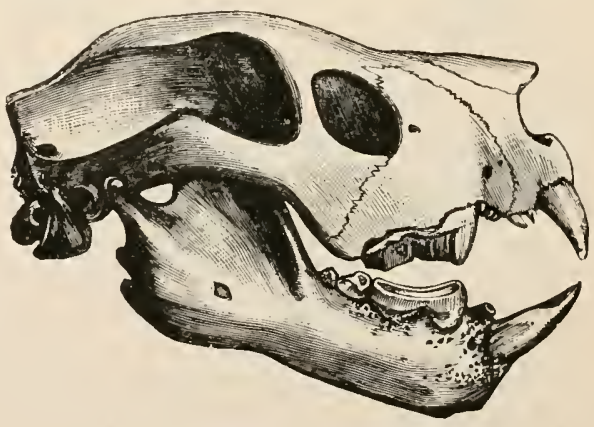

FIG. 1243.-Thylacoleo carnifex. Side view of skull. (After Flower.)

Among the Edentata the majority of fossil as of existing forms have been found in South America. But the family of the Cape Ant-eaters, at the present day confined to South Africa, is proved, 
by the discovery of remains in the Pliocene of the island of Samos in the Eastern Mediterranean and in the Eocene of Southern France, to have formerly had a wider distribution. The American fossil Edentata, all of Pleistocene age, comprise, in addition to extinct

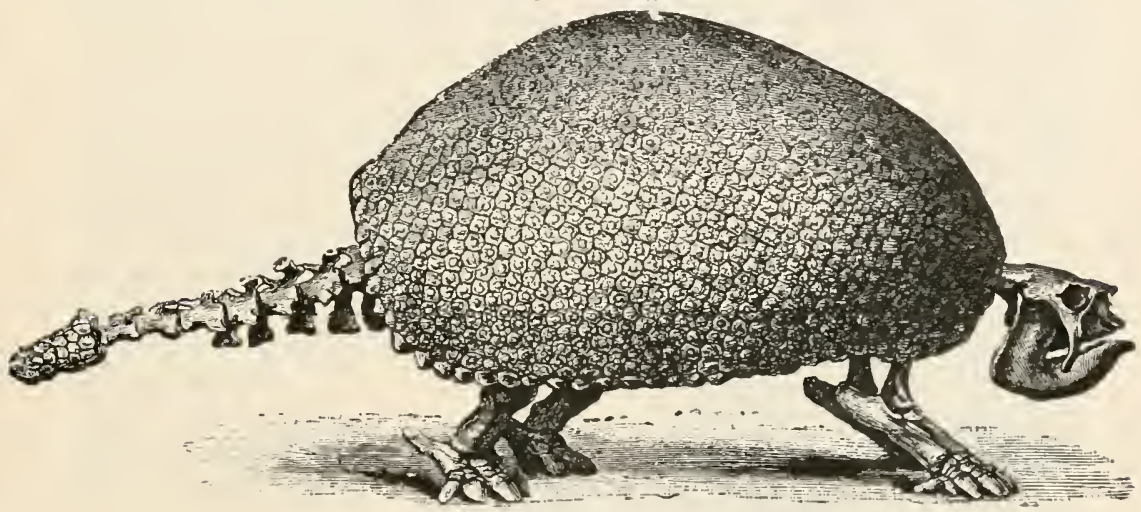

FIG. 1244.-Glyptodon clavipes. (After Owen.)

genera of Armadillos, some of gigantic size, and one genus of Sloths, representatives of two extinct families, the Glyptodontidoe and the Megatheriido. ${ }^{1}$ The former (Fig. 1244) are large Edentates resembling the Armadillos in the presence of a bony dermal carapace and a bony investment for the tail; but in the Glyptodontidæ the carapace has no movable rings, so that the animal could not roll itself up, and there is usually a ventral bony shield or plastron, never present in the Armadillos. Glyptodonts occur in North as well as in South America. 'The Mega-

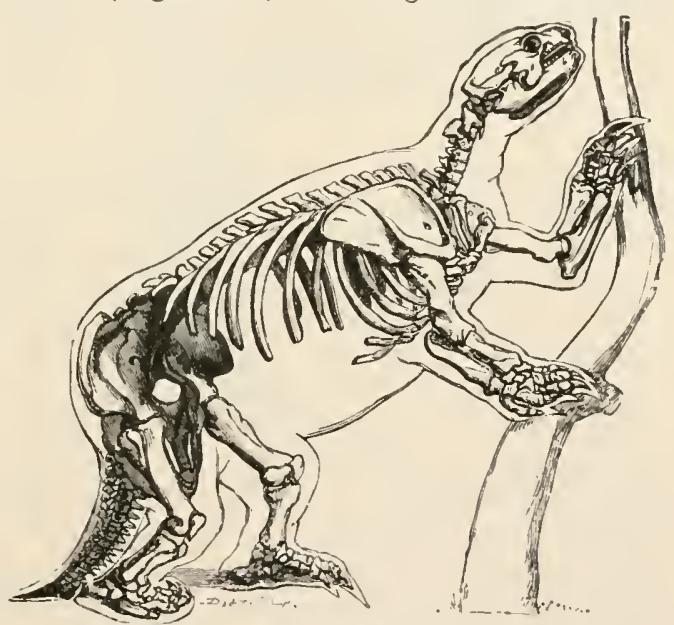

FIG. 1245.-Mylodon robustus. (Restoration, after Owen.) theriidæ (Fig. 1245) are Edentates, mostly of enormous size and massive build, which combine certain of the features now characteristic of the Ant-eaters (Myrmecophagidæ) and the Sloths (Brady-

1 Recent remains stated to belong to a Megatherium have been found in South America. 
podidx) respectively, the spinal column and limbs allying them with the former, and the crania and the teeth with the latter.

The Cetacea are represented in the Tertiary (Eocene and Miocene) of Europe, Egypt; and North America by an extinct sub-order-Archacoceti or Zeuglodonta, comprising only one known genus-Zeuglodon. Zeuglodon differs from existing Cetacea mainly in the possession of rooted heterodont teeth, and in the position of the narial aperture, which is situated comparatively far forwards; the limbs are not known; there were irregular dermal bony plates. The remains of both Whalebone Whales and Toothed Whales occur abundantly in Pliocene deposits, some belonging to extinct, others to existing genera. Toothed Whales occur also in Miocene formations, and there, as well as in the Pliocene and Pleistocene of Europe, North America, New Zealand, and Australia, are represented by an

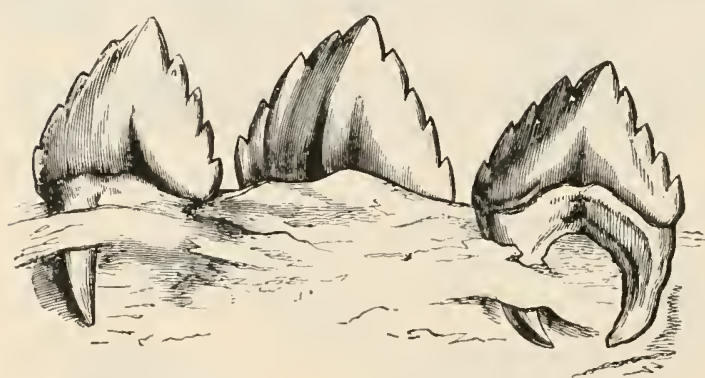

Fig. 1246.-Squalodon. Three of the lower true molars. (After Hlower.)

Halitherium is the best known. These were characterised by the possession of upper incisors, in some cases of canines, of enamelled pre-molars and molars, of a milk-dentition, and of small vestiges of femora. The family of the Dugongs is represented by a form nearly allied to the existing genus in the Pliocene of France, and probably by another genus in the Tertiary of California. The family of the Manatees is not known to be represented by any fossil forms. 'The recently extinct Rhytina ("Steller's Sea-Cow"), which lived within historic times in Behring's Straits, was the largest known member of the order, and sometimes attained a length of seven or eight metres.

The Tertiary Ungulata comprise an immense number of forms, including numerous extinct families, into an account of which it would be going beyond the scope of the present work to enter. In the Artiodactyle series there is to be traced a progressive union and coalescence of the third and fourth metacarpals to form the cannon-bone, a progressive reduction of the lateral digits, and a progressive development of horns or of tusks-absent or rudimentary in the earlier representatives of the sub-order. In the Perissodactyle series the reduction of the lateral toes reaches its 
maximum in the existing genus Equus. The history of this reduction, together with the development of other characteristic features, can be traced from pentadactyle forms with simple molars through a long series of gradations to the monodactyle Horses with their complexly folded molars. Similar genealogies, though not always so complete, can be traced for the Tapirs and Rhinoceroses, and for the Deer, Camels, and Pigs.

The order Proboscidea was represented in Tertiary and Pleistocene times, not only by forms allied to those now living -though sometimes, as in the Mammoths, of much greater size- but by an extinct family, the Dinotherida (Fig. 1247) (Miocene and Plio- cene of Europe and India), which possess a pair of downwardly - directed tusks in the lower jaw. The genus Pyrotherium, from the Patagonian Tertiary deposits, was a primitive Ungulate, with a pair of short tusk-like incisors in the lower jaw, which may have been related to the Proboscidea: and Meritherium, from the Eocene of Egypt, is probably also a primitive member of the same order.

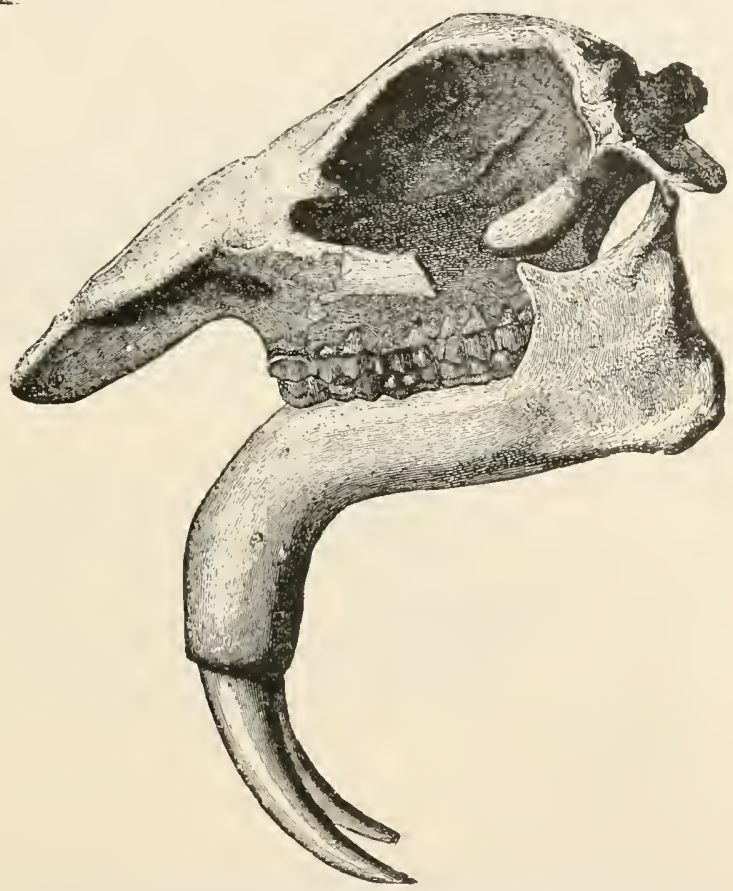

Fif, 1217-Dinotherium giganteum. Sile view of skull. isth natural size. (Erom Zittel's Palcontoloy', after Kaup.)

The Hyracoidea were represented in the Pliocene by an extinct genus (Pliohyrax); and Archoohyrax, from the Patagonian Tertiary, is perhaps also an allied form.

A separate sub-order, the Condylarthra, has been established for a number of Eocene Ungulates, which differ somewhat widely from all the other members of that group, and approach the Carnivora in some respects, though having certain resemblances to the Hyracoidea. The pre-molars and molars are short and usually bunodont, the pre-molars being simpler than the molars, the latter 
sometimes tritubercular, like those of many of the Carmivora; the incisors and canines also sometimes resemble those of the Carnivora. The humerus differs from that of the other Ungulata, and resembles that of the Carnivora in the presence of a foramen over the inner condyle. The arrangement of the carpals corresponds with what is observable in the Hyracoidea, and also, as in the latter group, the femmr has a third trochanter. The limbs are usually pentadactyle, with pointed ungual phalanges. The astragalus has, as in the Carnivora, a uniformly rounded distal articular surface. The fibula does not articulate with either the astragalus or the calcareum.

Another extinct primitive sub-order of the Ungulata is the Amblypoda, the members of which have been found, like the Condylarthra, in the Eocene of North America and of Europe. These resemble the Condylarthra and Hyracoidea in the scaphoid being opposite the trapezoid; both magnum and unciform articulate with the lunar. The fibula articulates with the calcaneum; the cuboid articulates with both the astragalus and the calcanenm; the feet are short, pentadactyle, and plantigrade. Canines are present in both upper and lower jaw; the pre-molars and molars are short and lophodont in type. Dinoceras and Tinoceras are characterised by the process of remarkable bony prominences on the upper surface of the skull. In the Tertiary deposits of Patagonia and Bolivia have been found the remains of another group of extinct Ungulates of low organisation, the Litopterna. These had the distal segments of the limbs elongated and constructed on the perissodactyle type, the number of the digits varying from five to one, the third being always the largest. The carpal and tarsal bones do not interlock as in the existing Ungulata vera. There is no foramen above the inner condyle, and clavicles are absent. A third trochanter is present. The brain-case is very much smaller than in the existing Ungulata vera. The dentition is complete or nearly so; the pre-molars and molars short and provided with roots.

The Ancylopoda are another group of primitive extinct Ungulates. The remains of some of these have only been found in the Patagonian Tertiary deposits, but others had a wide range both in the Old and New Worlds. They all differ from other groups of Ungulates in the form of the limbs : the weight of the body appears to have been borne on the outer edge of the manus and pes, and the digits were evidently provided, not with true hoofs, but with pointed claws. The teeth resemble those of the Perissodactyla.

The Typotheria and Toxodontia are two groups of extinct Ungulata the representatives of which have only so far been found in the South American Tertiary formations. The former differ from ordinary Ungulates in the possession of a clavicle and the presence of a foramen above the inner condyle of the humerus. 
The ulna and fibula are complete, and there are either four or five fully formed digits in each foot. The Toxodontia approach nearer the normal Ungulate type, the clavicle and the foramen over the inner condyle being both absent. They have a massive skull and short stout limbs, each with three digits.

The true Carnivora of the Tertiary period are, as compared with those of the present time, remarkable for the absence of the wellmarked distinction into groups such as are now to be recognised; numerous intermediate forms connect together the Dogs, Civets, Cats, Bears, and Weasels, which, in the existing fauna, appear separated from one another by differences of the most strongly marked character. Several extinct families are recognised, and one extinct order-the Creodonta. The latter present resemblances to the Insectivora on the one hand, and to the Polyprotodont

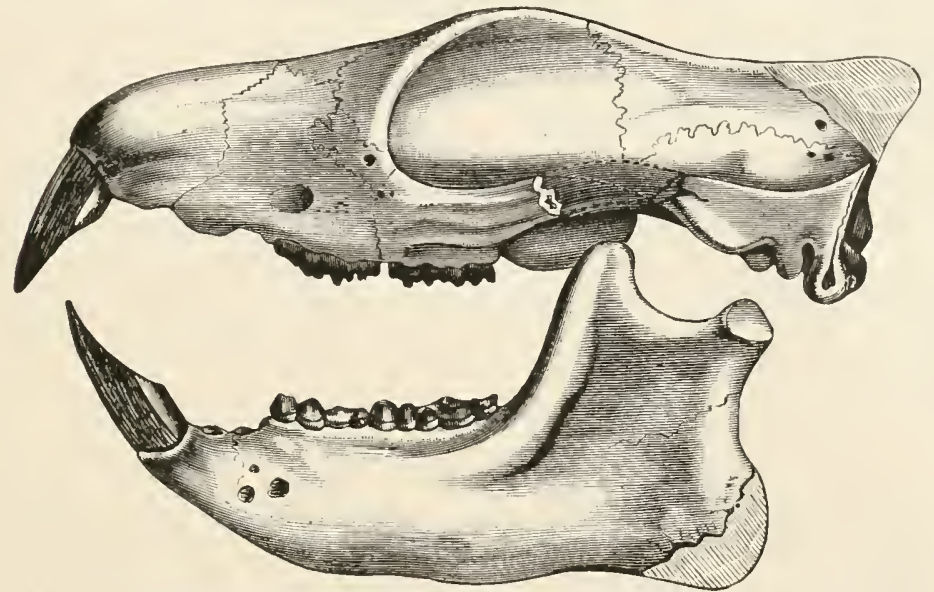

Fia. 124. - Tillotherium fodiens. Left lateral view of skull. (From Flower, aiter

Marsupials on the other, such as would appear to indicate a relationship with both of those groups.

A group of Eocene Mammals of uncertain affinities are the Tillodontia (Fig. 1248), which by some have been elevated to the rank of a distinct order. The Tillodontia appear to unite in a remarkable degree, in skull and dentition, ungulate, rodent, and carnivorous characteristics.

The Rodents were represented in the Tertiary period by all, or nearly all, the principal groups existing at the present day, together with several extinct families. Some of the Tertiary Rodents attained a much larger size than any living members of the order.

Among the Tertiary Insectivora, in addition to representatives of existing families, are a number of extinct forms. Through these it is possible to connect the living Insectivora with the Creodont Carnivora on the one hand, and with the Prosimii on the other. 
Chiroptera, not differing widely from existing forms, occurred as early as the Eocene.

Of the Primates, Prosimii occur from the Eocene onwards. A single extinct family is known, comprising Lemuroids which bear a closer resemblance to Insectivora than do the living members of the order. Of the Anthropoidea, the Hapalidx and Cebidx are only represented in the Miocene (Eocene?) and Pleistocene of South Amcrica; the Cercopithecidx in the Miocene and Pliocene of Europe and the Pliocene and Pleistocene of India by extinct genera (Mesopithecus, \&c.) and by species of the existing genera Macacus, Semnopithecus, and Cynocephalus, and in the Pliocene of India, France, and Italy by species of extinct genera. Among the Simiidæ the Gibbons occur in the Miocene of France, the Pliocene of Germany, and the Pleistocene of Borneo. An extinct genus, Dryopithecus, found in the Miocene of Europe, is perhaps related to the Gorilla; and a species of Orang (Simia), together with a form allied to the Chimpanzee, occurs in the Indian Pliocene.

In deposits of late Pliocene or early Pleistocene age in Java have been found the remains of an Anthropoid (Pithecanthropus) which has been supposed to be an intermediate form between the man-like Apes and Man. Traces of the existence of Man in the form of flints of undoubted human manufacture have been found in the Pliocene; but any such evidences are extremcly rare until we reach the Pleistocene.

\section{The Mutual Relationships of the Chordata.}

In discussing the relationships of the various groups of Chordata, it will be convenient to begin with Fishes, and to work from them upwards and downwards.

The question of the inter-relationships of the various groups of Fishes is a very puzzling one. As in other cases of the kind, there are three lines of evidence to be kept in mind-anatomical, embryological, and palæontological--the last being always, when available, the final court of appeal.

With regard to anatomical evidence, it seems fairly obvious that Fishes having neither limbs nor jaws are more primitive than forms in which those structures are present, unless undoubted evidence of degeneration can be produced; that a purely cartilaginous skeleton is more primitive than a bony one, and a notochord than a vertebral column, however simple; that a brain with distinct cerebral hemispheres is more advanced than one having an undivided prosencephalon; that an autostylic skull, being due to the concrescence of originally distinct parts, is more specialised than a hyostylic skull; that the loss of the spiracle and the presence of an operculum and of a highly differentiated 
hyoid arch are evidenees of speeialisation, as also is the presence of air-bladder or lung, spiral valve, conus arteriosis, or copulatory organs.

In embryology, eggs with much food-yolk are to be looked upon as more modified than those with little, mnless there is distinct evidence in the latter of reversion towards an aleeithal condition. Any special contrivanees for the nourishment and proteetion of the embryo, obviating the necessity for the production of immense nmmbers of eggs, are also marks of advanee.

On both these lines of evidenee the lowest plaee may safely be assigned to the Cyclostomes. In spite of all the attempts that have been made to homologise various eartilages in the neighbourhood of the mouth in the Lamprey with the elements of the anterior viseeral arehes in higher forms, there is no real evidence that the aneestors of the class had either jaws or limbs, and the most reasonable theory is that they are the deseendants-highly speeialised in eertain respects in aeeordanee with their peeuliar mode of life-of a primitive eraniate stoek.

With regard to the two largest gromps of Pisees-the Elasmobranehii and the Teleostei-the evidenee from anatomy and embryology is eonflieting. The Teleostei take the highest plaee in virtue of their skeleton, opereulum, air-bladder, and gills, as well as in their extraordinary adaptability to various environments; but the Elasmobranchs reaeh a distinetly higher grade of organisation in their enterie canal, heart, brain, and urinogenital organs, as well as in their large and well-protected eggs. The anatomy of Ganoids seems to show, however, that the spiral valve, eonus arteriosus, and typieal ovidncts (Mïllerian ducts) have been lost in the course of the evolution of the Teleostei, and that the simpler structure of these organs in that order is actually a eoneomitant of their extreme speeialisation.

The Holoeephali and the Dipnoi, while agreeing with Elasmobranehs in many important respects, show an advance in the presenee of an autostylie skull and of an opereulum, while the Dipnoi rise above all other Fishes in possessing not only lungs like Polypterus, but also posterior nares and a partially divided auricle. 'The lung appears to have been derived from an airbladder with pneumatic duct opening on the ventral wall of the œsophagus, as in Polypterus; by the dorsal shifting of the duet and its final atrophy the closed air-bladder of the higher Teleostei has arisen.

Coming to the results of Palæontology, many striking and unexpected faets have recently been bronght to light. There is reason to believe that Palæospondylus is a Cyelostome, but one with welldeveloped vertebræ; from which it must be assumed either that the vertebral eolumn of existing members of the elass is degenerate, or that Paloospondylus is a highly specialised offshoot of the 
primitive Cyclostome stock, in which a vertebral column had been independently acquired. The latter conclusion seems the more probable, and is supported by the fact that in all three orders of Ganoids there are some species with a persistent notochord, others with well ossified vertebræ; the conclusion being that the vertebral column is a polyphyletic structure-that is, has been evolved independently in various groups in accordance with similar conditions.

Among extinct Elasmobranchs the Acanthodea and Pleuracanthea had bones investing the cranium, and Cladoselache had no claspers. These facts seem to indicate as a probable ancestor of the Teleostomi and Dipnoi-the two sub-classes with ossified skeleton-a generalised Elasmobranch in which fusion of dermal ossicles into investing bones had begun, but in which the special reproductive phenomena of the existing members of the group-internal impregnation and few, large, well-protected eggs - had not yet been acquired. The origin of the Dipnoi from such a source is rendered more probable by the possession of the characteristic biserial fin or "archipterygium" by Pleuracanthus. The Holocephali and the existing Elasmobranchs may be considered as having arisen from the same primitive stock along diverging lines of descent. There is, however, at present no evidence to trace or to explain the fusion of the palatoquadrate with the cranium to form the characteristic autostylic skull of the Holocephali and Dipnoi.

The connection of the Ostracodermi with the better-known groups of Fishes is very uncertain. It has been proposed to class them with Cyclostomata on account of the absence-as far as our present knowledge goes-of jaws and limbs, and attempts have been made to show affinities with the Xiphosura and with larval Tunicates. They seem, however, to be undoubted Fishes, but with no clear relationship to any existing group. The Arthrodira appear to be most closely allied to the Dipnoi.

The question of the origin of Fishes from lower forms is involved in the greatest obscurity. Practically the only assistance in the solution of the problem is furnished by Amphioxus, which seems to indicate, as the ancestral stock of Vertebrates, fish-like animals having a skeleton in the form of a notochord, fin-rays, buccal cartilages, and branchial rods; a barely differentiated brain; no heart, but a contractile ventral vessel below the pharynx and a dorsal vessel immediately beneath the notochord; colourless blood; separate nephridia of the annulate type; a coelome developed as an enterocole; metamerically arranged gonads devoid of ducts; and alecithal eggs. The forward extension of the notochord, the inmense pharynx, the very numerous gill-slits, and the atrium are very probably characters special to the Acrania; but even 
putting them aside as of no phylogenetic importance, it is obvious that this group must have sprung from a point very low down the chordate stem. The morphological differences between Amphioxus and a Hag are, in fact, of a more fundamental character than those between a Hag and a Mammal.

Still lower must have been the point of origin of the Urochorda, with the notochord confined to the tail, the dorsal mouth, the absence of myomeres and of nephridia, and with only exceptional and ill-defined traces of segmentation. The huge pharynx with its innumerable stigmata is undoubtedly a secondary character; but the atrium, endostyle, dorsal lamina, and peripharyngeal bands seem to indicate an affinity with the Acrania. So also do the earlier stages of development; but the later stages, and especially the mode of origin of the atrium, are quite different in the two cases.

The propriety of including the Hemichorda among the Chordata is still sub judice. Allowing that any single organ may have a polyphyletic origin-i.e., may arise independently in different groups in accordance with similar needs-it seems highly improbable that three such peculiar and characteristic structures as notochord, hollow dorsal nervous system, and gill-slits can have arisen together more than once in the history of animals; and if it could be shown with certainty that these three characters were all present in the Hemichorda their place in the chordate phylum would be assured. But the cavity or cavities in the dorsal nervecord of Balanoglossus are inconstant, and are very different from the neurocœle of Urochorda and Vertebrata, which from the first extends through the whole length of a well-defined dorsal nervous system. In Cephalodiscus and Rhabdopleura, moreover, there is no trace of any such cavity.

The pharyngeal diverticulum of the Hemichorda, also, is a very different thing from the notochord of Urochorda and Vertebrata, nothing in the structure or development of which gives the slightest indication that it originally arose as a forward outgrowth of the anterior portion of the mid-gut. The diverticulum of Hemichorda is, in fact, obviously a support to the persistent prostomium of a fixed or sluggish animal, while that of Urochorda and Vertebrata forms a strengthening axis-either to the tail alone or to the whole body-of an active, elongated, animal swimming by lateral movements of the tail; and there seems to be no reason why two such different structures should not have had an independent origin. The supposed double "notochord" of Actinotrocha, the larva of Phoronis, is even more problematical.

Far more significant are the gill-slits, but even their evidence is hardly conclusive, since they are absent in Rhabdopleura and 
Actinotrocha, and in Cephalodiscus are a single pair of apertures having apparently no respiratory function. In Balanoglossus, however, they are very numerous and increase in number with the growth of the animal, as in Amphioxus, and the division of each by a "tongue" is very similar in the two cases. Further homologies have been suggested by comparing the snout of Amphioxus with the proboscis or pre-oral lobe of Hemichorda and its pre-oral pit with the proboscis-pore.

On the whole, although it is by no means certain that the "chordate" peculiarities of the Hemichorda may not have been independently evolved, it is convenient to retain them in the present phylum, pending further knowledge of their true affinities.

Various zoologists have supported the view that the nearest relatives of the Chordata among the Invertebrata were forms characterised, like all but the lowest of the former phylum, by the occurrence of metamerism, i.e., Annulata or Arthropoda; while others have looked upon the metamerism of the Chordata as independently evolved. Among the non-metameric groups which have been supposed to have been ancestral to the Chordata are the Nemertines-the proboscis-sheath being compared with the notochord, and the proboscis itself with the pituitary invagination. But this view has received little support. On the other hand, the supporters of the Annulate ancestry have been more numerous some seeing in the Chætopoda, others in the Hirudinea, the nearest Chordate relatives. The metamerism of the Annulata is regarded as supporting this theory, as well as the structure and arrangement of their circulatory, nervous, and excretory systems. The notochord might have been supposed to have originated in an Annulate from a typhlosole or from an intestinal diverticulum or siphon. But a very serious difficulty is met with when we proceed to try to derive the Chordate nervous system from that of the Annulata. Not only must the ancestral Annulate, in order to become transformed into the primitive Chordate, have reversed its habitual position, so that its neural surface became dorsal instead of ventral ; but its mouth, which pierced the central part of the nervous system, nust liave been abolished, while a new one was developed on the hæmal side of the head.

Elaborate comparisons have been instituted between the brain of Cyclostomes and Fishes and those of Crustacea and Xiphosura, and it has been sought to explain the neurocoele as the discarded Arthropod enteric canal. But if Amphioxus and the Urochorda, to say nothing of the Hemichorda, are branches from some low part of the chordate stem-a fact it seems hardly possible to doubt-it is obvious that there can be no direct connection with 
the highly specialised classes referred to. If, for instance, the lower Craniata sprang either from a Chætopod-like or from a Limulus-like ancestor, Amphioxus and the Tunicates must either have no connection at all with Vertebrates, or must have undergone a quite inconceivable amount of degeneration.

There appears, in fact, to be a good deal of evidence supporting the view that the first developed Chordates may not have had any such pronounced metamerism as Annulates and Arthropods possess, and it becomes possible to look for the immediate ancestral form among less highly organised Invertebrate groups. But on this matter, though there is abundant scope for ingenious speculation, no finality can be said to have been reached, and the ancestry of Vertebrates still remains an unsolved problem.

With regard to the higher classes, Amphibians may be held to have arisen from a Fish-type allied to the Dipnoi, the resemblances of which to the Amphibia are so great as to lead some authors to place them in a distinct class intermediate between Fishes and Amphibia. The chief difficulty in this case-and it is a serious one-is the derivation of the pentadactyle limb from the Fish's fin, a transformation of which no satisfactory explanation is at present offered either by anatomy, embryology, or palæontology.

Reptiles may be considered to have arisen from a generalised Amphibian stock, but there is no direct evidence on this point; and, apart from purely theoretical considerations, there is nothing to show how or why gills vanished so completely as to leave no trace of their existence apart from the branchial clefts, or by what steps the allantoic bladder became precociously enlarged into an embryonic respiratory organ. The precise mode in which the protecting amnion arose is also very doubtful, though from theoretical considerations it has been argued that its development in the Hedgehog (p. 574) indicates a more primitive condition than obtains in the other Mammalia or even in Sauropsida.

Birds appear to be undoubtedly derived from true Reptiles, although nothing is known of the actual ancestral form. In spite of the enormous adaptive differences between the warm-blooded, feathered, bipedal Bird and the cold-blooded, scaly, quadrupedal Reptile, the connection between the two is far closer than between any other two vertebrate classes.

Mammals also appear to have lad a reptilian origin: the numerous reptilian characters of the Monotremata certainly point in this direction, and the reproductive processes of that group help us to understand the stages by which the large-yolked egg of the ancestral form, with the embryo developed outside the body, may have given place to the secondarily alecithal egg of the typical Mammal, giving rise to a fœtus developed within 
the uterus, and nourished by a complete placenta. Though palæontology does not reveal to us the actual reptilian progenitors of the Mammalia, yet, as already pointed out, there are some of the Anomodontia (Theriodonta) which have pronounced mammalian resemblances in skeleton and teeth.

The following diagram may serve as a rough illustration of the view set forth in the preceding pages:-
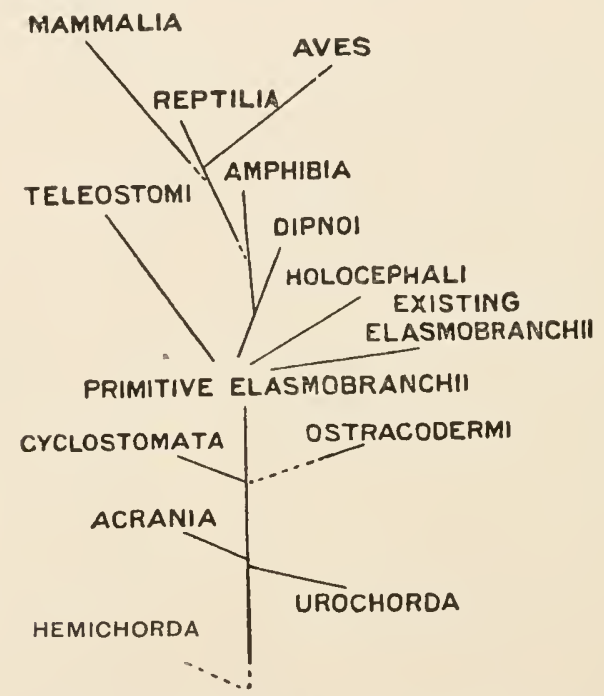

FIG. 1249.-Diagram illustrating the Mutual Relationships of the Chordata.

\section{On the Mutual Relations of the Phyla of Animals.}

It will be advantageous in concluding our survey of the animal kingdom to sum up with a few remarks as to the phylogeny of the primary groups or phyla, since that of the sub-divisions of each phylum has already, in nearly every instance, been discussed.

It cannot be too strongly emphasised that in the majority of cases it is useless to seek for the ancestors of any animal among existing forms. As far as we know, most living species are culminations-terminal branches of the great tree, not leading directly to any other form, but connected only at the fork of a branch. It is, as a rule, only among fossils that actual ancestral forms are to be looked for; hence the area of strict phylogenetic investigation is very limited, and in most instances the only evidence is to be sought in anatomy and embryology.

Not only are most existing species culminations, and therefor- 
off the direct line of ancestry of other species, but, as far as we can judge, the same is true of most genera and families, of a large majority of orders and classes, and even of most phyla. It would certainly seem that existing Chordata, Mollusca, Arthropoda, Annulata, Echinodermata, Nemathelminthes, and Platyhelminthes are all independent branches of the animal tree, having no connection with one another save through the trunk.

There are, however, existing groups which seem to represent actual stages in the existence of others. For instance, it can hardly be doubted that Amphibia are derived from Fishes and Birds from Reptiles; that if we could discover the unknown ancestors of those classes they would be classed definitely among Pisces and Reptilia respectively, though probably not belonging to any known order.

In the same way everything seems to point to the conclusion that all the higher phyla must have passed through some kind of cœlenterate stage, and, before that, some kind of protozoan stage, so that these two phyla may be said to represent actual steps in the evolution of the higher forms. It is therefore legitimate to assume, in the absence of direct evidence, that the ancestors of both the Cœlenterata and the Porifera were unicellular or "noncellular" forms, i.e., to be classed among the Protozoa, and that the ancestors of the nine higher or triploblastic phyla were diploblastic forms, i.e., to be classed among the Cœelenterata.

Most, if not all, of the triploblastic phyla appear to be terminal or culminating groups. There is no reason for thinking that either of the three highest phyla - the Chordata, Mollusca, and Arthropoda - ever passed through a stage which, if known, would be classed among Platyhelminthes, Nemathelminthes, Echinodermata, or Annulata. The wide occurrence of the trochophore, or some similar larval form, seems, however, to indicate a certain bond of union. The typical trochophore of Annulata and Mollusca, the echinopædium of Echinoderms, the ciliated larva of Molluscoida, the tornaria of Balanoglossus, and the adult Rotifer present, amid endless diversity in detail, common characters which, in the absence of better evidence, may be considered as indications of remote affinity. The Arthropoda alone among the higher phyla are devoid of even this slender connection with lower forms: there is no indication throughout the phylum of anything approaching to a trochophore; the crustacean nauplius is quite sui generis; and the larval forms of Insects and Arachnids simply suggest a homonomously segmented ancestor. This suggestion is supported by Peripatus, the cilia, hollow appendages, nephridia, and ladderlike nervous system of which seem to point to its derivation from a segmented "worm" not far removed from the annulate type. 
In accordance with these conclusions the mutual relationships of the phyla may be expressed in a diagrammatic form as follows :---

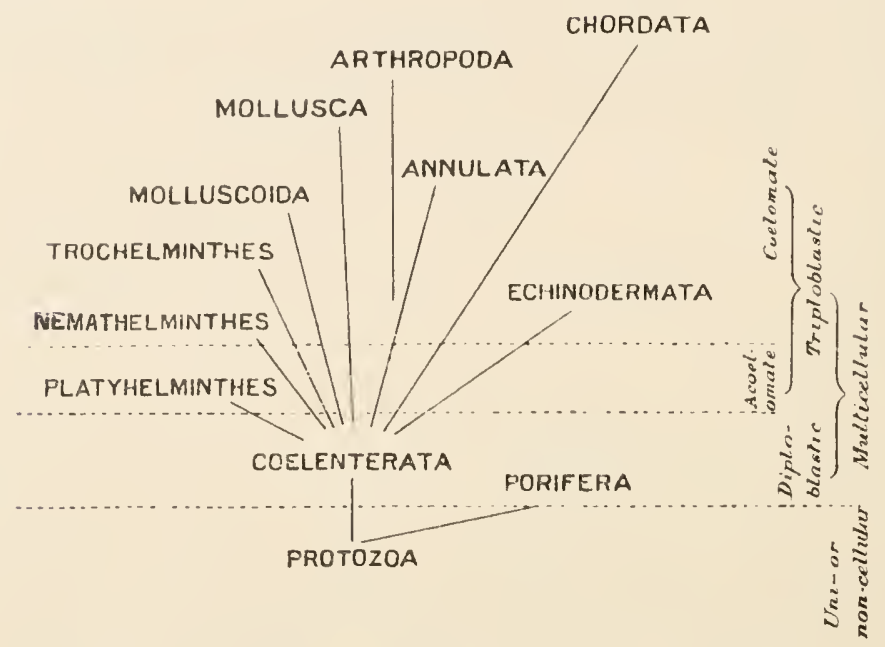

FIG, 1250,-Diagram illustrating the Mutual Relationships of the Phyla. 


\section{SECTION XIV}

\section{DISTRIBUTION}

In discussing the various groups of animals, the subject of their geographical and geological distribution has in every case been referred to, and the reader will already have noticed how strikingly the different parts of the earth's surface at the present day, and the different periods of its geological history, differ from one another in respect of their animal inhabitants. In order to bring forward the facts of distribution more prominently, the present section will be devoted to this important subject.

\section{Geographical Distribution.}

The facts and ideas of which the subject of Geographical Distribution takes cognisance are clearly brought out by comparing the fauna of Great Britain with that of the most distant of her colonies, New Zealand, including, in each case, the adjacent islands. The two countries are not widely different in size. The climate of each is temperate, Great Britain extending from about $50^{\circ}$ to $60^{\circ}$ north latitude, and having a mean annual temperature of about $48^{\circ} \mathrm{F}$., New Zealand extending from about $34^{\circ}$ to $48^{\circ}$ south latitude, and having a mean annual temperature of $55^{\circ}$. Both contain mountainous regions, forests, and arable land. The climate of both is humid, the rainfall of Great Britain being from about 25 to 30 inches in flat, 40 to 80 inches or more in mountainous districts, while the average rainfall for the whole of New Zealand is about 50 to 55 inches. The physical conditions of the two countries are thus very similar, the chief differences being the far higher summer temperature of the northern part of New Zealand, and the far lower winter temperature of the northern part of Great Britain.

But when we come to compare the faunæ of the two countries these similarities disappear. In Great Britain there are about forty species of native land Mammals, including Ungulata (Wild Cattle, Red Deer, Fallow Deer, \&c.), Carnivora (Fox, Badger, Wild Cat, Stoat, Marten, \&c.), Rodentia (Squirrel, Rabbit, Hare, \&c.), 
Insectivora (Hedgehog, Shrew, and Mole), and fifteen species of Bats. Moreover, within the historic period, the Wolf, Bear, Wild Boar, Reindeer, and Beaver were among the wild animals of Britain.

In New Zealand, on the other hand, the only land Mammals found in the islands previous to the advent of Europeans were two species of Bats (Chalinolobus morio and Mystacina tuberculata), the Maori Dog, which was certainly introduced by the Maories who colonised New Zealand from some of the Pacific Islands not many centuries ago, and the Maori Rat (Mus maorum), which perhaps owes its introduction to the same source. With the exception, then, of Seals, two Bats, and perhaps a Rat, are the only native Mammals of New Zealand.

The Birds of the two countries also offer a marked contrast. Great Britain has no native Ratitæ; in New Zealand there are now living five species of Apteryx, while within the historic period-three or four hundred years ago or less-there lived in the two islands half-a-dozen genera, and some five-and-twenty species of Moas (Dinornithidx). Great Britain has no Parrots; New Zealand has two species of Nestor, seven Parrakeets of the genus Cyanorhamphus, and the extraordinary Ground Parrot or Kakapo (Stringops). On the other hand the Finches (Fringillidæ) are wholly absent in New Zealand, but abundantly represented in Great Britain. Moreover, New Zealand is remarkable for the large number of flightless Birds included in its fauna: besides the Kiwis and Moas, there are Stringops (Ground Parrot); Ocydromus, Cabalus, and Notornis (Rallidæ); Nesonetta (the flightless Duck of the Auckland Islands); besides the extinct Giant Goose (Cnemiornis) and Giant Rail (Aptornis). In Great Britain none of the Birds are actually flightless.

As to Reptiles, the difference is less striking, but is still sufficiently marked, the most important facts being the entire absence of Snakes in New Zealand and the presence of Sphenodon, the only existing representative of the Rhynchocephalia. Lizards occur in both countries, and in both Crocodilia are wholly absent, and Chelonia occur only as occasional visitants.

Great Britain possesses four species of Tailed Amphibia (Newts), and the same number of Frogs and Toads. In New Zealand Urodeles are entirely absent, and there are only two rare species of Frogs.

The fresh-water Fishes of Britain are numerous and varied; the most important are the various species of Salmo (Salmon and Trout), the Perch, Carp, Grayling, Pike, Eels, \&c. In New Zealand there are only Eels, a small salmonoid, Retropinna, not found elsewhere, several species of Galaxias, an exclusively Australasian, South African, and South American Physostome, and a small indigenous genus (Neochanna) of the same family. The differences 
between the marine Fishes, though obvious enough, are less fundamental, a fair proportion of the New Zealand shore-fishes belonging to the same families, and in some cases even to the same genera and species, as those of Britain.

Among Mollusca the fresh-water Unio (fresh-water Mussel) is found in both countries, but New Zealand has no species of the common genus Helix (Land-snail), abundant in Great Britain, and its molluscan fauna generally is very peculiar.

The Insect-fauna of New Zealand is remarkable for the paucity of Butterflies - sixteen species against about seventy in Britainand for the abundance of Moths, mostly belonging to the Microlepidoptera and the Geometrina. The occurrence of Peripatus in New Zealand furnishes another strong point of contrast. Amongst fresh-water Crustacea, the British Astacus is represented by an allied genus Paranephrops. Among marine Crustacea many genera are common to the two countries, but there are numerous peculiar forms, and it is worthy of mention that the New Zealand species of Palinurus belongs to a more generalised type than the British species, having no stridulating organ.

The British Earthworms all belong to the familiar Lumbricidce (including Lumbricus) and Cryptodrilide; in New Zealand both these families are absent, and the majority of the Earthworms belong to the Megascolecidæ, including the genera Acanthodrilus, Octochatus, \&c. Lastly, there are found in New Zealand nearly forty species of Land Planarians and one terrestrial Nemertean; these groups are represented in the land-fauna of Britain only by one species of the former.

That these striking differences are quite independent of climate, food, \&c.-in other words, that the environment in the one country is in no way inimical to the fauna of the other-is shown by the zoological history of New Zealand since its colonisation. Apart from domestic animals, the Brown Rat (Mus decumanus) and the House Mouse (Mus domesticus) are now as common in New Zealand as in Britain; the Rabbit has become a plague, barely kept in check by constant effort stimulated by severe legislative enactments; Deer flourish as well in the mountains of Otago as in those of Scotland; the Birds first noticed by a visitor to the settled districts of the colony will probably be the Sparrow, Blackbird, Thrush, Starling, and Goldfinch; and Trout have become so thoroughly acclimatised in the streams and lakes, that in some districts the poorer settlers, like the British apprentices of old, decline to eat them. We thus learn to distinguish between the native or indigenous fauna of a country and the introduced fauna which owes its existence to human agency; in comparing the faunæ of any two countries, the latter element must of course be carefully eliminated. 
The contrast between our two selected countries is further emphasised when we compare the fauna of each with that of the nearest continental area-- the fauna of Great Britain with that of the Continent of Europe, the fauna of New Zealand with that of Australia.

With the exception of the Red Grouse (Lagopus scolicus), perhaps the Coal Tit (Parus brilannicus), and the Long-tailed Tit (Parus rosea), and some fifteen species of fresh-water Fishes, the British Vertebrates are all found on the European continent. On the other hand, there are many European species of Mammals, Birds, Reptiles, Amphibians, and Fishes which do not occur in Great Britain. The British fauna may, in fact, be described as an isolated and somewhat impoverished section of the general European fauna.

Now contrast New Zealand with Australia. Of the two New Zealand Bats, one (Chalinolobus morio) occurs also in Australia ; the other (Mystacina tuberculata) is endemic, that is, found nowhere else. Australia, instead of having a Mammalian fauna comprising only two Bats and a doubtful Rat, possesses a large number of characteristic Manmals, all except the Bats, Rats, and Mice, and the Dingo (Canis dingo), being either Monotremes or Marsupials. Out of about 200 species of New Zealand Birds, fully 100 are endemic; of the rest only about 50 are found in Australia. Conversely Australia has a large number of characteristic Birds, belonging to families wholly unrepresented in New Zealand, such as the Birds of Paradise and Bower Birds (Paradiseid(e), Cockatoos (Cacatuidce), Mound-makers (Megapodiider), the Lyre-Bird (Menura), the Emu, and the Cassowary. Among Reptiles, Sphenodon, i.e., the entire order Rhynchocephalia, is endemic in New Zealand, as also is the little Gecko Naultinus, while a small genus of Lizards, Lygosoma, is common to the two countries. Australia, on the other hand, besides possessing a large number of Lizards, such as the Monitors, is remarkable for the great number of its Snakes, a group quite unrepresented in New Zealand, and has two species of Crocodiles and several of Chelonia. Among Fishes, the presence of Ceratodus in Australia is specially remarkable. The fresh-water Crayfishes of each country are endemic, those of New Zealand belonging to the genus Paranephrops, those of Australia to Astacopsis, Cheraps, and Engaus. The majority of the Australian Earthworms belong to the families Perichatidee and Cryptodrilide, the latter including the Giant Earthworm of Gippsland (Megascolides); the Megascolecida are represented, but are not dominant, as in New Zealand.

Thus, while the zoological resemblances between Great Britain and the Continent of Europe are so close as almost to amount to identity, there is more difference, zoologically speaking, between New Zealand and Australia than between Europe and North America, or between England and Japan. 
The reason of this remarkable contrast is not far to seek. Geologieally speaking, Great Britain is a reeently detaehed portion of Europe, having been united with it during the latter part of the Glacial period (Pleistoeene), and, at the present moment, an elevation of the bed of the English Channel to the amount of 260 feet would bring about a re-union (Fig. 1251). Prior to this

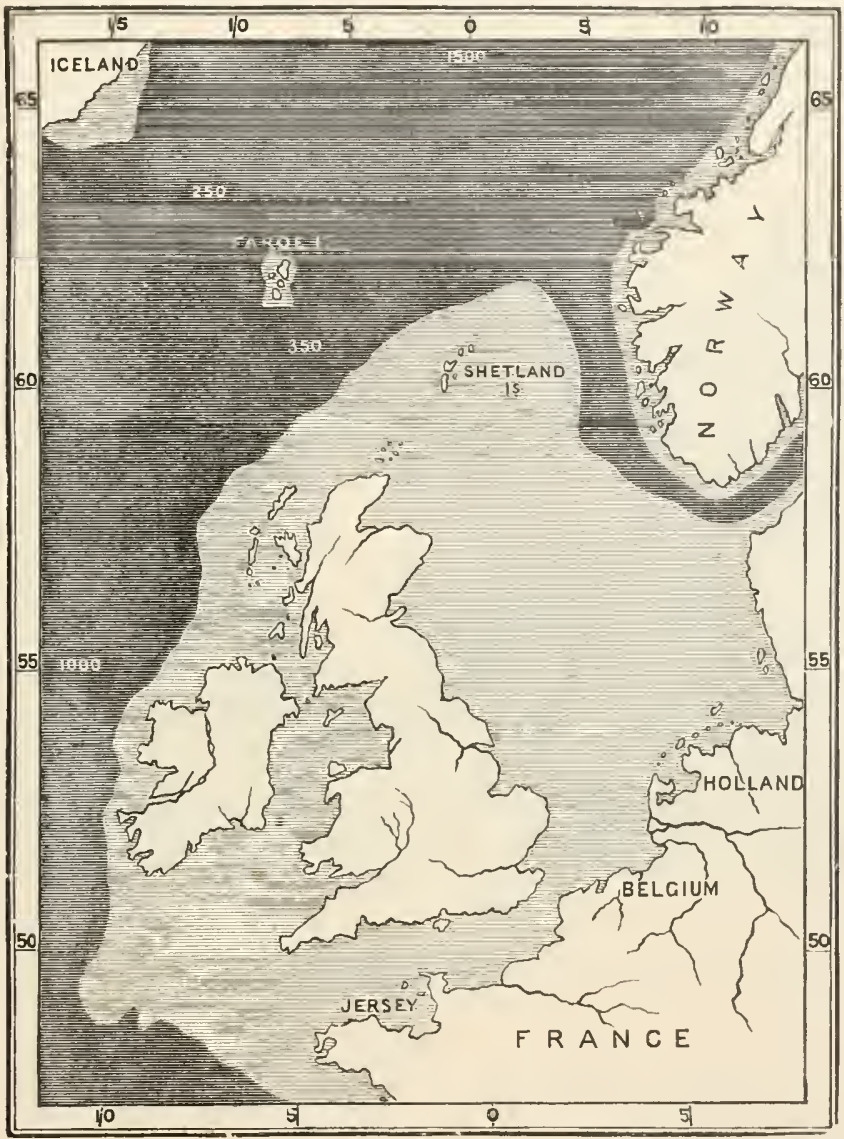

Fro, 1251.-Map showing the shallow bank connecting the British 1sles with the continent. The light shade inlicates a depth of less than 100 fathoms; the figures show the depth in fathoms. (From Wallace.)

union, moreover, it was largely submerged, so as to leave no traee of its Plioeene fauna. Thus, the British animals are all migrants from Europe, isolated by the post-glaeial separation from Europe, and the absenee of eertain European forms is due to the faet that the separation took place too early to allow of eomplete migration.

New Zealand, on the other hand, instead of being separated from 
the nearest continent by 21 miles of shallow sea, is divided from Australia by 1,000 miles of ocean-the Tasman Sea-varying from 2,000 to 2,600 fathoms $(12,000-15,600$ feet) in depth. It is almost certain that there was never any direct connection between the two countries, and the only indication of even an indirect connection is afforded by the existence of an area of comparatively shallow sea-i.e., under 1,000 fathoms-stretching between the North Island of New Zealand on the one hand and Northern Australia and New Guinea on the other (Fig. 1252). It would take, therefore, an upheaval of over 6,000 feet to join the two countries, and it may be taken as certain that if there ever was a

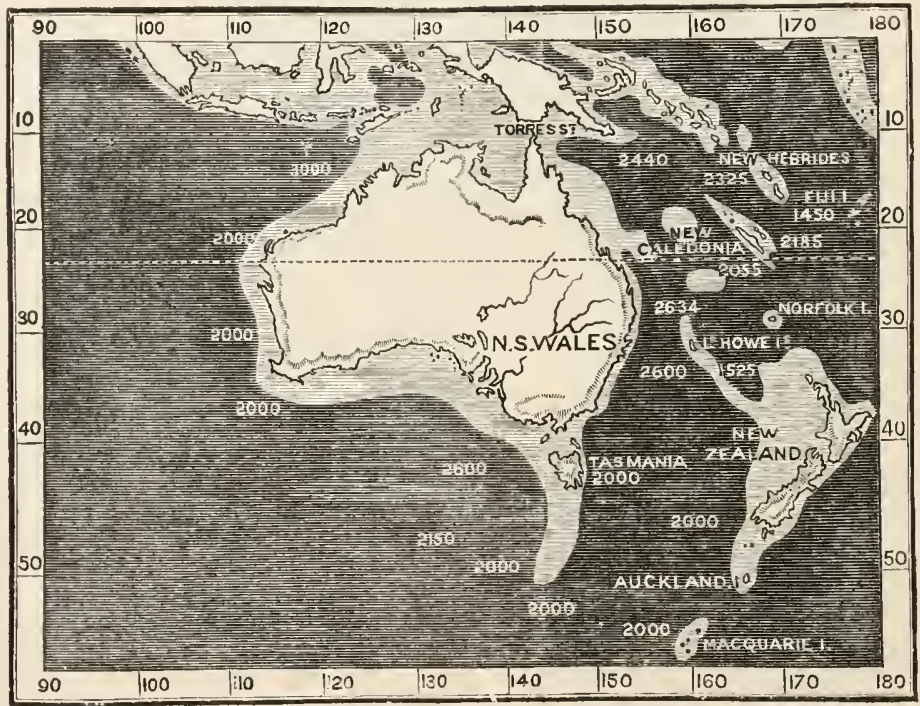

FIG. 1252.-Map showing depths of sea around Australia and New Zealand. The light shade indicates a depth of less than 1,000 fathoms; the dark shade indicates a depth of more than 1,000 fatlioms. (From Wallace.)

direct connection, either by continuous land or by a chain of islands, such connection could not have been later than the early part of the Mesozoic era.

It must also be noted that while the British fauna is related exclusively to that of Europe, the New Zealand fauna presents not only Australian but also Polynesian and South American affinities. Some of the Birds are represented by the same or closely allied species in New Caledonia, while the land Molluses and Insects, the Fresh-water Lamprey (Geotria), and the Earthworms, show South American affinities. In this connection it is interesting to find that there is a submerged bank of less depth than the surrounding ocean-under 2,000 fathoms-passing west- 
wards from South America, and including many of the Pacific Islands; and an area, also of less than 2,000 fathoms, in the Antarctic Ocean, sending offshoots northwards. The first of these may possibly indicate a former westward extension of South America, the second a former Antarctic land-area, perhaps more or less directly connected with the existing southern continents. The whole question is quite unsettled and extremely obscure, and is complicated by the fact that in one respect the New Zealand fauna shows Ethiopian affinities. There have been discovered in the Chatham Islands, a small group about 400 miles to the east of New Zealand, the remains of a long-beaked Rail (Diaphorapteryx), evidently not long extinct, the nearest ally of which is the Red Bird (Aphanapteryx) of Mauritius, known to have been exterminated by human agency. Moreover, the great Ratite Birds, the Epyornithidæ, of Madagascar show undoubted affinities with the Dinornithidæ.

The foregoing comparison of the faunæ of Great Britain and New Zealand leads us to the consideration of certain fundamental conceptions of zoo-geography.

Insular Faunæ.-We notice, in the first place, the striking contrast between the fauna of an island which has been recently detached from a great continental area and that of an island which has remained isolated for an immense and unknown period. In the one case the fauna has a strictly continental character, there having been insufficient time for modification since the separation took place. In the other case immigration has taken place from various sources over a vast period of time, during which modification has taken place to a sufficient extent to give rise to new or endemic species.

Habitat, Range, and Station.--Each kind of animal has, as a rule, its own habitat, fresh-water in one case, the sea between tide-marks in another, marsh, forest, snow-clad peaks, and so on. A similar habitat may characterise whole genera and even orders. Keeping always to its own habitat, the range of an animal may extend over a vast area. The marsh-loving Curlew, for instance, is found all over the world; the Cormorants (Phalacrocorax), Gulls (Larus), some Ducks (Anas), \&c., are also cosmopolitan or worldwide in distribution. On the other hand, the range of a species, genus, or order may be restricted to a single limited district. The genus Liopelma (New Zealand Frog) is found only in certain very limited areas; the species Salmo killinensis (Loch Killin Char) occurs only in Loch Killin in Inverness-shire; the Order Rhynchocephalia is confined to New Zealand. The entire range may be broken up, as it were, into a number of stations, depending sometimes on habitat, sometimes on unknown causes; the Tuatara, for instance, is found at present only in some half- 
dozen small islets, each of which is a station, while the whole of them constitute the range of the species.

Barriers.-A barrier in zoo-geography implies any permanent obstacle to the dispersal of a species. For instance, the Tasman Sea is an impassable barrier to the passage of most animals between Australia and New Zealand, strong-flying birds being the only species able to cross it. On the other hand, the number of Birds able to pass so narrow a strait as the British Channel is very considerable, while still narrower arms of the sea may be crossed by a large proportion of Mammals, Insects, \&c. Thus barriers are of unequal importance according to the particular animals under discussion; wide deserts and lofty snow-covered mountain ranges are impassable to most species; to some even a narrow river or arm of the sea is insuperable.

Means of Dispersal.--Most Mammals and many Reptiles can swim across rivers and arms of the sea if not too broad; thus narrow straits and rivers are of no significance as barriers to the dispersal of many animals. On the other hand, rivers of even moderate breadth are insuperable barriers to Monkeys, which are unable to swim.

Aërial animals can overcome many of the barriers impassable to flightless forms. Birds of strong flight often pass over immense stretches of ocean. For instance, a Cuckoo (Eudynamis taitensis) habitually winters in Fiji and other Pacific islands, and spends its summer in New Zealand, traversing the enormous distance of 1,500 miles twice a year. Many Insects also are able to fly great distances, especially when carried by gales.

Mechanical dispersal is an important factor in the case of many animals. Small Crustacea and Molluses may be carried great distances in the mud adhering to the feet of Birds. Infusoria, the eggs of Rotifers, and other microscopic forms may be transported in the dried condition by wind. Birds and Insects are frequently blown out to sea and carried for immense distances, and Mammals, Reptiles, \&c., may be widely distributed by being carried on driftwood, or on floating islands or "rafts" formed of large masses of matted vegetation, such as are often detached by storms in the tropics. Finally, the dispersal of many fixed or shore-haunting animals is ensured by their free-swimming larvæ.

Importance of the various Groups of Animals in ZooGeography.--In close dependence on the means of dispersal we have the fact that the various groups of animals are of very unequal value in the study of distribution. The greater the facilities for the transport of any species across a given barrier, the less significance will attach to its occurrence on both sides of the barrier. Conversely, when a species, having few or no facilities for dispersal, is found on opposite sides of an important barrier, the natural conclusion is either that the barrier is of comparatively 
recent formation, and that the two areas separated by it were once, so to speak, in zoological continuity, or that the species in question is a very ancient one, and was widely dispersed at a time when the arrangement of the land-surface was very different from what it is at the present day. For instance, the occurrence of strong-flying Birds, such as Gulls and Cormorants, in widely separated countrie. is a fact of no significance in determining the mutual relationships of the faunx of those countries. But the occurrence of the same species of Fresh-water Crayfish-to which the narrowest arm of the sea is an insuperable barrier-in Great Britain and the European Continent is explained only by the fact-of which there is independent evidence-that the English Channel is of recent formation. And when we find the various species of Peripatus dotted over the earth's surface in an apparently casual manner, we are forced to the conclusion that this genus must formerly have been very widely and continuously distributed and subsequently exterminated over the greater part of its range; since it is hardly possible to conceive of either the adult or the young of this creature, living in rotten wood in the recesses of the forest, having been transported between Australia and New Zealand, or between Africa and the West Indies.

Speaking generally, then, it may be said that discontinuity in the distribution of a species or other group is evidence of its antiquity. In addition to Peripatus, the Dipnoi and the Tapirs may be mentioned as examples.

It will be seen that terrestrial and fresh-water animals are of more importance, from the point of view of zoo-geography, than marine forms. Among the inhabitants of the sea, littoral species are of greater significance than pelagic or abyssal. Amongst land animals, those which are unable to swim, and those which cannot survive immersion in salt water, are of more importance than strong swimmers, or than such forms as are able to live for a prolonged period on driftwood, or in mud attached to the feet of Birds.

In connection with what has been said above about there being no special significance to be attached to the distribution of certain strong-flying Birds, it must be remarked that this is by no means true of migratory Birds. Many British Birds, such as the Swallow, Cuckoo, Swift, \&c., spend the summer in England, the winter in South Europe or Africa. One of the New Zealand Cuckoos winters in Australia, the others in Fiji or some other Pacific islands. Birds capable of such feats of flight might, one would think, soon overspread the globe; yet, as a matter of fact, each species is found to keep strictly to its own definite line of migration, even across 1,000-1,500 miles of sea.

Having now indicated the general character of the facts and problems connected with the subject of zoo-geography, we may 
proceed to give some account of the Zoo-geographical Regions into which the land-surface of the earth is divided (see Fig. 1253). It must be borne in mind that the determination of these regions depends largely upon the classes of animals upon which stress is laid, the peopling of any given portion of the earth by a particular class depending upon the time during which it has been in existence and its means of dispersal. Thus regions founded upon the distribution of Mollusca will differ from those depending on Reptiles or on Birds. The regions adopted here. are mainly founded on the distribution of Birds and Mammals.

The whole of Europe, Africa, and Arabia north of the Tropic of Cancer, and the whole of Asia except India, Burmah, Siam, and South-East China, together with Japan, Iceland, the Azores, and the Cape de Verde Islands, are so similar in their animal productions as to form a single division of the earth's surface called the Palæarctic Region. This region is bounded on the nortl, west, and east by ocean, but its southern limits are at first sight less obvious. It appears strange, for instance, that Northern Africa and Arabia should be included in this region, the Mediterranean being, as it were, ignored as a boundary. But the facts show that the great line of sandy deserts in the region of the Tropic of Cancer, the Sahara in Africa, and Roba el Khali in Arabia, form a far more efficient barrier to the dispersal of species than the Mediterranean, and it is probable that there- was direct land connection between Europe and North Africa during the Pleistocene period. In Asia the Himalayas form an effective barrier, which has existed since Tertiary times, between Thibet and India; an ill-defined line of country following the course of the Indus continues the boundary south-west to the shores of the Arabian Sea; and another ill-defined area passing south of the Yang-tseKiang, and travelling northward to Shanghai, constitutes the eastern end of the southern boundary of the region.

None of the larger groups of animals, no orders or even families, are absolutely confined to this region, the characteristics of whicl it is difficult to define without descending to genera and species. 'The Moles (Talpidae), Sheep and Goats (Ovidce), and Dormice, the Pheasants, Robins, Magpies, and many other Birds, are highly characteristic, and many species of Deer, Oxen, and Antelopes, Rodents, Passerines and other Birds, Reptiles, Amphibians-including Proteus-and fresh-water Fishes, are endemic.

The Palæarctic region includes, as we have seen, nearly all the northern portion of the eastern hemisphere; the corresponding part of the western hemisphere, viz., North America, with Greenland, constitutes the Nearctic Region. It also is bounded by the ocean on its northern, eastern, and western sides, while in the south an ill-defined tract of country, passing between Cape San 
Lucas on the west and the Rio Grande del Norte on the east, separates it from the Neotropical region.

The Nearctic differs from the Palæarctic region in the possession of several characteristic Mammals, such as Opossums (Didelphyidae), the Skunk, Racoon, \&c.; many Birds, such as the Blue-jays, and 'Turkey-buzzards, \&c.; Reptiles, such as Rattlesnakes and Iguanas; Amphibians, including the Axolotl, Necturus, Siren, and other large Urodeles; and numerous fresh-water Fishes. including Amia, Lepidosteus, Polyodon, and Scaphirhynchus. Only three entire families are endemic, two of Rodents, and one of Passerines.

On the other hand, the resemblances between the two northern regions are very close. Both possess Wild Cats, Hyænas, Foxes, Weasels, Bears, Elk, Deer, Wild Oxen, Beavers, Voles, Squirrels, Marmots, and Hares, the species of the one region being all closely allied to, and sometimes identical with, those of the other. Thrushes, Wrens, Tits, and Finches are also common to the two regions, and, generally speaking, the differences between them are, as we shall see, nothing like so striking as those between either of them and the region or regions bounding it to the south. Hence the Palæarctic and Nearetic regions are sometimes grouped together as a single Holarctic Region.

In the southern regions the characteristic features are much more striking. The Ethiopian Region is constituted by the whole of Africa and Arabia south of the Tropic of Cancer, together with Madagascar, Mauritius, Bourbon, Rodriguez, and the Seychelles. The region is bounded by sea on the west, south, and east, but on the north it is perfectly continuous with the Palæarctic region, and it certainly seems a very remarkable fact, until we remember what an impassable barrier is afforded by a sandy desert of great extent, that there should be more difference between the faunæ of northern and central Africa than between those of England and Japan, or of Alaska and Florida.

Among the animals most characteristic of the Ethiopian region and not found elsewhere are the Gorilla, the Chimpanzee, several Baboons, and the large majority of Lemurs, including the curious Aye-aye (Chiromys); several peculiar Insectivora, such as the Golden Moles (Chrysochloride), and the River Shrew (Pot $\iota$ mogale); the African Elephant, the Hippopotamus, two or three species of Rhinoceros, the Zebras and Quaggas, the Giraffe and Okapi, and more than seventy species of Antelopes; the Aardvark (Orycleropus), one of the most singular types of Edentata; the Plantain-eaters (Musiphagidee), the Secretary Bird (Serpentarius), and many other families and genera of Birds: numerous snakes and other Reptiles, and several freshwater Fishes, including the Dipnoan Protopterus, and the Ganoid Polypterus. The Lion, Leopard, and Ostrich are also characteristic, 
although not actually endemic, since the two former extend into the Palæarctic and Oriental regions, whilc the Ostrich occurs in Arabia and Syria. Almost equally remarkable are the negative peculiarities of the region, and especially the absence of Bears, Deer, and Oxen, and the extreme paucity of Goats, Sheep, truc Pigs (Sus), and Shrews.

The great island of Madagascar is characterised by the immonsc number of Lemurs, the absence of Monkeys, and the poverty of its carnivorous and ungulate fauna, the Lions, Antelopes, \&c., of the African continent being all absent. Most of its Mammals are endemic, only three out of twenty-eight (including Bats) being found in Africa. The Birds, also, are quite different from those of the African continent. It shows affinities with America in the presence of a peculiar family of Insectivora (Centetida), otherwise found only in the West Indies, and of certain Snakes; and its relationships with India are so marked that it has been proposed to account for them by assuming the former existence of a land connection, in Jurassic and Cretaceous times, extending northeastward across the Indian Ocean and represented at the present day by the Seychelles and other neighbouring islands. In the opinion of some authorities these peculiarities entitle Madagascar and the adjacent islands to rank as a distinct zoo-geographical region.

The Oriental Region consists of India, Burmah, Siam, southeastern China, and certain islands of the East Indian Archipelago, including Sumatra, Java, Borneo, and the Philippines. As we have seen, it is separated from the Palæarctic region by the Himalayas, continued on the west by a tract of country following the course of the Indus, and on the east by a region curving at first southwards and finally northwards to Shanghai. The southeastern boundary is an imaginary line, known as Wallace's line, which passes between the small islands of Bali and Lombok, then through the Straits of Macassar, between Borneo and Celebes, and finally to the east of the Philippines. The islands to the northwest of this line-conveniently distinguished as the Indo-Malayan Islands-belong to the Oriental region, those to the south-eastthe Austro-Malayan Islands - to the Australian region. Curiously enough, the zoological differences between the two groups of islands are morc marked between Bali and Lombok, separated by a deep channel of only about twenty miles in width, than between Borneo and Celebes, separated by the wholc width of the Straits of Macassar.

The most characteristic members of the Oriental fauna are the Orang-utan (Simia), the Gibbons (Hylolates and Siamanga), and numerous Lemurs; the Tiger, which, however, extends into the Palæarctic region, and several Bears and Civets; the Indian Elephant, the Indian Tapir, three species of Rhinoceros, and the 
Chevrotains or Mouse-deer (Tragulida); and several large and handsome Gallinaceous Birds, such as the Peacock, Argus Pheasant, and Jungle-fowl. The resemblances to the Ethiopian Region are numerous and striking, among the most important being the presence of the Elephant, Rhinoceros, the higher Apes, Lemurs, and Manis. On the other hand, the presence of Deer and Bears furnishes a characteristic difference.

The Australian Region includes Australia, Tasmania, and the Austro-Malayan Islands as defined above, from Celebes and Lombok on the west to the Solomon Islands on the east, the most important of them being the immense island of Papua or New Guinea. New Zealand and Polynesia are very generally included in this region, but it is more convenient, on the whole, to treat them apart.

The most striking feature of the region is the almost total absence of Eutheria, the Manmalian fauna belonging mainly to the Marsupials and Monotremes. The last-named order is entirely confined to this region, while Marsupials occur elsewhere only in America. The only exceptions are the Dingo or Australian Wild Dog, which is probably indigenous, the universally distributed groups of Rats, Mice, and Bats, and, in some of the islands bordering on the Oriental region, Deer, Civets, and Pigs. The abundance of Marsupials is very remarkable, all the leading groups of that sub-class, with the exception of the Didelphyidæ, or American Opossums, and Canolestes, being strictly endemic.

Equally striking is the number and peculiarity of the endemic Birds, the most important of which are the Emus and Cassowaries, the Mound-makers or Brush Turkeys (Talegallus, \&c.), the Birds of Paradise and Bower-birds, the Lyre-bird (Menura), the Cockatoos and Brush-tongued Lories. The great number and variety of Parrots, Kingfishers, and Pigeons is also a marked feature, as also is the absence of Pheasants, Woodpeckers, Finches, and other Birds abundant in the Oriental region. Snakes, Lizards, and Frogs are abundant, and in the rivers of Queensland occurs Ceratodus, one of the three existing genera of Dipnoi.

The New Zealand Region comprises the three islands of New Zealand (North, South, and Stewart's Islands), together with Norfolk, Lord Howe, and the Kermadec Islands to the north, the Chatham Islands to the east, and the Bounty, Antipodes, Auckland, Campbell, and Macquarie Islands to the south.

The characteristies of the New Zealand fauna have already been dealt with in some detail. The total absence of land Mammals, with the exception of two Bats and a Rat, the latter probably introduced; the large proportion of endemic Birds, many of 
which are flightless; the exclusive possession of more than half the known genera, and of a large majority of the species of Ratitx, and of the entire order Rhynchocephalia; the total absence of Ophidia, Chelonia, and Crocodilia; the paucity of Lacertilia and the almost total absence of Amphibia; all these faunal characters combine to make New Zealand one of the best marked and most peculiar tracts on the earth's surface.

One or two facts must be mentioned with regard to the smaller islands of the region. In Norfolk Island there existed until recently a flightless Rail, Notornis alba, belonging to a genus the only other species of which lives or lived in New Zealand. In Phillip Island, close to Norfolk Island, Nestor productus formerly occurred, a member of an endemic New Zealand family of Parrots. In Lord Howe Island there is a species of the endemic New Zealand flightless Rail Ocydromus. These three facts all point to a former partial or complete land connection between New Zealand and the islands in question. The remaining islands are closely related to New Zealand, but with greatly impoverished faunæ. In Macquarie Island, the southernmost land outside the Antarctic circle, there has recently been discovered an Earthworm with distinct South American affinities.

The Polynesian Region embraces the numerous groups of islands lying within the tropics to the east and north of the Austro-Malayan Islands. The most important groups are New Caledonia, the New Hebrides, Fiji, the Friendly Islands, Samoa, the Society Islands, and the Sandwich Islands. They are all typical oceanic islands, that is, they are of volcanic origin, have no stratified rocks, and show no indication of former connection with any continental area.

In correspondence with their isolated position, the faunæ of these islands, although exhibiting great variety from one group to another, all agree in the absence of Land Mammals, except Bats, and-with one or two exceptions- of Amphibians, in the small total number of species, and in the very large proportion of endemic species. The islands have evidently been peopled by waifs and strays from other lands, at periods so remote that most of the immigrants have assumed the character of distinct species, or even, especially in the isolated Sandwich Islands, of distinct genera.

On the whole, the affinities of the Polynesian fauna are distinctly Australian; they present, however, certain American characteristics, especially in the occurrence of Lizards, belonging to the American family of the Iguanidx, in Fiji. Amongst the most notable endemic forms are the Dodo-like Pigeon, Didunculus, in Samoa; the Kagu (Rhinochetus), a remarkable genus of Grallæ, in New Caledonia, and the Drepanidoe, a family of Passerines allied to the American Greenlets, in the Sandwich Islands. Polynesia 
cannot be said to form a well-defined region, the islands composing it being united largely on the ground of convenience.

In the Neotropical Region we have once more an immense tract of land, presenting such well-defined faunal characteristics as make it one of the best-marked of all the zoo-geographical regions. And this in spite of the fact that it is in free connection with the Nearctic region, the two being separated by an ill-defined transition-region formed by the northern part of Mexico. The Neotropical region includes, therefore, the tropical part of North America, as well as the whole South American Continent, the Antilles or West Indies, the Galapagos Islands, the Falkland lslands, and Juan Fernandez. Both geological and zoological evidence points to a complete separation of the two Americas during the Miocene and Pliocene periods.

The endemic animals of the region are very numerous and characteristic. They include, among Mammalia, the Prehensiletailed Monkeys (Cebidac) and the Marmosets (Hapalidce) ; the Chinchillas and Cavies, two peculiar families of Rodents; the Jaguar; the Llamas and Peccaries, and a species of Tapir; the Sloths, Armadillos and Ant-eaters-three entire families of Edentata. The Opossums (Didelphyida) are also very characteristic, though not actually endemic, since they extend into the Nearctic region. A single additional Marsupial (Canolestes) of uncertain affinities has been found in the extreme south. Among Birds the chief endemic forms are the three species of Rhea, constituting the entire order Rheæ; the Tinamous, forming the order Crypturi; the Toucans, Screamers, Oil-bird (Steatornis), Hoatzin (Opisthocomus), and many others. The Humming-birds, although extending into the Nearctic Region, are a characteristic group. Boas, Rattlesnakes, Iguanas, Crocodiles, and Caimans are abundant, and among the fresh-water Fish are the Electric Eel (Gymnotus), and Lepidosiren, one of the three existing genera of Dipnoi.

The negative characteristics of this region are also very remarkable. Except in Central America and the West Indies, there are no Insectivora; Civets, Oxen, Sheep, Antelopes, and true Swine $($ Suinc) are altogether absent, and there are very few species of Deer ; Crows and Ravens are also practically unrepresented.

In the West Indies there are no Edentata, Monkeys, or Carnivora, and there occurs a peculiar Insectivore, Solenodon, belonging to the Centetidæ, otherwise found only in Madagascar. The Galapagos Archipelago, a group of oceanic islands, about 600 miles to the west of the continent, has at the most two Mammals, a Bat and a Mouse; its Birds are very different from those of the mainland, and include many endemic species; and among the Reptiles are the gigantic Tortoises (Testudo), of which different species occur in the various islands. 
The general relations of the zoo-geographical regions may be expressed in a diagrammatic form as follows:- -

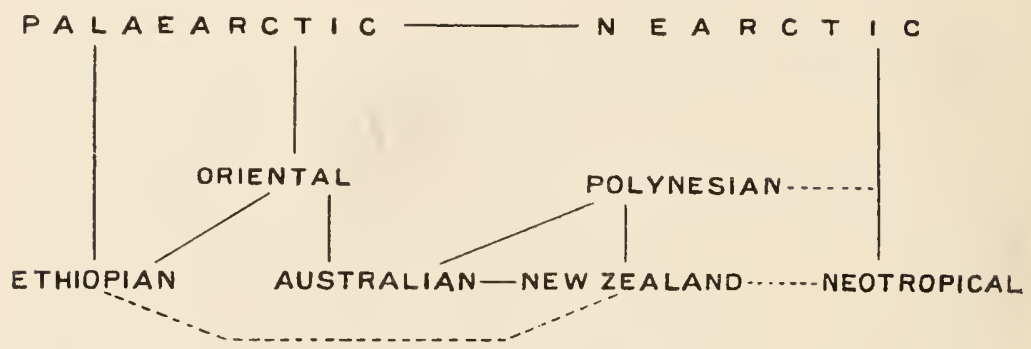

1F. 1253.-Diagram showing the general relations of the zoo-geographical regions.

\section{Bathymetrical Distribution.}

The foregoing pages have given a brief sketch of the facts connected with geographical or horizontal distribution. We now turn to bathymetrical or vertical distribution - the facts concerning the distribution of animals at various depths of the sea or of lakes, and at various heights of the land.

The region of greatest abundance of marine life, as regards both the number of genera and species and of individuals, is the littoral or shore-region. The rocks left dry by the retreating tide, the rock-pools exposed at low water, and the forests of kelp at the limit of low tide or a few fathoms below possess an extraordinarily rich and abundant fauna, including all the Calcareous Sponges and a large proportion of the remaining groups, Hydroid Zoophytes, Sea-anemones and Corals, Echinodermata, Turbellaria, Nemertinea, Polychrta, Polyzoa, Brachiopoda, decapod Crustacea, Pelecypoda, Gastropoda, Octopoda, and Teleostei. Numerous examples of other groups-Protozoa, the lower Crustacea, Insecta, and Elasmobranchii-are also littoral, and Penguins, Seals, and Sirenia may be included in the list.

Next in abundance to the littoral is the pelagic or oceansurface fauna, including animals which live habitually on the surface or at slight depths of the ocean, often far from land. Amongst them are many Foraminifera, such as Globigerina and Hastigerina, the Radiolaria, the Siphonophora, the majority of Medusæ, both hydrozoan and scyphozoan, nearly the whole class of Ctenophora, many Entomostraca and Schizopoda, the hemipterous Insect Halobates, the Pteropoda, Heteropoda, and some other Gastropods, such as Glaucus, most Cephalopods, Pyrosoma and the Salps, numerous Teleosts, such as Herrings, Flying-fish, Mackerel, \&c., the greater number of Sharks, and the majority of Cetacea. 
The pelagic Invertebrates are mostly distinguished by great transparency, and by being either colourless or of a blue or violet hue. Pelagic Fishes are usually grey or steel-blue above, white beneath, presenting none of the brilliant colours, varied markings, and extraordinary forms so often found among Shore-fishes.

It must be remembered that many littoral animals are pelagic in the larval condition, or during some phase of their life-history, e.g., many Sponges, fixed Hydrozoa and Actinozoa, Echinodermata, Annulata, Mollusca, Crustacea, and Fishes.

The abyssal or deep-sea fauna is far more abundant than might be supposed from the physical conditions-immense pressure and absence of light and of vegetation. In most parts of the world the bed of the ocean, at depths from 400 to 2,000-2,500 fathoms, is formed of a greyish mud called globigerina-ooze, consisting largely of the shells of Foraminifera, such as Globigerina, Orbulina, \&c., which have for the most part sunk to the bottom after death. Below 2,500 fathoms the sea-bottom is formed of a red clay, in which shells are absent, having apparently been dissolved during their descent to the greater depth.

Living on the sea-bottom, and most abundant on the globigerinaooze, are representatives of many groups of animals: Sponges, especially Hexactinellida; a few Medusæ and Corals; examples of all classes of Echinoderms, Stalked Crinoids and Holothurians being especially abundant; Crustaceans, particularly Schizopods and Prawns; and Teleosts. Crabs, Molluscs, and Annulates are rare.

Many abyssal animals are blind, including several of the Crustacea; many others are phosphorescent, and thus supply their own light in an otherwise dark environment. The deep-sea Teleosts are often of very grotesque appearance, with immense heads, wide mouths furnished with long, pointed teeth, extremely distensible stomachs, and phosphorescent organs arranged in rows along the body (see Fig. 894). Other forms, such as the Ribbonfish (Regalecus), attain a great size, and are toothless. When brought to the surface, the expansion of the gases in the interior of the deep-sea Teleosts often bursts the air-bladder, and produces a general disintegration of the tissues.

Plankton, Nekton, and Benthos.-Besides being arranged with regard to their relations to the shore, the surface of the ocean, and its bed, marine animals are also conveniently classified on the basis of their capacity for movement. Nany forms, such as Medusæ, Siphonophora, Ctenophora, Salps, and numerous pelagic larvæ are carried along passively by oceanic currents, their own powers of progression being of the feeblest. Such animals together constitute the Plankton, or "drifting-fauna." Others swim actively by means of fins or other appendages, such as the pelagic Teleosts and Elasmobranchs, Schizopods, Prawns, and 
Squids-they form the Nekton, or "swimming-fauna." Others, again, have no natatory organs, and are either permanently fixed, like Zoophytes and Stalked Crinoids; or move by creeping over the sea-bottom, like Starfishes, Holothurians, Chætopods, \&c..; such forms constitute the Benthos, or " bottom-fauna."

The Fresh-water Fauna presents certain characteristic features, and is divisible into fluviatile forms, inhabiting streams and rivers, and lacustrine forms, inhabiting lakes. It is very rich in Lobosa, Heliozoa, Flagellata, and Infusoria, but contains very few Foraminifera and no Radiolaria. Among Sponges there is only a single fresh-water family, the Spongillidæ : among Hydrozoa only four genera, Hydra, Cordylophora, Limnocodium, and Limnocnida ; and among Actinozoa and Ctenophora not a single species. There are also no fresh-water Echinoderms or Brachiopods, but many Turbellaria, a few Nemertinea, and numerous Nematoda. Among Polyzoa one genus of Endoprocta, the whole of the Phylactolæmata, and one or two genera of Gynmolæmata are fresh-water forms; so also are many of the Oligochæta, e.g., Nais and Tubifex, but very few Polychæta. Fresh-water Entomostraca are numerous and abundant, and belong to all orders except Cirripedia; among Malacostraca there are only some Amphipods and Isopods, Anaspides and its allies, and Fresh-water Shrimps, the various genera of Fresh-water Crayfishes, and a few Crabs. The larvæ of many Insects are aquatic, and there are several aquatic Spiders. Pelecypods and Gastropods furnish abundant fluviatile and lacustrine forms, although belonging to comparatively few genera; Cephalopods, on the other hand, are wholly absent from fresh waters, as also are the Tunicata. Among Fishes there are several species of Lampreys, and numerous Teleosts, the Siluroids and Salmonidæ being especially characteristic. There are no fresh-water Elasmobranchs, with the exception of one or two genera of Sting-Rays in the rivers of tropical America; but the Ganoids are a characteristic fresh-water group, although some forms, such as the Sturgeons, migrate to the sea at certain seasons. The Dipnoi are exclusively fluviatile, or live in swamps caused by river overflow, and the perennibranchiate Amphibia, as well as the larvæ of the caducibranchiate forms, are characteristic members of the fresh-water fauna. Many Chelonians and Crocodiles, such Birds as Ducks and Grebes, and such Mammals as Otters, the Hippopotamus, and Ornithorhynchus, may also be included in the fresh-water fauna, and some Dolphins are purely fluviatile.

The animal inhabitants of large lakes, like those of the sea, are divisible into littoral, pelagic, and deep-water forms: the pelagic forms are, in this case also, characterised by their extreme transparency. Mention must also be made of animals dwelling in deep subterranean caves, shut off from sunlight, such as Proteus, 
the blind Urodele of the caves of Carniola, the blind Fish (Amblyopsis spelceus) of the Mammoth caves of Kentucky, numerous Insects, \&c. These, like abyssal species, are blind, and usually colourless, and are obviously specialised derivatives of the ordinary fresh-water or land fauna.

In the Terrestrial Fauna, also, we find certain groups preponderant, others absent or nearly so. A terrestrial Amœeba has been described, and the Mycetozoa are all terrestrial, but no other Protozoa, nor any Sponges, Cœlentrates, or Echinoderms. Among Platyhelminthes we have the numerous species of Land-Planarians and the Land-Nemertines, and among Chætopods nearly the whole of the Earthworms. Several Crustacea are more or less completely adapted to terrestrial life, such as the Woodlice, Landcrabs, Cocoa-nut Crab, and Burrowing Crayfish. The Onychophora and Myriapoda are characteristic land-animals, so also are most Arachnida and many Insects. Among the Mollusea the only terrestrial forms are the majority of pulmonate Gastropoda. Among Fishes the Climbing Perch, Periophthalmus, and some others are imperfectly adapted to life on land, and the Caducibranch Urodeles, the Anura, and the Gymnophiona are all terrestrial or semiterrestrial. The Lacertilia, Sphenodon, the majority of Snakes, and the Tortoises are land-animals, and so also are many Birds, including all the Ratitæ, the Crypturi, Gallinæ, \&c., and the vast majority of Mammals.

Among terrestrial animals, those which habitually live on the open ground must be distinguished from arboreal forms, such as Tree-Kangaroos, Sloths, and Monkeys, which pass their lives among the branches of trees, and from cryptozoic forms, which live under stones, logs of wood, \&c., such as Land-Planarians, Peripatus, Centipedes, and Woodlice.

Lastly, we have the Aerial Fauna, including animals capable of sustaining themselves for an indefinite period in the air, such as most Insects, the large majority of Birds, and Bats. The Flying Fishes, Flying Dragons (Draco), Flying Phalangers, Flying Squirrels, and Flying Lemur (Galeopithecus) are semi-aerial.

The majority of land-animals live at or near the sea-level, and as we ascend mountains the fauna undergoes a gradual impoverishment as the snow-line is approached. The higher ranges of all great mountains have a characteristic Alpine Fauna. In the European Alps, the Chamois (Rupicapra), Alpine Hare (Lepus variabilis), and Marmot (Arctomys marmot) may be specially mentioned; in the Himalayas, Yaks (Poephagus), Musk-deer (Moschus), Goats and Ibexes (Capra), besides abundant Birds and Insects; in the Andes, the Condor (Sarcorhamphus); in the New Zealand Alps, the rapacious Kea or Mountain Parrot (Nestor notubilis). 


\section{Geological Distribution.}

In considering the distribution of animals in past time, we are met at the outset with the difficulty that our knowledge of the subject is, and must always remain, very imperfect and fragmentary. With few exceptions, only calcified, silicified, or strongly chitinised parts are preserved in the fossil state, so that whole classes of animals are absolutely unknown in that condition, and of the rest our whole information depends upon the more or less imperfect skeleton. Moreover, it is only under very favourable circumstances that even the hard parts are preserved; the chances are usually in favour of the animal being devoured or disintegrated before there is time for it to be silted over with mud or sand. And, lastly, many rocks have been so altered by the internal heat of the earth as to destroy any organic remains they may once have contained. Thus, while palæontology furnishes us with the only sure test of phylogenetic speculation, it is a test which, more often than not, is incapable of application, owing to the extreme imperfection of many parts of the geological record.

It is in the oldest of the stratified rocks that this imperfection is most severely felt. In the Laurentian period, forming the base of the sedimentary series (see Vol. I., p. 7), no animal or vegetable remains are known. In certain Canadian serpentine rocks belonging to this period there is found a remarkable structure which, under the microscope, bears a certain resemblance to the supplementary skeleton, with its canal-system, of an immense Foraminifer. On the assumption that it was the fossilised remains of a member of this order, it was called Eozöon canadense, but later researches seem to have shown conclusively that the supposed fossil is of purely mineral origin. Radiolarians and Foraminifera have been described from the Pre-Cambrian rocks of Brittany, but the nature of the bodies in question has not yet been established beyond dispute.

There are thus no undoubted fossil animals until the Cambrian period, where many existing groups appear to start suddenly into being. We find Radiolaria, Sponges, Graptolites, Polyzoa, Brachiopoda, Edriasteroidea, Carpoidea, Asteroidea, Chrotopoda (worm-tubes), Phyllocarida, Ostracoda, Trilobites, the generalised Insects known as Palæodictyoptera, iso- and heteromyarian Pelecypods, Gastropods (Prosobranchs and Pteropods), and tetrabranchiate Cephalopods (Orthoceras, \&c.) -all, it will be noticed, marine forms, with the exception of Insects.

Proceeding a stage onwards we find in the Silurian period, in addition to the above groups, Foraminifera, Actinozoa (rugose Corals), Ophiuroids, Echinoids, Crinoids, Cirripedes, Scorpions, Eurypterida, Amphineura, Scaphopoda, Elasmobranchii, and Ostracodermi. 
Thus, in the two earliest fossiliferous systems arc found representatives of all the skeleton-forming phyla, i.e., of all but Platyhelminthes, Nemathelminthes, and Trochelminthes. And, as far as our present knowledge goes, there is no indication of any connecting link between one phylum and another, the primary divisions of the animal kingdom having been apparently as wcll characterised at that enormously distant epoch as at the present day. Obviously all the older or more gencralised animal types, which, if we reason from analogy, we must suppose to have preceded the present well-marked phyla, have been destroyed by metamorphic action or otherwise, without lcaving a trace of their existence.

The Devonian period is remarkable for its abundant remains of Fishes; Crossopterygii, Chondrostei, and Dipnoi appear for the first time, and all three groups of Ostracodermi are abundant. Dccapod Crustacea, of the macrurous or Shrimp type, also make their appearance. In the Carboniferous period, notable for its immense forest-flora, there is a great development of airbreathing forms, such as Insects, Arachnids (Spiders), and Myriapods, as well as Stegocephala, the earliest amphibious Vertebrates. In the Permian rocks true air-breathing Vertebrates first make their appearance in the form of the reptilian orders Thermomorpha, Sauropterygia, and Rhynchocephalia. This period is also remarkable for the occurrence of Ceratodus, the oldest still existing genus of Vertebrates.

Thus by the end of the Palæozoic era, every important class of animals capable of leaving fossil remains is represented, with the exception of Mammalia and Birds. Moreover, the Trilobites, the Furypterida, the Palæodictyoptera, and the Ostracodermi come to an end during this era, no remains of them being known in rocks of secondary age.

In the succeeding Mesozoic era, the Triassic period introduces existing orders of Insects-Orthoptera, Neuroptera, and Coleoptera, as well as Xiphosura, siphoniate Pelecypods, opisthobranchiate Gastropods, and dibranchiate Cephalopods (Belemnites). The Palæozoic types of Tetrabranchs (Orthoceras, \&c.) have nearly disappeared, and the Ammonites have become important. Among Vertebrates are found Holostei, Chelonia, Ichthyopterygii, Crocodilia, and Dinosauria, the latter especially being a very prominent group, as well as several Mammalia (Microlestes, Hypsiprymnopsis, \&c.) of uncertain affinities.

In the Jurassic period the two highest orders of Insects, Hymenoptera and Iuepidoptera, are known for the first time, as well as the reptilian Ornithosauria, and the earliest known Bird (Archaopteryx). There are also several small Mammals (Plagiaulax, Amphitherium, Phascolotherium, \&c.) belonging either to the Prototheria or to the Metatheria, but occurring in Europe 
and North America, where there are at present-with the exception of the Opossums-no representatives of either order. This seems to indicate that the lower Mammals originated in the northern hemisphere and spread southwards.

In the Cretaceous period the Crabs-the most specialised of the higher Crustacea-and the Teleosts - the most specialised of Fishes-make their appearance. Of the last-named group, several Cretaceous genera survive and flourish to the present day, e.g., Clupea (Herring), Esox (Pike), Osmerus (Smelt), and Beryx. Ophidia are known for the first time, and Pythonomorpha, Dinosaurs, and Ornithosaurs are important. Mammals are practically unknown, but among Birds the Odontolcæ and the Ichthyornithes are characteristic. By the end of the period five entire groups of Reptiles - the Sauropterygia, Ichthyopterygia, Pythonomorpha, Dinosauria, and Ornithosauria-have become extinct, none of them being known to extend into Tertiary times.

Except in California and Patagonia there is a well-marked break between the Cretaceous and the Eocene periods, the fauna of the latter having a comparatively modern character. The Pelecypods and Gastropods belong to existing families and even to existing genera, and Belemnites have almost, and Ammonites quite, disappeared. The Fishes all belong to existing types; Stegocephala have given place to Urodela and Anura, and none of the Reptiles belong to extinct orders. Among Birds, the Penguins, Gulls, Rails, Owls, Picarians (Kingfishers, \&c.) and Passeres have appeared, as well as the extinct orders Stereornithes and Gastornithes, and the goose-like Odontopteryx.

But the most noticeable feature of the period is the rise and differentiation of the Mammalia. Among existing orders the Marsupialia (Opossums), Cetacea (Zeuglodon), Sirenia (Prorastomus, Eosiren), Ungulata, Carnivora, Insectivora, Chiroptera, and Primates (Lemurs) appear for the first time, as well as the extinct orders Creodonta, Condylarthra, Amblypoda, and Tillodontia, together with the Dinocerata, none of which extend beyond the Eocene period. In the lower Eocene none of the Mammals belong to existing genera, but in the upper Eocene are found Didelphys (Opossumı), Rhinoceros, Viverra (Civet), Mustela (Weasel), and possibly Canis. The period is also remarkable for the number of annectent or linking forms. There are, for instance, species connecting Dogs with Bears and with Civets, Civets with Hyænas, Hyænas with Cats, Pigs with Pecora, Deer with Chevrotains, Tapirs with Rhinoceroses and with Horses, and so on. It is perfectly clear that the orders, sub-orders, and families of Mammalia, as we now understand them, were, during the Eocene period, becoming gradually differentiated from common ancestral forms.

In the Miocene period the Proboscidea (Elephant and Mastodon) make their appearance, as well as Gibbon-like Anthropoidea 
(Pliopithecus, Hylobates and other genera), and some other Anthropoidea. Many existing families have arisen, such as Hedgehogs, Shrews, and Moles; Mice, Rabbits, and Porcupines; Whales and Dolphins; Tapirs, Hippopotami, Swine, and Antelopes; and species of Felis and Canis. The Rhinoceroses of the period still have no horns, and the antlers of the Deer are small or absent. The Tapir-like ancestors of the Equidæ found in the Eocene have developed into more Horse-like forms, and the ancestors of the Camels (Poëbrotherium) still retain upper incisors and distinct metacarpals. Numerous Marsupials lived in South America during this or the preceding period: many of these were small forms (Microbiotheridae) apparently allied to the living Canolestes; others (Sparassodonta ${ }^{1}$ ) were larger, carnivorous, with resemblances to the Tasmanian Thylacinus.

The Pliocene fauna has a still more modern aspect, a large proportion of the animals composing it belonging to existing genera, although most of the species are extinct. Indirect evidence, in the shape of chipped flints, of the existence of Man occurs in deposits assigned to this period. Complex antlers have appeared in the Deer, horns in the Rhinoceroses, and tusks in the Pigs. The occurrence of Giant Tortoises (Testudo) in the Pliocene of both Palæarctic and Nearctic regions, and of a Chimpanzee and a true Ostrich (Struthio) in deposits of this age in India and the Crimea, indicates the northern origin of these forms. Indeed it seems probable that most of the higher Vertebrata, except Penguins and the New-World Edentates, have originated in the Holarctic region.

In the Pleistocene period many existing species have made their appearance, but their geographical distribution is very different from that of the present day. For instance, the European fauna includes many forms now confined to the Ethiopian and Oriental regions, such as Apes, large Felidæ, Hyænas, Tapirs, Rhinoceroses, Hippopotami, Horses, and Elephants, all of which appear to have been driven southwards by the cold of the Glacial epoch. In some parts of the world the Pleistocene fauna includes remarkable and often gigantic forms now extinct-most notable being the great Edentates (Megatherium, Mylodon, Glyptodon, \&c.) of South America, the gigantic Marsupials (Diprotodon, $\Lambda^{\top}$ ototherium) of Australia, and the great flightless Birds (Dinornis, Epyornis, \&c.) of Madagascar and New Zealand. Nesopithecus, which occurs in the Pleistocene of Madagascar, is either a Monkey-like Lemur or a true Monkey: if it be the latter, its occurrence indicates a closer affinity between that island and Africa than their existing faunæ would indicate. Pithecanthropus, found in beds of late Pliocene or early Pleistocene age in Java, was perhaps a connecting link between the other Anthropoids and Man.

1 The marsupial affinities of the Sparassodonts, however, are very doubt. ful, and the resemblances to Thylacinus may $b$ ? entirely superficial.

VOL. II 
The Pleistocene passes insensibly into the Recent period, which has also witnessed some important zoological changes, especially the extinction of many interesting animal forms, for the most part by human agency. Among these may be particularly noticed Steller's Sea-cow (Rhytina), the Great Auk, the Dodo and Solitaire, several flightless Rails (Aptornis, Notornis, Aphanapteryx, \&c.), the Phillip Island Parrot, and, above all, the whole great race of Moas. 


\section{SECTION XV}

\section{THE PHILOSOPHY OF ZOOLOGY}

Is dealing with the structure and development of the various groups of animals, there has been occasion not infrequently to refer incidentally to various subjects of a general nature, such as evolution, heredity. and the like. Such topies, dealing, not with the concrete facts of the science, but with abstract generalisations deduced from the facts, may be grouped together under the general heading of the Philosophy of Zoology. The generalisations forming the subject-matter of the philosophy of zoology may, in some instances, be so clearly and directly deducible from the data concerned that it is scarcely possible for anyone conversant with the facts to refuse credence to the generalisation. But in other cases the conclusion is a matter of probability only, and one conclusion or another may be regarded as the more probable, according to the estimate formed of the relative importance to be attached to different sets of the facts or to different aspects of the facts. This will become clearer as we proceed; bnt at the outset it should be distinctly understood that what follows is not to be looked upon in the same light as the statements regarding the known phenomena of animal life which constitute the main substance of the preceding sections. Nearly all the subjects now to be touched upon are, to a greater or less extent, matters in which there may be variety of opinion among those conversant with the phenomena; they are all subjects which will bear discussion from various sides ; but, as discussion is here almost out of the question, it is possible to give little more than a brief statement of some of the current views on these questions as an introduction to the study of works specially dealing with them. ${ }^{1}$

The Philosophy of Zoology, or the Philosopliy of Biology (for it is here almost impossible to treat Zoology apart from its companion science of Botany), aims at an explanation of the facts of

1 See Appendix II, Nos. $2,3,4,14,15,16,17,19,21,22,23,36,37,42$, $45,55,62.63,65,66,67,72,74,75,77,78,79,81$. 
the science. It is observed that an animal possesses a certain structure, develops in a certain way, has certain affinities with other animals, has a certain geographical and geological range; and the attempt is made to find a satisfactory explanation of these facts.

Evolution.-Of these facts there is, to all intents and purposes, but one explanation requiring consideration here. The animal- and plant-life of the globe has come to be as it now is by a process of evolution which has been going on continuously from an early period in the history of the earth to the present time. The plantand animal-worlds, in other words, have been evolved by a gradual process of development, in the course of which the higher forms have originated from the lower. Evidence bearing on this doctrine has already been encountered in abundance-in fact the theory of evolution has to be looked upon as in many respects a guiding principle in the study of our science; and it has, accordingly, been necessary in many parts of previous sections to take its truth for granted. In discussing the relations of the various phyla to one another, the relations of the various classes of each phylum, and the position of the described examples within the classes; in referring to the homologies borne by the organs of the members of one class to those of the members of another, it has been necessary to assume the truth of a theory of evolution.

For the evidence, then, in favour of a doctrine of evolution the reader is referred to the substance of previous sections, where it will be found on almost every page. For his guidance some

landmarks may, however, be here pointed out.
Anatomical and Embryological Evidence.-A considerable body of the evidence in favour of the view that the higher animals have been derived from lower forms is obtained from the provinces of comparative anatomy and embryology. The mere fact that we are able conveniently to express the resemblances and differences in structure between different groups by the construction of such genealogical trees as have been given in some of the previous sections tells strongly in favour of a theory of descent; for, though it is by assuming evolution that such diagrams are constructed, the resemblances which they represent point strongly to common ancestry. A theory of evolution explains also the fact that there is running through a whole series of forms-let us say Fishes, Amphibians, Reptiles, Birds, and Mammals - a common type of structure, in which the same essential parts, though perhaps differently modified in accordance with differences in function, are to be found in the same mutual relations. It would be difficult, on any other view of the facts, to explain, for example, the occurrence in the wing of the Bird and of the Bat, the flipper of the Whale, and in the fore-leg of the Horse, of essentially the same bony elements. More difficult 
still would it be to explain the cases in which what is a functionally active and important part in one animal is to be found - though only as a mere vestige, apparently quite useless -in an allied form. Very many instances of this phenomenon will be found in the previous chapters. The wing of the Pigeon is an efficient organ of flight; in the New Zealand Kiwi or Apteryx it is a vestige, not visible externally, being covered over by the feathers and wholly without function; yet this vestige possesses essentially the same bony framework and the same muscles as the complete and functional wing of the Pigeon. Again, the teeth of the Rabbit are parts essential to the welfare and the very existence of the animal, and persist throughout life ; while in the WhaleboneWhale teeth are indeed developed in the foetal condition, but are thrown off before or shortly after birth, never being of any use for mastication or any other purpose. The conclusion that seems to follow from these facts is that it is at least highly probable that the Kiwi has vestiges of wings because it is descended from birds which, like the Pigeon, possessed functionally useful wings; and that the Whalebone-Whale has teeth in the fœetal state because it is descended from ancestors which possessed teeth in the adult condition.

The fact that the embryos of animals of one great phylum or class present a great resemblance to one another, and that the nearer the adult forms are in structure, the closer, usually, is the similarity in their developmental stages, tells strongly in favour of a theory of common descent. Thus the nauplius-stage is found in a considerable number of groups of Crustacea, but it is only between members of families whose structure is closely similar that there is a very near correspondence in the precise character of the nauplius and in the stages which the larva subsequently passes through.

Evidence of an allied character is afforded by the fact that in the course of its development one of the higher animals sometimes appears to exhibit in successive stages features which are permanent in forms lower in the scale. Thus the embryo of a Mammal presents at an early stage visceral arches and clefts comparable to the branchial arches and clefts of a Fish, and has a bloodcirculation in accordance with this ; while at a later stage it exhibits in these particulars some resemblance to an Amphibian, later on to a Reptile, and only when development is further advanced takes on its special Mammalian characters. Again, we have seen that such an Amphibian as the Frog is, in its early condition as a tadpole, to all intents and purposes a Fish. Such phenomena may be explained, according to the theory of evolution, by the supposition that the successive stages in the development of the individual animal tend to reproduce, though in a very abbreviated and often greatly modified shape, the stages through which the group to 
which the animal belongs has passed in the course of its evolution from lower forms. This supposition-the "biogenetic law," or "recapitulation theory," as it is termed-though it cannot be accepted without great modifications and reservations, yet covers a number of facts which distinctly demand a process of evolution for their explanation. ${ }^{1}$

The phenomenon of retrograde metamorphosis observable in many animals, for the most part parasitic in the adult condition, also affords evidence in favour of evolution. It would be difficult to give any other explanation than that afforded by a theory of descent, of the life-history of such animals as Sacculina (Vol. I., p. 583), the parasitic Copepoda (p. 583), or the Ascidians (Vol. II., p. 35). The relatively high organisation of the larva of Sacculina, for example, with its well-marked Crustacean features, can only be explained on the supposition that the shapeless, unsegmented adult has been derived by a process of retrograde development from more normally constructed ancestors.

Most Birds and Mammals, and many animals of lower groups, exhibit a more or less strongly marked sexual dimorphism, the males differing from the females in various other respects besides the character of the sexual organs. Such differences can only be explained on the supposition that they are the result of a gradual process of modification brought about in accordance with the more special adaptation of each sex to its special functions.

Palæontological Evidence.-A second body of evidence in favour of a theory of evolution comes from the side of Palæontology. It might, perhaps, on first considering the subject, be supposed that, had a process of evolution taken place, we ought to be able to find in the rocks belonging to the various geological formations a complete series of animal- and plant-remains representing all the stages in the evolution of the highest forms from the lowest. Beginning with those strata in which evidence of life first appears, we ought, it might be supposed, to be able to trace upwards, through all the series of fossil-bearing strata, continuous, unbroken lines of descent showing the gradual evolution of all the various forms of plant- and animal-life. But snch a supposition would leave out of account the extreme incompleteness of the record of

1 As an instance of the difficulties in the way of the acceptance of the biogenetic law as such, it may be pointed out here that the ovum is by no means equivalent to the simple cell with which the phylogenetic series inust. be supposed to have begun. On the contrary, the ovum of one of the higher animals must be an extremely complex structure, and in reality widely (lifferent from the Protozoan which, according to the biogenetic law, should be its prototype. The ovum of the higher animal is, it is true, a single cell; but it is a cell which comprises potentially within itself the entire complex adult organism, and is thus essentially an entirely different thing from the unicellular Protozoan. The same holds good of later developmental stages : they may resemble the adult condition of lower groups; but they differ from the latter in the same way as the ovum differs from the Protozoan. 
the history of life on the globe which is preserved to us in the rocks. In the first place, there are many groups of animals and plants which, owing to the absence of any hard supporting parts, are incapable of leaving any recognisable trace of their former existence in the form of fossils. Again, even in the case of such as have such hard parts, the conditions necessary for their preservation in deposits destined to be converted into rock cannot be of very frequent occurrence; and many forms might fail to be preserved simply owing to the non-occurrence of such conditions. In the case of land-animals, such as Mammals or Reptiles, for example, when one of them dies, it is for the most part torn to pieces, and even the bones destroyed by various carnivorous and carrion-feeding creatures. Only now and again would it happen that, by becoming buried in a morass, or swept away by a flood and buried under alluvial deposits, such forms might be preserved.

Again, great thicknesses of sedimentary strata, sometimes containing fossils, can be shown to have become removed by the agencies of denudation, or the various forces--such as the action of waves, tides, and currents in the sea, of rain and fresh-water streams on the land---by which rock-masses are constantly, where exposed, being worn away; while other rocks, subjected to the pressure of enormous superincumbent masses, and perhaps acted upon by intense heat and other agents of change, have been completely metamorphosed - their mineral constituents having become re-arranged and what organic remains they may have contained completely destroyed. Moreover, of the fossil-bearing rocks that remain unaltered, only a small part can be said to have been thoroughly explored for fossil-remains.

Yet, notwithstanding these causes of imperfection in the record of the succession of life on the earth preserved to us in the rocks, there is sufficient evidence to enable us to judge of the general character of the faunæ (and floræ) of the various geological periods. It is manifest, from what has already been stated throughout the earlier sections with regard to the geological history of each phylum and class, that there has been a general progress in successive eras from the simple to the more complex; the higher forms have, so far as the recorded facts enable us to judge, come into existence later than the lower. The Vertebrata may be taken as an example. There is no evidence of the existence of the highest class - the Mammalia - earlier than the Triassic period of the Mesozoic era. The case of the Birds appears at first sight anomalous: Birds appear for the first time in deposits of Jurassic age, and are therefore more recent than the oldest Mammals. Birds are, however, very higly specialised Vertebrates, and should it be proved that they appeared at a time when primitive Mammals already existed, the separate evolution of the two classes from lower forms would afford a sufficient explanation. Reptiles extend 
as far back as the Permian. Amphibians, in the shape of the Stegocephala, first appeared in the Devonian ; while all the earliest vertebrate remains in the Cambrian and Silurian formations appear to belong to the class of the Fishes. Within each of these classes a progress is usually traceable from older, more generalised types, along diverging lines, to the various specialised forms existing at the present day. In some cases, however,-notably in the Amphibia, Reptilia, and Aves - the orders first represented have become entirely extinct, and have been succeeded by others that made their appearance on the scene at a comparatively late period.

In certain cases among the Mammalia a number of closely-related stages have been discovered, showing, taken in their chronological order, a gradually increasing specialisation of structure. One of the best-known examples of this is that of the Horse, to which attention is directed in the section on the Mammalia (p. 586). No fewer than five parallel series of horselike Perissodactyles are traceable, which developed and culminated separately, the culminating member-viz., the genus Equus - of one only of these series surviving to the present day. And there are other families of Mammals, chiefly among the Ungulates (the family of the Pigs and various families of Ruminants), in which an equally complete history has been made out.

The direct evidence of the evolution of the Invertebrates is, in general, very imperfect. Some existing types of a comparatively highly organised character are to be recognised among the fossil remains in the oldest formations - the Cambrian - in which definite organic structures, if we except a few Radiolaria and Foraminifera, are traceable. There is no trace of primitive fossil members of the various invertebrate phyla, and the highly organised airbreathing Arthropods are represented both by Scorpions and by Insects as far back as the Silurian. Such remarkably complete geological histories as have been traced in some of the Mammalia are extremely rare in the Invertebrates. Such direct evidence, however, as is obtainable points to the probability of evolution, and it may be inferred that the absence of primitive generalised representatives of the invertebrate phyla is most probably due to the imperfect character of the geological record.

The Lamarckian Theory.-Supposing it to be regarded as proved that the organic world has come to be as we find it by a process of gradual evolution, we have next to inquire by what agencies this process of development has been brought about. "A slietch of the history of thought on this subject will be given in the section on the history of Zoology, and it will not be necessary here to refer to more than the most important points.

The first noteworthy attempt to solve the problem regarding the nature of the forces by means of which evolution has taken place was made, long before evolution was generally accepted among 
men of science, by Lamarck in his Philosophie Zoologique, published in 1809. Lamarck's view was that evolution of new forms has taken-and is taking-place, in great measure owing to the direct action of the conditions of life on the organism, but still more owing to the use and disuse of organs. The surroundings or environment of the animal or plant produce a direct effect on the individual-bring about slight modifications in one direction or another, and these slight differences are transmitted by inheritance to the next generation-such slight modifications going on, generation after generation, producing eventually a marked effect on the characters of the organism. The chief agencies that might be supposed to act in this way are climate, the nature of the country, and food. But, in addition to these, Lamarck attributes considerable influence to the use and disuse of organs. The exercise of a part tends to increase its size and efficiency, and such increase may be and frequently is, according to Lamarck, transmitted to the succeeding generation. In this way, in the course of a number of generations, very great changes might be brought about. To take an example which is often quoted, Lamarck accounts for the great length of the neck of the Giraffe as compared with other Ruminants by the supposition that it has been brought about by continuous efforts made by the animals through a long series of generations to reach higher and higher among the foliage of the trees from which they derive their main subsistence. Similarly, the disuse of a part, in Lamarck's view, gradually leads to its diminution, and perhaps ultimately to its complete disappearance. In this way he would explain the disappearance of the hind-limbs in the Cetacea, of both pairs of limbs in the Snakes, of the olfactory nerves in aquatic Mammals, and so on. Whether differences which are produced in the individual organism by surrounding conditions or by its own efforts may be transmitted by inheritance to succeeding generations is not yet a settled point: we shall have again to refer to this question - the question of the inheritance of acquired characters-at a later stage. That such inheritance, if it takes place, could account for the development of all the various groups of animals and plants is not held by many biologists at the present time.

Darwinian Theory.-It is to Charles Darwin that we owe the most thorough and consistent explanation of evolution that has hitherto been put forward--the explanation known as the theory of Natural Selection. The development of this theory and the share taken in it by Wallace will be sketched in the historical section. The two main supports of Darwin's theory are two sets of biological phenomena known respectively as the struggle for existence and variation, both of which have to be understood before it is possible to grasp the theory of natural selection.

Struggle for Existence.-In order that it may flourish, there are necessary for every species of plant and animal certain conditions. 
The plant must find a place with soil containing certain constituents, and with a certain degree of moisture and of sunlight. For spots presenting the necessary favourable conditions there is constantly going on a competition between individual plants of one species and between the members of different species. The nature of this struggle is well seen when a piece of garden-ground is allowed to run to waste. Its surface is soon overgrown by weeds of a variety of kinds, which kill out some of the original garden-plants. By and by the more hardy weeds kill out and replace such weaker forms as may first have obtained a footing, till an entirely new set of weeds may take the place of those that first appeared. Again, it was shown by Darwin that in turf which is kept cut close a much greater number of plants are enabled to grow than is the case if the turf is allowed to grow freely. If the turf is not kept cut some of the stronger plants gain predominance and kill out weaker forms. In a space of turf on which Darwin experimented, no less than half of the species present in the turf when kept pretty closely shaven perished when it was allowed to grow freely.

Plants, however, have not only to compete with one another for space and light and nourishment. They have also numerous animal foes to contend with. A large proportion of young seedling plants are destroyed by various Insects and by Snails and Slugs. One of Darwin's experiments bearing on this point was to clear and dig up a small plot of ground and watch the fate of the seedling plants that sprang up on it; he found as a result that some fourfifths were destroyed by Insects, Snails, and Slugs. But it is not the lower forms of animals alone that are thus destructive to plants. Many of the Mammalia, particularly, as we should expect, the herbivorous Ungulata, exercise a strong influence in this way. Cattle, and Goats especially, sometimes produce a marked effect on the flora of a country. The introduction of Goats has been observed gradually to destroy the forests of certain districts - the seedling plants being eaten as they appear, and thus no young trees being developed to take the place of those dying from old age or other causes. The mere enclosing of a piece of moorland by means of a fence was observed by Darwin to have resulted in the growth of a number of trees. In the unenclosed parts the young trees were never able to make any headway against the cattle by which they were constantly being browsed down.

Among animals, with which we are here more particularly concerned, as well as among plants, a struggle for existence goes on on all sides. To begin with, before there is any struggle for existence in the strict sense, there is-particularly in lower groupsa very great indiscriminate destruction of ova and young embryos. Most lower animals produce ova in great number-hundreds, more often thousands and tens of thousands, annually. Only a few of these reach maturity; a large proportion are destroyed indiscrimi- 
nately at one stage or another of their development, some failing to reach a spot favourable for their development, others becoming the food of other animals. But such of the young as are less adapted to escape the various dangers to be encountered, and less fitted to procure the necessary food, are more likely to be destroyed. This is one phase - and the most important, perhaps, of all-of the struggle for existence among animals. But there is also a struggle for existence not only between individual animals of the same kind, but between animals of different kinds. This struggle, in so far as it relates to the competition for food and shelter, is more severe between nearly-related species; for in such a case the food and the favourable conditions required are the same, or nearly so, in the two competitors. But there is also a struggle for existence of a constant and severe kind which goes on between carnivorous animals and the animals on which they prey-a struggle in which the defensive qualities of the latter, such as swiftness, power of eluding observation, power of resisting attack and the like, are opposed to the predatory powers of the former.

Variation.-It was by observing this struggle for existence constantly going on in nature, taken in connection with the phenomenon of variation, that Darwin was led to his principle of Natural Selection as accounting for evolution. Variations in domestic animals and cultivated plants are observed to take place in various directions. Taking advantage of this, man has been able to select, in the animals which he has domesticated and the plants which he has cultivated, those qualities which seemed most likely to be useful to him; he has thus been able to produce, from one and the same original wild stock, widely different varieties specially adapted for different purposes. Thus from one wild species of plant of the order Cruciferce-viz., Brassica oleracea-have apparently been produced all the varieties of cabbage, cauliflower, broccoli, Brussels sprouts, and other forms, each with a peculiar and strongly marked growth of its own. All the domestic vegetables afford us instances of the same thing, and so do all the cultivated fruits. The crab-apple or wild apple, for example, was the original of all the varieties of apple, amounting to about a thousand, cultivated at the present day-varieties presenting in many cases very great differences in size, colour, texture, flavour, time of ripening, and other qualities. In cultivated flowers the same holds good in an even higher degree.

The instances of variation observable among domestic animals are still more striking. The domestic Dog, for example, exhibits a large number of very marked varieties. Though all these seem to be fertile with one another, and to produce fertile offspring, it is generally supposed that they have been derived from several wild species with more or less hybridisation. But the enormous differences which are to be observed between some of the varieties 
have been produced to a great extent under domestication. These are not all mere superficial differences, but involve also the proportions and shape of the parts of the skeleton. The difference in the form of the skull and in the proportions of the bones of the limbs between a Greyhound and a Bulldog, for example, are very remarkable - so great, in fact, that if they were found to occur between two wild forms they would justify a zoologist in referring the two to distinct genera. Sheep and Cattle, Pigs and Horses, present similar, though not perhaps quite so strongly marked, varieties. One of the most remarkable cases of variation under domestication, and one to which Darwin paid a good deal of attention, is that of the domestic Pigeon. Of this there are a considerable number of varieties, known to fanciers as pouters, fantails, carriers, tumblers, and so on ; and it appears to be almost certain that these are descended from one wild species-the blue Rock-pigeon.

These varieties, and many more that might be mentioned, have been produced by man selecting those forms that tended to vary in a desired direction-have been produced, that is to say, by artificial selection, sometimes consciously exercised, sometimes, no doubt, unconsciously. This process has had a long period of time for its operation, many of our domestic animals and plants having been the objects of care and cultivation in Egypt and Western Asia certainly several thousand years ago ; in many cases the wild forms from which they were developed appear to have become totally extinct.

But variation occurs among animals and plants not only under domestication; it occurs also in a state of nature. Evidence of this has already been adduced in the account of certain of the examples of the various phyla ; and in the examination of specimens of these in the laboratory the student can hardly have failed to notice the occurrence of individual differences not due to differences in sex or age in animals of all classes. In this respect, in the strength of the tendency to individual variation, there is a very great inequality between different species of animals, some being extremely variable, some comparatively stable. Variations of extern ll parts have naturally, from the greater ease with which they may be observed, attracted most attention, but the examination of the internal parts in large numbers of individuals of the same species, when it has been carried out, has shown that variations in internal organs are also of great frequency.

Among the Protozoa, the Foraminifera are characterised by numerous and marked variations-so marked as "to include, not merely those differential characters which have usually been accounted specific, but also those upon which the greater part of the genera of this group have been founded, and even, in some instances, those of its orders." The Mollusca vary also very 
frequently and extensively, especially in the form and markings of the shell; and of some of the species which have been most completely studied in this respect a very large number of more or less strongly marked varieties have been recorded. Many of the Crustacea are also extremely variable in coloration and in the length and proportions of the various appendages. But, among the Arthropoda, it is in the Insecta, and more especially the Lepidoptera, that we find the most striking instances of variation. In the Vertebrata, also, variations in colour and proportions, as well as in internal organs, occur frequently in all classes.

Mutations.--It has been shown by de Vries that variations are not always of a comparatively minute character. According to de Vries, in addition to the small fluctuations on which Darwin mainly relied for the phylogenetic development of organisms, there are others which occur comparatively rarely, and are of a much more striking character. These larger variations, which de Vries distinguishes by the name of mutations, take the form of the sudden appearance of differences equivalent to the formation per saltum of new species; and it is by the successive appearance of such steps or leaps, and not by the more gradual process of Darwinian variation, that, according to de Vries, progress from the lower towards the higher is effected. The new forms developed in this sudden way may live side by side with the old, and thus isolation is not necessary for their perpetuation.

Natural Selection.-According to Darwin's theory of Natural Selection, nature, i.e., the conditions under which the organism exists, selects certain variations as they arise, very much as the breeder or the gardener selects variations in domestic animals or cultivated plants. Let us see how this selection is carried on. We have seen that there is going on, on all sides, a struggle for existence. It is at first difficult to realise the intensity of this struggle, for there is little appearance of it on the surface. If we consider, however, that a large proportion of living things prey on living things of other groups, and when we bear in mind the extremely small proportion which, in most cases, the surviving individuals of any group bear to the number of young produced, we come to understand that this struggle for existence must be general and intense.

Now in the case of a species living under tolerably uniform and stable conditions as regards climate, food-supply, and the like, the effects of this struggle will be the survival of the fittest. Of the young produced, only a small proportion (in most cases) reach maturity; some of these surviving forms have survived, perhaps, because they have happened to escape being preyed upon by enemies, while others have succumbed; but there can be little doubt that, in the long run, such individuals will survive as are best fitted to cope with the conditions to which they are subjected 
- such as are swiftest, let us say, in escaping pursuit; or such as, by their special shade of colour or the nature of their markings, elude the observation of an enemy; or such as, by reason of their thicker covering, can better endure extremes of cold. Such surviving individuals would, it is assumed, transmit their special properties to their progeny, and there would thus be a gradual approximation towards a better adaptation of the species to its surrounding conditions by virtue of this "survival of the fittest."

Let us suppose the conditions to change. Gradual changes in climate and other conditions are known to take place owing to subsidence or elevation of the land. But conditions might be changed in many other ways: some animal or plant previously used as food might become exterminated; or a new enemy might find its way into the district inhabited by the species. Then such individuals as presented variations which enabled them better to cope with the new surroundings would have the advantage over the others, and would have a much better chance of surviving and leaving progeny. The useful variations thus produced and transmitted to the progeny would tend to increase, generation after generation, until a form sufficiently distinct to be regarded as a new species had become developed from the original one.

The process of survival of the fittest has a reverse side, which has been termed the elimination of the unfit. Of the varieties that appear some are less completely adapted to their surroundings than the majority, and these (the conditions remaining the same) tend to become destroyed owing to their unfitness to cope with their enviromment. The result of this process of elimination (apart altogether from the selection of progressive variations by which evolution, according to the theory, proceeds) is to keep up a certain standard of efficiency in the organs of the members of the species. Under certain conditions this sustaining influence, as we may term it, of natural selection may be suspended ; the organism may be placed under conditions in which natural selection acts with reduced effect or does not act at all. There is, under such circumstances, no "elimination of the unfit"; and, as a result, fit and unfit survive indiscriminately, interbreed and produce offspring, the ultimate outcome in the course of generations being a gradual deterioration in the whole race.

This suspension of the influence of natural selection, with its results, has been termed cessation of selection, or panmixia. Panmixia acts more commonly on single organs than on the entire organism. 'Thus, if, owing to some change in surrounding conditions, an organ is no longer useful, it is no longer kept up to the previous degree of efficiency by the elimination of the individuals in which the organ in question is imperfectly developed, and, as these cross with one another, offspring is produced in which the organ is below the efficient standard; by a continuance of this process through a 
series of generations, it is supposed that the organ gradually dwindles in size, and may altogether disappear. Thus at that stage in the ancestral history of the Cetacea in which they had come to adopt a purely aquatic mode of life and no longer visited the shore, the hind-limbs, being no longer of service, would no longer be maintained by natural selection, and would gradually decrease in size until, finally, they entirely disappeared. In the case of these, as of many other rudimentary organs, however, it is probable that natural selection played a positive part in bringing about their diminution. Under the conditions supposed, the possession of hind-limbs would probably be an actual disadvantage to the animal, acting as an impediment to the swift progression through the water, and interfering with the free movements of the tail; and varicties with diminished hind-limbs would, therefore, possess an advantage over their fellows in the struggle for existence. There would then be, in a sense, a positive reversal of selection.

Darwin's theory of selection is concerned mainly with the small individual variations which are observed to occur, more frequently in some species, more rarely in others. Such variations are so slight and unimportant that it is difficult to understand how they could be of sufficient life-and-death value to give the individuals in which they occur sufficient advantage in the struggle for existence to enable them to survive, when others in which they are absent perish. Failing the extermination of the unmodified individuals, unless the appearance of the variation should happen to be coincident with the occurrence of other factors leading to the isolation of the individuals possessing the new variation from the stock in which they originated, the new varicty would tend to become swamped by intercrossing with the latter and would fail to be perpetuated. If, however, the individuals in which the new modification occurs should by some means-such as migration beyond a geographical barrier of some kind, or by the nature of the variation itself-be preserved from intercrossing with the stock, then, without the extermination of the latter being a necessary condition-without, that is to say, a life-and-death struggle - the new form might be preserved unaltered and perpetuated as a new and distinct variety, which further changes similarly brought about might raise to the rank of a species.

Detailed study of the geographical distribution of species and varieties in certain regions, more especially in the United States of America, has afforded much support to the view that the development of new forms takes place as a result of the appearance of varieties differing slightly from the parent stock and their isolation from the latter by geographical barriers; and by some writers evolution is even supposed to have proceeded solely, or almost solely, in this way with little, or entirely without, aid from natural selection. Another kind of isolation might be supposed to take 
the place of geographical in preventing the intercrossing of new varieties with the original stock. By means of sexual or physiological isolation, i.e., by the form in question becoming varied in such a way that it does not readily interbreed with the main stock, the new variety may be as effectively isolated as if separated from it by a geographical barrier.

That allied species and sub-species differ in their geographical range, and are often separated by geographical barriers, and that a physiological barrier may be set up between allied forms owing to union between them being impossible or sterile, are facts of great importance in the study of evolution, and the details of such cases are necessary data of the science. But that the whole of organic nature should have been evolved by variation and isolation alone seems to be highly improbable: such a view takes no account of progressive adaptation and orthogenetic development, and it would seem to call for the formation of an impossible succession of barriers.

A special phase of Natural Selection is distinguished under the title of Sexual Selection. By means of Sexual Selection it is attempted to explain the greater part of the secondary differences between the sexes which are so striking in many groups of animals. The special part which each sex has to play in the fertilising and deposition of the ova, in protecting and procuring food for the young, requires qualities, both anatomical and psychical, of a more or less widely divergent character in the male and female. Between the males of animals of many groups contests frequently take place, and this affords us an explanation of the presence or special development in many cases in that sex of various offensive and defensive weapons-horns, tusks, and the like. Similarly, we are able to understand the greater vigour, in the majority of cases, of the male, with concomitant greater intensity of coloration, and the development of various ornaments and excrescences not present in the female. In many groups of Insects, and in a large proportion of Birds, sexual differences in coloration are very marked. These are, in some instances, to be traced to the necessity for different protective resemblances required in the two sexes owing to different habits, or to the necessity for protective colorations and markings in the female and not in the male. In the case of Birds, when the sexes differ, as they do in a large proportion of the species, the male has always more brilliant coloration, and often possesses also special crests or frills, wattles and the like, not present or less developed in the female. The greater obscurity of the colouring of the female Bird appears to be adapted to rendering her less conspicuous to enemies, such as Birds of Prey, while sitting on the nest; and, in cases where the females are brightly coloured, the nest is covered over above, or is constructed in a hole in the ground. The brilliant colouring and other features distinguishing the males 
of many Birds may be in great part the by-product of higher vitality, and may thus be the indirect outcome of natural selection leading to the more vigorous males obtaining an advantage in contest with rivals. It is possible, also, that the choice of the female in selecting a mate may have been a factor in bringing about the special modifications in question. But the evidence which has been adduced for any such selection on the part of the female of a mate with some slight superiority in brilliancy of colouring, or in the development of crests and the like, over his rivals, is insufficient, and many observations tend to show that selection of this kind, though it may occur, is exceptional. In any case, as a general explanation of secondary sexual characters, sexual selection is not at the present time very widely accepted as adequate.

Protective and Aggressive Resemblance and Mimicry.One of the most important of the phenomena which are well explained by the theory of natural selection, and which may, therefore, well be taken as affording evidence in favour of that theory, are the phenomena of protective resemblance, warning characters, and of mimicry. In innumerable cases among all classes of animals there are found instances of a resemblance between the animal and its ordinary natural surroundings, which has the effect of rendering it inconspicuous and unlikely to attract the observation of an enemy, or of its prey. Such a resemblance is brought about sometimes merely by colour, very often by the arrangement of the colour in a pattern, this being frequently accompanied by modifications of shape, including sometimes the development of special excrescences or appendages. In some cases of protective resemblance the colour and even the markings change with a change of the surroundings. For details of such cases reference must be made to special works. Many Insects present elaborate markings which give them a close resemblance to a tuft of lichen or moss, a twig, a leaf, or other object, and resemblances of an equally striking character occur in other classes.

Some animals, more especially certain Insects, are protected by their nauseous character against being devoured by animals that would otherwise prey upon them; but often, no doubt, such nauseous Insects are attacked and killed before their unpalatable character is detected. It is thus manifestly of advantage to such animals that they should be readily recognisable, and should thus be passed over; and in many such cases the coloration is bright and conspicuous, or the animal is rendered conspicuous by other means (warning characters).

By mimicry is meant a superficial resemblance borne by one animal to a member of a different group. The best-known examples of mimicry occur among the Insects. It is manifestly of advantage to a Butterfly belonging to a group which is not nauseous to be readily mistaken for a nauseous form with con- 
spicuous warning colours and markings, and this appears to be the explanation of many cases of mimicry. Similarly, a variety of flower-frequenting Dipterous Insects which have no sting or other weapon bear a remarkable resemblance to Bees or Wasps, belonging to a distinct order (the Hymenoptera) - the resemblances embracing not only shape, colour, markings, and development of "hairs" on certain parts, but the movements of the wings and other parts and the humming sounds emitted, so that, on a superficial inspection, the mimicry appears complete.

Heredity.-The various characteristics of a plant or animal are transmitted to the succeeding generation. In the highest groups of animals this transmission is effected only through the intermediation of the sexual cells-ova and sperms-since they alone are capable of giving rise to a new generation. But in lower organisms the faculty of reproduction is more widely diffused among the component parts; in some lower multicellular plants each and every cell is capable of taking on the function of reproduction and giving rise to progeny similar in all respects to the parent; in other words, every cell in such cases must contain germinal substance. In other, somewhat higher, forms the germinal substance, though still widely diffused, may not be present, or capable of becoming active, in all parts, and may be confined to the cells of one or other of the layers. In the vegetable kingdom, even amongst the highest forms, the germinal substance can be shown to be widely diffused throughout the plant. Thus in many flowering plants, if we cut a shoot into lengths, the pieces are all capable of giving rise under suitable treatment to complete plants with flowers containing reproductive cells; and in many cases a leaf, or a portion of one, is capable of a similar development. In many animals a similar wide distribution of the germinal material may be shown to prevail. This appears most strikingly in forms that multiply by budding. In Hydra, for example, any part of the body seems capable of giving off buds, and in the buds, after they have become separate, ova and sperms are developed from the cells of the ectoderm. A similar phenomenon is to be observed in other Cœlenterates and in the Polyzoa and the Composite Ascidians, and also in certain cases among the Platyhelminthes and Annulata. In all these, and in other cases that might be mentioned, the germinal substance is not confined to the reproductive cells-new reproductive cells being capable of being formed from the substance of the cells of various tissue-layers.

The phenomena of regeneration are important in connection with this question of the site of the germinal substance. Many members, not only of the lowest phyla, but of the Echinodermata, the Annulata, the Arthropoda, the Mollusca and the Chordata, are able, as has been repeatedly mentioned, to replace, by a process resembling budding, parts that have been broken off: some of 
the eells of the adult body must, therefore, in these eases retain in a eertain degree the faculty of reproduction, and must contain germinal substanee. The germinal substance eoneerned in regeneration must, it is of importanee to note, be eapable of being stimulated into aetivity in a eertain definite direetion by an influenee brought to bear upon it from without.

In the Vertebrata the power of regeneration, if we leave out of aeeount the various epidermal structures, is exceptional; and where it oeeurs (most Amphibia, some Reptiles) it is confined to the limbs, jaws, lens of the eye, or the tail. In the highest Vertebrates there is no power of regenerating such parts when lost, and the eapacity for reproduetion is eonfined to the sexual cells.

A remarkable persistency charaeterises these reproductive eells. By their means there are handed down from one generation to another, with little alteration, all the eharaeteristies of the speeies of plant or animal. This speeial faculty of the reproductive eells is the faculty of heredity.

Heredity does not imply absolute fixedness of all the characteristies inherited by one generation from its predecessor. On the contrary, as already pointed out, variations are constantly presenting themselves. Some of the variations whieh animals exhibit are a direet result of the action of surrounding conditions, or of the use or disuse of parts, on the fully developed animal; we ean in some cases aetually cause the animal to ehange to a more or less marked extent by plaeing it under different conditions. Another set of variations produeed by the action of external influences on the organism only appears if the aetion takes plaee in the course of development at one stage or another between the oosperm and the adult. Of the oecurrence of both these forms of variation we have direct and positive evidenee. It is a familiar faet that increased exereise of a part tends to an inerease in the bulk of its museles. The colours and markings of eertain Fishes ean be altered at will (of course within eertain limits) by ehanging the material on the bottom of the aquarium in whieh they are eonfined; the eolours of many Caterpillars may be altered by changing the colour of their surroundings. A third set of variations probably also occurs, though direet evidence is wanting-namely, variations which may arise within the sexual eells before the union of ovum and sperm, or whieh may result from that umion. The former two sets of variations are generally spoken of as " aequired eharacters "-new eharacters aequired during the lifetime of the individual-but their nature would be more clearly indieated by terming them extrinsic variations, as contrasted with the intrinsic variations forming the last group.

The extrinsie variations being brought about by the action of external conditions, their eauses are very various. In every sueh case the organism responds to some persistent external influence $\mathrm{R} R 2$ 
by undergoing some more or less persistent change. Mutilations, the rapid mechanical removal or destruction of parts, are here, by the terms of the above definition, excluded from the class of variations altogether, since, though the change involved is frequently permanent, it is effected by an influence which is temporary in its character. This, as will be seen, is of importance in connection with the next question we have to deal with-the inheritance of acquired characters.

Can acquired characters or extrinsic variations be transmitted by inheritance? That they can be is of the essence of Lamarck's doctrine of development, which, in fact, may be described as a theory of development by means of the inheritance of extrinsic variations, or, as it is sometimes called, use-inheritance. But the maintenance of the view that extrinsic variations may be transmitted is not inconsistent with the acceptance of natural selection as a true cause of evolution. Evolution might be supposed to be due to the selection and inheritance of both intrinsic and extrinsic variations. From the nature of the case, evidence in favour of the inheritance of extrinsic variations on the one hand, and the occurrence of intrinsic on the other, is extremely difficult to obtain. One or the other must occur, or there would be no evolution. But to prove in any given case that a change is due to the one factor rather than to the other is extremely difficult. When a character not present in the parents appears in the offspring, there is, to begin with, great difficulty in proving that it is really new: characters not present in the parents are known to be frequently inherited from a more or less remote ancestor. But, if we suppose it to be established that the character is a new one (and absolutely new characters must appear, or we should have no evolution), then it would require a very accurate knowledge of all the circumstances to enable us to be certain whether the appearance of the eharacter is not due to the action of some external influence on the parent, either during development or in the adult state, rather than to a change arising within the reproductive cells. Instances are frequently brought forward which have been supposed to afford evidence of the transmission of mutilations from parent to offspring; but such a transmission must, from the nature of the case, always be extremely difficult to prove, and the majority, at least, of such cases are found, on a careful analysis, to be capable of other interpretations. On the other hand, though well-established cases of the inheritance of mutilations would greatly support the doctrine that acquired characters are transmissible, the negative results that have attended certain experiments on mutilations are of little value in the direction of proving that extrinsic variations cannot be transmitted, since, as has already been pointed out, such experiments in mutilation cannot be said to reproduce the conditions under which an extrinsic variation might be supposed to 
be transmitted; the mutilation is instantaneous; the variation must be supposed to be the result of long-continued action, which, it might be expected, would have a sufficiently profound effect to engraft it permanently on the organism.

It should be pointed out here that there is no absolutely hard and fast line to be drawn between the intrinsic and extrinsic variations, since changes in the sexual cells may very well be due, directly or indirectly, to influences exerted from without. The material from which reproductive cells may subsequently be fashioned is, in plants and in many animals, in such close and intimate union-so far as can be seen-with the other protoplasmic elements of the organism, that it seems highly probable that influences affecting the latter may in many cases affect also the former.

Another question that presents itself in conncction with heredity is: Can any special part of the germ-cell be fixed upon as the part spccially concerned in hereditary transmission? Is it by means of the nucleus alone that transmission takes place, or does the cytoplasm take a share in the process? The complicatcd changes which the nucleus undergoes during mitotic division (Vol. I., p. 18), with the definite form and (for each species) constant number of the chromosomes, and their precise halving during the process, tell strongly in favour of the view that the nucleus is the vehicle of transmission rather than the, apparently, less highly differentiated cytoplasm. But such evidence is far from amounting to positive proof.

To determine this point many series of embryological cxperiments have been carried out. Should it prove possible to fertilise by means of a sperm an ovum from which the nucleus had previonsly been removed, and as a result to obtain an embryo, it might be possible, by a process of exclusion, to get some light on the influence exerted on normal development by the nucleus of the ovum. For such experiments sperms and ova of the same species are not well adapted, since the differences between the individuals of a species, especially in the early stages, are not of a sufficiently strongly marked character to permit of any definite conclusions being arrived at regarding inheritance from one parent as against the other. Recourse has, therefore, been had to crossing bctween distinct species or distinct genera, or even between the members of distinct classes. In such cxperiments the Echinoderms have proved capable of affording the most convenient material, and have been very largely employed. It has been found to facilitate successful crossing between distinct kinds of Echinoderms if the ova experimented with are first treated by certain methods which have been found to prepare the way for the process of artificial parthenogenesis briefly referred to in the Introduction (Vol. I., p. 22). The ova so treated are then shaken violently in a tube, 
with sea-water, until they become to some extent broken up. From the fragments such large pieces as are found not to contain nuclei are picked out, and the sperms of the second kind of Echinoderm are added to the water in which these non-nucleated fragments are contained. In many cases it is found as a result that a sperm enters a non-nucleated fragment and an embryo becomes formed. It now has to be determined how far this embryo resembles and differs from the embryos of the two species from which the ovum and the sperm respectively have been derived. This is always a matter attended with a considerable amount of difficulty, since such embryos can rarely be reared beyond the gastrula stage. But evidence seems to have been obtained by means of this method that the nucleus has not a monopoly in the transmission of the parental characters, since in such experiments maternal features do appear in the embryo, and these, in the absence of a female nucleus, must have been transmitted by the agency of the cytoplasm.

Evidence tending in the same direction has been obtained as a result of experiments on the ova of Ctenophora (Vol. I., p. 214). If, before these are fertilised, a definite area of the cytoplasm be removed without the nucleus being interfered with in any way, the embryo which develops after fertilisation presents deficiencies in the organs of definite areas corresponding to the parts which have been removed.

It has been urged in connection with the question of heredity that what is transmitted from generation to generation is not so much matter as energy. The quantity of matter is always relatively small ; the important fact appears to be that this relatively small particle carries with it potential energy sufficient to effect the structural changes which precede the beginning of the process of assimilation, and to at least initiate that process. But we can hardly imagine a succession of complicated and very definite changes of structure, such as are involved in the development of an animal, taking place unless the germinal matter or germ-plasm in which they originate has a correspondingly complicated and definite structure.

The oosperm, having the faculty of reproducing the entire animal without (in many cases) any further influence emanating from the parent, must contain within itself something to represent each of the parts-even each group of cells-of the adult body. The oosperm of a Frog, for example (p. 280), simple though its structure appears to be, must contain potentially within itself all the characteristics of the adult animal, and not only these, but the characteristics of each successive stage in the formation of the tadpole and its metamorphosis into the adult Frog. Attempts have been made to explain how it is that the reproductive cells acquire this reproductive capacity. One of the most interesting of these is a theory which is termed pangenesis, the origination of which is due to Darwin. According to this theory, the cells of the various parts 
of the body throw off minute ultra-microscopic particles or "gemmules," and these find their way by various channels to the developing reproductive cells, in which they accumulate until each reproductive cell contains gemmules representing all parts of the body. When development takes place each gemmule develops into the part corresponding to that from which it has been derived.

If this theory afforded a true explanation of the facts of reproduction, there would necessarily be accummlated in the ovum gemmules representing not only every part of the body of the adult, but also every stage in the development of the embryo, and (since we see ancient ancestral characters occasionally reverted to) something to represent the special peculiarities of former generations. Now, it is a question if such an accumulation of gemmules, each necessarily several times the size of a chemical molecule, would not form a mass very much larger than an ovum. Such a doctrine would, moreover, hardly appear to be necessary in order to explain the facts. The accumulation in the ovum of the hereditary tendencies (as we may call them) may only in part take place during the lifetime of the individual : a good part of them-all, perhaps, except such as have been more recently acquired-might be contained in the ready-formed germinal material handed down from previous generations.

Against a hypothesis of pangenesis such as was formulated by Darwin, the mode of reproduction of many plants tells more strongly perhaps than any of the facts derived from the animal kingdom. Many of the higher flowering plants, for example, are capable of being propagated by means of a cutting of the stem or root, or even by a leaf. As the new plant developed from the cutting gives rise to flower and fruit, the cutting must contain germinal matter; and germinal matter must, therefore, be diffused throughout the cells of such a plant. Pangenesis, unmodified, would require that in such a case a large proportion of the ordinary cells of the plant should receive gemmules derived from all parts.

It is a moot point whether it is possible that any influence (such as is presupposed in pangenesis) can pass from the cells of the various parts of the body to the ova-whether there can be any communication of substance carrying with it tendencies to be transmitted to the next generation. It is certain, however, that an influence of a centrifugal character is exerted by the sexual cells. The absence of ovaries or testes has, in many cases, a marked effect on certain of the characters-an effect on the development and form of certain of the parts. This is seen not only in higher animals (Mammals and Birds), but also among some lower forms. In certain Crabs, for example, the presence of Sacculina, a parasitic Rhizocephalan nourished at the expense of the testes, which become destroyed, produces a very marked alteration in some of the external features. But, while this is the 
case, an influence exerted in the opposite direction-an influence transmitted from the other parts to the germ-cells-has not been proved, and from the nature of the case perhaps cannot be directly proved. Such an influence, it is hardly necessary to add, must be presupposed if we assent to the doctrine of the inheritance of acquired characters.

Rules or Laws of Heredity.-As in the case of the experiments referred to on p. 641, by which it has been sought to determine the respective values of nucleus and cytoplasm as bearers of the hereditary qualities, so also in the majority of the experiments designed to determine the laws by which the inheritance of paternal and maternal characters is regulated, it has been necessary to have recourse, not to fertilisation between individuals of the same variety, but to crossing between distinct varieties or between distinct species with the production of bastards or mongrels (varietyor species-crosses), since it is only in cases in which the differences between the maternal and paternal characters are strongly marked that it is possible to trace these characters in the descendants.

It is found that in such experiments in crossing between varieties the result may take one or other of the three following courses. In the first place the progeny may be intermediate in character between the two parents: the paternal and maternal characters, that is to say, may be mixed or blended without any tendency to the predominance of either: a cross between a black and a white variety, for example, in such a form of hybridisation becomes a grey. In a second set of cases, which are the rarest of all, the offspring exhibit paternal characters in one part or set of parts and maternal in another: thus a cross between a black variety and a white yields a piebald or a mottled black-and-white. 'This is the so-called mosaic form of bastard-inheritance. Lastly there are certain cases in which there is no blending and no mosaic formation, but the paternal and maternal characters remain separate, and appcar, or fail to appear, in the progeny in a certain regular order. This is the alternating or Mendelian form of bastard-inheritance.

It is with this last form of bastard-inheritance that the theory of Mendel, a product of the middle of the last century, but only comparatively recently re-discovered and given due recognition, is concerned. Mendel's original experiments were mainly with varieties of garden-peas. When he crossed two varieties he found that the offspring all presented the characters of one of the parent forms. Thus in the case of a cross between a tall and a short variety-whether the short was fertilised by the pollen of the tall or the tall fertilised by the pollen of the short-the progeny were tall : in a cross between yellow-seeded and green-seeded varieties the products were all yellow-seeded. Of the two opposed characters, that which reappears in the offspring is known as the dominant, that which does not appear as the recessive character: 
the varieties presenting these characters are accordingly spoken of respectively as either dominant or recessive. When the hybrids, all showing the dominant character, produce a second generation by self-fertilisation, or fertilisation among themselves, there is a reappearance of the recessive character in a certain fixed proportion of the progeny. In this second generation one quarter are pure recessives, one quarter pure dominants, and the rest of mixed character. The pure recessives in following (inbred) generations always remain true to the recessive character-the dominant having evidently become eliminated from their constitution; and the same holds good, mutatis mutandis, for the dominants. The intermediate forms present the dominant character, but when inbred they behave exactly like the original hybrid, that is to say, the progeny consist of pure dominants (a quarter), pure recessives (a quarter), and mixed or intermediates (a half). In future generations these proportions are regularly maintained.

It is inferred from their behaviour in inheritance that the Mendelian characters occur in pairs, the members of which have a fixed reciprocal relationship to one another, one of them being usually dominant, as in the case of tallness and shortness in the first example referred to: two such characters are said to be allelomorphic, or reciprocating, with regard to one another. The term segregation is applied to the process by which each becomes separated out in the pure dominants and pure recessives of the second and succeeding generations.

An important point in connection with the bearing of Mendelian inheritance in plants and animals on the problems of evolution is that the former provides a means by which new variations or mutations when they arise may be at once fixed and become perpetuated.

Orthogenesis.-The fact that, as shown by the evidence afforded by both existing and extinct forms, organisms vary in such a way as to follow definite lines leading to special adaptations (and sometimes to excessive development of parts) is held not to be explainable by a theory of selection of slight variations the direction of which is under no known guidance. This definiteness in the direction of evolution-orthogenesis -is a fact in nature of which there is evidence on all sides. In nearly all groups and nearly all systems of organs there is evidence of progressive development such as cannot be supposed to be due simply to the fortuitous appearance and selection of the Darwinian variations. A nervous system, simple, diffused, and superficial in the lower members of a group, becomes more highly elaborated, more complex, more deeply placed, in the higher. Light-perceiving organs, mere groups of pigmented elements, and nerve-cells with refractive bodies, in the lower forms, become represented in the higher by complex eyes with elaborate mechanism for the reception of images of external objects. Appen- 
dages undergo progressive modification, so that each pair becomes specially adapted for the performance of particular functions. Moreover, parallel and evidently independent lines of orthogenetic development are in many cases traceable in separate groups. As examples may be mentioned the series of stages in the development of complex eyes from simple rudiments, which are observable in the Annulata, the Arthropoda, and the Chordata: the parallel and quite unconnected stages in the reduction of the digits, leading to the greater perfection of the limbs as running organs, to be traced in the Perissodactyle and Artiodactyle series of the Ungulata. In many cases such orthogenetic development has led to excessive growth of parts - growth beyond the requirements of the organisman excess which has sometimes, apparently, led to extinction.

Since natural sclection has been judged by many to be inadequate to account for such straightforward progress in organisms and organs, various other theories of orthogenesis have been put forward. Such of these as merely postulate the existence in organisms of a principle or tendency to develop towards a more perfect condition fail to reach the standard of admissible scientific theories. Such a supposed tendency is not merely analogous to the tendency of the young organism to grow into the adult form-a phenomenon sufficiently difficult to account for by any nexus of causes and effects known to us: it must be something much more, since it must be not merely a tendency to repeat what has been received in inheritance, but must be in a highly important degree prophetic -must, in fact, be a tendency to develop to a point beyond that to which the hereditary impulse reaches. Other theories of orthogenesis rely upon the action of the environment for bringing about the results observed: these have to encounter the same fundamental difficulty as the Lamarckian theory itself-the difficulty of explaining how changes in the parents due to the effects of the environment can be impressed on the germ-cells in such a way as to become transmitted to the next generation. But experimental evidence in favour of the heritable character of various changes caused by external factors has been recently accumulating. And many of those who concern themselves with the study of evolution at the present day, while admitting the absence of any satisfactory theory of the mode of transmission to the germ-cells of changes of organisation in the adult of the nature of acquired characters, are yet inclined to the view that such a transmission does occur, and that without it it is quite impossible to account for the definite adaptive developments that have taken place, especially since there seems to be no convincing explanation, apart from such transmission, of the mode of origin of the first beginnings of structures destined, when further developed, to be of vital importance to the organism, but in their early stages not of sufficient value to be capable of determining its survival or extinction. 


\section{SECTION XVI}

\section{THE HISTORY OF ZOOLOGY}

ZooLogy, like other branches of Natural Science, has had two lines of progress, observation and generalisation. Without accurate and detailed knowledge of the facts and phenomena of animal life and structure, all theories of classification or of origin are so much idle speculation: in the absence of philosophic spirit suggesting hypotheses of greater or less magnitude, the mere accumulation of facts is an empirical and barren study.

Zoology as a science, therefore, can hardly be said to have existed until a sufficient proportion of the facts relating to animals had been observed and recorded accurately and systematically, and until some attempt had been made to classify these facts and to arrange animals into larger and smaller groups according to some definite plan.

This being the case, it may be said that the common knowledge of animals possessed by mankind in all ages, and constantly being developed and extended by lovers of external nature and by anatomists working from the medical standpoint, first became scientific and evolved itself into a system over 200 years ago, when John Ray, an English clergyman, first grasped the idea of species and published the earliest classification of animals founded upon anatomical characters. Although soon overshadowed by the greater genius of Linnæus, Ray may safely be called the father of modern zoological science, the only serious precursor of his Synopsis methodica animalium, published in 1693, being the voluminous De differentiis animalium of Edward Wotton, printed nearly 150 years earlier.

But although Zoology, as a science, was practically non-existent up to the period referred to, much valuable knowledge of animals had been accumulated, and was, as it were, merely waiting to be systematised. As in other branches of knowledge, the first steps were taken by the Greeks, and, in philosophical grasp, the zoological writings of Aristotle (384-322 B.C.) are far in advance of those of all other students of the subjects up to the times of Wotton and 
Ray. His treatises, especially The History of Animals, The Generation of Animal, and The Parts of Animals, contain an immense body of facts, many of them singularly accurate, others as curiously incorrect, a circumstance which no one will wonder at who, with all modern resources at his elbow, has tried to break fresh ground in any department of Zoology. Although he propounds no definite system of classification, he clearly recognises many of the more important animal groups, or, as he calls them, "genera." Vertebrata, for instance, are spoken of as animals with

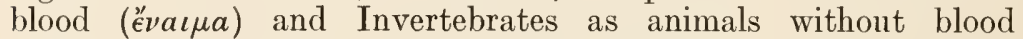
$\left(\ddot{a}_{\prime \prime}^{\prime} \iota \mu a\right)$, colourless blood not being recognised as such. Among animals with blood are included Viviparous Quadrupeds (Mammals), Birds, Oviparous Quadrupeds (Reptiles and Amphibians), Cetaceans, and Fishes: among bloodless forms, Malakia or soft animals (Cephalopods), Malacostraca or soft animals with shells (the higher Crustaceans), Entoma (Insects, Arachnids, Myriapods, and the higher Worms), and Ostracodermata or shelled animals (Echinoderms, Cirripedes, Pelecypods, Gastropods, and Tunicates). Starfishes, Medusæ, and Sponges are also referred to.

In the then existing state of knowledge it was impossible that even so profound a philosopher as Aristotle could erect a science of Zoology. No standard of nomenclature was established; there was no clear idea of what constitutes a species: in matters of structure, no distinction was drawn between nerves and tendons: in physiology the vessels and tendons were looked upon as the organs of movement, the muscles being considered as mere packing. Obviously, anything like real progress was barred by ignorance of animal structure and function, and it was absolutely necessary that exact anatomical knowledge should precede any thing approaching to successful generalisation.

It is, therefore, hardly to be wondered at that, up to the time of Ray, scientific Zoology owes more to those anatomists and physiologists whose main object was to advance the study of Medicine, than to the naturalists in the ordinary sense of the word. With the exception of the works of Galen (born A.D. 130), which contain numerous observations on the Anatomy of Mammals, anatomy, as well as Zoology in the broad sense, was practically at a standstill from the time of Aristotle to the sixteenth century, when Vesalius, by his observations, chiefly on the human subject, raised anatomy to a degree of accuracy hitherto undreamt of ; and Coiter, Bellonius, and Fabricius ab Aquapendente resumed the study of comparative anatomy, dormant since Aristotle. Somewhat later-in 1645-Severino published his Zootomia democretcea, the first book devoted exclusively to the general subject of comparative anatomy.

During the same period the general knowledge of animals was increasing, and a distinct epoch is marked by the learned and, fo 
the time, exhaustive Historia animalium of Conrad Gesner, published in 1551-58, and consisting of 4,500 folio pages, with numerous illustrations, some of them of considerable merit, some wonderfully inaccurate; some depicting various fabulous monsters, such as Winged Dragons, many-headed Hydras, and crowned Basilisks, the existence of which was not yet thoroughly discredited. The work is, however, rather an encyclopædia than the exposition of a science : it contains $n$ general ideas; there is still no conception of the subordination of groups, and no exact naming either of animals as a whole or of their various parts. Five chief groups of animals are recognised: Viviparous Quadrupeds, Oviparous Quadrupeds, Birds, Aquatic Animals, and Serpents. Within these divisions the various animals are described without any attempt at grouping. Among Aquatic Animals, for instance, Fishes, Amphibia, Cetacea, Molluses, Crustacea, Echinodermata, and Seaserpents are included.

In the seventeenth century great strides were made both in knowledge of structure and function, in generalisation, and in methods of investigation. Especially famous and fruitful-indeed one of the greatest scientific events of all time-was the discovery of the circulation of the blood, made by William Harvey in 1616, and announced in 1628 in a small pamphlet Exercitatio anatomica de Motu Cordis et Sanguinis. He demonstrated fully, partly by dissections, partly by experiments on living animals, the action of the heart as a pumping mechanism, the nature of its valves and of those of the veins, the presence of blood-not air, as was then supposed-in the arteries, the cause of the pulse, and the whole course of the circulation so far as it could be known previous to the discovery of the microscopic capillaries. Of hardly less importance is Harvey's embryological work: he made extended observations on the development of the Chick, and in his Exercitutiones de Generatione Animalium (1657) declared that all living things arise from a primordium, or ovum, and propounded the doctrine of epigenesis, according to which development is a process of gradual differentiation of the primordium, whereby " out of the inorganic arises the organic, out of the similar the dissimilar." The primordium itself he considered might " proceed from parents, or arise spontaneously, or out of putrefaction."

Harvey worked with no optical aid beyond a simple lens, and it is not surprising that his results are incomplete and often inaccurate. His successors had the advantage of the compound microscope, invented by Hans and Zacharias Janssen about 1590-1600, and sufficiently improved during the course of the seventeenth century to be an important engine of research in the hands of the earliest microscopists, Malpighi in Italy, Leeuwenhoek and Swammerdam in Holland, Robert Hook and Nehemiah Grew in England. Malpighi made numerous histological 
discoveries, with some of which-such as the Malpighian capsules of the kidneys and the Malpighian vessels of Insects-his name is still associated. He was also the first to study the development of the Chick under the microscope, and was one of the earliest supporters of the theory of preformation, ${ }^{1}$ according to which all the parts and organs of the adult are present in the germ, so that there is no differentiation, but only an unfolding. Leeuwenhoek discovered blood-corpuscles, striated muscle-fibres, dentinal canals, and epiderm-cells, observed the circulation of the blood in the tadpole's tail, and described many of the lesser forms of life, such as Infusoria, Rotifers, and Hydra. Swammerdam investigated the anatomy of Insects and Molluses, and the metamorphosis of Insects, and described the three "sexes" of Bees. The researches of Hook and Grew were mainly botanical ; both they and Malpighi discovered in the tissues of plants little spaces with firm walls and full of fluid; these they called cells, thus taking the first step in the structural analysis of the higher organisms.

Another discovery of fundamental importance was made in 1677 when Louis de Hamen observed and described the sperms of animals. These were at first thought to be the young, which only required to be nourished in the egg to grow into the embryo or fœtus, and were therefore considered to disprove the theory of the ovulists-such as Harvey, who made the egg the origin of the new generation-in favour of that of the spermatists, who believed the whole material to be furnished by the male parent.

Belonging also to this period are Redi's experiments on generation, in which he began the work of establishing the doctrine of biogenesis, according to which organisms originate only from preexisting organisms, and of demolishing that of abiogenesis, or "spontaneous generation," which, maintained from the time of Aristotle onwards, held that Flies, Lice, Worms, and other animals were directly generated in mud, putrefying flesh, dung, \&c., having, therefore, no living progenitors. Redi's contribution to this question lay in proving, for the first time, that the maggots, " bred" in putrefying meat, were the products of eggs laid thereon by Flies.

Thus the seventeenth century saw a great advance in the knowledge of animal structure and function, and the way was paved towards a rational classification. As we have already seen, Ray, towards the end of the century, gave Zoology as a whole a scientific form; he first grasped the ideas of species and of specific characters, acknowledged anatomy as the basis of classification, and introduced a greatly increased precision in the definition of species and other groups, and in terminology. He had, however,

1 Often known as the theory of evolution. As, however, the latter word is now universally used in a different sense, it is advisable to drop it in this connection, and to employ the synonym preformation. 
no clcar idea of genera, his genera being rather what we now call orders or families, and he showed an undue conservatism in retaining, as far as possiblc, the groups of Aristotle. His general classification of animals is as follows :

I. Animals with (red) blood [Verlebrata].

1. Respiration pulmonary.

A. Heart with two ventricles.

(a) Viviparous.

i. Aquatic (Cetacea].

ii. Terrestrial [olher Mammatia].

(b) Oviparous [Birds].

B. Heart with one ventricle.

Viviparous Quadrupeds and Serpents [i.e., Reptitia and Amphibia].

2. Respiration branchial [Fishes].

II. Animals without (red) blood [Inverlebrala].

1. Majora.
A. Mollia [Cephalopoda].
B. Crustacea.
C. Testacea [Gastropoda and Pelecypoda].

2. Minora.

Insecta [Insecla, Arachnida, Myriapoda, and Vermes].

It will be noticed that, while the classification of Vertebrates is fairly natural, being founded upon the rock of anatomy, the arrangement of Invertebrates is no advance upon that of Aristotle: the two main divisions depend upon mere size; and Crustacca, separated from the rest of the Arthropoda, are interposed between Cephalopods and the remaining Mollusca. In association with Ray must be mentioned his friend and fcllow-worker Francis Willughby, who made extensive contributions to Zoology.

The eighteenth century saw the imperfect efforts of Ray developed, and in some respects perfected, by Carl Linné, or Linnæus, universally recognised as the founder of modern systematic Zoology-or more accurately Biology, since his reforms equally affected Botany. Born in Sweden in 1707, two years after Ray's death, he published the first edition of lis Systema Naturu, in 1735 , as a small pamphlet. The twelfth edition (1766-68) was in three volumes, and was the last to receive the author's corrections, but from materials left at his death in 1778 an authoritative (thirteenth) edition in ten volumes was prepared by J. F. Gmelin.

It was Linnæus who first recognised the value of groups higher than species--genera, orders, classes, \&c., and employed them in a definite and uniform way, with due subordination of one to the other; it was he who invented binomial nomenclalure, the advantage of which in promoting precision in systematic work 
it is impossible to overestimate. He gave each species a brief diagnosis in Latin, so that any naturalist versed in his system could recognise whether an animal or plant which came under his notice was already described or not. In this way he, as it were, pigeonholed the facts of Biology, and so made the determination of the proper place of any new fact a comparatively simple matter. By universal consent, the Systema Naturce is taken as a starting-point by systematists. It is customary to place after the name of a species the initial or abbreviated name of the writer by whom the species was first distinguished and named. For instance, the Bass, a common British Teleost, was named Perca labrax by Linnæus. In 1828, Cuvier and Valenciennes, in their great work on Fishes, recognised that it was generically distinct from the Perch, and, retaining the generic name Perca for the latter, called the Bass Labrax lupus. In 1860, further investigations into the Perch family necessitated placing it in the genus Morone, and, according to the law of priority, the specific name lupus gives place to labrax, the latter lhaving been applied by Linnæus. The Bass is therefore correctly called Morone labrax, Linn., the more usual name, Labrax lupus, Cuv. and Val., becoming a synonym. In deciding all such questions of priority, the tenth edition (1758) of the Systema Nature is taken as a starting-point: all species distinguished by Linnæus, and not subsequently split up into two or more species, are distinguished by the abbreviation $L$. or Linn. For instance, Canis familiaris, Linn. is the Domestic Dog, Passer domesticus, Linn. the House Sparrow ; and names given by the older naturalists are neglected unless endorsed by Linnæus.

In many respects the system of Linnæus was eminently artificial ; he relied too much on single characters in classification, and did not take the totality of structure into sufficient consideration. He divided the aninal kingdom into the following six classes:-

1. Mammalia.

2. Aves.

3. Amphibia [including Reptilia and Amphibia].

4. Pisces.

5. Insecta [including all the Arthropoda].

6. Vermes [including Molluscs, Worms, Echinoderms, Coclenterates, and Protozoa].

It will be seen that all the classes are of natural groups, with the exception of the last, but that they are far from being of even approximately equal value. The first four are what we still call classes, but there is no attempt to unite them into a single group of higher order; and in this respect the classification of Linnæus falls behind that of Ray, who recognised the phylum Vertebrata under the name of animals with blood. The fifth class, on the other land-that of Insecta-is the equivalent of an entire phylum, 
while under the head of Vermes are included all the phyla recognised at the present day, except Chordata and Arthropoda.

Other naturalists of the eighteenth century must be briefly referred to. Bonnet introduced the idea of a "scale of beings" (échelle des êtres), conceiving the true classification to be a linear one, passing in a single series from the lowest to the highest forms. This conception was opposed by Pallas, who introduced the true conception of representing the relationships of the various groups nnder the form of a much-branched tree. Spallanzani made numerous investigations on reproduction, and, together with Bonnet, Buffon, and Haller, strongly supported the doctrine of preformation already referred to. Haller summed up the position by stating emphatically that there was no such thing as development or differentiation, no part of the body being made before another, but all parts simultaneously created. It followed, as a natural corollary from this view, that the germ destined to give rise to an animal-i.e., the ovum according to the ovulists, the sperm according to the spermatists-contained within itself the germ of the next generation, this of the next, and so on, ad infinitum, so that the first created male or female of each species contained within its sperms or ova the germs of all frture generations, enclosed one within the other, like a nest of Chinese boxes. Buffon, as the result of numerous experiments, came to the conclusion that the ovary secretes a seminal fluid containing moving particles analogous to sperms, and, from this erroneous observation, framed a theory which is an interesting anticipation of Darwin's Pangenesis (p. 642) - namely, that organic particles, derived from all parts of the body, occur in the seminal fluids of the two sexes, and that the union of these in the uterus "determines them to arrange themselves as they were in the individuals which furnished them."

The theory of preformation (as then understood) was practically demolished, and that of epigenesis, or new formation, established on a firm basis, by Caspar Friedreich Wolff, who at the age of twenty-six-in 1759 -gave the most accurate account of the development of the Chick hitherto known, and showed clearly that there was no preformation of the various parts, but a gradual differentiation from a layer of organised particles, or, as we should now say, from a cellular blastoderm.

Another great eighteenth century name is that of John Hunter, the most profound comparative anatomist and physiologist of his time. He was not a zoologist in the narrow sense of classifier, but his exquisite investigations on the various systems of organs and their functions throughout the animal kingdom furnished the science with a foundation of wide and exact knowledge which was of far more importance than the most cunningly devised system of classification. Important anatomical investigations were also made during this period by Vicq d'Azyr, who enunciated the 
principle of serial homology ; by Peter Camper, who investigated the pneumaticity of the bones of Birds, and was the first to apply exact methods of measurement to the human skull; by Alexander Monro, who greatly advanced our knowledge of the anatomy of fishes; and by Poli, whose Testacea utriusque Siciliae is the most famous of the older works on Mollusca. And in the domain of out-door zoology-the study of the actual life of animals with but little regard to their structure or classification, or to the broader scientific questions connected with them-special mention must be made of Gilbert White, whose Natural History and Antiquities of Selborne is a classic both in science and letters.

The latter part of the eighteenth century is also specially remarkable for the publication of the earliest scientific speculations on the origin of species. The idea of evolution is to be found in the works of more than one of the great Greek and Roman philosophers, such as Empedocles (495-415 B.c.) and Lucretius (99-55 в.c.); and the writings of some of the Fathers of the Church, such as Augustine (A.D. 353-430) and Thomas Aquinas (1225-1274), seem to show that they had no objection to "derivative creation," or evolution under direct Divine superintendence. But by about the middle of the sixteenth century the idea of the immutability of specially created species bad hardened into a dogma which it was unsafe to question; and, this state of things continuing, the earliest of the great evolutionists, Buffon, felt himself obliged to qualify all his speculations with a declaration, sincere or ironical, of his belief that species were immutable. Linnæus, reckoning all higher groups as subjective, contended for the real existence of species, saying "we recognise as many species as were originally created," and this opinion was held by the vast majority of naturalists, not only of his own time, but up to within forty or fifty years of the present day.

Buffon, born in the same year (1707) as Linnæus, was, in his methods and ideas, the exact opposite of his great systematising contemporary. He wrote charming accounts of the external characters and habits of animals, but declined to classify them, on the ground that all arrangements of the kind were arbitrary, and that it was easier, more useful, and more agreeable to consider the lower animals in relation to ourselves. On this principle, he begins his Histoire naturelle with Man, then takes up the various domestic Mammals, and afterwards proceeds to consider the less familiar forms. But he was essentially a philosophical zoologist ; besides enunciating a theory of heredity, he grasped the idea of homology, endeavoured to explain the facts of geographical distribution, and, in a tentative and guarded way, admitted the mutability of species, and advanced a hypothesis of their origin. His speculations refer mainly to the modification, or, as he calls it, degeneration, of domestic animals, and he sums up his position 
as to the factors of the process by saying " the temperature of the climate, the quality of nutriment, and the ills of slavery, these are the three causes of change, of alteration, and of degeneration in animals." In other words, he supports the theory of the direct action of the environment.

A bolder and more consistent evolutionist than Buffon was his contemporary, Erasmus Darwin (1731-1802), grandfather of the author of the Origin of Species. As a competent critic has said, "he was the first who proposed and consistently carried out a well-rounded theory with regard to the development of the living world." In his Zoonomia, published in 1794-6, after summarising the extraordinary adaptations to be seen in the animal kingdom, he asks, "Would it be too bold to imagine that all warm-blooded animals have arisen from one living filament [he was a spermatist] which the great First Cause endued with animality, with the power of acquiring new parts, attended with new propensities, directed by irritations, sensations, volitions, and associations; and thus possessing the faculty of continuing to improve by its own inherent activity, and of delivering down those improvements by generation to its posterity, world without end ?" And a little later he inquires: "Shall we conjecture that one and the same kind of living filament is and has been the cause of all organic life?" He anticipated Lamarck in the importance he attached to the principle of use and disuse, expressed his belief in the inheritance of acquired characters, and recognised the importance of sexual selection.

The study of Zoology was also greatly advanced during the eighteenth century by the voyages of Cook, Bougainville, and others. New countries were explored, the peculiarities of their faunæ recorded, and valuable data accumulated for the study of distribution. In this connection the names of Sir Joseph Banks, Solander, and the two Forsters-all attached to Cook's expeditions, of Sparrmann, and of Sir Hans Sloane, may be specially mentioned. The last-named was one of the greatest of collectors, and the founder of the British Museum.

The beginning of the nineteentl century was a period of great zoological activity, distinguished by the work of some of the most prominent leaders of the science.

J. B. P. A. de Lamarck (1744-1829) was not only a distinguished general zoologist and palæontologist, but may also be looked upon as the chief of the pre-Darwinian evolutionists. In his Philosophie Zoologique, published in 1809, he completely rejected the idea of the fixity of species, and endeavoured to explain the transformation of one form into another by the operation of known causes; of these he attached most importance to the principle of use and disuse, and he was a firm believer in use-inheritance. He was a uniformitarian in Geology, believing that the history 
of the earth and of its past inhabitants is to be explained by the action of the causes seen in operation to-day, and not by invoking great catastrophes or cataclysms by which changes of vast magnitude were suddenly produced. He considered, also, that the transformation of species took place by slow, orderly changes, Nature requiring only matter, space, and time in order to effect her various changes. He introduced the terms Vertebrata and Invertebrata, and, in the same year as Treviranus (1802), proposed the term Biology for the whole science of living things.

Lamarck at first believed in a linear classification of animals, but afterwards adopted the earliest known branching or phylogenetic classification - a crude attempt, but interesting as being the first of its kind. It is as follows :-

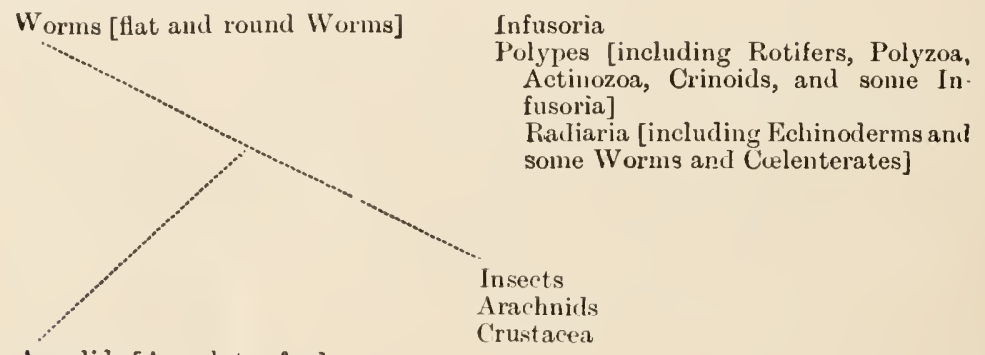

Annelids [Annulata, \&c.]

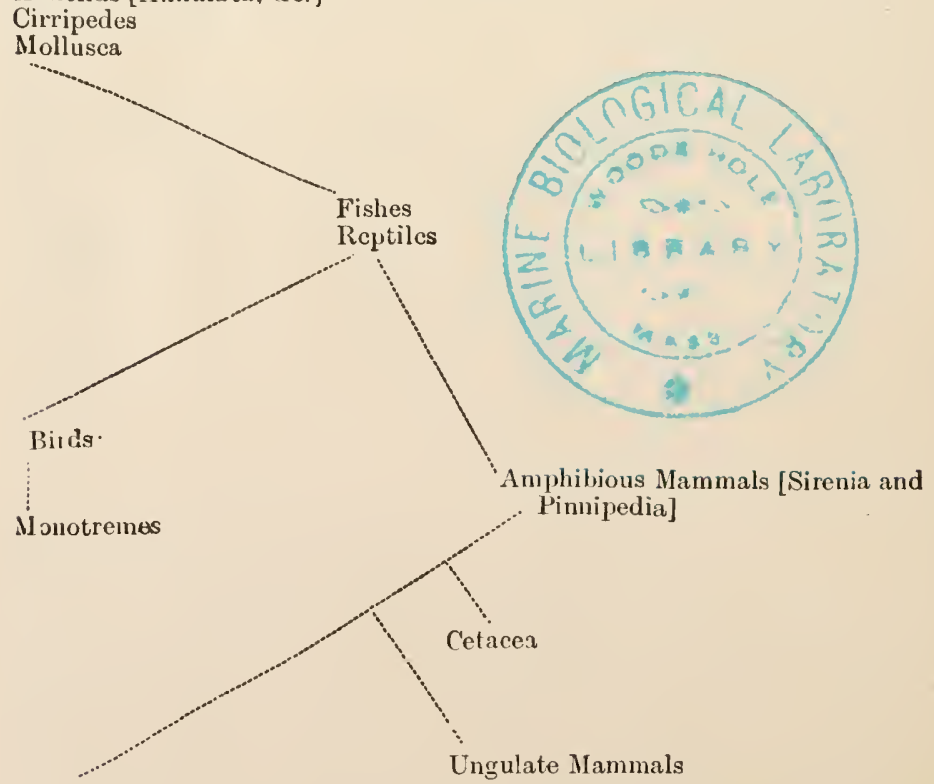

Unguiculate Mammals [Edentata, Rodentia, Marsupialia, Inscetivora, Carnivora, Chiroptera, and Primates]. 
The hypothesis of evolution was also supported by Lamarck's contemporary, Etienne Geoffroy St. Hilaire, who denied use-inheritance and considered the direct action of the environment as the sole cause of transformation. He also differed from Lamarck in believing in the occurrence of sudden changes, e.g., in the possibility of the emergence of a fully formed Bird from a Reptile's egg. In systematic zoology he established the orders Monotremata and Marsupialia: the members of the latter group had hitherto been distributed among Rodents and Primates.

Another keen supporter of evolution was the great poet Goethe (1739-1832), who also introduced the word Morphology, and made important contributions to the department of science thus named. He propounded the vertebral theory of the skull, presently to be referred to (p. 659), recognised the importance of vestigial organs, and predicted the presence of a premaxilla in Man-the absence of that bone in the adult human skull being hitherto considered as distinctively separating the genus Homo from the other Primates.

That the views of Lamarck and the other evolutionists produced so little effect upon contemporary science is largely due to the great and far-reaching influence of Georges Cuvier (1769-1832), one of the greatest of comparative anatomists, whose views dominated zoological science for half a century. He propounded the fruitful principle of correlation, according to which peculiarities in one part of the body are always associated with equally characteristic features in other parts-e.g., the ruminating stomach with cloven hoofs. He rejected the idea of a scale of being or unity of type, and, in his great work, the Règne Animal, abandoning the linear classification, divided animals into four Branches (embranchements), each with its own plan of organisation and independent of the rest. This conception, though not absolutely correct, marked a great advance in classification, as the following table shows.

Branch 1. Vertebrata.

"2. Molluscs [including Tunicata, Brachiopoda, and Cirripedia, as well as the true Mollusca].

3. Articulata [including Arthropoda and Annulata].

4. Radiata [including Echinodermata, Polyzoa, Nemathelminthes, Platyhelminthes, CœIenterata, Sponges, and Protozoa. The Rotifera are placed among the Protozoa, and Bacteria and the pedicellariæ of Echinoderms are also included].

Here, it will be seen, the Vertebrata as a whole, and not the separate classes of that phylum, are considered as the equivalent of one of the great invertebrate sub-divisions: the Linnæan Vermes are broken up, Mollusca being elevated to the rank of a 
primary sub-division, and the articulated worms associated with Arthropods; while Echinoderms are grouped with Cœlenterata on account of their radial symmetry, and the imperfectly understood lower Worms, Sponges, and Protozoa are included in the same branch.

Cuvier may also be said to have created the science of Palæontology by his investigations of the Tertiary Mammalia of France. As long ago as the sixth century B.C., Xenophanes had recognised fossils as the actual remains of animals, but the usual view was that they were merely mineral productions ; and one of the earliest observers in modern times to perceive their true nature was Scheuchzer, at the beginning of the eighteenth century, who considered them as evidences of a universal deluge. Cuvier, as well as the English geologist William Smith (1769-1839), showed that the older fossils belonged to entirely different species, genera, and even families, from the animals existing at the present day, the differences being greater in the deeper than in the more superficial formations. In this way the idea of a definite succession of life in time was introduced. Cuvier and his followers rejected, however, the notion of any genetic connection between the inhabitants of successive geological periods, and considered that the fauna of each epoch was exterminated by some cataclysm or convulsion of nature, and the earth subsequently re-peopled by a fresh creative act. This catastrophic view of the history of the earth received its death-blow in 1830-33, when Sir Charles Lyell (1797-1875) published his Principles of Geology-next to the Origin of Species the most famous contribution to natural science in modern times. By insisting on the evidences for contimuity in the history of the earth, he prepared men's minds for the idea of continuity in the history of its living inhabitants, and thus, more than any of the older evolutionists, paved the way for the reception of Darwin's views.

Apart from the work of Cuvier, the most important contributions to Zoology during the first half of the nineteenth century are in the domains of histology and embryology. In 1838 the cell-theory, according to which all parts of the body are built up either of cells or of tissues derived from cells, was propounded first for plants by Schleiden and shortly afterwards for animals by Schwann. Both, however, had an erroneous conception of the cell, considering the cell-wall as its essential part-whence the name cellula, a small chamber. But in 1846 the "plantslime," observed by Schleiden in the interior of the cell, was investigated with great thoroughness by von Mohl, and was called by him protoplasm, a name originally used by Purkinje in 1840 for the substance of which the youngest embryos of animals are composed. Albert Kölliker and others proved that animal-cells existed in which no cell-wall was present, and Dujardin showed 
that Amœbæ and other lowly organisms were formed entirely of protoplasm, or, as he called it, sarcode. These discoveries paved the way for the generalisations of Max Schultze and De Bary, that the essential constituent of the cell is protoplasm, and that the protoplasm of animals and plants is identical.

In embryology, the most important work of this time was that of K. E. von Baer, who, in 1827, discovered the ovum of Mammals. $\mathrm{He}$ also described three primary germ-layers-ectoderm, mesoderm, and endoderm-in the Vertebrate embryo, and showed that histological differentiation, or the formation of the permanent tissues from embryonic cells, proceeds hand in hand with morphological differentiation or the evolution of organs. He was thus led to enunciate what is known as von Baer's law, that development is a progress from the general to the special, and to frame the generalisation that embryos of animals belonging to various classes closely resemble one another in their earlier stages, but diverge more and more as development proceeds. His investigations led him to support Cuvier's view of the division of the animal kingdom into distinct and clearly separated types or branches.

It was during this period also that the real meaning of fertilisation was discovered, and the controversy between ovulists and spermatists finally set at rest. Artificial fertilisation had been tried in the last century, but up to 1842 the greatest physiologist and most accurate anatomist of his time, Johannes Müller, was unable to state positively whether or not the sperms were parasitic animalcules. But in 1843 Martin Barry observed the union of ovum and sperm in the Rabbit, and three years later Kölliker proved that the spernis were developed from the cells of the testis.

The period under consideration also saw the development of a school of speculative or deductive zoology. In 1790 Goethe conceived the idea that the skull of Vertebrates is made of modified vertebræ-in other words, that the skull is the highly differentiated anterior end of the backbone. This theory, which may be taken as a type of morphological speculation in the pre-evolutionary period, was re-enunciated and greatly elaborated in 1807 by Lorenz Oken, whose conclusions are worthy of mention, if only to show the dangers of the deductive method in natural science, and the lengths to which unbridled speculation may carry a presumably sane man. He did real service by demonstrating the secondary segmentations of the bony skull; the occipital segment being his "ear vertebræ," the parietal his "jaw vertebræ," and the frontal his "eye vertebræ." But he clearly went beyond the limits of legitimate speculation when he contended that the nasal cavity is a cephalic thorax and the mouth a cephalic abdomen; that the bones of the upper jaw are homologues of the fore-limbs, the lower jaw of the hind-limbs, and the teeth of the digits.

About the middle of the century the vertebral theory, freed 
from the most obvious absurdities of Oken, was resuscitated and developed by Sir Richard Owen (1803-93) in his Report on the Archetype and Homologies of the Vertebrate Skeleton, published in 1846. He also founded his generalisations on the structure of the adult or late embryonic skeleton in the higher groups, neglecting the unsegmented crania of Cyclostomes and Elasmobranchs and of the higher Vertebrate embryo. In his view, the limb-girdles are modified ribs, the shoulder-girdle belonging to the "occipital vertebra," while the limbs themselves are " diverging appendages," or uncinates.

Owen's chief services to Zoology were, however, his numerous and brilliant anatomical researches, such as those on Nautilus, on Apteryx, and on the structure and homologies of the teeth in the entire vertebrate series ; and his palæontological investigations, especially those on Archæopteryx, on the fossil Mammals of Australia, and on the Dinornithidæ and other flightless Birds. His conclusion, from the examination of a single fragmentary femur, that there had existed in New Zealand a Bird larger and heavier than the Ostrich-a fact then practically unknown-forms one of the most famous stories in natural history. His contributions to classification were not happy; he took the nervous system as the basis of his larger divisions, classifying Mammals, for instance, according to the presence or absence of a corpus callosum and of convolutions, and placing Man in a separate sub-class as the supposed sole possessor of a posterior cornu and hippocampus minor. He rendered great service to philosophical Zoology by pointing out the distinction between homology and analogy, and by the publication of his great text-book on the Anatomy and Physiology of Vertebrates.

The chief successor of Cuvier in France was Henri MilneEdwards (1800-85), who enunciated the principle of the division of physiological labour, and modified the classification of Cuvier in several particulars. He separated Tunicates from Mollusca proper and united them with Polyzoa under the name of Molluscoida, and he divided Vertebrates into Allantoidea and Anallantoidea, according to the presence or absence of an allantois ; in so doing he took the important step of separating Amphibia from Reptiles, a step in which De Blainville had been his only precursor. His learned Leçons de l'Anatomie et de la Physiologie comparées is a storehouse of information on the structure and functions of animals.

It was not until about the middle of the century that further increase in the knowledge of the lower animals resulted in the gradual dismemberment of Cuvier's unnatural Branch Radiata. Frey and Leuckart established the group Coelenterata, and placed Echinoderms apart; Wiegmann removed Rotifera from Protozoa to Vermes; Vaughan Thomson defined the Polyzoa, 
and Rudolphi, Leuckart, and von Siebold showed that the Flat-worms were in no sense Zoophytes. Sponges were considered by some as polypes, by others as plants; the current of water flowing in at the pores and out at the oscula was discovered by Robert Grant about 1820: later Bowerbank demonstrated the presence of cilia, and the full proof of their animal nature was made by the researches of Lieberkühn and Carter. The Foraminifera were classed as Cephalopoda until the 'thirties, when Dujardin determined their proper place by the discovery of the living protoplasmic body. Other important advances in classification were the separation of Cirripedia from Mollusca by Vaughan Thomson, and the withdrawal from intestinal worms of the parasitic Copepoda and of the Pentastomida. The Infusoria have also had a chequered history. Ehrenberg, in his magnificent work Die Infusionsthiere, looked upon the food-vacuoles as stomachs, and described a complex enteric canal connecting them; it is, therefore, not surprising that he considered them as belonging to the same group as Rotifers. Louis Agassiz, as late as 1859, considered Paramœcium, Opalina, \&c., to be the young of Planarians and Trematodes and Vorticella to be a Polyzoan, and it was only by the researches of Stein and others that the class of Infusoria was fully established as a natural group of unicellular organisins.

The Swiss zoologist Agassiz (1807-73), referred to in the preceding paragraph, is interesting not only as one of the foremost naturalists of his time and the founder of the large and active school of zoologists in the United States, where he spent the latter part of his life, but also as the last great biologist to maintain the fixity of species. In his Essay on Classification, published, curiously enough, in the same year (1859) as the Origin of Species, he supports the proposition that the various subordinate groups of animals, from phyla to species, are not mere "devices of the human mind to classify and arrange our knowledge in such a manner as to bring it more readily within our grasp and facilitate further investigations," but that they "have been instituted by the Divine Intelligence as the categories of His mode of thinking." In other words, that in our classifications we "have followed only, and reproduced, in our imperfect expressions, the plan whose foundations were laid in the dawn of creation."

In 1859 occurred what may fairly be called the most important event in the history of biological science, the publication of Charles Darwin's Origin of Species. The evolutionary theories of Buffon, Erasmus Darwin, Lamarck, and Geoffroy St. Hilaire had produced little effect upon contemporary zoology; and Robert Chambers's Vestiges of Creation (1844), although exciting great interest, was too crude and speculative to make many converts among men of science. But Darwin had the advantage of being not only a philosopher, but also a naturalist in the broadest 
sense-a systematist with a sufficient knowledge of anatomy, thoroughly conversant with the breeding of domestic animals and cultivated plants, a keen observer of external nature, both organic and inorganic, and with unrivalled experience as a traveller. It is not surprising, therefore, that the wealth of illustration, the close reasoning, and the philosophic spirit of the Origin converted the whole scientific world to the general doctrine of transformism within twenty years. The theory of Natural Selection, the Survival of the Fittest, or the Preservation of Favoured Races in the Struggle for Life, was first grasped by Darwin in 1838, but was not published until 1858, when it was announced simultaneously by himself and by Alfred Russel Wallace. Both these authors had, however, been anticipated by W. C. Wells in 1813, and by Patrick Matthew in 1831. Darwin's other works, especially The Variations of Animals and Plants under Domestication and The Descent of Man, rank among the most important contributions to philosophical Biology. With them must be mentioned the luminous Principles of Biology of Herbert Spencer, who consistently upheld the direct action of the environment as a factor in evolution. Wallace, on the other hand, is a pure selectionist, while Darwin held "that natural selection has been the main but not the exclusive means of modification."

The additions to zoological knowledge made by the voyagers of the eighteenth century have been referred to; even more important are the numerous great scientific expeditions of the nineteenth and twentieth centuries. Among the most prominent of these are the voyages of the French ships Astrolabe, Uranie, Bonité and Géographe, in which researches were carried on by Peron and La Sueur, Quoy and Gaimard, Eydoux and Souleyet, and Hombron and Jacquinot, and given to the world in splendidly illustrated folios. Still more famous is the voyage of H.M.S. Beagle (1831-36), in which Darwin gained his extraordinarily wide and accurate knowledge of natural history, and the narration of which is published in his Naturalist's Voyage. Other celebrated voyages are those of H.M.S. Rattlesnake (1846-50), of which T. H. Huxley was assistant-surgeon ; of H.M.SS. Erebus and Terror, accompanied by Sir J. D. Hooker ; of the American "Wilkes" expedition, with J. D. Dana as naturalist, and of the Austrian frigate Novara. But the most famous and complete of all scientific voyages was that of H.M.S. Challenger, in 1872-76, the five years' cruise of which was marked by discoveries of great importance by the scientific staff, Sir Wyville Thomson, John Murray, H. N. Moseley, and Willemoes-Suhm, while the zoological material collected on th s voyage was worked out by the leading zoologists in all parts of the world, and the results published in thirty handsome and fully-illustrated quarto volumes. 
On board the United States cruisers Blake and Albatross Alex. Agassiz made several cruises in the Gulf of Mexico (1877-80 and 1891), the Tropical Pacific (1899-1900), and the Eastern Pacific (1904-1905), the results of which have been in part published in The Memoirs and Bulletin of the Museum of Comparative Zoology of Harvard College. The results of the German Deep Sea Expedition of 1898, under the leadership of C. Chun, have been appearing in a long series of Memoirs since 1892; and those of the German Plankton Expedition (1889) since the same year, under the editorship of V. A. C. Hensen. An important scientific expedition to the Dutch East Indies was the cruise of the Siboga (1899-1900) under the directorship of Max Weber.

In land-travel, numerous journeys, and especially those of A. R. Wallace in the Malay Archipelago and Brazil, and of H. W. Bates in Brazil, have not only added immensely to our knowledge of the genera of the countries visited, but have enriched the science with the ideas of protective and aggressive characters, of mimicry, and of the relations of organism to environment generally.

The establishment of Zoological gardens in different parts of the world - notably in Paris and London, Stellingen, Berlin, Hamburg, and New York-has added greatly to our knowledge both of the habits and of the anatomy of animals, and a similar advance in the investigation of marine animals has followed upon the establishment of Zoological Stations or Marine Laboratories in various countries. The earliest and most important of these is the Naples Station, founded in 1870 by Anton Dohrn. The results of the researches there carried on form the most elaborate and sumptuous series of zoological monographs ever published. Other stations with similar aims have been instituted in all parts of the world: of these may be mentioned those of Plymouth, Wiméreux, Roscoff, Banyuls-sur-Mer, Kiel, Helder, Trieste, Woods Holl, and Tokyo: to these have been added in some countries stations for the study of the fresh-water and terrestrial faunas.

The establishment of Zoological (or Biological) Laboratories in connection with Universities is also a work of the last forty years, and has had an important influence both in diffusing a knowledge of the science and in stimulating research. Even more recent is the complete change of view as to the functions and arrangement of a Museum. Formerly it was looked upon as a collection of curiosities, in which everything was to be exhibited to the public. Now, thanks in great measure to Sir W. H. Flower in England, and Browne Goode in America, special collections are formed for study and research, while the cases accessible to the public are gradually becoming a series of actual illustrations of zoological science, in which not only the principles of classification, but the chief facts of structure, life-history, and habit are strikingly and 
adequately shown. Such reforms in the arrangement of museums and the advancement of their usefulness in many directions have been the objects aimed at by the Museums Association, an organisation of those interested in museums in all parts of the world, founded in 1889.

During the second half of the last century, Zoology as a whole has been greatly influenced by the writings of Thomas Henry Huxley and of Ernst Haeckel. Huxley (1825-1895) was the first to point out the homology of the ectoderm and endoderm of Cœlenterates with the two primary germ-layers of the vertebrate embryo. He also introduced the word zooid, demolished the vertebral theory of the skull, and placed the anatomy of the fossil Ganoids upon a satisfactory footing, as well as making many other important contributions to animal morphology. His Elements of Comparative Anatomy (1864) forms an important landmark in the history of modern Zoology, as giving the views of one of the keenest, most logical, and least speculative of biologists just before the time when the various improved histological and embryological methods began to revolutionise the science. Huxley's "eight primary categories or groups" are as follows :-

MOLLUSCA.

MOLLUSCOIDA

[including Brachiopoda, Polyzoa and

Tunicata].

CELENTERATA.
VERTEBRA'TA.

\section{ANNULOSA}

[including Arthropoda and Annulata]. [including Echinodermata, Rotifera, Platyhelminthes and Nemathelminthes]. INFUSORIA

[including Infusoria proper and Mastigophora].

\section{PROTOZOA}

[including Rhizopoda, Sporozoa, and Porifera].

The lower "Worms" are associated with Echinoderms, on account of the resemblance of the adult Rotifers, as well as of the larvæ of certain Flat Worms, to the echinopædium. Sponges are placed among the Protozoa, in accordance with the view that they are to be looked upon as colonies of unicellular zooids. Infusoria are separated from the remaining Protozoa, because the conjugation observed in them was misinterpreted, the meganucleus being considered as an ovary, the micronucleus as a testis.

Haeckel, apart from his elaborate and beautiful researches on the Radiolaria, Calcareous Sponges, and Hydrozoa, is remarkable as the first modern zoologist to attempt the classification of animals on a frankly evolutionary basis. We owe to him the terms phylogeny and ontogeny, cænogenesis and palingenesis, and the fruitful "gastræa-theory," according to which the gastrula is the ancestral form of all the Metazoa. His classifications take the 
form of genealogical trees, and he was the first to employ the method of introducing hypothetical ancestral forms, wherever they might be wanted to complete the connection between known groups. He may be said, in fact, to have founded a school of deductive zoology, the phylogenetic speculations of which are often as ingenious and suggestive as they are transient. The student must, however, bear in mind that Archi-molluses, Ideal Craniates, and Pro-mammalia are mere figments of the imagination, and have no more real existence than the "Divine Archetypes" of an earlier school of thought.

One result of the new views on species, very obvious in the writings of both Huxley and Haeckel, was the marked alteration in the position assigned to Man in the animal series. Linnæus considered Homo as a genus of his order Primates, equivalent to Simia, Lemur, \&c. ; but Cuvier took the retrograde step of erecting a distinct order, Bimana, to contain Man alone, the Apes and Lemurs forming the order Quadrumana. Ehrenberg went further, and divided the Animal Kingdom into Nations, i.e., Mankind, and Animals. Even as late as 1857 Owen, as we have already seen, made a distinct sub-class, Archencephala, for Man, the remaining Primates being included with the other higher mammalian orders in the sub-class Gyrencephala. This view of the isolated position of Man was connected with the theory of his late appearance in time, and the fact of his co-existence with the Mammoth and other extinct Mammals, first proved by Boucher de Perthes in 1836 by the discovery of flint axes 20-30 feet below the present surface, was for many years almost universally denied. But Lyell's Antiquity of Man (1863) placed the geological evidence on a sound footing, and the same was done for the morphological evidence by Huxley, who, in his Man's Place in Nature (1863), summed up the position by the statement, now universally conceded, "that the structural differences which separate Man from the Gorilla and the Chimpanzee are not so great as those which separate the Gorilla from the lower Apes." Finally, Darwin, in his Descent of Man (1871), discussed the question from every point of view, and concluded that "Man still bears in his bodily frame the indelible stamp of his lowly origin."

It was also during the third quarter of last century that the old doctrine of Abiogenesis or Spontaneous Generation, first assaulted by Redi, but maintained by many naturalists from Aristotle to Haeckel, was finally disposed of. The accurate methods of Louis Pasteur, Lord Lister, John Tyndall, and others, proved conclusively that the Bacteria, Monads, and other lowly organisms which occur in putrefying substances do not arise de novo, but are the product of germs in the floating dust of the air by the exclusion of which putrefaction may be absolutely prevented. 
During the last quarter of a century the progress of Zoology has been profoundly influenced by the improvements in microscopical methods, especially by the invention and perfection of the microtome, the method of serial section-cutting, and the various ways of preserving, imbedding, and staining tissues. The microtome began as a simple contrivance for holding small objects firmly while sections of them were cut by hand with a razor or other knife, and has developed into the various modern forms of the instrument in which the knife is fixed in a plane parallel to the surface of the object, and the latter is raised mechanically by small and equal increments as the sections are cut. In this way perfectly regular sections are obtained of an even thickness not exceeding the diameter of a cell. The method of imbedding began by simply holding an object, too small or too soft to be grasped by the fingers, between two pieces of carrot or pith, and has gradually been evolved into the present method of complete impregnation with paraffin or celloidin, by means of which imbedding material and object form a homogeneous mass. Simple preservation in alcohol has given place to elaborate fixing methods by means of chromic, picric, or osmic acids, platinum chloride, corrosive sublimate, etc., and gradual hardening in alcohols of increasing strength. Similarly, direct staining with an ammoniacal solution of carmine has developed into innumerable methods of differential staining, mostly with aniline dyes, by which the various tissues and the constituents of the cellchromatin, centrosomes, etc.- - are clearly brought into view. By the serial methods̀ successive sections of an embryo or small animal are mounted in regular order, so that the organs, tissues, etc., can be traced through the series. In this way the dislocation of parts produced by dissection is avoided, organs are seen in absolutely natural relations, and parts quite undiscernible either by dissection or by microscopic examination of the whole animal or of dissociated parts of it are clearly brought into view : the study of the structure upon which the sections are intended to throw light may be further facilitated by the fashioning of models in wax, reconstructed by putting together reproductions of the sections enlarged to scale. Morphological inquiry has, in fact, been brought within measurable distance of a precision limited only by the imperfections of our eyes and optical instruments. Similar accuracy in the topographical anatomy of the larger Animals, including Man, has been attained by freezing the whole subject and cutting it into sections with a saw.

These improved methods have necessitated a re-examination by their aid of every group in the animal kingdom, and, as a result, our knowledge of the structure of many animals, especially of the lower forms, of complex organs such as the vertebrate brain, of embryology, and of the minute structure of cells and tissues 
has been completely revolutionised. Spccially remarkable is the advance in our knowledge of the Protozoa, Sponges, Actinozoa, Echinoderms and Amphioxus. The new light which has bcen thrown on the affinities of Balanoglossus, Rhabdopleura, and Cephalodiscus is also worthy of special mention. Probably the greatest of comparatively recent cmbryological triumphs, belonging to the earlier part of the period now under discussion, is Kowalewsky's discovery of the notochord and hollow nervous system of the Tunicate larva, which resulted in the removal of the Urochorda from Molluscoida to Chordata, and in breaking down the sharp line between Vertebrates and Invertebrates.

But perhaps the most remarkable result of improved microscopical technique is the rise and development of a distinct department of histology, known as cytology, dealing with the minute structure of the protoplasm and nuclei and the various intra-cellular phenomena, such as mitosis. Our knowledge of this subject is entirely a product of the last forty-five years, and is due in great mcasure, in the first instance, to the researches of W. Fleming, E. Strasburger, and E. van Beneden. A modification of the cell-theory has also been necessitated by the proof that many animal tissues do not consist of distinct cells, but of a continuous mass of protoplasm with more or less regularly arranged nuclei, and are thcrefore strictly not multiccllular but non-cellular. As certain Protozoa, such as the Mycetozoa and Opalina, are also non-cellular, containing numerous nuclei in an undivided mass of protoplasm, the distinction between Protozoa and Metazoa appears to be less absolute than it was at one time considered.

The advance in palæontology during the same period has also been immense. In particular the researches of E. D. Cope, O. C. Marsh, and others in America, have added whole orders to Zoology--the Odontolcæ, Ichthyornithes, Stereornithes, Amblypoda and Dinocerata-and have resulted in the discovery of many new and strange forms among the Dinosauria, Elasmobranchs, Ganoids, and other groups, and in the tracing of the pedigrees of the Equidæ, Camelidæ, and other Mammalian families. Important though less striking discoveries have also been made among the fossil faunæ of Europe, India, South Africa, and Australia; while among Invertebrates the attempts to trace the pedigree of the Ammonites and Brachiopods are specially noteworthy.

In embryology an important landmark is furnished by $\mathbf{F}$. IM. Balfour's Comparative Embryology (1880-81); in distribution, by A. R. Wallace's Geographical Distribution of Animals (1876), each the first complete treatise on the subject in question. The zoo-geographical regions adopted by Wallace were originally proposed by P. L. Sclater in 1857. Similar landmarks for Zoology 
as a whole are Huxley's Anatomy of Vertebrated Animals (1871) and Anatomy of Invertebrated Animals (1877), Carl Gegenbaur's Elements of Comparative Anatomy (English edition, 1878), Claus's Text-Book of Zoology (1st English edition, 1884-5), Ray Lankester's Notes on Embryology and Classification (1877), and the same author's articles in the Encyclopodia Britannica (9th edition). Both Clans and Gegenbaur retain Vermes as a primary division; Lankester was the first to split up that unnatural assemblage into distinct phyla, and to include Balanoglossus and the Tunicata among Vertebrates, and Xiphosura and Eurypterida among Arachnida. He also associated Rotifers and Chrtopods with Arthropoda, and placed Hirudinea among the Platyhelminthes. A later development of the same author's views on morphology and classification is embodied in his Treatise on Zoology, of which eight volumes have now been published (see Appendix, 675). Of inestimable value in the advancement of the embryology of Vertebrates is the comprehensive Handbuch (1901-1906) of O. Hertwig, with sections by various other embryologists.

The student who is interested in the permutations and combinations of modern classification may be referred to the works just quoted as well as to the numerous text-books published of late years. The most important point to notice in this connection is the breaking down of the sharp boundaries between the four Cuvierian Branches and a return to something like the conception of unity of type, expressed, however, not as a linear series, but as a branch-work with the most complex and often puzzling interrelations.

Among the numerous recent contributions to philosophical Zoology it must suffice to mention the works on heredity and kindred subjects of August Weismann, the most prominent member of the ultra-Darwinian school, who deny use-inheritance and rely upon natural selection as the main, if not the sole, factor in evolution. The opposite view, which accepts the truth of useinheritance, is mainly supported by the American school of NeoLamarckians. Weismann has also resuscitated the theory of preformation under a modern form. He considers that the various parts of the adult organism are represented in the chromatin (germ-plasm) of the sex-cells by ultra-microscopic particles or determinants. These and allied topics are comprehensively treated from a different standpoint by $\mathrm{O}$. Hertwig in his Allgemeine Biologie (1909).

In a brief sketch like the present it is impossible to do more than refer, in general terms and without mention of names, to the vast amount of work now being done in every department of Zoology. The output of original research is greater than at any former time and is increasing rapidly, and every important addition to our knowledge necessitates a more or less thorough reconsidera- 
tion of the general and special problems of morphology and classification.

In addition to the continued study of adult structure and embryology of animals and plants with their classification and phylogeny and their distribution in space and time, the workers in Biology during the first two decades of the twentieth century have bestowed more attention than hitherto on (1) Experimental Biology and Embryology, (2) Genetics, and (3) Cytology. It is in the second of these departments-the revived and intensified study of heredity--that the impulse towards the first and third has mainly originated. A large part of the experimental work accomplished has had for its object the solution of genetic problems; and on the other hand the attempts to co-ordinate the minutix of structure in the germ-cells with known phenomena of heredity or theoretical explanations of such phenomena have given interest and importance to most recent cytological work. 



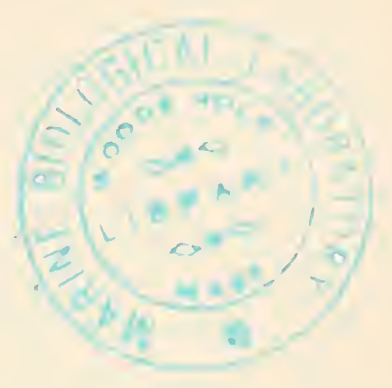

\section{APPENDIX}

\section{ZOOLOGICAL LITERATURE.}

THE following are lists of some of the publications which will be most useful to the student of Zoology, the first comprising books giving directions for practical work in the laboratory; the second, general works on the entire subject or on one or other of its main sub-divisions; the third, publications in which are given the titles, and in some cases abstracts of the contents, of new works as they appear. More or less comprehensive bibliographical lists are given in several of the works referred to in the second list (Nos. 8, 20, 32, $35,49,50,70,82$, etc.).

I. Books bearing specially on Laboratory work.

1. Арathy, S. Mikrotechnik der thierischen Morphologie, 1896-1901.

2. Brooks, W. K. Handbook of Invertebrate Zoology, 1890. [Amœba, Paramœcium, Vorticella, Calcareous Sponge, Zoophyte, Anthomedusa, Leptomedusa, Starfish, Sea-urchin, Embryology and Metamorphosis of Echinoderms, Earthworm, Leech, Crab, Crayfish or Lobster, Metamorphosis of Crab, Cyclops (including metamorphosis), Grasshopper, Mussel, Development of Lamellibranchs, Squid, Development of Squid.]

3. Ehrlich, P., Krause, R., Moss, M., Rosin, M., Weigert, K. Encyclopadie der mikroskopischen Technik, 2 vols., 2nd edition. 1910.

4. Foster, M., and Balfour, F. M. Elements of Embryology, 2nd edition, by A. Sedgwick and W. Heape, 1883. [Chick and Rabbit.]

5. Friedländer, R. Mikroskopische Technik, 6th edition, 1900.

6. Howes, G. B. Atlas of Practical Elementary Zootomy, 1902. [Amœba, Stentor, Paramorium, Spirostomum, Vorticella, Hydra, Fresh-water Aussel, Snail, Earthworm, Crayfish, Frog.] 
7. Huxley, T. H., and Martin, H. N. A Course of Practical Instruction in Elementary Biology, 2nd edition, by G. B. Howes and D. H. Scott, 1888. [Amøeba, Vorticella, Paramœcium, Opalina, Hydra, Earthworm, Crayfish, Mussel, Snail, Frog.]

8. Kढ̈kenthal, W. Leilfäden für das zoologische Praktikum, 4. Aufl., Jena, 1907.

9. Lee, A. B. Microtomists' Vade Mecum, 6th edition, 1905. [A compendium of laboratory methods.]

10. Marshall, A. M. The Frog: an Introduction to Anatomy, Histology, and Embryology, 6th edition, 1896.

11. Marshall, A. M., and Hurst, C. H. Practical Zoology, 7th edition, 1912. [Amœba, Vorticella, Paramœcium, Hydra, Liver-Fluke, Leech, Earthworm, Crayfish, Cockroach, Fresh-water Mussel, Snail, Amphioxus, Dogfish, Pigeon, Rabbit, Development of Chick.]

12. Parker, T. J. A Course of Instruction in Zootomy, 1884. [Lamprey, Skate, Cod, Lizard, Pigeon, Rabbit.]

13. Parker, T. J., and Parker, W. N. An Elementary Course of Practical Zoology, 5th edition, 1920. [Part I-Frog; Part IIAmœba, Sphærella, Paramœeium, Vorticella, Opalina, Monocystis, Hydra, Obelia, Earthworm, Nereis, Crayfish, Freshwater Mussel, Amphioxus, Dogfish, Rabbit ; Histology, Embryology, etc.]

14. Schnemer, K. C. Histologisches Praktikum der Thiere, 1908. 15. Vogt, C., and Jung, E. Traité d'Anatomie comparée pratique, 2 vols., 1883-94 (also a German edition). [Amœba, Foraminifer (Polystomella), Actinosphærium, Radiolarian (Actinometra), Paramoecium, Dicyema, Calcareous Sponge, Alcyonium, Aurelia, Hydra, Ctenophore (Bolina), Tænia, Distomum, Turbellarian, Nemertean Leech, Ascaris, Sipunculus, Rotifer (Brachionus), Earthworm, Lobworm (Arenicola), Feather-star, Starfish, Seaurchin, Holothurian, Polyzoan (Plumatella), Brachiopod, Mussel, Snail, Pteropod, Cuttle-fish, Crayfish, Centipede, Cockchafer, Spider, Salpa, Simple Ascidian, Amphioxus, Lamprey, Perch, Frog, Lizard, Pigeon, Rabbit.]

16. Wrider, B. G., and Gage, S. H. Anatomical Technology [Cat.]. 3rd edition, 1892 .

II. General Works and Text-books.

1. Balfour, F. M. Treatise on Comparative Embryology, 2 vols., 1880-81. Works, Memorial Edition, 4 vols., 1885.

2. Bateson, W. Materials for the Study of Variation, 1894.

3. Bateson, W. Mendel's Principles of Heredity, 1909.

4. Bedpard, F. Animal Coloration, 1892. 
5. BEDDard, F. Text-book of Zoogeography, 1895.

6. Boas, J. E. V. Lehrbuch der Zoologie, 1894. English Translation, 1891.

7. Bonnet, R. Lehrbuch der Entwickelungsgcschichte, 1907.

8. Bronn, H. G. Klassen und Ordnungen des Tierreichs. Protozoa (Bütschli), 1880-89; Porifera (Vosiane), 1887 ; Turbellaria (GRAFf) ; Trematoda (Braun) ; Cestoda (Braun) ; Ncmertina (Bürger); Tunicata (SEeliger); Asteroidea (LudwiG and Hamann); Ophiuroidca (Hamann); Echinoidea (Hamañ); Crinoidca (HamanN); Holothuroidea (Ludwig); Mollusca (Keferstein), 1862-66 ; Amphineura (Simroth); Gastropoda (Simroth) ; Crustacea (Gerstäcker) ; Myriapoda (Verhoeff) ; Amphibia (Hoffmann), 1873-78; Reptilia (Hoffuann), 1890 ; Aves (Gadow), 1891 ; Mammalia (Giebel and Leche).

9. Cambridge Natural History.

Vol. I. Protozoa (Hartog, M.) ; Porifcra (Sollas, I. B. J.); Coelenterates and Ctenophora (Hickson, S. J.); Echinoderms (MacBride, E. W.), 1906.

Vol. II. Flatworms and Mesozoa (Ganble, F. IV.); Nemertines (Sheldon, L.) ; Threadworms and Sagitta (Shrpley, A. E.); Rotifers (Hartog, M.) ; Polychaet Worms (Benham, W. B.) ; Earthworms and Leeches (BEDDARD, F. E.); Gephyren and Phoronis (Shipley, A. E.) ; Polyzot (Harmer, S. F.), 1896.

Vol. III. Molluscs (Cooke, A. H.) ; Brachiopods (recent), (SHipley, A. E.) ; Brachiopods (fossil), (REED, E. R. C.), 1895.

Vol. IV. Crustacea (Surth, G.) ; Trilobites (Woods, H.) ; Introduction to Arachnida and King-Crabs (SHipley, A. E.) ; Eurypterida (Woods, H.) ; Scorpions, Spiders, Mites, Ticks, etc. (Warburton, C.) ; Tardigrada (Shipley, A. E.) ; Pentastomida (Shipley, A. E.) ; Pycnogonida (Thompson, D'A. IV.).

Vol. V. Peripatus (Sedgwick, A.) ; Myriapods (Sinclair, F. G.) ; Insects, Part I. (Sharp, D.), 1895.

Vol. VI. Insects, Part II. (ShARP, D.), 1899.

Vol. VII. Hemichordata (Harmer, S. F.); Ascidians and Amphioxus (Herduan, IV. A.) ; Fishes (Bridge, T. IV., and Boulenger, G. A.), 1904.

Vol. VIII. Reptiles and Amphibia (GADow, H.), 1901.

Vol. IX. Birds (Evans, A. H.), 1897.

Vol. X. Mammalia (Beddard, F. E.), 1902.

10. Brooks, W. K. The Foundations of Zoology, 1899.

11. Bütschl, O. Vorlesungen über vergleichenden Anatomie, 1910, 1920.

12. Carus, V. Geschichte der Zoologic, 1872. (French Translation, Histoire de la Zoologie, 1880.) 
13. Cuvier, G. Règne Animal. Illustrated edition, 1849.

14. Darwin and Modern Science. Essays in commemoration of the Centenary of Charles Darwin, 1909.

15. Darwin, C. Origin of Speeies, 6th edition, 1880.

16. Darwin, C. Descent of Man, 1882.

17. Darwin, C. Animals and Plants under Domestication, 2 vols., 1888.

18. Dean, B. Fishes, Living and Fossil, 1895.

19. Delage, Y. L'Ilérédité et les grands problèmes de Biologie générale, 2nd edition, 1903.

20. Delage, Y., et Hérouard, E. Traité de Zoologie Concrète. I. La Cellule et les Protozoaires, 1896. II. Spongiaires et Coelentérés, 1899. III. Echinodermes, 1903. V. Vermidiens, 1897. VII. Prochordés, 1898.

21. Dendy, A. Outlines of Evolutionary Biology, 2nd edition, 1912.

22. Doncaster, L. Heredity in the Light of Recent Research, 1910.

23. Doncaster, L. Cytology, 1920.

24. Duval, M. Atlas d'Embryologie, 1889.

25. Emmer, T. Die Entstehung der Arten, 1888. English Translation, Organic Evolution, 1889.

26. Flower, W. H. Osteology of the Mammalia, 1885.

27. Flower, W. H., and Lydekker, R. Mammals, Living and Extinct, 1891.

28. GaUdry, J. Les enchaînements du monde animal dans les temps géologiques, 1878.

29. Gegenbaur, C. Elements of Comparative Anatomy. English Translation, 1878.

30. Gegenbaur, C. Vergleichende Anatomie der Wirbelthiere, 2 vols., 1898 and 1901.

31. Натьснек, B. Lehluch der Zoologie.

32. Heidenhain, M. Plusmu u. Zelle, 1907.

33. Heilprin, A. The Distribution of Animals, 1887.

34. Hertwig, O. Die Elcmente der Entwicklungslehre des Menschen und der Wirbelthiere, 1910.

35. Hertwig, O. Handluch der vergleichenden und experimentellen Entwickelungslehre der Wirbelthiere. Sections by various writers, 3 vols., 1901-1906.

36. Hertwic, O. The Biological Problem of To-day, 1896.

37. Hertwig, O. Allgemcine Biologie, 3rd edition, 1909.

38. Hertwia, R. Lehrbuch der Zoologie, 9th edition, 1910. English Translation of 3rd edition.

39. Hilzheimer, M. Ilandbuch der Biologie der Wirbelthiere, 1913.

40. Huxley, T. H. The Anatomy of Invertebrated Animals, 1877. 
41. Huxley, T. H. The Anatomy of Vertebrated Animals, 1871.

42. Jordan, D. S., and Kellogg, V. L. Evolution and Animal Life, 1907.

43. KeIBEL, F. Normentufeln zur Entwickelungsgeschichte der Wirbeltiere. [Pig, Fowl, Ceratodus, Lizard, Rabbit, Deer, Tarsius, Nycticebus, Man, Lapwing.] 1897-1909.

44. Kellicott, W. E. Text-book of Gencral Embryology, 1914.

4ta. Kellicott, W. E. Outlines of Chordate Development, 191 t.

45. Kelloga, V. L. Danwinism To-day, 1907.

46. KenR, J. G. Text-book of Embryology: Vertebrata, 1919.

47. Kingsley, J. S. Text-book of Vertebrate Zooloyy, 1899.

48. Korschelt, E., and Heider, K. Text-book of the Embryology "ff Invertebrates. English edition, 4 vols., 1895.

49. LANG, A. Lehrbuch der vergleichenden Anatomie der wibellosen Thiere, 1891. English Translation, Comparative Anatomy of Invertcbrates, 1891-6. Second German edition, Protosoa, 1901, Mollusea, 1900. Third German edition (Handbuch der Morphologie der wirbcllosen Thiere), 1912.

50. Lankester, E. R. A Treatise on Zoology. I. Protozoa (Lister, Minchin, Hickson). II. Porifera and Coelcntera (Minchin, Fowler, Bourne). III. Echinollcrma (Batuer). IV. Platyhelmia, Mesozoa and Nemertimi (Benham). V. Mollusea (Pelseneer). VII. Crustueen (Calman). IX. Cyclostomes and Fishes (GoodriciI). 1900-09.

51. Lilley, F. The Dcvelopment of the Chiek, 1908.

52. LydekKer, R. Royal Natural History. 6 vols. 1894-96.

53. MACBride, E. W. Text-book of Embryology. Invertcbrata, 1914.

54. Mlarshald, A. M. Vertcbrate Embryology, 1893. [Amphioxus, Frog, Chick, Rabbit, Human Embryo.]

55. Morgan, C. L. Animal Life and Intelligence, 1891.

55a. Morgan, C. L. Habit and Instinet, 1896.

56. Morgan, T. H. Experimental Zoology, 1906.

57. Nicholson, H. A., and Lydekner, R. Manual of Palcontology, 2 vols., 1889.

58. OpPEL, A. Lehrbueh der vergleichenden mikroskopischen Anatomie der Wirbelticie, 1896-1904.

59. Osbons, H. F. From the Greeks to Darwin, 1894.

60. Owen, R. Anatomy of Vertebrates, 4th edition, 1871.

61. Parker, T. J. Lessons in Elementury Biology, 3rd edition, reprinted, with corrections, 1919.

61a. Parker, T. J., and Haswell, W. N. A Manual of Zoology, reprinted 1908.

62. Poulton, E. B. Essays on Evolution, 1908. 
63. Punnett, R. C. Mendelism, 1911.

64. Rolleston, G., and Hatchett-Jackson, W. Forms of Animal Life, 1888.

65. RABL, C. Gesehiehte der biologisehen Theorien, 2 vols., 1909.

66. Reinke, J. Einleitung in die theoretische Biologie, 2nd edition, 1911.

67. Romanes, G. J. Darwin and After Darwin, 2 vols., 1892 and 1895. 68. Roux, W. Vorträge und Aufsätze über Entwickelungsmechanik der Organismen, 1901.

69. Sedgwick, A. A Student's Text-Book of Zoology. Vol. I. Protozoa to Chatognatha, 1898. Vol. II. Amphioxus and Vertebrata, 1905. Vol. III. Tunicata, Enteropneusta, Echinodermata, Arthropoda, 1909.

70. Sснімкеwiтsch, W. Lehrbuch der vergleiehendcn Anatomie der Wirbelthiere. German Translation from Russian, 1910.

71. Schneider, K. C. Lehrbuch der vergleiehenden Histologie der Thiere, 1902.

72. Thomson, J. A. Heredity, 1908.

73. Verworn, M. Allgemcinc Physiologie, 5th edition, 1909 ; English Translation, General Physiology, 1899.

74. Vries, H. De. Die Mutationstheorie, 2 vols., 1901. English Translation by Farmer and Darbishire, 1910.

75. WAGNER, M. Die Entstehung der Arten durch räumliehc Sonderung, 1889.

76. Wallace, A. R. Geographical Distribution of Animals, 2 vols., 1876.

77. Wallace, A. R. Darwinism, 1889.

78. Wallace, A. R. An Examination of Wcismamism, 1893.

79. Walter, H. E. Geneties, an Introduetion to the Study of Hcrcdity, 1913.

80. Weber, M. Dic Säugetiere, 1904.

81. Weismann, A. The Evolution Theory, 2 vols., 1904 (Trans. Thomson). 3rd German edition, 1913.

82. Wiedershim, R. Verglciehende Anatomie der Wirbeltiere, 7th edition, 1909.

83. Wiedersheim, R., and Parker, W. N. Comparative Anatomy of Vertebrates, 1907.

84. Willey, A. Amphioxus and the Anecstry of the Vertebratcs, 1894.

85. Wilson, E. B. The Cell in Development and Inheritanee, 2nd edition, 1900.

86. Woonward, A. S. Outlines of Vertebrate Palcontology, 1898.

87. ZiEgLer, E. H. Lehrbuch der Entwickelungsgesehichte der niederen Wirbeltiere, 1902. 
88. Zittel, K. von. Handluch der Paläontologie, 5 vols., 1876-93. English 'Translation, 2 vols., 1902.

89. Zittel, K. von. Grundzüge der Paläontologie, 2 Aufl., 1903.

III. Periodical Publications containing Current Bibliographies, etc.

1. American Naturalist, fortnightly. Contains original articles, usually of a popular character, and summaries of recent researches.

2. Anatomischer Anzeiger, fortnightly. Original papers (mostly short) and current bibliography.

3. Année Biologique, annual. Abstracts of works and papers on general Biology. 1895-1911.

4. Biologisches Centralblatt, fortnightly. Contains, in addition to original articles, résumés of recent publications.

5. Ergebnisse und Fortschritte der Zoologie. Résumés of the present state of knowledge on special subjects.

6. International Catalogue of Scientific Literature. Zoology and General Biology. Obtainable in sections. Full classified lists of all current writings with indication of new species and reference to new genera.

7. Journal of the Royal Microscopical Society, bi-monthly. Abstracts of papers on nearly all departments of Zoology.

8. Quartcrly Journal of Microscopical Science.

9. Science Progress, monthly. Contains abstracts of Zoological papers.

10. Zoological Record, annual. Bibliographical lists with some synopses of contents.

11. Zoologischer Anzeiger, fortnightly. Original papers (mostly short preliminary notices), and current bibliography (Bibliographia Zoologica, also to be had separately).

12. Zoologischer Jahresbericht, annual. Published by the Naples Zoological Station. Consists (1) of bibliographical lists, (2) of abstracts of the contents of most of the papers, 1879-1912.

13. Zoologisches Zentralblatt, fortnightly. Consists (1) of occasional summaries and criticism of recent work on special subjects, (2) of abstracts of individual books or papers in all departments of Zoology. 

INDEX 



\section{INDEX}

All numbers refer to pages : words in italics are names of families, genera and species: words in thick type are names of higher divisions: words in small capitals are names of examples. Numbers in thick type are numbers of pages on which there are figures: an asterisk after a number indicates a definition of the term or of the group.

A

A ard-varks, 465, 485, 579, 584See Orycteropus

Abdomen-Mammals, 70*, 433

Abdominal cavity, of Craniata, 70

Abdominal pore, $65,66,142,243$

Abdominal ribs, 293, 336, 405

Abdominal vein, 272, 273, 316,558

Abducent nerve, of Craniata, 101, 102

Abductor muscles, 266

Abiogenesis, 650

Abomasum, 553*

Abyssal fauna, $615^{*}$

Acanthias, 173

Acanthodei, 163*

Acanthodes, 164

Acanthodrilus, 601

Acanthopteri, 214*, 218, 219, 221, 225, 236

Accessory nerve, of Craniata, 101, 103

Accessory scapula, 417

Accipitres, 403*, 431

Acetabulum, of Craniata, 80, 82*

Acipenser, 212, 234-See Sturgeon

Acipenser ruthenus, 212

Acrania, $43 *$-See Amphioxus

Acrocoracoid process, 378

Acrodont teeth, 345*

Acromion process, of Pigeon, 378 : Rabbit, 441-See Pectoral arch

Acustico-lateral centre, 158

Ad-digital, 373

Adductor muscles, 266

Adelochorda, 2* : Affinities, 13

Adhesive papilla, of Ascidian larva, 34, 35

Adipose bodies, of Frog, 279 : Lizard,
Adipose fin, 194*

Adipose lobe, 194

Adrenals, of Craniata, 117 : Frog, 278 :

Pigeon, 385 : Rabbit, 459

Agithognathous arrangement, $415^{*}$

Epyornis, 399, 423, 429, $62 \mathrm{I}$

Epyornithes, 399*

Aerial fauna, $617 *$

Afferent branchial arteries, 90 : of Amphioxus, 49-See Vascular sys. tem

Affinities-See Relationships

After-shaft of Feather, 370, 410*, 411

Agamidoe, 358

Agassiz, Alex., 663

Agassiz, Louis, 66 I

Aggressive resemblance, 637

Aglossa, 285*, 295

Agoutis, 492

Air-bladder, 87* : of Trout, 204*, 205 :

Teleostomi, 229

Air-sacs, of Chamæleon, 347 : Pigeon, 386, 387 : Birds, 421

Air-space, of Bird's egg, 422, 423

Ala spuria, 368, 373*

Alar membrane, 368

Alaudidoe, 404

Albatrosses, 402, 417, 422

Albula, 231

Albumen, of Bird's egg, 394, 422, 423

Alca, 403

Alca impennis, 413

Alcedinidoe, 404, 423

Ali-sphenoid, ry*_-See Skull

Allantoic bladder, of Craniata, 116*

Allantoic placenta, 577

Allantois, Reptiles, 303, 354: Bird, 427, 428: Rabbit, 462 : Mammalia, 575 
Allelomorphic characters, 645

Alligator, 326, 343, 344, 346, 349, 357, 359

Alpine fauna, $617^{*}$

Altrices, 429*

Alveoli of lung, 454

Alveus, 561*

Alytcs obstetricans, 300,302

Amarccium, 22

Ambiens muscle, 383*

Amblyopsis spelaens, 617

Amblypoda, 588

Amblystoma, 284, 290, 294, 301, 302

American Ant-eaters, 465, 484, 509, $511,512,513,547,579$

Amia, 213, 218, 221, 222, 223, 227, $229,231,233,234,235,236$

Amia calva, 213

Ammocotes, $133^{*}$

Amnion, of Reptiles, 303, 354: Birds. 42\%, 428 : Rabbit, 461, 462 : Mammals. 572,573

Amniota, 303*

Amphibia, 64*, 256 : Example, 257: Distinctive characters and classi. fication, 283: General organisation, 285: External characters, 285: Exoskeleton, 289: Enrloskeleton, 289: Myology, 294: Digestive organs, 295: Respiratory organs, 295 : Circulatory organs, 296 : Nervous system and sense organs, 298 : Urinogenital organs, 299: Reproduction and development, 300 : Distribution, 302 : Mutual relation. ships, 302

Amphibolurus, 350, 355

Amphicalous, $142 *$

Amphioxidcs, 43, 59, 62

Amphioxidida, 43

Amphioxus, 43 : External fentures, 43: Body-wall, 44, 45: Skeleton, $45,46,47$ : Digestive and respiratory organs, $46,47,48$ : Atrium, 4\%, 49: Coelome, 4\%, 49: Bloodsystem, 49, 50: Excretory organs, 51: Nervous system, 51, 52, 53: Sensory organs, 52, 53, 54: Reproductive organs, 54: Development, $54,55,56,5 \%, 58,59,60,61,62$ : Distribution, 62 : Distinctive characters, 62, 63: Affinities, 63

Amphipnous, 228

Amphishrnians, 324, 332, 337, 347, 354,358

Amphistylic skull, 76*, 168

Amphitherium, 619

Ampkiuma, 284, 286, 287, 296, 298, 302

Amphiuma tridactyla, 286

Ampullæ, 111*, 112, 174*_-See Ear
Ampullary canals, 158*, 174

Anabas scandens, 228

Anacanthini, 21 **, 219, 221, 236

Anapophyses, of Rabbit, 435*

Anas, 403, 408, 415, 416, 605

Anas boschas, 416

Anatomical evidence of Evolution, 624

Anchinia, 22

Anchovy, 214

Ancylopoda, 588*

Angler, 219

Anguida, 358

Anguis, 328, 332, 350, 358

Angular, 199, 200*

Angular process, of mandible, 441See Skull

Angulo-splenial, 261, 262

Ankylosis, 195*

Annular cartilage, of Lamprey, 122, 123

Annulus ovalis, 450*

Annulus tympanicus, of Frog, 262, 278

Anomalurus, 476, 492

Anomodontia, 326

Anser, 403, 415

Anseres, 403*, 422, 429

Ant-eater, American, 465, 484, 509, 511, 512, 513, 547, 579: Bancled, 480: Cape, 465, 579 See Orycteropus : Scaly-See Manis: Two-toed, 484

Ant-eaters, 465

Antelopes, 468, 487, 580

Anterior clasper, 184

Anterior commissure, 206

Anterior vertebral plate, $167 *, 169$

Anthropoidea, 472*

Anthropopithecus, 473--See Chimpanzee

Anthropopithecus troglodytes, $\mathbf{5 3 8}$

\section{Antiarcha, 256}

Antibraclium, $304^{*}$

Antitrochanter, 380*

Antlers, 487

Antorbital, 170*

Anura, 284*, 285, 288, 289, 291, 293, $294,295,296,298,300,301,302$

Anwiella, 37

Aortre, 89, 90: Amphioxus, 49, 50See Vascular system

Aortic arches, 92, 93--See Vascular system

A patornis, 40:

Apes, 494

Aphanapteryx, 605, 622

Apical plate, of Tornaria, 7,8

Apoda, $285 *$

Appendicularia, 21, 24, 28, 29, 30, 41

Appendiculariida, 21

Appendix, vermiform, of Rabbit, 448

Aptcnodytes, 40: 
Apteria, 3\%2*, 409

Apteryges, 398

Aptery.x, 398, 399, 408, 414, 417, 419, $4: 0,421,422.423,426,429,600$, (i2):

A pteryx anstralis, 398, 399. 419

A pteryx mantelli, $414,415,41 \%$

A ptcry.x oweni, $\mathbf{4 2 0}$

Aptornis, 403, 413, 417, 600, 622

Aqueduet of Sylvius, 99*

Aqueductus vestibuli, 144, 158, 174

Aqueous chamber, of Eye, 108*

Aqueous humour, 108*

Aquila, 403, 423

Aquinas, Thomas, 654

Ara, 404, 416

Arachnoid membrane, 100

Arbor vite, of Rabbit, 458*

Arboreal famna. 617*

Archæoceti, 586

Arehoohinax, 587

Archeopteryx, 397, 405, 430, 619

Archapopteryx lithographica, 405, 406. $40 \%, 430,619$

Archropteryx sicmensii, 407

Archæornithes, $397 * 405$

Archenteron, $280 *, 281$

Archipallium, 561*

Arehipterygium, 163*, 250

Arciferr, $285 *$

Arctomys marmot, 617

Ardca, 403, 409, 410

Area opaca, 352, 424

Area pellucida, 352, 424

Area vasculosa, 426

Argentea, $206^{*}, 207,208$

Aristotle, 647

Armartillos, 465, 476, 484, 508, 509 . $510,511,512,513,516,547,579,58 \%$

Arteries, 89, 90 See Vascular system

Arthodira, 25:

Articular, $\%$ \%, 79*_-See Skull

Artiodactyla, 468*, 487, 521, 522, 526, $527,547,558$

Arvicola, 575

Arytenoirls, of Lizard, 318 : Reptilia, 347: Pigeon, 385: Rabbit, 453

Ascio1A, 14: Body-wall and Atrial cavity, 14,15 : Pharynx. 16, 1\%, 18 : Enteric canal, 18: Blood systen, 18, 19: Nerrous system, 19, 20 : Neural gland, 19, 20 : Excretory system, 20: Reproductire system, 20: Systematic position, 23: Development, 30

Ascidia mammillata, 35

Ascidiacea, 22*, 30

Ascidiæ compositæ, 22*, 24, 25

Ascidiæ simplices, $2.2 *, 24$

Asciclians, 1:3

Ascidiida, 23
Aspredo, 231

Asses, 468,580

Astacopsis, 60:2

Astracus, 601

Asteriseus, 208*, 209

Astragalo-seaphoid, 344

Astragalus, 259, 266, 344, 444 See Limb-skeleton

Astroscopus, 226

Ateles, 473

Athecata, 359

Atlas, 306*

Atrial aperture, $14^{*}, 15$

Atrial eanals, of Larvaeea, 23

Atrial cavity, of Ascidia, 14, 15, 16, 17

Atrial lobes, of Doliolum, 26

Atrial siphon, 15, 18

Atriopore, of Amphioxus, 43, 44, 4\%, 49

Atrium, of Ascidia, 16, 35: Amphioxus, $4 \%, 49$

Auditory eapsules, of C'raniata. 73*

Auditory foramina, 75

Auditory nerve, 101, 102-See Brain

Auclitory organ-See Ear

Auditory ossicles, $441^{*}$ - See Ear

Auditory region, of Craniata, 74*

Augustine, 654

Auks, 403, 422, 429, 431

Auriele, Ss-See Heart

Auricular appentix, 449

Australian region, $611 *$

Autostylic skull, $76^{*}, 185$

Aves, 64, 303, 366: Example, 367:

Distinctive characters and classi-

fication, 395 : General organisation, 405: Archreomithes, 405: External charaeters of Neornitles, 407 : Ptrrylosis, 409: Skeleton, 411: Myology, 420): Digestive organs, 420 : Respiratory and vocal organs, 421: Cireulatory organs, 421 : Nervous system and senso organs, 121: Urinogenital organs, 42.2 : Development, 42:2: Distribution, 4:9 : Ethology, 430 : Phylogeny, 430

Arocet, 408

Axis, 306*

Axis, basi-cranial, 498*

Axolotl, 284, 301, 302

Aye-Ayes, 47.2

Azygos veins, of Rabbit, 452: of U rodela, 29\%, 298

B ABOONS, 473, 499, 538, 539

Baer, K. E. von, 6.5)

Balana, 466

Balanida, 466

Balcenoptera musculus, 51 
Balcenoptera rostrata, 550,551

Balanoglossus, 2, 41, 42: External characters, 3, 4: Colome, 3: Digestive organs, 5 : Notochord or œsophageal diverticulum, 3, 6: Blood-vascular system, 4, 5: Nervous system, 4, 5, 6, 7: Reproductive system, 7: Development, 7, 8, 9: Metamorphosis, 8, 9

Baleen, $466^{*}, 550^{*}, 551$

Baleen whales, 466

Balfour, F. M., 667

Banded ant-eater, 480

Bandicoots, 464, 480, 507, 544, 5\%8

Banks, Sir I., 655

Barbel, 141*

Barbels, in Teleostomi, 212, 214, 218

Barbs, of feather, $3 \% 0^{*}, 3 \% 1$

Barbules, of feather, $3 \% 0^{*}, 3 \% 1$

Barriers, 606

Barry, Martin, 659

Basal plate, of Craniata, 73*, 74

Basalia, of Craniata, $80^{*}$

Basi-branchial, 74, 7\%, 145*, 199, 201

Basi-branchial plate, 169

Basi-branchiostegal, 201

Basi-cranial axis, 498*

Basi-cranial fontanelle, 122, 123*

Basi-dorsal, 143

Basi-facial axis, 498*

Basi-hyal, 74, 76*-Sce Skull

Basi-occipital, yy*_-See Skull

Basi-pterygium, 203, 204

Basi-pterygoid processes, of Birds, $378^{*}, 414,415$ : of Lizard, 308, 309

Basi-sphenoid,

Basi-temporals, 376, 37y

Basis cranii, $74 *$ - See Skull

Basking sharks, 173, 182

Bastards, 644

Bates, H. W., 663

Bathymetrical distribution, 614

Bats, 471, 493, 501, 553, 581-See Chiroptera

Bdellostoma, 134, 135, 136, 13\%, 138, 139

Bdellostoma forsteri, 135

Bdellostoma stouti, 137, 138

Beak, in Teleostomi, 218: of Pigeon, 367: of Neornithes, 408

Beaked whales, 466

Bears, 470, 491, 529, 530, 551, 552, 564,580

Beavers, 470, 491, 532

Bee-eaters, 404

Bellonius, 648

Belly, of muscle, 266*

Belodon, 359

Beneden, E. van, 667

Benthos, 616*

Beryx, 620
Bierps muscle, 268

Bieipital groove, 442

Bile, 85*

Bile ducts, 85-See Liver

Biogenesis, 650

Birds-See Aves

Birds of Paradise, 404, 411, 420, 602

Blackbirds, 404

Bladder, urinary, of Craniata, 116 : Trout, 208, 209 : Teleostomi, 232 :

Rabbit, 459, 460 : Mammals, 565

Blainville, 660

Blastocœle, 280*

Blastodermic vesicle, 569

Blastula, of Amphioxus, 54, 5.5

Blenny, 234

Blind-snakes, 324, 356

Blind-worm, 328, 332, 350, 358

Blood, 94-See Vascular system

Blood corpuseles, 50, 94-See Vaseular system

Blood vessels-See Vascular system

Boar, 548

Boas, 324,356

Boatswain-bird, 403

Body, of vertebra, 72*

Body-cavity-See Colomo

Body-wall, of Amphioxus, 44, 45: of Craniata, 67

Boltenia, 24

Bombinator, 293

Bones, of Craniata, 76

Bonnet, 653

Bony labyrinth, $394^{*}$

Bony Pike, 198, 213, 218

Botryllus, 22, 25

Bottle-nosed whales, 466, 501

Boucher de Perthes, 665

Bougainville, 655

Bovida, 468-See Oxen

Bowerbank, 661

Bower-birds, 429

Bow-fin, 190, 213

Brachial plexus, 277*

Brachium, 67, 304

Bradypodida, 465-See Sloths

Bradypus tridactylus, 512, 513, 514, 515,554

Brain, Amphioxus, 52 : of Craniata, 95, 97 : Lamprey, 126, 12\%, 128, $153,154,155,156,15 \%$ : Elasmobranchii, 173: Holocephali, 187, 188: Trout, 206, 20\%: Teleostomi, 231: Ceratodus, 246, 247 : Frog, 275, 276 : Amphibia, 298: Lizard, 318, 319, 320 : Reptilia, 349, 350 : Pigeon, 391, 392: Aves, 421, 422 : Rabbit, 454-458: Mammals, $5 \%$ y -562

Brain-case, of Craniata, 73*

Branchia, of Salpa, 2\%* 
Branchis, of Amphioxus, 47 : Lamprey, 126 : Dog-fish, 149 : Elasmobranchii, 173: Holocephali, 187 : Trout, 204: 'T'eleostomi, 227, 228: Ceratodus, 243, 244: Tadpole, 282 : Anphibia, 287,295

Branchiæ, external, 250, 281, 282, 295 : internal, 283, 295

Branchial apertures, of $A$ mphioxus, $46^{*}$, 47 : of Craniata, $65,86^{*}, 87$

Branchial arches, of Craniata, 75* : of Dog-fish, 145

Branchial basket, of Lamprey, 121, $124 *$

Branchial clefts, 2*-See Branchial slits

Branchial filaments, of Craniata, 86*

Branchial junctions, of Amphioxus, 47

Branchial lamellæe, of Amphioxus, 4\%*, 48

Branchial nerves, of Craniata, 101, 102, 103-See Brain

Branchial rays, 145*, 169

Branchial rods, of Amphioxus, 4\%, 48*

Iranchial slits, of Balanoglossus, 3, 4 : of Amphioxus, 46*, 4\%,60,61: of C'eploalodiscus, 11

Branchio-cardiac vessel, 1\%, 19

Branchiostegal membrane, 193, $21 \mathrm{~s}$

Branchiostegal rays, 193, 197

Brunchiostoma, 43-See Amphioxus

Brunchiostomidae, 43

Brassica oleracea, 631

Broad ligament, 323

Bronchi, of Lizard, 318: of Pigeon, 385,386 : of Rabbit, 453

Bronchioles, 454

Brown funnels, of Amphioxus, 47, 51

Brush-turkeys, 403

Buccal cavity, of Craniata, 82

Buccal funnel, of Lamprey, 120* : Myxine, 135

Buccal glands, 84*: Pigeon, 385 : Birds, 421

Budding, in Cephalodiscus, 11 : in Ascidians, 2t: Doliolum, 37, 39 : Salpa, 40

Buffon, 653, 654

Bufo vulgaris, 288

Bulbus aortæ, 89, 126, 205, 206, 271

Bulla tympani, 439, 498*

Bunodont, 545

Burchell's zebra, 488

Burnett Salmon, 239, 240

Burr, of antlers, $487^{*}$

Bursa Fabricii, 384, 385*

Bustards, 403

Butterfly-fish, 219

Button-quails, 403

\section{C}

$C_{A B A L U S, 600}$

Cacatua, 404

Cacutuidae, 602

Cadophore, 38*, 39

Caducibranchiata, $287 *, 291,302$

Coccilia, 285, 301

Cocilia pacínnema, 288

Crecilians, 256, 285

Cxecum, 313, 31\%, 448, 556

Caimans, $326,332,358$

Calamoichthys, 212, 236

Calamus, 369

Calamus seriptorius, $458^{*}$

Calcaneum, 259, 266, 444-Sce Limbskeleton

Calcar, 259, 266, 471*, 535*

Calcified cartilage, $76^{*}$

Callichthys, 229

Callithrix, 473

Callorhynchus, 183, 184, 185, 186, 18\%, $188,189,190,191$

Callosities, ischial, 494*

Cambrian, 618

Camelidae, 468-See Camels

Camels, 468, 487, 527, 548, 554, 555, 580

Campanula Halleri, 208*

Camper, Peter, 654

Camptotrichia, 240*

Canidoe, 470, 491-Seo Dogs

Canines, of Rabbit, 445-Sce Teeth

Canis, 483, 530

Canis dingo, 602

Canis familiaris, 529, 530, 543, 554, 559

Cannon bone, of Horse, 526 : Puminants, $52 \%$

Cape Ant-eaters, 465, 485, 509, 54\%, 579, 584

Capibara, 492

Capillaries, 50, 89, 90-Sec Vaseular systern

Capitellum, 442

Capitular facet, 305,435

Capitulum, 435*

Capra, 468, 487, 617

Caprimulgida, 404, 408

Capuchin Monkeys, 473

Capybara, 492

Carapace, of Chelonia, 329,336

Carboniferous period, 619

Carcharias, 172*

Carchariidae, 176

Carcharodon, 182

Cardiac nerve, of Craniata, 101, 103

Cardiac sac, of Balanoglossus, 4. 6*

Cardiac vein, 272, 273

Cardinal veins, 90 - See Vascular sys. tem

Cardio-visceral vessel, $17,19^{*}$ 
Carina sterni, $374,375,412,413$

Carinatæ, $400^{*}, 410,413,415,416$, $417,420,422,426,429,431$

Carnivora, 469*, 528, 529, 530, 531, $556,558,580,589$

Carnivora vera, 469*. 491, 528, 529, 530,551

Carotid arteries, 88, 90-See Vascular system

Carotid gland, 271*

Carp, 214, 230, 236

Carpals, of Craniata, 81*-See Limb. skeleton

Carpo-metacarpus, $3 \% \mathbf{9}$

Carriers, 367

Carter, 661

Cartilage-hones, $76^{*}$

Cartilages of Santorini, 453*

Caruncle, 428*

Casque, of Cassowary, 408*

Cassowaries, 398, 408, 411, 412, 417, 419,429

Castorida, 470

Casuarius-See Cassowaries

Cal-fishes, 214, 221, 225, 226, 230, 234, 236

Cathartes, 403

Cats, 470, 491, 529, 530, 551, 552, 580

Catume furcatus, 238

Caudal artery, 90

Caudal ganglion, of Appendicularia, 24. 28

Caudal swellings, 178, 179

Cauclal vein, 11-See Vascular system Cauclate lobe, 557*

Cavim arteriosum, 348*

Cavum pulmonale, 348*

Cavum venosum, $348^{*}$

Cebider, 473*, 494, 538, 581, 613

Cebus, 473

Cell, 650

Cell-theory, 658

Cement, 83

Centetes ecrudritus, $\mathbf{5 3 3}$

Centetider, 610

Cent ral canal, 95, 96*

Centrale, of Mlammals, 500 -See Limbskeleton

Centralia, of Craniata, 80, 81 *

Centrophorus calceus, $\mathbf{1 6}^{\text {ry }}$

Centrum, of Craniata, 72*

Cephataspis, 255, 256

Cephalaspis lyelli, 255

Cephalaspis murchisoni, 255

Cephalochorda, 43*

Cephatodiscus, 2, 9, 10, 11, 13

Cerato-branchial, of Craniata, 74, 76* - Sec Skull

Ceratomus rorsteri, 239: Extemal characters, 240 : Endo-skeleton, 241 : Digestive organs, 243 : Organs of respiration, 243, 244: Blood-vascular system, 244, 245: Brain, 246: Urinogenital organs, 247,248 :

Development, 247, 249

Cerato-hyal, 74, 76*-See Skull

Ceratotrichia, $746 *, 168$

Cercopithecidee, 473*, 494, 581

Cere, 368, 369*

Cerebellum, 97

Cerebral commissures, of Frog, 276, 277: Lizard, 319-See Brain

Cerebral flexure, of Craniata, $100^{*}$

Cerebral ganglion, of Appendicularia,

24,28

Cerebral hemispheres, 97-See Brain

Cerebral nerves, 100 Sec Brain

Cerebral vesicle, Amphioxus, 47, 52*

Cerebro-spinal cavity, of Craniata, $6 \mathrm{~S}^{*}, 69,95$

Cervical ribs, 307

Cervide, 468,487

Cervus elaphus, 520, 525, 526, 527

Cestracion galeritus, egg-case, 175,176

Cestracionts, 18:

Cetacea, $465 *, 476,486,495,496,501$, $513-51 \mathrm{~s}, 550,553,555,556,557$, $558,563,564,579,586$

Chalaza, $422 *$, 423

Chalcides, 354

Chatinotolus morio, 600, 602

Chameleo vulgaris, 328

Champleons, 324, 328, 332, 333, 337, $346,347,350,354,355,356$

Chambers, Robert, 661

Chambers, of eye, 108

Charadriiformes, 403

Charadrius, 403, 411

Chauna, 403, 409

Chelodina, 335

Chelone midas, 336, 341

Chelonia, 325*, 329, 332, 333, 335, $336,337,340,341,343,345,346$, $347,348,349,351,357,358,359$

Chevron-bones, 306*, 333

Chevrotains, 519

('hilobranchus, 235

Chimera, 182, 183, 184, 185, 186

Chimaeridae, 182

Chimpanzees, 473, 535, 538, 540, 581

Chirocentrus, 227

Chiromys, 472, 609

Chiroptera, $471^{*}, 493,534,553,558$, 581,590

Chlomydosaurus, 355

Chlamydoselachus, 164, 166, 169

Cholopus didactylus, 483*

Choloepus hoffmanni, 509, 513

Chondrichthyes, 140

Chondrocranium, 78*_-See Skull

Chondrostei, 212*, 218, 221, 222, 223, $224,227,231,234,235,236,23 \%, 238$ 
Chorda dorsalis-See Notochord

Chordx tendinere, 450

Chordata, I* $^{*}$

Chorion, of Ascidian, 31* : Rabbit, 462: Mammals, 572, 5\%3

Chorionic villi, of Rabbit, $462 *$

Choroid, 106, 107*

Choroid fissure, 109*

Choroid gland, $207 *, 208$

Choroid plexus, $100^{*}, 153^{*}$

Chrysochlorida, 609

Chrysophrys, 233

Chrysothrix, $4 \% 3$

Chmm, 663

Ciconia, 403, 408, 409, 115

Ciliary ganglion, 100, 101

Ciliary muscle, $107^{*}$

Ciliary processes, $106,107 *$

Ciliated funnel, of Ascidia, 20, 29

Circulatory system-See Vascular system

Cistudo lutaria, 336

Civets, 470,580

Cladoselache, 162, 163

Cladoselachii, 162*

Claspers, of Dog-fish, 141: Elasmobranchs, 166: Holocephali, 183, 184

Classification-See Distinctive characters and classification

Classification, of Arist otle, 648: Gesner, 649: Ray, 651 : Linnæus, 651, 652: Lamarck, 656 : Cuvier, 657 : Huxley, 664

Clans, 668

Clavellina, 32, 33

Clavicle, of Craniata, 80, 82*

Claws, 289,369

Cleithrum, 243,293

Climbing Perch, 228

Clitoris, of Reptilia, 351 : Rabbit, 459, 461 : Mammals, 567

Cloaca, of Craniata, $65 *$ - See Digestive system

Club-shaped gland, of Amphioxus, 59

Cnemial process, of Pigeon, $380^{*}$, 381

Cnemial ridge, 313

Cnemiornis, $413,417,600$

Cobitis, 235

Coccosteus decipiens, 253

Coceygeo-mesenteric, 389,390

Coecyx, 536*

Cochlea, 111*, 112, 321, 322-See Ear

Cockatoos, 404

Cod, 190, 214, 218, 223, 23:2, 233

Coliac artery, 90

Coeliac plexus, 458*

Coeliaco-mesenteric artery, 315,316

Calolepida, 254, 255*
Coclome. of Baltenoglossus, 3, 5 : Ascidir, 21 : Amphioxus, 4\%, 48, 49, 56, 57: Craniata, $68,69,119$ : Trout, 203, 205: Rabbit, 444, 445 Coelomic bays, $17 \%, 178$

C'onolestes, 579, 583, 611, 613, 621

Coffer-fishes, 216

Cogire, 466, 562

Coiter, 648

Colies, 404

Colii, 404

Collar, of Balanoglossus, 3, 4

Collar-pores, $4^{*}, 11$

Collocritir. 423

Colon, of Dogfish, 148, 149: of Rabbit, 448

Colours, of feathers, 410

Colours, courtship, 111

Colubrine Snakes, 345

Colugos, 492, 493

Columba, 404

ColumbA LIVIA, 367 : External char acters, 367,368 : Exnskeleton, 369, 370, 371, 372, 373: Endoskeleton, 373-382: Nuscular system, 382, 383 : Digestive organs, 383, 384 : Ductless glands, 385 : Respiratory and vocal organs, $385,386,38 \%, 388$ : Circulatory organs, 388, 389, 390 , 391 ; Nervous system, 391, 392 : Sensory organs, 393, 394: Urinogenital organs, 394, 395 : Systematic position, 404

Columbæ, 404*, 423, 429

Columbidee, 404

Columella auris, of Frog, 261, 263 : Lizard, 309*: Reptilia, 350 : Pigeon, $3 \% 8$

Columnæ carnex. $450 *$

Colymbus, 402, 431

Commissures, 319,320 , ete., aberrant, 319,320

Composite Ascidian-See Ascidice Compositre

Conclylar foramen, 438*

Condylarthra, 587

Condyle, of mandible, 437, 441: of skull, 436, $4: 38$

Cones of eye, 108

Contour feathers, $371 *$

Contra-deciduate placenta, 576

Conns arteriosus, ss

Cook, Captain, 6.5.)

Coots, 40!

Cope, E. D., 667

Coprodrem, of l'igeon, $384,385 *$

Copulatory saes, 322,323

Coraciide, 404

Coraco-humeralis, 268

Coracuid, of Craniata, 80, $82 *$-Sec Pectoral arch

U $\quad \mathbf{U}^{\prime 2}$

VOL. II 
Coraco-scapular angle, 378

Corium-See Dermis

Cormorants, 403, 409

Cornea, 106*

Cornu, hyoid of Craniata, 76-See Skull

Cornual cartilage, of Lamprey, 122, 123

Corona radiata, 569*

Coronal suture, $307 *, 439$

Coronary, 308, $310^{*}$

Coronary arteries, 450

Coronoid process, Lizard, 310-Sce

Skull of Mammals, 441

Corpora bigemina - See Optic lobes

Corpora cavernosa, 459, 460*, 461

Corpora quadrigemina, 458, 560*-See Brain of Mammals

Corpora restiformia, of Dogfish, 153* : Holocephali, 187-See Brain

Corpora striata, of Craniata, $99^{*}$ - Sec Brain

Corpus callosum, 454, 456, 560

Corpus geniculatum, 456, 457*

Corpus luteum, 569*

Corpus mammillare, 45\%, 458*-See Brain of Mammals

Corpus spongiosum, 459, 460*

Corpus sterni, 496

Corpus trapezoideum, of Rabbit, 458*

Corpus uteri, 567

Cortex, of hair, $474,4 \% 5$ : of Kidney, 564

Corvidee, 404, 432

Costal plate, $335^{*}$

Costal sternum, 265**

Costo-pulmonary muscles, 386

Cotyledonary placenta, 576

Cotyledons, $467 *$

Cotyloid, 443, 500

Cowper's glands, 460

Craig-fluke, 220

Cranes, 403, 409

Cranial eavity, of Craniata, 6s*

Cranial nerves-See Cerebral nerves, 100

Craniata, 43*, 63* : Classification, 63, 64: External characters, 65, 66, 67, 68: Body-wall and internal cavities, 6\%, 68, 69, 70 : Skeleton, 70-82: Digestive organs, 82, 83, $84,85,86$ : Respiratory organs, 86 , 87: Blood-vascular system, 88-94: Lymphaties, 89, 94, 95: Nervous system, 95-104: Sensory organs, 104-113 : Urinogenital organs, 113117 : Development, 11\%, 118 : Metamerism, 118, 119 : Distinctive characters, 119

Cranium of Craniata, $73^{*}$ _-See Skull

Crax, 403

Credontia, 589
Cremaster, 577

Cretaceous, 620

Cribriform plate, 438

Cricoid cartilage, of Lizard, 318 : Reptilia, 347 : Pigeon, 385 : Rabbit, 453

Cristæ acousticx, $111 *, 112$

Crocodilia, 326*, 331, 332, 333, 334,

$335,336,340,342,343,344,345$,

$346,347,348,349,350,351,352$,

$357,358,359,360$

Crop, of Pigeon, 383*, 384

Crossopterygii, 211*, 212, 218, 219, $222,223,224,225,227,229,235$. $236,237,238$

Crotalus, 338

Crowned pigeons, 404

Crows, 404, 432

Crura cerebri, of Craniata, $97 *$-See Brain

Crusta petrosa, $167 *$

Cryptobranchus, 284, 287

Cryptodrilida, 601,602

Cryptozoic fauna, 617*

Crypts, of uterus, 462,576

Crypturi, 403*, 414, 417, 429, 431

Ctenoid scales, 221*

Cubitals, 373*

Cuboid, 444

Cuckoos, 404

Cuculidae, 404

Cumulus proligerus, 568*

Cuneiform, 442, 443, 500, 501

Curlews, 403, $40 \mathrm{~s}$

Currasows, 403

Cutaneous glands, of Mammals, 476

Cuvier, 657, 658, 665

Cyanorhamplus, 600

Cyathozooid, $37^{*}$

Cycloid scales, 221*

Cyclomyaria, $21 *, 22,25,26,3 \%, 38,39$

Cyclopterus, 225

Cyclostomata, 64, 119*: Example, 119: Distinctive characters and classification, 133 : Comparison of Myxinoids with Lamprey, 134: General remarks, 138

Cycloturus, 484, 510, 512, 513

Cygnus, 403, 408, 409

Cynocephalus, 473, 499, 538, 539, 590

Cynocephalus anubis, 539

Cypselidce, 404, 408, 409

Cystic duet, of Craniata, 85*

\section{D}

$D$ ACTYLOPTERUS, 219

Dana, J. D., 662

Darwin, Charles, $629,630,661,662$

Darwin, Erasmus, 655 
Darwinian theory, 629

Dasypodido, 465-See Armadillos

Dasyproeta, 492

Dasypus sexcinetus, 484, 510, 511, 512, 513, 516

Dasyures, 464,480

Dasyuridce, 464, 479, 506, 508, 547, 577

Dasyurus, 508, 577

Dasyurus viverrinus, 480

D'Azyr, V., 653

Decidua, 462, 576

Deciduate placenta, 462,576

De Bary, 659

Deer, 468,487

Deer, Red, 520, 525, 526, 52\%

Delphinus, 466, 486, 54:3, 544

Deltoid ridge, $442 *$

Demersal eggs, $235^{*}$

Dendrohyrax, 469

Dental formula, 545*

Dental groove, 541, 542

Dental lamina, 541, 542

Dental papilla, 83, $84^{*}, 541,542$

Dental sac, $542 *$

Dentary, of Craniata, $7 \%, 80 *$

Dentine, $83^{*}, 84$

Dentine-forming layer, 542*

Dentition-See Teeth

De Perthes, B., 665

Depressor muscles, 267*

Dermal defences, 184

Dermal fin-rays, $80 *$

Dermal teeth, 140, 222

Dermatochelys, 336, 357, $35 \mathrm{~s}$

Dermis, of Amphioxus, 45 : Craniata, 67

Derotremata, 284*, 287

Desmognathous arrangement, 415*

Determinants, 668

Development, of Balanoglossus, \%, 8, 9 : Ascidian, $30,31,32,33,34,35$, 36, 37 : Pyrosoma, 37: Doliolum, 38, 39 : Salpa, 40: Amphioxus, 5562: Craniata, 11\%, 118 : Lamprey, 130, 131, 132, 133 : Elasmobranehii, $176,17 \%, 178,1 \% 9,180,181$ : Holocephali, 190, 191 : Trout, 209, 210 : Teleostomi, 234, 235: Ceratodus, 247, 248, 249 : Frog, 279, 280, 281, 282, 283: Amphibia, 300, 301, 302 : Reptilia, 351, 352, 353, 354: Aves, 422-429: Rabbit, 461, 462 : Mammals, 567-579

Devonian period, 619

Diaccele, 97

Diaphorapteryx, 605

Diaphragm, of Craniata, $70^{*}$ : of Rabbit, 445

Diastema, 445

Diazona, 22

Dicotyles, 468, 580
Didelphyidee, 464, 478, 479, 480, 506, $507,546,547,565,566,577,579$, $583,609,613$

Didelphys dorsigera, 566

Didelphys marsupialis, 546, 547

Didelphys virginiana, $\mathbf{4 8 0}$

Dididae, 404

Diduneulus, 612

Didus, 404, 417, 431

Diencephalon, of Craniata, 97*

Diffuse placenta, $576^{*}$

Digestive system, of Balanoglossus, 4, 5, 6: Ascidia, 18: Appendiculuria, 24, 28: Simple Ascidians, 24, 28: Composite Ascidians, 28: Salpa, 28: Doliolum, 28: Craniata, 82 : Lamprey, 124: Myxine, 136, 137 : Dogfish, 147, 148, 149: Elasmo. hranehii, 17:2 : Holocephali, 186 : Trout, 204, 205: Teleostomi, 226, 2:7 : Ceratodus, 243: Frog, 268, 269: Amphibia, 295: Lizard, 313, 314: Reptilia, 345, 346: Pigeon, 383, 384, 385: Aves, 420, 421: Rabbit, 445, 446, 447: Mammals, $541-557$

Digitals, $373^{*}$

Digitigrade, $364^{*}$

Digits, 67 - See Limbs

Dingo, 580, 611

Dinoceras, $5 \mathrm{~S} 8$

Dinornis robustus, 418,621

Dinornithes; $398 *, 417,423,429$

Dinornithida, 398, 429

Dinosauria, $326^{*}, 362,363,364$

Dinotherida, 587

Dinotherium giganteum, $\mathbf{5 8} \%$

Diomedea, $402,417,422$

Diphycercal fin, 184*, 2:3

Diphyodont, $463^{*}$

Diploblastic forms, 597

Dipneumona, 250*

Dipnoi, 64*, 239 : Example, 239 : Distinctive characters and classification, 249, 250: General remarks, 250

Dipodidae, 470, 531, 532

Diprotodon australis, 583, 584, 621

Diprotodont, 545

Diprotodontia, $46 \tilde{s}^{*}, 481,482,483,583$

Dipterus, 252

Dipus, 492

Discoidal placenta, 576

Dispersal, 606

Distalia, of Craniata, $\mathbf{8 0}, 81^{*}$

Distinctive characters and classification, of Acrania, 62, 63: Craniata, 64, 119 : Cyelostomata, 133 : Elasmobranchii, 161 : Teleostomi, 211 : Dipnoi, 249: Amplibia, 283 : Reptilia, 323: Aves, 395: Mammals, 462 
Distribution, of Acrania, 62

Distribution, geographical, 598: of Cephalodiseus, 13: Rhablopleura, 13: Urochorda, 41 : Cyelostomata, 139: Holocephali, Is2: Teleostomi, 235 : Dipnoi, 250 : Amplnibia, 302: Reptilia, 358, 359: Aves, 429: Mammalia, 579

Distribution, geological, 618 : of Cyelostomata, 139 : Elasmobranchii, 182: Holocephali, 190: 'Teleostomi, 236, 2:37 : Dipnoi, 252: Amplibia, 302: Reptilia, 359, 360: Aves, 430 : Maunmals, 581

Divers, 402,431

Divertieulum, asophageal, of Balanoglossus, 4, 6

Dodo, 404, 413, 417, 431, 6:2

Dogfish, 68 - See Seyllium and Hemiseyllium

Dogfishes, 165-1 \$2

Dogs, 470, 491, 499, 529, 530, 543, $551,552,559,580$

Dohrn, Anton, 663

Dolehinia, 22

Doliolida, 22

Doliolum, 22, 25, 26, 28, 29, 30, 37, $38,39,41$

Dolphins, $466,486,543,544$

Dominant characters, 644,645

Dersal aorta, 89 - See Vascular system

Dorsal fissure, 95, 96*

Dorsal lamina, 17, 18

Dorsal root, of spinal nerve, 96*, 101

Dorsal shield, Pteraspis, $: 54$

Dorsal tuberele, of Aseidia, 19, 20

Doves, 404

Down feathers, 369, 371*, 372, 409

Draco, $335,355,357,617$

Draco volans, 335, 355, 617

Drepanaspida, 254, $255 *$

Drepanaspis gemundcnensis, 254

Drepanida, 61:

Dromxognathous arrangement, $414^{*}$

Dromceus, 398, 408, 409, 413, 417, 419, $4: 9$

Dromatherium, 581

Dryopithecus, 590

Dryornis, 401

Duck-Bill, 464-See Ornithorlynnchus

Ducks, 403, 408, 415, 416

Ductless glands of Craniata, 85, 117

Ductus Botalli, 296, 298*

Ductus Cuvieri, 152*

Ductus endolymphaticus, 32:-

Dugong, 467, 496, 501, 518, 519, 550, $551,579,586$

Dujardin, 658

Dumb-bell-shaped bone, 502, 505*

Duodenum, 149* : of Pigeon, 384, 385 : Rabbit, $44 \%, 448$
Duplicidentata, 474

Dura mater, $100^{*}$

\section{$\mathrm{E}$}

1JAGLE-RAYS, 172

Eagles, 403, 415, 423

Ear, of Craniata, 110, 111, 112: of Lamprey, 130 : Myxine, 137: Dogfish, 158: Elasmobrauchii, 174: Trout, 208, 209: Frog, 29\%, 278: Lizard, 321 : Reptilia, 350: Pigeon, 369, 394: Aves, 422: Rabbit, 436, 459 : Mammals, 563, 564

Eared Seals, 470, 491, 580

Earless Seals, 470 - See Phocidr

Eedysis, 332

Eelieneis, 219

Eehidna, 464, 476, 477, 478, 479, 503, $504,505,541,545,554,558,560$, $561,562,57 \mathrm{~S}$

Eelidna aculcata, 47\%, 479, 504, 554, 560,561

Eetocuneiform, 444 See Limb-skeleton

Ecto-ethmoids, of Craniata, 7\%, 78*See Skull

Ectopterygoid, 308, 309

Edentata, 465*, 508-513, 541, 557, 565, $567,579,584,585$

Edestosaurus, 366

Eels, 190, 214, 218, 221, 230, 232, 233, 235,236

Efferent branchial arteries of $A m p h i$ oxus, 49, 50*-See Vascular System, S9

Efts, 284, 287

Egg-See Development

Egg-shell, of Dogfish, 161: Elasmobranchs, 175, 176: Holocephali, 190: Reptiles, 357 : Birds, 422 : Prototheria, 569

Ehrenberg, 661, 665

Elæoblast, 41

Elasmobranchii, 64*, 140* : Example, 140: Distinctive characters and classification, 161 : External characters, 165: Integument, 166 : Skeleton, 167: Skull, 168: Muscles, 171: Electric organs, 171: Luminous organs, 171 : Digestive system, 172: Respiratory organs, 173: Blood system, 173: Brain, 173: Organs of sense, 174: Urinogenital organs, 174: Development, 170 : Ethology and distribution, 181

Elastin, 146*

Electric Catfish, 226

Electric Eel, 226

Electric lobe, 171, 174* 
Electric organs, 171, 226

Electric rays, 165, 172, 174

Elephant, African, 524, 550

Elephants, 469, 476, 490, 541, 550, 580

Elephas, 469

Elcphas africames, 524, 550

Elevator museles, $207 *$

Elimination of the unfit, 634*

Embryological evirlence of evolution, 624

Embryon ectode1m, 5\% *

Embryon endoderin, 5\%

Embryonal knot, 569*

Embryonic membranes, of Bird, $42 \%$, 428

Embryonic rim, 177*

Embryonic shield, 352*, 424

Empedocles, 654

Enus, 398, 408, 409, 413, 417, 419, 129

Emys europaca, 340, 343, 346

Enamel, 83

Enamel membrane, 542

Enamel organ, 83, 84*, 541

Enamel pulp, 542

Encephalocole, 4\%, 52*

End-buds, 104*

Endemie, 60:*

Endochondral ossification, 76*

Endolymph, 111*

Endolymphatic duct, $111 *, 112$

Endoskeleton-See Skelet on

Endostyle, of Tornaria, 9: Ascidia, 16, 17*, 18 : Appendicularia, 23: Doliolum, 26 : Ascidian larva, 35, 36: of Amphioxus, 44, 46, 60, 62

Engceus, 602

Entepicondylar foramen, 512*

Enteric canal-See Digestive Organs

Enterocole, of Amphioxus, 57

Enteropneusta, 2*, 3, 4, 5, 6, 7, 8, 9

Entocuneiform, 44t-See Limb-skeleton

Entoplastron, 335*

Eocene, 620

Eosiren, 6:0

Eozöon canadcnse, 618

Epencephalon, 97

Ependyme, 97*, 99

Epiboly, 131*, 210, 280

Epibranchial, of Craniata, 74, 76*

Epicentrals, 222*

Epicœle, 97, 174

Epicoracoid, 264: Prototheria, 502, 505

Epicrium, 285

Epidermis, of Amphioxus, 45 : Craniata, 67

Epididymis, 159, 322, 323, 460

Epigast ric vein, 315, 316

Epiglottis, of Rabbit, 448 : Mam. malia, 558
Epiglottis, intra-11arial, 558

Epigonichthys, 43

Epihyal, of Craniata, 74, 76*, 199, 201

Epinemals, 22:2*

Epiotic, of Craniata, rg*

Epipharyngeal groove, Amphioxus, 46

Epiphyses, of Craniata, \$2* : Rabbit, 434: Mammials, 4!66

Epiphysis (cerebri), of Craniata, 99* : of Elasmobranelii, 173

Epiplastron, 335*

Epipleurals, 222*

Epipterygoid, 308, 309, 337

Epipubic bones, 502, 505*, 50\%, 508

Epipubic process, 170

Epipubis, 294, 295, 312, 50\% : Birds, 419

Episternum, of Lizard, 310*, 311

Rabbit, 436 : Prototheria, 503

Equida, 468, 488, 489

Equns burchelli, 488

Equus caballus, 521, 526, 52\%, 549

Erinaceidre, 471 - See Hedgehogs

Esox, 620

Ethiopian region, 609

Ethmoidal plane, 498*

Ethmo-turbinals, 43\%, 438* - See Skull

Ethology, of Elasmobranchii, 181: Ceratodus, 239: Reptiles, 351: Birds, 430

Euchorda, 42*

Eudynamis taitensis, 606

Eudyptes, 402

Eudyptes antipodum, 402

Eudyptes pachyrhynchus, 413

Euselachii, 164*

Eustachian aperture, 439

Eustachian tubes, of Frog, 268 : Rabbit, 439 : of Mammals, 563*, 564

Eustachian valve, 449, 557*

Eutheria, $465^{*}, 558,561,562,565,56 \%$, 569,577

Evolution, 624

Excretion, organs of, in Ascidia, 20 : Simple Aseidians, 30 : Amphioxus, 50, 51-See Urinogenital Organs

Ex-oceipital, 7\%*_- See Skull

Exocatus, 219

Exoskeleton, of Craniata, 70

Expiration, 193*

Extensor muscles, 266*

Extensores dorsi muscles, 266

External auditory meatus, :369

External characters, of Balanoglossus, 1: Ascidia, 14: Craniata, 65: Lamprey, 120: Dogfish, 140 : Elasmobranchii, 165: Holocephali, 183 : Trout, 192 : Telenstomi, 217 : Cratodus, 240: Frog, 25\%: Amphibia, 285: Lizard, 304: Reptilia, 
327: Pigeon, 367: Aves (Neornithes), 407 : Rabbit, 432 : Mammalia, 474

External colomic bay, 19y*

External elastic membrane, 70, 71

External gills, 87*, 235

External rectus muscle of eye, $\mathbf{1 1 0}$

Extra-branchials, 170

Extra-columella, 263, 378

Extremity, of long bone, 82*

Eycloux, 662

Eye, of Salpa, 29: of Amphioxus, 52, 53: Craniata, 106-110: Dogfish, 158: Elasmobranchii, 174: Trout, 206, 207, 208: Flat-fish, 219: Frog, 277: Amphibia, 298 : Lizard, 305, 321: Reptilia, 349: Pigeon, 368, 369 : Aves, 421 : Rabbit, 433, 458, 459 : Mammals, 563

Eye, development, 108, 109

Eyelids-Frog, 257 : Lizard, 305

Eye-muscles, 110

\section{F}

$\mathrm{I}_{\text {ABELLAi, } 444}$

Fabricius ab Aquapendente, 648

Facial ganglion, of Craniata, $10^{\circ}$

Facial nerve, of Craniata, 101, 102*

Falciform process, 208*

Falco, 403, 415

Falcons, 403, 415

Fallopian tubes, of Rabbit, 460, 461

Fan-tails, 367

Fasciæ dentatæ, 561*

Fat-bodies, of Frog, 278, 279

Faumas, 598

Feather-follicle, 371, 372*

Feather-germ, 3r1, 372*

Feather papilla, 3\%1, 372*

Feather-pulp, 371, 372*

Feather-tracts, 37\%*

Feathers, of Pigeon, 367, 369, 3\%, $3 \% 1$, 372, 373 : Archæopteryx, 405, 406, 407 : Neornithes, 410

Felide, 470, 491-See also Felis and Cats

Felis leo, 530

Felis tigris, 528, $\mathbf{5 2 9}$

Felting, of hair, 474

Femur, 80, 81*-See Limb-skeleton

Fenestra ovalis, 261, 27\%, 278, 321, 439 - See Ear

Fenestra rotunda, 321, 439

Fibula, 80, 81*-.See Limb-skeleton

Fibulare, of Craniata, 80, 81 *

File-fishes, 216, 221

Filo-plumes, 369, 371*, 409

Filum terminale, 277*

Fimbria, 456*
Finches, 404, 408, 429

Finlets, 218

Fin-rays, of Amplioxus, 46* : Dogfish, 145, 146 : Teleostomi, 224 : Teleostei, 219

Fins, of Amphioxus, 43, 44 : Craniata, 65, 66, 80: Lamprey, 121: Cyclostomata, 133, 134: Dogfish, 141 : Elasmobranchs, 170 : Holocephali, 1S4, 185 : 'Trout, 194 : Teleostomi, 201, 202: Teleostei, 218, 219: Ceratodus, 240: Dipnoi, 250

Fins, development of, 180

Fire-toad, 293

Firmisternia, 285*

Fishing-frog, 218, 219

Flamingoes, 403, 408

Flanges, of feather, $3 \% 0,371^{*}$

Flat fishes, 214, 219

Fleming, W., 677

Flexor muscles, 266*

Flexor perforans, 383

Flexor tarsi, 268

Flippers, 486

Floccular fossa, 439

Floceul, 458-Sce Brain

Flounder, 214

Flower, W. H., 663

Fluviatile fauna, 616

Flying-fish, 219

Flying Foxes, 471, 501, 534, 553, 581

Flying Lizard, 335, 355, 358, 617

Flying Mammals, 501

Flying Phalangers, 501

Flying Squirrels, 483, 492, 501

Fetal membranes, of Mammals, 571, $572,5 \% 3$

Follicle cells, of Ascidian, 30, 31 : Salpa, 39

Fontanelles, of Craniata, r4*: Lamprey, 123: Dogfish, 144: Trout, 197, 199 : Frog, 259, 261

Foot, 67-See Hind-limb

Foramen, of Monro, 97-See Brain

Foramen, ischiatic, 380

Foramen magnum, of Craniata, 14* -See Skull

Foramen ovale, of heart, 450

Foramen Panizzæ, 349*

Foramen triosseum, 379

Foramina, intervertebral, of Craniata, 71

Foramina (nerve), of Craniata, $74^{*}$ See Skull

Foramina, pneumatic, 379, 382

Fore-arm, 67, 304

Fore-brain, of Craniata, 97-See Brain

Fore-kidney, 113*, 115

Fore-limb, 67

Fornix, of Rabbit, 454, 456-See Brains of Mammals 
Forster, 655

Fossa, glenoid, of skull, 439: P'respinous, of scapula, 442: Postspinous, of scapula, 442

Fossa ovalis, 449, 450*

Fossa rhomboidalis, $153 *, 154$

Fossæ, of cranium, 440

Fourth ventricle, 97-Seo Brain

Fowls, 403, 409, 412, 415, 420, 421, $422,423,424,425,426$

Fratereula, 403

Fregata, 403

Fresh-water fauna, 616

Fresh-water Snakes, 324, 356

Frey, 660

Frigate-bird, 403

Fringillidoe, 404, 408, 430

Fritillaria, 24

Frogs, 256, 25\%, 284, 288, 289, 300 See Anura

Frontal clasper, 183, 184

Frontal segment, of Craniata, $7 \%$, 78*

Frontal sinuses, 559

Frontal suture, $307 *, 439$

Frontals, of Craniata, $9 \%, 78^{*}$ _Sec Skull

Fronto-parietals, 261, 262*

Fulera, 213, 22:2

Fulmars, 402

Fulmarus, 402

Fur, 491

Fur Seals, 491

Furcula, $378^{*}$

\section{G}

\section{GADUS MORRHUA, 214}

Gaimard, 662

Galaxias, 600

Galen, 648

Galeopitheeus, 492, 493, 617

Galesaurus planieeps, 360

Gall-bladder, of Craniata, 69, 85* : of

Dogfish, 148, 149: of Birds, 421 :

Rabbit, 448

Gallinæ, 403*, 429, 432

Gallus, 403

Gallus bankiva, 412, 415, 420, 423, 424, 425,426

Game birds, 403

Ganglia habenulæ, $12 y$

Ganglion, cœliac, 458

Ganglion impar, 458

Gannets, 403

Ganoidei, 213*, 219, 2.2. 224, 227, 229, $231,232,233,235,237,238,239$

Ganoid scales, 221*

Ganoin, 222

Gar-fish, 218

Gar-pike, 213
Gare-fowl, 429

Gasserian ganglion, 101, 155

Gasterosteus, 234

Gastornis, 399, 420

Gastornithes, $399 *, 430$

Gastræa theory, 66.1

Gastric glands, 83*

Gastric juice, $83^{*}$

Gastric nerve, of Craniata, 101, 103

Gastrochisma, 219

Gastrocnemius, muscle, 266,267

Gastro-cutancous pores, 6

Gastrula, of Amphioxus, 55: Craniata, 117

Gavia, 403, 411, 431

Gavial, 326, 359

Geckos, 324, 332, 333, 336, 350, 354, 358

Geese, 403, 408, 409, 415

Gegenbaur, C., 668

Genital pores, of Petromyzon, 130, 131 : of Trout; 209

Genu, 454

Geotria, 120, 134, 139, 604

Germinal disc, 175* : of Fowl, 42:2

Gesner, Conrad, 649

Giant fibres, Amphioxus, 53

Giant goose, 413

Giant nerve-cells, Amphioxus, 53

Giant Rail, 403

Giant Salamander, 287

Gibbons, 473, 494, 581

Gill-pouch, of Craniata, 86*

Gill-rakers, I 73, 201

Gill-rods, of Amphioxus, 46, 4\%, 48

Gill-slits-See Branchial slits

Gills-See Branchix: of Craniata, 86

Giraffa, 468

Giraftes, 468, 487, 580

Girdle bone, 261

Cizzard, 384

Glands, Cowper's, 459, 460

Glass-fish, 235*

Glenoid cavity, 80, 442

Glenoid fossa (of Skull), 439--Sce S'kull of Mammals

Glenoid surface, of Craniata, 80, $81 *$

Globe-fishes, 216, 221

Globigerina-ooze, 615

Globioeephalus, $\mathbf{5 1 8}$

Glomerulus, 114* : of Balanoglossus, 6 Glossopharyngeal nerve of Craniata, $101,102 *$ - See Brain

Glottis, 244*, 268, 289: Pigeon, 384, 385 : Rabbit, 453

Glycogen, 85*

Glyptodon clavipes, 585,621

Glyptodontida, 585

Glyptolepis, 236

Gmelin, J. F., 651

Goat-suckers, $404,40 \mathrm{~s}$ 
Goats, 468,487

Goethe, 657, 659

Gonads, of Craniata, 113, 115. $116-$

See Reproduction, Organs of

Goode, Brown, 6ti3

Gorilla, 473, 494, 495, 535, 538, 540, 581

Goura, 404

Graafian follicles, 461,568

Grallæ, 40:3*

Grant, R., 661

(irayling, 214

Gray's Whale, 562

(irebes, 402, 409

(Arew, Nehemiah, 649

Grey matter, 95,96*

Groove of Hatschek, 44, 5:3, 54*

(iround-parrot, 413, 431, 600

(irouse, 403

Crus, 403, 409)

Gudgeon, 214

Gullet-See Digestive organs

Gulls, 403, 411, 421, 429, 605

Gurnard, 215

Gustatory nerve, 101

Gymnarchus, 235

Gymnophiona, 285*, 288, 289, 291, $296,298,301,302$

Gymmotus, 226, 613

Gypaëtus, 410

Gypogcranus, 403, 609

$\mathrm{H}$ ABITA', 605

Haddock, 214, 218, 223, 235

Haeckel, Ernst, 664

Hrmal areh, of Craniata, 69, 72

Hæmal canal, of Craniata, 68*, 69, 72

Hæmal ridges, of Craniata, $71,7: 2$

Hamatopus, 403

Hags. 119, 134, 135, 136, 13\%, 138, 139

Hair-bulb, 475, 476

Hair-follicles, 474, 476

Hair-germ, $475,4 \% 6$

Hair-papilla, 475,476

Hairs, 4\%4, 475, 4\%6 : Develupment, $475,4 \% 6$

Hake, 214

Half-beak, 218

Halicore, 467-See Dugong

Halicore australis, 519

Halitherium, 586

Haller, 653

Hallux, of Craniata, 80, 81*

Halmaturus ualabatus, 478, 50\%

Hamen, Louis de, 650

Hammer-head Shark, 165

Hand, 67, 304

Hapale, 472, 581

Hapalida, 472*, 494, 538, 553, 5\$1, 613
Harderian gland, 321, 563

Hares, 470

Harriotta, 182, 183, 184

Harvey, William, 649

Hatschek, groove of, $44,53,54 *$

Hatschek's nephridium, 58*, 60

Hatteri $, 325,329,330,332,333,334$, $335,336,337,338,339,343,345$, $350,351,357,358$

Hawks, 421

Hawk's-bill Turtle, 358

Hearl, of Craniata, 65

Head-shieldls, of Lizard, 305, 331

Heart, of Balanoglossus, 6: Ascidian. 1\%, 18, 34: Craniata, 88 : Lamprey, 126 : Dogfish, 150 : Elasmohmanchii, 173: Holocephali, 187 : Trout, 204, 205, 206: Teleostomi, 231: Ceratoclus, 244, 245: Frog, 270, 271: Amphibia, 298: Lizard, 314, 315 : Reptilia, 347, 348, 349: Pigeon, 388, 389 : Aves, 421 : Rabbit, 448 , 449 : Mammals, 557

Herlgehogs, $471,476,493,574,575$

Helix, 601

IIelorterma, 345

Helodermidae, 358

Hemibranch, 86, s7*

Hemichorda, 2* : Affinities, 13

Hemimyaria, 22*

IIemipodes, 403

Hemiscyllium Monestum, 140 : General external features, 140,141 : Skeleton, 142-14\%: Enteric canal, $147,148,149$ : Organs of respiration, 149, 150: Blood-system, 150, 151, 152, 153: Nervous system, 153158: Organs of special sense, 158 : Urinogenital organs, 158, 159, 160 , 161

Hensen, V. A. C., 663

Hepatic artery, 91-See Vascular system

Hepatie cecum, Amphioxus, $4 \%$

Hepatie ducts, of Craniata, $85^{*}$

Hepatie portal system, 42, 49, 50, 91See Vaseular system

Hepatic portal vein, 50, 91--See Vascular system

Hepatic vein, Amphioxus, 50

Heptanehus, 164, 166, 167, 168, 169, $1 \% 0$

Heredity, 638, 644

Herodiones, 403*

Herons, 403, 409, 410

Herpestes, 552

Herring, 190, 214, 220, 231, 235

Hertwig, O., 668

Hesperornis, 400, 413, 417, 420

Hesperornis regalis, $\mathbf{4 0 0}$

Heterocercal tail, 141* 
Heterocolous vertebra, $373 *$

Heterodont teeth, $46 ; 3^{*}$

Heterodontus-See ('estracion

Heterostraci, $254^{*}, 255$

Heterotis, 23.5

Hexanchus, 164, 166, 167, 168, 16!

Hilaire, E. G., St., 657

Hilus, 45 乌* $^{*}$

Hind-brain, of Craniata, 97- See Brain Hind kidney, 113-See Metanephros

Hind-limb, 67-see Limbs

Hip-girlle, of Craniata, 82

Hippocampal commissure, of Frog, 277 : Lizard, 319 : Reptilia, 349: Birds, 422 : Mammals, 561

Hippocampal suleus, 456

Hippocampus, $319, \quad 349, \quad 456$ - See Brain

Hippocampus (Sca-horse), 21\%, 234

II ippopotamus, 468. 488, 489, 501, $523,524,525,526,580$

Hippopotamus umphibius, 488

Hirundinide, 104

Hoatzin, 403, 407, 408, 409, 613

Hock, $489 *$

Hoffman's Sloth, 495

Holarctic region, 609

Holoblastic segmentation, 569

Holobranch, 86, $87 *$

Holocephali, 64*, 182 : External cliaracters, 183 : Encloskeleton, 1S4: Digestive organs, 186 : Respiratory organs, 187: Heart, 157: Brain, 187, 188 : Urimogenital organs, 189 : Development, 190, 191: Fossil remains, 190

Holostei, 212*, 213, $218,221,222,223$, $227,229,231,232,233,234,235,238$

Hombrom, 66:

Hominidae, 473*

Homo supiens, 47:3-See Man

Homocercal tail-fins, $19 t$

Homodont teeth, $4633^{*}$

Hoofs, 486

Hook, Robert, $6+9$

Hooker, J. D., 66:2

Hooklets, of feather, $3 \% 0,371 *$

Houpoes, 404

Holnbills, 404, 40s, 420

Horns, of Ruminants, 487 : of Rhinoceros, 489

Horses, 468, 488, 489, 519, 521, 5.5, 526, 527, 549, 580, 587

House, of Appendicularia, $21^{*}$ : of Oikopleura, 23, 24

Howling monkeys, 473

Human species, 473

Humerus, 80, $\$ 1 *$

Humming-birds, 404, 423, 429

Hunter, John, 653

Huxley, T. H., 662, 664, 665, 668
Hycrna, $470,530, .580$

IIycrnidle, 470, 530, 5s0

Hydrocherus, 4!2

Hyla, 28s, 289, 2!):3

Hylobutes, 473, 494, 581, 610, 6.1

Hyoid arch, of Cramiata, $75^{*}-$ See s'kull

Hyoid bome, $80^{*}$

Hyoid cormu, of Craniata, $76 *$, ry, so

Hyoidean artery, :206;

Hyoidean suspensorium, 76

Hyomandibular, of Craniata, $76^{*}$, $7 \%$, S0: Dogfish, 145: Elasmolsanchii, I6S: Trout, 199, 200 : Teleostomi, 223

Hyomandibular nerve, of Craniata, 101, 10: - See Brain

Hyoplast ron, $33.35^{*}$

Hyostylic skull, 76*__See Skull

Hypapophysis, 306 *

Hyperoódon, 466,501

Hypnos, 168, 171

Нypo-branchial, of Craniata, 74, 76*

Hypoglossal nerve, of Craniata, 101, $10: 3 *$ _ See Brain

Hypo-hyal, of Craniata, 74, 76*, 199, 201

Hypo-ischium, 312,343

Hypophysis, of Ascidia, 19, 20 : Craniata, 99 -See Brain

Hypoplastron, $33.5 *$

Hypsiprymus rufescers, $5 \% 8$

Hyracidce, 469, 490, 580

Hyracoidea, 46.*, 524, 527, 5+9, 557, 580,587

Hyrax, 469, 495, 521, 524, 556,580

IIystricida, 492-See I'oreupimes

TBLS, 403, 408

Ibises, 403,408

Ichthyomyzon, 134, 139

Ichthyophis glutinosa, 292, 301

Ichthyopterygia, $: 326^{*}, 362$

Ichthyornis, $402,4: 0$

Ichthyornis victor, 401

Ichthyornithes, $402 *, 411,430,431,432$

Ichthyosaurus, 362

Ichthyotomi, 163*

Iguanas, 324, 333, 355, 358

Iquanodon, 363, $36 \pm$

Iguanodon bernissartensis, 363

Iguanodon mantelli, 363

Iliac artery, 88, 90

Iliac process, 171

Lliac region, $82^{*}$ : of Craniata-See Limb-skeleton

Iliac vein, 88,91

Ilium, $82 *$ - See Pelvic arch 
Impennes, $402 *, 411,413,422,431,432$ Incisors, of Rabbit, 445

Ineubation, 395*

Incus, 441, 564

Indigenous fauna, $601 *$

Inferior oblique muscle of eye, 110

Inferior rectus musele of eye, $\mathbf{1 1 0}$

Inferior temporal arch, 309

Inferior temporal fossa, 309

Inferior umbilicus, 369*

Infra-orbital glands, of Rabbit, 446

Infundibulum, of Craniata, 99-See Brain : of lung, 454

Inguinal canal, 460, 565

Innominate, 500-See Pelvic areh of Mammals

Innominate arteries, 151

Inseriptiones tendineæ, of Frog, 266, $26 \%$

Insectivora, $470^{*}, 492,532,533,552$, $558,563,580,581,589$

Inspiration, 193*

Insular faunas, 605

Integument, of Craniata, 67: Lamprey, 121 : Dogfish, 140: Elasmobranchii, 166 : Holocephali, 184 : Trout, 194: Teleostomi, 217:Ceratodus, 240: Frog, 258: Amphibia, 288: Lizard, 305: Reptilia, 331, 332 : Pigeon, 369, 3\%0, 3\%1, $3 \% 2$ : Aves, 409, 410 : Rabbit, 432: Mam. malia, $4 \% 4$

Inter-branchial septa, of Craniata, 86* : Dogfish, 149

Intercalary pieces, 167

Intercentra, 290, 307*, 333

Interclavicle, 293-See also Episternum

Intercostal arteries, 451, 452

Interdorsal plate, 143*

Inter-hyal, 199, 200

Intermedium, of Craniata, 80, $81 *$ See Limb-skeleton

Tntermuscular bones, 195

Internal cœlomic bay, 19y*

Internal rectus muscle of eye, 110

Inter-neural plate, $143 *$

Inter-opercular, 193, 19\%, 201

Interorbital region of skull, $74 *$

Interorbital septum, 198, 199, 307

Inter-parietal, $43 \%, 439$

Inter-renal bodies, $117^{*}$

Inter-spinous bones, 199, 201*

Inter-vertebral dises, of Crocodilia, 335 : Rabbit, 434* : Mammals, 496

Inter-vertebral foramina, 258

Intestinal glands, 83*

Intestine, of Craniata, 82

Intra-narial epiglottis, 558

Intrinsic muscles of syrinx, 386

Introduced fauna, 601*
Investing bones, $76 *$

Iris, 106, 107*

Ischiatic foramen, 380

Ischiatic symphysis, 312*

Isehium, of Craniata, 80, 81*_See Pelvic arch

Isthmus, 193*

Iter, 2\%6, 277- See Brain

$J$

.) ACANAS, 403

Jacobson's organ, of Craniata, 106: of Lizard, 320, 321 : of Reptilia, 349: of Rabbit, 445, 446 : of Mammals, 562

Jaequinot, 662

Janssen, Hans and Zacharias, $6+9$

Jaws, of Craniata, "4, 75*, 79-Se Skull

Jerboas, 470, 492, 531, 532

Jugal, 199, 200*, 440

Jugular eminence, 537*

Jugular plate, 213, 218

Jugular veins, $90-\mathrm{Sec}$ Vascular system

Jumping Shrews, 493

Jurassic period, 619

\section{K}

T.AGU, $612_{2}$

Kakapo, 413, 431, 600

Kangaroos, 465, 481, 482, 483, 506, $50 \%, 510,546,547,558,5 \%$

Kea, 617

Keel, of sternum, 375

Kidney, development of, 113, 114, 115

Kidneys-See Excretion, Organs of

Killers, 466, 486

King of the Herrings, 183

Kingfishers, 404, 423

Kiwis, 398, 417, 420, 421, 422, 423, 426, 429

Koalas, 482, 483, 499, 506, 508, 545, $562,577,578$

Kölliker, A., 658, 659

Kowalevskia, 23, 24, 28

Kowalewsky, 667

L

IABIA MAJORA, 461

Labial cartilages, of Craniata, 74, 76*: Dogfish, 145

Labrichthys psittacula, 215

Labyrinth, earotid, 271 : membranous - See Ear 
Labyrintliodonts, $256,288,295$

LACERTA, 304, 353 : External features, 304: Exo-skeleton, 305: Endoskeleton, 305-313: Digestive system, 313, 314: Vascular systrm, 314, 315, 316, 318: Organs of respiration, 315, 318: Brain, 318 , 319, 320 : Spinal cord, 320 : Organs: of special sense, 321,322 : Urinary and reproductive systems, 322,323 : Systematic position, $3 \geq 7$

Lacerta muralis, 348

Lacertidee, 327*, 358

Lacertilia, 324*, 327, 328, 329, 332, $333,335,336,337,338,342,345$, $346,347,348,349,351,353,354$, 358,359

Lacrymal bone, 307,308

Lacrymal gland; of Lizard, 321 : Mammalia, 563

Lacteals, $95^{*}$

Lacustrine fauna, 616

Lemargus, 172, 175, 176

Lagena, 208, 321, 322-See Ear

Lagenorhynchus, 544

Lagomyidae, 474

Lagopus scoticus, 602

Lainarck, $629,655,656$

Lamarckian theory, 628

Lambdoidal suture, 439

Lamina perpendicularis, $438 *$

Lamina terminalis, of Craniata, $99^{*}$ See Brain

Lamna cornubica, 165

Lampern, 120

Lamprey-See Petromyzon

Lanarkia spinosa, 254

Lancelet-See Amphioxus

Land tortoises, 325, 331, 357, 358

Languets, 24*, 25, 29

Lankester, E. R., 668

Lapillus, 208*, 209

Larks, 404

Larus, 403, 411, 421, 429, 605

Larvacea, $21 *, 23,28$

Laryngeal nerves, of Craniata, 103

Laryngo-tracheal chamber, 269, 295

Larynx, of Lizard, 318 : Reptilia, 346 : Bird, 385 : Rabbit, 453

La Sueur, 662

Lateral line, 105* : Petromyzon, 121: Dogfish, 140: Holocephali, 183, 184: 'Trout, 192, 194 : Ceratodus, 241: Amphibia, 299

Lateral line canal, 140: Elasmobranchs, 174

Lateral line organs, 105*, 174, 298

Lateral nerve, of Craniata, 102

Lateral plate, of Amplioxus, 59 : of mesoderm, 118

Lateral post-frontal, 308*
Lateral temporal fossa, $309 *$

Lateral vein, 91

Lateral ventricle, of Craniata, 97-See Brain

Laurentian period, 618

Leather-backed Tirtle, 336

Lceuwenloek, 649

Lcmur, 472-See Prosimii

Lens, 106, 108

Lens-capsule, 108

Lens involution, 109

Lepidosiren, 239, 250, 251

Lepidosteus, 213, 218, 221, 222, 223, $227,229,231,232,233,234,235$

Lepidosteus platystomus, 213

Lepidot richia, $202^{*}$

Lepidotus maximus, 238

Leporidce, 470, 474

Leptocephalus, 235

Leptoglossa, 327 *

Lepus cuniculus, 432 : External characters, 433, 434: Skeleton, 434 444 : Colome, 444, 445 : Digestive organs, 445-448: Circulatory organs. $446-$ 452 : Respiratory organs, 453, 454: Ductless glands, 454: Nervous system, 454, 455, 456, 457, 458: Organs of special sense, 458 : Urinogenital organs, 459, 460 : Development, 461: Systematic position, 474

Lepus variabilis, 617

Leuckart, 660, 661

Leucocytes, 94

Leydig's gland, 159*

Lieberkühn, 661

Lienogastric artery, 15]

Limb-girdles, of Craniata, 80, $81 *$ See Pectoral arch and Pelvic areh

Limb-skeleton, of Craniata, "79, 80 : Dogfish, 145: Elasmobranchii, 169: Holocephali, 186: Trout, 201, 202 : Teleostomi, 224: Dipnoi, 243: Frog, 265, 266: Amphibia, 294 : Lizard, $310,311,312,313$ : Reptilia, 342, 343, 344: Pigeon, $379,380,381$ : Aves, $417,419,420$ : Rabbit, 442, 443, 444: Naminalia, 500 : Prototheria, 505: Metatheria, 50s, 510 : Edentata, 511, 512, 513: Cetacea, 516: Sirenia, 519: Ungulata, 525: Carnivora, 530 : Rodentia, 532: Insectivora, 533: Chiroptera, 535 : Primates, 538, 539, 540 ,

Limbs, of Craniata, 67, 81 : Dogfish, 141: Elasmobranchii, 165, 166 : Holocephali, 184: Trout, 194: Teleostomi, 219: Ceratodus, 240: Dipnoi, 250: Frog, 257: Amphibia, 294: Lizard, 304, 305: Reptilia, 
328, 329, 331 : Pigeon, 367, 368 : Aves (Neornithes), 408, 409 : Rabbit, 433: Mammalia, 501

Limicolæ, 403*, 411

Limose, 403, 408

Linea alba, of Frog, $266,26 \%$

Ling, 214

Lingual cartilage, of Lamprey, 121, 123

Linnæus, 651, 652, 654, 665

Lion, $\mathbf{5 3 0}$

Liopelma, 605

Lip-fishes, 218

Liquor amnii, 572*

Liquor folliculi, 569*

Lister, Lord, 6655

Litopterna, 588

Littoral fauna, 614*

Liver, of Aseidians, 28: Euchorda, 42: Amphioxus, 47: Craniata, 69, 85* : Petromyzon, 125. 126 : Myxine, 136: Dogfislı, 148, 149: Elasmobranchii, 173: Trout, 205, 206 : Teleostomi, 227: Frog, 268, 269: Lizard, 313, 314: Pigeon, 384, 385 : Rabbit, 448 : Mammals, 556

Lizards-See Lacerta and Laeertilia

Llamas, 580

Loach, 214

Lobi inferiores, 153*, 154, 173

Lophins, 219

Lophobranchii, 216*, 221, 227

Lories, 404

Lorius, 404

Lucida, 2:2*

Lueretius, 654

Lumbo-sacral plexus, 277*

Lumbricida',601

Lumbricus, 601

Luminous organs, 171, 220, 221

Lump-fish, 2.25

Lunar, 44ㄹ, 443

Lung-fishes-See Dipnoi

Lumgs, of Craniata, 69, 87 : Cerutodus, 244: Frog, 268, 269: Tadpole. 283 : Amphibia, 295: Lizard, 315, 318 : Rrptilia, 347: Pigeon, 385, 386 : Aves, 4:21 : Rabbit, 453: Mammals, 558

Luth, 336, 357. 35S

Lutra, 491, 552

Lutrida, 470,491

Lyell, Sir C., 6.58, 665

Lygosoma, 602

Lymph, 94*

Lymph-eapillaries, 94*

Lymph-hearts, 95*, 275

Lymph-sinuses, 94*, 275

Lymph-space, $46,50^{*}$

Lymph-vessels-See Lymphatics

Lymphatic glands, 95*

Lymphaties, 89, 94*, 274, 275, 557
Lyra, 456*, 45\%

Lyre-birds, 404, 602, 611

M

\ICACUS, 473,590

Macaques, 473

Macaws, 404, 408, 416

Mackerel, 190, 215, 220

Macropodida, 465-See Kangaroos

Macropoma mantelli, 236

Macropus - See Kangaroos

Macropus bennettii, $\mathbf{5 1 0}$

Macropus major, 546, 562

Macroscelididee, 493

Macula acustiere, 111*

Madagasear, fauna, 610

Muritherium, 587

Magnum, 443 See Limb-skeleton of Mammals

Malapterurus, 266

Malar, $440 *$

Malleola $\mathrm{r}$ bone, $526^{*}$

Malleus, 441, 564

Malpighi, 649, 650

Malpighian eapsules, 113*, 114: of Bdellostoma, $13 \%$

Mamme-See Teats

Mammalia, 65*, 303, 432*: Example, 432: Distinctive characters and classification, 462: Integument and general extemal features, 474-494: Endoskeleton, 494-501 : Skeleton of l'rototheria, 501-506: Metatheria, 506-508: Edentata, 508: Cetacea, 513: Sirenia, 518: Ungulata, 519: Carnivora, 528: Rolentia, 531: Insectivora, 532 : Chiroptern, 533: Primates, 535: Digestive organs, 541: Vaseular system, 557: Organs of respiration, 558: Nervous system, 559 : Organs of special sense, 562 : Urinogenital organs, 564: Development, 567579 : Geographical dist ribution, 579 : Genlogical distribution, 581

Mammary foetus, 5\%

Mammary glands, 4\%"

Mammary prouch, $4 \% \%, 47 \mathrm{~s}$

Nammoths, 580, 581

Man, 473, 495, 499, 536, 537, 538, 539, $540,553,554$

Manatee, 467, 501, 519, 520, 550, 551, 579,621

Manatus, 467, 520

Manatus sencgalensis, $\mathbf{5 2 0}$

Mandible, of C'raniata, 79*_See Skull

Mandibular arch, of Craniata, 75*

Mandibular nerve, of Craniata, 101

Mandibular suspensorium, $76^{*}$ 
Manidle, 465, 485, 509, 547, 579

Manis, 476, 485, 509, 564

Manis yigantea. 485

Mantle of Ascidia, 15

Manubrium sterni, $4366^{*}$

Manus, 67, 304

Maori Dog. 600

Maori Rat, 600

Marginal plates, $335^{*}, 336$

Marmosets, 472-See Hapalidue

Marrow, 82*

Marsh, O. C., 607

Marsupial bones, 464, 505*, 507. 508

Mlarsupial Mole, 480, 481, 561, 5633

Marsupialia, $464^{*}, 477,4 \% 8,4 \% 9,480$, $481,482,483,500,506,50 \%, 508$. 509, 544, 545, 546, 556, 5.58, 560, 561, $562,564,565,566,569,576,5 \% 8,579$, 583. 621

Marsupium, 464, 4\%y

Mastodonsaurus, 295

Mastoid, 497, 5:37

Matthew, Patrick, 602

Maturation of ovum, of Amphioxus, 54.55

Maxilla, of Craniata, $7 \boldsymbol{y}, 79^{*}$-See Skiull

Maxillary antra, 5.59

Maxillary nerve, of Craniata, 101

Maxillo-turbinals, $440^{*}, 441$

Meckel's eartilage, of Craniata, 74, 75*, 79: of Dogfish, 145: of Elasmobranehs, 168: of Frog, 262

Mecliast inum, $449^{*}, 453$

Merlulla oblongata, $97 *$ - See Brain

Medullary eanal, 32*, 33, 34

Meclullary folds, of Ascidian, 32 : of Amphioxus, 56

Merlullary gronve, of Craniata, 32, 95

Merlullary keel, 131, 132*

Merdullary plate, of Ascidian, 32* : of Amphioxus, $\mathbf{5 6}$

Megachiroptera, 471*, 493. 534, 535

Megalobrtrachus, 284, 287

Megameres, $131 *, 280 *, 281$

Megaporliide, 602

Megraporlius, 40:3, 423, 6022

Megaseolides, 1002

Megatheriidle, 585, 586

Megatherium, 621

Megistanes, 395*, 422, 429, 432

Meibomian glands, 563

Meles, $\mathbf{5 5 2}$

Membrana gramulosa, 568*

Membrana semilunaris, 386

Membrane bones, $76 *$

Membranous cochlea, 459

Membranous labyrinth, $111^{*}, 112$-See Ear

Membranous vestibule, $111^{*}$

Meniscus, 373*
Mental prominence, 537*

Mento-meckelian, 261, 262

Menura, 404, 602, 611

Mergansers, 403

Mergus, 403

Heroblastic segmentation, 569

Meropida, 404

Merrythought, 37s

Mesencephalon-See Midl-brain, 97*

Mesenteric artery, 88,90

Mesenteries, dorsal and ventral, 4, 5

Mesentery, of Craniata, 69, $80^{*}$

Mesethmoicl, \%4*, ry, 78*_-See Skull

Mesoarium, of Dogfish, 159: Lizarl. $322,323^{*}$

Mesocoele, 97-See Brain

Meso-coracoirl, 20:2, 203

Mesocmeiform, 444-See Limb-skeleton of Mammals

Mesoderm, formation in Craniata, $\mathbf{1 1 7}$

Mesorlermal segments, of Craniata, 11\%, 118

Mesogaster, 313*

Mesonephric cluets, of Craniata, 113*, 115

Mesonephros, of Craniata, 113*, 115: of Cyclostomi, 134

Mesopithecus, 590

Mesoplodon, 466

Meso-pterygium, 14\%*, 169, 170

Meso-pterygoid, 199, 200

Mesorchium, of Dogfish, 159* : of Lizard, 322, 323*

Meso-rectum, 313*

Mesoscapular segment, $500^{*}$

Mesosternum, 496

Meso-tarsal joint, 381*

Metacarpals, of Craniata, 80, 81*

Metacarpals (featliers), 373

Mrtacole, 97-Ser Brain

Metacone, $544^{*}$

Metaconid, 545*

Metacromion, 442

Meta-cliscoidal placenta, 5:6*

Metagenesis of Thaliacea, 21

Metamerism, 118*

Metamorphosis, of Balanoglossus, 7 , 8. 9: of Asciclian, 22, 30: Frog, 282

Metamomhosis, retengressive, of Ascidian, $13,36.37$

Metanephric ducts, of Craniata, 113*, 115

Metanephros, of Craniata, 113*, 115

Metapleure, of Amphioxus, 43. 44*, 61

Metapophyses, of Rabbit, 434, 435*

Metapterygium, $147 *, 169,170$

Metapterygoid, 199, உ00

Metatarsals, of Craniata, 80, $81^{*}$

Metatheria, $464^{*}$ - Seo Marsupialia

Metenceplualon, $97^{*}$ - See Brain 
Mice, 470

Microbiotheride, 621

Microchiroptera, $471 *, 493,534$

Microlestes, 619

Micromeres, $280 *, 281$

Micropyle, 209

Mid-brain, of Craniata, 97*

Mid-digitals, 368,373

Mid-kidney, 113

Milne-Edwards, H., 660

Mimicry, 637

Minimus, of Craniata, 80, 81*

Miocene, 620

Mitral valve, 450

Mitsukurina, 182

Moas, 398, 414, 41 7, 423

Mohl, von, 658

Nolars, of Rabbit, 446

Mole, Marsupial, 480, 481, 561, 563

Moles, 471, 493, 533, 563, 575

Molge, 284, 287

Molgulida, 37

Momotidae, 404

Mongrels, 644

Monitors, 324, 336, 346, 355, 358

Monkeys-See Primates

Monophyodont Mammals, 463*

Monopneumona, 250*

Monotremata, 464*, 496, 55s, 560, 561, $562,563,564,565,569,5 \% 8,5 \% 9$

Monotremes-See Monotremata

Monro, Alexander, 654

Mordacia, 120, 134, 139

Morula, 577

Moschus, 617

Moseley, H. N., 662

Motmots, 404

Moulting, of feathers, 410

Mucous membrane, 83 *

Mud-fishes, 213, 239

Mud tortoises, 325

Müller, Johannes, 659

Müllerian duct, 115, 116*-See Reproductive system

Mullerornis, 399

Mullet, 215

Multituberculata, 5s1, 582

Murida, 470

Murray, John, 662

Mus, 575

Mus decumanus, 554, 601

MIus domesticus, 601

Mus maorum, 600

Mus musculus, $\mathbf{5 5 4}$

Muscle buds, 181*

Muscle-plates, 45

Muscles, of Amphioxus, 45 : Lamprey, 124: Elasmobranchii, 171 : Trout, 203: Frog, 266, 26\%: Amphibia, 294 : Pigeon, 382, 383: Aves, 420

Muscular layer, of Craniata, 67, 68
Muscular system, of Amphioxus, 45

Musculi papillares, $449,450 *$

Musculi pectinati, 314,449

Musculo-cutaneous vein, 271,293

Museums Association, 664

Musiphagida, 609

Musophagida, 404

Mustela, 554, 620

Musielida, 176, 470, 554

Mustelus antarcticus, 68

Mutations, $633^{*}$

Mycetes, 473

Myctodera, 284*

Myelencephalon, 97*

Myliobatis, 173

Mylodon robustus, 585,621

Myocœle, 59

Myocommas, of Amphioxus, 45* : Craniata, 68

Myomeres, of Amphioxus, 43, 44, 45* : Craniata, 68

Myotone, 56, 59*

Myrmecobius, 480

Myrmecophaga, 509, 511, 512

Myrmecophagide, 465

Mystacina tuberculata, 600,602

Mystacoceti, 466*, 516, 517, 51s

Myxine, 134, 135, 136, 13\%, 139

Myxine glutinosa, 135, 136

Myxinoidei, 134*

$\mathrm{N}$

$\mathrm{N}$ ARES-See Olfactory organ

Nasal spine, $537 *$

Nasals, ryg, $78^{*}$

Naso-buccal groove, 141

Naso-palatine canals, $446^{*}$

Naso-turbinals, $439^{*}$

Native Cats, 464-See Dasyures

Natural selection, $629,633^{*}$

Naultinus, 602

Navicular, 444*_-See Limb-skeleton (Mammalia)

Nearctic region, 608

Neck, of Craniata, 65

Necturus, 284, 285, 286, 291, 293, 294

Necturus maculatus, 285, 286

Nekton, 616*

Neoceratodus, 239

Neochanna, 600

Neo-pallium, 560

Neornithes, $397 *, 407,420$

Neotropical region, 613

Nephridium-See Excretion, Organs of

Nephrostome-See Excretion, ()rgans of

Nephrotome, 179*

Nerve components, 112

Nerve-foramina, of Craniata, 74 
Nerves, of Amphioxus, 52, 53 : Craniata, 100, 101-See under Brain and Spinal cord

Nervi terminales, $100^{*}$

Nervous system, of Balanoglossus, 6, 7 : Ascidia, 19, 20: Urochorda, 2s: Amphioxus, 51, 52, 53: Craniata, 95-104-See under Brain and Spinal cord

Ncsonetta, 600

Nesopithecus, 621

Nestling-downs, 409*

Nestor, 600

Nestor notabilis, 617

Nestor productus, 612

Nests, of Birds, 422,423 : of Stickleback, 234

Neural arch-See Vertebra

Neural canal, 51

Neural cavity, of Craniata, 68*, 69

Neural gland, 19, 20

Neural plate, $143 *$

Neural process, 143

Neural spine, 143*

Neural tube, Craniata, $\% 0,1 / 1 *$

Neurenteric canal, $33^{*}, 56,5 \%, 281,282$

Neurenteric passage, 178

Neurocœle, $2^{*}, 32,33,42,4 \%, 52,56,5 \%$

Neuroglia, 96 *

Neuromast organs, 104, 105*

Neuromasts, 104, 105*

Neuron, $42,47,48,51,53,56,95$

Neuropore, $32,52^{*}, 53,57,59,60$

New Zealand, comparison of its physical conditions and fauna with those of Great Britain, 599

New Zealand region, 611

Newts, 256, 284, 287

Nictitating membrane, of Elasmobranchii. 174: Frog, 257 : Lizard, 305: Pigeon, 368, 369: Rabbit, 433 : Mammalia, 563

Nidicolæe, $429 *$

Nidifugæ, 429*

Non-Ruminants, $468 *$

Nostril, 105

Notidanidee, 164, 173

Notochord, 1*: Balanoglossus, 6 : Cephalodiscus, 11: Rhabdopleura, 12: Appendicularia, 23, 24: Ascidian larva, 32, 33: Amphioxus, $44,45^{*}, 4 \%, 56,57,58$ : Craniata, $69, \% 0,71$ - See Vertebral column

Notochordal sheath, $46^{*}, 70,71$

Notochordal tissue, $46^{*}, 7_{0}, 71$

Notornis, $413,417,600,622$

Notornis alba, 612

Notoryctes, 480, 481, 561, 563

Notoryctes typhlops, $480, \mathbf{4 8 1}$

Nototherium, 621

Nototherium mitchelli, 584
Nototrema marsupium, 300

Nuchal plates, $335^{*}$

Nucleus, of Salpce, 28*

Nurse, Doliolum, 37*, 39

O

( ) BLique SePtum, $38 \%$ *

Obliquus externus, $266,26 \%$

Obliquus internus, $266, \mathbf{2 6 7}$

Obstetric Toad, 300

Obturator foramen, $443^{*}$

Obturator notch, 380

Occipital condyle, $185^{*}, 260,436,43 \%$, 498

Occipital plane, $498 *, 499$

Occipital region, $74 *$

Occipital ribs, $241^{*}$

Occipital segment, $7 \%, 78 *$

Oceanic islands, $612 *$

Occanites, 402

Octacnemus, 27, 28

Octochoetus, 601

Octopods, 614

Oculomotor ganglion, 100, 101

Oculomotor nerve, 100,101

Ocydromus, 403, 413, 417, 431, 600, 612

Odontoblasts, $84 *, 542$

Odontoceti, 466*, 486, 515, 517, 562, 586

Odontoid process, of Amphibia, 290 : Lizard, 306, 333

Odontolcæ, $401 *, 430,431,432$

Odontopteryx, 420

Esophageo-cutaneous duct, Myxinoid, 135

Oikopleura, 21, 23, 29

Oil-bird, 613

Oil-gland, 367, 368*

Olien, Lorenz, 659

Old-world Monkeys, 540

Olecranon, 311 *

Olfactory bulb, 97*, 153, 189, 206, $20 \%, 230,231$

Olfactory capsules, $73^{*}$

Olfactory lobe, $97 *$ - See Brain

Olfactory lobe, median : Amphioxus, 52

Olfactory nerve, 100

Olfactory organ, of Amphioxus, 52, 53: Craniata, 105, 106: Lamprey, 12\%, 128, 129, 130 : Myxinoids, 135 : Dogfish, 158: Elasmobranchii, 174 : Trout, 206: Ceratodus, 247: Frog, 277 : Amphibia, 298: Lizard, 321: Reptilia, 349 : Pigeon, 393 : Mammalia, 562

Olfactory peduncle, 188, 189*, 231

Olfactory region, of Skull, '74* 
Olfactory tracts, 231

Olfactory ventricle, $97 *$ _See Brain

Olivary body, 458*

Omentum, duodeno-hepatic, 313*

Omentum, gastro-hepatic, 313*

Omentum, great, 391

Omosternum, 263, 264*

Onychodactylus, 289

Opercular, 193*

Operculum, of Balanoglossus, 4 : Craniata, 65: Holocephali, 183, 184 : Trout, 192, 193 : Teleostomi, 218 : Dipnoi, 240, 241, 242 : Tadpole, 283

Ophidia, 324*, 329, 332, 333, 336, 33\%, $338,342,345,346,347,348,349$, $350,351,356,357,359$

Ophthalmic nerve, 101, 102, 155, 156

Ophthalmicus profundus, 155

Opisthocœlous vertebra, 222*, 289

Opisthocomus, 403, 407, 408, 613

Opisthotic, $7 y *$-See Skull

Opossums, 464, 478, 480, 506, 507, $546,565,566,579,583$

Optic capsules, $73^{*}$

Optic chiasma, 153

Optic cup, 109*

Optic foramen, $43 \%, 438^{*}$

Optic lobes, 98, 99*_-See Brain

Optic nerve, 100,101

Optic thalamus, 98, 99*-See Brain

Optic ventricle, 98, 99*_-See Brain

Optic vesicle, $109^{*}$

Optocœle, 98, $99^{*}$ _-See Brain

Ora serrata, 106, 108*

Oral cirri of Amphioxus, 43, 44*, 47

Oral hood, 43, 44*

Oral lobes of Doliolum, 26

Oral siphon, $15 *, 17$

Orang, 473, 535, 537, 538, 539, 540, 581

Orbicular, of Rabbit, 441

Orbit, 73*

Orbito-sphenoid, ry*_-See Skull

Orca, 466

Orca gladiator, 486

Oriental region, 610

Ornithorhynchus, 464, 475, 477, 478, $479,502,503,504,505,506,541,545$, 561,562

Ornithorhynchus anatinus, 479, 561

Ornithosauria-Sce Pterosauria

Orthagoriscus, 225

Orthoceras, 619

Orthogenesis, 645

Orthotomus. 423

Orycteropodida, 465-See Cape Ant. eaters

Orycteropus, 509, 54\%, 609

Orycteropus capensis, $\mathbf{4 8 5}$

Os cloacæ, $312^{*}$

Os cordis, 558*
Osmerus, 620

Ossa innominata, 312 *

Ossicula audit ûs, of Rabbit, 441

Ossification, centres of, $77,542 *$

Osteocranium, 78*

Osteo-dentine, $84^{*}$

Osteostraci, 255, 256

Ostracion, 216

Ostracodermi, 253*, 254, 255, 256

Ostrich, 398, 399, 408, 409, 417, 419, $422,425,429,431,432$

Otariidce, 470, 491, 580

Otis, 403

Otocyst, 24, 29, 34

Otoliths, 111

Otters, 470,491

Ovarian artery, 90

Ovarian vein, 91

Ovidce, 468, 608-See Sheep

Ovis aries, $\mathbf{5 2 3}$

Ovulists, 650

Owen, R., 660, 665

Owls, 404, 409, 417, 421, 432

Oxen, 468, 487, 553, 564, 579

Oyster-catchers, 403

\section{$\mathrm{P}$}

D andogenesis in Axolote, 302

Pachyornis, 417

Pacinian corpuscles, 103, 105*

Palæarctic region, 608

Palcohatteria, 359

Palceoniscus macropomus, $23 \%$

Palæontological evidence of evolution, 626

Pulceospondylus gunni, 138, 139

Pulamedea, 403

Palate, hard and soft, 446, 553

Palatine, 79 , $79 *$ _-See Skull

Palatine nerve, 101, 102*

Palatine teeth, Holocephali, 186, 187 :

Trout, 204, \&c. : Ceratodus, 243

Palato-pterygoid, 243

Palato-quadrate, 74, 75*, $7 \%$-See Skull

Palinurus, 601

Pallas, 653

Pallium, 99*

Pancreas, 69, 85*-See Digestive system

Pancreatic juice, $85 *$

Pangenesis, 642

Panmixia, 634

Parachordals, 73*

Paracœle, $97 *$-See Brain

Paracone. 544*

Paraconid, 545*

Paradiseidce, 404, 411, 429, 602

Parafibula, 508

Paramyxine, 134, 139 
Paranephrops, 601, 602

Paraphysis, 99*

Parapineal eye, 99*

Parapophyses, $195^{*}$

Paraquadrate, 262*, 339, 340

Parasphenoid, 7 \%, 78*, 261, 262-See Skull

Pareiosauria, 326

Parencephalon, 97*-See Brain

Parietal, 7\%, 78*-See Skull

Parietal foramen of Stegocephala, 293*

Parietal organ, 99*, 319, 320, 350, 351

Parietal segment, 7\%, 78*

Paroccipital, 43\%, 438

Parotic processes, 307

Parotid gland, 446

Parotoid glands, 28s*

Parra, 403

Parrakeets, 404, 417

Parrots, 404, 408, 409, 415, 421, 429

Partridge, 423

Parus britannicus, 602

Parus rosea, 602

Passeres, 404*, 415, 423, 429, 432

Pasteur, Louis, 665

Patagium, 492*, 501

Patella, 380*, 381

Patella ulnaris, 417

Paunch-See Rumen

Peccaries, 468, 580

Pecten, of Reptilia, 321, 349: Pigeon, 393 : Birds, 422

Pectoral arch, of Craniata, 80, 81* : Dogfish, 146*, 14\%: Elasmobranchii, 169, 170 : Trout, 203 : Teleostomi, 224: Ceratodus, 241, 243 : Frog, 263, 264: Amphibia, 293, 294: Lizard, 310, 311: Reptilia, 342, 343: Pigeon, 374, 378, 379: Aves, 416, 41\% (Neornithes): Rabbit, 441 : Mammalia, 498: Prototheria, 505: Metatheria, 506 : Edentata, 512, 513, 515: Cetacea, 516 : Sirenia, 519: Ungulata, 525: Carnivora, 530: Rodentia, 532 : Insectivora, 533: Chiroptera, 535 : Primates, 539

Pectoral fin-See Fin : Skeleton ofSee Limb-skeleton

Pectoralis muscle, $26 \%$ *

Pelagic eggs, 235*

Pelagic fauna, $614^{*}$

Pelagic fishes, 220

Pelagic larvæ of Eel, 235

Pelecanus, 403

Pelicans, 403

Pelvic arch, of Craniata, 80, 82*: Dogfish, 147: Elasmobranchii, 169, 170: Holocephali, 186 : Trout, 203,
204: Teleostomi, 225: Ceratodus, 243 : Frog, 265: Amphibia, 293, 294, 295 : Lizard, 3\%4, 379: Rep. tilia, 342, 343, 344: Pigeon, 374, 379: Aves, 417, 419 : Rabbit, 443 : Nammalia, 500: Prototheria, 505: Netatheria, 507: Edentata, 513, 515, 516 : Cetacea, 517: Sirenia, 521: Ungulata, 526: Carnivora, 530 : Rodentia, 532: Insectivora, 533 : Primates, 540

Pelvio fin-See Fin: Skeleton of-See Limb-skeleton

Pelvic vein, 272, 273

Pelvis of kidney, 459*

Pelvi-sternum, 294*

Penguins, 402, 409, 413, 417, 419, 422, 429,431

Penis, 351-See Urinogenital organs

Pennæ, 409*

Pentadactyle limb, 67*, 80, 81 : Skeleton of 80 : Origin of, 595

Perameles, 544, 577

Perameles obesula, $5 \% 8$

Peramelidoe, 464-Seo Bandicoots

Perch, 190, 215, 223, 229: climbing, 228

Perching mechanism, 382, 383

Perennibranchiata, 284*, 285, 286, 291, 295, 296, 298, 302

Peribranchial cavity-See Atrial cavity

Pericardial cavity, 69, $70^{*}$

Pericardio-peritoneal canal, 150

Pericardium, 70*

Perichotida, 602

Perichondrial ossification, 76*

Perichondrium, 76*

Perichordal tube, $71 *$

Perilymph, 112*

Perinæal glands, of Rabbit, 433, 460

Perinæum, 433*, 460, 556

Periophthalmus, 229

Periosteal ossification, 76 *

Periotic bone, 439, 497

Peripharyngeal bands, of Appendicularia, 23: of Amphioxus, 46

Peripharyngeal groove, 17

Peripharyngeal ridge, 17

Perissodactyla, $467 *, 489,521,525$, $527,549,557$

Peritoneum of Craniata, 69, 70*

Peri-vitelline membrane, 55

Permian period, 619

Peron, 662

Peronæus medius, 383

Persistent pulps, 543*

Pes-See Hind-limb

Pessulus, 386*

Petrels, 402, 412, 421, 423, 429

Petrogale penicillata, 508, 560

Petrogale xanthopus, 481 
Petromyzon, 119: Extemal characters, 120, 121 : Skeleton, 121, 122. 123, 124: Muscles, 124: Digestive organs, $124,125,126$ : Respiratory organs, 125, 126 : Circulatory system, 126: Nervous system, 126, $12 \%, 128$ : Sensory organs, 128, 129, 130 : Urinogenital organs, 130,131 : 1)evelopment, 130, 131, 132, 133

Petromyzon fluviatilis, 120. 133, 591

Petromyzon marimus, 121, 122. 125. $127,130,131$

Petromyzon planeri. 120

Petromyzontes, $134^{*}$

Pezophaps, 404, 413, 417

Phonicopterus, 403, 408

Phaëthon, 403

Phalacrocorax, 403, 409, 605

Phalangerida, 465, 483, 501, 506, 510, 562

Phalangers, 465-See Phalangeridæ

Phalanges, 80, 81*-See Limb

Phaneroglossa, 285

Pharyngeal bones, superior and inferior, 224 *

Pharyngo-branchial, \%4. 76*-See Skull

Pharyngognathi, 215*, 219, 224, 236

Pharyngo-hyal, 74 , 76*_-See Skull

Pharynx-See Digestive organs

Phascalarctos cinereus, 482, 545, 578

Phascolomyidae, 481, 482, 506, 509, 545

Phascolomys, 545, 566

Phascolomys mitchelli, 482

Phascolomys wombat, 509, 566

Plascolotherium buclilandi, 582, 619

Phasianus, 403, 411

Pheasants, 403,411

Phoca vituiina, 491, 531

Phocana, 466, 486, 501, 51\%, 543, 555. 556

Phocana communis, $51 \%$

Phocidce, 470, 491, 531, 5s0

Phororhacos, 401

Phorozooid, 39 *

- Phrenic veins, 452

Phylogeny, of Birds, 430

Pliyseter, 466, 501, 550

Physoclisti, 216*

Physostomi, 213*, 214, 216, 219, 221, $223,226,227,228,231,232,233,236$, 238

Pia mater, $100^{*}$

Picariæ, $404^{*}, 432$

Picas, 474

Pici, 404

Pigeon's milk, 395

Pigeons, 404-See Columba

Pigment-spot, 52, 53*

Pigs, 468, 488, 519, 522, 525, 526, 52\%, 548,580

Pike, 190, 214
Pineal apparatus, of Craniata, 65, 66 , 99*, 110*, 111: Petromyzon, 128

Pineal body, 99*

Pineal eye, 99, $110 *, 111,128$ : of Lizards, $319,320,350,351$

Pineal organ, $99^{*}, 153,154$

Pineal sense-organ, $65^{*}, 66$

Pinna, or ear, 433

Pinnipedia, 470*, 491, 501, 530, 531, $551,552,558,580$

Pipa americana, 285, 295, 300, 301, 302 Pipe-fish, 216, 234

Pisces, 64, 139* : Appendix, 253

Pisiform bone, 312, 442 - See Carpus

Pithecanthropus, 590,621

Pituitary body, of Craniata, 69, 71, 84*

Pituitary body, extra-cranial portion (Callorhynchus), 188, 159*

Pituitary diverticulum, 69,84 *

Pituitary foramen, $438 *$

Pituitary fossa, $438^{*}$

Pituitary pouch, Petromyzon, 128, 129, 130 : Myxinoids, 135

Placenta, Salpa, 30, 39*, 40 : Rabbit, 462: Mammalia, 576, 577

Placodontia, 326,360

Placoid scales, 141, 163, 166, 167

Placula, 31 *

Plagiaulax becklesi, 582, 619

Plankton, $615^{*}$

Plantain-eaters, 404

Plantigrade, $501 *$

Plastron, 329, 335

Platalea, 403, $40 \mathrm{~s}$

Platycercus, 404, 417

Platypus-See Ornithorhynchus

Platysomus strialus, $23 \%$

Plectognathi, 216*, 221, 231

Pleistocene period, 621

Plesiosaurus macrocephalus, 360, 361, 362

Pleura, 386*, 38\%

Pleuracanthei, 163*, 182

Pleuracanthus ducheni, 163

Pleural ribs, 195

Pleurodont, $345^{*}$

Pleuronectes cynoglossus, 220

Pleuronectida, 214, 219

Pleuropterygii, $162 *$

Pliocene period, 621

Pliohyrax, 587

Pliopithccus, 621

Ploughshare-bone--See Pygostyle

Plovers, 403, 411

Plumulæ, 409

Pneumatic duct, of Trout, 204, 205 : Teleostomi, 229

Pneumatic foramina, 3\%9, 382*

Pneumaticity of bones, Pigeon, 382 : Aves, 420

Pneumogastric, 101, $102 *, 156,15 \%$ 
Poducrpes, 402. 409

Poëbrotherium, $6 \mathbf{2} 1$

Poephagus, 617

Poison-glands, in Teleostei, 221. Ophidia, 345,356

Poli, 651

Pollex, 80, 81 *

Polynesian region. 612

Polyodon, 212, 215, 221, 236

Polyphyletic group, $431^{*}$

Polyprotodont, $5 \pm 6$ *

Polyprotodontia, $464^{*}, 579.58 \%$

Polypterus bichir, 190, 212, ㄴ15, 2-19. 222, 223, 224, 225, 227, 229, 235, 2: 26 , 609

Pons Varolii, 458*, 559, 560

Porcupines, 470, 47 j, 492. 532

Porpoises, 466-See Phoccena

Port Jackson Shark, 176, 1\%, 152

Portal veins, $152 *, 153$

Post-anal gut, $69, \mathrm{~S}$-2*

Post-axial, 2S7*, 305

Posteaval rein, 272

Post-clavicle, 203*

Posterior commissure, 206

Posterior temporal fossa, $309 *$

Post-orbital, 308

Post-patagium, 368*

Post-temporal, 203*

Potamogale, 609

Pouters, 367

Powder-down-patches, $409 *$, 410

Præcoces, 429*

Pre-anal plate, 305

Pre-axial, 257*, 305

Precaval vein, 90

Precocious voung, 397*

Pre-commissural arca, 561 *

Pre-formation, 650

Pre-frontal, 307, 308

Pre-hallux, 266, 294

Premaxilla, 7\%, 79*_See Skull

Premolars, 415-See Teeth

Pre-nasal, 523*

Pre-nasal region, $74 *$

Pre-olfactory nerves, 15.j

Pre-opercular, 193

Pre-oral pit, Amphioxus, 5s*, 59

Pre-patagium, $368^{*}$

Pre-pubic process, 170, 171

Prepuce, 460*

Pre-sphenoid, $7 \%, 78 *$ _-See Skull

Presternum, 436

Primates, 471*, 475, 535-541, 567, 580, 590

Primitive groove, $280^{*}, 124$

Primitive knot, 352, 353*

Primitive streak, of Ares, 354, 424

Pristiophorus, 165

Pristis, 165

Pristiurus, 182, 183
Pro-amnion, 424. 425*

P'ro-atlas, $33: 3 *$. 335

Proboscidea, 469*, 520, 521, 525, 528, 587

Proboscis, of Bulanoglossus, 3, 4 : Cephalodiscus, 11 : Rhabdopleura. 13

Proboscis-cavity, 3. 4, 9, 12

Proboscis-pore, 3, 4, 11, 12

Proboscis-skcleton, $4,6 *$

Procellaria, 412

Processus gracilis, $441 *$

Procolous vertebra. 258*

Pro-coracoid, 80, $81 *, 310,311$

Proctodrum-S॰c Digestive system

Pronation, 442

Pronephic duet, 113*, 114, 115

Promephros, 11:3*, 111, 115

Prongbuck, 580

Pro-otic, $y y^{*}$-See Skull

Propterygium, 147*, 169, 170

Prosenc sphalon, 97*, 153--See Brain

Prosimii, 47.2*, 494, 535, 539, 540, 5s1

Prosocole, 97*_. See Brain

Prostate, 459, 160

Protective:resemblance, 637

Proteus, 284, 257, 291, 298, 616

Proteus anguineus, 291

Protochordal plate, 353, 3.54*

Protocone, 5 $44^{*}$

Protoconid, 545*

Protoplasm, 65̃

Protopterus, 239, 250, 251, 252, 609

Protoselachii, 164*

Prototheria, $463,464^{*}, 4 \% \%, 478,4 \% 9$, $500,501-506,556,578,583$

Protovertebra, 56, 59, 11\%, $118^{*}, 179$

Protriton, 283

Proventriculus, of Pigeon, 384*

Psalterium, of brain, $456 *, 45 \%$ : of stomach, $553 *, 555$

Psammosteidee, 255*

Psephurus, 212, 236

Pseudis paradoxa, 301

Pseudobranchia, of Dogfish, 150 : Elasmobranchii, 173: 'Trout, 204:

Teleostomi, 227: Ceratodus, 244

Pseudocole, $155^{*}$

Pseudophycis bachus, 230

Psittaci, 404*, 429. 431

Psittacus, 404, 108, 409, 115, 121, 429

Ptarmigans, 411

Pteraspida, 254*

Pteraspis rostrata, 254

Pterichthys, 256

Pterobranchia, 3*, 9, 10, 11, 12, 13

Pterocles, 404

Pterocletes, $404^{*}$

Pterodactyles-See Pterosauria.

Pterodactylus, 364

Pteropido, 471 -Sec Flying Foxes 
Pteropus fuscus, $\mathbf{5 3 5}$

Pteropus jubatus, 534

Pterosauria, $327^{*}, 364^{*}, 365$

Pterotic, 19\%, 198

Pterygiophores, 69, 80*-See Fins

Pterygoid, ry, 79*-See Skull

Pterygopodia, 166*

Pterylæ, 3'2**

Pterylosis, of Pigeon, 372, 373*: Aves, 409, 410

Ptychodera, 5

Pubic symphysis, 312*

Pubis, 80, 82*-See Pelvic arch

Pubo-ischial region, $82^{*}$

Puffins, 429

Puffinus, 357, 402

Pulmonary aponeurosis, $386 *, 387$

Pulmonary artery and vein, 93

Pulmonary nerves, 103

Pupil, of eye, 107

Purkinje, $65 \mathrm{~s}$

Pygal plates, $335^{*}$

Pygochord, 6*

Pygopidae, 358

Pygopodes, 402*, 431

Pygopus lepidopus, 329

Pygostyle, of Pigeon, 374, 375*

Pyloric cæca, of Trout, 204, 205 :

Teleostomi, 227

Pyloric valve, 204

Pylorus, 148*

Pyrosoma, 22, 27, 28, 30, 37, 41

Pyrosomida, 22

Pyrotherium, 587

Pythonomorpha, 324*, 365, 366

Pythons, 324, 329, 333, 343, 356, 357
$Q_{\substack{\text { Uadrate, } \\ \text { Skull }}}$ Q $75^{*}, 7 \%, 79,337 \ldots$ See

Quadratojugal, 261, 262*

Quadrátojugal arch, 309

Quadrupedal attitude, $501 *$

Quagga, 609

Quills, 492*

Quoy, 662

$\mathrm{R}$

$\mathrm{R}$ Ааввітs, 470-See Lepus cuniculus Rachis, 369, 370*_See Feather Radiale, 80, 81*-See Limb-skeleton Radialia, radial cartilages, $\% 9,80^{*}$ See Limb-skeleton

Radio-ulna 259, 265*
Radius, 80, 81*-Sec Limb-skeleton

Rails, 403, 413

Rajida, $165^{*}$

Rallus, 403, 413

Rana pipiens, 257

RANA TEMPoraria and R. ESCUlenta, 25\%: External characters, 25\%: Endo-skeleton, 258, 259-266 : Muscular system, 266, 267: Digestive organs, 268, 269: Respiratory organs, 268, 269 : Circulatory organs, 2\%-2\%5: Nervous system, 275, 2\%6 : Sensory organs, 2\%\%, 278: Urinogenital organs, 278, 2\%9: De. velopment, 279, 280, 281, 282, 283 : Systematic position, 285

Range, $605^{*}$

Ranidce, 285*

Ranidens, 290

Rapacious Birds, 429

Ratitæ, 397*, 409, 410, 412, 413, 414, $416,417,420,429,431$

Rats, 470

Rattlesnakes, 324, 338, 356

Rauber's layer, 570*

Raven, 405

Ray, John, 647, 650

Rays, 165-182

Recapitulation Theory, 626

Recent period, 622

Receptaculum chyli, 557

Recessive characters, 644,645

Recognition-marks, 411

Rectal gland, Dogfish, 148, 149

Rectrices-See Pterylosis, 368, 373

Rectus abdominis, 266, 26\%

Red-bodies, 230*

Red Deer, 520, 525, 526, 52\%

Red-glands, 230*

Redi, 650

Reef-fishes, 218, $23 \mathrm{~s}$

Regalecus, 218, 225, 615

Regeneration, $63 \mathrm{~s}$

Reindeer, 487

Relationships, of Hemichorda, 13, 41 : Urochorda, 41 : Amphioxus, 63 : Cyclostomata, 138 : Amphibia, 302 : Aves, 430: Chordata, 590: Phyla of Animals, 596

Remiges-See Pterylosis, 373*

Renal artery, 90

Renal organs-See Excretion, Organs of, and Urinogenital organs

Renal portal system, $91^{*}$-See Vas. cular system

Renal veins, 91

Replacing bones, 76*

Reproductive organs, of Balanoglossus, 7: Cephalodiscus, 11: Ascidia, 20: Urochorda, 30: Amphioxus, 54, 55 -See Urinogenital organs 
Reptilia, 303* : Example, 304 : Distinctive characters and classification, 323: Extemal features, 327: Integument and exoskeleton, 331: Endoskeleton, 332 : Digestive organs, 345: Organs of respiration, 346: Organs of circulation, 347: Brain, 349: Sensory organs, 349: Reproductive organs, 351 : Development, 351 : Ethology, 354: Geo. graphical distribution, 358: Geological distribution, 359: Extinet groups of reptiles, 360 : Relationships, 590

Respiration, organs of, Amphioxus, 47: Craniata, 86, 87: Petromyzon, 125. 126 : Hemiscyllium, 149 : Elasmobranchii, 173 : Holocephali, 187 : Trout, 204: Teleostomi, 227 : Ceratodus, 244, 245: Frog, 269: Amphibia, 295: Lizard, 318 : Reptilia, $346,34 \%$ : Pigeon, 385, 386, 38\%, 388: Aves, 421: Rabbit, 453: Mammalia, 558

Respiratory heart, $93^{*}$

Respiratory tube of Petromyzon, 124, $125,126^{*}$

Respiratory valves, of Trout, 193

Restiform bodies, 187, 188

Rete mirabile, $173 *$

Reticulum, 553*, 554

Retina, Craniata, 106, 10\%, 108

Retropinna, 600

Reversal of selection, $635^{*}$

Rhabdopleura, 2, 9, 12, 13, 593

Rhamphorhynchus, 365

Rhamphotheca, 395*

Rhea, $398,408,417,429,431$

Rheæ, 398*, 422, 429

Rhina, 173

Rhinencephalon, 97*-See Brain

Rhinobatus, 176

Rhinoceros, 468, 476, 489, 490, 521, $522,525,526,527,528,580$

Rhinoceros indicus, 490

Rhinochetus, 612

Rhinocœle, 97*, 173-See Brain

Rhinoderma darwini, 300

Rhomboid scales, 221*

Rhynchocephalia, 325*, 329, 330, 333, ) 359

Rhytina, 467, 550, 579, 586, 622

Ribbon-fishes, $218,225,615$

Ribs, of Craniata, 69, 72 : Dogfish, 142, 143: Teleostomi, 222: Uro. dela, 290: Lizard, 306 : Reptilia, 333, 334, 335, 336 : Pigeon, 373 : Aves, 412: Rabbit, 435 : Mammalia, 495 : Edentata, 509 : Cetacea, 515 : Sirenia, 518 : Carnivora, 528: Chiroptera, 534
Rita buchanani, 214

River tortoises, $325,331,357$

Rock-pigeon, 367

Rock Wallaby, 481, 508, 560

Rodentia, 470*, 478, 531, 532, 552, $557,558,563,576,580,589$

Rods and cones, of retina, 10\%, 108*

Rollers, 404

Rorqual, 517

Rostrum of skull, Craniata, 74* : Dogfish, 144: Trout, 198: Pteraspis, 254: Aves, 376, 3yry

Rudolphi, 661

Rumen, 553*, 554

Ruminants, 468*,476, 487, 520, 525, $526,527,548,553,554,555$

Rupicapra, 617

\section{S}

SAcculus, $111 *, 112$

Saccus vasculosus, $99 *, 153 *, 154,173$, $206,20 \%$

Sacral ribs, 306

Sacral vertebra, 259, 260

Sacro-vertebral angle, 536*

Sacrum, 435*

Sagitta, 208*, 209

Sagittal crest, $521^{*}$

Sagittal suture, 439

Salamanders, 256, 284, 287, 288, 289, 292, 294, 295, 296, 29\%, 301, 302

Salamandra, 284, 294, 295, 296

Salamandra atra, 292, 301

Salamandra maculosa, 28\%, 288, 29'\%, 301

Salamandrina, 290

Saliva, 84*

Salivary glands, $84^{*}$-See Digestive system

SALMO FARIO, 192: External characters, 192, 193, 194: Skin and exoskeleton, 194, 195 : Endoskeleton, 195-203: ALuscles, 203: Cœlome, 203: Digestive organs, 204, 205 : Air-bladder, 204, 205 : Respiratory organs, 204: Circulatory organs, 204, 205, 206: Nervous system, 206, 20\%: Sensory organs, 206, 207, 208, 209: Urinogenital organs, 208, 209, 210: Development, 209, 210 : Systematic position, 216

Salmo ferox, 192

Salmo fontinalis, 192

Salmo killinensis, 605

Salmo salar, 192

Salmon, 190, 201, 214, 235

Salmonida, $216,235,236$ 
Salpa, 26, 2\%, 28, 29, 30, 39, 40

Salpa democratica, 26

Salpidoe, 22

Sand-grouse, 404

Sand-martins, 423

Sand-pride, 120

Sarcoplitus ursinus, 546

Sarcorhamphus, 617

Sargus, 227

Sauropsida, $303^{*}, 323$

Sauropterygia, $326^{*}, 360,361$

Saw-fish rays, 165

Saw-fish shark, 165

Scala tympani, 459

Scala vestibuli, 459

Scales, 194: of Pigeon. 369

Scaly Ant-eater, 485--Sce Manidæe

Scapanorhyncus, 182

Scaphirhynchus, 212, 236

Scaphognathus, 365

Scaphoid, 442, 443-See Limb-skeleton of Mlammalia

Scapula, 80, 81*, 8?-See Pectoral arch : accessory, 417

Scapular region, $81 *$. See Pcctoral areh

Schouchzer, 658

Schizocole, 118

Schizognathous arrangement, $415^{*}$

Schleiden, 658

Schneiderian membrane, 106*, 393

Schultze, Max, 659

Schwann, 658

Scincidæ-See Slkinks

Sciuridce, 470, 491, 532

Sclater, P. L., 667

Sclerotic, $73^{*}, 106,321,349,393$

Sclerotic plates, of Stegocephala, 293 : Lizard, 321: Reptilia, 349: Pigeon, 393

Screamers, 403

Scroll-valve of Elasmobranchii, 172

Scrotal sac of Rabbit, 433, 460

Scrotum, 565

Scutes, of Teleostomi, 221 : Stegocephali, 289: Reptilia, 329: Arma. dillos, 484

Scyllium canicula, 140-Seo Hemiscyllium

Scymnus, 16\%, 173

Sea-bream, 215

Sea-cows, 467-See Sirenia

Sea-horse, $216,21 \%, 218,219,234$

Sea-snakes, 324, 356

Sea-squirts, 14

Sea-turtles, 357

Seals, 470

Sebaceous glands, 474, 4\%5, 476

Sebastes percoides, 215

Secodont, 545*

Secondary cranium, 197
Secretary-bird, 403, 609

Segmental duct, 114*, 115

Segregation, $645^{*}$

Selache, 173, 182

Selachii, 164*

Selenodont, 545*

Sella turcica, ${ }^{4} 4$, ny*, 189

Semicircular canals, $111 *, 112$

Semi-lunar, 442

Semi-lunar valves, $2 \% 0,271$

Semi-plumes, 409*

Semnopithecus, 590

Sense-vesicle, 34, 35, 36

Sensory organs: Amphioxus, 52, 53, 54: Craniata, 104 -See Ear, Eye, Lateral line, Olfactory organ

Seps, 354

Septum auricularum, 270*, 296

Septum lucidum, 455, 456

Serous membrane, of Birds, 427428 : Mammalia, 462

Serpentarius, 609

Serranus, 233

Sesamoid bone, $380 *$

Severino, 648

Sexual selection, $636^{*}$

Shaft of long bone, $82 *$

Shagreen, 166

Shags, 403, 409

Shank, 67-See Hind-limb

Sharks, 165-182

Shearwaters, 357,402

Sheep, 468, 487, 523, 553, 579

Shell, of Chelonia, 329 : of Pigeon, 396

Shell-gland, Dogfish, 159, 160: Elasmobranchs, 175

Shell-membrane, 396, 422, 423, 577

Shore-fishes, 220

Shoulder-girdle-See Pectoral arch

Shrews, 471, 493

Siamanga, 610

Siebold, von, 661

Silurian period, 618

Siluroids, 214, 221, 222, 225, 226, 2:9, $230,234,236$

Simia, 473, 610

Simia satyrus, 539, 540

Simiidce, 473*, 494, 495, 535, 536, 537, 581

Sinus rhomboidalis, 392,393

Sinus venosus, 88- See Heart

Sinuses, 90, 151*

Siphons, oral and atrial, of Ascidia, 15,17

Siredon, 302

Siren, 284, 286, 287, 296

Siren lacertina, 286

Sirenia, 466*, 476, 486, 495, 496, 518, $519,550,558,563,579,586$

Skates, 165

Skeletogenous layer, $70,71 *$ 
Skeleton of Craniata-See Skull, Vertebral column, Ribs, Sternum, Pectoral arch, Pelvic arch, Limbskeleton

Skin, Craniata, 67

Skines, 324, 332, 354, 358

Skull, of Craniata, 173, 14, 75, 76, 19\%, 78: Petromyzon, 121, 122, 123 : Myxinoids, 136 : Dogfish, 143, 144 : Elasmobranchii, 168, 169 : Holocephali, 184, 185, 186, 18\% : Trout, $196,19 \%, 198,199,200,201$ : Teleostomi, 223 : Ceratodus, 241, 242 : Frog, 260, 261, 262 : Amphibia, 290, 291, 292, 293 : Lizard, 307, 308, 309: Reptilia, 33\%-341 : Pigeon, 3\%6, 3\%\%, 37S: Archropteryx, 40\%: Birds, 414 416 : Rabbit, 436-441 : Mammalia, 496, 49\%, 498, 499 : Prototheria, 502, 503, 504: Metatheria, 506-508: Edentata, 510, 511, 512: Cetacea, 515, 516, 51\%, 518 : Sirenia, 518, 519: Ungulata, 520-525 : Camivora, 528, 529, 530 : Rodentia, 532: Insectivora, 532, 533 : Chiroptera, 534, 535: Primates, 536-538

Slime-Eels, 134

Sloane, H., 655

Sloths, 465, 474, 483, 495, 500, 501, $509,511,512,513,514,515,547$, 556,579

Smelt, 214, 232, 620

Smith, William, 658

Snakes, 324 - See Ophidia : venomous, 356

Soft palate, $446 *, 553$

Soft tortoises, 325,332

Solander, 655

Sole, 190, 214, 235

Solenocytes, 51

Solenodon, 613

Solitaire, 404, 413, 417, 622

Somatic motor fibres, $113^{*}$

Somatic nerves, $96 *$

Somatic sensory fibres, $112 *$

Somites, 56, 57*

Soricidae, 471,493

Souleyet, 66:

South American Ostrich-See Rhea

Spallanzani, 653

Sparassodonts, 583, 621

Sparrmann, 65.5

Spelerpes, 290

Spencer, Herbert, 66:

Sperm-sac, of Elasmobranchii, 159, 175

Sperm Whales, 466, 501, 550

Spermatic artery, 90

Spermatic vein, 91

Spermatists, 650
Spermatophores, of Holocepliali, $\mathbf{1 8 9}$, 190 : Amphibia, 300

Sphargis, $336,357,358$

Sphenethmoid, 261*

Sphenodon punctatum, 325, 329, 330, $333,334,335,336,337,338,339$,

$343,345,350,351,357,35 \mathrm{~s}$

Sphenoidal fissure, 43.8*

Spheno-maxillary fissure, 537*

Sphenotic, 198, 199

Sphyrna, 165

Spider Monkeys, 473

Spigelian lobe, 556, 557*

Spinal accessory nerve, 321

Spinal column-See Vertebral column Spinal cord, of Urochorda, 34 : Amphi. oxus, 52: Craniata, 95, 96*

Spinal nerves, 53 : of Craniata, 96*

Spines, Spinous fin-rays of Teleostomi, 215, 219

Spiny Ant-eater, 464-See Ecridna

Spiracle, of Dogfish, $141^{*}$ : Teleostomi, 218

Spiracular cartilage, 168*, 169

Spiracular gill, 150*, 173

Spiral valve, of Petromyzon, 125, 126*, 130 : Dogfish, 148, 149: Elasmobranchs, 172: Teleostomi, 212 : Ceratodus, 243

Splanchnic nerves, 96 *

Splanchnotome, 59*

Spleen, 85*

Splenial-See Skull, 310*

Splenial teeth, 243

Splenium, 454*. 561*

Spontaneous generation-See Abio genesis

Spoon bills, 403, 403

Spur's, 409

Squalida, $164^{*}$

Squalodon, 586

Squalodontidce, 586

Squaloraja, 190

Squamata, $324^{*}$

Squamosal, 8\%, 80*_-See S'kull

Squamous suture, $436^{*}$

Squirrel Monkeys, 473

Squirrels, 470, 492, 532

Stapes, of Frog, 261, 263* : Urodela, 291: Rabbit, 441

Star-gazers, 218,226

Starlings, 404,430

Station, $605^{*}$

Statocyst-see Otocyst

Steatornis, 613

Steganopodes, $403^{*}$

Stegocephala, 256, 285*, 288, 289, 293, $295,299,302$

Stein, 661

Steller's Sea Cow, 586

Stereornithes, $401 *, 430$ 
Stereospondyly, 332*

Sterna, 403, 419

Sternal rib-See Rib

Sternebræ, $436^{*}, 496$

Sterno-tracheal muscles, 386

Sternum, of Craniata, 72: Heptanchus, 170: Frog, 263, 264: Amphibia, 293, 294 : Lizard, 307, 311 : Reptilia, 336 : Pigeon, 3\%4, 375: Birds, 412: Rabbit, 436 : Mammalia, 496: Prototheria, 502, 503: Edentata, 509: Cetacea, 515: Sirenia, 518: Ungulata, 521: Carnivora, 528: Rodentia, 532: Insectivora, 532 : Chiroptera, 534

Sternum, abdominal, 336

St. Hilaire, E. G., 657

Stickleback, 215, 234

Stigmata of Ascidia, 16*, 17

Sting-rays, $165,166,169$

Stolon, Rhabdopleura, 12 : Salpa, 2\%, 40 : Doliolum, 37, 38, 39

Stomach--See Digestive organs

Stomata, 94*

Stomias boa, 221

Stomodæum-See Digestive system

Storks, 403, 408, 409, 415

Storm-petrels, 402

Strasburger, E., 667

Stratum corneum--See Skin

Stratum malpighii--See Skin

Striges, 404*, 409, 417, 421, 432

Strigida, 404

Stringops, 413, 431, 600

Stroma of ovary, 116

Struthio, 399, 408, 409, 417, 419, 422, $426,429,431$

Struthiones, $399^{*}, 429$

Sturgeon, 190, 212, 218, 221, 222, 223, $227,234,235$

Sturnida, 404, 430

Styliform cartilages, 121, 124

Stylo-glossus, 438*

Stylo-hyal, 438*, 498

Styloid process, 121, 122, 123, 311, 537

Stylomastoid foramen, 436, 439

Struggle for existence, 629 *

Sub-atrial ridge, $61 *, 62$

Subclavian artery, 88, 90, 92, 150, 151

Subclavian vein, 92,152

Subclavius, 382,383

Subcutaneous sinus, 274

Sub-intestinal vein, 49, 50, 91, 94

Sub-lingual gland, 446

Sub-maxillary gland, 446

Sub-mucosa, 83*

Sub-neural gland, 19, 20, 29, 30

Sub-ocular arch, 121, 122, 123*

Sub-opercular, 193

Sub-orbitals, 19\%, 199
Subungulata, 468*

Subvertebral sinus, 274

Sucker of tadpole, 281, 28?

Sucking-fish, 219

Suinoe, 613

Sula, 403

Sun-fish, 216, 225, 231

Superior curved line, 537*

Superior oblique muscle of eye, 110

Superior rectus muscle, $\mathbf{1 1 0}$

Superior temporal arch, $309^{*}$

Supra-angular, 308, 310*-See Skull

Supra-clavicle, 203

Supra-ethmoid, 19\%, 199

Supra-occipital, ry**-See Skull

Supra-orbitals, 308

Supra-renals, $117^{*}$

Supra-scapula : Supra-scapular cartilage, 170--See Pectoral arch

Supra-temporal, 308

Surinam Toad, 300, 301, 302

Survival of the fittest, $633^{*}$

Sus, 468--See Pigs

Sus scrofa, 526, 52\%, 548

Suspensorium, 76*, 260, 261

Suspensory ligament, of eye, 106, 108 : Bird, 374

Sutures, $436^{*}$

Swallow, 404

Swammerdam, 649

Swan, 403, 408, 409

Sweat-glands, 474, 476

Swift, 403, 408, 409

Swim-bladder-See Air-bladder

Sword-fish, 218

Sylvian fissure, $454^{*}$

Sympathetic nerves: Craniata, 96* : Rabbit, 458

Symphysis, 145*

Symplectic, 199, 200

Synapticula, of Balanoglossus, 5: Amphioxus, 48

Syngnathus, 434

Synotus barbastellus, 493

Syn-sacrum of Pigeon, 375*

Syrinx of Pigeon, 385, 386 : Aves, 421

Syrrhaptes, 404

Systemic heart, 93

\section{$\mathbf{T}$}

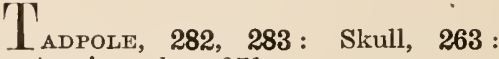
Aortic arches, 271

Tænia hippocampi, 456*, 561

Tail : Ascidian larva, 35: Amphioxus, 45 : Craniata, 65

Tail coverts-See Pterylosis, 373

Tailor-bird, 423

Talegallus, 611

Talpa, 493 
Talpidae, 471, 608-See Moles

Tapetum, 158*

Tapirs, 468, 489, 501, 519, 521, 522, $525,526,527,580$

Tapirus, 468

Tapirus indicus, 526

Tapirus terrestris, 489

Tarsals, of Craniata, 80, $81 *$ _-See Limb-skeleton

Tarsipes, 506

Tarsius, 472

Tarso-metatarsus, 368*, 381

Tasmanian Devil, 480, 546

Taste-buds, $105,446,553,563$

Taste, organ of, Craniata, 105*

Tatu, 484

Teats, of Rabbit, 433: Nammalia, 478*

Tee Tees, 473

Teeth, of Craniata, 84, 85 : Petromyzon, 120, 124: Myxine, 135: Elasmobranchs, 172: Holocephali, 186, 187: Trout, 204: Teleostomi, 226: Ceratodus, 242, 243 : Frog, 268 : Amphibia, 295: Lizard, 313: Rep. tilia, 345: Archæopteryx, 40\%: Rabbit, 43\%, 445 : Mammalia, 541553

Tejidce, 358

Telencephalon, $97 *$

Teleostei, 213*, 219, 220, 221, 222, $223,224,226,227,228,229,231$, $232,233,234,235,236,237,239$

Teleostomi, 64*, 190* : Example, 192 : Distinctive characters and classification, 211: External form, 217 : Exoskeleton, 221: Endoskeleton, 222 : Electric organs, 226: Digestive organs, 226: Respiratory ol'gans, 227: Air-bladder, 229 : Heart, 231: Brain, 231 : Urinogenital organs, 231: Reproduction and development, 234: Geographical distribution, 235: Distribution in time, 236

Temnospondyly, 332*

Temporal canal, 503*

Tendon, of muscle, $266^{*}$

Tenrec, $\mathbf{5 3 3}$

Tensores patagii, 382,383

Tentacles, of Pterobranchia, 9, 10, 11, 12: Ascidia, 15, 18: Myxine, 135

Tentorial plane, $498^{*}$

Terns, 403, 419

Terrestrial fauna, 617

Test, of Urochorda, 14*, 15

Test-cells of ovum, 31

T'estudo, 613

Testudo grceca, 333

Testudo loveridgii, 335

Tetrao, 403
Tetrazooids, $37 *$

Thaliacea, $21^{*}$

Thecodont teeth, $345^{*}, 463^{*}$

Theria, 464*, 498, 500

Theriodontia, 326

Theromorpha, $325 *, 360$

Thigh, 67-See Hind-limb

Thomson, Vaughan, 661

Thomson, Wyville, 662

Thoracic duct, 574

Thorax, 70*, 433

Thorn-backs, 165

Thread-cells, Myxine, 134

Three-toed Sloth-See Sloth

Thrushes, 404, 412

Thylacine, 480, 508, 583, 621

Thylacoleo carnifex, 584

Thylacus, 480, 508, 583, 621

Thymus, of Craniata, 86* : Elasmobranchii, 173: Frog, 269: Pigeon, 385 : Rabbit, 454

Thyro-hyal, 441

Thyroid, of Craniata, 85* : Elasmobranchii, 173: Frog, 269: Lizard, 316 : Pigeon, 385 : Rabbit, 454

Thyroid cartilage-See Larynx

Tibia, 80, 81*-See Limb-skeleton

Tibiale, 80, 81*_See Limb-skeleton

Tibio-fibula, 259, 265

Tibio-fibulare, 313

Tibio-tarsus, 380,381

Tiger, 528, 529

Tillodontia, 589

Tillotherium fodiens, $\mathbf{5 8 9}$

Tinamous, 403, 414, 417, 429, 431

Tinamus, 403, 414, 417, 429, 431

Tinoceras, 588

Toads, 256, 284, 285, 288, 295, 300

Tolypneutes, 484

Tongue, of Craniata, $84 *, 192$-See Digestive organs

Tooth-pulp, 84*

Toothed Whales, $466^{*}$

Tornaria, $\mathbf{7 , 8}, 9$

Torpedo, 171, 172

Tortoises, 64

Toucans, 408

Touch-cells, 103, 104*

Touch corpuscles, 103, 104*

Toxodontia, 588

Trabeculæ, $73^{*}$ : heart, 271

Trabecular regions, $74^{*}$

Trachea-See Respiratory organs

Trachinus, 221

Trachypterus, 225

Tragulidce, 611

Transverse process-See Vertebra

Trapezium, 443-See Limb-skeleton of Mammalia

Trapezoid, 443-See Limb-skeleton of Mammalia 
Tree-frogs, 288, 289, 293

Tree Kangaroos, 482

Tree-snakes, 324, 356

Treviranus, 656

Triassic period, 619

Trichechido, 470-See Walruses

Trichosurus, 56\%

Triconodont teeth, $544^{*}$

Trigeminal ganglion, 101*

Trigeminal ne rve, 101*, 154, 155, 156

Tritor, $186^{*}, 18 \%$

Trituberculate molar, 544*

Troclianter, lesser, great, 313, 444 : third, 444

Trochilidce, 404, 423, 429

Trochlea, 442

Trochlear nerve, 101*

Trogones, 404

Trogons, 404

Trophollast, $5 \%$ *

Tropidonotus natrix. $33 \%$

Trout, 192, 214, 220-Seo Salmo fario

Trunk, of Balancglossus, 3 : Craniata, 65 : Elephant, $490 *$

Trunk of spinal nerve, $96^{*}$

Trunk region, 195

Trunk vertebra, 195

Trygon, 181

Trygonorhina, 168, 176

Tuatara, 329-See Hatteria

Tuber cinereum, 457*

Tubercular facet, 435

Tuberculum olfactorium, 247*

Tumblers, 367

Tunic, of Urochorda, 14*

Tunicates-See Urochordata

Turicine, 14*

Turbinal bone, 321

Turbinals, 393

Turbinares, $402 *$, 412, 421, 423, 429

Turbot, 214, 235

Turdido, 404

Turdus, 412

Turkey-buzzards, 403

Turnix, 403

Turtles, 325, 331, 346, 348, 349, 357, 358

Turtur, 404

Tusks-See Teeth

Tympanic bone, 439, 497

Tympanic bulla, $43 \%, 438$

Tympanic cavity, 277, 278

Tympanic membrane, 257, 305*

Tympano-eustachian fossa, 309

Tympanum of syrinx, 385

Tyndall, J., 665

Typhlopidae, 338

Typhlosole, of Ascidia, 15, 18*: Petromyzon, 125, 130

Ty potheria, 588
$\mathrm{U}$

$U$ LNA $80,81 *$ _See Limb-skeleton

Ulnare, 80, 81*_-See Limb-skeleton

Umbilical cord, $577 *$

Umbilicus, inferior and superior, $369 *$

Unau, 483

Unciform, 443-See Limb-skeleton of Mammalia

Uncinates of Reptiles, 335* : Pigeons, 3\%4: Birds, 412

Ungulata, $467 *, 476,486,519-528$. $547,553,558,579,586$

Ungulata vera, $467 * 486,490,525$, $527,528,579$

Unguligrade fcet, $467 *$

Unio, 601

Upper arm, 67, 304

Upupidre, 404

Urachus, $577 *$

Urethra, 460

Urinary bladder, $116^{*}$

Urinary tubules, 113*, 114

Urinogenital organs, of Craniata, 113, 114, 115, 116, 117 : Petromyzon, 130 , 131: Myxinoids, 13\%: Dogfish, 15s, 159, 160, 161 : Elasmobranchii, 174 . 175: Holocephali, 189, 190 : Trout, 208, 209: Teleostomi, 231, 232, 233 : Ceratodus, 247, 248 : Frog, 278, 279 : Amphibia, 299, 300: Lizard, 322, 323: Reptilia, 351: Pigeon, 394, 395: Aves, 422: Rabbit, 459, 460, 461 : Mammalia, 564-56\%

Urinogenital organs, development of, 115,116

Urochord; 23*

Urochorda, 13* : Example, 14: Distinctive characters and classification, 20: Systematic position of Example, 23: General features, 23 : Enteric canal, 28: Heart, 28: Nervous system and sense organs, 28-30: Renal organ, 30: Reproductive system, 30: Development and metamorphosis, 30-40: Distribution, \&c., 40, 41: Affuities, 41, 42

Urodæum, 384, 385*

Urodela, 284*, 285, 287, 288, 289, 290, $291,293,294,295,296,298,299$, 300,302

Uro-hyal, 201

Urolophus cruciatus, 166

Urolophus testaceus, 169

Uropygium, $367 *$

Urostyle, of Salmo, 196 : Frog, 258, 259: Amphibia, 289

Ursida, 470, 529, 530, 551-See Bears

Ursus, 552

Ursus americanus, 530 
Ursus fero $x, \mathbf{5 3 0}$

Use-inheritance, 640

Uterine crypts, $462,576^{*}$

Uterus-See Urinogenital organs

Uterus masculinus, 459, 460*

Utriculus, $111 *, 112$

\section{V}

V AGINA, of Elasmobranchii, 175 : Rabbit, 460, 461 : Nammalia, 565, 567

Tagus ganglion, 102

Vagus nerve, 102*

Valve of Thebesius, $449^{*}$

Valve of Vieussens, $\mathbf{4 5 \%}, 458 *$

Vampire Bats, 581

Vane, 369

Varanus, 350

Variation, 630, 631*

Vasa efferentia, 119, 120*

Vascular system, of Balanoglossus, 4, 6: Ascidia, 1\%, 15, 19: Urochorda, 2s: Amphioxus, 49, 50: Craniata, 88-95 : Lamprey, 125, 126 : Dogfish, 150, 151, 152, 153 : Elasmobranchii, 173: Holocephali, 187: Trout; 204, 205 : Teleostomi, 231 : Ceratodus, 244, 245 : Frog, 2\%0-275: Amphibia, 296, 29\%, 298: Lizard, $314,315,316$ : Reptilia, 347, 348 , 349 : Pigeon, 388, 389, 390, 391 : Birds, 4:1 : Rabbit, 448-452 :.Mammalia, 557

Vaso-clentine, St*

Vaso-ganglion, 225*-_See Red-glands

Veins, Amphioxus, 49: Craniata, 90See Vascular system

Velar tentacles, Amphioxus, 46, 47

Velum, Amphioxus, 46, 47: Petromyzon, 124, 125

Velum interpositum, $45 \% *$

Velum transversum, $319,320^{*}$

"Velvet," 487*

Venomous snakes, 356

Ventral aorta, Amplioxus, 49 : Craniata, 88, 89 : Dogfish, 150, 151

Ventral fissure, 95, $96^{*}$

Ventral root of spinal nerve, 95, 96*

Ventral shield, of Pteraspis, 25t: Snakes, 331

Vent ricle, 88, 89-See Heart

Ventricles-See Brain, 97

Vermiform appendix, $\mathbf{4 4 8}$

Vermis, of cerebellum, 458*

Vertebra, 72*

Vertebral column, 2* : Euchorda, 42 : Craniata, 70, \%1, 72: Petromyzon, 121, 122: Myxinoids, 136: Dogfish, 142, 143, 144 : Elasmobranchii,
167, 169 : Holocephali, 1st, 185 : Trout, 195, 196 : Teleostomi, 222 : Ceratodus, 241: Frog, 258, 259: Amphibia, 289, 290 : Lizard, 305, 306: Reptilia, 332, 333, 334 : Pigeon, 373, 374, 3\%5: Birds (Neornithes), 411: Rabbit, 434, 435 : Mammalia, 494: Prototheria, 503: Metatheria, 506: Edentata, 508: Cetacea, 514, 515: Sirenia, 518: Ungulata, 519, 520, 521 : Carnivora, 528: Rodentia, 531 : Insectivora, 532: Chiroptera, 534: Primates, 535

Vertebral formula, 375

Vertebral plate, $117 *, 179,426$

Vertebral rib-See Rib

Vertebral theory of skull, 659,660

Vertebrarterial canal, 434

Vertebrarterial foramen, $373^{*}, 374$

Vertebrata, 43*

Vesalius, 648

Vesicles of Savi, 174*

Vespertilio, 471

Vestibule, of Amplicoxus, $44^{*}$ : Rabbit, $459,461 *$

Vexillum-See Feather, 369

Vibrissa, 433,476

Vicq d'Azyr, 653

Villi, of embryo Mammals, 574, 576

Vipers, 324,345

Virginian opossum, 480

Visceral arch, visceral bar, visceral skeleton-Craniata, 69, 74, 75*

Visceral motor fibres, $113^{*}$

Visceral sensory fibres, $113^{*}$

Viscero-branchial vessel, 19

Vitelline membrane, 55

Vitreous chamber, 106, 108*

Vitreous humour, 106, 108*

Viverra, 620

Viverridce, 470,580

Viviparous Blenny, 234

Vocal chords, 269

Vocal sacs, 258, 300

Voles, 609

Vomer, ry, 7S*_-See Skull

Vomerine teeth-Holocephali, 186*, 187\% : Trout, 204 : Urodela, 291

Vomero-palatine, 291, 292

Vultur, 403, 412

Vultures, 403, 412

Vulva of Rabbit, 433,461

IV ALLABY, 478, 50\%

Wallace, A. R., 662, 663, 667

Wallace's line, 610

Walruses, 470, 491, 552, 580 
Warning characters, $283,637 *$

Water-lizards, 355

Water-ncwt, 285

Water opossum, 479

Water-voles, 491

Weasels, 176, 470, 554

Weaver, 221

Weber, Max, 663

Weberian apparatus. 231*

Webs, 258, 409

Weismann, A., 668

Weka, 431

Wells, W. C., 662

Whalebone-See Baleen

Whale bone Whales, 446, 486, 501, 515, $517,541,550,586$

Whales-See Cetacea, 466

Wheel-organ of Amphioxus, 44*

White, Gilbert, 654

White matter, 95, 96*

Whiting, 214

Wiegmann, 660

Willemoes-Suhm, 662

Willughby, F., 651

Wing of Birds, 367, 368

Wing-coverts-See Pterylosis, 373

Wolff, C. F., 653

Wolffian body, 115

Wolffian duct, 115, 116*

Wombats, 465, 481, 482, 506, 509

Woodpeckers, 404, 409, 416, 421

Wotton, Edward, 647

Wrasse, 215, 218,227
$\mathrm{X}$

Ienophanes, 658

Xenopus, 285, 289, 294, 295

Xenosauridae, 358

Xiphiplastron, 335

Xiphisternum, 263, 264, 265

Y
Yak, 617
Yolk cells, 280*
Yolk-plug, 247, 249, 280, 281
Yolk-sac, 180, 209, 210, 249, 57
Yolk-sac placenta, 577

Z

Tebra, 468, 488, 489, 580
Zeuglodon, 586, 620

Zeuglodonta-See Archæoceti

Zoarces, 234

Zona radiata, 569*

Zonary placenta, 576*

Zonuridoe, 358

Zoo-geographical regions, 608, 614 : Relations of, 614

Zygana. 165

Zygantrum, 333*

Zygapophysis, 195*

Zygomatic arch, $496^{*}$

Zygomatic process, 439

Zygosphene, 333* 
PRINTED IN GREAT BRITAIN BY

THE CORNWALI PRESS, LTD., PARIS GARDEN, STAMFORD STREET, LONDON, S.E. I. 






$$
\text { (1) }
$$

